\title{
Chicken sperm transcriptome profiling by microarray analysis
}

\begin{tabular}{|r|l|}
\hline Journal: & Genome \\
\hline Manuscript ID & gen-2015-0106.R2 \\
\hline Manuscript Type: & Article \\
\hline Date Submitted by the Author: & $29-$ Nov-2015 \\
\hline Complete List of Authors: & $\begin{array}{l}\text { Singh, R. P.; Salim Ali Centre for Ornithology and natural History, Avian } \\
\text { Physiology and genetics } \\
\text { Shafeeque, C M; Salim Ali Centre for Ornithology and Natural History, ; } \\
\text { rajiv gandhi centre for biotechnology, HMG } \\
\text { Sharma, S; Central Avian Research Institute, Physiology and Reproduction } \\
\text { Singh, Renu; Indian Veterinary Research Institute, } \\
\text { Mohan, J; Central Avian Research Institute, Physiology and Reproduction } \\
\text { Sastry, K; Central Avian Research Institute, Physiology and Reproduction } \\
\text { Saxena, V; Central Avian Research Institute, } \\
\text { Azeez, P; Salim Ali Centre for Ornithology and natural History, Avian } \\
\text { Physiology and genetics }\end{array}$ \\
\hline Keyword: & \begin{tabular}{l} 
fertility biomarker, sperm RNA, embryonic development, egg, fertilization \\
\hline
\end{tabular} \\
\hline \multicolumn{2}{|c}{} \\
\hline
\end{tabular}

\section{SCHOLARONE ${ }^{\prime \prime}$ \\ Manuscripts}




\title{
Chicken sperm transcriptome profiling by microarray analysis
}

\author{
R.P. Singh ${ }^{1, *}$, C.M. Shafeeque ${ }^{1}$, S.K. Sharma ${ }^{2}$, R. Singh ${ }^{3}$, J. Mohan ${ }^{2}$, K.V.H.Sastry ${ }^{2}$, V. K. \\ Saxena $^{2}$, P. A. Azeez ${ }^{1}$ \\ ${ }^{1}$ Avian Physiology and Genetics Division, Sálim Ali Centre for Ornithology and Natural History, \\ Anaikatty-641108, Coimbatore, India \\ ${ }^{2}$ Central Avian Research Institute, Izatnagar, 243122, India. \\ ${ }^{3}$ Indian Veterinary Research Institute, Izatnagar, 243122, India.
}

Running title: RNA in chicken sperm

*Corresponding author current address

Smithsonian Conservation Biology Institute, Front Royal, VA 22630

E-mail address: SinghRP@si.edu

Phone- +1-540-305-5911 


\begin{abstract}
It has been confirmed that mammalian sperm contain thousands of functional RNAs, and some of them have vital roles in fertilization and early embryonic development. Therefore, we attempted to characterize transcriptome of the sperm of fertile chickens using microarray analysis. Spermatozoal RNA was pooled from ten fertile males and used for RNA preparation.Prior to performing the microarray, RNA quality was assessed using a bioanalyzer, and gDNA and somatic cell RNA contamination was assessed by CD4 and PTPRC gene amplification. The chicken sperm transcriptome was cross-examined by analysing sperm and testes RNA on a 4 x 44K chicken array, and results were verified by RT-PCR. Microarray analysis identified 17542 predominantly nuclear-encoded transcripts in chicken sperm. The majority $(87.3 \%)$ of the sperm transcripts were shared with the testes, while surprisingly, $12.7 \%$ transcripts were detected (raw signal intensity greater than 50) only in the sperm and not in the testes. The greatest proportion of up-regulated transcripts were responsible for signal transduction (63.20\%) followed by embryonic development $(56.76 \%)$ and cell structure (56.25\%). Of the 20 most abundant transcripts, 18 remain uncharacterized, whereas the least abundant genes were mostly associated with the ribosome. These findings lay a foundation for more detailed investigations on sperm RNAs in chickens to identify sperm-based biomarkers for fertility.
\end{abstract}

Key words: fertility biomarker, sperm RNA, embryonic development, egg, fertilization 


\section{Introduction}

Historically, it was believed that spermatozoa are just a vehicle that delivers the male genome to the oocyte. However, in the last decade research has shown that spermatozoa delivers more to the oocyte than just the paternal haploid genome. Upon fertilization, the spermatozoon provides a complete, highly structured, and epigenetically marked genome that, together with a defined complement of RNAs and proteins, serves a variety of divergent functions (Krawetz, 2005). A recent study reported an absence of fertility-related sperm RNA elements in men with idiopathic infertility, but whose sperm characteristics were normal (Jodar et al. 2015). Zhang et al. (2006) reported that infertile men possess a higher proportion of spermatozoa with an increased histone to protamine $(P R M)$ ratio than fertile controls. Similarly, human sperm devoid of phospholipase $\mathrm{C}$ zeta 1 (PLCZ1) fail to induce $\mathrm{Ca}^{2+}$ release and are unable to initiate the first step of embryo development (Yoon et al. 2008). Studies have reported that sperm antisense RNAs can epigenetically regulate early embryonic development and have a structural role in maintaining histone-bound sperm chromosomal regions (Lalancette et al. 2008; Krawetz et al. 2011; Liu et al. 2012). Besides their role in fertilization, Gapp et al. (2014) provided evidence for the idea that sperm RNA-dependent processes contribute to the transmission of acquired traits in mammals by experimentally proving that sperm RNAs are responsible for transgenerational inheritance of the effects of early trauma in mice.

The transcriptional profiling of sperm RNAs has been done for humans, mice and other domesticated animals such as bovine, swine and stallion (Goodrich et al. 2007; Gilbert et al. 2007; Yang et al. 2009; Das et al. 2013; Kawano et al. 2012; Card et al. 2013), and those results indicate a combination of coding, non-coding and miRNA molecules present in sperm. Gilbert et al. (2007) compared between bovine sperm and spermatid transcriptome and reported that sperm 
harbors a complex mixture of messengers implicated in a wide array of cell functions and representing a large subset of transcripts found in spermatids. Another study reported many fulllength transcripts in bovine sperm, the presence of which have already been reported in sperm of other species (Card et al. 2013), indicating that some candidate sperm transcripts might be conserved across species. Das et al. (2013) analysed the transcriptome of stallion sperm vs. testes by microarray and RNA-sequencing, and those results suggest that the rich repertoire of coding and non-coding RNAs in stallion sperm is not a random remnant from spermatogenesis in testes but a selectively retained and functionally coherent collection of RNAs. These results confirmed that sperm not only transfer the haploid male genome to the oocyte, but also deliver specific paternal messenger and non-coding RNAs to the egg at fertilization. However, the overall functional significance of these sperm RNAs in fertilization, early embryonic development or in any other function is yet to be understood and elucidated in most species. As a first step to examining the functional significance of sperm RNAs in any species, it is essential to characterise the species-specific sperm enriched RNA population, which will help in determining the molecular functions of sperm RNAs in silico based on biological pathway analysis. Therefore, this study was conceived to explore the chicken sperm RNA profiling by comparison of RNA transcripts between spermatozoa and testes using a microarray technique.

\section{Materials and methods}

All the procedures used in this study were reviewed and approved by the Central Avian Research Institute Animal ethics committee and were carried out in accordance with the revised framework of animals (Scientific Procedures) Act of 2002 of Government of India on animal welfare. 


\section{Semen and testes sample preparation}

Semen from ten fertile male broilers (IC3) of the same hatch (about 40 weeks of age) was collected by the abdominal massage method (Quinn and Burrows 1936), and evaluated for motility as described by Wheeler and Andrews (1943) and fertility by natural mating. The first few ejaculates from each male were discarded in order to obtain good quality semen. Semen samples that showed a motility score above 3 were used for further processing. The concentration of spermatozoa was determined spectrophotometrically at 550nm as described by Brillard and McDaniel (1985). To eliminate variation between individuals, an equal volume of semen from all males was pooled and considered as a single sample, and subjected for density gradient centrifugation. After semen collection, all ten males were euthanized, testes were removed aseptically and washed with RNase free chilled phosphate buffer saline. Approximately $1 \mathrm{mg}$ of testicular tissue from each male was collected, pooled and mixed with $1 \mathrm{~mL}$ of RNAlater and stored at $4^{0} \mathrm{C}$ for further use. Two biological replicates from one pooled sample of each sperm and testes RNA was used for microarray study.

\section{Somatic cell removal by density gradient centrifugation}

Pooled semen was subjected to density gradient centrifugation (DGC) using density gradient mediums, PureSperm (Nidacon International, Mölndal, Sweden), for the removal of somatic cells and immature diploid spermatocytes. In brief, $400 \mu \mathrm{L}$ neat semen was mixed well with $1000 \mu \mathrm{L}$ of $1 \mathrm{X}$ PBS and centrifuged at $1000 \mathrm{~g}$ for $5 \mathrm{~min}$ at room temperature (RT). The resultant pellet was then again dissolved with $1000 \mu \mathrm{L} 1 \mathrm{X}$ PBS, and sperm concentration was measured and adjusted. An aliquot of $1000 \mu \mathrm{L}\left(500-800 \times 10^{6}\right.$ sperm $)$ from this resultant semen solution was layered over $3 \mathrm{~mL}$ pre-warmed $\left(37^{\circ} \mathrm{C}\right) 40 \%$ PureSperm and centrifuged at $280 \mathrm{~g}$ for $30 \mathrm{~min}$ 
at RT. The bottom layer was carefully aspirated using a Pasteur pipette without disturbing the upper layer, transferred into a $2 \mathrm{~mL}$ centrifuge tube and washed twice with $1 \mathrm{X}$ PBS by centrifuging at $1000 \mathrm{~g}$ for $5 \mathrm{~min}$ at room temperature. Finally, the sperm pellet was suspended in $1 \mathrm{~mL} 1 \mathrm{X}$ PBS, and an aliquot of this purified sperm was examined by phase-contrast light microscopy (X 400) to check for the absence of somatic cells and immature spermatocytes. The sperm concentration in purified samples was adjusted to approximately $100-500 \times 10^{6} / \mathrm{mL}$, and used for either RNA isolation or mixed with an equal amount of RNAlater and stored at $4^{0} \mathrm{C}$ for further use.

\section{Total RNA isolation and quantity and quality assessment}

RNA isolation was carried out in an area dedicated to RNA work using RNase/DNase free water and RNase free glass- and plastic-ware. Total RNA was isolated from purified sperm and testes samples using a RNeasy Mini Kit with spin columns (Qiagen, Valencia, CA,USA) following manufacturer's instructions. The concentration and purity of the RNA extracted were evaluated using the Nanodrop Spectrophotometer (Thermo Scientific, Wilmington, DE, USA). The integrity of the extracted RNA was analysed on the Bioanalyzer (Agilent; 2100, Santa Clara, CA, USA). We considered RNA to be of good quality based on the 260/280 and 260/230 values (Nanodrop); additionally rRNA 28S/18S ratios and RNA integrity number (RIN) were also considered for testes RNA. Genomic DNA contamination in purified RNA samples was examined by reverse transcriptase PCR with intron-spanning primers for the $C D 4$ gene. Further, somatic cell RNA contamination in purified RNA samples was examined by reverse transcriptase PCR with intron-spanning primers for $C D 4$ and protein tyrosine phosphatase, receptor type, $\mathrm{C}(P T P R C)$ gene. 


\section{Microarray}

The RNA samples were labeled using Agilent Quick Amp Kit (Part number: 5190-0424). 500ng of total RNA was reverse transcribed using oligodT primer tagged to T7 promoter sequence. cDNA thus obtained was converted to double stranded cDNA in the same reaction. Further the cDNA was converted to cRNA by in-vitro transcription reaction by T7 RNA polymerase enzyme in presence of $\mathrm{Cy} 3$ dye. During cRNA synthesis $\mathrm{Cy} 3$ dye was incorporated into the newly synthesized cRNA strands. The cRNA obtained was then cleaned up using Qiagen RNeasy columns (Qiagen, Cat No: 74106). Both concentration and the amount of dye incorporated into cDNA were determined using Nanodrop. Specific activities of the labeled samples were determined. The labeling QC passed samples were hybridized. 1650ng of labeled cRNA were fragmented and hybridized on the chicken array (AMADID: 49081), containing 43604 chicken probes for 28078 genes, using the Gene Expression Hybridization kit (Part Number 5190-0404; Agilent) in Sure Hybridization Chambers (Agilent) at $65^{\circ} \mathrm{C}$ for 16 hours. Each hybridized slide was washed with wash buffer I for 1 minute at room temperature followed by wash buffer II for 1 minute at $37^{\circ} \mathrm{C}$ using Agilent Gene Expression wash buffers (Part No: 5188-5327). The hybridized, washed microarray slides were then scanned on a G2600D scanner (Agilent Technologies).

\section{Microarray data acquisition and analysis}

Images were quantified using Feature Extraction Software (Version-11.5, Agilent). Extracted raw data were analyzed using GeneSpring GX software from Agilent. Data were normalized in GeneSpring GX using the $50^{\text {th }}$ percentile shift (percentile shift normalization is a global normalization, where the locations of all the spot intensities in an array are adjusted). This 
normalization takes each column in an experiment independently, and computes the $n^{\text {th }}$ percentile of the expression values for this array, across all spots (where $\mathrm{n}$ has a range from 0 100 and $n=50$ is the median). It subtracts this value from the expression value of each entity, thereby normalizing each data point relative to specific control samples. Genes significantly upor down-regulated, showing a one-fold (log base2) or above change with respect to control samples were identified. The resulting gene lists underwent a clustering analysis (Cluster 3.0) to unravel Differentially Expressed Genes (DEGs), excluding transcripts showing a concordant value $<80 \%$. Genes were classified based on Gene ontology functional classification (DAVID).

\section{Validation of microarray results}

RNA samples were reverse transcribed with random hexamer primers using the 'RevertAid First strand cDNA synthesis kit' (MBI Fermentas, Hanover, MD, USA) according to the manufacturer's instructions. A total of $500 \mathrm{ng}$ RNA was used for cDNA synthesis. The reverse transcription reaction was carried out in a final volume of $20 \mu \mathrm{L}$. The resultant cDNA was stored at $-20{ }^{0} \mathrm{C}$ until used. The presence of testes and sperm specific genes (PRM and PLCZ1), and most and least abundant genes were examined using PCR with gene specific primers (Table 1). The amplification was carried out in $25 \mu \mathrm{L}$ volume containing $2.5 \mu \mathrm{L}$ PCR buffer (10X), $0.5 \mu \mathrm{L}$ dNTPs mix (10mM each), $0.5 \mu \mathrm{L}$ each of forward and reverse primer $(10 \mathrm{pmol} / \mu \mathrm{L}), 0.2 \mu \mathrm{L}$ Taq polymerase (5U/ $\mu \mathrm{L}$, Biotools, Spain), $2 \mu \mathrm{L}$ of cDNA (1:10 diluted) and 18.8 $\mu \mathrm{L}$ nuclease-free water. The PCR cycling conditions for all genes except $P R M$ were: initial denaturation of $95{ }^{\circ} \mathrm{C}$ for $5 \mathrm{~min}$, followed by 35 cycles of denaturation at $95{ }^{\circ} \mathrm{C}$ for $30 \mathrm{~s}$; annealing (Table 1) for $30 \mathrm{~s}$ and extension at $72{ }^{\circ} \mathrm{C}$ for $30 \mathrm{~s}$. The PCR cycling conditions for PRM followed those previously described elsewhere (Sharma et al. 2014). Negative controls were samples in which cDNA was 
not added. PCR products were resolved on $2.5 \%$ agarose gels and visualized by staining with ethidium bromide.

Semi-quantitative RT-PCR was chosen to estimate the transcripts amount in testes and sperm. To control for variation in the efficiencies of the RT step among different experimental samples, mRNA concentrations of $G A P D H$, a housekeeping gene, presumed to be expressed at constant amounts in chicken testes and sperm were also calculated, along with mRNA concentrations of targeted genes, by densitometry analysis using Image J software (NIH). Relative expression was determined as arbitrary units, defined as the ratio of mRNA level to the corresponding GAPDH mRNA level after subtraction of background intensity (value = (intensity; gene of interest intensity; background)/(intensity; GAPDH -intensity; background) (Singh et al. 2011). Mean values of three measurements for testes and sperm bands were used for analysis.

\section{Results}

\section{Sperm RNA quality}

The absorbance ratios at A260/ A280 and A260/230 for testes and sperm RNA samples isolated were between $1.86-2.10$ and 1.70-2.31, respectively (Table 2). The bioanalyzer profile of testes RNA showed two distinct peaks of $18 S$ and $28 S$ rRNA respectively (Fig. 1), at the same place as indicated in reference with a RNA integrity number (RIN) of 9.5. In contrast, the sperm RNA bioanalyzer profile exhibited only a single peak at 40-50 s intervals (Fig. 1). The absence of $18 S$ and $28 S$ rRNA peaks in sperm RNA indicated that the RNA was originated from sperm and was uncontaminated with somatic cell RNA. Further, PTPRC and CD4 genes were targeted to detect the traces of somatic cell RNA in sperm samples. The primers for CD4 gene were intronspanning in order to check for genomic DNA contamination also in RNA samples. The sperm 
RNA sample was found to be negative for PTPRC and CD4, whereas the testes sample was positive for PTPRC and CD4 (Fig. 2 \& 3). No genomic DNA contamination was detectable in the samples used for microarray.

\section{Microarray QC}

The quality of $\mathrm{Cy} 3$ dye labelled cRNA samples were evaluated by NanoDrop. The specific activity of the sperm and testes samples ranged from 14.11 to 18.74 (Table S1), which is considered to be good. It is recommended that specific activity greater than eight is good for hybridization (Diaz and Barisone, 2011). The hybridization images were manually verified and found to be devoid of uneven hybridization, streaks, blobs and other artefacts. Hybridization across the slides was good based on a number of features such as spot finding of the four corners of the array, spatial distribution of all outliers on the array, negative control and net signal statistics and histogram of signal plot (Data not shown).

The overall mRNA expression levels within a single hybridization are presented in a scatter plot, where each point represents the expression value of a gene in each of the two biological replicates, one plotted on the $\mathrm{x}$-axis and other on the $\mathrm{y}$. The scatter plot showed a linear relationship in expression of genes in both biological replicates (Fig. S1). These results were further substantiated by measuring the correlation coefficient based on the intensity values of expression (Table S2) and intra-array gene expression clustering (Fig. S2).

\section{Sperm transcript profile}

Gene expression microarray analysis revealed 17542 probes in chicken sperm and 21639 in the testes. The majority $(87.3 \%)$ of the sperm transcripts were shared with the testes, while surprisingly, $12.7 \%$ transcripts were detected (raw signal intensity greater than 50 ) only in the 
sperm and not in the testes (Fig. 4). The chicken sperm transcript contains predominantly nuclear-encoded mRNAs, including 13 mitochondrial-encoded RNAs. The differential analysis revealed 11785 genes up and 9946 genes down regulated compared to testes at signal to noise ratio $\geq 2$.

Gene ontology annotations grouped sperm transcripts into different groups as shown in Table 3. The most transcripts were related to nucleic acid binding (1744) followed by signal transduction (1598) and transcription (1318). The greatest numbers of up-regulated transcripts were responsible for signal transduction (63.20\%) followed by embryonic development $(56.76 \%)$ and cell structure (56.25\%). The lowest numbers of up-regulated transcripts were associated with spermatogenesis (11.62\%) and fertilization (18.18\%) (Figures $5 \& 6)$. All transcripts (100\%) related to ribosomal machinery were down regulated in sperm (Table S3).

A preliminary survey of the chicken sperm transcript profile for previously reported sperm RNA candidates identified several transcripts common in bovine, human, porcine, stallion and chicken (Table 4). These transcripts showed differential expression pattern in chicken spermatozoa. A list of the twenty most and least abundant RNAs in chicken sperm is presented in Tables 5 and 6 . Of these 20 most abundant transcripts, 18 were uncharacterized, whereas the 20 least abundant transcripts were mostly associated with the ribosome.

\section{Validation of microarray results}

For microarray result validation, specific candidate genes were selected according to their abundance in chicken sperm. Two known sperm specific-genes (PRM and PLCZ1) were also included for microarray results. The RT-PCR results show up-regulation of PRM, PLK2 and 
ENS1 gene, whereas down-regulation of PLCZ1, PABPC1 and RPL26L1 in sperm compared to testes, which are consistent with the microarray results (Fig. $7 \& 8$ ).

\section{Discussion}

This is the first time that the chicken spermatozoal transcript profile has been explored using microarray, which confirms the presence of thousands of predominantly nuclear-encoded transcripts. Our findings are in good agreement with previous research in the field on human, mouse, bovine and stallion (Das et al. 2010 \& 2013; Jodar et al. 2012; Gilbert et al. 2007; Yang et al. 2009; Kawano et al. 2012; Yoon et al. 2008; Krawetz 2005). The microarray results of two candidate sperm transcripts (PRM and PLCZ1) and two transcripts each from high and low abundant RNA in sperm were confirmed by RT-PCR. The PRM and PLCZ1 were chosen to make sure that the microarray template was germ cell originated. The expression of $P R M$ and PLCZ1 is known to be restricted in testes, thereby known as testes-specific marker genes. Since, sperm RNAs are thought to be remnants of spermatogenesis process; sperm also contains PRM and PLCZ1 transcripts. Earlier studies used PRM expression as marker for testes and sperm specific transcripts in mammalian species (Das et al. 2010 \& 2013; Jodar et al. 2012; Gilbert et al. 2007; Yang et al. 2009; Kawano et al. 2012; Yoon et al. 2008; Krawetz 2005). We had already confirmed the presence of these transcripts in chicken sperm (Shafeeque et al. 2014). Further, we have used intron-spanning primer of $C D 4$ gene to examine the genomic DNA contamination in RNA samples. CD4 gene amplification results showed that microarray templates were free from genomic DNA contamination. A similar technique has already been applied by other authors to check genomic DNA in RNA samples (Das et al. 2010; Jodar et al. 2012). We have examined the quality of RNA by measuring the absorbance ratios at A260/A280 and through bioanalyzer profiling, which is known to be the most sensitive technique to evaluate 
RNA integrity. The testes RNA showed two distinct peaks of $18 S$ and $28 S$ rRNA that are known as a hallmark of most tissue RNA (Krawetz 2005). In contrast, sperm RNA profile did not show $18 S$ and $28 S$ rRNA peaks. Das et al. (2010) and Shafeeque et al. (2014) reported the same results for stallion and chicken sperm RNA. The bioanalyzer profile of the sperm RNA samples exhibited a RIN value (7.3). However, since calculation of the RIN value is mainly based on the ratio of $18 S$ and $28 S$ rRNA, and sperm RNA contains trace amounts of rRNA, perhaps RIN values are not useful to reliably assess the quality of sperm RNA samples. In general, it is known that sperm are rRNA-depleted (Ostermeier et al. 2002; Krawetz 2005; Grunewald et al. 2005; Das et al. 2010 \& 2013), hence the absence of rRNA peaks in chicken sperm RNA samples indicated that the RNA originated from sperm and was uncontaminated with foreign RNA. This observation was further strengthened by visualising the $C D 4$ and PTPCR, somatic cell marker transcripts in sperm RNA samples. Both the somatic cell marker gene transcripts were found to be absent in chicken sperm RNA samples, indicating the absence of any foreign RNA. Similar results have been reported for stallion, human and chicken sperm (Das et al. 2010; Jodar et al. 2012; Shafeeque et al. 2014).

Our microarray results demonstrate that the majority of the transcripts $(87.3 \%)$ were common between sperm and testes, supporting the prevailing idea that sperm transcripts are solely historical records of spermatogenesis in testes (Krawetz 2005; Ostermeier et al. 2002). A previous study on stallion sperm reported $97 \%$ common transcripts between sperm and testes in the stallion (Das et al. 2013). The difference in the proportion of shared transcripts between sperm and testes is primarily due to species differences, because chicken sperm have relatively low cytoplasmic content ( $3 \%$ of initial spermatid cell volume in rooster sperm) compared to mammalian sperm (Sprando and Russell 1988). Further, our results show 12.7\% sperm-enriched 
transcripts in the chicken, which is higher than the previously reported $2.44 \%$ sperm-enriched transcripts in stallion sperm (Das et al. 2013). The difference in sperm-enriched transcripts may be due to species differences and the number of probes in oligoarray. Whereas the stallion study was conducted on equine oligoarray containing 14531 gene probes, we used a chicken oligoarray which contains approximately 44 thousand probes for 28077 genes. The sperm-enriched transcripts are considered to be foreign RNA taken up by sperm released from the testis during maturation in, or passage through,the epididymis. It is believed that microsomes such as epididymosomes may be used as a vehicle to provide such foreign RNA to the maturing sperm either during ejaculation or in the epididymis but this idea needs to be tested (Boerke et al. 2007). Gene ontology analysis revealed that the greatest number of sperm up-regulated transcripts were related to signal transduction followed by embryonic development and cell structure, as reported previously in bovine and stallion sperm (Das et al. 2013; Gilbert et al. 2007; Card et al. 2013). However, the lowest numbers of sperm up-regulated transcripts were associated with spermatogenesis and fertilization, indicating selective retention of transcripts in sperm. Das et al. (2013) has already speculated that certain transcriptional products of spermatogenesis are selectively retained in the sperm but not in the testes in stallion. All transcripts related to ribosomal machinery were down-regulated in sperm, indicating that translation was predominantly impaired in chicken sperm.

A preliminary survey of the chicken sperm transcript profile for previously reported sperm RNA candidates identified several transcripts common in bovine, human, porcine and chicken (Das et al. 2013; Gilbert et al. 2007; Card et al. 2013). The sperm RNA candidates includes PRM, CRISP2, CLGN and PLCZ1. These results indicate that some of the candidate sperm RNA transcripts are conserved across these species. Results of the top 20 most abundant transcripts 
showed all uncharacterized transcripts except for two genes (TANK\&SCARF1). These genes have no direct role in either fertilization or early embryonic development, but TANK plays an important role as an anti-apoptotic factor, and might be preventing apoptosis in the zygote. Whereas, SCARF1 regulates the uptake of chemically modified low density lipoproteins, including acetylated low density lipoprotein (Ac-LDL), and therefore may be involved in sperm energy metabolism.

In conclusion this study provides the first evidence of the presence of thousands of RNAs in chicken sperm; some of which have been already been identified as candidate sperm RNA in other species. The results will help in establishing the functional role of identified candidate sperm RNA in fertility and early embryonic development along with functional genomics studies of fertility to develop non-invasive fertility biomarkers.

\section{Declaration of interest}

All the authors hereby declare that there is no conflict of interest that could be perceived as prejudicing the impartiality of the research reported.

\section{Acknowledgements}

This work was supported by the Science and Engineering Research Board (SERB); Department of Science and Technology, Government of India (SERB/FT/LS-147/2011). We acknowledge Genotypic Technology Private Limited Bangalore for the microarray processing and data analysis reported in this publication. 


\section{References}

Boerke, A., Dieleman, S.J., and Gadella, B.M. 2007. A possible role for sperm RNA in early embryo development. Theriogenology 68S: S147-S155.

doi:10.1016/j.theriogenology.2007.05.058

Brillard, J.P., and McDaniel, G.R. 1985. The relativity and efficiency of various methods for estimating spermatozoa concentrations. Poult Sci. 64: 155-158. doi: 10.3382/ps.0640155.

Quinn, J.P., and Burrows, W.H. 1936. Artificial Insemination of Fowls. J Hered. 27: 31-37. Available from http://jhered.oxfordjournals.org/content/27/1/31.extract [accessed 15 December 2015].

Card, C.J., Anderson, E.J., Zamberlan, S., Krieger, K.E., Kaproth, M., and Sartini, B.L. 2013.Cryopreserved bovine spermatozoal transcript profile as revealed by high-throughput ribonucleic acid sequencing. Biol Reprod. 88(2): 1-9. doi: 10.1095/biolreprod.112.103788.

Das, P.J., McCarthy, F., Vishnoi, M., Paria, N., Gresham, C., Li, G., Kachroo, P., Sudderth, A.K., Teague, S., Love, C.C., Varner, D.D., Chowdhary, B.P., and Raudsepp, T. 2013. Stallion sperm transcriptome comprises functionally coherent coding and regulatory RNAs as revealed by microarray analysis and RNA-seq [online]. PLoS One. 8(2): e56535. doi:10.1371/journal.pone.0056535.

Das, P.J., Paria, N., Gustafson-Seabury, A., Vishnoi, M., Chaki, S.P., Love, C.C., Varner, D.D., Chowdhary, B.P., and Raudsepp, T. 2010. Total RNA isolation from stallion sperm and testis biopsies. Theriogenology 74: 1099-1106. doi:10.1016/j.theriogenology.2010.04.023. 
Diaz, E., and Barisone, G. A. 2011. DNA microarrays: sample quality control, array hybridization and scanning [online]. J. Vis. Exp. (49): e2546, doi:10.3791/2546 (2011).

Gapp, K., Jawaid, A., Sarkies, P., Bohacek, J., Pelczar, P., Prados, J., Farinelli, L., Miska, E., and Mansuy, I.M. 2014. Implication of sperm RNAs in transgenerational inheritance of the effects of early trauma in mice. Nat Neurosci. 17(5): 667-669. doi:10.1038/nn.3695.

Gilbert, I., Bissonnette, N., Boissonneault, G., Vallee, M., and Robert, C. 2007. Molecular analysis of the population of mRNA in bovine spermatozoa. Reproduction 133: 10731086. doi:10.1530/REP-06-0292.

Goodrich, R., Johnson, G., and Krawetz, S.A. 2007. The preparation of human spermatozoal RNA for clinical analysis. Arch Androl. 53: 161-167. Doi:10.1080/01485010701216526.

Grunewald, S., Paasch, U., Glander, H.J., and Anderegg, U. 2005. Mature human spermatozoa do not transcribe novel RNA. Andrologia 37: 69-71. Doi: 10.1111/j.14390272.2005.00656.x

Jodar, M., Kalko, S., Castillo, J., Ballescà, J.L., and Oliva, R. 2012. Differential RNAs in the sperm cells of asthenozoospermic patients. Hum Reprod. 27: 1431-8. doi: 10.1093/humrep/des021.

Kawano, M., Kawaji, H., Grandjean, V., Kiani, J., and Rassoulzadegan, M. 2012. Novel small noncoding RNAs in mouse spermatozoa, zygotes and early embryos [online]. PLoS ONE. 7(9): e44542. doi:10.1371/journal.pone.0044542. 
Krawetz, S.A., Kruger, A., Lalancette, C., Tagett, R., Anton, E., Draghici, S., and Diamond, M.P. (2011). A survey of small RNAs in human sperm. Hum Reprod. 26(12): 3401-3412. doi: 10.1093/humrep/der329.

Krawetz, S.A. 2005. Paternal contribution: new insights and future challenges. Nat Rev Genet. 6: 633- 642. doi:10.1038/nrg1654.

Lalancette, C., Miller, D., Li, Y., and Krawetz, S.A. 2008. Paternal contributions: new functional insights for spermatozoal RNA. J Cell Biochem. 104: 1570- 1579. Doi: 10.1002/jcb.21756.

Liu, W.M., Pang, R.T.K., Chiu, P. C. N., Wong, B.P.C., Lao, K., Lee, K.F., and Yeung, W.S.B. 2012. Sperm-borne microRNA-34c is required for the first cleavage division in mouse. PNAS 109: 490-494. doi:10.1073/pnas.1110368109.

Montjean, D., De La Grange, P., Gentien, D., Rapinat, A., Belloc, S., Cohen-Bacrie, P., Menezo, Y., and Benkhalifa, M. 2012. Sperm transcriptome profiling in oligozoospermia. J Assist Reprod Genet. 29: 3 -10. doi: 10.1007/s10815-011-9644-3.

Ostermeier, G.C., Dix, D.J., Miller, D., Khatri, P., and Krawetz, S.A. 2002. Spermatozoal RNA profiles of normal fertile men. Lancet 360: 772-777. doi:10.1016/S0140-6736(02)09899-9.

Ostermeier, G.C., Miller, D., Huntriss, J.D., Diamond, M.P., and Krawetz, S.A. 2004. Reproductive biology: delivering spermatozoan RNA to the oocyte. Nature 429: 154. doi:10.1038/429154a.

Shafeeque, C.M., Singh, R.P., Sharma, S.K., Mohan, J., Sastry, K.V., Kolluri, G., Saxena, V.K., Tyagi, J.S., Kataria, J.M., and Azeez, P.A. 2014. Development of a new method for sperm 
RNA purification in the chicken. Anim Reprod Sci. 149: 259-265.

doi:10.1016/j.anireprosci.2014.06.032.

Sharma, S.K., Shafeeque, C.M., Mohan, J., Azeez, P.A., and Singh, R.P. 2014. PCR amplification protocol for GC rich protamine gene from chicken testis cDNA. Advan Anim Vet Sci. 11(2): 559-605. doi.org/10.14737/journal.aavs/2014/2.11.599.605.

Singh, R.P., Sastry, K.V.H., Pandey, N.K., Shit, N., Agarawal, R., Singh, R., Sharma, S.K., Saxena, V.K., and Mohan, J. 2011. Molecular characterization and expression of Lactate Dehydrogenase A and B mRNA in testes of Japanese quail (Coturnix japonica). Asian Australas. J Anim Sci. 24(8): 1060-1068. doi: 10.5713/ajas.2011.10263.

Sprando, R.L., and Russell, L.D. 1988. Spermiogenesis in the red-ear turtle (Pseudemys scripta) and the domestic fowl (Gallus domesticus): a study of cytoplasmic events including cell volume changes and cytoplasmic elimination. J Morphol. 108: 95-118. Doi: 10.1002/jmor.1051980110

Wheeler, N.C., and Andrews, F.N. 1943. The influence of season on semen production in domestic fowl. Poult Sci. 3: 361-67. doi: 10.3382/ps.0220361.

Yang, C.C., Lin, Y.S., Hsu, C.C., Wu, S.C., Lin, E.C., and Cheng, W.T. 2009. Identification and sequencing of remnant messenger RNAs found in domestic swine (Sus scrofa) fresh ejaculated spermatozoa. Anim Reprod Sci. 113: 143-155. doi:

10.1016/j.anireprosci.2008.08.012.

Yoon, S.Y., Jellerette, T., Salicioni, A.M., Lee, H.C., Yoo, M.S., Coward, K., Parrington, J., Grow, D., Cibelli, J.B., Visconti, P.E., Mager, J., and Fissore, R.A. 2008. Human sperm 
devoid of PLC, zeta 1 fail to induce $\mathrm{Ca}^{(2+)}$ release and are unable to initiate the first step of embryo development. J Clin Invest. 118: 3671-3681. doi:10.1172/JCI36942.

Zhang, X., Gabriel, M.S., and Zini, A.A. 2006. Sperm nuclear histone to protamine ratio in fertile and infertile men: evidence of heterogeneous subpopulations of spermatozoa in the ejaculate. J Androl. 27: 414-420. Doi: 10.2164/jandrol.05171. 


\section{Caption of figures}

Figure 1: Image showing microarray electropherogram of testes and sperm RNA samples. (A) 18S and 28S rRNA reference; (B) Ladder; (C) Total sperm RNA; and (D) Total testes RNA.

Figure 2: Image showing the absence of $P T P R C$ RNA in sperm RNA (Lane 1: 100bp marker; Lane 2: non template control; Lane 3: testes RNA and Lane 4: sperm RNA).

Figure 3: Image showing the absence or presence of CD4 RNA and gDNA in sperm or testes samples. An intron-spanning CD4 primer set was used to detect the presence of genomic DNA contamination. Lane 1: 100 bp ladder. Lane 2: Non template control; Lane 3: Testes RNA spiked with genomic DNA to produce the amplicon of $248 \mathrm{bp}$ corresponding to genomic DNA and an amplicon of 162 bp corresponding to CD4 cDNA sperm RNA. Lane 4 : sperm RNA.

Figure 4: Venn diagram of the proportion of specific or common transcripts between testes and sperm with raw signal intensity greater than 50 by microarray analysis.

Figure 5: Heat map of genes that have a role in fertilization. Hierarchical clustering of genes is based on similar expression profiles in sperm samples vs. testes samples. Clustering analysis was performed using GeneSpringGX Software using Average Linkage rule with Pearson Uncentered Distance Metric. Red color in the cluster indicates up-regulation and green color in the cluster indicates down-regulation in Sperm cells when compared to Testes samples. The fold expression values represented in the cluster are in terms of log base 2.

Figure 6: Heat map of genes that has role in spermatogenesis. Hierarchical clustering of genes is based on similar expression profiles in sperm samples vs. testes samples. Clustering analysis was performed using GeneSpringGX Software using Average Linkage rule with Pearson Uncentered Distance Metric. Red color in the cluster indicates up-regulation and green color in the cluster 
indicates down-regulation in Sperm cells when compared to Testes samples. The fold expression values represented in the cluster are in terms of log base 2.

Figure 7: Validation of microarray results for selected transcripts by RT-PCR. Lanes 1, 2 and 3 contain PCR products amplified with sperm cDNA, whereas lanes 4, 5 and 6 contain PCR products amplified with testes cDNA. Gel electrophoresis was performed for all the genes in identical conditions.

Figure 8: RT-PCR analysis of selected transcripts in sperm and testes cDNA. Bars represent the mean of the normalized band density for each mRNA band obtained by densitometric analysis ( $m e a n \pm S E ; n=3$ ). Normalization was performed by dividing each band density value by the value of $\beta$-actin band density in the same sample. Asterisk $(*)$ indicates a significant difference $(\mathrm{p}<0.05)$ in mRNA expression. 
Table1: Primer pairs used for PCR.

\begin{tabular}{|c|c|c|c|c|}
\hline $\begin{array}{l}\text { Gene } \\
\text { name }\end{array}$ & $\begin{array}{l}\text { Accession } \\
\text { number }\end{array}$ & Primer Sequence (5'-3') & $\begin{array}{c}\text { Annealing } \\
\text { Temp. } \\
\left({ }^{\circ} \mathrm{C}\right) \\
\end{array}$ & Product size \\
\hline GAPDH & NM_204305 & $\begin{array}{l}\text { F-CGTGTTGTGGACTTGATGG } \\
\text { R-AACTGAGCGGTGGTGAAG }\end{array}$ & 58 & 120 \\
\hline PLCZ1 & AY843531 & $\begin{array}{l}\text { F-CGTAGTAGACAAGTATGC } \\
\text { R-ATTGGTGAAGTAAGAAGTT }\end{array}$ & 47.8 & 141 \\
\hline PRM & $\begin{array}{l}\text { M28100/ } \\
\text { L38713 }\end{array}$ & $\begin{array}{l}\text { F-CGCAGCAGGACCCGCAGCCG } \\
\text { R-CGGCGGCGGCGGCTCAGTAG }\end{array}$ & 66 & 187 \\
\hline $\mathrm{CD} 4$ & DQ202315 & $\begin{array}{l}\text { F-GAAAGATGGGACCTGTACTTGG } \\
\text { R-TCCAGGCTCAAGTCTGACAC }\end{array}$ & 58 & $\begin{array}{c}\mathrm{RNA} / \mathrm{cDNA} \\
=162 \\
\text { Genomic } \\
\text { DNA }=248\end{array}$ \\
\hline PTPRC & NM_204417 & $\begin{array}{l}\text { F- ACTATTGACCTTACTCCAT } \\
\text { R- GCCCATTTGTAGAACTTT }\end{array}$ & 49.7 & 109 \\
\hline PLK2 & XM_424739 & $\begin{array}{l}\text { F-TAGCCAAGCCAGGAGCATTAC } \\
\text { R-TCCAGACATCCACGCAATACC }\end{array}$ & 53.7 & 166 \\
\hline ENS-1 & NM_001080873 & $\begin{array}{l}\text { F-CCTTGTGCTTGCTATCTTCTC } \\
\text { R-CCTGTCCATCAGTGTCCTC }\end{array}$ & 53.7 & 191 \\
\hline PABPC1 & NM_001031597 & $\begin{array}{l}\text { F-AGAGAATGGCGAGTGTAAG } \\
\text { R-TTGGAATGGATGAGGTCTG }\end{array}$ & 59.5 & 197 \\
\hline RPL26L1 & NM_001277099 & $\begin{array}{l}\text { F-TCAGACCGCAGCAAGAAC } \\
\text { R-GACAACCTGGACTTCATCATC }\end{array}$ & 53.7 & 150 \\
\hline
\end{tabular}


Table 2: RNA quality and yield of RNA samples used for microarray analysis.

\begin{tabular}{cccc}
\hline Sample name & $\begin{array}{c}\text { Absorbance } \\
\text { value 260/280 }\end{array}$ & $\begin{array}{c}\text { Absorbance } \\
\text { value 260/230 }\end{array}$ & $\begin{array}{c}\text { RNA } \\
\text { concentration } \\
\mathrm{ng} / \mu \mathrm{l}\end{array}$ \\
\hline Testis RNA & 2.10 & 2.31 & 1643 \\
Sperm RNA & 1.86 & 1.7 & 231 \\
\hline
\end{tabular}


Table 3: Gene ontology functional groups of sperm up- and down-regulated transcripts.

\begin{tabular}{cccc}
\hline Function name & $\begin{array}{l}\text { Total genes } \\
\text { found }\end{array}$ & $\begin{array}{l}\text { Up-regulated } \\
\text { genes/\% }\end{array}$ & $\begin{array}{l}\text { Down-regulated } \\
\text { genes }\end{array}$ \\
\hline Nucleic acid binding & 1744 & $645(36.98)$ & 1099 \\
Signal transduction & 1598 & $1010(63.20)$ & 588 \\
Transcription & 1318 & $623(47.26)$ & 695 \\
Embryonic development & 377 & $214(56.76)$ & 163 \\
Cell structure & 16 & $9(56.25)$ & 7 \\
Spermatogenesis & 43 & $5(11.62)$ & 38 \\
Fertilization & 11 & $2(18.18)$ & 9 \\
Apoptosis & 471 & $226(47.98)$ & 245 \\
Oocyte & 167 & $52(31.13)$ & 115 \\
Cell cycle & 384 & $98(25.52)$ & 286 \\
Steroid & 195 & $104(53.33)$ & 91 \\
Protein modification & 855 & $388(45.38)$ & 467 \\
\hline
\end{tabular}


Table 4: A list of previously reported candidate sperm transcripts identified in the chicken sperm transcript profile.

\begin{tabular}{|c|c|c|c|c|}
\hline $\begin{array}{l}\text { Transcript } \\
\text { name }\end{array}$ & Accession no. & $\begin{array}{l}\text { Expression } \\
\text { in chicken } \\
\text { sperm }\end{array}$ & Species & REFERENCE \\
\hline PRM & M28100 & Up & $\begin{array}{l}\text { Human, Bovine, } \\
\text { Chicken }\end{array}$ & $\begin{array}{c}\text { Ziyyat et al., 1999; Gilbert } \\
\text { et al., 2007; Shafeeque et } \\
\text { al., } 2014\end{array}$ \\
\hline CRISP2 & ENSGALT00000026918 & Up & Bovine & $\begin{array}{l}\text { Arangasamy et al., 2011; } \\
\text { Zhao et al., } 2006\end{array}$ \\
\hline SPATA20 & XM_420103 & Up & Bovine & Gilbert et al., 2007 \\
\hline H2AFZ & NM_001031374 & Down & Bovine & Gilbert et al., 2007 \\
\hline EEF1A1 & NM_204157 & Down & Bovine & $\begin{array}{l}\text { Laalancette et al., 2008; } \\
\text { Zhao et al., 2006 }\end{array}$ \\
\hline ACTG1 & NM_001007824 & Down & Bovine & Gilbert et al., 2007 \\
\hline PLCZ1 & NM_001039273 & Down & Human, chicken & $\begin{array}{l}\text { Hamatani et al., 2012; } \\
\text { Shafeeque et al., } 2014\end{array}$ \\
\hline MYCBP & ENSGALT00000041046 & Down & Human & $\begin{array}{l}\text { Lambard et al., 2004; } \\
\text { Kumar et al., } 1993\end{array}$ \\
\hline PEBP1 & NM_001198642 & Down & Bovine & $\begin{array}{l}\text { Bissonette et al, 2009; } \\
\text { Arangasamy et al., } 2011\end{array}$ \\
\hline SPAG4 & XM_424281 & Down & Bovine & Gilbert et al., 2007 \\
\hline СCT8 & NM_001004389 & Down & Bovine & Arangasamy et al., 2011; \\
\hline PPIH & ENSGAL̄T00000007781 & Down & Bovine & Gilbert et al., 2007 \\
\hline STRBP & NM_001030680 & Down & Bovine & Gilbert et al., 2007 \\
\hline CHMP5 & ENSGAL̄T00000021491 & Down & Human & $\begin{array}{c}\text { Zhao et al., 2006; } \\
\text { Lalancette et al., 2008 }\end{array}$ \\
\hline CLGN & ENSGALT00000015978 & Down & Porcine & $\begin{array}{l}\text { Ostermeier et al., 2006; } \\
\text { Wang et al., 2004; } \\
\text { Kempisty et al., } 2008\end{array}$ \\
\hline EIF2B2 & NM_001006481 & Down & Bovine & Gilbert et al., 2007 \\
\hline CTTN & NM̄ 205468 & Down & Stallion & Das et al., 2013 \\
\hline ARID5B & NM_001031220 & Down & Stallion & Das et al., 2013 \\
\hline ATG12 & ENSGĀ̄T00000003696 & Down & Stallion & Das et al., 2013 \\
\hline GSTA1 & NM_001001777 & Down & Stallion & Das et al., 2013 \\
\hline PRPSAP1 & ENSGAL̄T00000002885 & Down & Stallion & Das et al., 2013 \\
\hline
\end{tabular}


Table 5: The twenty most abundant probes based on the fold change (NA-not available).

\begin{tabular}{|c|c|c|c|c|c|c|}
\hline Gene name & Accession number & $\begin{array}{l}\text { Fold } \\
\text { change }\end{array}$ & P value & $\begin{array}{c}\text { Chromosome } \\
\text { position }\end{array}$ & Size (bp) & Pathway \\
\hline CR389478 & BU441709 & 9.98 & $1.13 \mathrm{E}-03$ & 1 & 732 & Unknown \\
\hline$L 48903$ & L48903 & 10.37 & $1.12 \mathrm{E}-03$ & 1 & 297 & Unknown \\
\hline ENSGALT00000014963 & ENSGALT00000014963 & 10.38 & $9.47 \mathrm{E}-04$ & 25 & 483 & Unknown \\
\hline CR407005 & CR407005 & 10.56 & $8.66 \mathrm{E}-04$ & 2 & 869 & Unknown \\
\hline NP9665240 & NP9665240 & 10.72 & $3.07 \mathrm{E}-06$ & NA & NA & Unknown \\
\hline NP9669230 & NP9669230 & 10.41 & $5.76 \mathrm{E}-04$ & NA & NA & Unknown \\
\hline NP9668656 & NP9668656 & 10.63 & $3.29 \mathrm{E}-04$ & NA & NA & Unknown \\
\hline NP9669152 & NP9669152 & 10.57 & $6.11 \mathrm{E}-04$ & NA & NA & Unknown \\
\hline NP9665913 & NP9665913 & 9.93 & $9.26 \mathrm{E}-04$ & NA & NA & Unknown \\
\hline ENSGALT00000029799 & ENSGALT00000029799 & 11.39 & $1.25 \mathrm{E}-03$ & NA & NA & Unknown \\
\hline TANK & XM_422036 & 9.67 & $2.17 \mathrm{E}-04$ & 7 & 2695 & Apoptosis \\
\hline NP9668902 & NP9̄668902 & 10.87 & $1.61 \mathrm{E}-03$ & NA & NA & Unknown \\
\hline$B X 934641$ & TC442112 & 11.04 & $7.35 \mathrm{E}-04$ & 25 & 357 & Unknown \\
\hline NP9669128 & NP9669128 & 9.76 & $1.83 \mathrm{E}-04$ & NA & NA & Unknown \\
\hline NP9668791 & NP9668791 & 9.39 & $1.32 \mathrm{E}-03$ & NA & NA & Unknown \\
\hline NP9666246 & NP9666246 & 11.05 & $1.76 \mathrm{E}-03$ & NA & NA & Unknown \\
\hline NP9665148 & NP9665148 & 9.50 & $1.37 \mathrm{E}-03$ & NA & NA & Unknown \\
\hline NP9668920 & NP9668920 & 9.12 & $1.06 \mathrm{E}-02$ & NA & NA & Unknown \\
\hline TC429602 & TC429602 & 9.44 & $1.06 \mathrm{E}-04$ & NA & NA & Unknown \\
\hline SCARF1 & XM_001234822 & 10.32 & $3.25 \mathrm{E}-03$ & 19 & 2851 & Unknown \\
\hline
\end{tabular}


Table 6: The twenty least abundant probes based on the fold change (NA-not available).

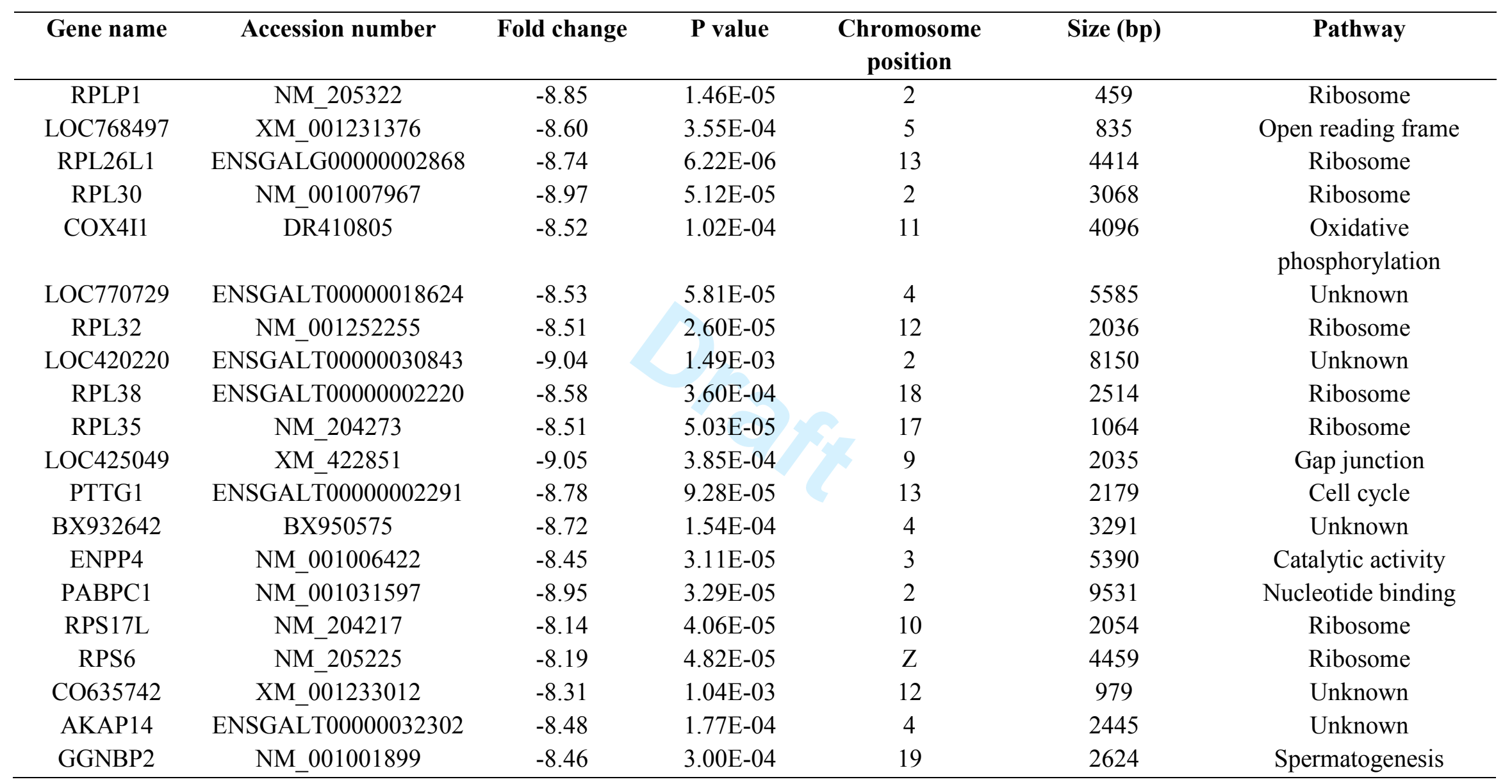



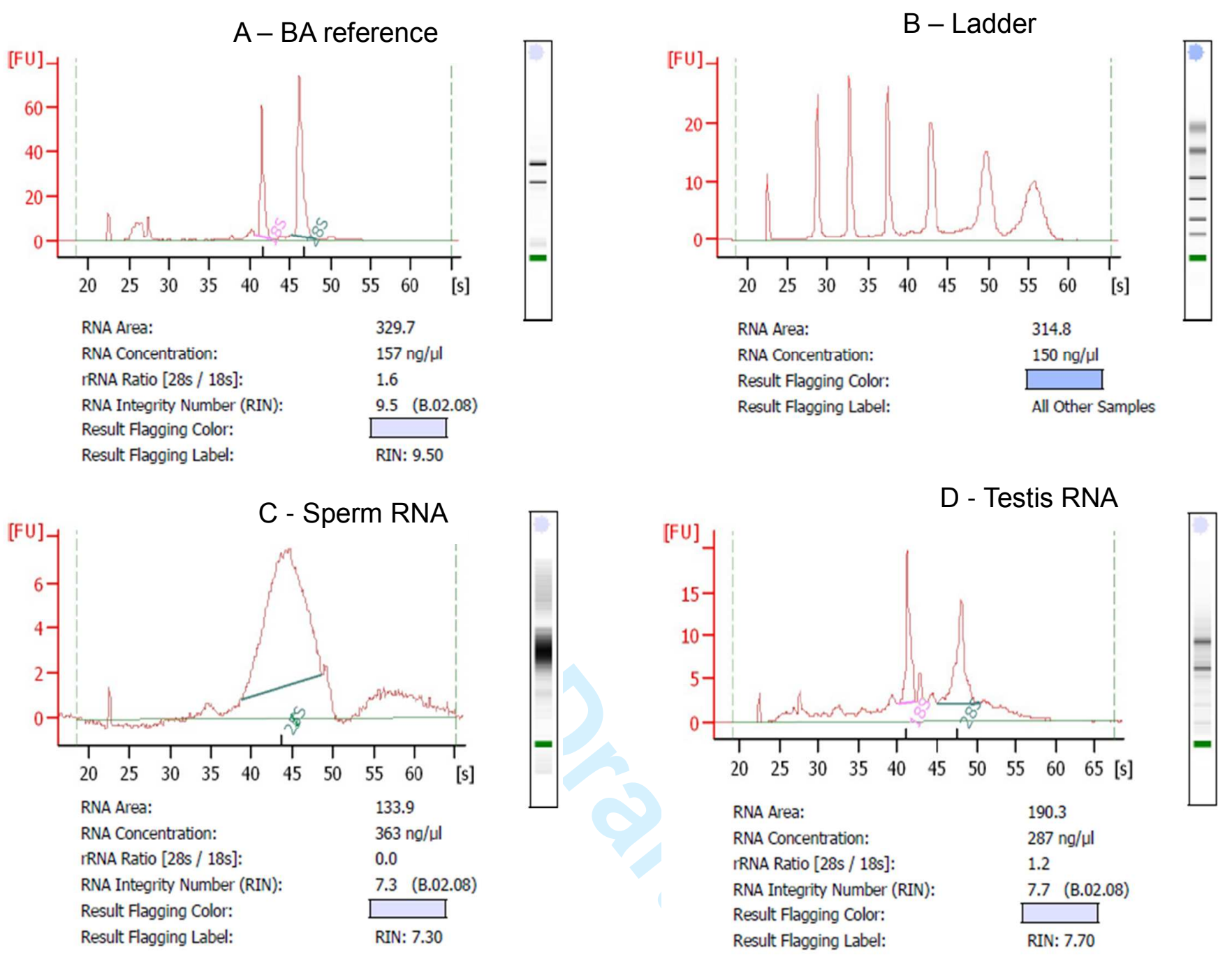

Fig. 1: Image showing microarray electropherogram of testis and sperm RNA samples. (A) $18 \mathrm{~S}$ and 28S rRNA reference; (B) Ladder; (C) Total sperm RNA; and (D) Total testis RNA. 


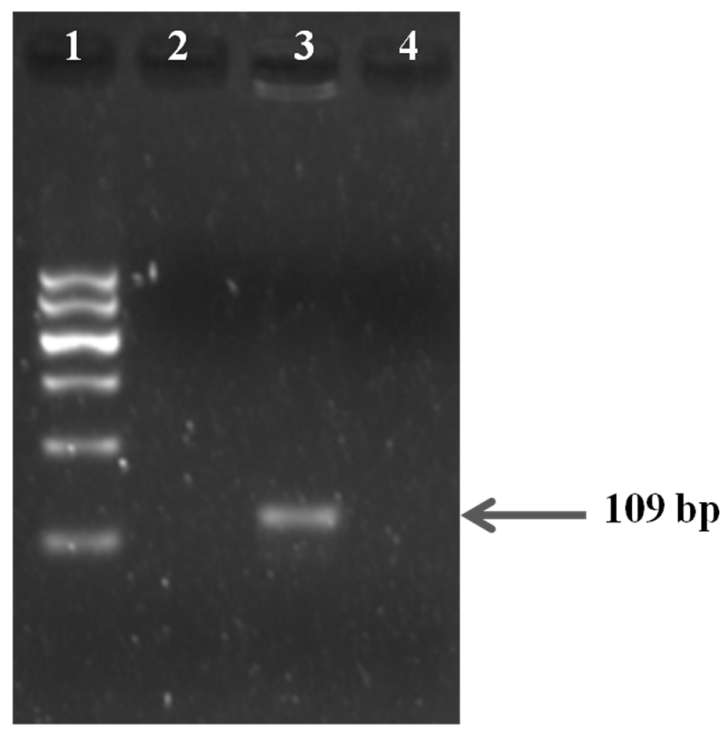

Fig. 2: Image showing the absence of PTPRC RNA in sperm RNA (Lane 1: 100bp marker; Lane 2: non template control; Lane 3:testis RNA and Lane 4: sperm RNA). 


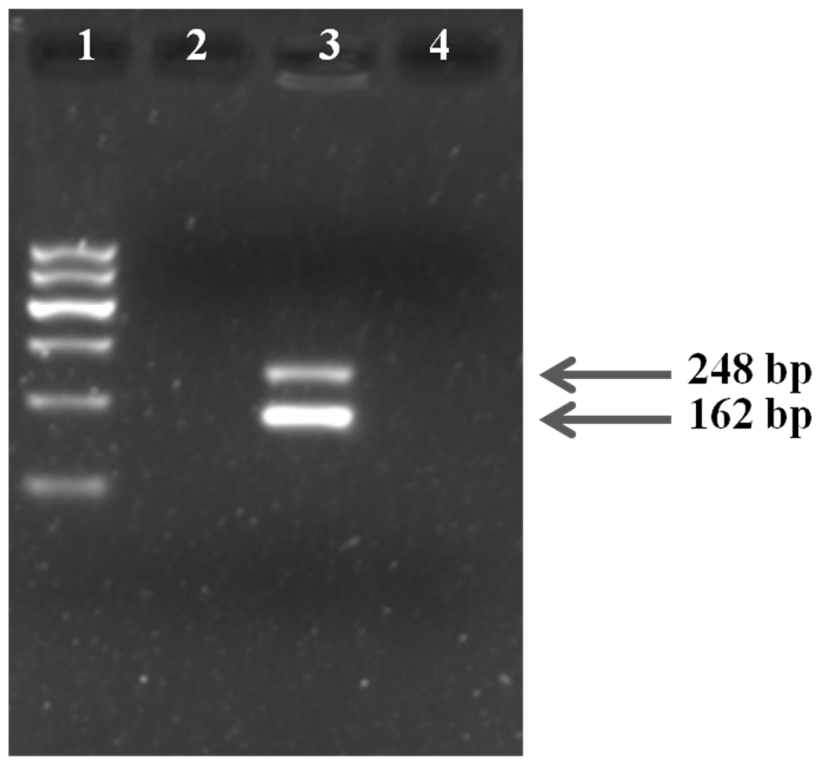

Fig. 3: Image showing the absence or presence of CD4 RNA and gDNA in sperm or testis samples. A intron-spanning CD4 primer set was used to detect the presence of genomic DNA contamination. Lane 1: 100 bp ladder. Lane 2: Non template control; Lane 3: Testes RNA spiked with genomic DNA to produce the amplicon of $248 \mathrm{bp}$ corresponding to genomic DNA and an amplicon of 162 bp corresponding to CD4 cDNA . Lane 3: sperm RNA. 


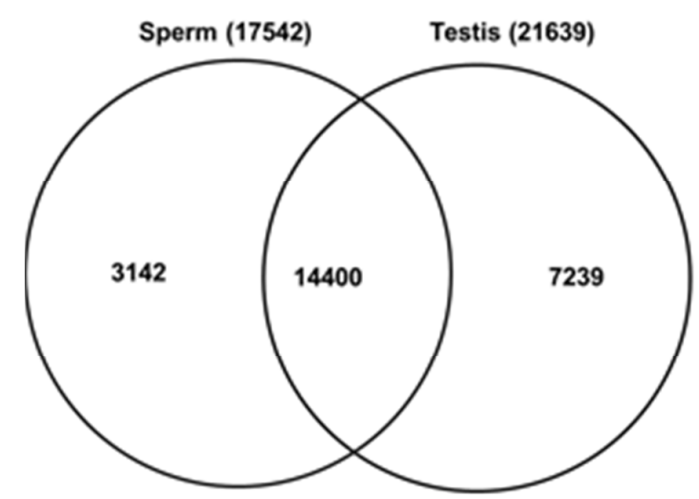

Fig. 4: Venn diagram of the proportion of specific or common transcripts between testis and sperm with raw signal intensity greater than 50 by microarray analysis. 


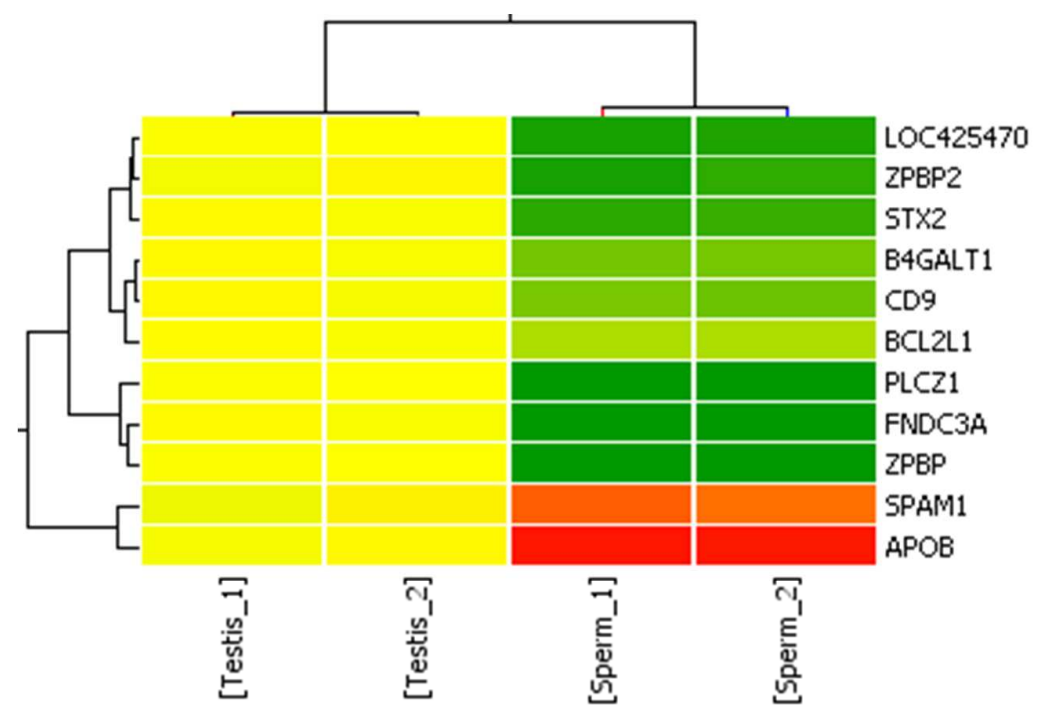

Fig. 5: Heat map of genes that have a role in fertilization. Hierarchical clustering of genes is based on similar expression profiles in sperm samples vs. testis samples. Red colour in the cluster indicates up regulation and green colour indicates down regulation in sperm when compared to testis sample. The fold expression values represented in the cluster are in terms of log base 2 . 


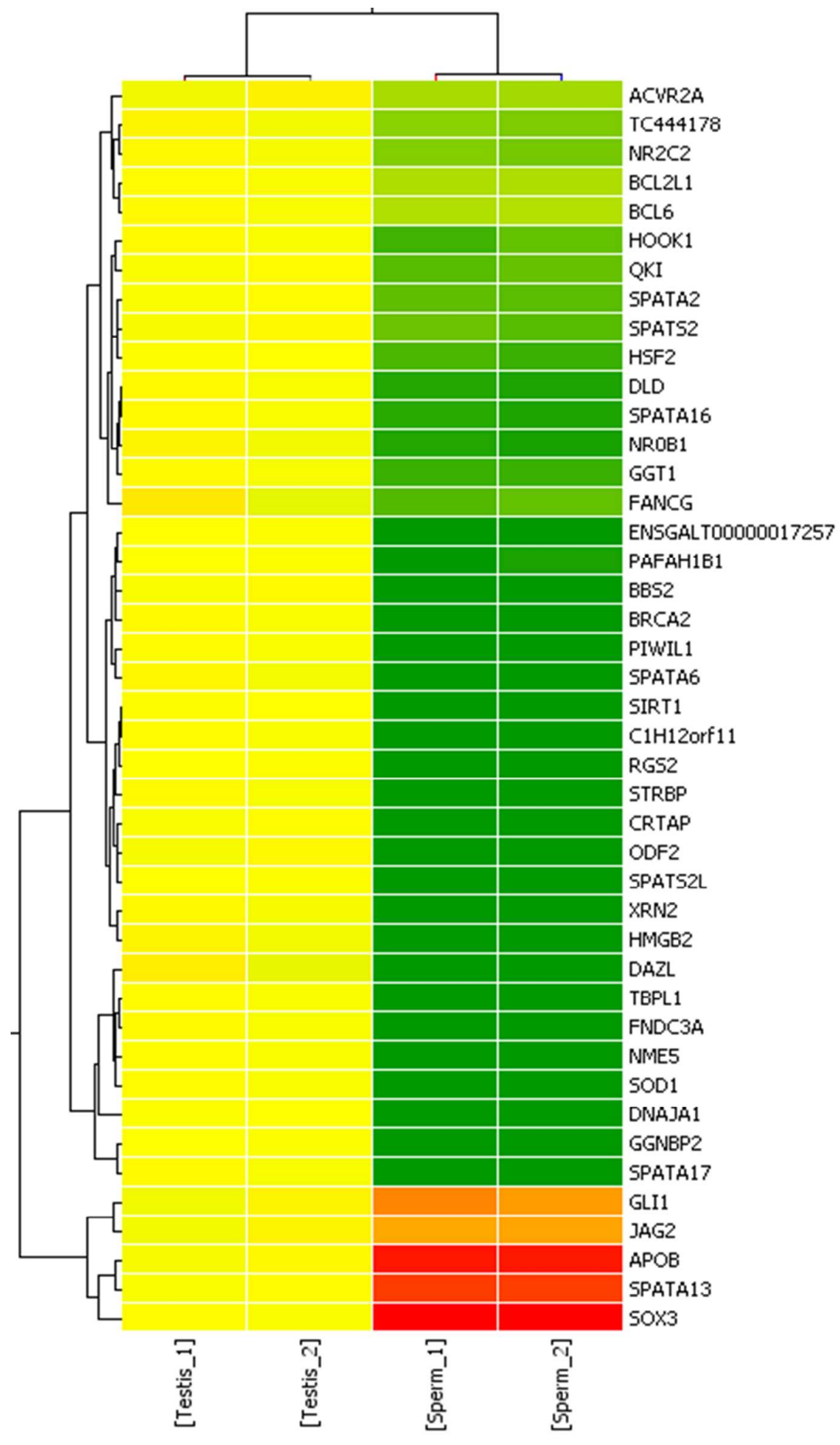

Fig. 6: Heat map of genes that have a role in spermatogenesis. Hierarchical clustering of genes is based on similar expression profiles in sperm samples vs. testis samples. Red colour in the cluster indicates up regulation and green colour indicates down regulation in sperm when compared to testis sample. The fold expression values represented in the cluster are in terms of log base 2 . 


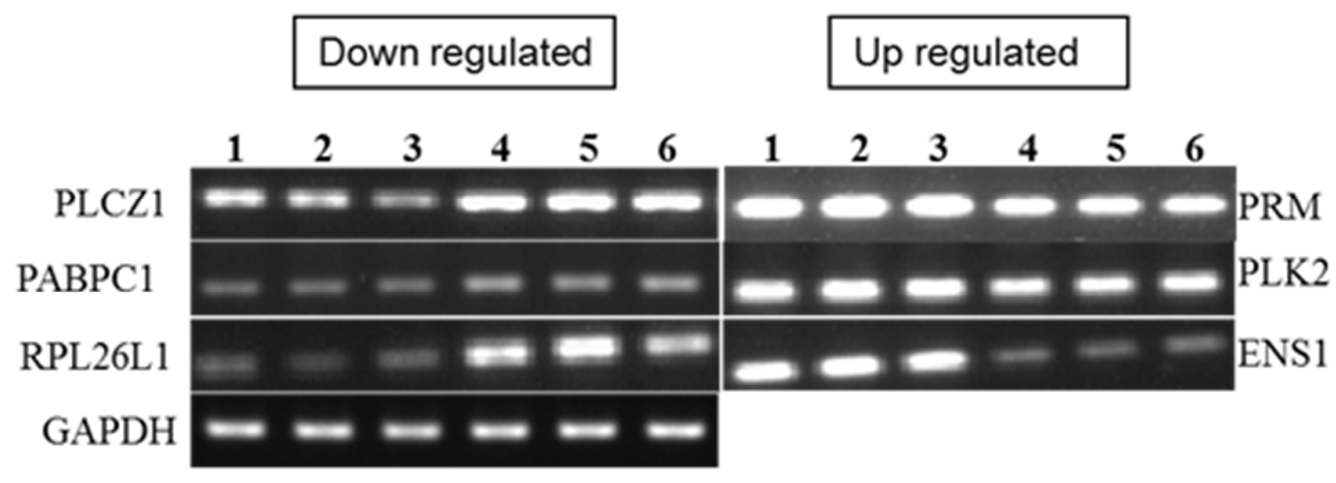

Fig. 7: Validation of microarray results for selected transcripts by RT-PCR. Lanes 1, 2 and 3 contain PCR products amplified with sperm cDNA, whereas lanes 4, 5 and 6 contain PCR products amplified with testis cDNA. Gel electrophoresis was performed for all the genes in identical conditions. 

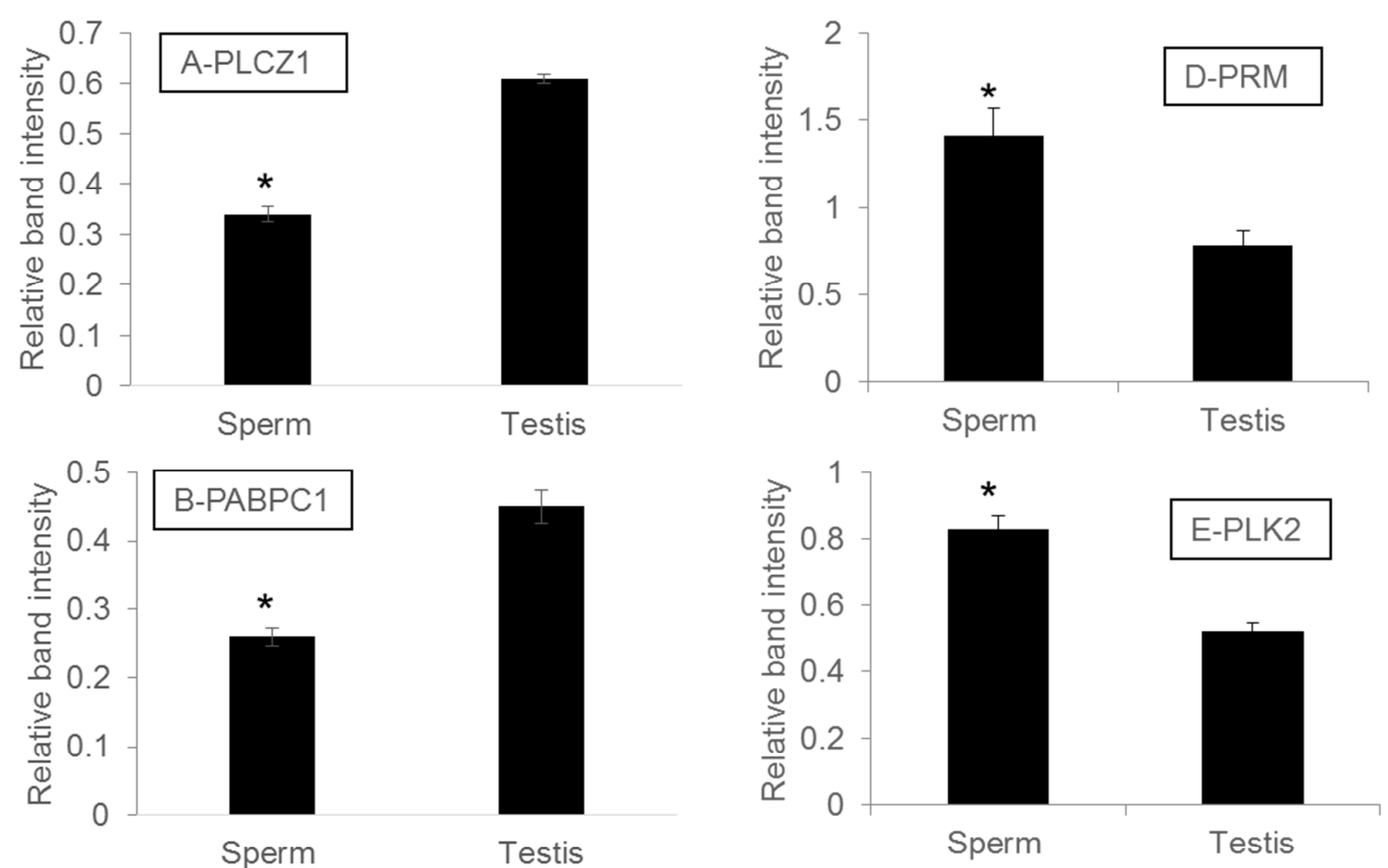

Testis
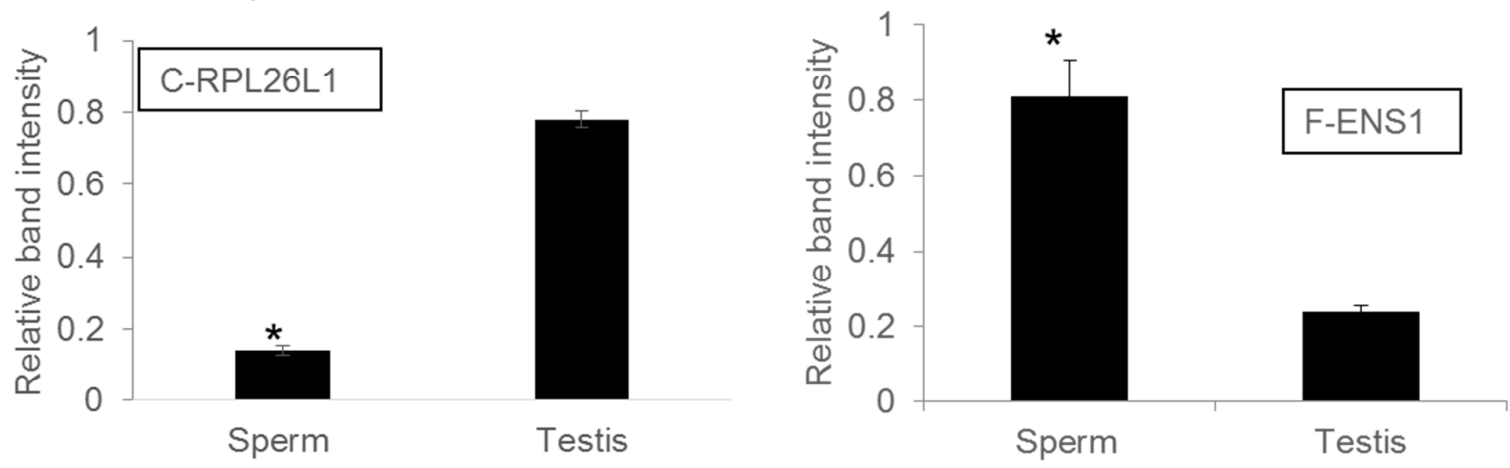

Fig. 8: RT-PCR analysis of selected transcripts in sperm and testis cDNA. Bars represent the mean of the normalized band density for each mRNA band obtained by densitometric analysis (mean $\pm \mathrm{SE} ; \mathrm{n}=3$ ). Normalization was performed by dividing each band density value by the value of GAPDH band density in the same sample. Asterisk (*) indicates a significant difference $(\mathrm{p}<0.05)$ in mRNA expression. 


\begin{tabular}{|c|c|c|c|}
\hline S. No. & Gene & Fold change & P-Value \\
\hline 1 & 41700 & -4.80 & $8.42 E-05$ \\
\hline 2 & 41701 & -5.26 & $8.26 \mathrm{E}-04$ \\
\hline 3 & 41703 & -5.90 & $1.60 \mathrm{E}-04$ \\
\hline 4 & 41705 & -2.76 & $1.03 E-02$ \\
\hline 5 & 41709 & -3.69 & 0.00439499 \\
\hline 6 & 41884 & -3.20 & $2.05 E-04$ \\
\hline 7 & 41885 & -2.38 & $1.59 \mathrm{E}-04$ \\
\hline 8 & 41888 & -4.44 & $4.15 E-04$ \\
\hline 9 & 41889 & -2.43 & $1.63 \mathrm{E}-03$ \\
\hline 10 & 41892 & -4.71 & $7.38 \mathrm{E}-06$ \\
\hline 11 & 41897 & -4.28 & $9.89 \mathrm{E}-03$ \\
\hline 12 & A2LD1 & -1.57 & $5.24 \mathrm{E}-02$ \\
\hline 13 & AACS & -3.87 & $7.65 \mathrm{E}-03$ \\
\hline 14 & AADACL4 & -6.35 & $1.55 \mathrm{E}-04$ \\
\hline 15 & AADAT & -4.32 & $2.81 \mathrm{E}-05$ \\
\hline 16 & AAED1 & -2.48 & $1.94 \mathrm{E}-03$ \\
\hline 17 & AAGAB & -4.18 & $5.31 \mathrm{E}-04$ \\
\hline 18 & AAMDC & -6.00 & $8.88 \mathrm{E}-05$ \\
\hline 19 & AAMP & -1.12 & $2.36 \mathrm{E}-02$ \\
\hline 20 & AAR2 & -2.15 & $1.33 \mathrm{E}-03$ \\
\hline 21 & AARS2 & -5.12 & $1.52 \mathrm{E}-04$ \\
\hline 22 & AARSD1 & -3.83 & $3.70 \mathrm{E}-04$ \\
\hline 23 & AASDH & -1.31 & $1.84 \mathrm{E}-02$ \\
\hline 24 & AASDHPPT & -4.09 & $6.41 \mathrm{E}-04$ \\
\hline 25 & ABAT & -5.99 & $1.03 E-04$ \\
\hline 26 & АВСB10 & -5.18 & 4.24E-05 \\
\hline 27 & $A B C B 6$ & -2.07 & $5.28 \mathrm{E}-07$ \\
\hline 28 & ABCB7 & -1.75 & $1.00 E-03$ \\
\hline 29 & $A B C C 1$ & -3.10 & $7.01 E-04$ \\
\hline 30 & $\mathrm{ABCC} 2$ & -1.93 & $5.05 E-03$ \\
\hline 31 & $\mathrm{ABCC} 4$ & -1.56 & $9.33 \mathrm{E}-03$ \\
\hline 32 & $A B C C 5$ & -3.17 & $8.47 \mathrm{E}-04$ \\
\hline 33 & ABCC9 & -5.35 & $3.70 \mathrm{E}-04$ \\
\hline 34 & $\mathrm{ABCD} 2$ & -2.18 & $1.64 \mathrm{E}-03$ \\
\hline 35 & $A B C D 3$ & -1.93 & $2.14 \mathrm{E}-02$ \\
\hline 36 & $\mathrm{ABCD} 4$ & -2.42 & $3.62 \mathrm{E}-03$ \\
\hline 37 & ABCE1 & -4.29 & $1.68 \mathrm{E}-03$ \\
\hline 38 & ABCF2 & -4.15 & $2.74 \mathrm{E}-03$ \\
\hline 39 & $\mathrm{ABCG} 2$ & -3.15 & $7.61 \mathrm{E}-03$ \\
\hline 40 & ABHD12 & -4.20 & $3.06 \mathrm{E}-05$ \\
\hline 41 & ABHD13 & -2.62 & 7.44E-03 \\
\hline 42 & ABHD2 & -3.62 & $1.06 \mathrm{E}-03$ \\
\hline 43 & ABHD3 & -1.82 & $1.62 \mathrm{E}-03$ \\
\hline 44 & $A B \mid 1$ & -2.57 & $3.67 E-03$ \\
\hline 45 & $A B \mid 2$ & -4.31 & $8.54 \mathrm{E}-04$ \\
\hline 46 & ABLIM1 & -4.22 & $3.98 \mathrm{E}-03$ \\
\hline 47 & ABTB1 & -1.02 & $1.08 \mathrm{E}-01$ \\
\hline 48 & ACAA1 & -2.37 & $5.02 \mathrm{E}-03$ \\
\hline 49 & ACAA2 & -5.57 & 3.90E-03 \\
\hline 50 & ACAD11 & -2.59 & $1.57 \mathrm{E}-03$ \\
\hline 51 & ACAD8 & -1.45 & $1.50 \mathrm{E}-03$ \\
\hline 52 & ACAD9 & -6.60 & $1.14 \mathrm{E}-04$ \\
\hline 53 & ACADL & -6.22 & $4.68 \mathrm{E}-04$ \\
\hline 54 & ACADS & -1.75 & 1.67E-02 \\
\hline 55 & ACADSB & -1.64 & $2.00 \mathrm{E}-02$ \\
\hline 56 & ACAP2 & -2.89 & $1.79 \mathrm{E}-03$ \\
\hline 57 & ACAT1 & -3.48 & $1.18 \mathrm{E}-05$ \\
\hline 58 & ACAT2 & -2.70 & $1.20 \mathrm{E}-02$ \\
\hline 59 & ACBD3 & -3.03 & $6.73 \mathrm{E}-02$ \\
\hline 60 & ACBD5 & -5.88 & $3.39 \mathrm{E}-04$ \\
\hline 61 & ACBD6 & -1.76 & $7.40 \mathrm{E}-03$ \\
\hline 62 & ACBD7 & $\begin{array}{l}-7.72 \\
\end{array}$ & $2.57 \mathrm{E}-04$ \\
\hline
\end{tabular}

\begin{tabular}{|c|c|c|c|}
\hline 63 & $A C D$ & -1.45 & 5.27E-03 \\
\hline 64 & ACER3 & -3.59 & $3.62 \mathrm{E}-04$ \\
\hline 65 & ACN9 & -3.62 & $8.10 \mathrm{E}-04$ \\
\hline 66 & ACO1 & -4.46 & $6.80 \mathrm{E}-04$ \\
\hline 67 & $\mathrm{ACO} 2$ & -4.72 & $1.11 \mathrm{E}-04$ \\
\hline 68 & АСОТ13 & -5.53 & $2.93 \mathrm{E}-03$ \\
\hline 69 & ACOT7 & -5.64 & $9.19 \mathrm{E}-05$ \\
\hline 70 & АсОT8 & -2.12 & $1.77 \mathrm{E}-03$ \\
\hline 71 & AсOT9 & -2.74 & $6.04 \mathrm{E}-04$ \\
\hline 72 & ACOX3 & -2.17 & $1.18 \mathrm{E}-02$ \\
\hline 73 & ACP1 & -6.21 & $2.85 \mathrm{E}-04$ \\
\hline 74 & ACP2 & -5.35 & $4.63 \mathrm{E}-05$ \\
\hline 75 & ACP6 & -4.28 & $5.83 \mathrm{E}-04$ \\
\hline 76 & ACPL2 & -1.58 & $5.29 \mathrm{E}-03$ \\
\hline 77 & ACPP & -1.49 & $1.42 \mathrm{E}-02$ \\
\hline 78 & ACR & -5.33 & $4.58 \mathrm{E}-05$ \\
\hline 79 & ACRC & -1.50 & $8.69 \mathrm{E}-03$ \\
\hline 80 & ACSBG1 & -1.06 & $3.92 \mathrm{E}-03$ \\
\hline 81 & ACSBG2 & -4.49 & $2.56 \mathrm{E}-04$ \\
\hline 82 & ACSF2 & -1.86 & $9.92 \mathrm{E}-04$ \\
\hline 83 & ACSL1 & -5.39 & 7.17E-05 \\
\hline 84 & ACSL3 & -5.67 & $6.00 \mathrm{E}-04$ \\
\hline 85 & ACSS1 & -2.36 & $3.15 \mathrm{E}-04$ \\
\hline 86 & ACSS2 & -2.49 & $8.64 \mathrm{E}-05$ \\
\hline 87 & ACSS3 & -5.62 & $7.91 E-04$ \\
\hline 88 & ACTA1 & -1.82 & 8.77E-04 \\
\hline 89 & ACTA2 & -2.52 & $1.58 \mathrm{E}-03$ \\
\hline 90 & АСТВ & -5.37 & $6.04 \mathrm{E}-05$ \\
\hline 91 & ACTG1 & -4.56 & $6.40 \mathrm{E}-03$ \\
\hline 92 & ACTL9 & -2.25 & $3.64 \mathrm{E}-03$ \\
\hline 93 & ACTN1 & -3.08 & $1.79 \mathrm{E}-03$ \\
\hline 94 & ACTR10 & -5.66 & $4.03 E-04$ \\
\hline 95 & ACTR1A & -2.55 & $6.88 \mathrm{E}-04$ \\
\hline 96 & ACTR2 & -2.34 & $8.26 \mathrm{E}-03$ \\
\hline 97 & ACTR3B & -1.30 & $2.48 \mathrm{E}-02$ \\
\hline 98 & ACTR5 & -3.90 & $1.53 \mathrm{E}-03$ \\
\hline 99 & ACTR6 & -4.51 & $1.74 \mathrm{E}-03$ \\
\hline 100 & ACTR8 & -4.12 & $4.39 \mathrm{E}-04$ \\
\hline 101 & ACVR1 & -4.50 & $1.18 \mathrm{E}-03$ \\
\hline 102 & ACVR2A & -1.38 & $1.88 \mathrm{E}-02$ \\
\hline 103 & ACYP1 & -7.52 & $5.79 \mathrm{E}-04$ \\
\hline 104 & ACYP2 & -4.72 & $6.65 \mathrm{E}-04$ \\
\hline 105 & ADA & -3.47 & $1.20 \mathrm{E}-03$ \\
\hline 106 & ADAD1 & -1.79 & $1.07 \mathrm{E}-01$ \\
\hline 107 & ADAM10 & -2.38 & $4.51 \mathrm{E}-03$ \\
\hline 108 & ADAM15 & -4.03 & $1.84 \mathrm{E}-03$ \\
\hline 109 & ADAM17 & -2.89 & $6.53 \mathrm{E}-04$ \\
\hline 110 & ADAM20 & -2.50 & $5.80 \mathrm{E}-04$ \\
\hline 111 & ADAM32 & -5.48 & $4.42 \mathrm{E}-04$ \\
\hline 112 & ADAM9 & -1.40 & $1.49 \mathrm{E}-02$ \\
\hline 113 & ADAMTS13 & -2.26 & $6.13 \mathrm{E}-03$ \\
\hline 114 & ADAMTS5 & -3.40 & $1.19 \mathrm{E}-03$ \\
\hline 115 & ADAP2 & -3.08 & $1.01 \mathrm{E}-02$ \\
\hline 116 & ADAR & -1.68 & $4.82 \mathrm{E}-02$ \\
\hline 117 & ADARB1 & -1.82 & $1.92 \mathrm{E}-02$ \\
\hline 118 & ADAT2 & -5.67 & $1.44 \mathrm{E}-04$ \\
\hline 119 & ADCK1 & -1.43 & $3.45 \mathrm{E}-03$ \\
\hline 120 & ADCK2 & -3.11 & $5.25 \mathrm{E}-04$ \\
\hline 121 & ADCY8 & -4.53 & $2.00 \mathrm{E}-03$ \\
\hline 122 & ADCY9 & -6.02 & $1.22 \mathrm{E}-03$ \\
\hline 123 & ADD1 & -4.85 & $7.70 \mathrm{E}-04$ \\
\hline 124 & ADD3 & -2.33 & $1.80 \mathrm{E}-02$ \\
\hline 125 & ADH5 & -4.18 & $1.00 \mathrm{E}-03$ \\
\hline
\end{tabular}

\begin{tabular}{|c|c|c|c|}
\hline 126 & ADH6 & -1.50 & $9.09 \mathrm{E}-03$ \\
\hline 127 & ADHFE1 & -1.74 & $3.43 \mathrm{E}-02$ \\
\hline 128 & ADIPOR1 & -4.41 & $6.63 \mathrm{E}-04$ \\
\hline 129 & ADIPOR2 & -2.75 & $1.03 \mathrm{E}-02$ \\
\hline 130 & ADK & -2.46 & 1.93E-01 \\
\hline 131 & ADNP & -4.87 & $1.36 \mathrm{E}-04$ \\
\hline 132 & ADNP2 & -1.62 & $6.64 \mathrm{E}-03$ \\
\hline 133 & ADRM1 & -4.34 & $1.00 \mathrm{E}-03$ \\
\hline 134 & ADSL & -4.56 & 1.47E-04 \\
\hline 135 & ADSS & -5.19 & $9.90 \mathrm{E}-04$ \\
\hline 136 & AEBP2 & -4.00 & $5.63 \mathrm{E}-04$ \\
\hline 137 & AEN & -1.44 & $1.34 \mathrm{E}-02$ \\
\hline 138 & AF090088 & -1.85 & $4.23 \mathrm{E}-03$ \\
\hline 139 & AF170930 & -5.33 & $1.20 \mathrm{E}-02$ \\
\hline 140 & AF188735 & -2.44 & $1.03 \mathrm{E}-02$ \\
\hline 141 & AF308592 & -4.87 & $1.92 \mathrm{E}-03$ \\
\hline 142 & AF309648 & -5.03 & $1.57 \mathrm{E}-04$ \\
\hline 143 & AFF1 & -2.99 & $5.42 \mathrm{E}-04$ \\
\hline 144 & AFF4 & -4.05 & $6.53 \mathrm{E}-04$ \\
\hline 145 & AFG3L2 & -5.10 & $8.95 \mathrm{E}-04$ \\
\hline 146 & AFMID & -3.33 & $2.73 \mathrm{E}-03$ \\
\hline 147 & AFTPH & -3.44 & $1.03 \mathrm{E}-03$ \\
\hline 148 & AGAP1 & -3.05 & $2.37 \mathrm{E}-04$ \\
\hline 149 & AGBL3 & -3.70 & $8.25 \mathrm{E}-04$ \\
\hline 150 & AGBL4 & -2.24 & $6.99 \mathrm{E}-04$ \\
\hline 151 & AGFG1 & -4.56 & $4.34 \mathrm{E}-04$ \\
\hline 152 & AGPAT5 & -2.15 & $4.06 \mathrm{E}-03$ \\
\hline 153 & AGPAT6 & -4.84 & $1.09 \mathrm{E}-04$ \\
\hline 154 & AGPAT9 & -5.77 & 1.37E-04 \\
\hline 155 & AGPHD1 & -2.39 & $1.35 \mathrm{E}-02$ \\
\hline 156 & AGPS & -1.55 & $6.55 \mathrm{E}-02$ \\
\hline 157 & AGTRAP & -2.47 & $3.33 \mathrm{E}-03$ \\
\hline 158 & AGXT2 & -1.37 & $1.47 \mathrm{E}-03$ \\
\hline 159 & AGXT2L2 & -2.67 & $1.97 \mathrm{E}-03$ \\
\hline 160 & AHCTF1 & -2.14 & $1.03 E-02$ \\
\hline 161 & AHCY & -2.11 & $8.27 \mathrm{E}-03$ \\
\hline 162 & AHCYL1 & -1.35 & $4.18 \mathrm{E}-04$ \\
\hline 163 & AHSA1 & -7.63 & $3.00 \mathrm{E}-05$ \\
\hline 164 & AHSA2 & -4.70 & $9.71 \mathrm{E}-03$ \\
\hline 165 & AIDA & -3.52 & $2.57 \mathrm{E}-03$ \\
\hline 166 & AIF1L & -2.72 & $2.64 \mathrm{E}-02$ \\
\hline 167 & AIFM1 & -4.82 & $2.69 \mathrm{E}-04$ \\
\hline 168 & AIG1 & -3.38 & $9.95 \mathrm{E}-04$ \\
\hline 169 & AIM1 & -3.39 & $1.92 \mathrm{E}-03$ \\
\hline 170 & AIMP1 & -3.20 & $3.78 \mathrm{E}-04$ \\
\hline 171 & AIMP2 & -4.72 & $2.79 \mathrm{E}-03$ \\
\hline 172 & AIP & -2.00 & $3.71 \mathrm{E}-04$ \\
\hline 173 & AJ301880 & -1.94 & $2.04 \mathrm{E}-02$ \\
\hline 174 & AJ301881 & -2.04 & $1.64 \mathrm{E}-02$ \\
\hline 175 & AJ393608 & -1.40 & $4.54 \mathrm{E}-02$ \\
\hline 176 & AJ443802 & -3.13 & 4.02E-03 \\
\hline 177 & AJ452155 & -1.43 & $8.38 \mathrm{E}-03$ \\
\hline 178 & AJ456460 & -1.83 & $1.68 \mathrm{E}-03$ \\
\hline 179 & AJ719505 & -1.61 & $3.42 \mathrm{E}-03$ \\
\hline 180 & AJ719778 & -2.41 & $2.13 \mathrm{E}-02$ \\
\hline 181 & AJ720157 & -4.95 & $5.79 \mathrm{E}-04$ \\
\hline 182 & AJ720254 & -2.67 & $4.10 \mathrm{E}-03$ \\
\hline 183 & AJ720285 & -2.26 & $5.14 \mathrm{E}-02$ \\
\hline 184 & AJ720512 & -1.83 & $2.19 \mathrm{E}-03$ \\
\hline 185 & AJ722492 & -3.97 & $2.31 \mathrm{E}-05$ \\
\hline 186 & AJ723596 & -1.62 & $1.19 \mathrm{E}-02$ \\
\hline 187 & AJ729193 & -2.29 & $2.54 \mathrm{E}-02$ \\
\hline 188 & AJ736817 & -3.29 & $5.90 \mathrm{E}-03$ \\
\hline
\end{tabular}




\begin{tabular}{|c|c|c|c|}
\hline 189 & AJ843259 & -1.30 & $4.34 \mathrm{E}-04$ \\
\hline 190 & AJ851519 & -1.81 & $1.33 \mathrm{E}-01$ \\
\hline 191 & AJ851720 & -4.46 & $1.15 \mathrm{E}-03$ \\
\hline 192 & AJ851804 & -8.22 & $1.02 \mathrm{E}-03$ \\
\hline 193 & AK1 & -3.74 & $6.75 E-05$ \\
\hline 194 & AK3 & -4.12 & $8.42 \mathrm{E}-04$ \\
\hline 195 & AK7 & -6.45 & 3.14E-05 \\
\hline 196 & AKAP1 & -2.94 & $6.13 \mathrm{E}-04$ \\
\hline 197 & AKAP10 & -2.97 & $1.68 \mathrm{E}-03$ \\
\hline 198 & AKAP12 & -5.76 & $5.94 \mathrm{E}-04$ \\
\hline 199 & AKAP14 & -8.48 & $1.77 \mathrm{E}-04$ \\
\hline 200 & AKAP17A & -2.01 & $2.42 \mathrm{E}-03$ \\
\hline 201 & AKD1 & -4.58 & $3.14 \mathrm{E}-04$ \\
\hline 202 & AKIP1 & -2.86 & $2.46 \mathrm{E}-02$ \\
\hline 203 & AKIRIN2 & -7.98 & $1.55 \mathrm{E}-05$ \\
\hline 204 & AKR1A1 & -1.27 & $5.21 \mathrm{E}-03$ \\
\hline 205 & AKR1B10 & -6.36 & $1.06 \mathrm{E}-05$ \\
\hline 206 & AKR7A2 & -4.51 & $6.83 \mathrm{E}-04$ \\
\hline 207 & AKTIP & -5.90 & 3.07E-05 \\
\hline 208 & AL588186 & -7.15 & $2.82 \mathrm{E}-05$ \\
\hline 209 & ALAS1 & -2.98 & 3.13E-03 \\
\hline 210 & ALDH1A2 & -2.06 & $1.91 \mathrm{E}-02$ \\
\hline 211 & ALDH2 & -4.77 & $6.71 \mathrm{E}-05$ \\
\hline 212 & ALDH3A2 & -3.92 & 3.51E-04 \\
\hline 213 & ALDH4A1 & -4.63 & $2.44 \mathrm{E}-03$ \\
\hline 214 & ALDH7A1 & -2.27 & $1.54 \mathrm{E}-03$ \\
\hline 215 & ALDH9A1 & -2.95 & $1.56 \mathrm{E}-03$ \\
\hline 216 & ALDOA & -1.98 & 1.15E-02 \\
\hline 217 & ALDOC & -4.43 & $6.75 \mathrm{E}-04$ \\
\hline 218 & ALG1 & -2.05 & $5.76 \mathrm{E}-04$ \\
\hline 219 & ALG10 & -3.13 & $1.75 \mathrm{E}-04$ \\
\hline 220 & ALG11 & -5.58 & $1.37 \mathrm{E}-04$ \\
\hline 221 & ALG13 & -4.50 & 2.03E-04 \\
\hline 222 & ALG14 & -3.84 & $2.21 \mathrm{E}-04$ \\
\hline 223 & ALG2 & -2.54 & $7.58 \mathrm{E}-03$ \\
\hline 224 & ALG5 & -3.59 & 5.15E-04 \\
\hline 225 & ALG6 & -4.34 & $5.30 \mathrm{E}-03$ \\
\hline 226 & ALG8 & -4.30 & 9.97E-04 \\
\hline 227 & ALKBH1 & -4.67 & $5.19 \mathrm{E}-04$ \\
\hline 228 & ALKBH2 & -3.00 & 7.81E-04 \\
\hline 229 & ALKBH4 & -3.74 & $3.54 \mathrm{E}-03$ \\
\hline 230 & ALKBH5 & -3.52 & $1.24 \mathrm{E}-03$ \\
\hline 231 & ALKBH8 & -3.33 & $2.99 \mathrm{E}-03$ \\
\hline 232 & ALMS1 & -3.62 & $1.14 \mathrm{E}-03$ \\
\hline 233 & ALOX5AP & -4.45 & 2.27E-03 \\
\hline 234 & AM284174 & -1.67 & $5.00 \mathrm{E}-02$ \\
\hline 235 & AMACR & -4.04 & $1.06 \mathrm{E}-04$ \\
\hline 236 & AMBP & -4.02 & 1.79E-04 \\
\hline 237 & AMBRA1 & -2.75 & 4.96E-04 \\
\hline 238 & AMD1 & -4.17 & $2.68 \mathrm{E}-04$ \\
\hline 239 & AMDHD1 & -2.01 & $1.32 \mathrm{E}-03$ \\
\hline 240 & AMDHD2 & -3.33 & $7.25 \mathrm{E}-04$ \\
\hline 241 & AMFR & -2.25 & 2.67E-02 \\
\hline 242 & AMH & -5.57 & $2.03 \mathrm{E}-04$ \\
\hline 243 & AMIGO2 & -1.45 & $1.92 \mathrm{E}-02$ \\
\hline 244 & AMN1 & -5.06 & $1.84 \mathrm{E}-04$ \\
\hline 245 & AMT & -1.28 & $5.61 \mathrm{E}-02$ \\
\hline 246 & AMY1A & -2.59 & $9.63 \mathrm{E}-03$ \\
\hline 247 & ANAPC1 & -5.00 & $2.83 \mathrm{E}-04$ \\
\hline 248 & ANAPC10 & -4.10 & $2.88 \mathrm{E}-04$ \\
\hline 249 & ANAPC16 & -6.15 & $5.38 \mathrm{E}-04$ \\
\hline 250 & ANAPC2 & -3.97 & 2.15E-04 \\
\hline 251 & ANAPC4 & -4.03 & $6.65 \mathrm{E}-04$ \\
\hline
\end{tabular}

\begin{tabular}{|c|c|c|c|}
\hline 252 & ANAPC5 & -1.79 & $1.77 \mathrm{E}-02$ \\
\hline 253 & ANAPC7 & -1.82 & $1.10 \mathrm{E}-01$ \\
\hline 254 & ANGEL1 & -4.17 & $6.80 \mathrm{E}-04$ \\
\hline 255 & ANGEL2 & -3.55 & $4.72 \mathrm{E}-04$ \\
\hline 256 & ANGPTL1 & -5.06 & $1.39 \mathrm{E}-03$ \\
\hline 257 & ANGPTL2 & -2.99 & $7.80 \mathrm{E}-03$ \\
\hline 258 & ANK2 & -4.32 & $6.59 \mathrm{E}-03$ \\
\hline 259 & ANK3 & -1.77 & $4.28 \mathrm{E}-03$ \\
\hline 260 & ANKH & -1.30 & $7.79 \mathrm{E}-03$ \\
\hline 261 & ANKHD1 & -2.73 & $1.64 \mathrm{E}-03$ \\
\hline 262 & ANKLE2 & -3.49 & $1.60 \mathrm{E}-03$ \\
\hline 263 & ANKMY1 & -6.79 & $6.42 \mathrm{E}-05$ \\
\hline 264 & ANKMY2 & -2.80 & $1.77 \mathrm{E}-03$ \\
\hline 265 & ANKRA2 & -2.77 & $2.28 \mathrm{E}-04$ \\
\hline 266 & ANKRD10 & -2.02 & $1.82 \mathrm{E}-03$ \\
\hline 267 & ANKRD11 & -3.87 & $2.56 \mathrm{E}-03$ \\
\hline 268 & ANKRD13A & -2.69 & $5.62 \mathrm{E}-03$ \\
\hline 269 & ANKRD13C & -3.47 & $1.97 \mathrm{E}-03$ \\
\hline 270 & ANKRD16 & -2.23 & $5.67 \mathrm{E}-03$ \\
\hline 271 & ANKRD22 & -2.35 & $2.31 \mathrm{E}-02$ \\
\hline 272 & ANKRD26 & -5.34 & $2.53 \mathrm{E}-04$ \\
\hline 273 & ANKRD28 & -1.32 & $1.34 \mathrm{E}-02$ \\
\hline 274 & ANKRD32 & -3.36 & $2.80 \mathrm{E}-04$ \\
\hline 275 & ANKRD42 & -2.20 & $1.15 \mathrm{E}-03$ \\
\hline 276 & ANKRD46 & -3.91 & $5.47 \mathrm{E}-06$ \\
\hline 277 & ANKRD49 & -2.90 & $7.85 \mathrm{E}-04$ \\
\hline 278 & ANKRD50 & -1.36 & $1.41 \mathrm{E}-02$ \\
\hline 279 & ANKRD54 & -1.94 & $8.02 \mathrm{E}-03$ \\
\hline 280 & ANKRD9 & -1.33 & 4.10E-04 \\
\hline 281 & ANKS3 & -2.77 & $6.97 \mathrm{E}-04$ \\
\hline 282 & ANKS6 & -1.11 & $5.25 \mathrm{E}-03$ \\
\hline 283 & ANKZF1 & -1.83 & $1.34 \mathrm{E}-02$ \\
\hline 284 & ANLN & -4.30 & 4.40E-04 \\
\hline 285 & ANO3 & -2.49 & $1.20 \mathrm{E}-04$ \\
\hline 286 & ANO4 & -4.06 & 5.37E-03 \\
\hline 287 & ANP32B & -2.00 & $1.64 \mathrm{E}-02$ \\
\hline 288 & ANP32E & -2.78 & $1.38 \mathrm{E}-03$ \\
\hline 289 & ANXA11 & -3.85 & $2.27 \mathrm{E}-03$ \\
\hline 290 & ANXA2 & -6.76 & $1.36 \mathrm{E}-04$ \\
\hline 291 & ANXA5 & -4.00 & $7.69 \mathrm{E}-04$ \\
\hline 292 & ANXA7 & -1.95 & $9.37 \mathrm{E}-03$ \\
\hline 293 & AOX1 & -1.88 & $6.65 \mathrm{E}-03$ \\
\hline 294 & AP1G1 & -4.64 & $6.63 \mathrm{E}-04$ \\
\hline 295 & AP1M1 & -2.94 & $3.23 \mathrm{E}-04$ \\
\hline 296 & AP1S2 & -4.17 & $1.29 \mathrm{E}-03$ \\
\hline 297 & AP1S3 & -4.50 & $3.00 \mathrm{E}-04$ \\
\hline 298 & AP2A2 & -2.27 & $3.56 \mathrm{E}-05$ \\
\hline 299 & AP2M1 & -4.97 & $1.87 \mathrm{E}-04$ \\
\hline 300 & AP3B1 & -3.35 & $3.81 \mathrm{E}-04$ \\
\hline 301 & AP3M1 & -6.07 & $8.22 \mathrm{E}-04$ \\
\hline 302 & AP3S1 & -4.66 & $5.66 \mathrm{E}-04$ \\
\hline 303 & AP3S2 & -3.83 & $2.39 \mathrm{E}-03$ \\
\hline 304 & AP4B1 & -2.54 & $3.79 \mathrm{E}-03$ \\
\hline 305 & AP4E1 & -1.08 & $1.11 \mathrm{E}-02$ \\
\hline 306 & AP4S1 & -3.15 & $3.95 \mathrm{E}-04$ \\
\hline 307 & APBA1 & -1.39 & $1.74 \mathrm{E}-02$ \\
\hline 308 & APEH & -3.83 & $2.33 \mathrm{E}-03$ \\
\hline 309 & API5 & -2.18 & $5.09 \mathrm{E}-05$ \\
\hline 310 & APITD1 & -7.47 & $4.26 \mathrm{E}-05$ \\
\hline 311 & APLF & -1.76 & $1.03 \mathrm{E}-02$ \\
\hline 312 & APLP2 & -4.29 & $4.04 \mathrm{E}-04$ \\
\hline 313 & APOA1 & -7.27 & $1.29 \mathrm{E}-04$ \\
\hline 314 & $\mathrm{APOH}$ & -3.46 & $3.96 \mathrm{E}-03$ \\
\hline
\end{tabular}

\begin{tabular}{|c|c|c|c|}
\hline 315 & APOO & -6.92 & $2.70 \mathrm{E}-03$ \\
\hline 316 & APOOL & -1.44 & $4.94 \mathrm{E}-02$ \\
\hline 317 & APOPT1 & -4.09 & $1.99 \mathrm{E}-03$ \\
\hline 318 & APP & -1.71 & $1.27 \mathrm{E}-02$ \\
\hline 319 & APTX & -7.94 & $1.25 \mathrm{E}-04$ \\
\hline 320 & AQP7 & -6.18 & $1.13 \mathrm{E}-03$ \\
\hline 321 & $A Q R$ & -2.82 & 7.47E-05 \\
\hline 322 & ARBP & -2.92 & 4.87E-03 \\
\hline 323 & ARCN1 & -4.74 & 1.40E-04 \\
\hline 324 & ARF1 & -6.69 & $2.34 \mathrm{E}-05$ \\
\hline 325 & ARF4 & -6.74 & $6.97 \mathrm{E}-05$ \\
\hline 326 & ARF6 & -1.73 & $8.10 \mathrm{E}-02$ \\
\hline 327 & ARFGAP1 & -2.59 & $5.33 \mathrm{E}-03$ \\
\hline 328 & ARFGAP2 & -2.76 & $9.35 \mathrm{E}-03$ \\
\hline 329 & ARFGAP3 & -5.21 & $1.06 \mathrm{E}-03$ \\
\hline 330 & ARFGEF1 & -1.66 & $1.36 \mathrm{E}-04$ \\
\hline 331 & ARFGEF2 & -1.87 & $6.55 \mathrm{E}-03$ \\
\hline 332 & ARFIP1 & -1.80 & $1.06 \mathrm{E}-02$ \\
\hline 333 & ARFRP1 & -4.68 & 2.62E-04 \\
\hline 334 & ARG2 & -4.78 & $1.06 \mathrm{E}-03$ \\
\hline 335 & ARGLU1 & -6.62 & $3.39 \mathrm{E}-04$ \\
\hline 336 & ARHGAP10 & -2.10 & 2.66E-03 \\
\hline 337 & ARHGAP11A & -4.08 & $2.01 \mathrm{E}-03$ \\
\hline 338 & ARHGAP12 & -3.54 & 2.33E-03 \\
\hline 339 & ARHGAP17 & -1.86 & $2.31 \mathrm{E}-03$ \\
\hline 340 & ARHGAP19 & -3.67 & $8.40 \mathrm{E}-05$ \\
\hline 341 & ARHGAP21 & -3.22 & $8.13 \mathrm{E}-05$ \\
\hline 342 & ARHGAP29 & -2.54 & $6.69 \mathrm{E}-03$ \\
\hline 343 & ARHGAP35 & -1.95 & $1.84 \mathrm{E}-02$ \\
\hline 344 & ARHGAP5 & -2.82 & $1.25 \mathrm{E}-03$ \\
\hline 345 & ARHGDIA & -1.73 & $4.01 \mathrm{E}-02$ \\
\hline 346 & ARHGEF12 & -1.57 & 4.33E-04 \\
\hline 347 & ARHGEF16 & -1.17 & $2.80 \mathrm{E}-03$ \\
\hline 348 & ARHGEF18 & -1.38 & 5.57E-03 \\
\hline 349 & ARHGEF19 & -1.53 & $1.19 \mathrm{E}-02$ \\
\hline 350 & ARHGEF26 & -2.17 & $3.24 \mathrm{E}-03$ \\
\hline 351 & ARHGEF9 & -5.29 & 7.67E-04 \\
\hline 352 & ARID2 & -4.26 & $3.31 \mathrm{E}-03$ \\
\hline 353 & ARID3B & -5.66 & $3.62 \mathrm{E}-05$ \\
\hline 354 & ARID4A & -3.13 & 4.22E-04 \\
\hline 355 & ARID5B & -1.29 & $2.68 \mathrm{E}-02$ \\
\hline 356 & ARIH1 & -6.13 & $1.74 \mathrm{E}-05$ \\
\hline 357 & ARIH2 & -3.27 & $1.08 \mathrm{E}-03$ \\
\hline 358 & ARL1 & -4.13 & $1.32 \mathrm{E}-03$ \\
\hline 359 & ARL13B & -6.60 & 4.89E-05 \\
\hline 360 & ARL14EP & -5.68 & 2.17E-05 \\
\hline 361 & ARL2BP & -1.33 & $1.52 \mathrm{E}-02$ \\
\hline 362 & ARL3 & -5.78 & $4.89 \mathrm{E}-04$ \\
\hline 363 & ARL4A & -1.18 & $3.65 \mathrm{E}-03$ \\
\hline 364 & ARL5B & -2.58 & $6.27 \mathrm{E}-04$ \\
\hline 365 & ARL6 & -4.00 & $5.58 \mathrm{E}-04$ \\
\hline 366 & ARLGIP1 & -5.04 & $8.12 \mathrm{E}-04$ \\
\hline 367 & ARL6IP4 & -3.30 & $2.22 \mathrm{E}-03$ \\
\hline 368 & ARL6IP5 & -4.19 & 2.99E-04 \\
\hline 369 & ARL8B & -4.73 & $8.85 \mathrm{E}-04$ \\
\hline 370 & ARMC1 & -3.70 & $2.23 \mathrm{E}-03$ \\
\hline 371 & ARMC10 & -2.26 & $8.62 \mathrm{E}-03$ \\
\hline 372 & ARMC2 & -4.58 & $2.65 \mathrm{E}-03$ \\
\hline 373 & ARMC3 & -2.82 & $6.02 \mathrm{E}-04$ \\
\hline 374 & ARMC6 & -4.16 & $4.42 \mathrm{E}-03$ \\
\hline 375 & ARMC7 & -3.40 & $2.38 \mathrm{E}-06$ \\
\hline 376 & ARMC8 & -2.32 & $6.94 \mathrm{E}-02$ \\
\hline 377 & ARMC9 & -1.74 & $7.19 \mathrm{E}-03$ \\
\hline
\end{tabular}




\begin{tabular}{|c|c|c|c|}
\hline 378 & ARNT & -3.25 & $6.32 \mathrm{E}-04$ \\
\hline 379 & ARNTL & -1.27 & $1.54 \mathrm{E}-03$ \\
\hline 380 & ARPC1A & -4.43 & $6.02 \mathrm{E}-03$ \\
\hline 381 & ARPC2 & -5.17 & $1.17 \mathrm{E}-04$ \\
\hline 382 & ARPC3 & -3.78 & $1.37 \mathrm{E}-03$ \\
\hline 383 & ARPC4 & -4.33 & $1.08 \mathrm{E}-04$ \\
\hline 384 & ARPC5 & -3.06 & $2.03 \mathrm{E}-03$ \\
\hline 385 & ARPC5L & -3.76 & $2.91 \mathrm{E}-04$ \\
\hline 386 & ARPP19 & -4.44 & 1.17E-04 \\
\hline 387 & ARRDC1 & -3.47 & $1.12 \mathrm{E}-03$ \\
\hline 388 & ARRDC2 & -2.01 & $6.20 \mathrm{E}-03$ \\
\hline 389 & ARRDC3 & -1.79 & 4.05E-03 \\
\hline 390 & ARSG & -1.73 & $1.40 \mathrm{E}-03$ \\
\hline 391 & ARVCF & -1.19 & $3.26 \mathrm{E}-02$ \\
\hline 392 & ASAH1 & -3.68 & $3.23 \mathrm{E}-04$ \\
\hline 393 & ASAP1 & -4.52 & $3.14 \mathrm{E}-04$ \\
\hline 394 & ASAP2 & -1.98 & $2.76 \mathrm{E}-02$ \\
\hline 395 & ASB14 & -1.42 & $5.04 \mathrm{E}-03$ \\
\hline 396 & ASB15 & -1.50 & $3.21 \mathrm{E}-02$ \\
\hline 397 & ASB5 & -1.64 & 3.47E-02 \\
\hline 398 & ASB8 & -1.21 & $1.77 \mathrm{E}-02$ \\
\hline 399 & ASCC2 & -3.51 & $1.79 \mathrm{E}-04$ \\
\hline 400 & ASF1A & -6.31 & $1.90 \mathrm{E}-05$ \\
\hline 401 & ASH2L & -4.26 & $8.79 \mathrm{E}-04$ \\
\hline 402 & ASMTL & -3.51 & $5.10 \mathrm{E}-04$ \\
\hline 403 & ASNS & -1.42 & $7.65 \mathrm{E}-02$ \\
\hline 404 & ASNSD1 & -5.41 & $1.78 \mathrm{E}-03$ \\
\hline 405 & ASPH & -3.18 & $1.77 \mathrm{E}-03$ \\
\hline 406 & ASPM & -3.55 & $7.18 \mathrm{E}-04$ \\
\hline 407 & ASXL3 & -2.31 & $1.19 \mathrm{E}-02$ \\
\hline 408 & ASZ1 & -3.20 & $1.75 \mathrm{E}-04$ \\
\hline 409 & ATAD1 & -3.20 & $8.49 \mathrm{E}-03$ \\
\hline 410 & ATAD2B & -4.50 & $2.54 \mathrm{E}-04$ \\
\hline 411 & ATAD5 & -3.10 & $5.02 \mathrm{E}-04$ \\
\hline 412 & ATF4 & -6.21 & $2.12 \mathrm{E}-04$ \\
\hline 413 & ATF7IP & -3.73 & $6.92 \mathrm{E}-04$ \\
\hline 414 & ATG12 & -4.43 & $7.28 \mathrm{E}-05$ \\
\hline 415 & ATG16L1 & -4.60 & $1.95 \mathrm{E}-05$ \\
\hline 416 & ATG3 & -4.02 & $4.58 \mathrm{E}-03$ \\
\hline 417 & ATG4B & -2.62 & $3.62 \mathrm{E}-03$ \\
\hline 418 & ATG5 & -2.13 & $6.05 \mathrm{E}-03$ \\
\hline 419 & ATG7 & -1.72 & $1.00 \mathrm{E}-02$ \\
\hline 420 & ATG9A & -1.95 & $2.32 \mathrm{E}-03$ \\
\hline 421 & ATIC & -5.18 & 4.77E-04 \\
\hline 422 & ATL1 & -2.83 & 4.10E-04 \\
\hline 423 & ATM & -2.12 & $1.28 \mathrm{E}-03$ \\
\hline 424 & ATOX1 & -5.21 & $1.73 \mathrm{E}-03$ \\
\hline 425 & ATP10D & -2.92 & $6.37 \mathrm{E}-03$ \\
\hline 426 & ATP11A & -2.37 & $3.24 \mathrm{E}-04$ \\
\hline 427 & ATP11B & -3.00 & $2.38 \mathrm{E}-03$ \\
\hline 428 & ATP12A & -1.56 & $1.25 \mathrm{E}-02$ \\
\hline 429 & ATP13A1 & -1.59 & 4.84E-04 \\
\hline 430 & ATP13A4 & -2.40 & $8.43 \mathrm{E}-03$ \\
\hline 431 & ATP1A1 & -4.55 & $7.60 \mathrm{E}-04$ \\
\hline 432 & ATP1B1 & -3.89 & $5.47 \mathrm{E}-03$ \\
\hline 433 & ATP1B3 & -2.84 & $1.40 \mathrm{E}-02$ \\
\hline 434 & ATP2A2 & -1.85 & $2.80 \mathrm{E}-02$ \\
\hline 435 & ATP2C1 & -3.08 & $5.45 \mathrm{E}-04$ \\
\hline 436 & ATP5A1 & -5.54 & $6.35 \mathrm{E}-04$ \\
\hline 437 & ATP5A1W & -4.21 & $8.25 \mathrm{E}-04$ \\
\hline 438 & ATP5B & -4.58 & $1.10 \mathrm{E}-03$ \\
\hline 439 & ATP5C1 & -8.41 & $5.02 \mathrm{E}-04$ \\
\hline 440 & ATP5E & -7.21 & $1.24 \mathrm{E}-04$ \\
\hline
\end{tabular}

\begin{tabular}{|c|c|c|c|}
\hline 441 & ATP5F1 & -6.49 & $6.61 \mathrm{E}-04$ \\
\hline 442 & ATP5G1 & -3.80 & $2.27 \mathrm{E}-03$ \\
\hline 443 & ATP5G3 & -7.46 & $8.11 \mathrm{E}-05$ \\
\hline 444 & ATP5H & -8.09 & $1.02 \mathrm{E}-04$ \\
\hline 445 & ATP5I & -8.29 & $1.26 \mathrm{E}-04$ \\
\hline 446 & ATP5J & -5.71 & $4.26 \mathrm{E}-03$ \\
\hline 447 & ATP5J2 & -5.88 & $3.21 \mathrm{E}-03$ \\
\hline 448 & ATP50 & -8.29 & $3.08 \mathrm{E}-04$ \\
\hline 449 & ATP5S & -4.11 & $2.13 \mathrm{E}-03$ \\
\hline 450 & ATP6 & -8.27 & $5.15 \mathrm{E}-03$ \\
\hline 451 & ATP6AP1 & -3.54 & $3.47 \mathrm{E}-03$ \\
\hline 452 & ATP6AP1L & -2.40 & $2.04 \mathrm{E}-03$ \\
\hline 453 & ATP6AP2 & -4.72 & $6.35 \mathrm{E}-04$ \\
\hline 454 & ATP6V0A1 & -1.54 & $1.51 \mathrm{E}-04$ \\
\hline 455 & ATP6VOA2 & -3.95 & $3.44 \mathrm{E}-03$ \\
\hline 456 & ATP6V0D1 & -1.36 & $4.73 \mathrm{E}-03$ \\
\hline 457 & ATP6V0E1 & -6.56 & $1.64 \mathrm{E}-04$ \\
\hline 458 & ATP6VOE2 & -2.63 & $6.66 \mathrm{E}-03$ \\
\hline 459 & ATP6V1A & -3.26 & $3.81 \mathrm{E}-04$ \\
\hline 460 & ATP6V1B2 & -5.63 & $3.48 \mathrm{E}-05$ \\
\hline 461 & ATP6V1D & -8.59 & $3.81 E-05$ \\
\hline 462 & ATP6V1E1 & -5.78 & $1.01 \mathrm{E}-04$ \\
\hline 463 & ATP6V1G1 & -8.58 & $1.21 \mathrm{E}-05$ \\
\hline 464 & ATP6V1H & -1.49 & $2.32 \mathrm{E}-03$ \\
\hline 465 & ATP7B & -1.06 & $3.64 \mathrm{E}-04$ \\
\hline 466 & ATP8 & -4.58 & $1.98 \mathrm{E}-02$ \\
\hline 467 & ATP8A2 & -2.14 & 7.67E-03 \\
\hline 468 & ATPAF1 & -3.14 & $6.60 \mathrm{E}-03$ \\
\hline 469 & ATPAF2 & -3.00 & $7.52 E-04$ \\
\hline 470 & ATRX & -5.20 & $2.29 \mathrm{E}-03$ \\
\hline 471 & ATXN1 & -1.99 & $1.45 \mathrm{E}-03$ \\
\hline 472 & ATXN10 & -4.05 & $1.55 \mathrm{E}-03$ \\
\hline 473 & ATXN2 & -1.32 & 5.07E-02 \\
\hline 474 & ATXN3 & -4.19 & $1.12 \mathrm{E}-03$ \\
\hline 475 & ATXN7 & -4.73 & $1.13 \mathrm{E}-04$ \\
\hline 476 & ATXN7L1 & -1.26 & $9.06 \mathrm{E}-04$ \\
\hline 477 & AURKA & -5.43 & 7.36E-04 \\
\hline 478 & AURKAIP1 & -6.38 & $4.50 \mathrm{E}-04$ \\
\hline 479 & AVEN & -8.10 & $7.55 \mathrm{E}-05$ \\
\hline 480 & AVL9 & -1.48 & $4.55 \mathrm{E}-03$ \\
\hline 481 & AXIN1 & -2.67 & $1.01 \mathrm{E}-03$ \\
\hline 482 & AZI1 & -3.33 & $1.15 \mathrm{E}-03$ \\
\hline 483 & AZIN1 & -3.76 & $1.65 \mathrm{E}-03$ \\
\hline 484 & $B 2 M$ & -5.45 & $1.67 \mathrm{E}-05$ \\
\hline 485 & B3GAT2 & -2.43 & $1.15 \mathrm{E}-02$ \\
\hline 486 & B3GAT3 & -2.50 & $4.83 \mathrm{E}-03$ \\
\hline 487 & B3GNT2 & -3.96 & $9.41 \mathrm{E}-04$ \\
\hline 488 & B3GNT5 & -6.23 & $2.27 \mathrm{E}-04$ \\
\hline 489 & B4GALT1 & -2.14 & $1.35 \mathrm{E}-03$ \\
\hline 490 & B4GALT2 & -4.55 & $1.15 \mathrm{E}-03$ \\
\hline 491 & B4GALT3 & -5.28 & $2.59 \mathrm{E}-04$ \\
\hline 492 & B4GALT4 & -3.01 & $2.90 \mathrm{E}-03$ \\
\hline 493 & B4GALT6 & -2.82 & $1.14 \mathrm{E}-03$ \\
\hline 494 & B4GALT7 & -4.11 & $2.82 \mathrm{E}-03$ \\
\hline 495 & B9D1 & -3.86 & $2.10 \mathrm{E}-05$ \\
\hline 496 & BACE2 & -1.94 & $5.57 \mathrm{E}-04$ \\
\hline 497 & $\mathrm{BACH} 1$ & -1.96 & $2.89 \mathrm{E}-02$ \\
\hline 498 & BAG1 & -3.52 & $2.72 \mathrm{E}-04$ \\
\hline 499 & BAG2 & -1.50 & $8.37 \mathrm{E}-02$ \\
\hline 500 & BAG3 & -2.38 & $3.20 \mathrm{E}-02$ \\
\hline 501 & BAG4 & -2.85 & $9.85 \mathrm{E}-04$ \\
\hline 502 & BAHD1 & -5.12 & $1.62 \mathrm{E}-05$ \\
\hline 503 & BAMBI & -3.11 & $1.60 \mathrm{E}-03$ \\
\hline
\end{tabular}

\begin{tabular}{|c|c|c|c|}
\hline 504 & BAP1 & -2.13 & $1.07 \mathrm{E}-02$ \\
\hline 505 & BARD1 & -2.02 & $1.88 \mathrm{E}-04$ \\
\hline 506 & BASP1 & -3.66 & $1.22 \mathrm{E}-02$ \\
\hline 507 & BAZ1A & -5.34 & $3.46 \mathrm{E}-05$ \\
\hline 508 & BAZ1B & -1.11 & $3.59 \mathrm{E}-04$ \\
\hline 509 & BAZ2B & -3.97 & 4.47E-03 \\
\hline 510 & BBS10 & -3.52 & $1.36 \mathrm{E}-04$ \\
\hline 511 & BBS12 & -3.05 & $1.89 \mathrm{E}-03$ \\
\hline 512 & BBS2 & -4.18 & 4.47E-04 \\
\hline 513 & BBS4 & -4.67 & $6.21 \mathrm{E}-04$ \\
\hline 514 & BBS5 & -2.91 & 4.05E-04 \\
\hline 515 & BBS7 & -1.39 & $1.03 \mathrm{E}-02$ \\
\hline 516 & BBS9 & -2.10 & $2.43 \mathrm{E}-03$ \\
\hline 517 & BBX & -3.78 & $1.39 \mathrm{E}-04$ \\
\hline 518 & BCAP29 & -7.77 & $8.64 \mathrm{E}-04$ \\
\hline 519 & BCAR1 & -4.94 & $9.26 \mathrm{E}-05$ \\
\hline 520 & BCAR3 & -6.08 & 2.03E-04 \\
\hline 521 & BCAS2 & -5.79 & $1.47 \mathrm{E}-04$ \\
\hline 522 & BCAS3 & -1.49 & $1.08 \mathrm{E}-02$ \\
\hline 523 & $\mathrm{BCHE}$ & -1.85 & $2.34 \mathrm{E}-03$ \\
\hline 524 & BCKDHA & -2.34 & $1.35 \mathrm{E}-03$ \\
\hline 525 & BCKDHB & -2.91 & $8.11 \mathrm{E}-04$ \\
\hline 526 & BCL10 & -1.68 & $5.41 \mathrm{E}-03$ \\
\hline 527 & BCL2L1 & -1.25 & $1.11 \mathrm{E}-03$ \\
\hline 528 & BCL6 & -1.18 & $7.12 \mathrm{E}-03$ \\
\hline 529 & BCL7A & -1.17 & $2.16 \mathrm{E}-02$ \\
\hline 530 & BCLAF1 & -1.27 & $4.73 \mathrm{E}-03$ \\
\hline 531 & $\mathrm{BCO} 2$ & -1.61 & $5.42 \mathrm{E}-03$ \\
\hline 532 & $\mathrm{BCR}$ & -2.99 & $1.56 \mathrm{E}-03$ \\
\hline 533 & BCS1L & -2.86 & $1.28 \mathrm{E}-03$ \\
\hline 534 & BD173581 & -3.72 & $9.59 \mathrm{E}-03$ \\
\hline 535 & BDH1 & -5.87 & $1.43 \mathrm{E}-04$ \\
\hline 536 & BDH2 & -2.92 & 2.97E-04 \\
\hline 537 & BEND3 & -1.04 & $8.71 \mathrm{E}-04$ \\
\hline 538 & BEND5 & -2.37 & $1.33 \mathrm{E}-02$ \\
\hline 539 & BEND7 & -6.19 & 4.57E-04 \\
\hline 540 & BET1L & -4.14 & 2.64E-04 \\
\hline 541 & BF845664 & -4.05 & $3.11 \mathrm{E}-02$ \\
\hline 542 & BFAR & -1.52 & 4.07E-03 \\
\hline 543 & BFSP1 & -1.18 & $2.58 \mathrm{E}-02$ \\
\hline 544 & BGLAP & -2.05 & $4.92 \mathrm{E}-02$ \\
\hline 545 & BICD1 & -1.98 & $1.14 \mathrm{E}-02$ \\
\hline 546 & BICD2 & -3.36 & $3.04 \mathrm{E}-03$ \\
\hline 547 & $\mathrm{BID}$ & -3.19 & 8.17E-04 \\
\hline 548 & BIN1 & -1.68 & $6.55 \mathrm{E}-02$ \\
\hline 549 & BIRC2 & -2.58 & 4.51E-04 \\
\hline 550 & BIRC5 & -7.33 & $6.57 \mathrm{E}-06$ \\
\hline 551 & BIRC6 & -2.18 & $6.62 \mathrm{E}-04$ \\
\hline 552 & BIVM & -2.72 & $6.31 \mathrm{E}-04$ \\
\hline 553 & BLB2 & -1.67 & $1.38 \mathrm{E}-02$ \\
\hline 554 & BLM & -3.12 & $8.16 \mathrm{E}-05$ \\
\hline 555 & BLMH & -3.20 & 4.60E-04 \\
\hline 556 & BLOC1S2 & -5.02 & $1.56 \mathrm{E}-04$ \\
\hline 557 & BLZF1 & -3.97 & $3.90 \mathrm{E}-04$ \\
\hline 558 & BM489687 & -3.00 & $5.08 \mathrm{E}-04$ \\
\hline 559 & BMP2K & -2.70 & $3.02 \mathrm{E}-04$ \\
\hline 560 & BMPR2 & -1.67 & $1.02 \mathrm{E}-03$ \\
\hline 561 & BMS1 & -1.84 & $3.84 \mathrm{E}-03$ \\
\hline 562 & BNIP2 & -4.55 & $5.48 \mathrm{E}-04$ \\
\hline 563 & BNIP3 & -3.02 & $2.28 \mathrm{E}-03$ \\
\hline 564 & BNIP3L & -2.36 & 3.53E-04 \\
\hline 565 & BOC & -2.62 & 4.41E-04 \\
\hline 566 & BOD1 & -4.56 & $1.56 \mathrm{E}-04$ \\
\hline
\end{tabular}




\begin{tabular}{|c|c|c|c|}
\hline 567 & BOLL & -1.22 & $6.70 \mathrm{E}-03$ \\
\hline 568 & BORA & -5.05 & $5.36 \mathrm{E}-04$ \\
\hline 569 & BPGM & -3.86 & 2.60E-04 \\
\hline 570 & BPHL & -1.54 & $1.14 \mathrm{E}-02$ \\
\hline 571 & BPNT1 & -3.05 & $2.02 \mathrm{E}-03$ \\
\hline 572 & BRAT1 & -3.11 & $5.90 \mathrm{E}-04$ \\
\hline 573 & BRCA1 & -3.26 & $3.74 \mathrm{E}-04$ \\
\hline 574 & BRCA2 & -4.01 & 3.05E-04 \\
\hline 575 & BRCC3 & -3.01 & 4.67E-04 \\
\hline 576 & BRD1 & -2.00 & 4.94E-03 \\
\hline 577 & BRD2 & -1.98 & 5.77E-03 \\
\hline 578 & BRD3 & -4.29 & 4.77E-04 \\
\hline 579 & BRD4 & -2.77 & $6.23 \mathrm{E}-04$ \\
\hline 580 & BRD7 & -7.54 & $5.95 \mathrm{E}-04$ \\
\hline 581 & BRD8 & -1.10 & $3.06 \mathrm{E}-03$ \\
\hline 582 & BRE & -4.96 & 2.47E-04 \\
\hline 583 & BRF1 & -3.24 & 1.43E-04 \\
\hline 584 & BRIP1 & -3.83 & $4.53 \mathrm{E}-04$ \\
\hline 585 & BRIX1 & -6.02 & $1.46 \mathrm{E}-04$ \\
\hline 586 & BRMS1L & -3.18 & 3.03E-03 \\
\hline 587 & BROX & -3.16 & $7.62 \mathrm{E}-04$ \\
\hline 588 & BRP44 & -6.19 & $3.32 \mathrm{E}-04$ \\
\hline 589 & BRP44L & -2.85 & $8.34 \mathrm{E}-03$ \\
\hline 590 & BRSK2 & -4.25 & $4.30 \mathrm{E}-03$ \\
\hline 591 & BRUNOL6 & -2.16 & $2.86 \mathrm{E}-03$ \\
\hline 592 & BRWD1 & -3.41 & $1.22 \mathrm{E}-04$ \\
\hline 593 & BSDC1 & -5.74 & $1.90 \mathrm{E}-05$ \\
\hline 594 & BSG & -4.58 & $7.70 \mathrm{E}-04$ \\
\hline 595 & BST1 & -1.66 & $7.23 \mathrm{E}-03$ \\
\hline 596 & BTBD1 & -2.51 & $8.08 \mathrm{E}-03$ \\
\hline 597 & BTBD10 & -4.43 & $1.33 \mathrm{E}-03$ \\
\hline 598 & BTBD7 & -2.43 & $1.51 \mathrm{E}-02$ \\
\hline 599 & BTBD9 & -1.15 & $6.77 \mathrm{E}-03$ \\
\hline 600 & BTC & -1.80 & $3.21 \mathrm{E}-03$ \\
\hline 601 & BTD & -6.43 & 3.02E-04 \\
\hline 602 & BTF3 & -6.60 & $4.21 \mathrm{E}-04$ \\
\hline 603 & BTF3L4 & -4.46 & 7.03E-04 \\
\hline 604 & BTG1 & -4.90 & $2.65 \mathrm{E}-03$ \\
\hline 605 & BU104491 & -1.21 & $1.56 \mathrm{E}-02$ \\
\hline 606 & BU108528 & -2.99 & $5.25 \mathrm{E}-03$ \\
\hline 607 & BU109100 & -1.54 & 2.10E-02 \\
\hline 608 & BU109404 & -4.33 & $1.88 \mathrm{E}-03$ \\
\hline 609 & BU111680 & -2.81 & $3.88 \mathrm{E}-03$ \\
\hline 610 & BU113518 & -2.98 & $3.72 E-05$ \\
\hline 611 & BU113971 & -3.56 & $1.21 \mathrm{E}-03$ \\
\hline 612 & BU114246 & -1.27 & $5.27 \mathrm{E}-03$ \\
\hline 613 & BU114269 & -3.60 & $2.25 \mathrm{E}-05$ \\
\hline 614 & BU115588 & -2.12 & $9.91 \mathrm{E}-03$ \\
\hline 615 & BU116401 & -1.34 & $6.84 \mathrm{E}-03$ \\
\hline 616 & BU116406 & -1.95 & $1.04 \mathrm{E}-02$ \\
\hline 617 & BU116459 & -1.53 & $3.05 E-03$ \\
\hline 618 & BU117124 & -2.17 & 2.99E-04 \\
\hline 619 & BU118729 & -1.27 & $3.06 \mathrm{E}-02$ \\
\hline 620 & BU119676 & -1.87 & $8.49 \mathrm{E}-03$ \\
\hline 621 & BU121308 & -3.91 & $2.28 \mathrm{E}-03$ \\
\hline 622 & BU128167 & -3.25 & 3.59E-03 \\
\hline 623 & BU130275 & -4.29 & 4.73E- 04 \\
\hline 624 & BU130630 & -2.93 & $1.80 \mathrm{E}-03$ \\
\hline 625 & BU134586 & -2.28 & $9.39 \mathrm{E}-03$ \\
\hline 626 & BU136639 & -7.32 & $9.35 \mathrm{E}-04$ \\
\hline 627 & BU137418 & -6.14 & $1.39 \mathrm{E}-03$ \\
\hline 628 & BU137447 & -1.97 & $1.51 \mathrm{E}-02$ \\
\hline 629 & BU139275 & -1.62 & 4.66E-03 \\
\hline
\end{tabular}

\begin{tabular}{|c|c|c|c|}
\hline 630 & BU140555 & -1.81 & $3.27 \mathrm{E}-03$ \\
\hline 631 & BU142555 & -1.20 & $9.05 \mathrm{E}-03$ \\
\hline 632 & BU144368 & -4.13 & $5.16 \mathrm{E}-04$ \\
\hline 633 & BU202730 & -3.71 & $1.33 \mathrm{E}-04$ \\
\hline 634 & BU203419 & -6.01 & $6.56 \mathrm{E}-05$ \\
\hline 635 & BU204424 & -3.45 & $1.92 \mathrm{E}-03$ \\
\hline 636 & BU211126 & -5.36 & $3.28 \mathrm{E}-04$ \\
\hline 637 & BU211163 & -1.24 & $3.85 \mathrm{E}-03$ \\
\hline 638 & BU213140 & -2.12 & $2.76 \mathrm{E}-03$ \\
\hline 639 & BU214537 & -2.52 & $1.43 \mathrm{E}-02$ \\
\hline 640 & BU215540 & -2.95 & 3.64E-03 \\
\hline 641 & BU216024 & -2.61 & $1.91 \mathrm{E}-02$ \\
\hline 642 & BU217054 & -5.28 & $2.06 \mathrm{E}-03$ \\
\hline 643 & BU217171 & -6.15 & $6.12 \mathrm{E}-05$ \\
\hline 644 & BU217492 & -1.33 & $2.90 \mathrm{E}-03$ \\
\hline 645 & BU219775 & -2.11 & $2.90 \mathrm{E}-03$ \\
\hline 646 & BU220187 & -3.03 & 7.97E-03 \\
\hline 647 & BU220292 & -3.09 & $9.48 \mathrm{E}-03$ \\
\hline 648 & BU221066 & -1.33 & $7.00 \mathrm{E}-03$ \\
\hline 649 & BU221176 & -1.32 & $1.58 \mathrm{E}-03$ \\
\hline 650 & BU222144 & -4.25 & $8.53 \mathrm{E}-04$ \\
\hline 651 & BU222871 & -3.73 & $8.82 \mathrm{E}-04$ \\
\hline 652 & BU228041 & -5.95 & 8.47E-04 \\
\hline 653 & BU230533 & -1.70 & $1.43 \mathrm{E}-02$ \\
\hline 654 & BU232990 & -2.65 & $1.63 \mathrm{E}-03$ \\
\hline 655 & BU233039 & -4.01 & $1.02 \mathrm{E}-03$ \\
\hline 656 & BU234200 & -5.48 & $1.98 \mathrm{E}-04$ \\
\hline 657 & BU234845 & -1.75 & $3.60 \mathrm{E}-03$ \\
\hline 658 & BU236609 & -3.20 & $9.73 \mathrm{E}-03$ \\
\hline 659 & BU237399 & -2.34 & $4.31 \mathrm{E}-03$ \\
\hline 660 & BU239725 & -1.85 & $6.06 \mathrm{E}-03$ \\
\hline 661 & BU240502 & -5.70 & 3.37E-05 \\
\hline 662 & BU242143 & -1.88 & $1.78 \mathrm{E}-02$ \\
\hline 663 & BU242606 & -1.65 & $1.03 \mathrm{E}-02$ \\
\hline 664 & BU242613 & -5.65 & $1.74 \mathrm{E}-05$ \\
\hline 665 & BU242939 & -3.31 & $1.18 \mathrm{E}-02$ \\
\hline 666 & BU245643 & -2.30 & $1.03 \mathrm{E}-03$ \\
\hline 667 & BU250162 & -3.46 & $7.82 \mathrm{E}-03$ \\
\hline 668 & BU254568 & -1.43 & $1.01 \mathrm{E}-01$ \\
\hline 669 & BU257618 & -3.88 & 7.43E-03 \\
\hline 670 & BU259793 & -3.44 & $1.01 \mathrm{E}-05$ \\
\hline 671 & BU262292 & -2.64 & $2.20 \mathrm{E}-03$ \\
\hline 672 & BU264475 & -2.16 & $1.57 \mathrm{E}-02$ \\
\hline 673 & BU269286 & -1.37 & $2.50 \mathrm{E}-03$ \\
\hline 674 & BU273126 & -1.74 & $3.89 \mathrm{E}-03$ \\
\hline 675 & BU273691 & -6.12 & $1.54 \mathrm{E}-04$ \\
\hline 676 & BU275217 & -3.26 & $8.60 \mathrm{E}-04$ \\
\hline 677 & BU275369 & -2.07 & $3.15 \mathrm{E}-03$ \\
\hline 678 & BU277859 & -4.76 & $2.40 \mathrm{E}-03$ \\
\hline 679 & BU278479 & -1.77 & $1.98 \mathrm{E}-02$ \\
\hline 680 & BU278743 & -1.92 & $1.98 \mathrm{E}-03$ \\
\hline 681 & BU279507 & -1.41 & 2.87E-02 \\
\hline 682 & BU280333 & -1.56 & $1.27 \mathrm{E}-02$ \\
\hline 683 & BU282626 & -1.51 & $2.33 \mathrm{E}-02$ \\
\hline 684 & BU288461 & -3.05 & $3.88 \mathrm{E}-03$ \\
\hline 685 & BU292776 & -2.45 & $8.65 \mathrm{E}-03$ \\
\hline 686 & BU295319 & -1.28 & $2.34 \mathrm{E}-02$ \\
\hline 687 & BU295520 & -1.95 & $2.49 \mathrm{E}-03$ \\
\hline 688 & BU299492 & -2.60 & $4.19 \mathrm{E}-03$ \\
\hline 689 & BU300843 & -2.01 & $8.26 \mathrm{E}-03$ \\
\hline 690 & BU303451 & -2.31 & $4.02 \mathrm{E}-03$ \\
\hline 691 & BU304798 & -1.73 & $6.21 \mathrm{E}-04$ \\
\hline 692 & BU306831 & -3.87 & $3.89 \mathrm{E}-03$ \\
\hline
\end{tabular}

\begin{tabular}{|c|c|c|c|}
\hline 693 & BU308973 & -2.31 & $3.46 \mathrm{E}-03$ \\
\hline 694 & BU309453 & -4.65 & $6.19 \mathrm{E}-05$ \\
\hline 695 & BU311548 & -4.66 & $7.51 \mathrm{E}-03$ \\
\hline 696 & BU316125 & -1.77 & 2.07E-04 \\
\hline 697 & BU319139 & -1.15 & $1.75 \mathrm{E}-02$ \\
\hline 698 & BU319249 & -2.27 & 7.46E-04 \\
\hline 699 & BU322109 & -1.69 & $6.28 \mathrm{E}-02$ \\
\hline 700 & BU322203 & -3.30 & $4.74 \mathrm{E}-03$ \\
\hline 701 & BU322364 & -3.98 & 1.43E-04 \\
\hline 702 & BU323306 & -2.13 & 4.07E-04 \\
\hline 703 & BU324289 & -5.20 & $1.35 \mathrm{E}-03$ \\
\hline 704 & BU327019 & -1.17 & 1.66E-02 \\
\hline 705 & BU330375 & -5.38 & $1.15 \mathrm{E}-03$ \\
\hline 706 & BU332430 & -3.81 & $3.18 \mathrm{E}-05$ \\
\hline 707 & BU334825 & -2.39 & $1.01 \mathrm{E}-02$ \\
\hline 708 & BU337284 & -3.72 & $1.19 \mathrm{E}-04$ \\
\hline 709 & BU338027 & -4.32 & $8.24 \mathrm{E}-04$ \\
\hline 710 & BU339296 & -4.61 & 4.46E-03 \\
\hline 711 & BU340250 & -1.67 & $7.96 \mathrm{E}-03$ \\
\hline 712 & BU343566 & -3.55 & $5.19 \mathrm{E}-03$ \\
\hline 713 & BU344120 & -4.80 & 3.29E-04 \\
\hline 714 & BU347374 & -6.15 & $6.26 \mathrm{E}-04$ \\
\hline 715 & BU349378 & -3.99 & 7.73E-04 \\
\hline 716 & BU350246 & -2.77 & 7.99E-03 \\
\hline 717 & BU350976 & -3.36 & 4.42E-04 \\
\hline 718 & BU351391 & -1.75 & $5.78 \mathrm{E}-02$ \\
\hline 719 & BU352584 & -2.27 & $5.20 \mathrm{E}-03$ \\
\hline 720 & BU354789 & -7.54 & $1.54 \mathrm{E}-04$ \\
\hline 721 & BU355364 & -2.73 & $8.48 \mathrm{E}-04$ \\
\hline 722 & BU368370 & -2.65 & 2.10E-03 \\
\hline 723 & BU370425 & -3.90 & $2.54 \mathrm{E}-03$ \\
\hline 724 & BU370618 & -1.11 & 3.95E-02 \\
\hline 725 & BU371327 & -3.28 & $1.11 \mathrm{E}-02$ \\
\hline 726 & BU371925 & -3.01 & $1.53 \mathrm{E}-02$ \\
\hline 727 & BU373399 & -2.08 & $2.86 \mathrm{E}-02$ \\
\hline 728 & BU375502 & -2.88 & 4.90E-05 \\
\hline 729 & BU377969 & -3.75 & $1.31 \mathrm{E}-03$ \\
\hline 730 & BU378738 & -4.33 & $2.41 \mathrm{E}-04$ \\
\hline 731 & BU382899 & -2.73 & $6.19 \mathrm{E}-03$ \\
\hline 732 & BU383595 & -2.05 & 4.57E-03 \\
\hline 733 & BU385229 & -2.49 & $2.48 \mathrm{E}-03$ \\
\hline 734 & BU385935 & -3.63 & $2.61 \mathrm{E}-02$ \\
\hline 735 & BU386892 & -3.08 & $6.96 \mathrm{E}-03$ \\
\hline 736 & BU388045 & -2.31 & $1.16 \mathrm{E}-02$ \\
\hline 737 & BU389255 & -3.39 & $9.29 \mathrm{E}-04$ \\
\hline 738 & BU389520 & -1.39 & $9.24 \mathrm{E}-03$ \\
\hline 739 & BU393834 & -1.25 & $3.72 \mathrm{E}-03$ \\
\hline 740 & BU399392 & -2.73 & $1.90 \mathrm{E}-02$ \\
\hline 741 & BU399899 & -1.78 & 7.00E-03 \\
\hline 742 & BU401633 & -3.60 & $1.14 \mathrm{E}-04$ \\
\hline 743 & BU406711 & -1.95 & $3.45 \mathrm{E}-03$ \\
\hline 744 & BU410862 & -3.45 & $5.30 \mathrm{E}-05$ \\
\hline 745 & BU412051 & -4.86 & $1.41 \mathrm{E}-04$ \\
\hline 746 & BU412468 & -1.17 & $3.75 \mathrm{E}-03$ \\
\hline 747 & BU413222 & -4.08 & $1.59 \mathrm{E}-03$ \\
\hline 748 & BU414688 & -3.63 & $1.46 \mathrm{E}-03$ \\
\hline 749 & BU416228 & -1.99 & $7.88 \mathrm{E}-03$ \\
\hline 750 & BU418491 & -2.77 & $6.24 \mathrm{E}-04$ \\
\hline 751 & BU419298 & -1.39 & $1.65 \mathrm{E}-02$ \\
\hline 752 & BU420213 & -2.40 & 4.99E-03 \\
\hline 753 & BU422033 & -1.31 & $5.21 \mathrm{E}-03$ \\
\hline 754 & BU422489 & -2.07 & $9.41 \mathrm{E}-04$ \\
\hline 755 & BU423835 & -3.00 & $1.91 \mathrm{E}-03$ \\
\hline
\end{tabular}




\begin{tabular}{|c|c|c|c|}
\hline 756 & BU424467 & -2.17 & $4.49 \mathrm{E}-03$ \\
\hline 757 & BU426033 & -4.16 & $6.18 \mathrm{E}-04$ \\
\hline 758 & BU427251 & -3.38 & $3.75 \mathrm{E}-03$ \\
\hline 759 & BU427545 & -3.06 & $2.99 \mathrm{E}-03$ \\
\hline 760 & BU432081 & -5.43 & $2.53 \mathrm{E}-04$ \\
\hline 761 & BU432383 & -1.97 & $1.84 \mathrm{E}-04$ \\
\hline 762 & BU434282 & -2.17 & $1.60 \mathrm{E}-03$ \\
\hline 763 & BU435083 & -5.49 & $9.44 \mathrm{E}-05$ \\
\hline 764 & BU435210 & -3.76 & $2.05 E-03$ \\
\hline 765 & BU435717 & -3.41 & $2.54 \mathrm{E}-04$ \\
\hline 766 & BU437151 & -2.38 & $7.34 \mathrm{E}-04$ \\
\hline 767 & BU438351 & $\begin{array}{l}-1.40 \\
\end{array}$ & $1.33 \mathrm{E}-03$ \\
\hline 768 & BU439396 & -3.70 & $1.02 \mathrm{E}-04$ \\
\hline 769 & BU442664 & -4.83 & $2.51 \mathrm{E}-05$ \\
\hline 770 & BU445593 & -5.08 & $5.57 \mathrm{E}-03$ \\
\hline 771 & BU445963 & -2.50 & $1.00 \mathrm{E}-02$ \\
\hline 772 & BU446528 & -4.84 & $4.05 E-04$ \\
\hline 773 & BU447721 & -6.30 & $1.33 \mathrm{E}-05$ \\
\hline 774 & BU447799 & -2.02 & $1.92 \mathrm{E}-03$ \\
\hline 775 & BU448880 & -2.69 & $1.35 \mathrm{E}-03$ \\
\hline 776 & BU457104 & -8.17 & $1.49 \mathrm{E}-04$ \\
\hline 777 & BU459548 & $\begin{array}{l}-7.13 \\
\end{array}$ & $2.30 \mathrm{E}-05$ \\
\hline 778 & BU461178 & $\begin{array}{l}-2.31 \\
\end{array}$ & $3.60 \mathrm{E}-02$ \\
\hline 779 & BU461476 & -5.58 & $7.08 \mathrm{E}-04$ \\
\hline 780 & BU462304 & -1.37 & $2.49 \mathrm{E}-04$ \\
\hline 781 & BU462330 & -2.42 & $2.76 E-02$ \\
\hline 782 & BU464906 & -2.32 & $1.09 \mathrm{E}-02$ \\
\hline 783 & BU465760 & -3.84 & $1.18 \mathrm{E}-03$ \\
\hline 784 & BU467036 & $\begin{array}{l}-3.19 \\
\end{array}$ & $2.37 \mathrm{E}-03$ \\
\hline 785 & BU483329 & -2.86 & $2.06 \mathrm{E}-04$ \\
\hline 786 & BUB1 & -5.60 & $1.01 E-03$ \\
\hline 787 & BUB1B & -4.53 & $2.56 \mathrm{E}-04$ \\
\hline 788 & BUB3 & $\begin{array}{l}-6.63 \\
\end{array}$ & $6.43 \mathrm{E}-05$ \\
\hline 789 & BUD13 & -3.93 & $6.77 \mathrm{E}-03$ \\
\hline 790 & BUD31 & -7.27 & $5.65 \mathrm{E}-05$ \\
\hline 791 & BVES & -4.56 & $1.08 \mathrm{E}-05$ \\
\hline 792 & BX256978 & -5.55 & $1.99 \mathrm{E}-03$ \\
\hline 793 & BX258241 & -2.65 & $7.64 \mathrm{E}-03$ \\
\hline 794 & BX258353 & -3.72 & $3.07 \mathrm{E}-03$ \\
\hline 795 & BX259218 & -5.06 & $7.94 \mathrm{E}-05$ \\
\hline 796 & BX259370 & -4.79 & $5.53 \mathrm{E}-05$ \\
\hline 797 & BX259812 & -5.92 & $1.23 \mathrm{E}-05$ \\
\hline 798 & BX259979 & -2.14 & $1.09 \mathrm{E}-02$ \\
\hline 799 & BX261305 & $\begin{array}{l}-3.87 \\
\end{array}$ & $2.45 \mathrm{E}-03$ \\
\hline 800 & BX262960 & -2.68 & $9.70 \mathrm{E}-03$ \\
\hline 801 & BX263196 & -2.04 & $9.65 \mathrm{E}-04$ \\
\hline 802 & BX266154 & -4.38 & $3.73 \mathrm{E}-03$ \\
\hline 803 & BX266529 & -3.91 & $3.62 \mathrm{E}-03$ \\
\hline 804 & BX266754 & $\begin{array}{l}-4.61 \\
\end{array}$ & $1.77 \mathrm{E}-04$ \\
\hline 805 & BX267869 & -2.14 & $3.70 \mathrm{E}-02$ \\
\hline 806 & BX267901 & -2.68 & $9.37 \mathrm{E}-04$ \\
\hline 807 & BX267911 & -7.01 & $5.86 \mathrm{E}-04$ \\
\hline 808 & BX270141 & $\begin{array}{l}-1.28 \\
\end{array}$ & $3.45 \mathrm{E}-02$ \\
\hline 809 & BX270748 & -1.88 & $1.13 \mathrm{E}-01$ \\
\hline 810 & BX271803 & -6.13 & $1.40 \mathrm{E}-03$ \\
\hline 811 & BX272269 & -4.00 & $2.39 \mathrm{E}-03$ \\
\hline 812 & BX273792 & -5.77 & $1.16 \mathrm{E}-04$ \\
\hline 813 & BX274617 & -3.07 & $1.51 \mathrm{E}-03$ \\
\hline 814 & BX275540 & -2.33 & $1.30 \mathrm{E}-02$ \\
\hline 815 & BX277450 & -2.37 & $2.66 \mathrm{E}-02$ \\
\hline 816 & BX277726 & -1.74 & $1.40 \mathrm{E}-02$ \\
\hline 817 & BX278173 & -5.49 & $1.93 \mathrm{E}-04$ \\
\hline 818 & BX929312 & -7.41 & $1.35 \mathrm{E}-03$ \\
\hline
\end{tabular}

\begin{tabular}{|c|c|c|c|}
\hline 819 & BX929336 & -1.50 & $3.39 E-03$ \\
\hline 820 & BX929356 & -4.53 & $2.43 E-03$ \\
\hline 821 & BX929359 & -7.64 & $2.56 \mathrm{E}-04$ \\
\hline 822 & BX929412 & \begin{tabular}{|l|}
-8.37 \\
\end{tabular} & $2.15 \mathrm{E}-03$ \\
\hline 823 & BX929445 & -6.55 & $1.88 \mathrm{E}-04$ \\
\hline 824 & BX929508 & \begin{tabular}{|l|}
-7.31 \\
\end{tabular} & $1.29 E-03$ \\
\hline 825 & BX929566 & -1.55 & $3.36 \mathrm{E}-03$ \\
\hline 826 & BX929600 & -2.94 & $3.43 E-03$ \\
\hline 827 & BX929608 & -3.42 & $9.81 E-04$ \\
\hline 828 & BX929625 & \begin{tabular}{|l|}
-2.89 \\
\end{tabular} & $4.90 \mathrm{E}-03$ \\
\hline 829 & BX929643 & -7.58 & $\begin{array}{r}4.73 E-04 \\
\end{array}$ \\
\hline 830 & BX929646 & -7.41 & $1.88 \mathrm{E}-03$ \\
\hline 831 & BX929653 & -3.02 & $3.46 \mathrm{E}-02$ \\
\hline 832 & BX929657 & \begin{tabular}{l|}
-2.91 \\
\end{tabular} & $8.39 \mathrm{E}-03$ \\
\hline 833 & BX929658 & -2.06 & $2.46 \mathrm{E}-02$ \\
\hline 834 & BX929659 & -1.68 & $1.63 \mathrm{E}-02$ \\
\hline 835 & BX929752 & -1.84 & $3.83 \mathrm{E}-02$ \\
\hline 836 & BX929780 & -2.75 & $4.06 \mathrm{E}-03$ \\
\hline 837 & BX929805 & -1.43 & $7.36 \mathrm{E}-03$ \\
\hline 838 & BX929816 & $\begin{array}{l}-7.58 \\
\end{array}$ & $2.27 E-05$ \\
\hline 839 & BX929823 & -3.64 & 6.67E-06 \\
\hline 840 & BX929830 & -1.95 & $9.74 E-03$ \\
\hline 841 & BX929854 & -2.45 & $1.12 \mathrm{E}-04$ \\
\hline 842 & BX929874 & -5.33 & $4.48 \mathrm{E}-03$ \\
\hline 843 & BX929896 & -4.90 & $7.68 \mathrm{E}-05$ \\
\hline 844 & BX929907 & $\begin{array}{l}-1.87 \\
\end{array}$ & $8.99 \mathrm{E}-03$ \\
\hline 845 & BX929911 & -4.55 & $1.46 \mathrm{E}-03$ \\
\hline 846 & BX929922 & -2.84 & $3.87 E-05$ \\
\hline 847 & BX929970 & -4.75 & $2.20 \mathrm{E}-03$ \\
\hline 848 & BX929986 & -1.38 & $2.02 E-02$ \\
\hline 849 & BX930001 & \begin{tabular}{|l|}
-1.44 \\
\end{tabular} & $7.42 E-03$ \\
\hline 850 & BX930013 & -3.65 & $3.62 E-03$ \\
\hline 851 & BX930022 & -2.97 & $4.80 \mathrm{E}-03$ \\
\hline 852 & BX930038 & -3.13 & $5.08 E-04$ \\
\hline 853 & BX930055 & -3.70 & $3.68 \mathrm{E}-03$ \\
\hline 854 & BX930110 & -2.71 & $2.26 \mathrm{E}-02$ \\
\hline 855 & BX930116 & -3.78 & $8.39 E-04$ \\
\hline 856 & BX930129 & -2.73 & $9.82 E-03$ \\
\hline 857 & BX930197 & -2.98 & $2.92 E-02$ \\
\hline 858 & BX930251 & $\begin{array}{l}-5.15 \\
\end{array}$ & $2.86 \mathrm{E}-04$ \\
\hline 859 & BX930253 & -3.35 & $1.09 E-03$ \\
\hline 860 & BX930276 & -4.34 & $5.75 E-04$ \\
\hline 861 & BX930288 & -2.20 & $1.69 E-03$ \\
\hline 862 & BX930337 & -5.68 & $3.25 \mathrm{E}-04$ \\
\hline 863 & BX930354 & -5.71 & $8.25 \mathrm{E}-04$ \\
\hline 864 & BX930362 & $\begin{array}{l}-2.53 \\
\end{array}$ & $9.31 E-04$ \\
\hline 865 & BX930423 & -1.23 & $5.83 E-03$ \\
\hline 866 & BX930460 & -2.38 & $1.47 \mathrm{E}-03$ \\
\hline 867 & BX930474 & -4.21 & $2.08 \mathrm{E}-02$ \\
\hline 868 & BX930483 & -8.27 & $6.80 \mathrm{E}-05$ \\
\hline 869 & BX930497 & -2.87 & $2.70 E-03$ \\
\hline 870 & BX930540 & -1.72 & $8.03 E-03$ \\
\hline 871 & BX930547 & -4.74 & $1.04 E-03$ \\
\hline 872 & BX930569 & -1.95 & $6.65 \mathrm{E}-03$ \\
\hline 873 & BX930638 & -6.99 & $7.02 E-04$ \\
\hline 874 & BX930641 & -4.31 & $6.17 \mathrm{E}-04$ \\
\hline 875 & BX930647 & -5.36 & $9.27 E-03$ \\
\hline 876 & BX930675 & -4.18 & $1.29 E-03$ \\
\hline 877 & BX930689 & -1.81 & $9.69 \mathrm{E}-02$ \\
\hline 878 & BX930718 & -3.62 & $1.88 \mathrm{E}-03$ \\
\hline 879 & BX930756 & -1.46 & $9.83 E-03$ \\
\hline 880 & BX930765 & -4.37 & $1.87 \mathrm{E}-04$ \\
\hline 881 & ВX930836 & -4.15 & $3.08 \mathrm{E}-03$ \\
\hline
\end{tabular}

\begin{tabular}{|c|c|c|c|}
\hline 882 & BX930863 & -3.01 & $4.78 \mathrm{E}-03$ \\
\hline 883 & BX930931 & -8.62 & $1.80 \mathrm{E}-03$ \\
\hline 884 & BX931089 & -6.53 & $4.35 \mathrm{E}-05$ \\
\hline 885 & BX931113 & -2.81 & $7.27 \mathrm{E}-03$ \\
\hline 886 & BX931120 & -1.91 & $7.66 \mathrm{E}-03$ \\
\hline 887 & BX931154 & -1.92 & $3.49 \mathrm{E}-03$ \\
\hline 888 & BX931202 & -1.24 & $8.34 \mathrm{E}-02$ \\
\hline 889 & BX931223 & -3.42 & $2.83 \mathrm{E}-03$ \\
\hline 890 & BX931282 & -7.45 & $5.39 \mathrm{E}-06$ \\
\hline 891 & BX931290 & -3.45 & $3.50 \mathrm{E}-04$ \\
\hline 892 & BX931315 & -3.33 & $5.18 \mathrm{E}-04$ \\
\hline 893 & BX931335 & -5.55 & 7.01E-04 \\
\hline 894 & BX931342 & -3.30 & $1.95 \mathrm{E}-02$ \\
\hline 895 & BX931362 & -7.21 & $3.56 \mathrm{E}-04$ \\
\hline 896 & BX931370 & -2.99 & $4.82 \mathrm{E}-04$ \\
\hline 897 & BX931384 & -2.40 & $2.06 \mathrm{E}-02$ \\
\hline 898 & BX931429 & -1.28 & $2.48 \mathrm{E}-05$ \\
\hline 899 & BX931431 & -5.30 & $1.23 \mathrm{E}-02$ \\
\hline 900 & BX931465 & -5.65 & $1.55 \mathrm{E}-03$ \\
\hline 901 & BX931488 & -3.12 & $1.45 \mathrm{E}-03$ \\
\hline 902 & BX931529 & -6.58 & $3.81 \mathrm{E}-04$ \\
\hline 903 & BX931573 & -2.51 & $4.43 \mathrm{E}-04$ \\
\hline 904 & BX931588 & -3.76 & $1.23 \mathrm{E}-05$ \\
\hline 905 & BX931611 & -5.87 & $1.95 \mathrm{E}-04$ \\
\hline 906 & BX931660 & -2.35 & $2.62 \mathrm{E}-03$ \\
\hline 907 & BX931678 & -5.10 & $1.58 \mathrm{E}-03$ \\
\hline 908 & BX931702 & -3.41 & $3.32 \mathrm{E}-03$ \\
\hline 909 & BX931721 & -6.13 & $6.34 \mathrm{E}-04$ \\
\hline 910 & BX931729 & -1.98 & $3.86 \mathrm{E}-03$ \\
\hline 911 & BX931754 & $\begin{array}{l}-1.41 \\
\end{array}$ & $8.83 \mathrm{E}-03$ \\
\hline 912 & BX931841 & -5.42 & $1.15 \mathrm{E}-03$ \\
\hline 913 & BX931846 & -4.31 & $6.74 \mathrm{E}-04$ \\
\hline 914 & BX931853 & -3.60 & $9.06 \mathrm{E}-03$ \\
\hline 915 & BX931896 & -3.30 & $1.59 \mathrm{E}-02$ \\
\hline 916 & BX931917 & -7.57 & $4.65 \mathrm{E}-03$ \\
\hline 917 & BX932001 & -8.31 & $\begin{array}{l}1.76 \mathrm{E}-04 \\
\end{array}$ \\
\hline 918 & BX932003 & -8.07 & $1.15 \mathrm{E}-04$ \\
\hline 919 & BX932036 & -5.88 & $1.08 \mathrm{E}-03$ \\
\hline 920 & BX932044 & -3.40 & $2.04 \mathrm{E}-03$ \\
\hline 921 & BX932052 & -5.73 & $1.51 \mathrm{E}-03$ \\
\hline 922 & BX932054 & -3.31 & $1.45 \mathrm{E}-03$ \\
\hline 923 & BX932063 & $\begin{array}{l}-7.23 \\
\end{array}$ & $7.12 \mathrm{E}-03$ \\
\hline 924 & BX932065 & -4.38 & 7.77E-04 \\
\hline 925 & BX932136 & -5.41 & $1.06 \mathrm{E}-03$ \\
\hline 926 & BX932151 & -6.93 & $2.63 \mathrm{E}-03$ \\
\hline 927 & BX932188 & -5.32 & $2.10 \mathrm{E}-04$ \\
\hline $\begin{array}{l}928 \\
\end{array}$ & BX932191 & -3.71 & $1.78 \mathrm{E}-03$ \\
\hline 929 & BX932205 & -5.49 & $2.67 \mathrm{E}-04$ \\
\hline 930 & BX932216 & -1.98 & $6.73 \mathrm{E}-03$ \\
\hline 931 & BX932246 & -1.77 & $1.36 \mathrm{E}-02$ \\
\hline 932 & BX932256 & -3.29 & $2.53 \mathrm{E}-04$ \\
\hline 933 & BX932291 & -2.94 & $5.86 \mathrm{E}-03$ \\
\hline 934 & BX932341 & -3.13 & $1.19 \mathrm{E}-03$ \\
\hline 935 & BX932364 & -5.94 & $8.56 \mathrm{E}-04$ \\
\hline 936 & BX932371 & -2.27 & $3.52 \mathrm{E}-02$ \\
\hline $\begin{array}{l}937 \\
\end{array}$ & BX932373 & -5.47 & $1.62 \mathrm{E}-04$ \\
\hline 938 & BX932413 & -3.66 & $4.98 \mathrm{E}-04$ \\
\hline 939 & BX932430 & -5.06 & $5.93 \mathrm{E}-04$ \\
\hline 940 & BX932451 & -1.58 & $9.12 \mathrm{E}-03$ \\
\hline 941 & BX932459 & -1.49 & $3.60 \mathrm{E}-03$ \\
\hline 942 & BX932460 & -1.97 & $8.78 \mathrm{E}-02$ \\
\hline 943 & BX932549 & -2.82 & $2.80 \mathrm{E}-04$ \\
\hline 944 & BX932555 & -2.18 & $5.43 \mathrm{E}-04$ \\
\hline
\end{tabular}




\begin{tabular}{|c|c|c|c|}
\hline 945 & BX932580 & -2.66 & $1.25 \mathrm{E}-03$ \\
\hline 946 & BX932594 & -5.04 & $9.28 \mathrm{E}-03$ \\
\hline 947 & BX932599 & -5.16 & $5.88 \mathrm{E}-04$ \\
\hline 948 & BX932600 & -8.57 & $1.82 \mathrm{E}-04$ \\
\hline 949 & BX932616 & -3.13 & $6.22 \mathrm{E}-03$ \\
\hline 950 & BX932629 & -8.35 & $2.71 \mathrm{E}-04$ \\
\hline 951 & BX932642 & -8.72 & $1.54 \mathrm{E}-04$ \\
\hline 952 & BX932650 & -3.17 & $2.82 \mathrm{E}-03$ \\
\hline 953 & BX932687 & -1.72 & $1.05 \mathrm{E}-03$ \\
\hline 954 & BX932708 & -3.17 & $6.30 \mathrm{E}-02$ \\
\hline 955 & BX932735 & -4.85 & $1.72 \mathrm{E}-03$ \\
\hline 956 & BX932774 & -2.50 & 4.51E-03 \\
\hline 957 & BX932786 & -1.62 & 4.15E-04 \\
\hline 958 & BX932795 & -1.26 & 4.12E-02 \\
\hline 959 & BX932816 & -2.89 & $1.12 \mathrm{E}-01$ \\
\hline 960 & BX932837 & -1.31 & $3.33 \mathrm{E}-03$ \\
\hline 961 & BX932840 & -1.65 & 4.27E-02 \\
\hline 962 & BX932849 & -2.20 & 1.37E-02 \\
\hline 963 & BX932862 & -6.15 & $2.89 \mathrm{E}-04$ \\
\hline 964 & BX932874 & -5.52 & 4.24E-04 \\
\hline 965 & BX932877 & -7.50 & 4.29E-04 \\
\hline 966 & BX932933 & -1.12 & $1.71 \mathrm{E}-02$ \\
\hline 967 & BX932982 & -1.50 & 4.33E-03 \\
\hline 968 & BX932985 & -1.89 & $5.80 \mathrm{E}-04$ \\
\hline 969 & BX932992 & -4.64 & 3.60E-03 \\
\hline 970 & BX933001 & -7.25 & 4.99E-04 \\
\hline 971 & BX933017 & -1.92 & $6.89 \mathrm{E}-03$ \\
\hline 972 & BX933023 & -2.58 & $3.08 \mathrm{E}-03$ \\
\hline 973 & BX933071 & -3.29 & $2.18 \mathrm{E}-03$ \\
\hline 974 & BX933147 & -1.18 & $7.41 \mathrm{E}-03$ \\
\hline 975 & BX933160 & -3.31 & $2.14 \mathrm{E}-02$ \\
\hline 976 & BX933174 & -3.30 & 2.13E-03 \\
\hline 977 & BX933193 & -7.57 & $1.23 \mathrm{E}-04$ \\
\hline 978 & BX933195 & -1.84 & 4.51E-02 \\
\hline 979 & BX933198 & -2.30 & 4.47E-02 \\
\hline 980 & BX933211 & -2.30 & $2.26 \mathrm{E}-02$ \\
\hline 981 & BX933251 & -3.96 & $8.06 \mathrm{E}-03$ \\
\hline 982 & BX933278 & -3.76 & $1.20 \mathrm{E}-03$ \\
\hline 983 & BX933376 & -5.54 & $1.57 \mathrm{E}-03$ \\
\hline 984 & BX933403 & -4.12 & $6.15 \mathrm{E}-04$ \\
\hline 985 & BX933422 & -2.17 & $1.37 \mathrm{E}-02$ \\
\hline 986 & BX933430 & -3.32 & $1.31 \mathrm{E}-03$ \\
\hline 987 & BX933456 & -3.26 & $9.99 \mathrm{E}-04$ \\
\hline 988 & BX933458 & -2.27 & $6.26 \mathrm{E}-03$ \\
\hline 989 & BX933470 & -1.19 & $3.32 \mathrm{E}-02$ \\
\hline 990 & BX933593 & -1.40 & $2.77 \mathrm{E}-02$ \\
\hline 991 & BX933633 & -3.48 & $2.74 \mathrm{E}-02$ \\
\hline 992 & BX933729 & -6.52 & $6.79 \mathrm{E}-04$ \\
\hline 993 & BX933749 & -1.56 & $8.68 \mathrm{E}-03$ \\
\hline 994 & BX933755 & -5.99 & $1.20 \mathrm{E}-04$ \\
\hline 995 & BX933777 & -2.11 & $1.04 \mathrm{E}-03$ \\
\hline 996 & BX933792 & -4.62 & 4.54E-04 \\
\hline 997 & BX933822 & -7.42 & 2.84E-04 \\
\hline 998 & BX933824 & -4.86 & 2.03E-04 \\
\hline 999 & BX933903 & -5.64 & $2.42 \mathrm{E}-02$ \\
\hline 1000 & BX933916 & -4.50 & $2.94 \mathrm{E}-03$ \\
\hline 1001 & BX933931 & -1.74 & $7.35 \mathrm{E}-03$ \\
\hline 1002 & BX934035 & -7.07 & 3.73E-04 \\
\hline 1003 & BX934048 & -7.33 & $8.48 \mathrm{E}-05$ \\
\hline 1004 & BX934051 & -1.12 & 7.15E-03 \\
\hline 1005 & BX934089 & -4.75 & 2.10E-03 \\
\hline 1006 & BX934095 & -6.49 & $8.16 \mathrm{E}-05$ \\
\hline 1007 & BX934107 & -4.32 & 1.13E-02 \\
\hline
\end{tabular}

\begin{tabular}{|c|c|c|c|}
\hline 1008 & BX934113 & -7.69 & $2.71 \mathrm{E}-03$ \\
\hline 1009 & BX934127 & -2.68 & 4.49E-03 \\
\hline 1010 & BX934164 & -3.19 & $1.69 \mathrm{E}-03$ \\
\hline 1011 & BX934201 & -4.88 & $2.52 \mathrm{E}-03$ \\
\hline 1012 & BX934247 & -3.75 & $1.88 \mathrm{E}-03$ \\
\hline 1013 & BX934273 & -3.08 & $3.91 \mathrm{E}-03$ \\
\hline 1014 & BX934293 & -4.59 & $1.97 \mathrm{E}-03$ \\
\hline 1015 & BX934316 & -1.85 & $3.92 \mathrm{E}-02$ \\
\hline 1016 & BX934379 & -1.16 & $9.13 \mathrm{E}-04$ \\
\hline 1017 & BX934422 & -4.60 & $2.41 \mathrm{E}-03$ \\
\hline 1018 & BX934434 & -1.94 & $1.40 \mathrm{E}-02$ \\
\hline 1019 & BX934492 & -6.90 & $2.24 \mathrm{E}-03$ \\
\hline 1020 & BX934615 & -4.77 & $2.56 \mathrm{E}-04$ \\
\hline 1021 & BX934739 & -4.52 & $3.61 \mathrm{E}-04$ \\
\hline 1022 & BX934745 & -5.15 & $1.04 \mathrm{E}-03$ \\
\hline 1023 & BX934757 & -2.73 & $2.50 \mathrm{E}-03$ \\
\hline 1024 & BX934779 & -3.61 & $2.24 \mathrm{E}-03$ \\
\hline 1025 & BX934859 & -2.29 & $2.99 \mathrm{E}-03$ \\
\hline 1026 & BX934897 & -3.14 & $7.11 \mathrm{E}-03$ \\
\hline 1027 & BX934918 & -7.03 & $1.68 \mathrm{E}-03$ \\
\hline 1028 & BX934943 & -2.82 & $8.95 \mathrm{E}-03$ \\
\hline 1029 & BX934945 & -4.11 & $9.26 \mathrm{E}-04$ \\
\hline 1030 & BX935016 & -5.95 & $3.79 \mathrm{E}-04$ \\
\hline 1031 & BX935057 & -5.50 & $1.42 \mathrm{E}-03$ \\
\hline 1032 & BX935059 & -6.24 & $9.34 \mathrm{E}-03$ \\
\hline 1033 & BX935093 & -1.53 & $1.30 \mathrm{E}-02$ \\
\hline 1034 & BX935100 & -1.99 & $6.59 \mathrm{E}-04$ \\
\hline 1035 & BX935104 & -3.18 & $8.33 \mathrm{E}-03$ \\
\hline 1036 & BX935110 & -1.69 & $3.62 \mathrm{E}-02$ \\
\hline 1037 & BX935137 & -8.44 & $2.22 \mathrm{E}-04$ \\
\hline 1038 & BX935301 & -3.73 & $3.46 \mathrm{E}-03$ \\
\hline 1039 & BX935369 & -5.21 & $5.67 \mathrm{E}-04$ \\
\hline 1040 & BX935396 & -6.53 & $9.89 \mathrm{E}-04$ \\
\hline 1041 & BX935398 & -3.85 & $3.35 \mathrm{E}-04$ \\
\hline 1042 & BX935421 & -4.14 & $1.12 \mathrm{E}-03$ \\
\hline 1043 & BX935424 & -4.55 & $1.63 \mathrm{E}-03$ \\
\hline 1044 & BX935455 & -3.59 & $9.27 \mathrm{E}-06$ \\
\hline 1045 & BX935460 & -3.53 & $4.88 \mathrm{E}-03$ \\
\hline 1046 & BX935465 & -4.35 & $1.09 \mathrm{E}-03$ \\
\hline 1047 & BX935490 & -2.41 & $6.11 \mathrm{E}-03$ \\
\hline 1048 & BX935537 & -2.30 & $2.66 \mathrm{E}-03$ \\
\hline 1049 & BX935585 & -3.34 & $1.59 \mathrm{E}-02$ \\
\hline 1050 & BX935592 & -5.89 & $3.82 E-04$ \\
\hline 1051 & BX935599 & -3.88 & $3.71 \mathrm{E}-03$ \\
\hline 1052 & BX935603 & -2.49 & $3.01 \mathrm{E}-02$ \\
\hline 1053 & BX935608 & -2.78 & 7.73E-04 \\
\hline 1054 & BX935611 & -1.77 & $2.32 \mathrm{E}-02$ \\
\hline 1055 & BX935614 & -2.10 & $9.56 \mathrm{E}-03$ \\
\hline 1056 & BX935617 & -6.82 & $1.30 \mathrm{E}-03$ \\
\hline 1057 & BX935654 & -6.10 & $4.73 \mathrm{E}-04$ \\
\hline 1058 & BX935655 & -5.05 & $2.86 \mathrm{E}-04$ \\
\hline 1059 & BX935662 & -2.23 & $2.46 \mathrm{E}-02$ \\
\hline 1060 & BX935670 & -2.40 & $3.10 \mathrm{E}-03$ \\
\hline 1061 & BX935682 & -1.42 & $1.56 \mathrm{E}-02$ \\
\hline 1062 & BX935684 & -3.86 & $4.30 \mathrm{E}-03$ \\
\hline 1063 & BX935690 & -1.35 & $4.88 \mathrm{E}-03$ \\
\hline 1064 & BX935718 & -1.54 & $2.71 \mathrm{E}-02$ \\
\hline 1065 & BX935724 & -2.35 & $7.41 \mathrm{E}-03$ \\
\hline 1066 & BX935738 & -6.69 & $8.39 \mathrm{E}-04$ \\
\hline 1067 & BX935740 & -1.45 & $3.26 \mathrm{E}-03$ \\
\hline 1068 & BX935828 & -1.40 & $7.21 \mathrm{E}-02$ \\
\hline 1069 & BX935876 & -2.56 & $1.20 \mathrm{E}-03$ \\
\hline 1070 & BX935919 & -1.10 & $4.15 \mathrm{E}-02$ \\
\hline
\end{tabular}

\begin{tabular}{|c|c|c|c|}
\hline 1071 & BX935933 & -5.51 & $2.16 \mathrm{E}-03$ \\
\hline 1072 & BX935950 & -1.41 & $9.12 \mathrm{E}-03$ \\
\hline 1073 & BX935964 & -2.91 & 8.23E-04 \\
\hline 1074 & BX935983 & -2.65 & $3.38 \mathrm{E}-03$ \\
\hline 1075 & BX935992 & -5.39 & 4.10E-04 \\
\hline 1076 & BX936015 & -4.45 & 3.44E-04 \\
\hline 1077 & BX936034 & -1.82 & $1.69 \mathrm{E}-04$ \\
\hline 1078 & BX936058 & -4.63 & $9.21 \mathrm{E}-04$ \\
\hline 1079 & BX936085 & -2.80 & 4.08E-03 \\
\hline 1080 & BX936099 & -2.04 & $1.26 \mathrm{E}-02$ \\
\hline 1081 & BX936147 & -4.66 & $3.93 \mathrm{E}-06$ \\
\hline 1082 & BX936170 & -3.48 & 1.47E-02 \\
\hline 1083 & BX936183 & -5.36 & $1.29 \mathrm{E}-03$ \\
\hline 1084 & BX936185 & -2.17 & $4.58 \mathrm{E}-03$ \\
\hline 1085 & BX936203 & -7.40 & $3.22 \mathrm{E}-04$ \\
\hline 1086 & BX936238 & -3.05 & 4.39E-04 \\
\hline 1087 & BX950246 & -6.66 & $9.75 \mathrm{E}-05$ \\
\hline 1088 & BX950297 & -6.77 & $9.13 \mathrm{E}-04$ \\
\hline 1089 & BX950304 & -3.13 & $6.22 \mathrm{E}-03$ \\
\hline 1090 & BX950335 & -4.44 & $2.49 \mathrm{E}-03$ \\
\hline 1091 & BX950348 & -4.91 & 4.60E-03 \\
\hline 1092 & BX950367 & -3.14 & 4.91E-04 \\
\hline 1093 & BX950369 & -2.07 & $4.02 \mathrm{E}-02$ \\
\hline 1094 & BX950411 & -6.92 & $2.06 \mathrm{E}-05$ \\
\hline 1095 & BX950494 & -5.10 & $3.24 \mathrm{E}-03$ \\
\hline 1096 & BX950520 & -3.73 & 5.37E-04 \\
\hline 1097 & BX950527 & -6.97 & $1.52 \mathrm{E}-04$ \\
\hline 1098 & BX950556 & -3.58 & $5.02 \mathrm{E}-03$ \\
\hline 1099 & BX950565 & -4.59 & $2.35 \mathrm{E}-03$ \\
\hline 1100 & BX950568 & -4.22 & $1.03 \mathrm{E}-03$ \\
\hline 1101 & BX950580 & -1.56 & 1.69E-04 \\
\hline 1102 & BX950605 & -8.39 & $7.81 \mathrm{E}-04$ \\
\hline 1103 & BX950615 & -1.86 & $8.51 \mathrm{E}-03$ \\
\hline 1104 & BX950634 & -7.22 & $3.61 \mathrm{E}-04$ \\
\hline 1105 & BX950648 & -6.46 & $5.50 \mathrm{E}-05$ \\
\hline 1106 & BX950682 & -4.15 & $7.06 \mathrm{E}-04$ \\
\hline 1107 & BX950768 & -4.14 & $1.42 \mathrm{E}-02$ \\
\hline 1108 & BX950770 & -4.82 & $4.67 \mathrm{E}-03$ \\
\hline 1109 & BX950780 & -6.37 & $3.13 \mathrm{E}-03$ \\
\hline 1110 & BX950807 & -1.32 & $1.11 \mathrm{E}-02$ \\
\hline 1111 & BX950820 & -6.19 & $2.26 \mathrm{E}-04$ \\
\hline 1112 & BX950834 & -8.00 & 5.57E-04 \\
\hline 1113 & BX950846 & -1.94 & $5.79 \mathrm{E}-03$ \\
\hline 1114 & BYSL & -1.12 & $1.38 \mathrm{E}-02$ \\
\hline 1115 & BZRAP1 & -2.64 & 3.14E-04 \\
\hline 1116 & BZW1 & -4.99 & 7.86E-04 \\
\hline 1117 & BZW2 & -6.60 & $5.37 \mathrm{E}-04$ \\
\hline 1118 & $\mathrm{C} 10 \mathrm{H} 15$ orf40 & -2.94 & $1.07 \mathrm{E}-03$ \\
\hline 1119 & $\mathrm{C} 10 \mathrm{H} 15$ orf60 & -3.61 & $8.29 \mathrm{E}-05$ \\
\hline 1120 & $\mathrm{C} 11 \mathrm{H} 16$ orf70 & -2.57 & $5.77 \mathrm{E}-03$ \\
\hline 1121 & $\mathrm{C} 11 \mathrm{H} 16$ orf80 & -4.84 & $1.37 \mathrm{E}-03$ \\
\hline 1122 & $\mathrm{C} 11 \mathrm{H} 16$ orf87 & -5.46 & $1.89 \mathrm{E}-04$ \\
\hline 1123 & C11H19orf12 & -2.98 & $1.55 \mathrm{E}-03$ \\
\hline 1124 & $\mathrm{C} 12 \mathrm{H} 3$ orf14 & -5.43 & $3.13 \mathrm{E}-04$ \\
\hline 1125 & C12H3orf37 & -2.98 & $3.34 \mathrm{E}-03$ \\
\hline 1126 & $\mathrm{C} 12 \mathrm{H} 3$ orf67 & -2.36 & $1.06 \mathrm{E}-02$ \\
\hline 1127 & C13H5orf15 & -5.14 & 5.35E-04 \\
\hline 1128 & C13H5orf25 & -6.51 & $2.83 \mathrm{E}-04$ \\
\hline 1129 & C13H5orf45 & -5.07 & 7.56E-04 \\
\hline 1130 & $\mathrm{C} 14 \mathrm{H} 16$ orf42 & -3.21 & $6.54 \mathrm{E}-03$ \\
\hline 1131 & C14H16orf5 & -4.21 & $1.14 \mathrm{E}-03$ \\
\hline 1132 & C14H16orf59 & -2.26 & $3.91 \mathrm{E}-03$ \\
\hline 1133 & C14H16orf62 & -4.57 & $3.29 \mathrm{E}-03$ \\
\hline
\end{tabular}




\begin{tabular}{|c|c|c|c|}
\hline 1134 & $\mathrm{C} 14 \mathrm{H} 17$ orf103 & -4.87 & $1.60 \mathrm{E}-04$ \\
\hline 1135 & C14H7orf50 & -1.57 & $1.11 \mathrm{E}-03$ \\
\hline 1136 & C14H7orf62 & -5.34 & $1.43 \mathrm{E}-03$ \\
\hline 1137 & C14H8orf33 & -4.40 & $8.08 \mathrm{E}-05$ \\
\hline 1138 & C14orf104 & -5.17 & $3.95 \mathrm{E}-04$ \\
\hline 1139 & C15H12orf43 & -3.84 & $1.68 \mathrm{E}-03$ \\
\hline 1140 & C15H12orf49 & -4.13 & $1.37 \mathrm{E}-03$ \\
\hline 1141 & C15H12orf65 & -4.63 & $5.32 \mathrm{E}-04$ \\
\hline 1142 & C15H14orf166B & $B-3.50$ & $2.84 \mathrm{E}-03$ \\
\hline 1143 & C15H22orf36 & -3.31 & $1.14 \mathrm{E}-02$ \\
\hline 1144 & C17H9orf114 & -5.05 & $9.59 \mathrm{E}-05$ \\
\hline 1145 & C17H9orf16 & -5.58 & $1.33 \mathrm{E}-04$ \\
\hline 1146 & C17H9orf78 & -4.73 & $7.03 \mathrm{E}-04$ \\
\hline 1147 & C17H9orf9 & -7.50 & $1.08 \mathrm{E}-04$ \\
\hline 1148 & C17H9orf96 & -6.87 & $2.39 \mathrm{E}-04$ \\
\hline 1149 & C18H17orf67 & -5.41 & $1.17 \mathrm{E}-03$ \\
\hline 1150 & C18H17orf75 & -2.89 & $1.34 \mathrm{E}-03$ \\
\hline 1151 & C18H17orf80 & -2.55 & $7.87 \mathrm{E}-04$ \\
\hline 1152 & С19H17orf108 & -2.38 & $1.47 \mathrm{E}-03$ \\
\hline 1153 & С19H17orf63 & -3.62 & $2.94 \mathrm{E}-03$ \\
\hline 1154 & C19H17orf78 & -8.49 & $1.13 \mathrm{E}-04$ \\
\hline 1155 & C19H17orf85 & -2.80 & $7.18 \mathrm{E}-04$ \\
\hline 1156 & C1D & -3.01 & $5.12 \mathrm{E}-04$ \\
\hline 1157 & C1GALT1 & -4.57 & $3.44 \mathrm{E}-04$ \\
\hline 1158 & C1GALT1C1 & -2.07 & $1.51 \mathrm{E}-03$ \\
\hline 1159 & C1H11orf54 & -3.26 & $1.64 \mathrm{E}-04$ \\
\hline 1160 & C1H11orf70 & -7.11 & $7.06 \mathrm{E}-04$ \\
\hline 1161 & C1H11orf73 & -5.71 & $1.83 \mathrm{E}-04$ \\
\hline 1162 & C1H12orf11 & -5.50 & $3.48 \mathrm{E}-04$ \\
\hline 1163 & C1H12orf23 & -5.16 & $3.15 E-04$ \\
\hline 1164 & C1H12orf35 & -2.56 & $1.20 \mathrm{E}-02$ \\
\hline 1165 & C1H12orf4 & -4.84 & $2.98 \mathrm{E}-05$ \\
\hline 1166 & C1H12orf45 & -2.73 & $9.85 \mathrm{E}-03$ \\
\hline 1167 & C1H12orf5 & -3.96 & $2.53 \mathrm{E}-05$ \\
\hline 1168 & C1H12orf50 & -3.05 & $2.14 \mathrm{E}-02$ \\
\hline 1169 & $\mathrm{C} 1 \mathrm{H} 12$ orf57 & -7.49 & $2.57 \mathrm{E}-04$ \\
\hline 1170 & C1H12orf73 & -2.24 & $5.68 \mathrm{E}-04$ \\
\hline 1171 & C1H13orf15 & -5.13 & 7.13E-04 \\
\hline 1172 & C1H1orf114 & -2.38 & $2.22 \mathrm{E}-04$ \\
\hline 1173 & C1H21orf33 & -3.29 & $1.61 \mathrm{E}-03$ \\
\hline 1174 & C1H21orf59 & -3.89 & $4.80 \mathrm{E}-04$ \\
\hline 1175 & C1H21orf91 & $\begin{array}{l}-3.05 \\
\end{array}$ & $2.31 \mathrm{E}-04$ \\
\hline 1176 & C1H22orf23 & -6.52 & $1.73 \mathrm{E}-04$ \\
\hline 1177 & C1H22orf28 & -3.41 & $2.76 \mathrm{E}-03$ \\
\hline 1178 & C1H22orf32 & -7.09 & $5.59 \mathrm{E}-05$ \\
\hline 1179 & $\mathrm{C} 1 \mathrm{H} 22$ orf33 & -6.59 & $2.91 E-04$ \\
\hline 1180 & C1H2orf29 & -5.94 & $4.65 \mathrm{E}-04$ \\
\hline 1181 & C1H3orf15 & -3.57 & $1.24 \mathrm{E}-03$ \\
\hline 1182 & C1H3orf17 & -2.18 & $1.58 \mathrm{E}-02$ \\
\hline 1183 & C1H3orf38 & -3.16 & 4.04E-04 \\
\hline 1184 & C1H7orf23 & -3.19 & $2.13 \mathrm{E}-03$ \\
\hline 1185 & C1H7orf55 & -1.73 & $3.40 \mathrm{E}-02$ \\
\hline 1186 & C1H7orf60 & -2.70 & $3.32 \mathrm{E}-03$ \\
\hline 1187 & C1HXorf22 & -3.10 & $2.71 \mathrm{E}-04$ \\
\hline 1188 & C1orf67 & -3.85 & $1.45 \mathrm{E}-03$ \\
\hline 1189 & C1QBP & -4.32 & $1.26 \mathrm{E}-05$ \\
\hline 1190 & C1S & -2.05 & $2.80 \mathrm{E}-03$ \\
\hline 1191 & C2OH20orf111 & -5.79 & $2.98 \mathrm{E}-04$ \\
\hline 1192 & C2OH20orf177 & -1.40 & $1.23 \mathrm{E}-03$ \\
\hline 1193 & C2OH20orf195 & -6.51 & $1.56 \mathrm{E}-04$ \\
\hline 1194 & $\mathrm{C} 20 \mathrm{OH} 20$ orf2O & -6.17 & $4.57 \mathrm{E}-04$ \\
\hline 1195 & С20H20orf24 & -2.81 & $1.73 \mathrm{E}-04$ \\
\hline 1196 & $\mathrm{C} 2 \mathrm{OH} 20$ orf85 & -7.45 & 5.46E-03 \\
\hline
\end{tabular}

\begin{tabular}{|c|c|c|c|}
\hline 1197 & C21H1orf158 & -5.99 & $1.77 \mathrm{E}-03$ \\
\hline 1198 & C21H1orf159 & -3.73 & $9.14 \mathrm{E}-04$ \\
\hline 1199 & C21H1orf174 & -2.49 & $1.80 \mathrm{E}-03$ \\
\hline 1200 & C21H1orf50 & -4.89 & $2.29 \mathrm{E}-03$ \\
\hline 1201 & C23H1orf109 & -3.50 & $2.31 \mathrm{E}-04$ \\
\hline 1202 & C23H1orf94 & -6.10 & $3.26 \mathrm{E}-03$ \\
\hline 1203 & C24H11orf1 & -5.89 & $6.36 \mathrm{E}-04$ \\
\hline 1204 & $\mathrm{C} 24 \mathrm{H} 11$ orf57 & -3.16 & $5.90 \mathrm{E}-05$ \\
\hline 1205 & C25H1orf43 & -4.40 & $2.23 \mathrm{E}-04$ \\
\hline 1206 & C26H1orf74 & -2.06 & $1.08 \mathrm{E}-02$ \\
\hline 1207 & C26H1orf88 & -5.44 & $3.35 \mathrm{E}-04$ \\
\hline 1208 & C26H6orf106 & -3.21 & $1.24 \mathrm{E}-02$ \\
\hline 1209 & C26H6orf130 & -4.04 & $1.32 \mathrm{E}-03$ \\
\hline 1210 & C27H17orf104 & -1.46 & $3.91 \mathrm{E}-03$ \\
\hline 1211 & C27H17orf105 & -8.37 & $1.33 \mathrm{E}-04$ \\
\hline 1212 & C28H19orf10 & -3.91 & $6.20 \mathrm{E}-03$ \\
\hline 1213 & $\mathrm{C} 28 \mathrm{H} 19$ orf44 & -3.43 & $4.79 \mathrm{E}-06$ \\
\hline 1214 & C28H19orf50 & -5.82 & $2.70 \mathrm{E}-04$ \\
\hline 1215 & C2CD5 & -3.04 & $8.16 \mathrm{E}-04$ \\
\hline 1216 & $\mathrm{C} 2 \mathrm{H} 18 \mathrm{orf21}$ & -3.86 & $1.12 \mathrm{E}-03$ \\
\hline 1217 & $\mathrm{C2}$ H18orf55 & -1.37 & $7.97 \mathrm{E}-02$ \\
\hline 1218 & C2H18orf8 & -4.27 & $1.73 \mathrm{E}-04$ \\
\hline 1219 & $\mathrm{C} 2 \mathrm{H} 1$ orf35 & -3.47 & $2.45 \mathrm{E}-03$ \\
\hline 1220 & $\mathrm{C} 2 \mathrm{H} 3$ orf23 & -2.16 & $1.42 \mathrm{E}-02$ \\
\hline 1221 & C2H3orf75 & -2.24 & $8.30 \mathrm{E}-03$ \\
\hline 1222 & C2H6orf145 & -1.24 & $1.65 \mathrm{E}-03$ \\
\hline 1223 & C2H6orf62 & -2.21 & $1.87 \mathrm{E}-02$ \\
\hline 1224 & C2H7orf10 & -2.86 & $2.99 \mathrm{E}-03$ \\
\hline 1225 & $\mathrm{C} 2 \mathrm{H} 7$ orf25 & -2.99 & $1.62 \mathrm{E}-03$ \\
\hline 1226 & C2H7orf41 & -4.76 & $2.84 \mathrm{E}-04$ \\
\hline 1227 & C2H7orf46 & -6.59 & $5.56 \mathrm{E}-04$ \\
\hline 1228 & C2H7orf57 & -6.21 & $8.79 \mathrm{E}-04$ \\
\hline 1229 & $\mathrm{C} 2 \mathrm{H} 8 \mathrm{orf37}$ & -4.41 & $5.76 \mathrm{E}-04$ \\
\hline 1230 & C2H8orf38 & -4.45 & $9.23 \mathrm{E}-04$ \\
\hline 1231 & С2H8orf45 & -3.55 & $6.32 \mathrm{E}-04$ \\
\hline 1232 & C2H8orf76 & -1.35 & $1.10 \mathrm{E}-02$ \\
\hline 1233 & С3H17orf59 & -5.04 & $1.65 \mathrm{E}-04$ \\
\hline 1234 & С3H1orf100 & -6.69 & $5.61 \mathrm{E}-04$ \\
\hline 1235 & C3H1orf101 & -2.23 & $1.06 \mathrm{E}-03$ \\
\hline 1236 & C3H1orf131 & -4.16 & $2.09 \mathrm{E}-04$ \\
\hline 1237 & С3н20orf3 & -4.84 & $6.11 \mathrm{E}-04$ \\
\hline 1238 & С3H20orf7 & -4.87 & $4.36 \mathrm{E}-04$ \\
\hline 1239 & С3H20orf72 & -6.40 & $2.17 \mathrm{E}-05$ \\
\hline 1240 & С3н2orf18 & -2.93 & $2.76 \mathrm{E}-04$ \\
\hline 1241 & С3н2orf56 & -3.04 & $1.05 \mathrm{E}-02$ \\
\hline 1242 & C3H6orf115 & -4.71 & $4.76 \mathrm{E}-03$ \\
\hline 1243 & C3H6orf12O & -3.57 & $2.22 \mathrm{E}-04$ \\
\hline 1244 & C3H6orf163 & -8.03 & $6.54 \mathrm{E}-04$ \\
\hline 1245 & C3H6orf165 & -4.73 & $3.39 \mathrm{E}-03$ \\
\hline 1246 & C3H6orf186 & -1.32 & $1.14 \mathrm{E}-03$ \\
\hline 1247 & C3H6orf192 & -4.13 & $4.66 \mathrm{E}-03$ \\
\hline 1248 & C3H6orf203 & -4.87 & $4.46 \mathrm{E}-04$ \\
\hline 1249 & C3H6orf211 & -5.76 & $2.72 \mathrm{E}-04$ \\
\hline 1250 & С3H6orf57 & -5.07 & $2.96 \mathrm{E}-03$ \\
\hline 1251 & С3н6orf70 & -2.13 & $1.15 \mathrm{E}-04$ \\
\hline 1252 & С3H8orf42 & -2.63 & $5.69 \mathrm{E}-04$ \\
\hline 1253 & C4 & -3.39 & $1.92 \mathrm{E}-03$ \\
\hline 1254 & С4H20orf194 & -1.30 & $4.36 \mathrm{E}-03$ \\
\hline 1255 & C4H4orf17 & -5.13 & $4.28 \mathrm{E}-04$ \\
\hline 1256 & C4H4orf21 & -4.29 & $7.00 \mathrm{E}-04$ \\
\hline 1257 & C4H4orf27 & -5.66 & $4.28 \mathrm{E}-05$ \\
\hline 1258 & C4H4orf29 & -1.83 & $6.23 \mathrm{E}-03$ \\
\hline 1259 & C4H4orf33 & $\begin{array}{l}-3.07 \\
\end{array}$ & $5.30 \mathrm{E}-03$ \\
\hline
\end{tabular}

\begin{tabular}{|c|c|c|c|}
\hline 1260 & C4H4orf34 & -3.81 & $1.35 \mathrm{E}-04$ \\
\hline 1261 & C4H4orf45 & -6.85 & $3.20 \mathrm{E}-04$ \\
\hline 1262 & C4H4orf47 & -9.87 & $2.19 \mathrm{E}-03$ \\
\hline 1263 & C4H4orf52 & -5.28 & $2.67 \mathrm{E}-04$ \\
\hline 1264 & C4H8orf40 & -5.11 & $2.53 \mathrm{E}-04$ \\
\hline 1265 & C4HXorf56 & -3.59 & $1.36 \mathrm{E}-05$ \\
\hline 1266 & C5 & -2.70 & $3.49 \mathrm{E}-04$ \\
\hline 1267 & C5H11orf10 & -6.68 & $1.77 \mathrm{E}-04$ \\
\hline 1268 & C5H11orf58 & -6.91 & $3.80 \mathrm{E}-04$ \\
\hline 1269 & C5H11orf74 & -8.22 & $8.23 \mathrm{E}-05$ \\
\hline 1270 & C5H14orf1 & -4.61 & $6.91 \mathrm{E}-04$ \\
\hline 1271 & C5H14orf101 & -3.27 & $1.47 \mathrm{E}-03$ \\
\hline 1272 & C5H14orf129 & -1.25 & $1.18 \mathrm{E}-02$ \\
\hline 1273 & C5H14orf149 & -2.14 & $4.85 \mathrm{E}-03$ \\
\hline 1274 & C5H14orf166 & -7.61 & $3.60 \mathrm{E}-05$ \\
\hline 1275 & C5H14orf166B & -1.70 & $1.99 \mathrm{E}-02$ \\
\hline 1276 & C5H14orf169 & -2.48 & $3.72 \mathrm{E}-02$ \\
\hline 1277 & C5H14orf38 & -3.70 & $1.73 \mathrm{E}-03$ \\
\hline 1278 & C5H14orf39 & -5.61 & $6.05 \mathrm{E}-04$ \\
\hline 1279 & C5H14orf49 & -3.80 & $1.91 \mathrm{E}-03$ \\
\hline 1280 & C5H15orf23 & -4.88 & $4.31 \mathrm{E}-04$ \\
\hline 1281 & C5H15orf24 & -3.42 & $9.19 \mathrm{E}-04$ \\
\hline 1282 & C5H15orf41 & -1.32 & $1.60 \mathrm{E}-02$ \\
\hline 1283 & C6H10orf107 & -6.73 & $4.23 \mathrm{E}-04$ \\
\hline 1284 & C6H10orf118 & -1.10 & $6.26 \mathrm{E}-03$ \\
\hline 1285 & C6H10orf32 & -4.05 & $3.94 \mathrm{E}-04$ \\
\hline 1286 & C6H10orf46 & -4.41 & $1.15 \mathrm{E}-03$ \\
\hline 1287 & C6H10orf57 & -5.25 & $8.17 \mathrm{E}-04$ \\
\hline 1288 & C6H10orf76 & -2.22 & $3.71 \mathrm{E}-03$ \\
\hline 1289 & C6H10orf88 & -5.15 & $1.20 \mathrm{E}-04$ \\
\hline 1290 & C7 & -3.26 & $1.12 \mathrm{E}-03$ \\
\hline 1291 & C7H2orf47 & -3.31 & $1.61 \mathrm{E}-03$ \\
\hline 1292 & C7H2orf66 & -3.44 & $2.02 \mathrm{E}-03$ \\
\hline 1293 & C7H2orf69 & -3.23 & $3.81 \mathrm{E}-03$ \\
\hline 1294 & $C 8 G$ & -3.17 & $2.81 \mathrm{E}-03$ \\
\hline 1295 & C8H1orf111 & -5.81 & $1.13 \mathrm{E}-03$ \\
\hline 1296 & C8H1orf123 & -6.68 & $1.50 \mathrm{E}-04$ \\
\hline 1297 & C8H1orf146 & -3.25 & $6.00 \mathrm{E}-03$ \\
\hline 1298 & C8H1orf173 & -2.16 & $2.90 \mathrm{E}-03$ \\
\hline 1299 & C8H1orf21 & -1.84 & $1.13 \mathrm{E}-03$ \\
\hline 1300 & C8H1orf228 & -1.22 & $6.43 \mathrm{E}-02$ \\
\hline 1301 & C8H1orf27 & -1.55 & $1.57 \mathrm{E}-02$ \\
\hline 1302 & C8H1orf52 & -4.27 & $9.92 \mathrm{E}-05$ \\
\hline 1303 & C9H21orf2 & -4.95 & $7.29 \mathrm{E}-04$ \\
\hline 1304 & CA2 & -4.14 & $1.73 \mathrm{E}-03$ \\
\hline 1305 & CA3 & -3.33 & $2.07 \mathrm{E}-02$ \\
\hline 1306 & CAAP1 & -5.27 & $4.51 \mathrm{E}-04$ \\
\hline 1307 & CAB39L & -2.88 & $1.07 \mathrm{E}-03$ \\
\hline 1308 & CABIN1 & -2.20 & $7.18 \mathrm{E}-04$ \\
\hline 1309 & CABP1 & -3.09 & $4.95 \mathrm{E}-04$ \\
\hline 1310 & CABYR & -4.48 & $2.40 \mathrm{E}-03$ \\
\hline 1311 & CACHD1 & -1.21 & $1.03 \mathrm{E}-02$ \\
\hline 1312 & CACNA2D1 & -3.08 & $2.59 \mathrm{E}-03$ \\
\hline 1313 & CACYBP & -5.53 & $8.69 \mathrm{E}-05$ \\
\hline 1314 & CADM1 & -2.13 & $7.18 \mathrm{E}-03$ \\
\hline 1315 & CALCOCO2 & -3.16 & $3.00 \mathrm{E}-03$ \\
\hline 1316 & CALD1 & -3.34 & $2.24 \mathrm{E}-03$ \\
\hline 1317 & CALM & -6.92 & $3.40 \mathrm{E}-04$ \\
\hline 1318 & CALM1 & -5.58 & $3.41 \mathrm{E}-04$ \\
\hline 1319 & CALR3 & -7.28 & $1.77 \mathrm{E}-03$ \\
\hline 1320 & CAMK1G & -1.10 & $3.53 \mathrm{E}-02$ \\
\hline 1321 & CAMK2D & -1.60 & $2.65 \mathrm{E}-03$ \\
\hline 1322 & CAMK2G & -2.38 & $1.33 \mathrm{E}-03$ \\
\hline
\end{tabular}




\begin{tabular}{|c|c|c|c|}
\hline 1323 & CAMK4 & -2.40 & $9.19 \mathrm{E}-03$ \\
\hline 1324 & CAMKК1 & -6.34 & $2.56 \mathrm{E}-04$ \\
\hline 1325 & CAMKMT & -3.33 & $9.72 \mathrm{E}-03$ \\
\hline 1326 & CAMLG & -3.02 & $1.52 \mathrm{E}-03$ \\
\hline 1327 & CAMSAP1 & -3.24 & $1.32 \mathrm{E}-03$ \\
\hline 1328 & CAND1 & -5.75 & $1.59 \mathrm{E}-04$ \\
\hline 1329 & CANX & -3.61 & $1.65 \mathrm{E}-04$ \\
\hline 1330 & CAPN10 & -2.05 & $1.26 \mathrm{E}-02$ \\
\hline 1331 & CAPN2 & -1.28 & $1.28 \mathrm{E}-02$ \\
\hline 1332 & CAPN6 & \begin{tabular}{|l|}
-1.41 \\
\end{tabular} & $1.49 \mathrm{E}-02$ \\
\hline 1333 & CAPRIN1 & -3.67 & $1.32 \mathrm{E}-03$ \\
\hline 1334 & CAPZA1 & -4.77 & $1.13 \mathrm{E}-03$ \\
\hline 1335 & CAPZA2 & -3.62 & $4.99 \mathrm{E}-03$ \\
\hline 1336 & CAPZA3 & \begin{tabular}{|l|} 
\\
\end{tabular} & $5.59 \mathrm{E}-05$ \\
\hline 1337 & CAPZB & -7.08 & $1.24 \mathrm{E}-04$ \\
\hline 1338 & CARD11 & $\begin{array}{l}-2.13 \\
\end{array}$ & $4.30 \mathrm{E}-04$ \\
\hline 1339 & CARD9 & -2.23 & $9.43 \mathrm{E}-03$ \\
\hline 1340 & CARKD & -4.86 & $4.71 \mathrm{E}-04$ \\
\hline 1341 & CARS & -4.55 & $9.55 \mathrm{E}-05$ \\
\hline 1342 & CARS2 & -3.31 & $3.03 \mathrm{E}-04$ \\
\hline 1343 & CASC1 & -3.37 & $4.95 \mathrm{E}-03$ \\
\hline 1344 & CASC3 & \begin{tabular}{|l|}
-3.21 \\
\end{tabular} & $7.54 \mathrm{E}-04$ \\
\hline 1345 & CASC4 & -6.04 & $3.31 \mathrm{E}-05$ \\
\hline 1346 & CASK & -3.71 & $6.20 \mathrm{E}-04$ \\
\hline 1347 & CASP1 & -5.13 & $1.34 \mathrm{E}-03$ \\
\hline 1348 & CASP18 & -2.14 & $3.94 \mathrm{E}-04$ \\
\hline 1349 & CASP2 & -2.85 & $9.47 \mathrm{E}-04$ \\
\hline 1350 & CASP3 & -1.44 & $5.34 \mathrm{E}-02$ \\
\hline 1351 & CASP6 & -1.31 & $2.79 \mathrm{E}-02$ \\
\hline 1352 & CASP8 & -1.45 & $1.06 \mathrm{E}-02$ \\
\hline 1353 & CASP9 & -3.06 & $1.08 \mathrm{E}-03$ \\
\hline 1354 & CAST & -1.22 & $4.34 \mathrm{E}-02$ \\
\hline 1355 & CAV2 & -2.99 & $2.99 \mathrm{E}-03$ \\
\hline 1356 & СBFA2T2 & -2.12 & $4.55 \mathrm{E}-03$ \\
\hline 1357 & CBFB & -2.60 & $6.09 \mathrm{E}-04$ \\
\hline 1358 & CBLL1 & -2.39 & $1.76 \mathrm{E}-03$ \\
\hline 1359 & CBLN1 & -3.76 & $3.47 \mathrm{E}-03$ \\
\hline 1360 & CBR1 & -2.15 & $9.48 \mathrm{E}-05$ \\
\hline 1361 & CBR4 & -2.77 & $2.42 \mathrm{E}-04$ \\
\hline 1362 & CBS & -3.16 & $1.66 \mathrm{E}-03$ \\
\hline 1363 & CBWD1 & -3.63 & $2.28 \mathrm{E}-03$ \\
\hline 1364 & CBX1 & $\begin{array}{l}-2.69 \\
\end{array}$ & $8.84 \mathrm{E}-03$ \\
\hline 1365 & CBX3 & -5.82 & $5.37 \mathrm{E}-05$ \\
\hline 1366 & CBY1 & -6.98 & $1.48 \mathrm{E}-04$ \\
\hline 1367 & CC2D1B & -1.85 & $1.46 \mathrm{E}-03$ \\
\hline 1368 & CC2D2A & -3.64 & $1.57 \mathrm{E}-03$ \\
\hline 1369 & CCAR1 & -3.68 & $1.77 \mathrm{E}-03$ \\
\hline 1370 & CCBL2 & -3.30 & $1.78 \mathrm{E}-04$ \\
\hline 1371 & CCBP2 & -1.40 & $1.78 \mathrm{E}-02$ \\
\hline 1372 & CCDC101 & -3.99 & $6.88 \mathrm{E}-04$ \\
\hline 1373 & CCDC103 & -6.85 & $2.50 \mathrm{E}-03$ \\
\hline 1374 & CCDC104 & -6.43 & $3.98 \mathrm{E}-04$ \\
\hline 1375 & CCDC107 & -7.24 & $3.75 \mathrm{E}-04$ \\
\hline 1376 & CCDC109B & -2.76 & $1.70 \mathrm{E}-03$ \\
\hline 1377 & CCDC111 & -3.60 & $1.99 \mathrm{E}-03$ \\
\hline 1378 & CCDC112 & -3.55 & $7.60 \mathrm{E}-04$ \\
\hline 1379 & CCDC12 & -2.69 & $5.71 E-03$ \\
\hline 1380 & CCDC121 & -5.53 & $5.18 \mathrm{E}-04$ \\
\hline 1381 & CCDC126 & -2.06 & $1.57 \mathrm{E}-03$ \\
\hline 1382 & $\mathrm{CCDC13}$ & -5.07 & $3.53 \mathrm{E}-04$ \\
\hline 1383 & CCDC132 & -2.04 & $9.98 \mathrm{E}-03$ \\
\hline 1384 & CCDC134 & -1.87 & $9.38 \mathrm{E}-04$ \\
\hline 1385 & CCDC14 & -4.01 & 1.9 \\
\hline
\end{tabular}

\begin{tabular}{|c|c|c|c|}
\hline 1386 & CCDC146 & -4.63 & $2.75 \mathrm{E}-03$ \\
\hline 1387 & CCDC147 & -4.35 & $1.45 \mathrm{E}-03$ \\
\hline 1388 & CCDC148 & -5.77 & $1.83 \mathrm{E}-04$ \\
\hline 1389 & CCDC15 & -6.12 & $5.17 \mathrm{E}-04$ \\
\hline 1390 & CCDC167 & -3.82 & $5.93 \mathrm{E}-03$ \\
\hline 1391 & CCDC17 & -1.64 & $5.93 \mathrm{E}-04$ \\
\hline 1392 & CCDC173 & -3.46 & $7.88 \mathrm{E}-03$ \\
\hline 1393 & CCDC174 & -3.02 & $3.23 \mathrm{E}-03$ \\
\hline 1394 & CCDC176 & -5.55 & $1.04 \mathrm{E}-03$ \\
\hline 1395 & CCDC18 & -2.64 & $2.37 \mathrm{E}-03$ \\
\hline 1396 & CCDC19 & -7.72 & $4.67 \mathrm{E}-04$ \\
\hline 1397 & CCDC25 & -4.42 & $1.06 \mathrm{E}-03$ \\
\hline 1398 & CCDC27 & -5.81 & $5.99 \mathrm{E}-05$ \\
\hline 1399 & CCDC28A & -4.13 & $3.34 \mathrm{E}-04$ \\
\hline 1400 & CCDC34 & -5.03 & 4.49E-04 \\
\hline 1401 & CCDC39 & -5.32 & $1.04 \mathrm{E}-04$ \\
\hline 1402 & CCDC40 & -8.09 & $4.46 \mathrm{E}-04$ \\
\hline 1403 & CCDC41 & -3.29 & 7.49E-04 \\
\hline 1404 & CCDC42 & -4.60 & $6.73 \mathrm{E}-03$ \\
\hline 1405 & CCDC43 & -3.09 & $5.65 \mathrm{E}-04$ \\
\hline 1406 & CCDC47 & -2.40 & $8.40 \mathrm{E}-03$ \\
\hline 1407 & CCDC53 & -1.66 & $5.19 \mathrm{E}-04$ \\
\hline 1408 & CCDC56 & -5.25 & $6.89 \mathrm{E}-06$ \\
\hline 1409 & CCDC58 & -1.26 & $4.12 \mathrm{E}-04$ \\
\hline 1410 & CCDC59 & -4.80 & $4.13 \mathrm{E}-04$ \\
\hline 1411 & CCDC6 & -2.39 & $2.55 \mathrm{E}-03$ \\
\hline 1412 & CCDC61 & -3.95 & $1.04 \mathrm{E}-03$ \\
\hline 1413 & CCDC63 & -2.65 & $4.34 \mathrm{E}-04$ \\
\hline 1414 & CCDC66 & -1.68 & $2.18 \mathrm{E}-03$ \\
\hline 1415 & $\mathrm{CCDC72}$ & -8.51 & $5.78 \mathrm{E}-06$ \\
\hline 1416 & CCDC75 & -4.72 & $1.45 \mathrm{E}-03$ \\
\hline 1417 & CCDC76 & -5.43 & $1.16 \mathrm{E}-03$ \\
\hline 1418 & CCDC77 & -5.20 & $4.20 \mathrm{E}-03$ \\
\hline 1419 & CCDC79 & -4.15 & $7.08 \mathrm{E}-04$ \\
\hline 1420 & CCDC80 & -1.94 & $9.07 \mathrm{E}-04$ \\
\hline 1421 & CCDC82 & -4.07 & $4.75 \mathrm{E}-03$ \\
\hline 1422 & CCDC83 & -5.83 & $1.44 \mathrm{E}-04$ \\
\hline 1423 & CCDC84 & -3.10 & $1.74 \mathrm{E}-02$ \\
\hline 1424 & CCDC86 & -3.99 & $1.32 \mathrm{E}-03$ \\
\hline 1425 & CCDC88C & -2.12 & $6.64 \mathrm{E}-03$ \\
\hline 1426 & CCDC89 & -5.12 & $1.93 \mathrm{E}-04$ \\
\hline 1427 & CCDC92 & -4.64 & $1.01 \mathrm{E}-03$ \\
\hline 1428 & CCDC93 & -3.58 & $4.47 \mathrm{E}-04$ \\
\hline 1429 & CCDC99 & -2.84 & $1.63 \mathrm{E}-02$ \\
\hline 1430 & CCK & -6.17 & $2.23 \mathrm{E}-04$ \\
\hline 1431 & CCL20 & -2.22 & $3.67 \mathrm{E}-03$ \\
\hline 1432 & CCM2 & -1.98 & $1.08 \mathrm{E}-03$ \\
\hline 1433 & CCNA1 & -8.05 & $1.38 \mathrm{E}-03$ \\
\hline 1434 & CCNA2 & -3.16 & $6.09 \mathrm{E}-03$ \\
\hline 1435 & CCNB2 & -5.18 & $9.26 \mathrm{E}-04$ \\
\hline 1436 & CCNB3 & -1.11 & $3.02 \mathrm{E}-03$ \\
\hline 1437 & CCNC & -1.88 & $9.73 \mathrm{E}-04$ \\
\hline 1438 & CCND1 & -2.57 & $1.98 \mathrm{E}-02$ \\
\hline 1439 & CCNDBP 1 & -4.11 & $2.45 \mathrm{E}-04$ \\
\hline 1440 & CCNG1 & -3.67 & $1.46 \mathrm{E}-02$ \\
\hline 1441 & $\mathrm{CCNH}$ & -8.22 & $1.20 \mathrm{E}-04$ \\
\hline 1442 & $\mathrm{CCNI}$ & -5.57 & $1.41 \mathrm{E}-03$ \\
\hline 1443 & CCNK & -6.69 & $9.33 \mathrm{E}-04$ \\
\hline 1444 & CCNL1 & -4.02 & $3.25 \mathrm{E}-04$ \\
\hline 1445 & CCNL2 & -3.03 & $1.54 \mathrm{E}-03$ \\
\hline 1446 & CCNYL1 & -3.70 & $4.22 \mathrm{E}-04$ \\
\hline 1447 & CCP110 & -2.89 & $2.78 \mathrm{E}-03$ \\
\hline 1448 & CCPG1 & -2.88 & 2.42E-04 \\
\hline
\end{tabular}

\begin{tabular}{|c|c|c|c|}
\hline 1449 & CCT2 & -6.41 & $5.98 E-05$ \\
\hline 1450 & CCT4 & -7.31 & $5.17 E-05$ \\
\hline 1451 & CCT5 & -4.92 & $2.42 \mathrm{E}-03$ \\
\hline 1452 & CCT6A & -6.87 & $1.25 \mathrm{E}-04$ \\
\hline 1453 & CCT7 & $\begin{array}{l}-7.09 \\
\end{array}$ & $2.58 \mathrm{E}-04$ \\
\hline 1454 & сСТ8 & -6.88 & $4.12 \mathrm{E}-04$ \\
\hline 1455 & CCZ1 & -2.62 & $2.16 \mathrm{E}-04$ \\
\hline 1456 & CD151 & -3.46 & $3.19 E-04$ \\
\hline 1457 & CD200 & -1.61 & $6.61 \mathrm{E}-03$ \\
\hline 1458 & CD24 & -2.03 & $1.93 E-02$ \\
\hline 1459 & $\begin{array}{ll}\text { CD247 } \\
\end{array}$ & \begin{tabular}{|l|}
-1.31 \\
\end{tabular} & $1.07 E-02$ \\
\hline 1460 & CD2AP & -4.51 & $6.76 E-04$ \\
\hline 1461 & CD3D & -3.45 & $1.52 \mathrm{E}-04$ \\
\hline 1462 & CD47 & -2.47 & $1.61 \mathrm{E}-02$ \\
\hline 1463 & CD729621 & -3.40 & $1.62 E-04$ \\
\hline 1464 & CD736984 & -5.61 & $4.00 E-03$ \\
\hline 1465 & CD74 & -4.56 & $3.58 \mathrm{E}-03$ \\
\hline 1466 & CD740706 & -1.83 & $1.57 \mathrm{E}-02$ \\
\hline 1467 & CD763379 & -2.47 & $8.51 E-05$ \\
\hline 1468 & CD763555 & -1.86 & $6.85 \mathrm{E}-02$ \\
\hline 1469 & CD765291 & -4.59 & $4.13 \mathrm{E}-03$ \\
\hline 1470 & CD81 & -2.11 & $2.24 \mathrm{E}-02$ \\
\hline 1471 & CD82 & -3.51 & $1.97 E-02$ \\
\hline 1472 & CD9 & -2.17 & $5.08 \mathrm{E}-03$ \\
\hline 1473 & CD99 & -4.16 & $4.64 \mathrm{E}-03$ \\
\hline 1474 & CDA & -2.90 & $4.21 \mathrm{E}-03$ \\
\hline 1475 & CDADC1 & -3.77 & $1.61 \mathrm{E}-03$ \\
\hline 1476 & CDC14B & -4.53 & 4.59E- 05 \\
\hline 1477 & CDC20 & -6.53 & $1.42 E-05$ \\
\hline 1478 & CDC23 & -3.89 & $1.23 \mathrm{E}-03$ \\
\hline 1479 & CDC25A & -3.87 & $1.11 \mathrm{E}-04$ \\
\hline 1480 & CDC26 & -4.86 & $5.23 \mathrm{E}-03$ \\
\hline 1481 & CDC27 & -5.58 & $7.28 \mathrm{E}-06$ \\
\hline 1482 & CDC2L1 & -3.09 & $3.08 E-04$ \\
\hline 1483 & CDC34 & -1.11 & $8.90 E-03$ \\
\hline 1484 & $\begin{array}{l}\text { CDC37 } \\
\end{array}$ & -3.85 & $7.41 E-04$ \\
\hline 1485 & CDC37L1 & -6.09 & 4.05E-04 \\
\hline 1486 & CDC40 & -6.08 & $9.51 \mathrm{E}-04$ \\
\hline 1487 & CDC42 & -6.74 & $2.82 E-06$ \\
\hline 1488 & CDC42BPB & -1.72 & $6.09 E-03$ \\
\hline 1489 & CDC42SE1 & -7.10 & $5.87 E-04$ \\
\hline 1490 & $\begin{array}{l}\text { CDC45 } \\
\end{array}$ & $\begin{array}{l}-1.56 \\
\end{array}$ & $1.29 E-03$ \\
\hline 1491 & CDC5L & -6.18 & $1.44 E-03$ \\
\hline 1492 & CDC7 & -1.38 & $1.05 E-02$ \\
\hline 1493 & CDCA2 & -4.56 & $4.77 E-04$ \\
\hline 1494 & CDCA4 & -5.68 & $8.18 \mathrm{E}-04$ \\
\hline 1495 & CDCA7 & -2.05 & $1.99 E-03$ \\
\hline 1496 & CDCA7L & -3.46 & $1.06 \mathrm{E}-03$ \\
\hline 1497 & CDCA8 & -3.02 & $7.45 E-04$ \\
\hline 1498 & CDH1-A & -1.84 & $1.06 E-02$ \\
\hline 1499 & $\mathrm{CDH} 2$ & -1.57 & $3.06 \mathrm{E}-02$ \\
\hline 1500 & CDH5 & -2.36 & $5.39 \mathrm{E}-03$ \\
\hline 1501 & CDK1 & -5.05 & $5.73 E-05$ \\
\hline 1502 & CDK10 & -4.21 & $5.34 \mathrm{E}-04$ \\
\hline 1503 & CDK13 & -4.32 & $3.83 \mathrm{E}-04$ \\
\hline 1504 & CDK17 & -2.26 & $2.48 \mathrm{E}-03$ \\
\hline 1505 & CDK19 & -3.07 & $9.21 \mathrm{E}-03$ \\
\hline 1506 & CDK2AP1 & -1.99 & $8.33 \mathrm{E}-04$ \\
\hline 1507 & CDK3 & -3.83 & $3.15 \mathrm{E}-03$ \\
\hline 1508 & CDK9 & -2.61 & 7.60E-04 \\
\hline 1509 & CDKAL1 & -3.16 & $3.06 E-04$ \\
\hline 1510 & CDKL1 & -3.03 & $4.39 \mathrm{E}-04$ \\
\hline 151 & CDKL2 & -4.42 & $1.02 E-03$ \\
\hline
\end{tabular}




\begin{tabular}{|c|c|c|c|}
\hline 1512 & CDKN1B & -4.46 & $2.36 \mathrm{E}-04$ \\
\hline 1513 & CDKN2A & -2.56 & $5.54 \mathrm{E}-04$ \\
\hline 1514 & CDKN3 & -3.64 & $4.01 \mathrm{E}-03$ \\
\hline 1515 & CDO1 & $\begin{array}{l}-2.97 \\
\end{array}$ & $3.24 \mathrm{E}-04$ \\
\hline 1516 & CDR2 & -1.24 & $1.67 \mathrm{E}-02$ \\
\hline 1517 & CDS1 & -5.93 & $5.33 \mathrm{E}-04$ \\
\hline 1518 & CDS2 & -2.31 & $2.32 \mathrm{E}-03$ \\
\hline 1519 & CDV3 & -5.18 & $6.83 \mathrm{E}-05$ \\
\hline 1520 & CEBPG & -2.80 & $1.72 \mathrm{E}-03$ \\
\hline 1521 & CEBPZ & -4.51 & $3.70 \mathrm{E}-04$ \\
\hline 1522 & CENPC1 & -6.93 & $1.63 \mathrm{E}-04$ \\
\hline 1523 & CENPF & -2.72 & $1.80 \mathrm{E}-03$ \\
\hline 1524 & CENPH & -4.92 & $1.12 \mathrm{E}-03$ \\
\hline 1525 & CENPK & -4.39 & $1.36 \mathrm{E}-04$ \\
\hline 1526 & CENPL & -3.85 & $9.16 \mathrm{E}-04$ \\
\hline 1527 & CENPM & -4.95 & $8.63 \mathrm{E}-05$ \\
\hline 1528 & CENPN & -4.24 & $2.08 \mathrm{E}-03$ \\
\hline 1529 & CENPP & -4.02 & $6.69 \mathrm{E}-04$ \\
\hline 1530 & CENPQ & -5.96 & $6.00 \mathrm{E}-04$ \\
\hline 1531 & CENPT & -6.37 & $1.75 \mathrm{E}-04$ \\
\hline 1532 & CENPV & -2.37 & $2.03 \mathrm{E}-02$ \\
\hline 1533 & CENPW & -5.53 & $4.76 \mathrm{E}-03$ \\
\hline 1534 & CEP104 & -6.31 & $1.88 \mathrm{E}-04$ \\
\hline 1535 & CEP112 & -3.98 & $4.27 \mathrm{E}-04$ \\
\hline 1536 & CEP120 & -5.47 & $1.12 \mathrm{E}-03$ \\
\hline 1537 & CEP135 & -4.28 & $4.53 \mathrm{E}-03$ \\
\hline 1538 & CEP170 & -5.79 & $7.67 \mathrm{E}-04$ \\
\hline 1539 & CEP19 & -6.22 & $6.75 \mathrm{E}-04$ \\
\hline 1540 & CEP192 & -5.23 & $2.32 \mathrm{E}-05$ \\
\hline 1541 & CEP290 & -5.34 & $3.97 \mathrm{E}-05$ \\
\hline 1542 & CEP350 & -3.17 & $1.99 \mathrm{E}-04$ \\
\hline 1543 & CEP44 & -6.32 & $6.31 \mathrm{E}-05$ \\
\hline 1544 & CEP55 & -5.27 & $5.58 \mathrm{E}-04$ \\
\hline 1545 & CEP57 & $\begin{array}{l}-3.26 \\
\end{array}$ & $2.79 \mathrm{E}-03$ \\
\hline 1546 & CEP63 & -2.89 & $3.29 \mathrm{E}-04$ \\
\hline 1547 & CEP70 & -5.27 & $3.96 \mathrm{E}-03$ \\
\hline 1548 & CEP85 & -2.23 & $2.87 \mathrm{E}-03$ \\
\hline 1549 & CEP89 & $\begin{array}{l}-4.76 \\
\end{array}$ & $1.15 \mathrm{E}-03$ \\
\hline 1550 & CEP95 & -1.55 & $5.70 \mathrm{E}-03$ \\
\hline 1551 & CEP97 & -2.34 & $4.56 \mathrm{E}-04$ \\
\hline 1552 & CERK & -4.75 & $1.02 \mathrm{E}-04$ \\
\hline 1553 & CERS1 & -2.02 & $7.76 \mathrm{E}-03$ \\
\hline 1554 & CERS5 & -1.28 & $1.23 \mathrm{E}-03$ \\
\hline 1555 & CERS6 & -2.04 & $2.05 \mathrm{E}-03$ \\
\hline 1556 & CETN1 & $\begin{array}{l}-6.15 \\
\end{array}$ & $1.16 \mathrm{E}-04$ \\
\hline 1557 & CETN2 & -5.41 & $1.48 \mathrm{E}-04$ \\
\hline 1558 & CETN3 & -7.44 & $1.73 \mathrm{E}-05$ \\
\hline 1559 & CF249937 & -2.63 & $2.15 \mathrm{E}-03$ \\
\hline 1560 & CF254431 & -2.46 & $2.46 \mathrm{E}-03$ \\
\hline 1561 & CF256373 & -2.61 & $2.20 \mathrm{E}-03$ \\
\hline 1562 & $\mathrm{CFC1B}$ & -3.77 & $1.06 \mathrm{E}-03$ \\
\hline 1563 & CFDP1 & -1.55 & $1.09 \mathrm{E}-02$ \\
\hline 1564 & $\mathrm{CFI}$ & \begin{tabular}{|l|}
-1.79 \\
\end{tabular} & $1.08 \mathrm{E}-02$ \\
\hline 1565 & CFL2 & -2.46 & $1.74 \mathrm{E}-02$ \\
\hline 1566 & CFLAR & -1.45 & $1.39 \mathrm{E}-02$ \\
\hline 1567 & CGA & -2.15 & $2.47 \mathrm{E}-03$ \\
\hline 1568 & CGNL1 & -2.80 & $4.45 \mathrm{E}-03$ \\
\hline 1569 & CGRRF1 & -6.80 & $2.44 \mathrm{E}-05$ \\
\hline 1570 & CHAC2 & -1.30 & $5.13 \mathrm{E}-04$ \\
\hline 1571 & CHAF1A & -3.74 & $2.74 E-04$ \\
\hline 1572 & CHAF1B & -4.01 & $3.41 \mathrm{E}-04$ \\
\hline 1573 & CHCHD1 & -3.38 & $8.01 E-04$ \\
\hline $15 / 4$ & CHCHD2 & -5.13 & $9.28 \mathrm{E}-03$ \\
\hline
\end{tabular}

\begin{tabular}{|c|c|c|c|}
\hline 1575 & $\mathrm{CHCHD} 3$ & -3.81 & $1.03 \mathrm{E}-04$ \\
\hline 1576 & CHCHD4 4 & -3.43 & $5.90 \mathrm{E}-04$ \\
\hline 1577 & CHCHD5 & -1.28 & $2.67 \mathrm{E}-02$ \\
\hline 1578 & CHCHD6 & -5.33 & $4.50 \mathrm{E}-04$ \\
\hline 1579 & CHD1 & -4.78 & $3.14 \mathrm{E}-05$ \\
\hline 1580 & CHD1L & \begin{tabular}{|l|}
-6.43 \\
\end{tabular} & $4.18 \mathrm{E}-04$ \\
\hline 1581 & CHD7 & $\begin{array}{l}-1.65 \\
\end{array}$ & $8.13 \mathrm{E}-03$ \\
\hline 1582 & CHD9 & -1.89 & $2.60 \mathrm{E}-03$ \\
\hline 1583 & CHERP & -3.96 & $1.16 \mathrm{E}-04$ \\
\hline 1584 & CHGB & -2.50 & $2.83 \mathrm{E}-04$ \\
\hline 1585 & CHIC2 & -3.29 & $2.41 \mathrm{E}-03$ \\
\hline 1586 & CHID1 & -4.82 & $3.82 \mathrm{E}-04$ \\
\hline 1587 & CHMP1A & -3.76 & $5.29 \mathrm{E}-03$ \\
\hline 1588 & CHMP1B & -2.33 & $1.55 \mathrm{E}-03$ \\
\hline 1589 & CHMP2A & -2.46 & $1.27 \mathrm{E}-03$ \\
\hline 1590 & CHMP2B & -2.45 & $1.38 \mathrm{E}-02$ \\
\hline 1591 & CHMP4B & \begin{tabular}{|l|}
-3.98 \\
\end{tabular} & $6.13 \mathrm{E}-03$ \\
\hline 1592 & CHMP5 & -5.42 & $1.87 \mathrm{E}-03$ \\
\hline 1593 & CHMP6 & -3.33 & $4.50 \mathrm{E}-05$ \\
\hline 1594 & CHMP7 & -3.42 & $1.94 \mathrm{E}-04$ \\
\hline 1595 & CHN1 & -3.50 & $1.03 \mathrm{E}-04$ \\
\hline 1596 & CHORDC1 & -4.29 & $1.62 \mathrm{E}-03$ \\
\hline 1597 & CHP1 & -5.95 & $3.53 \mathrm{E}-04$ \\
\hline 1598 & CHPT1 & -4.48 & $4.89 \mathrm{E}-04$ \\
\hline 1599 & CHRAC1 & -5.50 & $4.47 \mathrm{E}-04$ \\
\hline 1600 & $\begin{array}{l}\text { CHRDL1 } \\
\end{array}$ & -3.41 & $5.18 \mathrm{E}-03$ \\
\hline 1601 & CHST15 & -3.03 & $2.66 \mathrm{E}-03$ \\
\hline 1602 & CHST6 & -1.95 & $4.05 \mathrm{E}-03$ \\
\hline 1603 & CHSY1 & -2.27 & $4.26 \mathrm{E}-03$ \\
\hline 1604 & CHTOP & $\begin{array}{l}-1.69 \\
\end{array}$ & $2.34 \mathrm{E}-03$ \\
\hline 1605 & CHUK & \begin{tabular}{|l|}
-3.13 \\
\end{tabular} & $1.16 \mathrm{E}-03$ \\
\hline 1606 & CHURC1 & -5.84 & $2.72 \mathrm{E}-04$ \\
\hline 1607 & CIAPIN1 & -5.30 & $3.59 \mathrm{E}-05$ \\
\hline 1608 & CIB1 & -1.45 & $5.56 \mathrm{E}-04$ \\
\hline 1609 & CINP & -3.46 & $1.82 \mathrm{E}-04$ \\
\hline 1610 & CIR1 & -4.99 & $1.84 \mathrm{E}-04$ \\
\hline 1611 & CIRBP & -4.92 & $1.45 \mathrm{E}-05$ \\
\hline 1612 & CISD1 & $\begin{array}{l}-2.38 \\
\end{array}$ & $6.27 \mathrm{E}-03$ \\
\hline 1613 & CISD2 & -3.45 & $3.79 \mathrm{E}-04$ \\
\hline 1614 & CISH & -1.59 & $1.40 \mathrm{E}-02$ \\
\hline 1615 & CITED4 & -2.41 & $4.61 \mathrm{E}-03$ \\
\hline 1616 & CIZ1 & -2.42 & $7.31 \mathrm{E}-05$ \\
\hline 1617 & СК611406 & -2.91 & $8.52 \mathrm{E}-04$ \\
\hline 1618 & CK612070 & -2.47 & $1.47 \mathrm{E}-02$ \\
\hline 1619 & CK613298 & -2.02 & $6.58 \mathrm{E}-03$ \\
\hline 1620 & CK986952 & -2.23 & $2.82 \mathrm{E}-03$ \\
\hline 1621 & CKAP2 & -6.02 & $3.25 \mathrm{E}-04$ \\
\hline 1622 & $\begin{array}{l}\text { CKAP2L } \\
\end{array}$ & -1.74 & $3.21 \mathrm{E}-02$ \\
\hline 1623 & CKAP4 & -5.30 & $1.34 \mathrm{E}-05$ \\
\hline 1624 & CKAP5 & -2.10 & $4.77 \mathrm{E}-02$ \\
\hline 1625 & СКВ & \begin{tabular}{|l|}
-7.81 \\
\end{tabular} & $6.59 \mathrm{E}-03$ \\
\hline 1626 & CKMT1A & $\begin{array}{l}-2.43 \\
\end{array}$ & $4.53 \mathrm{E}-03$ \\
\hline 1627 & CKMT2 & $\begin{array}{l}-6.61 \\
\end{array}$ & $4.54 \mathrm{E}-04$ \\
\hline 1628 & CKR2 & -6.53 & $5.81 \mathrm{E}-05$ \\
\hline 1629 & CKS1B & -6.08 & $1.51 \mathrm{E}-04$ \\
\hline 1630 & CKS2 & $\begin{array}{l}-8.36 \\
\end{array}$ & $1.11 \mathrm{E}-03$ \\
\hline 1631 & CLCN3 & -1.27 & $1.01 \mathrm{E}-02$ \\
\hline 1632 & CLCN7 & -3.78 & $5.28 \mathrm{E}-04$ \\
\hline 1633 & CLCNKB & -1.95 & $4.51 \mathrm{E}-02$ \\
\hline 1634 & CLDN1 & -2.46 & $3.50 \mathrm{E}-03$ \\
\hline 1635 & CLDN15 & -2.80 & $1.35 \mathrm{E}-02$ \\
\hline 1636 & CLDND1 & -3.29 & $7.35 \mathrm{E}-05$ \\
\hline 1637 & CLEC16A & -1.39 & $1.83 \mathrm{E}-02$ \\
\hline
\end{tabular}

\begin{tabular}{|c|c|c|c|}
\hline 1638 & CLEC3B & -5.84 & $3.01 \mathrm{E}-03$ \\
\hline 1639 & CLGN & -8.13 & $6.50 \mathrm{E}-05$ \\
\hline 1640 & CLIC4 & -7.65 & $7.95 \mathrm{E}-05$ \\
\hline 1641 & CLINT1 & -1.98 & $3.17 \mathrm{E}-03$ \\
\hline 1642 & CLIP1 & -2.44 & $7.87 \mathrm{E}-03$ \\
\hline 1643 & CLK1 & -5.11 & $4.52 \mathrm{E}-03$ \\
\hline 1644 & CLK2 & $\begin{array}{l}-1.47 \\
\end{array}$ & $4.30 \mathrm{E}-03$ \\
\hline 1645 & CLK3 & -2.21 & $5.62 \mathrm{E}-03$ \\
\hline 1646 & CLK4 & -5.28 & $6.28 \mathrm{E}-05$ \\
\hline 1647 & CLMN & -6.66 & $3.62 \mathrm{E}-04$ \\
\hline 1648 & CLMP & -1.85 & $6.93 \mathrm{E}-02$ \\
\hline 1649 & CLN8 & -1.20 & $1.88 \mathrm{E}-03$ \\
\hline 1650 & CLNS1A & -6.74 & $2.70 \mathrm{E}-06$ \\
\hline 1651 & CLP1 & -2.97 & $1.66 \mathrm{E}-04$ \\
\hline 1652 & CLPTM1L & -3.60 & $3.69 \mathrm{E}-04$ \\
\hline 1653 & CLPX & -2.56 & $9.22 \mathrm{E}-04$ \\
\hline 1654 & CLSPN & -1.15 & $1.45 \mathrm{E}-03$ \\
\hline 1655 & CLSTN1 & -2.69 & $9.52 \mathrm{E}-03$ \\
\hline 1656 & CLTA & -6.95 & $3.14 \mathrm{E}-05$ \\
\hline 1657 & CLTB & -3.90 & $1.35 \mathrm{E}-04$ \\
\hline 1658 & CLTC & -3.39 & $3.93 \mathrm{E}-05$ \\
\hline 1659 & CLTCL1 & -2.51 & $4.67 \mathrm{E}-02$ \\
\hline 1660 & CLU & -2.80 & $1.12 \mathrm{E}-02$ \\
\hline 1661 & CMC1 & -4.08 & $2.40 \mathrm{E}-04$ \\
\hline 1662 & CMC2 & -3.96 & $4.52 \mathrm{E}-04$ \\
\hline 1663 & CMIP & -2.33 & $4.44 \mathrm{E}-03$ \\
\hline 1664 & CMPK1 & -3.59 & $6.52 \mathrm{E}-04$ \\
\hline 1665 & CMSS1 & -3.66 & $5.24 \mathrm{E}-04$ \\
\hline 1666 & CMTM3 & -1.95 & $6.73 \mathrm{E}-04$ \\
\hline 1667 & CN218106 & -4.20 & $1.24 \mathrm{E}-03$ \\
\hline 1668 & CN218725 & -1.43 & $4.67 \mathrm{E}-02$ \\
\hline 1669 & CN225732 & -3.27 & $1.02 \mathrm{E}-03$ \\
\hline 1670 & CN227175 & -6.02 & $2.22 \mathrm{E}-03$ \\
\hline 1671 & CN228445 & -3.59 & $2.76 \mathrm{E}-03$ \\
\hline 1672 & CN232510 & -1.67 & $6.18 \mathrm{E}-03$ \\
\hline $\begin{array}{l}1673 \\
\end{array}$ & CN236467 & $\begin{array}{l}-3.86 \\
\end{array}$ & $9.69 \mathrm{E}-04$ \\
\hline 1674 & CN236670 & -4.11 & $2.48 \mathrm{E}-03$ \\
\hline 1675 & CNBP & -6.48 & $7.48 \mathrm{E}-05$ \\
\hline 1676 & CNDP1 & -4.89 & $4.62 \mathrm{E}-04$ \\
\hline 1677 & CNDP2 & -4.56 & $1.87 \mathrm{E}-04$ \\
\hline 1678 & CNIH & -2.11 & $1.79 \mathrm{E}-02$ \\
\hline $\begin{array}{l}679 \\
\end{array}$ & $\mathrm{CNIH} 4$ & -5.71 & $5.10 \mathrm{E}-05$ \\
\hline 1680 & CNN3 & -1.49 & $1.61 \mathrm{E}-02$ \\
\hline 1681 & CNNM2 & -1.49 & $1.72 \mathrm{E}-03$ \\
\hline 1682 & CNOT1 & -1.58 & $4.25 \mathrm{E}-03$ \\
\hline 1683 & CNOT10 & -3.26 & $8.33 \mathrm{E}-04$ \\
\hline 1684 & CNOT2 & -2.40 & $4.55 \mathrm{E}-03$ \\
\hline 1685 & CNOT4 & -2.35 & $1.06 \mathrm{E}-03$ \\
\hline 1686 & CNOT7 & -2.01 & $1.00 \mathrm{E}-02$ \\
\hline 1687 & CNOT8 & -4.33 & $1.87 \mathrm{E}-03$ \\
\hline 1688 & CNP & -1.47 & $4.74 \mathrm{E}-04$ \\
\hline 1689 & CNPPD1 & -4.06 & $2.00 \mathrm{E}-03$ \\
\hline 1690 & CNST & -4.62 & $4.35 \mathrm{E}-03$ \\
\hline 1691 & CNTRL & -4.40 & $2.87 \mathrm{E}-03$ \\
\hline 1692 & CO420398 & -4.55 & $1.51 \mathrm{E}-03$ \\
\hline 1693 & 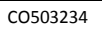 & -2.82 & $1.46 \mathrm{E}-03$ \\
\hline 1694 & CO635406 & -1.61 & $2.17 \mathrm{E}-02$ \\
\hline 1695 & CO635609 & -7.38 & $3.77 \mathrm{E}-03$ \\
\hline 1696 & CO635655 & -6.64 & $2.98 \mathrm{E}-04$ \\
\hline 1697 & C0635671 & -4.58 & $1.72 \mathrm{E}-04$ \\
\hline 1698 & CO635742 & -8.31 & $1.04 \mathrm{E}-03$ \\
\hline 1699 & 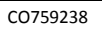 & -1.90 & $4.78 \mathrm{E}-04$ \\
\hline 1700 & CO759258 & -3.20 & $1.75 E-05$ \\
\hline
\end{tabular}




\begin{tabular}{|c|c|c|c|}
\hline 1701 & CO759280 & -4.55 & $1.09 \mathrm{E}-03$ \\
\hline 1702 & Co759332 & -4.31 & $2.02 E-04$ \\
\hline 1703 & C0759530 & -4.27 & $1.99 \mathrm{E}-04$ \\
\hline 1704 & Co759556 & -2.87 & $2.82 \mathrm{E}-04$ \\
\hline 1705 & C0760012 & -5.07 & $1.31 \mathrm{E}-03$ \\
\hline 1706 & CO760221 & -3.82 & $2.16 \mathrm{E}-03$ \\
\hline 1707 & C0760951 & -1.87 & $1.52 \mathrm{E}-03$ \\
\hline 1708 & CO760968 & -2.61 & $3.13 \mathrm{E}-03$ \\
\hline 1709 & CO761064 & -1.33 & $1.03 \mathrm{E}-02$ \\
\hline 1710 & CO761439 & -2.29 & $5.23 \mathrm{E}-03$ \\
\hline 1711 & CO761921 & -4.96 & 7.05E-04 \\
\hline 1712 & CO762094 & -4.31 & $1.46 \mathrm{E}-04$ \\
\hline 1713 & CO762098 & -1.83 & $4.35 \mathrm{E}-03$ \\
\hline 1714 & CO762224 & -3.46 & $1.30 \mathrm{E}-03$ \\
\hline 1715 & CO762861 & -3.20 & $2.02 \mathrm{E}-02$ \\
\hline 1716 & CO763026 & \begin{tabular}{|l|}
-3.78 \\
\end{tabular} & $1.11 \mathrm{E}-03$ \\
\hline 1717 & C0763237 & -3.20 & $1.54 \mathrm{E}-04$ \\
\hline 1718 & C0763508 & -2.46 & $1.51 \mathrm{E}-02$ \\
\hline 1719 & C0763836 & -5.28 & $6.49 \mathrm{E}-04$ \\
\hline 1720 & CO764067 & -8.41 & 7.00E-03 \\
\hline 1721 & C0764213 & -3.88 & $5.13 \mathrm{E}-04$ \\
\hline 1722 & 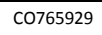 & \begin{tabular}{|l|}
-1.91 \\
\end{tabular} & $5.29 \mathrm{E}-03$ \\
\hline 1723 & C0766042 & -1.50 & $5.17 \mathrm{E}-03$ \\
\hline 1724 & Co766552 & -1.80 & $1.34 \mathrm{E}-03$ \\
\hline 1725 & C0766638 & -1.18 & $1.17 \mathrm{E}-03$ \\
\hline 1726 & C0766877 & -2.21 & $6.35 \mathrm{E}-03$ \\
\hline 1727 & C0767029 & -2.43 & $3.04 E-04$ \\
\hline 1728 & C0767272 & -1.60 & $7.27 \mathrm{E}-03$ \\
\hline 1729 & CO767662 & -1.14 & 7.97E-03 \\
\hline 1730 & CO767799 & -4.48 & $4.79 E-04$ \\
\hline 1731 & C0768102 & -2.10 & $9.79 E-03$ \\
\hline 1732 & C0768125 & -1.78 & $1.53 \mathrm{E}-03$ \\
\hline 1733 & CO768202 & -4.86 & $3.41 \mathrm{E}-04$ \\
\hline 1734 & CO768499 & -2.77 & $1.08 \mathrm{E}-03$ \\
\hline 1735 & C0770066 & -4.05 & $1.89 \mathrm{E}-04$ \\
\hline 1736 & CO770555 & -3.63 & $3.17 \mathrm{E}-03$ \\
\hline 1737 & C0770638 & -8.85 & $8.41 \mathrm{E}-06$ \\
\hline 1738 & C0770890 & -3.88 & $5.19 \mathrm{E}-04$ \\
\hline 1739 & Co771126 & -5.37 & 7.91E-04 \\
\hline 1740 & CO771485 & -4.79 & $3.28 \mathrm{E}-03$ \\
\hline 1741 & C0771662 & -2.36 & $2.64 \mathrm{E}-03$ \\
\hline 1742 & CO772032 & -5.37 & $4.02 E-03$ \\
\hline 1743 & C0772425 & -6.72 & $6.42 \mathrm{E}-04$ \\
\hline 1744 & C0773030 & -4.06 & $2.92 E-03$ \\
\hline 1745 & C0773370 & -5.82 & $1.20 \mathrm{E}-03$ \\
\hline 1746 & COA5 & -3.69 & $8.12 \mathrm{E}-05$ \\
\hline 1747 & COA6 & -4.05 & $1.25 \mathrm{E}-03$ \\
\hline 1748 & COBL & -3.55 & $1.60 \mathrm{E}-03$ \\
\hline 1749 & COBLL1 & -1.71 & $5.05 \mathrm{E}-03$ \\
\hline 1750 & COBRA1 & -3.75 & $5.30 \mathrm{E}-03$ \\
\hline 1751 & COG1 & -5.00 & $2.63 \mathrm{E}-04$ \\
\hline 1752 & COG2 & -5.03 & $2.78 \mathrm{E}-04$ \\
\hline 1753 & COG4 & -1.48 & $4.41 \mathrm{E}-03$ \\
\hline 1754 & COG5 & -1.31 & $1.73 \mathrm{E}-03$ \\
\hline 1755 & COG6 & -2.69 & $5.82 \mathrm{E}-05$ \\
\hline 1756 & COG7 & -5.13 & $2.18 \mathrm{E}-04$ \\
\hline 1757 & COIL & -5.80 & $2.62 \mathrm{E}-04$ \\
\hline 1758 & COL18A1 & -4.80 & $6.50 \mathrm{E}-04$ \\
\hline 1759 & COL2OA1 & -1.48 & $4.89 \mathrm{E}-02$ \\
\hline 1760 & COL3A1 & -2.68 & $5.19 \mathrm{E}-04$ \\
\hline 1761 & COLAA1 & -1.37 & $3.28 \mathrm{E}-02$ \\
\hline 1762 & COL4A3BP & -4.24 & $3.37 \mathrm{E}-03$ \\
\hline 1763 & & -2.04 & $3 . / 5$ \\
\hline
\end{tabular}

\begin{tabular}{|c|c|c|c|}
\hline 1764 & COL9A1 & -2.03 & $1.52 \mathrm{E}-02$ \\
\hline 1765 & COMMD1 & -4.46 & $3.86 \mathrm{E}-04$ \\
\hline 1766 & COMMD10 & -4.43 & $4.54 \mathrm{E}-04$ \\
\hline 1767 & COMMD2 & -4.55 & $8.83 \mathrm{E}-05$ \\
\hline 1768 & COMMD4 & -2.59 & $1.66 \mathrm{E}-03$ \\
\hline 1769 & COMMD6 & -4.45 & $7.66 \mathrm{E}-04$ \\
\hline 1770 & COMMD7 & -5.98 & $3.57 \mathrm{E}-05$ \\
\hline 1771 & COMMD8 & -4.97 & $2.19 \mathrm{E}-03$ \\
\hline 1772 & COPA & -3.93 & $6.20 \mathrm{E}-04$ \\
\hline 1773 & COPB1 & -5.93 & $5.62 \mathrm{E}-05$ \\
\hline 1774 & COPB2 & -6.60 & $6.89 \mathrm{E}-06$ \\
\hline 1775 & COPE & -6.26 & $6.60 \mathrm{E}-04$ \\
\hline 1776 & COPG & -2.18 & $1.11 \mathrm{E}-02$ \\
\hline 1777 & COPG2 & -4.55 & $2.40 \mathrm{E}-04$ \\
\hline 1778 & COPS2 & -4.44 & $1.14 \mathrm{E}-03$ \\
\hline 1779 & COPS3 & -8.41 & $2.05 E-04$ \\
\hline 1780 & COPS4 & -6.48 & $4.78 \mathrm{E}-04$ \\
\hline 1781 & COPS5 & -7.18 & $2.73 \mathrm{E}-04$ \\
\hline 1782 & COPS7A & -4.15 & $5.81 \mathrm{E}-05$ \\
\hline 1783 & COPS7B & -7.91 & $5.29 \mathrm{E}-04$ \\
\hline 1784 & COPS8 & -6.57 & $6.42 \mathrm{E}-06$ \\
\hline 1785 & COQ10A & -1.24 & $6.16 \mathrm{E}-04$ \\
\hline 1786 & $\mathrm{COQ}_{2}$ & -7.56 & $1.66 \mathrm{E}-04$ \\
\hline 1787 & $\mathrm{COQ}_{3}$ & -3.80 & $1.83 \mathrm{E}-03$ \\
\hline 1788 & COQ5 & -5.34 & $6.03 \mathrm{E}-06$ \\
\hline 1789 & coa6 & -2.30 & $4.10 \mathrm{E}-02$ \\
\hline 1790 & $\mathrm{COQ} 7$ & -7.99 & $2.44 \mathrm{E}-05$ \\
\hline 1791 & COQ9 & -4.03 & $9.82 \mathrm{E}-05$ \\
\hline 1792 & $\operatorname{cox} 1$ & -8.10 & $2.75 \mathrm{E}-03$ \\
\hline 1793 & $\operatorname{cox} 10$ & -2.91 & $7.41 \mathrm{E}-04$ \\
\hline 1794 & cox11 & -4.05 & $2.53 \mathrm{E}-03$ \\
\hline 1795 & $\operatorname{cox} 15$ & -1.62 & $3.07 \mathrm{E}-04$ \\
\hline 1796 & $\operatorname{cox} 17$ & -6.43 & $1.38 \mathrm{E}-04$ \\
\hline 1797 & cox18 & -2.70 & $6.65 \mathrm{E}-03$ \\
\hline 1798 & Cox19 & -5.92 & 4.64E-05 \\
\hline 1799 & $\operatorname{cox} 2$ & -7.79 & $6.00 \mathrm{E}-04$ \\
\hline 1800 & $\operatorname{cox} 20$ & -2.87 & $8.87 \mathrm{E}-05$ \\
\hline 1801 & $\operatorname{cox} 3$ & -7.32 & $1.66 \mathrm{E}-03$ \\
\hline 1802 & cox411 & -8.52 & $1.02 \mathrm{E}-04$ \\
\hline 1803 & COX5A & $\begin{array}{l}-7.67 \\
\end{array}$ & $1.21 \mathrm{E}-05$ \\
\hline 1804 & COX6A1 & -7.49 & $7.95 \mathrm{E}-06$ \\
\hline 1805 & COX6C & -6.03 & $3.42 \mathrm{E}-05$ \\
\hline 1806 & COX7A2 & -6.11 & $2.36 \mathrm{E}-04$ \\
\hline 1807 & COX7A2L & -7.60 & $4.58 \mathrm{E}-04$ \\
\hline 1808 & $\operatorname{cox} 7 \mathrm{C}$ & -6.00 & $1.03 \mathrm{E}-03$ \\
\hline 1809 & COX8A & -5.73 & $2.52 \mathrm{E}-03$ \\
\hline 1810 & CPA2 & -2.77 & $1.31 \mathrm{E}-02$ \\
\hline 1811 & CPEB2 & -4.17 & $6.11 \mathrm{E}-04$ \\
\hline 1812 & CPEB3 & -2.43 & $3.59 \mathrm{E}-03$ \\
\hline 1813 & CPNE1 & -1.93 & $2.16 \mathrm{E}-03$ \\
\hline 1814 & CPNE3 & -2.30 & $2.33 \mathrm{E}-03$ \\
\hline 1815 & CPNE8 & -3.07 & $3.46 \mathrm{E}-03$ \\
\hline 1816 & CPOX & -3.55 & $2.13 \mathrm{E}-03$ \\
\hline 1817 & $\mathrm{CPQ}$ & -1.40 & $5.96 \mathrm{E}-03$ \\
\hline 1818 & CPSF2 & -2.76 & $3.37 \mathrm{E}-04$ \\
\hline 1819 & CPSF3 & -4.54 & $5.80 \mathrm{E}-04$ \\
\hline 1820 & CPSF3L & -8.55 & $3.00 \mathrm{E}-05$ \\
\hline 1821 & CPSF4 & -3.56 & $4.60 \mathrm{E}-04$ \\
\hline 1822 & CPSF6 & -4.67 & $3.92 \mathrm{E}-04$ \\
\hline 1823 & CPT1A & -3.43 & $1.14 \mathrm{E}-04$ \\
\hline 1824 & CPT2 & -4.72 & $2.08 \mathrm{E}-03$ \\
\hline 1825 & CR338694 & -5.28 & $2.07 \mathrm{E}-04$ \\
\hline 1826 & CR338731 & -4.02 & $2.63 \mathrm{E}-03$ \\
\hline
\end{tabular}

\begin{tabular}{|c|c|c|c|}
\hline 1827 & CR338733 & -4.95 & $6.24 E-03$ \\
\hline 1828 & CR338740 & -4.32 & $1.90 E-03$ \\
\hline 1829 & CR338741 & -1.17 & $2.85 \mathrm{E}-03$ \\
\hline 1830 & CR338763 & -6.97 & $1.09 E-03$ \\
\hline 1831 & CR338764 & -2.29 & $8.88 \mathrm{E}-03$ \\
\hline 1832 & CR338780 & -5.39 & $1.09 E-03$ \\
\hline 1833 & CR338781 & -6.87 & $1.08 \mathrm{E}-03$ \\
\hline 1834 & CR338807 & -2.05 & $3.16 \mathrm{E}-03$ \\
\hline 1835 & CR338818 & -1.29 & $6.47 \mathrm{E}-02$ \\
\hline 1836 & CR338819 & $\begin{array}{l}-2.02 \\
\end{array}$ & $3.83 E-03$ \\
\hline 1837 & CR338837 & -4.17 & $2.38 E-04$ \\
\hline 1838 & CR338838 & \begin{tabular}{l|l|}
-2.09 \\
\end{tabular} & $5.71 E-03$ \\
\hline 1839 & CR338843 & -3.72 & $5.71 E-03$ \\
\hline 1840 & CR338869 & -3.01 & $4.36 \mathrm{E}-03$ \\
\hline 1841 & CR338874 & -2.89 & $7.80 \mathrm{E}-03$ \\
\hline 1842 & CR338900 & -3.20 & $1.61 E-03$ \\
\hline 1843 & CR338915 & -2.05 & $1.81 E-03$ \\
\hline 1844 & CR338916 & -2.44 & $2.46 \mathrm{E}-03$ \\
\hline 1845 & CR338923 & -2.16 & $5.48 \mathrm{E}-03$ \\
\hline 1846 & CR338928 & -4.00 & $9.29 E-04$ \\
\hline 1847 & CR338941 & -2.21 & $1.55 \mathrm{E}-02$ \\
\hline 1848 & CR338943 & -1.35 & $3.22 \mathrm{E}-03$ \\
\hline 1849 & CR338946 & -1.28 & $2.07 E-03$ \\
\hline 1850 & CR338956 & -3.65 & $1.41 E-03$ \\
\hline 1851 & CR339016 & -3.95 & $2.66 \mathrm{E}-04$ \\
\hline 1852 & CR352345 & -5.29 & $6.55 E-05$ \\
\hline 1853 & CR352346 & \begin{tabular}{|l|}
-2.11 \\
\end{tabular} & $7.83 E-03$ \\
\hline 1854 & CR352357 & -4.47 & $2.43 E-05$ \\
\hline 1855 & CR352359 & -1.34 & $1.66 \mathrm{E}-02$ \\
\hline 1856 & CR352361 & -2.06 & $9.73 \mathrm{E}-02$ \\
\hline 1857 & CR352363 & -6.12 & $1.34 E-03$ \\
\hline 1858 & CR352369 & -2.21 & $4.66 \mathrm{E}-03$ \\
\hline 1859 & CR352370 & -3.27 & $1.32 \mathrm{E}-03$ \\
\hline 1860 & CR352374 & -2.01 & $1.43 E-02$ \\
\hline 1861 & CR352376 & -2.96 & $1.33 E-03$ \\
\hline 1862 & CR352380 & -1.80 & $5.10 \mathrm{E}-02$ \\
\hline 1863 & CR352381 & -1.89 & $5.38 \mathrm{E}-03$ \\
\hline 1864 & CR352385 & -1.63 & $1.63 \mathrm{E}-02$ \\
\hline 1865 & CR352388 & -3.03 & $7.42 \mathrm{E}-03$ \\
\hline 1866 & CR352393 & -1.97 & $7.75 E-04$ \\
\hline 1867 & CR352404 & -4.56 & $3.25 E-04$ \\
\hline 1868 & CR352409 & -2.89 & $3.48 \mathrm{E}-03$ \\
\hline 1869 & CR352415 & -1.86 & $1.09 E-03$ \\
\hline 1870 & CR352419 & -3.87 & $5.53 \mathrm{E}-03$ \\
\hline 1871 & CR352432 & -3.76 & $5.38 \mathrm{E}-03$ \\
\hline 1872 & CR352433 & -3.69 & $7.99 \mathrm{E}-03$ \\
\hline 1873 & CR352434 & -7.28 & $5.55 E-04$ \\
\hline 1874 & CR352441 & -2.97 & $1.61 E-04$ \\
\hline 1875 & CR352447 & -1.09 & $7.09 E-03$ \\
\hline 1876 & CR352450 & -1.88 & $2.56 \mathrm{E}-02$ \\
\hline 1877 & CR352453 & -1.10 & $1.71 E-02$ \\
\hline 1878 & CR352457 & $\begin{array}{l}-1.92 \\
\end{array}$ & $1.14 E-02$ \\
\hline 1879 & CR352551 & -1.93 & $1.47 \mathrm{E}-02$ \\
\hline 1880 & CR352562 & -2.08 & $3.39 E-04$ \\
\hline 1881 & CR352573 & -5.36 & $3.31 E-04$ \\
\hline 1882 & CR352593 & -1.47 & $9.06 E-03$ \\
\hline 1883 & CR352594 & -7.83 & $5.46 \mathrm{E}-04$ \\
\hline 1884 & CR352615 & \begin{tabular}{|l|}
-3.93 \\
\end{tabular} & $\begin{array}{l}7.39 E-04 \\
\end{array}$ \\
\hline 1885 & CR352635 & -3.53 & $8.67 E-04$ \\
\hline 1886 & CR352638 & -2.70 & $4.06 E-04$ \\
\hline 1887 & CR352641 & -1.11 & $7.49 E-03$ \\
\hline 1888 & CR352645 & -2.29 & $1.79 E-04$ \\
\hline 889 & 2647 & -3.42 & \\
\hline
\end{tabular}




\begin{tabular}{|c|c|c|c|}
\hline 1890 & CR352651 & -2.23 & $6.49 \mathrm{E}-03$ \\
\hline 1891 & CR352656 & -1.20 & $9.59 \mathrm{E}-03$ \\
\hline 1892 & CR352689 & -1.51 & $3.40 \mathrm{E}-02$ \\
\hline 1893 & CR352690 & -6.84 & $1.70 \mathrm{E}-06$ \\
\hline 1894 & CR352708 & -1.96 & $1.80 \mathrm{E}-02$ \\
\hline 1895 & CR352717 & -2.39 & $6.37 \mathrm{E}-02$ \\
\hline 1896 & CR352728 & -2.66 & $4.84 \mathrm{E}-04$ \\
\hline 1897 & CR352729 & -3.33 & $1.66 \mathrm{E}-03$ \\
\hline 1898 & CR352731 & -1.39 & $5.59 \mathrm{E}-02$ \\
\hline 1899 & CR352732 & -1.48 & $1.39 \mathrm{E}-03$ \\
\hline 1900 & CR352739 & -5.90 & $4.53 \mathrm{E}-05$ \\
\hline 1901 & CR352744 & -3.80 & $2.14 \mathrm{E}-02$ \\
\hline 1902 & CR352747 & -6.32 & $8.68 \mathrm{E}-04$ \\
\hline 1903 & CR352749 & -5.88 & $4.30 \mathrm{E}-04$ \\
\hline 1904 & CR352764 & -4.86 & 8.64E-04 \\
\hline 1905 & CR352768 & -4.36 & $1.53 \mathrm{E}-03$ \\
\hline 1906 & CR352781 & -2.13 & $2.13 \mathrm{E}-03$ \\
\hline 1907 & CR352790 & -5.12 & $1.34 \mathrm{E}-03$ \\
\hline 1908 & CR352799 & -3.90 & $5.50 \mathrm{E}-04$ \\
\hline 1909 & CR352803 & -1.97 & $3.10 \mathrm{E}-03$ \\
\hline 1910 & CR352805 & -4.30 & $2.72 \mathrm{E}-02$ \\
\hline 1911 & CR352811 & -1.28 & $2.89 \mathrm{E}-02$ \\
\hline 1912 & CR352814 & -4.50 & $9.95 \mathrm{E}-04$ \\
\hline 1913 & CR352819 & -2.28 & $1.41 \mathrm{E}-02$ \\
\hline 1914 & CR352822 & -4.07 & $2.32 \mathrm{E}-03$ \\
\hline 1915 & CR352824 & -7.01 & $4.89 \mathrm{E}-03$ \\
\hline 1916 & CR352826 & -2.99 & $4.43 \mathrm{E}-03$ \\
\hline 1917 & CR352833 & -2.80 & $6.92 \mathrm{E}-02$ \\
\hline 1918 & CR352842 & -3.28 & $1.74 \mathrm{E}-03$ \\
\hline 1919 & CR352844 & -3.18 & $3.94 \mathrm{E}-03$ \\
\hline 1920 & CR352850 & -1.54 & $8.73 \mathrm{E}-03$ \\
\hline 1921 & CR352870 & $\begin{array}{l}-5.41 \\
\end{array}$ & $2.26 \mathrm{E}-03$ \\
\hline 1922 & CR352873 & -2.97 & $8.60 \mathrm{E}-03$ \\
\hline 1923 & CR352877 & -3.94 & $1.33 \mathrm{E}-03$ \\
\hline 1924 & CR352882 & -4.30 & $1.94 \mathrm{E}-04$ \\
\hline 1925 & CR352885 & -3.78 & $5.20 \mathrm{E}-03$ \\
\hline 1926 & CR352886 & -5.45 & $1.11 \mathrm{E}-03$ \\
\hline 1927 & CR352893 & -1.84 & $7.17 \mathrm{E}-03$ \\
\hline 1928 & CR352895 & -6.06 & $2.29 \mathrm{E}-04$ \\
\hline 1929 & CR352900 & -6.79 & $1.57 \mathrm{E}-04$ \\
\hline 1930 & CR352901 & -2.58 & $5.58 \mathrm{E}-03$ \\
\hline 1931 & CR352914 & -4.96 & $8.42 \mathrm{E}-04$ \\
\hline 1932 & CR352918 & \begin{tabular}{|l|}
-3.30 \\
\end{tabular} & $3.69 \mathrm{E}-04$ \\
\hline 1933 & CR352925 & -4.45 & $5.37 \mathrm{E}-04$ \\
\hline 1934 & CR352932 & -4.01 & $3.54 \mathrm{E}-03$ \\
\hline 1935 & CR352938 & -1.97 & $3.76 \mathrm{E}-02$ \\
\hline 1936 & CR352947 & \begin{tabular}{|l|}
-1.44 \\
\end{tabular} & $5.97 \mathrm{E}-03$ \\
\hline 1937 & CR352963 & \begin{tabular}{|l|}
-2.01 \\
\end{tabular} & $1.53 \mathrm{E}-02$ \\
\hline 1938 & CR352967 & -4.93 & $7.84 \mathrm{E}-05$ \\
\hline 1939 & CR352976 & -4.15 & $1.75 \mathrm{E}-03$ \\
\hline 1940 & CR352998 & -1.56 & $1.64 \mathrm{E}-03$ \\
\hline 1941 & CR353000 & $\begin{array}{l}-1.73 \\
\end{array}$ & $2.50 \mathrm{E}-02$ \\
\hline 1942 & CR353001 & -4.30 & $1.94 \mathrm{E}-04$ \\
\hline 1943 & CR353007 & -2.11 & $1.33 \mathrm{E}-03$ \\
\hline 1944 & CR353025 & \begin{tabular}{|l|}
-3.99 \\
\end{tabular} & $5.13 \mathrm{E}-04$ \\
\hline 1945 & CR353036 & -3.64 & $3.21 \mathrm{E}-04$ \\
\hline 1946 & CR353043 & -2.01 & $1.63 \mathrm{E}-02$ \\
\hline 1947 & CR353065 & -3.96 & $1.51 \mathrm{E}-03$ \\
\hline 1948 & CR353068 & -5.17 & $\begin{array}{l}7.54 \mathrm{E}-04 \\
\end{array}$ \\
\hline 1949 & CR353086 & -2.87 & 2.04E-02 \\
\hline 1950 & CR353135 & -3.82 & $1.58 \mathrm{E}-02$ \\
\hline 1951 & CR353144 & -2.26 & $7.40 \mathrm{E}-03$ \\
\hline 95 & CR353151 & -2.29 & $1.03 \mathrm{E}-02$ \\
\hline
\end{tabular}

\begin{tabular}{|c|c|c|c|}
\hline 1953 & CR353152 & -3.39 & $1.47 \mathrm{E}-03$ \\
\hline 1954 & CR353157 & -3.44 & $4.80 \mathrm{E}-03$ \\
\hline 1955 & CR353160 & -3.27 & $3.92 \mathrm{E}-03$ \\
\hline 1956 & CR353168 & -2.57 & $1.52 E-02$ \\
\hline 1957 & CR353171 & -2.87 & $3.23 \mathrm{E}-03$ \\
\hline 1958 & CR353181 & -1.15 & 3.45E-04 \\
\hline 1959 & CR353182 & -2.14 & $2.59 \mathrm{E}-02$ \\
\hline 1960 & CR353186 & -4.90 & $3.52 \mathrm{E}-02$ \\
\hline 1961 & CR353188 & -4.40 & $3.22 E-03$ \\
\hline 1962 & CR353200 & -4.28 & $2.11 E-04$ \\
\hline 1963 & CR353203 & -4.08 & $1.22 E-03$ \\
\hline 1964 & CR353208 & -1.42 & $1.53 \mathrm{E}-02$ \\
\hline 1965 & CR353214 & -1.40 & $2.21 \mathrm{E}-02$ \\
\hline 1966 & CR353216 & -5.07 & $4.27 \mathrm{E}-04$ \\
\hline 1967 & CR353219 & -6.04 & $5.62 E-04$ \\
\hline 1968 & CR353242 & -3.07 & $1.61 E-03$ \\
\hline 1969 & CR353246 & -1.71 & $5.47 \mathrm{E}-03$ \\
\hline 1970 & CR353249 & -2.32 & $1.08 \mathrm{E}-02$ \\
\hline 1971 & CR353256 & -3.75 & $1.40 \mathrm{E}-03$ \\
\hline 1972 & CR353257 & -4.24 & $1.31 E-03$ \\
\hline 1973 & CR353265 & -5.37 & $1.39 E-03$ \\
\hline 1974 & CR353281 & -3.16 & $8.08 E-03$ \\
\hline 1975 & CR353285 & -1.13 & $9.15 \mathrm{E}-03$ \\
\hline 1976 & CR353290 & -3.71 & $2.04 E-03$ \\
\hline 1977 & CR353308 & -3.47 & $9.10 \mathrm{E}-04$ \\
\hline 1978 & CR353350 & -8.31 & $6.62 E-05$ \\
\hline 1979 & CR353362 & -4.33 & $2.50 \mathrm{E}-03$ \\
\hline 1980 & CR353393 & -1.77 & $1.77 \mathrm{E}-02$ \\
\hline 1981 & CR353397 & -3.73 & $9.84 \mathrm{E}-04$ \\
\hline 1982 & CR353398 & -5.02 & $4.33 E-04$ \\
\hline 1983 & CR353401 & -2.70 & $3.52 \mathrm{E}-03$ \\
\hline 1984 & CR353411 & -3.21 & $4.17 \mathrm{E}-03$ \\
\hline 1985 & CR353414 & -2.73 & $1.49 \mathrm{E}-02$ \\
\hline 1986 & CR353421 & -2.02 & $9.19 E-03$ \\
\hline 1987 & CR353426 & -3.39 & $3.05 E-04$ \\
\hline 1988 & CR353427 & -4.08 & $6.17 \mathrm{E}-04$ \\
\hline 1989 & CR353434 & -4.30 & $9.02 E-04$ \\
\hline 1990 & CR353436 & -1.28 & $6.94 E-03$ \\
\hline 1991 & CR353438 & -5.96 & $5.64 E-04$ \\
\hline 1992 & CR353439 & $\begin{array}{l}-1.93 \\
\end{array}$ & $4.18 \mathrm{E}-02$ \\
\hline 1993 & CR353444 & -1.15 & $1.75 E-02$ \\
\hline 1994 & CR353449 & -1.80 & $3.03 E-03$ \\
\hline 1995 & CR353452 & -1.58 & $3.33 E-03$ \\
\hline 1996 & CR353459 & -6.23 & $2.39 E-04$ \\
\hline 1997 & CR353464 & -3.58 & $9.48 \mathrm{E}-04$ \\
\hline 1998 & CR353466 & -2.41 & $\begin{array}{l}7.51 E-03 \\
\end{array}$ \\
\hline 1999 & CR353489 & -2.86 & $1.53 E-04$ \\
\hline 2000 & CR353495 & -3.61 & $1.31 E-03$ \\
\hline 2001 & CR353509 & -3.38 & $1.66 \mathrm{E}-03$ \\
\hline 2002 & CR353511 & -2.64 & $6.63 \mathrm{E}-04$ \\
\hline 2003 & CR353513 & -4.10 & $1.29 E-03$ \\
\hline 2004 & CR353514 & -3.48 & $2.34 \mathrm{E}-04$ \\
\hline 2005 & CR353515 & -1.21 & $7.18 \mathrm{E}-03$ \\
\hline 2006 & CR353522 & -1.67 & $6.61 \mathrm{E}-03$ \\
\hline 2007 & CR353529 & -2.00 & $1.75 E-03$ \\
\hline 2008 & CR353533 & -2.07 & $1.57 \mathrm{E}-02$ \\
\hline 2009 & CR353541 & -5.19 & $6.70 E-04$ \\
\hline 2010 & CR353542 & -1.09 & $2.54 \mathrm{E}-03$ \\
\hline 2011 & CR353544 & -4.08 & $3.35 E-03$ \\
\hline 2012 & CR353546 & -3.32 & $7.43 E-04$ \\
\hline 2013 & CR353547 & -1.50 & $1.05 E-02$ \\
\hline 2014 & CR353548 & -2.48 & $4.01 E-03$ \\
\hline 2015 & CR353549 & -3.37 & $4.81 \mathrm{E}-04$ \\
\hline
\end{tabular}

\begin{tabular}{|c|c|c|c|}
\hline 2016 & CR353551 & -6.71 & $6.73 \mathrm{E}-04$ \\
\hline 2017 & CR353554 & -4.72 & $2.01 \mathrm{E}-02$ \\
\hline 2018 & CR353574 & -7.23 & $1.26 \mathrm{E}-04$ \\
\hline 2019 & CR353584 & -4.00 & $3.56 \mathrm{E}-03$ \\
\hline 2020 & CR353585 & -5.63 & $1.07 \mathrm{E}-04$ \\
\hline 2021 & CR353591 & -1.42 & $1.09 \mathrm{E}-03$ \\
\hline 2022 & CR353597 & -1.75 & $2.32 \mathrm{E}-02$ \\
\hline 2023 & CR353608 & -2.71 & $2.06 \mathrm{E}-03$ \\
\hline 2024 & CR353609 & -5.02 & $1.43 \mathrm{E}-03$ \\
\hline 2025 & CR353616 & -1.72 & $2.02 \mathrm{E}-03$ \\
\hline 2026 & \begin{tabular}{lc|} 
CR353622 \\
\end{tabular} & -4.84 & $2.39 \mathrm{E}-05$ \\
\hline 2027 & CR353624 & -2.58 & $2.54 \mathrm{E}-03$ \\
\hline 2028 & CR353625 & -4.33 & $5.87 \mathrm{E}-04$ \\
\hline 2029 & CR353639 & -5.24 & $1.19 \mathrm{E}-03$ \\
\hline 2030 & CR353664 & -1.16 & $4.30 \mathrm{E}-02$ \\
\hline 2031 & CR353684 & -2.63 & $2.60 \mathrm{E}-04$ \\
\hline 2032 & CR353734 & $\begin{array}{l}-7.14 \\
\end{array}$ & $2.01 \mathrm{E}-03$ \\
\hline 2033 & CR353739 & -7.45 & $3.26 \mathrm{E}-04$ \\
\hline 2034 & CR353740 & -5.66 & $8.14 \mathrm{E}-04$ \\
\hline 2035 & CR353748 & -4.62 & $2.08 \mathrm{E}-04$ \\
\hline 2036 & CR353800 & -5.84 & $7.35 \mathrm{E}-05$ \\
\hline 2037 & CR353803 & -3.63 & $8.64 \mathrm{E}-04$ \\
\hline 2038 & CR353842 & -2.00 & $1.21 \mathrm{E}-02$ \\
\hline 2039 & CR353857 & -6.00 & $1.33 \mathrm{E}-04$ \\
\hline 2040 & CR353877 & -2.81 & $2.93 \mathrm{E}-03$ \\
\hline 2041 & $\begin{array}{lc}\text { CR353881 } \\
\end{array}$ & -2.88 & $2.62 \mathrm{E}-04$ \\
\hline 2042 & CR353897 & -3.46 & $1.33 \mathrm{E}-03$ \\
\hline 2043 & CR353916 & -4.92 & $1.24 \mathrm{E}-04$ \\
\hline 2044 & CR353924 & -2.62 & $4.96 \mathrm{E}-03$ \\
\hline 2045 & CR353937 & -3.21 & $3.83 \mathrm{E}-03$ \\
\hline 2046 & CR353954 & -4.27 & $2.22 \mathrm{E}-03$ \\
\hline 2047 & CR353959 & -1.47 & $1.40 \mathrm{E}-02$ \\
\hline 2048 & CR353976 & -2.36 & $4.57 \mathrm{E}-03$ \\
\hline 2049 & CR353985 & -2.35 & $1.13 \mathrm{E}-03$ \\
\hline 2050 & CR354023 & -1.45 & $2.04 \mathrm{E}-02$ \\
\hline 2051 & $\begin{array}{ll}\text { CR354039 } \\
\end{array}$ & $\begin{array}{l}-2.03 \\
\end{array}$ & $1.28 \mathrm{E}-02$ \\
\hline 2052 & CR354042 & -1.66 & $1.37 \mathrm{E}-02$ \\
\hline 2053 & CR354044 & -3.27 & $6.38 \mathrm{E}-04$ \\
\hline 2054 & CR354076 & -2.73 & $8.57 \mathrm{E}-03$ \\
\hline 2055 & CR354078 & -3.48 & $5.69 \mathrm{E}-03$ \\
\hline 2056 & CR354080 & -1.16 & $4.47 \mathrm{E}-03$ \\
\hline 2057 & $\begin{array}{ll}\text { CR354084 } \\
\end{array}$ & -2.13 & $3.77 \mathrm{E}-03$ \\
\hline 2058 & CR354091 & -4.63 & $6.24 \mathrm{E}-06$ \\
\hline 2059 & CR354096 & -2.85 & $1.41 \mathrm{E}-03$ \\
\hline 2060 & CR354116 & -7.04 & $2.81 \mathrm{E}-05$ \\
\hline 2061 & CR354117 & -2.73 & $1.35 \mathrm{E}-02$ \\
\hline 2062 & CR354122 & -2.84 & $2.02 \mathrm{E}-04$ \\
\hline 2063 & CR354123 & -3.37 & $8.53 \mathrm{E}-03$ \\
\hline 2064 & CR354127 & -4.19 & $5.75 \mathrm{E}-04$ \\
\hline 2065 & CR354132 & -2.88 & $9.56 \mathrm{E}-04$ \\
\hline 2066 & CR354135 & -1.42 & $8.14 \mathrm{E}-04$ \\
\hline 2067 & CR354143 & -7.22 & $1.52 \mathrm{E}-04$ \\
\hline 2068 & CR354145 & -4.60 & $2.10 \mathrm{E}-03$ \\
\hline 2069 & CR354151 & -5.92 & 4.44E-04 \\
\hline 2070 & CR354172 & -1.55 & $5.88 \mathrm{E}-03$ \\
\hline 2071 & $\begin{array}{l}\text { CR354180 } \\
\end{array}$ & -1.67 & $6.06 \mathrm{E}-02$ \\
\hline 2072 & CR354183 & -2.42 & $4.83 \mathrm{E}-04$ \\
\hline 2073 & CR354192 & -8.05 & $5.07 \mathrm{E}-03$ \\
\hline 2074 & CR354195 & -1.50 & $1.37 \mathrm{E}-02$ \\
\hline 2075 & CR354203 & -4.55 & $2.51 \mathrm{E}-04$ \\
\hline 2076 & CR354213 & -5.67 & $1.30 \mathrm{E}-03$ \\
\hline 2077 & CR354222 & -1.49 & $3.94 \mathrm{E}-03$ \\
\hline 2078 & CR354230 & -3.92 & $3.57 \mathrm{E}-05$ \\
\hline
\end{tabular}




\begin{tabular}{|c|c|c|c|}
\hline 2079 & CR354259 & -3.67 & $6.81 \mathrm{E}-04$ \\
\hline 2080 & CR354271 & \begin{tabular}{|l|}
-2.03 \\
\end{tabular} & $6.38 \mathrm{E}-03$ \\
\hline 2081 & CR354275 & \begin{tabular}{|l|}
-1.38 \\
\end{tabular} & $2.05 \mathrm{E}-02$ \\
\hline 2082 & CR354278 & -2.56 & $1.10 \mathrm{E}-03$ \\
\hline 2083 & CR354291 & -4.00 & $5.82 \mathrm{E}-03$ \\
\hline 2084 & CR354305 & -5.44 & $6.46 \mathrm{E}-04$ \\
\hline 2085 & CR354306 & \begin{tabular}{|l|}
-7.90 \\
\end{tabular} & $3.00 \mathrm{E}-05$ \\
\hline 2086 & $\begin{array}{lcl}\text { CR354316 } & \\
\end{array}$ & -8.12 & $1.13 \mathrm{E}-03$ \\
\hline 2087 & CR354329 & -5.25 & $1.75 \mathrm{E}-04$ \\
\hline 2088 & CR354332 & -4.86 & $2.54 \mathrm{E}-04$ \\
\hline 2089 & CR354338 & -1.46 & $6.14 \mathrm{E}-03$ \\
\hline 2090 & CR354340 & $\begin{array}{l}-2.58 \\
\end{array}$ & $1.73 \mathrm{E}-02$ \\
\hline 2091 & 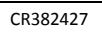 & \begin{tabular}{|l|}
-2.31 \\
\end{tabular} & $1.85 \mathrm{E}-02$ \\
\hline 2092 & $\begin{array}{lcl}\text { CR385096 } & \\
\end{array}$ & $\begin{array}{l}-6.13 \\
\end{array}$ & $9.43 \mathrm{E}-04$ \\
\hline 2093 & CR385102 & -3.47 & $2.58 \mathrm{E}-03$ \\
\hline 2094 & CR385106 & -4.02 & $7.69 \mathrm{E}-03$ \\
\hline 2095 & CR385108 & -2.67 & $1.47 \mathrm{E}-03$ \\
\hline 2096 & $\begin{array}{lc}\text { CR385119 } \\
\end{array}$ & -5.59 & $1.40 \mathrm{E}-03$ \\
\hline 2097 & CR385126 & -2.67 & $1.91 \mathrm{E}-07$ \\
\hline 2098 & CR385135 & -1.20 & $5.19 \mathrm{E}-03$ \\
\hline 2099 & CR385141 & -5.16 & $2.30 \mathrm{E}-04$ \\
\hline 2100 & CR385142 & \begin{tabular}{|l|}
-3.82 \\
\end{tabular} & $1.75 \mathrm{E}-03$ \\
\hline 2101 & CR385145 & -5.97 & $3.65 E-04$ \\
\hline 2102 & CR385155 & \begin{tabular}{|l|}
-5.81 \\
\end{tabular} & $5.63 \mathrm{E}-04$ \\
\hline 2103 & CR385160 & -1.71 & $1.59 \mathrm{E}-03$ \\
\hline 2104 & CR385161 & $\begin{array}{l}-1.47 \\
\end{array}$ & $2.08 \mathrm{E}-03$ \\
\hline 2105 & CR385162 & -2.89 & $6.83 \mathrm{E}-04$ \\
\hline 2106 & CR385164 & $\begin{array}{l}-2.69 \\
\end{array}$ & $5.91 \mathrm{E}-03$ \\
\hline 2107 & CR385166 & -5.94 & $2.12 \mathrm{E}-03$ \\
\hline 2108 & CR385169 & \begin{tabular}{|l|}
-2.59 \\
\end{tabular} & $4.37 \mathrm{E}-02$ \\
\hline 2109 & CR385171 & -1.39 & $3.04 E-02$ \\
\hline 2110 & $\begin{array}{lc}\text { CR385173 } \\
\end{array}$ & -3.25 & $1.45 \mathrm{E}-03$ \\
\hline 2111 & CR385195 & -2.13 & $3.62 \mathrm{E}-02$ \\
\hline 2112 & $\begin{array}{lcl}\text { CR385196 } \\
\end{array}$ & \begin{tabular}{|l|}
-2.78 \\
\end{tabular} & $2.02 E-03$ \\
\hline 2113 & CR385198 & -2.11 & $4.82 \mathrm{E}-03$ \\
\hline 2114 & CR385199 & -4.70 & $4.05 E-02$ \\
\hline 2115 & CR385217 & -3.74 & $2.37 \mathrm{E}-02$ \\
\hline 2116 & CR385225 & $\begin{array}{l}-1.46 \\
\end{array}$ & $2.55 \mathrm{E}-02$ \\
\hline 2117 & CR385228 & -3.38 & $2.65 \mathrm{E}-03$ \\
\hline 2118 & CR385236 & \begin{tabular}{|l|}
-1.92 \\
\end{tabular} & $3.32 \mathrm{E}-03$ \\
\hline 2119 & CR385245 & -3.39 & $1.24 \mathrm{E}-04$ \\
\hline 2120 & CR385250 & -1.55 & $1.37 \mathrm{E}-01$ \\
\hline 2121 & CR385262 & $\begin{array}{l}-1.48 \\
\end{array}$ & $2.10 \mathrm{E}-02$ \\
\hline 2122 & 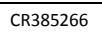 & \begin{tabular}{|l|}
-2.64 \\
\end{tabular} & $5.31 \mathrm{E}-03$ \\
\hline 2123 & $\begin{array}{ll}\text { CR385267 } \\
\end{array}$ & $\begin{array}{l}-2.42 \\
\end{array}$ & $2.35 \mathrm{E}-03$ \\
\hline 2124 & CR385268 & -3.28 & $8.99 \mathrm{E}-03$ \\
\hline 2125 & CR385287 & $\begin{array}{l}-4.88 \\
\end{array}$ & $1.31 \mathrm{E}-04$ \\
\hline 2126 & $\begin{array}{lc}\text { CR385297 } \\
\end{array}$ & \begin{tabular}{|l|}
-2.89 \\
\end{tabular} & $1.09 \mathrm{E}-04$ \\
\hline 2127 & \begin{tabular}{lc|} 
CR385306 \\
\end{tabular} & $\begin{array}{l}-1.29 \\
\end{array}$ & $4.58 \mathrm{E}-02$ \\
\hline 2128 & CR385307 & -4.69 & $2.01 \mathrm{E}-03$ \\
\hline 2129 & CR385310 & -4.45 & $4.95 \mathrm{E}-04$ \\
\hline 2130 & CR385317 & -7.36 & $5.11 \mathrm{E}-04$ \\
\hline 2131 & CR385325 & -2.32 & $3.27 \mathrm{E}-03$ \\
\hline 2132 & CR385330 & -5.47 & $3.09 \mathrm{E}-04$ \\
\hline 2133 & 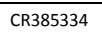 & \begin{tabular}{|l|}
-3.47 \\
\end{tabular} & $3.51 \mathrm{E}-04$ \\
\hline 2134 & CR385338 & -1.26 & $1.28 \mathrm{E}-02$ \\
\hline 2135 & CR385352 & \begin{tabular}{|l|}
-1.23 \\
\end{tabular} & $4.94 \mathrm{E}-03$ \\
\hline 2136 & CR385353 & -1.61 & $1.72 \mathrm{E}-02$ \\
\hline 2137 & CR385367 & $\begin{array}{l}-1.63 \\
\end{array}$ & $8.03 \mathrm{E}-03$ \\
\hline 2138 & CR385389 & -1.06 & $3.99 \mathrm{E}-03$ \\
\hline 2139 & CR385393 & -2.86 & $3.24 \mathrm{E}-03$ \\
\hline 2140 & CR385396 & -4.81 & $1.62 \mathrm{E}-03$ \\
\hline
\end{tabular}

\begin{tabular}{|c|c|c|c|}
\hline 2142 & CR385412 & -3.93 & $1.44 \mathrm{E}-02$ \\
\hline 2143 & CR385427 & -2.25 & $3.56 \mathrm{E}-04$ \\
\hline 2144 & CR385460 & -1.93 & $1.71 \mathrm{E}-03$ \\
\hline 2145 & CR385463 & -5.89 & $6.81 \mathrm{E}-04$ \\
\hline 2146 & CR385464 & -3.44 & $9.42 \mathrm{E}-04$ \\
\hline 2147 & CR385473 & -1.88 & $1.46 \mathrm{E}-02$ \\
\hline 2148 & CR385491 & -6.69 & $7.31 \mathrm{E}-04$ \\
\hline 2149 & CR385510 & -1.87 & $6.03 \mathrm{E}-03$ \\
\hline 2150 & CR385519 & -4.64 & $6.61 \mathrm{E}-04$ \\
\hline 2151 & CR385522 & -2.34 & $8.38 \mathrm{E}-04$ \\
\hline 2152 & CR385528 & -3.27 & $4.12 \mathrm{E}-03$ \\
\hline 2153 & CR385539 & -5.35 & $6.97 \mathrm{E}-03$ \\
\hline 2154 & CR385554 & -2.68 & $3.46 \mathrm{E}-03$ \\
\hline 2155 & CR385565 & -4.92 & $4.98 \mathrm{E}-04$ \\
\hline 2156 & CR385569 & -4.42 & $4.13 \mathrm{E}-03$ \\
\hline 2157 & CR385578 & -2.27 & $2.28 \mathrm{E}-02$ \\
\hline 2158 & CR385585 & -3.27 & $3.14 \mathrm{E}-03$ \\
\hline 2159 & CR385594 & -5.00 & $4.36 \mathrm{E}-03$ \\
\hline 2160 & CR385601 & -6.54 & $4.72 \mathrm{E}-04$ \\
\hline 2161 & CR385608 & -2.76 & $3.01 \mathrm{E}-04$ \\
\hline 2162 & CR385614 & -2.61 & $6.71 \mathrm{E}-04$ \\
\hline 2163 & CR385627 & -2.59 & $4.12 \mathrm{E}-03$ \\
\hline 2164 & CR385643 & -1.86 & $2.60 \mathrm{E}-02$ \\
\hline 2165 & CR385645 & -1.76 & $1.33 \mathrm{E}-03$ \\
\hline 2166 & CR385648 & -3.67 & $6.39 \mathrm{E}-03$ \\
\hline 2167 & CR385657 & -2.58 & $3.57 \mathrm{E}-03$ \\
\hline 2168 & CR385663 & -3.50 & $5.48 \mathrm{E}-04$ \\
\hline 2169 & CR385680 & -4.91 & $1.78 \mathrm{E}-03$ \\
\hline 2170 & CR385682 & -2.98 & $1.50 \mathrm{E}-03$ \\
\hline 2171 & CR385689 & -1.15 & $4.35 \mathrm{E}-03$ \\
\hline 2172 & CR385696 & -1.08 & $5.08 \mathrm{E}-02$ \\
\hline 2173 & CR385698 & -2.17 & $8.94 \mathrm{E}-03$ \\
\hline 2174 & CR385700 & -1.59 & $7.57 \mathrm{E}-03$ \\
\hline 2175 & CR385701 & -3.08 & $1.49 \mathrm{E}-03$ \\
\hline 2176 & CR385721 & -2.31 & $1.42 \mathrm{E}-04$ \\
\hline 2177 & CR385733 & -1.76 & $1.00 \mathrm{E}-02$ \\
\hline 2178 & CR385747 & -4.36 & $3.40 \mathrm{E}-03$ \\
\hline 2179 & CR385757 & -3.60 & $2.22 \mathrm{E}-03$ \\
\hline 2180 & CR385759 & -2.35 & $8.11 \mathrm{E}-04$ \\
\hline 2181 & CR385765 & -3.93 & $1.64 \mathrm{E}-03$ \\
\hline 2182 & CR385768 & -2.06 & $7.95 \mathrm{E}-03$ \\
\hline 2183 & CR385780 & -4.15 & $3.92 \mathrm{E}-05$ \\
\hline 2184 & CR385808 & -5.45 & $1.07 \mathrm{E}-04$ \\
\hline 2185 & CR385811 & -3.63 & $1.39 \mathrm{E}-03$ \\
\hline 2186 & CR385813 & -2.68 & $8.65 \mathrm{E}-04$ \\
\hline 2187 & CR385817 & -4.27 & $5.06 \mathrm{E}-04$ \\
\hline 2188 & CR385819 & -1.99 & $4.40 \mathrm{E}-02$ \\
\hline 2189 & CR385841 & -3.20 & $5.92 \mathrm{E}-04$ \\
\hline 2190 & CR385850 & -1.12 & $4.99 \mathrm{E}-03$ \\
\hline 2191 & CR385853 & -3.44 & $4.16 \mathrm{E}-04$ \\
\hline 2192 & CR385858 & -3.16 & $1.74 \mathrm{E}-03$ \\
\hline 2193 & CR385866 & -3.98 & 7.03E-04 \\
\hline 2194 & CR385869 & -1.79 & $1.51 \mathrm{E}-03$ \\
\hline 2195 & CR385870 & -3.94 & $2.24 \mathrm{E}-03$ \\
\hline 2196 & CR385879 & -1.45 & $1.76 \mathrm{E}-02$ \\
\hline 2197 & CR385880 & -1.69 & $7.98 \mathrm{E}-03$ \\
\hline 2198 & CR385885 & -1.94 & $1.21 \mathrm{E}-02$ \\
\hline 2199 & CR385912 & -1.38 & $1.90 \mathrm{E}-02$ \\
\hline 2200 & CR385950 & -2.91 & $9.00 \mathrm{E}-03$ \\
\hline 2201 & CR385956 & -4.76 & $8.78 \mathrm{E}-04$ \\
\hline 2202 & CR385972 & -1.74 & $1.50 \mathrm{E}-02$ \\
\hline 2203 & 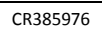 & -2.10 & $4.04 \mathrm{E}-04$ \\
\hline 2204 & CR385977 & -3.30 & $4.83 \mathrm{E}-04$ \\
\hline
\end{tabular}

\begin{tabular}{|c|c|c|c|}
\hline 2205 & CR385979 & -1.25 & $1.46 \mathrm{E}-03$ \\
\hline 2206 & CR385982 & -1.31 & $6.78 \mathrm{E}-03$ \\
\hline 2207 & CR385983 & -5.94 & $9.77 \mathrm{E}-04$ \\
\hline 2208 & CR385999 & -1.82 & $3.11 \mathrm{E}-03$ \\
\hline 2209 & CR386009 & -1.43 & $3.15 \mathrm{E}-02$ \\
\hline 2210 & CR386015 & -4.81 & $3.71 E-04$ \\
\hline 2211 & CR386026 & -4.63 & $2.26 \mathrm{E}-03$ \\
\hline 2212 & CR386037 & -2.34 & $1.69 \mathrm{E}-02$ \\
\hline 2213 & CR386047 & -4.54 & $1.38 E-03$ \\
\hline 2214 & CR386053 & -3.54 & $1.48 \mathrm{E}-03$ \\
\hline 2215 & CR386056 & -3.71 & $9.54 \mathrm{E}-04$ \\
\hline 2216 & CR386068 & -4.39 & $6.64 E-05$ \\
\hline 2217 & CR386075 & -2.54 & $2.56 \mathrm{E}-03$ \\
\hline 2218 & CR386094 & -2.15 & $2.02 E-03$ \\
\hline 2219 & CR386101 & -4.04 & $1.94 E-03$ \\
\hline 2220 & CR386103 & -1.43 & $7.58 \mathrm{E}-03$ \\
\hline 2221 & CR386113 & -5.41 & $1.99 \mathrm{E}-03$ \\
\hline 2222 & CR386119 & -4.71 & $9.60 E-05$ \\
\hline 2223 & CR386128 & -4.86 & $1.50 \mathrm{E}-04$ \\
\hline 2224 & CR386155 & -1.62 & $5.57 \mathrm{E}-04$ \\
\hline 2225 & CR386156 & -1.53 & $8.81 E-03$ \\
\hline 2226 & CR386166 & -1.42 & $5.24 \mathrm{E}-02$ \\
\hline 2227 & CR386170 & -2.12 & $5.89 \mathrm{E}-03$ \\
\hline 2228 & CR386175 & -3.26 & $2.82 E-04$ \\
\hline 2229 & CR386187 & -1.58 & $1.07 E-02$ \\
\hline 2230 & CR386188 & -3.95 & $8.57 \mathrm{E}-04$ \\
\hline 2231 & CR386190 & -2.35 & $5.00 E-03$ \\
\hline 2232 & CR386194 & -1.76 & $1.13 \mathrm{E}-02$ \\
\hline 2233 & CR386202 & -1.79 & $1.95 \mathrm{E}-02$ \\
\hline 2234 & CR386203 & -2.98 & $3.34 \mathrm{E}-03$ \\
\hline 2235 & CR386207 & -2.18 & $5.88 \mathrm{E}-03$ \\
\hline 2236 & CR386213 & -4.10 & $1.39 \mathrm{E}-03$ \\
\hline 2237 & CR386215 & -4.51 & $2.42 E-04$ \\
\hline 2238 & CR386224 & -4.03 & $1.95 E-03$ \\
\hline 2239 & CR386226 & -5.20 & $2.36 E-05$ \\
\hline 2240 & CR386231 & -1.31 & $4.00 E-02$ \\
\hline 2241 & CR386245 & -1.29 & $2.04 E-02$ \\
\hline 2242 & CR386247 & -3.63 & $1.95 E-03$ \\
\hline 2243 & CR386248 & -2.20 & $6.58 \mathrm{E}-03$ \\
\hline 2244 & CR386254 & -5.19 & $1.67 E-04$ \\
\hline 2245 & CR386257 & -3.14 & $4.56 \mathrm{E}-04$ \\
\hline 2246 & CR386261 & -1.47 & $2.39 \mathrm{E}-03$ \\
\hline 2247 & CR386268 & -2.39 & $1.01 E-02$ \\
\hline 2248 & CR386270 & -3.61 & $9.64 E-05$ \\
\hline 2249 & CR386273 & -2.28 & $1.50 \mathrm{E}-02$ \\
\hline 2250 & CR386280 & -3.97 & $2.46 \mathrm{E}-03$ \\
\hline 2251 & CR386282 & -2.00 & $2.59 \mathrm{E}-02$ \\
\hline 2252 & CR386284 & -3.39 & $5.03 E-04$ \\
\hline 2253 & CR386289 & -5.29 & $2.03 E-04$ \\
\hline 2254 & CR386291 & -2.21 & $2.59 \mathrm{E}-03$ \\
\hline 2255 & CR386297 & -1.78 & $5.54 \mathrm{E}-03$ \\
\hline 2256 & CR386304 & -2.38 & $5.65 \mathrm{E}-03$ \\
\hline 2257 & CR386306 & -2.34 & $5.99 E-04$ \\
\hline 2258 & CR386307 & -2.44 & $4.10 \mathrm{E}-04$ \\
\hline 2259 & CR386311 & -4.40 & $2.65 E-04$ \\
\hline 2260 & CR386312 & -1.38 & $5.77 \mathrm{E}-03$ \\
\hline 2261 & CR386323 & -2.09 & $2.55 \mathrm{E}-02$ \\
\hline 2262 & CR386327 & -3.18 & $1.16 \mathrm{E}-03$ \\
\hline 2263 & CR386334 & -2.22 & $6.03 E-05$ \\
\hline 2264 & CR386349 & -3.28 & 7.87E-04 \\
\hline 2265 & CR386379 & -3.60 & $2.98 \mathrm{E}-03$ \\
\hline 2266 & CR386387 & -2.62 & $3.11 E-03$ \\
\hline 2267 & CR386389 & -5.37 & $5.32 \mathrm{E}-05$ \\
\hline
\end{tabular}




\begin{tabular}{|c|c|c|c|}
\hline 2268 & CR386391 & -1.43 & $1.35 \mathrm{E}-02$ \\
\hline 2269 & CR386393 & -2.84 & $1.08 \mathrm{E}-04$ \\
\hline 2270 & CR386398 & -5.38 & $4.49 \mathrm{E}-04$ \\
\hline 2271 & CR386411 & -2.57 & $6.43 \mathrm{E}-03$ \\
\hline 2272 & CR386452 & -3.50 & $4.01 E-03$ \\
\hline 2273 & CR386456 & -2.25 & $5.01 \mathrm{E}-03$ \\
\hline 2274 & CR386468 & -4.33 & 9.58E-04 \\
\hline 2275 & CR386470 & -2.06 & $5.33 \mathrm{E}-03$ \\
\hline 2276 & CR386477 & -1.56 & $3.72 E-02$ \\
\hline 2277 & CR386492 & -4.02 & $1.96 \mathrm{E}-03$ \\
\hline 2278 & CR386493 & -1.12 & $3.53 \mathrm{E}-02$ \\
\hline 2279 & CR386494 & -1.28 & $2.33 \mathrm{E}-02$ \\
\hline 2280 & CR386498 & -1.25 & $3.11 E-03$ \\
\hline 2281 & CR386501 & -4.14 & 3.44E-04 \\
\hline 2282 & CR386506 & -3.55 & $1.11 \mathrm{E}-02$ \\
\hline 2283 & CR386515 & -6.95 & $1.70 \mathrm{E}-05$ \\
\hline 2284 & CR386526 & \begin{tabular}{|l|}
-2.14 \\
\end{tabular} & $5.03 E-03$ \\
\hline 2285 & CR386528 & \begin{tabular}{|l|}
-3.61 \\
\end{tabular} & $4.61 \mathrm{E}-04$ \\
\hline 2286 & CR386532 & -4.77 & 2.69E-04 \\
\hline 2287 & CR386543 & -2.54 & $1.24 \mathrm{E}-03$ \\
\hline 2288 & CR386560 & -2.82 & $1.72 E-02$ \\
\hline 2289 & CR386567 & -4.70 & $3.12 E-04$ \\
\hline 2290 & CR386568 & -2.32 & $2.21 \mathrm{E}-02$ \\
\hline 2291 & CR386570 & -3.97 & $8.01 E-04$ \\
\hline 2292 & CR386573 & -2.81 & $1.65 \mathrm{E}-04$ \\
\hline 2293 & CR386580 & -3.69 & $3.70 E-05$ \\
\hline 2294 & CR386584 & -3.62 & $1.96 \mathrm{E}-03$ \\
\hline 2295 & CR386592 & -4.84 & $3.24 E-04$ \\
\hline 2296 & CR386599 & \begin{tabular}{|c|}
-1.68 \\
\end{tabular} & 3.33E-02 \\
\hline 2297 & CR386601 & -4.65 & $1.03 E-03$ \\
\hline 2298 & CR386602 & -2.95 & $6.73 \mathrm{E}-03$ \\
\hline 2299 & CR386612 & -5.13 & $4.92 \mathrm{E}-04$ \\
\hline 2300 & CR386618 & -5.27 & $8.88 \mathrm{E}-04$ \\
\hline 2301 & $\begin{array}{lcl}\text { CR386619 } \\
\end{array}$ & -4.20 & $7.87 \mathrm{E}-02$ \\
\hline 2302 & CR386621 & -5.51 & $6.93 \mathrm{E}-04$ \\
\hline 2303 & CR386626 & -2.01 & $1.45 \mathrm{E}-02$ \\
\hline 2304 & CR386627 & -4.09 & $3.05 E-03$ \\
\hline 2305 & CR386629 & \begin{tabular}{|c|}
-3.89 \\
\end{tabular} & $1.94 \mathrm{E}-04$ \\
\hline 2306 & CR386661 & -1.79 & $2.11 \mathrm{E}-02$ \\
\hline 2307 & CR386670 & -3.17 & $7.78 E-03$ \\
\hline 2308 & CR386678 & -5.81 & $3.78 E-03$ \\
\hline 2309 & 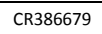 & -3.31 & $1.63 \mathrm{E}-03$ \\
\hline 2310 & CR386682 & -2.16 & $2.69 \mathrm{E}-03$ \\
\hline 2311 & CR386683 & -2.45 & $8.24 E-03$ \\
\hline 2312 & CR386701 & -1.11 & $4.60 \mathrm{E}-02$ \\
\hline 2313 & CR386711 & -4.55 & $1.13 \mathrm{E}-03$ \\
\hline 2314 & CR386729 & -2.86 & $1.10 \mathrm{E}-02$ \\
\hline 2315 & CR386730 & -2.70 & $2.31 E-03$ \\
\hline 2316 & CR386739 & -3.90 & $1.05 \mathrm{E}-04$ \\
\hline 2317 & CR386748 & -1.56 & $1.53 \mathrm{E}-02$ \\
\hline 2318 & CR386764 & -6.08 & $1.12 \mathrm{E}-04$ \\
\hline 2319 & CR386767 & -2.11 & $1.95 \mathrm{E}-03$ \\
\hline 2320 & CR386769 & -2.99 & $9.45 \mathrm{E}-04$ \\
\hline 2321 & CR386832 & -3.03 & $1.18 \mathrm{E}-03$ \\
\hline 2322 & CR386833 & -3.00 & $2.15 \mathrm{E}-04$ \\
\hline 2323 & CR386839 & -5.30 & $2.44 \mathrm{E}-03$ \\
\hline 2324 & CR386840 & -3.25 & $2.27 \mathrm{E}-03$ \\
\hline 2325 & CR386842 & -1.41 & $1.27 \mathrm{E}-03$ \\
\hline 2326 & CR386869 & -1.32 & $3.89 \mathrm{E}-02$ \\
\hline 2327 & CR386880 & -3.24 & $7.65 \mathrm{E}-04$ \\
\hline 2328 & CR386887 & -2.85 & $6.88 \mathrm{E}-05$ \\
\hline 2329 & CR386901 & -1.48 & $1.37 \mathrm{E}-03$ \\
\hline 2330 & & -1.93 & \\
\hline
\end{tabular}

\begin{tabular}{|c|c|c|c|}
\hline 2331 & CR386908 & -2.95 & $9.71 \mathrm{E}-03$ \\
\hline 2332 & CR386924 & -3.48 & $2.45 \mathrm{E}-03$ \\
\hline 2333 & CR386927 & -3.28 & $8.82 \mathrm{E}-04$ \\
\hline 2334 & CR386942 & -1.42 & $9.36 \mathrm{E}-03$ \\
\hline 2335 & \begin{tabular}{lc|} 
CR386946 \\
\end{tabular} & -3.66 & $3.88 \mathrm{E}-04$ \\
\hline 2336 & CR386949 & -3.93 & $1.44 \mathrm{E}-03$ \\
\hline 2337 & CR386954 & -4.46 & $4.09 \mathrm{E}-04$ \\
\hline 2338 & CR386967 & -2.08 & $2.03 \mathrm{E}-03$ \\
\hline 2339 & CR386980 & -3.82 & $5.48 \mathrm{E}-03$ \\
\hline 2340 & \begin{tabular}{lc|} 
CR386999 \\
\end{tabular} & -5.00 & $1.93 \mathrm{E}-03$ \\
\hline 2341 & CR387006 & -4.55 & $1.93 \mathrm{E}-03$ \\
\hline 2342 & CR387007 & -3.08 & $3.62 \mathrm{E}-04$ \\
\hline 2343 & CR387022 & -4.10 & $8.28 \mathrm{E}-04$ \\
\hline 2344 & $\begin{array}{l}\text { CR387028 } \\
\end{array}$ & \begin{tabular}{|l|}
-7.03 \\
\end{tabular} & $1.59 \mathrm{E}-04$ \\
\hline 2345 & CR387029 & -1.24 & $8.09 \mathrm{E}-03$ \\
\hline 2346 & \begin{tabular}{lc|} 
CR387038 \\
\end{tabular} & -1.80 & $2.80 \mathrm{E}-02$ \\
\hline 2347 & CR387039 & -2.15 & $1.17 \mathrm{E}-03$ \\
\hline 2348 & CR387051 & -3.78 & $2.83 \mathrm{E}-03$ \\
\hline 2349 & CR387056 & -2.11 & $1.82 \mathrm{E}-02$ \\
\hline 2350 & CR387057 & -1.87 & $5.90 \mathrm{E}-02$ \\
\hline 2351 & CR387058 & -4.97 & $9.11 \mathrm{E}-05$ \\
\hline 2352 & CR387062 & -2.27 & $1.49 \mathrm{E}-03$ \\
\hline 2353 & CR387063 & -4.02 & $1.45 \mathrm{E}-03$ \\
\hline 2354 & CR387069 & -3.46 & $3.66 \mathrm{E}-03$ \\
\hline 2355 & CR387081 & -2.65 & $1.05 \mathrm{E}-02$ \\
\hline 2356 & CR387083 & -6.49 & $3.05 E-05$ \\
\hline 2357 & CR387087 & -3.52 & $7.53 \mathrm{E}-04$ \\
\hline 2358 & CR387108 & -3.54 & $3.23 \mathrm{E}-03$ \\
\hline 2359 & CR387110 & -1.36 & $8.38 \mathrm{E}-04$ \\
\hline 2360 & CR387139 & -2.47 & $5.65 \mathrm{E}-03$ \\
\hline 2361 & CR387140 & -5.21 & $1.16 \mathrm{E}-04$ \\
\hline 2362 & 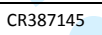 & -1.81 & $9.72 \mathrm{E}-03$ \\
\hline 2363 & CR387147 & -2.29 & $3.09 \mathrm{E}-03$ \\
\hline 2364 & 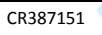 & -2.19 & $2.49 \mathrm{E}-02$ \\
\hline 2365 & CR387154 & -5.95 & $5.57 \mathrm{E}-04$ \\
\hline 2366 & CR387158 & -2.37 & $8.93 \mathrm{E}-03$ \\
\hline 2367 & CR387163 & -2.21 & $5.57 \mathrm{E}-03$ \\
\hline 2368 & CR387200 & -2.86 & $9.95 \mathrm{E}-04$ \\
\hline 2369 & CR387231 & -1.46 & $1.54 \mathrm{E}-02$ \\
\hline 2370 & \begin{tabular}{ll|} 
CR387238 \\
\end{tabular} & -3.12 & $7.88 \mathrm{E}-04$ \\
\hline 2371 & CR387243 & -3.45 & $7.95 \mathrm{E}-04$ \\
\hline 2372 & CR387246 & -2.80 & $3.61 \mathrm{E}-03$ \\
\hline 2373 & CR387248 & -1.76 & $1.36 \mathrm{E}-02$ \\
\hline 2374 & CR387255 & -3.41 & $3.87 \mathrm{E}-04$ \\
\hline 2375 & CR387256 & -4.91 & $1.43 \mathrm{E}-03$ \\
\hline 2376 & CR387258 & -1.87 & $4.36 \mathrm{E}-03$ \\
\hline 2377 & CR387269 & -3.84 & $3.68 \mathrm{E}-04$ \\
\hline 2378 & \begin{tabular}{lc|} 
CR387281 \\
\end{tabular} & -1.63 & $5.53 \mathrm{E}-02$ \\
\hline 2379 & CR387297 & -4.73 & $3.23 \mathrm{E}-03$ \\
\hline 2380 & CR387305 & -3.74 & $2.22 \mathrm{E}-04$ \\
\hline 2381 & CR387311 & -3.78 & $1.02 E-05$ \\
\hline 2382 & CR387312 & -3.26 & $1.58 \mathrm{E}-02$ \\
\hline 2383 & \begin{tabular}{lc|} 
CR387323 \\
\end{tabular} & -1.42 & $2.76 \mathrm{E}-03$ \\
\hline 2384 & CR387331 & -4.69 & $2.87 \mathrm{E}-03$ \\
\hline 2385 & CR387338 & -2.11 & $6.67 \mathrm{E}-04$ \\
\hline 2386 & CR387359 & -2.12 & $2.01 \mathrm{E}-03$ \\
\hline 2387 & CR387366 & -2.54 & $9.48 \mathrm{E}-04$ \\
\hline 2388 & CR387376 & -1.67 & $4.00 \mathrm{E}-03$ \\
\hline 2389 & CR387378 & -4.14 & $3.92 \mathrm{E}-03$ \\
\hline 2390 & CR387396 & -3.00 & $2.88 \mathrm{E}-04$ \\
\hline 2391 & CR387400 & -6.79 & $3.49 \mathrm{E}-04$ \\
\hline 2392 & CR387402 & -1.29 & $7.42 \mathrm{E}-03$ \\
\hline 2393 & $\begin{array}{ll}\text { CR387413 } \\
\end{array}$ & -1.80 & $2.83 \mathrm{E}-02$ \\
\hline
\end{tabular}

\begin{tabular}{|c|c|c|c|}
\hline 2394 & CR387417 & -4.06 & $1.04 \mathrm{E}-03$ \\
\hline 2395 & CR387429 & -1.75 & $1.98 \mathrm{E}-02$ \\
\hline 2396 & CR387431 & -7.30 & $3.36 \mathrm{E}-05$ \\
\hline 2397 & CR387438 & -1.66 & $2.19 \mathrm{E}-04$ \\
\hline 2398 & \begin{tabular}{ll|} 
CR387439 \\
\end{tabular} & -3.24 & $3.32 \mathrm{E}-03$ \\
\hline 2399 & CR387442 & -5.74 & $1.86 \mathrm{E}-04$ \\
\hline 2400 & CR387450 & -1.11 & $3.59 \mathrm{E}-04$ \\
\hline 2401 & CR387451 & -1.63 & $1.01 \mathrm{E}-02$ \\
\hline 2402 & CR387452 & -5.17 & $1.11 \mathrm{E}-03$ \\
\hline 2403 & CR387455 & -3.66 & $2.04 \mathrm{E}-03$ \\
\hline 2404 & $\begin{array}{ll}\text { CR387457 } \\
\end{array}$ & -1.46 & $5.40 \mathrm{E}-02$ \\
\hline 2405 & CR387468 & -7.08 & $2.91 \mathrm{E}-04$ \\
\hline 2406 & CR387473 & -1.84 & $2.88 \mathrm{E}-03$ \\
\hline 2407 & CR387479 & -2.50 & $8.69 \mathrm{E}-03$ \\
\hline 2408 & CR387493 & -1.49 & $1.51 \mathrm{E}-02$ \\
\hline 2409 & CR387499 & -4.85 & $9.27 \mathrm{E}-04$ \\
\hline 2410 & CR387500 & -6.40 & $7.99 \mathrm{E}-06$ \\
\hline 2411 & CR387502 & -3.50 & $2.75 \mathrm{E}-04$ \\
\hline 2412 & CR387504 & -3.71 & $1.17 \mathrm{E}-03$ \\
\hline 2413 & CR387515 & -1.10 & $1.41 \mathrm{E}-03$ \\
\hline 2414 & CR387517 & -5.12 & $9.16 \mathrm{E}-04$ \\
\hline 2415 & CR387523 & -3.69 & $5.85 \mathrm{E}-04$ \\
\hline 2416 & CR387527 & -1.67 & $9.52 \mathrm{E}-03$ \\
\hline 2417 & CR387530 & -3.77 & $6.00 \mathrm{E}-03$ \\
\hline 2418 & CR387539 & -3.19 & $6.14 \mathrm{E}-04$ \\
\hline 2419 & $\begin{array}{lc}\text { CR387543 } \\
\end{array}$ & -3.85 & $1.04 \mathrm{E}-02$ \\
\hline 2420 & CR387548 & $\begin{array}{l}-4.34 \\
\end{array}$ & $1.53 \mathrm{E}-03$ \\
\hline 2421 & CR387549 & -2.97 & 7.06E-04 \\
\hline 2422 & CR387551 & -3.54 & $1.32 \mathrm{E}-03$ \\
\hline 2423 & CR387574 & -1.21 & $3.00 \mathrm{E}-03$ \\
\hline 2424 & CR387583 & -6.22 & $7.62 \mathrm{E}-04$ \\
\hline 2425 & CR387625 & -2.19 & $2.74 \mathrm{E}-03$ \\
\hline 2426 & CR387628 & -4.47 & $2.79 \mathrm{E}-03$ \\
\hline 2427 & CR387632 & -2.40 & $1.21 \mathrm{E}-03$ \\
\hline 2428 & CR387638 & -1.75 & $7.57 \mathrm{E}-03$ \\
\hline 2429 & $\begin{array}{ll}\text { CR387642 } \\
\end{array}$ & -5.91 & $4.25 \mathrm{E}-04$ \\
\hline 2430 & CR387674 & -2.84 & $3.60 \mathrm{E}-03$ \\
\hline 2431 & CR387684 & -4.14 & $3.10 \mathrm{E}-03$ \\
\hline 2432 & CR387692 & -3.85 & $3.91 \mathrm{E}-03$ \\
\hline 2433 & CR387694 & -2.12 & $9.69 \mathrm{E}-04$ \\
\hline 2434 & CR387702 & -3.31 & $2.21 \mathrm{E}-03$ \\
\hline 2435 & $\begin{array}{ll}\text { CR387705 } \\
\end{array}$ & -5.55 & $\begin{array}{l}4.76 \mathrm{E}-03 \\
\end{array}$ \\
\hline 2436 & CR387716 & -1.93 & $2.40 \mathrm{E}-03$ \\
\hline 2437 & CR387724 & -1.58 & $1.80 \mathrm{E}-03$ \\
\hline 2438 & CR387725 & \begin{tabular}{|l|}
-3.18 \\
\end{tabular} & $9.49 \mathrm{E}-04$ \\
\hline 2439 & CR387726 & -3.52 & $1.56 \mathrm{E}-03$ \\
\hline 2440 & CR387732 & -4.56 & 7.04E-04 \\
\hline 2441 & CR387737 & -3.78 & $1.96 \mathrm{E}-03$ \\
\hline 2442 & CR387754 & -6.59 & $3.53 \mathrm{E}-04$ \\
\hline 2443 & CR387764 & -2.09 & $6.70 \mathrm{E}-03$ \\
\hline 2444 & CR387766 & -3.42 & $1.72 \mathrm{E}-04$ \\
\hline 2445 & CR387769 & -2.95 & $1.05 \mathrm{E}-03$ \\
\hline 2446 & CR387783 & -1.22 & $3.21 \mathrm{E}-02$ \\
\hline 2447 & CR387785 & -2.66 & $2.49 \mathrm{E}-03$ \\
\hline 2448 & CR387805 & -2.01 & $1.87 \mathrm{E}-02$ \\
\hline 2449 & CR387815 & -1.16 & $3.58 \mathrm{E}-02$ \\
\hline 2450 & CR387817 & -5.02 & $2.11 \mathrm{E}-03$ \\
\hline 2451 & CR387825 & -3.78 & $6.06 \mathrm{E}-04$ \\
\hline 2452 & CR387832 & -1.20 & $4.97 \mathrm{E}-03$ \\
\hline 2453 & CR387836 & -5.20 & $1.25 \mathrm{E}-03$ \\
\hline 2454 & CR387849 & -3.03 & $5.01 \mathrm{E}-03$ \\
\hline 2455 & CR387855 & -3.24 & $1.53 \mathrm{E}-03$ \\
\hline 2456 & CR387860 & -7.11 & $2.56 \mathrm{E}-03$ \\
\hline
\end{tabular}




\begin{tabular}{|c|c|c|c|}
\hline 2457 & CR387861 & -3.60 & $2.04 E-02$ \\
\hline 2458 & CR387872 & -3.05 & $2.87 \mathrm{E}-03$ \\
\hline 2459 & CR387879 & \begin{tabular}{|l|}
-1.47 \\
\end{tabular} & $8.19 \mathrm{E}-03$ \\
\hline 2460 & CR387888 & -1.99 & $3.12 \mathrm{E}-03$ \\
\hline 2461 & CR387898 & -2.52 & $1.53 \mathrm{E}-03$ \\
\hline 2462 & CR387899 & -3.11 & $1.96 \mathrm{E}-03$ \\
\hline 2463 & CR387903 & \begin{tabular}{|l|}
-1.88 \\
\end{tabular} & $6.11 \mathrm{E}-03$ \\
\hline 2464 & \begin{tabular}{lc|} 
CR388426 \\
\end{tabular} & -4.29 & $6.32 \mathrm{E}-04$ \\
\hline 2465 & CR388449 & -3.33 & $5.11 \mathrm{E}-04$ \\
\hline 2466 & CR388453 & -1.41 & $4.68 \mathrm{E}-02$ \\
\hline 2467 & CR388454 & -3.84 & $5.91 \mathrm{E}-04$ \\
\hline 2468 & CR388456 & -3.28 & $4.15 \mathrm{E}-04$ \\
\hline 2469 & $\begin{array}{ll}\text { CR388457 } \\
\end{array}$ & \begin{tabular}{|l|}
-2.74 \\
\end{tabular} & $4.03 \mathrm{E}-04$ \\
\hline 2470 & $\begin{array}{ll}\text { CR388459 } \\
\end{array}$ & -4.32 & $6.99 \mathrm{E}-04$ \\
\hline 2471 & CR388464 & -3.47 & $1.18 \mathrm{E}-03$ \\
\hline 2472 & CR388465 & -3.57 & $2.40 \mathrm{E}-03$ \\
\hline 2473 & CR388472 & -3.36 & $5.84 \mathrm{E}-04$ \\
\hline 2474 & \begin{tabular}{lc|} 
CR388478 \\
\end{tabular} & \begin{tabular}{|l|}
-3.88 \\
\end{tabular} & $2.40 \mathrm{E}-03$ \\
\hline 2475 & CR388483 & -2.21 & $3.63 \mathrm{E}-03$ \\
\hline 2476 & CR388489 & -3.21 & $5.09 \mathrm{E}-04$ \\
\hline 2477 & CR388491 & -3.04 & $3.39 \mathrm{E}-04$ \\
\hline 2478 & 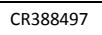 & \begin{tabular}{|l|}
-1.63 \\
\end{tabular} & $2.67 \mathrm{E}-02$ \\
\hline 2479 & CR388504 & -1.30 & $5.22 \mathrm{E}-03$ \\
\hline 2480 & $\begin{array}{ll}\text { CR388509 } \\
\end{array}$ & \begin{tabular}{|l|}
-1.38 \\
\end{tabular} & $1.95 \mathrm{E}-02$ \\
\hline 2481 & CR388514 & -1.53 & $4.87 \mathrm{E}-03$ \\
\hline 2482 & CR388524 & -3.95 & $2.37 \mathrm{E}-04$ \\
\hline 2483 & CR388526 & -3.73 & $2.14 \mathrm{E}-03$ \\
\hline 2484 & CR388531 & $\begin{array}{l}-1.55 \\
\end{array}$ & $3.08 \mathrm{E}-03$ \\
\hline 2485 & CR388539 & -1.75 & $2.36 \mathrm{E}-02$ \\
\hline 2486 & CR388542 & \begin{tabular}{l|l|}
-1.57 \\
\end{tabular} & $1.08 \mathrm{E}-02$ \\
\hline 2487 & CR388551 & -2.45 & $1.40 \mathrm{E}-03$ \\
\hline 2488 & 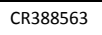 & -1.95 & $3.13 \mathrm{E}-03$ \\
\hline 2489 & CR388568 & -2.77 & $1.26 \mathrm{E}-03$ \\
\hline 2490 & CR388578 & \begin{tabular}{|l|}
-2.49 \\
\end{tabular} & $4.26 \mathrm{E}-04$ \\
\hline 2491 & CR388583 & -1.81 & $3.54 \mathrm{E}-04$ \\
\hline 2492 & CR388585 & -1.52 & $1.11 \mathrm{E}-02$ \\
\hline 2493 & CR388592 & -3.25 & $1.14 \mathrm{E}-02$ \\
\hline 2494 & CR388600 & -2.51 & $1.23 \mathrm{E}-02$ \\
\hline 2495 & CR388603 & -3.23 & $7.85 \mathrm{E}-05$ \\
\hline 2496 & CR388607 & \begin{tabular}{|l|}
-4.54 \\
\end{tabular} & $1.50 \mathrm{E}-03$ \\
\hline 2497 & CR388610 & -5.22 & $3.02 E-04$ \\
\hline 2498 & CR388611 & -7.22 & $1.96 \mathrm{E}-04$ \\
\hline 2499 & \begin{tabular}{lc|} 
CR388612 \\
\end{tabular} & \begin{tabular}{l|l|}
-2.58 \\
\end{tabular} & $2.30 \mathrm{E}-03$ \\
\hline 2500 & CR388617 & \begin{tabular}{|l|}
-1.13 \\
\end{tabular} & $4.39 \mathrm{E}-03$ \\
\hline 2501 & CR388623 & \begin{tabular}{|l|}
-3.24 \\
\end{tabular} & $1.90 \mathrm{E}-04$ \\
\hline 2502 & CR388641 & -3.39 & $1.34 \mathrm{E}-04$ \\
\hline 2503 & CR388647 & -1.75 & $1.04 \mathrm{E}-01$ \\
\hline 2504 & $\begin{array}{ll}\text { CR388654 } \\
\end{array}$ & \begin{tabular}{|l|}
-3.64 \\
\end{tabular} & $7.67 \mathrm{E}-04$ \\
\hline 2505 & $\begin{array}{ll}\text { CR388658 } \\
\end{array}$ & \begin{tabular}{|l|}
-3.66 \\
\end{tabular} & $1.70 \mathrm{E}-03$ \\
\hline 2506 & CR388659 & -5.28 & $1.28 \mathrm{E}-03$ \\
\hline 2507 & CR388666 & -1.61 & $3.51 \mathrm{E}-03$ \\
\hline 2508 & CR388672 & -3.50 & $2.62 \mathrm{E}-03$ \\
\hline 2509 & CR388683 & -4.53 & $2.25 \mathrm{E}-04$ \\
\hline 2510 & CR388685 & -2.95 & $2.08 \mathrm{E}-02$ \\
\hline 2511 & \begin{tabular}{lc|} 
CR388686 \\
\end{tabular} & $\begin{array}{l}-6.43 \\
\end{array}$ & $6.83 \mathrm{E}-05$ \\
\hline 2512 & CR388690 & -5.24 & 2.42E-04 \\
\hline 2513 & CR388700 & -4.35 & $2.17 \mathrm{E}-03$ \\
\hline 2514 & CR388705 & -2.97 & $1.48 \mathrm{E}-03$ \\
\hline 2515 & \begin{tabular}{lc|} 
CR388706 \\
\end{tabular} & \begin{tabular}{|l|}
-2.23 \\
\end{tabular} & $5.14 \mathrm{E}-04$ \\
\hline 2516 & CR388707 & -2.66 & $2.12 \mathrm{E}-05$ \\
\hline 2517 & CR388718 & -3.51 & $1.29 \mathrm{E}-03$ \\
\hline 2518 & CR388721 & -6.34 & $2.00 \mathrm{E}-05$ \\
\hline
\end{tabular}

\begin{tabular}{|c|c|c|c|}
\hline 2520 & CR388727 & -1.87 & $7.70 \mathrm{E}-03$ \\
\hline 2521 & CR388729 & -4.37 & $2.22 \mathrm{E}-03$ \\
\hline 2522 & CR388735 & -2.07 & $7.67 \mathrm{E}-04$ \\
\hline 2523 & CR388764 & -2.95 & $2.79 \mathrm{E}-03$ \\
\hline 2524 & CR388786 & -1.88 & $7.82 \mathrm{E}-03$ \\
\hline 2525 & CR388789 & -2.41 & $1.96 \mathrm{E}-04$ \\
\hline 2526 & CR388796 & -1.33 & $3.98 \mathrm{E}-02$ \\
\hline 2527 & CR388801 & -6.02 & $2.93 \mathrm{E}-04$ \\
\hline 2528 & CR388806 & -4.22 & $5.72 \mathrm{E}-04$ \\
\hline 2529 & CR388807 & -2.99 & $1.81 \mathrm{E}-02$ \\
\hline 2530 & CR388815 & -1.89 & $1.21 \mathrm{E}-02$ \\
\hline 2531 & CR388816 & -1.07 & $6.18 \mathrm{E}-02$ \\
\hline 2532 & CR388817 & -4.14 & $9.10 \mathrm{E}-07$ \\
\hline 2533 & CR388823 & -5.84 & $1.30 \mathrm{E}-03$ \\
\hline 2534 & CR388826 & -1.57 & $3.18 \mathrm{E}-02$ \\
\hline 2535 & CR388829 & -3.65 & $4.83 \mathrm{E}-04$ \\
\hline 2536 & CR388834 & -4.06 & $1.17 \mathrm{E}-03$ \\
\hline 2537 & CR388846 & -2.75 & $9.69 \mathrm{E}-04$ \\
\hline 2538 & CR388856 & -3.53 & $1.96 \mathrm{E}-03$ \\
\hline 2539 & CR388858 & -3.82 & $9.90 \mathrm{E}-04$ \\
\hline 2540 & CR388897 & -1.96 & $5.09 \mathrm{E}-02$ \\
\hline 2541 & CR388899 & -3.23 & $5.20 \mathrm{E}-03$ \\
\hline 2542 & CR388901 & -3.68 & $1.63 \mathrm{E}-03$ \\
\hline 2543 & CR388902 & -4.78 & $1.36 \mathrm{E}-03$ \\
\hline 2544 & CR388904 & -3.16 & $2.18 \mathrm{E}-04$ \\
\hline 2545 & CR388917 & -2.70 & $1.25 \mathrm{E}-02$ \\
\hline 2546 & CR388918 & -4.22 & $6.77 \mathrm{E}-03$ \\
\hline 2547 & CR388927 & -3.35 & $3.22 \mathrm{E}-03$ \\
\hline 2548 & CR388937 & -1.24 & $3.82 \mathrm{E}-03$ \\
\hline 2549 & CR388938 & -2.29 & $2.42 \mathrm{E}-03$ \\
\hline 2550 & CR388940 & -1.46 & $1.16 \mathrm{E}-02$ \\
\hline 2551 & CR388942 & -1.23 & $5.39 \mathrm{E}-03$ \\
\hline 2552 & CR388950 & -2.43 & $4.52 \mathrm{E}-03$ \\
\hline 2553 & CR388957 & -3.64 & $7.01 \mathrm{E}-03$ \\
\hline 2554 & CR388958 & -3.15 & $6.55 \mathrm{E}-05$ \\
\hline 2555 & CR388970 & -3.59 & $1.13 \mathrm{E}-03$ \\
\hline 2556 & CR388980 & -4.56 & $3.52 \mathrm{E}-04$ \\
\hline 2557 & CR388989 & -4.67 & $1.00 \mathrm{E}-03$ \\
\hline 2558 & CR388990 & -1.64 & $2.19 \mathrm{E}-03$ \\
\hline 2559 & CR388993 & -5.55 & $7.82 \mathrm{E}-04$ \\
\hline 2560 & CR388994 & -3.14 & 7.07E-05 \\
\hline 2561 & CR388995 & -2.80 & $9.25 \mathrm{E}-03$ \\
\hline 2562 & CR389003 & -3.53 & $1.97 \mathrm{E}-03$ \\
\hline 2563 & CR389004 & -1.59 & $3.16 \mathrm{E}-02$ \\
\hline 2564 & CR389010 & -5.33 & $1.21 \mathrm{E}-04$ \\
\hline 2565 & CR389017 & -2.42 & $1.63 \mathrm{E}-03$ \\
\hline 2566 & CR389018 & -2.88 & $8.41 \mathrm{E}-03$ \\
\hline 2567 & CR389022 & -4.11 & $8.75 \mathrm{E}-04$ \\
\hline 2568 & CR389036 & -3.42 & $3.47 \mathrm{E}-04$ \\
\hline 2569 & CR389041 & -1.35 & $1.55 \mathrm{E}-02$ \\
\hline 2570 & CR389049 & -4.13 & $1.19 \mathrm{E}-03$ \\
\hline 2571 & CR389060 & -1.49 & $4.00 \mathrm{E}-02$ \\
\hline 2572 & CR389064 & -1.72 & $1.07 \mathrm{E}-03$ \\
\hline 2573 & CR389070 & -2.86 & $1.97 \mathrm{E}-03$ \\
\hline 2574 & CR389072 & -2.56 & $9.83 \mathrm{E}-03$ \\
\hline 2575 & CR389091 & -3.29 & $2.26 \mathrm{E}-03$ \\
\hline 2576 & CR389092 & -2.96 & $1.28 \mathrm{E}-02$ \\
\hline 2577 & CR389094 & -4.22 & $5.66 \mathrm{E}-04$ \\
\hline 2578 & CR389099 & -3.01 & $1.24 \mathrm{E}-03$ \\
\hline 2579 & CR389116 & -4.97 & $1.30 \mathrm{E}-03$ \\
\hline 2580 & CR389124 & -2.35 & $1.09 \mathrm{E}-03$ \\
\hline 2581 & $\begin{array}{lc}\text { CR389126 } \\
\end{array}$ & -3.47 & $5.95 \mathrm{E}-04$ \\
\hline 2582 & CR389133 & -1.20 & $2.82 \mathrm{E}-02$ \\
\hline
\end{tabular}

\begin{tabular}{|c|c|c|c|}
\hline 2583 & CR389150 & -1.24 & $3.29 E-02$ \\
\hline 2584 & CR389158 & -3.73 & $\begin{array}{l}7.24 E-04 \\
\end{array}$ \\
\hline 2585 & CR389160 & -4.13 & $1.16 \mathrm{E}-03$ \\
\hline 2586 & CR389180 & -1.87 & $7.51 E-03$ \\
\hline 2587 & CR389182 & -2.60 & $7.85 E-04$ \\
\hline 2588 & CR389186 & -2.58 & $2.79 E-03$ \\
\hline 2589 & CR389189 & -2.84 & $1.51 \mathrm{E}-03$ \\
\hline 2590 & CR389195 & -5.90 & $1.23 E-03$ \\
\hline 2591 & CR389198 & -2.35 & $2.24 E-02$ \\
\hline 2592 & CR389221 & -2.65 & $1.37 E-04$ \\
\hline 2593 & CR389222 & -1.41 & $9.72 E-03$ \\
\hline 2594 & CR389223 & -1.38 & $5.63 \mathrm{E}-02$ \\
\hline 2595 & CR389234 & -4.11 & $2.04 E-03$ \\
\hline 2596 & CR389238 & -2.20 & $2.31 E-02$ \\
\hline 2597 & CR389242 & -2.68 & $2.35 E-03$ \\
\hline 2598 & CR389246 & -3.41 & $1.58 \mathrm{E}-03$ \\
\hline 2599 & CR389248 & -4.92 & $3.14 E-03$ \\
\hline 2600 & CR389260 & -3.39 & $2.57 \mathrm{E}-03$ \\
\hline 2601 & CR389266 & -3.20 & $5.37 \mathrm{E}-03$ \\
\hline 2602 & CR389270 & -6.70 & $8.30 \mathrm{E}-04$ \\
\hline 2603 & CR389278 & -2.37 & $2.22 E-05$ \\
\hline 2604 & CR389295 & -5.00 & $9.92 \mathrm{E}-03$ \\
\hline 2605 & CR389300 & -4.43 & $1.88 \mathrm{E}-03$ \\
\hline 2606 & CR389304 & -3.14 & $1.01 E-04$ \\
\hline 2607 & CR389305 & -3.19 & $3.06 E-03$ \\
\hline 2608 & CR389307 & -2.55 & $8.86 \mathrm{E}-03$ \\
\hline 2609 & CR389330 & -4.85 & $\begin{array}{l}.91 E-04 \\
\end{array}$ \\
\hline 2610 & CR389333 & -3.78 & $1.00 E-03$ \\
\hline 2611 & CR389353 & -2.67 & $3.22 E-03$ \\
\hline 2612 & CR389356 & -3.69 & $7.63 \mathrm{E}-04$ \\
\hline 2613 & CR389358 & -2.78 & $4.38 E-03$ \\
\hline 2614 & CR389369 & -1.34 & $2.34 \mathrm{E}-02$ \\
\hline 2615 & CR389371 & -4.06 & $1.11 \mathrm{E}-03$ \\
\hline 2616 & CR389374 & -3.59 & $8.01 E-04$ \\
\hline 2617 & CR389380 & -3.57 & $3.28 \mathrm{E}-03$ \\
\hline 2618 & CR389382 & -2.38 & $1.75 E-02$ \\
\hline 2619 & CR389385 & -2.61 & $7.27 E-03$ \\
\hline 2620 & CR389398 & -1.69 & $5.14 E-03$ \\
\hline 2621 & CR389410 & -3.50 & $2.51 E-04$ \\
\hline 2622 & CR389412 & -4.56 & $1.63 \mathrm{E}-03$ \\
\hline 2623 & CR389419 & -4.21 & $9.70 E-04$ \\
\hline 2624 & CR389421 & -1.40 & $3.53 \mathrm{E}-02$ \\
\hline 2625 & CR389434 & -4.16 & $6.42 E-03$ \\
\hline 2626 & CR389446 & -1.37 & $6.65 \mathrm{E}-03$ \\
\hline 2627 & CR389447 & -4.99 & $4.76 E-04$ \\
\hline 2628 & CR389451 & -2.96 & $2.38 \mathrm{E}-03$ \\
\hline 2629 & CR389454 & -2.86 & $1.02 E-02$ \\
\hline 2630 & CR389456 & -2.29 & $2.87 \mathrm{E}-02$ \\
\hline 2631 & CR389469 & -3.06 & $1.09 E-01$ \\
\hline 2632 & CR389476 & -3.17 & $1.36 \mathrm{E}-03$ \\
\hline 2633 & CR389477 & -1.98 & $3.30 \mathrm{E}-03$ \\
\hline 2634 & CR389494 & -1.39 & $6.31 \mathrm{E}-03$ \\
\hline 2635 & CR389495 & -2.79 & $3.24 E-03$ \\
\hline 2636 & CR389497 & -5.21 & $1.39 E-03$ \\
\hline 2637 & CR389505 & -1.89 & $6.40 \mathrm{E}-03$ \\
\hline 2638 & CR389506 & -3.71 & $3.12 \mathrm{E}-03$ \\
\hline 2639 & CR389521 & -2.29 & $3.54 \mathrm{E}-02$ \\
\hline 2640 & CR389524 & -1.62 & $6.17 \mathrm{E}-03$ \\
\hline 2641 & CR389533 & -3.11 & $5.39 E-04$ \\
\hline 2642 & CR389538 & -3.94 & $6.58 \mathrm{E}-04$ \\
\hline 2643 & CR389544 & -2.49 & $2.91 E-03$ \\
\hline 2644 & CR389546 & -2.62 & $1.22 E-02$ \\
\hline 645 & CR389558 & -2.62 & $1.10 \mathrm{E}-04$ \\
\hline
\end{tabular}




\begin{tabular}{|c|c|c|c|}
\hline 2646 & CR389567 & -4.22 & $8.36 \mathrm{E}-04$ \\
\hline 2647 & CR389571 & -1.36 & 7.69E-03 \\
\hline 2648 & CR389573 & \begin{tabular}{|c|}
-4.61 \\
\end{tabular} & $5.97 \mathrm{E}-04$ \\
\hline 2649 & CR389579 & -3.75 & $5.04 \mathrm{E}-04$ \\
\hline 2650 & CR389583 & -3.55 & $5.55 \mathrm{E}-04$ \\
\hline 2651 & CR389596 & -1.87 & $1.35 \mathrm{E}-03$ \\
\hline 2652 & CR389597 & -3.82 & $1.84 \mathrm{E}-04$ \\
\hline 2653 & CR389602 & -3.26 & $1.84 \mathrm{E}-04$ \\
\hline 2654 & CR389603 & \begin{tabular}{|c|}
-3.70 \\
\end{tabular} & $1.21 \mathrm{E}-03$ \\
\hline 2655 & CR389607 & -3.10 & $2.77 E-05$ \\
\hline 2656 & CR389614 & -2.92 & $1.36 \mathrm{E}-03$ \\
\hline 2657 & CR389616 & -1.47 & $2.06 \mathrm{E}-02$ \\
\hline 2658 & CR389623 & -1.76 & $4.18 \mathrm{E}-02$ \\
\hline 2659 & CR389627 & -1.35 & $1.03 \mathrm{E}-02$ \\
\hline 2660 & CR389636 & -2.80 & $2.90 E-03$ \\
\hline 2661 & CR389639 & \begin{tabular}{|l|}
-2.94 \\
\end{tabular} & $1.67 \mathrm{E}-03$ \\
\hline 2662 & CR389684 & -4.57 & $1.68 \mathrm{E}-04$ \\
\hline 2663 & CR389694 & -1.30 & $1.96 \mathrm{E}-02$ \\
\hline 2664 & CR389705 & -6.16 & $6.68 \mathrm{E}-03$ \\
\hline 2665 & CR389721 & -3.49 & $8.02 E-04$ \\
\hline 2666 & CR389728 & -1.64 & $9.25 \mathrm{E}-04$ \\
\hline 2667 & CR389732 & -3.81 & $6.92 E-04$ \\
\hline 2668 & CR389740 & -5.38 & $6.27 \mathrm{E}-04$ \\
\hline 2669 & CR389741 & -4.74 & $1.61 \mathrm{E}-05$ \\
\hline 2670 & CR389744 & -1.65 & $3.70 \mathrm{E}-03$ \\
\hline 2671 & CR389749 & -1.16 & $6.18 \mathrm{E}-03$ \\
\hline 2672 & CR389750 & -1.25 & $1.13 \mathrm{E}-02$ \\
\hline 2673 & CR389765 & \begin{tabular}{l|l|}
-2.91 \\
\end{tabular} & $1.49 E-05$ \\
\hline 2674 & CR389782 & -1.53 & $1.56 \mathrm{E}-02$ \\
\hline 2675 & CR389787 & -1.33 & $2.18 \mathrm{E}-03$ \\
\hline 2676 & CR389789 & -3.48 & $6.27 \mathrm{E}-04$ \\
\hline 2677 & CR389792 & -2.17 & $1.22 \mathrm{E}-02$ \\
\hline 2678 & CR389802 & -5.14 & $5.63 \mathrm{E}-03$ \\
\hline 2679 & CR389809 & -4.75 & $1.55 \mathrm{E}-03$ \\
\hline 2680 & CR389812 & -1.57 & 4.33E-02 \\
\hline 2681 & CR389818 & -4.71 & $3.83 \mathrm{E}-07$ \\
\hline 2682 & CR389819 & -5.66 & $2.12 \mathrm{E}-05$ \\
\hline 2683 & CR389821 & -2.35 & $1.06 \mathrm{E}-03$ \\
\hline 2684 & CR389822 & -2.21 & $9.49 \mathrm{E}-04$ \\
\hline 2685 & CR389833 & -5.72 & $6.90 \mathrm{E}-05$ \\
\hline 2686 & CR389834 & -5.69 & $2.37 \mathrm{E}-04$ \\
\hline 2687 & CR389853 & -4.33 & 2.67E-04 \\
\hline 2688 & CR389854 & -3.75 & $5.05 \mathrm{E}-05$ \\
\hline 2689 & CR389859 & -2.71 & $1.66 \mathrm{E}-03$ \\
\hline 2690 & CR389872 & -3.31 & $3.60 \mathrm{E}-03$ \\
\hline 2691 & CR389873 & -4.85 & 2.22E-03 \\
\hline 2692 & CR389887 & -1.26 & $2.15 \mathrm{E}-02$ \\
\hline 2693 & CR389889 & -4.12 & $1.27 E-03$ \\
\hline 2694 & CR389901 & -1.96 & $5.32 \mathrm{E}-03$ \\
\hline 2695 & CR389902 & -1.09 & $6.05 \mathrm{E}-03$ \\
\hline 2696 & CR389921 & -4.68 & $2.35 \mathrm{E}-04$ \\
\hline 2697 & CR389931 & -5.32 & $1.77 \mathrm{E}-03$ \\
\hline 2698 & CR389933 & -1.33 & $1.06 \mathrm{E}-02$ \\
\hline 2699 & CR389935 & -2.54 & $2.93 \mathrm{E}-03$ \\
\hline 2700 & CR389953 & -2.06 & $1.44 E-03$ \\
\hline 2701 & CR389954 & -3.03 & $1.40 \mathrm{E}-04$ \\
\hline 2702 & CR389965 & -3.87 & $1.09 E-03$ \\
\hline 2703 & CR389967 & -1.25 & $2.38 \mathrm{E}-02$ \\
\hline 2704 & CR389969 & -2.33 & $3.77 E-03$ \\
\hline 2705 & CR389970 & -1.13 & $2.02 \mathrm{E}-02$ \\
\hline 2706 & CR389977 & -2.64 & $1.96 \mathrm{E}-02$ \\
\hline 2707 & CR389978 & -3.27 & $1.80 \mathrm{E}-03$ \\
\hline 708 & 9984 & -1.25 & \\
\hline
\end{tabular}

\begin{tabular}{|c|c|c|c|}
\hline 2709 & CR389992 & -2.10 & $4.68 \mathrm{E}-03$ \\
\hline 2710 & CR389999 & -1.51 & $1.54 \mathrm{E}-03$ \\
\hline 2711 & CR390012 & -2.36 & $2.39 \mathrm{E}-03$ \\
\hline 2712 & CR390043 & -1.58 & $3.28 \mathrm{E}-04$ \\
\hline 2713 & CR390048 & -1.66 & $1.51 \mathrm{E}-03$ \\
\hline 2714 & CR390049 & -4.59 & $7.42 \mathrm{E}-04$ \\
\hline 2715 & CR390052 & -2.74 & $4.88 \mathrm{E}-03$ \\
\hline 2716 & CR390079 & -2.83 & $1.62 \mathrm{E}-02$ \\
\hline 2717 & CR390081 & -1.15 & $2.50 \mathrm{E}-02$ \\
\hline 2718 & CR390087 & -2.85 & $6.27 \mathrm{E}-04$ \\
\hline 2719 & CR390088 & -2.33 & $5.87 \mathrm{E}-03$ \\
\hline 2720 & CR390110 & -4.60 & $2.53 \mathrm{E}-03$ \\
\hline 2721 & CR390112 & -1.78 & $1.20 \mathrm{E}-02$ \\
\hline 2722 & CR390120 & -3.55 & $8.29 \mathrm{E}-03$ \\
\hline 2723 & CR390121 & -2.50 & $3.46 \mathrm{E}-04$ \\
\hline 2724 & CR390128 & -3.29 & $2.97 \mathrm{E}-03$ \\
\hline 2725 & CR390143 & -6.59 & $5.68 \mathrm{E}-05$ \\
\hline 2726 & CR390145 & -2.54 & $4.15 \mathrm{E}-03$ \\
\hline 2727 & CR390163 & -1.58 & $4.61 \mathrm{E}-02$ \\
\hline 2728 & CR390165 & -2.99 & $2.81 \mathrm{E}-03$ \\
\hline 2729 & CR390169 & -3.44 & $2.81 \mathrm{E}-03$ \\
\hline 2730 & CR390171 & -4.10 & $1.09 \mathrm{E}-03$ \\
\hline 2731 & CR390172 & -2.15 & $1.36 \mathrm{E}-02$ \\
\hline 2732 & CR390185 & -5.95 & $1.66 \mathrm{E}-03$ \\
\hline 2733 & CR390191 & -5.91 & $4.38 \mathrm{E}-04$ \\
\hline 2734 & CR390197 & -4.33 & $1.13 \mathrm{E}-02$ \\
\hline 2735 & CR390200 & -2.96 & 4.74E-03 \\
\hline 2736 & CR390202 & -1.49 & $2.52 \mathrm{E}-03$ \\
\hline 2737 & CR390211 & -3.03 & $1.36 \mathrm{E}-02$ \\
\hline 2738 & CR390216 & -1.95 & $4.36 \mathrm{E}-02$ \\
\hline 2739 & CR390227 & -3.04 & $6.91 \mathrm{E}-03$ \\
\hline 2740 & CR390230 & -2.64 & $4.75 \mathrm{E}-03$ \\
\hline 2741 & CR390234 & -4.50 & $5.78 \mathrm{E}-04$ \\
\hline 2742 & CR390260 & -1.77 & $4.24 \mathrm{E}-02$ \\
\hline 2743 & CR390264 & -1.39 & $2.39 \mathrm{E}-03$ \\
\hline 2744 & CR390274 & -2.38 & $4.60 \mathrm{E}-03$ \\
\hline 2745 & CR390276 & -4.49 & $2.16 \mathrm{E}-03$ \\
\hline 2746 & CR390304 & -2.07 & $1.34 \mathrm{E}-02$ \\
\hline 2747 & CR390310 & -3.39 & $1.22 \mathrm{E}-04$ \\
\hline 2748 & $\begin{array}{lc}\text { CR390315 } \\
\end{array}$ & -4.29 & $2.12 \mathrm{E}-03$ \\
\hline 2749 & CR390321 & -1.45 & $3.37 \mathrm{E}-03$ \\
\hline 2750 & CR390322 & -3.15 & $2.83 \mathrm{E}-02$ \\
\hline 2751 & CR390323 & -3.35 & $1.32 \mathrm{E}-03$ \\
\hline 2752 & CR390333 & -4.29 & $2.73 \mathrm{E}-05$ \\
\hline 2753 & CR390347 & -5.93 & $1.17 \mathrm{E}-03$ \\
\hline 2754 & \begin{tabular}{lc|} 
CR390358 \\
\end{tabular} & $\begin{array}{l}-4.63 \\
\end{array}$ & $9.08 \mathrm{E}-04$ \\
\hline 2755 & CR390361 & -1.67 & $1.85 \mathrm{E}-02$ \\
\hline 2756 & CR390373 & -3.36 & $4.54 \mathrm{E}-05$ \\
\hline 2757 & CR390391 & -2.09 & $1.25 \mathrm{E}-02$ \\
\hline 2758 & \begin{tabular}{lc|} 
CR390396 \\
\end{tabular} & $\begin{array}{l}-1.66 \\
\end{array}$ & $3.99 \mathrm{E}-03$ \\
\hline 2759 & CR390407 & -6.17 & $1.31 \mathrm{E}-04$ \\
\hline 2760 & CR390415 & -2.52 & $9.12 \mathrm{E}-04$ \\
\hline 2761 & CR390417 & -5.92 & $1.00 \mathrm{E}-03$ \\
\hline 2762 & CR390424 & -3.21 & $2.78 \mathrm{E}-03$ \\
\hline 2763 & CR390446 & -1.37 & $7.87 \mathrm{E}-02$ \\
\hline 2764 & \begin{tabular}{lc|} 
CR390456 \\
\end{tabular} & -5.21 & $1.34 \mathrm{E}-04$ \\
\hline 2765 & CR390463 & -1.65 & $2.06 \mathrm{E}-03$ \\
\hline 2766 & CR390478 & -2.43 & $3.60 \mathrm{E}-03$ \\
\hline 2767 & CR390484 & -3.81 & 7.30E-04 \\
\hline 2768 & CR390488 & -2.29 & $9.58 \mathrm{E}-03$ \\
\hline 2769 & CR390492 & -2.93 & $7.28 \mathrm{E}-03$ \\
\hline 2770 & CR390494 & -3.76 & $2.55 \mathrm{E}-03$ \\
\hline 2771 & CR390498 & -5.04 & $4.75 \mathrm{E}-03$ \\
\hline
\end{tabular}

\begin{tabular}{|c|c|c|c|}
\hline 2772 & CR390514 & -4.64 & $5.85 \mathrm{E}-04$ \\
\hline 2773 & CR390531 & -2.21 & $4.50 \mathrm{E}-03$ \\
\hline 2774 & CR390534 & -6.53 & $4.79 \mathrm{E}-04$ \\
\hline 2775 & CR390540 & -2.03 & $5.41 \mathrm{E}-03$ \\
\hline 2776 & \begin{tabular}{lc|} 
CR390556 \\
\end{tabular} & -1.77 & $9.25 \mathrm{E}-03$ \\
\hline 2777 & CR390577 & -1.30 & $2.14 \mathrm{E}-02$ \\
\hline 2778 & CR390591 & -1.51 & $9.82 \mathrm{E}-03$ \\
\hline 2779 & CR390592 & -1.94 & $2.41 \mathrm{E}-02$ \\
\hline 2780 & CR390600 & -3.08 & $6.03 \mathrm{E}-03$ \\
\hline 2781 & CR390601 & -1.91 & $2.11 \mathrm{E}-04$ \\
\hline 2782 & \begin{tabular}{lc|} 
CR390609 \\
\end{tabular} & -3.51 & $6.74 \mathrm{E}-03$ \\
\hline 2783 & CR390616 & -2.26 & $2.22 \mathrm{E}-02$ \\
\hline 2784 & CR390621 & -4.63 & $5.51 \mathrm{E}-04$ \\
\hline 2785 & CR390626 & -1.13 & $1.55 \mathrm{E}-02$ \\
\hline 2786 & CR390638 & -4.31 & $2.27 \mathrm{E}-02$ \\
\hline 2787 & CR390647 & -4.58 & $1.41 \mathrm{E}-03$ \\
\hline 2788 & CR390649 & -2.74 & $5.56 \mathrm{E}-03$ \\
\hline 2789 & CR390651 & -5.99 & $3.96 \mathrm{E}-04$ \\
\hline 2790 & CR390654 & -3.10 & $4.57 \mathrm{E}-03$ \\
\hline 2791 & CR390664 & -2.95 & $5.00 \mathrm{E}-03$ \\
\hline 2792 & CR390669 & -3.65 & $5.22 \mathrm{E}-04$ \\
\hline 2793 & CR390673 & -3.38 & $8.16 \mathrm{E}-03$ \\
\hline 2794 & CR390684 & -4.28 & $2.09 \mathrm{E}-04$ \\
\hline 2795 & CR390688 & -3.42 & $8.49 \mathrm{E}-04$ \\
\hline 2796 & CR390689 & -2.45 & $1.00 \mathrm{E}-03$ \\
\hline 2797 & \begin{tabular}{ll|} 
CR390721 \\
\end{tabular} & -1.29 & $1.95 \mathrm{E}-02$ \\
\hline 2798 & CR390732 & -2.12 & $6.41 \mathrm{E}-02$ \\
\hline 2799 & CR390739 & -1.98 & $1.66 \mathrm{E}-02$ \\
\hline 2800 & CR390752 & -1.33 & $3.74 \mathrm{E}-02$ \\
\hline 2801 & CR390759 & -2.90 & $4.58 \mathrm{E}-03$ \\
\hline 2802 & CR390794 & -5.07 & $3.49 \mathrm{E}-02$ \\
\hline 2803 & CR390795 & -1.12 & $1.16 \mathrm{E}-02$ \\
\hline 2804 & CR390797 & -1.48 & $1.66 \mathrm{E}-02$ \\
\hline 2805 & CR390803 & -2.86 & $1.22 \mathrm{E}-03$ \\
\hline 2806 & CR390808 & -2.30 & $9.94 \mathrm{E}-03$ \\
\hline 2807 & $\begin{array}{ll}\text { CR390817 } \\
\end{array}$ & -4.69 & $2.80 \mathrm{E}-03$ \\
\hline 2808 & CR390830 & -2.76 & $1.10 \mathrm{E}-03$ \\
\hline 2809 & CR390834 & -6.00 & $2.14 \mathrm{E}-03$ \\
\hline 2810 & CR390901 & -3.31 & $1.27 \mathrm{E}-02$ \\
\hline 2811 & CR390903 & -2.24 & $7.93 \mathrm{E}-03$ \\
\hline 2812 & CR390913 & -4.15 & $1.15 \mathrm{E}-03$ \\
\hline 2813 & $\begin{array}{ll}\text { CR390929 } \\
\end{array}$ & -2.09 & $1.92 \mathrm{E}-03$ \\
\hline 2814 & CR390942 & -2.95 & $2.54 \mathrm{E}-03$ \\
\hline 2815 & CR390943 & -1.31 & $4.97 \mathrm{E}-02$ \\
\hline 2816 & CR390946 & -1.13 & $9.63 \mathrm{E}-03$ \\
\hline 2817 & CR390949 & -3.06 & $7.96 \mathrm{E}-04$ \\
\hline 2818 & CR390955 & -1.15 & $5.96 \mathrm{E}-02$ \\
\hline 2819 & CR390956 & -1.52 & $5.34 \mathrm{E}-03$ \\
\hline 2820 & CR390959 & -1.63 & $1.55 \mathrm{E}-02$ \\
\hline 2821 & CR390967 & -5.25 & $1.75 \mathrm{E}-03$ \\
\hline 2822 & CR390976 & -5.52 & $9.46 \mathrm{E}-04$ \\
\hline 2823 & CR390977 & -3.22 & $1.35 \mathrm{E}-04$ \\
\hline 2824 & CR390978 & -1.56 & $1.08 \mathrm{E}-02$ \\
\hline 2825 & CR390980 & -3.24 & 7.19E-04 \\
\hline 2826 & CR390981 & -3.28 & $3.82 \mathrm{E}-04$ \\
\hline 2827 & $\begin{array}{ll}\text { CR390983 } \\
\end{array}$ & -3.52 & $5.47 \mathrm{E}-03$ \\
\hline 2828 & CR390985 & -5.20 & $6.19 \mathrm{E}-04$ \\
\hline 2829 & CR390997 & -1.61 & $4.12 \mathrm{E}-03$ \\
\hline 2830 & CR391001 & -1.54 & $9.91 \mathrm{E}-03$ \\
\hline 2831 & CR391007 & -3.53 & $2.31 \mathrm{E}-03$ \\
\hline 2832 & CR391013 & -2.24 & $3.04 \mathrm{E}-03$ \\
\hline 2833 & $\begin{array}{ll}\text { CR391025 } \\
\end{array}$ & -1.85 & $4.70 \mathrm{E}-02$ \\
\hline 2834 & CR391026 & -1.79 & $2.83 \mathrm{E}-03$ \\
\hline
\end{tabular}




\begin{tabular}{|c|c|c|c|}
\hline 2835 & CR391043 & -1.40 & $1.03 E-02$ \\
\hline 2836 & \begin{tabular}{lc|} 
CR391073 \\
\end{tabular} & \begin{tabular}{|l|}
-3.28 \\
\end{tabular} & $2.21 \mathrm{E}-04$ \\
\hline 2837 & CR391096 & -3.80 & $2.71 \mathrm{E}-03$ \\
\hline 2838 & CR391097 & -3.33 & $1.47 \mathrm{E}-04$ \\
\hline 2839 & CR391099 & -3.45 & $4.46 \mathrm{E}-03$ \\
\hline 2840 & CR391133 & -1.67 & $2.00 \mathrm{E}-03$ \\
\hline 2841 & CR391136 & \begin{tabular}{|l|}
-3.20 \\
\end{tabular} & $1.17 \mathrm{E}-03$ \\
\hline 2842 & CR391144 & -2.07 & $3.83 \mathrm{E}-04$ \\
\hline 2843 & CR391196 & -3.61 & $8.32 \mathrm{E}-05$ \\
\hline 2844 & CR391214 & -3.14 & $2.18 \mathrm{E}-03$ \\
\hline 2845 & CR391222 & -1.49 & $1.36 \mathrm{E}-03$ \\
\hline 2846 & CR391240 & \begin{tabular}{l|l|}
-2.26 \\
\end{tabular} & $9.25 \mathrm{E}-03$ \\
\hline 2847 & CR391257 & \begin{tabular}{|l|}
-3.22 \\
\end{tabular} & $6.69 \mathrm{E}-05$ \\
\hline 2848 & $\begin{array}{ll}\text { CR391266 } \\
\end{array}$ & \begin{tabular}{l|l|}
-2.54 \\
\end{tabular} & $1.51 \mathrm{E}-03$ \\
\hline 2849 & CR391273 & -2.17 & $2.62 \mathrm{E}-03$ \\
\hline 2850 & CR391275 & -1.32 & $3.50 \mathrm{E}-03$ \\
\hline 2851 & CR391302 & $\begin{array}{l}-1.24 \\
\end{array}$ & $3.66 \mathrm{E}-03$ \\
\hline 2852 & CR391322 & -1.33 & $1.11 \mathrm{E}-02$ \\
\hline 2853 & CR391327 & -1.24 & $2.53 \mathrm{E}-02$ \\
\hline 2854 & CR391331 & -3.29 & $1.13 \mathrm{E}-03$ \\
\hline 2855 & CR391356 & -3.59 & $1.93 \mathrm{E}-03$ \\
\hline 2856 & CR391368 & -1.71 & $6.34 \mathrm{E}-03$ \\
\hline 2857 & CR391389 & -4.21 & $8.88 \mathrm{E}-04$ \\
\hline 2858 & CR391390 & \begin{tabular}{|l|}
-1.78 \\
\end{tabular} & $1.39 \mathrm{E}-04$ \\
\hline 2859 & CR391394 & -4.03 & $3.26 \mathrm{E}-05$ \\
\hline 2860 & CR391397 & $\begin{array}{l}-3.75 \\
\end{array}$ & $3.82 \mathrm{E}-03$ \\
\hline 2861 & CR391399 & -3.59 & $2.61 \mathrm{E}-03$ \\
\hline 2862 & CR391402 & \begin{tabular}{|l|}
-2.41 \\
\end{tabular} & $2.18 \mathrm{E}-02$ \\
\hline 2863 & CR391403 & $\begin{array}{l}-3.54 \\
\end{array}$ & $1.10 \mathrm{E}-04$ \\
\hline 2864 & CR391406 & -3.19 & $6.53 \mathrm{E}-04$ \\
\hline 2865 & CR391410 & -1.75 & $3.06 \mathrm{E}-02$ \\
\hline 2866 & $\begin{array}{lc}\text { CR391411 } \\
\end{array}$ & \begin{tabular}{|l|l|} 
\\
\end{tabular} & $3.64 \mathrm{E}-05$ \\
\hline 2867 & CR391414 & -2.22 & $2.40 \mathrm{E}-03$ \\
\hline 2868 & CR391419 & $\begin{array}{l}-1.73 \\
\end{array}$ & $4.01 \mathrm{E}-02$ \\
\hline 2869 & CR391436 & -5.06 & 7.04E-04 \\
\hline 2870 & CR391440 & -1.76 & $6.00 \mathrm{E}-05$ \\
\hline 2871 & CR391442 & -2.34 & $1.75 E-02$ \\
\hline 2872 & CR391448 & \begin{tabular}{|l|}
-6.81 \\
\end{tabular} & $1.04 \mathrm{E}-04$ \\
\hline 2873 & CR391450 & -2.87 & $9.69 \mathrm{E}-04$ \\
\hline 2874 & CR391452 & -3.97 & $1.57 \mathrm{E}-03$ \\
\hline 2875 & CR391453 & -4.63 & $4.33 \mathrm{E}-04$ \\
\hline 2876 & CR391457 & -2.88 & $2.50 \mathrm{E}-03$ \\
\hline 2877 & $\begin{array}{lcl}\text { CR391460 } \\
\end{array}$ & \begin{tabular}{l|l|}
-2.17 \\
\end{tabular} & $3.08 \mathrm{E}-03$ \\
\hline 2878 & CR391464 & -1.72 & $7.87 \mathrm{E}-03$ \\
\hline 2879 & CR391465 & -2.57 & $5.10 \mathrm{E}-03$ \\
\hline 2880 & CR391477 & -2.67 & $5.26 \mathrm{E}-03$ \\
\hline 2881 & CR391480 & -2.26 & $3.32 \mathrm{E}-02$ \\
\hline 2882 & CR391482 & $\begin{array}{l}-1.22 \\
\end{array}$ & $2.65 \mathrm{E}-02$ \\
\hline 2883 & $\begin{array}{ll}\text { CR391486 } \\
\end{array}$ & $\begin{array}{l}-2.88 \\
\end{array}$ & $5.91 \mathrm{E}-03$ \\
\hline 2884 & CR391488 & -5.03 & $9.28 \mathrm{E}-04$ \\
\hline 2885 & CR391489 & -1.26 & $4.52 \mathrm{E}-03$ \\
\hline 2886 & CR391490 & $\begin{array}{l}-1.87 \\
\end{array}$ & $4.89 \mathrm{E}-03$ \\
\hline 2887 & CR391491 & -1.45 & $2.88 \mathrm{E}-03$ \\
\hline 2888 & CR391495 & -2.06 & $2.92 \mathrm{E}-03$ \\
\hline 2889 & CR391496 & \begin{tabular}{|l|}
-3.06 \\
\end{tabular} & $9.95 \mathrm{E}-05$ \\
\hline 2890 & CR391497 & -3.70 & 8.06E-04 \\
\hline 2891 & CR391504 & -3.61 & $2.60 \mathrm{E}-04$ \\
\hline 2892 & CR391505 & \begin{tabular}{|l|}
-2.19 \\
\end{tabular} & $7.95 \mathrm{E}-04$ \\
\hline 2893 & CR391507 & \begin{tabular}{|l|}
-2.51 \\
\end{tabular} & $3.76 \mathrm{E}-03$ \\
\hline 2894 & CR391508 & -2.47 & $2.82 \mathrm{E}-03$ \\
\hline 2895 & CR391514 & -3.82 & $3.96 \mathrm{E}-04$ \\
\hline 2896 & CR391523 & -3.86 & $1.42 \mathrm{E}-03$ \\
\hline
\end{tabular}

\begin{tabular}{|c|c|c|c|}
\hline 2898 & CR391527 & -1.33 & $1.09 \mathrm{E}-02$ \\
\hline 2899 & CR391529 & -2.08 & $1.36 \mathrm{E}-02$ \\
\hline 2900 & CR391532 & -3.02 & $4.39 \mathrm{E}-03$ \\
\hline 2901 & CR391535 & -2.25 & $5.49 \mathrm{E}-02$ \\
\hline 2902 & CR391536 & -2.59 & $4.28 \mathrm{E}-04$ \\
\hline 2903 & CR391554 & -2.21 & $1.33 \mathrm{E}-03$ \\
\hline 2904 & CR391556 & -2.20 & $7.55 \mathrm{E}-04$ \\
\hline 2905 & CR391563 & -3.28 & $2.53 \mathrm{E}-03$ \\
\hline 2906 & CR391569 & -2.33 & $2.10 \mathrm{E}-02$ \\
\hline 2907 & CR391586 & -3.45 & $5.65 \mathrm{E}-04$ \\
\hline 2908 & CR391591 & -3.33 & $2.07 \mathrm{E}-04$ \\
\hline 2909 & CR391593 & -1.92 & $7.54 \mathrm{E}-03$ \\
\hline 2910 & CR391600 & -1.77 & $1.95 \mathrm{E}-03$ \\
\hline 2911 & CR391608 & -2.00 & $5.08 \mathrm{E}-03$ \\
\hline 2912 & CR391611 & -1.47 & $4.55 \mathrm{E}-02$ \\
\hline 2913 & CR391615 & -5.99 & $3.42 \mathrm{E}-04$ \\
\hline 2914 & CR391616 & -1.93 & $8.59 \mathrm{E}-02$ \\
\hline 2915 & CR391620 & -4.54 & $7.99 \mathrm{E}-04$ \\
\hline 2916 & CR391622 & -5.38 & $2.11 \mathrm{E}-04$ \\
\hline 2917 & CR391646 & -2.58 & $1.02 \mathrm{E}-03$ \\
\hline 2918 & CR391649 & -2.94 & $2.94 \mathrm{E}-03$ \\
\hline 2919 & CR391651 & -5.97 & $6.01 \mathrm{E}-04$ \\
\hline 2920 & CR391652 & -2.79 & $1.39 \mathrm{E}-03$ \\
\hline 2921 & CR391654 & -4.92 & $4.53 \mathrm{E}-04$ \\
\hline 2922 & CR391663 & -8.13 & $1.76 \mathrm{E}-03$ \\
\hline 2923 & CR391666 & -2.35 & $1.21 \mathrm{E}-03$ \\
\hline 2924 & CR391667 & -3.20 & $1.29 \mathrm{E}-03$ \\
\hline 2925 & CR391668 & -1.30 & $3.61 \mathrm{E}-04$ \\
\hline 2926 & CR391670 & -1.99 & $6.36 \mathrm{E}-03$ \\
\hline 2927 & CR391676 & -1.50 & $6.49 \mathrm{E}-03$ \\
\hline 2928 & CR391681 & -3.52 & $1.66 \mathrm{E}-03$ \\
\hline 2929 & CR391703 & -4.37 & $1.19 \mathrm{E}-03$ \\
\hline 2930 & CR391713 & -6.52 & $2.81 \mathrm{E}-06$ \\
\hline 2931 & CR391714 & -3.21 & $1.79 E-04$ \\
\hline 2932 & CR391723 & -1.38 & 7.49E-03 \\
\hline 2933 & CR391747 & -4.29 & $4.31 \mathrm{E}-04$ \\
\hline 2934 & CR391751 & -4.99 & $9.41 \mathrm{E}-04$ \\
\hline 2935 & CR391753 & -1.49 & $8.13 \mathrm{E}-02$ \\
\hline 2936 & CR391759 & -3.45 & $2.43 \mathrm{E}-03$ \\
\hline 2937 & CR405727 & -2.09 & $4.93 \mathrm{E}-02$ \\
\hline 2938 & CR405742 & -4.78 & $2.60 \mathrm{E}-04$ \\
\hline 2939 & CR405764 & -2.94 & $4.13 \mathrm{E}-04$ \\
\hline 2940 & CR405790 & -2.55 & $2.91 \mathrm{E}-02$ \\
\hline 2941 & CR405835 & -6.09 & $8.78 \mathrm{E}-04$ \\
\hline 2942 & CR405837 & -1.51 & $6.95 \mathrm{E}-03$ \\
\hline 2943 & CR405852 & $\begin{array}{l}-1.14 \\
\end{array}$ & $9.17 \mathrm{E}-03$ \\
\hline 2944 & CR405854 & -1.39 & $1.19 \mathrm{E}-02$ \\
\hline 2945 & CR405866 & -1.46 & $5.18 \mathrm{E}-02$ \\
\hline 2946 & CR405890 & -2.41 & $7.86 \mathrm{E}-03$ \\
\hline 2947 & CR405893 & \begin{tabular}{l|l|}
-2.07 \\
\end{tabular} & $2.87 \mathrm{E}-03$ \\
\hline 2948 & CR405912 & -1.19 & $4.98 \mathrm{E}-03$ \\
\hline 2949 & CR405941 & -3.26 & $6.44 \mathrm{E}-03$ \\
\hline 2950 & CR405942 & -4.49 & $5.73 \mathrm{E}-03$ \\
\hline 2951 & CR405958 & -4.60 & $1.69 \mathrm{E}-04$ \\
\hline 2952 & CR405968 & -1.69 & $4.28 \mathrm{E}-03$ \\
\hline 2953 & CR405970 & $\begin{array}{l}-3.62 \\
\end{array}$ & $2.62 \mathrm{E}-03$ \\
\hline 2954 & CR405976 & -6.56 & $7.78 \mathrm{E}-05$ \\
\hline 2955 & CR405980 & -4.30 & $1.23 \mathrm{E}-03$ \\
\hline 2956 & CR405988 & -3.76 & $2.12 \mathrm{E}-03$ \\
\hline 2957 & CR405994 & -2.27 & $2.76 \mathrm{E}-03$ \\
\hline 2958 & CR406000 & -4.20 & $1.53 \mathrm{E}-03$ \\
\hline 2959 & CR406009 & -1.66 & $1.19 \mathrm{E}-02$ \\
\hline 2960 & CR406037 & -2.36 & $7.01 \mathrm{E}-03$ \\
\hline
\end{tabular}

\begin{tabular}{|c|c|c|c|}
\hline 2961 & CR406039 & -2.75 & $4.73 \mathrm{E}-03$ \\
\hline 2962 & CR406043 & -1.83 & $9.27 \mathrm{E}-03$ \\
\hline 2963 & CR406068 & -4.20 & $2.44 E-04$ \\
\hline 2964 & CR406087 & -1.57 & $1.66 E-02$ \\
\hline 2965 & CR406089 & $\begin{array}{l}-4.84 \\
\end{array}$ & $5.16 \mathrm{E}-04$ \\
\hline 2966 & CR406096 & -1.87 & $7.92 E-02$ \\
\hline 2967 & CR406099 & \begin{tabular}{|l|}
-3.88 \\
\end{tabular} & $\begin{array}{l}3.62 E-04 \\
\end{array}$ \\
\hline 2968 & CR406105 & -4.14 & $2.43 E-03$ \\
\hline 2969 & CR406108 & -1.33 & $6.07 E-03$ \\
\hline 2970 & CR406116 & -1.41 & $2.09 E-03$ \\
\hline 2971 & CR406121 & -1.22 & $2.29 \mathrm{E}-02$ \\
\hline 2972 & CR406125 & -4.49 & $2.39 E-03$ \\
\hline 2973 & CR406129 & -2.33 & $1.71 E-03$ \\
\hline 2974 & CR406130 & -5.75 & $7.98 E-05$ \\
\hline 2975 & CR406136 & -3.32 & $3.68 \mathrm{E}-03$ \\
\hline 2976 & CR406139 & -3.01 & $9.12 E-04$ \\
\hline 2977 & CR406146 & -5.52 & $9.54 E-05$ \\
\hline 2978 & CR406151 & -3.97 & $1.64 E-04$ \\
\hline 2979 & CR406163 & \begin{tabular}{l|l|}
-2.79 \\
\end{tabular} & $2.47 \mathrm{E}-03$ \\
\hline 2980 & CR406190 & -1.95 & $9.03 \mathrm{E}-03$ \\
\hline 2981 & CR406192 & -1.55 & $1.73 \mathrm{E}-02$ \\
\hline 2982 & CR406194 & -2.19 & $1.07 E-02$ \\
\hline 2983 & CR406196 & -2.85 & $5.66 \mathrm{E}-04$ \\
\hline 2984 & CR406198 & -1.81 & $3.56 \mathrm{E}-03$ \\
\hline 2985 & CR406202 & \begin{tabular}{|l|}
-3.53 \\
\end{tabular} & $1.53 \mathrm{E}-03$ \\
\hline 2986 & CR406208 & $\begin{array}{l}-5.13 \\
\end{array}$ & $\begin{array}{c}9.09 E-04 \\
\end{array}$ \\
\hline 2987 & CR406210 & -1.83 & $3.98 E-04$ \\
\hline 2988 & CR406214 & -2.84 & $4.23 \mathrm{E}-03$ \\
\hline 2989 & CR406226 & -1.62 & $1.66 \mathrm{E}-02$ \\
\hline 2990 & CR406269 & \begin{tabular}{|l|}
-3.78 \\
\end{tabular} & $1.47 E-04$ \\
\hline 2991 & CR406276 & -4.68 & $4.77 E-03$ \\
\hline 2992 & CR406289 & $\begin{array}{l}-2.63 \\
\end{array}$ & $6.65 \mathrm{E}-03$ \\
\hline 2993 & CR406292 & -2.36 & $4.72 E-04$ \\
\hline 2994 & CR406296 & -2.26 & $2.76 E-03$ \\
\hline 2995 & CR406307 & -4.11 & $2.33 \mathrm{E}-04$ \\
\hline 2996 & CR406309 & -2.61 & $6.16 \mathrm{E}-05$ \\
\hline 2997 & CR406318 & -2.88 & $3.54 \mathrm{E}-03$ \\
\hline 2998 & CR406331 & -3.16 & $3.29 \mathrm{E}-03$ \\
\hline 2999 & CR406342 & -1.32 & $1.82 E-02$ \\
\hline 3000 & CR406346 & $\begin{array}{l}-2.72 \\
\end{array}$ & $4.17 \mathrm{E}-03$ \\
\hline 3001 & CR406355 & -3.47 & $5.03 E-03$ \\
\hline 3002 & CR406357 & -2.51 & $7.40 \mathrm{E}-03$ \\
\hline 3003 & CR406365 & -2.80 & $9.26 \mathrm{E}-04$ \\
\hline 3004 & CR406366 & -3.97 & $2.24 E-05$ \\
\hline 3005 & CR406368 & -1.49 & $9.82 \mathrm{E}-03$ \\
\hline 3006 & CR406373 & -2.50 & $9.27 \mathrm{E}-03$ \\
\hline 3007 & CR406381 & -4.53 & $1.86 \mathrm{E}-03$ \\
\hline 3008 & CR406385 & -2.13 & $6.37 \mathrm{E}-03$ \\
\hline 3009 & CR406390 & -3.34 & $5.90 E-04$ \\
\hline 3010 & CR406397 & -2.83 & $1.57 \mathrm{E}-03$ \\
\hline 3011 & CR406398 & $\begin{array}{l}-2.53 \\
\end{array}$ & $7.26 \mathrm{E}-03$ \\
\hline 3012 & CR406400 & -1.56 & $1.04 E-02$ \\
\hline 3013 & CR406441 & -2.23 & $3.37 E-04$ \\
\hline 3014 & CR406446 & -3.11 & $8.28 \mathrm{E}-04$ \\
\hline 3015 & CR406452 & -1.38 & $1.98 \mathrm{E}-02$ \\
\hline 3016 & CR406455 & \begin{tabular}{|l|}
-2.81 \\
\end{tabular} & $6.79 \mathrm{E}-03$ \\
\hline 3017 & CR406456 & -3.52 & $2.76 \mathrm{E}-05$ \\
\hline 3018 & CR406474 & -2.74 & $1.36 \mathrm{E}-03$ \\
\hline 3019 & CR406485 & -3.13 & $2.03 E-03$ \\
\hline 3020 & CR406501 & -2.30 & $3.91 E-03$ \\
\hline 3021 & CR406504 & -1.31 & $1.01 E-03$ \\
\hline 3022 & CR406512 & -1.87 & $6.96 \mathrm{E}-03$ \\
\hline 023 & CR406521 & -1.54 & $4 E-03$ \\
\hline
\end{tabular}




\begin{tabular}{|c|c|c|c|}
\hline 3024 & CR406527 & -1.66 & $2.61 \mathrm{E}-03$ \\
\hline 3025 & CR406535 & -1.56 & $7.73 \mathrm{E}-03$ \\
\hline 3026 & CR406538 & -1.74 & $6.41 \mathrm{E}-04$ \\
\hline 3027 & CR406554 & -1.20 & $1.38 \mathrm{E}-02$ \\
\hline 3028 & CR406561 & -4.59 & $1.13 \mathrm{E}-03$ \\
\hline 3029 & CR406570 & -5.86 & $4.79 \mathrm{E}-05$ \\
\hline 3030 & CR406575 & -1.48 & $5.40 \mathrm{E}-02$ \\
\hline 3031 & CR406586 & -1.52 & $3.06 \mathrm{E}-02$ \\
\hline 3032 & CR406604 & -4.89 & $6.70 \mathrm{E}-04$ \\
\hline 3033 & CR406607 & -1.90 & $3.40 \mathrm{E}-02$ \\
\hline 3034 & CR406622 & -2.04 & $5.89 \mathrm{E}-03$ \\
\hline 3035 & CR406635 & -1.24 & $5.00 \mathrm{E}-03$ \\
\hline 3036 & CR406637 & -2.32 & $2.45 \mathrm{E}-02$ \\
\hline 3037 & CR406656 & -4.43 & $1.61 \mathrm{E}-03$ \\
\hline 3038 & CR406658 & -1.20 & $2.20 \mathrm{E}-02$ \\
\hline 3039 & CR406660 & \begin{tabular}{|l|l|}
-4.33 \\
\end{tabular} & $1.72 \mathrm{E}-03$ \\
\hline 3040 & CR406664 & -1.56 & $1.87 \mathrm{E}-02$ \\
\hline 3041 & CR406665 & -1.46 & $8.20 \mathrm{E}-02$ \\
\hline 3042 & CR406676 & -2.98 & $1.06 \mathrm{E}-03$ \\
\hline 3043 & CR406684 & -2.03 & $3.66 \mathrm{E}-03$ \\
\hline 3044 & CR406685 & -5.65 & $5.96 \mathrm{E}-04$ \\
\hline 3045 & $\begin{array}{ll}\text { CR406686 } \\
\end{array}$ & $\begin{array}{l}-1.53 \\
\end{array}$ & $3.69 \mathrm{E}-02$ \\
\hline 3046 & CR406688 & -4.05 & $2.06 \mathrm{E}-04$ \\
\hline 3047 & CR406692 & -4.30 & $1.87 \mathrm{E}-04$ \\
\hline 3048 & CR406696 & -5.01 & $1.21 \mathrm{E}-04$ \\
\hline 3049 & CR406697 & -1.19 & $2.10 \mathrm{E}-02$ \\
\hline 3050 & CR406706 & -2.22 & $7.45 \mathrm{E}-02$ \\
\hline 3051 & CR406716 & -5.57 & $6.94 \mathrm{E}-04$ \\
\hline 3052 & CR406732 & -3.13 & $8.77 E-04$ \\
\hline 3053 & CR406737 & -6.88 & $9.55 \mathrm{E}-05$ \\
\hline 3054 & CR406741 & -4.28 & $\begin{array}{r}3.26 \mathrm{E}-02 \\
\end{array}$ \\
\hline 3055 & CR406744 & -2.22 & $1.36 \mathrm{E}-02$ \\
\hline 3056 & CR406763 & -3.10 & $3.85 \mathrm{E}-03$ \\
\hline 3057 & CR406771 & -5.98 & $2.50 \mathrm{E}-04$ \\
\hline 3058 & CR406789 & -1.23 & $5.27 E-03$ \\
\hline 3059 & CR406796 & -2.83 & $2.79 \mathrm{E}-03$ \\
\hline 3060 & CR406798 & -4.92 & $1.89 \mathrm{E}-04$ \\
\hline 3061 & CR406800 & -2.81 & $2.32 \mathrm{E}-03$ \\
\hline 3062 & CR406804 & -1.72 & $1.98 \mathrm{E}-03$ \\
\hline 3063 & CR406809 & -6.07 & $3.67 \mathrm{E}-04$ \\
\hline 3064 & CR406813 & \begin{tabular}{|l|}
-3.86 \\
\end{tabular} & $3.55 \mathrm{E}-03$ \\
\hline 3065 & CR406823 & -1.18 & $5.93 \mathrm{E}-03$ \\
\hline 3066 & CR406826 & -1.23 & $1.63 \mathrm{E}-02$ \\
\hline 3067 & CR406829 & -2.58 & $2.47 \mathrm{E}-03$ \\
\hline 3068 & CR406836 & -1.65 & $1.23 \mathrm{E}-02$ \\
\hline 3069 & CR406838 & -1.96 & $1.48 \mathrm{E}-02$ \\
\hline 3070 & CR406841 & -4.81 & 8.05E-04 \\
\hline 3071 & CR406843 & -1.64 & $2.72 \mathrm{E}-02$ \\
\hline 3072 & CR406844 & -2.86 & $1.09 \mathrm{E}-02$ \\
\hline 3073 & CR406845 & -3.84 & $1.71 \mathrm{E}-03$ \\
\hline 3074 & CR406847 & -2.84 & $6.60 \mathrm{E}-04$ \\
\hline 3075 & CR406853 & -2.08 & 4.68E-03 \\
\hline 3076 & CR406868 & -3.93 & $8.51 \mathrm{E}-05$ \\
\hline 3077 & $\begin{array}{ll}\text { CR406873 } \\
\end{array}$ & \begin{tabular}{|l|}
-5.69 \\
\end{tabular} & $3.64 \mathrm{E}-04$ \\
\hline 3078 & CR406887 & -1.07 & $5.89 \mathrm{E}-02$ \\
\hline 3079 & CR406897 & -1.39 & $2.44 \mathrm{E}-03$ \\
\hline 3080 & CR406902 & -3.45 & $5.56 \mathrm{E}-04$ \\
\hline 3081 & CR406903 & -1.38 & $3.47 \mathrm{E}-03$ \\
\hline 3082 & CR406910 & -2.72 & $1.10 \mathrm{E}-03$ \\
\hline 3083 & CR406918 & -3.58 & $2.54 E-04$ \\
\hline 3084 & CR406935 & -3.61 & $3.88 \mathrm{E}-04$ \\
\hline 3085 & CR406956 & -4.01 & $2.31 \mathrm{E}-04$ \\
\hline 3086 & CR406962 & -3.15 & $1.50 \mathrm{t}-0$ \\
\hline
\end{tabular}

\begin{tabular}{|c|c|c|c|}
\hline 3087 & CR406965 & -4.66 & $2.62 E-04$ \\
\hline 3088 & CR406973 & \begin{tabular}{|l|}
-1.34 \\
\end{tabular} & $3.72 E-02$ \\
\hline 3089 & CR406991 & \begin{tabular}{|l|}
-1.14 \\
\end{tabular} & $1.92 E-02$ \\
\hline 3090 & CR407022 & -3.91 & $1.75 E-03$ \\
\hline 3091 & CR407023 & -5.41 & $5.22 \mathrm{E}-04$ \\
\hline 3092 & CR407050 & -5.14 & $6.92 E-04$ \\
\hline 3093 & $\begin{array}{lc}\text { CR407057 } \\
\end{array}$ & -1.97 & $2.88 \mathrm{E}-03$ \\
\hline 3094 & CR407063 & -7.35 & $3.43 E-04$ \\
\hline 3095 & CR407065 & -2.10 & $5.64 \mathrm{E}-03$ \\
\hline 3096 & CR407068 & -3.73 & $1.79 E-04$ \\
\hline 3097 & CR407069 & -3.53 & $1.63 \mathrm{E}-03$ \\
\hline 3098 & CR407094 & -3.31 & $1.32 E-04$ \\
\hline 3099 & CR407095 & -5.16 & $2.64 \mathrm{E}-04$ \\
\hline 3100 & CR407105 & $\begin{array}{l}2.67 \\
\end{array}$ & $1.83 \mathrm{E}-02$ \\
\hline 3101 & CR407120 & -2.87 & $2.25 \mathrm{E}-03$ \\
\hline 3102 & CR407126 & -1.20 & $1.22 \mathrm{E}-03$ \\
\hline 3103 & CR407146 & -2.52 & $3.16 \mathrm{E}-03$ \\
\hline 3104 & CR407164 & -1.92 & $3.74 E-03$ \\
\hline 3105 & CR407178 & \begin{tabular}{|l|}
-3.49 \\
\end{tabular} & $3.21 \mathrm{E}-03$ \\
\hline 3106 & CR407192 & -4.07 & $9.97 E-04$ \\
\hline 3107 & CR407207 & -4.09 & $6.96 \mathrm{E}-04$ \\
\hline 3108 & CR407208 & -1.71 & $3.95 \mathrm{E}-03$ \\
\hline 3109 & CR407209 & -4.71 & $4.10 \mathrm{E}-05$ \\
\hline 3110 & CR407214 & -3.43 & $7.06 \mathrm{E}-03$ \\
\hline 3111 & CR407222 & -1.38 & $5.99 \mathrm{E}-02$ \\
\hline 3112 & $\begin{array}{ll}\text { CR407224 } \\
\end{array}$ & -2.14 & $8.03 \mathrm{E}-03$ \\
\hline 3113 & CR407227 & -2.01 & $1.41 \mathrm{E}-03$ \\
\hline 3114 & CR407229 & -4.47 & $3.45 \mathrm{E}-03$ \\
\hline 3115 & CR407242 & -3.71 & $3.62 E-03$ \\
\hline 3116 & CR407243 & \begin{tabular}{l|l|}
-5.37 \\
\end{tabular} & $1.21 \mathrm{E}-03$ \\
\hline 3117 & CR407244 & -4.80 & $1.10 \mathrm{E}-03$ \\
\hline 3118 & $\begin{array}{lc}\text { CR407248 } \\
\end{array}$ & -1.96 & $4.09 \mathrm{E}-03$ \\
\hline 3119 & CR407251 & -3.53 & $2.78 \mathrm{E}-03$ \\
\hline 3120 & \begin{tabular}{lc|} 
CR407254 \\
\end{tabular} & -4.25 & $6.33 \mathrm{E}-04$ \\
\hline 3121 & CR407256 & -3.02 & $1.55 \mathrm{E}-03$ \\
\hline 3122 & CR407259 & -2.84 & $7.05 E-04$ \\
\hline 3123 & CR407269 & -2.19 & $7.39 \mathrm{E}-03$ \\
\hline 3124 & CR407283 & -3.08 & $4.15 \mathrm{E}-03$ \\
\hline 3125 & CR407284 & -4.87 & $4.43 E-04$ \\
\hline 3126 & \begin{tabular}{lc|} 
CR407286 \\
\end{tabular} & $\begin{array}{l}-1.15 \\
\end{array}$ & $4.34 \mathrm{E}-03$ \\
\hline 3127 & CR407293 & -4.44 & $3.43 \mathrm{E}-04$ \\
\hline 3128 & $\begin{array}{ll}\text { CR407297 } \\
\end{array}$ & -6.00 & $3.83 \mathrm{E}-04$ \\
\hline 3129 & CR407300 & -4.89 & $2.55 \mathrm{E}-03$ \\
\hline 3130 & CR407306 & -4.92 & $1.28 \mathrm{E}-05$ \\
\hline 3131 & CR407307 & \begin{tabular}{|c|}
-3.19 \\
\end{tabular} & $1.03 E-02$ \\
\hline 3132 & CR407315 & -1.75 & $2.18 \mathrm{E}-02$ \\
\hline 3133 & CR407326 & -1.41 & $7.65 \mathrm{E}-03$ \\
\hline 3134 & CR407328 & -1.70 & $1.60 \mathrm{E}-02$ \\
\hline 3135 & CR407338 & -3.42 & $5.53 \mathrm{E}-03$ \\
\hline 3136 & CR407377 & -6.75 & $4.57 \mathrm{E}-04$ \\
\hline 3137 & CR407379 & \begin{tabular}{|l|}
-1.64 \\
\end{tabular} & $2.92 E-04$ \\
\hline 3138 & CR407386 & -3.14 & $7.31 \mathrm{E}-04$ \\
\hline 3139 & $\begin{array}{lc}\text { CR407387 } \\
\end{array}$ & -2.26 & 4.44E-04 \\
\hline 3140 & CR407408 & -3.46 & $1.59 \mathrm{E}-04$ \\
\hline 3141 & CR407409 & -2.88 & $5.38 \mathrm{E}-03$ \\
\hline 3142 & CR407413 & -3.16 & $5.72 \mathrm{E}-04$ \\
\hline 3143 & CR407415 & -3.31 & $2.07 E-03$ \\
\hline 3144 & CR407426 & -1.50 & $3.35 \mathrm{E}-03$ \\
\hline 3145 & CR407428 & -3.60 & $1.18 \mathrm{E}-04$ \\
\hline 3146 & CR407433 & -3.98 & $1.65 \mathrm{E}-05$ \\
\hline 3147 & CR407447 & -1.98 & $1.90 \mathrm{E}-02$ \\
\hline 3148 & CR407487 & -3.92 & $1.90 \mathrm{E}-04$ \\
\hline 3149 & CR407491 & -6.36 & $1.42 E-03$ \\
\hline
\end{tabular}

\begin{tabular}{|c|c|c|c|}
\hline 3150 & CR407503 & -1.65 & $1.48 \mathrm{E}-02$ \\
\hline 3151 & CR407504 & -5.33 & $1.21 \mathrm{E}-03$ \\
\hline 3152 & CR407511 & -3.43 & $7.21 \mathrm{E}-03$ \\
\hline 3153 & CR407515 & -6.00 & $1.04 \mathrm{E}-03$ \\
\hline 3154 & CR407516 & -1.77 & $7.93 \mathrm{E}-02$ \\
\hline 3155 & CR407521 & -2.76 & $3.01 \mathrm{E}-03$ \\
\hline 3156 & $\begin{array}{lc}\text { CR407528 } \\
\end{array}$ & -6.53 & $2.78 \mathrm{E}-04$ \\
\hline 3157 & CR407533 & -1.25 & $1.94 \mathrm{E}-02$ \\
\hline 3158 & CR407572 & -2.95 & $4.22 \mathrm{E}-03$ \\
\hline 3159 & CR407574 & -6.75 & $3.62 \mathrm{E}-04$ \\
\hline 3160 & \begin{tabular}{lc|} 
CR522884 \\
\end{tabular} & -2.67 & $8.77 \mathrm{E}-03$ \\
\hline 3161 & CR522885 & -3.46 & $5.30 \mathrm{E}-03$ \\
\hline 3162 & CR522889 & -2.11 & $2.69 \mathrm{E}-03$ \\
\hline 3163 & CR522901 & -2.62 & $1.47 \mathrm{E}-03$ \\
\hline 3164 & CR522902 & -1.96 & $3.49 \mathrm{E}-02$ \\
\hline 3165 & CR522911 & -2.99 & $3.82 \mathrm{E}-04$ \\
\hline 3166 & CR522924 & -4.00 & $1.22 \mathrm{E}-04$ \\
\hline 3167 & CR522933 & -1.78 & $3.35 \mathrm{E}-04$ \\
\hline 3168 & CR522937 & -1.48 & $2.35 \mathrm{E}-02$ \\
\hline 3169 & CR522950 & -3.66 & $1.44 \mathrm{E}-02$ \\
\hline 3170 & CR522954 & -2.96 & $1.03 \mathrm{E}-02$ \\
\hline 3171 & CR522957 & -5.43 & $\begin{array}{l}1.17 \mathrm{E}-03 \\
\end{array}$ \\
\hline 3172 & CR522961 & -2.67 & $1.08 \mathrm{E}-02$ \\
\hline 3173 & CR522971 & -1.74 & $1.17 \mathrm{E}-03$ \\
\hline 3174 & CR522976 & -2.57 & $2.70 \mathrm{E}-04$ \\
\hline 3175 & CR522983 & -2.50 & $1.14 \mathrm{E}-03$ \\
\hline 3176 & CR522985 & $\begin{array}{l}-2.79 \\
\end{array}$ & $1.88 \mathrm{E}-03$ \\
\hline 3177 & CR522990 & -3.16 & $2.96 \mathrm{E}-04$ \\
\hline 3178 & CR522994 & -1.72 & $1.74 \mathrm{E}-02$ \\
\hline 3179 & CR522995 & -2.52 & $1.16 \mathrm{E}-03$ \\
\hline 3180 & CR523002 & -4.68 & $1.80 \mathrm{E}-04$ \\
\hline 3181 & CR523006 & -1.75 & $1.97 \mathrm{E}-03$ \\
\hline 3182 & CR523007 & -1.55 & $9.80 \mathrm{E}-05$ \\
\hline 3183 & CR523012 & -3.57 & $6.32 \mathrm{E}-04$ \\
\hline 3184 & CR523022 & -1.89 & $6.89 \mathrm{E}-02$ \\
\hline 3185 & $\begin{array}{ll}\text { CR523025 } \\
\end{array}$ & -4.59 & $7.89 \mathrm{E}-05$ \\
\hline 3186 & CR523027 & -3.76 & $2.80 \mathrm{E}-03$ \\
\hline 3187 & CR523037 & -3.14 & $3.38 \mathrm{E}-03$ \\
\hline 3188 & CR523038 & -4.62 & $2.63 \mathrm{E}-03$ \\
\hline 3189 & CR523042 & -2.07 & $3.28 \mathrm{E}-03$ \\
\hline 3190 & CR523045 & -2.49 & $1.58 \mathrm{E}-03$ \\
\hline 3191 & $\begin{array}{ll}\text { CR523047 } \\
\end{array}$ & -4.29 & $1.30 \mathrm{E}-04$ \\
\hline 3192 & CR523052 & -6.58 & $6.03 \mathrm{E}-04$ \\
\hline 3193 & CR523053 & -3.11 & $4.93 \mathrm{E}-04$ \\
\hline 3194 & CR523056 & -3.05 & $8.99 \mathrm{E}-04$ \\
\hline 3195 & CR523067 & -1.35 & $1.82 \mathrm{E}-03$ \\
\hline 3196 & CR523068 & -4.50 & $3.76 \mathrm{E}-04$ \\
\hline 3197 & CR523071 & -3.37 & $1.31 \mathrm{E}-03$ \\
\hline 3198 & CR523073 & -1.42 & $4.67 \mathrm{E}-02$ \\
\hline 3199 & CR523074 & -1.29 & $2.52 \mathrm{E}-02$ \\
\hline 3200 & CR523084 & -3.01 & $1.15 \mathrm{E}-03$ \\
\hline 3201 & CR523085 & -1.20 & $6.40 \mathrm{E}-05$ \\
\hline 3202 & CR523088 & -2.77 & $2.23 \mathrm{E}-03$ \\
\hline 3203 & CR523093 & -2.47 & $6.94 \mathrm{E}-03$ \\
\hline 3204 & CR523102 & -4.52 & $5.79 \mathrm{E}-04$ \\
\hline 3205 & CR523112 & -2.26 & $6.23 \mathrm{E}-03$ \\
\hline 3206 & CR523119 & -5.62 & $7.57 \mathrm{E}-04$ \\
\hline 3207 & CR523137 & -1.69 & $1.41 \mathrm{E}-03$ \\
\hline 3208 & CR523138 & -4.01 & $1.54 \mathrm{E}-03$ \\
\hline 3209 & CR523145 & -3.36 & $2.62 \mathrm{E}-02$ \\
\hline 3210 & CR523149 & -5.36 & $1.45 \mathrm{E}-03$ \\
\hline 3211 & $\begin{array}{ll}\text { CR523153 } \\
\end{array}$ & -2.47 & $1.22 \mathrm{E}-02$ \\
\hline 3212 & CR523167 & $\begin{array}{l}-3.75 \\
\end{array}$ & $1.42 \mathrm{E}-02$ \\
\hline
\end{tabular}




\begin{tabular}{|c|c|c|c|}
\hline 3213 & CR523182 & -6.21 & $1.72 E-05$ \\
\hline 3214 & CR523188 & -1.69 & $2.26 \mathrm{E}-02$ \\
\hline 3215 & CR523193 & -1.22 & $1.00 \mathrm{E}-02$ \\
\hline 3216 & CR523195 & -2.47 & $\begin{array}{l}7.54 \mathrm{E}-04 \\
\end{array}$ \\
\hline 3217 & CR523206 & -1.59 & $7.24 \mathrm{E}-02$ \\
\hline 3218 & CR523239 & -1.75 & $1.87 \mathrm{E}-02$ \\
\hline 3219 & CR523258 & -3.66 & $3.76 \mathrm{E}-04$ \\
\hline 3220 & CR523259 & -3.01 & $3.80 \mathrm{E}-03$ \\
\hline 3221 & CR523262 & -4.49 & $5.71 \mathrm{E}-04$ \\
\hline 3222 & CR523268 & \begin{tabular}{|l|}
-2.91 \\
\end{tabular} & $1.01 \mathrm{E}-03$ \\
\hline 3223 & CR523273 & -3.27 & $8.35 \mathrm{E}-04$ \\
\hline 3224 & CR523277 & -3.99 & $1.15 \mathrm{E}-04$ \\
\hline 3225 & CR523278 & -3.38 & $3.39 \mathrm{E}-03$ \\
\hline 3226 & CR523281 & -2.24 & $3.41 \mathrm{E}-02$ \\
\hline 3227 & CR523303 & -3.00 & $9.29 \mathrm{E}-03$ \\
\hline 3228 & CR523341 & \begin{tabular}{|l|}
-3.53 \\
\end{tabular} & $8.72 \mathrm{E}-04$ \\
\hline 3229 & CR523357 & -1.09 & $5.92 \mathrm{E}-03$ \\
\hline 3230 & CR523359 & -1.99 & $6.47 \mathrm{E}-03$ \\
\hline 3231 & CR523361 & -2.01 & $6.63 \mathrm{E}-03$ \\
\hline 3232 & CR523362 & -2.13 & $3.03 \mathrm{E}-02$ \\
\hline 3233 & CR523377 & -3.09 & $1.34 \mathrm{E}-03$ \\
\hline 3234 & CR523380 & $\begin{array}{l}-1.82 \\
\end{array}$ & $7.14 \mathrm{E}-03$ \\
\hline 3235 & CR523382 & -3.52 & $2.05 E-03$ \\
\hline 3236 & CR523406 & -4.08 & $5.15 \mathrm{E}-03$ \\
\hline 3237 & CR523407 & -4.64 & $4.79 E-03$ \\
\hline 3238 & CR523409 & -2.97 & $3.48 \mathrm{E}-03$ \\
\hline 3239 & CR523411 & -3.54 & $3.25 \mathrm{E}-03$ \\
\hline 3240 & CR523418 & -2.97 & $3.44 \mathrm{E}-02$ \\
\hline 3241 & CR523420 & -3.54 & $1.69 \mathrm{E}-02$ \\
\hline 3242 & CR523421 & -5.53 & $5.37 \mathrm{E}-05$ \\
\hline 3243 & CR523427 & -4.01 & $1.53 \mathrm{E}-03$ \\
\hline 3244 & CR523432 & -4.26 & $9.13 \mathrm{E}-05$ \\
\hline 3245 & CR523436 & -2.99 & 7.07E-04 \\
\hline 3246 & CR523445 & \begin{tabular}{|l|}
-2.94 \\
\end{tabular} & $2.19 \mathrm{E}-04$ \\
\hline 3247 & CR523449 & -5.64 & $6.78 \mathrm{E}-05$ \\
\hline 3248 & CR523450 & -2.20 & 7.14E-04 \\
\hline 3249 & CR523451 & -1.97 & $\begin{array}{r}2.47 \mathrm{E}-03 \\
\end{array}$ \\
\hline 3250 & CR523452 & -1.38 & $2.49 \mathrm{E}-03$ \\
\hline 3251 & CR523456 & -3.35 & $2.93 \mathrm{E}-03$ \\
\hline 3252 & CR523466 & -1.54 & $8.73 \mathrm{E}-03$ \\
\hline 3253 & CR523467 & -2.24 & $7.62 \mathrm{E}-04$ \\
\hline 3254 & CR523468 & -3.92 & $2.85 \mathrm{E}-04$ \\
\hline 3255 & CR523471 & -1.56 & $1.52 \mathrm{E}-02$ \\
\hline 3256 & CR523474 & -2.46 & $5.08 \mathrm{E}-03$ \\
\hline 3257 & CR523476 & -2.13 & $7.56 \mathrm{E}-03$ \\
\hline 3258 & CR523481 & -1.71 & $2.25 \mathrm{E}-03$ \\
\hline 3259 & CR523495 & -6.71 & 7.46E-04 \\
\hline 3260 & CR523496 & -2.16 & $2.13 \mathrm{E}-03$ \\
\hline 3261 & CR523505 & -3.34 & $2.82 \mathrm{E}-03$ \\
\hline 3262 & CR523506 & -4.56 & $9.66 \mathrm{E}-05$ \\
\hline 3263 & CR523507 & -3.89 & $1.29 \mathrm{E}-04$ \\
\hline 3264 & CR523515 & -6.67 & $8.93 \mathrm{E}-04$ \\
\hline 3265 & CR523529 & -1.92 & $6.25 \mathrm{E}-03$ \\
\hline 3266 & CR523535 & $\begin{array}{l}-1.19 \\
\end{array}$ & $2.84 \mathrm{E}-02$ \\
\hline 3267 & CR523538 & -1.52 & $1.99 \mathrm{E}-02$ \\
\hline 3268 & CR523543 & -2.37 & $1.92 \mathrm{E}-02$ \\
\hline 3269 & CR523550 & -1.74 & $2.32 \mathrm{E}-03$ \\
\hline 3270 & CR523554 & -3.19 & $4.26 \mathrm{E}-03$ \\
\hline 3271 & CR523555 & -5.61 & $6.47 \mathrm{E}-04$ \\
\hline 3272 & CR523560 & -2.74 & $4.82 \mathrm{E}-03$ \\
\hline 3273 & CR523588 & -4.06 & $4.05 E-03$ \\
\hline 3274 & CR523597 & -3.37 & $2.30 \mathrm{E}-03$ \\
\hline 3275 & СК523599 & -3.14 & $2 . / 1 \mathrm{I}-0$ \\
\hline
\end{tabular}

\begin{tabular}{|c|c|c|c|}
\hline 3276 & CR523603 & -1.13 & $1.53 \mathrm{E}-02$ \\
\hline 3277 & CR523607 & -2.88 & $5.39 \mathrm{E}-03$ \\
\hline 3278 & CR523610 & -3.49 & $5.23 \mathrm{E}-04$ \\
\hline 3279 & CR523615 & -2.47 & $4.08 \mathrm{E}-03$ \\
\hline 3280 & CR523616 & -2.03 & $9.04 \mathrm{E}-03$ \\
\hline 3281 & CR523620 & -6.45 & $2.66 \mathrm{E}-04$ \\
\hline 3282 & CR523636 & -3.99 & 4.07E-04 \\
\hline 3283 & CR523640 & -5.84 & 4.06E-04 \\
\hline 3284 & CR523644 & -1.78 & $5.81 \mathrm{E}-05$ \\
\hline 3285 & CR523650 & -2.16 & $2.82 \mathrm{E}-03$ \\
\hline 3286 & CR523658 & -1.37 & $2.30 \mathrm{E}-02$ \\
\hline 3287 & CR523661 & -3.51 & $3.72 \mathrm{E}-05$ \\
\hline 3288 & CR523681 & -2.70 & $8.54 \mathrm{E}-03$ \\
\hline 3289 & CR523699 & -4.02 & $2.52 \mathrm{E}-04$ \\
\hline 3290 & CR523701 & -2.88 & $7.06 \mathrm{E}-03$ \\
\hline 3291 & CR523719 & -1.93 & $5.33 \mathrm{E}-04$ \\
\hline 3292 & CR523723 & -3.83 & $1.18 \mathrm{E}-04$ \\
\hline 3293 & CR523726 & -6.41 & $1.13 \mathrm{E}-04$ \\
\hline 3294 & CR523729 & -1.96 & $1.39 \mathrm{E}-02$ \\
\hline 3295 & CR523735 & -2.01 & $2.61 \mathrm{E}-03$ \\
\hline 3296 & CR523739 & -2.99 & $6.20 \mathrm{E}-04$ \\
\hline 3297 & CR523743 & -4.13 & $1.97 \mathrm{E}-03$ \\
\hline 3298 & CR523745 & -2.45 & $9.63 \mathrm{E}-03$ \\
\hline 3299 & CR523750 & -3.48 & $7.40 \mathrm{E}-04$ \\
\hline 3300 & CR523760 & -1.75 & $1.65 \mathrm{E}-03$ \\
\hline 3301 & CR523766 & -3.19 & $2.08 \mathrm{E}-03$ \\
\hline 3302 & CR523771 & -1.37 & $5.70 \mathrm{E}-02$ \\
\hline 3303 & CR523778 & -5.63 & $4.27 \mathrm{E}-05$ \\
\hline 3304 & CR523794 & -3.70 & $5.35 \mathrm{E}-04$ \\
\hline 3305 & CR523795 & -5.77 & $3.18 \mathrm{E}-04$ \\
\hline 3306 & CR523806 & -1.55 & $3.53 \mathrm{E}-02$ \\
\hline 3307 & CR523813 & -2.70 & $7.22 \mathrm{E}-04$ \\
\hline 3308 & CR523816 & -1.79 & $2.27 \mathrm{E}-02$ \\
\hline 3309 & CR523844 & -1.30 & $3.19 \mathrm{E}-02$ \\
\hline 3310 & CR523871 & -3.49 & $1.08 \mathrm{E}-03$ \\
\hline 3311 & CR523878 & -2.18 & $1.65 \mathrm{E}-02$ \\
\hline 3312 & CR523883 & -2.19 & $1.29 \mathrm{E}-04$ \\
\hline 3313 & CR523903 & -2.61 & $3.82 \mathrm{E}-04$ \\
\hline 3314 & CR523929 & -3.41 & $5.73 \mathrm{E}-04$ \\
\hline 3315 & CR523934 & -4.19 & $9.42 \mathrm{E}-05$ \\
\hline 3316 & CR523941 & -1.44 & $1.16 \mathrm{E}-02$ \\
\hline 3317 & CR523950 & -3.07 & $6.46 \mathrm{E}-03$ \\
\hline 3318 & CR523971 & -1.26 & $7.21 \mathrm{E}-04$ \\
\hline 3319 & CR523978 & -3.71 & $3.48 \mathrm{E}-04$ \\
\hline 3320 & CR523983 & -4.50 & $6.74 \mathrm{E}-04$ \\
\hline 3321 & CR523991 & -3.79 & $5.79 E-04$ \\
\hline 3322 & CR524006 & -2.28 & $1.09 \mathrm{E}-02$ \\
\hline 3323 & CR524027 & -2.43 & $6.78 \mathrm{E}-04$ \\
\hline 3324 & CR524031 & -1.85 & $6.97 \mathrm{E}-04$ \\
\hline 3325 & CR524053 & -4.85 & $6.97 \mathrm{E}-04$ \\
\hline 3326 & CR524058 & -1.59 & $1.46 \mathrm{E}-02$ \\
\hline 3327 & CR524063 & -5.07 & $1.26 \mathrm{E}-04$ \\
\hline 3328 & CR524078 & -3.21 & $3.04 \mathrm{E}-04$ \\
\hline 3329 & CR524091 & -3.00 & $3.21 \mathrm{E}-04$ \\
\hline 3330 & CR524093 & -2.50 & $2.82 \mathrm{E}-03$ \\
\hline 3331 & CR524097 & -1.60 & $9.87 \mathrm{E}-03$ \\
\hline 3332 & CR524099 & -1.50 & $5.56 \mathrm{E}-05$ \\
\hline 3333 & CR524109 & -1.57 & $3.37 \mathrm{E}-03$ \\
\hline 3334 & CR524113 & -1.65 & $3.71 \mathrm{E}-03$ \\
\hline 3335 & CR524118 & -1.47 & $5.04 \mathrm{E}-04$ \\
\hline 3336 & CR524122 & -3.00 & $1.15 \mathrm{E}-03$ \\
\hline 3337 & CR524125 & -5.52 & $1.09 \mathrm{E}-03$ \\
\hline 3338 & CR524132 & -1.60 & $3.29 \mathrm{E}-02$ \\
\hline
\end{tabular}

\begin{tabular}{|c|c|c|c|}
\hline 3339 & CR524142 & -1.32 & $4.68 \mathrm{E}-03$ \\
\hline 3340 & CR524148 & -3.05 & $4.91 E-03$ \\
\hline 3341 & CR524149 & -4.60 & $1.91 \mathrm{E}-03$ \\
\hline 3342 & CR524162 & -5.52 & $5.55 \mathrm{E}-06$ \\
\hline 3343 & CR524164 & -2.04 & $6.85 \mathrm{E}-03$ \\
\hline 3344 & CR524165 & -2.30 & $6.00 E-03$ \\
\hline 3345 & CR524171 & -3.29 & $\begin{array}{l}1.07 E-03 \\
\end{array}$ \\
\hline 3346 & CR524193 & -1.97 & $2.56 \mathrm{E}-04$ \\
\hline 3347 & CR524194 & -1.11 & $7.73 E-03$ \\
\hline 3348 & CR524196 & -2.21 & $7.65 \mathrm{E}-03$ \\
\hline 3349 & CR524201 & -1.92 & $2.76 E-03$ \\
\hline 3350 & CR524215 & -4.09 & $3.51 E-04$ \\
\hline 3351 & CR524234 & -4.31 & $1.02 E-03$ \\
\hline 3352 & CR524242 & -1.34 & $1.99 E-02$ \\
\hline 3353 & CR524257 & -4.04 & $1.19 \mathrm{E}-03$ \\
\hline 3354 & CR524259 & -4.20 & $2.39 E-03$ \\
\hline 3355 & CR524260 & -3.96 & $2.53 \mathrm{E}-03$ \\
\hline 3356 & CR524264 & -2.16 & $3.20 \mathrm{E}-03$ \\
\hline 3357 & CR524266 & -3.59 & $9.98 E-04$ \\
\hline 3358 & CR524270 & -4.33 & $\begin{array}{l}7.63 E-04 \\
\end{array}$ \\
\hline 3359 & CR524275 & -4.82 & $3.65 E-04$ \\
\hline 3360 & CR524282 & -4.13 & $3.94 E-04$ \\
\hline 3361 & CR524288 & -2.30 & $6.05 E-04$ \\
\hline 3362 & CR524290 & -4.62 & $8.91 E-04$ \\
\hline 3363 & CR524300 & -2.21 & $5.11 \mathrm{E}-04$ \\
\hline 3364 & CR524313 & -3.73 & $5.29 \mathrm{E}-03$ \\
\hline 3365 & CR524315 & -4.93 & $2.88 \mathrm{E}-04$ \\
\hline 3366 & CR524325 & -1.54 & $5.15 E-03$ \\
\hline 3367 & CR524329 & -2.63 & $6.51 \mathrm{E}-03$ \\
\hline 3368 & CR524337 & -1.65 & $2.30 \mathrm{E}-03$ \\
\hline 3369 & CR524339 & -1.42 & $3.79 E-03$ \\
\hline 3370 & CR524351 & -3.42 & $6.36 \mathrm{E}-05$ \\
\hline 3371 & CR524352 & -1.51 & $5.82 E-02$ \\
\hline 3372 & CR524355 & -3.38 & $4.38 E-04$ \\
\hline 3373 & CR524365 & -3.53 & $4.91 E-04$ \\
\hline 3374 & CR524369 & -2.24 & $2.51 \mathrm{E}-03$ \\
\hline 3375 & CR524375 & -1.58 & $3.98 \mathrm{E}-03$ \\
\hline 3376 & CR524376 & -1.49 & $3.27 E-02$ \\
\hline 3377 & CR524387 & -1.11 & $1.81 E-02$ \\
\hline 3378 & CR524389 & -4.02 & $2.08 \mathrm{E}-03$ \\
\hline 3379 & CR524390 & -3.58 & $2.14 E-05$ \\
\hline 3380 & CR524404 & -5.63 & $7.55 \mathrm{E}-04$ \\
\hline 3381 & CR524406 & -3.78 & $6.34 \mathrm{E}-04$ \\
\hline 3382 & CR524412 & -2.06 & $1.76 E-02$ \\
\hline 3383 & CR524429 & $\begin{array}{l}-1.72 \\
\end{array}$ & $4.25 \mathrm{E}-03$ \\
\hline 3384 & CR524436 & -6.22 & $2.13 E-05$ \\
\hline 3385 & CR524462 & -2.26 & $6.47 \mathrm{E}-03$ \\
\hline 3386 & CR524463 & -1.53 & $1.18 \mathrm{E}-02$ \\
\hline 3387 & CR524466 & -3.05 & $9.37 E-03$ \\
\hline 3388 & CR732735 & -3.57 & $6.94 E-03$ \\
\hline 3389 & CR732778 & -1.91 & $7.61 \mathrm{E}-03$ \\
\hline 3390 & CR732803 & -6.89 & $7.98 E-04$ \\
\hline 3391 & CR732824 & -1.40 & $3.73 E-02$ \\
\hline 3392 & CR733020 & -2.06 & $4.85 E-02$ \\
\hline 3393 & CR733064 & -4.86 & $1.93 E-04$ \\
\hline 3394 & CR733118 & -3.23 & $4.19 \mathrm{E}-03$ \\
\hline 3395 & CR733127 & -1.27 & $7.35 \mathrm{E}-03$ \\
\hline 3396 & CR733131 & -1.24 & $1.64 \mathrm{E}-02$ \\
\hline 3397 & CR733143 & -1.50 & $3.42 E-02$ \\
\hline 3398 & CR733216 & -5.08 & $2.11 \mathrm{E}-03$ \\
\hline 3399 & CR733231 & -7.68 & $3.51 E-04$ \\
\hline 3400 & CR733258 & -1.98 & $2.90 E-02$ \\
\hline 3401 & CR733265 & -3.89 & $3.56 \mathrm{E}-04$ \\
\hline
\end{tabular}




\begin{tabular}{|c|c|c|c|}
\hline 3402 & CR733307 & -4.30 & 4.63E- 03 \\
\hline 3403 & CR733330 & -1.70 & $6.89 \mathrm{E}-02$ \\
\hline 3404 & CR733334 & -3.24 & $1.40 \mathrm{E}-03$ \\
\hline 3405 & $\begin{array}{l}\text { CR733347 } \\
\end{array}$ & $\begin{array}{ll}-3.17 \\
\end{array}$ & $1.39 \mathrm{E}-03$ \\
\hline 3406 & CR733352 & -4.17 & $1.07 E-03$ \\
\hline 3407 & CR733387 & -2.59 & $8.68 \mathrm{E}-05$ \\
\hline 3408 & CR733416 & -2.58 & $3.49 \mathrm{E}-03$ \\
\hline 3409 & CRABP1 & -3.08 & $8.98 \mathrm{E}-03$ \\
\hline 3410 & CRCP & -5.60 & $2.05 \mathrm{E}-04$ \\
\hline 3411 & CRDS2 & -2.66 & $1.15 \mathrm{E}-03$ \\
\hline 3412 & CREB3 & -3.54 & $2.93 \mathrm{E}-04$ \\
\hline 3413 & CREB3L1 & -1.23 & $1.04 \mathrm{E}-02$ \\
\hline 3414 & CREBL2 & -3.56 & $3.10 \mathrm{E}-04$ \\
\hline 3415 & CRELD2 & -4.62 & 2.27E-04 \\
\hline 3416 & CREM & -2.28 & $9.78 \mathrm{E}-03$ \\
\hline 3417 & CRH & $\begin{array}{ll}-1.87 \\
\end{array}$ & $9.77 E-02$ \\
\hline 3418 & CRIM1 & -2.03 & $5.30 \mathrm{E}-03$ \\
\hline 3419 & $\begin{array}{ll}\text { CRIPT } \\
\end{array}$ & -5.37 & $9.31 \mathrm{E}-04$ \\
\hline 3420 & CRKL & -5.06 & 9.19E-04 \\
\hline 3421 & CRLF3 & -5.35 & $3.72 \mathrm{E}-04$ \\
\hline 3422 & CRLS1 & -3.12 & 4.59E-04 \\
\hline 3423 & CRNKL1 & -3.99 & $1.26 \mathrm{E}-03$ \\
\hline 3424 & CROT & -7.49 & $1.74 E-04$ \\
\hline 3425 & CRTAP & -5.09 & $1.01 \mathrm{E}-03$ \\
\hline 3426 & CRTC1 & -5.99 & $8.29 \mathrm{E}-05$ \\
\hline 3427 & CRY1 & -3.31 & 2.82E-04 \\
\hline 3428 & CRY2 & -4.19 & 4.16E-04 \\
\hline 3429 & CRYGN & -1.46 & $4.26 \mathrm{E}-02$ \\
\hline 3430 & CRYL1 & -2.39 & $3.42 \mathrm{E}-04$ \\
\hline 3431 & CRYM & -2.16 & $2.19 E-02$ \\
\hline 3432 & CRYZ & -2.14 & $2.10 \mathrm{E}-03$ \\
\hline 3433 & CRYZL1 & -6.21 & 4.18E-04 \\
\hline 3434 & CSDA & -3.02 & $4.78 E-03$ \\
\hline 3435 & CSDE1 & -3.66 & 6.63E-05 \\
\hline 3436 & CSE1L & -4.57 & $3.55 \mathrm{E}-03$ \\
\hline 3437 & CSGALNACT2 & -2.08 & $7.80 \mathrm{E}-03$ \\
\hline 3438 & CSNK1A1 & -5.99 & $1.00 E-03$ \\
\hline 3439 & CSNK1D & -2.30 & $5.75 \mathrm{E}-04$ \\
\hline 3440 & CSNK1E & -4.76 & $1.76 \mathrm{E}-03$ \\
\hline 3441 & CSNK1G1 & -3.85 & $1.21 \mathrm{E}-03$ \\
\hline 3442 & CSNK2A1 & -4.27 & $1.41 \mathrm{E}-04$ \\
\hline 3443 & CSRNP1 & -3.19 & $1.33 \mathrm{E}-02$ \\
\hline 3444 & CSRNP3 & -6.69 & 4.47E-04 \\
\hline 3445 & CSRP2 & -4.18 & $1.80 \mathrm{E}-03$ \\
\hline 3446 & CSRP2BP & -4.07 & $9.20 \mathrm{E}-05$ \\
\hline 3447 & CST3 & -6.89 & $1.89 \mathrm{E}-04$ \\
\hline 3448 & CSTB & -5.70 & $8.25 \mathrm{E}-04$ \\
\hline 3449 & CSTF2 & -2.70 & $8.17 \mathrm{E}-03$ \\
\hline 3450 & CSTF3 & -6.41 & $3.55 E-04$ \\
\hline 3451 & СТВP1 & -3.17 & 4.29E-04 \\
\hline 3452 & CTCF & -1.97 & $2.77 E-02$ \\
\hline 3453 & CTDP1 & -3.41 & $1.08 \mathrm{E}-05$ \\
\hline 3454 & CTDSPL & -2.50 & $1.93 \mathrm{E}-02$ \\
\hline 3455 & CTDSPL2 & -1.87 & $2.74 E-02$ \\
\hline 3456 & CTGF & -3.26 & $1.98 \mathrm{E}-02$ \\
\hline 3457 & СтH & -1.78 & 1.97E-02 \\
\hline 3458 & CTHRC1 & -4.12 & $1.78 \mathrm{E}-03$ \\
\hline 3459 & CTNNA1 & -3.20 & $3.51 \mathrm{E}-04$ \\
\hline 3460 & CTNNA3 & -1.47 & $2.07 E-03$ \\
\hline 3461 & CTNNB1 & -5.82 & $6.38 \mathrm{E}-04$ \\
\hline 3462 & CTNNBIP1 & -2.50 & $1.02 E-03$ \\
\hline 3463 & CTNNBL1 & -3.40 & $2.72 \mathrm{E}-04$ \\
\hline 3464 & CTNND1 & -1.65 & $1.80 \mathrm{E}-02$ \\
\hline
\end{tabular}

\begin{tabular}{|c|c|c|c|}
\hline 3465 & CTPS2 & -4.38 & $6.45 \mathrm{E}-04$ \\
\hline 3466 & CTR9 & -3.95 & $1.54 \mathrm{E}-04$ \\
\hline 3467 & CTSA & -5.96 & $6.24 \mathrm{E}-04$ \\
\hline 3468 & CTSH & -1.05 & $7.86 \mathrm{E}-03$ \\
\hline 3469 & CTSL2 & -4.03 & $2.70 \mathrm{E}-04$ \\
\hline 3470 & CTSS & -1.21 & $4.40 \mathrm{E}-02$ \\
\hline 3471 & CTSZ & -1.11 & $1.37 \mathrm{E}-03$ \\
\hline 3472 & CTTN & -2.46 & $3.79 \mathrm{E}-03$ \\
\hline 3473 & CTTNBP2 & -4.53 & $1.85 \mathrm{E}-04$ \\
\hline 3474 & CTTNBP2NL & -2.50 & $8.33 \mathrm{E}-04$ \\
\hline 3475 & CUL1 & -2.58 & $4.47 \mathrm{E}-03$ \\
\hline 3476 & CUL2 & -1.22 & $6.71 \mathrm{E}-04$ \\
\hline 3477 & CUL3 & -6.83 & $7.45 \mathrm{E}-05$ \\
\hline 3478 & CUL4A & -3.87 & $1.90 \mathrm{E}-03$ \\
\hline 3479 & CUL5 & -2.58 & $6.88 \mathrm{E}-04$ \\
\hline 3480 & CUTA & -2.22 & $3.97 \mathrm{E}-02$ \\
\hline 3481 & CUTC & -3.79 & $1.12 \mathrm{E}-04$ \\
\hline 3482 & CUX1 & -4.13 & $8.00 \mathrm{E}-03$ \\
\hline 3483 & CV854043 & -2.25 & $4.46 \mathrm{E}-03$ \\
\hline 3484 & CV855125 & -2.65 & $4.89 \mathrm{E}-04$ \\
\hline 3485 & CV855835 & -4.53 & $7.15 \mathrm{E}-04$ \\
\hline 3486 & CV856329 & -4.67 & $4.44 \mathrm{E}-04$ \\
\hline 3487 & CV856945 & -2.98 & $1.53 \mathrm{E}-02$ \\
\hline 3488 & CV858534 & -3.77 & $1.24 \mathrm{E}-04$ \\
\hline 3489 & CV858761 & -4.74 & $8.52 \mathrm{E}-04$ \\
\hline 3490 & CV859025 & -1.73 & $1.92 \mathrm{E}-02$ \\
\hline 3491 & CV859088 & -3.65 & $3.35 \mathrm{E}-04$ \\
\hline 3492 & CV859173 & -3.67 & $3.11 \mathrm{E}-03$ \\
\hline 3493 & CV859265 & -2.58 & $3.43 \mathrm{E}-03$ \\
\hline 3494 & CV859284 & -2.45 & $2.56 \mathrm{E}-05$ \\
\hline 3495 & CV859334 & -1.93 & $8.33 \mathrm{E}-04$ \\
\hline 3496 & CV859391 & -1.39 & $3.94 \mathrm{E}-02$ \\
\hline 3497 & CV859444 & -2.75 & $2.34 \mathrm{E}-04$ \\
\hline 3498 & CV859557 & -2.72 & $7.15 \mathrm{E}-04$ \\
\hline 3499 & CV859752 & -4.18 & $2.83 \mathrm{E}-04$ \\
\hline 3500 & CV859797 & -3.36 & $3.15 \mathrm{E}-03$ \\
\hline 3501 & CV859945 & -1.37 & $1.81 \mathrm{E}-03$ \\
\hline 3502 & CV860146 & -3.56 & $1.87 \mathrm{E}-03$ \\
\hline 3503 & CV860159 & -3.09 & $1.36 \mathrm{E}-03$ \\
\hline 3504 & CV860274 & -1.52 & $5.09 \mathrm{E}-03$ \\
\hline 3505 & CV860626 & -3.58 & $3.78 \mathrm{E}-03$ \\
\hline 3506 & CV860651 & -3.30 & $8.57 \mathrm{E}-02$ \\
\hline 3507 & CV860692 & -1.67 & $1.31 \mathrm{E}-02$ \\
\hline 3508 & CV860933 & -4.58 & $8.78 \mathrm{E}-04$ \\
\hline 3509 & CV861196 & -3.29 & $6.13 \mathrm{E}-04$ \\
\hline 3510 & CV861241 & -1.34 & $1.45 \mathrm{E}-02$ \\
\hline 3511 & CV861265 & -1.57 & $6.86 \mathrm{E}-03$ \\
\hline 3512 & CV861302 & -3.88 & $1.54 \mathrm{E}-04$ \\
\hline 3513 & CV861605 & -3.15 & $9.38 \mathrm{E}-04$ \\
\hline 3514 & CV861615 & -3.03 & $3.59 \mathrm{E}-04$ \\
\hline 3515 & CV861629 & -1.23 & $1.42 \mathrm{E}-02$ \\
\hline 3516 & CV861667 & -2.00 & $6.83 \mathrm{E}-03$ \\
\hline 3517 & CV861885 & -2.69 & $1.88 \mathrm{E}-03$ \\
\hline 3518 & CV862250 & -1.45 & $2.04 \mathrm{E}-03$ \\
\hline 3519 & CV862376 & -2.92 & $1.44 \mathrm{E}-02$ \\
\hline 3520 & CV862635 & -4.14 & $6.23 \mathrm{E}-04$ \\
\hline 3521 & CV862799 & -5.97 & $9.17 \mathrm{E}-04$ \\
\hline 3522 & CWC15 & -5.68 & $4.25 \mathrm{E}-05$ \\
\hline 3523 & CWC22 & -5.17 & $4.12 \mathrm{E}-04$ \\
\hline 3524 & CWC25 & -1.41 & $1.97 \mathrm{E}-02$ \\
\hline 3525 & CWC27 & -3.68 & $1.04 \mathrm{E}-04$ \\
\hline 3526 & CWF19L2 & $\begin{array}{l}-3.73 \\
\end{array}$ & $6.47 \mathrm{E}-05$ \\
\hline 3527 & CXADR & -4.68 & $5.79 \mathrm{E}-03$ \\
\hline
\end{tabular}

\begin{tabular}{|c|c|c|c|}
\hline 3528 & CXCL13L2 & -1.41 & $4.71 \mathrm{E}-03$ \\
\hline 3529 & CXCR7 & -2.84 & $3.63 \mathrm{E}-04$ \\
\hline 3530 & CXXC5 & -1.11 & $8.87 \mathrm{E}-03$ \\
\hline 3531 & CYB5A & -3.73 & $4.39 \mathrm{E}-04$ \\
\hline 3532 & СУВ5В & -1.57 & $1.78 \mathrm{E}-02$ \\
\hline 3533 & CYB5D2 & -3.69 & $5.61 \mathrm{E}-04$ \\
\hline 3534 & CYB5R2 & -2.39 & $5.43 \mathrm{E}-03$ \\
\hline 3535 & CYBSRL & -2.56 & $2.48 \mathrm{E}-04$ \\
\hline 3536 & CYBASC3 & -1.37 & $7.20 \mathrm{E}-03$ \\
\hline 3537 & CYCS & -2.96 & $1.94 \mathrm{E}-02$ \\
\hline 3538 & CYP11A1 & -2.12 & $6.51 \mathrm{E}-02$ \\
\hline 3539 & CYP20A1 & -1.41 & $2.49 \mathrm{E}-03$ \\
\hline 3540 & СYР39A1 & -2.40 & $3.11 \mathrm{E}-03$ \\
\hline 3541 & CYP46A1 & -5.41 & $2.02 \mathrm{E}-04$ \\
\hline 3542 & CYP51A1 & -2.12 & $2.26 \mathrm{E}-03$ \\
\hline 3543 & CYP7A1 & -1.30 & $2.89 \mathrm{E}-02$ \\
\hline 3544 & СYP8B1 & -3.63 & $3.03 \mathrm{E}-02$ \\
\hline 3545 & CYSTM1 & -2.93 & $6.59 \mathrm{E}-04$ \\
\hline 3546 & СYTB & -8.51 & $2.98 \mathrm{E}-03$ \\
\hline 3547 & CYTH1 & -2.07 & $3.22 \mathrm{E}-04$ \\
\hline 3548 & CZH18orf25 & -1.40 & $4.04 \mathrm{E}-03$ \\
\hline 3549 & CZH20orf134 & -4.02 & $1.15 \mathrm{E}-03$ \\
\hline 3550 & CZH5orf28 & -3.35 & $1.25 \mathrm{E}-03$ \\
\hline 3551 & CZH5orf3O & -1.78 & $5.74 \mathrm{E}-02$ \\
\hline 3552 & CZH5orf44 & -4.47 & $6.66 \mathrm{E}-05$ \\
\hline 3553 & CZH5orf51 & -3.94 & $1.60 \mathrm{E}-03$ \\
\hline 3554 & CZH5orf63 & -3.12 & $4.25 \mathrm{E}-03$ \\
\hline 3555 & CZH9orf100 & -1.32 & $3.47 \mathrm{E}-03$ \\
\hline 3556 & CZH9orf103 & -2.96 & $1.17 \mathrm{E}-03$ \\
\hline 3557 & CZH9orf150 & -3.53 & $2.67 \mathrm{E}-04$ \\
\hline 3558 & CZH9orf24 & -6.78 & $7.46 \mathrm{E}-04$ \\
\hline 3559 & CZH9orf41 & -2.11 & $2.09 \mathrm{E}-03$ \\
\hline 3560 & CZH9orf64 & -1.88 & $2.07 \mathrm{E}-03$ \\
\hline 3561 & CZH9orf95 & -3.00 & $4.49 \mathrm{E}-03$ \\
\hline 3562 & D2HGDH & -1.62 & $1.48 \mathrm{E}-02$ \\
\hline 3563 & D90399 & -2.25 & $3.14 \mathrm{E}-02$ \\
\hline 3564 & DAAM1 & -1.64 & $1.47 \mathrm{E}-02$ \\
\hline 3565 & DAAM2 & -3.65 & $1.26 \mathrm{E}-03$ \\
\hline 3566 & DAB2IP & -2.76 & $2.26 \mathrm{E}-03$ \\
\hline 3567 & DACH2 & -3.66 & $1.34 \mathrm{E}-03$ \\
\hline 3568 & DAD1 & -7.19 & $4.35 \mathrm{E}-04$ \\
\hline 3569 & DAGLA & -1.25 & $3.21 \mathrm{E}-01$ \\
\hline 3570 & DAP & -2.00 & $2.94 \mathrm{E}-02$ \\
\hline 3571 & DAP3 & -3.98 & $4.90 \mathrm{E}-03$ \\
\hline 3572 & DAPP1 & -3.94 & $6.39 \mathrm{E}-04$ \\
\hline 3573 & DARS & -3.95 & $3.26 \mathrm{E}-03$ \\
\hline 3574 & DASRAA & -3.77 & $2.39 \mathrm{E}-03$ \\
\hline 3575 & DAZAP1 & -6.65 & $6.43 \mathrm{E}-04$ \\
\hline 3576 & DAZL & -6.94 & $1.70 \mathrm{E}-03$ \\
\hline 3577 & DBI & -4.64 & $1.76 \mathrm{E}-03$ \\
\hline 3578 & DBNDD1 & -2.77 & $1.16 \mathrm{E}-01$ \\
\hline 3579 & DBT & -1.65 & $1.01 \mathrm{E}-03$ \\
\hline 3580 & DBX2 & -3.67 & 4.96E-04 \\
\hline 3581 & DCAF12 & -2.51 & $5.83 \mathrm{E}-03$ \\
\hline 3582 & DCAF13 & -2.71 & $1.11 \mathrm{E}-04$ \\
\hline 3583 & DCAF4 & -5.93 & $2.76 \mathrm{E}-04$ \\
\hline 3584 & DCAF5 & -1.77 & $3.35 \mathrm{E}-02$ \\
\hline 3585 & DCAF7 & -3.81 & $1.25 \mathrm{E}-04$ \\
\hline 3586 & DCAKD & -3.31 & $1.45 \mathrm{E}-03$ \\
\hline 3587 & DCBLD2 & -4.70 & $6.35 \mathrm{E}-04$ \\
\hline 3588 & DCK & -4.40 & $8.61 \mathrm{E}-04$ \\
\hline 3589 & DCLRE1A & $\begin{array}{l}-2.70 \\
\end{array}$ & $2.20 \mathrm{E}-03$ \\
\hline 3590 & DCLRE1B & -2.38 & $3.48 \mathrm{E}-04$ \\
\hline
\end{tabular}




\begin{tabular}{|c|c|c|c|}
\hline 3591 & $\mathrm{DCN}$ & -1.19 & $3.27 \mathrm{E}-03$ \\
\hline 3592 & DCP1A & -2.59 & $8.59 \mathrm{E}-03$ \\
\hline 3593 & DCPS & -2.25 & $7.15 \mathrm{E}-03$ \\
\hline 3594 & DCTD & -3.65 & $2.22 \mathrm{E}-03$ \\
\hline 3595 & DCTN1 & -1.08 & $4.42 \mathrm{E}-03$ \\
\hline 3596 & DCTN3 & -6.17 & 2.37E-05 \\
\hline 3597 & DCTN4 & -4.27 & 3.34E-04 \\
\hline 3598 & DCTN5 & -3.18 & $1.65 \mathrm{E}-03$ \\
\hline 3599 & DCTN6 & -6.43 & $3.48 \mathrm{E}-04$ \\
\hline 3600 & DCUN1D1 & -4.60 & $1.96 \mathrm{E}-04$ \\
\hline 3601 & DCUN1D2 & -4.12 & $1.51 \mathrm{E}-02$ \\
\hline 3602 & DCUN1D3 & -2.00 & $1.79 \mathrm{E}-03$ \\
\hline 3603 & DCUN1D4 & -2.52 & $3.59 \mathrm{E}-02$ \\
\hline 3604 & DCUN1D5 & -7.22 & $2.59 \mathrm{E}-04$ \\
\hline 3605 & DCXR & -3.55 & $3.02 \mathrm{E}-04$ \\
\hline 3606 & DDA1 & -3.21 & $1.68 \mathrm{E}-03$ \\
\hline 3607 & DDB1 & -2.28 & $1.14 \mathrm{E}-03$ \\
\hline 3608 & DDHD1 & -2.43 & $1.29 \mathrm{E}-02$ \\
\hline 3609 & DDHD2 & -4.08 & $3.44 \mathrm{E}-03$ \\
\hline 3610 & DDOST & -1.91 & $6.93 \mathrm{E}-03$ \\
\hline 3611 & DDRGK1 & -6.56 & $7.86 \mathrm{E}-05$ \\
\hline 3612 & DDT & -6.80 & 5.19E-04 \\
\hline 3613 & DDX1 & -6.42 & $1.30 \mathrm{E}-05$ \\
\hline 3614 & DDX10 & -2.11 & 7.15E-03 \\
\hline 3615 & DDX11 & -3.98 & $1.90 \mathrm{E}-03$ \\
\hline 3616 & DDX17 & -4.26 & 4.01E-04 \\
\hline 3617 & DDX20 & -3.18 & 7.69E-03 \\
\hline 3618 & DDX24 & -1.65 & 1.47E-02 \\
\hline 3619 & DDX25 & -6.32 & $4.52 \mathrm{E}-04$ \\
\hline 3620 & DDX26B & -2.20 & $4.14 \mathrm{E}-03$ \\
\hline 3621 & DDX27 & -3.82 & $8.98 \mathrm{E}-04$ \\
\hline 3622 & DDX31 & -2.12 & 4.64E-03 \\
\hline 3623 & DDX3X & -5.51 & $6.24 \mathrm{E}-04$ \\
\hline 3624 & DDX4 & -7.15 & 4.13E-06 \\
\hline 3625 & DDX42 & -3.94 & $5.02 \mathrm{E}-05$ \\
\hline 3626 & DDX43 & -4.34 & 5.03E-04 \\
\hline 3627 & DDX46 & -3.03 & $1.26 \mathrm{E}-02$ \\
\hline 3628 & DDX49 & -1.61 & $3.75 \mathrm{E}-03$ \\
\hline 3629 & DDX5 & -3.31 & $5.43 \mathrm{E}-05$ \\
\hline 3630 & DDX50 & -4.34 & 4.89E-04 \\
\hline 3631 & DDX51 & -2.82 & 7.85E-04 \\
\hline 3632 & DDX52 & -3.72 & $9.64 \mathrm{E}-04$ \\
\hline 3633 & DDX55 & -2.92 & $3.15 \mathrm{E}-04$ \\
\hline 3634 & DDX59 & -1.32 & $6.20 \mathrm{E}-03$ \\
\hline 3635 & DEAF1 & -2.15 & $3.04 \mathrm{E}-03$ \\
\hline 3636 & DECR1 & -4.21 & $1.94 \mathrm{E}-03$ \\
\hline 3637 & DEGS1 & -3.20 & $9.76 \mathrm{E}-05$ \\
\hline 3638 & DEK & -2.78 & $3.61 \mathrm{E}-02$ \\
\hline 3639 & DENND1B & -4.60 & $1.28 \mathrm{E}-04$ \\
\hline 3640 & DENND2C & -1.39 & $8.19 \mathrm{E}-03$ \\
\hline 3641 & DENND4C & -1.11 & 4.49E-03 \\
\hline 3642 & DENND5A & -3.65 & $5.96 \mathrm{E}-03$ \\
\hline 3643 & DENND6A & -2.88 & $4.01 \mathrm{E}-03$ \\
\hline 3644 & DENR & -6.48 & $1.68 \mathrm{E}-05$ \\
\hline 3645 & DEPDC1 & -4.34 & $2.64 \mathrm{E}-03$ \\
\hline 3646 & DEPDC1B & -2.40 & $1.37 \mathrm{E}-02$ \\
\hline 3647 & DEPDC5 & -1.50 & $3.45 \mathrm{E}-03$ \\
\hline 3648 & DEPDC7 & -6.05 & 5.49E-04 \\
\hline 3649 & DERA & -3.15 & 3.77E-03 \\
\hline 3650 & DERL1 & -4.94 & $7.80 \mathrm{E}-04$ \\
\hline 3651 & DERL2 & -4.28 & $3.08 \mathrm{E}-04$ \\
\hline 3652 & DERL3 & -2.98 & $1.77 \mathrm{E}-03$ \\
\hline 3653 & DFFA & -2.99 & 9.93E-04 \\
\hline
\end{tabular}

\begin{tabular}{|c|c|c|c|}
\hline 3654 & DFFB & -3.68 & $1.84 \mathrm{E}-03$ \\
\hline 3655 & DFNA5 & -5.26 & 1.94E-04 \\
\hline 3656 & DGCR14 & -4.23 & 3.46E-04 \\
\hline 3657 & DGCR6 & -1.94 & $1.03 \mathrm{E}-03$ \\
\hline 3658 & DGCR8 & -2.46 & $5.35 \mathrm{E}-03$ \\
\hline 3659 & DGKE & -3.92 & $1.06 \mathrm{E}-03$ \\
\hline 3660 & DGKQ & -3.10 & $5.57 \mathrm{E}-03$ \\
\hline 3661 & DHCR24 & -2.23 & $5.80 \mathrm{E}-03$ \\
\hline 3662 & DHCR7 & -4.53 & $2.58 \mathrm{E}-03$ \\
\hline 3663 & DHDDS & -1.85 & 7.11E-04 \\
\hline 3664 & DHFR & -3.45 & $6.04 \mathrm{E}-05$ \\
\hline 3665 & DHODH & -1.61 & 3.91E-04 \\
\hline 3666 & DHRS7 & -4.75 & 2.27E-04 \\
\hline 3667 & DHRS7B & -5.40 & $4.71 \mathrm{E}-04$ \\
\hline 3668 & DHX15 & -5.51 & $1.18 \mathrm{E}-06$ \\
\hline 3669 & DHX36 & -6.99 & $6.27 \mathrm{E}-05$ \\
\hline 3670 & DHX37 & -1.41 & 3.77E-03 \\
\hline 3671 & DHX38 & -3.88 & $2.41 \mathrm{E}-03$ \\
\hline 3672 & DHX40 & -2.35 & $9.13 \mathrm{E}-03$ \\
\hline 3673 & DIABLO & -1.51 & $1.44 \mathrm{E}-03$ \\
\hline 3674 & DIAPH1 & -4.66 & $1.86 \mathrm{E}-03$ \\
\hline 3675 & DIAPH3 & -4.56 & 5.11E-04 \\
\hline 3676 & DIEXF & -4.12 & $2.58 \mathrm{E}-03$ \\
\hline 3677 & DIMT1L & -2.75 & $1.84 \mathrm{E}-03$ \\
\hline 3678 & DIRC2 & -1.82 & 4.45E-02 \\
\hline 3679 & DIS3 & -4.34 & 1.07E-04 \\
\hline 3680 & DIS3L2 & -1.12 & $1.53 \mathrm{E}-03$ \\
\hline 3681 & DISP1 & -1.74 & $7.70 \mathrm{E}-03$ \\
\hline 3682 & DIXDC1 & -1.92 & $6.16 \mathrm{E}-02$ \\
\hline 3683 & DKC1 & -2.65 & $8.12 \mathrm{E}-03$ \\
\hline 3684 & DKK3 & -5.74 & 2.83E-04 \\
\hline 3685 & DLD & -3.48 & $7.55 E-04$ \\
\hline 3686 & DLGAP4 & -3.69 & $9.28 \mathrm{E}-03$ \\
\hline 3687 & DLGAP5 & -5.60 & 4.85E-05 \\
\hline 3688 & DLST & -2.63 & $1.35 \mathrm{E}-03$ \\
\hline 3689 & DMD & -1.38 & $1.14 \mathrm{E}-02$ \\
\hline 3690 & DMRT1 & -3.19 & $9.30 \mathrm{E}-03$ \\
\hline 3691 & DMTF1 & -1.71 & $4.89 \mathrm{E}-03$ \\
\hline 3692 & DN851424 & -1.34 & 2.04E-02 \\
\hline 3693 & DNA2 & -6.11 & 4.41E-05 \\
\hline 3694 & DNAAF1 & -5.92 & 3.34E-04 \\
\hline 3695 & DNAH10 & -1.88 & $1.71 \mathrm{E}-03$ \\
\hline 3696 & DNAH12 & -3.01 & $5.39 \mathrm{E}-03$ \\
\hline 3697 & DNAH17 & -4.29 & $1.89 \mathrm{E}-04$ \\
\hline 3698 & DNAH3 & -3.60 & $8.21 \mathrm{E}-04$ \\
\hline 3699 & DNAI2 & -5.71 & 4.23E-05 \\
\hline 3700 & DNAJA1 & -5.49 & $1.75 \mathrm{E}-02$ \\
\hline 3701 & DNAJA2 & -5.57 & 2.70E-04 \\
\hline 3702 & DNAJA3 & -2.91 & 4.44E-02 \\
\hline 3703 & DNAJA4 & -4.09 & $1.82 \mathrm{E}-03$ \\
\hline 3704 & DNAJB12 & -2.94 & 3.64E-03 \\
\hline 3705 & DNAJB13 & -2.57 & $8.83 E-03$ \\
\hline 3706 & DNAJB14 & -4.88 & $6.20 \mathrm{E}-04$ \\
\hline 3707 & DNAJB4 & -4.70 & 2.17E-04 \\
\hline 3708 & DNAJB6 & -4.01 & $9.31 \mathrm{E}-04$ \\
\hline 3709 & DNAJB9 & -2.82 & $5.98 \mathrm{E}-04$ \\
\hline 3710 & DNAJC1 & -4.03 & $1.63 \mathrm{E}-03$ \\
\hline 3711 & DNAJC10 & -3.63 & 2.19E-03 \\
\hline 3712 & DNAJC11 & -2.86 & 3.60E-04 \\
\hline 3713 & DNAJC12 & -3.84 & $1.18 \mathrm{E}-04$ \\
\hline 3714 & DNAJC13 & -2.74 & 3.89E-04 \\
\hline 3715 & DNAJC15 & -7.61 & $1.17 \mathrm{E}-04$ \\
\hline 3716 & DNAJC17 & -4.05 & $1.89 \mathrm{E}-02$ \\
\hline
\end{tabular}

\begin{tabular}{|c|c|c|c|}
\hline 3717 & DNAJC18 & -4.52 & $1.07 \mathrm{E}-03$ \\
\hline 3718 & DNAJC2 & -7.12 & 2.50E-06 \\
\hline 3719 & DNAJC21 & -2.69 & $2.61 \mathrm{E}-03$ \\
\hline 3720 & DNAJC24 & -7.09 & $5.91 \mathrm{E}-04$ \\
\hline 3721 & DNAJC25 & -3.19 & $6.63 \mathrm{E}-04$ \\
\hline 3722 & DNAJC27 & -1.55 & $8.92 \mathrm{E}-03$ \\
\hline 3723 & DNAJC3 & -3.49 & $1.67 \mathrm{E}-02$ \\
\hline 3724 & DNAJC30 & -2.62 & $8.83 \mathrm{E}-04$ \\
\hline 3725 & DNAJC5 & -1.92 & 4.44E-03 \\
\hline 3726 & DNAJC8 & -5.39 & 4.58E-06 \\
\hline 3727 & DNAJC9 & -6.43 & $1.45 \mathrm{E}-05$ \\
\hline 3728 & DNAL1 & -2.12 & $1.99 \mathrm{E}-03$ \\
\hline 3729 & DNAL4 & -5.16 & 2.97E-03 \\
\hline 3730 & DNALI1 & -6.05 & $5.60 \mathrm{E}-04$ \\
\hline 3731 & DNER & -2.95 & $2.39 \mathrm{E}-04$ \\
\hline 3732 & DNLZ & -2.19 & 5.59E-03 \\
\hline 3733 & DNM1 & -3.13 & $1.14 \mathrm{E}-03$ \\
\hline 3734 & DNM1L & -3.48 & $1.78 \mathrm{E}-03$ \\
\hline 3735 & DNPEP & -3.74 & 7.47E-04 \\
\hline 3736 & DOCK7 & -1.89 & $2.32 \mathrm{E}-03$ \\
\hline 3737 & $\mathrm{DOHH}$ & -3.42 & $1.54 \mathrm{E}-03$ \\
\hline 3738 & DOLK & -3.04 & $1.07 \mathrm{E}-02$ \\
\hline 3739 & DONSON & -4.14 & $4.24 \mathrm{E}-04$ \\
\hline 3740 & DOPEY1 & -1.52 & 4.53E-02 \\
\hline 3741 & DPAGT1 & -2.45 & 2.90E-02 \\
\hline 3742 & DPCD & -5.30 & $2.16 \mathrm{E}-03$ \\
\hline 3743 & DPH1 & -1.91 & $1.37 \mathrm{E}-03$ \\
\hline 3744 & $\mathrm{DPH} 2$ & -3.95 & $1.06 \mathrm{E}-04$ \\
\hline 3745 & DPH5 & -4.28 & $1.32 \mathrm{E}-04$ \\
\hline 3746 & DPP4 & -1.62 & $3.75 \mathrm{E}-02$ \\
\hline 3747 & DPP7 & -3.63 & $1.28 \mathrm{E}-03$ \\
\hline 3748 & DPP8 & -3.46 & $3.38 \mathrm{E}-04$ \\
\hline 3749 & DPY19L1 & -4.67 & $8.73 \mathrm{E}-05$ \\
\hline 3750 & DPY30 & -5.85 & $1.28 \mathrm{E}-04$ \\
\hline 3751 & DPYS & -6.66 & $6.56 \mathrm{E}-05$ \\
\hline 3752 & DPYSL3 & -3.75 & $2.29 \mathrm{E}-03$ \\
\hline 3753 & DPYSL4 & -1.72 & 5.95E-02 \\
\hline 3754 & DR1 & -6.64 & $2.21 \mathrm{E}-05$ \\
\hline 3755 & DR410336 & -1.85 & $1.31 \mathrm{E}-03$ \\
\hline 3756 & DR410398 & -4.10 & $2.42 \mathrm{E}-03$ \\
\hline 3757 & DR410535 & -2.86 & 4.45E-04 \\
\hline 3758 & DR410876 & -4.80 & $1.30 \mathrm{E}-04$ \\
\hline 3759 & DR411196 & -2.51 & $3.59 \mathrm{E}-03$ \\
\hline 3760 & DR411245 & -5.26 & $4.61 \mathrm{E}-03$ \\
\hline 3761 & DR411270 & -4.59 & 2.16E-03 \\
\hline 3762 & DR411532 & -2.55 & 4.43E-03 \\
\hline 3763 & DR411613 & -2.10 & $1.37 \mathrm{E}-04$ \\
\hline 3764 & DR411789 & -5.41 & $3.05 \mathrm{E}-03$ \\
\hline 3765 & DR411838 & -2.75 & $7.46 \mathrm{E}-03$ \\
\hline 3766 & DR411968 & -6.62 & 1.93E-04 \\
\hline 3767 & DR412230 & -3.06 & 7.13E-04 \\
\hline 3768 & DR412563 & -5.37 & $1.27 \mathrm{E}-03$ \\
\hline 3769 & DR412887 & -6.83 & $2.55 \mathrm{E}-04$ \\
\hline 3770 & DR413278 & -1.65 & $1.57 \mathrm{E}-04$ \\
\hline 3771 & DR413425 & -3.52 & $3.74 \mathrm{E}-03$ \\
\hline 3772 & DR413643 & -2.84 & $2.22 \mathrm{E}-03$ \\
\hline 3773 & DR413961 & -6.76 & 2.33E-04 \\
\hline 3774 & DR414083 & -5.16 & $3.48 \mathrm{E}-03$ \\
\hline 3775 & DR414223 & -3.46 & $5.15 \mathrm{E}-03$ \\
\hline 3776 & DR414315 & -3.17 & 3.59E-03 \\
\hline 3777 & DR414494 & -5.37 & 3.05E-03 \\
\hline 3778 & DR414565 & -6.04 & $6.20 \mathrm{E}-05$ \\
\hline 3779 & DR415129 & -3.46 & $1.17 \mathrm{E}-03$ \\
\hline
\end{tabular}




\begin{tabular}{|c|c|c|c|}
\hline 3780 & DR415393 & -1.37 & $1.89 \mathrm{E}-02$ \\
\hline 3781 & DR415668 & -2.14 & $6.16 \mathrm{E}-03$ \\
\hline 3782 & DR415886 & \begin{tabular}{|l|}
-3.37 \\
\end{tabular} & $1.95 \mathrm{E}-02$ \\
\hline 3783 & DR415989 & -2.89 & $5.65 \mathrm{E}-03$ \\
\hline 3784 & DR416056 & -1.92 & $7.04 E-03$ \\
\hline 3785 & DR416088 & -2.41 & $7.49 \mathrm{E}-04$ \\
\hline 3786 & DR416112 & -2.13 & $2.37 E-03$ \\
\hline 3787 & DR416800 & -2.81 & $5.33 \mathrm{E}-03$ \\
\hline 3788 & DR417302 & -2.64 & $9.49 \mathrm{E}-04$ \\
\hline 3789 & DR417655 & \begin{tabular}{|l|}
-2.18 \\
\end{tabular} & 2.44E-02 \\
\hline 3790 & DR417798 & -2.24 & $2.50 \mathrm{E}-02$ \\
\hline 3791 & DR417811 & -2.24 & $6.51 \mathrm{E}-04$ \\
\hline 3792 & DR417814 & -3.40 & $1.49 \mathrm{E}-03$ \\
\hline 3793 & DR417916 & -1.39 & $3.48 \mathrm{E}-02$ \\
\hline 3794 & DR417963 & -3.32 & $8.12 \mathrm{E}-03$ \\
\hline 3795 & DR418155 & \begin{tabular}{|l|}
-2.88 \\
\end{tabular} & $8.50 \mathrm{E}-04$ \\
\hline 3796 & DR418477 & -3.11 & $1.29 \mathrm{E}-04$ \\
\hline 3797 & DR418821 & -1.26 & $4.23 \mathrm{E}-03$ \\
\hline 3798 & DR419086 & -2.47 & $5.95 \mathrm{E}-04$ \\
\hline 3799 & DR419108 & -5.07 & $1.19 \mathrm{E}-03$ \\
\hline 3800 & DR419169 & -2.83 & $1.85 \mathrm{E}-03$ \\
\hline 3801 & DR419300 & \begin{tabular}{|l|}
-1.71 \\
\end{tabular} & $2.08 \mathrm{E}-02$ \\
\hline 3802 & DR419490 & -2.11 & $1.48 \mathrm{E}-04$ \\
\hline 3803 & DR419542 & -2.40 & $1.19 \mathrm{E}-02$ \\
\hline 3804 & DR419625 & -8.19 & $1.36 \mathrm{E}-03$ \\
\hline 3805 & DR419728 & -3.12 & $2.72 \mathrm{E}-03$ \\
\hline 3806 & DR420403 & -2.22 & $4.26 \mathrm{E}-03$ \\
\hline 3807 & DR420538 & -5.22 & $2.55 \mathrm{E}-03$ \\
\hline 3808 & DR420690 & -1.98 & 3.27E-03 \\
\hline 3809 & DR420743 & -2.25 & $2.34 \mathrm{E}-02$ \\
\hline 3810 & DR420879 & -4.43 & $1.77 E-03$ \\
\hline 3811 & DR424806 & \begin{tabular}{|l|}
-2.01 \\
\end{tabular} & $2.54 \mathrm{E}-03$ \\
\hline 3812 & DR426316 & -4.67 & $1.34 \mathrm{E}-03$ \\
\hline 3813 & DR427235 & -5.47 & $9.05 E-05$ \\
\hline 3814 & DR429050 & -2.11 & $1.84 \mathrm{E}-03$ \\
\hline 3815 & DR429224 & -3.69 & $7.83 \mathrm{E}-04$ \\
\hline 3816 & DR429499 & -3.93 & $1.45 \mathrm{E}-04$ \\
\hline 3817 & DR429689 & -3.76 & $1.02 \mathrm{E}-03$ \\
\hline 3818 & DR431170 & -5.15 & $1.01 E-04$ \\
\hline 3819 & DR431746 & -2.69 & $3.94 E-03$ \\
\hline 3820 & DR973990 & -2.37 & $1.13 \mathrm{E}-02$ \\
\hline 3821 & DRAM2 & -3.53 & $2.03 E-04$ \\
\hline 3822 & DRD4 & -1.35 & $5.42 \mathrm{E}-03$ \\
\hline 3823 & DRG1 & -6.20 & 2.62E-04 \\
\hline 3824 & DROSHA & -2.86 & $3.20 \mathrm{E}-03$ \\
\hline 3825 & DSCC1 & -1.73 & $8.04 E-03$ \\
\hline 3826 & DSCR3 & -1.23 & $2.30 \mathrm{E}-02$ \\
\hline 3827 & DSN1 & -5.22 & $2.54 E-03$ \\
\hline 3828 & DSP & -2.83 & $8.28 \mathrm{E}-04$ \\
\hline 3829 & DSTN & -3.86 & $5.47 \mathrm{E}-03$ \\
\hline 3830 & DSTYK & -1.35 & 2.87E-02 \\
\hline 3831 & DTD1 & \begin{tabular}{|l|}
-7.20 \\
\end{tabular} & $1.80 \mathrm{E}-04$ \\
\hline 3832 & DTL & -2.75 & $1.13 E-04$ \\
\hline 3833 & DTNA & -1.78 & $1.52 \mathrm{E}-03$ \\
\hline 3834 & DTNB & -3.87 & $9.79 \mathrm{E}-04$ \\
\hline 3835 & DTNBP1 & -2.50 & $5.06 \mathrm{E}-03$ \\
\hline 3836 & DTWD1 & -5.19 & $7.89 E-05$ \\
\hline 3837 & DTX2 & -4.61 & $2.44 E-04$ \\
\hline 3838 & DTX3L & -3.69 & $7.29 \mathrm{E}-04$ \\
\hline 3839 & DTYMK & -4.24 & $1.13 \mathrm{E}-03$ \\
\hline 3840 & DUPD1 & -5.48 & $2.01 E-03$ \\
\hline 3841 & DUS1L & -3.89 & $3.87 E-04$ \\
\hline & & -2.79 & 1.6 \\
\hline
\end{tabular}

\begin{tabular}{|c|c|c|c|}
\hline 3843 & DUS3L & -3.18 & 3.40E-04 \\
\hline 3844 & DUS4L & -3.72 & $4.62 \mathrm{E}-04$ \\
\hline 3845 & DUSP1 & -2.52 & $1.97 \mathrm{E}-03$ \\
\hline 3846 & DUSP12 & -4.99 & $3.98 \mathrm{E}-04$ \\
\hline 3847 & DUSP14 & -1.95 & $4.62 \mathrm{E}-03$ \\
\hline 3848 & DUSP18 & -7.10 & $2.89 \mathrm{E}-04$ \\
\hline 3849 & DUSP19 & -2.07 & $2.13 \mathrm{E}-02$ \\
\hline 3850 & DUSP22 & -1.84 & $2.68 \mathrm{E}-03$ \\
\hline 3851 & DUSP3 & -4.15 & $5.59 \mathrm{E}-03$ \\
\hline 3852 & DUT & -4.00 & $1.89 \mathrm{E}-04$ \\
\hline 3853 & DYDC1 & -7.93 & $3.27 \mathrm{E}-04$ \\
\hline 3854 & DYM & -3.68 & $2.18 \mathrm{E}-03$ \\
\hline 3855 & DYNC111 & -2.86 & $1.95 \mathrm{E}-04$ \\
\hline 3856 & DYNC112 & -2.18 & $1.37 \mathrm{E}-03$ \\
\hline 3857 & DYNC1LI1 & -1.83 & $1.84 \mathrm{E}-04$ \\
\hline 3858 & DYNC1LI2 & -1.54 & $1.17 \mathrm{E}-02$ \\
\hline 3859 & DYNC2H1 & -2.76 & $3.70 \mathrm{E}-03$ \\
\hline 3860 & DYNC2L1 1 & -1.68 & $1.10 \mathrm{E}-02$ \\
\hline 3861 & DYNLL1 & -6.23 & $2.05 \mathrm{E}-04$ \\
\hline 3862 & DYNLL2 & -4.53 & $1.07 \mathrm{E}-04$ \\
\hline 3863 & DYNLRB1 & -2.98 & $3.66 \mathrm{E}-03$ \\
\hline 3864 & DYNLT1 & -8.37 & $9.57 \mathrm{E}-06$ \\
\hline 3865 & DYNLT3 & -2.27 & $1.03 \mathrm{E}-02$ \\
\hline 3866 & DYRK1A & -4.49 & $2.29 \mathrm{E}-03$ \\
\hline 3867 & DYRK2 & -1.74 & $3.38 \mathrm{E}-02$ \\
\hline 3868 & DYRK3 & -6.74 & $1.49 \mathrm{E}-04$ \\
\hline 3869 & DYX1C1 & -4.40 & $1.71 \mathrm{E}-03$ \\
\hline 3870 & DZANK1 & -3.33 & 4.13E-04 \\
\hline 3871 & DZIP1 & -4.17 & $3.75 \mathrm{E}-03$ \\
\hline 3872 & E2F1 & -2.97 & $1.10 \mathrm{E}-03$ \\
\hline 3873 & E2F4 & -1.92 & 4.17E-03 \\
\hline 3874 & E2F5 & -3.62 & $1.40 \mathrm{E}-03$ \\
\hline 3875 & E2F6 & -1.81 & $4.58 \mathrm{E}-03$ \\
\hline 3876 & E2F8 & -4.71 & $3.72 \mathrm{E}-04$ \\
\hline 3877 & E4F1 & -4.03 & $1.66 \mathrm{E}-03$ \\
\hline 3878 & EAF1 & -1.36 & $6.54 \mathrm{E}-03$ \\
\hline 3879 & EAPP & -4.69 & $1.17 \mathrm{E}-03$ \\
\hline 3880 & EARS2 & -2.05 & $6.82 \mathrm{E}-04$ \\
\hline 3881 & EBAG9 & -5.50 & $6.27 \mathrm{E}-04$ \\
\hline 3882 & EBNA1BP2 & -4.79 & $2.34 \mathrm{E}-04$ \\
\hline 3883 & ECD & -2.20 & $3.45 \mathrm{E}-03$ \\
\hline 3884 & ECE1 & -2.76 & $5.25 \mathrm{E}-03$ \\
\hline 3885 & ECHDC2 & -3.95 & $9.37 \mathrm{E}-04$ \\
\hline 3886 & ECHDC3 & -6.76 & $7.98 \mathrm{E}-04$ \\
\hline 3887 & ECHS1 & -3.22 & $5.43 \mathrm{E}-04$ \\
\hline 3888 & ECI1 & -1.20 & $8.84 \mathrm{E}-03$ \\
\hline 3889 & $\mathrm{ECl} 2$ & -1.80 & $4.25 \mathrm{E}-02$ \\
\hline 3890 & EDARADD & -2.76 & $3.85 \mathrm{E}-03$ \\
\hline 3891 & EDEM3 & -2.76 & $1.59 \mathrm{E}-03$ \\
\hline 3892 & EDF1 & -6.55 & $2.80 \mathrm{E}-04$ \\
\hline 3893 & EDNRA & -1.86 & $8.68 \mathrm{E}-04$ \\
\hline 3894 & EEA1 & -3.65 & $6.62 \mathrm{E}-04$ \\
\hline 3895 & EEF1A1 & -5.49 & $1.14 \mathrm{E}-03$ \\
\hline 3896 & EEF1B2 & -7.44 & $2.70 \mathrm{E}-04$ \\
\hline 3897 & EEF1D & -7.89 & $1.17 \mathrm{E}-04$ \\
\hline 3898 & EEF1E1 & -4.29 & $3.83 \mathrm{E}-05$ \\
\hline 3899 & EEF1G & -5.89 & $6.41 \mathrm{E}-03$ \\
\hline 3900 & EEF2 & -4.64 & $6.91 \mathrm{E}-03$ \\
\hline 3901 & EEPD1 & -2.66 & $2.70 \mathrm{E}-03$ \\
\hline 3902 & EFCAB1 & -7.76 & $7.97 \mathrm{E}-04$ \\
\hline 3903 & EFCAB11 & -5.20 & $1.89 \mathrm{E}-04$ \\
\hline 3904 & EFCAB4B & -2.52 & $5.33 \mathrm{E}-04$ \\
\hline 3905 & EFCABG & -6.45 & $4.00 \mathrm{E}-04$ \\
\hline
\end{tabular}

\begin{tabular}{|c|c|c|c|}
\hline 3906 & EFEMP1 & -2.64 & $2.25 \mathrm{E}-03$ \\
\hline 3907 & EFHA1 & -1.99 & $3.23 \mathrm{E}-02$ \\
\hline 3908 & EFHA2 & -2.77 & $7.64 \mathrm{E}-04$ \\
\hline 3909 & EFHB & -8.03 & $2.26 \mathrm{E}-04$ \\
\hline 3910 & EFHC1 & -7.26 & $3.15 \mathrm{E}-05$ \\
\hline 3911 & EFHC2 & -8.00 & $2.72 \mathrm{E}-05$ \\
\hline 3912 & EFHD1 & -3.25 & $2.14 \mathrm{E}-03$ \\
\hline 3913 & EFR3A & -3.62 & $2.02 \mathrm{E}-04$ \\
\hline 3914 & EFTUD1 & -3.90 & $1.36 \mathrm{E}-03$ \\
\hline 3915 & EFTUD2 & -2.91 & $2.30 \mathrm{E}-02$ \\
\hline 3916 & EGFL6 & -3.12 & $3.03 \mathrm{E}-03$ \\
\hline 3917 & EGLN3 & -4.50 & $5.13 \mathrm{E}-03$ \\
\hline 3918 & EGR1 & -1.57 & $5.82 \mathrm{E}-02$ \\
\hline 3919 & EHD3 & -3.40 & $3.26 \mathrm{E}-03$ \\
\hline 3920 & EHF & -2.34 & $1.73 \mathrm{E}-03$ \\
\hline 3921 & EI24 & -3.57 & $3.02 \mathrm{E}-03$ \\
\hline 3922 & EIF1 & -6.37 & $1.95 \mathrm{E}-04$ \\
\hline 3923 & EIF1AD & -6.17 & $1.25 \mathrm{E}-03$ \\
\hline 3924 & EIF1AY & -5.48 & $1.67 \mathrm{E}-04$ \\
\hline 3925 & EIF1B & -6.64 & $1.83 \mathrm{E}-05$ \\
\hline 3926 & EIF $2 A$ & -5.83 & $2.10 \mathrm{E}-03$ \\
\hline 3927 & EIF2AK1 & -3.47 & $2.01 \mathrm{E}-03$ \\
\hline 3928 & EIF2B2 & -5.23 & $9.60 \mathrm{E}-04$ \\
\hline 3929 & EIF $2 B 3$ & -3.31 & $7.86 \mathrm{E}-03$ \\
\hline 3930 & EIF $2 B 4$ & -2.42 & $3.52 \mathrm{E}-03$ \\
\hline 3931 & $\mathrm{EIF} 2 \mathrm{C3}$ & -3.45 & $2.04 \mathrm{E}-04$ \\
\hline 3932 & $\mathrm{EIF} 2 \mathrm{C} 4$ & -1.30 & $6.56 \mathrm{E}-04$ \\
\hline 3933 & EIF2D & -4.28 & $3.85 \mathrm{E}-04$ \\
\hline 3934 & EIF2S1 & -6.66 & $2.75 \mathrm{E}-05$ \\
\hline 3935 & EIF2S2 & $\begin{array}{l}-6.17 \\
\end{array}$ & $2.83 \mathrm{E}-05$ \\
\hline 3936 & EIF2S3 & -7.30 & $7.27 \mathrm{E}-06$ \\
\hline 3937 & EIF3A & $\begin{array}{l}-2.07 \\
\end{array}$ & $2.53 \mathrm{E}-02$ \\
\hline 3938 & EIF3D & -5.10 & $6.65 \mathrm{E}-05$ \\
\hline 3939 & EIF3E & \begin{tabular}{|l|}
-7.60 \\
\end{tabular} & $7.30 \mathrm{E}-05$ \\
\hline 3940 & EIF3F & -5.76 & $1.59 \mathrm{E}-04$ \\
\hline 3941 & EIF3H & $\begin{array}{l}-7.78 \\
\end{array}$ & $3.15 \mathrm{E}-05$ \\
\hline 3942 & E|F3| & -3.81 & $1.18 \mathrm{E}-02$ \\
\hline 3943 & EIF3J & -5.37 & $8.52 \mathrm{E}-05$ \\
\hline 3944 & EIF3L & -7.64 & $4.81 \mathrm{E}-04$ \\
\hline 3945 & EIF3M & -8.21 & $1.15 \mathrm{E}-04$ \\
\hline 3946 & EIF4A2 & -4.42 & $3.56 \mathrm{E}-03$ \\
\hline 3947 & EIF4A3 & -6.59 & $1.28 \mathrm{E}-03$ \\
\hline 3948 & EIF4E & -5.85 & $4.63 \mathrm{E}-05$ \\
\hline 3949 & EIF4E3 & -2.68 & $2.20 \mathrm{E}-03$ \\
\hline 3950 & EIF4EBP2 & -3.61 & 4.64E-04 \\
\hline 3951 & EIF4G2 & -6.57 & $2.26 \mathrm{E}-04$ \\
\hline 3952 & EIF4G3 & -3.47 & $8.55 \mathrm{E}-03$ \\
\hline 3953 & EIF4H & -3.06 & $1.15 \mathrm{E}-02$ \\
\hline 3954 & EIF5 & -7.65 & $1.37 \mathrm{E}-04$ \\
\hline 3955 & EIF5A2 & -3.48 & $1.15 \mathrm{E}-02$ \\
\hline 3956 & EIF5B & -3.83 & $7.40 \mathrm{E}-04$ \\
\hline 3957 & EIF6 & -3.84 & $5.58 \mathrm{E}-04$ \\
\hline 3958 & ELAC2 & -2.16 & $5.89 \mathrm{E}-02$ \\
\hline 3959 & ELAVL1 & -4.19 & $8.07 E-05$ \\
\hline 3960 & ELAVL4 & -2.36 & $5.27 \mathrm{E}-03$ \\
\hline 3961 & ELK3 & -1.62 & $1.28 \mathrm{E}-03$ \\
\hline 3962 & ELL & -1.74 & $8.98 \mathrm{E}-03$ \\
\hline 3963 & ELL2 & -3.80 & $1.32 \mathrm{E}-03$ \\
\hline 3964 & ELMO1 & -2.33 & $4.72 \mathrm{E}-03$ \\
\hline 3965 & ELMO2 & -4.03 & $5.27 \mathrm{E}-04$ \\
\hline 3966 & ELMOD2 & -4.13 & 4.17E-04 \\
\hline 3967 & $\begin{array}{l}\text { ELOVL1 } \\
\end{array}$ & -3.21 & $5.19 \mathrm{E}-06$ \\
\hline 3968 & ELOVL5 & -5.60 & $9.00 \mathrm{E}-04$ \\
\hline
\end{tabular}




\begin{tabular}{|c|c|c|}
\hline 3969 & ELOVL6 & $7.68 \mathrm{E}-03$ \\
\hline 3970 & -5.32 & $1.15 \mathrm{E}-03$ \\
\hline 3971 & -3.77 & $7.47 \mathrm{E}-04$ \\
\hline 3972 & -4.41 & $2.86 \mathrm{E}-06$ \\
\hline 3973 & -5.12 & $1.06 \mathrm{E}-03$ \\
\hline 3974 & EMC1 & $2.11 \mathrm{E}-02$ \\
\hline 3975 & EMG1 & $3.58 \mathrm{E}-03$ \\
\hline 3976 & EMILIN2 & $1.23 \mathrm{E}-02$ \\
\hline 3977 & EML1 & $1.74 \mathrm{E}-02$ \\
\hline 3978 & EML4 & $2.58 \mathrm{E}-03$ \\
\hline 3979 & EMP2 & $1.13 \mathrm{E}-03$ \\
\hline 3980 & ENAH & $2.34 \mathrm{E}-04$ \\
\hline 3981 & ENDOG & $6.11 \mathrm{E}-02$ \\
\hline 3982 & ENDOV & $7.60 \mathrm{E}-05$ \\
\hline 3983 & ENKUR & $3.12 \mathrm{E}-05$ \\
\hline 3984 & ENO1 & $7.68 \mathrm{E}-05$ \\
\hline 3985 & ENO4 & $1.31 \mathrm{E}-03$ \\
\hline 3986 & ENOX2 & $1.60 \mathrm{E}-04$ \\
\hline 3987 & ENPP2 & $5.02 \mathrm{E}-04$ \\
\hline 3988 & ENPP4 & $3.11 \mathrm{E}-05$ \\
\hline 3989 & ENSA & $2.00 \mathrm{E}-03$ \\
\hline 3990 & $\begin{array}{l}\text { ENSGALT00000000053 } \\
6.38 \mathrm{E}-02\end{array}$ & -1.79 \\
\hline 3991 & $\begin{array}{l}\text { ENSGALTO0000000076 } \\
4.45 E-03\end{array}$ & -3.14 \\
\hline 3992 & $\begin{array}{l}\text { ENSGALT00000000113 } \\
5.27 E-04\end{array}$ & -4.97 \\
\hline 3993 & $\begin{array}{l}\text { ENSGALTO0000000129 } \\
1.28 E-04\end{array}$ & -6.67 \\
\hline 3994 & $\begin{array}{l}\text { ENSGALTO0000000145 } \\
7.77 E-05\end{array}$ & -6.47 \\
\hline 3995 & $\begin{array}{l}\text { ENSGALT00000000159 } \\
1.53 E-03\end{array}$ & -3.53 \\
\hline 3996 & $\begin{array}{l}\text { ENSGALTO0000000234 } \\
2.05 E-02\end{array}$ & -2.36 \\
\hline 3997 & $\begin{array}{l}\text { ENSGALTO0000000252 } \\
5.94 \mathrm{E}-03\end{array}$ & -1.96 \\
\hline 3998 & $\begin{array}{l}\text { ENSGALTO0000000296 } \\
2.24 E-04\end{array}$ & -6.68 \\
\hline 3999 & $\begin{array}{l}\text { ENSGALTO0000000338 } \\
1.42 E-03\end{array}$ & -2.98 \\
\hline 4000 & $\begin{array}{l}\text { ENSGALT00000000355 } \\
6.11 E-04\end{array}$ & -4.55 \\
\hline 4001 & $\begin{array}{l}\text { ENSGALTO0000000385 } \\
1.57 E-04\end{array}$ & -3.75 \\
\hline 4002 & $\begin{array}{l}\text { ENSGALT00000000388 } \\
1.41 \mathrm{E}-03\end{array}$ & -3.43 \\
\hline 4003 & $\begin{array}{l}\text { ENSGALTO0000000417 } \\
4.09 E-03\end{array}$ & -2.77 \\
\hline 4004 & $\begin{array}{l}\text { ENSGALT00000000502 } \\
1.00 E-03\end{array}$ & -3.73 \\
\hline 4005 & $\begin{array}{l}\text { ENSGALT00000000505 } \\
3.59 \text { E-04 }\end{array}$ & -3.23 \\
\hline 4006 & $\begin{array}{l}\text { ENSGALTO0000000522 } \\
2.36 E-03\end{array}$ & -3.36 \\
\hline 4007 & $\begin{array}{l}\text { ENSGALT00000000552 } \\
2.66 E-03\end{array}$ & -1.46 \\
\hline 4008 & $\begin{array}{l}\text { ENSGALTO0000000596 } \\
\text { 3.42E-03 }\end{array}$ & -4.87 \\
\hline 4009 & $\begin{array}{l}\text { ENSGALT00000000616 } \\
4.41 E-04\end{array}$ & -5.43 \\
\hline 4010 & $\begin{array}{l}\text { ENSGALTO0000000626 } \\
4.09 E-05\end{array}$ & -3.50 \\
\hline 4011 & $\begin{array}{l}\text { ENSGALTO0000000678 } \\
5.23 E-05\end{array}$ & -5.09 \\
\hline 4012 & $\begin{array}{l}\text { ENSGALT00000000679 } \\
3.25 E-03\end{array}$ & -3.43 \\
\hline 4013 & $\begin{array}{l}\text { ENSGALT00000000797 } \\
2.13 \mathrm{E}-03\end{array}$ & -3.02 \\
\hline 4014 & $\begin{array}{l}\text { ENSGALT00000000841 } \\
2.99 \mathrm{E}-03\end{array}$ & -2.70 \\
\hline 4015 & $\begin{array}{l}\text { ENSGALTO0000000890 } \\
1.55 E-03\end{array}$ & -2.99 \\
\hline
\end{tabular}

\begin{tabular}{|c|c|c|}
\hline 4016 & $\begin{array}{l}\text { ENSGALTO0000000920 } \\
3.75 E-03\end{array}$ & -6.58 \\
\hline 4017 & $\begin{array}{l}\text { ENSGALTO0000000970 } \\
1.23 E-02\end{array}$ & -3.03 \\
\hline 4018 & $\begin{array}{l}\text { ENSGALTO0000001010 } \\
1.42 E-03\end{array}$ & -2.87 \\
\hline 4019 & $\begin{array}{l}\text { ENSGALTO0000001059 } \\
8.43 E-03\end{array}$ & -1.47 \\
\hline 4020 & $\begin{array}{l}\text { ENSGALTO0000001087 } \\
1.95 E-04\end{array}$ & -3.58 \\
\hline 4021 & $\begin{array}{l}\text { ENSGALTO0000001127 } \\
1.13 \mathrm{E}-05\end{array}$ & -4.70 \\
\hline 4022 & $\begin{array}{l}\text { ENSGALTO0000001302 } \\
3.44 E-05\end{array}$ & -2.26 \\
\hline 4023 & $\begin{array}{l}\text { ENSGALTO0000001466 } \\
2.84 E-03\end{array}$ & -1.35 \\
\hline 4024 & $\begin{array}{l}\text { ENSGALT00000001593 } \\
1.71 E-03\end{array}$ & -6.53 \\
\hline 4025 & $\begin{array}{l}\text { ENSGALTO0000001678 } \\
3.24 E-05\end{array}$ & -5.28 \\
\hline 4026 & $\begin{array}{l}\text { ENSGALTO0000001801 } \\
\text { 3.39E-04 }\end{array}$ & -2.30 \\
\hline 4027 & $\begin{array}{l}\text { ENSGALTO0000001885 } \\
1.22 \mathrm{E}-04\end{array}$ & -3.91 \\
\hline 4028 & $\begin{array}{l}\text { ENSGALTO0000001925 } \\
3.88 \mathrm{E}-04\end{array}$ & -2.53 \\
\hline 4029 & $\begin{array}{l}\text { ENSGALT00000001982 } \\
\text { 3.44E-04 }\end{array}$ & -4.00 \\
\hline 4030 & $\begin{array}{l}\text { ENSGALTO0000002005 } \\
4.75 E-04\end{array}$ & -4.53 \\
\hline 4031 & $\begin{array}{l}\text { ENSGALTO0000002034 } \\
3.75 E-03\end{array}$ & -3.24 \\
\hline 4032 & $\begin{array}{l}\text { ENSGALTO0000002155 } \\
5.80 \mathrm{E}-03\end{array}$ & -2.90 \\
\hline 4033 & $\begin{array}{l}\text { ENSGALTO0000002299 } \\
1.09 E-05\end{array}$ & -6.08 \\
\hline 4034 & $\begin{array}{l}\text { ENSGALTO0000002410 } \\
3.11 \mathrm{-}-04\end{array}$ & -7.22 \\
\hline 4035 & $\begin{array}{l}\text { ENSGALTO0000002439 } \\
\text { 3.62E-05 }\end{array}$ & -5.53 \\
\hline 4036 & $\begin{array}{l}\text { ENSGALT00000002562 } \\
8.92 E-04\end{array}$ & -3.48 \\
\hline 4037 & $\begin{array}{l}\text { ENSGALTO0000002612 } \\
1.60 \mathrm{E}-03\end{array}$ & -3.92 \\
\hline 4038 & $\begin{array}{l}\text { ENSGALT00000002661 } \\
9.60 \mathrm{E}-03\end{array}$ & -1.14 \\
\hline 4039 & $\begin{array}{l}\text { ENSGALTO0000002758 } \\
7.83 E-03\end{array}$ & -2.05 \\
\hline 4040 & $\begin{array}{l}\text { ENSGALTO0000002759 } \\
1.42 E-02\end{array}$ & -2.13 \\
\hline 4041 & $\begin{array}{l}\text { ENSGALT00000002763 } \\
9.95 E-05\end{array}$ & -4.33 \\
\hline 4042 & $\begin{array}{l}\text { ENSGALTO0000002781 } \\
1.76 E-04\end{array}$ & -8.17 \\
\hline 4043 & $\begin{array}{l}\text { ENSGALTO0000002785 } \\
4.08 E-04\end{array}$ & -6.16 \\
\hline 4044 & $\begin{array}{l}\text { ENSGALTO0000002789 } \\
2.00 E-02\end{array}$ & -3.31 \\
\hline 4045 & $\begin{array}{l}\text { ENSGALTO0000002870 } \\
6.39 E-05\end{array}$ & -5.91 \\
\hline 4046 & $\begin{array}{l}\text { ENSGALTO0000002880 } \\
2.55 E-04\end{array}$ & -3.73 \\
\hline 4047 & $\begin{array}{l}\text { ENSGALTO0000003212 } \\
8.15 E-05\end{array}$ & -3.54 \\
\hline 4048 & $\begin{array}{l}\text { ENSGALTO0000003426 } \\
7.39 E-03\end{array}$ & -4.98 \\
\hline 4049 & $\begin{array}{l}\text { ENSGALTO0000003481 } \\
2.26 E-02\end{array}$ & -2.32 \\
\hline 4050 & $\begin{array}{l}\text { ENSGALT00000003569 } \\
4.58 \mathrm{E}-05\end{array}$ & -3.64 \\
\hline 4051 & $\begin{array}{l}\text { ENSGALT00000003611 } \\
6.58 \mathrm{E}-02\end{array}$ & -1.71 \\
\hline 4052 & $\begin{array}{l}\text { ENSGALTO0000003620 } \\
2.74 E-02\end{array}$ & -1.51 \\
\hline 4053 & $\begin{array}{l}\text { ENSGALT00000003676 } \\
2.07 E-02\end{array}$ & -1.41 \\
\hline 4054 & $\begin{array}{l}\text { ENSGALTO0000003682 } \\
1.23 E-03\end{array}$ & -2.97 \\
\hline
\end{tabular}

\begin{tabular}{|c|c|c|}
\hline 4055 & $\begin{array}{l}\text { ENSGALTO0000003688 } \\
9.12 E-04\end{array}$ & -3.54 \\
\hline 4056 & $\begin{array}{l}\text { ENSGALTO0000003720 } \\
5.05 E-03\end{array}$ & -5.84 \\
\hline 4057 & $\begin{array}{l}\text { ENSGALTO0000003739 } \\
1.22 E-02\end{array}$ & -1.14 \\
\hline 4058 & $\begin{array}{l}\text { ENSGALTO0000003778 } \\
1.67 E-03\end{array}$ & -5.19 \\
\hline 4059 & $\begin{array}{l}\text { ENSGALTO0000003831 } \\
2.93 E-02\end{array}$ & -1.26 \\
\hline 4060 & $\begin{array}{l}\text { ENSGALTO0000003959 } \\
2.77 E-03\end{array}$ & -3.25 \\
\hline 4061 & $\begin{array}{l}\text { ENSGALTO0000003960 } \\
2.90 E-03\end{array}$ & -3.47 \\
\hline 4062 & $\begin{array}{l}\text { ENSGALTO0000003983 } \\
5.43 E-03\end{array}$ & -2.19 \\
\hline 4063 & $\begin{array}{l}\text { ENSGALT00000004194 } \\
8.83 \mathrm{E}-03\end{array}$ & -2.81 \\
\hline 4064 & $\begin{array}{l}\text { ENSGALTO0000004266 } \\
1.55 E-03\end{array}$ & -3.48 \\
\hline 4065 & $\begin{array}{l}\text { ENSGALT00000004283 } \\
9.41 \mathrm{E}-03\end{array}$ & -1.17 \\
\hline 4066 & $\begin{array}{l}\text { ENSGALTO0000004308 } \\
9.20 \mathrm{E}-03\end{array}$ & -2.51 \\
\hline 4067 & $\begin{array}{l}\text { ENSGALTO0000004323 } \\
9.91 E-05\end{array}$ & -2.69 \\
\hline 4068 & $\begin{array}{l}\text { ENSGALT00000004366 } \\
6.76 E-04\end{array}$ & -2.06 \\
\hline 4069 & $\begin{array}{l}\text { ENSGALTO0000004380 } \\
1.17 \mathrm{E}-04\end{array}$ & -5.95 \\
\hline 4070 & $\begin{array}{l}\text { ENSGALTO0000004394 } \\
2.11 \mathrm{E}-03\end{array}$ & -5.45 \\
\hline 4071 & $\begin{array}{l}\text { ENSGALT00000004410 } \\
2.34 \mathrm{E}-02\end{array}$ & -1.60 \\
\hline 4072 & $\begin{array}{l}\text { ENSGALTO0000004517 } \\
1.71 E-03\end{array}$ & -7.18 \\
\hline 4073 & $\begin{array}{l}\text { ENSGALTO0000004526 } \\
1.75 E-02\end{array}$ & -2.60 \\
\hline 4074 & $\begin{array}{l}\text { ENSGALTO0000004533 } \\
\text { 7.64E-04 }\end{array}$ & -5.32 \\
\hline 4075 & $\begin{array}{l}\text { ENSGALTO0000004545 } \\
2.39 E-04\end{array}$ & -5.88 \\
\hline 4076 & $\begin{array}{l}\text { ENSGALTO0000004546 } \\
1.55 E-03\end{array}$ & -2.40 \\
\hline 4077 & $\begin{array}{l}\text { ENSGALTO0000004668 } \\
6.35 E-02\end{array}$ & -1.99 \\
\hline 4078 & $\begin{array}{l}\text { ENSGALTO0000004712 } \\
2.61 E-04\end{array}$ & -5.77 \\
\hline 4079 & $\begin{array}{l}\text { ENSGALTO0000004713 } \\
4.18 \mathrm{E}-03\end{array}$ & -2.47 \\
\hline 4080 & $\begin{array}{l}\text { ENSGALTO0000004810 } \\
\text { 3.29E-04 }\end{array}$ & -6.11 \\
\hline 4081 & $\begin{array}{l}\text { ENSGALTO0000004814 } \\
2.06 E-02\end{array}$ & -1.43 \\
\hline 4082 & $\begin{array}{l}\text { ENSGALTO0000004817 } \\
2.21 E-03\end{array}$ & -1.50 \\
\hline 4083 & $\begin{array}{l}\text { ENSGALTO0000004870 } \\
1.43 E-03\end{array}$ & -4.29 \\
\hline 4084 & $\begin{array}{l}\text { ENSGALTO0000004872 } \\
1.87 E-03\end{array}$ & -2.98 \\
\hline 4085 & $\begin{array}{l}\text { ENSGALTO0000004875 } \\
2.31 E-03\end{array}$ & -4.66 \\
\hline 4086 & $\begin{array}{l}\text { ENSGALT00000004909 } \\
2.01 E-03\end{array}$ & -2.98 \\
\hline 4087 & $\begin{array}{l}\text { ENSGALTO0000004978 } \\
\text { 3.12E-03 }\end{array}$ & -3.64 \\
\hline 4088 & $\begin{array}{l}\text { ENSGALTO0000004989 } \\
1.03 E-03\end{array}$ & -4.05 \\
\hline 4089 & $\begin{array}{l}\text { ENSGALTO0000005016 } \\
2.76 E-03\end{array}$ & -1.81 \\
\hline 4090 & $\begin{array}{l}\text { ENSGALTO0000005087 } \\
1.30 \mathrm{E}-03\end{array}$ & -2.03 \\
\hline 4091 & $\begin{array}{l}\text { ENSGALTO0000005096 } \\
2.81 E-03\end{array}$ & -5.30 \\
\hline 4092 & $\begin{array}{l}\text { ENSGALTO0000005121 } \\
2.93 E-04\end{array}$ & -4.17 \\
\hline 4093 & $\begin{array}{l}\text { ENSGALTO0000005160 } \\
2.70 E-03\end{array}$ & -4.78 \\
\hline
\end{tabular}




\begin{tabular}{|c|c|c|}
\hline 4094 & $\begin{array}{l}\text { ENSGALTO0000005208 } \\
3.07 E-04\end{array}$ & -4.28 \\
\hline 4095 & $\begin{array}{l}\text { ENSGALT00000005216 } \\
5.18 \mathrm{E}-03\end{array}$ & -3.51 \\
\hline 4096 & $\begin{array}{l}\text { ENSGALT00000005228 } \\
\text { 3.65E- } 03\end{array}$ & -3.04 \\
\hline 4097 & $\begin{array}{l}\text { ENSGALTO0000005259 } \\
5.74 E-04\end{array}$ & -7.20 \\
\hline 4098 & $\begin{array}{l}\text { ENSGALT00000005278 } \\
5.69 \mathrm{E}-04\end{array}$ & -2.86 \\
\hline 4099 & $\begin{array}{l}\text { ENSGALT00000005286 } \\
6.78 \mathrm{E}-02\end{array}$ & -1.65 \\
\hline 4100 & $\begin{array}{l}\text { ENSGALT00000005305 } \\
5.98 \mathrm{E}-03\end{array}$ & -1.77 \\
\hline 4101 & $\begin{array}{l}\text { ENSGALT00000005427 } \\
1.92 \mathrm{E}-03\end{array}$ & -4.88 \\
\hline 4102 & $\begin{array}{l}\text { ENSGALT00000005448 } \\
2.30 \text { - } 03\end{array}$ & -3.65 \\
\hline 4103 & $\begin{array}{l}\text { ENSGALT00000005451 } \\
1.47 \mathrm{E}-03\end{array}$ & -2.62 \\
\hline 4104 & $\begin{array}{l}\text { ENSGALT00000005458 } \\
3.92 \mathrm{E}-05\end{array}$ & -7.07 \\
\hline 4105 & $\begin{array}{l}\text { ENSGALT00000005471 } \\
1.44 E-04\end{array}$ & -6.86 \\
\hline 4106 & $\begin{array}{l}\text { ENSGALT00000005480 } \\
8.54 \mathrm{E}-03\end{array}$ & -4.45 \\
\hline 4107 & $\begin{array}{l}\text { ENSGALT00000005488 } \\
1.49 \mathrm{E}-03\end{array}$ & -3.75 \\
\hline 4108 & $\begin{array}{l}\text { ENSGALT00000005549 } \\
1.67 \mathrm{-} 01\end{array}$ & -1.61 \\
\hline 4109 & $\begin{array}{l}\text { ENSGALT00000005602 } \\
2.41 E-04\end{array}$ & -5.19 \\
\hline 4110 & $\begin{array}{l}\text { ENSGALT00000005620 } \\
7.00 E-04\end{array}$ & -3.63 \\
\hline 4111 & $\begin{array}{l}\text { ENSGALT00000005629 } \\
1.25 \mathrm{E}-03\end{array}$ & -1.97 \\
\hline 4112 & $\begin{array}{l}\text { ENSGALT00000005683 } \\
7.18 \mathrm{E}-04\end{array}$ & -2.75 \\
\hline 4113 & $\begin{array}{l}\text { ENSGALT00000005691 } \\
1.38 \mathrm{E}-04\end{array}$ & -6.26 \\
\hline 4114 & $\begin{array}{l}\text { ENSGALT00000005700 } \\
1.83 E-02\end{array}$ & -5.32 \\
\hline 4115 & $\begin{array}{l}\text { ENSGALT00000005708 } \\
1.48 \mathrm{E}-02\end{array}$ & -2.05 \\
\hline 4116 & $\begin{array}{l}\text { ENSGALTO0000005733 } \\
2.45 E-03\end{array}$ & -4.39 \\
\hline 4117 & $\begin{array}{l}\text { ENSGALT00000005741 } \\
1.13 E-02\end{array}$ & -2.89 \\
\hline 4118 & $\begin{array}{l}\text { ENSGALT00000005810 } \\
5.86 \mathrm{E}-04\end{array}$ & -2.08 \\
\hline 4119 & $\begin{array}{l}\text { ENSGALT00000005815 } \\
9.86 \mathrm{E}-03\end{array}$ & -2.18 \\
\hline 4120 & $\begin{array}{l}\text { ENSGALT00000005822 } \\
3.85 \mathrm{E}-03\end{array}$ & -2.56 \\
\hline 4121 & $\begin{array}{l}\text { ENSGALTO0000006001 } \\
\text { 3.53E-04 }\end{array}$ & -2.94 \\
\hline 4122 & $\begin{array}{l}\text { ENSGALT00000006070 } \\
2.12 E-04\end{array}$ & -5.90 \\
\hline 4123 & $\begin{array}{l}\text { ENSGALTO0000006088 } \\
1.40 E-02\end{array}$ & -1.50 \\
\hline 4124 & $\begin{array}{l}\text { ENSGALT00000006159 } \\
1.90 \mathrm{E}-03\end{array}$ & -3.00 \\
\hline 4125 & $\begin{array}{l}\text { ENSGALT00000006223 } \\
1.54 E-03\end{array}$ & -4.25 \\
\hline 4126 & $\begin{array}{l}\text { ENSGALTO0000006290 } \\
1.04 E-03\end{array}$ & -4.33 \\
\hline 4127 & $\begin{array}{l}\text { ENSGALTO0000006360 } \\
1.04 E-04\end{array}$ & -4.75 \\
\hline 4128 & $\begin{array}{l}\text { ENSGALTO0000006408 } \\
1.69 E-03\end{array}$ & -1.79 \\
\hline 4129 & $\begin{array}{l}\text { ENSGALT00000006419 } \\
6.86 \mathrm{E}-03\end{array}$ & -2.60 \\
\hline 4130 & $\begin{array}{l}\text { ENSGALT00000006430 } \\
5.31 \mathrm{E}-03\end{array}$ & -2.68 \\
\hline 4131 & $\begin{array}{l}\text { ENSGALTO0000006504 } \\
6.14 E-02\end{array}$ & -1.15 \\
\hline 4132 & $\begin{array}{l}\text { ENSGALTO0000006557 } \\
\text { 3.64E-04 }\end{array}$ & -6.19 \\
\hline
\end{tabular}

\begin{tabular}{|c|c|c|}
\hline 4133 & $\begin{array}{l}\text { ENSGALTO0000006634 } \\
6.96 E-03\end{array}$ & -2.42 \\
\hline 4134 & $\begin{array}{l}\text { ENSGALT00000006738 } \\
8.66 E-03\end{array}$ & -2.22 \\
\hline 4135 & $\begin{array}{l}\text { ENSGALTO0000006741 } \\
6.58 E-04\end{array}$ & -7.56 \\
\hline 4136 & $\begin{array}{l}\text { ENSGALTO0000006755 } \\
5.49 E-04\end{array}$ & -7.29 \\
\hline 4137 & $\begin{array}{l}\text { ENSGALTO0000006770 } \\
1.37 E-02\end{array}$ & -1.16 \\
\hline 4138 & $\begin{array}{l}\text { ENSGALTO0000006789 } \\
2.58 E-04\end{array}$ & -5.17 \\
\hline 4139 & $\begin{array}{l}\text { ENSGALTO0000006996 } \\
3.53 \mathrm{E}-04\end{array}$ & -2.89 \\
\hline 4140 & $\begin{array}{l}\text { ENSGALTO0000007036 } \\
2.27 E-03\end{array}$ & -3.33 \\
\hline 4141 & $\begin{array}{l}\text { ENSGALT00000007043 } \\
2.76 E-03\end{array}$ & -3.21 \\
\hline 4142 & $\begin{array}{l}\text { ENSGALT00000007055 } \\
1.24 E-04\end{array}$ & -5.34 \\
\hline 4143 & $\begin{array}{l}\text { ENSGALTO0000007068 } \\
2.48 E-03\end{array}$ & -3.28 \\
\hline 4144 & $\begin{array}{l}\text { ENSGALTO0000007158 } \\
1.35 E-04\end{array}$ & -6.46 \\
\hline 4145 & $\begin{array}{l}\text { ENSGALTO0000007164 } \\
2.28 \mathrm{E}-03\end{array}$ & -1.69 \\
\hline 4146 & $\begin{array}{l}\text { ENSGALTO0000007176 } \\
5.57 E-04\end{array}$ & -2.12 \\
\hline 4147 & $\begin{array}{l}\text { ENSGALTO0000007178 } \\
1.62 E-04\end{array}$ & -4.99 \\
\hline 4148 & $\begin{array}{l}\text { ENSGALTO0000007245 } \\
1.11 \mathrm{E}-02\end{array}$ & -1.22 \\
\hline 4149 & $\begin{array}{l}\text { ENSGALTO0000007342 } \\
6.64 \mathrm{E}-03\end{array}$ & -2.97 \\
\hline 4150 & $\begin{array}{l}\text { ENSGALTO0000007349 } \\
1.35 E-04\end{array}$ & -3.52 \\
\hline 4151 & $\begin{array}{l}\text { ENSGALTO0000007438 } \\
1.56 E-03\end{array}$ & -2.37 \\
\hline 4152 & $\begin{array}{l}\text { ENSGALT00000007481 } \\
2.15 E-04\end{array}$ & -4.72 \\
\hline 4153 & $\begin{array}{l}\text { ENSGALTO0000007511 } \\
2.75 E-03\end{array}$ & -1.65 \\
\hline 4154 & $\begin{array}{l}\text { ENSGALTO0000007571 } \\
2.08 E-03\end{array}$ & -4.10 \\
\hline 4155 & $\begin{array}{l}\text { ENSGALTO0000007601 } \\
7.79 \mathrm{E}-04\end{array}$ & -1.48 \\
\hline 4156 & $\begin{array}{l}\text { ENSGALTO0000007624 } \\
3.72 E-03\end{array}$ & -2.26 \\
\hline 4157 & $\begin{array}{l}\text { ENSGALTO0000007787 } \\
1.60 E-02\end{array}$ & -1.43 \\
\hline 4158 & $\begin{array}{l}\text { ENSGALTO0000007790 } \\
5.90 \mathrm{E}-04\end{array}$ & -5.29 \\
\hline 4159 & $\begin{array}{l}\text { ENSGALTO0000007793 } \\
1.68 \mathrm{E}-02\end{array}$ & -3.27 \\
\hline 4160 & $\begin{array}{l}\text { ENSGALTO0000007806 } \\
7.09 E-06\end{array}$ & -1.77 \\
\hline 4161 & $\begin{array}{l}\text { ENSGALTO0000007854 } \\
4.03 E-02\end{array}$ & -1.34 \\
\hline 4162 & $\begin{array}{l}\text { ENSGALTO0000007941 } \\
2.71 E-05\end{array}$ & -7.54 \\
\hline 4163 & $\begin{array}{l}\text { ENSGALTO0000007970 } \\
1.61 E-02\end{array}$ & -1.72 \\
\hline 4164 & $\begin{array}{l}\text { ENSGALTO0000008076 } \\
2.36 E-03\end{array}$ & -1.31 \\
\hline 4165 & $\begin{array}{l}\text { ENSGALTO0000008187 } \\
1.02 E-02\end{array}$ & -1.25 \\
\hline 4166 & $\begin{array}{l}\text { ENSGALTO0000008219 } \\
7.35 E-05\end{array}$ & -4.31 \\
\hline 4167 & $\begin{array}{l}\text { ENSGALTO0000008225 } \\
1.30 E-03\end{array}$ & -2.36 \\
\hline 4168 & $\begin{array}{l}\text { ENSGALT00000008337 } \\
4.03 E-04\end{array}$ & -1.23 \\
\hline 4169 & $\begin{array}{l}\text { ENSGALTO0000008376 } \\
4.20 E-03\end{array}$ & -1.44 \\
\hline 4170 & $\begin{array}{l}\text { ENSGALTO0000008386 } \\
6.24 E-04\end{array}$ & -5.58 \\
\hline 4171 & $\begin{array}{l}\text { ENSGALT00000008482 } \\
2.47 E-03\end{array}$ & -2.59 \\
\hline
\end{tabular}

\begin{tabular}{|c|c|c|}
\hline 4172 & $\begin{array}{l}\text { ENSGALTO0000008627 } \\
1.28 E-05\end{array}$ & -2.95 \\
\hline 4173 & $\begin{array}{l}\text { ENSGALTO0000008677 } \\
3.22 E-04\end{array}$ & -8.03 \\
\hline 4174 & $\begin{array}{l}\text { ENSGALTO0000008690 } \\
6.95 E-04\end{array}$ & -2.10 \\
\hline 4175 & $\begin{array}{l}\text { ENSGALTO0000008816 } \\
3.09 E-06\end{array}$ & -7.48 \\
\hline 4176 & $\begin{array}{l}\text { ENSGALTO0000008821 } \\
2.86 E-03\end{array}$ & -2.34 \\
\hline 4177 & $\begin{array}{l}\text { ENSGALTO0000008822 } \\
2.03 E-03\end{array}$ & -3.86 \\
\hline 4178 & $\begin{array}{l}\text { ENSGALTO0000008851 } \\
1.66 E-04\end{array}$ & -7.09 \\
\hline 4179 & $\begin{array}{l}\text { ENSGALTO0000008872 } \\
2.95 E-05\end{array}$ & -3.77 \\
\hline 4180 & $\begin{array}{l}\text { ENSGALTO0000008873 } \\
1.16 E-03\end{array}$ & -1.59 \\
\hline 4181 & $\begin{array}{l}\text { ENSGALT00000008888 } \\
1.15 E-02\end{array}$ & -1.61 \\
\hline 4182 & $\begin{array}{l}\text { ENSGALT00000008901 } \\
\text { 5.43E- } 03\end{array}$ & -2.43 \\
\hline 4183 & $\begin{array}{l}\text { ENSGALTO0000008953 } \\
\text { 5.55E-02 }\end{array}$ & -2.05 \\
\hline 4184 & $\begin{array}{l}\text { ENSGALT00000009018 } \\
2.17 E-04\end{array}$ & -4.36 \\
\hline 4185 & $\begin{array}{l}\text { ENSGALTO0000009049 } \\
9.67 E-06\end{array}$ & -3.87 \\
\hline 4186 & $\begin{array}{l}\text { ENSGALT00000009067 } \\
7.20 \mathrm{E}-02\end{array}$ & -1.56 \\
\hline 4187 & $\begin{array}{l}\text { ENSGALTO0000009092 } \\
5.32 E-04\end{array}$ & -5.26 \\
\hline 4188 & $\begin{array}{l}\text { ENSGALTO0000009112 } \\
7.25 E-04\end{array}$ & -5.85 \\
\hline 4189 & $\begin{array}{l}\text { ENSGALT00000009122 } \\
\text { 3.09E-03 }\end{array}$ & -3.55 \\
\hline 4190 & $\begin{array}{l}\text { ENSGALTO0000009152 } \\
2.09 \mathrm{E}-05\end{array}$ & -5.87 \\
\hline 4191 & $\begin{array}{l}\text { ENSGALTO0000009174 } \\
4.01 E-04\end{array}$ & -2.36 \\
\hline 4192 & $\begin{array}{l}\text { ENSGALTO0000009209 } \\
\text { 7.59E-04 }\end{array}$ & -7.91 \\
\hline 4193 & $\begin{array}{l}\text { ENSGALTO0000009283 } \\
1.30 E-05\end{array}$ & -6.30 \\
\hline 4194 & $\begin{array}{l}\text { ENSGALTO0000009292 } \\
3.65 E-05\end{array}$ & -5.09 \\
\hline 4195 & $\begin{array}{l}\text { ENSGALTO0000009358 } \\
3.12 E-03\end{array}$ & -4.39 \\
\hline 4196 & $\begin{array}{l}\text { ENSGALT00000009363 } \\
7.75 E-04\end{array}$ & -3.98 \\
\hline 4197 & $\begin{array}{l}\text { ENSGALTO0000009435 } \\
2.68 E-05\end{array}$ & -7.30 \\
\hline 4198 & $\begin{array}{l}\text { ENSGALTO0000009484 } \\
\text { 5.31E-05 }\end{array}$ & -4.52 \\
\hline 4199 & $\begin{array}{l}\text { ENSGALT00000009519 } \\
1.88 \mathrm{E}-01\end{array}$ & -1.49 \\
\hline 4200 & $\begin{array}{l}\text { ENSGALTO0000009595 } \\
\text { 3.14E-02 }\end{array}$ & -1.73 \\
\hline 4201 & $\begin{array}{l}\text { ENSGALT00000009600 } \\
\text { 3.33E-04 }\end{array}$ & -8.34 \\
\hline 4202 & $\begin{array}{l}\text { ENSGALTO0000009669 } \\
\text { 3.02E-04 }\end{array}$ & -2.15 \\
\hline 4203 & $\begin{array}{l}\text { ENSGALTO0000009670 } \\
\text { 3.22E-03 }\end{array}$ & -2.79 \\
\hline 4204 & $\begin{array}{l}\text { ENSGALTO0000009734 } \\
1.79 \mathrm{E}-05\end{array}$ & -3.77 \\
\hline 4205 & $\begin{array}{l}\text { ENSGALTO0000009765 } \\
\text { 7.68E-05 }\end{array}$ & -6.82 \\
\hline 4206 & $\begin{array}{l}\text { ENSGALTO0000009804 } \\
5.10 \mathrm{E}-04\end{array}$ & -4.8 \\
\hline 4207 & $\begin{array}{l}\text { ENSGALTO0000009829 } \\
2.13 E-03\end{array}$ & -1.54 \\
\hline 4208 & $\begin{array}{l}\text { ENSGALTO0000009875 } \\
2.85 E-04\end{array}$ & -3.45 \\
\hline 4209 & $\begin{array}{l}\text { ENSGALT00000009958 } \\
2.73 E-03\end{array}$ & -6.11 \\
\hline 4210 & $\begin{array}{l}\text { ENSGALTO00000010020 } \\
1.54 E-05\end{array}$ & -7.27 \\
\hline
\end{tabular}




\begin{tabular}{|c|c|c|}
\hline 4211 & $\begin{array}{l}\text { ENSGALTO0000010062 } \\
1.01 E-03\end{array}$ & -3.21 \\
\hline 4212 & $\begin{array}{l}\text { ENSGALTO0000010115 } \\
9.35 E-04\end{array}$ & -3.38 \\
\hline 4213 & $\begin{array}{l}\text { ENSGALTO0000010150 } \\
2.53 E-04\end{array}$ & -4.56 \\
\hline 4214 & $\begin{array}{l}\text { ENSGALTO0000010171 } \\
1.96 E-03\end{array}$ & -4.38 \\
\hline 4215 & $\begin{array}{l}\text { ENSGALTO0000010207 } \\
2.61 E-04\end{array}$ & -6.52 \\
\hline 4216 & $\begin{array}{l}\text { ENSGALT00000010228 } \\
7.64 E-04\end{array}$ & -5.93 \\
\hline 4217 & $\begin{array}{l}\text { ENSGALTO0000010235 } \\
5.20 \text { - } 04\end{array}$ & -7.63 \\
\hline 4218 & $\begin{array}{l}\text { ENSGALT00000010237 } \\
1.13 E-03\end{array}$ & -5.09 \\
\hline 4219 & $\begin{array}{l}\text { ENSGALTO0000010291 } \\
1.26 E-02\end{array}$ & -1.93 \\
\hline 4220 & $\begin{array}{l}\text { ENSGALTO0000010322 } \\
2.43 \mathrm{E}-03\end{array}$ & -3.27 \\
\hline 4221 & $\begin{array}{l}\text { ENSGALTO0000010350 } \\
1.27 E-02\end{array}$ & -1.29 \\
\hline 4222 & $\begin{array}{l}\text { ENSGALTO0000010386 } \\
4.34 E-04\end{array}$ & -6.36 \\
\hline 4223 & $\begin{array}{l}\text { ENSGALTO0000010418 } \\
1.67 E-02\end{array}$ & -1.17 \\
\hline 4224 & $\begin{array}{l}\text { ENSGALTO0000010432 } \\
5.22 E-03\end{array}$ & -2.00 \\
\hline 4225 & $\begin{array}{l}\text { ENSGALTO0000010448 } \\
1.30 \mathrm{E}-04\end{array}$ & -7.17 \\
\hline 4226 & $\begin{array}{l}\text { ENSGALT00000010530 } \\
2.53 \mathrm{E}-03\end{array}$ & -4.70 \\
\hline 4227 & $\begin{array}{l}\text { ENSGALTO0000010536 } \\
7.95 E-02\end{array}$ & -1.14 \\
\hline 4228 & $\begin{array}{l}\text { ENSGALT00000010542 } \\
1.27 E-04\end{array}$ & -2.99 \\
\hline 4229 & $\begin{array}{l}\text { ENSGALT00000010576 } \\
1.68 \mathrm{E}-04\end{array}$ & -5.90 \\
\hline 4230 & $\begin{array}{l}\text { ENSGALT00000010588 } \\
1.22 E-03\end{array}$ & -5.08 \\
\hline 4231 & $\begin{array}{l}\text { ENSGALT00000010589 } \\
1.91 \mathrm{E}-04\end{array}$ & -5.94 \\
\hline 4232 & $\begin{array}{l}\text { ENSGALTO0000010627 } \\
9.00 E-03\end{array}$ & -1.32 \\
\hline 4233 & $\begin{array}{l}\text { ENSGALTO0000010642 } \\
2.53 E-03\end{array}$ & -3.32 \\
\hline 4234 & $\begin{array}{l}\text { ENSGALTO0000010681 } \\
2.74 E-03\end{array}$ & -2.79 \\
\hline 4235 & $\begin{array}{l}\text { ENSGALT00000010684 } \\
8.03 E-04\end{array}$ & -1.91 \\
\hline 4236 & $\begin{array}{l}\text { ENSGALT00000010711 } \\
3.28 \mathrm{E}-03\end{array}$ & -1.12 \\
\hline 4237 & $\begin{array}{l}\text { ENSGALTO0000010733 } \\
8.63 E-04\end{array}$ & -5.01 \\
\hline 4238 & $\begin{array}{l}\text { ENSGALTO0000010789 } \\
3.43 E-04\end{array}$ & -4.96 \\
\hline 4239 & $\begin{array}{l}\text { ENSGALTO0000010951 } \\
5.99 \mathrm{E}-03\end{array}$ & -2.13 \\
\hline 4240 & $\begin{array}{l}\text { ENSGALTO0000010978 } \\
2.33 E-02\end{array}$ & -1.26 \\
\hline 4241 & $\begin{array}{l}\text { ENSGALT00000011071 } \\
9.32 \mathrm{E}-04\end{array}$ & -7.69 \\
\hline 4242 & $\begin{array}{l}\text { ENSGALTO0000011207 } \\
7.70 E-04\end{array}$ & -2.31 \\
\hline 4243 & $\begin{array}{l}\text { ENSGALTO0000011211 } \\
3.78 \mathrm{E}-03\end{array}$ & -3.36 \\
\hline 4244 & $\begin{array}{l}\text { ENSGALTO0000011225 } \\
2.85 E-03\end{array}$ & -3.04 \\
\hline 4245 & $\begin{array}{l}\text { ENSGALTO0000011235 } \\
2.41 E-04\end{array}$ & -6.36 \\
\hline 4246 & $\begin{array}{l}\text { ENSGALTO0000011237 } \\
2.92 E-02\end{array}$ & -1.96 \\
\hline 4247 & $\begin{array}{l}\text { ENSGALTO0000011292 } \\
1.09 E-04\end{array}$ & -6.14 \\
\hline 4248 & $\begin{array}{l}\text { ENSGALTO0000011493 } \\
3.37 E-04\end{array}$ & -5.72 \\
\hline 4249 & $\begin{array}{l}\text { ENSGALTO0000011495 } \\
1.19 E-03\end{array}$ & -6.03 \\
\hline
\end{tabular}

\begin{tabular}{|c|c|c|}
\hline 4250 & $\begin{array}{l}\text { ENSGALT00000011538 } \\
3.37 \mathrm{E}-02\end{array}$ & -1.74 \\
\hline 4251 & $\begin{array}{l}\text { ENSGALT00000011556 } \\
3.47 E-04\end{array}$ & -4.99 \\
\hline 4252 & $\begin{array}{l}\text { ENSGALT00000011561 } \\
6.45 \mathrm{E}-03\end{array}$ & -3.58 \\
\hline 4253 & $\begin{array}{l}\text { ENSGALT00000011586 } \\
2.98 \mathrm{E}-04\end{array}$ & -4.66 \\
\hline 4254 & $\begin{array}{l}\text { ENSGALT00000011588 } \\
2.22 E-04\end{array}$ & -7.88 \\
\hline 4255 & $\begin{array}{l}\text { ENSGALT00000011589 } \\
1.46 \mathrm{E}-03\end{array}$ & -4.63 \\
\hline 4256 & $\begin{array}{l}\text { ENSGALTO0000011609 } \\
8.09 \mathrm{E}-03\end{array}$ & -1.26 \\
\hline 4257 & $\begin{array}{l}\text { ENSGALT00000011610 } \\
8.63 E-03\end{array}$ & -4.17 \\
\hline 4258 & $\begin{array}{l}\text { ENSGALTO0000011640 } \\
4.00 E-04\end{array}$ & -4.86 \\
\hline 4259 & $\begin{array}{l}\text { ENSGALT00000011789 } \\
1.52 E-03\end{array}$ & -4.01 \\
\hline 4260 & $\begin{array}{l}\text { ENSGALTO0000011808 } \\
9.58 \mathrm{E}-04\end{array}$ & -5.63 \\
\hline 4261 & $\begin{array}{l}\text { ENSGALT00000011831 } \\
1.95 E-02\end{array}$ & -3.43 \\
\hline 4262 & $\begin{array}{l}\text { ENSGALT00000011863 } \\
1.36 \mathrm{E}-04\end{array}$ & -5.12 \\
\hline 4263 & $\begin{array}{l}\text { ENSGALT00000011945 } \\
7.12 \mathrm{E}-04\end{array}$ & -2.29 \\
\hline 4264 & $\begin{array}{l}\text { ENSGALT00000011997 } \\
\text { 7.78E-04 }\end{array}$ & -5.97 \\
\hline 4265 & $\begin{array}{l}\text { ENSGALT00000012074 } \\
1.17 E-03\end{array}$ & -2.62 \\
\hline 4266 & $\begin{array}{l}\text { ENSGALT00000012081 } \\
\text { 4.51E-04 }\end{array}$ & -3.61 \\
\hline 4267 & $\begin{array}{l}\text { ENSGALTO0000012108 } \\
9.31 \mathrm{E}-03\end{array}$ & -2.28 \\
\hline 4268 & $\begin{array}{l}\text { ENSGALTO0000012146 } \\
\text { 3.32E-04 }\end{array}$ & -6.82 \\
\hline 4269 & $\begin{array}{l}\text { ENSGALT00000012154 } \\
4.73 E-03\end{array}$ & -1.04 \\
\hline 4270 & $\begin{array}{l}\text { ENSGALT00000012179 } \\
1.42 \mathrm{E}-03\end{array}$ & -1.82 \\
\hline 4271 & $\begin{array}{l}\text { ENSGALTO0000012241 } \\
2.00 \mathrm{E}-03\end{array}$ & -3.39 \\
\hline 4272 & $\begin{array}{l}\text { ENSGALT00000012292 } \\
2.17 \mathrm{E}-02\end{array}$ & -3.11 \\
\hline 4273 & $\begin{array}{l}\text { ENSGALT00000012300 } \\
\text { 5.11E-04 }\end{array}$ & -2.54 \\
\hline 4274 & $\begin{array}{l}\text { ENSGALT00000012323 } \\
\text { 4.21E-04 }\end{array}$ & -3.09 \\
\hline 4275 & $\begin{array}{l}\text { ENSGALT00000012339 } \\
6.89 E-03\end{array}$ & -4.94 \\
\hline 4276 & $\begin{array}{l}\text { ENSGALTO0000012360 } \\
9.00 \mathrm{0}-03\end{array}$ & -1.37 \\
\hline 4277 & $\begin{array}{l}\text { ENSGALTO0000012364 } \\
1.65 E-01\end{array}$ & -2.02 \\
\hline 4278 & $\begin{array}{l}\text { ENSGALT00000012438 } \\
1.34 \mathrm{E}-03\end{array}$ & -1.17 \\
\hline 4279 & $\begin{array}{l}\text { ENSGALT00000012445 } \\
\text { 3.03E-04 }\end{array}$ & -3.26 \\
\hline 4280 & $\begin{array}{l}\text { ENSGALTO0000012612 } \\
2.14 \mathrm{E}-03\end{array}$ & -3.66 \\
\hline 4281 & $\begin{array}{l}\text { ENSGALT00000012644 } \\
7.24 E-03\end{array}$ & -3.51 \\
\hline 4282 & $\begin{array}{l}\text { ENSGALT00000012655 } \\
1.55 E-03\end{array}$ & -2.01 \\
\hline 4283 & $\begin{array}{l}\text { ENSGALT00000012727 } \\
1.29 \mathrm{E}-03\end{array}$ & -5.46 \\
\hline 4284 & $\begin{array}{l}\text { ENSGALT00000012748 } \\
1.14 \mathrm{E}-02\end{array}$ & -2.91 \\
\hline 4285 & $\begin{array}{l}\text { ENSGALT00000012756 } \\
2.44 E-03\end{array}$ & -5.27 \\
\hline 4286 & $\begin{array}{l}\text { ENSGALT00000012763 } \\
1.85 E-02\end{array}$ & -2.53 \\
\hline 4287 & $\begin{array}{l}\text { ENSGALT00000012809 } \\
5.10 \mathrm{E}-03\end{array}$ & -1.89 \\
\hline 4288 & $\begin{array}{l}\text { ENSGALT00000012865 } \\
7.99 \mathrm{E}-03\end{array}$ & -1.21 \\
\hline
\end{tabular}

\begin{tabular}{|c|c|c|}
\hline 4289 & $\begin{array}{l}\text { ENSGALTO0000012888 } \\
1.02 E-03\end{array}$ & -3.45 \\
\hline 4290 & $\begin{array}{l}\text { ENSGALT00000013011 } \\
9.83 \mathrm{E}-03\end{array}$ & -1.94 \\
\hline 4291 & $\begin{array}{l}\text { ENSGALTO0000013077 } \\
1.31 E-03\end{array}$ & -2.33 \\
\hline 4292 & $\begin{array}{l}\text { ENSGALT00000013157 } \\
2.34 \mathrm{E}-04\end{array}$ & -2.41 \\
\hline 4293 & $\begin{array}{l}\text { ENSGALT00000013167 } \\
1.32 \text { E-03 }\end{array}$ & -3.29 \\
\hline 4294 & $\begin{array}{l}\text { ENSGALT00000013169 } \\
\text { 3.05E-03 }\end{array}$ & -3.84 \\
\hline 4295 & $\begin{array}{l}\text { ENSGALT00000013285 } \\
7.82 E-04\end{array}$ & -4.71 \\
\hline 4296 & $\begin{array}{l}\text { ENSGALTO0000013351 } \\
4.01 E-03\end{array}$ & -1.23 \\
\hline 4297 & $\begin{array}{l}\text { ENSGALTO0000013446 } \\
8.33 E-05\end{array}$ & -3.63 \\
\hline 4298 & $\begin{array}{l}\text { ENSGALTO0000013484 } \\
2.04 E-03\end{array}$ & -2.67 \\
\hline 4299 & $\begin{array}{l}\text { ENSGALTO0000013532 } \\
3.39 E-03\end{array}$ & -3.43 \\
\hline 4300 & $\begin{array}{l}\text { ENSGALTO0000013675 } \\
\text { 3.35E-02 }\end{array}$ & -1.12 \\
\hline 4301 & $\begin{array}{l}\text { ENSGALTO0000013682 } \\
9.25 E-04\end{array}$ & -2.31 \\
\hline 4302 & $\begin{array}{l}\text { ENSGALT00000013683 } \\
7.41 E-03\end{array}$ & -5.66 \\
\hline 4303 & $\begin{array}{l}\text { ENSGALTO0000013703 } \\
5.37 E-04\end{array}$ & -6.00 \\
\hline 4304 & $\begin{array}{l}\text { ENSGALTO0000013724 } \\
2.70 \text {-03 }\end{array}$ & -1.15 \\
\hline 4305 & $\begin{array}{l}\text { ENSGALTO0000013770 } \\
1.32 \text { E-02 }\end{array}$ & -1.37 \\
\hline 4306 & $\begin{array}{l}\text { ENSGALTO0000013824 } \\
4.27 E-04\end{array}$ & -4.48 \\
\hline 4307 & $\begin{array}{l}\text { ENSGALTO0000013833 } \\
1.71 \mathrm{E}-03\end{array}$ & -2.56 \\
\hline 4308 & $\begin{array}{l}\text { ENSGALTO0000013868 } \\
3.62 E-03\end{array}$ & -4.75 \\
\hline 4309 & $\begin{array}{l}\text { ENSGALTO0000013882 } \\
7.15 E-04\end{array}$ & -3.44 \\
\hline 4310 & $\begin{array}{l}\text { ENSGALTO0000013914 } \\
1.99 \mathrm{E}-03\end{array}$ & -4.00 \\
\hline 4311 & $\begin{array}{l}\text { ENSGALTO0000014081 } \\
1.22 E-03\end{array}$ & -3.27 \\
\hline 4312 & $\begin{array}{l}\text { ENSGALT00000014101 } \\
9.25 \mathrm{E}-04\end{array}$ & -3.27 \\
\hline 4313 & $\begin{array}{l}\text { ENSGALTO0000014145 } \\
6.82 E-06\end{array}$ & -3.91 \\
\hline 4314 & $\begin{array}{l}\text { ENSGALTO0000014381 } \\
3.42 E-04\end{array}$ & -4.72 \\
\hline 4315 & $\begin{array}{l}\text { ENSGALTO0000014383 } \\
6.98 \mathrm{E}-03\end{array}$ & -2.46 \\
\hline 4316 & $\begin{array}{l}\text { ENSGALTO0000014479 } \\
9.50 E-04\end{array}$ & -4.70 \\
\hline 4317 & $\begin{array}{l}\text { ENSGALT00000014487 } \\
1.28 \mathrm{E}-03\end{array}$ & -1.32 \\
\hline 4318 & $\begin{array}{l}\text { ENSGALTO0000014506 } \\
1.86 E-02\end{array}$ & -3.85 \\
\hline 4319 & $\begin{array}{l}\text { ENSGALTO0000014545 } \\
8.07 E-04\end{array}$ & -4.44 \\
\hline 4320 & $\begin{array}{l}\text { ENSGALTO0000014574 } \\
1.35 E-05\end{array}$ & -4.17 \\
\hline 4321 & $\begin{array}{l}\text { ENSGALTO0000014592 } \\
8.77 \mathrm{E}-04\end{array}$ & -6.32 \\
\hline 4322 & $\begin{array}{l}\text { ENSGALTO0000014608 } \\
6.35 E-03\end{array}$ & -2.86 \\
\hline 4323 & $\begin{array}{l}\text { ENSGALTO0000014614 } \\
2.18 E-04\end{array}$ & -4.55 \\
\hline 4324 & $\begin{array}{l}\text { ENSGALTO0000014632 } \\
4.56 E-05\end{array}$ & -7.54 \\
\hline 4325 & $\begin{array}{l}\text { ENSGALTO0000014766 } \\
6.52 E-04\end{array}$ & -7.73 \\
\hline 4326 & $\begin{array}{l}\text { ENSGALTO0000014840 } \\
2.48 E-04\end{array}$ & -2.79 \\
\hline 4327 & $\begin{array}{l}\text { ENSGALT00000014845 } \\
3.67 E-04\end{array}$ & -4.36 \\
\hline
\end{tabular}




\begin{tabular}{|c|c|c|}
\hline 4328 & $\begin{array}{l}\text { ENSGALTO0000014852 } \\
1.10 \mathrm{-} 02\end{array}$ & -2.55 \\
\hline 4329 & $\begin{array}{l}\text { ENSGALT00000014910 } \\
2.57 \mathrm{E}-03\end{array}$ & -3.64 \\
\hline 4330 & $\begin{array}{l}\text { ENSGALT00000015018 } \\
1.55 \mathrm{E}-02\end{array}$ & -3.48 \\
\hline 4331 & $\begin{array}{l}\text { ENSGALTO0000015026 } \\
1.49 E-02\end{array}$ & -1.27 \\
\hline 4332 & $\begin{array}{l}\text { ENSGALT00000015161 } \\
8.25 \mathrm{E}-03\end{array}$ & -5.08 \\
\hline 4333 & $\begin{array}{l}\text { ENSGALT00000015199 } \\
6.03 E-03\end{array}$ & -6.45 \\
\hline 4334 & $\begin{array}{l}\text { ENSGALT00000015265 } \\
2.24 E-03\end{array}$ & -5.71 \\
\hline 4335 & $\begin{array}{l}\text { ENSGALT00000015314 } \\
1.69 \mathrm{E}-03\end{array}$ & -5.33 \\
\hline 4336 & $\begin{array}{l}\text { ENSGALT00000015333 } \\
5.50 \mathrm{E}-03\end{array}$ & -2.10 \\
\hline 4337 & $\begin{array}{l}\text { ENSGALT00000015359 } \\
1.42 E-03\end{array}$ & -4.35 \\
\hline 4338 & $\begin{array}{l}\text { ENSGALT00000015496 } \\
5.11 \mathrm{E}-03\end{array}$ & -2.47 \\
\hline 4339 & $\begin{array}{l}\text { ENSGALT00000015530 } \\
\text { 7.42E-05 }\end{array}$ & -1.67 \\
\hline 4340 & $\begin{array}{l}\text { ENSGALT00000015535 } \\
6.83 \mathrm{E}-04\end{array}$ & -1.78 \\
\hline 4341 & $\begin{array}{l}\text { ENSGALT00000015561 } \\
4.98 \mathrm{E}-04\end{array}$ & -5.92 \\
\hline 4342 & $\begin{array}{l}\text { ENSGALT00000015575 } \\
1.74 E-05\end{array}$ & -4.08 \\
\hline 4343 & $\begin{array}{l}\text { ENSGALT00000015662 } \\
3.15 \mathrm{E}-03\end{array}$ & -3.83 \\
\hline 4344 & $\begin{array}{l}\text { ENSGALT00000015675 } \\
1.35 E-03\end{array}$ & -1.07 \\
\hline 4345 & $\begin{array}{l}\text { ENSGALT00000015676 } \\
2.39 \mathrm{E}-03\end{array}$ & -3.19 \\
\hline 4346 & $\begin{array}{l}\text { ENSGALT00000015752 } \\
1.82 E-04\end{array}$ & -4.06 \\
\hline 4347 & $\begin{array}{l}\text { ENSGALT00000015761 } \\
2.96 \mathrm{E}-03\end{array}$ & -3.15 \\
\hline 4348 & $\begin{array}{l}\text { ENSGALTO0000015764 } \\
8.98 \mathrm{E}-04\end{array}$ & -2.23 \\
\hline 4349 & $\begin{array}{l}\text { ENSGALT00000015782 } \\
1.05 E-03\end{array}$ & -4.20 \\
\hline 4350 & $\begin{array}{l}\text { ENSGALTO0000015796 } \\
4.03 E-03\end{array}$ & -1.72 \\
\hline 4351 & $\begin{array}{l}\text { ENSGALTO0000015806 } \\
\text { 4.58E-06 }\end{array}$ & -4.66 \\
\hline 4352 & $\begin{array}{l}\text { ENSGALT00000015851 } \\
6.83 \mathrm{E}-05\end{array}$ & -7.06 \\
\hline 4353 & $\begin{array}{l}\text { ENSGALT00000015965 } \\
2.34 \mathrm{E}-02\end{array}$ & -2.21 \\
\hline 4354 & $\begin{array}{l}\text { ENSGALT00000015972 } \\
6.15 \mathrm{E}-04\end{array}$ & -5.38 \\
\hline 4355 & $\begin{array}{l}\text { ENSGALTO0000016123 } \\
1.33 \text { - } 03\end{array}$ & -2.68 \\
\hline 4356 & $\begin{array}{l}\text { ENSGALT00000016159 } \\
1.68 \mathrm{E}-02\end{array}$ & -1.97 \\
\hline 4357 & $\begin{array}{l}\text { ENSGALTO0000016328 } \\
6.24 E-04\end{array}$ & -1.57 \\
\hline 4358 & $\begin{array}{l}\text { ENSGALTO0000016350 } \\
3.81 E-03\end{array}$ & -2.66 \\
\hline 4359 & $\begin{array}{l}\text { ENSGALTO0000016352 } \\
1.80 \mathrm{E}-03\end{array}$ & -3.86 \\
\hline 4360 & $\begin{array}{l}\text { ENSGALTO0000016356 } \\
1.25 E-03\end{array}$ & -2.41 \\
\hline 4361 & $\begin{array}{l}\text { ENSGALT00000016414 } \\
1.86 E-04\end{array}$ & -5.20 \\
\hline 4362 & $\begin{array}{l}\text { ENSGALTO0000016453 } \\
2.27 E-03\end{array}$ & -5.01 \\
\hline 4363 & $\begin{array}{l}\text { ENSGALTO0000016562 } \\
2.27 E-04\end{array}$ & -3.23 \\
\hline 4364 & $\begin{array}{l}\text { ENSGALT00000016572 } \\
1.39 E-02\end{array}$ & -3.33 \\
\hline 4365 & $\begin{array}{l}\text { ENSGALTO0000016926 } \\
\text { 3.08E-03 }\end{array}$ & -2.85 \\
\hline 4366 & $\begin{array}{l}\text { ENSGALTO0000016936 } \\
\text { 3.64E-03 }\end{array}$ & -4.88 \\
\hline
\end{tabular}

\begin{tabular}{|c|c|c|}
\hline 4367 & $\begin{array}{l}\text { ENSGALTO0000016952 } \\
6.83 E-03\end{array}$ & -1.32 \\
\hline 4368 & $\begin{array}{l}\text { ENSGALTO0000017008 } \\
1.31 \mathrm{E}-03\end{array}$ & -2.31 \\
\hline 4369 & $\begin{array}{l}\text { ENSGALTO0000017125 } \\
1.61 E-03\end{array}$ & -4.18 \\
\hline 4370 & $\begin{array}{l}\text { ENSGALTO0000017131 } \\
2.54 E-02\end{array}$ & -1.97 \\
\hline 4371 & $\begin{array}{l}\text { ENSGALTO0000017148 } \\
\text { 7.97E-04 }\end{array}$ & -3.93 \\
\hline 4372 & $\begin{array}{l}\text { ENSGALTO0000017162 } \\
1.66 E-04\end{array}$ & -4.82 \\
\hline 4373 & $\begin{array}{l}\text { ENSGALTO0000017186 } \\
5.30 E-03\end{array}$ & -1.83 \\
\hline 4374 & $\begin{array}{l}\text { ENSGALTO0000017187 } \\
4.53 E-03\end{array}$ & -1.53 \\
\hline 4375 & $\begin{array}{l}\text { ENSGALT00000017257 } \\
1.02 \mathrm{E}-03\end{array}$ & -4.10 \\
\hline 4376 & $\begin{array}{l}\text { ENSGALTO0000017329 } \\
2.08 E-02\end{array}$ & -2.74 \\
\hline 4377 & $\begin{array}{l}\text { ENSGALTO0000017346 } \\
5.17 E-03\end{array}$ & -2.15 \\
\hline 4378 & $\begin{array}{l}\text { ENSGALTO0000017499 } \\
4.30 \text {-04 }\end{array}$ & -4.12 \\
\hline 4379 & $\begin{array}{l}\text { ENSGALTO0000017510 } \\
1.42 E-03\end{array}$ & -7.88 \\
\hline 4380 & $\begin{array}{l}\text { ENSGALTO0000017545 } \\
2.95 E-04\end{array}$ & -4.22 \\
\hline 4381 & $\begin{array}{l}\text { ENSGALTO0000017549 } \\
6.17 \mathrm{E}-04\end{array}$ & -6.92 \\
\hline 4382 & $\begin{array}{l}\text { ENSGALT00000017586 } \\
3.52 E-03\end{array}$ & -2.28 \\
\hline 4383 & $\begin{array}{l}\text { ENSGALTO0000017594 } \\
4.26 E-03\end{array}$ & -3.20 \\
\hline 4384 & $\begin{array}{l}\text { ENSGALTO0000017618 } \\
6.95 E-04\end{array}$ & -2.07 \\
\hline 4385 & $\begin{array}{l}\text { ENSGALTO0000017760 } \\
3.19 \mathrm{E}-04\end{array}$ & -3.01 \\
\hline 4386 & $\begin{array}{l}\text { ENSGALTO0000017886 } \\
1.46 E-03\end{array}$ & -2.09 \\
\hline 4387 & $\begin{array}{l}\text { ENSGALTO0000017892 } \\
3.68 \mathrm{E}-02\end{array}$ & -1.32 \\
\hline 4388 & $\begin{array}{l}\text { ENSGALTO0000017897 } \\
1.33 \text { E-03 }\end{array}$ & -5.72 \\
\hline 4389 & $\begin{array}{l}\text { ENSGALT00000017916 } \\
5.85 \mathrm{E}-03\end{array}$ & -3.08 \\
\hline 4390 & $\begin{array}{l}\text { ENSGALTO0000018018 } \\
3.78 E-04\end{array}$ & -3.46 \\
\hline 4391 & $\begin{array}{l}\text { ENSGALTO0000018029 } \\
1.58 \mathrm{E}-03\end{array}$ & -1.51 \\
\hline 4392 & $\begin{array}{l}\text { ENSGALT00000018063 } \\
1.62 E-05\end{array}$ & -3.14 \\
\hline 4393 & $\begin{array}{l}\text { ENSGALTO0000018250 } \\
1.04 E-03\end{array}$ & -5.75 \\
\hline 4394 & $\begin{array}{l}\text { ENSGALTO0000018253 } \\
2.73 E-03\end{array}$ & -3.06 \\
\hline 4395 & $\begin{array}{l}\text { ENSGALTO0000018455 } \\
2.07 E-03\end{array}$ & -3.45 \\
\hline 4396 & $\begin{array}{l}\text { ENSGALTO0000018474 } \\
4.93 \mathrm{E}-03\end{array}$ & -1.90 \\
\hline 4397 & $\begin{array}{l}\text { ENSGALTO0000018539 } \\
4.54 E-03\end{array}$ & -4.11 \\
\hline 4398 & $\begin{array}{l}\text { ENSGALTO0000018548 } \\
6.53 \mathrm{E}-04\end{array}$ & -4.33 \\
\hline 4399 & $\begin{array}{l}\text { ENSGALTO0000018584 } \\
2.68 \mathrm{E}-04\end{array}$ & -3.31 \\
\hline 4400 & $\begin{array}{l}\text { ENSGALTO0000018637 } \\
8.64 \text { E-04 }\end{array}$ & -2.78 \\
\hline 4401 & $\begin{array}{l}\text { ENSGALTO0000018638 } \\
1.06 E-03\end{array}$ & -2.96 \\
\hline 4402 & $\begin{array}{l}\text { ENSGALTO0000018667 } \\
5.10 \mathrm{E}-04\end{array}$ & -5.88 \\
\hline 4403 & $\begin{array}{l}\text { ENSGALTO0000018685 } \\
3.34 E-03\end{array}$ & -2.20 \\
\hline 4404 & $\begin{array}{l}\text { ENSGALTO0000018700 } \\
7.12 E-03\end{array}$ & -1.40 \\
\hline 4405 & $\begin{array}{l}\text { ENSGALTO0000018701 } \\
1.38 E-03\end{array}$ & -5.78 \\
\hline
\end{tabular}

\begin{tabular}{|c|c|c|}
\hline 4406 & $\begin{array}{l}\text { ENSGALTO0000018879 } \\
6.60 E-04\end{array}$ & -7.23 \\
\hline 4407 & $\begin{array}{l}\text { ENSGALTO0000018981 } \\
1.89 \mathrm{E}-02\end{array}$ & -1.44 \\
\hline 4408 & $\begin{array}{l}\text { ENSGALTO0000018997 } \\
1.70 \text {-04 }\end{array}$ & -3.99 \\
\hline 4409 & $\begin{array}{l}\text { ENSGALTO0000019023 } \\
8.21 E-04\end{array}$ & -3.84 \\
\hline 4410 & $\begin{array}{l}\text { ENSGALTO0000019030 } \\
6.30 \mathrm{E}-04\end{array}$ & -7.26 \\
\hline 4411 & $\begin{array}{l}\text { ENSGALTO0000019190 } \\
3.38 \mathrm{E}-02\end{array}$ & -1.90 \\
\hline 4412 & $\begin{array}{l}\text { ENSGALT00000019277 } \\
7.59 \mathrm{-}-04\end{array}$ & -5.21 \\
\hline 4413 & $\begin{array}{l}\text { ENSGALTO0000019339 } \\
1.07 E-04\end{array}$ & -5.75 \\
\hline 4414 & $\begin{array}{l}\text { ENSGALTO0000019385 } \\
2.07 E-04\end{array}$ & -7.08 \\
\hline 4415 & $\begin{array}{l}\text { ENSGALT00000019429 } \\
7.75 E-03\end{array}$ & -1.77 \\
\hline 4416 & $\begin{array}{l}\text { ENSGALTO0000019431 } \\
4.52 \mathrm{E}-04\end{array}$ & -5.39 \\
\hline 4417 & $\begin{array}{l}\text { ENSGALT00000019558 } \\
2.26 \mathrm{-}-04\end{array}$ & -2.10 \\
\hline 4418 & $\begin{array}{l}\text { ENSGALTO0000019682 } \\
6.86 E-03\end{array}$ & -1.24 \\
\hline 4419 & $\begin{array}{l}\text { ENSGALTO0000019873 } \\
4.40 \mathrm{E}-02\end{array}$ & -1.41 \\
\hline 4420 & $\begin{array}{l}\text { ENSGALTO0000019911 } \\
8.32 E-04\end{array}$ & -4.68 \\
\hline 4421 & $\begin{array}{l}\text { ENSGALTO0000019979 } \\
7.48 \mathrm{E}-04\end{array}$ & -6.41 \\
\hline 4422 & $\begin{array}{l}\text { ENSGALT00000020008 } \\
5.97 E-03\end{array}$ & -1.50 \\
\hline 4423 & $\begin{array}{l}\text { ENSGALTO0000020010 } \\
1.50 \mathrm{-}-04\end{array}$ & -7.66 \\
\hline 4424 & $\begin{array}{l}\text { ENSGALTO0000020016 } \\
3.18 E-02\end{array}$ & -1.43 \\
\hline 4425 & $\begin{array}{l}\text { ENSGALTO0000020032 } \\
\text { 4.16E-04 }\end{array}$ & -6.38 \\
\hline 4426 & $\begin{array}{l}\text { ENSGALTO0000020176 } \\
2.99 \mathrm{E}-05\end{array}$ & -6.87 \\
\hline 4427 & $\begin{array}{l}\text { ENSGALTO0000020359 } \\
1.92 E-05\end{array}$ & -3.31 \\
\hline 4428 & $\begin{array}{l}\text { ENSGALTO0000020410 } \\
5.10 \mathrm{E}-03\end{array}$ & -1.75 \\
\hline 4429 & $\begin{array}{l}\text { ENSGALTO0000020434 } \\
7.63 E-03\end{array}$ & -3.73 \\
\hline 4430 & $\begin{array}{l}\text { ENSGALTO0000020442 } \\
2.58 \mathrm{E}-03\end{array}$ & -2.06 \\
\hline 4431 & $\begin{array}{l}\text { ENSGALTO0000020539 } \\
\text { 3.07E-03 }\end{array}$ & -3.40 \\
\hline 4432 & $\begin{array}{l}\text { ENSGALTO0000020877 } \\
3.40 \text {-04 }\end{array}$ & -6.60 \\
\hline 4433 & $\begin{array}{l}\text { ENSGALTO0000021064 } \\
2.58 E-04\end{array}$ & -5.10 \\
\hline 4434 & $\begin{array}{l}\text { ENSGALTO0000021128 } \\
7.17 \mathrm{E}-03\end{array}$ & -1.47 \\
\hline 4435 & $\begin{array}{l}\text { ENSGALTO0000021143 } \\
8.34 \text { - } 04\end{array}$ & -4.38 \\
\hline 4436 & $\begin{array}{l}\text { ENSGALTO0000021246 } \\
1.89 \mathrm{E}-03\end{array}$ & -1.73 \\
\hline 4437 & $\begin{array}{l}\text { ENSGALTO0000021306 } \\
1.19 \mathrm{E}-03\end{array}$ & -2.29 \\
\hline 4438 & $\begin{array}{l}\text { ENSGALTO0000021367 } \\
5.55 E-03\end{array}$ & -3.20 \\
\hline 4439 & $\begin{array}{l}\text { ENSGALTO0000021438 } \\
2.79 E-04\end{array}$ & -2.64 \\
\hline 4440 & $\begin{array}{l}\text { ENSGALTO0000021448 } \\
\text { 4.25E-04 }\end{array}$ & -2.20 \\
\hline 4441 & $\begin{array}{l}\text { ENSGALTO0000021457 } \\
4.16 \mathrm{E}-03\end{array}$ & -2.60 \\
\hline 4442 & $\begin{array}{l}\text { ENSGALTO0000021535 } \\
2.08 E-03\end{array}$ & -6.27 \\
\hline 4443 & $\begin{array}{l}\text { ENSGALT00000021541 } \\
1.24 E-03\end{array}$ & -2.11 \\
\hline 4444 & $\begin{array}{l}\text { ENSGALTO0000021576 } \\
1.83 E-03\end{array}$ & -4.18 \\
\hline
\end{tabular}




\begin{tabular}{|c|c|c|}
\hline 4445 & $\begin{array}{l}\text { ENSGALTO0000021577 } \\
3.70 \mathrm{-}-03\end{array}$ & -5.20 \\
\hline 4446 & $\begin{array}{l}\text { ENSGALT00000021605 } \\
2.71 E-04\end{array}$ & -3.52 \\
\hline 4447 & $\begin{array}{l}\text { ENSGALT00000021705 } \\
1.13 E-03\end{array}$ & -6.10 \\
\hline 4448 & $\begin{array}{l}\text { ENSGALTO0000021796 } \\
4.71 \mathrm{E}-03\end{array}$ & -3.99 \\
\hline 4449 & $\begin{array}{l}\text { ENSGALT00000021843 } \\
4.27 E-03\end{array}$ & -3.10 \\
\hline 4450 & $\begin{array}{l}\text { ENSGALTO0000021846 } \\
2.70 \mathrm{0}-02\end{array}$ & -3.30 \\
\hline 4451 & $\begin{array}{l}\text { ENSGALT00000021875 } \\
2.05 E-03\end{array}$ & -4.69 \\
\hline 4452 & $\begin{array}{l}\text { ENSGALTO0000021922 } \\
1.64 E-04\end{array}$ & -2.44 \\
\hline 4453 & $\begin{array}{l}\text { ENSGALTO0000021947 } \\
6.17 \mathrm{E}-04\end{array}$ & -2.11 \\
\hline 4454 & $\begin{array}{l}\text { ENSGALT00000021967 } \\
3.21 E-03\end{array}$ & -1.36 \\
\hline 4455 & $\begin{array}{l}\text { ENSGALTO0000021988 } \\
1.71 \mathrm{E}-03\end{array}$ & -1.28 \\
\hline 4456 & $\begin{array}{l}\text { ENSGALTO0000022068 } \\
1.49 \mathrm{E}-03\end{array}$ & -3.96 \\
\hline 4457 & $\begin{array}{l}\text { ENSGALTO0000022123 } \\
5.60 \mathrm{E}-04\end{array}$ & -4.13 \\
\hline 4458 & $\begin{array}{l}\text { ENSGALT00000022162 } \\
1.63 E-05\end{array}$ & -5.18 \\
\hline 4459 & $\begin{array}{l}\text { ENSGALTO0000022311 } \\
9.18 \mathrm{E}-04\end{array}$ & -3.59 \\
\hline 4460 & $\begin{array}{l}\text { ENSGALTO0000022351 } \\
3.90 \mathrm{0}-04\end{array}$ & -8.12 \\
\hline 4461 & $\begin{array}{l}\text { ENSGALTO0000022378 } \\
8.21 E-04\end{array}$ & -4.18 \\
\hline 4462 & $\begin{array}{l}\text { ENSGALTO0000022437 } \\
1.07 E-03\end{array}$ & -6.23 \\
\hline 4463 & $\begin{array}{l}\text { ENSGALTO0000022454 } \\
1.24 E-02\end{array}$ & -1.82 \\
\hline 4464 & $\begin{array}{l}\text { ENSGALT00000022468 } \\
1.51 E-02\end{array}$ & -1.10 \\
\hline 4465 & $\begin{array}{l}\text { ENSGALTO0000022477 } \\
2.40 \mathrm{E}-03\end{array}$ & -3.35 \\
\hline 4466 & $\begin{array}{l}\text { ENSGALTO0000022479 } \\
2.46 E-04\end{array}$ & -4.21 \\
\hline 4467 & $\begin{array}{l}\text { ENSGALTO0000022497 } \\
\text { 4.42E-04 }\end{array}$ & -4.09 \\
\hline 4468 & $\begin{array}{l}\text { ENSGALTO0000022498 } \\
1.22 E-02\end{array}$ & -1.42 \\
\hline 4469 & $\begin{array}{l}\text { ENSGALT00000022503 } \\
2.79 E-03\end{array}$ & -4.95 \\
\hline 4470 & $\begin{array}{l}\text { ENSGALTO0000022532 } \\
3.24 E-02\end{array}$ & -2.26 \\
\hline 4471 & $\begin{array}{l}\text { ENSGALTO0000022540 } \\
5.23 E-03\end{array}$ & -1.53 \\
\hline 4472 & $\begin{array}{l}\text { ENSGALTO0000022584 } \\
8.67 \mathrm{E}-03\end{array}$ & -1.90 \\
\hline 4473 & $\begin{array}{l}\text { ENSGALTO0000022585 } \\
7.45 E-04\end{array}$ & -4.44 \\
\hline 4474 & $\begin{array}{l}\text { ENSGALT00000022607 } \\
2.43 E-04\end{array}$ & -5.31 \\
\hline 4475 & $\begin{array}{l}\text { ENSGALTO0000022610 } \\
4.98 \mathrm{-}-03\end{array}$ & -1.86 \\
\hline 4476 & $\begin{array}{l}\text { ENSGALTO0000022647 } \\
4.05 E-03\end{array}$ & -2.12 \\
\hline 4477 & $\begin{array}{l}\text { ENSGALTO0000022686 } \\
1.76 E-04\end{array}$ & -3.97 \\
\hline 4478 & $\begin{array}{l}\text { ENSGALTO0000022732 } \\
5.66 \mathrm{E}-04\end{array}$ & -3.22 \\
\hline 4479 & $\begin{array}{l}\text { ENSGALTO0000022745 } \\
6.50 E-04\end{array}$ & -4.56 \\
\hline 4480 & $\begin{array}{l}\text { ENSGALTO0000022768 } \\
5.25 E-04\end{array}$ & -5.37 \\
\hline 4481 & $\begin{array}{l}\text { ENSGALTO0000022773 } \\
2.62 E-03\end{array}$ & -6.26 \\
\hline 4482 & $\begin{array}{l}\text { ENSGALTO0000022794 } \\
2.56 \mathrm{E}-03\end{array}$ & -1.72 \\
\hline 4483 & $\begin{array}{l}\text { ENSGALTO0000022810 } \\
1.24 \mathrm{E}-02\end{array}$ & -1.75 \\
\hline
\end{tabular}

\begin{tabular}{|c|c|c|}
\hline 4484 & $\begin{array}{l}\text { ENSGALT00000022828 } \\
1.46 E-02\end{array}$ & -3.74 \\
\hline 4485 & $\begin{array}{l}\text { ENSGALTO0000022847 } \\
1.64 E-03\end{array}$ & -1.54 \\
\hline 4486 & $\begin{array}{l}\text { ENSGALTO0000022855 } \\
2.42 E-04\end{array}$ & -6.31 \\
\hline 4487 & $\begin{array}{l}\text { ENSGALTO0000022884 } \\
3.21 \mathrm{E}-02\end{array}$ & -1.36 \\
\hline 4488 & $\begin{array}{l}\text { ENSGALTO0000022893 } \\
2.32 E-02\end{array}$ & -1.87 \\
\hline 4489 & $\begin{array}{l}\text { ENSGALTO0000022896 } \\
2.80 E-03\end{array}$ & -2.95 \\
\hline 4490 & $\begin{array}{l}\text { ENSGALTO0000022916 } \\
\text { 4.69E-04 }\end{array}$ & -4.16 \\
\hline 4491 & $\begin{array}{l}\text { ENSGALTO0000023031 } \\
3.98 E-03\end{array}$ & -1.32 \\
\hline 4492 & $\begin{array}{l}\text { ENSGALTO0000023032 } \\
5.42 E-04\end{array}$ & -4.77 \\
\hline 4493 & $\begin{array}{l}\text { ENSGALTO0000023052 } \\
6.50 \mathrm{E}-04\end{array}$ & -2.38 \\
\hline 4494 & $\begin{array}{l}\text { ENSGALTO0000023056 } \\
7.68 E-04\end{array}$ & -4.50 \\
\hline 4495 & $\begin{array}{l}\text { ENSGALT00000023057 } \\
1.71 E-03\end{array}$ & -2.37 \\
\hline 4496 & $\begin{array}{l}\text { ENSGALTO0000023081 } \\
6.53 \mathrm{E}-03\end{array}$ & -1.56 \\
\hline 4497 & $\begin{array}{l}\text { ENSGALT00000023123 } \\
1.97 E-02\end{array}$ & -3.41 \\
\hline 4498 & $\begin{array}{l}\text { ENSGALTO0000023134 } \\
3.27 E-04\end{array}$ & -5.75 \\
\hline 4499 & $\begin{array}{l}\text { ENSGALT00000023152 } \\
1.71 E-04\end{array}$ & -6.28 \\
\hline 4500 & $\begin{array}{l}\text { ENSGALTO0000023153 } \\
6.80 \mathrm{E}-03\end{array}$ & -6.81 \\
\hline 4501 & $\begin{array}{l}\text { ENSGALTO0000023186 } \\
1.18 \mathrm{E}-02\end{array}$ & -2.76 \\
\hline 4502 & $\begin{array}{l}\text { ENSGALTO0000023194 } \\
1.76 E-03\end{array}$ & -4.72 \\
\hline 4503 & $\begin{array}{l}\text { ENSGALTO0000023197 } \\
7.13 E-04\end{array}$ & -2.35 \\
\hline 4504 & $\begin{array}{l}\text { ENSGALTO0000023206 } \\
\text { 3.20E-03 }\end{array}$ & -2.75 \\
\hline 4505 & $\begin{array}{l}\text { ENSGALTO0000023217 } \\
1.28 \mathrm{E}-03\end{array}$ & -4.19 \\
\hline 4506 & $\begin{array}{l}\text { ENSGALT00000023240 } \\
1.38 \mathrm{E}-02\end{array}$ & -1.41 \\
\hline 4507 & $\begin{array}{l}\text { ENSGALTO0000023256 } \\
2.51 E-02\end{array}$ & -1.27 \\
\hline 4508 & $\begin{array}{l}\text { ENSGALTO0000023302 } \\
3.35 E-03\end{array}$ & -3.95 \\
\hline 4509 & $\begin{array}{l}\text { ENSGALTO0000023342 } \\
3.51 E-03\end{array}$ & -3.11 \\
\hline 4510 & $\begin{array}{l}\text { ENSGALTO0000023364 } \\
2.57 E-03\end{array}$ & -4.61 \\
\hline 4511 & $\begin{array}{l}\text { ENSGALTO0000023372 } \\
7.47 E-03\end{array}$ & -3.07 \\
\hline 4512 & $\begin{array}{l}\text { ENSGALTO0000023374 } \\
4.00 E-03\end{array}$ & -1.98 \\
\hline 4513 & $\begin{array}{l}\text { ENSGALTO0000023376 } \\
3.35 E-03\end{array}$ & -1.69 \\
\hline 4514 & $\begin{array}{l}\text { ENSGALTO0000023425 } \\
1.88 \mathrm{E}-04\end{array}$ & -7.89 \\
\hline 4515 & $\begin{array}{l}\text { ENSGALTO0000023436 } \\
6.83 E-05\end{array}$ & -5.52 \\
\hline 4516 & $\begin{array}{l}\text { ENSGALT00000023458 } \\
\text { 5.62E-03 }\end{array}$ & -2.38 \\
\hline 4517 & $\begin{array}{l}\text { ENSGALTO0000023480 } \\
3.73 E-04\end{array}$ & -4.32 \\
\hline 4518 & $\begin{array}{l}\text { ENSGALTO0000023489 } \\
2.71 \mathrm{E}-03\end{array}$ & -1.64 \\
\hline 4519 & $\begin{array}{l}\text { ENSGALTO0000023514 } \\
8.15 E-05\end{array}$ & -6.09 \\
\hline 4520 & $\begin{array}{l}\text { ENSGALTO0000023554 } \\
1.02 E-03\end{array}$ & -5.05 \\
\hline 4521 & $\begin{array}{l}\text { ENSGALT00000023634 } \\
4.81 E-03\end{array}$ & -2.15 \\
\hline 4522 & $\begin{array}{l}\text { ENSGALTO0000023636 } \\
\text { 6.65E-04 }\end{array}$ & -4.34 \\
\hline
\end{tabular}

\begin{tabular}{|c|c|c|}
\hline 4523 & $\begin{array}{l}\text { ENSGALTO0000023640 } \\
1.31 E-03\end{array}$ & -2.02 \\
\hline 4524 & $\begin{array}{l}\text { ENSGALT00000023745 } \\
2.93 E-02\end{array}$ & -1.34 \\
\hline 4525 & $\begin{array}{l}\text { ENSGALTO0000023839 } \\
1.92 E-03\end{array}$ & -3.51 \\
\hline 4526 & $\begin{array}{l}\text { ENSGALTO0000023847 } \\
5.29 \mathrm{E}-05\end{array}$ & -5.62 \\
\hline 4527 & $\begin{array}{l}\text { ENSGALTO0000023851 } \\
4.56 \mathrm{E}-03\end{array}$ & -1.26 \\
\hline 4528 & $\begin{array}{l}\text { ENSGALT00000023876 } \\
1.32 E-02\end{array}$ & -3.07 \\
\hline 4529 & $\begin{array}{l}\text { ENSGALTO0000023898 } \\
3.40 E-05\end{array}$ & -4.77 \\
\hline 4530 & $\begin{array}{l}\text { ENSGALTO0000023923 } \\
6.42 E-04\end{array}$ & -3.46 \\
\hline 4531 & $\begin{array}{l}\text { ENSGALTO0000023925 } \\
4.52 E-04\end{array}$ & -5.03 \\
\hline 4532 & $\begin{array}{l}\text { ENSGALTO0000023926 } \\
1.06 E-03\end{array}$ & -3.27 \\
\hline 4533 & $\begin{array}{l}\text { ENSGALT00000024008 } \\
\text { 3.75E-02 }\end{array}$ & -1.52 \\
\hline 4534 & $\begin{array}{l}\text { ENSGALTO0000024014 } \\
9.87 \mathrm{E}-04\end{array}$ & -3.12 \\
\hline 4535 & $\begin{array}{l}\text { ENSGALTO0000024023 } \\
8.94 \mathrm{E}-04\end{array}$ & -4.86 \\
\hline 4536 & $\begin{array}{l}\text { ENSGALT00000024062 } \\
3.42 E-04\end{array}$ & -3.51 \\
\hline 4537 & $\begin{array}{l}\text { ENSGALTO0000024072 } \\
2.20 \mathrm{E}-03\end{array}$ & -2.69 \\
\hline 4538 & $\begin{array}{l}\text { ENSGALT00000024101 } \\
2.20 \mathrm{E}-02\end{array}$ & -1.28 \\
\hline 4539 & $\begin{array}{l}\text { ENSGALT00000024368 } \\
5.94 \mathrm{E}-05\end{array}$ & -2.78 \\
\hline 4540 & $\begin{array}{l}\text { ENSGALTO0000024483 } \\
5.06 E-05\end{array}$ & -3.71 \\
\hline 4541 & $\begin{array}{l}\text { ENSGALTO0000024577 } \\
1.26 E-04\end{array}$ & -6.61 \\
\hline 4542 & $\begin{array}{l}\text { ENSGALTO0000024580 } \\
3.71 E-04\end{array}$ & -3.20 \\
\hline 4543 & $\begin{array}{l}\text { ENSGALTO0000024606 } \\
1.57 E-03\end{array}$ & -1.24 \\
\hline 4544 & $\begin{array}{l}\text { ENSGALTO0000024666 } \\
2.41 E-03\end{array}$ & -7.94 \\
\hline 4545 & $\begin{array}{l}\text { ENSGALTO0000024733 } \\
3.80 E-03\end{array}$ & -5.26 \\
\hline 4546 & $\begin{array}{l}\text { ENSGALTO0000024752 } \\
5.27 \mathrm{E}-04\end{array}$ & -1.68 \\
\hline 4547 & $\begin{array}{l}\text { ENSGALTO0000024828 } \\
6.40 \mathrm{E}-04\end{array}$ & -5.28 \\
\hline 4548 & $\begin{array}{l}\text { ENSGALTO0000024885 } \\
1.00 E-02\end{array}$ & -1.10 \\
\hline 4549 & $\begin{array}{l}\text { ENSGALTO0000024895 } \\
5.53 \mathrm{E}-04\end{array}$ & -5.16 \\
\hline 4550 & $\begin{array}{l}\text { ENSGALTO0000024919 } \\
7.81 E-02\end{array}$ & -1.34 \\
\hline 4551 & $\begin{array}{l}\text { ENSGALT00000024965 } \\
2.19 E-02\end{array}$ & -3.03 \\
\hline 4552 & $\begin{array}{l}\text { ENSGALTO0000024992 } \\
2.08 E-01\end{array}$ & -1.03 \\
\hline 4553 & $\begin{array}{l}\text { ENSGALTO0000025043 } \\
1.17 E-04\end{array}$ & -8.57 \\
\hline 4554 & $\begin{array}{l}\text { ENSGALTO0000025166 } \\
2.88 E-02\end{array}$ & -1.59 \\
\hline 4555 & $\begin{array}{l}\text { ENSGALTO0000025170 } \\
1.21 E-02\end{array}$ & -1.87 \\
\hline 4556 & $\begin{array}{l}\text { ENSGALTO0000025192 } \\
1.21 E-04\end{array}$ & -4.99 \\
\hline 4557 & $\begin{array}{l}\text { ENSGALTO0000025254 } \\
5.32 E-05\end{array}$ & -5.23 \\
\hline 4558 & $\begin{array}{l}\text { ENSGALT00000025344 } \\
3.86 \mathrm{E}-03\end{array}$ & -3.27 \\
\hline 4559 & $\begin{array}{l}\text { ENSGALTO0000025396 } \\
4.09 \mathrm{E}-02\end{array}$ & -1.98 \\
\hline 4560 & $\begin{array}{l}\text { ENSGALTO0000025439 } \\
1.50 E-03\end{array}$ & -3.00 \\
\hline 4561 & $\begin{array}{l}\text { ENSGALTO0000025452 } \\
1.24 E-04\end{array}$ & -6.68 \\
\hline
\end{tabular}




\begin{tabular}{|c|c|c|}
\hline 4562 & $\begin{array}{l}\text { ENSGALTO0000025550 } \\
1.64 E-03\end{array}$ & -5.25 \\
\hline 4563 & $\begin{array}{l}\text { ENSGALTO0000025716 } \\
7.34 \mathrm{E}-03\end{array}$ & -2.42 \\
\hline 4564 & $\begin{array}{l}\text { ENSGALTO0000025774 } \\
7.88 \mathrm{E}-05\end{array}$ & -8.61 \\
\hline 4565 & $\begin{array}{l}\text { ENSGALTO0000025786 } \\
\text { 5.49E-06 }\end{array}$ & -4.67 \\
\hline 4566 & $\begin{array}{l}\text { ENSGALTO0000025796 } \\
2.58 E-05\end{array}$ & -6.93 \\
\hline 4567 & $\begin{array}{l}\text { ENSGALT00000025800 } \\
1.65 E-03\end{array}$ & -4.26 \\
\hline 4568 & $\begin{array}{l}\text { ENSGALTO0000025828 } \\
7.15 E-04\end{array}$ & -4.63 \\
\hline 4569 & $\begin{array}{l}\text { ENSGALTO0000025829 } \\
5.85 E-03\end{array}$ & -2.60 \\
\hline 4570 & $\begin{array}{l}\text { ENSGALT00000025838 } \\
3.57 E-05\end{array}$ & -1.83 \\
\hline 4571 & $\begin{array}{l}\text { ENSGALTO0000025840 } \\
2.78 E-05\end{array}$ & -5.48 \\
\hline 4572 & $\begin{array}{l}\text { ENSGALTO0000025842 } \\
8.55 E-04\end{array}$ & -5.93 \\
\hline 4573 & $\begin{array}{l}\text { ENSGALTO0000025850 } \\
1.21 \mathrm{E}-02\end{array}$ & -2.11 \\
\hline 4574 & $\begin{array}{l}\text { ENSGALTO0000025883 } \\
9.55 E-06\end{array}$ & -7.93 \\
\hline 4575 & $\begin{array}{l}\text { ENSGALTO0000025947 } \\
4.46 E-04\end{array}$ & -3.47 \\
\hline 4576 & $\begin{array}{l}\text { ENSGALTO0000025976 } \\
3.61 E-04\end{array}$ & -6.54 \\
\hline 4577 & $\begin{array}{l}\text { ENSGALT00000025991 } \\
7.69 \mathrm{E}-05\end{array}$ & -2.44 \\
\hline 4578 & $\begin{array}{l}\text { ENSGALTO0000026017 } \\
1.12 E-02\end{array}$ & -1.59 \\
\hline 4579 & $\begin{array}{l}\text { ENSGALTO0000026039 } \\
5.76 E-03\end{array}$ & -3.63 \\
\hline 4580 & $\begin{array}{l}\text { ENSGALT00000026078 } \\
1.06 E-03\end{array}$ & -5.29 \\
\hline 4581 & $\begin{array}{l}\text { ENSGALTO0000026119 } \\
5.47 E-04\end{array}$ & -3.90 \\
\hline 4582 & $\begin{array}{l}\text { ENSGALT00000026211 } \\
3.63 \text { E-04 }\end{array}$ & -2.54 \\
\hline 4583 & $\begin{array}{l}\text { ENSGALTO0000026231 } \\
2.36 \mathrm{E}-03\end{array}$ & -2.38 \\
\hline 4584 & $\begin{array}{l}\text { ENSGALTO0000026251 } \\
1.57 E-03\end{array}$ & -5.86 \\
\hline 4585 & $\begin{array}{l}\text { ENSGALT00000026267 } \\
2.89 \mathrm{E}-03\end{array}$ & -3.38 \\
\hline 4586 & $\begin{array}{l}\text { ENSGALTO0000026454 } \\
1.38 E-04\end{array}$ & -3.34 \\
\hline 4587 & $\begin{array}{l}\text { ENSGALT00000026483 } \\
7.76 E-04\end{array}$ & -4.82 \\
\hline 4588 & $\begin{array}{l}\text { ENSGALTO0000026506 } \\
2.81 E-03\end{array}$ & -1.35 \\
\hline 4589 & $\begin{array}{l}\text { ENSGALTO0000026609 } \\
6.72 E-03\end{array}$ & -4.37 \\
\hline 4590 & $\begin{array}{l}\text { ENSGALTO0000026662 } \\
9.04 E-04\end{array}$ & -6.54 \\
\hline 4591 & $\begin{array}{l}\text { ENSGALTO0000026790 } \\
\text { 3.34E-04 }\end{array}$ & -3.37 \\
\hline 4592 & $\begin{array}{l}\text { ENSGALT00000026810 } \\
3.18 \mathrm{E}-02\end{array}$ & -1.75 \\
\hline 4593 & $\begin{array}{l}\text { ENSGALT00000026862 } \\
2.08 E-04\end{array}$ & -5.58 \\
\hline 4594 & $\begin{array}{l}\text { ENSGALTO0000026863 } \\
2.84 E-04\end{array}$ & -3.36 \\
\hline 4595 & $\begin{array}{l}\text { ENSGALT00000026884 } \\
7.74 E-03\end{array}$ & -2.31 \\
\hline 4596 & $\begin{array}{l}\text { ENSGALTO0000026953 } \\
\text { 3.78E-03 }\end{array}$ & -2.13 \\
\hline 4597 & $\begin{array}{l}\text { ENSGALT00000026965 } \\
5.25 E-03\end{array}$ & -3.48 \\
\hline 4598 & $\begin{array}{l}\text { ENSGALT00000027007 } \\
7.05 E-04\end{array}$ & -1.86 \\
\hline 4599 & $\begin{array}{l}\text { ENSGALTO0000027008 } \\
5.30 E-04\end{array}$ & -2.78 \\
\hline 4600 & $\begin{array}{l}\text { ENSGALTO0000027044 } \\
5.64 E-05\end{array}$ & -7.32 \\
\hline
\end{tabular}

\begin{tabular}{|c|c|c|}
\hline 4601 & $\begin{array}{l}\text { ENSGALTO0000027045 } \\
4.25 E-03\end{array}$ & -3.24 \\
\hline 4602 & $\begin{array}{l}\text { ENSGALTO0000027054 } \\
\text { 3.99E-03 }\end{array}$ & -2.67 \\
\hline 4603 & $\begin{array}{l}\text { ENSGALTO0000027092 } \\
2.87 E-03\end{array}$ & -1.40 \\
\hline 4604 & $\begin{array}{l}\text { ENSGALTO0000027144 } \\
4.13 E-02\end{array}$ & -1.30 \\
\hline 4605 & $\begin{array}{l}\text { ENSGALTO0000027361 } \\
1.45 E-02\end{array}$ & -2.67 \\
\hline 4606 & $\begin{array}{l}\text { ENSGALTO0000027363 } \\
1.02 E-02\end{array}$ & -1.71 \\
\hline 4607 & $\begin{array}{l}\text { ENSGALTO0000027411 } \\
1.62 E-02\end{array}$ & -1.52 \\
\hline 4608 & $\begin{array}{l}\text { ENSGALTO0000027451 } \\
2.61 E-02\end{array}$ & -2.95 \\
\hline 4609 & $\begin{array}{l}\text { ENSGALT00000027467 } \\
3.16 \mathrm{E}-04\end{array}$ & -7.10 \\
\hline 4610 & $\begin{array}{l}\text { ENSGALTO0000027493 } \\
5.88 \mathrm{E}-03\end{array}$ & -4.47 \\
\hline 4611 & $\begin{array}{l}\text { ENSGALTO0000027506 } \\
8.05 E-04\end{array}$ & -1.50 \\
\hline 4612 & $\begin{array}{l}\text { ENSGALTO0000027544 } \\
1.68 E-04\end{array}$ & -5.42 \\
\hline 4613 & $\begin{array}{l}\text { ENSGALTO0000027601 } \\
1.30 E-03\end{array}$ & -2.17 \\
\hline 4614 & $\begin{array}{l}\text { ENSGALTO0000027694 } \\
1.76 E-03\end{array}$ & -3.65 \\
\hline 4615 & $\begin{array}{l}\text { ENSGALTO0000027711 } \\
2.97 E-04\end{array}$ & -3.40 \\
\hline 4616 & $\begin{array}{l}\text { ENSGALTO0000027794 } \\
5.30 \mathrm{E}-03\end{array}$ & -1.56 \\
\hline 4617 & $\begin{array}{l}\text { ENSGALTO0000027840 } \\
6.10 \mathrm{E}-04\end{array}$ & -8.97 \\
\hline 4618 & $\begin{array}{l}\text { ENSGALTO0000027882 } \\
1.15 E-02\end{array}$ & -2.98 \\
\hline 4619 & $\begin{array}{l}\text { ENSGALTO0000027884 } \\
1.44 E-02\end{array}$ & -1.28 \\
\hline 4620 & $\begin{array}{l}\text { ENSGALTO0000027939 } \\
1.37 E-03\end{array}$ & -2.53 \\
\hline 4621 & $\begin{array}{l}\text { ENSGALTO0000027945 } \\
1.26 \text {-04 }\end{array}$ & -5.83 \\
\hline 4622 & $\begin{array}{l}\text { ENSGALTO0000027948 } \\
5.92 E-04\end{array}$ & -2.54 \\
\hline 4623 & $\begin{array}{l}\text { ENSGALT00000027971 } \\
\text { 5.63E-04 }\end{array}$ & -4.63 \\
\hline 4624 & $\begin{array}{l}\text { ENSGALTO0000028049 } \\
4.21 \mathrm{E}-03\end{array}$ & -2.93 \\
\hline 4625 & $\begin{array}{l}\text { ENSGALTO0000028050 } \\
9.64 E-04\end{array}$ & -5.36 \\
\hline 4626 & $\begin{array}{l}\text { ENSGALTO0000028227 } \\
1.76 E-04\end{array}$ & -4.93 \\
\hline 4627 & $\begin{array}{l}\text { ENSGALTO0000028305 } \\
1.03 E-04\end{array}$ & -6.42 \\
\hline 4628 & $\begin{array}{l}\text { ENSGALTO0000028395 } \\
4.48 \mathrm{E}-03\end{array}$ & -1.82 \\
\hline 4629 & $\begin{array}{l}\text { ENSGALTO0000029228 } \\
4.98 \mathrm{E}-04\end{array}$ & -3.02 \\
\hline 4630 & $\begin{array}{l}\text { ENSGALTO0000029296 } \\
2.31 E-03\end{array}$ & -4.52 \\
\hline 4631 & $\begin{array}{l}\text { ENSGALTO0000029359 } \\
5.13 \mathrm{E}-05\end{array}$ & -4.57 \\
\hline 4632 & $\begin{array}{l}\text { ENSGALTO0000029361 } \\
1.16 E-04\end{array}$ & -5.05 \\
\hline 4633 & $\begin{array}{l}\text { ENSGALTO0000029382 } \\
2.29 \mathrm{E}-04\end{array}$ & -2.43 \\
\hline 4634 & $\begin{array}{l}\text { ENSGALTO0000029394 } \\
2.98 \mathrm{E}-04\end{array}$ & -4.46 \\
\hline 4635 & $\begin{array}{l}\text { ENSGALTO0000029529 } \\
8.86 E-04\end{array}$ & -3.68 \\
\hline 4636 & $\begin{array}{l}\text { ENSGALTO0000029552 } \\
1.43 E-02\end{array}$ & -2.07 \\
\hline 4637 & $\begin{array}{l}\text { ENSGALTO0000029577 } \\
1.24 E-02\end{array}$ & -1.72 \\
\hline 4638 & $\begin{array}{l}\text { ENSGALTO0000029613 } \\
4.16 E-03\end{array}$ & -1.37 \\
\hline 4639 & $\begin{array}{l}\text { ENSGALTO0000029629 } \\
1.87 E-03\end{array}$ & -4.35 \\
\hline
\end{tabular}

\begin{tabular}{|c|c|c|}
\hline 4640 & $\begin{array}{l}\text { ENSGALTO0000029647 } \\
2.76 E-04\end{array}$ & -2.89 \\
\hline 4641 & $\begin{array}{l}\text { ENSGALTO0000029695 } \\
2.14 E-02\end{array}$ & -1.88 \\
\hline 4642 & $\begin{array}{l}\text { ENSGALTO0000029709 } \\
1.32 E-03\end{array}$ & -1.48 \\
\hline 4643 & $\begin{array}{l}\text { ENSGALTO0000029733 } \\
3.58 E-04\end{array}$ & -4.50 \\
\hline 4644 & $\begin{array}{l}\text { ENSGALTO0000029737 } \\
1.20 \mathrm{E}-02\end{array}$ & -2.25 \\
\hline 4645 & $\begin{array}{l}\text { ENSGALTO0000029744 } \\
1.87 E-02\end{array}$ & -1.89 \\
\hline 4646 & $\begin{array}{l}\text { ENSGALTO0000029763 } \\
3.64 E-03\end{array}$ & -2.28 \\
\hline 4647 & $\begin{array}{l}\text { ENSGALTO0000029798 } \\
6.60 \mathrm{E}-04\end{array}$ & -3.49 \\
\hline 4648 & $\begin{array}{l}\text { ENSGALTO0000029816 } \\
2.13 \mathrm{E}-03\end{array}$ & -4.07 \\
\hline 4649 & $\begin{array}{l}\text { ENSGALTO0000029869 } \\
1.27 E-02\end{array}$ & -2.21 \\
\hline 4650 & $\begin{array}{l}\text { ENSGALT00000029943 } \\
1.08 \mathrm{E}-03\end{array}$ & -4.37 \\
\hline 4651 & $\begin{array}{l}\text { ENSGALT00000030060 } \\
4.30 \mathrm{E}-05\end{array}$ & -7.31 \\
\hline 4652 & $\begin{array}{l}\text { ENSGALTO0000030134 } \\
1.59 \mathrm{E}-03\end{array}$ & -3.70 \\
\hline 4653 & $\begin{array}{l}\text { ENSGALT00000030281 } \\
1.22 \text { E-03 }\end{array}$ & -5.65 \\
\hline 4654 & $\begin{array}{l}\text { ENSGALTO0000030391 } \\
1.80 \mathrm{E}-03\end{array}$ & -1.98 \\
\hline 4655 & $\begin{array}{l}\text { ENSGALTO0000030462 } \\
1.88 E-04\end{array}$ & -6.06 \\
\hline 4656 & $\begin{array}{l}\text { ENSGALTO0000030545 } \\
4.53 \mathrm{E}-03\end{array}$ & -2.06 \\
\hline 4657 & $\begin{array}{l}\text { ENSGALTO0000030615 } \\
3.30 E-02\end{array}$ & -1.13 \\
\hline 4658 & $\begin{array}{l}\text { ENSGALTO0000030645 } \\
1.50 \mathrm{E}-03\end{array}$ & -3.90 \\
\hline 4659 & $\begin{array}{l}\text { ENSGALTO0000030669 } \\
1.16 E-02\end{array}$ & -2.44 \\
\hline 4660 & $\begin{array}{l}\text { ENSGALTO0000030816 } \\
2.80 E-04\end{array}$ & -7.75 \\
\hline 4661 & $\begin{array}{l}\text { ENSGALTO0000030823 } \\
1.03 E-03\end{array}$ & -5.87 \\
\hline 4662 & $\begin{array}{l}\text { ENSGALTO0000030914 } \\
1.69 \mathrm{E}-03\end{array}$ & -1.92 \\
\hline 4663 & $\begin{array}{l}\text { ENSGALTO0000030948 } \\
2.88 \mathrm{E}-04\end{array}$ & -4.11 \\
\hline 4664 & $\begin{array}{l}\text { ENSGALT00000030959 } \\
3.72 E-02\end{array}$ & -1.80 \\
\hline 4665 & $\begin{array}{l}\text { ENSGALTO0000030981 } \\
1.18 \mathrm{E}-02\end{array}$ & -2.63 \\
\hline 4666 & $\begin{array}{l}\text { ENSGALTO0000031123 } \\
4.88 \mathrm{E}-03\end{array}$ & -1.63 \\
\hline 4667 & $\begin{array}{l}\text { ENSGALTO0000031143 } \\
4.54 E-04\end{array}$ & -5.09 \\
\hline 4668 & $\begin{array}{l}\text { ENSGALT00000031165 } \\
8.14 \mathrm{E}-03\end{array}$ & -2.72 \\
\hline 4669 & $\begin{array}{l}\text { ENSGALTO0000031192 } \\
8.57 E-04\end{array}$ & -7.54 \\
\hline 4670 & $\begin{array}{l}\text { ENSGALTO0000031350 } \\
8.78 E-04\end{array}$ & -4.50 \\
\hline 4671 & $\begin{array}{l}\text { ENSGALTO0000031351 } \\
5.61 E-03\end{array}$ & -3.08 \\
\hline 4672 & $\begin{array}{l}\text { ENSGALTO0000031457 } \\
9.25 E-03\end{array}$ & -3.72 \\
\hline 4673 & $\begin{array}{l}\text { ENSGALTO0000031469 } \\
1.67 E-02\end{array}$ & -1.64 \\
\hline 4674 & $\begin{array}{l}\text { ENSGALTO0000031486 } \\
2.65 E-02\end{array}$ & -1.12 \\
\hline 4675 & $\begin{array}{l}\text { ENSGALTO0000031552 } \\
1.22 E-02\end{array}$ & -1.18 \\
\hline 4676 & $\begin{array}{l}\text { ENSGALTO0000031553 } \\
2.28 \mathrm{E}-02\end{array}$ & -1.55 \\
\hline 4677 & $\begin{array}{l}\text { ENSGALT00000031708 } \\
6.07 E-04\end{array}$ & -5.43 \\
\hline 4678 & $\begin{array}{l}\text { ENSGALTO0000031915 } \\
1.26 E-03\end{array}$ & -4.29 \\
\hline
\end{tabular}




\begin{tabular}{|c|c|c|}
\hline 4679 & $\begin{array}{l}\text { ENSGALT00000031916 } \\
1.34 \text {-04 }\end{array}$ & -7.65 \\
\hline 4680 & $\begin{array}{l}\text { ENSGALTO0000031940 } \\
2.54 E-02\end{array}$ & -1.55 \\
\hline 4681 & $\begin{array}{l}\text { ENSGALT00000031955 } \\
\text { 3.92E-02 }\end{array}$ & -1.76 \\
\hline 4682 & $\begin{array}{l}\text { ENSGALTO0000031956 } \\
3.24 E-03\end{array}$ & -6.40 \\
\hline 4683 & $\begin{array}{l}\text { ENSGALTO0000031996 } \\
1.79 \mathrm{-}-03\end{array}$ & -4.53 \\
\hline 4684 & $\begin{array}{l}\text { ENSGALT00000032023 } \\
6.85 E-04\end{array}$ & -6.85 \\
\hline 4685 & $\begin{array}{l}\text { ENSGALT00000032091 } \\
1.99 E-04\end{array}$ & -5.23 \\
\hline 4686 & $\begin{array}{l}\text { ENSGALT00000032098 } \\
1.63 E-02\end{array}$ & -1.83 \\
\hline 4687 & $\begin{array}{l}\text { ENSGALTO0000032239 } \\
4.00 \mathrm{0}-03\end{array}$ & -4.65 \\
\hline 4688 & $\begin{array}{l}\text { ENSGALT00000032282 } \\
1.75 E-02\end{array}$ & -4.34 \\
\hline 4689 & $\begin{array}{l}\text { ENSGALTO0000032288 } \\
1.47 \mathrm{E}-04\end{array}$ & -5.40 \\
\hline 4690 & $\begin{array}{l}\text { ENSGALTO0000032339 } \\
3.62 E-04\end{array}$ & -4.72 \\
\hline 4691 & $\begin{array}{l}\text { ENSGALTO0000032350 } \\
7.15 E-02\end{array}$ & -1.48 \\
\hline 4692 & $\begin{array}{l}\text { ENSGALT00000032365 } \\
6.47 E-03\end{array}$ & -4.23 \\
\hline 4693 & $\begin{array}{l}\text { ENSGALT00000032438 } \\
1.07 E-02\end{array}$ & -5.33 \\
\hline 4694 & $\begin{array}{l}\text { ENSGALTO0000032505 } \\
2.48 E-03\end{array}$ & -2.36 \\
\hline 4695 & $\begin{array}{l}\text { ENSGALT00000032561 } \\
2.27 E-03\end{array}$ & -4.42 \\
\hline 4696 & $\begin{array}{l}\text { ENSGALTO0000032563 } \\
2.70 E-03\end{array}$ & -4.36 \\
\hline 4697 & $\begin{array}{l}\text { ENSGALTO0000032826 } \\
4.36 E-04\end{array}$ & -3.61 \\
\hline 4698 & $\begin{array}{l}\text { ENSGALTO0000032827 } \\
4.55 E-03\end{array}$ & -3.25 \\
\hline 4699 & $\begin{array}{l}\text { ENSGALTO0000032830 } \\
7.29 E-04\end{array}$ & -5.27 \\
\hline 4700 & $\begin{array}{l}\text { ENSGALT00000032887 } \\
\text { 3.53E-02 }\end{array}$ & -2.66 \\
\hline 4701 & $\begin{array}{l}\text { ENSGALTO0000032906 } \\
2.72 E-02\end{array}$ & -2.50 \\
\hline 4702 & $\begin{array}{l}\text { ENSGALT00000033073 } \\
2.46 E-03\end{array}$ & -6.02 \\
\hline 4703 & $\begin{array}{l}\text { ENSGALTO0000033142 } \\
7.65 E-04\end{array}$ & -2.60 \\
\hline 4704 & $\begin{array}{l}\text { ENSGALTO0000033157 } \\
9.14 \mathrm{-}-04\end{array}$ & -1.40 \\
\hline 4705 & $\begin{array}{l}\text { ENSGALT00000033253 } \\
1.70 E-02\end{array}$ & -1.39 \\
\hline 4706 & $\begin{array}{l}\text { ENSGALTO0000033325 } \\
1.06 E-03\end{array}$ & -3.13 \\
\hline 4707 & $\begin{array}{l}\text { ENSGALTO0000033479 } \\
4.10 E-04\end{array}$ & -8.98 \\
\hline 4708 & $\begin{array}{l}\text { ENSGALT00000033571 } \\
1.03 E-02\end{array}$ & -1.68 \\
\hline 4709 & $\begin{array}{l}\text { ENSGALTO0000033572 } \\
2.97 E-04\end{array}$ & -5.13 \\
\hline 4710 & $\begin{array}{l}\text { ENSGALTO0000033583 } \\
2.54 \mathrm{E}-02\end{array}$ & -1.47 \\
\hline 4711 & $\begin{array}{l}\text { ENSGALTO0000033596 } \\
1.07 E-02\end{array}$ & -3.01 \\
\hline 4712 & $\begin{array}{l}\text { ENSGALTO0000033705 } \\
1.28 E-03\end{array}$ & -2.79 \\
\hline 4713 & $\begin{array}{l}\text { ENSGALTO0000033787 } \\
9.90 \mathrm{E}-05\end{array}$ & -8.20 \\
\hline 4714 & $\begin{array}{l}\text { ENSGALTO0000033804 } \\
2.35 E-04\end{array}$ & -5.63 \\
\hline 4715 & $\begin{array}{l}\text { ENSGALTO0000033814 } \\
9.75 E-04\end{array}$ & -3.59 \\
\hline 4716 & $\begin{array}{l}\text { ENSGALTO0000033820 } \\
1.23 E-02\end{array}$ & -3.19 \\
\hline 4717 & $\begin{array}{l}\text { ENSGALTO0000033882 } \\
2.10 \mathrm{E}-03\end{array}$ & -6.96 \\
\hline
\end{tabular}

\begin{tabular}{|c|c|c|}
\hline 4718 & $\begin{array}{l}\text { ENSGALTO0000033912 } \\
1.42 E-02\end{array}$ & -5.02 \\
\hline 4719 & $\begin{array}{l}\text { ENSGALTO0000034014 } \\
2.05 E-04\end{array}$ & -5.25 \\
\hline 4720 & $\begin{array}{l}\text { ENSGALTO0000034019 } \\
\text { 4.64E-05 }\end{array}$ & -5.45 \\
\hline 4721 & $\begin{array}{l}\text { ENSGALTO0000034023 } \\
1.11 \mathrm{E}-01\end{array}$ & -2.08 \\
\hline 4722 & $\begin{array}{l}\text { ENSGALT00000034037 } \\
2.45 E-01\end{array}$ & -1.28 \\
\hline 4723 & $\begin{array}{l}\text { ENSGALTO0000034065 } \\
8.75 E-05\end{array}$ & -6.28 \\
\hline 4724 & $\begin{array}{l}\text { ENSGALT00000034107 } \\
2.16 E-04\end{array}$ & -6.00 \\
\hline 4725 & $\begin{array}{l}\text { ENSGALTO0000034148 } \\
5.81 E-06\end{array}$ & -6.53 \\
\hline 4726 & $\begin{array}{l}\text { ENSGALTO0000034280 } \\
2.10 \mathrm{E}-03\end{array}$ & -2.20 \\
\hline 4727 & $\begin{array}{l}\text { ENSGALTO0000034311 } \\
4.63 E-04\end{array}$ & -6.27 \\
\hline 4728 & $\begin{array}{l}\text { ENSGALTO0000034312 } \\
1.32 E-02\end{array}$ & -3.74 \\
\hline 4729 & $\begin{array}{l}\text { ENSGALTO0000034313 } \\
1.38 \mathrm{E}-02\end{array}$ & -4.08 \\
\hline 4730 & $\begin{array}{l}\text { ENSGALTO0000034377 } \\
2.66 \mathrm{E}-03\end{array}$ & -5.35 \\
\hline 4731 & $\begin{array}{l}\text { ENSGALT00000034384 } \\
4.03 E-03\end{array}$ & -2.61 \\
\hline 4732 & $\begin{array}{l}\text { ENSGALTO0000034401 } \\
2.53 \mathrm{E}-03\end{array}$ & -2.01 \\
\hline 4733 & $\begin{array}{l}\text { ENSGALTO0000034423 } \\
4.11 E-03\end{array}$ & -2.51 \\
\hline 4734 & $\begin{array}{l}\text { ENSGALTO0000034442 } \\
6.11 \mathrm{E}-05\end{array}$ & -7.04 \\
\hline 4735 & $\begin{array}{l}\text { ENSGALTO0000034473 } \\
2.23 E-03\end{array}$ & -2.07 \\
\hline 4736 & $\begin{array}{l}\text { ENSGALTO0000034549 } \\
2.28 E-02\end{array}$ & -1.34 \\
\hline 4737 & $\begin{array}{l}\text { ENSGALTO0000034557 } \\
3.47 \mathrm{E}-03\end{array}$ & -2.81 \\
\hline 4738 & $\begin{array}{l}\text { ENSGALT00000034587 } \\
\text { 3.38E-04 }\end{array}$ & -4.41 \\
\hline 4739 & $\begin{array}{l}\text { ENSGALTO0000034819 } \\
1.84 \mathrm{E}-04\end{array}$ & -5.95 \\
\hline 4740 & $\begin{array}{l}\text { ENSGALT00000034881 } \\
1.47 E-03\end{array}$ & -4.77 \\
\hline 4741 & $\begin{array}{l}\text { ENSGALTO0000034912 } \\
2.13 \mathrm{E}-03\end{array}$ & -1.90 \\
\hline 4742 & $\begin{array}{l}\text { ENSGALT00000035098 } \\
3.05 E-02\end{array}$ & -1.64 \\
\hline 4743 & $\begin{array}{l}\text { ENSGALTO0000035294 } \\
1.44 E-02\end{array}$ & -2.52 \\
\hline 4744 & $\begin{array}{l}\text { ENSGALTO0000035353 } \\
1.05 E-02\end{array}$ & -1.55 \\
\hline 4745 & $\begin{array}{l}\text { ENSGALTO0000035355 } \\
4.86 E-03\end{array}$ & -4.74 \\
\hline 4746 & $\begin{array}{l}\text { ENSGALTO0000035364 } \\
4.64 E-04\end{array}$ & -7.49 \\
\hline 4747 & $\begin{array}{l}\text { ENSGALTO0000035369 } \\
4.46 E-03\end{array}$ & -4.60 \\
\hline 4748 & $\begin{array}{l}\text { ENSGALTO0000035375 } \\
1.05 E-02\end{array}$ & -2.40 \\
\hline 4749 & $\begin{array}{l}\text { ENSGALTO0000035378 } \\
1.03 E-02\end{array}$ & -2.70 \\
\hline 4750 & $\begin{array}{l}\text { ENSGALTO0000035404 } \\
1.54 E-03\end{array}$ & -3.91 \\
\hline 4751 & $\begin{array}{l}\text { ENSGALTO0000035419 } \\
5.27 E-03\end{array}$ & -1.16 \\
\hline 4752 & $\begin{array}{l}\text { ENSGALTO0000035429 } \\
2.88 \mathrm{E}-03\end{array}$ & -2.83 \\
\hline 4753 & $\begin{array}{l}\text { ENSGALTO0000035431 } \\
7.37 \mathrm{E}-05\end{array}$ & -4.35 \\
\hline 4754 & $\begin{array}{l}\text { ENSGALTO0000035433 } \\
3.92 E-03\end{array}$ & -3.70 \\
\hline 4755 & $\begin{array}{l}\text { ENSGALT00000035434 } \\
1.59 \mathrm{E}-02\end{array}$ & -4.12 \\
\hline 4756 & $\begin{array}{l}\text { ENSGALTO0000035443 } \\
8.87 E-04\end{array}$ & -3.43 \\
\hline
\end{tabular}

\begin{tabular}{|c|c|c|}
\hline 4757 & $\begin{array}{l}\text { ENSGALTO0000035493 } \\
1.25 E-02\end{array}$ & -2.65 \\
\hline 4758 & $\begin{array}{l}\text { ENSGALTO0000035572 } \\
1.72 E-02\end{array}$ & -1.68 \\
\hline 4759 & $\begin{array}{l}\text { ENSGALTO0000035573 } \\
1.69 \mathrm{E}-03\end{array}$ & -3.47 \\
\hline 4760 & $\begin{array}{l}\text { ENSGALTO0000035621 } \\
2.62 E-03\end{array}$ & -7.10 \\
\hline 4761 & $\begin{array}{l}\text { ENSGALTO0000035653 } \\
1.04 E-02\end{array}$ & -1.47 \\
\hline 4762 & $\begin{array}{l}\text { ENSGALTO0000035666 } \\
3.12 E-02\end{array}$ & -1.50 \\
\hline 4763 & $\begin{array}{l}\text { ENSGALTO0000035677 } \\
\text { 3.62E-03 }\end{array}$ & -1.72 \\
\hline 4764 & $\begin{array}{l}\text { ENSGALT00000035697 } \\
1.87 \mathrm{E}-02\end{array}$ & -1.71 \\
\hline 4765 & $\begin{array}{l}\text { ENSGALTO0000035703 } \\
\text { 5.30E-04 }\end{array}$ & -1.37 \\
\hline 4766 & $\begin{array}{l}\text { ENSGALTO0000035712 } \\
1.50 E-03\end{array}$ & -7.35 \\
\hline 4767 & $\begin{array}{l}\text { ENSGALT00000035726 } \\
2.12 E-05\end{array}$ & -8.65 \\
\hline 4768 & $\begin{array}{l}\text { ENSGALTO0000035730 } \\
2.02 E-02\end{array}$ & -1.16 \\
\hline 4769 & $\begin{array}{l}\text { ENSGALTO0000035742 } \\
5.18 \mathrm{E}-04\end{array}$ & -4.31 \\
\hline 4770 & $\begin{array}{l}\text { ENSGALTO0000035764 } \\
5.42 E-03\end{array}$ & -1.46 \\
\hline 4771 & $\begin{array}{l}\text { ENSGALTO0000035780 } \\
3.99 E-03\end{array}$ & -3.83 \\
\hline 4772 & $\begin{array}{l}\text { ENSGALT00000035786 } \\
1.23 \mathrm{E}-02\end{array}$ & -1.31 \\
\hline 4773 & $\begin{array}{l}\text { ENSGALT00000035822 } \\
2.43 \mathrm{E}-02\end{array}$ & -1.17 \\
\hline 4774 & $\begin{array}{l}\text { ENSGALTO0000035893 } \\
5.50 E-04\end{array}$ & -6.91 \\
\hline 4775 & $\begin{array}{l}\text { ENSGALT00000035955 } \\
4.63 E-05\end{array}$ & -7.09 \\
\hline 4776 & $\begin{array}{l}\text { ENSGALTO0000035962 } \\
2.89 E-02\end{array}$ & -2.43 \\
\hline 4777 & $\begin{array}{l}\text { ENSGALTO0000036002 } \\
8.48 E-04\end{array}$ & -1.57 \\
\hline 4778 & $\begin{array}{l}\text { ENSGALTO0000036032 } \\
1.17 \mathrm{E}-03\end{array}$ & -1.51 \\
\hline 4779 & $\begin{array}{l}\text { ENSGALTO0000036076 } \\
3.44 E-03\end{array}$ & -4.81 \\
\hline 4780 & $\begin{array}{l}\text { ENSGALTO0000036121 } \\
3.05 E-03\end{array}$ & -2.13 \\
\hline 4781 & $\begin{array}{l}\text { ENSGALTO0000036129 } \\
6.58 \mathrm{E}-02\end{array}$ & -1.32 \\
\hline 4782 & $\begin{array}{l}\text { ENSGALTO0000036153 } \\
1.76 E-03\end{array}$ & -2.53 \\
\hline 4783 & $\begin{array}{l}\text { ENSGALTO0000036196 } \\
1.47 \mathrm{E}-03\end{array}$ & -1.44 \\
\hline 4784 & $\begin{array}{l}\text { ENSGALT00000036227 } \\
3.49 \mathrm{E}-02\end{array}$ & -1.46 \\
\hline 4785 & $\begin{array}{l}\text { ENSGALTO0000036267 } \\
1.70 E-04\end{array}$ & -6.59 \\
\hline 4786 & $\begin{array}{l}\text { ENSGALTO0000036273 } \\
9.87 E-03\end{array}$ & -1.45 \\
\hline 4787 & $\begin{array}{l}\text { ENSGALTO0000036276 } \\
4.42 E-02\end{array}$ & -2.45 \\
\hline 4788 & $\begin{array}{l}\text { ENSGALTO0000036278 } \\
4.68 E-03\end{array}$ & -2.16 \\
\hline 4789 & $\begin{array}{l}\text { ENSGALT00000036286 } \\
8.27 E-03\end{array}$ & -4.41 \\
\hline 4790 & $\begin{array}{l}\text { ENSGALTO0000036352 } \\
1.74 E-03\end{array}$ & -6.57 \\
\hline 4791 & $\begin{array}{l}\text { ENSGALTO0000036398 } \\
2.07 E-03\end{array}$ & -2.16 \\
\hline 4792 & $\begin{array}{l}\text { ENSGALTO00000036407 } \\
1.04 E-02\end{array}$ & -3.42 \\
\hline 4793 & $\begin{array}{l}\text { ENSGALTO0000036420 } \\
4.27 E-04\end{array}$ & -2.10 \\
\hline 4794 & $\begin{array}{l}\text { ENSGALTO0000036444 } \\
7.43 E-04\end{array}$ & -9.25 \\
\hline 4795 & $\begin{array}{l}\text { ENSGALTO0000036513 } \\
6.24 \mathrm{E}-03\end{array}$ & -2.05 \\
\hline
\end{tabular}




\begin{tabular}{|c|c|c|}
\hline 4796 & $\begin{array}{l}\text { ENSGALTO0000036521 } \\
1.28 \mathrm{E}-03\end{array}$ & -1.18 \\
\hline 4797 & $\begin{array}{l}\text { ENSGALT00000036561 } \\
1.53 E-02\end{array}$ & -1.20 \\
\hline 4798 & $\begin{array}{l}\text { ENSGALT00000036568 } \\
9.74 E-05\end{array}$ & -5.03 \\
\hline 4799 & $\begin{array}{l}\text { ENSGALT00000036586 } \\
4.85 E-04\end{array}$ & -3.11 \\
\hline 4800 & $\begin{array}{l}\text { ENSGALT00000036642 } \\
1.79 E-02\end{array}$ & -1.44 \\
\hline 4801 & $\begin{array}{l}\text { ENSGALTO0000036647 } \\
2.29 \mathrm{E}-03\end{array}$ & -6.19 \\
\hline 4802 & $\begin{array}{l}\text { ENSGALT00000036656 } \\
8.13 \mathrm{E}-03\end{array}$ & -1.41 \\
\hline 4803 & $\begin{array}{l}\text { ENSGALT00000036658 } \\
2.35 E-04\end{array}$ & -2.86 \\
\hline 4804 & $\begin{array}{l}\text { ENSGALT00000036676 } \\
1.67 E-03\end{array}$ & -4.11 \\
\hline 4805 & $\begin{array}{l}\text { ENSGALT00000036685 } \\
2.66 \mathrm{E}-02\end{array}$ & -1.53 \\
\hline 4806 & $\begin{array}{l}\text { ENSGALT00000036688 } \\
9.37 \mathrm{-}-04\end{array}$ & -4.06 \\
\hline 4807 & $\begin{array}{l}\text { ENSGALT00000036729 } \\
6.36 \mathrm{E}-03\end{array}$ & -2.06 \\
\hline 4808 & $\begin{array}{l}\text { ENSGALT00000036731 } \\
2.68 \mathrm{E}-03\end{array}$ & -1.85 \\
\hline 4809 & $\begin{array}{l}\text { ENSGALT00000036734 } \\
4.01 E-03\end{array}$ & -1.58 \\
\hline 4810 & $\begin{array}{l}\text { ENSGALT00000036736 } \\
1.51 \mathrm{E}-02\end{array}$ & -2.40 \\
\hline 4811 & $\begin{array}{l}\text { ENSGALT00000036786 } \\
2.38 \mathrm{E}-03\end{array}$ & -4.09 \\
\hline 4812 & $\begin{array}{l}\text { ENSGALT00000036788 } \\
8.00 \mathrm{0}-06\end{array}$ & -7.52 \\
\hline 4813 & $\begin{array}{l}\text { ENSGALT00000036800 } \\
1.23 E-03\end{array}$ & -2.39 \\
\hline 4814 & $\begin{array}{l}\text { ENSGALT00000036849 } \\
1.22 E-02\end{array}$ & -1.35 \\
\hline 4815 & $\begin{array}{l}\text { ENSGALT00000036897 } \\
1.40 \mathrm{E}-03\end{array}$ & -3.87 \\
\hline 4816 & $\begin{array}{l}\text { ENSGALT00000036899 } \\
7.21 E-04\end{array}$ & -3.33 \\
\hline 4817 & $\begin{array}{l}\text { ENSGALT00000036912 } \\
8.56 \mathrm{E}-03\end{array}$ & -2.03 \\
\hline 4818 & $\begin{array}{l}\text { ENSGALT00000036918 } \\
6.30 \mathrm{E}-03\end{array}$ & -2.58 \\
\hline 4819 & $\begin{array}{l}\text { ENSGALT00000036939 } \\
1.21 \mathrm{E}-03\end{array}$ & -1.26 \\
\hline 4820 & $\begin{array}{l}\text { ENSGALT00000036943 } \\
6.48 \mathrm{E}-01\end{array}$ & -2.17 \\
\hline 4821 & $\begin{array}{l}\text { ENSGALT00000036945 } \\
\text { 2.68E-03 }\end{array}$ & -4.07 \\
\hline 4822 & $\begin{array}{l}\text { ENSGALT00000036955 } \\
3.84 E-04\end{array}$ & -4.67 \\
\hline 4823 & $\begin{array}{l}\text { ENSGALT00000036966 } \\
6.08 E-02\end{array}$ & -2.59 \\
\hline 4824 & $\begin{array}{l}\text { ENSGALT00000037099 } \\
6.08 \mathrm{E}-03\end{array}$ & -2.03 \\
\hline 4825 & $\begin{array}{l}\text { ENSGALT00000037100 } \\
2.90 \mathrm{-}-02\end{array}$ & -1.59 \\
\hline 4826 & $\begin{array}{l}\text { ENSGALT00000037178 } \\
3.67 E-03\end{array}$ & -1.64 \\
\hline 4827 & $\begin{array}{l}\text { ENSGALT00000037199 } \\
7.60 \mathrm{E}-03\end{array}$ & -2.45 \\
\hline 4828 & $\begin{array}{l}\text { ENSGALT00000037242 } \\
3.26 \mathrm{-} 02\end{array}$ & -1.39 \\
\hline 4829 & $\begin{array}{l}\text { ENSGALT00000037284 } \\
4.35 E-03\end{array}$ & -1.63 \\
\hline 4830 & $\begin{array}{l}\text { ENSGALT00000037285 } \\
1.84 \mathrm{E}-04\end{array}$ & -6.92 \\
\hline 4831 & $\begin{array}{l}\text { ENSGALT00000037310 } \\
4.66 E-03\end{array}$ & -3.93 \\
\hline 4832 & $\begin{array}{l}\text { ENSGALT00000037321 } \\
3.15 \mathrm{E}-03\end{array}$ & -1.17 \\
\hline 4833 & $\begin{array}{l}\text { ENSGALT00000037360 } \\
1.42 E-03\end{array}$ & -3.50 \\
\hline 4834 & $\begin{array}{l}\text { ENSGALT00000037363 } \\
1.25 \mathrm{E}-03\end{array}$ & -3.22 \\
\hline
\end{tabular}

\begin{tabular}{|c|c|c|}
\hline 4835 & $\begin{array}{l}\text { ENSGALTO0000037388 } \\
9.51 E-03\end{array}$ & -1.31 \\
\hline 4836 & $\begin{array}{l}\text { ENSGALTO0000037420 } \\
3.43 E-02\end{array}$ & -1.35 \\
\hline 4837 & $\begin{array}{l}\text { ENSGALTO0000037421 } \\
2.91 \mathrm{E}-02\end{array}$ & -1.22 \\
\hline 4838 & $\begin{array}{l}\text { ENSGALTO0000037423 } \\
3.60 \mathrm{E}-03\end{array}$ & -3.53 \\
\hline 4839 & $\begin{array}{l}\text { ENSGALTO0000037444 } \\
2.52 E-02\end{array}$ & -2.11 \\
\hline 4840 & $\begin{array}{l}\text { ENSGALTO0000037512 } \\
3.04 E-04\end{array}$ & -4.93 \\
\hline 4841 & $\begin{array}{l}\text { ENSGALT00000037514 } \\
2.95 E-02\end{array}$ & -1.23 \\
\hline 4842 & $\begin{array}{l}\text { ENSGALTO0000037541 } \\
1.39 \mathrm{E}-02\end{array}$ & -1.63 \\
\hline 4843 & $\begin{array}{l}\text { ENSGALTO0000037579 } \\
1.41 E-03\end{array}$ & -4.08 \\
\hline 4844 & $\begin{array}{l}\text { ENSGALT00000037580 } \\
6.83 E-03\end{array}$ & -1.37 \\
\hline 4845 & $\begin{array}{l}\text { ENSGALTO0000037616 } \\
1.22 E-03\end{array}$ & -3.62 \\
\hline 4846 & $\begin{array}{l}\text { ENSGALT00000037637 } \\
2.58 \mathrm{E}-03\end{array}$ & -2.69 \\
\hline 4847 & $\begin{array}{l}\text { ENSGALTO0000037641 } \\
1.44 E-03\end{array}$ & -4.06 \\
\hline 4848 & $\begin{array}{l}\text { ENSGALTO0000037644 } \\
4.43 E-04\end{array}$ & -3.74 \\
\hline 4849 & $\begin{array}{l}\text { ENSGALT00000037665 } \\
2.21 \mathrm{E}-03\end{array}$ & -3.70 \\
\hline 4850 & $\begin{array}{l}\text { ENSGALT00000037711 } \\
2.63 E-04\end{array}$ & -3.08 \\
\hline 4851 & $\begin{array}{l}\text { ENSGALTO0000037712 } \\
6.72 E-03\end{array}$ & -4.88 \\
\hline 4852 & $\begin{array}{l}\text { ENSGALTO0000037717 } \\
9.39 \mathrm{E}-04\end{array}$ & -3.63 \\
\hline 4853 & $\begin{array}{l}\text { ENSGALTO0000037742 } \\
2.75 E-04\end{array}$ & -2.92 \\
\hline 4854 & $\begin{array}{l}\text { ENSGALTO0000037769 } \\
2.23 E-03\end{array}$ & -2.00 \\
\hline 4855 & $\begin{array}{l}\text { ENSGALTO0000037777 } \\
9.69 E-03\end{array}$ & -1.67 \\
\hline 4856 & $\begin{array}{l}\text { ENSGALTO0000037783 } \\
2.64 E-04\end{array}$ & -5.11 \\
\hline 4857 & $\begin{array}{l}\text { ENSGALT00000037784 } \\
1.20 \mathrm{E}-03\end{array}$ & -2.59 \\
\hline 4858 & $\begin{array}{l}\text { ENSGALTO0000037789 } \\
2.95 E-03\end{array}$ & -1.81 \\
\hline 4859 & $\begin{array}{l}\text { ENSGALTO0000037839 } \\
9.18 \mathrm{E}-02\end{array}$ & -1.27 \\
\hline 4860 & $\begin{array}{l}\text { ENSGALTO0000037856 } \\
1.06 E-02\end{array}$ & -1.46 \\
\hline 4861 & $\begin{array}{l}\text { ENSGALTO0000037871 } \\
4.60 \mathrm{E}-04\end{array}$ & -6.61 \\
\hline 4862 & $\begin{array}{l}\text { ENSGALTO0000037914 } \\
\text { 7.96E-04 }\end{array}$ & -4.53 \\
\hline 4863 & $\begin{array}{l}\text { ENSGALTO0000037985 } \\
2.03 E-03\end{array}$ & -2.25 \\
\hline 4864 & $\begin{array}{l}\text { ENSGALT00000037995 } \\
1.26 E-01\end{array}$ & -2.34 \\
\hline 4865 & $\begin{array}{l}\text { ENSGALTO0000038028 } \\
1.57 E-02\end{array}$ & -1.89 \\
\hline 4866 & $\begin{array}{l}\text { ENSGALTO0000038124 } \\
1.72 E-03\end{array}$ & -7.64 \\
\hline 4867 & $\begin{array}{l}\text { ENSGALTO0000038166 } \\
2.55 E-04\end{array}$ & -2.89 \\
\hline 4868 & $\begin{array}{l}\text { ENSGALTO0000038177 } \\
1.64 E-04\end{array}$ & -5.88 \\
\hline 4869 & $\begin{array}{l}\text { ENSGALTO0000038226 } \\
1.39 E-02\end{array}$ & -2.59 \\
\hline 4870 & $\begin{array}{l}\text { ENSGALTO0000038278 } \\
1.23 \mathrm{E}-03\end{array}$ & -4.54 \\
\hline 4871 & $\begin{array}{l}\text { ENSGALTO0000038279 } \\
3.83 E-04\end{array}$ & -6.72 \\
\hline 4872 & $\begin{array}{l}\text { ENSGALTO0000038290 } \\
1.38 \mathrm{E}-04\end{array}$ & -8.23 \\
\hline 4873 & $\begin{array}{l}\text { ENSGALTO0000038297 } \\
1.18 \mathrm{E}-03\end{array}$ & -7.23 \\
\hline
\end{tabular}

\begin{tabular}{|c|c|c|}
\hline 4874 & $\begin{array}{l}\text { ENSGALTO0000038318 } \\
4.40 E-03\end{array}$ & -3.03 \\
\hline 4875 & $\begin{array}{l}\text { ENSGALTO0000038319 } \\
2.85 E-03\end{array}$ & -2.47 \\
\hline 4876 & $\begin{array}{l}\text { ENSGALTO0000038326 } \\
2.35 E-02\end{array}$ & -1.72 \\
\hline 4877 & $\begin{array}{l}\text { ENSGALTO0000038355 } \\
3.83 E-04\end{array}$ & -7.74 \\
\hline 4878 & $\begin{array}{l}\text { ENSGALTO0000038364 } \\
5.64 E-03\end{array}$ & -3.86 \\
\hline 4879 & $\begin{array}{l}\text { ENSGALT00000038365 } \\
7.95 E-03\end{array}$ & -3.67 \\
\hline 4880 & $\begin{array}{l}\text { ENSGALT00000038375 } \\
2.53 E-02\end{array}$ & -1.93 \\
\hline 4881 & $\begin{array}{l}\text { ENSGALTO0000038413 } \\
1.41 \mathrm{E}-03\end{array}$ & -3.82 \\
\hline 4882 & $\begin{array}{l}\text { ENSGALTO0000038441 } \\
1.31 E-02\end{array}$ & -1.86 \\
\hline 4883 & $\begin{array}{l}\text { ENSGALTO0000038453 } \\
1.06 E-02\end{array}$ & -2.56 \\
\hline 4884 & $\begin{array}{l}\text { ENSGALT00000038455 } \\
1.77 \mathrm{E}-03\end{array}$ & -4.15 \\
\hline 4885 & $\begin{array}{l}\text { ENSGALTO0000038486 } \\
1.61 E-03\end{array}$ & -6.11 \\
\hline 4886 & $\begin{array}{l}\text { ENSGALTO0000038514 } \\
5.81 E-03\end{array}$ & -1.03 \\
\hline 4887 & $\begin{array}{l}\text { ENSGALT00000038525 } \\
2.57 \text { E-01 }\end{array}$ & -1.20 \\
\hline 4888 & $\begin{array}{l}\text { ENSGALTO0000038597 } \\
1.42 E-02\end{array}$ & -1.53 \\
\hline 4889 & $\begin{array}{l}\text { ENSGALT00000038600 } \\
2.44 \mathrm{E}-03\end{array}$ & -2.63 \\
\hline 4890 & $\begin{array}{l}\text { ENSGALTO0000038602 } \\
8.81 E-04\end{array}$ & -2.96 \\
\hline 4891 & $\begin{array}{l}\text { ENSGALTO0000038613 } \\
1.64 E-03\end{array}$ & -4.42 \\
\hline 4892 & $\begin{array}{l}\text { ENSGALTO0000038631 } \\
2.30 \mathrm{E}-04\end{array}$ & -5.17 \\
\hline 4893 & $\begin{array}{l}\text { ENSGALTO0000038643 } \\
3.51 E-02\end{array}$ & -2.04 \\
\hline 4894 & $\begin{array}{l}\text { ENSGALT00000038655 } \\
6.74 E-01\end{array}$ & -1.61 \\
\hline 4895 & $\begin{array}{l}\text { ENSGALTO0000038678 } \\
1.05 E-03\end{array}$ & -1.22 \\
\hline 4896 & $\begin{array}{l}\text { ENSGALTO0000038693 } \\
2.63 E-03\end{array}$ & -4.50 \\
\hline 4897 & $\begin{array}{l}\text { ENSGALTO0000038739 } \\
1.99 \mathrm{E}-04\end{array}$ & -5.68 \\
\hline 4898 & $\begin{array}{l}\text { ENSGALTO0000038785 } \\
1.05 E-03\end{array}$ & -2.28 \\
\hline 4899 & $\begin{array}{l}\text { ENSGALTO0000038814 } \\
1.11 \mathrm{E}-04\end{array}$ & -2.28 \\
\hline 4900 & $\begin{array}{l}\text { ENSGALTO0000038832 } \\
1.31 E-03\end{array}$ & -2.91 \\
\hline 4901 & $\begin{array}{l}\text { ENSGALTO0000038851 } \\
5.11 \mathrm{E}-03\end{array}$ & -1.84 \\
\hline 4902 & $\begin{array}{l}\text { ENSGALT00000038859 } \\
3.65 E-03\end{array}$ & -1.93 \\
\hline 4903 & $\begin{array}{l}\text { ENSGALTO0000038868 } \\
2.28 E-03\end{array}$ & -4.22 \\
\hline 4904 & $\begin{array}{l}\text { ENSGALT00000038870 } \\
1.01 E-04\end{array}$ & -4.63 \\
\hline 4905 & $\begin{array}{l}\text { ENSGALTO0000038873 } \\
1.01 E-03\end{array}$ & -5.97 \\
\hline 4906 & $\begin{array}{l}\text { ENSGALTO0000038915 } \\
7.01 E-03\end{array}$ & -4.76 \\
\hline 4907 & $\begin{array}{l}\text { ENSGALTO0000038945 } \\
5.46 E-05\end{array}$ & -6.79 \\
\hline 4908 & $\begin{array}{l}\text { ENSGALTO0000038948 } \\
6.93 E-04\end{array}$ & -5.39 \\
\hline 4909 & $\begin{array}{l}\text { ENSGALT00000038958 } \\
6.21 E-03\end{array}$ & -2.21 \\
\hline 4910 & $\begin{array}{l}\text { ENSGALTO0000038970 } \\
3.43 E-03\end{array}$ & -1.05 \\
\hline 4911 & $\begin{array}{l}\text { ENSGALT00000039021 } \\
5.24 E-04\end{array}$ & -5.73 \\
\hline 4912 & $\begin{array}{l}\text { ENSGALTO0000039024 } \\
4.28 \mathrm{E}-03\end{array}$ & -3.30 \\
\hline
\end{tabular}




\begin{tabular}{|c|c|c|}
\hline 4913 & $\begin{array}{l}\text { ENSGALTO0000039025 } \\
6.66 \mathrm{-}-03\end{array}$ & -1.75 \\
\hline 4914 & $\begin{array}{l}\text { ENSGALTO0000039044 } \\
6.17 \mathrm{E}-03\end{array}$ & -2.52 \\
\hline 4915 & $\begin{array}{l}\text { ENSGALTO0000039073 } \\
2.56 \text { E-04 }\end{array}$ & -4.42 \\
\hline 4916 & $\begin{array}{l}\text { ENSGALTO0000039131 } \\
6.33 E-03\end{array}$ & -1.71 \\
\hline 4917 & $\begin{array}{l}\text { ENSGALTO0000039135 } \\
1.21 E-02\end{array}$ & -1.25 \\
\hline 4918 & $\begin{array}{l}\text { ENSGALT00000039149 } \\
2.16 \mathrm{E}-04\end{array}$ & -5.56 \\
\hline 4919 & $\begin{array}{l}\text { ENSGALTO0000039151 } \\
2.32 E-02\end{array}$ & -1.39 \\
\hline 4920 & $\begin{array}{l}\text { ENSGALT00000039152 } \\
1.86 E-02\end{array}$ & -2.66 \\
\hline 4921 & $\begin{array}{l}\text { ENSGALTO0000039154 } \\
1.31 \mathrm{E}-03\end{array}$ & -2.10 \\
\hline 4922 & $\begin{array}{l}\text { ENSGALTO0000039169 } \\
7.85 E-06\end{array}$ & -5.90 \\
\hline 4923 & $\begin{array}{l}\text { ENSGALTO0000039177 } \\
4.46 E-03\end{array}$ & -4.00 \\
\hline 4924 & $\begin{array}{l}\text { ENSGALTO0000039187 } \\
5.88 \mathrm{E}-03\end{array}$ & -2.66 \\
\hline 4925 & $\begin{array}{l}\text { ENSGALTO0000039205 } \\
\text { 3.70E-02 }\end{array}$ & -1.16 \\
\hline 4926 & $\begin{array}{l}\text { ENSGALTO0000039217 } \\
\text { 3.44E-04 }\end{array}$ & -4.41 \\
\hline 4927 & $\begin{array}{l}\text { ENSGALTO0000039223 } \\
1.07 E-03\end{array}$ & -3.99 \\
\hline 4928 & $\begin{array}{l}\text { ENSGALTO0000039231 } \\
1.00 \mathrm{E}-03\end{array}$ & -3.35 \\
\hline 4929 & $\begin{array}{l}\text { ENSGALTO0000039270 } \\
2.96 E-03\end{array}$ & -2.07 \\
\hline 4930 & $\begin{array}{l}\text { ENSGALT00000039308 } \\
4.56 E-03\end{array}$ & -2.46 \\
\hline 4931 & $\begin{array}{l}\text { ENSGALT00000039326 } \\
6.79 E-05\end{array}$ & -2.38 \\
\hline 4932 & $\begin{array}{l}\text { ENSGALT00000039329 } \\
1.42 E-02\end{array}$ & -1.57 \\
\hline 4933 & $\begin{array}{l}\text { ENSGALTO0000039359 } \\
2.34 E-03\end{array}$ & -1.66 \\
\hline 4934 & $\begin{array}{l}\text { ENSGALTO0000039388 } \\
5.26 E-03\end{array}$ & -2.90 \\
\hline 4935 & $\begin{array}{l}\text { ENSGALT00000039406 } \\
\text { 3.63E-02 }\end{array}$ & -1.26 \\
\hline 4936 & $\begin{array}{l}\text { ENSGALT00000039437 } \\
1.70 \mathrm{E}-02\end{array}$ & -1.30 \\
\hline 4937 & $\begin{array}{l}\text { ENSGALT00000039464 } \\
8.35 E-04\end{array}$ & -4.83 \\
\hline 4938 & $\begin{array}{l}\text { ENSGALT00000039480 } \\
7.12 \mathrm{E}-03\end{array}$ & -1.76 \\
\hline 4939 & $\begin{array}{l}\text { ENSGALTO0000039509 } \\
2.86 E-04\end{array}$ & -6.37 \\
\hline 4940 & $\begin{array}{l}\text { ENSGALTO0000039511 } \\
\text { 4.49E-04 }\end{array}$ & -6.52 \\
\hline 4941 & $\begin{array}{l}\text { ENSGALTO0000039514 } \\
9.95 E-04\end{array}$ & -8.09 \\
\hline 4942 & $\begin{array}{l}\text { ENSGALTO0000039520 } \\
4.67 E-04\end{array}$ & -6.46 \\
\hline 4943 & $\begin{array}{l}\text { ENSGALT00000039524 } \\
\text { 4.03E-04 }\end{array}$ & -6.75 \\
\hline 4944 & $\begin{array}{l}\text { ENSGALTO0000039532 } \\
8.38 E-04\end{array}$ & -2.73 \\
\hline 4945 & $\begin{array}{l}\text { ENSGALT00000039568 } \\
1.18 \mathrm{E}-02\end{array}$ & -2.47 \\
\hline 4946 & $\begin{array}{l}\text { ENSGALTO0000039594 } \\
4.40 E-02\end{array}$ & -1.86 \\
\hline 4947 & $\begin{array}{l}\text { ENSGALTO0000039612 } \\
\text { 3.25E-04 }\end{array}$ & -7.01 \\
\hline 4948 & $\begin{array}{l}\text { ENSGALT00000039614 } \\
6.92 E-04\end{array}$ & -5.69 \\
\hline 4949 & $\begin{array}{l}\text { ENSGALTO0000039617 } \\
1.46 E-03\end{array}$ & -2.84 \\
\hline 4950 & $\begin{array}{l}\text { ENSGALT00000039659 } \\
2.71 E-03\end{array}$ & -1.88 \\
\hline 4951 & $\begin{array}{l}\text { ENSGALT00000039665 } \\
2.87 E-02\end{array}$ & -2.50 \\
\hline
\end{tabular}

\begin{tabular}{|c|c|c|}
\hline 4952 & $\begin{array}{l}\text { ENSGALTO0000039666 } \\
6.81 E-04\end{array}$ & -1.35 \\
\hline 4953 & $\begin{array}{l}\text { ENSGALTO0000039691 } \\
2.13 \mathrm{E}-04\end{array}$ & -4.33 \\
\hline 4954 & $\begin{array}{l}\text { ENSGALTO0000039723 } \\
3.02 E-03\end{array}$ & -2.71 \\
\hline 4955 & $\begin{array}{l}\text { ENSGALTO0000039732 } \\
6.13 E-03\end{array}$ & -2.89 \\
\hline 4956 & $\begin{array}{l}\text { ENSGALTO0000039740 } \\
7.54 E-03\end{array}$ & -1.79 \\
\hline 4957 & $\begin{array}{l}\text { ENSGALTO0000039742 } \\
1.15 E-03\end{array}$ & -3.23 \\
\hline 4958 & $\begin{array}{l}\text { ENSGALTO0000039756 } \\
5.79 E-03\end{array}$ & -3.71 \\
\hline 4959 & $\begin{array}{l}\text { ENSGALTO0000039771 } \\
1.07 E-03\end{array}$ & -1.25 \\
\hline 4960 & $\begin{array}{l}\text { ENSGALTO0000039773 } \\
1.34 E-04\end{array}$ & -5.29 \\
\hline 4961 & $\begin{array}{l}\text { ENSGALTO0000039780 } \\
9.47 E-04\end{array}$ & -3.32 \\
\hline 4962 & $\begin{array}{l}\text { ENSGALTO0000039794 } \\
2.36 E-03\end{array}$ & -3.41 \\
\hline 4963 & $\begin{array}{l}\text { ENSGALTO0000039847 } \\
2.53 \mathrm{E}-03\end{array}$ & -1.29 \\
\hline 4964 & $\begin{array}{l}\text { ENSGALTO0000039851 } \\
7.46 E-04\end{array}$ & -3.35 \\
\hline 4965 & $\begin{array}{l}\text { ENSGALTO0000039942 } \\
3.07 E-03\end{array}$ & -4.33 \\
\hline 4966 & $\begin{array}{l}\text { ENSGALTO0000039951 } \\
2.30 \mathrm{E}-02\end{array}$ & -3.47 \\
\hline 4967 & $\begin{array}{l}\text { ENSGALTO0000040052 } \\
2.53 E-02\end{array}$ & -1.15 \\
\hline 4968 & $\begin{array}{l}\text { ENSGALTO0000040115 } \\
1.84 \text { E-02 }\end{array}$ & -2.37 \\
\hline 4969 & $\begin{array}{l}\text { ENSGALTO0000040180 } \\
1.07 E-02\end{array}$ & -5.26 \\
\hline 4970 & $\begin{array}{l}\text { ENSGALTO0000040187 } \\
2.35 E-02\end{array}$ & -1.29 \\
\hline 4971 & $\begin{array}{l}\text { ENSGALTO0000040193 } \\
1.85 E-05\end{array}$ & -5.36 \\
\hline 4972 & $\begin{array}{l}\text { ENSGALT00000040225 } \\
1.19 \mathrm{E}-02\end{array}$ & -2.29 \\
\hline 4973 & $\begin{array}{l}\text { ENSGALTO0000040242 } \\
2.34 E-02\end{array}$ & -1.26 \\
\hline 4974 & $\begin{array}{l}\text { ENSGALTO0000040244 } \\
3.55 E-03\end{array}$ & -4.32 \\
\hline 4975 & $\begin{array}{l}\text { ENSGALTO0000040271 } \\
3.45 E-04\end{array}$ & -6.82 \\
\hline 4976 & $\begin{array}{l}\text { ENSGALTO0000040314 } \\
1.03 E-04\end{array}$ & -6.34 \\
\hline 4977 & $\begin{array}{l}\text { ENSGALTO0000040352 } \\
3.28 E-04\end{array}$ & -6.42 \\
\hline 4978 & $\begin{array}{l}\text { ENSGALTO0000040393 } \\
3.74 E-04\end{array}$ & -7.98 \\
\hline 4979 & $\begin{array}{l}\text { ENSGALTO0000040398 } \\
3.31 E-03\end{array}$ & -1.91 \\
\hline 4980 & $\begin{array}{l}\text { ENSGALT00000040424 } \\
6.92 E-04\end{array}$ & -5.66 \\
\hline 4981 & $\begin{array}{l}\text { ENSGALTO0000040440 } \\
5.76 E-03\end{array}$ & -1.37 \\
\hline 4982 & $\begin{array}{l}\text { ENSGALTO0000040493 } \\
9.53 \mathrm{E}-04\end{array}$ & -2.65 \\
\hline 4983 & $\begin{array}{l}\text { ENSGALT00000040503 } \\
3.22 \text { E-02 }\end{array}$ & -1.30 \\
\hline 4984 & $\begin{array}{l}\text { ENSGALTO0000040513 } \\
7.89 \mathrm{E}-03\end{array}$ & -2.40 \\
\hline 4985 & $\begin{array}{l}\text { ENSGALT00000040525 } \\
2.25 E-04\end{array}$ & -8.13 \\
\hline 4986 & $\begin{array}{l}\text { ENSGALTO0000040573 } \\
1.94 \mathrm{E}-03\end{array}$ & -2.10 \\
\hline 4987 & $\begin{array}{l}\text { ENSGALT00000040608 } \\
7.76 E-06\end{array}$ & -7.37 \\
\hline 4988 & $\begin{array}{l}\text { ENSGALT00000040633 } \\
2.02 E-04\end{array}$ & -5.77 \\
\hline 4989 & $\begin{array}{l}\text { ENSGALTO0000040671 } \\
\text { 3.73E-02 }\end{array}$ & -1.50 \\
\hline 4990 & $\begin{array}{l}\text { ENSGALT00000040696 } \\
1.27 E-03\end{array}$ & -5.21 \\
\hline
\end{tabular}

\begin{tabular}{|c|c|c|}
\hline 4991 & $\begin{array}{l}\text { ENSGALTO0000040732 } \\
3.40 E-03\end{array}$ & -3.32 \\
\hline 4992 & $\begin{array}{l}\text { ENSGALTO0000040733 } \\
6.31 E-05\end{array}$ & -1.09 \\
\hline 4993 & $\begin{array}{l}\text { ENSGALTO0000040735 } \\
1.03 E-02\end{array}$ & -1.46 \\
\hline 4994 & $\begin{array}{l}\text { ENSGALTO0000040740 } \\
1.12 E-02\end{array}$ & -2.06 \\
\hline 4995 & $\begin{array}{l}\text { ENSGALT00000040808 } \\
1.13 E-03\end{array}$ & -2.75 \\
\hline 4996 & $\begin{array}{l}\text { ENSGALTO0000040817 } \\
1.33 \mathrm{E}-04\end{array}$ & -5.45 \\
\hline 4997 & $\begin{array}{l}\text { ENSGALTO0000040818 } \\
2.31 E-03\end{array}$ & -2.19 \\
\hline 4998 & $\begin{array}{l}\text { ENSGALTO0000040829 } \\
2.04 E-03\end{array}$ & -3.72 \\
\hline 4999 & $\begin{array}{l}\text { ENSGALTO0000040831 } \\
7.77 E-05\end{array}$ & -6.82 \\
\hline 5000 & $\begin{array}{l}\text { ENSGALTO0000040895 } \\
1.59 \mathrm{E}-03\end{array}$ & -2.97 \\
\hline 5001 & $\begin{array}{l}\text { ENSGALTO0000040947 } \\
1.80 \text { - } 03\end{array}$ & -1.91 \\
\hline 5002 & $\begin{array}{l}\text { ENSGALT00000040966 } \\
\text { 4.31E-03 }\end{array}$ & -3.31 \\
\hline 5003 & $\begin{array}{l}\text { ENSGALTO0000040970 } \\
1.32 E-02\end{array}$ & -1.76 \\
\hline 5004 & $\begin{array}{l}\text { ENSGALT00000040998 } \\
6.08 \mathrm{E}-04\end{array}$ & -1.38 \\
\hline 5005 & $\begin{array}{l}\text { ENSGALTO0000040999 } \\
2.19 \mathrm{E}-02\end{array}$ & -2.04 \\
\hline 5006 & $\begin{array}{l}\text { ENSGALTO0000041037 } \\
1.26 E-03\end{array}$ & -3.15 \\
\hline 5007 & $\begin{array}{l}\text { ENSGALTO0000041068 } \\
1.47 E-03\end{array}$ & -4.42 \\
\hline 5008 & $\begin{array}{l}\text { ENSGALTO0000041087 } \\
1.59 \mathrm{E}-03\end{array}$ & -3.46 \\
\hline 5009 & $\begin{array}{l}\text { ENSGALTO0000041103 } \\
4.34 E-04\end{array}$ & -4.86 \\
\hline 5010 & $\begin{array}{l}\text { ENSGALTO0000041107 } \\
5.71 E-03\end{array}$ & -1.55 \\
\hline 5011 & $\begin{array}{l}\text { ENSGALTO0000041137 } \\
1.02 E-02\end{array}$ & -2.12 \\
\hline 5012 & $\begin{array}{l}\text { ENSGALTO0000041158 } \\
2.20 \mathrm{E}-04\end{array}$ & -5.48 \\
\hline 5013 & $\begin{array}{l}\text { ENSGALTO0000041245 } \\
1.42 E-03\end{array}$ & -2.25 \\
\hline 5014 & $\begin{array}{l}\text { ENSGALTO0000041248 } \\
7.77 E-04\end{array}$ & -1.63 \\
\hline 5015 & $\begin{array}{l}\text { ENSGALTO0000041272 } \\
7.07 E-04\end{array}$ & -2.14 \\
\hline 5016 & $\begin{array}{l}\text { ENSGALTO0000041273 } \\
4.11 \mathrm{E}-04\end{array}$ & -4.98 \\
\hline 5017 & $\begin{array}{l}\text { ENSGALTO0000041320 } \\
7.38 \mathrm{E}-03\end{array}$ & -2.02 \\
\hline 5018 & $\begin{array}{l}\text { ENSGALTO0000041328 } \\
7.62 E-04\end{array}$ & -5.25 \\
\hline 5019 & $\begin{array}{l}\text { ENSGALTO0000041350 } \\
4.08 E-02\end{array}$ & -1.88 \\
\hline 5020 & $\begin{array}{l}\text { ENSGALTO0000041367 } \\
4.30 E-02\end{array}$ & -1.68 \\
\hline 5021 & $\begin{array}{l}\text { ENSGALTO0000041373 } \\
1.22 E-04\end{array}$ & -1.66 \\
\hline 5022 & $\begin{array}{l}\text { ENSGALTO0000041391 } \\
2.75 E-04\end{array}$ & -3.44 \\
\hline 5023 & $\begin{array}{l}\text { ENSGALTO0000041392 } \\
1.33 E-02\end{array}$ & -3.04 \\
\hline 5024 & $\begin{array}{l}\text { ENSGALTO0000041403 } \\
4.90 \mathrm{0}-02\end{array}$ & -2.63 \\
\hline 5025 & ENTPD4 & 4.46E-04 \\
\hline 5026 & ENTPD5 & $9.91 \mathrm{E}-04$ \\
\hline 5027 & ENTPD6 & $2.76 \mathrm{E}-03$ \\
\hline 5028 & EPB41L4A & $6.73 \mathrm{E}-04$ \\
\hline 5029 & EPCAM & $6.22 \mathrm{E}-04$ \\
\hline 5030 & EPHA1 & $3.41 \mathrm{E}-03$ \\
\hline 5031 & EPHB2 & $1.73 \mathrm{E}-03$ \\
\hline 5032 & $\begin{array}{ll}\text { EPHX1 } & -2.57\end{array}$ & $9.47 \mathrm{E}-05$ \\
\hline
\end{tabular}




\begin{tabular}{|c|c|c|c|}
\hline 5033 & EPM2A & -1.90 & $3.03 E-03$ \\
\hline 5034 & EPN2 & -5.53 & $3.18 \mathrm{E}-04$ \\
\hline 5035 & EPRS & -5.80 & $6.78 \mathrm{E}-04$ \\
\hline 5036 & EPS15 & -4.44 & $4.87 \mathrm{E}-04$ \\
\hline 5037 & EPS15L1 & -1.66 & $1.91 \mathrm{E}-02$ \\
\hline 5038 & EPS8 & -2.17 & $8.64 \mathrm{E}-03$ \\
\hline 5039 & EPSTI1 & -1.07 & $9.97 \mathrm{E}-02$ \\
\hline 5040 & ERAL1 & -4.47 & $1.62 \mathrm{E}-04$ \\
\hline 5041 & ERAP1 & -3.31 & $2.58 \mathrm{E}-03$ \\
\hline 5042 & ERBB4 & -1.85 & $3.78 \mathrm{E}-02$ \\
\hline 5043 & ERC1 & -3.07 & $5.64 \mathrm{E}-04$ \\
\hline 5044 & ERCC3 & -5.58 & $4.34 \mathrm{E}-04$ \\
\hline 5045 & ERCC4 & -1.31 & $1.01 \mathrm{E}-02$ \\
\hline 5046 & ERCC5 & -3.06 & $3.25 \mathrm{E}-04$ \\
\hline 5047 & ERCC6 & -2.83 & $4.55 \mathrm{E}-03$ \\
\hline 5048 & ERCC6L2 & $\begin{array}{l}-1.68 \\
\end{array}$ & $2.46 \mathrm{E}-03$ \\
\hline 5049 & ERCC8 & -1.76 & $1.67 \mathrm{E}-03$ \\
\hline 5050 & ERGIC2 & -2.36 & $2.89 \mathrm{E}-03$ \\
\hline 5051 & ERGIC3 & -2.10 & $1.11 \mathrm{E}-02$ \\
\hline 5052 & ERH & -4.90 & $1.51 \mathrm{E}-04$ \\
\hline 5053 & ERI1 & -6.26 & $1.08 \mathrm{E}-04$ \\
\hline 5054 & ERICH1 & \begin{tabular}{|l|}
-1.83 \\
\end{tabular} & $1.20 \mathrm{E}-02$ \\
\hline 5055 & ERLIN1 & -3.62 & $2.49 \mathrm{E}-04$ \\
\hline 5056 & ERLIN2 & -4.75 & $2.91 \mathrm{E}-04$ \\
\hline 5057 & ERO1L & -4.83 & $2.50 \mathrm{E}-04$ \\
\hline 5058 & ERP29 & -4.43 & $1.77 \mathrm{E}-03$ \\
\hline 5059 & ERP44 & -3.59 & $1.29 \mathrm{E}-03$ \\
\hline 5060 & ERRFI1 & -2.61 & $4.26 \mathrm{E}-03$ \\
\hline 5061 & ESCO1 & -4.82 & $2.57 \mathrm{E}-04$ \\
\hline 5062 & ESD & -2.95 & $2.62 \mathrm{E}-02$ \\
\hline 5063 & ESF1 & -3.22 & $1.00 \mathrm{E}-03$ \\
\hline 5064 & ESRRB & -2.71 & $1.95 E-02$ \\
\hline 5065 & ETAA1 & -3.10 & $7.60 \mathrm{E}-03$ \\
\hline 5066 & ETF1 & -5.28 & $6.05 E-05$ \\
\hline 5067 & ETFA & -3.07 & $2.26 \mathrm{E}-03$ \\
\hline 5068 & ETFDH & -1.79 & $6.58 \mathrm{E}-04$ \\
\hline 5069 & ETNK1 & -2.55 & $3.29 \mathrm{E}-04$ \\
\hline 5070 & ETNK2 & -1.27 & $4.57 \mathrm{E}-03$ \\
\hline 5071 & EU048536 & -6.49 & $1.81 \mathrm{E}-03$ \\
\hline 5072 & EU082129 & -2.68 & $2.65 \mathrm{E}-03$ \\
\hline 5073 & EU240280 & -3.39 & $6.83 \mathrm{E}-03$ \\
\hline 5074 & EU240284 & \begin{tabular}{l|}
-2.51 \\
\end{tabular} & $1.61 \mathrm{E}-04$ \\
\hline 5075 & EU240299 & -1.73 & $5.85 \mathrm{E}-03$ \\
\hline 5076 & EVI5 & -1.85 & $6.43 \mathrm{E}-03$ \\
\hline 5077 & EVL & -2.01 & $1.60 \mathrm{E}-02$ \\
\hline 5078 & EWSR1 & -4.63 & $1.04 \mathrm{E}-01$ \\
\hline 5079 & EXD1 & -1.89 & $8.91 \mathrm{E}-03$ \\
\hline 5080 & EXD2 & -1.59 & $3.70 \mathrm{E}-03$ \\
\hline 5081 & EXD3 & -2.83 & $2.92 \mathrm{E}-03$ \\
\hline 5082 & EXO1 & -4.56 & $1.39 \mathrm{E}-04$ \\
\hline 5083 & EXOC1 & -1.83 & $7.80 \mathrm{E}-03$ \\
\hline 5084 & EXOC3 & -1.46 & $4.93 \mathrm{E}-02$ \\
\hline 5085 & EХOСЗ८4 & -1.84 & $1.40 \mathrm{E}-02$ \\
\hline 5086 & EXOC4 & -4.38 & $9.56 \mathrm{E}-04$ \\
\hline 5087 & EXOC5 & -5.73 & $4.71 \mathrm{E}-06$ \\
\hline 5088 & EXOC6 & -1.13 & $4.57 \mathrm{E}-03$ \\
\hline 5089 & EXOC7 & -4.01 & $3.28 \mathrm{E}-04$ \\
\hline 5090 & EXOC8 & -4.09 & $9.02 E-04$ \\
\hline 5091 & EXOG & -2.52 & $3.84 \mathrm{E}-04$ \\
\hline 5092 & EXOSC1 & -2.01 & $1.60 \mathrm{E}-02$ \\
\hline 5093 & EXOSC10 & -3.85 & $1.66 \mathrm{E}-03$ \\
\hline 5094 & EXOSC2 & -2.48 & $9.66 \mathrm{E}-03$ \\
\hline 5095 & Exoscs & -5.20 & $3.95 \mathrm{E}-04$ \\
\hline
\end{tabular}

\begin{tabular}{|c|c|c|c|}
\hline 5096 & EXOSC7 & -5.11 & $1.52 \mathrm{E}-05$ \\
\hline 5097 & EXOSC9 & -4.41 & $1.71 \mathrm{E}-04$ \\
\hline 5098 & EZH2 & -2.09 & $1.94 \mathrm{E}-03$ \\
\hline 5099 & EZR & -2.71 & $2.05 \mathrm{E}-03$ \\
\hline 5100 & F10 & -1.29 & $1.34 \mathrm{E}-01$ \\
\hline 5101 & F11R & -1.21 & $1.97 \mathrm{E}-03$ \\
\hline 5102 & F2RL1 & -2.23 & $1.42 \mathrm{E}-03$ \\
\hline 5103 & F9 & -4.89 & $2.27 \mathrm{E}-04$ \\
\hline 5104 & FA2H & -1.12 & $4.22 \mathrm{E}-03$ \\
\hline 5105 & FAAH & -2.35 & $6.81 \mathrm{E}-05$ \\
\hline 5106 & FABP6 & -3.55 & $2.53 \mathrm{E}-03$ \\
\hline 5107 & FADS1 & -3.76 & $2.65 \mathrm{E}-04$ \\
\hline 5108 & FAF1 & -2.97 & $9.17 \mathrm{E}-04$ \\
\hline 5109 & FAF2 & \begin{tabular}{|l|}
-2.33 \\
\end{tabular} & $5.54 \mathrm{E}-04$ \\
\hline 5110 & FAHD2A & -5.46 & $2.45 \mathrm{E}-03$ \\
\hline 5111 & FAIM & -5.86 & $2.29 \mathrm{E}-04$ \\
\hline 5112 & FAM102B & -1.86 & $7.67 \mathrm{E}-04$ \\
\hline 5113 & FAM103A1 & -4.13 & $1.44 \mathrm{E}-03$ \\
\hline 5114 & FAM104A & -6.00 & $1.28 \mathrm{E}-04$ \\
\hline 5115 & FAM107A & -2.43 & $8.35 \mathrm{E}-03$ \\
\hline 5116 & FAM108B1 & -4.23 & $4.22 \mathrm{E}-04$ \\
\hline 5117 & FAM114A2 & -3.33 & $1.42 \mathrm{E}-03$ \\
\hline 5118 & FAM118B & -3.58 & $2.54 \mathrm{E}-03$ \\
\hline 5119 & FAM120A & -2.05 & $1.59 \mathrm{E}-03$ \\
\hline 5120 & FAM120B & -3.13 & $4.34 \mathrm{E}-03$ \\
\hline 5121 & FAM122A & -1.59 & $1.29 \mathrm{E}-02$ \\
\hline 5122 & FAM122B & -2.40 & $8.38 \mathrm{E}-04$ \\
\hline 5123 & FAM123B & -1.89 & $2.82 \mathrm{E}-02$ \\
\hline 5124 & FAM125A & -4.57 & $9.63 \mathrm{E}-04$ \\
\hline 5125 & FAM125B & -2.57 & $4.32 \mathrm{E}-04$ \\
\hline 5126 & FAM126A & -8.16 & $5.26 \mathrm{E}-04$ \\
\hline 5127 & FAM129A & -2.39 & $3.98 \mathrm{E}-03$ \\
\hline 5128 & FAM132A & -2.30 & $2.40 \mathrm{E}-02$ \\
\hline 5129 & FAM133B & $\begin{array}{l}-6.37 \\
\end{array}$ & $1.54 \mathrm{E}-04$ \\
\hline 5130 & FAM134A & -5.26 & $7.53 \mathrm{E}-04$ \\
\hline 5131 & FAM134C & -2.46 & $2.37 \mathrm{E}-03$ \\
\hline 5132 & FAM135A & -4.02 & $8.32 \mathrm{E}-05$ \\
\hline 5133 & FAM13A & -1.61 & $2.19 \mathrm{E}-03$ \\
\hline 5134 & FAM13B & -4.26 & $1.52 \mathrm{E}-03$ \\
\hline 5135 & FAM149B1 & -2.42 & $1.95 \mathrm{E}-03$ \\
\hline 5136 & FAM150B & -2.99 & $2.59 \mathrm{E}-03$ \\
\hline 5137 & FAM162A & -3.37 & $8.50 \mathrm{E}-04$ \\
\hline 5138 & FAM162B & -4.29 & $6.70 \mathrm{E}-04$ \\
\hline 5139 & FAM164C & -6.03 & $2.20 \mathrm{E}-03$ \\
\hline 5140 & FAM165B & -4.56 & $2.65 \mathrm{E}-03$ \\
\hline 5141 & FAM166A & -1.86 & $1.80 \mathrm{E}-03$ \\
\hline 5142 & FAM169B & -2.86 & $6.18 \mathrm{E}-03$ \\
\hline 5143 & FAM171B & $\begin{array}{l}-5.54 \\
\end{array}$ & $4.33 \mathrm{E}-03$ \\
\hline 5144 & FAM173A & -3.44 & $4.94 \mathrm{E}-04$ \\
\hline 5145 & FAM174A & -5.11 & $1.66 \mathrm{E}-04$ \\
\hline 5146 & FAM174B & -1.04 & $1.23 \mathrm{E}-02$ \\
\hline 5147 & FAM175A & -2.18 & $1.30 \mathrm{E}-04$ \\
\hline 5148 & Fam175b & -2.80 & $1.81 \mathrm{E}-04$ \\
\hline 5149 & FAM177A1 & -4.94 & $7.00 \mathrm{E}-05$ \\
\hline 5150 & FAM179B & -2.40 & $6.48 \mathrm{E}-03$ \\
\hline 5151 & FAM183A & $\begin{array}{l}-7.74 \\
\end{array}$ & $1.29 \mathrm{E}-03$ \\
\hline 5152 & FAM184A & -4.27 & $6.27 \mathrm{E}-04$ \\
\hline 5153 & FAM185A & -2.82 & $3.44 \mathrm{E}-03$ \\
\hline 5154 & FAM188A & -2.43 & $4.35 \mathrm{E}-04$ \\
\hline 5155 & FAM18A & $\begin{array}{l}-7.77 \\
\end{array}$ & $5.07 \mathrm{E}-05$ \\
\hline 5156 & FAM192A & -5.42 & $9.46 \mathrm{E}-05$ \\
\hline 5157 & FAM193A & -3.13 & $1.83 \mathrm{E}-03$ \\
\hline 5158 & FAM194B & -6.02 & $4.50 \mathrm{E}-05$ \\
\hline
\end{tabular}

\begin{tabular}{|c|c|c|c|}
\hline 5159 & FAM19A1 & -1.31 & $2.02 \mathrm{E}-02$ \\
\hline 5160 & FAM204A & -6.25 & $7.41 \mathrm{E}-04$ \\
\hline 5161 & FAM206A & -6.16 & $1.46 \mathrm{E}-03$ \\
\hline 5162 & FAM207A & -4.23 & $4.51 \mathrm{E}-04$ \\
\hline 5163 & FAM208A & -3.10 & $4.45 \mathrm{E}-04$ \\
\hline 5164 & FAM20B & -3.59 & $5.65 \mathrm{E}-04$ \\
\hline 5165 & FAM210A & -3.64 & $5.49 \mathrm{E}-04$ \\
\hline 5166 & FAM213A & -4.48 & $3.89 \mathrm{E}-03$ \\
\hline 5167 & FAM214A & -3.64 & $1.72 \mathrm{E}-04$ \\
\hline 5168 & FAM21A & -3.19 & $7.17 \mathrm{E}-04$ \\
\hline 5169 & FAM32A & $\begin{array}{l}-7.14 \\
\end{array}$ & $6.08 \mathrm{E}-04$ \\
\hline 5170 & FAM3B & -2.24 & $4.12 \mathrm{E}-03$ \\
\hline 5171 & FAM3C & -2.73 & $3.77 \mathrm{E}-03$ \\
\hline 5172 & FAM45A & -1.35 & $4.31 \mathrm{E}-03$ \\
\hline 5173 & FAM46D & -7.27 & $1.12 \mathrm{E}-03$ \\
\hline 5174 & FAM48A & -1.75 & $2.69 \mathrm{E}-03$ \\
\hline 5175 & FAM49A & -3.38 & $2.60 \mathrm{E}-04$ \\
\hline 5176 & FAM49B & -3.44 & $7.02 \mathrm{E}-04$ \\
\hline 5177 & FAM53A & -5.58 & $1.22 \mathrm{E}-03$ \\
\hline 5178 & FAM60A & -2.20 & $4.75 \mathrm{E}-03$ \\
\hline 5179 & FAM62C & -1.31 & $1.08 \mathrm{E}-02$ \\
\hline 5180 & FAM63B & -2.69 & $\begin{array}{l}1.17 \mathrm{E}-03 \\
\end{array}$ \\
\hline 5181 & FAM64A & -4.74 & $2.41 \mathrm{E}-04$ \\
\hline 5182 & FAM65B & -2.09 & $8.66 \mathrm{E}-04$ \\
\hline 5183 & FAM69A & -2.65 & $9.13 \mathrm{E}-04$ \\
\hline $\begin{array}{l}5184 \\
\end{array}$ & FAM69C & -1.34 & $4.19 \mathrm{E}-03$ \\
\hline 5185 & FAM70A & -1.58 & $2.84 \mathrm{E}-03$ \\
\hline 5186 & FAM70B & -1.26 & $2.52 \mathrm{E}-02$ \\
\hline 5187 & FAM72A & -3.88 & $8.73 \mathrm{E}-05$ \\
\hline 5188 & FAM73A & -1.80 & $2.82 \mathrm{E}-03$ \\
\hline 5189 & FAM76A & -3.67 & $2.83 \mathrm{E}-05$ \\
\hline 5190 & FAM76B & -1.32 & $4.77 \mathrm{E}-03$ \\
\hline 5191 & FAM81A & -3.26 & $1.99 \mathrm{E}-03$ \\
\hline 5192 & FAM82B & -5.73 & $4.52 \mathrm{E}-04$ \\
\hline 5193 & FAM83A & -1.46 & $2.85 \mathrm{E}-03$ \\
\hline 5194 & FAM83D & -1.33 & $4.57 \mathrm{E}-02$ \\
\hline 5195 & FAM $84 \mathrm{~A}$ & -2.87 & $3.78 \mathrm{E}-02$ \\
\hline 5196 & FAM8A1 & -1.33 & $1.47 \mathrm{E}-02$ \\
\hline 5197 & FAM91A1 & -3.27 & $3.21 \mathrm{E}-04$ \\
\hline 5198 & FAM92A1 & -6.88 & $9.60 \mathrm{E}-05$ \\
\hline 5199 & FAM96A & -6.66 & $1.65 \mathrm{E}-06$ \\
\hline 5200 & FAM96B & -5.09 & $2.71 \mathrm{E}-04$ \\
\hline 5201 & FAM98A & -3.97 & $2.26 \mathrm{E}-03$ \\
\hline 5202 & FANCA & -4.30 & $1.06 \mathrm{E}-04$ \\
\hline 5203 & FANCC & -4.03 & $1.39 \mathrm{E}-03$ \\
\hline 5204 & FANCE & -1.94 & $3.78 \mathrm{E}-02$ \\
\hline 5205 & FANCG & -2.54 & $2.03 \mathrm{E}-02$ \\
\hline 5206 & FANCI & -1.60 & $4.21 \mathrm{E}-04$ \\
\hline 5207 & FANCL & -5.74 & $4.21 \mathrm{E}-04$ \\
\hline 5208 & FAR1 & -2.38 & $8.58 \mathrm{E}-04$ \\
\hline 5209 & FARS2 & -3.21 & $1.95 \mathrm{E}-03$ \\
\hline 5210 & FARSA & -3.14 & $8.46 \mathrm{E}-03$ \\
\hline 5211 & FARSB & -3.50 & $1.20 \mathrm{E}-04$ \\
\hline 5212 & FASN & -3.04 & $3.51 \mathrm{E}-04$ \\
\hline 5213 & FASTKD2 & -3.37 & $1.60 \mathrm{E}-04$ \\
\hline 5214 & FASTKD3 & -2.02 & $4.78 \mathrm{E}-03$ \\
\hline 5215 & FBF1 & -4.56 & $3.11 \mathrm{E}-03$ \\
\hline 5216 & FBLN1 & -1.95 & $4.39 \mathrm{E}-02$ \\
\hline 5217 & FBXL13 & -5.21 & $9.36 \mathrm{E}-04$ \\
\hline 5218 & FBXL18 & -1.14 & $5.53 \mathrm{E}-03$ \\
\hline 5219 & FBXL2 & -2.50 & $1.35 \mathrm{E}-03$ \\
\hline 5220 & FBXL20 & -1.97 & $7.10 \mathrm{E}-03$ \\
\hline 5221 & FBXL21 & -2.84 & $1.12 \mathrm{E}-03$ \\
\hline
\end{tabular}




\begin{tabular}{|c|c|c|c|}
\hline 5222 & FBXL3 & -3.79 & $1.24 \mathrm{E}-03$ \\
\hline 5223 & FBXL5 & -3.71 & $2.89 \mathrm{E}-03$ \\
\hline 5224 & FBXL7 & -1.70 & $1.55 \mathrm{E}-01$ \\
\hline 5225 & FBXL8 & -2.02 & $2.27 \mathrm{E}-03$ \\
\hline 5226 & FBX011 & -1.98 & $3.05 E-03$ \\
\hline 5227 & FBX015 & -5.17 & $1.90 \mathrm{E}-03$ \\
\hline 5228 & FBX016 & -6.21 & $2.60 \mathrm{E}-03$ \\
\hline 5229 & FBX018 & -1.59 & $1.21 \mathrm{E}-02$ \\
\hline 5230 & FBXO21 & -5.97 & $3.02 \mathrm{E}-04$ \\
\hline 5231 & FBXO22 & \begin{tabular}{l|l|}
-2.67 \\
\end{tabular} & $8.85 \mathrm{E}-04$ \\
\hline 5232 & FBX025 & -3.02 & $1.52 \mathrm{E}-03$ \\
\hline 5233 & $\mathrm{FBX028}$ & -2.90 & $3.23 \mathrm{E}-03$ \\
\hline 5234 & FBX030 & -1.17 & $2.83 \mathrm{E}-03$ \\
\hline 5235 & FBXO34 & -4.43 & $3.29 \mathrm{E}-06$ \\
\hline 5236 & FBX039 & -5.91 & $1.33 \mathrm{E}-04$ \\
\hline 5237 & FBXO4 & $\begin{array}{l}-1.67 \\
\end{array}$ & $1.80 \mathrm{E}-02$ \\
\hline 5238 & FBXO43 & -4.13 & $8.90 \mathrm{E}-04$ \\
\hline 5239 & FBX047 & -1.65 & $8.56 \mathrm{E}-03$ \\
\hline 5240 & $\mathrm{FBX048}$ & -5.12 & $3.54 \mathrm{E}-05$ \\
\hline 5241 & FBXO6 & -2.52 & $1.34 \mathrm{E}-02$ \\
\hline 5242 & FBXO7 & -7.18 & $2.35 \mathrm{E}-05$ \\
\hline 5243 & $\begin{array}{l}\text { FBXO8 } \\
\end{array}$ & $\begin{array}{l}-2.99 \\
\end{array}$ & $1.56 \mathrm{E}-03$ \\
\hline 5244 & FBXO9 & -3.65 & $7.69 \mathrm{E}-04$ \\
\hline 5245 & FBXW11 & -2.58 & $6.62 \mathrm{E}-05$ \\
\hline 5246 & FBXW5 & -1.91 & $1.84 \mathrm{E}-02$ \\
\hline 5247 & FBXW7 & -2.67 & $1.44 \mathrm{E}-03$ \\
\hline 5248 & FBXW8 & -2.27 & $8.05 \mathrm{E}-03$ \\
\hline 5249 & FCF1 & -5.18 & $1.74 \mathrm{E}-04$ \\
\hline 5250 & $\mathrm{FCHO} 2$ & -1.44 & $1.92 \mathrm{E}-02$ \\
\hline 5251 & FCHSD2 & -1.39 & $1.35 \mathrm{E}-02$ \\
\hline 5252 & FCN2 & -3.60 & $1.21 \mathrm{E}-03$ \\
\hline 5253 & FDFT1 & -4.76 & $1.11 \mathrm{E}-02$ \\
\hline 5254 & FDX1 & -5.81 & $2.96 \mathrm{E}-05$ \\
\hline 5255 & FEM1B & -5.28 & $1.18 \mathrm{E}-04$ \\
\hline 5256 & FEN1 & -3.41 & $7.76 \mathrm{E}-03$ \\
\hline 5257 & FER & -2.59 & $2.15 \mathrm{E}-03$ \\
\hline 5258 & FER1L5 & -4.50 & $4.89 \mathrm{E}-03$ \\
\hline 5259 & FERMT1 & -5.55 & $2.33 \mathrm{E}-04$ \\
\hline 5260 & FEZ2 & -4.04 & $2.12 \mathrm{E}-03$ \\
\hline 5261 & FGB & -3.20 & $2.12 \mathrm{E}-03$ \\
\hline 5262 & FGD3 & -1.42 & $2.70 \mathrm{E}-03$ \\
\hline 5263 & $\begin{array}{l}\text { FGF14 } \\
\end{array}$ & -1.62 & $1.26 \mathrm{E}-02$ \\
\hline 5264 & FGF18 & -2.07 & $8.24 E-02$ \\
\hline 5265 & FGF19 & -1.72 & $5.12 \mathrm{E}-03$ \\
\hline 5266 & FGF2 & -3.09 & $7.72 E-03$ \\
\hline 5267 & FGFBP2 & -3.15 & $3.83 \mathrm{E}-03$ \\
\hline 5268 & FGFR1OP & -3.12 & $2.23 \mathrm{E}-03$ \\
\hline 5269 & FGFR1OP2 & -4.13 & $\begin{array}{l}7.64 \mathrm{E}-04 \\
\end{array}$ \\
\hline 5270 & FGFRL1 & -2.50 & $8.71 E-05$ \\
\hline 5271 & FGG & -2.22 & $4.86 \mathrm{E}-04$ \\
\hline 5272 & FGGY & -4.24 & $5.40 \mathrm{E}-04$ \\
\hline 5273 & FGL1 & -3.17 & $1.05 E-02$ \\
\hline 5274 & $\mathrm{FH}$ & -6.92 & $5.93 \mathrm{E}-04$ \\
\hline 5275 & FHIT & -4.15 & $2.32 \mathrm{E}-04$ \\
\hline 5276 & FHL2 & -1.45 & $6.32 E-03$ \\
\hline 5277 & FIBIN & -1.39 & $3.34 \mathrm{E}-03$ \\
\hline 5278 & FICD & -2.37 & $3.62 \mathrm{E}-04$ \\
\hline 5279 & FIG4 & -2.02 & $8.35 \mathrm{E}-03$ \\
\hline 5280 & FIGNL1 & -4.67 & $1.76 \mathrm{E}-04$ \\
\hline 5281 & FIP1L1 & -3.66 & $5.74 \mathrm{E}-03$ \\
\hline 5282 & FITM2 & -1.45 & $8.76 E-03$ \\
\hline 5283 & FKBP14 & -4.22 & $8.57 \mathrm{E}-04$ \\
\hline 5284 & FKBP1A & -5.75 & $4.15 \mathrm{E}-03$ \\
\hline
\end{tabular}

\begin{tabular}{|c|c|c|c|}
\hline 5285 & FKBP3 & -7.65 & $7.29 \mathrm{E}-05$ \\
\hline 5286 & FKBP4 & -6.12 & $2.73 \mathrm{E}-04$ \\
\hline 5287 & FKBP5 & -1.80 & $7.43 \mathrm{E}-02$ \\
\hline 5288 & FKBP6 & -3.11 & $2.53 \mathrm{E}-02$ \\
\hline 5289 & FKBP7 & -1.84 & $1.17 \mathrm{E}-02$ \\
\hline 5290 & FKBP9 & -2.64 & $6.04 \mathrm{E}-03$ \\
\hline 5291 & FLCN & -2.37 & $4.37 \mathrm{E}-04$ \\
\hline 5292 & FMN1 & -1.99 & $6.74 \mathrm{E}-04$ \\
\hline 5293 & FMOD & -1.51 & $8.23 \mathrm{E}-02$ \\
\hline 5294 & FMR1 & -4.28 & $2.09 \mathrm{E}-04$ \\
\hline 5295 & FN3KRP & -4.33 & $4.96 \mathrm{E}-04$ \\
\hline 5296 & FNBP1L & -1.26 & $1.94 \mathrm{E}-03$ \\
\hline 5297 & FNBP4 & -4.81 & $1.55 \mathrm{E}-04$ \\
\hline 5298 & FNDC3A & -6.95 & $1.05 \mathrm{E}-04$ \\
\hline 5299 & FNDC7 & -5.55 & $1.12 \mathrm{E}-03$ \\
\hline 5300 & FNTA & -7.36 & $1.56 \mathrm{E}-04$ \\
\hline 5301 & FOCAD & -1.35 & $4.55 \mathrm{E}-03$ \\
\hline 5302 & FOPNL & -6.34 & $2.84 \mathrm{E}-04$ \\
\hline 5303 & FOXK2 & -4.70 & $6.02 \mathrm{E}-03$ \\
\hline 5304 & FOXN3 & -4.76 & $2.34 \mathrm{E}-04$ \\
\hline 5305 & FRA10AC1 & -5.63 & $4.94 \mathrm{E}-04$ \\
\hline 5306 & FRG1 & -3.22 & $1.33 \mathrm{E}-03$ \\
\hline 5307 & FRMD3 & -2.03 & $3.32 \mathrm{E}-02$ \\
\hline 5308 & FRS2 & -5.57 & $4.20 \mathrm{E}-04$ \\
\hline 5309 & FRZB & -1.45 & $5.60 \mathrm{E}-04$ \\
\hline 5310 & FSCN2 & -1.12 & $1.20 \mathrm{E}-02$ \\
\hline 5311 & FSD1L & -7.63 & $1.52 \mathrm{E}-04$ \\
\hline 5312 & FSHR & -2.35 & $2.55 \mathrm{E}-03$ \\
\hline 5313 & FSIP1 & -2.63 & $3.33 \mathrm{E}-02$ \\
\hline 5314 & FTH1 & -5.99 & $1.37 \mathrm{E}-03$ \\
\hline 5315 & FTSJ2 & -2.17 & $3.15 \mathrm{E}-02$ \\
\hline 5316 & FTSJ3 & -3.94 & $2.21 \mathrm{E}-04$ \\
\hline 5317 & FUBP1 & -4.35 & $5.62 \mathrm{E}-04$ \\
\hline 5318 & FUCA1 & -3.77 & $2.73 \mathrm{E}-04$ \\
\hline 5319 & FUNDC1 & -7.26 & $1.12 \mathrm{E}-03$ \\
\hline 5320 & FUS & -2.57 & $2.96 \mathrm{E}-03$ \\
\hline 5321 & FUT10 & -3.20 & $1.34 \mathrm{E}-03$ \\
\hline 5322 & FUT11 & -3.81 & $1.62 \mathrm{E}-04$ \\
\hline 5323 & FUT8 & -1.62 & $1.89 \mathrm{E}-03$ \\
\hline 5324 & FXN & -4.35 & $2.89 \mathrm{E}-04$ \\
\hline 5325 & FXR1 & -4.45 & $4.59 \mathrm{E}-04$ \\
\hline 5326 & FXYD6 & -1.85 & $4.35 \mathrm{E}-04$ \\
\hline 5327 & FYCO1 & -2.09 & $3.64 \mathrm{E}-03$ \\
\hline 5328 & FYTTD1 & -4.99 & $1.45 \mathrm{E}-03$ \\
\hline 5329 & FZD3 & -4.98 & $3.55 \mathrm{E}-04$ \\
\hline 5330 & FZD6 & -2.24 & $2.96 \mathrm{E}-03$ \\
\hline 5331 & FZD9 & -1.78 & $2.12 \mathrm{E}-03$ \\
\hline 5332 & G2E3 & -2.50 & $2.32 \mathrm{E}-04$ \\
\hline 5333 & G3BP1 & -3.79 & $6.91 \mathrm{E}-04$ \\
\hline 5334 & G3BP2 & -3.68 & $4.98 \mathrm{E}-03$ \\
\hline 5335 & GABARAPL2 & -4.92 & $3.24 \mathrm{E}-04$ \\
\hline 5336 & GABPA & -1.05 & $1.84 \mathrm{E}-03$ \\
\hline 5337 & GABPB1 & -1.85 & $3.76 \mathrm{E}-03$ \\
\hline 5338 & GABRA4 & -2.22 & $3.27 \mathrm{E}-03$ \\
\hline 5339 & GADD45A & -2.80 & $1.69 \mathrm{E}-02$ \\
\hline 5340 & GADD45B & -3.71 & $1.35 \mathrm{E}-04$ \\
\hline 5341 & GALC & -1.43 & $1.94 \mathrm{E}-02$ \\
\hline 5342 & GALK2 & -3.54 & $5.00 \mathrm{E}-04$ \\
\hline 5343 & GALM & -1.29 & $1.19 \mathrm{E}-02$ \\
\hline 5344 & GALNT1 & -2.88 & $3.67 \mathrm{E}-03$ \\
\hline 5345 & GALNT11 & -1.47 & $5.24 \mathrm{E}-03$ \\
\hline 5346 & GALNT3 & $\begin{array}{l}-1.53 \\
\end{array}$ & $6.47 \mathrm{E}-03$ \\
\hline 5347 & GALNT7 & -2.04 & $7.40 \mathrm{E}-03$ \\
\hline
\end{tabular}

\begin{tabular}{|c|c|c|c|}
\hline 5348 & GALNT9 & -1.37 & $4.53 E-04$ \\
\hline 5349 & GALNTL1 & -1.77 & $9.20 \mathrm{E}-03$ \\
\hline 5350 & GALNTL4 & $\begin{array}{l}-1.46 \\
\end{array}$ & $2.85 \mathrm{E}-02$ \\
\hline 5351 & GAN & -1.16 & $1.34 \mathrm{E}-01$ \\
\hline 5352 & GAPDH & \begin{tabular}{|l|}
-7.51 \\
\end{tabular} & $1.54 \mathrm{E}-04$ \\
\hline 5353 & GAPVD1 & -3.92 & $2.69 \mathrm{E}-04$ \\
\hline 5354 & GAR1 & -1.07 & $8.24 \mathrm{E}-04$ \\
\hline 5355 & GARNL3 & -3.07 & $9.10 E-03$ \\
\hline 5356 & GARS & -4.16 & $1.84 \mathrm{E}-03$ \\
\hline 5357 & GART & -2.82 & $4.20 \mathrm{E}-03$ \\
\hline 5358 & GAS6 & -4.68 & $1.16 \mathrm{E}-03$ \\
\hline 5359 & GAS8 & -5.72 & $1.53 \mathrm{E}-04$ \\
\hline 5360 & GATA1 & -1.91 & $7.59 \mathrm{E}-03$ \\
\hline 5361 & GATAD1 & -4.69 & $4.93 E-04$ \\
\hline 5362 & GATAD2A & -4.15 & $6.35 E-04$ \\
\hline 5363 & GATC & -1.45 & $6.04 E-02$ \\
\hline 5364 & GATSL2 & -3.33 & $2.19 \mathrm{E}-03$ \\
\hline 5365 & GBAS & -5.11 & $1.42 \mathrm{E}-03$ \\
\hline 5366 & GBE & -3.30 & $4.11 E-02$ \\
\hline 5367 & GBF1 & -4.49 & $8.84 E-05$ \\
\hline 5368 & GBGT1 & -2.26 & $3.07 E-04$ \\
\hline 5369 & GC & -1.17 & $4.70 E-03$ \\
\hline 5370 & GC1 & -1.49 & $6.97 E-02$ \\
\hline 5371 & GCAT & -2.83 & $1.40 E-03$ \\
\hline 5372 & GCC2 & -2.16 & $1.45 E-03$ \\
\hline 5373 & GCFC1 & \begin{tabular}{|l|}
-1.49 \\
\end{tabular} & $3.08 E-02$ \\
\hline 5374 & GCG & \begin{tabular}{l|l|}
-2.12 \\
\end{tabular} & $3.46 \mathrm{E}-03$ \\
\hline 5375 & GCH1 & -2.08 & $9.83 \mathrm{E}-04$ \\
\hline 5376 & GCLC & -4.23 & $6.75 E-04$ \\
\hline 5377 & GCNT2 & -3.03 & $4.41 E-02$ \\
\hline 5378 & GCOM1 & -4.44 & $3.91 \mathrm{E}-04$ \\
\hline 5379 & GCSH & -3.23 & $2.79 E-03$ \\
\hline 5380 & GDF10 & -1.93 & $9.23 \mathrm{E}-04$ \\
\hline 5381 & GDI2 & -3.30 & $8.29 E-04$ \\
\hline 5382 & GDPD1 & -4.52 & $7.56 \mathrm{E}-04$ \\
\hline 5383 & GEMIN4 & $\begin{array}{l}-2.58 \\
\end{array}$ & $3.74 E-05$ \\
\hline 5384 & GEMIN5 & -1.62 & $3.18 \mathrm{E}-03$ \\
\hline 5385 & GEMIN6 & -5.37 & $4.59 \mathrm{E}-04$ \\
\hline 5386 & GET4 & -4.72 & $1.40 \mathrm{E}-04$ \\
\hline 5387 & GFER & -3.42 & $9.80 E-04$ \\
\hline 5388 & GFI1 & -3.79 & $1.11 \mathrm{E}-04$ \\
\hline 5389 & GFM1 & \begin{tabular}{|l|}
-3.37 \\
\end{tabular} & $2.98 E-04$ \\
\hline 5390 & GFPT1 & -2.60 & $1.94 E-03$ \\
\hline 5391 & GGA3 & -3.46 & $9.10 \mathrm{E}-04$ \\
\hline 5392 & GGCL1 & -1.56 & $1.31 E-03$ \\
\hline 5393 & GGNBP2 & -8.46 & $3.00 E-04$ \\
\hline 5394 & GGPS1 & -2.72 & $1.88 \mathrm{E}-04$ \\
\hline 5395 & GGT1 & \begin{tabular}{|l|}
-3.06 \\
\end{tabular} & $7.19 E-04$ \\
\hline 5396 & GHITM & -5.77 & $1.99 \mathrm{E}-03$ \\
\hline 5397 & GID8 & -1.64 & $1.20 \mathrm{E}-02$ \\
\hline 5398 & GIN1 & -1.63 & $3.41 E-03$ \\
\hline 5399 & GINM1 & -4.76 & $1.10 \mathrm{E}-03$ \\
\hline 5400 & GINS1 & -2.50 & $1.00 E-02$ \\
\hline 5401 & GIP & -1.19 & $9.59 \mathrm{E}-03$ \\
\hline 5402 & GIT2 & -2.18 & $1.28 \mathrm{E}-04$ \\
\hline 5403 & GJA1 & -2.57 & $6.42 E-03$ \\
\hline 5404 & GJC1 & -3.39 & $1.42 \mathrm{E}-03$ \\
\hline 5405 & GK & -6.03 & $5.46 E-05$ \\
\hline 5406 & GK5 & -5.74 & $3.70 E-04$ \\
\hline 5407 & GKAP1 & -8.79 & $3.00 E-05$ \\
\hline 5408 & GLB1L & -2.16 & $6.28 \mathrm{E}-03$ \\
\hline 5409 & GLCCI1 & -1.51 & $2.85 E-02$ \\
\hline 410 & GLG & -2.23 & 1.67E-03 \\
\hline
\end{tabular}




\begin{tabular}{|c|c|c|c|}
\hline 5411 & GLIPR1L2 & -7.86 & $2.56 \mathrm{E}-04$ \\
\hline 5412 & GLO1 & -3.27 & $5.80 \mathrm{E}-03$ \\
\hline 5413 & GLP1R & -2.69 & $2.19 \mathrm{E}-02$ \\
\hline 5414 & GLRX3 & -6.64 & $1.72 \mathrm{E}-04$ \\
\hline 5415 & GLRX5 & -2.81 & $1.88 \mathrm{E}-03$ \\
\hline 5416 & GLT1D1 & -1.89 & $1.77 \mathrm{E}-02$ \\
\hline 5417 & GLT8D1 & -7.31 & 3.39E-05 \\
\hline 5418 & GLTP & -2.67 & $2.00 \mathrm{E}-02$ \\
\hline 5419 & GLTPD1 & -1.32 & $8.23 \mathrm{E}-03$ \\
\hline 5420 & GLUL & -2.30 & $3.27 \mathrm{E}-03$ \\
\hline 5421 & GLYR1 & -2.03 & $3.31 \mathrm{E}-03$ \\
\hline 5422 & GMCL1 & -1.27 & 4.79E-02 \\
\hline 5423 & GMFB & -3.36 & $4.73 \mathrm{E}-03$ \\
\hline 5424 & GMNN & -4.85 & 5.83E-04 \\
\hline 5425 & GMPPB & -2.45 & $2.50 \mathrm{E}-03$ \\
\hline 5426 & GMPR & -4.50 & $1.34 \mathrm{E}-03$ \\
\hline 5427 & GMPS & -5.94 & $4.05 \mathrm{E}-03$ \\
\hline 5428 & GNA11 & -3.37 & $6.89 \mathrm{E}-04$ \\
\hline 5429 & GNA13 & -2.25 & 4.97E-03 \\
\hline 5430 & GNAI1 & -3.73 & $1.70 \mathrm{E}-03$ \\
\hline 5431 & GNAI3 & -3.07 & $3.38 \mathrm{E}-03$ \\
\hline 5432 & GNAL & -3.71 & 2.89E-03 \\
\hline 5433 & GNAS & -3.84 & $2.55 \mathrm{E}-03$ \\
\hline 5434 & GNAT2 & -2.04 & 3.68E-02 \\
\hline 5435 & GNAT3 & -2.41 & 5.43E-03 \\
\hline 5436 & GNAZ & -1.59 & 4.35E-03 \\
\hline 5437 & GNB1 & -4.42 & $9.69 \mathrm{E}-04$ \\
\hline 5438 & GNB1L & -3.89 & $2.93 \mathrm{E}-03$ \\
\hline 5439 & GNB2L1 & -7.24 & $1.35 \mathrm{E}-04$ \\
\hline 5440 & GNE & -3.65 & 2.37E-04 \\
\hline 5441 & GNG12 & -4.01 & $8.98 \mathrm{E}-04$ \\
\hline 5442 & GNG4 & -7.29 & $3.44 \mathrm{E}-04$ \\
\hline 5443 & GNG5 & -6.92 & 4.69E-05 \\
\hline 5444 & GNL2 & -6.51 & 9.50E-07 \\
\hline 5445 & GNL3 & -5.19 & 5.62E-05 \\
\hline 5446 & GNMT & -1.19 & $8.72 \mathrm{E}-03$ \\
\hline 5447 & GNPAT & -3.34 & $3.26 \mathrm{E}-03$ \\
\hline 5448 & GNPDA1 & -3.40 & $3.70 \mathrm{E}-04$ \\
\hline 5449 & GNPDA2 & -3.58 & $1.53 \mathrm{E}-04$ \\
\hline 5450 & GNPNAT1 & -3.92 & 9.56E-04 \\
\hline 5451 & GNPTG & -1.42 & $3.65 \mathrm{E}-03$ \\
\hline 5452 & GNRH1 & -1.54 & $2.12 \mathrm{E}-03$ \\
\hline 5453 & GNS & -4.44 & $1.59 \mathrm{E}-03$ \\
\hline 5454 & GOLGA1 & -3.47 & $2.28 \mathrm{E}-04$ \\
\hline 5455 & GOLGA3 & -4.13 & $1.09 \mathrm{E}-02$ \\
\hline 5456 & GOLGA4 & -2.91 & 4.60E-04 \\
\hline 5457 & GOLGA5 & -1.75 & 3.55E-04 \\
\hline 5458 & GOLGA7 & -5.12 & $1.38 \mathrm{E}-04$ \\
\hline 5459 & GOLIM4 & -1.88 & $1.05 \mathrm{E}-02$ \\
\hline 5460 & GOLM1 & -6.87 & $3.12 \mathrm{E}-04$ \\
\hline 5461 & GOLT1B & -2.38 & $3.15 \mathrm{E}-03$ \\
\hline 5462 & GON4L & -4.35 & $6.49 \mathrm{E}-06$ \\
\hline 5463 & GOPC & -3.08 & $6.39 \mathrm{E}-03$ \\
\hline 5464 & GORAB & -4.26 & $1.20 \mathrm{E}-03$ \\
\hline 5465 & GORASP1 & -2.18 & $2.44 \mathrm{E}-03$ \\
\hline 5466 & GORASP2 & -4.58 & $1.16 \mathrm{E}-04$ \\
\hline 5467 & GOSR1 & -2.89 & $2.26 \mathrm{E}-04$ \\
\hline 5468 & GOT1 & -7.05 & $9.41 \mathrm{E}-05$ \\
\hline 5469 & GOT2 & -1.94 & 4.64E-03 \\
\hline 5470 & GPAT2 & -4.98 & $3.53 \mathrm{E}-05$ \\
\hline 5471 & GPATCH1 & -2.52 & $1.41 \mathrm{E}-03$ \\
\hline 5472 & GPATCH2L & -1.68 & 7.95E-04 \\
\hline 5473 & GPBP1 & -2.89 & 2.64E-04 \\
\hline
\end{tabular}

\begin{tabular}{|c|c|c|c|}
\hline 5474 & GPBP1L1 & -2.41 & 4.60E-03 \\
\hline 5475 & GPC6 & -1.59 & $5.96 \mathrm{E}-02$ \\
\hline 5476 & GPCPD1 & -5.74 & $3.65 \mathrm{E}-04$ \\
\hline 5477 & GPD1 & -6.62 & $1.22 \mathrm{E}-05$ \\
\hline 5478 & GPD2 & -2.25 & $4.01 \mathrm{E}-02$ \\
\hline 5479 & GPI & -5.34 & $3.10 \mathrm{E}-04$ \\
\hline 5480 & GPN2 & -3.71 & $1.13 \mathrm{E}-03$ \\
\hline 5481 & GPN3 & -5.22 & $2.15 \mathrm{E}-03$ \\
\hline 5482 & GPR107 & -2.37 & $6.70 \mathrm{E}-03$ \\
\hline 5483 & GPR137B & -3.24 & $5.11 \mathrm{E}-03$ \\
\hline 5484 & GPR149 & -3.48 & $8.27 \mathrm{E}-04$ \\
\hline 5485 & GPR155 & -2.80 & $3.16 \mathrm{E}-03$ \\
\hline 5486 & GPR158 & -1.51 & $6.15 \mathrm{E}-03$ \\
\hline 5487 & GPR160 & -2.28 & $1.80 \mathrm{E}-03$ \\
\hline 5488 & GPR162 & -2.27 & $1.35 \mathrm{E}-02$ \\
\hline 5489 & GPR176 & -2.47 & $1.09 \mathrm{E}-04$ \\
\hline 5490 & GPR37 & -2.20 & $1.02 \mathrm{E}-02$ \\
\hline 5491 & GPS1 & -5.73 & $1.02 \mathrm{E}-04$ \\
\hline 5492 & GPSM2 & -4.38 & 7.00E-04 \\
\hline 5493 & GPX3 & -4.66 & $4.23 \mathrm{E}-04$ \\
\hline 5494 & GPX4 & -4.93 & $2.87 \mathrm{E}-03$ \\
\hline 5495 & GPX8 & -1.96 & $1.07 \mathrm{E}-02$ \\
\hline 5496 & GQ365760 & -1.32 & $1.03 \mathrm{E}-02$ \\
\hline 5497 & GRAMD1C & -5.44 & 4.01E-04 \\
\hline 5498 & GRAMD3 & -1.59 & 7.00E-03 \\
\hline 5499 & GRB10 & -5.13 & $9.55 \mathrm{E}-04$ \\
\hline 5500 & GRB14 & -2.59 & 4.27E-03 \\
\hline 5501 & GRB2 & -3.22 & $1.20 \mathrm{E}-03$ \\
\hline 5502 & GRHL1 & -5.70 & $5.45 \mathrm{E}-04$ \\
\hline 5503 & GRHPR & -1.63 & $3.60 \mathrm{E}-03$ \\
\hline 5504 & GRIN2B & -2.30 & $2.57 \mathrm{E}-03$ \\
\hline 5505 & GRK4 & -4.72 & $1.26 \mathrm{E}-04$ \\
\hline 5506 & GRK5 & -1.51 & $1.05 \mathrm{E}-03$ \\
\hline 5507 & GRK6 & -1.06 & $6.03 E-03$ \\
\hline 5508 & GRPEL1 & -2.54 & $3.11 \mathrm{E}-03$ \\
\hline 5509 & GRSF1 & -7.37 & $2.53 \mathrm{E}-04$ \\
\hline 5510 & GRTP1 & -4.36 & $6.41 \mathrm{E}-04$ \\
\hline 5511 & GSK3B & -4.14 & $2.28 \mathrm{E}-03$ \\
\hline 5512 & GSN & -2.94 & $9.22 \mathrm{E}-05$ \\
\hline 5513 & GSPT1 & -2.27 & $1.82 \mathrm{E}-03$ \\
\hline 5514 & GSR & -3.44 & $1.63 \mathrm{E}-04$ \\
\hline 5515 & GSTA & -3.06 & $8.96 \mathrm{E}-03$ \\
\hline 5516 & GSTA1 & -1.06 & $2.57 \mathrm{E}-01$ \\
\hline 5517 & GSTCD & -1.74 & $2.72 \mathrm{E}-02$ \\
\hline 5518 & GSTK1 & -3.24 & $1.88 \mathrm{E}-03$ \\
\hline 5519 & GSTM2 & -1.44 & $9.59 \mathrm{E}-03$ \\
\hline 5520 & GSTO1 & -5.18 & $4.68 \mathrm{E}-04$ \\
\hline 5521 & GSTT1 & -1.45 & $1.19 \mathrm{E}-02$ \\
\hline 5522 & GSTZ1 & -2.84 & $4.11 \mathrm{E}-04$ \\
\hline 5523 & GTF2A1 & -2.38 & $9.92 \mathrm{E}-05$ \\
\hline 5524 & GTF2A1L & -7.36 & 2.04E-04 \\
\hline 5525 & GTF2A2 & -6.79 & $8.74 \mathrm{E}-05$ \\
\hline 5526 & GTF2B & -4.35 & $5.26 \mathrm{E}-04$ \\
\hline 5527 & GTF2E2 & -3.94 & $1.14 \mathrm{E}-04$ \\
\hline 5528 & GTF2F2 & -1.49 & $3.34 \mathrm{E}-02$ \\
\hline 5529 & GTF2H3 & -2.83 & $4.62 \mathrm{E}-03$ \\
\hline 5530 & GTF2H4 & -3.51 & $7.81 \mathrm{E}-04$ \\
\hline 5531 & GTF2H5 & -1.42 & $1.39 \mathrm{E}-02$ \\
\hline 5532 & GTF2IRD1 & -1.98 & $1.22 \mathrm{E}-03$ \\
\hline 5533 & GTF3A & -1.47 & $9.38 \mathrm{E}-04$ \\
\hline 5534 & GTF3C3 & -4.30 & $2.06 \mathrm{E}-05$ \\
\hline 5535 & GTF3C5 & -3.98 & $1.41 \mathrm{E}-04$ \\
\hline 5536 & GTPBP1 & -3.82 & $1.46 \mathrm{E}-04$ \\
\hline
\end{tabular}

\begin{tabular}{|c|c|c|c|}
\hline 5537 & GTPBP10 & -2.34 & $2.61 \mathrm{E}-03$ \\
\hline 5538 & GTPBP2 & -1.47 & $8.50 \mathrm{E}-03$ \\
\hline 5539 & GTPBP4 & -5.67 & $2.98 \mathrm{E}-04$ \\
\hline 5540 & GTPBP5 & -2.34 & $1.19 \mathrm{E}-02$ \\
\hline 5541 & GTPBP6 & -2.35 & $1.23 \mathrm{E}-03$ \\
\hline 5542 & GTPBP8 & -2.02 & $1.90 \mathrm{E}-03$ \\
\hline 5543 & GTSE1 & -2.52 & $5.38 \mathrm{E}-03$ \\
\hline 5544 & GTSF1 & -6.05 & $3.45 \mathrm{E}-04$ \\
\hline 5545 & GUCA2A & -1.74 & $2.98 \mathrm{E}-03$ \\
\hline 5546 & GUF1 & -2.15 & $1.03 \mathrm{E}-02$ \\
\hline 5547 & GUK1 & -3.18 & $6.65 \mathrm{E}-03$ \\
\hline 5548 & GULP1 & -2.56 & $1.62 \mathrm{E}-02$ \\
\hline 5549 & GUSB & -1.41 & $1.40 \mathrm{E}-02$ \\
\hline 5550 & GXYLT1 & -4.04 & $4.90 \mathrm{E}-04$ \\
\hline 5551 & GYG1 & -3.56 & $9.65 \mathrm{E}-04$ \\
\hline 5552 & GZF1 & -2.19 & $3.82 \mathrm{E}-03$ \\
\hline 5553 & H2AFJ & -3.09 & $2.18 \mathrm{E}-03$ \\
\hline 5554 & H2AFY & -5.32 & 8.97E-04 \\
\hline 5555 & H2AFY2 & -5.00 & 7.07E-05 \\
\hline 5556 & H2AFZ & -8.54 & 2.62E-04 \\
\hline 5557 & $\mathrm{H} 3 \mathrm{~F} 3 \mathrm{C}$ & -7.17 & $1.22 \mathrm{E}-04$ \\
\hline 5558 & HAAO & -3.57 & $6.66 \mathrm{E}-03$ \\
\hline 5559 & HABP4 & -3.99 & $3.73 \mathrm{E}-03$ \\
\hline 5560 & HACE1 & -4.10 & $5.15 \mathrm{E}-04$ \\
\hline 5561 & HADHA & -1.99 & $3.32 \mathrm{E}-03$ \\
\hline 5562 & HADHB & -5.88 & $2.59 \mathrm{E}-04$ \\
\hline 5563 & HAGH & -1.79 & 7.10E-02 \\
\hline 5564 & HAGHL & -1.26 & $5.41 \mathrm{E}-04$ \\
\hline 5565 & HARBI1 & -2.55 & $2.74 \mathrm{E}-03$ \\
\hline 5566 & HAT1 & -4.93 & $2.01 \mathrm{E}-03$ \\
\hline 5567 & HAUS1 & -5.28 & $9.15 \mathrm{E}-05$ \\
\hline 5568 & HAUS2 & -7.24 & $2.42 \mathrm{E}-05$ \\
\hline 5569 & HAUS3 & -3.20 & $1.16 \mathrm{E}-02$ \\
\hline 5570 & HAUS6 & -1.67 & $1.73 \mathrm{E}-02$ \\
\hline 5571 & HAUS8 & -2.52 & $3.79 \mathrm{E}-03$ \\
\hline 5572 & HBG2 & -1.16 & $8.95 \mathrm{E}-02$ \\
\hline 5573 & HBP1 & -2.74 & $6.66 \mathrm{E}-04$ \\
\hline 5574 & HBS1L & -3.28 & $3.35 \mathrm{E}-05$ \\
\hline 5575 & HCCS & -4.15 & $5.01 \mathrm{E}-04$ \\
\hline 5576 & HCN2 & -3.20 & $5.64 \mathrm{E}-03$ \\
\hline 5577 & HDAC1 & -3.11 & 3.15E-03 \\
\hline 5578 & HDAC11 & -5.40 & $1.01 \mathrm{E}-03$ \\
\hline 5579 & HDAC2 & -3.95 & 2.05E-04 \\
\hline 5580 & HDAC4 & -1.56 & $6.85 \mathrm{E}-03$ \\
\hline 5581 & HDAC8 & -4.12 & 4.09E-04 \\
\hline 5582 & HDDC2 & -5.34 & 4.57E-04 \\
\hline 5583 & HDHD1 & -7.18 & $2.18 \mathrm{E}-04$ \\
\hline 5584 & HDHD2 & -2.64 & $2.58 \mathrm{E}-03$ \\
\hline 5585 & HDHD3 & -5.10 & $1.36 \mathrm{E}-04$ \\
\hline 5586 & HDLBP & -5.71 & $9.26 \mathrm{E}-05$ \\
\hline 5587 & HDX & -3.22 & $6.15 \mathrm{E}-04$ \\
\hline 5588 & HEATR2 & -3.66 & $9.80 \mathrm{E}-04$ \\
\hline 5589 & HEATR3 & -3.28 & $1.69 \mathrm{E}-03$ \\
\hline 5590 & HEATR5A & -1.50 & $1.50 \mathrm{E}-03$ \\
\hline 5591 & HEATR6 & -4.58 & $8.66 \mathrm{E}-05$ \\
\hline 5592 & HEBP1 & -3.14 & $1.67 \mathrm{E}-03$ \\
\hline 5593 & HECTD1 & -1.25 & $9.23 \mathrm{E}-03$ \\
\hline 5594 & HECTD3 & -3.41 & $3.19 \mathrm{E}-04$ \\
\hline 5595 & HECW1 & -1.22 & $1.12 \mathrm{E}-02$ \\
\hline 5596 & HELLS & -5.83 & $1.95 \mathrm{E}-04$ \\
\hline 5597 & HELQ & -2.51 & $4.28 \mathrm{E}-02$ \\
\hline 5598 & HEMGN & -2.90 & $1.72 \mathrm{E}-02$ \\
\hline 5599 & HEMK1 & -2.19 & $9.72 \mathrm{E}-04$ \\
\hline
\end{tabular}




\begin{tabular}{|c|c|c|c|}
\hline 5600 & HERC1 & -2.28 & $1.51 \mathrm{E}-03$ \\
\hline 5601 & HERC2 & -3.19 & $9.38 \mathrm{E}-05$ \\
\hline 5602 & HERC4 & -3.46 & $3.67 \mathrm{E}-04$ \\
\hline 5603 & HERPUD1 & -2.17 & $3.49 \mathrm{E}-03$ \\
\hline 5604 & HEXA & -4.44 & $5.43 \mathrm{E}-04$ \\
\hline 5605 & HEXB & -2.54 & $6.70 \mathrm{E}-03$ \\
\hline 5606 & HEXDC & -2.78 & 7.07E-03 \\
\hline 5607 & HGSNAT & -3.64 & 3.45E-04 \\
\hline 5608 & HIAT1 & -5.08 & $1.14 \mathrm{E}-03$ \\
\hline 5609 & HIBADH & -3.79 & 3.20E-04 \\
\hline 5610 & $\mathrm{HIBCH}$ & -3.58 & 9.03E-05 \\
\hline 5611 & HIC1 & -1.27 & $9.22 \mathrm{E}-03$ \\
\hline 5612 & HIF1A & -2.61 & $5.74 \mathrm{E}-03$ \\
\hline 5613 & HIGD1A & -1.24 & 2.64E-02 \\
\hline 5614 & HIGD2A & -6.48 & $1.38 \mathrm{E}-05$ \\
\hline 5615 & HINT1 & -5.63 & $6.67 \mathrm{E}-04$ \\
\hline 5616 & HINT3 & -3.66 & 4.20E-05 \\
\hline 5617 & HINTW & -4.13 & $1.32 \mathrm{E}-04$ \\
\hline 5618 & HIP1 & -4.15 & 4.80E-04 \\
\hline 5619 & HIPK2 & -1.84 & 7.96E-03 \\
\hline 5620 & HIPK3 & -2.47 & 3.12E-05 \\
\hline 5621 & HIST1H1C & -2.30 & 3.87E-02 \\
\hline 5622 & HIST1H2BO & -5.63 & $5.18 \mathrm{E}-04$ \\
\hline 5623 & HIST2H2AC & -2.88 & 4.41E-04 \\
\hline 5624 & HIVEP2 & -1.74 & $1.52 \mathrm{E}-02$ \\
\hline 5625 & HIVEP3 & -3.52 & 2.68E-02 \\
\hline 5626 & HJURP & -4.61 & $1.25 \mathrm{E}-03$ \\
\hline 5627 & HK1 & -2.19 & $1.39 \mathrm{E}-03$ \\
\hline 5628 & HMBOX1 & -4.74 & $5.60 \mathrm{E}-03$ \\
\hline 5629 & HMBS & -5.85 & $8.95 \mathrm{E}-05$ \\
\hline 5630 & HMCN1 & -2.62 & $4.11 \mathrm{E}-03$ \\
\hline 5631 & HMG2OA & -2.19 & $2.16 \mathrm{E}-04$ \\
\hline 5632 & HMGB1 & -5.13 & $9.15 \mathrm{E}-04$ \\
\hline 5633 & HMGB2 & -6.06 & $8.46 \mathrm{E}-04$ \\
\hline 5634 & HMGB3 & -2.38 & $2.92 \mathrm{E}-02$ \\
\hline 5635 & HMGCLL1 & -3.72 & 7.84E-04 \\
\hline 5636 & HMGCR & -2.06 & $3.17 \mathrm{E}-03$ \\
\hline 5637 & HMGCS1 & -3.32 & $5.64 \mathrm{E}-03$ \\
\hline 5638 & HMGCS2 & -6.54 & $9.44 \mathrm{E}-05$ \\
\hline 5639 & HMGN1 & -4.58 & 2.97E-05 \\
\hline 5640 & HMGN2 & -8.33 & 4.41E-05 \\
\hline 5641 & HMGN3 & -3.13 & 4.81E-04 \\
\hline 5642 & HMGN5 & -3.86 & $1.40 \mathrm{E}-03$ \\
\hline 5643 & HMGXB4 & -2.50 & $1.61 \mathrm{E}-03$ \\
\hline 5644 & HMHA1 & -1.41 & $2.40 \mathrm{E}-02$ \\
\hline 5645 & HMMR & -2.41 & 4.56E- 03 \\
\hline 5646 & HMOX2 & -4.27 & $1.08 \mathrm{E}-03$ \\
\hline 5647 & HN1 & -7.13 & 1.97E-04 \\
\hline 5648 & HNRNPA2B1 & -6.18 & 1.17E-05 \\
\hline 5649 & HNRNPA3 & -3.70 & $2.29 \mathrm{E}-03$ \\
\hline 5650 & HNRNPAB & -6.04 & $9.09 \mathrm{E}-05$ \\
\hline 5651 & HNRNPD & -5.29 & 3.14E-05 \\
\hline 5652 & HNRNPH1 & -5.21 & $7.73 E-04$ \\
\hline 5653 & HNRNPH3 & -4.26 & $7.64 \mathrm{E}-06$ \\
\hline 5654 & HNRNPK & -5.78 & $2.60 \mathrm{E}-04$ \\
\hline 5655 & HNRNPM & -2.26 & $1.23 \mathrm{E}-03$ \\
\hline 5656 & HNRNPR & -3.94 & $1.53 \mathrm{E}-04$ \\
\hline 5657 & HNRNPU & -6.27 & $6.06 \mathrm{E}-05$ \\
\hline 5658 & HNRPDL & -4.20 & $2.02 \mathrm{E}-04$ \\
\hline 5659 & HNRPK & -3.97 & $1.81 \mathrm{E}-03$ \\
\hline 5660 & HNRPLL & -4.15 & $2.14 \mathrm{E}-04$ \\
\hline 5661 & HOMER3 & -2.46 & $1.35 \mathrm{E}-03$ \\
\hline 5662 & HOOK1 & -2.69 & $1.18 \mathrm{E}-02$ \\
\hline
\end{tabular}

\begin{tabular}{|c|c|c|c|}
\hline 5663 & НООКЗ & -6.31 & $1.03 \mathrm{E}-03$ \\
\hline 5664 & HOPX & -1.31 & $1.02 \mathrm{E}-01$ \\
\hline 5665 & HORMAD1 & -5.25 & $1.28 \mathrm{E}-03$ \\
\hline 5666 & HORMAD2 & -2.28 & $4.81 E-04$ \\
\hline 5667 & HP1BP3 & -4.71 & $1.18 \mathrm{E}-04$ \\
\hline 5668 & HPD & -3.08 & $4.04 \mathrm{E}-04$ \\
\hline 5669 & HPRT1 & -2.41 & $1.95 \mathrm{E}-03$ \\
\hline 5670 & HRSP12 & -2.46 & $1.97 \mathrm{E}-02$ \\
\hline 5671 & HS1BP3 & -3.59 & $2.33 \mathrm{E}-03$ \\
\hline 5672 & HS3ST2 & -1.15 & $4.29 \mathrm{E}-03$ \\
\hline 5673 & HS3ST3A1 & -2.09 & $3.82 \mathrm{E}-03$ \\
\hline 5674 & HS6ST1 & -2.07 & $1.31 \mathrm{E}-03$ \\
\hline 5675 & HS6ST2 & -3.18 & $2.23 \mathrm{E}-04$ \\
\hline 5676 & HSBP1 & -6.78 & $6.19 \mathrm{E}-04$ \\
\hline 5677 & HSD17B11 & -3.95 & $1.22 \mathrm{E}-04$ \\
\hline 5678 & HSD17B12 & -4.49 & $2.45 \mathrm{E}-04$ \\
\hline 5679 & HSD17B4 & -7.97 & $3.88 \mathrm{E}-05$ \\
\hline 5680 & HSD17B7 & -3.21 & $8.04 \mathrm{E}-04$ \\
\hline 5681 & HSDL1 & -1.64 & $2.30 \mathrm{E}-03$ \\
\hline 5682 & HSF2 & -2.92 & $2.17 \mathrm{E}-03$ \\
\hline 5683 & HSF5 & -4.08 & $2.61 \mathrm{E}-04$ \\
\hline 5684 & HSP25 & -2.44 & $9.18 \mathrm{E}-02$ \\
\hline 5685 & HSP90AA1 & -7.45 & $4.98 \mathrm{E}-04$ \\
\hline 5686 & HSP90AB1 & -3.66 & $7.78 \mathrm{E}-04$ \\
\hline 5687 & HSP90B1 & -5.75 & $6.99 \mathrm{E}-05$ \\
\hline 5688 & HSPA2 & -4.03 & $1.05 \mathrm{E}-02$ \\
\hline 5689 & HSPA4 & -3.75 & $1.06 \mathrm{E}-04$ \\
\hline 5690 & HSPA4L & -8.26 & $1.10 \mathrm{E}-04$ \\
\hline 5691 & HSPA5 & -6.46 & $1.99 \mathrm{E}-04$ \\
\hline 5692 & HSPA8 & -4.98 & $1.43 \mathrm{E}-02$ \\
\hline 5693 & HSPA9 & -5.29 & $8.75 \mathrm{E}-04$ \\
\hline 5694 & HSPB11 & -3.11 & 4.70E-03 \\
\hline 5695 & HSPB8 & -1.42 & $7.65 \mathrm{E}-02$ \\
\hline 5696 & HSPBAP1 & -5.79 & $2.88 \mathrm{E}-04$ \\
\hline 5697 & HSPD1 & -4.09 & $9.24 \mathrm{E}-04$ \\
\hline 5698 & HSPE1 & -7.16 & $4.51 \mathrm{E}-04$ \\
\hline 5699 & HSPH1 & -4.36 & $3.74 \mathrm{E}-03$ \\
\hline 5700 & HTATSF1 & -2.28 & $2.98 \mathrm{E}-03$ \\
\hline 5701 & HTR1D & -1.68 & $1.12 \mathrm{E}-03$ \\
\hline 5702 & HTRA2 & -3.84 & $3.62 \mathrm{E}-03$ \\
\hline 5703 & HTRA3 & -2.65 & $9.00 \mathrm{E}-04$ \\
\hline 5704 & HUS1 & -2.46 & $5.46 \mathrm{E}-03$ \\
\hline 5705 & HYDIN & -2.73 & $2.17 \mathrm{E}-03$ \\
\hline 5706 & HYLS1 & -7.52 & $9.04 \mathrm{E}-05$ \\
\hline 5707 & HYOU1 & -2.53 & $1.05 \mathrm{E}-03$ \\
\hline 5708 & IAH1 & -4.06 & 4.03E-04 \\
\hline 5709 & IARS & -5.12 & $4.52 \mathrm{E}-04$ \\
\hline 5710 & IARS2 & -5.01 & $1.64 \mathrm{E}-04$ \\
\hline 5711 & IBTK & -4.26 & $2.71 \mathrm{E}-03$ \\
\hline 5712 & ICA1 & -3.82 & $2.92 \mathrm{E}-01$ \\
\hline 5713 & ICMT & -1.58 & 1.47E-02 \\
\hline 5714 & ICT1 & -6.93 & $4.58 \mathrm{E}-05$ \\
\hline 5715 & ID1 & -2.10 & $1.54 \mathrm{E}-02$ \\
\hline 5716 & ID2 & -2.77 & 2.04E-03 \\
\hline 5717 & ID4 & -1.55 & $4.72 \mathrm{E}-02$ \\
\hline 5718 & IDH1 & -2.74 & $1.81 \mathrm{E}-03$ \\
\hline 5719 & IDH2 & -3.48 & $1.03 \mathrm{E}-03$ \\
\hline 5720 & IDH3A & -2.39 & $1.14 \mathrm{E}-05$ \\
\hline 5721 & IDH3B & -4.76 & $5.39 \mathrm{E}-04$ \\
\hline 5722 & IDI1 & -7.08 & $5.33 \mathrm{E}-04$ \\
\hline 5723 & IDUA & -1.16 & $1.71 \mathrm{E}-02$ \\
\hline 5724 & IFFO1 & -1.96 & $1.68 \mathrm{E}-03$ \\
\hline 5725 & IFI27L2 & -1.50 & $1.21 \mathrm{E}-02$ \\
\hline
\end{tabular}

\begin{tabular}{|c|c|c|c|}
\hline 5726 & IFI35 & -2.39 & $3.24 \mathrm{E}-04$ \\
\hline 5727 & $\mathrm{IFIH1}$ & -3.06 & $1.05 \mathrm{E}-03$ \\
\hline 5728 & IFNGR2 & -4.03 & $8.91 \mathrm{E}-04$ \\
\hline 5729 & IFRD1 & -3.06 & $2.01 \mathrm{E}-03$ \\
\hline 5730 & IFT140 & -3.25 & 2.43E-02 \\
\hline 5731 & IFT172 & -3.09 & 8.06E-04 \\
\hline 5732 & IFT20 & -6.34 & $7.36 \mathrm{E}-05$ \\
\hline 5733 & IFT27 & -6.52 & $4.90 \mathrm{E}-04$ \\
\hline 5734 & IFT46 & -6.40 & 4.38E-05 \\
\hline 5735 & IFT52 & -5.80 & $3.44 \mathrm{E}-03$ \\
\hline 5736 & IFT57 & -7.62 & $1.30 \mathrm{E}-04$ \\
\hline 5737 & IFT80 & -5.73 & $3.56 \mathrm{E}-04$ \\
\hline 5738 & IFT81 & -3.40 & $1.05 \mathrm{E}-03$ \\
\hline 5739 & IFT88 & -3.41 & $1.49 \mathrm{E}-04$ \\
\hline 5740 & IGBP1 & -4.81 & $6.63 \mathrm{E}-04$ \\
\hline 5741 & IGF1R & -1.68 & 2.24E-03 \\
\hline 5742 & IGFBP7 & -6.63 & $3.91 \mathrm{E}-05$ \\
\hline 5743 & IGHMBP2 & -4.45 & 1.33E-04 \\
\hline 5744 & IGSF21 & -1.83 & $9.02 \mathrm{E}-03$ \\
\hline 5745 & IK & -6.10 & $2.25 \mathrm{E}-04$ \\
\hline 5746 & IKBIP & -4.02 & 3.25E-04 \\
\hline 5747 & IKBKAP & -3.19 & $5.42 \mathrm{E}-03$ \\
\hline 5748 & IKBKB & -2.11 & $4.51 \mathrm{E}-03$ \\
\hline 5749 & IKZF5 & -4.15 & $2.31 \mathrm{E}-04$ \\
\hline 5750 & IL12RB2 & -2.39 & $9.39 \mathrm{E}-04$ \\
\hline 5751 & IL15 & -1.20 & $1.05 \mathrm{E}-03$ \\
\hline 5752 & IL17D & -3.39 & $3.64 \mathrm{E}-03$ \\
\hline 5753 & IL1RAPL2 & -1.29 & $7.79 \mathrm{E}-03$ \\
\hline 5754 & IL23R & -2.20 & $1.42 \mathrm{E}-02$ \\
\hline 5755 & IL2RG & -1.43 & $1.53 \mathrm{E}-03$ \\
\hline 5756 & IL9 & -1.44 & $2.54 \mathrm{E}-02$ \\
\hline 5757 & ILDR1 & -2.75 & $3.53 \mathrm{E}-03$ \\
\hline 5758 & ILF2 & -2.52 & 3.37E-03 \\
\hline 5759 & ILK & -1.78 & $3.28 \mathrm{E}-02$ \\
\hline 5760 & ILKAP & -1.98 & $1.40 \mathrm{E}-03$ \\
\hline 5761 & IMMP1L & -3.67 & $1.70 \mathrm{E}-04$ \\
\hline 5762 & IMMP2L & -3.01 & $1.26 \mathrm{E}-03$ \\
\hline 5763 & IMMT & -4.18 & $8.27 \mathrm{E}-04$ \\
\hline 5764 & IMPA1 & -5.06 & $7.78 \mathrm{E}-04$ \\
\hline 5765 & IMPACT & -5.63 & 7.41E-04 \\
\hline 5766 & IMPAD1 & -1.52 & $1.09 \mathrm{E}-02$ \\
\hline 5767 & IMPDH2 & -4.64 & $1.68 \mathrm{E}-04$ \\
\hline 5768 & IMPG2 & -4.60 & $3.71 \mathrm{E}-03$ \\
\hline 5769 & INCENP & -2.97 & 4.20E-05 \\
\hline 5770 & ING1 & -3.59 & $3.68 \mathrm{E}-03$ \\
\hline 5771 & ING2 & -4.38 & 1.56E-04 \\
\hline 5772 & ING3 & -3.50 & 7.76E-04 \\
\hline 5773 & ING4 & -5.39 & 9.57E-05 \\
\hline 5774 & INIP & -3.45 & $8.27 \mathrm{E}-03$ \\
\hline 5775 & INO80 & -1.57 & 2.60E-04 \\
\hline 5776 & INO80C & -3.80 & $5.39 \mathrm{E}-03$ \\
\hline 5777 & INPP4A & -3.26 & $6.41 \mathrm{E}-04$ \\
\hline 5778 & INPP5B & -2.08 & $4.56 \mathrm{E}-03$ \\
\hline 5779 & INPP5E & -2.38 & 8.47E-04 \\
\hline 5780 & INPP5K & -2.53 & $1.54 \mathrm{E}-03$ \\
\hline 5781 & INSIG1 & -1.37 & $2.78 \mathrm{E}-03$ \\
\hline 5782 & INSIG2 & -1.77 & $1.03 \mathrm{E}-02$ \\
\hline 5783 & INTS10 & -4.99 & 9.56E-05 \\
\hline 5784 & INTS12 & -2.76 & $1.99 \mathrm{E}-04$ \\
\hline 5785 & INTS2 & -1.31 & $1.92 \mathrm{E}-02$ \\
\hline 5786 & INTS7 & -2.63 & 7.33E-04 \\
\hline 5787 & INTS8 & -4.27 & $1.04 \mathrm{E}-03$ \\
\hline 5788 & INTS9 & -2.14 & $4.74 \mathrm{E}-03$ \\
\hline
\end{tabular}




\begin{tabular}{|c|c|c|c|}
\hline 5789 & INVS & -3.72 & $1.44 \mathrm{E}-03$ \\
\hline 5790 & IPGK2 & -6.28 & $5.01 E-04$ \\
\hline 5791 & IPO5 & -4.33 & $8.27 \mathrm{E}-04$ \\
\hline 5792 & IPO7 & -2.33 & $9.05 \mathrm{E}-03$ \\
\hline 5793 & IP08 & -2.77 & $2.75 \mathrm{E}-03$ \\
\hline 5794 & IPO9 & -3.60 & $1.39 \mathrm{E}-05$ \\
\hline 5795 & IPPK & -3.67 & $7.80 \mathrm{E}-05$ \\
\hline 5796 & IQCA1 & -4.55 & $1.18 \mathrm{E}-04$ \\
\hline 5797 & IQCB1 & -3.16 & $1.62 \mathrm{E}-04$ \\
\hline 5798 & IQCC & -1.65 & $2.16 \mathrm{E}-02$ \\
\hline 5799 & IQCD & -5.31 & $3.90 \mathrm{E}-04$ \\
\hline 5800 & IQCE & -4.37 & $1.25 \mathrm{E}-05$ \\
\hline 5801 & IQCG & $\begin{array}{l}-7.08 \\
\end{array}$ & $6.07 \mathrm{E}-05$ \\
\hline 5802 & IQCK & -2.56 & $4.82 \mathrm{E}-04$ \\
\hline 5803 & IQGAP1 & -3.23 & $3.88 \mathrm{E}-03$ \\
\hline 5804 & IQUB & -6.84 & $1.10 \mathrm{E}-04$ \\
\hline 5805 & IRAK1BP1 & -6.42 & $2.04 E-04$ \\
\hline 5806 & IRAK2 & -4.20 & $1.24 \mathrm{E}-04$ \\
\hline 5807 & IRAK4 & -1.93 & $1.70 \mathrm{E}-02$ \\
\hline 5808 & IREB2 & -3.96 & $5.81 \mathrm{E}-04$ \\
\hline 5809 & IRF2 & -2.11 & $1.01 E-02$ \\
\hline 5810 & IRF2BPL & -2.90 & $7.98 \mathrm{E}-03$ \\
\hline 5811 & IRF6 & $\begin{array}{l}-3.62 \\
\end{array}$ & $1.69 \mathrm{E}-03$ \\
\hline 5812 & ISCA1 & -5.19 & $6.10 \mathrm{E}-04$ \\
\hline 5813 & ISCU & -4.72 & $2.95 \mathrm{E}-04$ \\
\hline 5814 & ISM1 & $\begin{array}{l}-1.66 \\
\end{array}$ & $2.55 \mathrm{E}-03$ \\
\hline 5815 & ISOC1 & -3.26 & $2.24 \mathrm{E}-03$ \\
\hline 5816 & ISY1 & -3.84 & $1.51 \mathrm{E}-03$ \\
\hline 5817 & ITFG1 & $\begin{array}{l}-1.81 \\
\end{array}$ & $2.77 \mathrm{E}-02$ \\
\hline 5818 & ITFG2 & -1.44 & $2.86 \mathrm{E}-02$ \\
\hline 5819 & ITFG3 & -4.63 & $2.71 \mathrm{E}-04$ \\
\hline 5820 & ITGA3 & -1.15 & $2.10 \mathrm{E}-02$ \\
\hline 5821 & ITGA6 & -1.07 & $6.53 \mathrm{E}-03$ \\
\hline 5822 & ITGB1 & -3.05 & $9.91 \mathrm{E}-04$ \\
\hline 5823 & ITGB1BP1 & -3.72 & $2.02 E-04$ \\
\hline 5824 & ITGB1BP3 & -8.28 & $1.06 E-03$ \\
\hline 5825 & ITGB3BP & -4.01 & $7.86 \mathrm{E}-03$ \\
\hline 5826 & ITGB5 & -2.17 & $9.76 \mathrm{E}-03$ \\
\hline 5827 & ITM2A & -7.83 & $9.68 \mathrm{E}-04$ \\
\hline 5828 & ITM2B & -4.49 & $1.04 \mathrm{E}-03$ \\
\hline 5829 & ITPA & -6.67 & $7.42 \mathrm{E}-04$ \\
\hline 5830 & ITPK1 & -1.41 & $4.33 \mathrm{E}-02$ \\
\hline 5831 & ITPKB & -2.73 & $7.58 \mathrm{E}-03$ \\
\hline 5832 & ITSN1 & -3.93 & $1.59 \mathrm{E}-03$ \\
\hline 5833 & IVD & -2.08 & $1.93 \mathrm{E}-04$ \\
\hline 5834 & IVNS1ABP & -2.95 & $3.99 \mathrm{E}-04$ \\
\hline 5835 & JAK1 & -2.48 & $6.64 \mathrm{E}-03$ \\
\hline 5836 & JAKMIP2 & -5.37 & $1.84 \mathrm{E}-03$ \\
\hline 5837 & JAM2 & -3.29 & $1.13 \mathrm{E}-03$ \\
\hline 5838 & JAM3 & -1.20 & $3.49 \mathrm{E}-02$ \\
\hline 5839 & JAZF1 & -3.72 & $5.38 \mathrm{E}-04$ \\
\hline 5840 & JHDM1D & -3.36 & $2.68 \mathrm{E}-03$ \\
\hline 5841 & JKAMP & -4.52 & $2.68 \mathrm{E}-04$ \\
\hline 5842 & JMJD1C & -3.45 & $4.63 \mathrm{E}-03$ \\
\hline 5843 & JMJD4 & -1.71 & $2.69 \mathrm{E}-03$ \\
\hline 5844 & JMJD5 & -2.58 & $7.06 E-03$ \\
\hline 5845 & JMJD6 & -3.33 & $4.31 \mathrm{E}-03$ \\
\hline 5846 & JMJD7 & -2.90 & $3.70 \mathrm{E}-04$ \\
\hline 5847 & JMJD8 & -4.40 & $3.65 \mathrm{E}-03$ \\
\hline 5848 & JOSD1 & -2.52 & $4.84 \mathrm{E}-03$ \\
\hline 5849 & JPH1 & -3.24 & $7.99 \mathrm{E}-04$ \\
\hline 5850 & JTB & -5.39 & $3.70 \mathrm{E}-02$ \\
\hline (5) & JUN & -3.46 & $3.65 \mathrm{E}-03$ \\
\hline
\end{tabular}

\begin{tabular}{|c|c|c|c|}
\hline 5852 & KANK1 & -2.63 & $6.03 \mathrm{E}-03$ \\
\hline 5853 & KARS & -5.69 & $1.57 \mathrm{E}-04$ \\
\hline 5854 & KAT2A & -3.29 & $3.28 \mathrm{E}-03$ \\
\hline 5855 & KAT2B & -2.18 & $2.53 \mathrm{E}-04$ \\
\hline 5856 & KATNA1 & -2.10 & $1.02 \mathrm{E}-02$ \\
\hline 5857 & KATNAL1 & -3.96 & $8.00 \mathrm{E}-05$ \\
\hline 5858 & KATNAL2 & -1.87 & $4.63 \mathrm{E}-04$ \\
\hline 5859 & KATNB1 & -6.71 & $7.90 \mathrm{E}-05$ \\
\hline 5860 & KBTBD2 & -3.46 & $1.23 \mathrm{E}-03$ \\
\hline 5861 & KBTBD4 & -3.08 & $2.58 \mathrm{E}-03$ \\
\hline 5862 & KCMF1 & -5.84 & $2.72 \mathrm{E}-04$ \\
\hline 5863 & KCNAB1 & -1.24 & $7.18 \mathrm{E}-03$ \\
\hline 5864 & KCNK1 & -1.78 & $2.57 \mathrm{E}-02$ \\
\hline 5865 & KCNK5 & -2.00 & $5.65 \mathrm{E}-03$ \\
\hline 5866 & KCNMB1 & -1.67 & $4.77 \mathrm{E}-03$ \\
\hline 5867 & KCNMB4 & -1.36 & $4.72 \mathrm{E}-03$ \\
\hline 5868 & KCNN2 & -2.68 & $2.63 \mathrm{E}-03$ \\
\hline 5869 & KCNT2 & -2.14 & $1.98 \mathrm{E}-04$ \\
\hline 5870 & KCTD2 & -5.68 & $8.91 \mathrm{E}-05$ \\
\hline 5871 & KCTD7 & -1.21 & $3.32 \mathrm{E}-03$ \\
\hline 5872 & KCTD9 & -4.08 & $5.89 \mathrm{E}-04$ \\
\hline 5873 & KDELC1 & -4.37 & $2.02 \mathrm{E}-04$ \\
\hline 5874 & KDELR2 & -4.27 & $3.61 \mathrm{E}-04$ \\
\hline 5875 & KDM1A & -2.50 & $1.53 \mathrm{E}-03$ \\
\hline 5876 & KDM3A & -4.70 & $1.68 \mathrm{E}-04$ \\
\hline 5877 & KDM3B & -5.44 & $2.20 \mathrm{E}-03$ \\
\hline 5878 & KDM4A & -3.65 & $2.93 \mathrm{E}-03$ \\
\hline 5879 & KDM5A & -1.17 & $1.56 \mathrm{E}-02$ \\
\hline 5880 & KDM5B & -1.79 & $2.63 \mathrm{E}-02$ \\
\hline 5881 & KDSR & $\begin{array}{l}-1.38 \\
\end{array}$ & $3.66 \mathrm{E}-03$ \\
\hline 5882 & KHDRBS1 & -2.26 & $3.32 \mathrm{E}-02$ \\
\hline 5883 & KHDRBS3 & -5.28 & $8.43 \mathrm{E}-05$ \\
\hline 5884 & KIAA0020 & -3.04 & $6.61 \mathrm{E}-04$ \\
\hline 5885 & KIAA0100 & -1.39 & $4.35 \mathrm{E}-03$ \\
\hline 5886 & KIAA0196 & -2.40 & $2.41 \mathrm{E}-03$ \\
\hline 5887 & KIAA0232 & -3.63 & $3.67 \mathrm{E}-04$ \\
\hline 5888 & KIAA0317 & -1.30 & $7.39 \mathrm{E}-03$ \\
\hline 5889 & KIAA0319L & -4.86 & $1.71 \mathrm{E}-05$ \\
\hline 5890 & KIAA0368 & -2.01 & $2.08 \mathrm{E}-02$ \\
\hline 5891 & KIAA0391 & -2.21 & $8.95 \mathrm{E}-04$ \\
\hline 5892 & KIAA0586 & -4.09 & $1.62 \mathrm{E}-04$ \\
\hline 5893 & KIAA0776 & -4.89 & $2.03 \mathrm{E}-03$ \\
\hline 5894 & KIAA0825 & -1.67 & $7.29 \mathrm{E}-03$ \\
\hline 5895 & KIAA0907 & \begin{tabular}{|l|}
-4.61 \\
\end{tabular} & $2.44 \mathrm{E}-04$ \\
\hline 5896 & KIAA0930 & -3.50 & $2.56 \mathrm{E}-03$ \\
\hline 5897 & KIAA1109 & -4.00 & $7.44 \mathrm{E}-05$ \\
\hline 5898 & KIAA1143 & -4.30 & $6.07 \mathrm{E}-04$ \\
\hline 5899 & KIAA1199 & -2.50 & $1.34 \mathrm{E}-02$ \\
\hline 5900 & KIAA1279 & -1.46 & $2.78 \mathrm{E}-04$ \\
\hline 5901 & KIAA1429 & -4.49 & $2.70 \mathrm{E}-04$ \\
\hline 5902 & KIAA1430 & \begin{tabular}{|l|}
-4.31 \\
\end{tabular} & $3.46 \mathrm{E}-04$ \\
\hline 5903 & KIAA1432 & -2.61 & $1.72 \mathrm{E}-03$ \\
\hline 5904 & KIAA1456 & -3.96 & $1.66 \mathrm{E}-03$ \\
\hline 5905 & KIAA1462 & -1.61 & $5.06 \mathrm{E}-03$ \\
\hline 5906 & KIAA1467 & -1.13 & $3.12 \mathrm{E}-02$ \\
\hline 5907 & KIAA1468 & -1.64 & $1.72 \mathrm{E}-03$ \\
\hline 5908 & KIAA1524 & -4.82 & $2.04 \mathrm{E}-04$ \\
\hline 5909 & KIAA1609 & -3.11 & $2.27 \mathrm{E}-03$ \\
\hline 5910 & KIAA1704 & -4.82 & $8.26 \mathrm{E}-04$ \\
\hline 5911 & KIAA1715 & -1.95 & $2.51 \mathrm{E}-03$ \\
\hline 5912 & KIAA1731 & -6.44 & $1.41 \mathrm{E}-03$ \\
\hline 5913 & KIAA1737 & -1.87 & $1.36 \mathrm{E}-02$ \\
\hline 5914 & KIAA2018 & -2.19 & $2.46 \mathrm{E}-03$ \\
\hline
\end{tabular}

\begin{tabular}{|c|c|c|c|}
\hline 5915 & KIAA2026 & -2.72 & $5.35 \mathrm{E}-04$ \\
\hline 5916 & KIDINS220 & -5.41 & $1.32 \mathrm{E}-05$ \\
\hline 5917 & KIF11 & -4.78 & $9.82 \mathrm{E}-04$ \\
\hline 5918 & KIF13A & -2.55 & $4.94 \mathrm{E}-04$ \\
\hline 5919 & KIF15 & -5.76 & $2.89 \mathrm{E}-04$ \\
\hline 5920 & KIF16B & -5.26 & $2.87 \mathrm{E}-04$ \\
\hline 5921 & $\begin{array}{l}\text { KIF18A } \\
\text {. }\end{array}$ & -4.72 & $8.39 \mathrm{E}-04$ \\
\hline 5922 & KIF1A & -2.14 & $8.01 \mathrm{E}-03$ \\
\hline 5923 & KIF1B & -3.80 & $1.26 \mathrm{E}-03$ \\
\hline 5924 & KIF $20 \mathrm{~A}$ & -4.39 & $1.73 \mathrm{E}-04$ \\
\hline 5925 & KIF 23 & -5.03 & $4.69 \mathrm{E}-04$ \\
\hline 5926 & KIF24 & -7.17 & $8.21 \mathrm{E}-05$ \\
\hline 5927 & KIF 27 & -4.48 & 7.05E-04 \\
\hline 5928 & KIF $2 B$ & -4.08 & $7.46 \mathrm{E}-04$ \\
\hline 5929 & KIF $2 \mathrm{C}$ & -7.05 & $2.66 \mathrm{E}-04$ \\
\hline 5930 & KIF3A & -5.01 & $1.41 \mathrm{E}-04$ \\
\hline 5931 & КІF3B & -2.84 & $2.37 \mathrm{E}-03$ \\
\hline 5932 & KIF4A & -6.34 & $2.97 \mathrm{E}-04$ \\
\hline 5933 & KIF5B & -4.81 & $2.18 \mathrm{E}-03$ \\
\hline 5934 & KIF5C & -1.59 & $2.06 \mathrm{E}-02$ \\
\hline 5935 & KIFC1 & -4.05 & $5.40 \mathrm{E}-03$ \\
\hline 5936 & KLC1 & -3.33 & $\begin{array}{l}9.91 \mathrm{E}-04 \\
\end{array}$ \\
\hline 5937 & KLF11 & -4.68 & $1.98 \mathrm{E}-04$ \\
\hline 5938 & KLF3 & -1.30 & $1.49 \mathrm{E}-02$ \\
\hline 5939 & KLF5 & -2.53 & $7.48 \mathrm{E}-03$ \\
\hline 5940 & KLHDC1 & -3.88 & $4.35 \mathrm{E}-04$ \\
\hline 5941 & KLHDC2 & -5.87 & $2.46 \mathrm{E}-06$ \\
\hline 5942 & KLHDC3 & -4.58 & $1.03 \mathrm{E}-04$ \\
\hline 5943 & KLHDC4 & -3.52 & $4.46 \mathrm{E}-04$ \\
\hline 5944 & KLHDC8B & -1.30 & $1.03 \mathrm{E}-02$ \\
\hline 5945 & KLHL10 & -7.46 & $5.81 \mathrm{E}-04$ \\
\hline 5946 & $\begin{array}{l}\text { KLHL18 } \\
\end{array}$ & -1.17 & $2.67 \mathrm{E}-02$ \\
\hline 5947 & KLHL20 & -1.08 & $5.22 \mathrm{E}-03$ \\
\hline 5948 & KLHL26 & -2.66 & $5.44 \mathrm{E}-04$ \\
\hline 5949 & KLHL28 & -2.90 & $3.55 \mathrm{E}-04$ \\
\hline 5950 & $\begin{array}{l}\text { KLHL7 } \\
\end{array}$ & -1.82 & $2.87 \mathrm{E}-03$ \\
\hline 5951 & KLHL8 & -2.37 & $7.72 \mathrm{E}-04$ \\
\hline 5952 & KNTC1 & -3.07 & $8.52 \mathrm{E}-04$ \\
\hline 5953 & KPNA1 & -1.05 & $7.84 \mathrm{E}-04$ \\
\hline 5954 & KPNA2 & -1.30 & $1.07 \mathrm{E}-02$ \\
\hline 5955 & KPNA3 & -3.41 & $7.94 \mathrm{E}-03$ \\
\hline 5956 & $\begin{array}{l}\text { KPNA4 } \\
\end{array}$ & -6.28 & $5.08 \mathrm{E}-05$ \\
\hline 5957 & KPNAG & -4.26 & $5.95 \mathrm{E}-03$ \\
\hline 5958 & $\begin{array}{l}\text { KRIT1 } \\
\end{array}$ & -5.33 & $9.94 \mathrm{E}-05$ \\
\hline 5959 & KRR1 & -5.86 & $1.88 \mathrm{E}-03$ \\
\hline 5960 & KRT15 & -2.37 & $1.95 \mathrm{E}-03$ \\
\hline 5961 & KRT23 & -1.17 & $3.39 \mathrm{E}-02$ \\
\hline 5962 & KSR1 & -2.12 & $5.65 \mathrm{E}-03$ \\
\hline 5963 & KTI12 & -1.85 & $5.63 \mathrm{E}-03$ \\
\hline 5964 & KTN1 & -1.71 & $9.42 \mathrm{E}-03$ \\
\hline 5965 & L06098 & -3.50 & $9.05 \mathrm{E}-02$ \\
\hline 5966 & L3MBTL2 & -4.24 & $1.35 \mathrm{E}-03$ \\
\hline 5967 & L3MBTL4 & -3.75 & $1.35 \mathrm{E}-03$ \\
\hline 5968 & L48911 & -3.46 & $3.16 \mathrm{E}-02$ \\
\hline 5969 & L48915 & -2.80 & $8.39 \mathrm{E}-03$ \\
\hline 5970 & $\begin{array}{l}\text { L48920 } \\
\end{array}$ & $\begin{array}{l}-7.39 \\
\end{array}$ & $8.69 \mathrm{E}-04$ \\
\hline 5971 & LACE1 & -4.25 & $8.68 \mathrm{E}-04$ \\
\hline 5972 & LACTB & -3.01 & $7.40 \mathrm{E}-04$ \\
\hline 5973 & LAMA3 & -1.80 & $3.20 \mathrm{E}-02$ \\
\hline 5974 & LAMA5 & -2.89 & $2.08 \mathrm{E}-03$ \\
\hline 5975 & LAMB2 & -2.68 & $3.44 \mathrm{E}-03$ \\
\hline 5976 & LAMB4 & -1.49 & $1.96 \mathrm{E}-02$ \\
\hline 5977 & LAMP1 & -3.65 & $4.40 \mathrm{E}-04$ \\
\hline
\end{tabular}




\begin{tabular}{|c|c|c|c|}
\hline 5978 & LAMTOR3 & -5.18 & $4.20 \mathrm{E}-04$ \\
\hline 5979 & LANCL1 & -4.11 & $9.42 \mathrm{E}-04$ \\
\hline 5980 & LANCL2 & -4.22 & $8.14 \mathrm{E}-04$ \\
\hline 5981 & LAP3 & -4.67 & $7.56 \mathrm{E}-05$ \\
\hline 5982 & LAPTM4A & -6.75 & $1.12 \mathrm{E}-04$ \\
\hline 5983 & LARP1B & -3.83 & $2.62 \mathrm{E}-03$ \\
\hline 5984 & LARPAB & -3.04 & $2.80 \mathrm{E}-03$ \\
\hline 5985 & LARP7 & -6.22 & $1.20 \mathrm{E}-04$ \\
\hline 5986 & LARS & -1.80 & $4.10 \mathrm{E}-02$ \\
\hline 5987 & LARS2 & -2.51 & $5.07 \mathrm{E}-04$ \\
\hline 5988 & LASP1 & -3.13 & $8.72 \mathrm{E}-03$ \\
\hline 5989 & LATS1 & -3.14 & $6.69 \mathrm{E}-04$ \\
\hline 5990 & LBH & -2.92 & $3.69 \mathrm{E}-03$ \\
\hline 5991 & LBR & \begin{tabular}{|l|}
-7.44 \\
\end{tabular} & $4.98 \mathrm{E}-05$ \\
\hline 5992 & LBX3 & -4.15 & $2.19 \mathrm{E}-02$ \\
\hline 5993 & LCA5 & \begin{tabular}{|l|l|}
-7.13 \\
\end{tabular} & $1.56 \mathrm{E}-04$ \\
\hline 5994 & LCAT & -1.83 & $8.75 \mathrm{E}-03$ \\
\hline 5995 & LCLAT1 & -3.24 & $5.67 \mathrm{E}-04$ \\
\hline 5996 & LCMT1 & -3.99 & $4.93 \mathrm{E}-03$ \\
\hline 5997 & LCORL & -2.33 & $1.60 \mathrm{E}-03$ \\
\hline 5998 & LDHA & -6.64 & $2.70 \mathrm{E}-05$ \\
\hline 5999 & LDHB & \begin{tabular}{|l|}
-7.41 \\
\end{tabular} & $2.99 \mathrm{E}-04$ \\
\hline 6000 & LDLRAD4 & -4.67 & $2.25 \mathrm{E}-03$ \\
\hline 6001 & LEFTY2 & -1.47 & $1.37 \mathrm{E}-03$ \\
\hline 6002 & LEMD3 & -1.39 & $5.15 \mathrm{E}-02$ \\
\hline 6003 & LEPREL2 & -1.84 & $9.18 \mathrm{E}-04$ \\
\hline 6004 & LEPROT & -3.36 & $9.56 \mathrm{E}-04$ \\
\hline 6005 & \begin{tabular}{|l|l} 
LEPROTL1 \\
\end{tabular} & \begin{tabular}{|l|}
-1.89 \\
\end{tabular} & $2.55 \mathrm{E}-03$ \\
\hline 6006 & LETM1 & -4.40 & $1.41 \mathrm{E}-04$ \\
\hline 6007 & LETM2 & -2.78 & $1.21 \mathrm{E}-04$ \\
\hline 6008 & LGALS2 & -3.63 & $4.15 \mathrm{E}-03$ \\
\hline 6009 & LGALSL & -1.81 & $8.09 \mathrm{E}-05$ \\
\hline 6010 & LGI1 & -1.52 & $2.85 \mathrm{E}-02$ \\
\hline 6011 & LGMN & -2.50 & $1.23 \mathrm{E}-03$ \\
\hline 6012 & LGR4 & -3.00 & $1.98 \mathrm{E}-04$ \\
\hline 6013 & LHCGR & -2.68 & $2.16 \mathrm{E}-03$ \\
\hline 6014 & LHFPL5 & -2.53 & $1.67 \mathrm{E}-03$ \\
\hline 6015 & LAS & -3.61 & $1.02 \mathrm{E}-03$ \\
\hline 6016 & LIFR & -1.35 & $5.82 \mathrm{E}-02$ \\
\hline 6017 & LIG3 & -3.01 & $1.54 \mathrm{E}-03$ \\
\hline 6018 & LIG4 & -4.70 & $1.55 \mathrm{E}-04$ \\
\hline $\begin{array}{ll}6019 \\
\end{array}$ & LIMA1 & \begin{tabular}{l|l|}
-2.66 \\
\end{tabular} & $8.59 \mathrm{E}-04$ \\
\hline 6020 & LMD1 & -2.04 & $2.26 \mathrm{E}-02$ \\
\hline 6021 & LIN52 & -5.59 & $1.16 \mathrm{E}-03$ \\
\hline 6022 & LIN54 & -5.97 & $9.59 \mathrm{E}-05$ \\
\hline 6023 & LIN7C & -5.27 & $3.92 \mathrm{E}-04$ \\
\hline 6024 & LIN9 & -4.03 & $8.79 \mathrm{E}-04$ \\
\hline 6025 & LINGO1 & -1.35 & $4.24 \mathrm{E}-03$ \\
\hline 6026 & LINS & -1.66 & $5.78 \mathrm{E}-03$ \\
\hline 6027 & LIPC & -1.24 & $7.93 \mathrm{E}-02$ \\
\hline 6028 & LIPT1 & -2.38 & $3.99 \mathrm{E}-04$ \\
\hline 6029 & LITAF & -4.60 & $3.16 \mathrm{E}-04$ \\
\hline 6030 & LLPH & -6.67 & $4.03 E-04$ \\
\hline 6031 & LMAN1 & -6.60 & $2.56 \mathrm{E}-05$ \\
\hline 6032 & LMBR1 & -4.54 & $4.21 \mathrm{E}-03$ \\
\hline 6033 & LMBRD1 & -4.27 & $1.51 \mathrm{E}-04$ \\
\hline 6034 & LMF1 & -2.89 & $5.90 \mathrm{E}-04$ \\
\hline 6035 & LMF2 & -2.32 & $1.08 \mathrm{E}-02$ \\
\hline 6036 & LMNB2 & -1.98 & $2.45 \mathrm{E}-03$ \\
\hline 6037 & LMO7 & -1.91 & $2.37 \mathrm{E}-03$ \\
\hline 6038 & LNP1 & -2.21 & $5.36 \mathrm{E}-03$ \\
\hline 6039 & LOC100502566 & -2.79 & $5.62 \mathrm{E}-02$ \\
\hline 5040 & LOC1008 & -2.40 & $3.07 \mathrm{E}-03$ \\
\hline
\end{tabular}

\begin{tabular}{|c|c|c|c|}
\hline 6041 & LOC100857702 & -2.51 & $9.11 \mathrm{E}-04$ \\
\hline 6042 & LOC100857911 & -3.02 & $1.47 \mathrm{E}-03$ \\
\hline 6043 & LOC100857946 & -2.14 & $2.84 \mathrm{E}-04$ \\
\hline 6044 & LOC100858157 & -2.22 & $3.39 \mathrm{E}-03$ \\
\hline 6045 & LOC100858441 & -1.85 & $2.50 \mathrm{E}-03$ \\
\hline 6046 & LOC100858553 & -2.28 & $1.28 \mathrm{E}-03$ \\
\hline 6047 & LOC100858662 & -1.63 & $1.29 \mathrm{E}-03$ \\
\hline 6048 & LOC100858846 & -3.41 & $4.92 \mathrm{E}-03$ \\
\hline 6049 & LOC100859245 & -1.65 & $1.73 \mathrm{E}-03$ \\
\hline 6050 & LOC100859298 & -3.31 & $1.31 \mathrm{E}-02$ \\
\hline 6051 & LOC100859334 & -3.62 & $2.95 \mathrm{E}-04$ \\
\hline 6052 & LOC100859802 & -4.52 & $4.44 \mathrm{E}-04$ \\
\hline 6053 & LOC100859840 & -2.57 & $6.91 \mathrm{E}-04$ \\
\hline 6054 & LOC100859855 & -2.42 & $1.24 \mathrm{E}-02$ \\
\hline 6055 & LOC395159 & -3.94 & $6.34 \mathrm{E}-04$ \\
\hline 6056 & LOC395676 & -2.43 & $1.57 \mathrm{E}-03$ \\
\hline 6057 & LOC395787 & -1.39 & $2.19 \mathrm{E}-03$ \\
\hline 6058 & LOC395926 & -2.91 & $2.77 \mathrm{E}-02$ \\
\hline 6059 & LOC395991 & -2.55 & $1.15 \mathrm{E}-02$ \\
\hline 6060 & LOC396120 & -1.35 & $7.07 \mathrm{E}-03$ \\
\hline 6061 & LOC396380 & -6.79 & $7.20 \mathrm{E}-05$ \\
\hline 6062 & LOC415456 & -2.82 & $2.41 \mathrm{E}-04$ \\
\hline 6063 & LOC415464 & -3.86 & $2.97 \mathrm{E}-02$ \\
\hline 6064 & LOC415529 & -1.47 & $2.44 \mathrm{E}-03$ \\
\hline 6065 & LOC415708 & -1.41 & $7.29 \mathrm{E}-03$ \\
\hline 6066 & LOC415756 & -3.34 & $2.11 \mathrm{E}-03$ \\
\hline 6067 & LOC415859 & -2.75 & $1.58 \mathrm{E}-02$ \\
\hline 6068 & LOC415871 & -3.95 & $2.64 \mathrm{E}-04$ \\
\hline 6069 & LOC415872 & -4.96 & $4.80 \mathrm{E}-04$ \\
\hline 6070 & LOC415950 & -6.00 & $2.87 \mathrm{E}-05$ \\
\hline 6071 & LOC415976 & -1.72 & $5.80 \mathrm{E}-02$ \\
\hline 6072 & LOC416055 & -5.44 & $7.05 E-05$ \\
\hline 6073 & LOC416390 & -6.85 & $2.19 \mathrm{E}-04$ \\
\hline 6074 & LOC416415 & -2.69 & $4.56 \mathrm{E}-03$ \\
\hline 6075 & LOC416500 & -1.05 & $2.01 \mathrm{E}-03$ \\
\hline 6076 & LOC416530 & -4.06 & $3.55 \mathrm{E}-03$ \\
\hline 6077 & LOC416668 & -2.09 & $8.17 \mathrm{E}-04$ \\
\hline 6078 & LOC416916 & -2.71 & $1.04 \mathrm{E}-03$ \\
\hline 6079 & LOC416931 & -2.58 & $3.04 \mathrm{E}-02$ \\
\hline 6080 & LOC416951 & -4.03 & $2.19 \mathrm{E}-02$ \\
\hline 6081 & LOC416968 & -2.15 & $2.65 \mathrm{E}-04$ \\
\hline 6082 & LOC416993 & -2.41 & $3.24 \mathrm{E}-03$ \\
\hline 6083 & LOC416995 & -2.34 & $5.90 \mathrm{E}-03$ \\
\hline 6084 & LOC417263 & \begin{tabular}{ll|}
-7.04 \\
\end{tabular} & $4.00 \mathrm{E}-04$ \\
\hline 6085 & LOC417325 & -1.31 & $6.80 \mathrm{E}-02$ \\
\hline 6086 & LOC417340 & -3.06 & $1.23 \mathrm{E}-03$ \\
\hline 6087 & LOC417380 & -7.62 & $5.60 \mathrm{E}-04$ \\
\hline 6088 & LOC417386 & -2.49 & $4.27 \mathrm{E}-03$ \\
\hline 6089 & LOC417531 & -8.24 & $1.31 \mathrm{E}-04$ \\
\hline 6090 & LOC417551 & -4.53 & $1.35 \mathrm{E}-03$ \\
\hline 6091 & LOC417800 & -1.73 & $2.50 \mathrm{E}-02$ \\
\hline 6092 & LOC417948 & -2.32 & $3.48 \mathrm{E}-02$ \\
\hline 6093 & LOC417954 & -1.74 & $1.03 \mathrm{E}-01$ \\
\hline 6094 & LOC418170 & -5.73 & $2.07 \mathrm{E}-04$ \\
\hline 6095 & LOC418249 & -1.19 & $2.98 \mathrm{E}-03$ \\
\hline 6096 & LOC418362 & -1.32 & $3.91 \mathrm{E}-03$ \\
\hline 6097 & LOC418395 & -5.42 & $4.41 \mathrm{E}-04$ \\
\hline 6098 & LOC418713 & -2.70 & $1.53 \mathrm{E}-03$ \\
\hline 6099 & LOC418756 & -4.15 & $6.97 \mathrm{E}-04$ \\
\hline 6100 & LOC418811 & -6.41 & $4.68 \mathrm{E}-05$ \\
\hline 6101 & LOC418918 & -1.48 & $8.31 \mathrm{E}-02$ \\
\hline 6102 & LOC418927 & -4.01 & $8.95 \mathrm{E}-04$ \\
\hline 6103 & LOC419074 & -2.57 & $2.35 \mathrm{E}-03$ \\
\hline
\end{tabular}

\begin{tabular}{|c|c|c|c|}
\hline 6104 & LOC419136 & -1.58 & $5.75 E-02$ \\
\hline 6105 & LOC419390 & -1.72 & $1.13 E-02$ \\
\hline 6106 & LOC419404 & $\begin{array}{l}-4.17 \\
\end{array}$ & $9.70 E-05$ \\
\hline 6107 & LOC419409 & -2.62 & $5.12 \mathrm{E}-03$ \\
\hline 6108 & LOC419511 & \begin{tabular}{|l|}
-4.21 \\
\end{tabular} & $5.48 \mathrm{E}-04$ \\
\hline 6109 & LOC419545 & -1.40 & $1.38 \mathrm{E}-03$ \\
\hline 6110 & LOC419602 & -2.30 & $8.01 E-03$ \\
\hline 6111 & LOC419693 & -1.83 & $7.28 \mathrm{E}-03$ \\
\hline 6112 & LOC419782 & -6.24 & $2.69 \mathrm{E}-03$ \\
\hline 6113 & LOC419830 & -7.40 & $4.88 \mathrm{E}-04$ \\
\hline 6114 & $\begin{array}{l}\text { LOC420108 } \\
\end{array}$ & $\begin{array}{l}-1.78 \\
\end{array}$ & $3.37 E-02$ \\
\hline 6115 & LOC420153 & -2.53 & 4.49E-04 \\
\hline 6116 & LOC420220 & -9.04 & $1.49 \mathrm{E}-03$ \\
\hline 6117 & LOC420411 & -3.80 & $3.57 E-04$ \\
\hline 6118 & LOC420454 & -2.45 & $5.15 \mathrm{E}-03$ \\
\hline 6119 & LOC420466 & -1.51 & $3.84 \mathrm{E}-02$ \\
\hline 6120 & LOC420516 & -3.37 & $1.44 E-03$ \\
\hline 6121 & LOC420552 & -2.43 & $3.60 E-03$ \\
\hline 6122 & LOC420553 & -1.12 & $2.34 \mathrm{E}-03$ \\
\hline 6123 & LOC420579 & -2.60 & $4.43 E-03$ \\
\hline 6124 & LOC420606 & \begin{tabular}{|l|}
-1.71 \\
\end{tabular} & 1.47E-01 \\
\hline 6125 & LOC420707 & -2.67 & $1.32 E-03$ \\
\hline 6126 & LOC420716 & -1.45 & $1.89 E-03$ \\
\hline 6127 & LOC420726 & -3.78 & $1.22 \mathrm{E}-02$ \\
\hline 6128 & LOC420734 & $\begin{array}{l}-7.94 \\
\end{array}$ & $3.09 E-04$ \\
\hline 6129 & LOC420793 & -3.80 & $8.08 E-04$ \\
\hline 6130 & LOC420807 & -4.41 & $3.93 E-04$ \\
\hline 6131 & LOC420860 & -3.69 & $4.39 E-03$ \\
\hline 6132 & LOC420915 & -2.00 & $4.21 \mathrm{E}-03$ \\
\hline 6133 & LOC420992 & -1.04 & $1.93 E-02$ \\
\hline 6134 & LOC421099 & -5.21 & $7.58 \mathrm{E}-04$ \\
\hline 6135 & LOC421212 & -4.66 & $7.06 E-05$ \\
\hline 6136 & LOC421259 & -4.67 & $1.77 E-03$ \\
\hline 6137 & LOC421419 & -2.38 & $7.98 E-05$ \\
\hline 6138 & LOC421447 & -1.88 & $5.92 E-03$ \\
\hline 6139 & LOC421506 & -3.24 & $2.55 \mathrm{E}-03$ \\
\hline 6140 & LOC421792 & -5.20 & $1.09 E-04$ \\
\hline 6141 & LOC421805 & -4.16 & $2.11 E-04$ \\
\hline 6142 & LOC421975 & -5.24 & $6.53 \mathrm{E}-04$ \\
\hline 6143 & LOC421978 & -6.55 & $3.80 \mathrm{E}-04$ \\
\hline 6144 & LOC422008 & -8.62 & $3.71 E-04$ \\
\hline 6145 & LOC422051 & -4.56 & $3.36 \mathrm{E}-03$ \\
\hline 6146 & LOC422071 & -6.52 & $7.99 \mathrm{E}-04$ \\
\hline 6147 & LOC422090 & -3.69 & $4.95 E-04$ \\
\hline 6148 & LOC422151 & -1.44 & $6.43 E-04$ \\
\hline 6149 & LOC422198 & -5.97 & $2.16 \mathrm{E}-04$ \\
\hline 6150 & LOC422212 & -3.79 & $1.06 E-03$ \\
\hline 6151 & LOC422221 & $\begin{array}{l}-2.55 \\
\end{array}$ & $9.35 E-03$ \\
\hline 6152 & LOC422249 & -4.30 & $1.25 \mathrm{E}-03$ \\
\hline 6153 & LOC422270 & -1.92 & $1.82 E-02$ \\
\hline 6154 & LOC422304 & -4.90 & $2.38 E-04$ \\
\hline 6155 & LOC422426 & -8.34 & $7.95 E-05$ \\
\hline 6156 & LOC422442 & -2.82 & $2.49 E-03$ \\
\hline 6157 & LOC422448 & -2.32 & $2.76 \mathrm{E}-02$ \\
\hline 6158 & LOC422513 & -4.45 & $2.70 \mathrm{E}-03$ \\
\hline 6159 & LOC422577 & -1.63 & $3.53 \mathrm{E}-02$ \\
\hline 6160 & LOC422609 & -2.50 & $6.11 E-04$ \\
\hline 6161 & LOC422619 & -1.65 & $2.07 E-03$ \\
\hline 6162 & LOC422681 & -2.03 & $2.09 E-02$ \\
\hline 6163 & LOC422821 & -2.03 & $4.38 E-04$ \\
\hline 6164 & LOC422887 & -2.57 & $4.48 E-03$ \\
\hline 6165 & LOC422895 & -5.10 & $1.13 \mathrm{E}-03$ \\
\hline 166 & LOC & -3.60 & \\
\hline
\end{tabular}




\begin{tabular}{|c|c|c|c|}
\hline 6167 & LOC422928 & -4.94 & $2.68 \mathrm{E}-02$ \\
\hline 6168 & LOC422993 & -1.55 & $1.12 \mathrm{E}-02$ \\
\hline 6169 & LOC423006 & -4.99 & $2.23 \mathrm{E}-04$ \\
\hline 6170 & LOC423008 & -3.51 & $2.63 \mathrm{E}-03$ \\
\hline 6171 & LOC423256 & -2.41 & $1.62 \mathrm{E}-02$ \\
\hline 6172 & LOC423277 & -4.65 & $3.35 \mathrm{E}-04$ \\
\hline 6173 & LOC423306 & -1.85 & $2.58 \mathrm{E}-03$ \\
\hline 6174 & LOC423317 & -2.99 & $7.98 \mathrm{E}-03$ \\
\hline 6175 & LOC423321 & -5.92 & $1.04 E-04$ \\
\hline 6176 & LOC423425 & -2.85 & $1.44 \mathrm{E}-03$ \\
\hline 6177 & LOC423462 & -6.96 & $1.04 \mathrm{E}-03$ \\
\hline 6178 & LOC423499 & -1.68 & $3.83 \mathrm{E}-04$ \\
\hline 6179 & LOC423740 & -2.53 & $4.29 \mathrm{E}-03$ \\
\hline 6180 & LOC423793 & -5.08 & $1.37 \mathrm{E}-03$ \\
\hline 6181 & LOC423967 & -3.36 & $3.42 \mathrm{E}-03$ \\
\hline 6182 & LOC424014 & -4.33 & $2.23 \mathrm{E}-03$ \\
\hline 6183 & LOC424109 & -2.20 & $1.54 \mathrm{E}-02$ \\
\hline 6184 & LOC424111 & -3.42 & $1.25 \mathrm{E}-03$ \\
\hline 6185 & LOC424201 & -2.92 & $9.50 \mathrm{E}-03$ \\
\hline 6186 & LOC424459 & -1.09 & $1.45 \mathrm{E}-02$ \\
\hline 6187 & LOC424676 & -1.66 & $1.70 \mathrm{E}-02$ \\
\hline 6188 & LOC424816 & -2.95 & $1.07 \mathrm{E}-02$ \\
\hline 6189 & LOC424925 & -7.17 & $2.06 \mathrm{E}-04$ \\
\hline 6190 & LOC425001 & -3.05 & $1.68 \mathrm{E}-03$ \\
\hline 6191 & LOC425011 & -2.83 & $6.94 \mathrm{E}-03$ \\
\hline 6192 & LOC425015 & -1.91 & $3.91 \mathrm{E}-03$ \\
\hline 6193 & LOC425441 & -5.16 & $1.37 \mathrm{E}-03$ \\
\hline 6194 & LOC425470 & -3.53 & $3.88 \mathrm{E}-04$ \\
\hline 6195 & LOC425531 & -3.58 & $3.72 \mathrm{E}-04$ \\
\hline 6196 & LOC426015 & -5.57 & $2.51 \mathrm{E}-03$ \\
\hline 6197 & LOC426023 & -1.68 & $1.28 \mathrm{E}-02$ \\
\hline 6198 & LOC426034 & -2.52 & $9.06 \mathrm{E}-03$ \\
\hline 6199 & LOC426092 & -1.80 & $6.30 \mathrm{E}-03$ \\
\hline 6200 & LOC426097 & -4.57 & $2.22 \mathrm{E}-03$ \\
\hline 6201 & LOC426106 & -1.62 & $4.66 \mathrm{E}-02$ \\
\hline 6202 & LOC426128 & -1.80 & $9.08 \mathrm{E}-03$ \\
\hline 6203 & LOC426187 & -2.89 & $1.36 \mathrm{E}-03$ \\
\hline 6204 & LOC426295 & -5.53 & $4.03 \mathrm{E}-04$ \\
\hline 6205 & LOC426385 & -1.10 & 7.44E-04 \\
\hline 6206 & LOC426514 & -2.36 & $2.17 \mathrm{E}-04$ \\
\hline 6207 & LOC426710 & -1.46 & $1.68 \mathrm{E}-02$ \\
\hline 6208 & LOC426801 & -1.21 & $5.64 \mathrm{E}-03$ \\
\hline 6209 & LOC426880 & -4.36 & $8.52 \mathrm{E}-05$ \\
\hline 6210 & LOC427001 & -2.15 & $4.90 \mathrm{E}-04$ \\
\hline 6211 & LOC427161 & -4.44 & $1.38 \mathrm{E}-04$ \\
\hline 6212 & LOC427241 & -1.84 & $2.91 \mathrm{E}-03$ \\
\hline 6213 & LOC427259 & -1.32 & $1.43 \mathrm{E}-02$ \\
\hline 6214 & LOC427369 & -8.04 & $5.01 \mathrm{E}-04$ \\
\hline 6215 & LOC427419 & -2.76 & $4.32 E-05$ \\
\hline 6216 & LOC427826 & -4.27 & $3.68 \mathrm{E}-03$ \\
\hline 6217 & LOC427882 & -3.42 & $3.72 \mathrm{E}-03$ \\
\hline 6218 & LOC427896 & -2.71 & $7.25 \mathrm{E}-03$ \\
\hline 6219 & LOC429054 & -1.40 & $2.03 \mathrm{E}-02$ \\
\hline 6220 & LOC429115 & -5.17 & $1.01 \mathrm{E}-04$ \\
\hline 6221 & LOC429566 & -6.42 & $3.81 \mathrm{E}-03$ \\
\hline 6222 & LOC430766 & -4.10 & $1.80 \mathrm{E}-02$ \\
\hline 6223 & LOC430910 & -5.69 & $3.30 \mathrm{E}-04$ \\
\hline 6224 & LOC693257 & -6.16 & $3.73 \mathrm{E}-04$ \\
\hline 6225 & LOC693265 & -4.73 & $1.59 \mathrm{E}-04$ \\
\hline 6226 & LOC768418 & -1.55 & $3.97 \mathrm{E}-03$ \\
\hline 6227 & LOC768497 & -8.60 & $3.55 \mathrm{E}-04$ \\
\hline 6228 & LOC768565 & -1.74 & $1.71 \mathrm{E}-02$ \\
\hline 6229 & LOC768701 & -3.62 & 8.68 \\
\hline
\end{tabular}

\begin{tabular}{|c|c|c|c|}
\hline 6230 & LOC768709 & -3.50 & $8.37 \mathrm{E}-03$ \\
\hline 6231 & LOC768803 & -5.75 & $1.44 \mathrm{E}-03$ \\
\hline 6232 & LOC768819 & -6.76 & $1.27 \mathrm{E}-03$ \\
\hline 6233 & LOC768945 & -1.48 & $4.69 \mathrm{E}-02$ \\
\hline 6234 & LOC769134 & -6.23 & $2.32 \mathrm{E}-06$ \\
\hline 6235 & LOC7692777 & -2.91 & $1.67 \mathrm{E}-02$ \\
\hline 6236 & LOC769329 & -2.05 & $5.55 \mathrm{E}-02$ \\
\hline 6237 & LOC769463 & -1.80 & $2.87 \mathrm{E}-02$ \\
\hline 6238 & LOC770271 & -4.50 & $3.59 \mathrm{E}-04$ \\
\hline 6239 & LOC770483 & -6.24 & $1.14 \mathrm{E}-03$ \\
\hline 6240 & LOC770490 & -1.94 & $1.25 \mathrm{E}-03$ \\
\hline 6241 & LOC770616 & -4.46 & $2.88 \mathrm{E}-03$ \\
\hline 6242 & LOC770634 & -2.28 & $6.17 \mathrm{E}-03$ \\
\hline 6243 & LOC770653 & -1.29 & $4.04 \mathrm{E}-02$ \\
\hline 6244 & LOC770729 & -8.53 & $5.81 \mathrm{E}-05$ \\
\hline 6245 & LOC770771 & -5.46 & $2.17 \mathrm{E}-04$ \\
\hline 6246 & LOC770804 & -5.25 & $6.24 \mathrm{E}-04$ \\
\hline 6247 & LOC770838 & -6.69 & $3.03 \mathrm{E}-04$ \\
\hline 6248 & LOC770857 & -3.72 & $4.14 \mathrm{E}-03$ \\
\hline 6249 & LOC771022 & -4.90 & $8.55 \mathrm{E}-04$ \\
\hline 6250 & LOC771069 & -1.84 & $4.30 \mathrm{E}-02$ \\
\hline 6251 & LOC771098 & -3.30 & $2.07 \mathrm{E}-03$ \\
\hline 6252 & LOC771160 & -2.10 & $6.78 \mathrm{E}-03$ \\
\hline 6253 & LOC771178 & -4.65 & $7.37 \mathrm{E}-03$ \\
\hline 6254 & LOC771207 & -3.18 & $9.07 \mathrm{E}-04$ \\
\hline 6255 & LOC771339 & -6.04 & $1.28 \mathrm{E}-06$ \\
\hline 6256 & LOC771388 & -2.32 & $4.80 \mathrm{E}-03$ \\
\hline 6257 & LOC771527 & -3.75 & $7.15 \mathrm{E}-03$ \\
\hline 6258 & LOC771702 & -4.53 & $1.52 \mathrm{E}-03$ \\
\hline 6259 & LOC771713 & -3.83 & $1.56 \mathrm{E}-03$ \\
\hline 6260 & LOC771935 & -3.02 & $1.12 \mathrm{E}-02$ \\
\hline 6261 & LOC771947 & -7.12 & $5.62 E-05$ \\
\hline 6262 & LOC771956 & -2.94 & $2.00 \mathrm{E}-03$ \\
\hline 6263 & LOC772071 & -2.59 & $5.08 \mathrm{E}-02$ \\
\hline 6264 & LOC772245 & -1.35 & $4.37 \mathrm{E}-02$ \\
\hline 6265 & LOC772269 & -2.77 & $8.59 \mathrm{E}-04$ \\
\hline 6266 & LOC775963 & -3.25 & $7.75 \mathrm{E}-04$ \\
\hline 6267 & LOC776176 & -7.00 & $4.99 \mathrm{E}-04$ \\
\hline 6268 & LOC776458 & -1.22 & $1.60 \mathrm{E}-02$ \\
\hline 6269 & LOC776720 & -5.60 & $3.36 \mathrm{E}-04$ \\
\hline 6270 & LOH12CR1 & -2.86 & $2.25 \mathrm{E}-04$ \\
\hline 6271 & LONP1 & -1.82 & $5.56 \mathrm{E}-04$ \\
\hline 6272 & LONP2 & -3.48 & $1.25 \mathrm{E}-03$ \\
\hline 6273 & LOXL2 & -2.23 & $8.58 \mathrm{E}-04$ \\
\hline 6274 & LPAR1 & -3.07 & $4.11 \mathrm{E}-04$ \\
\hline 6275 & LPAR6 & -1.94 & $3.79 \mathrm{E}-02$ \\
\hline 6276 & LPCAT1 & -4.38 & $9.02 E-04$ \\
\hline 6277 & LPCAT2 & -5.52 & $1.07 E-04$ \\
\hline 6278 & LPCAT3 & -3.11 & $5.46 \mathrm{E}-04$ \\
\hline 6279 & LPIN2 & -6.25 & $4.15 \mathrm{E}-04$ \\
\hline 6280 & LPPR5 & -2.68 & $1.03 \mathrm{E}-05$ \\
\hline 6281 & LRCH1 & -2.38 & $2.72 \mathrm{E}-03$ \\
\hline 6282 & LRIF1 & -2.91 & $1.38 \mathrm{E}-02$ \\
\hline 6283 & LRMP & -1.47 & $1.20 \mathrm{E}-02$ \\
\hline 6284 & LRP2BP & -4.68 & $8.17 \mathrm{E}-04$ \\
\hline 6285 & LRPAP1 & -5.20 & $4.67 \mathrm{E}-03$ \\
\hline 6286 & LRPPRC & -3.35 & $1.20 \mathrm{E}-02$ \\
\hline 6287 & LRRC23 & -2.86 & $1.59 \mathrm{E}-04$ \\
\hline 6288 & LRRC28 & -3.82 & $1.84 \mathrm{E}-05$ \\
\hline 6289 & LRRC40 & -5.47 & $1.32 \mathrm{E}-04$ \\
\hline 6290 & LRRC45 & -4.19 & $1.65 \mathrm{E}-03$ \\
\hline 6291 & LRRC46 & -2.26 & $6.28 \mathrm{E}-04$ \\
\hline 6292 & LRRC47 & -1.20 & $2.60 \mathrm{E}-02$ \\
\hline
\end{tabular}

\begin{tabular}{|c|c|c|c|}
\hline 6293 & LRRC48 & -7.46 & $1.49 \mathrm{E}-04$ \\
\hline 6294 & $\begin{array}{l}\text { LRRC49 } \\
\end{array}$ & -3.35 & $2.05 \mathrm{E}-03$ \\
\hline 6295 & LRRC57 & -1.33 & $1.64 \mathrm{E}-02$ \\
\hline 6296 & LRRC59 & -4.03 & $1.39 \mathrm{E}-03$ \\
\hline 6297 & LRRC6 & -6.34 & $2.88 \mathrm{E}-04$ \\
\hline 6298 & LRRC61 & -2.30 & $6.93 \mathrm{E}-03$ \\
\hline 6299 & $\begin{array}{l}\text { LRRC72 } \\
\end{array}$ & $\begin{array}{l}-7.38 \\
\end{array}$ & $1.94 \mathrm{E}-04$ \\
\hline 6300 & LRRC73 & -3.61 & $3.45 \mathrm{E}-05$ \\
\hline 6301 & LRRC8B & -3.02 & $4.52 \mathrm{E}-02$ \\
\hline 6302 & \begin{tabular}{ll|} 
LRRCC1 \\
\end{tabular} & -4.57 & $1.03 \mathrm{E}-03$ \\
\hline 6303 & LRRFIP2 & -4.02 & $1.11 \mathrm{E}-03$ \\
\hline 6304 & LRRIQ4 & -7.18 & $7.80 \mathrm{E}-04$ \\
\hline 6305 & LSAMP & -3.94 & $2.64 \mathrm{E}-04$ \\
\hline 6306 & LSG1 & -6.28 & $1.63 \mathrm{E}-03$ \\
\hline 6307 & LSM1 & -4.48 & $9.45 \mathrm{E}-04$ \\
\hline 6308 & LSM10 & -2.69 & $1.64 \mathrm{E}-03$ \\
\hline 6309 & LSM14A & -4.13 & $1.51 \mathrm{E}-04$ \\
\hline 6310 & LSM3 & -3.88 & $2.27 \mathrm{E}-03$ \\
\hline 6311 & LSM4 & -3.18 & $1.11 \mathrm{E}-02$ \\
\hline $\begin{array}{l}6312 \\
\end{array}$ & LSM5 & -5.17 & $1.52 \mathrm{E}-04$ \\
\hline 6313 & LSM6 & -4.65 & $3.16 \mathrm{E}-03$ \\
\hline 6314 & LSMD1 & -3.25 & $2.10 \mathrm{E}-03$ \\
\hline 6315 & LSS & -3.28 & $7.21 \mathrm{E}-04$ \\
\hline 6316 & LTA4H & -5.13 & $4.90 \mathrm{E}-05$ \\
\hline 6317 & LTK & -2.08 & $7.57 \mathrm{E}-02$ \\
\hline 6318 & LTN1 & -3.54 & $8.54 \mathrm{E}-04$ \\
\hline 6319 & LTV1 & -3.35 & $\begin{array}{l}1.28 \mathrm{E}-03 \\
\end{array}$ \\
\hline 6320 & LUC7L2 & -3.08 & $5.80 \mathrm{E}-04$ \\
\hline 6321 & LUC7L3 & -5.83 & $5.12 \mathrm{E}-05$ \\
\hline $\begin{array}{l}6322 \\
\end{array}$ & LUZP1 & -4.25 & $4.13 \mathrm{E}-04$ \\
\hline 6323 & LY6E & -1.98 & $1.22 \mathrm{E}-02$ \\
\hline 6324 & LYAR & -6.99 & $6.05 \mathrm{E}-04$ \\
\hline 6325 & LYN & -1.31 & $1.69 \mathrm{E}-03$ \\
\hline 6326 & LYPD6B & -1.61 & $2.12 \mathrm{E}-02$ \\
\hline 6327 & LYPLAL1 & -2.82 & $4.02 E-04$ \\
\hline $\begin{array}{l}6328 \\
\end{array}$ & $\begin{array}{l}\text { LYRM1 } \\
\end{array}$ & -3.05 & $2.18 \mathrm{E}-03$ \\
\hline 6329 & LYRM2 & -3.62 & $2.01 \mathrm{E}-03$ \\
\hline 6330 & LYRM5 & -4.72 & 7.94E-04 \\
\hline 6331 & LYRM7 & -5.03 & $1.27 \mathrm{E}-05$ \\
\hline 6332 & LYSMD2 & -6.62 & $1.78 \mathrm{E}-04$ \\
\hline 6333 & LYVE1 & -2.29 & $3.41 \mathrm{E}-03$ \\
\hline $\begin{array}{ll}6334 \\
\end{array}$ & LYZ & -2.35 & $1.04 \mathrm{E}-03$ \\
\hline 6335 & LZIC & -3.73 & $4.27 \mathrm{E}-04$ \\
\hline 6336 & LZTFL1 & -6.00 & $2.83 \mathrm{E}-03$ \\
\hline 6337 & M28628 & -3.12 & $2.61 \mathrm{E}-03$ \\
\hline 6338 & M63624 & -5.04 & $9.24 \mathrm{E}-04$ \\
\hline 6339 & M6PR & -2.13 & $7.18 \mathrm{E}-04$ \\
\hline 6340 & MACROD2 & -2.92 & $\begin{array}{l}7.99 \mathrm{E}-03 \\
\end{array}$ \\
\hline 6341 & MAD2L1 & -4.57 & $1.78 \mathrm{E}-03$ \\
\hline 6342 & MAD2L1BP & -2.70 & $2.61 \mathrm{E}-04$ \\
\hline 6343 & MAD2L2 & -3.20 & $5.07 \mathrm{E}-03$ \\
\hline 6344 & MAEA & -4.44 & $8.99 \mathrm{E}-04$ \\
\hline 6345 & MAEL & -4.03 & $2.03 \mathrm{E}-03$ \\
\hline 6346 & MAGI3 & -1.23 & $9.15 \mathrm{E}-03$ \\
\hline 6347 & $\mathrm{MAGOH}$ & -6.76 & $2.04 \mathrm{E}-04$ \\
\hline 6348 & MAGT1 & -4.77 & $7.29 \mathrm{E}-04$ \\
\hline 6349 & MAK & -5.29 & $1.63 \mathrm{E}-03$ \\
\hline 6350 & MAK16 & -1.30 & $1.59 \mathrm{E}-03$ \\
\hline 6351 & MAL2 & -1.50 & $2.11 \mathrm{E}-03$ \\
\hline 6352 & MALSU1 & -1.89 & $6.28 \mathrm{E}-03$ \\
\hline 6353 & MAML1 & -1.12 & $2.67 \mathrm{E}-03$ \\
\hline (6354 & MAN1B1 & -2.14 & $1.02 \mathrm{E}-02$ \\
\hline 6355 & MAN1C1 & -2.84 & $4.09 \mathrm{E}-03$ \\
\hline
\end{tabular}




\begin{tabular}{|c|c|c|c|}
\hline 6356 & MAN2B2 & -3.78 & $4.01 \mathrm{E}-03$ \\
\hline 6357 & MANBA & -4.91 & $4.23 \mathrm{E}-03$ \\
\hline 6358 & MANF & -5.62 & $1.27 \mathrm{E}-03$ \\
\hline 6359 & MANSC1 & -2.41 & $2.44 \mathrm{E}-03$ \\
\hline 6360 & MAP1A & -1.89 & $2.91 \mathrm{E}-04$ \\
\hline 6361 & MAP1LC3B & -4.72 & $2.80 \mathrm{E}-03$ \\
\hline 6362 & MAP1LC3C & -3.56 & $5.78 \mathrm{E}-04$ \\
\hline 6363 & МАР2К1 & -4.50 & $1.96 \mathrm{E}-04$ \\
\hline 6364 & MAP2K4 & -2.23 & $1.48 \mathrm{E}-02$ \\
\hline 6365 & MAP2K5 & -2.76 & $7.28 \mathrm{E}-04$ \\
\hline 6366 & МАРЗК15 & -1.95 & $1.64 \mathrm{E}-02$ \\
\hline 6367 & MAP3K7 & -3.17 & $9.40 \mathrm{E}-07$ \\
\hline 6368 & MAP4K3 & -2.81 & $6.56 \mathrm{E}-04$ \\
\hline 6369 & MAP9 & -5.79 & 2.44E-04 \\
\hline 6370 & MAPK1 & -2.49 & 4.30E-03 \\
\hline 6371 & MAPK11 & -1.74 & $2.42 \mathrm{E}-03$ \\
\hline 6372 & MAPK12 & -2.50 & $1.96 \mathrm{E}-03$ \\
\hline 6373 & MAPK14 & -5.17 & $3.40 \mathrm{E}-04$ \\
\hline 6374 & MAPK1IP1L & -1.59 & $2.01 \mathrm{E}-02$ \\
\hline 6375 & MAPK6 & -3.10 & $3.78 \mathrm{E}-04$ \\
\hline 6376 & MAPK8IP3 & -1.94 & $7.88 \mathrm{E}-04$ \\
\hline 6377 & MAPKAPK2 & -1.92 & 3.67E-03 \\
\hline 6378 & MAPKAPK5 & -2.42 & $1.44 \mathrm{E}-03$ \\
\hline 6379 & MAPRE1 & -3.27 & $2.26 \mathrm{E}-02$ \\
\hline 6380 & MAPRE2 & -3.29 & $2.53 \mathrm{E}-03$ \\
\hline 6381 & MAPT & -6.77 & $5.76 \mathrm{E}-04$ \\
\hline 6382 & MARCKS & -1.85 & $3.01 \mathrm{E}-02$ \\
\hline 6383 & MARCKSL1 & -6.94 & $1.44 \mathrm{E}-04$ \\
\hline 6384 & MARF1 & -2.43 & 2.87E-03 \\
\hline 6385 & MARK1 & -2.24 & $1.52 \mathrm{E}-02$ \\
\hline 6386 & MARK3 & -3.44 & $5.79 \mathrm{E}-04$ \\
\hline 6387 & MARS2 & -1.70 & $2.54 \mathrm{E}-02$ \\
\hline 6388 & MARVELD2 & -3.24 & $4.06 \mathrm{E}-04$ \\
\hline 6389 & MAST2 & -2.74 & $2.21 \mathrm{E}-03$ \\
\hline 6390 & MASTL & -1.29 & $2.76 \mathrm{E}-03$ \\
\hline 6391 & MAT2B & -3.59 & $9.71 \mathrm{E}-04$ \\
\hline 6392 & MATN2 & -3.22 & $1.31 \mathrm{E}-03$ \\
\hline 6393 & MATN3 & -4.13 & 7.07E-03 \\
\hline 6394 & MATR3 & -4.50 & 4.03E-05 \\
\hline 6395 & MAU2 & -4.47 & $7.78 \mathrm{E}-04$ \\
\hline 6396 & MBD2 & -4.30 & $1.28 \mathrm{E}-03$ \\
\hline 6397 & MBD4 & -7.22 & $1.91 \mathrm{E}-04$ \\
\hline 6398 & MBIP & -3.50 & 4.60E-04 \\
\hline 6399 & MBLAC2 & -2.64 & $3.66 \mathrm{E}-03$ \\
\hline 6400 & MCAT & -5.38 & $2.12 \mathrm{E}-04$ \\
\hline 6401 & MCCC1 & -3.07 & $7.36 \mathrm{E}-03$ \\
\hline 6402 & MCEE & -3.52 & $5.19 \mathrm{E}-04$ \\
\hline 6403 & MCF2 & -1.54 & 4.33E-02 \\
\hline 6404 & MCFD2 & -4.35 & $1.56 \mathrm{E}-03$ \\
\hline 6405 & MCL1 & -1.66 & $1.19 \mathrm{E}-01$ \\
\hline 6406 & MCM2 & -4.86 & $7.25 \mathrm{E}-05$ \\
\hline 6407 & MCM3 & -3.28 & $2.14 \mathrm{E}-03$ \\
\hline 6408 & MCM3AP & -4.94 & $2.61 \mathrm{E}-04$ \\
\hline 6409 & MCM6 & -1.99 & $5.77 \mathrm{E}-04$ \\
\hline 6410 & MCM8 & -4.32 & 2.26E-04 \\
\hline 6411 & MCMBP & -3.21 & $9.41 \mathrm{E}-04$ \\
\hline 6412 & MCOLN2 & -1.31 & $7.28 \mathrm{E}-03$ \\
\hline 6413 & MCPH1 & -3.99 & $5.44 \mathrm{E}-04$ \\
\hline 6414 & MCTS1 & -5.97 & $3.49 \mathrm{E}-04$ \\
\hline 6415 & MDH1 & -4.90 & $4.93 \mathrm{E}-03$ \\
\hline 6416 & MDH1B & -6.87 & $1.46 \mathrm{E}-03$ \\
\hline 6417 & $\mathrm{MDH} 2$ & -2.48 & 2.67E-03 \\
\hline 6418 & MDM1 & -1.74 & $4.21 \mathrm{E}-03$ \\
\hline
\end{tabular}

\begin{tabular}{|c|c|c|c|}
\hline 6419 & MDM4 & -5.95 & $1.61 \mathrm{E}-03$ \\
\hline 6420 & ME1 & -2.26 & $1.74 \mathrm{E}-02$ \\
\hline 6421 & ME3 & -1.25 & $1.83 \mathrm{E}-02$ \\
\hline 6422 & MEAF6 & -5.04 & $1.54 \mathrm{E}-04$ \\
\hline 6423 & MED1 & -1.21 & $4.17 \mathrm{E}-03$ \\
\hline 6424 & MED10 & -4.75 & $2.66 \mathrm{E}-04$ \\
\hline 6425 & MED12 & -3.44 & $1.07 \mathrm{E}-04$ \\
\hline 6426 & MED16 & -1.58 & $7.02 E-04$ \\
\hline 6427 & MED17 & -3.47 & $1.16 \mathrm{E}-03$ \\
\hline 6428 & MED19 & -4.34 & $3.23 \mathrm{E}-03$ \\
\hline 6429 & MED20 & -5.53 & $8.76 \mathrm{E}-05$ \\
\hline 6430 & MED21 & -3.11 & $7.30 \mathrm{E}-04$ \\
\hline 6431 & MED22 & -2.53 & $8.06 \mathrm{E}-03$ \\
\hline 6432 & MED23 & -3.69 & $2.98 \mathrm{E}-04$ \\
\hline 6433 & MED24 & -2.48 & $2.91 \mathrm{E}-02$ \\
\hline 6434 & MED27 & -3.79 & $1.98 \mathrm{E}-04$ \\
\hline 6435 & MED28 & -5.52 & $1.40 \mathrm{E}-03$ \\
\hline 6436 & MED30 & -5.75 & $1.10 \mathrm{E}-03$ \\
\hline 6437 & MED31 & -4.22 & $2.03 \mathrm{E}-03$ \\
\hline 6438 & MED4 & -3.46 & $5.06 \mathrm{E}-03$ \\
\hline 6439 & MED6 & -3.47 & $1.88 \mathrm{E}-03$ \\
\hline 6440 & MED8 & -3.81 & $1.61 \mathrm{E}-04$ \\
\hline 6441 & MED9 & -2.90 & $3.75 \mathrm{E}-03$ \\
\hline 6442 & MEF2BNB & -4.29 & $3.86 \mathrm{E}-04$ \\
\hline 6443 & MEI1 & -4.89 & $6.43 \mathrm{E}-04$ \\
\hline 6444 & MELK & -4.03 & $2.82 \mathrm{E}-04$ \\
\hline 6445 & MEMO1 & -3.87 & $1.52 \mathrm{E}-03$ \\
\hline 6446 & MEP1A & -2.37 & $4.64 \mathrm{E}-04$ \\
\hline 6447 & MEP1B & -1.32 & $1.61 \mathrm{E}-02$ \\
\hline 6448 & MESDC1 & -1.60 & $1.31 \mathrm{E}-02$ \\
\hline 6449 & MESDC2 & -6.72 & $2.64 \mathrm{E}-04$ \\
\hline 6450 & MEST & -1.57 & $5.33 \mathrm{E}-02$ \\
\hline 6451 & METAP2 & -5.89 & $1.81 \mathrm{E}-03$ \\
\hline 6452 & METTL10 & -4.07 & $2.75 \mathrm{E}-03$ \\
\hline 6453 & METTL11A & -4.19 & 8.90E-04 \\
\hline 6454 & METTL14 & -3.30 & $5.46 \mathrm{E}-03$ \\
\hline 6455 & METTL16 & -2.38 & $1.11 \mathrm{E}-03$ \\
\hline 6456 & METTL19 & -2.61 & $1.71 \mathrm{E}-03$ \\
\hline 6457 & METTL21A & -3.90 & $5.46 \mathrm{E}-06$ \\
\hline 6458 & METTL21D & -4.98 & $5.80 \mathrm{E}-05$ \\
\hline 6459 & METTL25 & -1.73 & $2.49 \mathrm{E}-02$ \\
\hline 6460 & METTL2A & -5.36 & $3.85 \mathrm{E}-04$ \\
\hline 6461 & METTL5 & -4.89 & $5.88 \mathrm{E}-04$ \\
\hline 6462 & METTL7A & -4.71 & $9.24 \mathrm{E}-04$ \\
\hline 6463 & METTL9 & -4.13 & $1.78 \mathrm{E}-04$ \\
\hline 6464 & MEX3D & -1.33 & $1.32 \mathrm{E}-02$ \\
\hline 6465 & MFAP1 & -2.65 & $1.35 \mathrm{E}-03$ \\
\hline 6466 & MFAP3 & -3.81 & 3.94E-04 \\
\hline 6467 & MFN1 & -4.37 & $1.04 \mathrm{E}-03$ \\
\hline 6468 & MFSD1 & -2.64 & $1.63 \mathrm{E}-03$ \\
\hline 6469 & MFSD10 & -6.88 & $1.55 \mathrm{E}-03$ \\
\hline 6470 & MFSD9 & -1.96 & $6.02 \mathrm{E}-03$ \\
\hline 6471 & MGAT5 & -3.03 & $3.51 \mathrm{E}-03$ \\
\hline 6472 & MGEA5 & -3.13 & $1.41 \mathrm{E}-03$ \\
\hline 6473 & MGLL & -2.22 & $2.35 \mathrm{E}-03$ \\
\hline 6474 & MGMT & -5.06 & $1.78 \mathrm{E}-04$ \\
\hline 6475 & MGST1 & -6.68 & $3.73 \mathrm{E}-05$ \\
\hline 6476 & MGST2 & -1.67 & $8.10 \mathrm{E}-03$ \\
\hline 6477 & MIB1 & -2.72 & $8.59 \mathrm{E}-04$ \\
\hline 6478 & MICALL1 & -3.38 & $8.98 \mathrm{E}-04$ \\
\hline 6479 & MICU1 & -2.43 & $1.21 \mathrm{E}-02$ \\
\hline 6480 & MID1IP1 & -3.76 & $4.98 \mathrm{E}-05$ \\
\hline 6481 & MINA & -2.71 & 4.77E-04 \\
\hline
\end{tabular}

\begin{tabular}{|c|c|c|c|}
\hline 6482 & MINOS1 & -4.95 & $8.33 \mathrm{E}-04$ \\
\hline 6483 & MIPEP & -5.61 & $9.83 \mathrm{E}-04$ \\
\hline 6484 & MIS12 & -2.66 & $1.50 \mathrm{E}-02$ \\
\hline 6485 & MIS18A & -2.01 & 7.07E-03 \\
\hline 6486 & MKI67 & -5.17 & $8.86 \mathrm{E}-05$ \\
\hline 6487 & MKI67IP & -5.29 & 3.97E-04 \\
\hline 6488 & MKKS & -4.43 & $2.92 \mathrm{E}-04$ \\
\hline 6489 & MKLN1 & -5.60 & $2.73 \mathrm{E}-04$ \\
\hline 6490 & MKNK1 & -3.70 & $7.83 \mathrm{E}-05$ \\
\hline 6491 & MKS1 & -3.38 & $3.20 \mathrm{E}-05$ \\
\hline 6492 & MLF1 & -7.19 & $8.11 \mathrm{E}-03$ \\
\hline 6493 & MLF1IP & -4.58 & $1.34 \mathrm{E}-04$ \\
\hline 6494 & MLF2 & -4.07 & $1.00 \mathrm{E}-03$ \\
\hline 6495 & MLH1 & -1.67 & $7.03 E-04$ \\
\hline 6496 & MLL3 & -2.93 & $2.76 \mathrm{E}-03$ \\
\hline 6497 & MLL5 & -4.73 & $1.15 \mathrm{E}-04$ \\
\hline 6498 & MLLT10 & -2.40 & $9.34 \mathrm{E}-04$ \\
\hline 6499 & MLLT3 & -3.46 & $1.64 \mathrm{E}-04$ \\
\hline 6500 & MLLT4 & -3.34 & $9.01 \mathrm{E}-04$ \\
\hline 6501 & MLST8 & -2.11 & $3.56 \mathrm{E}-03$ \\
\hline 6502 & MLX & -3.41 & $6.40 \mathrm{E}-05$ \\
\hline 6503 & MLXIP & -1.28 & $1.64 \mathrm{E}-02$ \\
\hline 6504 & MMAA & -2.80 & $2.41 \mathrm{E}-03$ \\
\hline 6505 & MMACHC & -3.00 & $7.76 \mathrm{E}-03$ \\
\hline 6506 & MMADHC & -4.90 & $1.93 \mathrm{E}-03$ \\
\hline 6507 & MMD & -4.72 & $4.08 \mathrm{E}-05$ \\
\hline 6508 & MMGT1 & -2.97 & $9.04 \mathrm{E}-04$ \\
\hline 6509 & MMS19 & -3.09 & $1.33 \mathrm{E}-03$ \\
\hline 6510 & MMS22L & -2.04 & $1.69 \mathrm{E}-03$ \\
\hline 6511 & MNAT1 & -2.87 & $2.04 \mathrm{E}-04$ \\
\hline 6512 & MND1 & -6.72 & $4.65 \mathrm{E}-04$ \\
\hline 6513 & MOB2 & -6.82 & $1.06 \mathrm{E}-03$ \\
\hline 6514 & МOB4 & -3.62 & $6.81 \mathrm{E}-04$ \\
\hline 6515 & MOBKL2B & -1.10 & $1.67 \mathrm{E}-02$ \\
\hline 6516 & MOCS2 & -3.51 & $9.19 \mathrm{E}-04$ \\
\hline 6517 & MOCS3 & -2.47 & $3.80 \mathrm{E}-03$ \\
\hline 6518 & MON1A & -1.98 & $9.47 \mathrm{E}-03$ \\
\hline 6519 & MON2 & -1.35 & $1.83 \mathrm{E}-03$ \\
\hline 6520 & MORC3 & -3.39 & $2.89 \mathrm{E}-04$ \\
\hline 6521 & MORF4L1 & -6.88 & $1.53 \mathrm{E}-04$ \\
\hline 6522 & MORN4 & -1.24 & $8.41 \mathrm{E}-03$ \\
\hline 6523 & MORN5 & -3.71 & $5.48 \mathrm{E}-04$ \\
\hline 6524 & MOSPD1 & -4.19 & $2.83 \mathrm{E}-03$ \\
\hline 6525 & MOSPD2 & -2.80 & $2.01 \mathrm{E}-04$ \\
\hline 6526 & MOXD1 & -1.18 & $2.00 \mathrm{E}-01$ \\
\hline 6527 & MPDZ & -1.58 & $5.39 \mathrm{E}-03$ \\
\hline 6528 & MPG & -2.17 & $6.27 \mathrm{E}-03$ \\
\hline 6529 & MPHOSPH10 & -3.63 & $3.52 \mathrm{E}-04$ \\
\hline 6530 & MPHOSPH6 & -4.28 & $3.28 \mathrm{E}-04$ \\
\hline 6531 & MPHOSPH8 & -4.00 & $2.29 \mathrm{E}-04$ \\
\hline 6532 & MPHOSPH9 & -1.28 & $9.83 \mathrm{E}-03$ \\
\hline 6533 & MPI & -4.30 & $8.52 \mathrm{E}-03$ \\
\hline 6534 & MPLKIP & -1.06 & $1.54 \mathrm{E}-02$ \\
\hline 6535 & MPP1 & -4.50 & 4.05E-04 \\
\hline 6536 & MPP3 & -4.29 & 4.50E-04 \\
\hline 6537 & MPP5 & -1.86 & $5.34 \mathrm{E}-03$ \\
\hline 6538 & MPP6 & -5.42 & 1.19E-04 \\
\hline 6539 & MPP7 & -4.36 & $1.15 \mathrm{E}-02$ \\
\hline 6540 & MPPE1 & -1.30 & $4.42 \mathrm{E}-02$ \\
\hline 6541 & MPPED1 & -1.39 & $2.70 \mathrm{E}-03$ \\
\hline 6542 & MPZL3 & -1.94 & $6.14 \mathrm{E}-02$ \\
\hline 6543 & MRAS & -4.15 & $7.02 \mathrm{E}-04$ \\
\hline 6544 & MRE11A & -3.20 & $3.93 \mathrm{E}-03$ \\
\hline
\end{tabular}




\begin{tabular}{|c|c|c|c|}
\hline 6545 & MRM1 & -1.15 & $2.82 \mathrm{E}-03$ \\
\hline 6546 & MRP63 & -7.07 & $1.70 \mathrm{E}-04$ \\
\hline 6547 & MRPL1 & -2.32 & $1.05 \mathrm{E}-02$ \\
\hline 6548 & MRPL10 & -1.34 & $3.96 \mathrm{E}-02$ \\
\hline 6549 & MRPL12 & -5.58 & $2.18 \mathrm{E}-05$ \\
\hline 6550 & MRPL13 & -5.82 & $8.43 \mathrm{E}-04$ \\
\hline 6551 & MRPL14 & -6.59 & $2.15 \mathrm{E}-03$ \\
\hline 6552 & MRPL15 & -5.17 & $8.48 \mathrm{E}-04$ \\
\hline 6553 & MRPL16 & -5.30 & $5.73 \mathrm{E}-04$ \\
\hline 6554 & MRPL17 & -4.46 & $3.77 \mathrm{E}-02$ \\
\hline 6555 & MRPL18 & -7.39 & $1.20 \mathrm{E}-04$ \\
\hline 6556 & MRPL19 & -3.05 & $1.79 \mathrm{E}-03$ \\
\hline 6557 & MRPL2 & -3.24 & $1.06 \mathrm{E}-02$ \\
\hline 6558 & MRPL20 & -4.11 & $2.05 \mathrm{E}-03$ \\
\hline 6559 & MRPL21 & -5.65 & $5.26 \mathrm{E}-04$ \\
\hline 6560 & MRPL22 & -2.69 & $6.31 \mathrm{E}-04$ \\
\hline 6561 & MRPL23 & -6.87 & $3.41 \mathrm{E}-04$ \\
\hline 6562 & MRPL24 & -4.63 & $2.38 \mathrm{E}-03$ \\
\hline 6563 & MRPL27 & -6.27 & $1.05 \mathrm{E}-04$ \\
\hline 6564 & MRPL28 & -4.16 & $8.10 \mathrm{E}-03$ \\
\hline 6565 & MRPL3 & -4.85 & $1.86 \mathrm{E}-03$ \\
\hline 6566 & MRPL30 & -5.90 & $5.15 \mathrm{E}-04$ \\
\hline 6567 & MRPL32 & -4.04 & $5.23 \mathrm{E}-04$ \\
\hline 6568 & MRPL33 & -4.34 & $1.75 \mathrm{E}-04$ \\
\hline 6569 & MRPL37 & -4.65 & $2.54 \mathrm{E}-04$ \\
\hline 6570 & MRPL38 & -5.13 & $2.37 \mathrm{E}-04$ \\
\hline 6571 & MRPL39 & -6.00 & $1.50 \mathrm{E}-04$ \\
\hline 6572 & MRPL41 & -4.78 & $3.17 \mathrm{E}-05$ \\
\hline 6573 & MRPL42 & -5.26 & $4.82 \mathrm{E}-04$ \\
\hline 6574 & MRPL44 & -6.54 & $2.89 \mathrm{E}-04$ \\
\hline 6575 & MRPL45 & -4.42 & $2.02 \mathrm{E}-03$ \\
\hline 6576 & MRPL46 & -5.01 & $5.42 \mathrm{E}-04$ \\
\hline 6577 & MRPL48 & -5.08 & $3.23 \mathrm{E}-04$ \\
\hline 6578 & MRPL50 & -6.23 & $5.19 \mathrm{E}-04$ \\
\hline 6579 & MRPL51 & -4.19 & $1.21 \mathrm{E}-03$ \\
\hline 6580 & MRPL53 & -5.70 & $1.96 \mathrm{E}-03$ \\
\hline 6581 & MRPS10 & -3.99 & $2.56 \mathrm{E}-03$ \\
\hline 6582 & MRPS11 & -8.35 & $2.33 \mathrm{E}-05$ \\
\hline 6583 & MRPS12 & -5.78 & $2.47 \mathrm{E}-04$ \\
\hline 6584 & MRPS14 & -4.69 & $2.32 \mathrm{E}-03$ \\
\hline 6585 & MRPS16 & -5.45 & $6.12 \mathrm{E}-05$ \\
\hline 6586 & MRPS17 & -7.87 & $1.84 \mathrm{E}-04$ \\
\hline 6587 & MRPS18A & -5.00 & $1.08 \mathrm{E}-03$ \\
\hline 6588 & MRPS18C & -5.69 & $2.25 \mathrm{E}-03$ \\
\hline 6589 & MRPS2 & -2.56 & $9.10 \mathrm{E}-03$ \\
\hline 6590 & MRPS21 & -3.85 & $7.29 \mathrm{E}-04$ \\
\hline 6591 & MRPS22 & -4.88 & $2.32 \mathrm{E}-04$ \\
\hline 6592 & MRPS23 & -5.81 & $8.71 \mathrm{E}-04$ \\
\hline 6593 & MRPS25 & -5.68 & $3.96 \mathrm{E}-03$ \\
\hline 6594 & MRPS26 & -5.13 & $1.50 \mathrm{E}-03$ \\
\hline 6595 & MRPS27 & -2.39 & $2.41 \mathrm{E}-03$ \\
\hline 6596 & MRPS28 & -7.45 & $5.98 \mathrm{E}-06$ \\
\hline 6597 & MRPS30 & -5.06 & $1.39 \mathrm{E}-03$ \\
\hline 6598 & MRPS31 & -4.99 & $1.56 \mathrm{E}-04$ \\
\hline 6599 & MRPS33 & -3.10 & $2.61 \mathrm{E}-02$ \\
\hline 6600 & MRPS35 & -4.34 & 4.96E-04 \\
\hline 6601 & MRPS36 & -5.11 & $1.92 \mathrm{E}-03$ \\
\hline 6602 & MRPS5 & -5.65 & $2.44 \mathrm{E}-04$ \\
\hline 6603 & MRPS6 & -2.76 & $1.66 \mathrm{E}-02$ \\
\hline 6604 & MRPS9 & -5.29 & $5.84 \mathrm{E}-04$ \\
\hline 6605 & MRRF & -3.69 & 4.23E-04 \\
\hline 6606 & MRS2 & -1.49 & $8.98 \mathrm{E}-03$ \\
\hline 6607 & MSANTD1 & -1.14 & $8.83 \mathrm{E}-03$ \\
\hline
\end{tabular}

\begin{tabular}{|c|c|c|c|}
\hline 6608 & MSANTD2 & -3.24 & 3.70E-04 \\
\hline 6609 & MSANTD3 & -4.63 & $6.91 \mathrm{E}-06$ \\
\hline 6610 & MSANTD4 & -5.59 & $1.01 \mathrm{E}-03$ \\
\hline 6611 & MSH2 & -3.18 & 7.29E-04 \\
\hline 6612 & MSH4 & -4.14 & $1.82 \mathrm{E}-03$ \\
\hline 6613 & MSH6 & -1.38 & $1.04 \mathrm{E}-02$ \\
\hline 6614 & MSI1 & -2.07 & $8.47 \mathrm{E}-02$ \\
\hline 6615 & MSI2 & -6.00 & $9.32 \mathrm{E}-04$ \\
\hline 6616 & MSL1 & -2.21 & 9.17E-03 \\
\hline 6617 & MSL2 & -2.74 & $6.53 \mathrm{E}-04$ \\
\hline 6618 & MSN & -2.47 & $1.15 \mathrm{E}-03$ \\
\hline 6619 & MSRA & -2.31 & $5.58 \mathrm{E}-04$ \\
\hline 6620 & MSRB3 & -2.13 & $8.32 \mathrm{E}-03$ \\
\hline 6621 & MT3 & -4.62 & $3.08 \mathrm{E}-04$ \\
\hline 6622 & MT4 & -7.01 & $2.72 \mathrm{E}-05$ \\
\hline 6623 & MTA1 & -3.59 & $3.82 \mathrm{E}-03$ \\
\hline 6624 & MTA3 & -4.08 & $2.25 \mathrm{E}-04$ \\
\hline 6625 & MTAP & -4.38 & $1.59 \mathrm{E}-03$ \\
\hline 6626 & $\mathrm{MTCH} 2$ & -1.28 & $5.92 \mathrm{E}-02$ \\
\hline 6627 & MTERFD1 & -4.88 & $1.86 \mathrm{E}-04$ \\
\hline 6628 & MTF2 & -3.60 & $2.88 \mathrm{E}-04$ \\
\hline 6629 & MTFMT & -4.06 & $1.16 \mathrm{E}-03$ \\
\hline 6630 & MTFP1 & -2.37 & 3.34E-02 \\
\hline 6631 & MTFR1 & -6.21 & $4.52 \mathrm{E}-05$ \\
\hline 6632 & MTFR1L & -4.86 & 1.17E-03 \\
\hline 6633 & MTG1 & -2.57 & $3.50 \mathrm{E}-03$ \\
\hline 6634 & MTHFD1 & -4.76 & $4.42 \mathrm{E}-04$ \\
\hline 6635 & MTHFD2 & -2.70 & $1.05 \mathrm{E}-04$ \\
\hline 6636 & MTHFD2L & -1.30 & 5.16E-02 \\
\hline 6637 & MTHFS & -2.36 & $1.35 \mathrm{E}-02$ \\
\hline 6638 & MTHFSD & -3.48 & 7.06E-04 \\
\hline 6639 & MTIF2 & -3.29 & $2.48 \mathrm{E}-03$ \\
\hline 6640 & MTIF3 & -4.41 & 5.57E-05 \\
\hline 6641 & MTMR1 & -3.18 & $2.56 \mathrm{E}-04$ \\
\hline 6642 & MTMR10 & -1.50 & $3.64 \mathrm{E}-03$ \\
\hline 6643 & MTMR14 & -1.79 & $2.02 \mathrm{E}-03$ \\
\hline 6644 & MTMR2 & -2.35 & 3.31E-03 \\
\hline 6645 & MTMR3 & -2.13 & $1.32 \mathrm{E}-04$ \\
\hline 6646 & MTMR8 & -1.74 & $2.15 \mathrm{E}-02$ \\
\hline 6647 & MTMR9 & -5.94 & $3.91 \mathrm{E}-04$ \\
\hline 6648 & MTOR & -4.34 & $6.78 \mathrm{E}-04$ \\
\hline 6649 & MTPAP & -1.38 & $4.20 \mathrm{E}-03$ \\
\hline 6650 & MTPN & -4.22 & $1.98 \mathrm{E}-03$ \\
\hline 6651 & MTR & -2.26 & $3.32 \mathrm{E}-03$ \\
\hline 6652 & MTRF1 & -4.25 & $5.84 \mathrm{E}-04$ \\
\hline 6653 & MTRF1L & -3.73 & $6.78 \mathrm{E}-04$ \\
\hline 6654 & MTSS1 & -1.67 & $2.31 \mathrm{E}-02$ \\
\hline 6655 & MTX2 & -5.21 & $3.83 \mathrm{E}-03$ \\
\hline 6656 & MTX3 & -2.41 & $2.98 \mathrm{E}-03$ \\
\hline 6657 & MUDENG & -3.63 & $5.48 \mathrm{E}-03$ \\
\hline 6658 & MUL1 & -1.79 & $1.32 \mathrm{E}-02$ \\
\hline 6659 & MUT & -1.97 & $1.93 \mathrm{E}-03$ \\
\hline 6660 & MUTED & -5.07 & $2.90 \mathrm{E}-05$ \\
\hline 6661 & MVP & -2.38 & $9.00 \mathrm{E}-04$ \\
\hline 6662 & MXD1 & -1.41 & $6.58 \mathrm{E}-02$ \\
\hline 6663 & MXD4 & -1.38 & 3.57E-02 \\
\hline 6664 & MXI1 & -5.05 & 4.73E-04 \\
\hline 6665 & MXRA8 & -2.94 & $1.45 \mathrm{E}-03$ \\
\hline 6666 & MYBBP1A & -1.20 & $2.51 \mathrm{E}-02$ \\
\hline 6667 & MYBL1 & -4.75 & 5.45E-04 \\
\hline 6668 & MYBL2 & -3.78 & $8.85 \mathrm{E}-04$ \\
\hline 6669 & MYCBP & -6.77 & $2.12 \mathrm{E}-03$ \\
\hline 6670 & MYCBP2 & -1.49 & $1.73 \mathrm{E}-03$ \\
\hline
\end{tabular}

\begin{tabular}{|c|c|c|c|}
\hline 6671 & MYCN & -1.93 & 4.67E-03 \\
\hline 6672 & MYD88 & -3.17 & $6.24 \mathrm{E}-04$ \\
\hline 6673 & MYEF2 & -3.10 & $7.46 \mathrm{E}-04$ \\
\hline 6674 & MYEOV2 & -6.81 & $1.22 \mathrm{E}-04$ \\
\hline 6675 & MYF6 & -2.49 & $6.15 \mathrm{E}-04$ \\
\hline 6676 & MYH10 & -4.19 & $6.65 \mathrm{E}-05$ \\
\hline 6677 & MYH11 & -3.57 & $5.65 \mathrm{E}-03$ \\
\hline 6678 & MYH7B & -1.15 & $3.55 \mathrm{E}-03$ \\
\hline 6679 & MYL10 & -2.46 & $5.12 \mathrm{E}-04$ \\
\hline 6680 & MYL12A & -4.01 & $3.21 \mathrm{E}-04$ \\
\hline 6681 & MYL3 & -2.28 & $2.12 \mathrm{E}-03$ \\
\hline 6682 & MYL4 & -6.33 & $3.33 \mathrm{E}-04$ \\
\hline 6683 & MYL6 & -1.57 & $1.95 \mathrm{E}-03$ \\
\hline 6684 & MYLIP & -2.06 & $1.61 \mathrm{E}-03$ \\
\hline 6685 & MYLK & -1.45 & $2.41 \mathrm{E}-02$ \\
\hline 6686 & MYO1C & -2.21 & $6.16 \mathrm{E}-03$ \\
\hline 6687 & MYO5A & -2.88 & $1.68 \mathrm{E}-03$ \\
\hline 6688 & MYPN & -2.00 & 4.94E-03 \\
\hline 6689 & MYRIP & -2.50 & $5.36 \mathrm{E}-03$ \\
\hline 6690 & MYST2 & -5.30 & $5.53 \mathrm{E}-04$ \\
\hline 6691 & MYST3 & -1.74 & $1.29 \mathrm{E}-03$ \\
\hline 6692 & MZT1 & -6.63 & 2.67E-03 \\
\hline 6693 & N4BP1 & -5.51 & $1.25 \mathrm{E}-04$ \\
\hline 6694 & N4BP2L2 & -3.20 & $5.02 \mathrm{E}-04$ \\
\hline 6695 & N6AMT1 & -4.18 & 7.66E-04 \\
\hline 6696 & N6AMT2 & -3.13 & $3.28 \mathrm{E}-03$ \\
\hline 6697 & NAA15 & -3.85 & 7.35E-04 \\
\hline 6698 & NAA20 & -6.35 & 5.57E-04 \\
\hline 6699 & NAA25 & -1.63 & $7.95 \mathrm{E}-03$ \\
\hline 6700 & NAA30 & -1.87 & $2.32 \mathrm{E}-02$ \\
\hline 6701 & NAA35 & -5.10 & $6.55 \mathrm{E}-04$ \\
\hline 6702 & NAA38 & -6.22 & $8.42 \mathrm{E}-04$ \\
\hline 6703 & NAA40 & -5.47 & $3.26 \mathrm{E}-03$ \\
\hline 6704 & NAA50 & -1.62 & $1.11 \mathrm{E}-02$ \\
\hline 6705 & NAB1 & -5.36 & $9.60 \mathrm{E}-04$ \\
\hline 6706 & NACA & -4.41 & 2.07E-03 \\
\hline 6707 & NACAD & -2.51 & $2.64 \mathrm{E}-03$ \\
\hline 6708 & NACC2 & -2.52 & $1.18 \mathrm{E}-03$ \\
\hline 6709 & NADK & -4.28 & $1.47 \mathrm{E}-03$ \\
\hline 6710 & NADSYN1 & -2.36 & $2.33 \mathrm{E}-02$ \\
\hline 6711 & NAE1 & -5.28 & 4.51E-04 \\
\hline 6712 & NAF1 & -2.66 & $9.67 \mathrm{E}-04$ \\
\hline 6713 & NAGPA & -1.52 & $5.11 \mathrm{E}-03$ \\
\hline 6714 & NAIF1 & -1.56 & 7.06E-02 \\
\hline 6715 & NAMPT & -2.04 & $1.27 \mathrm{E}-03$ \\
\hline 6716 & NANP & -1.98 & $1.50 \mathrm{E}-03$ \\
\hline 6717 & NAP1L1 & -6.33 & 3.37E-04 \\
\hline 6718 & NAP1L4 & -6.37 & $3.62 \mathrm{E}-04$ \\
\hline 6719 & NAPEPLD & -6.09 & 2.27E-04 \\
\hline 6720 & NAPG & -2.76 & $7.73 \mathrm{E}-03$ \\
\hline 6721 & NARFL & -4.67 & 4.04E-04 \\
\hline 6722 & NARG2 & -1.91 & $4.66 \mathrm{E}-03$ \\
\hline 6723 & NARS & -2.57 & $1.73 \mathrm{E}-03$ \\
\hline 6724 & NARS2 & -2.34 & $1.39 \mathrm{E}-02$ \\
\hline 6725 & NASP & -3.96 & $5.77 \mathrm{E}-03$ \\
\hline 6726 & NAT10 & -3.57 & 7.47E-03 \\
\hline 6727 & NAT9 & -2.74 & $1.18 \mathrm{E}-02$ \\
\hline 6728 & NBAS & -3.01 & $6.73 \mathrm{E}-04$ \\
\hline 6729 & NBL1 & -1.91 & $9.07 \mathrm{E}-03$ \\
\hline 6730 & NBN & -5.37 & 3.07E-03 \\
\hline 6731 & NBR1 & -3.12 & $8.00 \mathrm{E}-03$ \\
\hline 6732 & NCAPD2 & -2.86 & $2.18 \mathrm{E}-03$ \\
\hline 6733 & NCAPG2 & -3.65 & $1.38 \mathrm{E}-03$ \\
\hline
\end{tabular}




\begin{tabular}{|c|c|c|c|}
\hline 6734 & NCBP1 & -4.08 & $2.18 \mathrm{E}-03$ \\
\hline 6735 & NCBP2 & -5.77 & $6.82 \mathrm{E}-05$ \\
\hline 6736 & NCDN & -3.92 & 8.07E-04 \\
\hline 6737 & NCEH1 & -2.00 & $6.68 \mathrm{E}-03$ \\
\hline 6738 & NCK2 & -2.34 & $2.35 \mathrm{E}-04$ \\
\hline 6739 & NCKAP1 & -1.62 & 7.90E-03 \\
\hline 6740 & NCKIPSD & -3.95 & 3.64E-04 \\
\hline 6741 & $\mathrm{NCL}$ & -5.51 & $1.40 \mathrm{E}-04$ \\
\hline 6742 & NCOA3 & -1.96 & $1.05 \mathrm{E}-03$ \\
\hline 6743 & NCOA4 & -6.80 & $2.24 \mathrm{E}-04$ \\
\hline 6744 & NCOA6 & -1.16 & $5.32 \mathrm{E}-04$ \\
\hline 6745 & NCOA7 & -1.17 & 1.62E-02 \\
\hline 6746 & NCOR1 & -4.29 & $3.24 \mathrm{E}-04$ \\
\hline 6747 & ND1 & -7.24 & $1.11 \mathrm{E}-02$ \\
\hline 6748 & ND2 & -5.15 & $5.44 \mathrm{E}-03$ \\
\hline 6749 & ND3 & -6.69 & $6.91 \mathrm{E}-03$ \\
\hline 6750 & ND4 & -5.17 & $1.94 \mathrm{E}-02$ \\
\hline 6751 & ND4L & -3.11 & $1.52 \mathrm{E}-02$ \\
\hline 6752 & ND5 & -6.79 & $3.26 \mathrm{E}-03$ \\
\hline 6753 & ND6 & -5.54 & $1.44 \mathrm{E}-02$ \\
\hline 6754 & NDC80 & -2.95 & $1.24 \mathrm{E}-02$ \\
\hline 6755 & NDE1 & -2.89 & $8.63 \mathrm{E}-04$ \\
\hline 6756 & NDEL1 & -3.57 & 5.39E-04 \\
\hline 6757 & NDFIP1 & -6.01 & 2.70E-04 \\
\hline 6758 & NDFIP2 & -6.33 & 4.96E-04 \\
\hline 6759 & NDNL2 & -7.26 & $9.31 \mathrm{E}-04$ \\
\hline 6760 & NDOR1 & -1.31 & $6.70 \mathrm{E}-03$ \\
\hline 6761 & NDRG1 & -1.64 & 2.29E-02 \\
\hline 6762 & NDRG3 & -3.77 & $3.31 \mathrm{E}-03$ \\
\hline 6763 & NDST3 & -1.35 & $3.09 \mathrm{E}-02$ \\
\hline 6764 & NDUFA1 & -6.00 & $9.68 \mathrm{E}-04$ \\
\hline 6765 & NDUFA10 & -4.58 & $2.28 \mathrm{E}-04$ \\
\hline 6766 & NDUFA11 & -6.40 & 5.55E-04 \\
\hline 6767 & NDUFA12 & -7.54 & $2.74 \mathrm{E}-05$ \\
\hline 6768 & NDUFA2 & -7.35 & $1.04 \mathrm{E}-04$ \\
\hline 6769 & NDUFA4 & -6.56 & $1.21 \mathrm{E}-03$ \\
\hline 6770 & NDUFA5 & -5.89 & 3.93E-04 \\
\hline 6771 & NDUFA6 & -6.90 & $1.02 \mathrm{E}-03$ \\
\hline 6772 & NDUFA7 & -7.56 & $8.01 \mathrm{E}-04$ \\
\hline 6773 & NDUFA8 & -8.52 & 3.43E-05 \\
\hline 6774 & NDUFA9 & -4.15 & $5.64 \mathrm{E}-04$ \\
\hline 6775 & NDUFAB1 & -6.60 & $2.08 \mathrm{E}-04$ \\
\hline 6776 & NDUFAF1 & -6.07 & $5.99 \mathrm{E}-04$ \\
\hline 6777 & NDUFAF2 & -6.07 & $1.51 \mathrm{E}-04$ \\
\hline 6778 & NDUFAF4 & -7.53 & $5.24 \mathrm{E}-04$ \\
\hline 6779 & NDUFB1 & -8.02 & $1.74 \mathrm{E}-04$ \\
\hline 6780 & NDUFB10 & -7.90 & 4.68E-04 \\
\hline 6781 & NDUFB2 & -3.43 & $1.50 \mathrm{E}-03$ \\
\hline 6782 & NDUFB3 & -5.58 & $1.71 \mathrm{E}-04$ \\
\hline 6783 & NDUFB5 & -4.84 & $1.01 \mathrm{E}-04$ \\
\hline 6784 & NDUFB6 & -8.25 & $3.25 \mathrm{E}-04$ \\
\hline 6785 & NDUFB8 & -4.16 & $1.03 \mathrm{E}-03$ \\
\hline 6786 & NDUFB9 & -7.27 & $1.52 \mathrm{E}-04$ \\
\hline 6787 & NDUFC2 & -7.23 & $5.48 \mathrm{E}-05$ \\
\hline 6788 & NDUFS1 & -6.10 & $1.80 \mathrm{E}-04$ \\
\hline 6789 & NDUFS3 & -7.52 & 5.95E-04 \\
\hline 6790 & NDUFS4 & -9.07 & $1.71 \mathrm{E}-04$ \\
\hline 6791 & NDUFS5 & -4.82 & $2.35 \mathrm{E}-04$ \\
\hline 6792 & NDUFS6 & -7.44 & $4.78 \mathrm{E}-04$ \\
\hline 6793 & NDUFS8 & -6.47 & $2.62 \mathrm{E}-04$ \\
\hline 6794 & NDUFV1 & -3.90 & $1.33 \mathrm{E}-04$ \\
\hline 6795 & NDUFV2 & -6.55 & $9.48 \mathrm{E}-06$ \\
\hline 6796 & NECAP2 & -2.78 & $1.65 \mathrm{E}-02$ \\
\hline
\end{tabular}

\begin{tabular}{|c|c|c|c|}
\hline 6797 & NEDD1 & -5.92 & 4.76E-04 \\
\hline 6798 & NEFL & -2.41 & $2.57 \mathrm{E}-03$ \\
\hline 6799 & NEK2 & -3.47 & $8.73 \mathrm{E}-04$ \\
\hline 6800 & NEK3 & -1.64 & $2.76 \mathrm{E}-03$ \\
\hline 6801 & NEK6 & -2.86 & $1.56 \mathrm{E}-03$ \\
\hline 6802 & NEK7 & -4.26 & $1.72 \mathrm{E}-04$ \\
\hline 6803 & NELF & -2.18 & $2.02 \mathrm{E}-02$ \\
\hline 6804 & NELL2 & -4.34 & $1.79 \mathrm{E}-04$ \\
\hline 6805 & NEMF & -7.09 & $1.42 \mathrm{E}-04$ \\
\hline 6806 & NENF & -2.43 & $6.51 \mathrm{E}-03$ \\
\hline 6807 & NET1 & -5.58 & $1.21 \mathrm{E}-04$ \\
\hline 6808 & NEUROD1 & -2.23 & 3.77E-02 \\
\hline 6809 & NF2 & -7.67 & $5.92 E-05$ \\
\hline 6810 & NFAM1 & -5.04 & 4.97E-04 \\
\hline 6811 & NFIL3 & -2.68 & $1.84 \mathrm{E}-03$ \\
\hline 6812 & NFIX & -2.74 & $7.01 \mathrm{E}-04$ \\
\hline 6813 & NFKB1 & -2.35 & $1.29 \mathrm{E}-03$ \\
\hline 6814 & NFKBIA & -3.59 & $1.70 \mathrm{E}-03$ \\
\hline 6815 & NFS1 & -3.43 & 4.07E-03 \\
\hline 6816 & NFU1 & -7.11 & 4.25E-05 \\
\hline 6817 & NFYA & -1.47 & $4.46 \mathrm{E}-03$ \\
\hline 6818 & NFYB & -3.81 & $4.52 \mathrm{E}-04$ \\
\hline 6819 & NFYC & -4.21 & $1.04 \mathrm{E}-02$ \\
\hline 6820 & NHP2 & -2.61 & $2.03 \mathrm{E}-01$ \\
\hline 6821 & NHP2L1 & -2.66 & $2.63 \mathrm{E}-02$ \\
\hline 6822 & NID1 & -3.11 & $1.43 \mathrm{E}-05$ \\
\hline 6823 & NIF3L1 & -3.57 & $1.22 \mathrm{E}-03$ \\
\hline 6824 & NINJ2 & -3.53 & $1.11 \mathrm{E}-03$ \\
\hline 6825 & NIP7 & -3.97 & $8.83 \mathrm{E}-04$ \\
\hline 6826 & NIPA1 & -1.02 & 7.77E-04 \\
\hline 6827 & NIPA2 & -2.00 & $2.11 \mathrm{E}-03$ \\
\hline 6828 & NIPAL3 & -2.72 & $8.01 \mathrm{E}-03$ \\
\hline 6829 & NIPBL & -1.15 & $7.38 \mathrm{E}-04$ \\
\hline 6830 & NIPSNAP1 & -2.26 & $1.36 \mathrm{E}-02$ \\
\hline 6831 & NIPSNAP3A & -1.88 & $5.12 \mathrm{E}-04$ \\
\hline 6832 & $\mathrm{NISCH}$ & -1.52 & $3.19 \mathrm{E}-03$ \\
\hline 6833 & NIT2 & -6.89 & $2.74 \mathrm{E}-04$ \\
\hline 6834 & NKAP & -4.42 & 3.47E-04 \\
\hline 6835 & NKIRAS1 & -1.88 & $2.32 \mathrm{E}-03$ \\
\hline 6836 & NKIRAS2 & -2.47 & $4.11 \mathrm{E}-03$ \\
\hline 6837 & NKRF & -1.62 & $6.95 \mathrm{E}-03$ \\
\hline 6838 & NKTR & -2.60 & $5.35 \mathrm{E}-03$ \\
\hline 6839 & NLK & -1.18 & $2.36 \mathrm{E}-03$ \\
\hline 6840 & NMB & -5.85 & $6.29 \mathrm{E}-04$ \\
\hline 6841 & NMBR & -1.87 & $3.06 \mathrm{E}-02$ \\
\hline 6842 & NMD3 & -2.62 & $3.18 \mathrm{E}-04$ \\
\hline 6843 & NME2 & -7.58 & $4.35 \mathrm{E}-04$ \\
\hline 6844 & NME3 & -1.82 & $3.87 \mathrm{E}-03$ \\
\hline 6845 & NME4 & -2.38 & $1.07 \mathrm{E}-01$ \\
\hline 6846 & NME5 & -7.29 & $4.25 \mathrm{E}-04$ \\
\hline 6847 & NME6 & -5.07 & $6.08 \mathrm{E}-04$ \\
\hline 6848 & $\mathrm{NMI}$ & -4.25 & $2.27 \mathrm{E}-03$ \\
\hline 6849 & NMT1 & -5.18 & 3.70E-04 \\
\hline 6850 & NMT2 & -2.96 & $1.27 \mathrm{E}-03$ \\
\hline 6851 & NNF1 & -2.66 & $1.29 \mathrm{E}-03$ \\
\hline 6852 & NOA1 & -1.49 & $5.22 \mathrm{E}-02$ \\
\hline 6853 & NOB1 & -5.47 & $4.31 \mathrm{E}-04$ \\
\hline 6854 & NOL10 & -3.84 & $1.74 \mathrm{E}-04$ \\
\hline 6855 & NOL11 & -4.58 & $6.44 \mathrm{E}-05$ \\
\hline 6856 & NOL7 & -4.73 & $1.91 \mathrm{E}-04$ \\
\hline 6857 & NOM1 & -1.85 & 4.67E-03 \\
\hline 6858 & NONO & -4.80 & $1.58 \mathrm{E}-04$ \\
\hline 6859 & NOP14 & -4.54 & $2.03 \mathrm{E}-04$ \\
\hline
\end{tabular}

\begin{tabular}{|c|c|c|c|}
\hline 6860 & NOP16 & -3.93 & $8.43 \mathrm{E}-04$ \\
\hline 6861 & NOP2 & -2.00 & $1.08 \mathrm{E}-03$ \\
\hline 6862 & NOP56 & -2.62 & $1.28 \mathrm{E}-03$ \\
\hline 6863 & NOP58 & -4.06 & $1.34 \mathrm{E}-03$ \\
\hline 6864 & NOVA1 & -1.90 & $6.83 \mathrm{E}-03$ \\
\hline 6865 & NP9665207 & -1.33 & $2.45 \mathrm{E}-02$ \\
\hline 6866 & NP9665338 & -5.22 & $2.52 \mathrm{E}-02$ \\
\hline 6867 & NP9666011 & -2.80 & $7.86 \mathrm{E}-02$ \\
\hline 6868 & NP9666165 & -2.51 & $6.36 \mathrm{E}-02$ \\
\hline 6869 & NP9666776 & -2.73 & 3.83E-02 \\
\hline 6870 & NP9666844 & -3.41 & $1.90 \mathrm{E}-04$ \\
\hline 6871 & NP9666891 & -3.43 & $4.00 \mathrm{E}-03$ \\
\hline 6872 & NP9667176 & -3.17 & $1.53 \mathrm{E}-03$ \\
\hline 6873 & NP9667974 & -1.26 & $6.25 \mathrm{E}-02$ \\
\hline 6874 & NP9667992 & -1.38 & $1.24 \mathrm{E}-02$ \\
\hline 6875 & NP9668036 & -2.78 & $1.59 \mathrm{E}-01$ \\
\hline 6876 & NP9668076 & -6.61 & $1.98 \mathrm{E}-04$ \\
\hline 6877 & NP9668396 & -2.25 & $2.59 \mathrm{E}-03$ \\
\hline 6878 & NP9668692 & -1.55 & 4.57E-02 \\
\hline 6879 & NP9669405 & -3.11 & $1.88 \mathrm{E}-04$ \\
\hline 6880 & NP9670087 & -3.21 & $2.11 \mathrm{E}-02$ \\
\hline 6881 & NP9670332 & -3.56 & $1.18 \mathrm{E}-03$ \\
\hline 6882 & NP9670503 & -4.32 & 4.89E-04 \\
\hline 6883 & NP9670715 & -5.65 & 7.07E-03 \\
\hline 6884 & NP9670951 & -5.23 & $1.45 \mathrm{E}-04$ \\
\hline 6885 & NP9671000 & -1.67 & $5.41 \mathrm{E}-03$ \\
\hline 6886 & NP9671157 & -2.31 & $1.35 \mathrm{E}-02$ \\
\hline 6887 & NP9671209 & -2.57 & $8.03 \mathrm{E}-03$ \\
\hline 6888 & NP9671587 & -1.93 & 8.07E-03 \\
\hline 6889 & NP9672026 & -2.29 & $1.01 \mathrm{E}-03$ \\
\hline 6890 & NP9672261 & -3.47 & 2.76E-04 \\
\hline 6891 & NP9672321 & -1.72 & $4.78 \mathrm{E}-03$ \\
\hline 6892 & NP9672471 & -2.19 & $1.26 \mathrm{E}-02$ \\
\hline 6893 & NP9672894 & -1.54 & $1.93 \mathrm{E}-02$ \\
\hline 6894 & NP9673267 & -2.59 & $9.49 \mathrm{E}-04$ \\
\hline 6895 & NP9673446 & -2.35 & $5.42 \mathrm{E}-02$ \\
\hline 6896 & NP9673556 & -3.06 & $9.72 \mathrm{E}-04$ \\
\hline 6897 & NP9673563 & -1.45 & $1.56 \mathrm{E}-02$ \\
\hline 6898 & NP9673909 & -5.35 & 8.43E-04 \\
\hline 6899 & NP9674358 & -5.87 & 4.29E- 04 \\
\hline 6900 & NP9674417 & -2.11 & $1.33 \mathrm{E}-02$ \\
\hline 6901 & NP9674761 & -3.33 & $6.17 \mathrm{E}-02$ \\
\hline 6902 & NP9675060 & -2.96 & $2.23 \mathrm{E}-02$ \\
\hline 6903 & NP9675172 & -1.65 & $1.06 \mathrm{E}-01$ \\
\hline 6904 & NP9675550 & -1.32 & $2.26 \mathrm{E}-02$ \\
\hline 6905 & NP9675894 & -5.42 & $1.15 \mathrm{E}-04$ \\
\hline 6906 & NP9676001 & -2.11 & $1.86 \mathrm{E}-03$ \\
\hline 6907 & NP9676025 & -1.56 & $5.63 \mathrm{E}-02$ \\
\hline 6908 & NP9676219 & -2.27 & $1.80 \mathrm{E}-01$ \\
\hline 6909 & NP9677157 & -3.25 & $1.24 \mathrm{E}-03$ \\
\hline 6910 & NP9677271 & -2.98 & 4.37E-03 \\
\hline 6911 & NP9677553 & -1.27 & $9.23 \mathrm{E}-02$ \\
\hline 6912 & NP9677820 & -2.76 & $4.30 \mathrm{E}-03$ \\
\hline 6913 & NP9678050 & -2.69 & $1.65 \mathrm{E}-02$ \\
\hline 6914 & NP9678544 & -1.55 & $5.68 \mathrm{E}-03$ \\
\hline 6915 & NP9678639 & -8.12 & $4.24 \mathrm{E}-04$ \\
\hline 6916 & NP9679065 & -5.24 & 4.30E-04 \\
\hline 6917 & NP9679459 & -2.18 & $3.48 \mathrm{E}-03$ \\
\hline 6918 & NP9679748 & -2.84 & $3.81 \mathrm{E}-03$ \\
\hline 6919 & NP9680503 & -7.31 & $1.87 \mathrm{E}-04$ \\
\hline 6920 & NP9680619 & -1.67 & $5.62 \mathrm{E}-02$ \\
\hline 6921 & NPC1 & -3.04 & $9.67 \mathrm{E}-03$ \\
\hline 6922 & NPC2 & -3.50 & $2.61 \mathrm{E}-04$ \\
\hline
\end{tabular}




\begin{tabular}{|c|c|c|c|}
\hline 6923 & NPEPL1 & -1.38 & $5.02 \mathrm{E}-03$ \\
\hline 6924 & NPEPPS & -3.07 & $9.37 \mathrm{E}-03$ \\
\hline 6925 & NPHP1 & -5.31 & $3.94 \mathrm{E}-04$ \\
\hline 6926 & NPHP3 & -2.32 & $1.59 \mathrm{E}-04$ \\
\hline 6927 & NPLOC4 & -2.95 & $1.79 \mathrm{E}-03$ \\
\hline 6928 & NPM1 & -3.23 & $1.71 \mathrm{E}-03$ \\
\hline 6929 & NPR1 & -2.30 & $2.25 \mathrm{E}-03$ \\
\hline 6930 & NPRL3 & -2.96 & $1.56 \mathrm{E}-02$ \\
\hline 6931 & NPTN & -4.18 & $2.86 \mathrm{E}-04$ \\
\hline 6932 & NPY & -4.10 & 7.03E-03 \\
\hline 6933 & $\mathrm{NQO} 2$ & -4.52 & $6.62 \mathrm{E}-04$ \\
\hline 6934 & NROB1 & -3.53 & $2.65 \mathrm{E}-03$ \\
\hline 6935 & NROB2 & -3.54 & $2.94 \mathrm{E}-03$ \\
\hline 6936 & NR13 & -4.17 & $3.02 \mathrm{E}-04$ \\
\hline 6937 & NR1D2 & -3.87 & $7.49 \mathrm{E}-05$ \\
\hline 6938 & $\mathrm{NR} 1 \mathrm{H} 3$ & -1.77 & $1.71 \mathrm{E}-02$ \\
\hline 6939 & NR2C1 & -1.77 & $2.19 \mathrm{E}-03$ \\
\hline 6940 & NR2C2 & -2.01 & $5.54 \mathrm{E}-03$ \\
\hline 6941 & NR2F2 & -1.34 & $8.46 \mathrm{E}-03$ \\
\hline 6942 & NR4A3 & -4.23 & $3.42 \mathrm{E}-04$ \\
\hline 6943 & NR5A1 & -2.36 & $1.32 \mathrm{E}-02$ \\
\hline 6944 & NRAS & -3.18 & $5.20 \mathrm{E}-04$ \\
\hline 6945 & NRBF2 & -2.81 & $7.56 \mathrm{E}-03$ \\
\hline 6946 & NRD1 & -8.36 & $6.17 \mathrm{E}-04$ \\
\hline 6947 & NRDE2 & -5.57 & $1.49 \mathrm{E}-04$ \\
\hline 6948 & NREP & -3.47 & $2.35 \mathrm{E}-03$ \\
\hline 6949 & NRG4 & -6.01 & $3.15 \mathrm{E}-03$ \\
\hline 6950 & NRK & -2.39 & $5.16 \mathrm{E}-04$ \\
\hline 6951 & NRSN1 & -2.44 & $5.53 \mathrm{E}-04$ \\
\hline 6952 & NSA2 & -6.57 & $4.14 \mathrm{E}-04$ \\
\hline 6953 & NSF & -2.85 & $2.59 \mathrm{E}-03$ \\
\hline 6954 & NSFL1C & -2.79 & $2.38 \mathrm{E}-03$ \\
\hline 6955 & NSG1 & -2.88 & $9.88 \mathrm{E}-03$ \\
\hline 6956 & NSL1 & -4.30 & $8.56 \mathrm{E}-04$ \\
\hline 6957 & NSMAF & -3.42 & $9.44 \mathrm{E}-04$ \\
\hline 6958 & NSMCE1 & -4.75 & $1.37 \mathrm{E}-03$ \\
\hline 6959 & NSMCE2 & -6.52 & $5.01 \mathrm{E}-04$ \\
\hline 6960 & NSMCE4A & -6.96 & $1.52 \mathrm{E}-04$ \\
\hline 6961 & NSRP1 & -4.31 & $1.24 \mathrm{E}-03$ \\
\hline 6962 & NSUN2 & -2.23 & $6.80 \mathrm{E}-04$ \\
\hline 6963 & NSUN3 & -4.44 & $9.48 \mathrm{E}-05$ \\
\hline 6964 & NSUNG & -1.46 & $7.68 \mathrm{E}-03$ \\
\hline 6965 & NSUN7 & -3.07 & $4.31 \mathrm{E}-03$ \\
\hline 6966 & NT5C2 & -1.86 & $4.02 \mathrm{E}-02$ \\
\hline 6967 & NT5C3L & -4.32 & $8.19 \mathrm{E}-03$ \\
\hline 6968 & NT5DC2 & -3.43 & $1.53 \mathrm{E}-03$ \\
\hline 6969 & NTAN1 & -2.41 & $1.27 \mathrm{E}-04$ \\
\hline 6970 & NTHL1 & -2.35 & $5.79 \mathrm{E}-04$ \\
\hline 6971 & NTM & -3.76 & $3.06 \mathrm{E}-04$ \\
\hline 6972 & NTPCR & -1.44 & $2.33 \mathrm{E}-02$ \\
\hline 6973 & NUB1 & -6.72 & $3.87 \mathrm{E}-05$ \\
\hline 6974 & NUBP1 & -6.41 & $1.88 \mathrm{E}-05$ \\
\hline 6975 & NUBP2 & -1.76 & $6.47 \mathrm{E}-03$ \\
\hline 6976 & NUCB2 & -4.82 & $1.73 \mathrm{E}-04$ \\
\hline 6977 & NUCKS1 & -3.29 & 7.06E-03 \\
\hline 6978 & NUDC & -6.73 & $2.95 \mathrm{E}-05$ \\
\hline 6979 & NUDCD2 & -1.12 & $8.39 \mathrm{E}-03$ \\
\hline 6980 & NUDCD3 & -3.69 & $7.65 \mathrm{E}-04$ \\
\hline 6981 & NUDT1 & -4.87 & $1.55 \mathrm{E}-03$ \\
\hline 6982 & NUDT12 & -2.44 & $7.89 \mathrm{E}-03$ \\
\hline 6983 & NUDT13 & -3.64 & $4.99 \mathrm{E}-03$ \\
\hline 6984 & NUDT14 & -5.49 & 2.09E-04 \\
\hline 6985 & NUDT15 & -3.08 & $5.94 \mathrm{E}-03$ \\
\hline
\end{tabular}

\begin{tabular}{|c|c|c|c|}
\hline 6986 & NUDT16L1 & -3.47 & $1.83 \mathrm{E}-03$ \\
\hline 6987 & NUDT19 & -2.85 & $1.41 \mathrm{E}-03$ \\
\hline 6988 & NUDT2 & -3.46 & $2.65 \mathrm{E}-04$ \\
\hline 6989 & NUDT4 & -1.44 & $8.04 \mathrm{E}-05$ \\
\hline 6990 & NUDT5 & -4.57 & $8.16 \mathrm{E}-05$ \\
\hline 6991 & NUDT7 & -3.39 & $2.98 \mathrm{E}-03$ \\
\hline 6992 & NUDT9 & -4.59 & $7.86 \mathrm{E}-04$ \\
\hline 6993 & NUF2 & -2.05 & $5.32 \mathrm{E}-02$ \\
\hline 6994 & NUFIP1 & -4.01 & $4.04 \mathrm{E}-03$ \\
\hline 6995 & NUMB & -1.44 & 5.97E-02 \\
\hline 6996 & NUP107 & -5.25 & $2.13 \mathrm{E}-04$ \\
\hline 6997 & NUP133 & -2.54 & $2.09 \mathrm{E}-03$ \\
\hline 6998 & NUP153 & -4.14 & $1.14 \mathrm{E}-03$ \\
\hline 6999 & NUP155 & -4.75 & $2.21 \mathrm{E}-04$ \\
\hline 7000 & NUP188 & -3.24 & $2.27 \mathrm{E}-03$ \\
\hline 7001 & NUP205 & -1.20 & $2.04 \mathrm{E}-03$ \\
\hline 7002 & NUP210L & -4.26 & $1.24 \mathrm{E}-03$ \\
\hline 7003 & NUP35 & -6.11 & $5.25 \mathrm{E}-04$ \\
\hline 7004 & NUP37 & -4.97 & $1.92 \mathrm{E}-04$ \\
\hline 7005 & NUP43 & -4.23 & $5.05 \mathrm{E}-04$ \\
\hline 7006 & NUP50 & -4.06 & $1.90 \mathrm{E}-03$ \\
\hline 7007 & NUP54 & -2.92 & $8.75 \mathrm{E}-03$ \\
\hline 7008 & NUP62 & -3.90 & $8.55 \mathrm{E}-05$ \\
\hline 7009 & NUP85 & -6.90 & $8.13 \mathrm{E}-05$ \\
\hline 7010 & NUP88 & -3.36 & $1.53 \mathrm{E}-02$ \\
\hline 7011 & NUP93 & -3.24 & 5.55E-04 \\
\hline 7012 & NUP98 & -1.07 & $8.71 \mathrm{E}-03$ \\
\hline 7013 & NUPL1 & -4.22 & 2.69E-04 \\
\hline 7014 & NUPL2 & -1.54 & 4.63E-04 \\
\hline 7015 & NUS1 & -2.46 & $2.82 \mathrm{E}-04$ \\
\hline 7016 & NUSAP1 & -4.10 & $2.16 \mathrm{E}-04$ \\
\hline 7017 & NUTF2 & -6.87 & 4.79E-05 \\
\hline 7018 & NVL & -2.66 & $7.45 \mathrm{E}-03$ \\
\hline 7019 & NXT2 & -3.03 & $2.93 \mathrm{E}-02$ \\
\hline 7020 & OAT & -6.23 & 7.05E-03 \\
\hline 7021 & OAZ1 & -7.83 & $1.68 \mathrm{E}-04$ \\
\hline 7022 & OAZ2 & -5.18 & $1.11 \mathrm{E}-03$ \\
\hline 7023 & OBFC1 & -1.54 & $1.39 \mathrm{E}-04$ \\
\hline 7024 & OBFC2A & -2.13 & $6.14 \mathrm{E}-05$ \\
\hline 7025 & OCC-1 & -3.59 & $8.49 \mathrm{E}-04$ \\
\hline 7026 & OCIAD1 & -5.20 & $1.46 \mathrm{E}-03$ \\
\hline 7027 & ODC1 & -6.10 & $8.32 \mathrm{E}-05$ \\
\hline 7028 & ODF2 & -4.53 & $6.79 \mathrm{E}-04$ \\
\hline 7029 & ODF2L & -8.13 & $6.04 \mathrm{E}-05$ \\
\hline 7030 & OFD1 & -2.58 & $1.80 \mathrm{E}-03$ \\
\hline 7031 & OGDH & -1.97 & $6.53 \mathrm{E}-03$ \\
\hline 7032 & OGDHL & -3.46 & $1.03 \mathrm{E}-03$ \\
\hline 7033 & OGT & -4.18 & $1.38 \mathrm{E}-04$ \\
\hline 7034 & OIP5 & -1.32 & $2.41 \mathrm{E}-03$ \\
\hline 7035 & OLA1 & -5.94 & $1.45 \mathrm{E}-04$ \\
\hline 7036 & OLFML3 & -2.77 & $2.60 \mathrm{E}-04$ \\
\hline 7037 & OMA1 & -5.94 & $2.85 \mathrm{E}-04$ \\
\hline 7038 & OPA1 & -2.32 & $6.58 \mathrm{E}-03$ \\
\hline 7039 & OPHN1 & -4.82 & $1.69 \mathrm{E}-04$ \\
\hline 7040 & OPN5 & -2.69 & $1.19 \mathrm{E}-02$ \\
\hline 7041 & OPTN & -5.47 & $1.56 \mathrm{E}-04$ \\
\hline 7042 & ORAI2 & -4.96 & $8.93 \mathrm{E}-05$ \\
\hline 7043 & ORAOV1 & -3.92 & $1.85 \mathrm{E}-04$ \\
\hline 7044 & ORC1 & -5.12 & $2.39 \mathrm{E}-03$ \\
\hline 7045 & ORC2 & -3.65 & $2.83 \mathrm{E}-03$ \\
\hline 7046 & ORC3 & -2.73 & $1.79 \mathrm{E}-03$ \\
\hline 7047 & ORC4 & -5.57 & $9.61 \mathrm{E}-05$ \\
\hline 7048 & ORC5 & -2.15 & $1.74 \mathrm{E}-02$ \\
\hline
\end{tabular}

\begin{tabular}{|c|c|c|c|}
\hline 7049 & ORC6 & -2.99 & $1.57 \mathrm{E}-03$ \\
\hline 7050 & ORMDL1 & -3.34 & $2.51 \mathrm{E}-03$ \\
\hline 7051 & ORMDL2 & -3.99 & $2.19 \mathrm{E}-03$ \\
\hline 7052 & OSBPL10 & -3.13 & $1.68 \mathrm{E}-03$ \\
\hline 7053 & OSBPL1A & -7.91 & 2.13E-04 \\
\hline 7054 & OSBPL3 & -1.33 & $1.13 \mathrm{E}-02$ \\
\hline 7055 & OSBPL8 & -3.78 & $3.20 \mathrm{E}-04$ \\
\hline 7056 & OSBPL9 & -3.60 & $1.37 \mathrm{E}-03$ \\
\hline 7057 & OSCP1 & -4.00 & $9.96 \mathrm{E}-04$ \\
\hline 7058 & OSGEPL1 & -6.90 & 7.04E-04 \\
\hline 7059 & OST4 & -6.96 & $5.43 \mathrm{E}-04$ \\
\hline 7060 & OSTBETA & -6.58 & $1.16 \mathrm{E}-02$ \\
\hline 7061 & OSTC & -6.11 & $1.18 \mathrm{E}-05$ \\
\hline 7062 & OSTF1 & -3.71 & $1.06 \mathrm{E}-03$ \\
\hline 7063 & OTC & -1.25 & $8.99 \mathrm{E}-04$ \\
\hline 7064 & OTOP1 & -1.78 & $6.01 \mathrm{E}-03$ \\
\hline 7065 & OTUD3 & -4.37 & 2.29E-05 \\
\hline 7066 & OTUD6B & -5.24 & 4.85E-03 \\
\hline 7067 & OXA1L & -3.08 & $1.21 \mathrm{E}-02$ \\
\hline 7068 & OXCT1 & -3.28 & $9.64 \mathrm{E}-04$ \\
\hline 7069 & OXNAD1 & -4.12 & 7.37E-04 \\
\hline 7070 & OXR1 & -1.33 & $2.90 \mathrm{E}-02$ \\
\hline 7071 & OXSM & -2.01 & 7.85E-03 \\
\hline 7072 & $\mathrm{P} 2 \mathrm{RX} 1$ & -3.94 & $1.32 \mathrm{E}-03$ \\
\hline 7073 & P2RX4 & -1.34 & 4.66E-03 \\
\hline 7074 & P2RX5 & -1.39 & $7.64 \mathrm{E}-03$ \\
\hline 7075 & P33MONOX & -4.28 & $5.81 \mathrm{E}-04$ \\
\hline 7076 & P4HA1 & -2.00 & $2.61 \mathrm{E}-03$ \\
\hline 7077 & P4HB & -4.74 & $6.00 \mathrm{E}-04$ \\
\hline 7078 & PA2G4 & -2.98 & $3.01 \mathrm{E}-03$ \\
\hline 7079 & РАВPC1 & -8.95 & 3.29E-05 \\
\hline 7080 & РАBPC4 & -3.89 & 2.50E-04 \\
\hline 7081 & PACRG & -4.70 & 1.99E-06 \\
\hline 7082 & PACRGL & -4.51 & 7.06E-04 \\
\hline 7083 & PACS2 & -3.38 & $2.74 \mathrm{E}-02$ \\
\hline 7084 & PACSIN2 & -5.51 & 2.06E-04 \\
\hline 7085 & PACSIN3 & -3.54 & 4.80E-04 \\
\hline 7086 & PAFAH1B1 & -3.79 & $3.31 \mathrm{E}-03$ \\
\hline 7087 & PAICS & -2.25 & $3.12 \mathrm{E}-03$ \\
\hline 7088 & PAIP2 & -7.22 & $4.70 \mathrm{E}-04$ \\
\hline 7089 & PAK1 & -1.56 & $1.85 \mathrm{E}-02$ \\
\hline 7090 & PAK1IP1 & -7.53 & 1.17E-04 \\
\hline 7091 & PALB2 & -4.45 & 2.62E-04 \\
\hline 7092 & PALLD & -2.33 & $4.18 \mathrm{E}-03$ \\
\hline 7093 & PAM16 & -5.44 & $2.27 \mathrm{E}-03$ \\
\hline 7094 & PAN3 & -4.67 & 9.57E-04 \\
\hline 7095 & PANK1 & -1.59 & $1.08 \mathrm{E}-03$ \\
\hline 7096 & PANK2 & -2.24 & $8.60 \mathrm{E}-04$ \\
\hline 7097 & PANK3 & -3.09 & $1.89 \mathrm{E}-02$ \\
\hline 7098 & PANK4 & -1.23 & $1.41 \mathrm{E}-03$ \\
\hline 7099 & PAPD7 & -2.95 & $1.59 \mathrm{E}-03$ \\
\hline 7100 & PAPOLA & -4.72 & $8.77 \mathrm{E}-04$ \\
\hline 7101 & PAPSS1 & -4.10 & $2.58 \mathrm{E}-04$ \\
\hline 7102 & PAQR8 & -1.57 & 2.93E-02 \\
\hline 7103 & PARD3B & -1.16 & 7.35E-03 \\
\hline 7104 & PARD6B & -2.09 & $1.22 \mathrm{E}-04$ \\
\hline 7105 & PARG & -3.26 & $5.13 \mathrm{E}-04$ \\
\hline 7106 & PARK7 & -7.56 & 3.19E-04 \\
\hline 7107 & PARL & -5.53 & $9.42 \mathrm{E}-05$ \\
\hline 7108 & PARN & -1.29 & $3.81 \mathrm{E}-03$ \\
\hline 7109 & PARP1 & -6.36 & 2.55E-04 \\
\hline 7110 & PARP11 & -5.69 & $3.70 \mathrm{E}-04$ \\
\hline 7111 & PARP12 & -8.24 & $6.57 \mathrm{E}-04$ \\
\hline
\end{tabular}




\begin{tabular}{|c|c|c|c|}
\hline 7112 & PARP16 & -4.35 & $2.79 \mathrm{E}-04$ \\
\hline 7113 & $\begin{array}{l}\text { PARP4 } \\
\end{array}$ & \begin{tabular}{|l|}
-2.37 \\
\end{tabular} & $4.28 \mathrm{E}-04$ \\
\hline 7114 & PARP6 & -2.62 & $2.97 \mathrm{E}-02$ \\
\hline 7115 & $\begin{array}{l}\text { PARP8 } \\
\end{array}$ & -5.53 & $7.21 \mathrm{E}-05$ \\
\hline 7116 & PARP9 & -1.94 & $9.10 \mathrm{E}-03$ \\
\hline 7117 & PARS2 & -2.24 & $1.57 \mathrm{E}-03$ \\
\hline 7118 & $\begin{array}{l}\text { PARVA } \\
\end{array}$ & -3.80 & $4.87 \mathrm{E}-04$ \\
\hline 7119 & PARVB & -2.02 & $5.38 \mathrm{E}-03$ \\
\hline 7120 & PASK & -5.55 & $5.49 \mathrm{E}-05$ \\
\hline 7121 & PAWR & -2.06 & $5.54 \mathrm{E}-03$ \\
\hline 7122 & PAXIP1 & $\begin{array}{l}-3.08 \\
\end{array}$ & $8.63 \mathrm{E}-04$ \\
\hline 7123 & PBDC1 & -5.65 & $1.81 \mathrm{E}-03$ \\
\hline 7124 & PBK & $\begin{array}{l}-7.83 \\
\end{array}$ & $4.10 \mathrm{E}-05$ \\
\hline 7125 & PBLD & -1.69 & $4.55 \mathrm{E}-03$ \\
\hline 7126 & PBRM1 & -2.76 & $7.39 \mathrm{E}-06$ \\
\hline 7127 & PBX3 & -2.82 & $6.06 \mathrm{E}-04$ \\
\hline 7128 & PCBD1 & \begin{tabular}{|l|}
-1.21 \\
\end{tabular} & $1.86 \mathrm{E}-02$ \\
\hline 7129 & PCBD2 & $\begin{array}{ll}-5.02 \\
\end{array}$ & $3.17 \mathrm{E}-04$ \\
\hline 7130 & PCCA & \begin{tabular}{|l|}
-2.41 \\
\end{tabular} & $1.21 \mathrm{E}-04$ \\
\hline 7131 & PCCB & -5.68 & $1.83 \mathrm{E}-05$ \\
\hline 7132 & PCDH15 & -2.79 & $3.51 \mathrm{E}-03$ \\
\hline 7133 & PCDH18 & $\begin{array}{l}-1.78 \\
\end{array}$ & $5.09 \mathrm{E}-03$ \\
\hline 7134 & PCDP1 & -3.59 & $3.67 \mathrm{E}-03$ \\
\hline 7135 & PCGF3 & \begin{tabular}{|l|}
-1.41 \\
\end{tabular} & $8.84 \mathrm{E}-03$ \\
\hline 7136 & PCGF6 & -1.44 & $1.91 \mathrm{E}-02$ \\
\hline 7137 & PCID2 & $\begin{array}{l}-1.27 \\
\end{array}$ & $3.50 \mathrm{E}-03$ \\
\hline 7138 & PCM1 & -5.39 & $6.28 \mathrm{E}-05$ \\
\hline 7139 & PCMT1 & $\begin{array}{l}-6.53 \\
\end{array}$ & $9.21 \mathrm{E}-04$ \\
\hline 7140 & PCMTD1 & $\begin{array}{l}-2.56 \\
\end{array}$ & $5.26 \mathrm{E}-06$ \\
\hline 7141 & 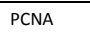 & $\begin{array}{l}-4.04 \\
\end{array}$ & $6.03 \mathrm{E}-04$ \\
\hline 7142 & PCNP & -2.71 & $1.01 \mathrm{E}-05$ \\
\hline 7143 & $\begin{array}{l}\text { PCNT } \\
\end{array}$ & $\begin{array}{l}-4.48 \\
\end{array}$ & $3.32 \mathrm{E}-04$ \\
\hline 7144 & PCOLCE2 & -3.28 & $8.58 \mathrm{E}-03$ \\
\hline 7145 & PCSK1 & $\begin{array}{l}-4.47 \\
\end{array}$ & $1.21 \mathrm{E}-04$ \\
\hline 7146 & PCSK5 & -1.51 & $6.42 \mathrm{E}-03$ \\
\hline 7147 & PCSK7 & -1.69 & $3.39 \mathrm{E}-03$ \\
\hline 7148 & РCTP & -1.26 & $4.38 \mathrm{E}-02$ \\
\hline 7149 & PCYOX1 & \begin{tabular}{|l|}
-3.21 \\
\end{tabular} & $3.54 \mathrm{E}-03$ \\
\hline 7150 & PCYT1B & -8.78 & $3.23 \mathrm{E}-04$ \\
\hline 7151 & PCYT2 & -4.35 & $1.18 \mathrm{E}-03$ \\
\hline 7152 & PDAP1 & $\begin{array}{l}-2.96 \\
\end{array}$ & $1.20 \mathrm{E}-03$ \\
\hline 7153 & PDCD10 & $\begin{array}{l}-5.64 \\
\end{array}$ & $2.21 \mathrm{E}-04$ \\
\hline 7154 & PDCD2 & $\begin{array}{l}-6.62 \\
\end{array}$ & $3.68 \mathrm{E}-04$ \\
\hline 7155 & $\begin{array}{l}\text { PDCD2L } \\
\end{array}$ & \begin{tabular}{|l|}
-3.64 \\
\end{tabular} & $5.66 \mathrm{E}-04$ \\
\hline 7156 & PDCD4 & $\begin{array}{l}-1.48 \\
\end{array}$ & $2.61 \mathrm{E}-03$ \\
\hline 7157 & PDCD5 & -3.75 & $1.02 E-02$ \\
\hline 7158 & PDCD6 & -4.15 & $1.88 \mathrm{E}-02$ \\
\hline 7159 & PDCDGIP & \begin{tabular}{|l|}
-2.78 \\
\end{tabular} & $\begin{array}{l}7.70 \mathrm{E}-04 \\
\end{array}$ \\
\hline 7160 & $\begin{array}{l}\text { PDCD7 } \\
\end{array}$ & \begin{tabular}{|l|}
-3.96 \\
\end{tabular} & $5.52 \mathrm{E}-04$ \\
\hline 7161 & PDCL & -2.07 & $3.23 \mathrm{E}-03$ \\
\hline 7162 & PDCL2 & -8.86 & $2.62 \mathrm{E}-04$ \\
\hline 7163 & PDDC1 & $\begin{array}{l}-1.97 \\
\end{array}$ & $3.66 \mathrm{E}-03$ \\
\hline 7164 & PDE10A & -2.79 & $1.52 \mathrm{E}-03$ \\
\hline 7165 & PDE4B & -1.11 & $7.84 \mathrm{E}-03$ \\
\hline 7166 & PDE4D & $\begin{array}{l}-1.41 \\
\end{array}$ & $1.26 \mathrm{E}-03$ \\
\hline 7167 & PDE6D & -4.83 & $2.08 \mathrm{E}-03$ \\
\hline 7168 & $\begin{array}{l}\text { PDE8A } \\
\end{array}$ & \begin{tabular}{|l|}
-1.27 \\
\end{tabular} & $2.54 \mathrm{E}-03$ \\
\hline 7169 & PDGFD & \begin{tabular}{|l|}
-1.42 \\
\end{tabular} & $3.92 \mathrm{E}-02$ \\
\hline 7170 & $\begin{array}{l}\text { PDGFRL } \\
\end{array}$ & \begin{tabular}{|l|}
-3.68 \\
\end{tabular} & $\begin{array}{l}7.77 E-04 \\
\end{array}$ \\
\hline 7171 & PDHA1 & -5.51 & $7.55 \mathrm{E}-04$ \\
\hline 7172 & PDHB & -4.73 & $3.99 \mathrm{E}-05$ \\
\hline 7173 & PDHX & -3.08 & $1.02 E-02$ \\
\hline
\end{tabular}

\begin{tabular}{|c|c|c|c|}
\hline 7175 & PDIA4 & -3.04 & $7.54 \mathrm{E}-04$ \\
\hline 7176 & PDIA5 & -3.31 & $6.34 \mathrm{E}-03$ \\
\hline 7177 & PDIAG & -4.57 & $2.84 \mathrm{E}-04$ \\
\hline 7178 & PDIK1L & -3.51 & $6.10 \mathrm{E}-04$ \\
\hline 7179 & PDK1 & -2.30 & $9.49 \mathrm{E}-03$ \\
\hline 7180 & PDK3 & -4.63 & $6.58 \mathrm{E}-04$ \\
\hline 7181 & PDLIM1 & -2.82 & $5.47 \mathrm{E}-03$ \\
\hline 7182 & PDLIM5 & -1.45 & $1.33 \mathrm{E}-02$ \\
\hline 7183 & PDP1 & -3.49 & $1.66 \mathrm{E}-03$ \\
\hline 7184 & PDPK1 & -4.52 & $1.11 \mathrm{E}-03$ \\
\hline 7185 & PDRG1 & -5.09 & $3.96 \mathrm{E}-04$ \\
\hline 7186 & PDS5A & -3.15 & $2.56 \mathrm{E}-04$ \\
\hline 7187 & PDS5B & -2.94 & $6.35 \mathrm{E}-03$ \\
\hline $\begin{array}{l}7188 \\
\end{array}$ & PDSS1 & -1.46 & $9.34 \mathrm{E}-03$ \\
\hline 7189 & PDSS2 & -6.08 & $1.57 \mathrm{E}-04$ \\
\hline 7190 & PDXDC1 & -1.34 & $1.65 \mathrm{E}-03$ \\
\hline 7191 & PDXK & -5.45 & $1.48 \mathrm{E}-03$ \\
\hline 7192 & PDZD11 & -4.13 & $1.05 \mathrm{E}-04$ \\
\hline 7193 & PDZD8 & -6.38 & $1.56 \mathrm{E}-04$ \\
\hline 7194 & PEAK1 & -1.14 & $1.45 \mathrm{E}-02$ \\
\hline 7195 & PEBP1 & -6.69 & $1.41 \mathrm{E}-04$ \\
\hline 7196 & PECR & -4.77 & $1.29 \mathrm{E}-03$ \\
\hline 7197 & PEF1 & -3.26 & $2.06 \mathrm{E}-03$ \\
\hline 7198 & PELI1 & -3.83 & $1.47 \mathrm{E}-04$ \\
\hline 7199 & PELI2 & -4.41 & $7.17 \mathrm{E}-04$ \\
\hline 7200 & $\begin{array}{l}\text { PELO } \\
\end{array}$ & -1.54 & $3.91 \mathrm{E}-02$ \\
\hline 7201 & PENK & -6.86 & $1.55 \mathrm{E}-03$ \\
\hline 7202 & PEPD & -2.66 & $3.00 \mathrm{E}-02$ \\
\hline 7203 & PER2 & -3.61 & $1.15 \mathrm{E}-03$ \\
\hline 7204 & PET112 & -1.92 & $3.58 \mathrm{E}-03$ \\
\hline 7205 & PEX1 & -3.33 & $1.98 \mathrm{E}-03$ \\
\hline 7206 & PEX10 & -5.00 & $3.22 \mathrm{E}-04$ \\
\hline 7207 & PEX11A & -2.46 & $2.74 \mathrm{E}-03$ \\
\hline 7208 & PEX11G & -5.54 & $2.60 \mathrm{E}-04$ \\
\hline 7209 & PEX12 & -2.29 & $8.51 \mathrm{E}-04$ \\
\hline 7210 & PEX13 & -3.19 & $2.97 \mathrm{E}-04$ \\
\hline 7211 & PEX14 & -2.06 & $1.47 \mathrm{E}-03$ \\
\hline 7212 & PEX16 & -2.81 & $2.26 \mathrm{E}-03$ \\
\hline 7213 & PEX2 & -3.16 & $2.62 \mathrm{E}-04$ \\
\hline $\begin{array}{l}7214 \\
\end{array}$ & $\begin{array}{l}\text { PEX3 } \\
\end{array}$ & -4.80 & $4.17 \mathrm{E}-04$ \\
\hline 7215 & PEX6 & -3.90 & $3.66 \mathrm{E}-03$ \\
\hline 7216 & PEX7 & -3.60 & $7.81 \mathrm{E}-04$ \\
\hline 7217 & PFDN1 & -5.44 & $1.11 \mathrm{E}-04$ \\
\hline 7218 & PFDN4 & \begin{tabular}{|l|}
-5.61 \\
\end{tabular} & $1.11 \mathrm{E}-04$ \\
\hline 7219 & PFKM & -1.48 & $1.86 \mathrm{E}-03$ \\
\hline 7220 & $\begin{array}{l}\text { PFKP } \\
\end{array}$ & $\begin{array}{l}-7.30 \\
\end{array}$ & $9.85 \mathrm{E}-05$ \\
\hline 7221 & PFN2 & -4.53 & $2.49 \mathrm{E}-03$ \\
\hline 7222 & PFN3 & -4.13 & $1.86 \mathrm{E}-02$ \\
\hline 7223 & PFN4 & -5.71 & $1.03 \mathrm{E}-03$ \\
\hline $\begin{array}{l}7224 \\
\end{array}$ & PGAM1 & -6.26 & $1.27 \mathrm{E}-03$ \\
\hline 7225 & PGBD5 & -4.53 & $2.00 \mathrm{E}-04$ \\
\hline 7226 & PGD & -2.69 & $1.44 \mathrm{E}-03$ \\
\hline 7227 & PGGT1B & -2.40 & $6.97 \mathrm{E}-04$ \\
\hline 7228 & PGK1 & -5.26 & $8.25 \mathrm{E}-04$ \\
\hline 7229 & PGM1 & -1.23 & $5.97 \mathrm{E}-03$ \\
\hline 7230 & PGM2 & -1.76 & $5.85 \mathrm{E}-03$ \\
\hline 7231 & PGP & -4.72 & $1.04 \mathrm{E}-03$ \\
\hline 7232 & PGPEP1 & -1.50 & $2.33 \mathrm{E}-03$ \\
\hline 7233 & PGR & -1.32 & $2.94 \mathrm{E}-03$ \\
\hline 7234 & PGRMC1 & -6.10 & $1.02 \mathrm{E}-03$ \\
\hline 7235 & PGRMC2 & -5.19 & $6.01 \mathrm{E}-04$ \\
\hline 7236 & $\begin{array}{l}\text { PGS1 } \\
\end{array}$ & -3.32 & $2.79 \mathrm{E}-03$ \\
\hline 7237 & PHACTR3 & -1.67 & $1.53 \mathrm{E}-03$ \\
\hline
\end{tabular}

\begin{tabular}{|c|c|c|c|}
\hline 7238 & PHAX & -3.76 & $1.14 E-03$ \\
\hline 7239 & PHB & -3.38 & $\begin{array}{l}7.82 E-04 \\
\end{array}$ \\
\hline 7240 & PHB2 & -5.99 & $1.45 \mathrm{E}-04$ \\
\hline 7241 & PHC1 & -2.12 & $2.66 \mathrm{E}-03$ \\
\hline 7242 & PHC3 & -1.80 & $4.34 \mathrm{E}-03$ \\
\hline 7243 & PHF13 & -1.42 & $2.15 \mathrm{E}-02$ \\
\hline 7244 & $\begin{array}{l}\text { PHF15 } \\
\end{array}$ & -3.39 & $1.36 \mathrm{E}-03$ \\
\hline 7245 & PHF17 & -4.10 & $3.00 E-03$ \\
\hline 7246 & PHF2O & -4.91 & $7.24 E-05$ \\
\hline 7247 & PHF20L1 & -2.45 & $3.51 E-05$ \\
\hline 7248 & PHF3 & -3.19 & $5.50 \mathrm{E}-04$ \\
\hline 7249 & PHF5A & -4.94 & 4.44E-04 \\
\hline 7250 & PHF6 & -2.07 & $2.34 \mathrm{E}-03$ \\
\hline 7251 & PHKA2 & -2.73 & $4.01 E-03$ \\
\hline 7252 & PHKB & -3.13 & $3.51 E-03$ \\
\hline 7253 & PHKG1 & -5.06 & $2.19 \mathrm{E}-03$ \\
\hline 7254 & PHOSPHO2 & -1.14 & $2.05 E-02$ \\
\hline 7255 & PHPT1 & -4.51 & $6.71 E-05$ \\
\hline 7256 & PHTF1 & -2.54 & $3.55 \mathrm{E}-04$ \\
\hline 7257 & $\begin{array}{l}\text { PHTF2 } \\
\end{array}$ & -1.76 & $9.52 \mathrm{E}-03$ \\
\hline 7258 & PHYH & -4.86 & $4.98 \mathrm{E}-03$ \\
\hline 7259 & PHYHIPL & -1.75 & $8.74 E-03$ \\
\hline 7260 & PI4K2B & -3.76 & $2.49 \mathrm{E}-02$ \\
\hline 7261 & PI4KA & -1.45 & $3.14 E-03$ \\
\hline 7262 & PIAS1 & -2.69 & $5.76 E-03$ \\
\hline 7263 & PIAS2 & -1.27 & $9.80 \mathrm{E}-03$ \\
\hline 7264 & PIBF1 & $\begin{array}{l}-2.71 \\
\end{array}$ & $3.26 \mathrm{E}-04$ \\
\hline 7265 & PICK1 & -6.09 & $1.82 E-04$ \\
\hline 7266 & PIF1 & -1.48 & $9.94 E-03$ \\
\hline 7267 & PIGG & -2.22 & $9.47 E-04$ \\
\hline 7268 & PIGK & -4.66 & $4.06 E-04$ \\
\hline 7269 & PIGL & -3.32 & $8.46 \mathrm{E}-04$ \\
\hline 7270 & PIGM & -3.46 & $3.22 E-03$ \\
\hline 7271 & PIGN & -2.27 & $1.07 E-03$ \\
\hline 7272 & PIGP & -5.63 & $4.03 E-04$ \\
\hline 7273 & PIGW & -2.11 & $2.02 E-02$ \\
\hline 7274 & PIGX & -2.87 & $1.26 \mathrm{E}-02$ \\
\hline 7275 & PIH1D2 & -3.12 & $8.07 E-03$ \\
\hline 7276 & PIH1D3 & -6.61 & $1.57 E-04$ \\
\hline 7277 & PIK3AP1 & -1.85 & $1.64 E-03$ \\
\hline 7278 & РІК3С2A & -1.54 & $6.10 \mathrm{E}-03$ \\
\hline 7279 & PIK3CA & -2.93 & $1.53 \mathrm{E}-03$ \\
\hline 7280 & РІКЗСВ & -4.80 & $8.64 E-04$ \\
\hline 7281 & PIK3R3 & -4.75 & $7.27 E-04$ \\
\hline 7282 & PIK3R4 & -1.36 & $8.54 \mathrm{E}-03$ \\
\hline 7283 & PIN4 & -6.91 & $5.28 \mathrm{E}-05$ \\
\hline 7284 & PINX1 & -1.62 & $3.28 \mathrm{E}-03$ \\
\hline 7285 & PIP4K2A & -3.94 & $\begin{array}{l}7.65 E-04 \\
\end{array}$ \\
\hline 7286 & PIP4K2B & -1.35 & $3.02 E-02$ \\
\hline 7287 & PIP5K1B & -3.37 & $7.91 E-05$ \\
\hline 7288 & PIP5K1C & -1.46 & $1.69 \mathrm{E}-03$ \\
\hline 7289 & $\begin{array}{l}\text { PITHD1 } \\
\end{array}$ & -2.78 & $4.23 \mathrm{E}-03$ \\
\hline 7290 & PITPNB & -2.00 & $1.44 E-02$ \\
\hline 7291 & PITPNC1 & -4.00 & $1.83 E-05$ \\
\hline 7292 & PITPNM3 & -1.33 & $4.89 \mathrm{E}-02$ \\
\hline 7293 & $\begin{array}{l}\text { PITRM1 } \\
\end{array}$ & -4.90 & $5.38 \mathrm{E}-04$ \\
\hline 7294 & PITX2 & -4.33 & $6.07 \mathrm{E}-03$ \\
\hline 7295 & PIWIL1 & -4.36 & $\begin{array}{c}6.65 \mathrm{E}-03 \\
\end{array}$ \\
\hline 7296 & PJA2 & -5.62 & $3.30 E-04$ \\
\hline 7297 & PKD2 & -1.57 & $8.38 \mathrm{E}-02$ \\
\hline 7298 & PKIG & -5.74 & $1.35 E-04$ \\
\hline 7299 & PKM2 & -1.98 & $9.88 \mathrm{E}-03$ \\
\hline 7300 & PKN2 & -1.06 & $9.67 \mathrm{E}-03$ \\
\hline
\end{tabular}




\begin{tabular}{|c|c|c|c|}
\hline 7301 & PKP4 & -2.22 & $3.79 E-03$ \\
\hline 7302 & PLA2R1 & -1.47 & $3.08 \mathrm{E}-03$ \\
\hline 7303 & PLAA & -5.49 & $1.92 E-04$ \\
\hline 7304 & PLAC8 & $\begin{array}{l}-4.43 \\
\end{array}$ & $1.38 \mathrm{E}-03$ \\
\hline 7305 & PLAU & -2.22 & $1.62 \mathrm{E}-03$ \\
\hline 7306 & PLBD1 & -1.89 & $2.81 \mathrm{E}-02$ \\
\hline 7307 & PLBD2 & -1.36 & $6.22 \mathrm{E}-03$ \\
\hline 7308 & PLCB1 & -2.48 & $1.20 \mathrm{E}-02$ \\
\hline 7309 & PLCXD2 & -4.92 & $3.23 \mathrm{E}-04$ \\
\hline 7310 & PLCZ1 & \begin{tabular}{|l|}
-7.12 \\
\end{tabular} & $5.32 \mathrm{E}-03$ \\
\hline 7311 & PLDN & -2.44 & 7.17E-03 \\
\hline 7312 & PLEKHA3 & -4.57 & 4.56E- 03 \\
\hline 7313 & PLEKHA5 & -3.75 & $1.20 \mathrm{E}-03$ \\
\hline 7314 & PLEKHA8 & -3.87 & $1.86 \mathrm{E}-04$ \\
\hline 7315 & PLEKHF2 & -1.63 & 4.19E-03 \\
\hline 7316 & PLEKHJ1 & $\begin{array}{l}-2.36 \\
\end{array}$ & $1.51 \mathrm{E}-02$ \\
\hline 7317 & PLEKHM1 & -2.36 & $6.88 \mathrm{E}-04$ \\
\hline 7318 & PLK1 & -1.40 & $1.07 E-02$ \\
\hline 7319 & PLK1S1 & -4.22 & $3.09 E-04$ \\
\hline 7320 & PLOD1 & -3.78 & $2.68 \mathrm{E}-03$ \\
\hline 7321 & PLRG1 & -5.09 & $1.50 \mathrm{E}-03$ \\
\hline 7322 & $\begin{array}{l}\text { PLS1 } \\
\end{array}$ & $\begin{array}{l}-1.72 \\
\end{array}$ & $9.59 \mathrm{E}-04$ \\
\hline 7323 & PM20D1 & -5.29 & 4.45E-04 \\
\hline 7324 & PMM2 & -1.81 & 7.44E-05 \\
\hline 7325 & PMP22 & -1.29 & $1.11 \mathrm{E}-03$ \\
\hline 7326 & PMPCA & -3.45 & 3.54E- 03 \\
\hline 7327 & PMPCB & -5.26 & $1.31 \mathrm{E}-04$ \\
\hline 7328 & PMS1 & -4.53 & $8.20 \mathrm{E}-05$ \\
\hline 7329 & PNISR & -2.92 & $1.72 \mathrm{E}-04$ \\
\hline 7330 & PNLDC1 & -4.43 & $5.45 E-04$ \\
\hline 7331 & PNN & -5.56 & $6.09 \mathrm{E}-04$ \\
\hline 7332 & PNO1 & -5.70 & 3.44E-04 \\
\hline 7333 & PNPLA3 & -2.54 & $7.52 \mathrm{E}-05$ \\
\hline 7334 & PNPLA4 & -1.87 & 3.66E-02 \\
\hline 7335 & PNPLA7 & -1.46 & $1.53 \mathrm{E}-03$ \\
\hline 7336 & PNPLA8 & -6.16 & $8.96 \mathrm{E}-04$ \\
\hline 7337 & PNRC1 & -4.55 & $4.23 \mathrm{E}-03$ \\
\hline 7338 & $\begin{array}{l}\text { PNRC2 } \\
\end{array}$ & -3.66 & $1.20 \mathrm{E}-03$ \\
\hline 7339 & POC1A & -2.30 & $2.42 \mathrm{E}-02$ \\
\hline 7340 & POC5 & -3.26 & $3.25 \mathrm{E}-03$ \\
\hline 7341 & POF1B & -2.65 & $4.11 \mathrm{E}-03$ \\
\hline 7342 & POFUT1 & -1.23 & $4.52 \mathrm{E}-03$ \\
\hline 7343 & POFUT2 & -2.10 & $8.58 \mathrm{E}-04$ \\
\hline 7344 & POGLUT1 & -2.05 & 2.32E-04 \\
\hline 7345 & POLA1 & -2.17 & $1.88 \mathrm{E}-03$ \\
\hline 7346 & POLD3 & -4.60 & $1.84 \mathrm{E}-04$ \\
\hline 7347 & POLDIP3 & -4.51 & $6.45 E-04$ \\
\hline 7348 & POLE2 & -5.46 & $1.73 \mathrm{E}-03$ \\
\hline 7349 & POLE3 & -3.58 & $2.01 \mathrm{E}-03$ \\
\hline 7350 & POLG2 & -3.68 & 3.56E-04 \\
\hline 7351 & POLN & -2.34 & $1.04 E-02$ \\
\hline 7352 & POLRIC & -4.80 & $3.89 \mathrm{E}-04$ \\
\hline 7353 & POLR2B & -6.11 & $5.38 \mathrm{E}-06$ \\
\hline 7354 & POLR2C & -3.31 & $1.18 \mathrm{E}-02$ \\
\hline 7355 & POLR2D & -5.48 & $1.90 \mathrm{E}-05$ \\
\hline 7356 & POLR2E & -5.01 & 8.71E-04 \\
\hline 7357 & POLR2F & -6.97 & $5.59 \mathrm{E}-05$ \\
\hline 7358 & POLR2H & -4.77 & $6.90 \mathrm{E}-05$ \\
\hline 7359 & POLR2I & -5.24 & 1.17E-04 \\
\hline 7360 & POLR2K & -4.97 & $8.98 \mathrm{E}-05$ \\
\hline 7361 & POLR2L & -5.99 & $1.24 \mathrm{E}-04$ \\
\hline 7362 & POLR3A & -1.75 & $3.66 \mathrm{E}-02$ \\
\hline 363 & PLLR3B & -2.69 & $2.26 \mathrm{t}-03$ \\
\hline
\end{tabular}

\begin{tabular}{|c|c|c|c|}
\hline 7364 & POLR3E & -1.28 & $6.47 \mathrm{E}-04$ \\
\hline 7365 & POLR3F & -3.75 & $1.95 \mathrm{E}-03$ \\
\hline 7366 & POLR3H & -4.03 & $1.51 \mathrm{E}-04$ \\
\hline 7367 & PON2 & -4.69 & $9.88 \mathrm{E}-04$ \\
\hline 7368 & POP4 & -4.74 & $9.09 \mathrm{E}-04$ \\
\hline 7369 & POP5 & -4.74 & $2.59 \mathrm{E}-03$ \\
\hline 7370 & POT1 & -2.37 & $2.34 \mathrm{E}-02$ \\
\hline 7371 & PPA1 & -5.08 & $4.08 \mathrm{E}-04$ \\
\hline 7372 & PPA2 & -3.50 & $1.84 \mathrm{E}-03$ \\
\hline 7373 & PPAP2A & \begin{tabular}{|l|}
-3.47 \\
\end{tabular} & $1.14 \mathrm{E}-03$ \\
\hline 7374 & PPAPDC1B & $\begin{array}{l}-1.77 \\
\end{array}$ & $2.84 \mathrm{E}-04$ \\
\hline 7375 & PPARA & -3.67 & $9.01 E-05$ \\
\hline 7376 & PPARD & -2.56 & 2.50E-04 \\
\hline 7377 & PPAT & -2.31 & $3.24 \mathrm{E}-03$ \\
\hline 7378 & PPDPF & -3.49 & $6.83 \mathrm{E}-04$ \\
\hline 7379 & PPEF1 & -4.54 & $1.14 \mathrm{E}-03$ \\
\hline 7380 & PPFIA1 & -4.01 & $6.92 \mathrm{E}-05$ \\
\hline 7381 & PPFIBP1 & -3.72 & $5.06 \mathrm{E}-04$ \\
\hline 7382 & PPHLN1 & -3.06 & $1.42 \mathrm{E}-04$ \\
\hline 7383 & PPIA & -6.84 & $1.93 \mathrm{E}-04$ \\
\hline 7384 & PPIB & -5.90 & $2.86 \mathrm{E}-04$ \\
\hline 7385 & PPIC & -2.30 & $7.94 \mathrm{E}-03$ \\
\hline 7386 & PPID & -7.09 & $1.25 \mathrm{E}-04$ \\
\hline 7387 & PPIE & -3.73 & $3.71 \mathrm{E}-04$ \\
\hline 7388 & PPIF & -2.21 & $3.82 \mathrm{E}-03$ \\
\hline 7389 & PPIG & -5.33 & $4.72 \mathrm{E}-05$ \\
\hline 7390 & PPIH & -4.28 & 7.44E- 05 \\
\hline 7391 & PPIL2 & -3.75 & $2.78 \mathrm{E}-04$ \\
\hline 7392 & PPIL3 & -3.69 & $6.14 \mathrm{E}-04$ \\
\hline 7393 & PPIL4 & -4.14 & $9.96 \mathrm{E}-04$ \\
\hline 7394 & $\begin{array}{l}\text { PPIL6 } \\
\end{array}$ & -6.00 & $2.63 \mathrm{E}-04$ \\
\hline 7395 & PPIP5K2 & -6.39 & $3.56 \mathrm{E}-04$ \\
\hline 7396 & PPM1A & -1.78 & $5.34 \mathrm{E}-04$ \\
\hline 7397 & $\begin{array}{l}\text { PPM1B } \\
\end{array}$ & \begin{tabular}{|l|}
-3.74 \\
\end{tabular} & $4.20 \mathrm{E}-04$ \\
\hline 7398 & PPM1D & -4.01 & $9.50 \mathrm{E}-05$ \\
\hline 7399 & PPM1K & -2.65 & $1.14 \mathrm{E}-02$ \\
\hline 7400 & PPM1L & -1.29 & $1.00 \mathrm{E}-02$ \\
\hline 7401 & PPME1 & -2.01 & $4.03 \mathrm{E}-03$ \\
\hline 7402 & PPP1CC & -5.35 & $4.10 \mathrm{E}-04$ \\
\hline 7403 & $\begin{array}{l}\text { PPP1R12A } \\
\end{array}$ & -3.36 & $1.72 \mathrm{E}-03$ \\
\hline 7404 & PPP1R13B & -5.57 & $2.87 \mathrm{E}-04$ \\
\hline 7405 & $\begin{array}{l}\text { PPP1R17 } \\
\end{array}$ & $\begin{array}{l}-1.63 \\
\end{array}$ & $3.39 \mathrm{E}-03$ \\
\hline 7406 & PPP1R2 & -5.97 & 4.44E-04 \\
\hline 7407 & PPP1R21 & -4.40 & $2.12 \mathrm{E}-03$ \\
\hline 7408 & PPP1R36 & -7.07 & $9.42 \mathrm{E}-05$ \\
\hline 7409 & PPP1R7 & -7.97 & $6.02 \mathrm{E}-05$ \\
\hline 7410 & $\begin{array}{l}\text { PPP1R8 } \\
\end{array}$ & -1.19 & $1.95 \mathrm{E}-02$ \\
\hline 7411 & PPP1R9B & -3.97 & $1.47 \mathrm{E}-03$ \\
\hline 7412 & PPP2CA & -2.93 & $1.60 \mathrm{E}-03$ \\
\hline 7413 & РРР2СВ & $\begin{array}{l}-3.18 \\
\end{array}$ & $7.74 \mathrm{E}-04$ \\
\hline 7414 & PPP2R2C & -4.45 & $4.83 \mathrm{E}-03$ \\
\hline 7415 & PPP2R2D & $\begin{array}{l}-3.77 \\
\end{array}$ & $5.20 \mathrm{E}-05$ \\
\hline 7416 & PPP2R3B & -3.49 & $6.15 \mathrm{E}-05$ \\
\hline 7417 & PPP2R3C & -4.41 & $2.92 \mathrm{E}-04$ \\
\hline 7418 & PPP2R5A & -3.33 & $5.46 \mathrm{E}-04$ \\
\hline 7419 & PPP2R5E & -1.87 & $7.83 \mathrm{E}-03$ \\
\hline 7420 & PPP3CA & -4.66 & $3.15 \mathrm{E}-03$ \\
\hline 7421 & PPP3R1 & -4.38 & $3.58 \mathrm{E}-04$ \\
\hline 7422 & PPP4R2 & -5.93 & $2.07 \mathrm{E}-03$ \\
\hline 7423 & PPP6C & -1.41 & $9.75 \mathrm{E}-03$ \\
\hline 7424 & PPP6R3 & -3.92 & $1.91 \mathrm{E}-03$ \\
\hline 7425 & PPPDE2 & -5.81 & $2.87 \mathrm{E}-03$ \\
\hline 7426 & PPRC1 & -1.90 & $1.55 \mathrm{E}-03$ \\
\hline
\end{tabular}

\begin{tabular}{|c|c|c|c|}
\hline 7427 & PPT1 & -4.41 & $3.08 \mathrm{E}-03$ \\
\hline 7428 & PPWD1 & -4.94 & $8.03 \mathrm{E}-04$ \\
\hline 7429 & PQLC2 & -3.16 & $2.97 \mathrm{E}-03$ \\
\hline 7430 & PRADC1 & -3.39 & $7.56 \mathrm{E}-05$ \\
\hline 7431 & PRC1 & -2.43 & $3.13 \mathrm{E}-03$ \\
\hline 7432 & PRCC & -1.20 & $1.24 \mathrm{E}-02$ \\
\hline $\begin{array}{l}7433 \\
\end{array}$ & PRDM4 & -2.89 & $7.87 \mathrm{E}-04$ \\
\hline 7434 & PRDX1 & -5.91 & $5.87 \mathrm{E}-05$ \\
\hline 7435 & PRDX3 & -3.30 & $7.34 \mathrm{E}-04$ \\
\hline 7436 & PRDX4 & -6.43 & $9.46 \mathrm{E}-05$ \\
\hline 7437 & PRDX6 & $\begin{array}{l}-7.19 \\
\end{array}$ & $8.51 \mathrm{E}-05$ \\
\hline 7438 & PRELID1 & -7.19 & $1.22 \mathrm{E}-04$ \\
\hline 7439 & PREP & -3.53 & $3.82 \mathrm{E}-03$ \\
\hline 7440 & PRIM2 & -1.65 & $4.38 \mathrm{E}-03$ \\
\hline 7441 & PRKAA1 & -1.51 & $1.06 \mathrm{E}-04$ \\
\hline 7442 & PRKAB1 & -3.72 & $2.58 \mathrm{E}-03$ \\
\hline 7443 & PRKAB2 & -4.33 & $1.98 \mathrm{E}-04$ \\
\hline 7444 & PRKACB & -2.00 & $5.00 \mathrm{E}-03$ \\
\hline 7445 & PRKAG2 & -4.01 & $2.51 \mathrm{E}-04$ \\
\hline 7446 & $\begin{array}{l}\text { PRKAR1A } \\
\end{array}$ & -5.30 & $1.50 \mathrm{E}-04$ \\
\hline 7447 & PRKAR1B & -2.25 & $6.85 \mathrm{E}-03$ \\
\hline 7448 & PRKCD & -2.90 & $6.05 \mathrm{E}-04$ \\
\hline 7449 & PRKCZ & -2.20 & $5.59 \mathrm{E}-03$ \\
\hline 7450 & PRKRIP1 & -3.60 & $1.66 \mathrm{E}-04$ \\
\hline 7451 & PRKRIR & -1.38 & $5.39 \mathrm{E}-02$ \\
\hline 7452 & $\begin{array}{l}\text { PRLR } \\
\end{array}$ & -1.94 & $1.25 \mathrm{E}-02$ \\
\hline 7453 & PRMT10 & -4.36 & $9.93 \mathrm{E}-04$ \\
\hline 7454 & PRMT3 & -1.93 & $9.50 \mathrm{E}-04$ \\
\hline 7455 & PRMT7 & -4.69 & $7.93 \mathrm{E}-04$ \\
\hline 7456 & $\begin{array}{l}\text { PRMT8 } \\
\end{array}$ & -1.11 & $8.92 \mathrm{E}-03$ \\
\hline 7457 & PROSC & -2.82 & $8.06 \mathrm{E}-04$ \\
\hline 7458 & PROSER1 & -2.13 & $2.85 \mathrm{E}-03$ \\
\hline 7459 & PROSER2 & -2.83 & $2.43 \mathrm{E}-03$ \\
\hline $\begin{array}{l}7460 \\
\end{array}$ & PRPF3 & \begin{tabular}{|l|}
-3.67 \\
\end{tabular} & $1.64 \mathrm{E}-03$ \\
\hline 7461 & PRPF38A & -4.12 & $1.93 \mathrm{E}-04$ \\
\hline 7462 & PRPF38B & -1.77 & $2.48 \mathrm{E}-03$ \\
\hline 7463 & PRPF4 & -2.15 & $1.25 \mathrm{E}-03$ \\
\hline 7464 & PRPF40A & -7.07 & $9.21 \mathrm{E}-05$ \\
\hline 7465 & PRPF4B & -2.35 & $9.08 \mathrm{E}-03$ \\
\hline 7466 & PRPF8 & -3.30 & $6.69 \mathrm{E}-04$ \\
\hline 7467 & PRPS1 & -3.04 & $3.82 \mathrm{E}-05$ \\
\hline $\begin{array}{l}7468 \\
\end{array}$ & PRPSAP1 & -1.61 & $1.07 \mathrm{E}-02$ \\
\hline 7469 & PRPSAP2 & -5.29 & $1.40 \mathrm{E}-04$ \\
\hline 7470 & PRR11 & -4.54 & $2.53 \mathrm{E}-04$ \\
\hline 7471 & PRRC1 & -2.55 & $2.18 \mathrm{E}-03$ \\
\hline 7472 & PRSS12 & -4.01 & $1.20 \mathrm{E}-03$ \\
\hline 7473 & PRSS55 & -5.90 & $1.25 \mathrm{E}-03$ \\
\hline 7474 & PSAP & -6.88 & $1.28 \mathrm{E}-04$ \\
\hline 7475 & PSAT1 & -1.33 & $3.42 \mathrm{E}-03$ \\
\hline 7476 & PSD3 & -2.95 & $5.25 \mathrm{E}-03$ \\
\hline 7477 & PSEN1 & -2.92 & $3.80 \mathrm{E}-04$ \\
\hline 7478 & PSIP1 & -6.33 & $2.33 \mathrm{E}-04$ \\
\hline 7479 & PSMA1 & -7.31 & $8.66 \mathrm{E}-04$ \\
\hline 7480 & PSMA2 & -5.29 & $2.48 \mathrm{E}-04$ \\
\hline 7481 & PSMA3 & -7.27 & $1.10 \mathrm{E}-04$ \\
\hline 7482 & $\begin{array}{l}\text { PSMA4 } \\
\end{array}$ & $\begin{array}{l}-7.14 \\
\end{array}$ & $7.63 \mathrm{E}-05$ \\
\hline 7483 & PSMA5 & -5.51 & $7.09 \mathrm{E}-05$ \\
\hline 7484 & PSMA6 & -6.69 & $3.46 \mathrm{E}-05$ \\
\hline 7485 & PSMA7 & -6.47 & $2.89 \mathrm{E}-04$ \\
\hline 7486 & PSMB1 & -5.80 & $3.47 \mathrm{E}-04$ \\
\hline 7487 & PSMB2 & -3.73 & $5.63 \mathrm{E}-04$ \\
\hline 7488 & PSMB3 & -4.75 & $1.09 \mathrm{E}-04$ \\
\hline 7489 & PSMB4 & -7.42 & $1.88 \mathrm{E}-05$ \\
\hline
\end{tabular}




\begin{tabular}{|c|c|c|c|}
\hline 7490 & PSMB5 & -3.62 & $4.36 \mathrm{E}-04$ \\
\hline 7491 & PSMC1 & -6.16 & $8.17 \mathrm{E}-05$ \\
\hline 7492 & PSMC2 & -7.82 & $9.50 \mathrm{E}-05$ \\
\hline 7493 & PSMC3 & -5.03 & $1.62 \mathrm{E}-04$ \\
\hline 7494 & PSMC5 & -6.71 & $8.23 \mathrm{E}-06$ \\
\hline 7495 & PSMC6 & -5.99 & $4.97 \mathrm{E}-04$ \\
\hline 7496 & PSMD1 & -3.92 & $1.36 \mathrm{E}-02$ \\
\hline 7497 & PSMD10 & -5.56 & $1.74 \mathrm{E}-04$ \\
\hline 7498 & PSMD12 & -5.98 & $5.39 \mathrm{E}-05$ \\
\hline 7499 & PSMD14 & -2.54 & $1.98 \mathrm{E}-04$ \\
\hline 7500 & PSMD2 & -1.64 & $1.97 \mathrm{E}-03$ \\
\hline 7501 & PSMD3 & -5.23 & $6.74 E-06$ \\
\hline 7502 & PSMD4 & -2.97 & $1.89 \mathrm{E}-03$ \\
\hline 7503 & PSMD5 & -1.95 & $2.14 \mathrm{E}-02$ \\
\hline 7504 & PSMD6 & -4.62 & $1.20 \mathrm{E}-03$ \\
\hline 7505 & PSMD7 & \begin{tabular}{|l|} 
\\
\end{tabular} & $1.01 \mathrm{E}-04$ \\
\hline 7506 & PSMD9 & -2.64 & $9.25 \mathrm{E}-05$ \\
\hline 7507 & PSME3 & -5.67 & $3.56 \mathrm{E}-04$ \\
\hline 7508 & PSME4 & -4.30 & $6.48 \mathrm{E}-04$ \\
\hline 7509 & PSMF1 & -5.03 & $5.97 \mathrm{E}-06$ \\
\hline 7510 & PSMG1 & -6.10 & $1.08 \mathrm{E}-04$ \\
\hline 7511 & $\begin{array}{l}\text { PSMG2 } \\
\end{array}$ & $\begin{array}{l}-6.67 \\
\end{array}$ & $3.61 \mathrm{E}-04$ \\
\hline 7512 & PSMG3 & -3.26 & $4.54 \mathrm{E}-04$ \\
\hline 7513 & PSPC1 & -5.30 & $3.48 \mathrm{E}-04$ \\
\hline 7514 & PSPH & -5.73 & $5.40 \mathrm{E}-05$ \\
\hline 7515 & PTBP1 & -2.72 & $3.33 \mathrm{E}-04$ \\
\hline 7516 & PTCD1 & -2.74 & $5.74 \mathrm{E}-04$ \\
\hline 7517 & PTCD2 & -4.51 & $1.94 \mathrm{E}-03$ \\
\hline 7518 & PTDSS1 & -2.71 & $3.01 \mathrm{E}-03$ \\
\hline 7519 & PTEN & -6.39 & $3.79 E-04$ \\
\hline 7520 & PTER & -1.35 & $1.87 \mathrm{E}-02$ \\
\hline 7521 & PTGDS & -2.50 & $3.64 \mathrm{E}-02$ \\
\hline 7522 & PTGES2 & -1.97 & $1.23 \mathrm{E}-03$ \\
\hline 7523 & PTGR1 & -4.80 & $3.76 \mathrm{E}-04$ \\
\hline 7524 & PTK7 & -2.14 & $2.62 E-04$ \\
\hline 7525 & PTMA & -4.75 & $8.12 \mathrm{E}-03$ \\
\hline 7526 & PTMS & -1.65 & 5.63E-04 \\
\hline 7527 & PTN & -3.61 & $1.88 \mathrm{E}-03$ \\
\hline 7528 & PTP4A1 & -3.34 & $1.69 \mathrm{E}-03$ \\
\hline 7529 & PTP4A2 & -4.79 & $7.80 \mathrm{E}-03$ \\
\hline 7530 & PTP4A3 & -2.01 & $5.98 \mathrm{E}-03$ \\
\hline 7531 & $\begin{array}{l}\text { PTPDC1 } \\
\end{array}$ & \begin{tabular}{|l|}
-3.41 \\
\end{tabular} & $1.37 \mathrm{E}-04$ \\
\hline 7532 & $\begin{array}{l}\text { PTPLA } \\
\end{array}$ & \begin{tabular}{|l|}
-1.83 \\
\end{tabular} & $2.14 \mathrm{E}-03$ \\
\hline 7533 & PTPLAD1 & -5.26 & $1.02 \mathrm{E}-04$ \\
\hline 7534 & PTPLB & -4.11 & $5.97 \mathrm{E}-04$ \\
\hline 7535 & PTPN11 & -3.62 & $9.47 \mathrm{E}-04$ \\
\hline 7536 & PTPN2 & -3.32 & $2.30 \mathrm{E}-04$ \\
\hline 7537 & PTPN4 & -2.16 & $6.82 \mathrm{E}-03$ \\
\hline 7538 & PTPN5 & -5.54 & $1.49 \mathrm{E}-03$ \\
\hline 7539 & PTPRG & -1.26 & $1.98 \mathrm{E}-02$ \\
\hline 7540 & PTPRT & -1.15 & $7.69 \mathrm{E}-03$ \\
\hline 7541 & PTRH2 & -5.50 & $2.32 \mathrm{E}-04$ \\
\hline 7542 & PTRHD1 & -3.80 & $3.51 \mathrm{E}-03$ \\
\hline 7543 & PTS & -2.88 & $6.69 \mathrm{E}-04$ \\
\hline 7544 & PTTG1 & -8.78 & $9.28 \mathrm{E}-05$ \\
\hline 7545 & PTTG1IP & -1.76 & $5.65 E-03$ \\
\hline 7546 & PUF60 & -5.90 & $2.08 \mathrm{E}-04$ \\
\hline 7547 & PUM1 & -3.23 & $7.32 \mathrm{E}-04$ \\
\hline 7548 & PUM2 & -4.12 & $2.08 \mathrm{E}-03$ \\
\hline 7549 & PURG & -2.39 & 7.49E-04 \\
\hline 7550 & PUS1 & -2.71 & $2.47 \mathrm{E}-03$ \\
\hline 7551 & PUS10 & -3.03 & $5.98 \mathrm{E}-04$ \\
\hline 7552 & PUS & -3.90 & . \\
\hline
\end{tabular}

\begin{tabular}{|c|c|c|c|}
\hline 7553 & PUS7L & -2.75 & $2.09 \mathrm{E}-03$ \\
\hline 7554 & PVRL3 & -5.44 & $1.15 \mathrm{E}-04$ \\
\hline 7555 & PWP1 & -3.09 & $1.69 \mathrm{E}-04$ \\
\hline 7556 & PWP2 & -1.15 & $7.62 E-03$ \\
\hline 7557 & PXK & -3.03 & $5.91 \mathrm{E}-04$ \\
\hline 7558 & PXMP4 & -2.33 & $6.38 \mathrm{E}-02$ \\
\hline 7559 & PYCRL & -2.07 & $1.72 \mathrm{E}-03$ \\
\hline 7560 & PYROXD1 & -3.72 & $4.10 \mathrm{E}-03$ \\
\hline 7561 & QARS & -3.26 & $3.46 \mathrm{E}-04$ \\
\hline 7562 & QDPR & -4.79 & $5.97 \mathrm{E}-04$ \\
\hline 7563 & QKI & -2.48 & $2.51 \mathrm{E}-03$ \\
\hline 7564 & QPCT & -2.12 & $3.75 \mathrm{E}-03$ \\
\hline 7565 & QRICH1 & -1.69 & $1.57 \mathrm{E}-03$ \\
\hline 7566 & QRSL1 & -3.84 & $1.05 \mathrm{E}-03$ \\
\hline 7567 & QSER1 & -1.98 & $1.64 \mathrm{E}-03$ \\
\hline 7568 & QSoX2 & -2.59 & $6.78 \mathrm{E}-07$ \\
\hline 7569 & QTRTD1 & -2.24 & $8.40 \mathrm{E}-03$ \\
\hline 7570 & R3HCC1 & -3.66 & $6.02 \mathrm{E}-04$ \\
\hline 7571 & R3HDM1 & -3.36 & $7.56 \mathrm{E}-04$ \\
\hline 7572 & R3HDM2 & -3.09 & $7.96 \mathrm{E}-05$ \\
\hline 7573 & RAB10 & -2.16 & $1.12 \mathrm{E}-02$ \\
\hline 7574 & RAB11A & -5.79 & $1.64 \mathrm{E}-04$ \\
\hline 7575 & RAB11B & -2.99 & $5.03 \mathrm{E}-03$ \\
\hline 7576 & RAB11FIP1 & -1.39 & $1.72 \mathrm{E}-02$ \\
\hline 7577 & RAB11FIP2 & -2.03 & $2.14 \mathrm{E}-03$ \\
\hline 7578 & RAB11FIP4 & -6.26 & $2.82 \mathrm{E}-04$ \\
\hline 7579 & RAB11FIP5 & -3.21 & $7.09 \mathrm{E}-04$ \\
\hline 7580 & RAB12 & -1.82 & $2.90 \mathrm{E}-03$ \\
\hline 7581 & RAB14 & -1.64 & $9.03 \mathrm{E}-04$ \\
\hline 7582 & RAB17 & -1.38 & $3.08 \mathrm{E}-03$ \\
\hline 7583 & RAB18 & -4.47 & $2.56 \mathrm{E}-04$ \\
\hline 7584 & RAB1A & -5.89 & $1.07 E-04$ \\
\hline 7585 & RAB21 & -2.36 & $6.06 \mathrm{E}-05$ \\
\hline 7586 & RAB22A & -1.46 & $2.81 \mathrm{E}-02$ \\
\hline 7587 & RAB23 & -3.39 & $3.33 \mathrm{E}-04$ \\
\hline 7588 & RAB24 & -4.02 & $7.40 \mathrm{E}-04$ \\
\hline 7589 & RAB28 & -3.48 & $1.58 \mathrm{E}-03$ \\
\hline 7590 & RAB2A & -4.25 & $8.66 \mathrm{E}-05$ \\
\hline 7591 & RAB33B & -2.09 & $5.38 \mathrm{E}-03$ \\
\hline 7592 & RAB35 & -2.63 & $1.14 \mathrm{E}-03$ \\
\hline 7593 & RAB36 & -3.76 & $3.90 \mathrm{E}-03$ \\
\hline 7594 & RAB3B & -1.83 & $9.80 \mathrm{E}-03$ \\
\hline 7595 & RAB3GAP1 & -2.49 & $5.72 \mathrm{E}-03$ \\
\hline 7596 & RAB3GAP2 & -1.72 & $1.91 \mathrm{E}-02$ \\
\hline 7597 & RAB3IP & -3.64 & $7.17 \mathrm{E}-04$ \\
\hline 7598 & RAB40B & -3.59 & $8.72 \mathrm{E}-04$ \\
\hline 7599 & RAB4A & -1.70 & $1.41 \mathrm{E}-02$ \\
\hline 7600 & RAB5A & -2.81 & $1.25 \mathrm{E}-02$ \\
\hline 7601 & RAB5C & -2.24 & $3.30 \mathrm{E}-02$ \\
\hline 7602 & RAB6A & -3.44 & $3.95 \mathrm{E}-03$ \\
\hline 7603 & RAB7A & -4.89 & $1.41 \mathrm{E}-04$ \\
\hline 7604 & RAB8A & -4.29 & $9.31 \mathrm{E}-04$ \\
\hline 7605 & RAB $8 B$ & -5.94 & $1.86 \mathrm{E}-04$ \\
\hline 7606 & RAB9A & -4.94 & $1.45 \mathrm{E}-03$ \\
\hline 7607 & RABEP1 & -4.50 & $6.82 \mathrm{E}-0 \mathrm{0c}$ \\
\hline 7608 & RABEPK & -5.80 & $1.16 \mathrm{E}-04$ \\
\hline 7609 & RABGAP1 & -2.21 & $9.49 \mathrm{E}-03$ \\
\hline 7610 & RABGAP1L & -1.19 & $4.36 \mathrm{E}-03$ \\
\hline 7611 & RABGEF1 & -2.32 & $4.40 \mathrm{E}-03$ \\
\hline 7612 & RABGGTB & -3.07 & $2.09 \mathrm{E}-04$ \\
\hline 7613 & RABL2B & $\begin{array}{l}-1.67 \\
\end{array}$ & $1.79 \mathrm{E}-02$ \\
\hline 7614 & RABL3 & -1.86 & $2.67 \mathrm{E}-02$ \\
\hline 7615 & RABL5 & -6.15 & $1.30 \mathrm{E}-06$ \\
\hline
\end{tabular}

\begin{tabular}{|c|c|c|c|}
\hline 7616 & RAC1 & -6.28 & $9.92 E-04$ \\
\hline 7617 & RAC3 & -2.59 & $2.69 \mathrm{E}-03$ \\
\hline 7618 & RACGAP1 & -7.69 & $1.57 E-04$ \\
\hline 7619 & RAD1 & -4.15 & $2.75 E-03$ \\
\hline 7620 & RAD17 & -3.41 & $1.04 E-03$ \\
\hline 7621 & RAD18 & -5.21 & $\begin{array}{l}4.06 E-04 \\
.\end{array}$ \\
\hline 7622 & RAD21 & -4.52 & $2.92 \mathrm{E}-04$ \\
\hline 7623 & RAD23B & -1.87 & $2.62 E-03$ \\
\hline 7624 & RAD50 & -1.78 & $3.20 \mathrm{E}-03$ \\
\hline 7625 & RAD51 & -5.96 & $1.42 \mathrm{E}-04$ \\
\hline 7626 & RAD51C & \begin{tabular}{|l|}
-3.14 \\
\end{tabular} & $1.69 E-03$ \\
\hline 7627 & RAD51D & -2.88 & $6.45 E-05$ \\
\hline 7628 & RAD52 & -3.72 & $7.33 E-05$ \\
\hline 7629 & RAD54B & -2.83 & $1.37 E-03$ \\
\hline 7630 & RAD54L2 & -3.42 & $1.84 E-05$ \\
\hline 7631 & RAE1 & -2.02 & $1.73 E-03$ \\
\hline 7632 & RAGE & -4.28 & $1.88 \mathrm{E}-03$ \\
\hline 7633 & RAI2 & -3.48 & $3.77 E-03$ \\
\hline 7634 & RALA & -6.92 & $3.55 E-04$ \\
\hline 7635 & RALB & -5.64 & $8.19 E-04$ \\
\hline 7636 & RALBP1 & -5.23 & $2.08 E-04$ \\
\hline 7637 & RALGAPA1 & -1.21 & $1.46 \mathrm{E}-02$ \\
\hline 7638 & RALGAPA2 & -1.05 & $5.82 E-03$ \\
\hline 7639 & RALGAPB & -2.59 & $3.05 E-03$ \\
\hline 7640 & RALGPS2 & -4.63 & $6.06 \mathrm{E}-04$ \\
\hline 7641 & RAN & \begin{tabular}{|l|} 
\\
\end{tabular} & $3.24 E-05$ \\
\hline 7642 & RANBP1 & -6.11 & $1.01 E-04$ \\
\hline 7643 & RANBP10 & -2.36 & $1.74 E-03$ \\
\hline 7644 & RANBP2 & -4.43 & $1.58 \mathrm{E}-03$ \\
\hline 7645 & RANBP3 & -2.11 & $3.09 E-03$ \\
\hline 7646 & RANBP9 & -4.93 & $1.80 \mathrm{E}-03$ \\
\hline 7647 & RANGAP1 & -3.84 & $3.49 E-03$ \\
\hline 7648 & RAP1A & -2.46 & $1.10 \mathrm{E}-02$ \\
\hline 7649 & RAP1B & -2.24 & $4.19 E-03$ \\
\hline 7650 & RAP1GAP & -2.69 & $4.87 \mathrm{E}-04$ \\
\hline 7651 & RAP1GDS1 & -4.05 & $2.11 \mathrm{E}-04$ \\
\hline 7652 & RAP2A & -3.11 & $7.84 \mathrm{E}-03$ \\
\hline 7653 & RARRES1 & -4.43 & 1.19E-04 \\
\hline 7654 & RARS & -4.26 & $9.17 E-04$ \\
\hline 7655 & RARS2 & -3.97 & $1.92 E-03$ \\
\hline 7656 & RASA1 & -3.52 & 2.42E-04 \\
\hline 7657 & RASA2 & \begin{tabular}{|l|}
-1.07 \\
\end{tabular} & $3.33 \mathrm{E}-03$ \\
\hline 7658 & RASA3 & -3.98 & $7.80 \mathrm{E}-04$ \\
\hline 7659 & RASAL2 & -4.90 & $1.99 \mathrm{E}-04$ \\
\hline 7660 & RASD1 & -1.27 & $1.46 \mathrm{E}-01$ \\
\hline 7661 & RASGEF1A & -3.91 & $3.21 \mathrm{E}-03$ \\
\hline 7662 & RASSF1 & -2.51 & $9.78 E-03$ \\
\hline 7663 & RASSF3 & \begin{tabular}{|l|}
-3.18 \\
\end{tabular} & $1.66 \mathrm{E}-03$ \\
\hline 7664 & RASSF8 & -3.48 & $8.90 E-04$ \\
\hline 7665 & RB1 & -1.66 & $5.86 \mathrm{E}-02$ \\
\hline 7666 & RB1CC1 & -2.38 & $8.84 \mathrm{E}-04$ \\
\hline 7667 & RBBP4 & -5.17 & $3.18 \mathrm{E}-04$ \\
\hline 7668 & RBBP5 & -2.65 & $2.00 E-03$ \\
\hline 7669 & RBBP6 & -4.09 & $1.46 E-03$ \\
\hline 7670 & RBBP7 & -5.08 & $3.09 E-03$ \\
\hline 7671 & RBBP8 & -2.88 & $1.90 \mathrm{E}-03$ \\
\hline 7672 & RBFA & -2.53 & $3.30 \mathrm{E}-03$ \\
\hline 7673 & RBFOX2 & -2.72 & $8.64 E-03$ \\
\hline 7674 & RBKS & -1.49 & $2.28 \mathrm{E}-02$ \\
\hline 7675 & RBM12 & -2.31 & $9.36 \mathrm{E}-03$ \\
\hline 7676 & RBM12B & -3.80 & $6.26 \mathrm{E}-04$ \\
\hline 7677 & RBM14 & -1.86 & $7.56 \mathrm{E}-03$ \\
\hline 678 & RBM1 & -5.05 & 3.94E-04 \\
\hline
\end{tabular}




\begin{tabular}{|c|c|c|c|}
\hline 7679 & RBM18 & -5.37 & $2.68 \mathrm{E}-04$ \\
\hline 7680 & RBM19 & -3.40 & $9.32 \mathrm{E}-05$ \\
\hline 7681 & RBM22 & -5.37 & 3.03E-04 \\
\hline 7682 & RBM25 & -3.95 & $1.07 E-03$ \\
\hline 7683 & RBM26 & -2.34 & $1.09 \mathrm{E}-02$ \\
\hline 7684 & RBM39 & -2.19 & $1.50 \mathrm{E}-02$ \\
\hline 7685 & RBM43 & -3.13 & $1.79 \mathrm{E}-04$ \\
\hline 7686 & RBM45 & -1.98 & $3.71 \mathrm{E}-03$ \\
\hline 7687 & RBM46 & -6.53 & $1.52 \mathrm{E}-04$ \\
\hline 7688 & RBM48 & -3.92 & 4.39E-04 \\
\hline 7689 & RBM5 & -5.15 & 2.54E-04 \\
\hline 7690 & RBM6 & -2.77 & $2.18 \mathrm{E}-02$ \\
\hline 7691 & RBM7 & -4.15 & $2.11 \mathrm{E}-03$ \\
\hline 7692 & RBMX & -3.40 & $1.07 \mathrm{E}-03$ \\
\hline 7693 & RBP5 & -5.27 & $1.25 \mathrm{E}-03$ \\
\hline 7694 & RBX1 & -6.89 & $1.74 \mathrm{E}-04$ \\
\hline 7695 & $\mathrm{RC} 3 \mathrm{H} 2$ & -1.23 & $1.38 \mathrm{E}-02$ \\
\hline 7696 & RCAN3 & -3.82 & $3.70 \mathrm{E}-03$ \\
\hline 7697 & RCBTB1 & -4.05 & $2.22 \mathrm{E}-05$ \\
\hline 7698 & RCCD1 & -2.67 & $1.90 \mathrm{E}-03$ \\
\hline 7699 & RCHY1 & -3.89 & 4.67E-03 \\
\hline 7700 & RCL1 & -5.27 & $8.84 \mathrm{E}-04$ \\
\hline 7701 & RCN2 & -4.24 & $1.97 \mathrm{E}-03$ \\
\hline 7702 & RCOR1 & -2.38 & $6.01 \mathrm{E}-04$ \\
\hline 7703 & RD3 & -2.55 & $6.51 \mathrm{E}-03$ \\
\hline 7704 & RDH12 & -2.96 & $1.66 \mathrm{E}-03$ \\
\hline 7705 & RDH14 & -2.59 & $8.75 \mathrm{E}-03$ \\
\hline 7706 & RDX & -3.62 & 6.97E-04 \\
\hline 7707 & RECQL & -3.33 & $2.18 \mathrm{E}-04$ \\
\hline 7708 & REEP5 & -3.65 & 2.17E-03 \\
\hline 7709 & REEP6 & -4.78 & $1.51 \mathrm{E}-03$ \\
\hline 7710 & RELL1 & -1.80 & $1.85 \mathrm{E}-02$ \\
\hline 7711 & REPS1 & -1.39 & $5.32 \mathrm{E}-02$ \\
\hline 7712 & RER1 & -3.46 & $6.40 \mathrm{E}-03$ \\
\hline 7713 & RERE & -1.92 & $8.66 \mathrm{E}-02$ \\
\hline 7714 & RERG & -3.66 & 2.57E-03 \\
\hline 7715 & REST & -4.54 & $4.75 E-05$ \\
\hline 7716 & REV1 & -4.33 & 7.96E-04 \\
\hline 7717 & REXO1 & -5.45 & 4.57E-05 \\
\hline 7718 & REXO2 & -6.45 & $6.20 \mathrm{E}-05$ \\
\hline 7719 & REXO4 & -2.32 & $1.27 \mathrm{E}-02$ \\
\hline 7720 & RFC1 & -5.91 & 5.07E-04 \\
\hline 7721 & RFC2 & -2.73 & $5.88 \mathrm{E}-03$ \\
\hline 7722 & RFC3 & -5.95 & $2.24 \mathrm{E}-03$ \\
\hline 7723 & RFC4 & -5.33 & $2.06 \mathrm{E}-04$ \\
\hline 7724 & RFFL & -2.37 & $2.70 \mathrm{E}-04$ \\
\hline 7725 & RFK & -3.65 & 3.68E-05 \\
\hline 7726 & RFNG & -3.36 & 2.37E-04 \\
\hline 7727 & RFT1 & -3.79 & $1.48 \mathrm{E}-03$ \\
\hline 7728 & RFTN1 & -4.22 & 5.04E-04 \\
\hline 7729 & RFX3 & -5.14 & $1.49 \mathrm{E}-04$ \\
\hline 7730 & RFX6 & -3.16 & $6.12 \mathrm{E}-04$ \\
\hline 7731 & RFXANK & -2.83 & $1.14 \mathrm{E}-03$ \\
\hline 7732 & RG9MTD1 & -3.05 & $5.53 \mathrm{E}-03$ \\
\hline 7733 & RG9MTD2 & -7.16 & 3.99E-04 \\
\hline 7734 & RGL1 & -4.76 & $3.98 \mathrm{E}-04$ \\
\hline 7735 & RGN & -3.70 & $1.86 \mathrm{E}-03$ \\
\hline 7736 & RGS10 & -2.73 & $1.26 \mathrm{E}-03$ \\
\hline 7737 & RGS12 & -1.49 & $3.81 \mathrm{E}-03$ \\
\hline 7738 & RGS2 & -5.26 & $1.65 \mathrm{E}-04$ \\
\hline 7739 & RHEB & -2.53 & $2.34 \mathrm{E}-03$ \\
\hline 7740 & RHOA & -4.73 & $5.50 \mathrm{E}-04$ \\
\hline 7741 & RHOB & -3.31 & $2.98 \mathrm{E}-03$ \\
\hline
\end{tabular}

\begin{tabular}{|c|c|c|c|}
\hline 7742 & RHOQ & -1.57 & $7.20 \mathrm{E}-04$ \\
\hline 7743 & RHOT1 & -3.98 & 7.73E-04 \\
\hline 7744 & RHOT2 & -1.48 & $4.70 \mathrm{E}-03$ \\
\hline 7745 & RHPN1 & -2.02 & $2.61 \mathrm{E}-03$ \\
\hline 7746 & RIBC2 & -7.08 & $1.60 \mathrm{E}-04$ \\
\hline 7747 & RIC3 & -8.43 & $3.21 \mathrm{E}-04$ \\
\hline 7748 & RIC8B & -4.80 & $9.43 \mathrm{E}-05$ \\
\hline 7749 & RICTOR & -2.76 & $3.55 \mathrm{E}-03$ \\
\hline 7750 & RIF1 & -3.05 & $1.94 \mathrm{E}-04$ \\
\hline 7751 & RILPL1 & -2.25 & $3.41 E-02$ \\
\hline 7752 & RILPL2 & -4.72 & $2.91 \mathrm{E}-03$ \\
\hline 7753 & RIN2 & -1.61 & $1.69 \mathrm{E}-04$ \\
\hline 7754 & RIOK2 & -4.65 & $2.33 \mathrm{E}-04$ \\
\hline 7755 & RIOK3 & -2.97 & $2.50 \mathrm{E}-03$ \\
\hline 7756 & RIT1 & -1.68 & $1.00 \mathrm{E}-02$ \\
\hline 7757 & RLBP1 & -1.98 & 4.43E-03 \\
\hline 7758 & RLF & -2.59 & $3.21 \mathrm{E}-04$ \\
\hline 7759 & RLIM & -2.77 & $1.73 \mathrm{E}-03$ \\
\hline 7760 & RMI1 & -5.97 & $6.69 \mathrm{E}-04$ \\
\hline 7761 & RMND1 & -5.70 & $3.99 \mathrm{E}-04$ \\
\hline 7762 & RMND5A & -2.81 & $3.35 \mathrm{E}-03$ \\
\hline 7763 & RNASEH1 & -4.05 & 7.86E-04 \\
\hline 7764 & RNASEH2B & -1.79 & 9.87E-04 \\
\hline 7765 & RND3 & -1.74 & $1.01 \mathrm{E}-03$ \\
\hline 7766 & RNF10 & -2.73 & $5.78 \mathrm{E}-05$ \\
\hline 7767 & RNF103 & -5.08 & $8.83 \mathrm{E}-04$ \\
\hline 7768 & RNF111 & -1.79 & 1.99E-04 \\
\hline 7769 & RNF113A & -5.55 & $1.09 \mathrm{E}-04$ \\
\hline 7770 & RNF114 & -4.78 & $5.23 \mathrm{E}-05$ \\
\hline 7771 & RNF13 & -6.16 & 4.00E-04 \\
\hline 7772 & RNF130 & -5.69 & 5.87E-04 \\
\hline 7773 & RNF139 & -5.82 & $4.35 \mathrm{E}-05$ \\
\hline 7774 & RNF14 & -4.28 & $1.94 \mathrm{E}-04$ \\
\hline 7775 & RNF141 & -3.65 & $4.70 \mathrm{E}-03$ \\
\hline 7776 & RNF144A & -2.92 & $3.18 \mathrm{E}-03$ \\
\hline 7777 & RNF149 & -4.53 & $1.28 \mathrm{E}-04$ \\
\hline 7778 & RNF151 & -3.10 & $2.33 \mathrm{E}-03$ \\
\hline 7779 & RNF152 & -3.76 & $3.26 \mathrm{E}-04$ \\
\hline 7780 & RNF166 & -2.47 & $1.03 \mathrm{E}-02$ \\
\hline 7781 & RNF169 & -1.48 & 1.17E-02 \\
\hline 7782 & RNF170 & -1.38 & $1.82 \mathrm{E}-02$ \\
\hline 7783 & RNF185 & -1.82 & $6.92 \mathrm{E}-02$ \\
\hline 7784 & RNF20 & -5.76 & $7.34 \mathrm{E}-05$ \\
\hline 7785 & RNF214 & -3.44 & 4.77E-03 \\
\hline 7786 & RNF216 & -6.01 & $3.20 \mathrm{E}-03$ \\
\hline 7787 & RNF217 & -5.11 & $3.54 \mathrm{E}-04$ \\
\hline 7788 & RNF219 & -1.81 & $1.28 \mathrm{E}-02$ \\
\hline 7789 & RNF32 & -1.89 & $1.13 \mathrm{E}-02$ \\
\hline 7790 & RNF34 & -1.66 & $3.46 \mathrm{E}-02$ \\
\hline 7791 & RNF38 & -1.68 & $4.13 \mathrm{E}-02$ \\
\hline 7792 & RNF4 & -4.36 & $5.56 \mathrm{E}-03$ \\
\hline 7793 & RNF41 & -3.43 & $2.34 \mathrm{E}-03$ \\
\hline 7794 & RNF7 & -1.91 & $6.33 \mathrm{E}-03$ \\
\hline 7795 & RNF8 & -1.55 & $9.80 \mathrm{E}-03$ \\
\hline 7796 & RNFT1 & -2.92 & $1.10 \mathrm{E}-03$ \\
\hline 7797 & RNH1 & -4.84 & $3.61 \mathrm{E}-03$ \\
\hline 7798 & RNLS & -2.88 & $4.51 \mathrm{E}-03$ \\
\hline 7799 & RNMT & -2.94 & $1.67 \mathrm{E}-03$ \\
\hline 7800 & RNMTL1 & -1.94 & $1.90 \mathrm{E}-03$ \\
\hline 7801 & RNPC3 & -1.98 & $8.19 \mathrm{E}-03$ \\
\hline 7802 & RNPEPL1 & -1.33 & $2.70 \mathrm{E}-03$ \\
\hline 7803 & RNPS1 & -1.15 & $1.79 \mathrm{E}-03$ \\
\hline 7804 & ROCK1 & -3.05 & 4.40E-05 \\
\hline
\end{tabular}

\begin{tabular}{|c|c|c|c|}
\hline 7805 & ROCK2 & -2.33 & $2.30 \mathrm{E}-03$ \\
\hline 7806 & ROGDI & -2.74 & $6.94 \mathrm{E}-03$ \\
\hline 7807 & ROMO1 & -6.38 & $4.22 \mathrm{E}-04$ \\
\hline 7808 & ROPN1L & -8.56 & $6.72 \mathrm{E}-04$ \\
\hline 7809 & RORA & -2.51 & $2.62 \mathrm{E}-03$ \\
\hline 7810 & RP2 & -4.14 & $1.23 \mathrm{E}-03$ \\
\hline 7811 & RP9 & -2.24 & $5.18 \mathrm{E}-02$ \\
\hline 7812 & RPA1 & -4.75 & $2.05 \mathrm{E}-04$ \\
\hline 7813 & RPA2 & -4.34 & $8.54 \mathrm{E}-05$ \\
\hline 7814 & RPA3 & -5.99 & 4.07E-04 \\
\hline 7815 & RPAIN & -5.21 & $1.87 \mathrm{E}-03$ \\
\hline 7816 & RPAP1 & -1.59 & $2.06 \mathrm{E}-02$ \\
\hline 7817 & RPAP2 & -2.59 & $5.48 \mathrm{E}-04$ \\
\hline 7818 & RPAP3 & -5.05 & $1.57 \mathrm{E}-04$ \\
\hline 7819 & RPE & -3.78 & $1.04 \mathrm{E}-03$ \\
\hline 7820 & RPF1 & -3.77 & $3.66 \mathrm{E}-05$ \\
\hline 7821 & RPF2 & -4.64 & $1.94 \mathrm{E}-03$ \\
\hline 7822 & RPH3A & -2.82 & $8.05 \mathrm{E}-04$ \\
\hline 7823 & RPIA & -1.85 & $2.23 \mathrm{E}-03$ \\
\hline 7824 & RPL10A & -7.39 & $5.14 \mathrm{E}-04$ \\
\hline 7825 & RPL10L & -6.92 & $4.57 \mathrm{E}-03$ \\
\hline 7826 & RPL11 & -7.44 & 2.24E-04 \\
\hline 7827 & RPL12 & -8.08 & $1.53 \mathrm{E}-04$ \\
\hline 7828 & RPL13 & -8.06 & $1.89 \mathrm{E}-05$ \\
\hline 7829 & RPL14 & -8.23 & $3.10 \mathrm{E}-05$ \\
\hline 7830 & RPL15 & -7.53 & $8.39 \mathrm{E}-05$ \\
\hline 7831 & RPL18A & -7.73 & $9.22 \mathrm{E}-04$ \\
\hline 7832 & RPL19 & -7.42 & $2.59 \mathrm{E}-04$ \\
\hline 7833 & RPL21 & -7.02 & $9.09 \mathrm{E}-05$ \\
\hline 7834 & RPL22 & -4.76 & $2.39 \mathrm{E}-03$ \\
\hline 7835 & RPL23 & -7.71 & 4.96E-04 \\
\hline 7836 & RPL23A & -8.46 & $2.09 \mathrm{E}-05$ \\
\hline 7837 & RPL24 & -8.33 & 2.15E-04 \\
\hline 7838 & RPL26L1 & -8.74 & $6.22 \mathrm{E}-06$ \\
\hline 7839 & RPL27 & -7.21 & 2.27E-04 \\
\hline 7840 & RPL27A & -7.82 & $1.83 \mathrm{E}-04$ \\
\hline 7841 & RPL29 & -8.46 & $3.18 \mathrm{E}-04$ \\
\hline 7842 & RPL3 & -7.06 & $4.10 \mathrm{E}-04$ \\
\hline 7843 & RPL30 & -8.97 & $5.12 \mathrm{E}-05$ \\
\hline 7844 & RPL32 & -8.51 & $2.60 \mathrm{E}-05$ \\
\hline 7845 & RPL35 & -8.51 & 5.03E-05 \\
\hline 7846 & RPL35A & -7.83 & $4.50 \mathrm{E}-04$ \\
\hline 7847 & RPL36 & -7.61 & $7.45 \mathrm{E}-05$ \\
\hline 7848 & RPL37 & -8.80 & $3.76 \mathrm{E}-04$ \\
\hline 7849 & RPL37A & -7.91 & $7.16 \mathrm{E}-05$ \\
\hline 7850 & RPL38 & -8.58 & $3.60 \mathrm{E}-04$ \\
\hline 7851 & RPL39 & -5.05 & $6.35 \mathrm{E}-03$ \\
\hline 7852 & RPL3L & -5.37 & $1.30 \mathrm{E}-03$ \\
\hline 7853 & RPL4 & -7.97 & 3.53E-05 \\
\hline 7854 & RPL5 & -8.28 & $2.95 \mathrm{E}-05$ \\
\hline 7855 & RPL6 & -6.75 & $1.86 \mathrm{E}-04$ \\
\hline 7856 & RPL7 & -7.22 & $1.17 \mathrm{E}-04$ \\
\hline 7857 & RPL7A & -7.10 & $5.21 \mathrm{E}-05$ \\
\hline 7858 & RPL7L1 & -5.82 & $1.73 \mathrm{E}-03$ \\
\hline 7859 & RPL8 & -7.69 & $1.21 \mathrm{E}-05$ \\
\hline 7860 & RPL9 & -8.66 & $8.56 \mathrm{E}-05$ \\
\hline 7861 & RPLPO & -6.04 & $1.07 \mathrm{E}-03$ \\
\hline 7862 & RPLP1 & -8.85 & $1.46 \mathrm{E}-05$ \\
\hline 7863 & RPLP2 & -5.87 & $1.79 \mathrm{E}-04$ \\
\hline 7864 & RPN1 & -5.14 & $3.51 \mathrm{E}-04$ \\
\hline 7865 & RPN2 & -4.42 & $5.66 \mathrm{E}-04$ \\
\hline 7866 & RPP14 & -2.99 & $9.91 \mathrm{E}-05$ \\
\hline 7867 & RPP38 & -2.69 & 4.70E-03 \\
\hline
\end{tabular}




\begin{tabular}{|c|c|c|c|}
\hline 7868 & RPP40 & -3.22 & $2.19 \mathrm{E}-03$ \\
\hline 7869 & RPRD1A & -1.33 & $8.22 \mathrm{E}-04$ \\
\hline 7870 & RPRD1B & -2.48 & $3.73 \mathrm{E}-04$ \\
\hline 7871 & RPS10 & -5.07 & $1.34 \mathrm{E}-04$ \\
\hline 7872 & RPS11 & -7.63 & $2.11 \mathrm{E}-05$ \\
\hline 7873 & RPS12 & -8.03 & $3.06 \mathrm{E}-05$ \\
\hline 7874 & RPS13 & -6.43 & 4.45E-04 \\
\hline 7875 & RPS14 & -7.43 & 3.03E-05 \\
\hline 7876 & RPS15 & -7.29 & $5.11 \mathrm{E}-04$ \\
\hline 7877 & RPS15A & -7.69 & $1.21 \mathrm{E}-04$ \\
\hline 7878 & RPS16 & -7.94 & $1.68 \mathrm{E}-04$ \\
\hline 7879 & RPS17L & -8.14 & $4.06 \mathrm{E}-05$ \\
\hline 7880 & RPS19BP1 & -3.92 & $1.33 \mathrm{E}-04$ \\
\hline 7881 & RPS20 & -8.44 & $1.12 \mathrm{E}-04$ \\
\hline 7882 & RPS21 & -8.87 & $6.75 \mathrm{E}-04$ \\
\hline 7883 & RPS23 & -7.79 & $1.65 \mathrm{E}-05$ \\
\hline 7884 & RPS24 & -7.76 & $1.12 \mathrm{E}-04$ \\
\hline 7885 & RPS25 & -7.66 & $1.45 \mathrm{E}-04$ \\
\hline 7886 & RPS26 & -5.36 & $2.21 \mathrm{E}-02$ \\
\hline 7887 & RPS27A & -8.32 & $1.43 \mathrm{E}-04$ \\
\hline 7888 & RPS27L & -6.76 & $9.35 \mathrm{E}-05$ \\
\hline 7889 & RPS28 & -5.49 & $1.68 \mathrm{E}-04$ \\
\hline 7890 & RPS29 & -7.31 & $1.29 \mathrm{E}-03$ \\
\hline 7891 & RPS3 & -7.71 & $9.65 \mathrm{E}-05$ \\
\hline 7892 & RPS3A & -8.65 & $2.66 \mathrm{E}-05$ \\
\hline 7893 & RPS4X & -8.25 & $5.22 \mathrm{E}-05$ \\
\hline 7894 & RPS6 & -8.19 & 4.82E-05 \\
\hline 7895 & RPS6KA2 & -2.20 & 4.77E-03 \\
\hline 7896 & RPS6KA6 & -1.41 & $6.62 \mathrm{E}-02$ \\
\hline 7897 & RPS6KC1 & -2.27 & $6.96 \mathrm{E}-03$ \\
\hline 7898 & RPS7 & -7.53 & 4.40E-04 \\
\hline 7899 & RPS8 & -8.18 & $1.06 \mathrm{E}-04$ \\
\hline 7900 & RPSA & -7.43 & $3.57 \mathrm{E}-05$ \\
\hline 7901 & RPUSD4 & -1.47 & $6.84 \mathrm{E}-02$ \\
\hline 7902 & RQCD1 & -3.86 & $2.62 \mathrm{E}-03$ \\
\hline 7903 & RRAGB & -4.00 & $1.80 \mathrm{E}-04$ \\
\hline 7904 & RRAGC & -3.55 & $3.64 \mathrm{E}-04$ \\
\hline 7905 & RRAS2 & -5.46 & $2.09 \mathrm{E}-04$ \\
\hline 7906 & RRM1 & -4.79 & $3.22 \mathrm{E}-04$ \\
\hline 7907 & RRM2 & -3.13 & $1.62 \mathrm{E}-03$ \\
\hline 7908 & RRM2B & -3.67 & $2.05 \mathrm{E}-03$ \\
\hline 7909 & RRN3 & -4.88 & $8.40 \mathrm{E}-04$ \\
\hline 7910 & RRNAD1 & -2.80 & $1.00 \mathrm{E}-03$ \\
\hline 7911 & RRP15 & -5.30 & $8.73 \mathrm{E}-05$ \\
\hline 7912 & RRP1B & -2.28 & $2.06 \mathrm{E}-01$ \\
\hline 7913 & RRP7A & -4.97 & $8.83 \mathrm{E}-04$ \\
\hline 7914 & RS1 & -1.68 & $1.88 \mathrm{E}-02$ \\
\hline 7915 & RSBN1 & -1.99 & $2.47 \mathrm{E}-03$ \\
\hline 7916 & RSBN1L & -2.76 & $3.47 \mathrm{E}-03$ \\
\hline 7917 & RSF1 & -2.76 & $5.26 \mathrm{E}-03$ \\
\hline 7918 & RSL1D1 & -3.50 & $5.90 \mathrm{E}-04$ \\
\hline 7919 & RSL24D1 & -5.06 & $3.72 \mathrm{E}-04$ \\
\hline 7920 & RSPH1 & -8.39 & $2.19 \mathrm{E}-04$ \\
\hline 7921 & RSPH10B2 & -4.42 & $8.52 \mathrm{E}-04$ \\
\hline 7922 & RSPH3 & -5.60 & $3.78 \mathrm{E}-04$ \\
\hline 7923 & RSPH4A & -4.36 & $1.80 \mathrm{E}-03$ \\
\hline 7924 & RSPH9 & -6.19 & $3.33 \mathrm{E}-05$ \\
\hline 7925 & RSPRY1 & -3.37 & $1.73 \mathrm{E}-03$ \\
\hline 7926 & RSRC1 & -5.81 & 4.47E-03 \\
\hline 7927 & RSU1 & -4.07 & $2.52 \mathrm{E}-03$ \\
\hline 7928 & RTCD1 & -3.97 & $1.25 \mathrm{E}-03$ \\
\hline 7929 & RTDR1 & -4.91 & $2.98 \mathrm{E}-03$ \\
\hline 7930 & RTF1 & -4.46 & $3.01 \mathrm{E}-05$ \\
\hline
\end{tabular}

\begin{tabular}{|c|c|c|c|}
\hline 7931 & RTFDC1 & -7.77 & $1.02 \mathrm{E}-04$ \\
\hline 7932 & RTKN2 & -1.24 & $1.97 \mathrm{E}-03$ \\
\hline 7933 & RTN4IP1 & -2.34 & $2.83 \mathrm{E}-03$ \\
\hline 7934 & RTTN & -2.34 & $2.45 \mathrm{E}-02$ \\
\hline 7935 & RUFY1 & -5.72 & $9.69 \mathrm{E}-04$ \\
\hline 7936 & RUFY2 & -2.62 & $6.38 \mathrm{E}-04$ \\
\hline 7937 & RUFY3 & -2.70 & $7.28 \mathrm{E}-03$ \\
\hline 7938 & RUNDC3B & -1.15 & $1.34 \mathrm{E}-03$ \\
\hline 7939 & RUNX1T1 & -3.19 & $1.72 \mathrm{E}-03$ \\
\hline 7940 & RUVBL1 & -5.65 & $2.42 \mathrm{E}-03$ \\
\hline 7941 & RWDD1 & -1.77 & $5.35 \mathrm{E}-04$ \\
\hline 7942 & RWDD2B & -5.04 & $1.43 \mathrm{E}-03$ \\
\hline 7943 & RWDD3 & -3.28 & $3.08 \mathrm{E}-03$ \\
\hline 7944 & RWDD4 & -4.99 & $6.34 \mathrm{E}-04$ \\
\hline 7945 & RYK & -3.62 & $4.62 \mathrm{E}-03$ \\
\hline 7946 & S100A10 & -6.18 & 4.43E-04 \\
\hline 7947 & S100A11 & -2.63 & $6.17 \mathrm{E}-03$ \\
\hline 7948 & S100B & -5.26 & $2.99 \mathrm{E}-03$ \\
\hline 7949 & SAFB2 & -5.17 & $2.82 \mathrm{E}-04$ \\
\hline 7950 & SAG & -1.08 & $6.58 \mathrm{E}-02$ \\
\hline 7951 & SAMD13 & -3.97 & $2.90 \mathrm{E}-03$ \\
\hline 7952 & SAMD15 & -5.62 & $1.64 \mathrm{E}-04$ \\
\hline 7953 & SAMHD1 & -1.58 & $6.25 \mathrm{E}-03$ \\
\hline 7954 & SAMM50 & -5.71 & $4.02 \mathrm{E}-04$ \\
\hline 7955 & SAP130 & -1.60 & $3.26 \mathrm{E}-02$ \\
\hline 7956 & SAP18 & -6.24 & $1.46 \mathrm{E}-06$ \\
\hline 7957 & SAP30 & -6.23 & 4.53E-04 \\
\hline 7958 & SAP30BP & -1.42 & $2.15 \mathrm{E}-03$ \\
\hline 7959 & SAR1B & -2.33 & $3.25 \mathrm{E}-03$ \\
\hline 7960 & SARNP & -2.83 & $1.97 \mathrm{E}-03$ \\
\hline 7961 & SARS & -1.94 & $3.83 \mathrm{E}-03$ \\
\hline 7962 & SART3 & -3.27 & $9.77 \mathrm{E}-04$ \\
\hline 7963 & SASS6 & -4.01 & $4.22 \mathrm{E}-04$ \\
\hline 7964 & SAV1 & -6.57 & $2.56 \mathrm{E}-05$ \\
\hline 7965 & SAYSD1 & -5.13 & $5.74 \mathrm{E}-04$ \\
\hline 7966 & SBDS & -3.91 & $3.44 \mathrm{E}-04$ \\
\hline 7967 & SBNO1 & -6.69 & $2.41 \mathrm{E}-04$ \\
\hline 7968 & SC4MOL & -5.26 & 4.62E-05 \\
\hline 7969 & SCAF4 & -1.75 & $2.48 \mathrm{E}-03$ \\
\hline 7970 & SCAF8 & -1.10 & $6.51 \mathrm{E}-04$ \\
\hline 7971 & SCAI & -1.61 & $1.23 \mathrm{E}-03$ \\
\hline 7972 & SCAMP1 & -6.35 & 7.87E-05 \\
\hline 7973 & SCAMP2 & -3.22 & $3.05 \mathrm{E}-03$ \\
\hline 7974 & SCAMP5 & -1.93 & $3.19 \mathrm{E}-02$ \\
\hline 7975 & SCAPER & -4.03 & $5.23 \mathrm{E}-04$ \\
\hline 7976 & SCARB2 & -4.12 & $8.26 \mathrm{E}-04$ \\
\hline 7977 & SCCPDH & -6.36 & $2.65 \mathrm{E}-05$ \\
\hline 7978 & SCD5 & -4.19 & $2.13 \mathrm{E}-03$ \\
\hline 7979 & SCFD1 & -4.87 & 4.05E-04 \\
\hline 7980 & SCHIP1 & -1.30 & $4.16 \mathrm{E}-02$ \\
\hline 7981 & SCLT1 & -6.23 & $9.45 \mathrm{E}-04$ \\
\hline 7982 & SCML2 & -2.04 & $8.96 \mathrm{E}-03$ \\
\hline 7983 & SCN1A & -2.09 & 3.97E-03 \\
\hline 7984 & $\mathrm{scoc}$ & -2.81 & 7.17E-04 \\
\hline 7985 & SCP2 & -6.51 & $4.78 \mathrm{E}-04$ \\
\hline 7986 & SCPEP1 & -4.15 & $2.40 \mathrm{E}-03$ \\
\hline 7987 & SCRN1 & -4.17 & $2.72 \mathrm{E}-03$ \\
\hline 7988 & SCRN3 & -4.56 & $5.98 \mathrm{E}-04$ \\
\hline 7989 & SCTR & -1.30 & $1.08 \mathrm{E}-02$ \\
\hline 7990 & SCX & -1.94 & $1.70 \mathrm{E}-02$ \\
\hline 7991 & SCYL2 & -4.85 & $1.18 \mathrm{E}-03$ \\
\hline 7992 & SCYL3 & -3.21 & $4.46 E-03$ \\
\hline 7993 & SDAD1 & -4.13 & $2.33 \mathrm{E}-04$ \\
\hline
\end{tabular}

\begin{tabular}{|c|c|c|c|}
\hline 7994 & SDC2 & -3.43 & 2.93E-04 \\
\hline 7995 & SDCBP & -6.35 & 4.20E-04 \\
\hline 7996 & SDCCAG3 & -2.50 & $3.67 \mathrm{E}-03$ \\
\hline 7997 & SDCCAG8 & -3.32 & 5.53E-04 \\
\hline 7998 & SDE2 & -4.89 & $6.96 \mathrm{E}-04$ \\
\hline 7999 & SDF2 & -6.74 & $3.16 \mathrm{E}-06$ \\
\hline 8000 & SDF2L1 & -2.73 & $3.99 \mathrm{E}-04$ \\
\hline 8001 & SDF4 & -4.71 & $8.31 \mathrm{E}-04$ \\
\hline 8002 & SDHA & -6.77 & $6.22 \mathrm{E}-04$ \\
\hline 8003 & SDHB & -6.25 & 1.53E-04 \\
\hline 8004 & SDHD & -2.14 & $2.75 \mathrm{E}-04$ \\
\hline 8005 & SDPR & -1.24 & $1.20 \mathrm{E}-03$ \\
\hline 8006 & SDR16C5 & -3.96 & $1.30 \mathrm{E}-04$ \\
\hline 8007 & SDR42E1 & -2.33 & $9.34 \mathrm{E}-04$ \\
\hline 8008 & SDR42E2 & -7.04 & 1.19E-04 \\
\hline 8009 & SDSL & -2.26 & 1.17E-03 \\
\hline 8010 & SEC11A & -4.91 & $6.11 \mathrm{E}-04$ \\
\hline 8011 & SEC11C & -4.82 & 1.13E-04 \\
\hline 8012 & SEC13 & -7.26 & $6.73 \mathrm{E}-04$ \\
\hline 8013 & SEC14L1 & -3.63 & $1.91 \mathrm{E}-03$ \\
\hline 8014 & SEC22A & -7.41 & 5.77E-04 \\
\hline 8015 & SEC22B & -4.78 & $7.91 \mathrm{E}-04$ \\
\hline 8016 & SEC22C & -1.63 & $4.61 \mathrm{E}-03$ \\
\hline 8017 & SEC23A & -1.24 & $1.25 \mathrm{E}-03$ \\
\hline 8018 & SEC23B & -3.96 & $6.17 \mathrm{E}-04$ \\
\hline 8019 & SEC23IP & -3.04 & $1.71 \mathrm{E}-03$ \\
\hline 8020 & SEC24B & -3.04 & $1.93 \mathrm{E}-03$ \\
\hline 8021 & SEC24C & -1.59 & $5.11 \mathrm{E}-02$ \\
\hline 8022 & SEC24D & -5.37 & 7.19E-04 \\
\hline 8023 & SEC31A & -5.29 & 2.80E-04 \\
\hline 8024 & SEC31B & -4.65 & $2.48 \mathrm{E}-04$ \\
\hline 8025 & SEC61B & -8.14 & $1.98 \mathrm{E}-05$ \\
\hline 8026 & SEC61G & -4.82 & $1.81 \mathrm{E}-03$ \\
\hline 8027 & SEC62 & -3.93 & $5.14 \mathrm{E}-04$ \\
\hline 8028 & SEC63 & -3.44 & 4.60E-04 \\
\hline 8029 & SECISBP2 & -4.06 & $5.26 \mathrm{E}-04$ \\
\hline 8030 & SEL1L & -5.57 & 2.50E-04 \\
\hline 8031 & SELENBP1 & -1.83 & $2.22 \mathrm{E}-03$ \\
\hline 8032 & SELK & -7.37 & $5.38 \mathrm{E}-05$ \\
\hline 8033 & SELRC1 & -5.03 & 2.46E-04 \\
\hline 8034 & SELT & -4.77 & 8.57E-04 \\
\hline 8035 & SEMA4B & -1.57 & $9.82 \mathrm{E}-03$ \\
\hline 8036 & SEMA6D & -3.37 & 3.86E-04 \\
\hline 8037 & SENP1 & -5.70 & $3.08 \mathrm{E}-04$ \\
\hline 8038 & SENP5 & -2.53 & 1.99E-04 \\
\hline 8039 & SENP6 & -3.14 & 2.97E-03 \\
\hline 8040 & SEPHS1 & -3.59 & $1.10 \mathrm{E}-03$ \\
\hline 8041 & SEPP1 & -4.09 & $1.18 \mathrm{E}-03$ \\
\hline 8042 & SEPSECS & -3.18 & $1.77 \mathrm{E}-03$ \\
\hline 8043 & SEPW1 & -7.34 & $2.80 \mathrm{E}-03$ \\
\hline 8044 & SERBP1 & -5.05 & 4.84E-04 \\
\hline 8045 & SERF1A & -7.48 & $1.68 \mathrm{E}-04$ \\
\hline 8046 & SERF2 & -7.07 & 2.04E-04 \\
\hline 8047 & SERHL2 & -3.40 & $1.66 \mathrm{E}-03$ \\
\hline 8048 & SERINC1 & -4.35 & $1.68 \mathrm{E}-03$ \\
\hline 8049 & SERINC3 & -3.77 & $1.29 \mathrm{E}-03$ \\
\hline 8050 & SERPINB6 & -4.43 & 7.00E-04 \\
\hline 8051 & SERPINE2 & -5.14 & $3.30 \mathrm{E}-05$ \\
\hline 8052 & SERPINF1 & -3.79 & $2.23 \mathrm{E}-04$ \\
\hline 8053 & SERPINH1 & -2.88 & $1.21 \mathrm{E}-02$ \\
\hline 8054 & SERPINI1 & -6.48 & $5.88 \mathrm{E}-04$ \\
\hline 8055 & SESN1 & -2.54 & $2.28 \mathrm{E}-03$ \\
\hline 8056 & SESTD1 & -2.70 & $2.29 \mathrm{E}-03$ \\
\hline
\end{tabular}




\begin{tabular}{|c|c|c|c|}
\hline 8057 & SET & -3.74 & $1.67 \mathrm{E}-03$ \\
\hline 8058 & SETD3 & -4.49 & $1.73 \mathrm{E}-03$ \\
\hline 8059 & SETD4 & -2.59 & $1.63 \mathrm{E}-03$ \\
\hline 8060 & SETD5 & -4.95 & $1.93 \mathrm{E}-04$ \\
\hline 8061 & SETD6 & -3.88 & $7.65 \mathrm{E}-04$ \\
\hline 8062 & SETD7 & -1.59 & $7.79 \mathrm{E}-03$ \\
\hline 8063 & SETD9 & -6.70 & $4.95 \mathrm{E}-04$ \\
\hline 8064 & SETX & -2.54 & $2.39 \mathrm{E}-04$ \\
\hline 8065 & SF3A1 & -4.12 & $3.09 \mathrm{E}-04$ \\
\hline 8066 & SF3A3 & -3.23 & $1.55 \mathrm{E}-02$ \\
\hline 8067 & SF3B1 & -5.61 & $1.48 \mathrm{E}-05$ \\
\hline 8068 & SF3B14 & -5.02 & $4.51 \mathrm{E}-04$ \\
\hline 8069 & SF3B3 & -5.37 & $5.57 \mathrm{E}-05$ \\
\hline 8070 & SF $3 B 5$ & -2.28 & $5.89 \mathrm{E}-03$ \\
\hline 8071 & SFMBT1 & -1.55 & $2.00 \mathrm{E}-02$ \\
\hline 8072 & SFRP2 & -1.97 & $1.10 \mathrm{E}-02$ \\
\hline 8073 & SFRP4 & -2.20 & $7.90 \mathrm{E}-03$ \\
\hline 8074 & SFSWAP & -2.99 & $7.74 \mathrm{E}-04$ \\
\hline 8075 & SFT2D1 & -6.74 & $3.09 \mathrm{E}-03$ \\
\hline 8076 & SFXN1 & -3.53 & $1.26 \mathrm{E}-04$ \\
\hline 8077 & SFXN2 & -1.08 & $2.73 \mathrm{E}-02$ \\
\hline 8078 & SFXN4 & $\begin{array}{l}-2.67 \\
\end{array}$ & $1.60 \mathrm{E}-03$ \\
\hline 8079 & SFXN5 & $\begin{array}{l}-1.93 \\
\end{array}$ & $1.21 \mathrm{E}-05$ \\
\hline 8080 & SGCB & -4.34 & $4.90 \mathrm{E}-03$ \\
\hline 8081 & SGCE & -4.60 & $2.19 \mathrm{E}-04$ \\
\hline 8082 & SGK3 & -2.65 & $2.81 \mathrm{E}-02$ \\
\hline 8083 & SGOL1 & -3.96 & $1.04 \mathrm{E}-03$ \\
\hline 8084 & SGOL2 & -6.00 & $7.64 \mathrm{E}-05$ \\
\hline 8085 & SGPL1 & $\begin{array}{l}-2.93 \\
\end{array}$ & $2.08 \mathrm{E}-03$ \\
\hline 8086 & SGTA & -3.40 & $1.06 E-03$ \\
\hline 8087 & SGTB & -2.42 & $2.99 \mathrm{E}-02$ \\
\hline 8088 & SH3BGR & -2.07 & $5.64 \mathrm{E}-03$ \\
\hline 8089 & SH3BGRL & -4.96 & $4.87 \mathrm{E}-04$ \\
\hline 8090 & SH3BGRL2 & -2.11 & $1.65 \mathrm{E}-03$ \\
\hline 8091 & SH3GL1 & -4.70 & $1.19 \mathrm{E}-03$ \\
\hline 8092 & SH3GL3 & -1.68 & $1.24 \mathrm{E}-03$ \\
\hline 8093 & SH3GLB1 & -7.34 & $2.41 \mathrm{E}-04$ \\
\hline 8094 & SH3GLB2 & $\begin{array}{l}-4.79 \\
\end{array}$ & $8.61 \mathrm{E}-05$ \\
\hline 8095 & SH3YL1 & -4.70 & $5.85 \mathrm{E}-04$ \\
\hline 8096 & SHC4 & -1.11 & $4.95 \mathrm{E}-03$ \\
\hline 8097 & SHFM1 & -2.46 & $1.95 \mathrm{E}-03$ \\
\hline 8098 & SHISA5 & -3.34 & $5.41 \mathrm{E}-03$ \\
\hline 8099 & SHOC2 & -2.51 & $1.29 \mathrm{E}-02$ \\
\hline 8100 & SHPRH & $\begin{array}{l}-4.31 \\
\end{array}$ & $1.16 \mathrm{E}-03$ \\
\hline 8101 & SHQ1 & -2.74 & $3.19 \mathrm{E}-03$ \\
\hline 8102 & SIAH1 & -2.75 & $2.69 \mathrm{E}-04$ \\
\hline 8103 & SIAH2 & -5.71 & $2.77 \mathrm{E}-04$ \\
\hline 8104 & SIK1 & -3.68 & $1.86 \mathrm{E}-04$ \\
\hline 8105 & SIKE1 & -3.53 & $3.06 E-04$ \\
\hline 8106 & SIL1 & -1.80 & $8.66 \mathrm{E}-04$ \\
\hline 8107 & SIN3B & -1.02 & $6.69 \mathrm{E}-04$ \\
\hline 8108 & SIPA1L1 & -3.46 & $2.78 \mathrm{E}-03$ \\
\hline 8109 & SIRT1 & -5.52 & $1.85 \mathrm{E}-04$ \\
\hline 8110 & SIRT2 & $\begin{array}{l}-1.79 \\
\end{array}$ & $6.06 \mathrm{E}-02$ \\
\hline 8111 & SIRT3 & -3.91 & $3.84 \mathrm{E}-03$ \\
\hline 8112 & SIRT4 & -5.83 & $1.97 \mathrm{E}-03$ \\
\hline 8113 & SIRT6 & -1.30 & $3.68 \mathrm{E}-04$ \\
\hline 8114 & SIVA1 & -6.49 & $3.25 \mathrm{E}-05$ \\
\hline 8115 & SKA2 & -6.62 & $2.34 \mathrm{E}-04$ \\
\hline 8116 & SKAP2 & -3.61 & $5.97 \mathrm{E}-04$ \\
\hline 8117 & SKI & -1.37 & $6.77 \mathrm{E}-03$ \\
\hline 8118 & SKIL & -3.06 & $5.78 \mathrm{E}-04$ \\
\hline 8119 & SKIVZL2 & -5.49 & $1.64 \mathrm{E}-04$ \\
\hline
\end{tabular}

\begin{tabular}{|c|c|c|c|}
\hline 8120 & SKP1 & -7.45 & $1.38 \mathrm{E}-05$ \\
\hline 8121 & SKP2 & -1.61 & $9.52 \mathrm{E}-03$ \\
\hline 8122 & SLAIN1 & -1.61 & $1.93 \mathrm{E}-03$ \\
\hline 8123 & SLBP & -5.56 & $2.59 \mathrm{E}-04$ \\
\hline 8124 & SLC10A7 & -1.40 & $3.32 \mathrm{E}-03$ \\
\hline 8125 & SLC16A1 & -3.60 & $3.44 E-04$ \\
\hline 8126 & SLC16A3 & -1.70 & $3.57 \mathrm{E}-03$ \\
\hline 8127 & SLC17A5 & -3.86 & $6.76 E-04$ \\
\hline 8128 & SLC19A1 & -1.48 & $1.36 \mathrm{E}-02$ \\
\hline 8129 & SLC1A4 & -1.46 & $1.14 \mathrm{E}-03$ \\
\hline 8130 & SLC1A7 & -2.65 & $5.42 E-02$ \\
\hline 8131 & SLC2OA2 & -1.42 & $7.99 E-03$ \\
\hline 8132 & SLC22A 23 & -1.98 & $3.94 E-03$ \\
\hline 8133 & SLC22A5 & -4.58 & $5.05 \mathrm{E}-03$ \\
\hline 8134 & SLC23A2 & -1.14 & $1.46 \mathrm{E}-02$ \\
\hline 8135 & SLC24A5 & -1.83 & $5.14 \mathrm{E}-02$ \\
\hline 8136 & SLC25A13 & -2.77 & $1.73 E-03$ \\
\hline 8137 & SLC25A14 & -3.35 & $6.12 E-04$ \\
\hline 8138 & SLC25A15 & -4.60 & $2.33 \mathrm{E}-05$ \\
\hline 8139 & SLC25A16 & -2.55 & $4.50 \mathrm{E}-03$ \\
\hline 8140 & SLC25A17 & -4.47 & $1.16 \mathrm{E}-04$ \\
\hline 8141 & SLC25A20 & -4.51 & $2.61 E-03$ \\
\hline 8142 & SLC25A21 & -4.95 & $\begin{array}{l}4.77 E-04 \\
\end{array}$ \\
\hline 8143 & SLC25A22 & -1.43 & $1.75 E-02$ \\
\hline 8144 & SLC25A24 & -3.37 & $1.51 \mathrm{E}-03$ \\
\hline 8145 & SLC25A25 & -1.18 & $2.31 \mathrm{E}-02$ \\
\hline 8146 & SLC25A28 & -4.48 & $1.23 \mathrm{E}-03$ \\
\hline 8147 & SLC25A3 & -5.40 & $2.78 E-04$ \\
\hline 8148 & SLC25A32 & -1.74 & $1.05 E-02$ \\
\hline 8149 & SLC25A33 & -4.93 & $3.51 E-05$ \\
\hline 8150 & SLC25A36 & -4.44 & $2.40 \mathrm{E}-04$ \\
\hline 8151 & SLC25A37 & -3.88 & $2.86 \mathrm{E}-03$ \\
\hline 8152 & SLC25A39 & -3.74 & $1.23 \mathrm{E}-04$ \\
\hline 8153 & SLC25A4 & -3.04 & $4.08 E-03$ \\
\hline 8154 & SLC25A40 & -3.06 & $1.34 \mathrm{E}-03$ \\
\hline 8155 & SLC25A43 & -1.05 & $3.48 \mathrm{E}-03$ \\
\hline 8156 & SLC25A46 & -4.51 & $1.19 E-03$ \\
\hline 8157 & SLC25A5 & -6.73 & $8.82 E-04$ \\
\hline 8158 & SLC25A6 & -1.71 & $1.11 \mathrm{E}-03$ \\
\hline 8159 & SLC26A3 & $\begin{array}{l}-1.47 \\
\end{array}$ & $\begin{array}{l}1.64 E-04 \\
\end{array}$ \\
\hline 8160 & SLC27A1 & -1.45 & $2.50 \mathrm{E}-02$ \\
\hline 8161 & SLC27A6 & -2.26 & $9.64 \mathrm{E}-03$ \\
\hline 8162 & SLC28A3 & -3.71 & $2.07 E-02$ \\
\hline 8163 & SLC2A12 & -1.59 & $2.43 E-03$ \\
\hline 8164 & SLC2A3 & -8.19 & $2.11 E-05$ \\
\hline 8165 & SLCZA5 & $\begin{array}{l}-1.47 \\
\end{array}$ & $\begin{array}{l}2.64 E-04 \\
\end{array}$ \\
\hline 8166 & SLCZAB & -3.20 & $4.98 E-04$ \\
\hline 8167 & SLC30A1 & -1.81 & $1.02 E-02$ \\
\hline 8168 & SLC3OA4 & -4.95 & $2.39 E-05$ \\
\hline 8169 & SLC30A5 & -4.23 & $7.33 E-04$ \\
\hline 8170 & SLC30A6 & -4.96 & $1.88 \mathrm{E}-03$ \\
\hline 8171 & SLC30A7 & -3.94 & $6.76 E-04$ \\
\hline 8172 & SLC33A1 & -3.45 & $1.97 E-03$ \\
\hline 8173 & SLC35A1 & -1.78 & $1.87 \mathrm{E}-03$ \\
\hline 8174 & SLC35A3 & -1.25 & $5.84 \mathrm{E}-04$ \\
\hline 8175 & SLC35A5 & -4.45 & $2.18 \mathrm{E}-04$ \\
\hline 8176 & SLC35B1 & -4.73 & $7.38 \mathrm{E}-04$ \\
\hline 8177 & SLC35B3 & -3.40 & $5.11 E-04$ \\
\hline 8178 & SLC35B4 & -1.33 & $7.93 E-03$ \\
\hline 8179 & SLC35C1 & -1.65 & $5.08 \mathrm{E}-04$ \\
\hline 8180 & SLC35F1 & -2.40 & $3.61 E-03$ \\
\hline 8181 & SLC35F2 & -1.35 & $4.68 \mathrm{E}-03$ \\
\hline 8182 & SLC35F3 & -1.59 & $3.55 \mathrm{E}-03$ \\
\hline
\end{tabular}

\begin{tabular}{|c|c|c|c|}
\hline 8183 & SLC35G2 & -3.53 & $6.16 \mathrm{E}-04$ \\
\hline 8184 & SLC37A2 & -1.08 & $7.29 \mathrm{E}-04$ \\
\hline 8185 & SLC37A4 & -1.38 & $4.92 \mathrm{E}-03$ \\
\hline 8186 & SLC38A2 & -4.37 & $8.15 \mathrm{E}-04$ \\
\hline 8187 & SLC38A6 6 & -1.44 & $3.33 \mathrm{E}-02$ \\
\hline 8188 & SLC39A13 & -3.33 & $1.82 \mathrm{E}-03$ \\
\hline 8189 & SLC39A3 & $\begin{array}{l}-2.03 \\
\end{array}$ & $8.42 \mathrm{E}-03$ \\
\hline 8190 & SLC39A6 & -3.62 & $3.29 \mathrm{E}-03$ \\
\hline 8191 & SLC39A9 & -4.15 & $3.67 \mathrm{E}-04$ \\
\hline 8192 & SLC3A1 & -3.91 & $4.75 \mathrm{E}-04$ \\
\hline 8193 & SLC40A1 & -2.24 & $4.60 \mathrm{E}-04$ \\
\hline 8194 & SLC41A2 & -2.60 & $2.59 \mathrm{E}-03$ \\
\hline 8195 & SLC44A3 & -3.36 & 4.44E-03 \\
\hline 8196 & SLC45A4 & -1.13 & $1.95 \mathrm{E}-02$ \\
\hline 8197 & SLC46A3 & -2.99 & $7.76 \mathrm{E}-04$ \\
\hline 8198 & SLC48A1 & -4.97 & $2.91 \mathrm{E}-03$ \\
\hline 8199 & SLCAA3 & -6.82 & $1.09 \mathrm{E}-03$ \\
\hline 8200 & SLCAA8 & -4.43 & $2.05 \mathrm{E}-03$ \\
\hline 8201 & SLC5A8 & -2.33 & $6.49 \mathrm{E}-05$ \\
\hline 8202 & SLC6A7 & -1.06 & $3.77 \mathrm{E}-04$ \\
\hline 8203 & SLCGA9 & -5.03 & 4.07E-04 \\
\hline 8204 & SLC7A5 & -3.38 & $2.10 \mathrm{E}-03$ \\
\hline 8205 & SLC7A6 & -1.41 & $7.26 \mathrm{E}-04$ \\
\hline 8206 & SLC7A6OS & -2.14 & $2.12 \mathrm{E}-02$ \\
\hline 8207 & SLC7A9 & -1.36 & $4.01 \mathrm{E}-03$ \\
\hline 8208 & $\begin{array}{l}\text { SLMAP } \\
\end{array}$ & -2.42 & $1.81 \mathrm{E}-04$ \\
\hline 8209 & SLMO1 & -6.75 & $9.89 \mathrm{E}-04$ \\
\hline 8210 & SLMO2 & -3.76 & $5.91 \mathrm{E}-04$ \\
\hline 8211 & SLTM & -1.97 & $6.50 \mathrm{E}-03$ \\
\hline 8212 & SLU7 & -3.24 & $6.17 \mathrm{E}-05$ \\
\hline 8213 & SLX4 & -3.47 & $7.62 \mathrm{E}-03$ \\
\hline 8214 & SLXYIP & -2.67 & $1.25 \mathrm{E}-03$ \\
\hline 8215 & SMAD3 & -1.62 & $3.88 \mathrm{E}-03$ \\
\hline 8216 & SMAD6 & -3.08 & $1.15 \mathrm{E}-03$ \\
\hline 8217 & SMARCA1 & -1.55 & $7.41 \mathrm{E}-03$ \\
\hline 8218 & SMARCA2 & -5.31 & $7.49 \mathrm{E}-04$ \\
\hline 8219 & SMARCA4 & -3.84 & $6.48 \mathrm{E}-03$ \\
\hline 8220 & SMARCA5 & -6.10 & $7.15 \mathrm{E}-04$ \\
\hline 8221 & SMARCB1 & -2.13 & $2.20 \mathrm{E}-02$ \\
\hline 8222 & SMARCD2 & -1.40 & $5.39 \mathrm{E}-02$ \\
\hline 8223 & SMARCE1 & -4.94 & $4.45 \mathrm{E}-04$ \\
\hline 8224 & SMC1B & -2.74 & $1.37 \mathrm{E}-02$ \\
\hline 8225 & SMC2 & -5.68 & 7.04E-05 \\
\hline 8226 & SMC3 & -4.67 & $2.44 \mathrm{E}-04$ \\
\hline 8227 & SMC4 & -5.50 & $4.97 \mathrm{E}-04$ \\
\hline 8228 & SMC5 & -5.78 & $7.68 \mathrm{E}-04$ \\
\hline 8229 & SMC6 & -4.91 & $1.57 \mathrm{E}-03$ \\
\hline 8230 & SMCHD1 & -2.37 & $6.34 \mathrm{E}-04$ \\
\hline 8231 & SMEK2 & -1.94 & $3.26 \mathrm{E}-02$ \\
\hline 8232 & SMG7 & -1.50 & $8.63 \mathrm{E}-04$ \\
\hline 8233 & SMG8 & -2.17 & $4.36 \mathrm{E}-03$ \\
\hline 8234 & SMIM12 & -6.01 & $4.15 \mathrm{E}-04$ \\
\hline 8235 & SMIM15 & -3.52 & $7.60 \mathrm{E}-04$ \\
\hline 8236 & SMN & -4.23 & $3.61 \mathrm{E}-04$ \\
\hline 8237 & SMNDC1 & -3.07 & $4.55 \mathrm{E}-04$ \\
\hline 8238 & SMO & -3.49 & $4.81 \mathrm{E}-04$ \\
\hline 8239 & SMPD4 & -1.22 & $1.34 \mathrm{E}-03$ \\
\hline 8240 & SMS & -3.64 & $7.33 \mathrm{E}-04$ \\
\hline 8241 & SMU1 & -4.51 & $2.33 \mathrm{E}-04$ \\
\hline 8242 & SMYD1 & -1.20 & $6.15 \mathrm{E}-03$ \\
\hline 8243 & SMYD2 & -3.74 & $3.38 \mathrm{E}-03$ \\
\hline 8244 & SMYD3 & -3.55 & $4.15 \mathrm{E}-03$ \\
\hline 8245 & SMYD4 & -4.35 & $4.12 \mathrm{E}-04$ \\
\hline
\end{tabular}




\begin{tabular}{|c|c|c|c|}
\hline 8246 & SNAPC1 & -3.35 & $2.58 \mathrm{E}-03$ \\
\hline 8247 & SNAPC3 & -1.57 & $2.83 \mathrm{E}-04$ \\
\hline 8248 & SNAPC5 & -4.23 & $3.79 \mathrm{E}-03$ \\
\hline 8249 & SNCA & -2.02 & $1.80 \mathrm{E}-01$ \\
\hline 8250 & SNF8 & -3.73 & $1.23 \mathrm{E}-03$ \\
\hline 8251 & SNHG8 & -3.10 & $1.35 \mathrm{E}-03$ \\
\hline 8252 & SNIP1 & -2.93 & $1.38 \mathrm{E}-02$ \\
\hline 8253 & SNRNP25 & -5.64 & $2.18 \mathrm{E}-04$ \\
\hline 8254 & SNRNP27 & -5.94 & $1.18 \mathrm{E}-03$ \\
\hline 8255 & SNRNP35 & -2.85 & $3.04 \mathrm{E}-03$ \\
\hline 8256 & SNRNP40 & -1.82 & $1.23 \mathrm{E}-03$ \\
\hline 8257 & SNRNP48 & -3.29 & $1.70 \mathrm{E}-03$ \\
\hline 8258 & SNRPA1 & -4.90 & $9.62 \mathrm{E}-05$ \\
\hline 8259 & SNRPB & -6.79 & $1.01 \mathrm{E}-04$ \\
\hline 8260 & SNRPB2 & -5.24 & 2.56E-03 \\
\hline 8261 & SNRPC & -6.51 & $5.21 \mathrm{E}-05$ \\
\hline 8262 & SNRPD1 & -2.33 & $3.28 \mathrm{E}-03$ \\
\hline 8263 & SNRPD3 & -5.00 & $3.71 \mathrm{E}-03$ \\
\hline 8264 & SNRPE & -6.16 & 2.00E-04 \\
\hline 8265 & SNRPF & -5.46 & $1.26 \mathrm{E}-03$ \\
\hline 8266 & SNW1 & -3.97 & $1.76 \mathrm{E}-03$ \\
\hline 8267 & SNX1 & -4.71 & 4.74E-04 \\
\hline 8268 & SNX10 & -2.95 & 7.42E-03 \\
\hline 8269 & SNX14 & -4.23 & 3.68E-05 \\
\hline 8270 & SNX17 & -1.20 & 2.97E-02 \\
\hline 8271 & SNX2 & -4.61 & $1.72 \mathrm{E}-04$ \\
\hline 8272 & SNX21 & -1.28 & 1.27E-02 \\
\hline 8273 & SNX24 & -2.60 & $1.11 \mathrm{E}-03$ \\
\hline 8274 & SNX25 & -2.96 & $2.36 \mathrm{E}-03$ \\
\hline 8275 & SNX3 & -6.66 & $1.93 \mathrm{E}-05$ \\
\hline 8276 & SNX4 & -2.62 & $4.22 \mathrm{E}-05$ \\
\hline 8277 & SNX5 & -5.31 & 4.18E-05 \\
\hline 8278 & SNX6 & -4.38 & $2.32 \mathrm{E}-04$ \\
\hline 8279 & SNX7 & -2.44 & $3.64 \mathrm{E}-04$ \\
\hline 8280 & SOAT1 & -3.87 & $2.92 \mathrm{E}-03$ \\
\hline 8281 & SOBP & -2.74 & $1.35 \mathrm{E}-02$ \\
\hline 8282 & SOCS4 & -1.31 & $1.23 \mathrm{E}-02$ \\
\hline 8283 & SOCS5 & -1.79 & $5.72 \mathrm{E}-05$ \\
\hline 8284 & SOD1 & -7.27 & $6.76 \mathrm{E}-05$ \\
\hline 8285 & SOD2 & -4.76 & $1.20 \mathrm{E}-04$ \\
\hline 8286 & SORL1 & -2.30 & $1.51 \mathrm{E}-02$ \\
\hline 8287 & Sox30 & -3.58 & $3.27 \mathrm{E}-05$ \\
\hline 8288 & sox5 & -2.44 & $1.25 \mathrm{E}-03$ \\
\hline 8289 & SP1 & -1.65 & $3.39 \mathrm{E}-03$ \\
\hline 8290 & SP3 & -2.06 & $8.48 \mathrm{E}-04$ \\
\hline 8291 & SP4 & -4.79 & $1.17 \mathrm{E}-03$ \\
\hline 8292 & SP5 & -3.35 & $1.85 \mathrm{E}-03$ \\
\hline 8293 & SPACA1 & -4.72 & 3.70E-03 \\
\hline 8294 & SPAG16 & -2.18 & $1.19 \mathrm{E}-02$ \\
\hline 8295 & SPAG4 & -1.73 & $1.02 \mathrm{E}-02$ \\
\hline 8296 & SPAG6 & -6.41 & 7.46E-05 \\
\hline 8297 & SPAG9 & -2.76 & $3.11 \mathrm{E}-03$ \\
\hline 8298 & SPARC & -2.44 & 1.15E-02 \\
\hline 8299 & SPAST & -1.57 & $3.30 \mathrm{E}-02$ \\
\hline 8300 & SPATA16 & -3.42 & $1.35 \mathrm{E}-03$ \\
\hline 8301 & SPATA17 & -8.65 & 1.33E-04 \\
\hline 8302 & SPATA18 & -7.14 & $2.02 \mathrm{E}-03$ \\
\hline 8303 & SPATA2 & -2.52 & 7.37E-04 \\
\hline 8304 & SPATA5 & -3.46 & $8.05 \mathrm{E}-04$ \\
\hline 8305 & SPATAG & -4.56 & $2.13 \mathrm{E}-03$ \\
\hline 8306 & SPATS2 & -2.45 & $6.02 \mathrm{E}-03$ \\
\hline 8307 & SPATS2L & -4.82 & 1.46E-04 \\
\hline 8308 & SPC25 & -8.09 & $1.11 \mathrm{E}-04$ \\
\hline
\end{tabular}

\begin{tabular}{|c|c|c|c|}
\hline 8309 & SPCS1 & -7.49 & 2.47E-04 \\
\hline 8310 & SPCS2 & -5.77 & $6.32 \mathrm{E}-04$ \\
\hline 8311 & SPCS3 & -3.28 & 3.78E-04 \\
\hline 8312 & SPDYA & -6.16 & $1.55 \mathrm{E}-04$ \\
\hline 8313 & SPECC1 & -4.83 & 4.13E-04 \\
\hline 8314 & SPEN & -1.56 & $2.28 \mathrm{E}-02$ \\
\hline 8315 & SPERT & -7.67 & $1.16 \mathrm{E}-03$ \\
\hline 8316 & SPG21 & -5.94 & $6.60 \mathrm{E}-04$ \\
\hline 8317 & SPG7 & -5.61 & $5.48 \mathrm{E}-04$ \\
\hline 8318 & SPHKAP & -3.64 & $1.94 \mathrm{E}-04$ \\
\hline 8319 & SPICE1 & -1.79 & $2.54 \mathrm{E}-02$ \\
\hline 8320 & SPINK4 & -2.75 & $1.73 \mathrm{E}-04$ \\
\hline 8321 & SPINW & -2.65 & $6.79 \mathrm{E}-03$ \\
\hline 8322 & SPINZ & -3.70 & $1.10 \mathrm{E}-04$ \\
\hline 8323 & SPO11 & -4.00 & 4.50E-04 \\
\hline 8324 & SPON1 & -2.11 & 4.04E-03 \\
\hline 8325 & SPON2 & -2.56 & $1.78 \mathrm{E}-02$ \\
\hline 8326 & SPOPL & -1.54 & $9.69 \mathrm{E}-03$ \\
\hline 8327 & SPPL2A & -3.53 & $1.45 \mathrm{E}-04$ \\
\hline 8328 & SPPL2B & -4.27 & $6.46 \mathrm{E}-04$ \\
\hline 8329 & SPRED1 & -2.97 & $2.63 \mathrm{E}-03$ \\
\hline 8330 & SPRYD7 & -4.34 & $1.41 \mathrm{E}-03$ \\
\hline 8331 & SPSB1 & -1.60 & 7.69E-02 \\
\hline 8332 & SPSB3 & -3.30 & $4.78 \mathrm{E}-05$ \\
\hline 8333 & SPSB4 & -2.87 & $1.44 \mathrm{E}-03$ \\
\hline 8334 & SPTAN1 & -4.33 & 7.33E-05 \\
\hline 8335 & SPTBN1 & -4.32 & 4.98E-05 \\
\hline 8336 & SPTLC2 & -2.96 & 1.33E-04 \\
\hline 8337 & SPTSSA & -2.54 & $6.49 \mathrm{E}-03$ \\
\hline 8338 & SPTY2D1 & -3.51 & $1.26 \mathrm{E}-03$ \\
\hline 8339 & SQLE & -4.98 & 3.76E-04 \\
\hline 8340 & SQRDL & -1.32 & 3.47E-03 \\
\hline 8341 & SQSTM1 & -4.92 & $3.70 \mathrm{E}-05$ \\
\hline 8342 & SREK1IP1 & -3.73 & $2.34 \mathrm{E}-04$ \\
\hline 8343 & SRFBP1 & -3.54 & $6.31 \mathrm{E}-04$ \\
\hline 8344 & SRGAP1 & -4.90 & $5.62 E-05$ \\
\hline 8345 & SRI & -5.71 & $1.48 \mathrm{E}-03$ \\
\hline 8346 & SRP14 & -6.24 & $1.31 \mathrm{E}-04$ \\
\hline 8347 & SRP19 & -4.31 & 4.39E-03 \\
\hline 8348 & SRP54 & -6.22 & $1.76 E-04$ \\
\hline 8349 & SRP68 & -2.26 & $3.76 \mathrm{E}-03$ \\
\hline 8350 & SRP72 & -4.12 & $2.64 \mathrm{E}-03$ \\
\hline 8351 & SRP9 & -4.26 & 2.11E-04 \\
\hline 8352 & SRPK1 & -5.83 & $1.39 \mathrm{E}-04$ \\
\hline 8353 & SRPK2 & -4.09 & 3.31E-04 \\
\hline 8354 & SRPX & -2.27 & $1.48 \mathrm{E}-02$ \\
\hline 8355 & SRRM1 & -4.14 & $1.97 \mathrm{E}-03$ \\
\hline 8356 & SRRM2 & -5.18 & 7.73E-04 \\
\hline 8357 & SRSF1 & -4.56 & $7.64 \mathrm{E}-03$ \\
\hline 8358 & SRSF10 & -5.69 & 4.05E-04 \\
\hline 8359 & SRSF11 & -3.68 & $9.65 \mathrm{E}-04$ \\
\hline 8360 & SRSF2 & -3.89 & 5.60E-04 \\
\hline 8361 & SRSF3 & -6.43 & $1.96 \mathrm{E}-04$ \\
\hline 8362 & SRSF4 & -4.32 & $4.70 \mathrm{E}-04$ \\
\hline 8363 & SRSF5 & -2.39 & $2.66 \mathrm{E}-03$ \\
\hline 8364 & SRSF5A & -4.78 & $1.23 \mathrm{E}-04$ \\
\hline 8365 & SRSF6 & -4.63 & 3.93E-05 \\
\hline 8366 & SRSF7 & -7.29 & $1.54 \mathrm{E}-04$ \\
\hline 8367 & SS18 & -2.43 & $1.17 E-03$ \\
\hline 8368 & SS18L2 & -4.35 & $1.26 \mathrm{E}-05$ \\
\hline 8369 & SSB & -5.68 & $8.62 E-05$ \\
\hline 8370 & SSBP1 & -5.34 & $1.21 \mathrm{E}-03$ \\
\hline 8371 & SSBP2 & -1.61 & $2.79 \mathrm{E}-03$ \\
\hline
\end{tabular}

\begin{tabular}{|c|c|c|c|}
\hline 8372 & SSBP3 & -1.73 & $5.36 \mathrm{E}-04$ \\
\hline 8373 & SSNA1 & -5.88 & 3.69E-04 \\
\hline 8374 & SSR1 & -4.39 & $3.11 \mathrm{E}-04$ \\
\hline 8375 & SSR2 & -5.41 & $1.61 \mathrm{E}-03$ \\
\hline 8376 & SSR3 & -4.82 & $3.80 \mathrm{E}-05$ \\
\hline 8377 & SSRP1 & -3.49 & $1.00 \mathrm{E}-04$ \\
\hline 8378 & SSU72 & -3.68 & 4.09E-04 \\
\hline 8379 & SSX2IP & -6.22 & $3.35 \mathrm{E}-04$ \\
\hline 8380 & ST13 & -5.33 & 1.23E-04 \\
\hline 8381 & ST3GAL5 & -4.49 & 1.77E-04 \\
\hline 8382 & ST3GAL6 & -3.84 & $2.09 \mathrm{E}-03$ \\
\hline 8383 & ST5 & -2.65 & $1.20 \mathrm{E}-02$ \\
\hline 8384 & ST6GALNAC1 & -4.09 & $5.42 \mathrm{E}-04$ \\
\hline 8385 & ST7 & -4.76 & $1.14 \mathrm{E}-03$ \\
\hline 8386 & ST7L & -5.49 & 7.33E-07 \\
\hline 8387 & STAG1 & -2.65 & $2.26 \mathrm{E}-03$ \\
\hline 8388 & STAG3 & -2.79 & $2.51 \mathrm{E}-03$ \\
\hline 8389 & STAM & -1.16 & $2.41 \mathrm{E}-02$ \\
\hline 8390 & STAP1 & -1.77 & $1.18 \mathrm{E}-02$ \\
\hline 8391 & STARD3NL & -3.28 & $5.83 \mathrm{E}-05$ \\
\hline 8392 & STARD4 & -2.20 & 3.03E-03 \\
\hline 8393 & STAT3 & -3.17 & 5.27E-05 \\
\hline 8394 & STAT4 & -1.15 & $5.64 \mathrm{E}-02$ \\
\hline 8395 & STAU1 & -4.32 & $2.13 \mathrm{E}-02$ \\
\hline 8396 & STIL & -1.81 & $2.16 \mathrm{E}-03$ \\
\hline 8397 & STIM1 & -2.43 & 4.07E-05 \\
\hline 8398 & STK10 & -2.15 & $1.40 \mathrm{E}-03$ \\
\hline 8399 & STK11 & -1.77 & 4.96E-02 \\
\hline 8400 & STK11IP & -3.02 & $3.91 \mathrm{E}-03$ \\
\hline 8401 & STK17A & -3.68 & $6.74 \mathrm{E}-04$ \\
\hline 8402 & STK17B & -2.95 & $1.05 \mathrm{E}-04$ \\
\hline 8403 & STK3 & -1.25 & $1.26 \mathrm{E}-02$ \\
\hline 8404 & STK31 & -4.82 & 3.60E-04 \\
\hline 8405 & STK38L & -2.79 & $9.30 \mathrm{E}-03$ \\
\hline 8406 & STK4 & -2.68 & $2.49 \mathrm{E}-03$ \\
\hline 8407 & STK40 & -5.24 & $3.22 \mathrm{E}-04$ \\
\hline 8408 & STMN1 & -8.73 & $1.26 \mathrm{E}-03$ \\
\hline 8409 & STMN4 & -7.21 & 7.49E-04 \\
\hline 8410 & STOM & -2.55 & 4.84E-03 \\
\hline 8411 & STON2 & -2.71 & $2.62 \mathrm{E}-03$ \\
\hline 8412 & STOX2 & -3.27 & $8.81 \mathrm{E}-03$ \\
\hline 8413 & STRA8 & -3.30 & $7.36 \mathrm{E}-04$ \\
\hline 8414 & STRADA & -5.93 & 4.53E-04 \\
\hline 8415 & STRAP & -4.50 & $6.77 \mathrm{E}-05$ \\
\hline 8416 & STRBP & -5.08 & 4.80E-04 \\
\hline 8417 & STRIP2 & -1.92 & $3.24 \mathrm{E}-03$ \\
\hline 8418 & STRN3 & -6.11 & $8.26 \mathrm{E}-04$ \\
\hline 8419 & STT3B & -3.47 & $1.60 \mathrm{E}-03$ \\
\hline 8420 & STX16 & -3.82 & 2.53E-04 \\
\hline 8421 & STX17 & -1.63 & $1.39 \mathrm{E}-02$ \\
\hline 8422 & STX18 & -4.91 & $3.32 \mathrm{E}-03$ \\
\hline 8423 & STX1A & -2.70 & $1.34 \mathrm{E}-03$ \\
\hline 8424 & STX2 & -3.20 & $1.22 \mathrm{E}-03$ \\
\hline 8425 & STX6 & -1.33 & $2.08 \mathrm{E}-03$ \\
\hline 8426 & STXBP3 & -2.30 & $3.62 \mathrm{E}-03$ \\
\hline 8427 & STYK1 & -2.64 & $5.82 \mathrm{E}-03$ \\
\hline 8428 & STYX & -6.77 & $5.46 \mathrm{E}-05$ \\
\hline 8429 & STYXL1 & -3.62 & $2.80 \mathrm{E}-04$ \\
\hline 8430 & SUB1 & -3.54 & $2.61 \mathrm{E}-04$ \\
\hline 8431 & SUCLA2 & -2.73 & $1.23 \mathrm{E}-03$ \\
\hline 8432 & SUCLG1 & -3.07 & $1.54 \mathrm{E}-04$ \\
\hline 8433 & SUCLG2 & -2.47 & $6.95 \mathrm{E}-04$ \\
\hline 8434 & SUDS3 & -2.85 & $8.73 \mathrm{E}-04$ \\
\hline
\end{tabular}




\begin{tabular}{|c|c|c|c|}
\hline 8435 & SUFU & -4.11 & $1.84 \mathrm{E}-04$ \\
\hline 8436 & SUGP1 & -1.75 & $6.32 \mathrm{E}-04$ \\
\hline 8437 & SUGT1 & \begin{tabular}{|l|} 
\\
\end{tabular} & $2.02 E-04$ \\
\hline 8438 & SULF2 & $\begin{array}{l}-2.10 \\
\end{array}$ & $5.70 \mathrm{E}-03$ \\
\hline 8439 & SULT1E1 & -6.21 & $1.17 E-02$ \\
\hline 8440 & SULTAA1 & -3.21 & $2.02 E-04$ \\
\hline 8441 & SUMF2 & -4.28 & $1.34 \mathrm{E}-03$ \\
\hline 8442 & SUMO2 & -5.28 & $1.82 \mathrm{E}-04$ \\
\hline 8443 & SUMO3 & -7.60 & $4.91 E-05$ \\
\hline 8444 & SUN1 & -4.46 & 4.57E-05 \\
\hline 8445 & SUPT4H1 & -4.99 & $1.40 \mathrm{E}-04$ \\
\hline 8446 & SUPTSH & \begin{tabular}{|l|}
-3.16 \\
\end{tabular} & $1.07 E-03$ \\
\hline 8447 & SUPV3L1 & -2.17 & $3.69 \mathrm{E}-02$ \\
\hline 8448 & SURF1 & -2.46 & 7.37E-03 \\
\hline 8449 & SURF2 & -3.55 & $1.02 E-03$ \\
\hline 8450 & SURF4 & -4.73 & $3.45 E-05$ \\
\hline 8451 & SURF6 & -5.60 & $3.96 \mathrm{E}-03$ \\
\hline 8452 & SUSD3 & -2.35 & $1.42 E-03$ \\
\hline 8453 & SUSD4 & -1.89 & $2.17 E-02$ \\
\hline 8454 & SUV39H2 & -3.62 & $5.80 \mathrm{E}-04$ \\
\hline 8455 & SUV42OH1 & -1.82 & 5.04E-02 \\
\hline 8456 & SUZ12 & -3.37 & $3.21 E-04$ \\
\hline 8457 & SWAP70 & -3.27 & $1.18 \mathrm{E}-03$ \\
\hline 8458 & SWI5 & -6.54 & $2.95 \mathrm{E}-04$ \\
\hline 8459 & SYAP1 & -5.09 & $3.73 \mathrm{E}-05$ \\
\hline 8460 & SYBU & -2.20 & $3.36 \mathrm{E}-03$ \\
\hline 8461 & SYCE3 & -7.66 & 4.60E-04 \\
\hline 8462 & $\begin{array}{l}\text { SYCP3 } \\
\end{array}$ & \begin{tabular}{|l|}
-7.58 \\
\end{tabular} & $2.85 \mathrm{E}-06$ \\
\hline 8463 & SYF2 & -5.04 & $3.05 E-04$ \\
\hline 8464 & SYNE1 & -1.67 & $3.59 \mathrm{E}-03$ \\
\hline 8465 & SYNGR2 & -3.47 & $1.58 \mathrm{E}-04$ \\
\hline 8466 & $\begin{array}{l}\text { SYNGR3 } \\
\end{array}$ & -1.52 & $2.60 \mathrm{E}-05$ \\
\hline 8467 & SYNJ2BP & -5.07 & $9.47 E-04$ \\
\hline 8468 & SYNRG & $\begin{array}{l}-1.69 \\
\end{array}$ & $5.69 \mathrm{E}-03$ \\
\hline 8469 & SYPL1 & -1.98 & $3.25 \mathrm{E}-03$ \\
\hline 8470 & SYS1 & -3.37 & $5.23 \mathrm{E}-04$ \\
\hline 8471 & SYT11 & -1.33 & $1.84 \mathrm{E}-03$ \\
\hline 8472 & SYT8 & -1.47 & $2.41 \mathrm{E}-03$ \\
\hline 8473 & TAB2 & -1.47 & $3.86 \mathrm{E}-02$ \\
\hline 8474 & TAB3 & -1.66 & $8.94 E-02$ \\
\hline 8475 & TACC3 & -3.56 & $2.32 \mathrm{E}-02$ \\
\hline 8476 & TACR3 & -1.34 & $1.92 E-02$ \\
\hline 8477 & TADA1L & -2.22 & $2.83 \mathrm{E}-04$ \\
\hline 8478 & TADA2A & \begin{tabular}{|c|}
-3.99 \\
\end{tabular} & $9.52 \mathrm{E}-04$ \\
\hline 8479 & TADA3 & -1.43 & $1.11 \mathrm{E}-02$ \\
\hline 8480 & TAF1 & -4.73 & $3.28 \mathrm{E}-04$ \\
\hline 8481 & TAF11 & -2.41 & $4.86 \mathrm{E}-03$ \\
\hline 8482 & TAF13 & -4.61 & $4.09 E-03$ \\
\hline 8483 & TAF15 & -5.53 & $8.55 E-05$ \\
\hline 8484 & TAF1A & -3.99 & 3.33E-04 \\
\hline 8485 & TAF1B & -2.03 & $3.73 E-03$ \\
\hline 8486 & TAF2 & -3.21 & $4.58 \mathrm{E}-03$ \\
\hline 8487 & TAF3 & -4.52 & $1.83 \mathrm{E}-04$ \\
\hline 8488 & TAF5 & -3.11 & $2.87 \mathrm{E}-04$ \\
\hline 8489 & TAF7 & -2.05 & $2.23 \mathrm{E}-03$ \\
\hline 8490 & TAF9 & -2.28 & 4.62E-04 \\
\hline 8491 & TAGLN3 & -1.33 & $1.14 \mathrm{E}-02$ \\
\hline 8492 & TALDO1 & -2.55 & $3.10 \mathrm{E}-03$ \\
\hline 8493 & TAMM41 & -1.81 & $3.97 E-03$ \\
\hline 8494 & TANC1 & -1.23 & 4.05E-03 \\
\hline 8495 & TANGO2 & -5.94 & $5.80 \mathrm{E}-04$ \\
\hline 8496 & TAPBP & -2.10 & $6.08 \mathrm{E}-02$ \\
\hline $4 y$ & IAPT & -2.19 & 1.27E-03 \\
\hline
\end{tabular}

\begin{tabular}{|c|c|c|c|}
\hline 8498 & TARDBP & -3.98 & $5.02 \mathrm{E}-04$ \\
\hline 8499 & TARS & -4.45 & $3.02 \mathrm{E}-04$ \\
\hline 8500 & TATDN1 & -5.21 & $4.76 \mathrm{E}-04$ \\
\hline 8501 & TATDN3 & -2.89 & $3.61 \mathrm{E}-03$ \\
\hline 8502 & TAX1BP1 & -3.37 & $5.88 \mathrm{E}-03$ \\
\hline 8503 & TAX1BP3 & -2.67 & $1.31 \mathrm{E}-02$ \\
\hline 8504 & TBC1D1 & -2.79 & $6.49 \mathrm{E}-03$ \\
\hline 8505 & TBC1D13 & -4.31 & $1.94 \mathrm{E}-04$ \\
\hline 8506 & TBC1D14 & -2.82 & $6.75 \mathrm{E}-03$ \\
\hline 8507 & TBC1D15 & -3.75 & $1.75 \mathrm{E}-04$ \\
\hline 8508 & TBC1D22A & -3.95 & $6.59 \mathrm{E}-04$ \\
\hline 8509 & TBC1D23 & -3.46 & $1.56 \mathrm{E}-03$ \\
\hline 8510 & TBC1D7 & -4.06 & $2.79 \mathrm{E}-03$ \\
\hline 8511 & TBC1D8B & -3.45 & $6.88 \mathrm{E}-04$ \\
\hline 8512 & TBC1D9B & -3.09 & $1.01 \mathrm{E}-04$ \\
\hline 8513 & TBCA & -5.21 & $5.78 \mathrm{E}-04$ \\
\hline 8514 & TBCC & -3.14 & $9.41 \mathrm{E}-05$ \\
\hline 8515 & TBCCD1 & -2.28 & $1.17 \mathrm{E}-03$ \\
\hline 8516 & TBCD & -5.45 & $4.75 \mathrm{E}-04$ \\
\hline 8517 & TBCE & -5.93 & $2.72 \mathrm{E}-04$ \\
\hline 8518 & TBCK & -1.59 & $6.07 \mathrm{E}-03$ \\
\hline 8519 & TBK1 & -2.17 & $3.61 \mathrm{E}-03$ \\
\hline 8520 & TBL1XR1 & -1.79 & $1.19 \mathrm{E}-02$ \\
\hline 8521 & TBL3 & -2.29 & $2.38 \mathrm{E}-03$ \\
\hline 8522 & TBP & -3.10 & $1.76 \mathrm{E}-03$ \\
\hline 8523 & TBPL1 & -6.76 & $3.93 \mathrm{E}-05$ \\
\hline 8524 & TBRG4 & -2.88 & $1.38 \mathrm{E}-03$ \\
\hline 8525 & TC373484 & -1.44 & $1.40 \mathrm{E}-03$ \\
\hline 8526 & TC373980 & -1.45 & $3.56 \mathrm{E}-02$ \\
\hline 8527 & TC374189 & -3.68 & $1.97 \mathrm{E}-03$ \\
\hline 8528 & TC375054 & -3.49 & 4.45E-03 \\
\hline 8529 & TC375593 & -2.78 & 4.49E-04 \\
\hline 8530 & TC375681 & -4.72 & $1.40 \mathrm{E}-03$ \\
\hline 8531 & TC375708 & -4.26 & $1.08 \mathrm{E}-04$ \\
\hline 8532 & TC375780 & -7.03 & $8.74 \mathrm{E}-04$ \\
\hline 8533 & TC376077 & -4.45 & $5.21 \mathrm{E}-03$ \\
\hline 8534 & TC376248 & -2.31 & $3.94 \mathrm{E}-03$ \\
\hline 8535 & TC376510 & -3.88 & $6.88 \mathrm{E}-04$ \\
\hline 8536 & TC376617 & -1.11 & $2.79 \mathrm{E}-03$ \\
\hline 8537 & $\begin{array}{l}\text { TC376858 } \\
\end{array}$ & -5.73 & $1.05 \mathrm{E}-04$ \\
\hline 8538 & TC377048 & -3.64 & $\begin{array}{r}2.42 \mathrm{E}-04 \\
\end{array}$ \\
\hline 8539 & TC377127 & -1.29 & $3.61 \mathrm{E}-03$ \\
\hline 8540 & TC377153 & -3.46 & $3.11 \mathrm{E}-03$ \\
\hline 8541 & TC377200 & -4.51 & $1.88 \mathrm{E}-03$ \\
\hline 8542 & TC377266 & -4.41 & $3.84 \mathrm{E}-03$ \\
\hline 8543 & TC377267 & -3.11 & $1.51 \mathrm{E}-02$ \\
\hline 8544 & TC377386 & -5.84 & $2.67 \mathrm{E}-04$ \\
\hline 8545 & TC377463 & -3.37 & $1.02 \mathrm{E}-03$ \\
\hline 8546 & TC377465 & -3.20 & $2.94 \mathrm{E}-03$ \\
\hline 8547 & TC377467 & -2.57 & $1.99 \mathrm{E}-02$ \\
\hline 8548 & TC377491 & -3.66 & $3.02 \mathrm{E}-03$ \\
\hline 8549 & TC377517 & -6.99 & $2.08 \mathrm{E}-04$ \\
\hline 8550 & TC377538 & -1.39 & $1.85 \mathrm{E}-02$ \\
\hline 8551 & TC377588 & -7.05 & $5.87 \mathrm{E}-04$ \\
\hline 8552 & TC377621 & -3.05 & $1.77 \mathrm{E}-03$ \\
\hline 8553 & TC377656 & -1.93 & $6.41 \mathrm{E}-02$ \\
\hline 8554 & TC377657 & -1.62 & $3.98 \mathrm{E}-04$ \\
\hline 8555 & TC377658 & -5.30 & $3.29 \mathrm{E}-04$ \\
\hline 8556 & TC377663 & -2.13 & $4.56 \mathrm{E}-03$ \\
\hline 8557 & TC377664 & -2.12 & $5.54 \mathrm{E}-03$ \\
\hline 8558 & TC377684 & -3.68 & $7.94 \mathrm{E}-05$ \\
\hline 8559 & TC377694 & -1.49 & $4.44 \mathrm{E}-03$ \\
\hline 8560 & TC377695 & -4.14 & $3.00 \mathrm{E}-04$ \\
\hline
\end{tabular}

\begin{tabular}{|c|c|c|c|}
\hline 8561 & TC377715 & -5.54 & $1.27 \mathrm{E}-03$ \\
\hline 8562 & TC377740 & -1.95 & $1.74 \mathrm{E}-03$ \\
\hline 8563 & TC377741 & -2.62 & $5.49 \mathrm{E}-03$ \\
\hline 8564 & TC377748 & -3.30 & $4.27 \mathrm{E}-02$ \\
\hline 8565 & TC377761 & -6.04 & $1.40 \mathrm{E}-04$ \\
\hline 8566 & TC377762 & -4.62 & $3.97 \mathrm{E}-04$ \\
\hline 8567 & TC377805 & -4.46 & $4.95 \mathrm{E}-03$ \\
\hline 8568 & TC377808 & -2.08 & $4.09 \mathrm{E}-03$ \\
\hline 8569 & TC377820 & -5.80 & $3.42 \mathrm{E}-05$ \\
\hline 8570 & TC377828 & -1.17 & $1.81 \mathrm{E}-03$ \\
\hline 8571 & TC377837 & -4.58 & $1.00 \mathrm{E}-02$ \\
\hline 8572 & TC377841 & -1.31 & $5.39 \mathrm{E}-03$ \\
\hline 8573 & TC377866 & -3.82 & $1.21 \mathrm{E}-04$ \\
\hline 8574 & TC377877 & -2.78 & $2.11 \mathrm{E}-03$ \\
\hline 8575 & TC377899 & -2.11 & $5.21 \mathrm{E}-03$ \\
\hline 8576 & TC377974 & -2.58 & $2.95 \mathrm{E}-04$ \\
\hline 8577 & TC377983 & -3.13 & $8.26 \mathrm{E}-04$ \\
\hline 8578 & TC377984 & -3.02 & $1.30 \mathrm{E}-03$ \\
\hline 8579 & TC377996 & -4.25 & $1.97 \mathrm{E}-04$ \\
\hline 8580 & TC378006 & -2.13 & $5.38 \mathrm{E}-03$ \\
\hline 8581 & TC378019 & -4.65 & $4.87 \mathrm{E}-04$ \\
\hline 8582 & TC378055 & -4.07 & $4.75 \mathrm{E}-03$ \\
\hline 8583 & TC378056 & -6.74 & 4.49E-04 \\
\hline 8584 & TC378109 & -3.47 & $5.86 \mathrm{E}-04$ \\
\hline 8585 & TC378123 & -1.82 & $1.41 \mathrm{E}-05$ \\
\hline 8586 & TC378126 & -1.95 & $9.12 \mathrm{E}-03$ \\
\hline 8587 & TC378159 & -3.09 & $1.36 \mathrm{E}-03$ \\
\hline 8588 & TC378181 & -1.61 & $2.00 \mathrm{E}-02$ \\
\hline 8589 & TC378197 & -3.07 & $1.19 \mathrm{E}-03$ \\
\hline 8590 & TC378228 & -3.39 & $2.06 \mathrm{E}-03$ \\
\hline 8591 & TC378238 & -5.10 & $5.93 \mathrm{E}-05$ \\
\hline 8592 & TC378243 & -5.65 & $8.41 \mathrm{E}-05$ \\
\hline 8593 & TC378249 & -6.98 & $2.17 \mathrm{E}-04$ \\
\hline 8594 & TC378269 & -4.11 & $7.37 \mathrm{E}-04$ \\
\hline 8595 & TC378281 & -4.40 & $3.76 \mathrm{E}-04$ \\
\hline 8596 & TC378307 & $\begin{array}{l}-2.76 \\
\end{array}$ & $5.13 \mathrm{E}-03$ \\
\hline 8597 & TC378314 & -4.97 & $8.24 \mathrm{E}-05$ \\
\hline 8598 & TC378336 & -2.54 & $3.92 \mathrm{E}-03$ \\
\hline 8599 & TC378372 & -4.88 & $8.09 \mathrm{E}-04$ \\
\hline 8600 & TC378386 & -3.33 & $4.80 \mathrm{E}-03$ \\
\hline 8601 & TC378393 & -4.31 & $2.56 \mathrm{E}-04$ \\
\hline 8602 & TC378396 & $\begin{array}{l}-1.36 \\
\end{array}$ & $2.78 \mathrm{E}-02$ \\
\hline 8603 & TC378432 & -4.83 & $1.11 \mathrm{E}-04$ \\
\hline 8604 & TC378464 & -5.41 & $3.32 \mathrm{E}-03$ \\
\hline 8605 & TC378472 & -3.23 & $1.39 \mathrm{E}-03$ \\
\hline 8606 & TC378487 & -1.30 & $1.21 \mathrm{E}-02$ \\
\hline 8607 & TC378493 & -2.48 & $3.28 \mathrm{E}-03$ \\
\hline 8608 & TC378499 & -1.24 & $7.21 \mathrm{E}-02$ \\
\hline 8609 & TC378525 & -2.37 & $2.10 \mathrm{E}-02$ \\
\hline 8610 & TC378546 & -2.96 & $3.21 \mathrm{E}-03$ \\
\hline 8611 & TC378580 & -4.00 & $4.96 \mathrm{E}-04$ \\
\hline 8612 & TC378587 & -1.19 & $8.63 \mathrm{E}-04$ \\
\hline 8613 & TC378622 & -1.51 & $7.48 \mathrm{E}-04$ \\
\hline 8614 & TC378643 & -3.37 & $1.91 \mathrm{E}-03$ \\
\hline 8615 & TC378644 & -5.70 & $4.21 \mathrm{E}-04$ \\
\hline 8616 & TC378687 & -1.19 & $5.55 \mathrm{E}-02$ \\
\hline 8617 & TC378717 & -4.31 & $5.93 \mathrm{E}-04$ \\
\hline 8618 & TC378718 & -2.46 & $1.07 \mathrm{E}-03$ \\
\hline 8619 & TC378724 & -2.72 & $5.60 \mathrm{E}-03$ \\
\hline 8620 & TC378748 & -5.53 & $1.35 \mathrm{E}-03$ \\
\hline 8621 & TC378774 & -4.00 & $8.17 \mathrm{E}-04$ \\
\hline 8622 & TC378777 & $\begin{array}{l}-2.76 \\
\end{array}$ & $2.08 \mathrm{E}-04$ \\
\hline 8623 & TC378813 & -3.50 & $8.25 \mathrm{E}-04$ \\
\hline
\end{tabular}




\begin{tabular}{|c|c|c|c|}
\hline 8624 & TC378829 & -6.35 & $1.00 \mathrm{E}-04$ \\
\hline 8625 & TC378841 & -5.53 & $8.10 \mathrm{E}-06$ \\
\hline 8626 & TC378854 & -3.07 & $1.57 \mathrm{E}-02$ \\
\hline 8627 & TC378868 & -4.64 & $9.68 \mathrm{E}-04$ \\
\hline 8628 & TC378873 & -3.09 & $1.73 \mathrm{E}-03$ \\
\hline 8629 & TC378896 & -3.87 & $3.01 \mathrm{E}-03$ \\
\hline 8630 & TC378912 & -3.86 & $1.16 \mathrm{E}-03$ \\
\hline 8631 & TC378935 & -5.85 & $6.65 \mathrm{E}-05$ \\
\hline 8632 & TC378939 & -5.50 & $3.90 \mathrm{E}-04$ \\
\hline 8633 & TC378943 & -1.78 & $1.55 \mathrm{E}-02$ \\
\hline 8634 & TC378965 & -6.53 & 7.50E-05 \\
\hline 8635 & TC378982 & -3.32 & 1.13E-02 \\
\hline 8636 & TC378996 & -2.42 & $1.82 \mathrm{E}-03$ \\
\hline 8637 & TC379005 & -1.01 & $7.58 \mathrm{E}-04$ \\
\hline 8638 & TC379017 & -4.14 & $1.39 \mathrm{E}-03$ \\
\hline 8639 & TC379019 & -3.02 & $9.67 \mathrm{E}-03$ \\
\hline 8640 & TC379024 & -2.44 & 7.33E-03 \\
\hline 8641 & TC379063 & -3.46 & $1.09 \mathrm{E}-03$ \\
\hline 8642 & TC379087 & -1.14 & $6.52 \mathrm{E}-03$ \\
\hline 8643 & TC379096 & -4.51 & $1.14 \mathrm{E}-03$ \\
\hline 8644 & TC379113 & -3.00 & 9.56E-04 \\
\hline 8645 & TC379135 & -1.64 & $5.80 \mathrm{E}-02$ \\
\hline 8646 & TC379144 & -2.68 & $1.77 \mathrm{E}-03$ \\
\hline 8647 & TC379150 & -3.20 & $1.19 \mathrm{E}-03$ \\
\hline 8648 & TC379161 & -5.19 & $1.04 \mathrm{E}-03$ \\
\hline 8649 & TC379174 & -3.18 & $6.43 \mathrm{E}-04$ \\
\hline 8650 & TC379225 & -4.75 & $2.20 \mathrm{E}-03$ \\
\hline 8651 & ТС379250 & -2.97 & $1.58 \mathrm{E}-03$ \\
\hline 8652 & TC379254 & -3.03 & 7.33E-04 \\
\hline 8653 & TC379259 & -3.76 & $1.74 \mathrm{E}-03$ \\
\hline 8654 & TC379314 & -6.56 & 5.63E-04 \\
\hline 8655 & TC379318 & -1.84 & $1.20 \mathrm{E}-02$ \\
\hline 8656 & TC379370 & -6.37 & 4.99E-05 \\
\hline 8657 & TC379371 & -2.92 & $1.02 \mathrm{E}-03$ \\
\hline 8658 & TC379402 & -3.75 & 7.74E-04 \\
\hline 8659 & TC379406 & -3.57 & $6.99 \mathrm{E}-04$ \\
\hline 8660 & TC379421 & -2.78 & $9.06 \mathrm{E}-04$ \\
\hline 8661 & TC379425 & -2.87 & $2.52 \mathrm{E}-02$ \\
\hline 8662 & TC379426 & -1.86 & $2.54 \mathrm{E}-03$ \\
\hline 8663 & ТС379444 & -5.24 & 2.80E-05 \\
\hline 8664 & TC379445 & -1.67 & $2.03 \mathrm{E}-03$ \\
\hline 8665 & TC379461 & -3.48 & $3.66 \mathrm{E}-03$ \\
\hline 8666 & TC379498 & -7.74 & $5.02 \mathrm{E}-04$ \\
\hline 8667 & TC379515 & -4.51 & $2.89 \mathrm{E}-05$ \\
\hline 8668 & TC379579 & -3.64 & $5.16 \mathrm{E}-03$ \\
\hline 8669 & ТC379597 & -4.30 & $1.06 \mathrm{E}-03$ \\
\hline 8670 & TC379605 & -3.83 & $2.31 \mathrm{E}-04$ \\
\hline 8671 & TC379610 & -3.23 & 2.17E-03 \\
\hline 8672 & ТС379629 & -4.14 & $1.21 \mathrm{E}-04$ \\
\hline 8673 & TC379650 & -1.31 & $1.64 \mathrm{E}-02$ \\
\hline 8674 & TC379659 & -1.68 & $1.66 \mathrm{E}-02$ \\
\hline 8675 & TC379676 & -3.39 & $1.18 \mathrm{E}-03$ \\
\hline 8676 & TC379682 & -4.40 & 2.57E-04 \\
\hline 8677 & ТC379715 & -2.26 & $3.96 \mathrm{E}-03$ \\
\hline 8678 & ТС379719 & -5.81 & $6.02 \mathrm{E}-04$ \\
\hline 8679 & TC379745 & -2.81 & 5.05E-03 \\
\hline 8680 & TC379746 & -2.61 & $1.12 \mathrm{E}-02$ \\
\hline 8681 & TC379751 & -1.72 & 4.30E-02 \\
\hline 8682 & TC379754 & -3.60 & $6.15 \mathrm{E}-04$ \\
\hline 8683 & TC379765 & -4.89 & $1.99 \mathrm{E}-04$ \\
\hline 8684 & TC379789 & -4.43 & $6.11 \mathrm{E}-05$ \\
\hline 8685 & ТC379817 & -2.70 & $1.31 \mathrm{E}-03$ \\
\hline 8686 & TC379824 & -1.36 & $1.71 \mathrm{E}-04$ \\
\hline
\end{tabular}

\begin{tabular}{|c|c|c|c|}
\hline 8687 & TC379846 & -1.15 & $1.34 \mathrm{E}-01$ \\
\hline 8688 & TC379878 & -3.77 & $1.28 \mathrm{E}-04$ \\
\hline 8689 & TC379894 & -6.28 & $9.33 \mathrm{E}-04$ \\
\hline 8690 & TC379896 & -4.79 & $2.99 \mathrm{E}-03$ \\
\hline 8691 & TC379899 & -3.72 & $1.91 \mathrm{E}-03$ \\
\hline 8692 & TC379943 & -5.48 & $2.02 \mathrm{E}-03$ \\
\hline 8693 & TC379975 & -1.58 & $5.82 \mathrm{E}-02$ \\
\hline 8694 & TC380055 & -4.47 & $4.17 \mathrm{E}-04$ \\
\hline 8695 & TC380060 & -5.94 & 4.19E-04 \\
\hline 8696 & TC380104 & -4.42 & $3.90 \mathrm{E}-04$ \\
\hline 8697 & TC380131 & -5.09 & $4.92 \mathrm{E}-04$ \\
\hline 8698 & TC380132 & -1.96 & $1.57 \mathrm{E}-02$ \\
\hline 8699 & TC380147 & -7.20 & $1.39 \mathrm{E}-04$ \\
\hline 8700 & TC380177 & -1.67 & $2.06 \mathrm{E}-03$ \\
\hline 8701 & TC380188 & -4.36 & $1.90 \mathrm{E}-04$ \\
\hline 8702 & TC380191 & -1.06 & $4.65 \mathrm{E}-03$ \\
\hline 8703 & TC380197 & -2.32 & $1.06 \mathrm{E}-02$ \\
\hline 8704 & TC380258 & -2.03 & $3.67 E-03$ \\
\hline 8705 & TC380276 & -3.71 & $1.42 \mathrm{E}-03$ \\
\hline 8706 & TC380282 & -4.02 & $2.20 \mathrm{E}-03$ \\
\hline 8707 & TC380292 & -4.95 & $3.68 \mathrm{E}-04$ \\
\hline 8708 & TC380293 & -2.24 & $5.93 \mathrm{E}-06$ \\
\hline 8709 & TC380341 & -7.42 & $4.11 \mathrm{E}-04$ \\
\hline 8710 & TC380347 & -4.15 & 5.47E-04 \\
\hline 8711 & TC380407 & -3.87 & 7.09E-03 \\
\hline 8712 & TC380414 & -3.18 & $1.82 \mathrm{E}-02$ \\
\hline 8713 & TC380436 & -3.00 & $1.43 \mathrm{E}-04$ \\
\hline 8714 & TC380440 & -2.16 & $1.33 \mathrm{E}-02$ \\
\hline 8715 & TC380445 & -2.07 & $2.27 \mathrm{E}-04$ \\
\hline 8716 & TC380496 & -6.14 & $3.05 \mathrm{E}-04$ \\
\hline 8717 & TC380506 & -4.58 & $4.46 \mathrm{E}-03$ \\
\hline 8718 & TC380516 & -1.63 & $2.07 \mathrm{E}-03$ \\
\hline 8719 & TC380524 & -3.84 & $1.57 \mathrm{E}-03$ \\
\hline 8720 & TC380525 & -3.30 & $9.48 \mathrm{E}-05$ \\
\hline 8721 & TC380539 & -4.54 & $1.29 \mathrm{E}-05$ \\
\hline 8722 & TC380544 & -3.61 & $2.75 \mathrm{E}-03$ \\
\hline 8723 & TC380557 & -4.04 & $3.50 \mathrm{E}-03$ \\
\hline 8724 & TC380576 & -5.30 & $1.23 \mathrm{E}-03$ \\
\hline 8725 & TC380587 & -2.91 & $5.19 \mathrm{E}-03$ \\
\hline 8726 & TC380603 & -3.01 & $1.33 \mathrm{E}-04$ \\
\hline 8727 & TC380604 & -5.11 & $1.98 \mathrm{E}-05$ \\
\hline 8728 & TC380614 & -6.13 & $1.17 \mathrm{E}-03$ \\
\hline 8729 & TC380633 & -1.11 & $6.83 \mathrm{E}-03$ \\
\hline 8730 & TC380634 & -2.47 & $1.68 \mathrm{E}-02$ \\
\hline 8731 & TC380647 & -4.32 & $8.61 \mathrm{E}-04$ \\
\hline 8732 & TC380703 & -2.94 & $3.71 \mathrm{E}-04$ \\
\hline 8733 & TC380714 & -2.47 & $1.12 \mathrm{E}-03$ \\
\hline 8734 & TC380718 & -3.33 & $2.97 \mathrm{E}-03$ \\
\hline 8735 & TC380729 & -2.63 & $6.06 \mathrm{E}-03$ \\
\hline 8736 & TC380746 & -3.42 & $2.00 E-04$ \\
\hline 8737 & TC380751 & -6.07 & $1.41 \mathrm{E}-04$ \\
\hline 8738 & TC380757 & -6.05 & $6.79 \mathrm{E}-04$ \\
\hline 8739 & TC380778 & -5.14 & $4.84 \mathrm{E}-04$ \\
\hline 8740 & TC380787 & -2.65 & $1.07 \mathrm{E}-03$ \\
\hline 8741 & TC380803 & -1.33 & $6.99 \mathrm{E}-04$ \\
\hline 8742 & TC380809 & -1.26 & $4.12 \mathrm{E}-02$ \\
\hline 8743 & TC380813 & -1.68 & $1.64 \mathrm{E}-03$ \\
\hline 8744 & TC380817 & -3.74 & $6.66 \mathrm{E}-03$ \\
\hline 8745 & TC380874 & -2.02 & $5.52 \mathrm{E}-03$ \\
\hline 8746 & TC380893 & -5.09 & $3.77 \mathrm{E}-04$ \\
\hline 8747 & TC380912 & -1.24 & $5.40 \mathrm{E}-03$ \\
\hline 8748 & TC380925 & -3.18 & $2.49 \mathrm{E}-02$ \\
\hline 8749 & TC381012 & -1.24 & $5.16 \mathrm{E}-03$ \\
\hline
\end{tabular}

\begin{tabular}{|c|c|c|c|}
\hline 8750 & TC381014 & -4.51 & $1.07 \mathrm{E}-03$ \\
\hline 8751 & TC381032 & -2.80 & $9.52 \mathrm{E}-04$ \\
\hline 8752 & TC381061 & -1.13 & $1.63 \mathrm{E}-03$ \\
\hline 8753 & TC381096 & -2.65 & $1.38 \mathrm{E}-04$ \\
\hline 8754 & TC381132 & -1.08 & $9.83 \mathrm{E}-03$ \\
\hline 8755 & TC381155 & -3.45 & 3.33E-02 \\
\hline 8756 & TC381170 & -6.24 & 5.97E-04 \\
\hline 8757 & TC381203 & -4.46 & $9.12 \mathrm{E}-04$ \\
\hline 8758 & TC381217 & -6.02 & $6.41 \mathrm{E}-04$ \\
\hline 8759 & TC381290 & -5.58 & $8.45 \mathrm{E}-04$ \\
\hline 8760 & TC381294 & -2.60 & $1.23 \mathrm{E}-03$ \\
\hline 8761 & TC381340 & -2.64 & $9.13 \mathrm{E}-04$ \\
\hline 8762 & TC381354 & -1.90 & $1.77 \mathrm{E}-03$ \\
\hline 8763 & TC381362 & -5.13 & $1.71 \mathrm{E}-04$ \\
\hline 8764 & TC381428 & -5.72 & 3.67E-04 \\
\hline 8765 & TC381480 & -4.08 & $4.06 \mathrm{E}-04$ \\
\hline 8766 & TC381554 & -4.05 & $5.40 \mathrm{E}-03$ \\
\hline 8767 & TC381558 & -6.26 & $1.02 \mathrm{E}-04$ \\
\hline 8768 & TC381591 & -2.91 & $5.59 \mathrm{E}-04$ \\
\hline 8769 & TC381595 & -3.44 & $3.99 \mathrm{E}-03$ \\
\hline 8770 & TC381601 & -5.31 & $6.48 \mathrm{E}-04$ \\
\hline 8771 & TC381614 & -1.77 & $3.26 \mathrm{E}-03$ \\
\hline 8772 & TC381638 & -5.09 & $4.88 \mathrm{E}-04$ \\
\hline 8773 & TC381721 & -5.60 & $7.89 \mathrm{E}-04$ \\
\hline 8774 & TC381809 & -1.84 & $1.25 \mathrm{E}-02$ \\
\hline 8775 & TC381844 & -5.98 & 3.60E-04 \\
\hline 8776 & TC381925 & -2.14 & $1.98 \mathrm{E}-03$ \\
\hline 8777 & TC381928 & -1.63 & $1.91 \mathrm{E}-02$ \\
\hline 8778 & TC381960 & -2.02 & $1.03 \mathrm{E}-03$ \\
\hline 8779 & TC381979 & -4.71 & $7.52 \mathrm{E}-03$ \\
\hline 8780 & TC381988 & -5.51 & $5.94 \mathrm{E}-04$ \\
\hline 8781 & TC382017 & -2.24 & $1.67 \mathrm{E}-02$ \\
\hline 8782 & TC382072 & -4.75 & $1.30 \mathrm{E}-03$ \\
\hline 8783 & TC382098 & -3.93 & $5.71 \mathrm{E}-04$ \\
\hline 8784 & TC382109 & -2.26 & $3.38 \mathrm{E}-04$ \\
\hline 8785 & TC382115 & -3.20 & $1.12 \mathrm{E}-03$ \\
\hline 8786 & TC382116 & -1.73 & $1.67 \mathrm{E}-02$ \\
\hline 8787 & TC382118 & -1.21 & $2.73 \mathrm{E}-03$ \\
\hline 8788 & TC382129 & -1.67 & $3.73 \mathrm{E}-02$ \\
\hline 8789 & TC382157 & -3.87 & $1.28 \mathrm{E}-03$ \\
\hline 8790 & TC382313 & -2.89 & 2.44E-04 \\
\hline 8791 & TC382344 & -4.90 & $6.13 \mathrm{E}-05$ \\
\hline 8792 & TC382357 & -5.52 & $3.09 \mathrm{E}-03$ \\
\hline 8793 & TC382370 & -2.62 & $1.06 \mathrm{E}-03$ \\
\hline 8794 & TC382379 & -3.91 & $1.21 \mathrm{E}-05$ \\
\hline 8795 & TC382384 & -3.08 & $2.33 \mathrm{E}-03$ \\
\hline 8796 & TC382480 & -1.55 & 1.30E-02 \\
\hline 8797 & TC382491 & -1.80 & $2.39 \mathrm{E}-02$ \\
\hline 8798 & TC382501 & -3.64 & $2.05 \mathrm{E}-04$ \\
\hline 8799 & TC382591 & -2.02 & $1.74 \mathrm{E}-02$ \\
\hline 8800 & TC382619 & -2.16 & $2.17 \mathrm{E}-03$ \\
\hline 8801 & TC382642 & -1.79 & $3.40 \mathrm{E}-03$ \\
\hline 8802 & TC382646 & -1.34 & $1.95 \mathrm{E}-03$ \\
\hline 8803 & TC382681 & -2.93 & $2.42 \mathrm{E}-03$ \\
\hline 8804 & TC382715 & -6.13 & 4.74E-04 \\
\hline 8805 & TC382723 & -4.35 & $5.41 \mathrm{E}-04$ \\
\hline 8806 & TC382764 & -5.77 & $1.82 \mathrm{E}-05$ \\
\hline 8807 & TC382829 & -1.33 & $2.05 \mathrm{E}-02$ \\
\hline 8808 & TC382831 & -1.61 & $2.24 \mathrm{E}-03$ \\
\hline 8809 & TC382838 & -1.88 & 7.69E-04 \\
\hline 8810 & TC382842 & -3.62 & $1.93 \mathrm{E}-03$ \\
\hline 8811 & TC382954 & -4.17 & $2.55 \mathrm{E}-04$ \\
\hline 8812 & TC382962 & -2.51 & $3.77 \mathrm{E}-03$ \\
\hline
\end{tabular}




\begin{tabular}{|c|c|c|c|}
\hline 8813 & TC382998 & -1.17 & $1.00 \mathrm{E}-02$ \\
\hline 8814 & TC383002 & -2.28 & $1.27 \mathrm{E}-03$ \\
\hline 8815 & TC383011 & -5.36 & $1.38 \mathrm{E}-03$ \\
\hline 8816 & TC383028 & -1.30 & $3.78 \mathrm{E}-02$ \\
\hline 8817 & TC383039 & -1.16 & $7.08 \mathrm{E}-03$ \\
\hline 8818 & TC383055 & -2.30 & $1.51 \mathrm{E}-02$ \\
\hline 8819 & TC383086 & -1.45 & $1.24 \mathrm{E}-02$ \\
\hline 8820 & TC383115 & $\begin{array}{l}-1.66 \\
\end{array}$ & $1.88 \mathrm{E}-02$ \\
\hline 8821 & TC383192 & -6.55 & $4.30 \mathrm{E}-04$ \\
\hline 8822 & TC383249 & -2.85 & $3.67 \mathrm{E}-03$ \\
\hline 8823 & TC383254 & -3.98 & $2.26 \mathrm{E}-03$ \\
\hline 8824 & TC383289 & -4.60 & $4.76 \mathrm{E}-04$ \\
\hline 8825 & TC383349 & $\begin{array}{l}-1.36 \\
\end{array}$ & $8.23 \mathrm{E}-03$ \\
\hline 8826 & TC383351 & -3.15 & $1.26 \mathrm{E}-02$ \\
\hline 8827 & TC383377 & -6.47 & $1.45 \mathrm{E}-04$ \\
\hline 8828 & TC383407 & -4.93 & $2.48 \mathrm{E}-03$ \\
\hline 8829 & TC383411 & -2.50 & $7.33 \mathrm{E}-03$ \\
\hline 8830 & TC383450 & -3.18 & $3.37 \mathrm{E}-03$ \\
\hline 8831 & TC383580 & -2.00 & $9.88 \mathrm{E}-04$ \\
\hline 8832 & TC383582 & -3.39 & $6.52 \mathrm{E}-05$ \\
\hline 8833 & TC383588 & -5.32 & $1.22 \mathrm{E}-03$ \\
\hline 8834 & TC383602 & $\begin{array}{l}-6.01 \\
\end{array}$ & $1.75 \mathrm{E}-03$ \\
\hline 8835 & TC383629 & -3.23 & $8.83 \mathrm{E}-02$ \\
\hline 8836 & TC383673 & -1.45 & $4.93 \mathrm{E}-02$ \\
\hline 8837 & TC383685 & -2.46 & $8.50 \mathrm{E}-03$ \\
\hline 8838 & TC383732 & -5.93 & $3.21 \mathrm{E}-03$ \\
\hline 8839 & TC383738 & -2.97 & $2.58 \mathrm{E}-03$ \\
\hline 8840 & TC383780 & -3.11 & $1.05 \mathrm{E}-03$ \\
\hline 8841 & TC383840 & -6.78 & $6.74 \mathrm{E}-04$ \\
\hline 8842 & TC383846 & -8.58 & $3.02 \mathrm{E}-04$ \\
\hline 8843 & TC383916 & -3.88 & $8.24 \mathrm{E}-03$ \\
\hline 8844 & TC383926 & $\begin{array}{l}-2.53 \\
\end{array}$ & $8.71 \mathrm{E}-04$ \\
\hline 8845 & TC383952 & -4.35 & $9.59 \mathrm{E}-04$ \\
\hline 8846 & TC383976 & -4.09 & $3.16 \mathrm{E}-04$ \\
\hline 8847 & TC383994 & -2.82 & $6.88 \mathrm{E}-03$ \\
\hline 8848 & TC384028 & -1.03 & $1.09 \mathrm{E}-04$ \\
\hline 8849 & TC384081 & -3.66 & $2.18 \mathrm{E}-03$ \\
\hline 8850 & TC384142 & -3.91 & $5.47 \mathrm{E}-03$ \\
\hline 8851 & TC384154 & -1.73 & $3.01 \mathrm{E}-03$ \\
\hline 8852 & TC384207 & -2.14 & $5.22 \mathrm{E}-03$ \\
\hline 8853 & TC384224 & \begin{tabular}{|l|}
-3.43 \\
\end{tabular} & $1.13 \mathrm{E}-03$ \\
\hline 8854 & TC384302 & -1.69 & $1.22 \mathrm{E}-01$ \\
\hline 8855 & TC384500 & -1.99 & $3.74 \mathrm{E}-03$ \\
\hline 8856 & TC384527 & -5.55 & 5.04E-04 \\
\hline 8857 & TC384532 & -1.20 & $6.77 \mathrm{E}-02$ \\
\hline 8858 & TC384570 & -2.34 & $3.06 \mathrm{E}-03$ \\
\hline 8859 & TC384674 & -4.06 & $6.36 \mathrm{E}-05$ \\
\hline 8860 & TC384694 & $\begin{array}{l}-4.93 \\
\end{array}$ & $1.13 \mathrm{E}-04$ \\
\hline 8861 & TC384770 & -1.40 & $4.34 \mathrm{E}-03$ \\
\hline 8862 & TC384808 & -3.64 & $4.83 \mathrm{E}-04$ \\
\hline 8863 & TC384895 & -1.26 & $3.12 \mathrm{E}-02$ \\
\hline 8864 & TC384930 & -3.10 & $6.21 \mathrm{E}-03$ \\
\hline 8865 & TC385000 & -2.45 & $4.01 \mathrm{E}-03$ \\
\hline 8866 & TC385071 & -2.44 & $2.63 \mathrm{E}-03$ \\
\hline 8867 & TC385173 & -1.72 & $1.03 \mathrm{E}-01$ \\
\hline 8868 & TC385220 & -2.99 & $1.54 \mathrm{E}-05$ \\
\hline 8869 & TC385246 & $\begin{array}{l}-1.80 \\
\end{array}$ & $2.21 \mathrm{E}-02$ \\
\hline 8870 & TC385346 & $\begin{array}{l}-1.47 \\
\end{array}$ & $2.24 \mathrm{E}-02$ \\
\hline 8871 & TC385360 & -3.82 & $1.35 \mathrm{E}-03$ \\
\hline 8872 & TC385392 & -1.53 & $1.40 \mathrm{E}-02$ \\
\hline 8873 & TC385626 & -4.88 & $5.74 \mathrm{E}-04$ \\
\hline 8874 & TC385636 & -2.27 & $2.83 \mathrm{E}-02$ \\
\hline $8 / 5$ & ТС 385656 & -4.75 & $3.33 \mathrm{E}-06$ \\
\hline
\end{tabular}

\begin{tabular}{|c|c|c|c|}
\hline 8876 & TC385714 & -2.03 & $7.83 \mathrm{E}-03$ \\
\hline 8877 & TC386024 & -1.94 & $3.49 \mathrm{E}-02$ \\
\hline 8878 & TC386064 & -3.63 & $5.51 \mathrm{E}-04$ \\
\hline 8879 & TC386275 & -2.75 & $4.15 \mathrm{E}-03$ \\
\hline 8880 & TC386292 & -1.93 & $2.13 \mathrm{E}-03$ \\
\hline 8881 & TC386337 & -1.55 & $6.91 \mathrm{E}-03$ \\
\hline 8882 & TC386351 & -2.56 & $6.05 \mathrm{E}-03$ \\
\hline 8883 & TC386594 & -1.57 & $2.03 \mathrm{E}-02$ \\
\hline 8884 & TC386681 & -6.07 & $1.51 \mathrm{E}-05$ \\
\hline 8885 & TC386732 & -5.81 & $5.19 \mathrm{E}-05$ \\
\hline 8886 & TC387639 & -4.66 & $1.99 \mathrm{E}-03$ \\
\hline 8887 & TC387843 & -3.69 & $3.82 \mathrm{E}-03$ \\
\hline 8888 & TC388220 & -5.30 & $6.76 \mathrm{E}-04$ \\
\hline 8889 & TC388238 & -4.12 & $5.27 \mathrm{E}-04$ \\
\hline 8890 & TC388361 & -4.69 & $1.27 \mathrm{E}-03$ \\
\hline 8891 & TC388430 & -4.16 & $5.83 \mathrm{E}-04$ \\
\hline 8892 & TC388515 & -2.90 & $1.37 \mathrm{E}-03$ \\
\hline 8893 & TC388521 & -6.77 & $9.88 \mathrm{E}-04$ \\
\hline 8894 & TC388706 & -5.46 & $2.60 \mathrm{E}-04$ \\
\hline 8895 & TC388707 & -1.17 & $1.51 \mathrm{E}-03$ \\
\hline 8896 & TC388832 & -2.03 & $6.28 \mathrm{E}-04$ \\
\hline 8897 & TC388863 & -3.51 & 6.64E-04 \\
\hline 8898 & TC389056 & -2.73 & $6.30 \mathrm{E}-03$ \\
\hline 8899 & TC389097 & -2.42 & $1.44 \mathrm{E}-02$ \\
\hline 8900 & TC389283 & -2.74 & $2.50 \mathrm{E}-03$ \\
\hline 8901 & TC389337 & -2.45 & $2.08 \mathrm{E}-02$ \\
\hline 8902 & TC389421 & -5.47 & $1.90 \mathrm{E}-04$ \\
\hline 8903 & TC389428 & -3.36 & $1.09 \mathrm{E}-04$ \\
\hline 8904 & TC389495 & -2.31 & $1.55 \mathrm{E}-03$ \\
\hline 8905 & TC389618 & -4.35 & $9.60 \mathrm{E}-04$ \\
\hline 8906 & TC389683 & -6.35 & 3.18E-04 \\
\hline 8907 & TC389795 & -2.57 & $2.05 \mathrm{E}-02$ \\
\hline 8908 & TC389871 & -3.11 & $1.59 \mathrm{E}-03$ \\
\hline 8909 & TC389872 & -3.29 & $2.65 \mathrm{E}-04$ \\
\hline 8910 & TC390042 & -4.93 & $1.97 \mathrm{E}-03$ \\
\hline 8911 & TC390057 & -4.67 & $3.80 \mathrm{E}-03$ \\
\hline 8912 & TC390443 & -1.90 & $5.03 \mathrm{E}-03$ \\
\hline 8913 & TC390532 & -2.02 & $3.77 \mathrm{E}-03$ \\
\hline 8914 & TC390567 & -2.87 & $4.72 \mathrm{E}-03$ \\
\hline 8915 & TC390883 & $\begin{array}{l}-2.73 \\
\end{array}$ & $1.25 \mathrm{E}-03$ \\
\hline 8916 & TC391063 & -1.98 & $2.02 \mathrm{E}-03$ \\
\hline 8917 & TC391148 & -4.53 & $1.66 \mathrm{E}-03$ \\
\hline 8918 & TC391293 & -2.83 & $1.09 \mathrm{E}-03$ \\
\hline 8919 & TC391420 & -3.29 & $3.44 \mathrm{E}-03$ \\
\hline 8920 & TC391643 & -3.51 & $4.54 \mathrm{E}-03$ \\
\hline 8921 & TC392128 & -2.48 & $5.32 \mathrm{E}-03$ \\
\hline 8922 & TC392270 & -4.44 & $1.13 \mathrm{E}-03$ \\
\hline 8923 & TC392635 & -3.99 & $1.93 \mathrm{E}-02$ \\
\hline 8924 & TC392720 & -1.25 & $2.35 \mathrm{E}-02$ \\
\hline 8925 & TC392777 & -3.81 & $1.05 E-03$ \\
\hline 8926 & TC392812 & -1.06 & $5.61 \mathrm{E}-03$ \\
\hline 8927 & TC393126 & -4.28 & $1.37 \mathrm{E}-04$ \\
\hline 8928 & TC393138 & -3.80 & $3.23 \mathrm{E}-03$ \\
\hline 8929 & TC393205 & -2.68 & $1.19 \mathrm{E}-02$ \\
\hline 8930 & TC393216 & -1.57 & $3.16 \mathrm{E}-03$ \\
\hline 8931 & TC393310 & -1.92 & $1.07 \mathrm{E}-05$ \\
\hline 8932 & TC393597 & -1.41 & $4.29 \mathrm{E}-02$ \\
\hline 8933 & TC393629 & -3.05 & $8.75 \mathrm{E}-03$ \\
\hline 8934 & TC394459 & -6.81 & $5.02 E-04$ \\
\hline 8935 & TC394553 & -2.53 & $1.04 \mathrm{E}-02$ \\
\hline 8936 & TC394733 & -3.63 & $2.94 \mathrm{E}-03$ \\
\hline 8937 & TC394891 & -1.17 & $2.44 \mathrm{E}-03$ \\
\hline 8938 & ТC395447 & -1.30 & $1.18 \mathrm{E}-03$ \\
\hline
\end{tabular}

\begin{tabular}{|c|c|c|c|}
\hline 8939 & TC395666 & -3.50 & $3.81 \mathrm{E}-04$ \\
\hline 8940 & TC395803 & -5.21 & $2.07 \mathrm{E}-04$ \\
\hline 8941 & TC396009 & -2.39 & $5.96 \mathrm{E}-02$ \\
\hline 8942 & TC396061 & -3.57 & $5.49 \mathrm{E}-04$ \\
\hline 8943 & TC396102 & -4.82 & $3.03 \mathrm{E}-04$ \\
\hline 8944 & TC396299 & -1.37 & $3.92 \mathrm{E}-02$ \\
\hline 8945 & TC396352 & -3.98 & $5.04 \mathrm{E}-03$ \\
\hline 8946 & TC396426 & -1.63 & $2.31 \mathrm{E}-02$ \\
\hline 8947 & TC397084 & -4.39 & $9.31 \mathrm{E}-04$ \\
\hline 8948 & TC397253 & -4.61 & $2.34 \mathrm{E}-04$ \\
\hline 8949 & TC397341 & -1.78 & $3.07 \mathrm{E}-04$ \\
\hline 8950 & TC397354 & -3.60 & $2.41 \mathrm{E}-04$ \\
\hline 8951 & TC398219 & -3.45 & $1.50 \mathrm{E}-04$ \\
\hline 8952 & TC398431 & -6.49 & $5.73 \mathrm{E}-04$ \\
\hline 8953 & TC398475 & -2.17 & $6.71 \mathrm{E}-03$ \\
\hline 8954 & TC398668 & -3.23 & $1.30 \mathrm{E}-03$ \\
\hline 8955 & TC398920 & -4.69 & $6.41 \mathrm{E}-04$ \\
\hline 8956 & TC399074 & -1.75 & $1.92 \mathrm{E}-03$ \\
\hline 8957 & TC399158 & -1.35 & $7.95 \mathrm{E}-02$ \\
\hline 8958 & TC399280 & -1.56 & $9.06 \mathrm{E}-02$ \\
\hline 8959 & TC399475 & -4.76 & $9.55 \mathrm{E}-04$ \\
\hline 8960 & TC399666 & -2.33 & $3.99 \mathrm{E}-04$ \\
\hline 8961 & TC399732 & -4.83 & $3.49 \mathrm{E}-04$ \\
\hline 8962 & TC399776 & -1.57 & $1.62 \mathrm{E}-02$ \\
\hline 8963 & TC400353 & -7.28 & $3.32 \mathrm{E}-04$ \\
\hline 8964 & TC400608 & -1.87 & $1.17 \mathrm{E}-02$ \\
\hline 8965 & TC400994 & -7.08 & $5.17 \mathrm{E}-03$ \\
\hline 8966 & TC401006 & -3.39 & $3.61 \mathrm{E}-03$ \\
\hline 8967 & TC401126 & -1.12 & $2.15 \mathrm{E}-02$ \\
\hline 8968 & TC401635 & -4.16 & $1.31 \mathrm{E}-03$ \\
\hline 8969 & TC402133 & -3.10 & $1.17 \mathrm{E}-03$ \\
\hline 8970 & TC402136 & -2.92 & $2.28 \mathrm{E}-03$ \\
\hline 8971 & TC402270 & -4.47 & $2.48 \mathrm{E}-03$ \\
\hline 8972 & TC402586 & -2.87 & $9.34 \mathrm{E}-03$ \\
\hline 8973 & TC402644 & -5.07 & $6.43 \mathrm{E}-04$ \\
\hline 8974 & TC402957 & -2.31 & $1.91 \mathrm{E}-03$ \\
\hline 8975 & TC403002 & -2.35 & $2.89 \mathrm{E}-03$ \\
\hline 8976 & TC403049 & -1.32 & $2.44 \mathrm{E}-03$ \\
\hline 8977 & TC403175 & -1.73 & $1.07 \mathrm{E}-03$ \\
\hline 8978 & TC403764 & -1.98 & $2.86 \mathrm{E}-03$ \\
\hline 8979 & TC403838 & -5.34 & $5.35 \mathrm{E}-05$ \\
\hline 8980 & TC403923 & -2.19 & $4.11 \mathrm{E}-03$ \\
\hline 8981 & TC403989 & -3.38 & $4.38 \mathrm{E}-04$ \\
\hline 8982 & TC404016 & -5.47 & $1.36 \mathrm{E}-03$ \\
\hline 8983 & TC404395 & -2.26 & $1.86 \mathrm{E}-02$ \\
\hline 8984 & TC404502 & -1.49 & $1.83 \mathrm{E}-02$ \\
\hline 8985 & TC404701 & -2.76 & $1.22 \mathrm{E}-03$ \\
\hline 8986 & TC405014 & -2.34 & $3.59 \mathrm{E}-03$ \\
\hline 8987 & TC405282 & -3.90 & $1.86 \mathrm{E}-03$ \\
\hline 8988 & TC405592 & -6.59 & $2.35 \mathrm{E}-04$ \\
\hline 8989 & TC405764 & -3.09 & $5.28 \mathrm{E}-04$ \\
\hline 8990 & TC406324 & -4.55 & $8.07 \mathrm{E}-05$ \\
\hline 8991 & TC406846 & -4.03 & $1.32 \mathrm{E}-03$ \\
\hline 8992 & TC407293 & -4.02 & $2.10 \mathrm{E}-04$ \\
\hline 8993 & TC407560 & -4.65 & $5.26 \mathrm{E}-04$ \\
\hline 8994 & TC407872 & -1.38 & $3.68 \mathrm{E}-03$ \\
\hline 8995 & TC407881 & -2.07 & $3.76 \mathrm{E}-04$ \\
\hline 8996 & TC408045 & -3.10 & $6.26 \mathrm{E}-03$ \\
\hline 8997 & TC408244 & -1.61 & $5.28 \mathrm{E}-03$ \\
\hline 8998 & TC408316 & -2.07 & $3.56 \mathrm{E}-03$ \\
\hline 8999 & TC408479 & -4.76 & $3.96 \mathrm{E}-04$ \\
\hline 9000 & TC408745 & -7.92 & $1.77 \mathrm{E}-04$ \\
\hline 9001 & TC408847 & -1.42 & $3.06 \mathrm{E}-02$ \\
\hline
\end{tabular}




\begin{tabular}{|c|c|c|c|}
\hline 9002 & TC409047 & -2.64 & $1.83 \mathrm{E}-02$ \\
\hline 9003 & TC409229 & -3.64 & $1.33 \mathrm{E}-03$ \\
\hline 9004 & TC409572 & -1.79 & $1.60 \mathrm{E}-02$ \\
\hline 9005 & TC409664 & -2.02 & $1.95 E-03$ \\
\hline 9006 & TC409734 & -2.30 & $8.07 E-03$ \\
\hline 9007 & TC410019 & -3.03 & $5.98 \mathrm{E}-04$ \\
\hline 9008 & TC410073 & -1.40 & $1.85 \mathrm{E}-02$ \\
\hline 9009 & TC410529 & -3.35 & $3.48 \mathrm{E}-02$ \\
\hline 9010 & TC410822 & -2.82 & $8.98 \mathrm{E}-02$ \\
\hline 9011 & TC411312 & -3.15 & $9.11 \mathrm{E}-04$ \\
\hline 9012 & TC411737 & -5.54 & $6.17 \mathrm{E}-05$ \\
\hline 9013 & TC413029 & -1.74 & $2.07 E-03$ \\
\hline 9014 & TC413047 & -3.75 & $7.39 \mathrm{E}-04$ \\
\hline 9015 & TC413091 & -1.93 & $4.97 \mathrm{E}-03$ \\
\hline 9016 & TC413917 & -1.51 & $4.25 \mathrm{E}-02$ \\
\hline 9017 & TC413996 & -1.43 & $1.53 \mathrm{E}-02$ \\
\hline 9018 & TC414094 & -4.82 & $2.18 \mathrm{E}-02$ \\
\hline 9019 & TC415803 & -1.30 & $3.32 \mathrm{E}-03$ \\
\hline 9020 & TC415869 & -4.26 & $4.90 \mathrm{E}-04$ \\
\hline 9021 & TC416104 & -1.69 & $1.46 \mathrm{E}-02$ \\
\hline 9022 & TC416420 & -5.70 & $8.00 \mathrm{E}-04$ \\
\hline 9023 & TC416933 & -4.32 & $1.22 \mathrm{E}-03$ \\
\hline 9024 & TC417507 & -8.06 & $2.53 \mathrm{E}-04$ \\
\hline 9025 & TC417518 & -5.25 & $1.80 \mathrm{E}-06$ \\
\hline 9026 & TC417783 & -1.72 & $7.56 \mathrm{E}-03$ \\
\hline 9027 & TC417876 & -3.94 & 7.65E-04 \\
\hline 9028 & TC417921 & -4.30 & $6.04 \mathrm{E}-03$ \\
\hline 9029 & TC418171 & $\begin{array}{l}-1.18 \\
\end{array}$ & $1.29 \mathrm{E}-02$ \\
\hline 9030 & TC418277 & -6.45 & $1.00 E-03$ \\
\hline 9031 & TC418732 & -2.67 & $6.70 E-03$ \\
\hline 9032 & TC418801 & -1.81 & $2.86 \mathrm{E}-02$ \\
\hline 9033 & TC419189 & -1.23 & $4.05 E-03$ \\
\hline 9034 & TC419350 & -7.16 & $1.13 \mathrm{E}-04$ \\
\hline 9035 & TC419433 & -4.75 & $1.93 \mathrm{E}-04$ \\
\hline 9036 & TC419481 & -2.25 & $4.02 E-04$ \\
\hline 9037 & TC420146 & -3.78 & $4.80 \mathrm{E}-04$ \\
\hline 9038 & TC420212 & -3.42 & $6.42 \mathrm{E}-03$ \\
\hline 9039 & TC420771 & -2.50 & $3.75 E-03$ \\
\hline 9040 & TC421147 & -3.97 & $6.65 \mathrm{E}-03$ \\
\hline 9041 & TC421445 & -5.71 & 2.46E-05 \\
\hline 9042 & TC421504 & -3.81 & $1.37 \mathrm{E}-02$ \\
\hline 9043 & TC421873 & -4.35 & $2.88 \mathrm{E}-04$ \\
\hline 9044 & TC421958 & -2.05 & $1.08 \mathrm{E}-02$ \\
\hline 9045 & TC422612 & -1.46 & $9.15 E-03$ \\
\hline 9046 & TC422833 & -7.77 & 4.04E-04 \\
\hline 9047 & TC422844 & -3.89 & $6.96 \mathrm{E}-04$ \\
\hline 9048 & TC423055 & -6.80 & $9.60 \mathrm{E}-04$ \\
\hline 9049 & TC423437 & $\begin{array}{l}-1.78 \\
\end{array}$ & $1.66 \mathrm{E}-02$ \\
\hline 9050 & TC423926 & -5.59 & $8.77 \mathrm{E}-03$ \\
\hline 9051 & TC424683 & -1.88 & $5.71 E-02$ \\
\hline 9052 & TC425082 & -2.77 & $5.72 \mathrm{E}-03$ \\
\hline 9053 & TC425426 & -5.01 & $1.86 \mathrm{E}-04$ \\
\hline 9054 & TC425574 & -2.73 & $1.94 \mathrm{E}-03$ \\
\hline 9055 & TC425664 & -4.88 & $2.43 \mathrm{E}-04$ \\
\hline 9056 & TC426194 & -5.94 & $5.39 \mathrm{E}-05$ \\
\hline 9057 & TC426384 & -1.98 & $8.15 \mathrm{E}-03$ \\
\hline 9058 & TC426449 & -2.48 & $5.33 \mathrm{E}-03$ \\
\hline 9059 & TC427584 & -4.75 & $2.09 \mathrm{E}-04$ \\
\hline 9060 & TC427873 & -1.67 & $6.70 \mathrm{E}-03$ \\
\hline 9061 & TC427877 & -1.53 & $3.61 \mathrm{E}-03$ \\
\hline 9062 & TC429003 & -3.38 & $4.88 \mathrm{E}-04$ \\
\hline 9063 & TC429214 & -4.29 & $3.52 \mathrm{E}-04$ \\
\hline 0064 & $29 / 94$ & -3.41 & \\
\hline
\end{tabular}

\begin{tabular}{|c|c|c|c|}
\hline 9065 & TC430203 & -5.02 & 4.14E-04 \\
\hline 9066 & TC430306 & -4.30 & $1.14 \mathrm{E}-03$ \\
\hline 9067 & TC430417 & -4.67 & $1.05 \mathrm{E}-03$ \\
\hline 9068 & TC430657 & -4.72 & $3.03 E-05$ \\
\hline 9069 & TC430852 & -2.06 & $5.42 \mathrm{E}-02$ \\
\hline 9070 & TC431023 & -2.41 & $2.60 \mathrm{E}-03$ \\
\hline 9071 & TC431042 & -4.66 & $2.23 \mathrm{E}-03$ \\
\hline 9072 & TC431160 & -1.33 & $1.39 \mathrm{E}-02$ \\
\hline 9073 & TC431230 & -3.37 & $5.71 \mathrm{E}-04$ \\
\hline 9074 & TC431356 & $\begin{array}{l}-1.26 \\
\end{array}$ & $1.28 \mathrm{E}-03$ \\
\hline 9075 & TC431486 & -3.57 & $3.83 \mathrm{E}-04$ \\
\hline 9076 & TC431974 & -1.89 & $5.75 \mathrm{E}-02$ \\
\hline 9077 & TC432113 & -1.56 & $5.66 \mathrm{E}-03$ \\
\hline 9078 & TC432715 & \begin{tabular}{|l|}
-4.61 \\
\end{tabular} & $1.03 \mathrm{E}-03$ \\
\hline 9079 & TC432820 & -1.52 & $2.23 \mathrm{E}-02$ \\
\hline 9080 & TC432834 & -2.10 & $2.80 \mathrm{E}-03$ \\
\hline 9081 & TC432852 & -1.47 & $3.43 \mathrm{E}-02$ \\
\hline 9082 & TC433069 & -1.90 & $1.29 \mathrm{E}-04$ \\
\hline 9083 & TC433336 & -1.73 & $2.67 \mathrm{E}-02$ \\
\hline 9084 & TC433358 & -4.95 & $1.76 \mathrm{E}-03$ \\
\hline 9085 & TC433908 & -6.91 & $2.27 \mathrm{E}-04$ \\
\hline 9086 & TC434062 & -1.37 & $3.09 \mathrm{E}-03$ \\
\hline 9087 & TC434876 & -2.41 & $7.09 \mathrm{E}-02$ \\
\hline 9088 & TC434958 & -1.61 & $8.59 \mathrm{E}-03$ \\
\hline 9089 & TC434983 & -5.41 & $2.14 \mathrm{E}-05$ \\
\hline 9090 & TC435313 & \begin{tabular}{|l|} 
\\
\end{tabular} & $3.85 \mathrm{E}-04$ \\
\hline 9091 & TC435460 & -4.51 & $1.67 \mathrm{E}-03$ \\
\hline 9092 & TC435669 & -4.30 & $7.38 \mathrm{E}-03$ \\
\hline 9093 & TC435999 & -1.85 & $1.71 \mathrm{E}-03$ \\
\hline 9094 & TC436067 & -6.54 & $6.52 \mathrm{E}-05$ \\
\hline 9095 & TC436313 & -2.26 & $4.35 \mathrm{E}-02$ \\
\hline 9096 & TC436567 & -6.19 & $7.76 \mathrm{E}-04$ \\
\hline 9097 & TC436863 & -3.12 & $9.63 \mathrm{E}-03$ \\
\hline 9098 & TC436965 & -3.32 & $5.63 \mathrm{E}-04$ \\
\hline 9099 & TC437323 & -4.34 & $1.08 \mathrm{E}-02$ \\
\hline 9100 & TC437475 & -2.00 & $2.09 \mathrm{E}-02$ \\
\hline 9101 & TC438108 & -2.53 & $6.28 \mathrm{E}-05$ \\
\hline 9102 & TC438286 & -6.31 & $7.32 \mathrm{E}-04$ \\
\hline 9103 & TC438399 & -6.51 & $3.68 \mathrm{E}-04$ \\
\hline 9104 & TC438773 & -1.28 & $1.41 \mathrm{E}-02$ \\
\hline 9105 & TC439011 & -2.54 & $7.88 \mathrm{E}-03$ \\
\hline 9106 & TC440014 & -1.19 & $1.10 \mathrm{E}-03$ \\
\hline 9107 & TC440759 & -5.97 & $6.62 \mathrm{E}-04$ \\
\hline 9108 & TC440776 & -2.30 & $2.40 \mathrm{E}-03$ \\
\hline 9109 & TC440997 & -3.68 & $4.36 \mathrm{E}-04$ \\
\hline 9110 & TC441114 & -6.07 & $1.93 \mathrm{E}-03$ \\
\hline 9111 & TC441145 & -4.87 & $3.08 \mathrm{E}-03$ \\
\hline 9112 & TC441172 & -4.13 & $5.67 \mathrm{E}-05$ \\
\hline 9113 & TC441441 & -1.80 & $5.08 \mathrm{E}-03$ \\
\hline 9114 & TC442213 & -1.74 & $4.67 \mathrm{E}-02$ \\
\hline 9115 & TC442986 & -4.17 & $8.25 \mathrm{E}-04$ \\
\hline 9116 & TC443247 & -5.11 & $2.82 \mathrm{E}-04$ \\
\hline 9117 & TC443504 & -1.88 & $3.52 \mathrm{E}-05$ \\
\hline 9118 & TC444002 & -2.00 & $2.93 \mathrm{E}-03$ \\
\hline 9119 & TC444019 & -2.74 & $7.94 \mathrm{E}-03$ \\
\hline 9120 & TC444178 & -1.89 & $1.19 \mathrm{E}-02$ \\
\hline 9121 & TC444206 & -5.04 & $7.52 \mathrm{E}-04$ \\
\hline 9122 & TCEA1 & -4.45 & $2.21 \mathrm{E}-04$ \\
\hline 9123 & TCEA2 & -6.48 & $4.39 \mathrm{E}-05$ \\
\hline 9124 & TCEANC & -4.22 & $8.63 \mathrm{E}-05$ \\
\hline 9125 & TCEB1 & -4.16 & $7.76 \mathrm{E}-05$ \\
\hline 9126 & TCEB3 & -3.87 & $4.25 \mathrm{E}-04$ \\
\hline 9127 & TCERG1 & -4.00 & $2.47 \mathrm{E}-03$ \\
\hline
\end{tabular}

\begin{tabular}{|c|c|c|c|}
\hline 9128 & TCF12 & -3.01 & $7.24 E-04$ \\
\hline 9129 & TCF20 & -1.61 & $7.16 \mathrm{E}-03$ \\
\hline 9130 & TCF3 & -2.23 & $3.07 E-03$ \\
\hline 9131 & TCFL5 & -5.84 & $6.74 E-04$ \\
\hline 9132 & TCOF1 & -3.81 & $1.67 E-04$ \\
\hline 9133 & TCP1 & -7.82 & $1.71 E-05$ \\
\hline 9134 & TCP11 & $\begin{array}{l}-8.04 \\
\end{array}$ & $5.30 \mathrm{E}-05$ \\
\hline 9135 & TCP11L1 & -4.05 & $3.92 E-04$ \\
\hline 9136 & TCTE1 & -2.83 & $9.63 E-03$ \\
\hline 9137 & TCTE3 & -5.52 & $1.89 \mathrm{E}-03$ \\
\hline 9138 & TCTEX1D2 & -2.55 & $2.46 E-02$ \\
\hline 9139 & TCTN1 & -3.42 & $5.87 E-03$ \\
\hline 9140 & TDG & -2.21 & $2.56 \mathrm{E}-04$ \\
\hline 9141 & TDP1 & -4.43 & $8.63 E-05$ \\
\hline 9142 & TDP2 & -3.41 & $1.18 \mathrm{E}-04$ \\
\hline 9143 & TDRD12 & -2.00 & $1.98 \mathrm{E}-02$ \\
\hline 9144 & TDRD3 & -5.84 & $8.92 E-04$ \\
\hline 9145 & TDRD5 & -3.26 & $8.10 \mathrm{E}-03$ \\
\hline 9146 & TDRD9 & -2.99 & $1.06 E-04$ \\
\hline 9147 & TEAD4 & -3.97 & $2.02 E-04$ \\
\hline 9148 & TECPR2 & -4.84 & $2.97 E-05$ \\
\hline 9149 & TEF & -3.86 & $2.72 E-05$ \\
\hline 9150 & TEKT1 & -8.01 & $3.63 E-04$ \\
\hline 9151 & TEKT2 & -6.30 & $\begin{array}{l}4.46 E-04 \\
\end{array}$ \\
\hline 9152 & TEKT3 & -3.81 & $3.60 E-03$ \\
\hline 9153 & $\begin{array}{l}\text { TEKT4 } \\
\end{array}$ & -8.50 & $5.97 \mathrm{E}-07$ \\
\hline 9154 & TEKT5 & -6.35 & $3.48 E-05$ \\
\hline 9155 & TERF1 & -4.17 & $2.05 E-05$ \\
\hline 9156 & TERF2 & -1.95 & $2.82 E-03$ \\
\hline 9157 & TERF2IP & -2.21 & $1.09 E-02$ \\
\hline 9158 & TESC & -2.08 & $1.08 E-02$ \\
\hline 9159 & TEX10 & -5.15 & $3.22 E-04$ \\
\hline 9160 & TEX2 & -1.83 & $4.45 E-03$ \\
\hline 9161 & TEX30 & -3.69 & $2.40 E-04$ \\
\hline 9162 & TFAM & -4.99 & $3.79 E-04$ \\
\hline 9163 & TFAP2A & -2.86 & $1.67 E-03$ \\
\hline 9164 & TFB1M & -4.85 & $5.90 E-04$ \\
\hline 9165 & TFB2M & -5.32 & $7.43 E-04$ \\
\hline 9166 & TFDP1 & -2.95 & $2.67 E-04$ \\
\hline 9167 & TFG & -5.52 & $1.10 \mathrm{E}-04$ \\
\hline 9168 & TFIP11 & -3.18 & $1.31 \mathrm{E}-01$ \\
\hline 9169 & $\begin{array}{l}\text { TFPI } \\
\end{array}$ & -2.12 & $8.22 E-04$ \\
\hline 9170 & TFPI2 & -4.33 & $7.29 E-04$ \\
\hline 9171 & TGDS & -2.96 & $1.21 \mathrm{E}-03$ \\
\hline 9172 & TGFBR1 & -2.40 & $1.84 \mathrm{E}-03$ \\
\hline 9173 & TGIF1 & -1.38 & $6.92 E-02$ \\
\hline 9174 & TGM2 & -4.60 & $9.80 \mathrm{E}-04$ \\
\hline 9175 & TGM4 & -2.31 & $\begin{array}{l}6.14 E-04 \\
\end{array}$ \\
\hline 9176 & TGS1 & -2.12 & $1.74 E-03$ \\
\hline 9177 & TH1L & -3.76 & $1.48 E-04$ \\
\hline 9178 & THAP1 & -3.26 & $5.28 \mathrm{E}-02$ \\
\hline 9179 & THAP5 & -6.48 & $5.14 E-04$ \\
\hline 9180 & THBS1 & -3.04 & $8.62 E-05$ \\
\hline 9181 & THBS4 & -2.96 & $2.55 E-04$ \\
\hline 9182 & THG1L & -4.79 & $5.61 \mathrm{E}-03$ \\
\hline 9183 & THNSL1 & -1.03 & $6.02 E-03$ \\
\hline 9184 & THNSL2 & -3.59 & $3.71 E-04$ \\
\hline 9185 & THOC1 & -4.17 & $2.50 \mathrm{E}-03$ \\
\hline 9186 & THOC2 & -4.77 & $3.47 E-04$ \\
\hline 9187 & ТНОС3 & -2.31 & $4.83 \mathrm{E}-04$ \\
\hline 9188 & THOC4 & -5.86 & $6.92 E-04$ \\
\hline 9189 & THOC5 & -4.21 & $5.39 E-04$ \\
\hline 9190 & ТНОС7 & -5.02 & $9.93 \mathrm{E}-04$ \\
\hline
\end{tabular}




\begin{tabular}{|c|c|c|c|}
\hline 9191 & THRAP3 & -3.28 & $8.58 \mathrm{E}-04$ \\
\hline 9192 & THSD4 & -1.58 & $5.89 \mathrm{E}-03$ \\
\hline 9193 & THSD7B & -1.76 & $1.20 \mathrm{E}-02$ \\
\hline 9194 & THUMPD1 & -2.54 & $4.45 \mathrm{E}-04$ \\
\hline 9195 & THUMPD3 & -4.90 & $2.03 \mathrm{E}-04$ \\
\hline 9196 & THYN1 & -5.94 & $1.23 \mathrm{E}-03$ \\
\hline 9197 & TIA1 & -3.38 & $8.99 \mathrm{E}-04$ \\
\hline 9198 & TIMM10 & -2.10 & $4.93 \mathrm{E}-02$ \\
\hline 9199 & TIMM17A & -4.71 & $5.99 \mathrm{E}-03$ \\
\hline 9200 & TIMM23 & -4.52 & $4.90 \mathrm{E}-03$ \\
\hline 9201 & TIMM44 & -4.00 & $7.67 \mathrm{E}-04$ \\
\hline 9202 & TIMM8A & -3.61 & $8.89 \mathrm{E}-05$ \\
\hline 9203 & TIMM9 & -5.55 & $3.36 \mathrm{E}-04$ \\
\hline 9204 & TIMMDC1 & -3.39 & $2.04 \mathrm{E}-03$ \\
\hline 9205 & TIMP3 & -2.52 & $6.36 \mathrm{E}-03$ \\
\hline 9206 & TIMP4 & -1.68 & $2.03 \mathrm{E}-02$ \\
\hline 9207 & TIPIN & -4.13 & $2.36 \mathrm{E}-03$ \\
\hline 9208 & TIPRL & -4.40 & $2.05 \mathrm{E}-03$ \\
\hline 9209 & TIRAP & -4.31 & $3.69 \mathrm{E}-03$ \\
\hline 9210 & TK1 & -2.14 & $2.05 \mathrm{E}-03$ \\
\hline 9211 & TK2 & -3.41 & $9.45 \mathrm{E}-05$ \\
\hline 9212 & TLE3 & -3.29 & $3.22 \mathrm{E}-03$ \\
\hline 9213 & TLK1 & -1.95 & $6.07 \mathrm{E}-05$ \\
\hline 9214 & TLK2 & -3.82 & $5.74 \mathrm{E}-05$ \\
\hline 9215 & TLR6 & -1.08 & $2.75 \mathrm{E}-02$ \\
\hline 9216 & TLX3 & $\begin{array}{l}-6.90 \\
\end{array}$ & $1.33 \mathrm{E}-02$ \\
\hline 9217 & TM2D2 & -4.70 & $3.05 \mathrm{E}-03$ \\
\hline 9218 & TM4SF4 & \begin{tabular}{|l|}
-3.01 \\
\end{tabular} & $8.80 \mathrm{E}-04$ \\
\hline 9219 & TM7SF3 & -1.39 & $1.24 \mathrm{E}-02$ \\
\hline 9220 & TM9SF2 & -4.75 & $9.60 \mathrm{E}-04$ \\
\hline 9221 & TM9SF3 & -2.88 & $2.13 \mathrm{E}-04$ \\
\hline 9222 & TMA16 & $\begin{array}{l}-4.35 \\
\end{array}$ & $2.52 \mathrm{E}-04$ \\
\hline 9223 & TMBIM1 1 & -4.25 & $1.41 \mathrm{E}-03$ \\
\hline 9224 & TMBIM4 & $\begin{array}{l}-6.16 \\
\end{array}$ & $2.02 \mathrm{E}-05$ \\
\hline 9225 & TMC7 & -5.22 & $4.18 \mathrm{E}-05$ \\
\hline 9226 & TMCC3 & -2.22 & $2.34 \mathrm{E}-02$ \\
\hline 9227 & TMCO1 & -5.23 & $6.95 \mathrm{E}-04$ \\
\hline 9228 & TMCO3 & -2.02 & $3.77 \mathrm{E}-03$ \\
\hline 9229 & TMCO6 & $\begin{array}{l}-3.48 \\
\end{array}$ & $4.87 \mathrm{E}-03$ \\
\hline 9230 & TMCO7 & -2.69 & $1.29 \mathrm{E}-03$ \\
\hline 9231 & TMED10 & -3.11 & $2.86 \mathrm{E}-03$ \\
\hline 9232 & TMED2 & $\begin{array}{l}-2.91 \\
\end{array}$ & $7.25 \mathrm{E}-03$ \\
\hline 9233 & TMED5 & -2.52 & $1.05 \mathrm{E}-02$ \\
\hline 9234 & TMEFF2 & -5.07 & $9.53 \mathrm{E}-04$ \\
\hline 9235 & TMEM101 & -3.10 & $3.10 \mathrm{E}-03$ \\
\hline 9236 & TMEM104 & -2.05 & $2.81 \mathrm{E}-03$ \\
\hline 9237 & TMEM106B & -2.23 & $5.73 \mathrm{E}-03$ \\
\hline 9238 & TMEM111 & -6.34 & $6.65 \mathrm{E}-04$ \\
\hline 9239 & TMEM114 & -2.19 & $7.78 \mathrm{E}-03$ \\
\hline 9240 & TMEM120A & -3.86 & $7.48 \mathrm{E}-03$ \\
\hline 9241 & TMEM123 & -4.04 & $6.82 \mathrm{E}-04$ \\
\hline 9242 & TMEM128 & -5.09 & $1.20 \mathrm{E}-04$ \\
\hline 9243 & TMEM129 & -3.86 & $7.38 \mathrm{E}-04$ \\
\hline 9244 & TMEM130 & -2.63 & $2.67 \mathrm{E}-03$ \\
\hline 9245 & TMEM131 & $\begin{array}{l}-2.66 \\
\end{array}$ & $2.25 \mathrm{E}-03$ \\
\hline 9246 & TMEM138 & -5.61 & $5.88 \mathrm{E}-05$ \\
\hline 9247 & TMEM140 & $\begin{array}{l}-1.43 \\
\end{array}$ & $1.96 \mathrm{E}-02$ \\
\hline 9248 & TMEM141 & -2.30 & $1.09 \mathrm{E}-02$ \\
\hline 9249 & TMEM144 & -1.99 & $1.92 \mathrm{E}-03$ \\
\hline 9250 & TMEM14A & -5.11 & $2.68 \mathrm{E}-05$ \\
\hline 9251 & TMEM14C & -4.71 & $6.43 \mathrm{E}-03$ \\
\hline 9252 & TMEM159 & -4.66 & $2.78 \mathrm{E}-05$ \\
\hline
\end{tabular}

\begin{tabular}{|c|c|c|c|}
\hline 9254 & TMEM167A & -3.89 & $5.76 E-04$ \\
\hline 9255 & TMEM167B & -4.05 & $1.01 E-04$ \\
\hline 9256 & TMEM168 & -1.36 & $3.45 E-03$ \\
\hline 9257 & TMEM17 & -2.21 & $2.90 E-04$ \\
\hline 9258 & TMEM170A & -2.13 & $5.67 E-05$ \\
\hline 9259 & TMEM170B & -2.43 & $8.71 E-03$ \\
\hline 9260 & TMEM171 & -1.09 & $5.39 E-05$ \\
\hline 9261 & TMEM173 & -1.34 & $5.93 \mathrm{E}-03$ \\
\hline 9262 & TMEM175 & -4.39 & $1.89 E-04$ \\
\hline 9263 & TMEM178B & -2.95 & $3.99 \mathrm{E}-02$ \\
\hline 9264 & TMEM18 & -5.59 & $1.42 \mathrm{E}-03$ \\
\hline 9265 & TMEM180 & -4.44 & $6.29 E-04$ \\
\hline 9266 & TMEM183A & -4.58 & $3.93 E-04$ \\
\hline 9267 & TMEM184C & -2.80 & $2.70 \mathrm{E}-03$ \\
\hline 9268 & TMEM185A & -4.29 & $2.74 E-04$ \\
\hline 9269 & TMEM188 & -4.26 & $1.45 E-05$ \\
\hline 9270 & TMEM189 & -3.29 & $2.78 E-03$ \\
\hline 9271 & TMEM199 & -7.96 & $6.95 E-05$ \\
\hline 9272 & TMEM206 & -5.41 & $3.03 E-03$ \\
\hline 9273 & TMEM208 & -6.14 & $1.18 \mathrm{E}-04$ \\
\hline 9274 & TMEM209 & -2.58 & $6.97 E-04$ \\
\hline 9275 & TMEM214 & -2.62 & $8.40 E-05$ \\
\hline 9276 & TMEM216 & -1.18 & $9.60 \mathrm{E}-03$ \\
\hline 9277 & TMEM222 & -1.43 & $7.22 E-03$ \\
\hline 9278 & TMEM231 & -1.07 & $2.85 \mathrm{E}-03$ \\
\hline 9279 & TMEM234 & -4.13 & $1.39 \mathrm{E}-03$ \\
\hline 9280 & TMEM237 & -2.09 & $9.09 E-03$ \\
\hline 9281 & TMEM248 & -2.60 & $1.18 \mathrm{E}-03$ \\
\hline 9282 & TMEM26 & -1.71 & $6.82 E-02$ \\
\hline 9283 & TMEM27 & -3.91 & $3.55 \mathrm{E}-03$ \\
\hline 9284 & TMEM30A & -3.91 & $4.67 E-04$ \\
\hline 9285 & TMEM33 & -2.93 & $7.34 E-04$ \\
\hline 9286 & TMEM35 & -1.03 & $2.50 \mathrm{E}-02$ \\
\hline 9287 & TMEM38A & -1.52 & $9.18 \mathrm{E}-03$ \\
\hline 9288 & TMEM38B & -3.96 & 4.74E-04 \\
\hline 9289 & TMEM39B & -4.91 & $3.54 E-04$ \\
\hline 9290 & TMEM41B & -1.78 & $1.22 E-03$ \\
\hline 9291 & TMEM43 & -5.16 & $2.58 \mathrm{E}-03$ \\
\hline 9292 & TMEM48 & -6.86 & $4.02 E-04$ \\
\hline 9293 & TMEM5 & -4.07 & $6.17 \mathrm{E}-03$ \\
\hline 9294 & TMEM50B & -3.24 & $2.20 \mathrm{E}-03$ \\
\hline 9295 & TMEM53 & -1.74 & $5.35 E-03$ \\
\hline 9296 & TMEM55A & -4.03 & $6.68 \mathrm{E}-04$ \\
\hline 9297 & TMEM57 & -1.82 & $4.00 E-02$ \\
\hline 9298 & TMEM59 & -6.17 & $1.49 E-04$ \\
\hline 9299 & TMEM60 & \begin{tabular}{ll|l}
-1.74 \\
\end{tabular} & $4.91 E-05$ \\
\hline 9300 & TMEM62 & -1.48 & $6.91 E-03$ \\
\hline 9301 & TMEM63A & -4.47 & $2.34 \mathrm{E}-04$ \\
\hline 9302 & TMEM64 & -2.94 & $1.72 E-03$ \\
\hline 9303 & TMEM65 & -3.83 & $2.66 \mathrm{E}-04$ \\
\hline 9304 & TMEM66 & -6.77 & $2.49 E-05$ \\
\hline 9305 & TMEM67 & -2.93 & $1.81 E-03$ \\
\hline 9306 & TMEM68 & -4.50 & $3.19 E-03$ \\
\hline 9307 & TMEM70 & -5.43 & $2.89 \mathrm{E}-04$ \\
\hline 9308 & TMEM79 & -3.52 & $1.63 \mathrm{E}-03$ \\
\hline 9309 & TMEM80 & -2.39 & $1.36 \mathrm{E}-03$ \\
\hline 9310 & TMEM81 & -2.06 & $9.95 E-03$ \\
\hline 9311 & TMEM87A & -4.65 & $2.69 E-04$ \\
\hline 9312 & TMEM93 & -4.54 & $1.83 E-04$ \\
\hline 9313 & TMEM97 & -2.98 & $1.95 E-03$ \\
\hline 9314 & TMEM9B & -3.84 & $7.50 \mathrm{E}-03$ \\
\hline 9315 & TMF1 & -4.56 & $1.09 E-03$ \\
\hline 9316 & TMLHE & -1.87 & $2.17 \mathrm{E}-03$ \\
\hline
\end{tabular}

\begin{tabular}{|c|c|c|c|}
\hline 9317 & TMOD3 & -1.80 & $4.38 \mathrm{E}-03$ \\
\hline 9318 & TMPO & -2.66 & $2.82 \mathrm{E}-04$ \\
\hline 9319 & TMPRSS7 & -1.56 & $2.76 \mathrm{E}-02$ \\
\hline 9320 & TMSB4X & -6.24 & $6.95 \mathrm{E}-04$ \\
\hline 9321 & TMTC3 & -1.89 & $1.99 \mathrm{E}-03$ \\
\hline 9322 & TMX2 & -3.51 & $2.88 \mathrm{E}-04$ \\
\hline $\begin{array}{l}323 \\
\end{array}$ & TMX3 & -3.87 & $1.18 \mathrm{E}-03$ \\
\hline 9324 & TMX4 & -2.99 & $1.63 \mathrm{E}-03$ \\
\hline 9325 & TNFAIP6 & -2.78 & $3.05 E-03$ \\
\hline 9326 & TNFAIP8 & -1.61 & $2.28 \mathrm{E}-03$ \\
\hline 9327 & TNFAIP8L1 & -3.29 & $2.66 \mathrm{E}-03$ \\
\hline 9328 & TNFRSF11B & -1.33 & $1.29 \mathrm{E}-02$ \\
\hline 9329 & TNFRSF21 & -1.73 & $9.99 \mathrm{E}-02$ \\
\hline 9330 & TNFRSF8 & -2.70 & $5.99 \mathrm{E}-02$ \\
\hline 9331 & TNKS & -6.86 & $3.37 \mathrm{E}-04$ \\
\hline 9332 & TNKS2 & -3.69 & $1.71 \mathrm{E}-03$ \\
\hline 9333 & TNN & -1.79 & $4.83 \mathrm{E}-03$ \\
\hline 9334 & TNNC1 & -1.97 & $5.93 \mathrm{E}-03$ \\
\hline 9335 & TNNT3 & -3.85 & $3.33 \mathrm{E}-03$ \\
\hline 9336 & TNPO3 & -3.08 & $5.07 \mathrm{E}-03$ \\
\hline 9337 & TNRC15 & -4.31 & $1.39 \mathrm{E}-03$ \\
\hline 9338 & TNRC6A & -1.52 & $1.20 \mathrm{E}-02$ \\
\hline 9339 & TNRC6C & -2.92 & $4.47 \mathrm{E}-03$ \\
\hline 9340 & TOB1 & -1.87 & $1.69 \mathrm{E}-03$ \\
\hline 9341 & TOB2 & -2.22 & $4.10 \mathrm{E}-03$ \\
\hline 9342 & TOLLIP & -5.04 & $2.13 \mathrm{E}-03$ \\
\hline 9343 & TOM1L2 & -3.45 & $8.61 \mathrm{E}-03$ \\
\hline 9344 & томм20 & -6.22 & $3.27 \mathrm{E}-04$ \\
\hline 9345 & TOMM22 & -1.60 & $2.62 \mathrm{E}-02$ \\
\hline 9346 & TOMM34 & -1.96 & $4.54 \mathrm{E}-04$ \\
\hline 9347 & TOMM5 & -5.77 & $9.81 \mathrm{E}-05$ \\
\hline 9348 & TOMM6 & $\begin{array}{l}-7.74 \\
\end{array}$ & $1.38 \mathrm{E}-03$ \\
\hline 9349 & TOMM7 & -5.99 & $1.26 \mathrm{E}-04$ \\
\hline 9350 & TOMM70A & -2.68 & $1.41 \mathrm{E}-02$ \\
\hline 9351 & TOP1 & -4.95 & $1.55 \mathrm{E}-04$ \\
\hline 9352 & TOP2A & -6.39 & $1.84 \mathrm{E}-05$ \\
\hline 9353 & TOP2B & -5.79 & $4.15 \mathrm{E}-04$ \\
\hline 9354 & TOP3A & -2.97 & $4.40 \mathrm{E}-03$ \\
\hline 9355 & TOR1A & -2.66 & $1.13 \mathrm{E}-02$ \\
\hline 9356 & TP5313 & -3.44 & $4.88 \mathrm{E}-04$ \\
\hline 9357 & TP53INP1 & -3.01 & $2.14 \mathrm{E}-03$ \\
\hline 9358 & TP53RK & -3.11 & $1.32 \mathrm{E}-03$ \\
\hline 9359 & TP53TG5 & -7.21 & $1.53 \mathrm{E}-04$ \\
\hline 9360 & TPCN1 & -1.21 & $3.17 \mathrm{E}-02$ \\
\hline 9361 & TPCN3 & -1.31 & $3.11 \mathrm{E}-03$ \\
\hline 9362 & TPD52 & -6.01 & $1.95 \mathrm{E}-04$ \\
\hline 9363 & TPD52L2 & -6.59 & $8.54 \mathrm{E}-06$ \\
\hline 9364 & TPH1 & -1.18 & $7.89 \mathrm{E}-02$ \\
\hline 9365 & TPH2 & -3.70 & $4.86 \mathrm{E}-03$ \\
\hline 9366 & TPI1 & -6.96 & $3.95 \mathrm{E}-05$ \\
\hline 9367 & TPM1 & -2.31 & $3.17 \mathrm{E}-03$ \\
\hline 9368 & TPM2 & -4.70 & $1.04 \mathrm{E}-02$ \\
\hline 9369 & TPM3 & -1.91 & $5.34 \mathrm{E}-03$ \\
\hline 9370 & TPMT & -5.62 & $8.74 E-05$ \\
\hline 9371 & TPP2 & -3.35 & $5.24 \mathrm{E}-04$ \\
\hline 9372 & TPPP2 & -5.10 & $3.07 \mathrm{E}-03$ \\
\hline 9373 & TPR & -3.73 & $1.19 \mathrm{E}-06$ \\
\hline 9374 & TPRG1L & -5.91 & $3.95 \mathrm{E}-05$ \\
\hline 9375 & TPRKB & -3.89 & $1.12 \mathrm{E}-03$ \\
\hline 9376 & TPST1 & -3.04 & $7.01 \mathrm{E}-03$ \\
\hline 9377 & TPST2 & -3.09 & $8.57 \mathrm{E}-05$ \\
\hline 9378 & TPT1 & -5.88 & $1.18 \mathrm{E}-02$ \\
\hline 9379 & TPTE2 & -4.89 & $3.47 \mathrm{E}-03$ \\
\hline
\end{tabular}




\begin{tabular}{|c|c|c|c|}
\hline 9380 & TPX2 & -6.10 & $1.47 \mathrm{E}-04$ \\
\hline 9381 & TRA2A & -4.80 & $8.34 \mathrm{E}-04$ \\
\hline 9382 & TRA2B & -3.71 & 4.00E-03 \\
\hline 9383 & TRABD & -3.73 & $7.02 \mathrm{E}-03$ \\
\hline 9384 & TRAF3 & -1.77 & $1.19 \mathrm{E}-03$ \\
\hline 9385 & TRAF3IP2 & -2.67 & 9.93E-05 \\
\hline 9386 & TRAF5 & -2.14 & $1.12 \mathrm{E}-03$ \\
\hline 9387 & TRAF6 & -4.58 & $1.26 \mathrm{E}-03$ \\
\hline 9388 & TRAF7 & -2.96 & $9.25 \mathrm{E}-04$ \\
\hline 9389 & TRAFD1 & -3.35 & $2.93 \mathrm{E}-03$ \\
\hline 9390 & TRAIP & -3.00 & $7.73 \mathrm{E}-03$ \\
\hline 9391 & TRAM1 & -4.88 & $2.01 \mathrm{E}-03$ \\
\hline 9392 & TRAP1 & -4.38 & 2.29E-05 \\
\hline 9393 & TRAPPC11 & -4.09 & $3.32 \mathrm{E}-03$ \\
\hline 9394 & TRAPPC2 & -2.92 & 3.17E-03 \\
\hline 9395 & TRAPPC2L & -4.91 & 2.49E-04 \\
\hline 9396 & TRAPPC3 & -6.45 & 5.97E-05 \\
\hline 9397 & TRAPPC4 & -4.37 & 5.02E-04 \\
\hline 9398 & TRAPPC5 & -2.40 & 5.09E-02 \\
\hline 9399 & TRAPPC6B & -6.78 & $5.04 \mathrm{E}-04$ \\
\hline 9400 & TRAPPC8 & -3.85 & 4.62E-03 \\
\hline 9401 & TRAPPC9 & -2.25 & $2.29 \mathrm{E}-03$ \\
\hline 9402 & TRDMT1 & -1.29 & 5.07E-04 \\
\hline 9403 & TRDV3 & -2.08 & 5.39E-03 \\
\hline 9404 & TRIAP1 & -5.29 & $9.11 \mathrm{E}-04$ \\
\hline 9405 & TRIB2 & -1.55 & 2.23E-02 \\
\hline 9406 & TRIM23 & -1.80 & 8.57E-03 \\
\hline 9407 & TRIM24 & -4.71 & 2.19E-04 \\
\hline 9408 & TRIM33 & -1.41 & 5.47E-02 \\
\hline 9409 & TRIM36 & -7.26 & 7.49E-04 \\
\hline 9410 & TRIM37 & -6.45 & $2.18 \mathrm{E}-05$ \\
\hline 9411 & TRIM42 & -4.84 & $1.81 \mathrm{E}-04$ \\
\hline 9412 & TRIM59 & -2.34 & $3.86 \mathrm{E}-04$ \\
\hline 9413 & TRIM63 & -3.16 & $1.28 \mathrm{E}-02$ \\
\hline 9414 & TRIP11 & -3.58 & $2.70 \mathrm{E}-04$ \\
\hline 9415 & TRIP12 & -2.19 & $6.15 \mathrm{E}-03$ \\
\hline 9416 & TRIP13 & -4.95 & 5.87E-03 \\
\hline 9417 & TRIP4 & -3.70 & $1.42 \mathrm{E}-02$ \\
\hline 9418 & TRIT1 & -2.97 & $1.96 \mathrm{E}-03$ \\
\hline 9419 & TRMT11 & -1.71 & $8.27 \mathrm{E}-04$ \\
\hline 9420 & TRMT1L & -2.75 & $2.12 \mathrm{E}-03$ \\
\hline 9421 & TRMT2A & -5.73 & $1.04 \mathrm{E}-04$ \\
\hline 9422 & TRMT5 & -4.21 & $1.35 \mathrm{E}-03$ \\
\hline 9423 & TRMT61A & -1.92 & 4.63E-04 \\
\hline 9424 & TRMU & -5.28 & $2.08 \mathrm{E}-03$ \\
\hline 9425 & TRNAU1AP & -5.82 & $2.38 \mathrm{E}-04$ \\
\hline 9426 & TRNT1 & -3.69 & $1.19 \mathrm{E}-03$ \\
\hline 9427 & TRPC1 & -1.95 & $1.31 \mathrm{E}-03$ \\
\hline 9428 & TRPC3 & -3.35 & 7.57E-04 \\
\hline 9429 & TRPC4AP & -2.03 & $1.42 \mathrm{E}-02$ \\
\hline 9430 & TRPS1 & -2.16 & $5.07 \mathrm{E}-03$ \\
\hline 9431 & TRPV1 & -4.45 & $6.91 \mathrm{E}-04$ \\
\hline 9432 & TRSPAP1 & -1.93 & $1.04 \mathrm{E}-03$ \\
\hline 9433 & TRUB1 & -3.40 & $4.71 \mathrm{E}-03$ \\
\hline 9434 & TRUB2 & -1.38 & $1.51 \mathrm{E}-02$ \\
\hline 9435 & TSC22D1 & -3.12 & $3.32 \mathrm{E}-03$ \\
\hline 9436 & TSC22D3 & -2.45 & $3.64 \mathrm{E}-03$ \\
\hline 9437 & TSEN15 & -4.27 & $1.03 \mathrm{E}-04$ \\
\hline 9438 & TSEN2 & -3.71 & $2.05 \mathrm{E}-03$ \\
\hline 9439 & TSGA14 & -7.40 & $4.72 \mathrm{E}-04$ \\
\hline 9440 & TSN & -3.15 & 3.99E-03 \\
\hline 9441 & TSNAXIP1 & -4.70 & $1.93 \mathrm{E}-03$ \\
\hline 9442 & TSPAN13 & -4.92 & $1.39 \mathrm{E}-03$ \\
\hline
\end{tabular}

\begin{tabular}{|c|c|c|c|}
\hline 9443 & TSPAN15 & -1.70 & $8.70 \mathrm{E}-03$ \\
\hline 9444 & TSPAN3 & -7.13 & $1.04 \mathrm{E}-03$ \\
\hline 9445 & TSPAN6 & -5.71 & $1.62 \mathrm{E}-05$ \\
\hline 9446 & TSPO & -3.76 & $1.47 \mathrm{E}-04$ \\
\hline 9447 & TSR1 & -1.92 & $3.60 \mathrm{E}-03$ \\
\hline 9448 & TSSC4 & -3.64 & $4.14 \mathrm{E}-03$ \\
\hline 9449 & TSSK3 & -4.52 & $6.98 \mathrm{E}-04$ \\
\hline 9450 & TSSK6 & -7.00 & $9.71 \mathrm{E}-05$ \\
\hline 9451 & TSTD2 & -4.44 & $1.11 \mathrm{E}-03$ \\
\hline 9452 & ТாВК2 & -2.58 & $8.33 \mathrm{E}-04$ \\
\hline 9453 & Пत1 & -3.99 & $1.99 \mathrm{E}-04$ \\
\hline 9454 & Тत12 & -6.92 & $1.78 \mathrm{E}-04$ \\
\hline 9455 & Тत13 & -1.48 & $1.50 \mathrm{E}-02$ \\
\hline 9456 & Тत14 & -3.30 & $1.28 \mathrm{E}-03$ \\
\hline 9457 & Tत15 & -3.72 & $4.32 \mathrm{E}-03$ \\
\hline 9458 & ПТс19 & -2.73 & $1.39 \mathrm{E}-04$ \\
\hline 9459 & $\pi \mathrm{C} 21 \mathrm{~B}$ & -5.39 & $1.22 \mathrm{E}-03$ \\
\hline 9460 & Тत226 & -1.84 & $1.21 \mathrm{E}-02$ \\
\hline 9461 & ТС27 & -4.26 & $1.55 \mathrm{E}-04$ \\
\hline 9462 & Тत2 29 & -4.33 & $5.69 \mathrm{E}-04$ \\
\hline 9463 & ТТС $30 \mathrm{~A}$ & -6.19 & $1.14 \mathrm{E}-04$ \\
\hline 9464 & ТС32 & -7.54 & $2.00 E-03$ \\
\hline 9465 & ТС33 & -2.47 & $1.57 \mathrm{E}-03$ \\
\hline 9466 & ТTC35 & -6.18 & $1.59 \mathrm{E}-04$ \\
\hline 9467 & Псз6 & -2.48 & $3.98 \mathrm{E}-01$ \\
\hline 9468 & ТСЗ37 & -3.76 & $2.02 \mathrm{E}-04$ \\
\hline 9469 & ТТСЗ9А & -4.40 & $4.14 \mathrm{E}-03$ \\
\hline 9470 & ТТСз9В & -3.85 & $2.00 \mathrm{E}-04$ \\
\hline 9471 & TTC7A & -3.12 & $2.20 \mathrm{E}-03$ \\
\hline 9472 & ПТСВ & -4.09 & $3.81 \mathrm{E}-03$ \\
\hline 9473 & ТС8 & -6.84 & $6.17 \mathrm{E}-04$ \\
\hline 9474 & TाF2 & -2.79 & $6.27 \mathrm{E}-03$ \\
\hline 9475 & $\pi / 1$ & -1.59 & $7.98 \mathrm{E}-03$ \\
\hline 9476 & $\pi 12$ & -2.42 & $4.45 \mathrm{E}-03$ \\
\hline 9477 & TLL1 & -2.09 & $1.59 \mathrm{E}-03$ \\
\hline 9478 & TTLL11 & -3.84 & $1.59 \mathrm{E}-03$ \\
\hline 9479 & TLL12 & -5.65 & $4.85 \mathrm{E}-04$ \\
\hline 9480 & TTLL5 & -5.88 & 4.14E-05 \\
\hline 9481 & TTPAL & -2.65 & $8.11 \mathrm{E}-03$ \\
\hline 9482 & ТТН3 & -1.54 & $8.11 \mathrm{E}-04$ \\
\hline 9483 & TUBA1C & -2.78 & $4.41 \mathrm{E}-03$ \\
\hline 9484 & TUBA3E & -6.11 & $4.84 \mathrm{E}-04$ \\
\hline 9485 & TUBA4A & -5.69 & $1.71 \mathrm{E}-04$ \\
\hline 9486 & TUBA8 & -6.86 & $1.57 \mathrm{E}-03$ \\
\hline 9487 & TUBB1 & -1.54 & $4.60 \mathrm{E}-03$ \\
\hline 9488 & TUBB2B & -2.71 & $1.21 \mathrm{E}-03$ \\
\hline 9489 & TUBB2C & -7.00 & $7.45 \mathrm{E}-04$ \\
\hline 9490 & TUBB3 & -5.55 & $6.82 \mathrm{E}-06$ \\
\hline 9491 & TUBB6 & -2.12 & $3.28 \mathrm{E}-03$ \\
\hline 9492 & TUBE1 & -2.39 & $3.15 \mathrm{E}-03$ \\
\hline 9493 & TUBG1 & -4.21 & $4.79 \mathrm{E}-03$ \\
\hline 9494 & TUBGCP2 & -2.55 & $2.45 \mathrm{E}-03$ \\
\hline 9495 & TUBGCP3 & -5.25 & $4.91 \mathrm{E}-03$ \\
\hline 9496 & TUBGCP4 & -1.81 & $3.12 \mathrm{E}-02$ \\
\hline 9497 & TUSC2 & -6.15 & $3.74 \mathrm{E}-04$ \\
\hline 9498 & TUSC3 & -2.40 & $5.13 \mathrm{E}-03$ \\
\hline 9499 & TUT1 & -1.15 & $2.58 \mathrm{E}-02$ \\
\hline 9500 & TVP23B & -5.41 & $1.82 \mathrm{E}-04$ \\
\hline 9501 & TWF2 & -1.48 & $2.07 \mathrm{E}-03$ \\
\hline 9502 & TWISTNB & -3.18 & $8.62 \mathrm{E}-04$ \\
\hline 9503 & TWSG1 & -1.73 & $1.84 \mathrm{E}-02$ \\
\hline 9504 & TXLNA & -1.40 & $2.33 \mathrm{E}-02$ \\
\hline 9505 & TXLNG & -3.94 & $4.08 \mathrm{E}-04$ \\
\hline
\end{tabular}

\begin{tabular}{|c|c|c|c|}
\hline 9506 & TXN & -7.24 & $1.34 \mathrm{E}-04$ \\
\hline 9507 & TXN2 & -5.90 & 4.05E-04 \\
\hline 9508 & TXNDC12 & -4.08 & 3.00E-04 \\
\hline 9509 & TXNDC17 & -3.78 & $7.39 \mathrm{E}-04$ \\
\hline 9510 & TXNDC3 & -5.48 & 9.04E-04 \\
\hline 9511 & TXNDC5 & -3.16 & $2.68 \mathrm{E}-03$ \\
\hline 9512 & TXNDC9 & -6.33 & $9.67 \mathrm{E}-04$ \\
\hline 9513 & TXNL1 & -4.45 & $3.36 \mathrm{E}-05$ \\
\hline 9514 & TXNL4A & -4.00 & $2.56 \mathrm{E}-03$ \\
\hline 9515 & TXNL4B & -3.11 & $3.39 \mathrm{E}-03$ \\
\hline 9516 & TXNRD1 & -4.48 & $4.74 \mathrm{E}-04$ \\
\hline 9517 & TXNRD2 & -2.04 & $2.57 \mathrm{E}-03$ \\
\hline 9518 & TXNRD3 & -4.04 & 7.47E-04 \\
\hline 9519 & TYMS & -3.91 & $5.53 \mathrm{E}-04$ \\
\hline 9520 & TYRO3 & -2.89 & $9.76 \mathrm{E}-04$ \\
\hline 9521 & TYW5 & -3.08 & 2.70E-04 \\
\hline 9522 & U25026 & -2.11 & $1.84 \mathrm{E}-03$ \\
\hline 9523 & U2AF1 & -3.17 & $1.25 \mathrm{E}-03$ \\
\hline 9524 & U2SURP & -3.03 & $3.11 \mathrm{E}-03$ \\
\hline 9525 & U31667 & -6.56 & $4.68 \mathrm{E}-06$ \\
\hline 9526 & U93866 & -1.69 & $1.29 \mathrm{E}-02$ \\
\hline 9527 & UBA2 & -3.78 & $2.48 \mathrm{E}-04$ \\
\hline 9528 & UBA52 & -6.71 & 5.55E-05 \\
\hline 9529 & UBAC2 & -2.32 & $9.81 \mathrm{E}-04$ \\
\hline 9530 & UBAP1 & -3.32 & $6.69 \mathrm{E}-04$ \\
\hline 9531 & UBAP2 & -2.18 & $3.56 \mathrm{E}-03$ \\
\hline 9532 & UBC & -5.56 & $4.32 \mathrm{E}-05$ \\
\hline 9533 & UBE2A & -5.00 & $1.30 \mathrm{E}-04$ \\
\hline 9534 & UBE2CBP & -3.50 & 7.65E-04 \\
\hline 9535 & UBE2D1 & -6.81 & $1.60 \mathrm{E}-03$ \\
\hline 9536 & UBE2D2 & -4.01 & $1.07 \mathrm{E}-03$ \\
\hline 9537 & UBE2D3 & -4.61 & $8.34 \mathrm{E}-05$ \\
\hline 9538 & UBE2E1 & -1.89 & $7.91 \mathrm{E}-03$ \\
\hline 9539 & UBE2F & -5.46 & $1.40 \mathrm{E}-03$ \\
\hline 9540 & UBE2G1 & -2.00 & $3.61 \mathrm{E}-03$ \\
\hline 9541 & UBE2G2 & -6.49 & $4.51 \mathrm{E}-06$ \\
\hline 9542 & UBE2H & -1.91 & 8.17E-03 \\
\hline 9543 & UBE2I & -5.92 & 4.20E-05 \\
\hline 9544 & UBE2J1 & -1.93 & $1.13 \mathrm{E}-02$ \\
\hline 9545 & UBE2J2 & -2.44 & $1.10 \mathrm{E}-03$ \\
\hline 9546 & UBE2K & -5.40 & $1.40 \mathrm{E}-04$ \\
\hline 9547 & UBE2L3 & -5.58 & $1.54 \mathrm{E}-04$ \\
\hline 9548 & UBE2N & -2.60 & $2.45 \mathrm{E}-04$ \\
\hline 9549 & UBE2O & -2.83 & $1.60 \mathrm{E}-03$ \\
\hline 9550 & UBE2Q2 2 & -4.34 & $2.36 \mathrm{E}-05$ \\
\hline 9551 & UBE2QL1 & -1.21 & $9.82 \mathrm{E}-04$ \\
\hline 9552 & UBE2R2 & -3.41 & $5.78 \mathrm{E}-04$ \\
\hline 9553 & UBE2T & -5.32 & 4.53E-04 \\
\hline 9554 & UBE2V2 & -2.49 & $1.69 \mathrm{E}-03$ \\
\hline 9555 & UBE2W & -2.95 & 4.05E-03 \\
\hline 9556 & UBE2Z & -3.59 & $8.98 \mathrm{E}-04$ \\
\hline 9557 & UBE3A & -3.92 & $8.24 \mathrm{E}-03$ \\
\hline 9558 & UBE3C & -1.38 & $6.56 \mathrm{E}-04$ \\
\hline 9559 & UBE4A & -2.80 & 4.00E-03 \\
\hline 9560 & UBFD1 & -1.58 & $1.56 \mathrm{E}-03$ \\
\hline 9561 & UBI & -1.78 & $1.37 \mathrm{E}-03$ \\
\hline 9562 & UBIAD1 & -1.68 & 8.45E-04 \\
\hline 9563 & UBL3 & -2.72 & $8.75 \mathrm{E}-03$ \\
\hline 9564 & UBLCP1 & -3.67 & $3.98 \mathrm{E}-04$ \\
\hline 9565 & UBOX5 & -4.76 & 2.04E-04 \\
\hline 9566 & UBP1 & -4.87 & $3.98 \mathrm{E}-04$ \\
\hline 9567 & UBQLN1 & -2.66 & $2.82 \mathrm{E}-03$ \\
\hline 9568 & UBQLN4 & -3.74 & 5.37E-04 \\
\hline
\end{tabular}




\begin{tabular}{|c|c|c|c|}
\hline 9569 & UBR1 & -3.37 & $3.25 \mathrm{E}-04$ \\
\hline 9570 & UBR2 & -2.87 & $2.55 \mathrm{E}-03$ \\
\hline 9571 & UBR7 & -3.75 & $8.57 E-04$ \\
\hline 9572 & UBTD2 & -2.80 & $8.18 \mathrm{E}-03$ \\
\hline 9573 & UBXN11 & -3.42 & $4.79 \mathrm{E}-03$ \\
\hline 9574 & UBXN2A & -3.10 & $2.75 \mathrm{E}-03$ \\
\hline 9575 & UBXN2B & -4.97 & $1.03 \mathrm{E}-04$ \\
\hline 9576 & UBXN4 & -6.04 & $2.46 \mathrm{E}-04$ \\
\hline 9577 & UBXN6 & -5.26 & $1.76 \mathrm{E}-04$ \\
\hline 9578 & UCHL1 & -7.23 & $1.13 \mathrm{E}-04$ \\
\hline 9579 & UCHL3 & -6.46 & $2.11 \mathrm{E}-04$ \\
\hline 9580 & UCHL5 & -4.61 & 2.64E-04 \\
\hline 9581 & UCK1 & -2.67 & $3.98 \mathrm{E}-03$ \\
\hline 9582 & UFD1L & -6.01 & 7.29E-04 \\
\hline 9583 & UFM1 & -4.88 & $1.79 \mathrm{E}-05$ \\
\hline 9584 & UGGT1 & -3.20 & $1.49 \mathrm{E}-03$ \\
\hline 9585 & UGGT2 & -3.81 & $3.19 \mathrm{E}-05$ \\
\hline 9586 & UGP2 & -3.61 & $6.18 \mathrm{E}-04$ \\
\hline 9587 & UGT1A1 & -1.59 & $6.12 \mathrm{E}-03$ \\
\hline 9588 & UGT8 & -2.65 & $1.39 \mathrm{E}-02$ \\
\hline 9589 & UHМК1 & -7.04 & $2.89 \mathrm{E}-05$ \\
\hline 9590 & UHRF1 & -3.93 & $2.51 \mathrm{E}-03$ \\
\hline 9591 & UHRF1BP1L & -4.32 & $2.23 \mathrm{E}-04$ \\
\hline 9592 & UMPS & -3.50 & $1.79 \mathrm{E}-03$ \\
\hline 9593 & UNC119B & -2.53 & $1.24 \mathrm{E}-03$ \\
\hline 9594 & UNC50 & -4.53 & $1.41 \mathrm{E}-04$ \\
\hline 9595 & UNC5C & -1.26 & $1.04 \mathrm{E}-03$ \\
\hline 9596 & UNC5D & -2.34 & $2.38 \mathrm{E}-02$ \\
\hline 9597 & UPB1 & -6.97 & 2.01E-04 \\
\hline 9598 & UPF1 & -3.86 & $2.02 \mathrm{E}-03$ \\
\hline 9599 & UPF2 & -1.30 & 4.96E-02 \\
\hline 9600 & UPF3A & -4.33 & 7.27E-04 \\
\hline 9601 & UPP1 & -3.60 & $5.44 \mathrm{E}-03$ \\
\hline 9602 & UPP2 & -2.77 & 8.17E-03 \\
\hline 9603 & UPRT & -3.78 & 2.01E-05 \\
\hline 9604 & Uacc & -3.55 & 9.54E-04 \\
\hline 9605 & UQCR10 & -5.27 & $1.75 \mathrm{E}-04$ \\
\hline 9606 & UQCR11 & -5.02 & $1.20 \mathrm{E}-04$ \\
\hline 9607 & UQCRC1 & -5.33 & $3.51 \mathrm{E}-05$ \\
\hline 9608 & UQCRC2 & -4.93 & 7.19E-04 \\
\hline 9609 & UQCRFS1 & -7.04 & 2.23E-04 \\
\hline 9610 & UQCRH & -8.45 & $6.79 \mathrm{E}-04$ \\
\hline 9611 & UQCRQ & -5.17 & 3.53E-04 \\
\hline 9612 & URM1 & -3.10 & $8.72 \mathrm{E}-04$ \\
\hline 9613 & UROD & -1.23 & $8.34 \mathrm{E}-03$ \\
\hline 9614 & UROS & -2.50 & $1.32 \mathrm{E}-03$ \\
\hline 9615 & URP & -5.72 & 7.43E-05 \\
\hline 9616 & USE1 & -2.58 & 9.99E-05 \\
\hline 9617 & USO1 & -4.01 & $8.80 \mathrm{E}-04$ \\
\hline 9618 & USP1 & -1.48 & $1.04 \mathrm{E}-02$ \\
\hline 9619 & USP10 & -1.82 & $4.66 \mathrm{E}-03$ \\
\hline 9620 & USP12 & -2.12 & $1.03 E-03$ \\
\hline 9621 & USP12P1 & -1.60 & $1.81 \mathrm{E}-02$ \\
\hline 9622 & USP14 & -2.93 & $1.88 \mathrm{E}-03$ \\
\hline 9623 & USP15 & -1.48 & $1.76 \mathrm{E}-02$ \\
\hline 9624 & USP16 & -4.15 & $9.24 \mathrm{E}-04$ \\
\hline 9625 & USP18 & -1.59 & $6.50 \mathrm{E}-03$ \\
\hline 9626 & USP2 & -4.73 & $3.44 \mathrm{E}-04$ \\
\hline 9627 & USP20 & -1.98 & $1.19 \mathrm{E}-02$ \\
\hline 9628 & USP22 & -3.05 & 4.58E-04 \\
\hline 9629 & USP25 & -2.16 & $3.39 \mathrm{E}-03$ \\
\hline 9630 & USP28 & -5.27 & $1.18 \mathrm{E}-04$ \\
\hline 9631 & USP3 & -2.16 & $1.79 \mathrm{E}-03$ \\
\hline
\end{tabular}

\begin{tabular}{|c|c|c|c|}
\hline 9632 & USP30 & -1.92 & $6.43 \mathrm{E}-03$ \\
\hline 9633 & USP32 & -1.43 & $5.89 \mathrm{E}-02$ \\
\hline 9634 & USP33 & -4.28 & $1.77 \mathrm{E}-05$ \\
\hline 9635 & USP34 & -1.91 & $3.15 \mathrm{E}-04$ \\
\hline 9636 & USP38 & -6.93 & $1.60 \mathrm{E}-04$ \\
\hline 9637 & USP4 & -3.36 & $2.21 \mathrm{E}-03$ \\
\hline 9638 & USP42 & -4.34 & $2.94 \mathrm{E}-03$ \\
\hline 9639 & USP45 & -1.44 & $2.42 \mathrm{E}-03$ \\
\hline 9640 & USP46 & -2.56 & $2.94 \mathrm{E}-03$ \\
\hline 9641 & USP47 & -4.91 & $6.43 \mathrm{E}-04$ \\
\hline 9642 & USP48 & -3.90 & $5.39 \mathrm{E}-05$ \\
\hline 9643 & USP49 & -2.90 & $2.16 \mathrm{E}-03$ \\
\hline 9644 & USP5 & -1.45 & $5.43 \mathrm{E}-03$ \\
\hline 9645 & USP6NL & -3.01 & $8.50 \mathrm{E}-03$ \\
\hline 9646 & USP7 & -4.41 & $7.90 \mathrm{E}-04$ \\
\hline 9647 & USP9X & -5.35 & $2.67 \mathrm{E}-03$ \\
\hline 9648 & USPL1 & -3.73 & $3.35 \mathrm{E}-04$ \\
\hline 9649 & UTP11L & -5.42 & $3.58 \mathrm{E}-05$ \\
\hline 9650 & UTP15 & -3.94 & $5.82 \mathrm{E}-04$ \\
\hline 9651 & UTP18 & -3.76 & $3.37 \mathrm{E}-04$ \\
\hline 9652 & UTP23 & -6.22 & $1.57 \mathrm{E}-04$ \\
\hline 9653 & UTP3 & -3.37 & $2.83 \mathrm{E}-06$ \\
\hline 9654 & UTP6 & -3.69 & $5.05 \mathrm{E}-03$ \\
\hline 9655 & UXS1 & -2.82 & $5.26 \mathrm{E}-03$ \\
\hline 9656 & V00414 & -6.64 & $1.58 \mathrm{E}-04$ \\
\hline 9657 & VAC14 & -3.78 & $3.21 \mathrm{E}-03$ \\
\hline 9658 & VAMP3 & -2.07 & $3.19 \mathrm{E}-03$ \\
\hline 9659 & VAMP4 & -4.78 & $3.82 \mathrm{E}-05$ \\
\hline 9660 & VAMP7 & -2.47 & $5.90 \mathrm{E}-04$ \\
\hline 9661 & VAPB & -1.84 & $1.37 \mathrm{E}-02$ \\
\hline 9662 & VASH2 & -5.29 & $6.32 \mathrm{E}-04$ \\
\hline 9663 & VBP1 & -7.70 & $4.45 \mathrm{E}-04$ \\
\hline 9664 & VCL & -1.78 & $1.78 \mathrm{E}-02$ \\
\hline 9665 & VCP & -3.86 & $1.36 \mathrm{E}-02$ \\
\hline 9666 & VCPIP1 & -3.46 & $6.03 \mathrm{E}-03$ \\
\hline 9667 & VDAC1 & -4.02 & $8.43 \mathrm{E}-04$ \\
\hline 9668 & VDAC2 & -7.86 & $3.65 \mathrm{E}-06$ \\
\hline 9669 & VDAC3 & -2.98 & $2.33 \mathrm{E}-03$ \\
\hline 9670 & VEZF1 & -2.11 & 3.77E-04 \\
\hline 9671 & VGLL1 & -3.93 & $9.13 \mathrm{E}-03$ \\
\hline 9672 & VGLL4 & -2.48 & $3.23 \mathrm{E}-03$ \\
\hline 9673 & VIM & -1.60 & $1.08 \mathrm{E}-02$ \\
\hline 9674 & VIMP & -5.86 & $7.53 \mathrm{E}-05$ \\
\hline 9675 & VIPAR & -1.32 & $6.38 \mathrm{E}-03$ \\
\hline 9676 & VIPR2 & -1.25 & $5.07 \mathrm{E}-02$ \\
\hline 9677 & VLDLR & -4.07 & $1.89 \mathrm{E}-03$ \\
\hline 9678 & VMA21 & -2.71 & $3.66 \mathrm{E}-03$ \\
\hline 9679 & VMP1 & -4.33 & $3.79 \mathrm{E}-04$ \\
\hline 9680 & VOPP1 & -2.71 & $2.27 \mathrm{E}-03$ \\
\hline 9681 & VPRBP & -2.63 & $1.23 \mathrm{E}-02$ \\
\hline 9682 & VPREB3 & -1.76 & $8.44 \mathrm{E}-03$ \\
\hline 9683 & VPS11 & -1.52 & $2.42 \mathrm{E}-03$ \\
\hline 9684 & VPS13B & -3.65 & $7.78 \mathrm{E}-03$ \\
\hline 9685 & VPS13C & -1.46 & $1.14 \mathrm{E}-02$ \\
\hline 9686 & VPS13D & -3.72 & $7.86 \mathrm{E}-04$ \\
\hline 9687 & VPS18 & -3.10 & $8.00 \mathrm{E}-04$ \\
\hline 9688 & VPS24 & -6.23 & $1.51 \mathrm{E}-04$ \\
\hline 9689 & VPS26A & -3.52 & $6.56 \mathrm{E}-04$ \\
\hline 9690 & VPS26B & -1.31 & $2.52 \mathrm{E}-02$ \\
\hline 9691 & VPS29 & -2.57 & $2.88 \mathrm{E}-04$ \\
\hline 9692 & VPS33A & -2.49 & $7.80 \mathrm{E}-03$ \\
\hline 9693 & VPS33B & -2.93 & $2.43 \mathrm{E}-03$ \\
\hline 9694 & VPS35 & -4.09 & $5.05 \mathrm{E}-04$ \\
\hline
\end{tabular}

\begin{tabular}{|c|c|c|c|}
\hline 9695 & VPS36 & -2.08 & $2.96 \mathrm{E}-03$ \\
\hline 9696 & VPS37A & -3.14 & $1.14 \mathrm{E}-03$ \\
\hline 9697 & VPS37C & -2.81 & $1.88 \mathrm{E}-03$ \\
\hline 9698 & VPS39 & -4.14 & $1.50 \mathrm{E}-04$ \\
\hline 9699 & VPS41 & -5.29 & $6.33 \mathrm{E}-05$ \\
\hline 9700 & VPS4B & -4.07 & $1.58 \mathrm{E}-03$ \\
\hline 9701 & VPS72 & -1.80 & $2.43 \mathrm{E}-03$ \\
\hline 9702 & VPS8 & -2.92 & $2.15 \mathrm{E}-04$ \\
\hline 9703 & VRK1 & -1.65 & $4.82 \mathrm{E}-03$ \\
\hline 9704 & VRK3 & -4.57 & 9.07E-04 \\
\hline 9705 & VSNL1 & -3.32 & $6.38 \mathrm{E}-04$ \\
\hline 9706 & VSX1 & -1.12 & 5.57E-03 \\
\hline 9707 & VTA1 & -5.87 & $3.46 \mathrm{E}-04$ \\
\hline 9708 & VTG2 & -2.46 & $1.50 \mathrm{E}-03$ \\
\hline 9709 & VTI1A & -3.28 & $1.76 \mathrm{E}-03$ \\
\hline 9710 & VTI1B & -4.56 & $4.98 \mathrm{E}-04$ \\
\hline 9711 & VWA9 & -4.00 & $4.13 \mathrm{E}-04$ \\
\hline 9712 & VWC2 & -3.40 & 4.67E-03 \\
\hline 9713 & WAC & -3.38 & $3.62 \mathrm{E}-04$ \\
\hline 9714 & WAPAL & -2.36 & $6.77 \mathrm{E}-03$ \\
\hline 9715 & WARS & -2.09 & $6.92 \mathrm{E}-04$ \\
\hline 9716 & WASF1 & -1.90 & 4.18E-03 \\
\hline 9717 & WASF3 & -2.30 & $2.82 \mathrm{E}-03$ \\
\hline 9718 & WASH1 & -3.65 & $1.11 \mathrm{E}-04$ \\
\hline 9719 & WASL & -4.19 & $1.73 \mathrm{E}-02$ \\
\hline 9720 & WBP11 & -3.44 & $5.25 \mathrm{E}-03$ \\
\hline 9721 & WBP2 & -4.28 & $8.14 \mathrm{E}-04$ \\
\hline 9722 & WBP4 & -2.22 & $5.68 \mathrm{E}-03$ \\
\hline 9723 & WBSCR16 & -3.66 & $5.64 \mathrm{E}-03$ \\
\hline 9724 & WBSCR22 & -2.35 & $3.61 \mathrm{E}-02$ \\
\hline 9725 & WDFY1 & -2.46 & $2.76 \mathrm{E}-03$ \\
\hline 9726 & WDFY4 & -1.12 & $3.28 \mathrm{E}-02$ \\
\hline 9727 & WDHD1 & -1.77 & $3.12 \mathrm{E}-03$ \\
\hline 9728 & WDPCP & -2.30 & $8.21 \mathrm{E}-03$ \\
\hline 9729 & WDR1 & -3.61 & $2.45 \mathrm{E}-04$ \\
\hline 9730 & WDR11 & -1.71 & $2.11 \mathrm{E}-03$ \\
\hline 9731 & WDR12 & -3.42 & $9.02 \mathrm{E}-04$ \\
\hline 9732 & WDR24 & -2.70 & $2.36 \mathrm{E}-03$ \\
\hline 9733 & WDR25 & -4.47 & $2.14 \mathrm{E}-04$ \\
\hline 9734 & WDR26 & -3.93 & $4.12 \mathrm{E}-04$ \\
\hline 9735 & WDR33 & -2.49 & $6.42 \mathrm{E}-04$ \\
\hline 9736 & WDR34 & -4.97 & $3.30 \mathrm{E}-04$ \\
\hline 9737 & WDR35 & -5.26 & $1.41 \mathrm{E}-04$ \\
\hline 9738 & WDR36 & -3.22 & $7.42 \mathrm{E}-03$ \\
\hline 9739 & WDR37 & -1.68 & $3.11 \mathrm{E}-03$ \\
\hline 9740 & WDR4 & -2.00 & $4.63 \mathrm{E}-03$ \\
\hline 9741 & WDR41 & -2.19 & $2.08 \mathrm{E}-02$ \\
\hline 9742 & WDR43 & -3.21 & 9.55E-04 \\
\hline 9743 & WDR45L & -3.87 & $1.03 \mathrm{E}-03$ \\
\hline 9744 & WDR47 & -1.39 & $6.91 \mathrm{E}-04$ \\
\hline 9745 & WDR48 & -2.17 & $5.06 \mathrm{E}-03$ \\
\hline 9746 & WDR52 & -6.47 & $5.08 \mathrm{E}-05$ \\
\hline 9747 & WDR53 & -1.81 & $6.41 \mathrm{E}-03$ \\
\hline 9748 & WDR59 & -2.51 & $1.10 \mathrm{E}-03$ \\
\hline 9749 & WDR61 & -3.93 & $2.23 \mathrm{E}-04$ \\
\hline 9750 & WDR63 & -6.36 & $1.70 \mathrm{E}-04$ \\
\hline 9751 & WDR65 & -7.24 & $2.46 \mathrm{E}-04$ \\
\hline 9752 & WDR66 & -4.53 & $3.26 \mathrm{E}-04$ \\
\hline 9753 & WDR67 & -3.10 & $2.68 \mathrm{E}-04$ \\
\hline 9754 & WDR69 & -9.02 & $9.92 \mathrm{E}-04$ \\
\hline 9755 & WDR70 & -2.02 & $7.81 \mathrm{E}-03$ \\
\hline 9756 & WDR73 & -4.56 & $3.32 \mathrm{E}-04$ \\
\hline 9757 & WDR75 & -4.84 & 4.90E-04 \\
\hline
\end{tabular}




\begin{tabular}{|c|c|c|c|}
\hline 9758 & WDR77 & -3.85 & $4.93 \mathrm{E}-04$ \\
\hline 9759 & WDR81 & -2.63 & $3.94 \mathrm{E}-02$ \\
\hline 9760 & WDR82 & -3.64 & $7.58 \mathrm{E}-04$ \\
\hline 9761 & WDR91 & -4.51 & $2.27 \mathrm{E}-04$ \\
\hline 9762 & WDR92 & -5.17 & $6.31 \mathrm{E}-04$ \\
\hline 9763 & WDTC1 & -4.16 & $1.30 \mathrm{E}-04$ \\
\hline 9764 & WEE1 & -8.04 & $5.00 \mathrm{E}-04$ \\
\hline 9765 & WFS1 & -3.59 & $6.35 \mathrm{E}-04$ \\
\hline 9766 & WHDC1 & -2.92 & $4.92 \mathrm{E}-03$ \\
\hline 9767 & WHSC2 & -4.04 & $1.45 \mathrm{E}-03$ \\
\hline 9768 & WIF1 & -1.95 & 4.35E-04 \\
\hline 9769 & WIPI2 & -3.93 & $1.27 \mathrm{E}-03$ \\
\hline 9770 & WISP1 & -3.75 & 4.16E-04 \\
\hline 9771 & WLS & -3.81 & $1.18 \mathrm{E}-04$ \\
\hline 9772 & WRAP73 & -4.11 & $1.24 \mathrm{E}-02$ \\
\hline 9773 & WRB & -3.44 & $2.73 \mathrm{E}-04$ \\
\hline 9774 & WRNIP1 & -2.34 & $4.20 \mathrm{E}-03$ \\
\hline 9775 & WSB2 & -4.53 & $8.59 \mathrm{E}-04$ \\
\hline 9776 & WT1 & -2.75 & $6.54 \mathrm{E}-03$ \\
\hline 9777 & WTAP & -4.55 & $6.08 \mathrm{E}-04$ \\
\hline 9778 & WTIP & -2.42 & 4.26E-03 \\
\hline 9779 & WWC1 & -2.71 & $1.99 \mathrm{E}-03$ \\
\hline 9780 & WWP1 & -2.07 & 4.35E-04 \\
\hline 9781 & X59368 & -4.76 & 5.51E-04 \\
\hline 9782 & ХАB1 & -3.50 & $1.19 \mathrm{E}-03$ \\
\hline 9783 & XAF1 & -6.61 & 2.56E-04 \\
\hline 9784 & XBP1 & -4.09 & $8.68 \mathrm{E}-03$ \\
\hline 9785 & XDH & -3.05 & 9.97E-05 \\
\hline 9786 & XIAP & -1.60 & $3.92 \mathrm{E}-03$ \\
\hline 9787 & XPA & -3.94 & $1.41 \mathrm{E}-03$ \\
\hline 9788 & XPC & -2.39 & $6.20 \mathrm{E}-03$ \\
\hline 9789 & XPNPEP1 & -2.48 & $1.40 \mathrm{E}-02$ \\
\hline 9790 & XPNPEP3 & -1.93 & $1.15 \mathrm{E}-02$ \\
\hline 9791 & XPO1 & -2.44 & $1.98 \mathrm{E}-03$ \\
\hline 9792 & ХРОТ & -2.29 & $4.36 \mathrm{E}-03$ \\
\hline 9793 & XPR1 & -1.86 & 4.84E-04 \\
\hline 9794 & XRCC2 & -3.55 & 2.93E-04 \\
\hline 9795 & XRCC3 & -2.16 & 2.93E-04 \\
\hline 9796 & XRCC6 & -3.25 & $9.13 \mathrm{E}-04$ \\
\hline 9797 & XRCC6BP1 & -5.03 & $8.08 \mathrm{E}-05$ \\
\hline 9798 & XRN1 & -3.33 & $1.28 \mathrm{E}-02$ \\
\hline 9799 & XRN2 & -5.69 & $7.29 \mathrm{E}-04$ \\
\hline 9800 & XYLT2 & -3.63 & $1.39 \mathrm{E}-03$ \\
\hline 9801 & YAE1D1 & -3.57 & 4.06E-05 \\
\hline 9802 & YAF2 & -2.79 & 6.59E-04 \\
\hline 9803 & YAP1 & -2.95 & $2.48 \mathrm{E}-03$ \\
\hline 9804 & YARS & -4.58 & $3.56 \mathrm{E}-04$ \\
\hline 9805 & YARS2 & -3.16 & $1.98 \mathrm{E}-04$ \\
\hline 9806 & YBX1 & -7.90 & $6.58 \mathrm{E}-04$ \\
\hline 9807 & YDJC & -3.42 & $1.45 \mathrm{E}-03$ \\
\hline 9808 & YEATS4 & -5.82 & $1.62 \mathrm{E}-04$ \\
\hline 9809 & YIPF1 & -4.72 & $1.94 \mathrm{E}-04$ \\
\hline 9810 & YIPF3 & -4.97 & $6.97 \mathrm{E}-05$ \\
\hline 9811 & YIPF4 & -3.39 & $6.93 \mathrm{E}-04$ \\
\hline 9812 & YIPF5 & -4.68 & $3.01 \mathrm{E}-03$ \\
\hline 9813 & YLPM1 & -2.06 & $2.36 \mathrm{E}-03$ \\
\hline 9814 & YME1L1 & -4.92 & $5.25 \mathrm{E}-04$ \\
\hline 9815 & YOD1 & -1.45 & $1.04 \mathrm{E}-03$ \\
\hline 9816 & YPEL1 & -3.60 & 1.07E-03 \\
\hline 9817 & YPEL2 & -1.52 & $3.75 \mathrm{E}-02$ \\
\hline 9818 & YPEL5 & -6.74 & 5.11E- 05 \\
\hline 9819 & YRDC & -5.31 & $2.84 \mathrm{E}-04$ \\
\hline 9820 & YSK4 & -3.24 & $1.70 \mathrm{E}-02$ \\
\hline
\end{tabular}

\begin{tabular}{|c|c|c|c|}
\hline 9821 & YTHDC1 & -1.56 & $3.66 \mathrm{E}-02$ \\
\hline 9822 & YTHDC2 & -2.88 & 7.69E-04 \\
\hline 9823 & YTHDF1 & -3.50 & $4.31 \mathrm{E}-04$ \\
\hline 9824 & YTHDF2 & -2.35 & $1.55 \mathrm{E}-02$ \\
\hline 9825 & YTHDF3 & -2.52 & $8.53 \mathrm{E}-03$ \\
\hline 9826 & YWHAB & -3.14 & $2.64 \mathrm{E}-03$ \\
\hline 9827 & YWHAE & -3.78 & $6.70 \mathrm{E}-04$ \\
\hline 9828 & YWHAG & -1.39 & $3.05 E-02$ \\
\hline 9829 & YWHAH & -5.58 & $3.02 \mathrm{E}-04$ \\
\hline 9830 & YWHAQ & -5.87 & 5.97E-05 \\
\hline 9831 & YWHAZ & -6.43 & $4.05 \mathrm{E}-05$ \\
\hline 9832 & YY1 & -4.39 & $7.05 \mathrm{E}-04$ \\
\hline 9833 & ZAK & -2.94 & $1.14 \mathrm{E}-03$ \\
\hline 9834 & ZAR1 & -2.06 & $1.88 \mathrm{E}-02$ \\
\hline 9835 & ZAR1L & -4.26 & $2.16 \mathrm{E}-04$ \\
\hline 9836 & ZBTB10 & -2.92 & $3.33 \mathrm{E}-03$ \\
\hline 9837 & ZВТВ2 & -3.95 & $1.46 \mathrm{E}-03$ \\
\hline 9838 & ZBTB20 & -2.39 & $3.08 \mathrm{E}-03$ \\
\hline 9839 & ZBTB26 & -3.36 & $5.27 \mathrm{E}-04$ \\
\hline 9840 & ZВTB43 & -1.99 & $3.70 \mathrm{E}-03$ \\
\hline 9841 & ZBTB44 & -2.04 & $3.84 \mathrm{E}-03$ \\
\hline 9842 & ZBTB46 & -3.41 & $6.74 \mathrm{E}-03$ \\
\hline 9843 & ZВТВ48 & -1.92 & $3.68 \mathrm{E}-03$ \\
\hline 9844 & ZВТВ49 & -1.76 & $2.38 \mathrm{E}-03$ \\
\hline 9845 & $\mathrm{ZC} 2 \mathrm{HC} 1 \mathrm{~A}$ & -3.05 & $1.45 \mathrm{E}-03$ \\
\hline 9846 & $\mathrm{ZC} 2 \mathrm{HC} 1 \mathrm{~B}$ & -8.06 & $5.71 \mathrm{E}-04$ \\
\hline 9847 & ZC3H11A & -1.51 & $5.85 \mathrm{E}-02$ \\
\hline 9848 & ZC3H13 & -5.04 & $2.49 \mathrm{E}-04$ \\
\hline 9849 & ZС3H14 & -3.68 & $1.63 \mathrm{E}-06$ \\
\hline 9850 & ZС3H15 & -7.45 & $7.23 \mathrm{E}-05$ \\
\hline 9851 & ZС3H18 & -1.37 & $3.88 \mathrm{E}-02$ \\
\hline 9852 & ZС3H3 & -1.44 & $2.40 \mathrm{E}-03$ \\
\hline 9853 & ZC3H6 & -3.02 & $1.51 \mathrm{E}-04$ \\
\hline 9854 & ZC3H7A & -2.65 & $3.24 \mathrm{E}-04$ \\
\hline 9855 & ZC3HAV1 & -3.28 & $2.46 \mathrm{E}-04$ \\
\hline 9856 & $\mathrm{ZC} 4 \mathrm{H} 2$ & -3.35 & $1.20 \mathrm{E}-03$ \\
\hline 9857 & ZCCHC10 & -2.19 & $2.10 \mathrm{E}-02$ \\
\hline 9858 & ZCCHC11 & -3.78 & 4.81E-05 \\
\hline 9859 & ZCCHC17 & -4.59 & 8.67E-04 \\
\hline 9860 & $\mathrm{ZCCHC4}$ & -2.52 & $2.33 \mathrm{E}-02$ \\
\hline 9861 & ZCCHC6 & -2.20 & $3.44 \mathrm{E}-03$ \\
\hline 9862 & $\mathrm{ZCCHC7}$ & -2.59 & $3.63 \mathrm{E}-05$ \\
\hline 9863 & $\mathrm{ZCCHC8}$ & -3.76 & $4.94 \mathrm{E}-05$ \\
\hline 9864 & ZCCHC9 & -3.28 & $6.42 \mathrm{E}-04$ \\
\hline 9865 & ZCRB1 & -5.11 & 4.26E-04 \\
\hline 9866 & ZDBF2 & -5.66 & $7.82 \mathrm{E}-04$ \\
\hline 9867 & ZDHHC1 & -1.27 & $1.49 \mathrm{E}-02$ \\
\hline 9868 & ZDHHC13 & -5.52 & $2.27 E-04$ \\
\hline 9869 & ZDHHC16 & -1.71 & $7.05 E-02$ \\
\hline 9870 & ZDHHC17 & -2.56 & $2.25 \mathrm{E}-04$ \\
\hline 9871 & ZDHHC2 & -1.30 & $1.22 \mathrm{E}-02$ \\
\hline 9872 & ZDHHC21 & -3.37 & $1.97 \mathrm{E}-04$ \\
\hline 9873 & ZDHHC6 & -4.62 & $1.30 \mathrm{E}-04$ \\
\hline 9874 & ZDHHC8 & -1.18 & $3.89 \mathrm{E}-03$ \\
\hline 9875 & ZDHHC9 & -4.97 & $5.21 \mathrm{E}-05$ \\
\hline 9876 & ZEB1 & -1.83 & $3.18 \mathrm{E}-02$ \\
\hline 9877 & ZER1 & -2.27 & $1.79 \mathrm{E}-03$ \\
\hline 9878 & ZFAND1 & -4.57 & $5.46 \mathrm{E}-04$ \\
\hline 9879 & ZFAND2A & -3.25 & 2.14E-04 \\
\hline 9880 & ZFAND3 & -6.50 & $2.54 \mathrm{E}-04$ \\
\hline 9881 & ZFAND5 & -6.07 & 3.31E-04 \\
\hline 9882 & ZFAND6 & -1.99 & $9.90 \mathrm{E}-03$ \\
\hline 9883 & ZFAT & -1.48 & $2.27 \mathrm{E}-04$ \\
\hline
\end{tabular}

\begin{tabular}{|c|c|c|c|}
\hline 9884 & $\mathrm{ZFC3H1}$ & -4.19 & $1.53 \mathrm{E}-03$ \\
\hline 9885 & ZFHX3 & -2.41 & $2.73 \mathrm{E}-02$ \\
\hline 9886 & $\mathrm{ZFX}$ & -2.48 & $9.85 \mathrm{E}-05$ \\
\hline 9887 & ZFYVE19 & -2.92 & $5.23 \mathrm{E}-03$ \\
\hline 9888 & ZFYVE20 & -1.67 & $1.65 \mathrm{E}-02$ \\
\hline 9889 & ZFYVE21 & -4.08 & 4.44E-04 \\
\hline 9890 & ZFYVE28 & -2.63 & $6.18 \mathrm{E}-03$ \\
\hline 9891 & ZFYVE9 & -3.55 & 3.56E-04 \\
\hline 9892 & ZIC1 & -4.30 & $8.28 \mathrm{E}-04$ \\
\hline 9893 & ZMAT2 & -4.97 & $6.19 \mathrm{E}-05$ \\
\hline 9894 & ZMYM4 & -2.56 & $2.94 \mathrm{E}-02$ \\
\hline 9895 & ZNF143 & -3.23 & $2.62 \mathrm{E}-04$ \\
\hline 9896 & ZNF217 & -3.70 & $1.13 \mathrm{E}-03$ \\
\hline 9897 & ZNF236 & -1.81 & $3.32 \mathrm{E}-02$ \\
\hline 9898 & ZNF238 & -2.78 & $3.12 \mathrm{E}-05$ \\
\hline 9899 & ZNF259 & -1.39 & $1.17 \mathrm{E}-03$ \\
\hline 9900 & ZNF277 & -2.95 & $3.49 \mathrm{E}-05$ \\
\hline 9901 & ZNF280D & -2.17 & $1.71 \mathrm{E}-02$ \\
\hline 9902 & ZNF292 & -3.46 & 2.69E-04 \\
\hline 9903 & ZNF318 & -3.23 & $5.92 \mathrm{E}-04$ \\
\hline 9904 & ZNF326 & -5.23 & $1.55 \mathrm{E}-04$ \\
\hline 9905 & ZNF330 & -2.06 & $1.45 \mathrm{E}-03$ \\
\hline 9906 & ZNF335 & -2.54 & $2.82 \mathrm{E}-03$ \\
\hline 9907 & ZNF341 & -4.74 & $9.21 \mathrm{E}-04$ \\
\hline 9908 & ZNF346 & -3.76 & 4.88E-03 \\
\hline 9909 & ZNF365 & -3.03 & $1.23 \mathrm{E}-03$ \\
\hline 9910 & ZNF367 & -3.05 & 3.87E-03 \\
\hline 9911 & ZNF407 & -1.16 & $2.13 \mathrm{E}-02$ \\
\hline 9912 & ZNF410 & -3.95 & $2.21 \mathrm{E}-04$ \\
\hline 9913 & ZNF507 & -2.64 & $2.78 \mathrm{E}-03$ \\
\hline 9914 & ZNF511 & -1.23 & $1.52 \mathrm{E}-02$ \\
\hline 9915 & ZNF512 & -2.72 & $9.89 \mathrm{E}-04$ \\
\hline 9916 & ZNF513 & -1.39 & $1.69 \mathrm{E}-02$ \\
\hline 9917 & ZNF568 & -2.93 & $1.11 \mathrm{E}-03$ \\
\hline 9918 & ZNF592 & -3.34 & $6.80 \mathrm{E}-03$ \\
\hline 9919 & ZNF593 & -6.46 & $7.51 \mathrm{E}-05$ \\
\hline 9920 & ZNF598 & -3.54 & $1.07 \mathrm{E}-03$ \\
\hline 9921 & ZNF609 & -1.31 & $1.01 \mathrm{E}-02$ \\
\hline 9922 & ZNF618 & -4.72 & 5.47E-03 \\
\hline 9923 & ZNF622 & -4.37 & $1.00 \mathrm{E}-03$ \\
\hline 9924 & ZNF639 & -4.52 & 4.63E-04 \\
\hline 9925 & ZNF692 & -1.69 & $9.84 \mathrm{E}-03$ \\
\hline 9926 & ZNF704 & -1.76 & $5.86 \mathrm{E}-02$ \\
\hline 9927 & ZNF706 & -4.86 & $1.76 \mathrm{E}-04$ \\
\hline 9928 & ZNF777 & -2.29 & $1.34 \mathrm{E}-03$ \\
\hline 9929 & ZNF804A & -3.80 & $7.49 \mathrm{E}-03$ \\
\hline 9930 & ZNF821 & -3.75 & $2.26 \mathrm{E}-04$ \\
\hline 9931 & ZNF830 & -2.74 & 9.07E-03 \\
\hline 9932 & ZNHIT6 & -3.41 & 6.69E-04 \\
\hline 9933 & ZNRF2 & -3.76 & $5.28 \mathrm{E}-04$ \\
\hline 9934 & ZNRF4 & -7.06 & 3.84E-05 \\
\hline 9935 & ZP4 & -2.49 & $1.04 \mathrm{E}-03$ \\
\hline 9936 & ZРBP & -7.60 & 1.12E-04 \\
\hline 9937 & ZPBP2 & -3.44 & $5.40 \mathrm{E}-03$ \\
\hline 9938 & ZPD & -3.30 & $5.61 \mathrm{E}-04$ \\
\hline 9939 & ZRANB2 & -3.06 & $6.74 \mathrm{E}-03$ \\
\hline 9940 & ZRSR2 & -4.83 & $1.21 \mathrm{E}-03$ \\
\hline 9941 & ZSWIM7 & $\begin{array}{l}-7.47 \\
\end{array}$ & $1.99 \mathrm{E}-04$ \\
\hline 9942 & ZUFSP & -2.59 & $6.41 \mathrm{E}-03$ \\
\hline 9943 & ZW10 & -5.61 & $1.31 \mathrm{E}-04$ \\
\hline 9944 & ZXDC & -3.19 & $2.59 \mathrm{E}-04$ \\
\hline 9945 & $Z Y X$ & -3.72 & $1.54 \mathrm{E}-04$ \\
\hline 9946 & ZZZZ & -2.49 & $2.36 \mathrm{E}-03$ \\
\hline
\end{tabular}


Table S1: NanoDrop analysis of labelled cRNA

\begin{tabular}{|c|c|c|c|c|c|}
\hline Sample & Dye & Pmol/ $\mu \mathrm{l}$ & $\begin{array}{c}\text { Concentration } \\
(\mathrm{ng} / \mu \mathrm{l})\end{array}$ & $260 / 280$ & $\begin{array}{c}\text { Specific } \\
\text { activity }\end{array}$ \\
\hline Testis_1 & Cy3 & 3.43 & 243.12 & 2.28 & 14.11 \\
\hline Testis_2 & Cy3 & 2.55 & 177.90 & 2.30 & 14.33 \\
\hline Sperm_1 & Cy3 & 11.93 & 645.76 & 2.32 & 18.47 \\
\hline Sperm_2 & Cy3 & 4.73 & 295.58 & 2.16 & 16.00 \\
\hline
\end{tabular}

Figure S1: The scatter plots of gene expression values in two biological replicated showing linear relationship (A: testis replicates; B: sperm replicates)
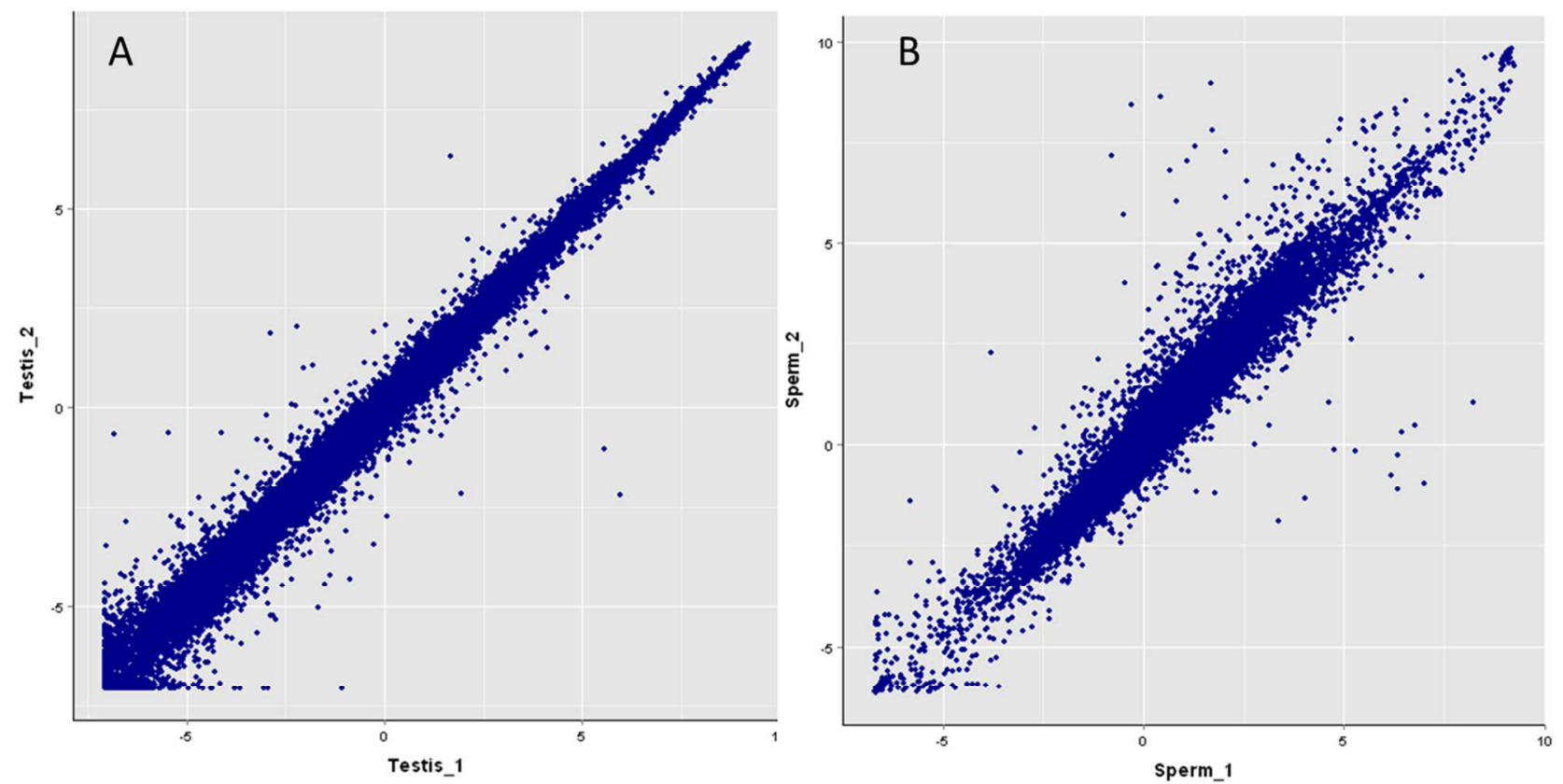

Table S2:: Correlation coefficient based on the expression intensity values in testis and sperm samples.

\begin{tabular}{|c|c|c|c|c|}
\hline Array Name & Testis_1 & Testis_2 & Sperm_1 & Sperm_2 \\
\hline Testis_1 & 1 & & & \\
\hline Testis_2 & 0.995499 & 1 & & \\
\hline Sperm_1 & & & 1 & \\
\hline Sperm_2 & & & 0.916439 & 1 \\
\hline
\end{tabular}




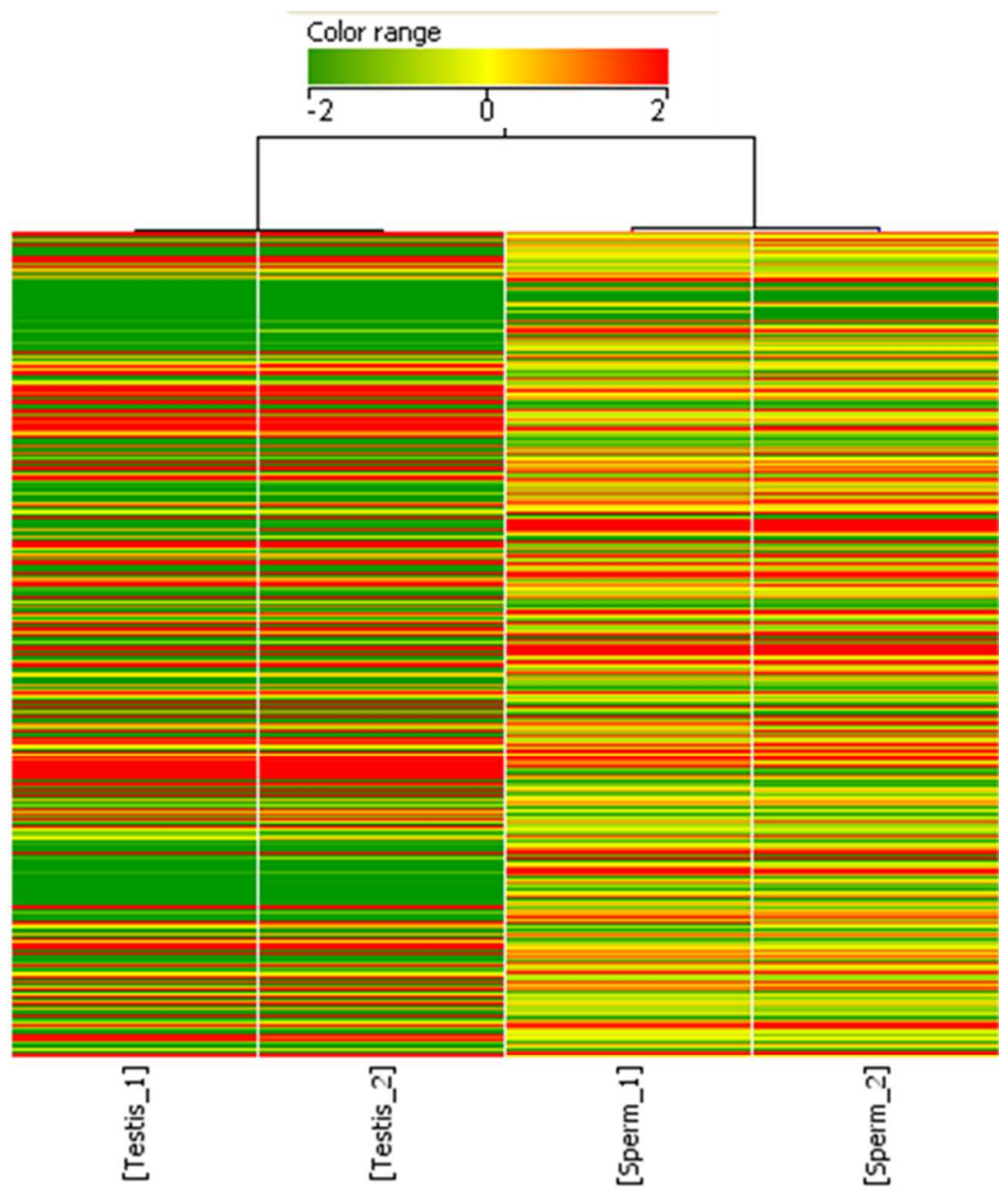

Figure S2: Image showing clusters of genes for intra-array quality control. Intra-array normalization deals with variability within a single array. In intra array normalization, gprocessed signals (dye normalized background subtracted signal intensity) was log transformed and then for each of the array the $75^{\text {th }}$ percentile value was calculated separately. In each sample the log transformed intensity values for each probe was subtracted by the calculated $75^{\text {th }}$ percentile value of the respective array and expression values were obtained. 
Table S3: A list of down regulated transcripts related to ribosomal machinery in sperm.

\begin{tabular}{|c|c|c|c|}
\hline S. No & Gene Name & Fold Expression & P-Value \\
\hline 1 & RPL30 & -8.97 & $5.12 \mathrm{E}-05$ \\
\hline 2 & RPLP1 & -8.85 & $1.46 \mathrm{E}-05$ \\
\hline 3 & RPL37 & -8.80 & $3.76 \mathrm{E}-04$ \\
\hline 4 & RPL26L1 & -8.74 & $6.22 \mathrm{E}-06$ \\
\hline 5 & RPL9 & -8.66 & $8.56 \mathrm{E}-05$ \\
\hline 6 & ENSGALT00000035726 & -8.65 & $2.12 \mathrm{E}-05$ \\
\hline 7 & RPL32 & -8.51 & 2.60E-05 \\
\hline 8 & RPL35 & -8.51 & 5.03E-05 \\
\hline 9 & RPL29 & -8.46 & $3.18 \mathrm{E}-04$ \\
\hline 10 & RPS20 & -8.44 & $1.12 \mathrm{E}-04$ \\
\hline 11 & RPL24 & -8.33 & 2.15E-04 \\
\hline 12 & RPS27A & -8.32 & $1.43 \mathrm{E}-04$ \\
\hline 13 & RPL5 & -8.28 & $2.95 \mathrm{E}-05$ \\
\hline 14 & RPS4X & -8.25 & $5.22 \mathrm{E}-05$ \\
\hline 15 & RPS6 & -8.19 & $4.82 \mathrm{E}-05$ \\
\hline 16 & RPS8 & -8.18 & $1.06 \mathrm{E}-04$ \\
\hline 17 & RPS17L & -8.14 & 4.06E-05 \\
\hline 18 & RPL13 & -8.06 & $1.89 \mathrm{E}-05$ \\
\hline 19 & RPS12 & -8.03 & $3.06 \mathrm{E}-05$ \\
\hline 20 & RPL4 & -7.97 & 3.53E-05 \\
\hline 21 & RPL37A & -7.91 & 7.16E-05 \\
\hline
\end{tabular}




\begin{tabular}{|c|c|c|c|}
\hline 22 & MRPS17 & -7.87 & 1.84E-04 \\
\hline 23 & RPL35A & -7.83 & 4.50E-04 \\
\hline 24 & RPS23 & -7.79 & $1.65 \mathrm{E}-05$ \\
\hline 25 & RPL18A & -7.73 & $9.22 \mathrm{E}-04$ \\
\hline 26 & RPS3 & -7.71 & 9.65E-05 \\
\hline 27 & RPS11 & -7.63 & 2.11E-05 \\
\hline 28 & RPL36 & -7.61 & 7.45E-05 \\
\hline 29 & RPS7 & -7.53 & 4.40E-04 \\
\hline 30 & MRPS28 & -7.45 & $5.98 \mathrm{E}-06$ \\
\hline 31 & RPL11 & -7.44 & 2.24E-04 \\
\hline 32 & RPS14 & -7.43 & 3.03E-05 \\
\hline 33 & RPSA & -7.43 & 3.57E-05 \\
\hline 34 & RPL19 & -7.42 & 2.59E-04 \\
\hline 35 & MRPL18 & -7.39 & $1.20 \mathrm{E}-04$ \\
\hline 36 & RPL10A & -7.39 & $5.14 \mathrm{E}-04$ \\
\hline 37 & RPS29 & -7.31 & 1.29E-03 \\
\hline 38 & RPS15 & -7.29 & $5.11 \mathrm{E}-04$ \\
\hline 39 & ENSGALT00000002410 & -7.22 & $3.11 \mathrm{E}-04$ \\
\hline 40 & RPL7 & -7.22 & 1.17E-04 \\
\hline 41 & RPL27 & -7.21 & 2.27E-04 \\
\hline 42 & ENSGALT00000035955 & -7.09 & 4.63E-05 \\
\hline 43 & MRP63 & -7.07 & $1.70 \mathrm{E}-04$ \\
\hline 44 & RPL3 & -7.06 & 4.10E-04 \\
\hline
\end{tabular}




\begin{tabular}{|c|c|c|c|}
\hline 45 & RPL21 & -7.02 & $9.09 \mathrm{E}-05$ \\
\hline 46 & MRPL23 & -6.87 & $3.41 \mathrm{E}-04$ \\
\hline 47 & UBA52 & -6.71 & $5.55 \mathrm{E}-05$ \\
\hline 48 & MRPL44 & -6.54 & $2.89 \mathrm{E}-04$ \\
\hline 49 & RPS13 & -6.43 & $4.45 \mathrm{E}-04$ \\
\hline 50 & MRPL27 & -6.27 & $1.05 \mathrm{E}-04$ \\
\hline 51 & MRPL50 & -6.23 & $5.19 \mathrm{E}-04$ \\
\hline 52 & ENSGALT00000033073 & -6.02 & $2.46 \mathrm{E}-03$ \\
\hline 53 & MRPL39 & -6.00 & $1.50 \mathrm{E}-04$ \\
\hline 54 & MRPL30 & -5.90 & $5.15 \mathrm{E}-04$ \\
\hline 55 & RPLP2 & -5.87 & $1.79 \mathrm{E}-04$ \\
\hline 56 & RPL7L1 & -5.82 & $1.73 \mathrm{E}-03$ \\
\hline 57 & MRPS23 & -5.81 & $8.71 \mathrm{E}-04$ \\
\hline 58 & MRPS12 & -5.78 & 2.47E-04 \\
\hline 59 & MRPL53 & -5.70 & $1.96 \mathrm{E}-03$ \\
\hline 60 & MRPS25 & -5.68 & $3.96 \mathrm{E}-03$ \\
\hline 61 & MRPL21 & -5.65 & $5.26 \mathrm{E}-04$ \\
\hline 62 & MRPS5 & -5.65 & 2.44E-04 \\
\hline 63 & MRPL12 & -5.58 & $2.18 \mathrm{E}-05$ \\
\hline 64 & MRPL16 & -5.30 & $5.73 \mathrm{E}-04$ \\
\hline 65 & RRP15 & -5.30 & $8.73 \mathrm{E}-05$ \\
\hline 66 & MRPL15 & -5.17 & $8.48 \mathrm{E}-04$ \\
\hline 67 & MRPL38 & -5.13 & 2.37E-04 \\
\hline
\end{tabular}




\begin{tabular}{|c|c|c|c|}
\hline 68 & MRPS26 & -5.13 & $1.50 \mathrm{E}-03$ \\
\hline 69 & MRPS36 & -5.11 & $1.92 \mathrm{E}-03$ \\
\hline 70 & MRPL48 & -5.08 & $3.23 E-04$ \\
\hline 71 & MRPS30 & -5.06 & 1.39E-03 \\
\hline 72 & RSL24D1 & -5.06 & $3.72 \mathrm{E}-04$ \\
\hline 73 & MRPS18A & -5.00 & $1.08 \mathrm{E}-03$ \\
\hline 74 & RRP7A & -4.97 & 8.83E-04 \\
\hline 75 & MRPS22 & -4.88 & $2.32 \mathrm{E}-04$ \\
\hline 76 & MRPL3 & -4.85 & $1.86 \mathrm{E}-03$ \\
\hline 77 & MRPS14 & -4.69 & 2.32E-03 \\
\hline 78 & MRPL37 & -4.65 & 2.54E-04 \\
\hline 79 & MRPL24 & -4.63 & $2.38 \mathrm{E}-03$ \\
\hline 80 & $\mathrm{ZCCHC} 17$ & -4.59 & 8.67E-04 \\
\hline 81 & MRPL45 & -4.42 & $2.02 \mathrm{E}-03$ \\
\hline 82 & MRPL33 & -4.34 & $1.75 \mathrm{E}-04$ \\
\hline 83 & MRPS35 & -4.34 & 4.96E-04 \\
\hline 84 & ENSGALT00000002763 & -4.33 & 9.95E-05 \\
\hline 85 & MRPL51 & -4.19 & $1.21 \mathrm{E}-03$ \\
\hline 86 & MRPL28 & -4.16 & 8.10E-03 \\
\hline 87 & MRPL32 & -4.04 & $5.23 \mathrm{E}-04$ \\
\hline 88 & MRPS10 & -3.99 & $2.56 \mathrm{E}-03$ \\
\hline 89 & DAP3 & -3.98 & 4.90E-03 \\
\hline 90 & MRPS21 & -3.85 & 7.29E-04 \\
\hline
\end{tabular}




\begin{tabular}{|c|c|c|c|}
\hline 91 & TC409229 & -3.64 & $1.33 \mathrm{E}-03$ \\
\hline 92 & RSL1D1 & -3.50 & $5.90 \mathrm{E}-04$ \\
\hline 93 & ENSGALT00000035573 & -3.47 & 1.69E-03 \\
\hline 94 & MRPL2 & -3.24 & $1.06 \mathrm{E}-02$ \\
\hline 95 & NPM1 & -3.23 & $1.71 \mathrm{E}-03$ \\
\hline 96 & MRPS33 & -3.10 & 2.61E-02 \\
\hline 97 & MRPL19 & -3.05 & 1.79E-03 \\
\hline 98 & RRNAD1 & -2.80 & 1.00E-03 \\
\hline 99 & MRPS6 & -2.76 & $1.66 \mathrm{E}-02$ \\
\hline 100 & MRPL22 & -2.69 & $6.31 \mathrm{E}-04$ \\
\hline 101 & MRPS2 & -2.56 & $9.10 \mathrm{E}-03$ \\
\hline 102 & MRPS27 & -2.39 & 2.41E-03 \\
\hline 103 & MRPL1 & -2.32 & $1.05 \mathrm{E}-02$ \\
\hline 104 & RRP1B & -2.28 & 2.06E-01 \\
\hline 105 & RPS6KC1 & -2.27 & $6.96 \mathrm{E}-03$ \\
\hline 106 & RPS6KA2 & -2.20 & 4.77E-03 \\
\hline 107 & MALSU1 & -1.89 & $6.28 \mathrm{E}-03$ \\
\hline 108 & TC399074 & -1.75 & $1.92 \mathrm{E}-03$ \\
\hline 109 & RPS6KA6 & -1.41 & $6.62 \mathrm{E}-02$ \\
\hline 110 & MRPL10 & -1.34 & $3.96 \mathrm{E}-02$ \\
\hline
\end{tabular}


Table S4: RT-qPCR values of $A C T B$ and GAPDH in testis and sperm RNA samples

\begin{tabular}{|c|c|c|}
\hline \multirow{2}{*}{ Gene name } & \multicolumn{2}{|c|}{ Ct values } \\
\cline { 2 - 3 } & Testis RNA & Sperm RNA \\
\hline ACTB & 22.88 & 34.93 \\
\hline GAPDH & 24.44 & 25.07 \\
\hline
\end{tabular}




\begin{tabular}{|c|c|c|c|c|c|c|c|c|c|c|c|}
\hline S.No. & Gene Fold & hange & P-Value & 68 & ADAMTS9 & 1.04 & $2.15 \mathrm{E}-02$ & 136 & AJ719919 & 2.45 & $3.36 \mathrm{E}-03$ \\
\hline 1 & 41699 & 1.74 & $2.05 E-02$ & 69 & ADAMTSL1 & 1.55 & $6.57 \mathrm{E}-02$ & 137 & AJ720282 & 3.38 & $2.10 \mathrm{E}-03$ \\
\hline 2 & 41702 & 1.81 & $1.34 \mathrm{E}-02$ & 70 & ADAMTSL3 & 3.79 & $3.96 \mathrm{E}-04$ & 138 & AJ720749 & 1.88 & $1.89 \mathrm{E}-02$ \\
\hline 3 & 41706 & 3.04 & $3.82 \mathrm{E}-03$ & 71 & ADARB2 & 6.77 & $1.02 \mathrm{E}-03$ & 139 & AJ720835 & 6.50 & $4.04 E-03$ \\
\hline 4 & 41894 & 2.44 & $1.29 \mathrm{E}-04$ & 72 & ADCY1 & 4.36 & $1.29 \mathrm{E}-02$ & 140 & AJ721074 & 1.14 & 7.47E-03 \\
\hline 5 & M28100 & 1.19 & $1.89 \mathrm{E}-01$ & 73 & ADCY5 & 2.80 & $5.93 \mathrm{E}-03$ & 141 & AJ722285 & 4.41 & $7.25 \mathrm{E}-05$ \\
\hline 6 & $\mathrm{~A} 1 \mathrm{CF}$ & 1.96 & $1.09 \mathrm{E}-02$ & 74 & ADIPOQ & 1.98 & $2.39 \mathrm{E}-03$ & 142 & AJ729814 & 2.11 & $1.13 \mathrm{E}-02$ \\
\hline 7 & A4GALT & 7.07 & $1.14 \mathrm{E}-03$ & 75 & ADMP & 2.11 & $3.69 \mathrm{E}-03$ & 143 & AJ736013 & 5.01 & $1.86 \mathrm{E}-03$ \\
\hline 8 & A4GNT & 6.42 & $1.29 \mathrm{E}-04$ & 76 & ADORA1 & 1.13 & $5.88 \mathrm{E}-03$ & 144 & AKR1B1L & 1.78 & $3.03 E-03$ \\
\hline 9 & AADACL2 & 4.83 & $4.42 E-03$ & 77 & ADORA2A & 2.95 & $1.46 \mathrm{E}-03$ & 145 & AKR1D1 & 3.14 & $1.45 \mathrm{E}-02$ \\
\hline 10 & AADACL3 & 3.81 & $8.11 \mathrm{E}-03$ & 78 & ADORA2B & 2.56 & $5.35 \mathrm{E}-04$ & 146 & AКT3 & 4.47 & $6.33 \mathrm{E}-04$ \\
\hline 11 & AAK1 & 1.67 & $9.68 \mathrm{E}-03$ & 79 & ADORA3 & 3.28 & $8.20 \mathrm{E}-03$ & 147 & AL587215 & 3.66 & $2.15 \mathrm{E}-02$ \\
\hline 12 & AANAT & 1.38 & $6.85 \mathrm{E}-02$ & 80 & ADPRHL1 & 2.60 & $2.41 \mathrm{E}-02$ & 148 & ALAD & 2.35 & $3.86 \mathrm{E}-04$ \\
\hline 13 & AB178596 & 3.40 & $1.84 \mathrm{E}-02$ & 81 & ADPRHL2 & 1.43 & $9.89 \mathrm{E}-03$ & 149 & ALDH3B1 & 3.74 & $2.72 \mathrm{E}-03$ \\
\hline 14 & AB193010 & 5.66 & $5.58 \mathrm{E}-03$ & 82 & ADRA1A & 3.34 & $1.10 \mathrm{E}-03$ & 150 & ALDH8A1 & 7.53 & $5.93 \mathrm{E}-03$ \\
\hline 15 & AB193015 & 5.10 & $6.32 \mathrm{E}-03$ & 83 & ADRA1B & 3.94 & $4.30 \mathrm{E}-03$ & 151 & ALG9 & 1.54 & $1.60 \mathrm{E}-01$ \\
\hline 16 & AB232994 & 4.67 & $1.59 \mathrm{E}-05$ & 84 & ADRA2A & 2.35 & $2.72 \mathrm{E}-04$ & 152 & ALK & 2.72 & $2.51 \mathrm{E}-03$ \\
\hline 17 & AB232995 & 2.46 & $1.47 \mathrm{E}-02$ & 85 & ADRA2B & 3.58 & $1.88 \mathrm{E}-03$ & 153 & ALPK3 & 3.03 & $3.83 E-03$ \\
\hline 18 & AB232999 & 1.51 & $1.67 \mathrm{E}-02$ & 86 & ADRA2C & 4.33 & $1.03 \mathrm{E}-02$ & 154 & ALPL & 1.66 & 4.49E-04 \\
\hline 19 & AB233000 & 5.09 & $7.96 \mathrm{E}-03$ & 87 & ADRB1 & 3.65 & $6.32 \mathrm{E}-04$ & 155 & ALX4 & 2.06 & $4.08 \mathrm{E}-03$ \\
\hline 20 & AB233001 & 3.18 & $2.57 \mathrm{E}-03$ & 88 & ADRB2 & 4.63 & $1.25 \mathrm{E}-02$ & 156 & AM067177 & 3.55 & $7.66 \mathrm{E}-03$ \\
\hline 21 & AB233004 & 3.29 & $7.95 \mathrm{E}-02$ & 89 & AF003093 & 2.22 & $1.83 \mathrm{E}-03$ & 157 & AM067499 & 4.86 & $3.33 E-04$ \\
\hline 22 & AB233009 & 2.98 & $3.34 \mathrm{E}-03$ & 90 & AF026562 & 2.74 & $2.62 \mathrm{E}-02$ & 158 & AM068168 & 1.29 & $1.56 \mathrm{E}-03$ \\
\hline 23 & AB233011 & 4.87 & 5.07E-03 & 91 & Af175441 & 6.00 & $2.82 \mathrm{E}-03$ & 159 & AM068169 & 3.57 & $8.44 \mathrm{E}-03$ \\
\hline 24 & AB233015 & 5.72 & $8.08 \mathrm{E}-03$ & 92 & AF175443 & 5.60 & $6.07 \mathrm{E}-04$ & 160 & AM070520 & 3.42 & $7.16 \mathrm{E}-03$ \\
\hline 25 & AB233016 & 4.73 & $8.05 \mathrm{E}-03$ & 93 & AF175447 & 6.12 & $2.06 \mathrm{E}-03$ & 161 & AM076726 & 6.52 & $2.30 \mathrm{E}-05$ \\
\hline 26 & AB233022 & 5.90 & $4.76 \mathrm{E}-03$ & 94 & AF175454 & 6.78 & $5.37 \mathrm{E}-04$ & 162 & AM773244 & 2.98 & $1.70 \mathrm{E}-02$ \\
\hline 27 & AB233025 & 4.15 & $1.01 \mathrm{E}-02$ & 95 & AF175456 & 2.55 & $5.03 \mathrm{E}-02$ & 163 & AM773245 & 4.87 & $8.27 E-03$ \\
\hline 28 & AB376099 & 5.56 & $4.17 \mathrm{E}-03$ & 96 & AF175458 & 5.72 & $2.47 \mathrm{E}-03$ & 164 & AM773248 & 4.03 & $1.04 \mathrm{E}-02$ \\
\hline 29 & AB617822 & 2.06 & $5.85 \mathrm{E}-03$ & 97 & AF182318 & 6.39 & $5.45 \mathrm{E}-03$ & 165 & AM773249 & 4.93 & $1.53 \mathrm{E}-03$ \\
\hline 30 & AB710349 & 1.73 & $1.02 E-02$ & 98 & AF208400 & 1.35 & $2.64 \mathrm{E}-02$ & 166 & AM773254 & 4.52 & $8.47 E-03$ \\
\hline 31 & ABCA4 & 1.79 & $3.52 \mathrm{E}-03$ & 99 & AF375474 & 1.17 & $9.12 \mathrm{E}-02$ & 167 & AM773255 & 5.75 & $6.54 \mathrm{E}-03$ \\
\hline 32 & ABCA5 & 3.31 & $3.42 \mathrm{E}-03$ & 100 & AFAP1 & 1.61 & $2.72 \mathrm{E}-03$ & 168 & AM773257 & 6.65 & $1.68 \mathrm{E}-03$ \\
\hline 33 & ABCA8 & 5.19 & $1.65 \mathrm{E}-03$ & 101 & AFAP1L2 & 1.71 & $6.35 \mathrm{E}-02$ & 169 & AM773258 & 5.40 & $7.46 \mathrm{E}-03$ \\
\hline 34 & ABCA9 & 2.93 & $6.38 \mathrm{E}-03$ & 102 & AGAP3 & 3.41 & $2.49 \mathrm{E}-06$ & 170 & AM773259 & 1.56 & $7.96 \mathrm{E}-03$ \\
\hline 35 & ABCB9 & 4.63 & $5.65 \mathrm{E}-03$ & 103 & AGBL1 & 2.29 & $1.70 \mathrm{E}-02$ & 171 & AM773262 & 2.37 & $2.28 \mathrm{E}-02$ \\
\hline 36 & ABCC3 & 1.20 & $5.15 \mathrm{E}-03$ & 104 & AGBL2 & 4.32 & $9.24 \mathrm{E}-05$ & 172 & AM773264 & 3.03 & $2.31 \mathrm{E}-02$ \\
\hline 37 & $\mathrm{ABCC} 8$ & 1.20 & $1.34 \mathrm{E}-02$ & 105 & AGMAT & 2.37 & $2.21 \mathrm{E}-02$ & 173 & AM773265 & 3.26 & $1.18 \mathrm{E}-02$ \\
\hline 38 & ABCF3 & 2.07 & $5.70 \mathrm{E}-04$ & 106 & AGPAT3 & 7.07 & $2.50 \mathrm{E}-03$ & 174 & AM773266 & 2.46 & $5.30 \mathrm{E}-03$ \\
\hline 39 & ABCG5 & 2.32 & $4.72 \mathrm{E}-03$ & 107 & AGR2 & 1.19 & $2.59 \mathrm{E}-02$ & 175 & AM773269 & 4.46 & $6.32 \mathrm{E}-03$ \\
\hline 40 & ACAD10 & 1.48 & $5.45 \mathrm{E}-03$ & 108 & AGR3 & 6.35 & $7.74 \mathrm{E}-05$ & 176 & AM773270 & 5.30 & $6.50 \mathrm{E}-04$ \\
\hline 41 & ACAN & 2.50 & $2.03 E-04$ & 109 & AGRP & 1.68 & $1.27 \mathrm{E}-03$ & 177 & AM773271 & 5.44 & $7.53 \mathrm{E}-04$ \\
\hline 42 & ACCN2 & 6.23 & $1.55 \mathrm{E}-03$ & 110 & AGT & 4.92 & $6.57 \mathrm{E}-03$ & 178 & AM773275 & 2.49 & $2.25 \mathrm{E}-02$ \\
\hline 43 & ACCN5 & 5.37 & $5.36 \mathrm{E}-03$ & 111 & AGTR1 & 4.49 & $3.90 \mathrm{E}-03$ & 179 & AM773278 & 3.44 & $6.78 \mathrm{E}-02$ \\
\hline 44 & ACE & 2.76 & $5.38 \mathrm{E}-02$ & 112 & AGTR2 & 2.81 & $6.97 \mathrm{E}-03$ & 180 & AMICA1 & 2.44 & $2.83 \mathrm{E}-03$ \\
\hline 45 & ACE2 & 2.38 & $2.05 \mathrm{E}-02$ & 113 & AGXT & 2.77 & $5.76 \mathrm{E}-02$ & 181 & AMMECR1 & 1.83 & $1.36 \mathrm{E}-02$ \\
\hline 46 & ACER1 & 2.48 & $3.02 \mathrm{E}-02$ & 114 & AGXT2L1 & 5.07 & $2.20 \mathrm{E}-04$ & 182 & AMN & 5.45 & $1.43 \mathrm{E}-03$ \\
\hline 47 & ACHE & 2.25 & $9.55 \mathrm{E}-04$ & 115 & AHCYL2 & 1.97 & $1.41 \mathrm{E}-02$ & 183 & AMOTL1 & 5.57 & $1.00 \mathrm{E}-02$ \\
\hline 48 & ACOT11 & 1.24 & $3.65 \mathrm{E}-03$ & 116 & AHNAK & 4.57 & $9.51 \mathrm{E}-03$ & 184 & AMPD1 & 4.22 & $5.20 \mathrm{E}-03$ \\
\hline 49 & ACSF3 & 2.70 & $1.92 \mathrm{E}-03$ & 117 & AHNAK2 & 5.03 & $1.01 \mathrm{E}-04$ & 185 & AMY2A & 2.68 & $4.66 \mathrm{E}-03$ \\
\hline 50 & ACSL5 & 2.57 & $1.42 \mathrm{E}-03$ & 118 & AHSG & 1.95 & $2.25 \mathrm{E}-03$ & 186 & AMZ1 & 1.48 & $4.21 \mathrm{E}-02$ \\
\hline 51 & ACSL6 & 4.99 & $2.43 \mathrm{E}-03$ & 119 & AIFM3 & 2.33 & $4.28 \mathrm{E}-02$ & 187 & ANG4 & 1.72 & $1.22 \mathrm{E}-01$ \\
\hline 52 & ACSM3 & 1.22 & $7.19 \mathrm{E}-04$ & 120 & AIM1L & 1.81 & $1.37 \mathrm{E}-01$ & 188 & ANGPT1 & 3.40 & $1.02 E-02$ \\
\hline 53 & ACSM5 & 1.60 & $4.15 \mathrm{E}-03$ & 121 & AIPL1 & 3.47 & $1.51 \mathrm{E}-03$ & 189 & ANGPTL4 & 5.44 & $1.53 \mathrm{E}-02$ \\
\hline 54 & ACTBL2 & 5.30 & $1.21 E-04$ & 122 & AIRE & 4.78 & $7.42 \mathrm{E}-03$ & 190 & ANGPTL7 & 6.00 & $4.55 E-04$ \\
\hline 55 & ADAM11 & 4.90 & $7.52 \mathrm{E}-04$ & 123 & AJ002599 & 7.66 & $2.73 \mathrm{E}-03$ & 191 & ANKIB1 & 3.99 & $3.48 \mathrm{E}-02$ \\
\hline 56 & ADAM12 & 5.23 & $2.18 \mathrm{E}-05$ & 124 & AJ002601 & 4.63 & $1.76 \mathrm{E}-03$ & 192 & ANKRD1 & 5.18 & $1.64 \mathrm{E}-03$ \\
\hline 57 & ADAM19 & 5.63 & $6.51 \mathrm{E}-05$ & 125 & AJ392630 & 5.72 & $1.78 \mathrm{E}-03$ & 193 & ANKRD2 & 3.62 & $3.50 E-04$ \\
\hline 58 & ADAM22 & 1.40 & $4.84 \mathrm{E}-02$ & 126 & AJ395369 & 2.74 & $8.42 \mathrm{E}-03$ & 194 & ANKRD29 & 1.40 & $4.93 \mathrm{E}-03$ \\
\hline 59 & ADAM28 & 2.02 & $2.38 \mathrm{E}-03$ & 127 & AJ396326 & 3.81 & $5.52 \mathrm{E}-03$ & 195 & ANKRD33 & 5.91 & $4.29 \mathrm{E}-04$ \\
\hline 60 & ADAM33 & 3.46 & $1.43 \mathrm{E}-03$ & 128 & AJ397685 & 6.92 & $2.20 \mathrm{E}-05$ & 196 & ANKRD33B & 3.86 & $4.14 \mathrm{E}-04$ \\
\hline 61 & ADAMTS1 & 1.92 & $2.36 \mathrm{E}-03$ & 129 & AJ444710 & 5.10 & $8.77 \mathrm{E}-04$ & 197 & ANKRD34B & 3.69 & $3.19 \mathrm{E}-03$ \\
\hline 62 & ADAMTS10 & 5.99 & $6.73 E-03$ & 130 & AJ447538 & 2.37 & $6.64 \mathrm{E}-02$ & 198 & ANKRD34C & 4.66 & $3.03 E-03$ \\
\hline 63 & ADAMTS14 & 6.43 & $1.72 \mathrm{E}-04$ & 131 & AJ449198 & 4.19 & $1.41 \mathrm{E}-03$ & 199 & ANKRD43 & 2.64 & $5.01 \mathrm{E}-03$ \\
\hline 64 & ADAMTS15 & 1.83 & $6.73 \mathrm{E}-03$ & 132 & AJ449718 & 1.57 & $3.49 \mathrm{E}-02$ & 200 & ANKRD52 & 1.86 & $2.23 \mathrm{E}-02$ \\
\hline 65 & ADAMTS19 & 2.20 & $2.98 \mathrm{E}-02$ & 133 & AJ452752 & 3.69 & $5.44 \mathrm{E}-04$ & 201 & ANKS1A & 2.65 & $6.82 \mathrm{E}-03$ \\
\hline 66 & ADAMTS20 & 1.94 & $2.44 \mathrm{E}-02$ & 134 & AJ455130 & 2.55 & $3.21 \mathrm{E}-03$ & 202 & ANKS1B & 1.26 & $1.04 \mathrm{E}-02$ \\
\hline 67 & ADAMTS8 & 4.15 & $3.81 \mathrm{E}-03$ & 135 & AJ456143 & 1.22 & $4.39 \mathrm{E}-02$ & 203 & ANKS4B & 5.37 & $1.81 \mathrm{E}-04$ \\
\hline
\end{tabular}




\begin{tabular}{|c|c|c|c|c|c|c|c|c|c|c|c|}
\hline 204 & ANO1 & 2.88 & $1.22 \mathrm{E}-02$ & 272 & ASB13 & 3.97 & $6.27 \mathrm{E}-03$ & 340 & BEGAIN & 8.02 & $5.28 \mathrm{E}-03$ \\
\hline 205 & ANO6 & 3.83 & $1.87 E-03$ & 273 & ASB18 & 6.60 & $1.07 E-03$ & 341 & BEST1 & 2.71 & $5.81 \mathrm{E}-03$ \\
\hline 206 & ANO8 & 2.97 & 3.83E-03 & 274 & ASB2 & 4.74 & $3.10 \mathrm{E}-03$ & 342 & BEST3 & 1.79 & $5.86 \mathrm{E}-03$ \\
\hline 207 & ANTXR1 & 5.21 & $9.03 E-04$ & 275 & ASB4 & 3.08 & $3.29 \mathrm{E}-04$ & 343 & BEST4 & 3.21 & $3.11 \mathrm{E}-02$ \\
\hline 208 & ANTXRL & 2.10 & $8.46 \mathrm{E}-03$ & 276 & ASB9 & 4.74 & 8.97E-03 & 344 & BF1 & 3.51 & $2.92 \mathrm{E}-02$ \\
\hline 209 & ANXA1 & 3.06 & $6.38 \mathrm{E}-03$ & 277 & ASCL1 & 1.65 & $3.02 E-02$ & 345 & $\mathrm{BF} 2$ & 4.55 & $1.30 \mathrm{E}-02$ \\
\hline 210 & ANXA10 & 2.23 & 4.87E-02 & 278 & ASCL2 & 5.92 & $5.47 E-02$ & 346 & B-G & 2.91 & $2.62 \mathrm{E}-02$ \\
\hline 211 & ANXA6 & 1.30 & $5.04 E-02$ & 279 & ASCL4 & 2.89 & 2.30E-02 & 347 & BG2 & 3.17 & $9.06 \mathrm{E}-04$ \\
\hline 212 & АOAH & 2.52 & $1.28 \mathrm{E}-02$ & 280 & ASL2 & 2.88 & $3.72 \mathrm{E}-03$ & 348 & BG709695 & 4.20 & $3.59 \mathrm{E}-03$ \\
\hline 213 & AP3M2 & 2.06 & 7.17E-03 & 281 & ASMT & 2.58 & $5.29 \mathrm{E}-03$ & 349 & BHLHA15 & 2.80 & $5.76 \mathrm{E}-03$ \\
\hline 214 & AP4M1 & 1.31 & $7.89 \mathrm{E}-03$ & 282 & ASPHD2 & 3.33 & 4.80E-03 & 350 & BHLHE4O & 2.43 & $2.62 \mathrm{E}-03$ \\
\hline 215 & APAF1 & 2.10 & $2.23 \mathrm{E}-04$ & 283 & ASTL & 2.99 & $1.09 \mathrm{E}-02$ & 351 & B1065499 & 2.60 & $6.48 \mathrm{E}-02$ \\
\hline 216 & APBB1IP & 2.36 & $6.04 E-03$ & 284 & ASTN2 & 2.02 & $1.67 E-02$ & 352 & B1066217 & 4.14 & $2.00 \mathrm{E}-03$ \\
\hline 217 & APBB3 & 5.76 & $1.69 \mathrm{E}-03$ & 285 & ATF3 & 2.74 & $1.28 \mathrm{E}-03$ & 353 & B1066329 & 4.60 & $3.00 \mathrm{E}-03$ \\
\hline 218 & APC2 & 2.86 & $2.43 \mathrm{E}-02$ & 286 & ATF7 & 2.89 & $1.59 \mathrm{E}-02$ & 354 & BIK & 2.16 & $1.41 \mathrm{E}-02$ \\
\hline 219 & APH1A & 1.61 & $1.64 \mathrm{E}-02$ & 287 & ATG14 & 1.37 & 4.54E-02 & 355 & BIRC7 & 1.10 & $4.46 \mathrm{E}-03$ \\
\hline 220 & APLNR & 1.85 & $2.62 E-02$ & 288 & ATG2B & 1.97 & $7.85 E-03$ & 356 & BKJ & 4.62 & $2.50 \mathrm{E}-04$ \\
\hline 221 & APOA4 & 2.19 & $1.47 \mathrm{E}-02$ & 289 & ATHL1 & 2.25 & $7.12 E-04$ & 357 & B-LA & 2.49 & $3.67 E-02$ \\
\hline 222 & APOA5 & 1.78 & $1.41 \mathrm{E}-03$ & 290 & ATMIN & 1.84 & $1.28 \mathrm{E}-02$ & 358 & BLB1 & 2.78 & $1.56 \mathrm{E}-02$ \\
\hline 223 & APOB & 3.66 & $9.56 \mathrm{E}-04$ & 291 & ATOH1 & 3.47 & 3.04E-02 & 359 & BLK & 3.13 & $1.73 \mathrm{E}-03$ \\
\hline 224 & APOBEC2 & 2.49 & $3.24 \mathrm{E}-03$ & 292 & ATOH7 & 1.81 & $1.32 \mathrm{E}-02$ & 360 & BLNK & 2.58 & $1.60 \mathrm{E}-03$ \\
\hline 225 & APOD & 4.01 & $6.04 \mathrm{E}-04$ & 293 & ATP13A2 & 3.25 & $1.30 \mathrm{E}-03$ & 361 & BLOC1S3 & 4.01 & $1.50 \mathrm{E}-03$ \\
\hline 226 & APOLD1 & 4.60 & $5.15 \mathrm{E}-03$ & 294 & АТР13АЗ & 2.72 & $1.53 \mathrm{E}-03$ & 362 & BMA1 & 1.47 & $1.50 \mathrm{E}-02$ \\
\hline 227 & APOVLDLII & 3.45 & $2.34 \mathrm{E}-04$ & 295 & ATP13A5 & 6.30 & $8.04 E-04$ & 363 & B-MA2 & 2.81 & $2.25 \mathrm{E}-03$ \\
\hline 228 & AQP12 & 2.94 & 4.17E-04 & 296 & ATP2B4 & 1.39 & $3.76 E-03$ & 364 & BMP1 & 1.44 & $5.86 \mathrm{E}-03$ \\
\hline 229 & AQP2 & 5.24 & $3.01 \mathrm{E}-03$ & 297 & ATP6V0A4 & 1.30 & $1.57 \mathrm{E}-02$ & 365 & BMP15 & 3.93 & $3.14 \mathrm{E}-05$ \\
\hline 230 & AQP3 & 4.19 & $7.71 \mathrm{E}-04$ & 298 & ATP6VOD2 & 2.79 & $2.81 \mathrm{E}-03$ & 366 & BMP3 & 1.37 & $1.62 \mathrm{E}-01$ \\
\hline 231 & AQP4 & 4.69 & $1.74 \mathrm{E}-04$ & 299 & ATP7A & 1.31 & $3.25 \mathrm{E}-03$ & 367 & BMP4 & 3.38 & $9.92 \mathrm{E}-04$ \\
\hline 232 & AQP5 & 6.39 & $2.41 \mathrm{E}-03$ & 300 & ATP9A & 3.76 & $4.93 E-05$ & 368 & BMP5 & 4.45 & $3.33 \mathrm{E}-03$ \\
\hline 233 & AQP8 & 1.77 & $3.71 \mathrm{E}-03$ & 301 & ATPBD4 & 1.15 & $2.56 \mathrm{E}-03$ & 369 & BMP7 & 3.48 & $2.56 \mathrm{E}-02$ \\
\hline 234 & ARAP3 & 1.30 & $2.54 \mathrm{E}-03$ & 302 & ATRNL1 & 1.95 & $2.11 \mathrm{E}-02$ & 370 & BMPER & 2.51 & $2.62 \mathrm{E}-03$ \\
\hline 235 & ARC & 4.84 & $4.87 \mathrm{E}-02$ & 303 & AUTS2 & 3.04 & $7.32 \mathrm{E}-04$ & 371 & BNC1 & 3.19 & $1.35 \mathrm{E}-03$ \\
\hline 236 & AREGB & 3.67 & $2.70 \mathrm{E}-03$ & 304 & AVPR1A & 3.67 & $5.85 E-03$ & 372 & BNC2 & 4.49 & $4.70 \mathrm{E}-04$ \\
\hline 237 & ARHGAP1 & 2.00 & $4.10 \mathrm{E}-03$ & 305 & AVPR1B & 5.45 & $1.33 \mathrm{E}-03$ & 373 & B-NK & 4.89 & $2.84 \mathrm{E}-03$ \\
\hline 238 & ARHGAP20 & 1.85 & $4.48 \mathrm{E}-06$ & 306 & AVPR2 & 4.16 & $4.72 E-03$ & 374 & BOK & 6.02 & $2.70 \mathrm{E}-04$ \\
\hline 239 & ARHGAP22 & 1.44 & $3.50 \mathrm{E}-03$ & 307 & AVR2 & 1.71 & $3.30 \mathrm{E}-02$ & 375 & BPI & 3.90 & $2.62 \mathrm{E}-03$ \\
\hline 240 & ARHGAP24 & 1.47 & $1.05 \mathrm{E}-02$ & 308 & AW773267 & 3.48 & $1.39 \mathrm{E}-02$ & 376 & BPIFB8 & 3.84 & $1.72 \mathrm{E}-03$ \\
\hline 241 & ARHGAP25 & 2.86 & $3.83 \mathrm{E}-04$ & 309 & AY046570 & 5.41 & $2.60 \mathrm{E}-03$ & 377 & BRF2 & 4.73 & $9.23 \mathrm{E}-03$ \\
\hline 242 & ARHGAP28 & 2.93 & $2.03 \mathrm{E}-02$ & 310 & AY332744 & 5.46 & $6.06 \mathrm{E}-03$ & 378 & BRPF3 & 1.78 & $1.05 \mathrm{E}-02$ \\
\hline 243 & ARHGAP40 & 1.91 & $2.13 \mathrm{E}-02$ & 311 & AY591907 & 3.22 & $7.48 \mathrm{E}-03$ & 379 & BRS3 & 5.81 & $3.00 \mathrm{E}-04$ \\
\hline 244 & ARHGEF11 & 2.84 & 1.49E-04 & 312 & AY640311 & 4.57 & $1.17 E-03$ & 380 & BRUNOL5 & 6.36 & $4.55 \mathrm{E}-03$ \\
\hline 245 & ARHGEF33 & 4.18 & $1.29 \mathrm{E}-02$ & 313 & AY885261 & 2.67 & $1.22 \mathrm{E}-03$ & 381 & BSN & 3.44 & $2.16 \mathrm{E}-04$ \\
\hline 246 & ARHGEF37 & 2.31 & 7.49E-03 & 314 & AY994087 & 2.20 & 4.37E-03 & 382 & $B S X$ & 2.50 & $2.42 \mathrm{E}-02$ \\
\hline 247 & ARHGEF38 & 2.52 & 2.49E-02 & 315 & B3GALT1 & 3.90 & $7.68 \mathrm{E}-03$ & 383 & BTBD11 & 1.53 & $2.11 \mathrm{E}-02$ \\
\hline 248 & ARHGEF4 & 1.85 & $1.02 E-02$ & 316 & B3GALT5 & 2.84 & $2.01 E-03$ & 384 & BTBD17 & 1.36 & $2.91 \mathrm{E}-02$ \\
\hline 249 & ARHGEF6 & 2.26 & $4.05 \mathrm{E}-02$ & 317 & B3GAT1 & 4.37 & $1.11 \mathrm{E}-03$ & 385 & BTG2 & 3.18 & $4.81 \mathrm{E}-03$ \\
\hline 250 & ARID1A & 1.82 & $2.48 \mathrm{E}-02$ & 318 & B3GNT7 & 4.35 & $1.94 \mathrm{E}-03$ & 386 & BTLA & 1.15 & $1.02 \mathrm{E}-02$ \\
\hline 251 & ARID1B & 2.81 & $3.15 \mathrm{E}-03$ & 319 & B3GNT9 & 3.91 & $1.29 \mathrm{E}-04$ & 387 & BTN1A1 & 2.32 & $1.61 \mathrm{E}-02$ \\
\hline 252 & ARID5A & 1.59 & $1.26 \mathrm{E}-02$ & 320 & B3GNTL1 & 1.63 & $8.62 \mathrm{E}-03$ & 388 & BTRC & 1.58 & $3.06 \mathrm{E}-02$ \\
\hline 253 & ARL10 & 1.48 & $3.01 \mathrm{E}-02$ & 321 & B4GALT5 & 3.10 & $5.08 \mathrm{E}-03$ & 389 & BU104496 & 2.04 & $3.32 \mathrm{E}-03$ \\
\hline 254 & ARL11 & 4.29 & $1.79 \mathrm{E}-03$ & 322 & BAAT & 1.19 & $3.24 \mathrm{E}-03$ & 390 & BU104907 & 5.53 & $1.47 \mathrm{E}-03$ \\
\hline 255 & ARL16 & 3.76 & $5.84 \mathrm{E}-04$ & 323 & BACE1 & 2.57 & $9.34 \mathrm{E}-03$ & 391 & BU104967 & 4.53 & $2.35 \mathrm{E}-03$ \\
\hline 256 & ARL2 & 1.85 & $1.24 \mathrm{E}-02$ & 324 & BAG5 & 2.78 & $1.70 E-02$ & 392 & BU107712 & 1.51 & $1.54 \mathrm{E}-03$ \\
\hline 257 & ARL4C & 2.92 & $1.22 \mathrm{E}-02$ & 325 & BAHCC1 & 3.22 & $1.26 \mathrm{E}-03$ & 393 & BU108272 & 1.94 & $8.30 \mathrm{E}-03$ \\
\hline 258 & ARL8A & 4.81 & $7.28 \mathrm{E}-04$ & 326 & BAI2 & 6.20 & $2.19 E-03$ & 394 & BU110607 & 2.63 & $1.21 \mathrm{E}-02$ \\
\hline 259 & ARNTL2 & 2.31 & $3.23 \mathrm{E}-02$ & 327 & BAI3 & 1.74 & $2.35 \mathrm{E}-02$ & 395 & BU112156 & 2.52 & $4.33 \mathrm{E}-03$ \\
\hline 260 & ARR3 & 5.41 & $8.73 \mathrm{E}-04$ & 328 & BAIAP2 & 5.02 & $8.33 E-04$ & 396 & BU112196 & 1.49 & $1.61 \mathrm{E}-02$ \\
\hline 261 & ARSA & 3.12 & $2.00 \mathrm{E}-03$ & 329 & BARX2 & 3.70 & $2.02 E-02$ & 397 & BU116348 & 1.73 & $2.08 \mathrm{E}-03$ \\
\hline 262 & ARSD & 2.24 & $3.36 \mathrm{E}-03$ & 330 & BATF & 4.49 & $3.36 \mathrm{E}-03$ & 398 & BU117764 & 3.43 & $2.09 \mathrm{E}-02$ \\
\hline 263 & ARSH & 1.38 & $2.68 \mathrm{E}-02$ & 331 & BCDIN3D & 1.31 & $1.65 \mathrm{E}-02$ & 399 & BU117855 & 2.11 & $8.01 \mathrm{E}-03$ \\
\hline 264 & ARSI & 5.72 & $2.06 \mathrm{E}-03$ & 332 & BCL11A & 3.92 & $5.05 E-03$ & 400 & BU119689 & 3.80 & $7.72 \mathrm{E}-03$ \\
\hline 265 & ART1 & 2.16 & $2.30 \mathrm{E}-02$ & 333 & BCL11B & 5.28 & $1.39 \mathrm{E}-03$ & 401 & BU120493 & 2.94 & $1.84 \mathrm{E}-04$ \\
\hline 266 & ART4 & 6.99 & $3.81 \mathrm{E}-03$ & 334 & BCL2A1 & 3.08 & $5.73 E-03$ & 402 & BU122542 & 4.35 & $5.29 \mathrm{E}-03$ \\
\hline 267 & ART5 & 2.17 & $7.52 \mathrm{E}-03$ & 335 & BCL9 & 2.64 & $1.61 \mathrm{E}-02$ & 403 & BU123298 & 2.27 & $1.58 \mathrm{E}-03$ \\
\hline 268 & ART7B & 4.97 & $1.18 \mathrm{E}-03$ & 336 & BCL9L & 1.71 & $1.07 E-02$ & 404 & BU123609 & 2.69 & $1.07 \mathrm{E}-02$ \\
\hline 269 & ARV1 & 1.28 & $2.38 \mathrm{E}-02$ & 337 & BDKRB1 & 3.90 & $1.93 \mathrm{E}-02$ & 405 & BU123685 & 2.94 & $3.46 \mathrm{E}-04$ \\
\hline 270 & ASAH2 & 3.71 & 7.57E-03 & 338 & BDKRB2 & 3.69 & $6.72 \mathrm{E}-04$ & 406 & BU123752 & 2.40 & $5.30 \mathrm{E}-02$ \\
\hline 271 & ASB1 & 1.90 & $8.75 \mathrm{E}-03$ & 339 & BDNF & 1.95 & $1.33 \mathrm{E}-02$ & 407 & BU124345 & 1.24 & $2.63 \mathrm{E}-03$ \\
\hline
\end{tabular}




\begin{tabular}{|c|c|c|c|c|c|c|c|c|c|c|c|}
\hline 408 & BU124761 & 2.43 & $4.91 E-03$ & 476 & BU308037 & 1.89 & $1.51 \mathrm{E}-02$ & 544 & BU 444312 & 1.95 & $1.53 \mathrm{E}-04$ \\
\hline 409 & BU125157 & 3.39 & $1.43 \mathrm{E}-03$ & 477 & BU310423 & 1.93 & $2.48 \mathrm{E}-03$ & 545 & BU447010 & 2.54 & 2.92E-02 \\
\hline 410 & BU131051 & 3.92 & $3.74 \mathrm{E}-03$ & 478 & BU312095 & 1.89 & $4.29 \mathrm{E}-02$ & 546 & BU 448424 & 1.40 & $5.20 \mathrm{E}-03$ \\
\hline 411 & BU131321 & 3.84 & $2.06 \mathrm{E}-04$ & 479 & BU315004 & 2.67 & $4.62 \mathrm{E}-02$ & 547 & BU450216 & 1.56 & $4.22 \mathrm{E}-02$ \\
\hline 412 & BU132303 & 2.65 & $1.08 \mathrm{E}-02$ & 480 & BU315457 & 1.69 & $3.37 \mathrm{E}-02$ & 548 & BU453976 & 1.66 & 7.37E-03 \\
\hline 413 & BU134784 & 4.92 & $1.16 \mathrm{E}-02$ & 481 & BU316204 & 1.30 & $9.10 \mathrm{E}-04$ & 549 & BU454962 & 3.03 & $6.22 \mathrm{E}-04$ \\
\hline 414 & BU135594 & 2.08 & $1.27 \mathrm{E}-02$ & 482 & BU316679 & 3.09 & $1.60 \mathrm{E}-02$ & 550 & BU455514 & 7.25 & $5.37 E-04$ \\
\hline 415 & BU138204 & 2.99 & $5.73 \mathrm{E}-03$ & 483 & BU317892 & 1.27 & $2.24 \mathrm{E}-04$ & 551 & BU455976 & 3.07 & $2.08 \mathrm{E}-03$ \\
\hline 416 & BU141905 & 4.55 & $7.04 E-03$ & 484 & BU318920 & 1.30 & $1.78 \mathrm{E}-02$ & 552 & BU456537 & 4.24 & $2.33 \mathrm{E}-03$ \\
\hline 417 & BU144237 & 1.26 & $2.88 \mathrm{E}-03$ & 485 & BU319628 & 3.51 & $5.59 \mathrm{E}-04$ & 553 & BU458939 & 4.90 & $4.88 \mathrm{E}-04$ \\
\hline 418 & BU144418 & 1.59 & $1.85 \mathrm{E}-02$ & 486 & BU324978 & 3.13 & $2.16 \mathrm{E}-02$ & 554 & BU459921 & 2.22 & $6.59 \mathrm{E}-03$ \\
\hline 419 & BU200094 & 3.45 & $2.81 \mathrm{E}-04$ & 487 & BU326672 & 1.75 & $7.65 \mathrm{E}-03$ & 555 & BU461005 & 6.62 & 4.16E-04 \\
\hline 420 & BU201425 & 1.42 & $4.96 \mathrm{E}-02$ & 488 & BU326817 & 2.85 & $1.27 \mathrm{E}-03$ & 556 & BU461157 & 4.32 & $1.06 \mathrm{E}-02$ \\
\hline 421 & BU201899 & 4.46 & $2.54 \mathrm{E}-03$ & 489 & BU326944 & 3.06 & $6.57 \mathrm{E}-02$ & 557 & BU461339 & 5.28 & $5.70 \mathrm{E}-03$ \\
\hline 422 & BU203936 & 4.72 & $2.39 \mathrm{E}-03$ & 490 & BU327801 & 2.15 & $1.43 \mathrm{E}-02$ & 558 & BU 462383 & 3.30 & $8.98 \mathrm{E}-03$ \\
\hline 423 & BU206349 & 4.14 & $2.53 \mathrm{E}-03$ & 491 & BU328025 & 3.14 & $1.56 \mathrm{E}-03$ & 559 & BU462443 & 2.38 & $5.50 \mathrm{E}-03$ \\
\hline 424 & BU208007 & 3.89 & $9.36 \mathrm{E}-03$ & 492 & BU329695 & 2.24 & $6.49 \mathrm{E}-02$ & 560 & BU462843 & 3.64 & $1.19 \mathrm{E}-03$ \\
\hline 425 & BU216519 & 4.32 & $1.30 \mathrm{E}-03$ & 493 & BU335749 & 4.23 & $6.54 \mathrm{E}-03$ & 561 & BU462933 & 1.64 & $6.65 \mathrm{E}-03$ \\
\hline 426 & BU216729 & 1.19 & $2.97 \mathrm{E}-03$ & 494 & BU336605 & 2.36 & $8.65 \mathrm{E}-02$ & 562 & BU463196 & 2.72 & $8.63 \mathrm{E}-03$ \\
\hline 427 & BU217250 & 1.47 & $3.36 \mathrm{E}-02$ & 495 & BU340614 & 2.51 & $2.53 \mathrm{E}-05$ & 563 & BU463320 & 1.46 & $2.56 \mathrm{E}-02$ \\
\hline 428 & BU218651 & 2.21 & $2.76 \mathrm{E}-03$ & 496 & BU340657 & 1.97 & $8.16 \mathrm{E}-03$ & 564 & BU464939 & 1.76 & $3.06 \mathrm{E}-02$ \\
\hline 429 & BU218730 & 2.98 & $1.18 \mathrm{E}-03$ & 497 & BU344626 & 1.72 & $4.12 \mathrm{E}-02$ & 565 & BU467863 & 7.21 & $9.05 E-04$ \\
\hline 430 & BU222413 & 1.39 & $3.61 \mathrm{E}-02$ & 498 & BU347949 & 5.34 & 2.09E-03 & 566 & BU468825 & 1.44 & $1.35 \mathrm{E}-02$ \\
\hline 431 & BU225747 & 3.02 & $2.25 \mathrm{E}-03$ & 499 & BU350064 & 4.27 & $1.39 \mathrm{E}-02$ & 567 & BU468854 & 2.88 & $6.49 \mathrm{E}-03$ \\
\hline 432 & BU227140 & 3.80 & $5.04 \mathrm{E}-02$ & 500 & BU350714 & 1.10 & $1.46 \mathrm{E}-03$ & 568 & BU469805 & 4.68 & $1.58 \mathrm{E}-03$ \\
\hline 433 & BU228965 & 1.30 & $1.77 \mathrm{E}-02$ & 501 & BU353109 & 2.09 & $1.78 \mathrm{E}-02$ & 569 & BU 470522 & 4.66 & $8.41 E-03$ \\
\hline 434 & BU231018 & 1.44 & $2.42 \mathrm{E}-02$ & 502 & BU354922 & 3.75 & $2.49 \mathrm{E}-02$ & 570 & BU 473004 & 2.29 & $5.48 \mathrm{E}-02$ \\
\hline 435 & BU231446 & 5.91 & $2.34 \mathrm{E}-03$ & 503 & BU356078 & 4.88 & $7.09 \mathrm{E}-04$ & 571 & BU482205 & 1.79 & $2.17 E-02$ \\
\hline 436 & BU233544 & 4.18 & $1.15 \mathrm{E}-02$ & 504 & BU359585 & 1.43 & $1.48 \mathrm{E}-02$ & 572 & BU485799 & 6.91 & $3.89 \mathrm{E}-03$ \\
\hline 437 & BU233706 & 1.62 & $7.95 E-03$ & 505 & BU364686 & 2.52 & $9.04 \mathrm{E}-03$ & 573 & BU486057 & 2.25 & $2.11 \mathrm{E}-03$ \\
\hline 438 & BU233812 & 3.85 & $2.54 \mathrm{E}-03$ & 506 & BU364817 & 6.51 & $1.73 \mathrm{E}-03$ & 574 & BX257537 & 5.41 & $8.83 E-04$ \\
\hline 439 & BU240107 & 2.14 & $5.31 \mathrm{E}-02$ & 507 & BU365339 & 2.87 & $4.42 \mathrm{E}-02$ & 575 & BX258330 & 4.39 & $2.07 E-03$ \\
\hline 440 & BU240738 & 4.59 & $1.69 \mathrm{E}-03$ & 508 & BU365462 & 1.81 & $6.01 \mathrm{E}-03$ & 576 & BX258816 & 4.17 & $7.08 \mathrm{E}-04$ \\
\hline 441 & BU241776 & 2.91 & $1.70 \mathrm{E}-02$ & 509 & BU365573 & 3.05 & $2.72 \mathrm{E}-04$ & 577 & BX260390 & 4.61 & $1.34 \mathrm{E}-03$ \\
\hline 442 & BU247163 & 3.23 & $2.28 \mathrm{E}-03$ & 510 & BU367801 & 1.47 & $7.39 \mathrm{E}-03$ & 578 & BX260400 & 2.70 & $1.03 E-02$ \\
\hline 443 & BU247582 & 2.77 & $1.16 \mathrm{E}-03$ & 511 & BU372304 & 1.48 & $1.59 \mathrm{E}-02$ & 579 & BX260631 & 6.80 & $2.29 \mathrm{E}-03$ \\
\hline 444 & BU250077 & 5.19 & $7.12 \mathrm{E}-03$ & 512 & BU373338 & 3.76 & $5.62 \mathrm{E}-03$ & 580 & BX261457 & 3.05 & $1.30 \mathrm{E}-03$ \\
\hline 445 & BU250980 & 3.51 & $9.46 \mathrm{E}-03$ & 513 & BU374316 & 2.45 & $9.24 \mathrm{E}-03$ & 581 & BX261858 & 4.25 & $2.46 \mathrm{E}-04$ \\
\hline 446 & BU252373 & 1.24 & $1.90 \mathrm{E}-02$ & 514 & BU375915 & 2.61 & $2.18 \mathrm{E}-03$ & 582 & BX262098 & 2.94 & $1.64 \mathrm{E}-02$ \\
\hline 447 & BU253273 & 1.99 & $1.65 \mathrm{E}-03$ & 515 & BU377450 & 3.19 & $1.40 \mathrm{E}-02$ & 583 & BX262726 & 2.39 & $1.54 \mathrm{E}-02$ \\
\hline 448 & BU256099 & 2.27 & $5.79 \mathrm{E}-03$ & 516 & BU380356 & 9.45 & $4.16 \mathrm{E}-05$ & 584 & BX263536 & 7.01 & $8.30 \mathrm{E}-04$ \\
\hline 449 & BU257729 & 4.12 & $2.32 \mathrm{E}-04$ & 517 & BU380534 & 4.82 & $6.35 \mathrm{E}-04$ & 585 & BX263618 & 3.01 & $4.61 \mathrm{E}-02$ \\
\hline 450 & BU263572 & 4.76 & 4.63E-03 & 518 & BU381671 & 1.70 & $1.71 \mathrm{E}-02$ & 586 & BX268607 & 1.66 & $2.19 \mathrm{E}-02$ \\
\hline 451 & BU264482 & 2.63 & $7.54 E-04$ & 519 & BU382710 & 5.71 & $7.34 \mathrm{E}-04$ & 587 & BX271739 & 2.47 & $6.03 E-03$ \\
\hline 452 & BU270017 & 2.30 & $3.98 \mathrm{E}-03$ & 520 & BU384146 & 2.30 & $3.83 \mathrm{E}-03$ & 588 & BX273250 & 4.41 & 4.11E-02 \\
\hline 453 & BU272111 & 3.02 & $4.21 E-04$ & 521 & BU384182 & 2.12 & $2.67 \mathrm{E}-03$ & 589 & BX273552 & 7.62 & $2.48 \mathrm{E}-03$ \\
\hline 454 & BU273151 & 1.62 & $1.09 \mathrm{E}-02$ & 522 & BU384771 & 2.14 & $7.40 \mathrm{E}-04$ & 590 & BX274565 & 5.36 & $5.05 E-03$ \\
\hline 455 & BU273897 & 5.17 & $7.60 \mathrm{E}-03$ & 523 & BU385860 & 5.26 & $9.07 \mathrm{E}-03$ & 591 & BX275584 & 1.51 & $3.36 \mathrm{E}-03$ \\
\hline 456 & BU274011 & 4.00 & $1.15 \mathrm{E}-02$ & 524 & BU387323 & 8.10 & $3.63 \mathrm{E}-05$ & 592 & BX276285 & 1.44 & $2.72 \mathrm{E}-03$ \\
\hline 457 & BU274445 & 2.93 & $7.66 \mathrm{E}-04$ & 525 & BU387623 & 4.18 & $3.61 \mathrm{E}-04$ & 593 & BX276404 & 2.66 & 4.12E-04 \\
\hline 458 & BU274619 & 2.99 & $2.87 \mathrm{E}-03$ & 526 & BU388091 & 3.30 & 2.66E-03 & 594 & BX277627 & 2.40 & 4.37E-04 \\
\hline 459 & BU274821 & 5.50 & $7.77 \mathrm{E}-04$ & 527 & BU393019 & 5.47 & $6.50 \mathrm{E}-04$ & 595 & BX278186 & 1.49 & $8.70 \mathrm{E}-03$ \\
\hline 460 & BU277984 & 2.00 & $1.11 \mathrm{E}-01$ & 528 & BU398913 & 1.29 & $1.19 \mathrm{E}-01$ & 596 & BX929271 & 3.56 & 4.38E-04 \\
\hline 461 & BU281570 & 2.00 & $2.31 \mathrm{E}-03$ & 529 & BU401146 & 2.24 & $1.44 \mathrm{E}-02$ & 597 & BX929283 & 1.31 & $1.09 \mathrm{E}-02$ \\
\hline 462 & BU281863 & 3.24 & $2.13 \mathrm{E}-03$ & 530 & BU404556 & 1.42 & $5.99 \mathrm{E}-02$ & 598 & BX929311 & 4.91 & 3.77E-04 \\
\hline 463 & BU282632 & 2.61 & $1.75 \mathrm{E}-03$ & 531 & BU406302 & 1.23 & $1.35 \mathrm{E}-02$ & 599 & BX929324 & 3.56 & 4.61E-05 \\
\hline 464 & BU282887 & 2.83 & $1.46 \mathrm{E}-03$ & 532 & BU410749 & 4.48 & $6.67 \mathrm{E}-03$ & 600 & BX929334 & 2.01 & $1.45 \mathrm{E}-03$ \\
\hline 465 & BU284480 & 2.97 & $1.10 \mathrm{E}-03$ & 533 & BU410899 & 1.18 & $7.76 \mathrm{E}-02$ & 601 & BX929335 & 4.21 & $9.74 E-03$ \\
\hline 466 & BU284885 & 2.71 & $8.43 \mathrm{E}-03$ & 534 & BU 412532 & 2.64 & $9.28 \mathrm{E}-03$ & 602 & BX929396 & 5.57 & $2.86 \mathrm{E}-03$ \\
\hline 467 & BU285976 & 4.37 & $6.25 \mathrm{E}-03$ & 535 & BU412664 & 6.07 & $5.83 \mathrm{E}-03$ & 603 & BX929413 & 2.73 & $3.50 \mathrm{E}-03$ \\
\hline 468 & BU285991 & 3.36 & $8.52 \mathrm{E}-03$ & 536 & BU417032 & 3.83 & $2.42 \mathrm{E}-02$ & 604 & BX929425 & 4.91 & $9.13 \mathrm{E}-02$ \\
\hline 469 & BU288827 & 2.32 & $1.17 \mathrm{E}-03$ & 537 & BU424807 & 1.55 & $1.79 \mathrm{E}-02$ & 605 & BX929487 & 1.15 & $1.67 E-04$ \\
\hline 470 & BU291924 & 4.36 & $5.50 \mathrm{E}-04$ & 538 & BU424835 & 1.66 & $1.53 \mathrm{E}-03$ & 606 & BX929500 & 1.78 & $7.70 \mathrm{E}-03$ \\
\hline 471 & BU291956 & 2.66 & $2.89 \mathrm{E}-02$ & 539 & BU424935 & 5.79 & $5.47 \mathrm{E}-04$ & 607 & BX929501 & 5.25 & $4.25 \mathrm{E}-03$ \\
\hline 472 & BU295709 & 4.24 & $3.66 \mathrm{E}-03$ & 540 & BU425927 & 3.33 & $6.19 \mathrm{E}-03$ & 608 & BX929504 & 4.09 & $4.53 \mathrm{E}-03$ \\
\hline 473 & BU300822 & 1.91 & $3.34 \mathrm{E}-03$ & 541 & BU426884 & 2.04 & $1.44 \mathrm{E}-02$ & 609 & BX929507 & 4.51 & $1.20 \mathrm{E}-03$ \\
\hline 474 & BU302836 & 3.21 & $2.22 \mathrm{E}-04$ & 542 & BU435651 & 2.67 & 7.01E-03 & 610 & BX929516 & 1.79 & 2.47E-02 \\
\hline 475 & BU302898 & 1.58 & $1.75 \mathrm{E}-03$ & 543 & BU437509 & 3.13 & $5.74 \mathrm{E}-03$ & 611 & BX929538 & 3.06 & 7.47E-04 \\
\hline
\end{tabular}




\begin{tabular}{|c|c|c|c|c|c|c|c|c|c|c|c|}
\hline 612 & BX929585 & 4.63 & $1.76 \mathrm{E}-02$ & 680 & BX930588 & 5.52 & $8.94 \mathrm{E}-04$ & 748 & BX931575 & 2.87 & $1.84 \mathrm{E}-02$ \\
\hline 613 & BX929597 & 4.44 & $1.11 \mathrm{E}-03$ & 681 & BX930600 & 2.23 & $2.66 \mathrm{E}-04$ & 749 & BX931614 & 2.19 & $2.67 \mathrm{E}-02$ \\
\hline 614 & BX929609 & 2.84 & $1.59 \mathrm{E}-02$ & 682 & BX930614 & 7.33 & $2.41 \mathrm{E}-04$ & 750 & BX931627 & 5.67 & $5.80 \mathrm{E}-05$ \\
\hline 615 & BX929612 & 5.30 & $1.55 \mathrm{E}-04$ & 683 & BX930625 & 3.80 & 1.16E-02 & 751 & BX931669 & 4.43 & $5.57 \mathrm{E}-04$ \\
\hline 616 & BX929614 & 3.21 & $1.89 \mathrm{E}-02$ & 684 & BX930633 & 1.32 & $2.22 \mathrm{E}-02$ & 752 & BX931693 & 4.99 & $8.32 \mathrm{E}-03$ \\
\hline 617 & BX929655 & 4.32 & $9.45 \mathrm{E}-03$ & 685 & BX930645 & 4.85 & $1.19 \mathrm{E}-03$ & 753 & BX931732 & 2.12 & $4.01 \mathrm{E}-02$ \\
\hline 618 & BX929656 & 1.42 & $1.83 \mathrm{E}-02$ & 686 & BX930695 & 3.07 & $2.32 \mathrm{E}-03$ & 754 & BX931761 & 1.44 & $6.04 \mathrm{E}-02$ \\
\hline 619 & BX929671 & 1.80 & $3.59 \mathrm{E}-03$ & 687 & BX930737 & 1.78 & $2.22 \mathrm{E}-02$ & 755 & BX931773 & 4.02 & $4.34 \mathrm{E}-03$ \\
\hline 620 & BX929680 & 1.56 & $4.96 \mathrm{E}-02$ & 688 & BX930812 & 4.31 & $1.63 E-03$ & 756 & BX931785 & 3.59 & $1.48 \mathrm{E}-02$ \\
\hline 621 & BX929693 & 5.47 & $7.46 \mathrm{E}-04$ & 689 & BX930854 & 2.95 & $3.31 \mathrm{E}-02$ & 757 & BX931787 & 5.06 & $2.42 \mathrm{E}-04$ \\
\hline 622 & BX929695 & 1.06 & $7.21 \mathrm{E}-03$ & 690 & BX930875 & 4.95 & $1.76 \mathrm{E}-03$ & 758 & BX931794 & 4.23 & $4.48 \mathrm{E}-03$ \\
\hline 623 & BX929711 & 3.55 & $2.59 \mathrm{E}-04$ & 691 & BX930894 & 4.78 & 3.76E-03 & 759 & BX931799 & 5.45 & $7.59 \mathrm{E}-05$ \\
\hline 624 & BX929718 & 2.37 & $1.86 \mathrm{E}-02$ & 692 & BX930903 & 1.26 & $2.98 \mathrm{E}-03$ & 760 & BX931805 & 1.35 & $2.22 \mathrm{E}-02$ \\
\hline 625 & BX929759 & 4.33 & $6.26 \mathrm{E}-04$ & 693 & BX930936 & 3.53 & $9.89 \mathrm{E}-04$ & 761 & BX931815 & 2.21 & $4.42 \mathrm{E}-03$ \\
\hline 626 & BX929781 & 3.54 & $8.64 \mathrm{E}-05$ & 694 & BX930969 & 3.74 & 3.42E-04 & 762 & BX931821 & 1.48 & $1.45 \mathrm{E}-02$ \\
\hline 627 & BX929785 & 2.59 & $2.06 \mathrm{E}-03$ & 695 & BX931015 & 6.75 & $6.84 \mathrm{E}-06$ & 763 & BX931825 & 1.74 & $1.88 \mathrm{E}-02$ \\
\hline 628 & BX929796 & 2.76 & $5.74 \mathrm{E}-03$ & 696 & BX931019 & 3.95 & $5.65 \mathrm{E}-03$ & 764 & BX931881 & 4.68 & $3.15 \mathrm{E}-03$ \\
\hline 629 & BX929817 & 4.96 & $1.13 \mathrm{E}-03$ & 697 & BX931020 & 2.84 & $5.71 \mathrm{E}-02$ & 765 & BX931955 & 2.45 & $2.93 \mathrm{E}-02$ \\
\hline 630 & BX929834 & 2.11 & $2.27 \mathrm{E}-03$ & 698 & BX931022 & 4.25 & $2.01 \mathrm{E}-03$ & 766 & BX931956 & 2.88 & $4.86 \mathrm{E}-04$ \\
\hline 631 & BX929837 & 5.17 & $8.20 \mathrm{E}-03$ & 699 & BX931029 & 5.04 & $1.21 \mathrm{E}-02$ & 767 & BX931960 & 4.31 & 3.49E-03 \\
\hline 632 & BX929879 & 1.44 & $6.25 \mathrm{E}-02$ & 700 & BX931032 & 4.82 & $4.35 \mathrm{E}-03$ & 768 & BX931966 & 1.37 & $3.30 \mathrm{E}-02$ \\
\hline 633 & BX929912 & 5.39 & $2.43 \mathrm{E}-05$ & 701 & BX931034 & 3.18 & $2.07 \mathrm{E}-03$ & 769 & BX931974 & 3.31 & $6.30 \mathrm{E}-02$ \\
\hline 634 & BX929928 & 1.48 & $1.60 \mathrm{E}-02$ & 702 & BX931035 & 4.28 & $7.82 \mathrm{E}-03$ & 770 & BX931980 & 5.10 & $1.57 \mathrm{E}-02$ \\
\hline 635 & BX929956 & 1.68 & $1.03 \mathrm{E}-02$ & 703 & BX931038 & 3.19 & $6.80 \mathrm{E}-03$ & 771 & BX932007 & 1.79 & $2.40 \mathrm{E}-03$ \\
\hline 636 & BX930016 & 3.45 & $4.89 \mathrm{E}-04$ & 704 & BX931053 & 4.96 & $4.74 \mathrm{E}-03$ & 772 & BX932018 & 3.29 & $1.87 \mathrm{E}-02$ \\
\hline 637 & BX930030 & 4.33 & $1.49 \mathrm{E}-03$ & 705 & BX931075 & 2.97 & $1.03 E-02$ & 773 & BX932021 & 1.48 & $3.44 \mathrm{E}-02$ \\
\hline 638 & BX930045 & 6.59 & $2.97 \mathrm{E}-03$ & 706 & BX931080 & 4.26 & 2.10E-03 & 774 & BX932023 & 5.77 & $1.89 \mathrm{E}-05$ \\
\hline 639 & BX930062 & 3.17 & $9.49 \mathrm{E}-03$ & 707 & BX931081 & 4.05 & $7.67 \mathrm{E}-03$ & 775 & BX932031 & 4.89 & $2.24 \mathrm{E}-03$ \\
\hline 640 & BX930066 & 1.23 & $2.58 \mathrm{E}-03$ & 708 & BX931082 & 2.25 & $8.04 \mathrm{E}-03$ & 776 & BX932037 & 2.10 & $1.98 \mathrm{E}-02$ \\
\hline 641 & BX930072 & 4.41 & 4.66E-02 & 709 & BX931093 & 5.18 & $5.46 \mathrm{E}-04$ & 777 & BX932127 & 2.73 & $2.94 \mathrm{E}-02$ \\
\hline 642 & BX930091 & 7.01 & 3.06E-03 & 710 & BX931097 & 1.11 & $4.95 \mathrm{E}-03$ & 778 & BX932131 & 2.78 & $9.02 \mathrm{E}-03$ \\
\hline 643 & BX930128 & 3.59 & $7.11 \mathrm{E}-03$ & 711 & BX931102 & 1.27 & $2.80 \mathrm{E}-02$ & 779 & BX932134 & 2.97 & $1.39 \mathrm{E}-02$ \\
\hline 644 & BX930132 & 1.34 & 4.33E-02 & 712 & BX931104 & 6.40 & 4.39E-04 & 780 & BX932141 & 1.65 & $6.95 E-02$ \\
\hline 645 & BХ930163 & 5.63 & $1.67 \mathrm{E}-03$ & 713 & BX931114 & 5.60 & $5.46 \mathrm{E}-03$ & 781 & BX932152 & 3.20 & $1.34 \mathrm{E}-03$ \\
\hline 646 & BX930176 & 5.97 & $4.24 \mathrm{E}-03$ & 714 & BX931124 & 2.82 & $1.21 \mathrm{E}-02$ & 782 & BX932156 & 1.61 & $1.54 \mathrm{E}-03$ \\
\hline 647 & BX930202 & 6.47 & $3.59 \mathrm{E}-04$ & 715 & BX931126 & 4.70 & $1.27 \mathrm{E}-02$ & 783 & BX932171 & 3.94 & $2.16 \mathrm{E}-04$ \\
\hline 648 & BX930204 & 2.69 & $2.37 \mathrm{E}-02$ & 716 & BX931127 & 4.45 & $1.33 \mathrm{E}-02$ & 784 & BX932173 & 1.10 & $2.79 \mathrm{E}-03$ \\
\hline 649 & BX930205 & 2.20 & $1.78 \mathrm{E}-03$ & 717 & BX931134 & 3.71 & $9.76 \mathrm{E}-03$ & 785 & BX932237 & 5.13 & $2.68 \mathrm{E}-03$ \\
\hline 650 & BX930207 & 1.36 & $1.81 \mathrm{E}-03$ & 718 & BX931135 & 6.74 & $1.84 \mathrm{E}-03$ & 786 & BX932257 & 2.30 & $2.01 \mathrm{E}-03$ \\
\hline 651 & BX930211 & 2.80 & $1.67 \mathrm{E}-02$ & 719 & BX931178 & 5.40 & $2.62 \mathrm{E}-03$ & 787 & BX932294 & 1.75 & $5.22 \mathrm{E}-04$ \\
\hline 652 & BX930227 & 8.56 & $1.67 \mathrm{E}-03$ & 720 & BX931209 & 4.00 & $8.15 \mathrm{E}-03$ & 788 & BX932304 & 5.38 & $2.13 \mathrm{E}-03$ \\
\hline 653 & BX930235 & 1.19 & $8.08 \mathrm{E}-03$ & 721 & BX931211 & 4.04 & $1.83 E-03$ & 789 & BX932362 & 4.93 & $5.47 \mathrm{E}-04$ \\
\hline 654 & BX930246 & 3.23 & $2.59 \mathrm{E}-03$ & 722 & BX931212 & 2.09 & $3.41 \mathrm{E}-03$ & 790 & BX932369 & 1.94 & $3.54 \mathrm{E}-02$ \\
\hline 655 & BX930274 & 6.67 & $5.80 \mathrm{E}-03$ & 723 & BX931221 & 3.96 & $9.72 \mathrm{E}-03$ & 791 & BX932377 & 3.30 & $9.72 \mathrm{E}-03$ \\
\hline 656 & BX930296 & 1.83 & $8.49 \mathrm{E}-03$ & 724 & BX931225 & 2.92 & $1.00 \mathrm{E}-03$ & 792 & BX932380 & 1.09 & $3.09 \mathrm{E}-03$ \\
\hline 657 & BX930306 & 2.32 & 4.27E- 03 & 725 & BX931226 & 6.72 & $2.52 \mathrm{E}-03$ & 793 & BX932401 & 1.75 & $4.77 \mathrm{E}-02$ \\
\hline 658 & BX930316 & 3.15 & $1.74 \mathrm{E}-04$ & 726 & BX931232 & 8.37 & $1.45 \mathrm{E}-02$ & 794 & BX932417 & 1.18 & $1.68 \mathrm{E}-02$ \\
\hline 659 & BX930317 & 4.75 & $2.74 \mathrm{E}-04$ & 727 & BX931267 & 1.10 & $5.32 \mathrm{E}-02$ & 795 & BX932450 & 1.48 & $5.76 \mathrm{E}-02$ \\
\hline 660 & BX930327 & 5.05 & $8.35 \mathrm{E}-04$ & 728 & BX931268 & 1.54 & $5.15 \mathrm{E}-02$ & 796 & BX932465 & 2.62 & $8.61 \mathrm{E}-03$ \\
\hline 661 & BX930364 & 2.29 & $1.77 \mathrm{E}-01$ & 729 & BX931269 & 2.02 & $8.52 \mathrm{E}-03$ & 797 & BX932474 & 4.63 & $4.70 \mathrm{E}-03$ \\
\hline 662 & BX930365 & 3.83 & $2.44 \mathrm{E}-03$ & 730 & BX931283 & 3.50 & 3.47E-03 & 798 & BX932482 & 2.86 & $3.57 \mathrm{E}-03$ \\
\hline 663 & BX930383 & 6.72 & 3.69E-03 & 731 & BX931295 & 2.83 & $3.40 \mathrm{E}-02$ & 799 & BX932494 & 1.37 & $1.85 \mathrm{E}-03$ \\
\hline 664 & BX930393 & 2.26 & $1.18 \mathrm{E}-02$ & 732 & BX931327 & 2.25 & $8.60 \mathrm{E}-02$ & 800 & BX932515 & 1.72 & $2.64 \mathrm{E}-02$ \\
\hline 665 & BX930414 & 4.75 & $8.37 \mathrm{E}-05$ & 733 & BX931346 & 2.98 & $2.34 \mathrm{E}-03$ & 801 & BX932535 & 4.65 & $6.94 \mathrm{E}-03$ \\
\hline 666 & BX930420 & 1.71 & $2.83 \mathrm{E}-03$ & 734 & BX931349 & 1.45 & 4.11E-02 & 802 & BX932558 & 2.15 & $3.22 \mathrm{E}-02$ \\
\hline 667 & BX930432 & 5.18 & $2.34 \mathrm{E}-02$ & 735 & BX931365 & 2.88 & $2.26 \mathrm{E}-02$ & 803 & BX932567 & 3.72 & $3.11 \mathrm{E}-03$ \\
\hline 668 & BX930433 & 3.49 & $6.79 \mathrm{E}-03$ & 736 & BX931377 & 3.05 & $1.73 \mathrm{E}-02$ & 804 & BX932573 & 1.79 & $1.56 \mathrm{E}-03$ \\
\hline 669 & BX930447 & 2.13 & $4.20 \mathrm{E}-03$ & 737 & BX931426 & 2.32 & $1.78 \mathrm{E}-02$ & 805 & BX932593 & 4.79 & $8.39 \mathrm{E}-03$ \\
\hline 670 & BX930450 & 5.49 & $5.66 \mathrm{E}-03$ & 738 & BX931439 & 3.02 & $4.95 \mathrm{E}-02$ & 806 & BX932609 & 5.30 & $1.24 \mathrm{E}-02$ \\
\hline 671 & BX930478 & 1.13 & 3.35E-02 & 739 & BX931466 & 6.31 & $8.74 \mathrm{E}-03$ & 807 & BX932640 & 6.08 & $1.73 \mathrm{E}-03$ \\
\hline 672 & BX930479 & 5.09 & $3.12 \mathrm{E}-03$ & 740 & BX931476 & 1.24 & $8.79 \mathrm{E}-02$ & 808 & BX932669 & 2.54 & $5.05 E-03$ \\
\hline 673 & BX930482 & 2.83 & $2.12 \mathrm{E}-02$ & 741 & BX931491 & 2.35 & $1.73 \mathrm{E}-02$ & 809 & BX932677 & 1.58 & $1.83 \mathrm{E}-02$ \\
\hline 674 & BX930526 & 4.47 & $1.12 \mathrm{E}-01$ & 742 & BX931506 & 1.98 & $8.15 E-03$ & 810 & BX932691 & 7.93 & $4.16 \mathrm{E}-03$ \\
\hline 675 & BX930530 & 6.19 & $1.27 \mathrm{E}-02$ & 743 & BX931526 & 5.09 & $2.24 \mathrm{E}-03$ & 811 & BX932705 & 5.34 & $1.63 \mathrm{E}-02$ \\
\hline 676 & BX930553 & 3.34 & $8.24 \mathrm{E}-05$ & 744 & BX931534 & 1.37 & $5.44 \mathrm{E}-02$ & 812 & BX932743 & 2.60 & 8.07E-04 \\
\hline 677 & BX930571 & 3.86 & $4.19 \mathrm{E}-04$ & 745 & BX931546 & 2.91 & $1.26 \mathrm{E}-02$ & 813 & BX932770 & 2.72 & $3.74 \mathrm{E}-04$ \\
\hline 678 & BX930572 & 5.96 & $1.59 \mathrm{E}-03$ & 746 & BX931553 & 1.71 & $3.81 \mathrm{E}-03$ & 814 & BX932771 & 1.22 & $5.68 \mathrm{E}-03$ \\
\hline 679 & BX930578 & 3.07 & $1.25 \mathrm{E}-02$ & 747 & BX931555 & 2.91 & 3.91E-04 & 815 & BX932778 & 4.43 & $1.98 \mathrm{E}-02$ \\
\hline
\end{tabular}




\begin{tabular}{|c|c|c|c|c|c|c|c|c|c|c|c|}
\hline 816 & BX932784 & 2.35 & $2.78 \mathrm{E}-03$ & 884 & BX933494 & 1.50 & $3.50 \mathrm{E}-02$ & 952 & BX934301 & 2.86 & 3.93E-03 \\
\hline 817 & BX932787 & 6.26 & $5.32 \mathrm{E}-05$ & 885 & BX933496 & 2.29 & $1.23 \mathrm{E}-02$ & 953 & BX934310 & 2.00 & 3.14E- 03 \\
\hline 818 & BX932806 & 3.08 & $7.15 \mathrm{E}-04$ & 886 & BX933498 & 5.30 & $5.84 \mathrm{E}-03$ & 954 & BX934334 & 1.51 & $8.63 \mathrm{E}-03$ \\
\hline 819 & BX932807 & 3.50 & $3.00 \mathrm{E}-03$ & 887 & BX933512 & 1.77 & $3.80 \mathrm{E}-02$ & 955 & BX934354 & 4.77 & $2.72 \mathrm{E}-02$ \\
\hline 820 & BX932812 & 1.25 & $5.64 \mathrm{E}-02$ & 888 & BX933518 & 3.46 & $1.70 \mathrm{E}-03$ & 956 & BX934355 & 4.23 & $6.74 E-03$ \\
\hline 821 & BX932818 & 4.74 & $6.42 E-04$ & 889 & BX933522 & 1.57 & $2.34 \mathrm{E}-03$ & 957 & BX934357 & 4.98 & 3.42E-04 \\
\hline 822 & BX932820 & 2.93 & $3.69 \mathrm{E}-04$ & 890 & BX933526 & 5.84 & $3.69 \mathrm{E}-04$ & 958 & BX934359 & 1.63 & $1.49 \mathrm{E}-02$ \\
\hline 823 & BX932826 & 4.81 & $9.43 \mathrm{E}-05$ & 891 & BX933527 & 2.35 & $6.34 \mathrm{E}-02$ & 959 & BX934380 & 2.41 & 3.76E-02 \\
\hline 824 & BX932833 & 4.54 & $4.02 E-03$ & 892 & BX933539 & 5.01 & $2.94 \mathrm{E}-03$ & 960 & BX934383 & 3.81 & $4.78 \mathrm{E}-03$ \\
\hline 825 & BX932838 & 4.59 & $3.33 \mathrm{E}-03$ & 893 & BX933541 & 4.95 & $3.91 \mathrm{E}-03$ & 961 & BX934385 & 3.67 & $6.57 \mathrm{E}-04$ \\
\hline 826 & BX932843 & 5.35 & $6.04 \mathrm{E}-03$ & 894 & BX933548 & 2.60 & $1.89 \mathrm{E}-02$ & 962 & BX934386 & 5.66 & $4.76 \mathrm{E}-03$ \\
\hline 827 & BX932851 & 4.42 & $1.56 \mathrm{E}-03$ & 895 & BX933556 & 2.14 & 7.17E-03 & 963 & BX934396 & 3.99 & $5.70 \mathrm{E}-03$ \\
\hline 828 & BX932868 & 1.26 & $3.46 \mathrm{E}-02$ & 896 & BX933560 & 3.86 & $6.74 \mathrm{E}-03$ & 964 & BX934401 & 1.58 & $1.86 \mathrm{E}-02$ \\
\hline 829 & BX932870 & 4.60 & $3.40 \mathrm{E}-03$ & 897 & BX933562 & 4.87 & $1.14 \mathrm{E}-03$ & 965 & BX934412 & 5.07 & $1.03 \mathrm{E}-02$ \\
\hline 830 & BX932872 & 2.72 & $2.26 \mathrm{E}-02$ & 898 & BX933586 & 5.72 & $3.39 \mathrm{E}-03$ & 966 & BX934436 & 4.09 & 4.42E-06 \\
\hline 831 & BX932881 & 3.44 & $1.38 \mathrm{E}-02$ & 899 & BX933661 & 2.54 & $8.53 \mathrm{E}-05$ & 967 & BX934437 & 4.32 & $1.41 \mathrm{E}-03$ \\
\hline 832 & BX932891 & 3.06 & $2.02 E-03$ & 900 & BX933682 & 2.13 & 3.27E-03 & 968 & BX934455 & 2.52 & 4.32E-04 \\
\hline 833 & BX932900 & 4.11 & $2.47 \mathrm{E}-03$ & 901 & BX933694 & 7.97 & $1.81 \mathrm{E}-03$ & 969 & BX934474 & 3.50 & $1.22 \mathrm{E}-02$ \\
\hline 834 & BX932958 & 1.85 & $4.07 E-02$ & 902 & BX933696 & 2.78 & $5.43 \mathrm{E}-02$ & 970 & BX934508 & 2.60 & $3.62 \mathrm{E}-03$ \\
\hline 835 & BX932966 & 1.23 & $1.46 \mathrm{E}-02$ & 903 & BX933706 & 1.35 & $6.55 \mathrm{E}-03$ & 971 & BX934529 & 3.16 & $1.29 \mathrm{E}-02$ \\
\hline 836 & BX932978 & 4.40 & $6.07 \mathrm{E}-03$ & 904 & BX933711 & 2.37 & $9.53 \mathrm{E}-03$ & 972 & BX934539 & 2.16 & $3.97 \mathrm{E}-03$ \\
\hline 837 & BX933013 & 1.50 & $5.55 \mathrm{E}-02$ & 905 & BX933714 & 1.53 & $5.97 \mathrm{E}-02$ & 973 & BX934546 & 1.45 & $4.79 E-03$ \\
\hline 838 & BX933037 & 1.60 & $8.45 \mathrm{E}-03$ & 906 & BX933716 & 2.48 & $4.15 \mathrm{E}-02$ & 974 & BX934571 & 2.11 & $9.15 \mathrm{E}-02$ \\
\hline 839 & BX933043 & 4.55 & $4.94 E-04$ & 907 & BX933759 & 3.45 & $7.76 \mathrm{E}-03$ & 975 & BX934636 & 4.19 & $2.15 \mathrm{E}-03$ \\
\hline 840 & BX933049 & 1.59 & $3.91 \mathrm{E}-02$ & 908 & BX933770 & 1.89 & $1.07 \mathrm{E}-03$ & 976 & BX934640 & 1.48 & $9.02 E-02$ \\
\hline 841 & BX933051 & 3.40 & $7.04 \mathrm{E}-04$ & 909 & BX933782 & 3.34 & $1.02 \mathrm{E}-02$ & 977 & BX934641 & 11.04 & $7.35 E-04$ \\
\hline 842 & BX933057 & 4.72 & $2.47 \mathrm{E}-05$ & 910 & BX933785 & 3.18 & $4.68 \mathrm{E}-04$ & 978 & BX934663 & 2.94 & $3.27 E-03$ \\
\hline 843 & BX933078 & 4.07 & $4.39 \mathrm{E}-03$ & 911 & BX933803 & 5.34 & $3.72 \mathrm{E}-02$ & 979 & BX934692 & 3.99 & 2.11E-03 \\
\hline 844 & BX933086 & 2.65 & $1.38 \mathrm{E}-03$ & 912 & BX933808 & 2.17 & $6.32 \mathrm{E}-02$ & 980 & BX934695 & 2.41 & $3.09 \mathrm{E}-03$ \\
\hline 845 & BX933113 & 4.71 & $2.30 \mathrm{E}-03$ & 913 & BX933810 & 4.13 & $1.60 \mathrm{E}-02$ & 981 & BX934696 & 4.73 & $6.94 \mathrm{E}-03$ \\
\hline 846 & BX933120 & 2.81 & $6.99 \mathrm{E}-02$ & 914 & BX933816 & 5.65 & $3.32 \mathrm{E}-03$ & 982 & BX934701 & 5.74 & $2.31 \mathrm{E}-03$ \\
\hline 847 & BX933177 & 4.30 & $7.70 E-04$ & 915 & BX933836 & 3.12 & $1.04 \mathrm{E}-03$ & 983 & BX934722 & 6.57 & $6.06 \mathrm{E}-03$ \\
\hline 848 & BX933194 & 3.15 & $3.76 \mathrm{E}-04$ & 916 & BX933839 & 3.75 & $3.09 \mathrm{E}-03$ & 984 & BX934726 & 5.50 & $2.24 \mathrm{E}-03$ \\
\hline 849 & BX933205 & 3.48 & $2.53 \mathrm{E}-03$ & 917 & BX933845 & 4.53 & $4.65 \mathrm{E}-02$ & 985 & BX934740 & 3.16 & 4.64E-04 \\
\hline 850 & BX933209 & 3.95 & $3.56 \mathrm{E}-03$ & 918 & BX933854 & 3.11 & $2.69 \mathrm{E}-03$ & 986 & BX934741 & 6.52 & $4.60 \mathrm{E}-03$ \\
\hline 851 & BX933210 & 6.46 & $1.26 \mathrm{E}-03$ & 919 & BX933860 & 3.66 & $5.65 \mathrm{E}-03$ & 987 & BX934756 & 2.00 & 5.49E- 03 \\
\hline 852 & BX933257 & 2.61 & $4.04 E-03$ & 920 & BX933871 & 4.16 & $5.31 \mathrm{E}-03$ & 988 & BX934773 & 2.99 & $2.30 \mathrm{E}-03$ \\
\hline 853 & BX933258 & 2.37 & $2.09 \mathrm{E}-02$ & 921 & BX933874 & 4.72 & $8.24 \mathrm{E}-03$ & 989 & BX934780 & 4.64 & $5.34 \mathrm{E}-03$ \\
\hline 854 & BX933275 & 4.28 & $1.81 \mathrm{E}-03$ & 922 & BX933879 & 2.95 & $1.06 \mathrm{E}-02$ & 990 & BX934783 & 2.75 & $1.76 \mathrm{E}-02$ \\
\hline 855 & BX933287 & 3.50 & $1.64 \mathrm{E}-04$ & 923 & BX933898 & 3.44 & $8.43 \mathrm{E}-02$ & 991 & BX934791 & 3.65 & $3.47 E-02$ \\
\hline 856 & BX933288 & 2.92 & $2.65 \mathrm{E}-03$ & 924 & BX933908 & 5.01 & $1.28 \mathrm{E}-03$ & 992 & BX934809 & 2.55 & $5.96 \mathrm{E}-03$ \\
\hline 857 & BX933289 & 5.49 & $1.50 \mathrm{E}-04$ & 925 & BX933936 & 4.89 & $7.49 \mathrm{E}-03$ & 993 & BX934820 & 1.24 & $1.06 \mathrm{E}-01$ \\
\hline 858 & BX933291 & 4.51 & $3.71 E-05$ & 926 & BX933965 & 2.87 & $2.33 \mathrm{E}-03$ & 994 & BX934821 & 2.23 & $4.23 E-03$ \\
\hline 859 & BX933295 & 5.65 & $1.66 \mathrm{E}-02$ & 927 & BX934018 & 1.70 & $2.79 \mathrm{E}-02$ & 995 & BX934830 & 4.21 & 5.86E-04 \\
\hline 860 & BX933296 & 3.43 & $1.31 \mathrm{E}-03$ & 928 & BX934067 & 1.35 & $8.63 \mathrm{E}-03$ & 996 & BX934835 & 2.40 & $4.68 \mathrm{E}-02$ \\
\hline 861 & BX933299 & 2.53 & $1.63 \mathrm{E}-02$ & 929 & BX934084 & 3.43 & $5.03 \mathrm{E}-03$ & 997 & BX934842 & 1.98 & $2.32 \mathrm{E}-02$ \\
\hline 862 & BX933302 & 2.64 & $2.60 \mathrm{E}-05$ & 930 & BX934096 & 3.83 & $3.42 \mathrm{E}-03$ & 998 & BX934845 & 6.55 & $2.84 \mathrm{E}-03$ \\
\hline 863 & BX933311 & 6.59 & $2.75 E-02$ & 931 & BX934101 & 1.23 & $1.19 \mathrm{E}-02$ & 999 & BX934855 & 4.58 & $6.28 \mathrm{E}-03$ \\
\hline 864 & BX933312 & 5.98 & $1.32 \mathrm{E}-03$ & 932 & BX934110 & 1.24 & $2.61 \mathrm{E}-02$ & 1000 & BX934865 & 2.01 & 3.93E-03 \\
\hline 865 & BX933317 & 4.37 & $4.15 \mathrm{E}-03$ & 933 & BX934130 & 5.88 & $4.76 \mathrm{E}-04$ & 1001 & BX934870 & 3.26 & $1.34 \mathrm{E}-03$ \\
\hline 866 & BX933320 & 3.75 & $1.01 E-03$ & 934 & BX934140 & 2.11 & $2.83 \mathrm{E}-02$ & 1002 & BX934898 & 3.70 & 2.54E-02 \\
\hline 867 & BX933321 & 3.36 & $1.20 \mathrm{E}-02$ & 935 & BX934141 & 9.67 & $9.35 \mathrm{E}-04$ & 1003 & BX934903 & 1.00 & $1.38 \mathrm{E}-02$ \\
\hline 868 & BX933322 & 3.61 & $8.45 \mathrm{E}-03$ & 936 & BX934157 & 2.98 & $2.25 \mathrm{E}-03$ & 1004 & BX934985 & 2.22 & $6.80 \mathrm{E}-04$ \\
\hline 869 & BX933326 & 7.52 & $7.83 \mathrm{E}-04$ & 937 & BX934160 & 2.57 & $1.13 \mathrm{E}-03$ & 1005 & BX934995 & 3.99 & $8.57 E-03$ \\
\hline 870 & BX933335 & 2.26 & $4.90 \mathrm{E}-03$ & 938 & BX934176 & 3.60 & $4.03 \mathrm{E}-04$ & 1006 & BX934996 & 1.76 & $1.58 \mathrm{E}-02$ \\
\hline 871 & BX933356 & 1.95 & $5.00 \mathrm{E}-03$ & 939 & BX934179 & 2.97 & $7.14 \mathrm{E}-03$ & 1007 & BX935007 & 2.73 & 5.53E-02 \\
\hline 872 & BX933362 & 2.03 & $4.59 \mathrm{E}-03$ & 940 & BX934181 & 2.45 & $5.86 \mathrm{E}-03$ & 1008 & BX935012 & 3.32 & $8.63 \mathrm{E}-03$ \\
\hline 873 & BX933363 & 3.77 & $1.30 \mathrm{E}-02$ & 941 & BX934183 & 1.51 & $2.27 \mathrm{E}-02$ & 1009 & BX935018 & 4.85 & 7.07E-03 \\
\hline 874 & BX933381 & 6.37 & 3.63E-04 & 942 & BX934193 & 4.00 & 7.06E-04 & 1010 & BX935028 & 4.09 & $1.23 \mathrm{E}-03$ \\
\hline 875 & BX933386 & 4.42 & $2.26 \mathrm{E}-03$ & 943 & BX934202 & 3.48 & $7.76 \mathrm{E}-02$ & 1011 & BX935060 & 1.28 & $5.65 E-02$ \\
\hline 876 & BX933394 & 3.61 & $1.42 \mathrm{E}-02$ & 944 & BX934213 & 3.26 & $1.10 \mathrm{E}-02$ & 1012 & BX935083 & 4.10 & $2.81 \mathrm{E}-03$ \\
\hline 877 & BX933401 & 5.03 & $1.73 \mathrm{E}-02$ & 945 & BX934223 & 5.69 & $9.18 \mathrm{E}-04$ & 1013 & BX935087 & 3.66 & $5.31 \mathrm{E}-03$ \\
\hline 878 & BX933451 & 7.21 & $4.80 \mathrm{E}-04$ & 946 & BX934228 & 2.81 & $8.00 \mathrm{E}-03$ & 1014 & BX935127 & 2.73 & $3.26 \mathrm{E}-03$ \\
\hline 879 & BX933460 & 4.73 & $5.35 \mathrm{E}-03$ & 947 & BX934248 & 6.62 & $8.27 \mathrm{E}-04$ & 1015 & BX935172 & 5.74 & $1.67 E-02$ \\
\hline 880 & BX933467 & 3.69 & $1.70 \mathrm{E}-02$ & 948 & BX934251 & 4.56 & $2.32 \mathrm{E}-04$ & 1016 & BX935178 & 3.49 & $2.17 E-02$ \\
\hline 881 & BX933471 & 4.04 & $1.71 E-02$ & 949 & BX934268 & 4.16 & $1.85 \mathrm{E}-03$ & 1017 & BX935194 & 2.77 & $2.64 \mathrm{E}-03$ \\
\hline 882 & BX933472 & 3.33 & $1.58 \mathrm{E}-03$ & 950 & BX934275 & 3.48 & $2.20 \mathrm{E}-03$ & 1018 & BX935197 & 4.02 & $2.10 \mathrm{E}-02$ \\
\hline 883 & BX933485 & 2.58 & 7.15E-02 & 951 & BX934285 & 3.25 & $1.32 \mathrm{E}-02$ & 1019 & BX935202 & 3.21 & $6.16 \mathrm{E}-03$ \\
\hline
\end{tabular}




\begin{tabular}{|c|c|c|c|c|c|c|c|c|c|c|c|}
\hline 1020 & BX935224 & 3.56 & $1.35 \mathrm{E}-02$ & 1088 & BX950249 & 5.92 & $1.21 \mathrm{E}-04$ & 1156 & C17H9orf140 & 3.17 & $2.52 \mathrm{E}-03$ \\
\hline 1021 & BX935250 & 6.33 & $4.36 \mathrm{E}-04$ & 1089 & BX950250 & 1.98 & $2.85 \mathrm{E}-03$ & 1157 & C17H9orf167 & 4.93 & $7.66 \mathrm{E}-03$ \\
\hline 1022 & BX935277 & 3.71 & $1.69 \mathrm{E}-02$ & 1090 & BX950256 & 2.88 & $4.69 \mathrm{E}-03$ & 1158 & C17H9orf91 & 1.21 & $1.28 \mathrm{E}-02$ \\
\hline 1023 & BX935293 & 7.04 & $4.21 \mathrm{E}-02$ & 1091 & BX950264 & 7.36 & $4.09 \mathrm{E}-03$ & 1159 & C18H17orf28 & 2.56 & $2.80 \mathrm{E}-03$ \\
\hline 1024 & BX935356 & 1.21 & $2.33 \mathrm{E}-02$ & 1092 & BX950265 & 2.03 & $4.83 \mathrm{E}-02$ & 1160 & C1H11orf87 & 2.71 & $3.87 \mathrm{E}-03$ \\
\hline 1025 & BX935362 & 3.60 & $6.31 \mathrm{E}-03$ & 1093 & BX950272 & 4.45 & $6.56 \mathrm{E}-03$ & 1161 & C1H12orf28 & 2.12 & $1.67 \mathrm{E}-04$ \\
\hline 1026 & BX935383 & 1.30 & $1.46 \mathrm{E}-02$ & 1094 & BX950306 & 4.51 & $2.54 \mathrm{E}-03$ & 1162 & C1H12orf69 & 5.64 & $1.44 \mathrm{E}-03$ \\
\hline 1027 & BX935414 & 2.35 & $8.16 \mathrm{E}-03$ & 1095 & BX950310 & 2.56 & $2.58 \mathrm{E}-02$ & 1163 & $\mathrm{C} 1 \mathrm{H} 21$ orf62 & 3.84 & $2.47 \mathrm{E}-03$ \\
\hline 1028 & BX935422 & 5.16 & $2.35 \mathrm{E}-03$ & 1096 & BX950352 & 1.40 & $9.85 E-04$ & 1164 & $\mathrm{C} 1 \mathrm{H} 21$ orf63 & 1.59 & $2.10 \mathrm{E}-02$ \\
\hline 1029 & BX935439 & 1.71 & $4.27 \mathrm{E}-02$ & 1097 & BX950357 & 1.39 & $9.13 \mathrm{E}-03$ & 1165 & C1H7orf53 & 3.58 & $1.43 \mathrm{E}-03$ \\
\hline 1030 & BX935443 & 4.98 & $3.01 \mathrm{E}-03$ & 1098 & BX950365 & 3.40 & $9.48 \mathrm{E}-03$ & 1166 & C1HXorf36 & 1.77 & $1.17 \mathrm{E}-02$ \\
\hline 1031 & BX935458 & 5.49 & $1.21 \mathrm{E}-03$ & 1099 & BX950370 & 2.76 & $2.32 \mathrm{E}-02$ & 1167 & C1QA & 1.63 & $3.81 E-02$ \\
\hline 1032 & BX935462 & 5.74 & $1.52 \mathrm{E}-02$ & 1100 & BX950377 & 2.53 & $1.74 \mathrm{E}-01$ & 1168 & C1QL2 & 3.15 & $6.42 \mathrm{E}-03$ \\
\hline 1033 & BX935471 & 7.84 & 3.64E-04 & 1101 & BX950390 & 3.17 & $1.48 \mathrm{E}-01$ & 1169 & C1QL4 & 2.15 & $1.46 \mathrm{E}-02$ \\
\hline 1034 & BX935513 & 1.82 & $9.48 \mathrm{E}-02$ & 1102 & BX950405 & 2.14 & $2.08 \mathrm{E}-03$ & 1170 & C1QTNF1 & 3.13 & $3.82 E-02$ \\
\hline 1035 & BX935525 & 4.83 & $2.22 \mathrm{E}-03$ & 1103 & BX950448 & 3.53 & $6.98 \mathrm{E}-03$ & 1171 & C1QTNF3 & 1.95 & $2.79 \mathrm{E}-03$ \\
\hline 1036 & BX935533 & 2.95 & $2.44 \mathrm{E}-03$ & 1104 & BX950459 & 3.20 & $5.53 \mathrm{E}-02$ & 1172 & C1QTNF5 & 3.18 & $1.79 \mathrm{E}-03$ \\
\hline 1037 & BX935559 & 3.20 & $2.21 \mathrm{E}-02$ & 1105 & BX950463 & 2.34 & $1.55 \mathrm{E}-01$ & 1173 & C1QTNF6 & 6.00 & $1.33 \mathrm{E}-03$ \\
\hline 1038 & BX935570 & 3.74 & $1.48 \mathrm{E}-03$ & 1106 & BX950484 & 4.23 & $4.64 \mathrm{E}-03$ & 1174 & C1QTNF8 & 5.97 & $9.92 \mathrm{E}-03$ \\
\hline 1039 & BX935607 & 4.31 & $8.71 \mathrm{E}-04$ & 1107 & BX950492 & 3.40 & $8.38 \mathrm{E}-04$ & 1175 & C1QTNF9 & 5.40 & 5.35E-04 \\
\hline 1040 & BX935626 & 1.62 & $5.76 \mathrm{E}-03$ & 1108 & BX950495 & 1.30 & $3.46 \mathrm{E}-02$ & 1176 & $\mathrm{C} 20 \mathrm{H} 20$ orf123 & 6.30 & $2.99 \mathrm{E}-05$ \\
\hline 1041 & BX935643 & 1.72 & $2.24 \mathrm{E}-02$ & 1109 & BX950500 & 2.15 & $1.61 \mathrm{E}-03$ & 1177 & $\mathrm{C} 20 \mathrm{H} 20$ orf151 & 3.50 & $2.33 \mathrm{E}-03$ \\
\hline 1042 & BX935646 & 2.77 & $5.08 \mathrm{E}-03$ & 1110 & BX950512 & 2.72 & $1.51 \mathrm{E}-02$ & 1178 & $\mathrm{C} 20 \mathrm{H} 20$ orf160 & 3.86 & $1.48 \mathrm{E}-03$ \\
\hline 1043 & BX935652 & 4.13 & $6.38 \mathrm{E}-04$ & 1111 & BX950525 & 3.11 & $2.14 \mathrm{E}-04$ & 1179 & $\mathrm{C} 21 \mathrm{H} 1$ orf70 & 6.00 & $3.15 \mathrm{E}-03$ \\
\hline 1044 & BX935653 & 4.07 & $2.26 \mathrm{E}-04$ & 1112 & BX950533 & 5.24 & $7.55 \mathrm{E}-04$ & 1180 & $\mathrm{C} 22 \mathrm{H} 2 \mathrm{orf} 42$ & 1.63 & $3.22 \mathrm{E}-02$ \\
\hline 1045 & BX935658 & 3.25 & $1.02 \mathrm{E}-03$ & 1113 & BX950541 & 1.70 & $3.84 \mathrm{E}-03$ & 1181 & C23H1orf216 & 4.75 & $1.56 \mathrm{E}-03$ \\
\hline 1046 & BX935659 & 4.28 & $1.99 \mathrm{E}-03$ & 1114 & BX950549 & 5.02 & 4.47E-03 & 1182 & C26H1orf116 & 3.48 & $1.71 \mathrm{E}-03$ \\
\hline 1047 & BX935661 & 2.16 & $2.17 \mathrm{E}-02$ & 1115 & BX950553 & 5.29 & $6.87 \mathrm{E}-03$ & 1183 & C26H1orf183 & 3.77 & $1.11 \mathrm{E}-03$ \\
\hline 1048 & BX935667 & 6.64 & $6.47 \mathrm{E}-05$ & 1116 & BX950572 & 2.96 & $6.89 \mathrm{E}-03$ & 1184 & C2CD2L & 2.13 & $1.80 \mathrm{E}-02$ \\
\hline 1049 & BX935672 & 3.27 & $5.89 \mathrm{E}-03$ & 1117 & BX950576 & 1.88 & $8.99 \mathrm{E}-04$ & 1185 & $\mathrm{C} 2 \mathrm{CD} 4 \mathrm{C}$ & 5.19 & $3.48 \mathrm{E}-03$ \\
\hline 1050 & BX935677 & 1.87 & $2.64 \mathrm{E}-02$ & 1118 & BX950595 & 6.24 & $6.80 \mathrm{E}-04$ & 1186 & $\mathrm{C} 2 \mathrm{H} 10$ orf112 & 1.33 & 5.33E-02 \\
\hline 1051 & BX935692 & 3.47 & $1.75 \mathrm{E}-03$ & 1119 & BX950596 & 7.62 & $2.24 \mathrm{E}-05$ & 1187 & $\mathrm{C} 2 \mathrm{H} 18$ orf63 & 3.27 & $7.61 \mathrm{E}-04$ \\
\hline 1052 & BX935706 & 3.43 & $9.88 \mathrm{E}-03$ & 1120 & BX950608 & 2.90 & $9.44 \mathrm{E}-04$ & 1188 & C2H6orf105 & 5.24 & $6.48 \mathrm{E}-03$ \\
\hline 1053 & BX935715 & 3.07 & $6.28 \mathrm{E}-03$ & 1121 & BX950609 & 2.07 & $2.92 \mathrm{E}-03$ & 1189 & $\mathrm{C} 2 \mathrm{H} 8$ orf22 & 2.89 & $5.62 \mathrm{E}-03$ \\
\hline 1054 & BX935717 & 2.16 & $1.60 \mathrm{E}-02$ & 1122 & BX950618 & 3.31 & $9.61 \mathrm{E}-04$ & 1190 & $\mathrm{C} 2 \mathrm{H} 8 \mathrm{orf} 34$ & 1.79 & $1.81 \mathrm{E}-02$ \\
\hline 1055 & BX935720 & 2.27 & $2.25 \mathrm{E}-02$ & 1123 & BX950623 & 3.57 & $2.16 \mathrm{E}-03$ & 1191 & C2H9orf152 & 6.73 & $3.33 \mathrm{E}-02$ \\
\hline 1056 & BX935721 & 5.95 & $1.02 E-03$ & 1124 & BX950650 & 1.30 & $8.95 \mathrm{E}-02$ & 1192 & C3 & 2.38 & $2.10 \mathrm{E}-03$ \\
\hline 1057 & BX935723 & 3.93 & $1.05 \mathrm{E}-03$ & 1125 & BX950652 & 2.89 & $8.31 \mathrm{E}-03$ & 1193 & C3AR1 & 2.83 & $3.37 \mathrm{E}-03$ \\
\hline 1058 & BX935743 & 4.73 & 7.67E-05 & 1126 & BX950658 & 1.46 & $9.41 \mathrm{E}-03$ & 1194 & C3H1orf115 & 1.14 & $3.93 \mathrm{E}-02$ \\
\hline 1059 & BX935758 & 4.26 & $1.29 \mathrm{E}-03$ & 1127 & BX950660 & 3.84 & $3.89 \mathrm{E}-02$ & 1195 & C3H1orf95 & 5.09 & $6.08 \mathrm{E}-03$ \\
\hline 1060 & BX935766 & 2.94 & $1.68 \mathrm{E}-02$ & 1128 & BX950686 & 1.14 & $2.28 \mathrm{E}-02$ & 1196 & С3H20orf103 & 1.14 & $1.34 \mathrm{E}-03$ \\
\hline 1061 & BX935945 & 5.37 & $1.36 \mathrm{E}-04$ & 1129 & BX950697 & 1.76 & $7.59 \mathrm{E}-02$ & 1197 & С3H2orf71 & 1.89 & $5.25 \mathrm{E}-03$ \\
\hline 1062 & BX935982 & 7.32 & $1.69 \mathrm{E}-03$ & 1130 & BX950706 & 2.61 & $2.96 \mathrm{E}-03$ & 1198 & C3H6orf168 & 1.82 & $1.81 \mathrm{E}-02$ \\
\hline 1063 & BX935985 & 2.84 & $5.00 \mathrm{E}-03$ & 1131 & BX950708 & 2.34 & $2.57 \mathrm{E}-03$ & 1199 & С3H6orf191 & 7.49 & $8.69 \mathrm{E}-05$ \\
\hline 1064 & BX935988 & 6.42 & $1.91 \mathrm{E}-03$ & 1132 & BX950709 & 6.20 & $1.40 \mathrm{E}-02$ & 1200 & C4H4orf19 & 4.88 & $2.95 \mathrm{E}-03$ \\
\hline 1065 & BX935994 & 4.38 & $3.72 \mathrm{E}-02$ & 1133 & BX950720 & 4.07 & $2.68 \mathrm{E}-03$ & 1201 & C5AR1 & 2.46 & $2.36 \mathrm{E}-03$ \\
\hline 1066 & BX935999 & 2.19 & $2.54 \mathrm{E}-03$ & 1134 & BX950727 & 1.11 & $1.01 \mathrm{E}-02$ & 1202 & C5H11orf16 & 5.28 & $4.52 \mathrm{E}-02$ \\
\hline 1067 & BX936014 & 1.56 & $1.42 \mathrm{E}-01$ & 1135 & BX950740 & 4.61 & 4.16E-03 & 1203 & C5H11orf24 & 2.96 & $5.83 \mathrm{E}-04$ \\
\hline 1068 & BX936024 & 2.75 & $9.93 \mathrm{E}-04$ & 1136 & BX950756 & 2.25 & $4.21 \mathrm{E}-02$ & 1204 & C5H11orf41 & 1.81 & $2.38 \mathrm{E}-02$ \\
\hline 1069 & BX936025 & 3.48 & $9.35 \mathrm{E}-03$ & 1137 & BX950767 & 1.34 & $1.19 \mathrm{E}-02$ & 1205 & C5H14orf159 & 2.41 & $1.07 E-02$ \\
\hline 1070 & BX936055 & 1.67 & $3.86 \mathrm{E}-02$ & 1138 & BX950775 & 2.87 & 3.45E-02 & 1206 & C5H14orf37 & 1.79 & $1.03 E-02$ \\
\hline 1071 & BX936071 & 4.43 & $3.75 \mathrm{E}-02$ & 1139 & BX950786 & 3.61 & $3.45 \mathrm{E}-04$ & 1207 & C5H15orf52 & 2.57 & $6.33 \mathrm{E}-03$ \\
\hline 1072 & BX936110 & 2.55 & $1.10 \mathrm{E}-02$ & 1140 & BX950787 & 3.40 & $3.50 \mathrm{E}-03$ & 1208 & C6 & 6.33 & $3.97 \mathrm{E}-03$ \\
\hline 1073 & BX936132 & 4.08 & $3.04 \mathrm{E}-03$ & 1141 & BX950795 & 4.37 & $2.56 \mathrm{E}-03$ & 1209 & C6H10orf11 & 2.59 & $3.98 \mathrm{E}-02$ \\
\hline 1074 & BX936137 & 5.34 & $2.16 \mathrm{E}-03$ & 1142 & BX950798 & 1.67 & $2.41 \mathrm{E}-02$ & 1210 & C6H10orf2 & 3.76 & $4.81 \mathrm{E}-05$ \\
\hline 1075 & BX936155 & 4.44 & $1.80 \mathrm{E}-03$ & 1143 & BX950817 & 2.64 & $5.10 \mathrm{E}-03$ & 1211 & $\mathrm{C} 6 \mathrm{H} 10$ orf26 & 2.40 & $5.35 \mathrm{E}-03$ \\
\hline 1076 & BX936174 & 6.10 & $6.43 \mathrm{E}-04$ & 1144 & BX950830 & 3.04 & $1.24 \mathrm{E}-03$ & 1212 & C6H10orf28 & 1.34 & $1.38 \mathrm{E}-02$ \\
\hline 1077 & BX936192 & 3.63 & $5.60 \mathrm{E}-03$ & 1145 & BX950831 & 5.09 & $1.39 \mathrm{E}-03$ & 1213 & C6H10orf54 & 3.58 & $1.81 \mathrm{E}-03$ \\
\hline 1078 & BX936231 & 4.34 & $4.76 \mathrm{E}-03$ & 1146 & BX950832 & 8.00 & $1.16 \mathrm{E}-03$ & 1214 & C6H10orf71 & 3.82 & $6.57 \mathrm{E}-03$ \\
\hline 1079 & BX936249 & 2.50 & $8.70 \mathrm{E}-04$ & 1147 & BX950836 & 4.91 & $7.94 \mathrm{E}-04$ & 1215 & C8A & 5.72 & $2.77 \mathrm{E}-03$ \\
\hline 1080 & BX936259 & 5.08 & $3.00 \mathrm{E}-05$ & 1148 & BX950837 & 5.44 & $7.12 \mathrm{E}-03$ & 1216 & C8H1orf168 & 3.00 & $3.60 \mathrm{E}-03$ \\
\hline 1081 & BX950231 & 5.43 & $2.03 \mathrm{E}-03$ & 1149 & BX950840 & 1.85 & $5.99 \mathrm{E}-03$ & 1217 & C8H1orf190 & 1.43 & $1.85 \mathrm{E}-02$ \\
\hline 1082 & BX950233 & 2.26 & $1.83 \mathrm{E}-02$ & 1150 & BX950847 & 2.24 & $6.62 \mathrm{E}-02$ & 1218 & $\mathrm{C9H} 2 \mathrm{orf54}$ & 5.06 & $2.47 \mathrm{E}-03$ \\
\hline 1083 & BX950237 & 5.12 & $5.42 \mathrm{E}-03$ & 1151 & BX950849 & 2.17 & $2.55 \mathrm{E}-02$ & 1219 & C9H3orf58 & 1.96 & $2.49 \mathrm{E}-03$ \\
\hline 1084 & BX950239 & 3.93 & $2.38 \mathrm{E}-03$ & \multicolumn{2}{|c|}{$152 \quad \mathrm{C} 10 \mathrm{H} 15$ orf59 } & 4.22 & $1.20 \mathrm{E}-02$ & 1220 & CA10 & 2.97 & $6.07 \mathrm{E}-03$ \\
\hline 1085 & BX950240 & 2.75 & $1.85 \mathrm{E}-02$ & 1153 & C14H16orf45 & 1.87 & $1.52 \mathrm{E}-02$ & 1221 & CA12 & 5.39 & $2.69 \mathrm{E}-03$ \\
\hline 1086 & BX950242 & 4.08 & $4.15 \mathrm{E}-03$ & 1154 & C14H16orf79 & 1.31 & $9.15 \mathrm{E}-03$ & 1222 & CA4 & 3.21 & $9.59 \mathrm{E}-03$ \\
\hline 1087 & BX950243 & 2.33 & $1.38 \mathrm{E}-03$ & 1155 & C14H7orf26 & 1.84 & $2.10 \mathrm{E}-02$ & 1223 & CA5B & 1.83 & $1.61 \mathrm{E}-01$ \\
\hline
\end{tabular}




\begin{tabular}{|c|c|c|c|c|c|c|c|c|c|c|c|}
\hline 1224 & CA6 & 4.34 & $4.70 \mathrm{E}-05$ & 1292 & CCR4 & 5.12 & $1.74 \mathrm{E}-02$ & 1360 & CDK6 & 1.54 & $1.55 \mathrm{E}-03$ \\
\hline 1225 & CABLES2 & 2.11 & $5.05 \mathrm{E}-03$ & 1293 & CCR5 & 4.13 & $1.12 \mathrm{E}-03$ & 1361 & CDKN2B & 3.56 & $1.11 \mathrm{E}-03$ \\
\hline 1226 & CABP7 & 5.06 & $1.24 \mathrm{E}-04$ & 1294 & CCR6 & 5.98 & $7.12 \mathrm{E}-03$ & 1362 & CDR2L & 3.89 & $1.40 \mathrm{E}-03$ \\
\hline 1227 & CACFD1 & 3.69 & $2.39 \mathrm{E}-03$ & 1295 & CCR7 & 3.84 & $1.68 \mathrm{E}-03$ & 1363 & CDX1 & 3.27 & $6.39 \mathrm{E}-03$ \\
\hline 1228 & CACNA1B & 2.81 & $2.95 \mathrm{E}-03$ & 1296 & CCR8-L & 3.31 & $1.51 \mathrm{E}-02$ & 1364 & $\operatorname{CDX} 2$ & 6.07 & $3.21 \mathrm{E}-03$ \\
\hline 1229 & CACNA1D & 2.59 & $2.73 \mathrm{E}-02$ & 1297 & CCR9 & 4.65 & $8.55 \mathrm{E}-04$ & 1365 & CDX4 & 3.33 & $1.18 \mathrm{E}-02$ \\
\hline 1230 & CACNA1E & 2.65 & 3.05E-03 & 1298 & $\operatorname{cCS}$ & 2.06 & $1.31 \mathrm{E}-02$ & 1366 & CDYL & 1.20 & 9.06E-03 \\
\hline 1231 & CACNA2D2 & 4.95 & $4.38 \mathrm{E}-04$ & 1299 & CD14 & 7.27 & 3.24E-05 & 1367 & CDYL2 & 2.15 & 2.96E-02 \\
\hline 1232 & CACNB2 & 2.84 & $1.13 \mathrm{E}-02$ & 1300 & CD163 & 2.97 & $5.27 \mathrm{E}-03$ & 1368 & CEBPA & 4.01 & $9.64 \mathrm{E}-02$ \\
\hline 1233 & CACNG1 & 4.80 & $1.15 \mathrm{E}-03$ & 1301 & $\mathrm{CD} 1 \mathrm{~B}$ & 4.74 & $3.09 \mathrm{E}-02$ & 1369 & CEBPB & 1.46 & $1.08 \mathrm{E}-02$ \\
\hline 1234 & CACNG2 & 4.34 & $1.32 \mathrm{E}-03$ & 1302 & CD1C & 6.18 & $4.29 \mathrm{E}-03$ & 1370 & CECR2 & 5.25 & 9.59E-04 \\
\hline 1235 & CACNG3 & 4.83 & $1.39 \mathrm{E}-03$ & 1303 & CD2 & 2.39 & $1.39 \mathrm{E}-02$ & 1371 & CELA1 & 3.52 & $9.13 \mathrm{E}-02$ \\
\hline 1236 & CACNG4 & 4.08 & $9.25 \mathrm{E}-03$ & 1304 & CD200R1 & 2.91 & $7.55 \mathrm{E}-04$ & 1372 & CELF4 & 1.24 & $1.30 \mathrm{E}-02$ \\
\hline 1237 & CACNG7 & 5.13 & $1.29 \mathrm{E}-04$ & 1305 & CD200R1L & 3.96 & $1.27 \mathrm{E}-03$ & 1373 & CELSR3 & 4.37 & $3.42 \mathrm{E}-03$ \\
\hline 1238 & CACTIN & 1.30 & 3.03E-03 & 1306 & CD215054 & 2.56 & $8.14 \mathrm{E}-03$ & 1374 & CEND1 & 3.83 & $1.36 \mathrm{E}-03$ \\
\hline 1239 & CAD & 4.07 & $2.89 \mathrm{E}-03$ & 1307 & CD219003 & 3.41 & $2.41 \mathrm{E}-03$ & 1375 & CEP57L1 & 4.42 & $3.27 \mathrm{E}-03$ \\
\hline 1240 & CALB1 & 3.35 & $6.68 \mathrm{E}-03$ & 1308 & CD219033 & 3.79 & $9.15 \mathrm{E}-04$ & 1376 & CEP68 & 2.56 & $1.29 \mathrm{E}-03$ \\
\hline 1241 & CALB2 & 2.36 & 3.48E-03 & 1309 & CD226 & 4.70 & $1.08 \mathrm{E}-03$ & 1377 & CER1 & 4.69 & 4.89E-03 \\
\hline 1242 & CALCR & 5.15 & 3.00E-04 & 1310 & CD274 & 3.43 & $2.17 \mathrm{E}-03$ & 1378 & CERCAM & 3.18 & $1.14 \mathrm{E}-02$ \\
\hline 1243 & CALHM1 & 4.95 & $8.82 \mathrm{E}-04$ & 1311 & CD28 & 4.72 & $2.21 \mathrm{E}-03$ & 1379 & CES1 & 4.35 & $1.35 \mathrm{E}-03$ \\
\hline 1244 & CALHM2 & 2.52 & $1.98 \mathrm{E}-03$ & 1312 & CD300A & 6.29 & $6.95 \mathrm{E}-04$ & 1380 & CF255803 & 2.17 & $5.64 \mathrm{E}-03$ \\
\hline 1245 & CALHM3 & 3.82 & $6.07 \mathrm{E}-04$ & 1313 & CD300L-S1 & 5.17 & $1.69 \mathrm{E}-03$ & 1381 & CF256356 & 2.69 & $3.26 \mathrm{E}-03$ \\
\hline 1246 & CALM3 & 2.49 & $5.71 \mathrm{E}-04$ & 1314 & CD320 & 1.36 & $4.58 \mathrm{E}-02$ & 1382 & CF257125 & 2.05 & $4.95 \mathrm{E}-03$ \\
\hline 1247 & CALML4 & 1.66 & 7.19E-02 & 1315 & CD34 & 2.84 & $8.37 \mathrm{E}-03$ & 1383 & CGNRH-R & 5.59 & $1.23 \mathrm{E}-03$ \\
\hline 1248 & CAMK1 & 1.09 & $2.97 \mathrm{E}-03$ & 1316 & CD38 & 2.63 & $5.94 \mathrm{E}-03$ & 1384 & CHAC1 & 2.19 & $6.14 \mathrm{E}-04$ \\
\hline 1249 & САMKK2 & 3.49 & $9.85 \mathrm{E}-03$ & 1317 & CD40 & 2.66 & $1.52 \mathrm{E}-02$ & 1385 & CHAD & 3.77 & 7.92E-04 \\
\hline 1250 & CAMKV & 2.78 & $9.34 \mathrm{E}-03$ & 1318 & CD40LG & 2.24 & 4.12E-03 & 1386 & CHAT & 5.85 & $1.24 \mathrm{E}-04$ \\
\hline 1251 & CAMP & 2.74 & $2.23 \mathrm{E}-03$ & 1319 & CD44 & 2.52 & $1.32 \mathrm{E}-03$ & 1387 & CHAT1 & 5.99 & $4.55 \mathrm{E}-03$ \\
\hline 1252 & CAPN3 & 1.95 & $2.98 \mathrm{E}-02$ & 1320 & CD5 & 1.83 & $1.64 \mathrm{E}-03$ & 1388 & CHAT2 & 6.83 & $5.38 \mathrm{E}-04$ \\
\hline 1253 & CAPN9 & 2.55 & $8.73 \mathrm{E}-04$ & 1321 & CD55 & 4.01 & $5.11 \mathrm{E}-03$ & 1389 & $\mathrm{CHDH}$ & 3.29 & 3.48E-04 \\
\hline 1254 & CAPSL & 2.66 & $6.62 \mathrm{E}-03$ & 1322 & CD7 & 5.02 & $2.10 \mathrm{E}-04$ & 1390 & CHDSD & 4.23 & $2.73 \mathrm{E}-02$ \\
\hline 1255 & CASKIN1 & 5.41 & $2.12 \mathrm{E}-03$ & 1323 & $\mathrm{CD} 72$ & 5.98 & $3.94 \mathrm{E}-04$ & 1391 & CHEK2 & 5.09 & 9.33E-04 \\
\hline 1256 & CASP14 & 5.74 & $8.15 \mathrm{E}-04$ & 1324 & CD727080 & 5.86 & $2.73 \mathrm{E}-03$ & 1392 & CHGA & 3.25 & 3.09E-04 \\
\hline 1257 & CASP7 & 2.17 & $1.85 \mathrm{E}-02$ & 1325 & CD734044 & 2.43 & $1.37 \mathrm{E}-03$ & 1393 & CHIA & 1.23 & $1.40 \mathrm{E}-02$ \\
\hline 1258 & CASQ2 & 2.99 & $1.70 \mathrm{E}-02$ & 1326 & CD737244 & 1.19 & $2.19 \mathrm{E}-03$ & 1394 & CHIR2D-956 & 5.66 & $2.91 \mathrm{E}-04$ \\
\hline 1259 & CAT & 5.64 & $4.85 \mathrm{E}-03$ & 1327 & CD737500 & 1.46 & $1.67 \mathrm{E}-03$ & 1395 & CHIR-A2 & 5.07 & $3.98 \mathrm{E}-04$ \\
\hline 1260 & CATHB1 & 4.24 & $5.58 \mathrm{E}-04$ & 1328 & CD738021 & 1.80 & $2.46 \mathrm{E}-02$ & 1396 & CHIR-AB1 & 8.12 & $2.71 \mathrm{E}-04$ \\
\hline 1261 & CAV1 & 2.37 & $5.27 \mathrm{E}-03$ & 1329 & CD762402 & 2.07 & $1.34 \mathrm{E}-02$ & 1397 & CHIR-B2 & 7.42 & $1.13 \mathrm{E}-02$ \\
\hline 1262 & CAV3 & 3.77 & $6.86 \mathrm{E}-03$ & 1330 & CD762671 & 5.90 & $1.29 \mathrm{E}-04$ & 1398 & CHIR-B3 & 4.94 & $1.62 \mathrm{E}-03$ \\
\hline 1263 & CB016838 & 1.26 & $1.36 \mathrm{E}-01$ & 1331 & CD763711 & 3.14 & 2.18E-03 & 1399 & CHIR-B4 & 5.96 & 8.67E-04 \\
\hline 1264 & CB017893 & 3.85 & 2.65E-03 & 1332 & CD764185 & 2.24 & 3.29E-03 & 1400 & CHIR-B5 & 5.12 & $4.56 \mathrm{E}-03$ \\
\hline 1265 & СВFA2Т3 & 2.26 & $1.29 \mathrm{E}-02$ & 1333 & CD765191 & 1.22 & $2.43 \mathrm{E}-02$ & 1401 & CHL1 & 6.52 & $3.61 \mathrm{E}-03$ \\
\hline 1266 & CBL & 3.69 & $1.66 \mathrm{E}-02$ & 1334 & CD79B & 4.05 & $3.28 \mathrm{E}-06$ & 1402 & CHN2 & 1.61 & $3.09 \mathrm{E}-02$ \\
\hline 1267 & CBLB & 1.09 & $6.28 \mathrm{E}-04$ & 1335 & CD86 & 3.48 & $1.24 \mathrm{E}-02$ & 1403 & CHODL & 3.39 & $1.82 \mathrm{E}-04$ \\
\hline 1268 & CBLN2 & 4.90 & $4.88 \mathrm{E}-03$ & 1336 & CD8A & 3.94 & $5.30 \mathrm{E}-03$ & 1404 & CHPF & 3.92 & $2.85 \mathrm{E}-03$ \\
\hline 1269 & CBLN4 & 5.55 & $1.50 \mathrm{E}-03$ & 1337 & CD8B & 2.28 & $1.33 \mathrm{E}-02$ & 1405 & CHRD & 3.02 & 7.19E-03 \\
\hline 1270 & CBX2 & 3.55 & $4.24 \mathrm{E}-03$ & 1338 & CDC42EP1 & 3.58 & 7.93E-03 & 1406 & CHRM3 & 3.20 & 7.00E-03 \\
\hline 1271 & CBX8 & 5.07 & $9.89 \mathrm{E}-04$ & 1339 & CDC42EP3 & 1.56 & $4.13 \mathrm{E}-03$ & 1407 & CHRM4 & 3.55 & $7.25 \mathrm{E}-03$ \\
\hline 1272 & CCBE1 & 1.79 & $1.90 \mathrm{E}-03$ & 1340 & CDCP2 & 5.04 & $2.13 \mathrm{E}-03$ & 1408 & CHRNA1 & 5.15 & $1.09 \mathrm{E}-03$ \\
\hline 1273 & CCBL1 & 1.15 & $4.86 \mathrm{E}-03$ & 1341 & CDD & 3.54 & $2.69 \mathrm{E}-03$ & 1409 & CHRNA10 & 4.17 & 1.77E-04 \\
\hline 1274 & CCDC108 & 2.66 & $3.08 \mathrm{E}-02$ & 1342 & $\mathrm{CDH} 10$ & 5.83 & 4.56E-03 & 1410 & CHRNA2 & 3.29 & $1.28 \mathrm{E}-02$ \\
\hline 1275 & CCDC113 & 3.31 & $4.08 \mathrm{E}-03$ & 1343 & $\mathrm{CDH} 12$ & 6.37 & $4.41 \mathrm{E}-03$ & 1411 & CHRNA4 & 2.19 & $8.87 \mathrm{E}-03$ \\
\hline 1276 & CCDC124 & 2.26 & $1.84 \mathrm{E}-03$ & 1344 & $\mathrm{CDH} 17$ & 1.84 & $1.56 \mathrm{E}-03$ & 1412 & CHRNA7 & 5.03 & $5.71 \mathrm{E}-03$ \\
\hline 1277 & CCDC141 & 4.08 & $2.69 \mathrm{E}-04$ & 1345 & $\mathrm{CDH} 19$ & 5.10 & $2.34 \mathrm{E}-03$ & 1413 & CHRNB3 & 2.94 & $4.60 \mathrm{E}-03$ \\
\hline 1278 & CCDC28B & 1.81 & $6.40 \mathrm{E}-03$ & 1346 & $\mathrm{CDH} 2 \mathrm{O}$ & 2.25 & $9.43 \mathrm{E}-03$ & 1414 & CHRNB4 & 3.43 & $2.86 \mathrm{E}-04$ \\
\hline 1279 & CCDC50 & 2.29 & $5.84 \mathrm{E}-02$ & 1347 & $\mathrm{CDH} 22$ & 5.25 & $2.29 \mathrm{E}-02$ & 1415 & CHRND & 5.60 & $5.19 \mathrm{E}-04$ \\
\hline 1280 & CCDC51 & 2.79 & $2.11 \mathrm{E}-03$ & 1348 & $\mathrm{CDH} 23$ & 3.30 & $1.67 \mathrm{E}-03$ & 1416 & CHRNG & 2.19 & $5.24 \mathrm{E}-03$ \\
\hline 1281 & CCKBR & 5.19 & $5.27 \mathrm{E}-03$ & 1349 & $\mathrm{CDH} 3$ & 3.07 & $5.12 \mathrm{E}-03$ & 1417 & CHST1 & 3.36 & $1.51 \mathrm{E}-02$ \\
\hline 1282 & CCL1 & 2.10 & $1.02 \mathrm{E}-01$ & 1350 & $\mathrm{CDH} 4$ & 1.48 & $6.68 \mathrm{E}-02$ & 1418 & CHST12 & 2.28 & 3.47E-03 \\
\hline 1283 & CCL17 & 4.62 & $2.28 \mathrm{E}-03$ & 1351 & $\mathrm{CDH6}$ & 1.29 & $1.06 \mathrm{E}-01$ & 1419 & CHST13 & 1.55 & $1.01 \mathrm{E}-02$ \\
\hline 1284 & CCL19 & 4.45 & $2.33 \mathrm{E}-02$ & 1352 & $\mathrm{CDH7}$ & 5.46 & $2.84 \mathrm{E}-04$ & 1420 & CHST14 & 3.17 & $2.39 \mathrm{E}-03$ \\
\hline 1285 & CCL4 & 3.92 & $1.82 \mathrm{E}-04$ & 1353 & $\mathrm{CDH} 8$ & 2.52 & 3.35E-03 & 1421 & CHST2 & 3.52 & $2.75 \mathrm{E}-03$ \\
\hline 1286 & CCL5 & 3.37 & $1.17 \mathrm{E}-03$ & 1354 & $\mathrm{CDH} 9$ & 6.41 & $4.88 \mathrm{E}-03$ & 1422 & CHST3 & 5.46 & $7.28 \mathrm{E}-03$ \\
\hline 1287 & CCLI5 & 2.99 & $7.08 \mathrm{E}-04$ & 1355 & CDHR2 & 1.85 & $9.58 \mathrm{E}-03$ & 1423 & CHUNK-1 & 3.87 & 7.19E-04 \\
\hline 1288 & CCNJ & 1.86 & $9.72 \mathrm{E}-03$ & 1356 & CDK12 & 2.03 & $1.49 \mathrm{E}-02$ & 1424 & CIB2 & 1.11 & $3.01 \mathrm{E}-03$ \\
\hline 1289 & CCNJL & 4.11 & $1.26 \mathrm{E}-04$ & 1357 & CDK14 & 3.29 & $3.16 \mathrm{E}-03$ & 1425 & CILP & 4.17 & 4.75E-04 \\
\hline 1290 & CCNY & 2.87 & $9.05 E-03$ & 1358 & CDK2 & 1.60 & $2.68 \mathrm{E}-02$ & 1426 & CIRH1A & 1.64 & $2.07 E-03$ \\
\hline 1291 & CCR2 & 2.53 & $1.83 \mathrm{E}-02$ & 1359 & CDK5RAP3 & 1.75 & $7.60 \mathrm{E}-03$ & 1427 & CK609977 & 7.49 & $2.91 \mathrm{E}-03$ \\
\hline
\end{tabular}




\begin{tabular}{|c|c|c|c|c|c|c|c|c|c|c|c|}
\hline 1428 & CKM & 2.72 & 4.47E-03 & 1496 & CO635716 & 3.24 & $1.11 \mathrm{E}-02$ & 1564 & CR338699 & 4.26 & $1.25 \mathrm{E}-02$ \\
\hline 1429 & $\mathrm{CL2}$ & 1.93 & $5.72 \mathrm{E}-03$ & 1497 & C0635731 & 1.32 & $3.62 \mathrm{E}-03$ & 1565 & CR338701 & 6.92 & $1.09 \mathrm{E}-03$ \\
\hline 1430 & CLDN11 & 1.55 & $6.56 \mathrm{E}-02$ & 1498 & CO759325 & 1.84 & 3.03E-03 & 1566 & CR338702 & 4.54 & $2.27 E-03$ \\
\hline 1431 & CLDN12 & 2.07 & $1.44 \mathrm{E}-02$ & 1499 & CO759834 & 1.58 & $1.22 \mathrm{E}-02$ & 1567 & CR338705 & 4.76 & $7.61 \mathrm{E}-04$ \\
\hline 1432 & CLDN14 & 3.86 & $2.59 \mathrm{E}-04$ & 1500 & CO760685 & 2.83 & $1.35 \mathrm{E}-02$ & 1568 & CR338706 & 1.28 & $4.20 \mathrm{E}-02$ \\
\hline 1433 & CLDN16 & 4.07 & $1.20 \mathrm{E}-02$ & 1501 & CO761139 & 4.79 & $1.32 \mathrm{E}-03$ & 1569 & CR338707 & 2.22 & $3.10 \mathrm{E}-02$ \\
\hline 1434 & CLDN2 & 2.58 & $2.01 E-03$ & 1502 & C0763152 & 1.31 & $8.18 \mathrm{E}-02$ & 1570 & CR338708 & 3.90 & $2.54 \mathrm{E}-03$ \\
\hline 1435 & CLDN20 & 5.61 & $5.51 \mathrm{E}-03$ & 1503 & CO763296 & 1.41 & $3.99 \mathrm{E}-02$ & 1571 & CR338709 & 5.16 & $8.91 E-03$ \\
\hline 1436 & CLDN25 & 5.56 & $1.42 \mathrm{E}-03$ & 1504 & C0763813 & 1.24 & $1.61 \mathrm{E}-02$ & 1572 & CR338710 & 5.96 & $5.73 E-03$ \\
\hline 1437 & CLDN3 & 1.68 & $4.28 \mathrm{E}-03$ & 1505 & CO766732 & 3.15 & $2.20 \mathrm{E}-04$ & 1573 & CR338716 & 5.19 & $3.25 \mathrm{E}-03$ \\
\hline 1438 & CLDN4 & 5.87 & $6.04 \mathrm{E}-04$ & 1506 & CO767189 & 5.69 & $3.17 \mathrm{E}-06$ & 1574 & CR338717 & 4.78 & $3.69 \mathrm{E}-03$ \\
\hline 1439 & CLDN5 & 1.90 & $6.81 \mathrm{E}-02$ & 1507 & C0767778 & 4.01 & $9.91 \mathrm{E}-05$ & 1575 & CR338719 & 4.27 & $1.87 \mathrm{E}-03$ \\
\hline 1440 & CLDN8 & 6.68 & $1.70 \mathrm{E}-03$ & 1508 & CO767816 & 4.33 & $5.70 \mathrm{E}-03$ & 1576 & CR338721 & 4.92 & $4.19 E-03$ \\
\hline 1441 & CLEC2D & 4.01 & $1.66 \mathrm{E}-03$ & 1509 & CO772450 & 1.44 & $5.06 \mathrm{E}-02$ & 1577 & CR338722 & 1.24 & $3.18 \mathrm{E}-03$ \\
\hline 1442 & CLEC3A & 8.59 & $4.67 \mathrm{E}-04$ & 1510 & $\mathrm{COCH}$ & 4.94 & $1.52 \mathrm{E}-02$ & 1578 & CR338724 & 5.92 & $5.25 \mathrm{E}-03$ \\
\hline 1443 & CLIC2 & 2.13 & $3.55 \mathrm{E}-03$ & 1511 & COL11A1 & 1.49 & $3.67 \mathrm{E}-03$ & 1579 & CR338725 & 4.96 & $6.15 \mathrm{E}-04$ \\
\hline 1444 & CLIC3 & 1.94 & $1.38 \mathrm{E}-02$ & 1512 & COL12A1 & 2.54 & $7.53 \mathrm{E}-03$ & 1580 & CR338729 & 3.15 & 2.47E-03 \\
\hline 1445 & CLIC5 & 4.17 & $9.29 \mathrm{E}-04$ & 1513 & COL22A1 & 1.36 & 3.07E-02 & 1581 & CR338736 & 5.67 & $2.96 \mathrm{E}-04$ \\
\hline 1446 & CLIC6 & 3.25 & $5.69 \mathrm{E}-04$ & 1514 & COL23A1 & 1.75 & $7.50 \mathrm{E}-03$ & 1582 & CR338737 & 1.58 & $6.70 \mathrm{E}-03$ \\
\hline 1447 & CLPS & 4.39 & $3.96 \mathrm{E}-04$ & 1515 & COL24A1 & 1.51 & $1.24 \mathrm{E}-01$ & 1583 & CR338738 & 1.42 & $5.05 E-02$ \\
\hline 1448 & CLRN1 & 3.21 & $6.34 \mathrm{E}-03$ & 1516 & COL25A1 & 4.83 & $6.20 \mathrm{E}-04$ & 1584 & CR338742 & 1.99 & $2.27 E-02$ \\
\hline 1449 & CLRN3 & 3.60 & $2.74 \mathrm{E}-03$ & 1517 & COL27A1 & 3.48 & $1.14 \mathrm{E}-02$ & 1585 & CR338743 & 6.68 & $2.23 \mathrm{E}-03$ \\
\hline 1450 & CLUL1 & 3.86 & $1.40 \mathrm{E}-03$ & 1518 & COL2A1 & 2.38 & $3.66 \mathrm{E}-02$ & 1586 & CR338747 & 2.78 & $2.16 \mathrm{E}-02$ \\
\hline 1451 & CLVS1 & 3.83 & $5.46 \mathrm{E}-03$ & 1519 & COL4A2 & 1.41 & $1.32 \mathrm{E}-02$ & 1587 & CR338749 & 5.20 & $4.05 \mathrm{E}-04$ \\
\hline 1452 & CMBL & 5.56 & $2.11 \mathrm{E}-03$ & 1520 & COL4A3 & 2.67 & $4.23 \mathrm{E}-03$ & 1588 & CR338750 & 1.34 & $9.48 \mathrm{E}-03$ \\
\hline 1453 & CMPK2 & 2.30 & $2.48 \mathrm{E}-02$ & 1521 & COL4A6 & 2.24 & $7.97 E-04$ & 1589 & CR338754 & 5.40 & $1.50 \mathrm{E}-05$ \\
\hline 1454 & CMTM6 & 3.70 & $1.34 \mathrm{E}-02$ & 1522 & COL6A3 & 1.54 & $4.63 \mathrm{E}-02$ & 1590 & CR338758 & 4.41 & $1.08 \mathrm{E}-02$ \\
\hline 1455 & CN210139 & 3.18 & $1.90 \mathrm{E}-02$ & 1523 & COL8A2 & 4.13 & $5.12 \mathrm{E}-02$ & 1591 & CR338762 & 4.18 & $6.76 \mathrm{E}-03$ \\
\hline 1456 & CN210811 & 1.77 & $6.65 \mathrm{E}-02$ & 1524 & COL9A2 & 3.03 & $4.66 \mathrm{E}-02$ & 1592 & CR338767 & 2.70 & $2.91 \mathrm{E}-03$ \\
\hline 1457 & CN210842 & 1.69 & $4.36 \mathrm{E}-02$ & 1525 & COLEC11 & 1.96 & $1.39 \mathrm{E}-03$ & 1593 & CR338770 & 3.94 & $2.71 \mathrm{E}-02$ \\
\hline 1458 & CN218494 & 3.88 & $3.20 \mathrm{E}-05$ & 1526 & COLQ & 2.45 & $1.42 \mathrm{E}-02$ & 1594 & CR338772 & 4.70 & $7.96 \mathrm{E}-03$ \\
\hline 1459 & CN221000 & 2.08 & $1.76 \mathrm{E}-02$ & 1527 & COMMD9 & 6.45 & $3.21 \mathrm{E}-03$ & 1595 & CR338773 & 7.01 & 2.03E-03 \\
\hline 1460 & CN221487 & 4.49 & $1.20 \mathrm{E}-02$ & 1528 & COPN5L1 & 3.60 & $5.20 \mathrm{E}-05$ & 1596 & CR338775 & 4.31 & $1.28 \mathrm{E}-03$ \\
\hline 1461 & CN221750 & 3.52 & $2.31 \mathrm{E}-04$ & 1529 & COPN5L2 & 4.31 & $1.35 \mathrm{E}-03$ & 1597 & CR338777 & 1.31 & $3.01 \mathrm{E}-02$ \\
\hline 1462 & CN226347 & 4.62 & $2.16 \mathrm{E}-03$ & 1530 & COR1 & 2.97 & $8.61 \mathrm{E}-03$ & 1598 & CR338782 & 2.59 & $7.19 E-03$ \\
\hline 1463 & CN227706 & 4.53 & $6.20 \mathrm{E}-03$ & 1531 & COR4 & 4.36 & $4.50 \mathrm{E}-04$ & 1599 & CR338788 & 3.90 & $5.35 \mathrm{E}-03$ \\
\hline 1464 & CN229808 & 3.57 & $2.78 \mathrm{E}-04$ & 1532 & COR6 & 4.63 & $1.79 \mathrm{E}-03$ & 1600 & CR338792 & 2.14 & $4.39 \mathrm{E}-02$ \\
\hline 1465 & CN231830 & 4.09 & $2.28 \mathrm{E}-03$ & 1533 & COR7A & 5.68 & $1.59 \mathrm{E}-04$ & 1601 & CR338798 & 3.99 & $4.71 \mathrm{E}-03$ \\
\hline 1466 & CN232637 & 4.29 & $1.03 \mathrm{E}-03$ & 1534 & COR8 & 3.19 & $9.29 \mathrm{E}-03$ & 1602 & CR338800 & 2.38 & $9.57 E-03$ \\
\hline 1467 & CN232948 & 4.36 & $2.11 \mathrm{E}-04$ & 1535 & CORO7 & 4.66 & $2.76 \mathrm{E}-03$ & 1603 & CR338806 & 5.03 & 5.34E-04 \\
\hline 1468 & CN234658 & 4.80 & $7.46 \mathrm{E}-04$ & 1536 & COTL1 & 2.85 & $1.34 \mathrm{E}-02$ & 1604 & CR338811 & 5.58 & $3.15 \mathrm{E}-03$ \\
\hline 1469 & CN235660 & 1.33 & $6.96 \mathrm{E}-03$ & 1537 & $\mathrm{CP}$ & 3.73 & $7.26 \mathrm{E}-03$ & 1605 & CR338812 & 5.10 & $8.15 E-04$ \\
\hline 1470 & CNGA1 & 5.55 & $9.91 \mathrm{E}-03$ & 1538 & CPA1 & 3.45 & $3.94 \mathrm{E}-03$ & 1606 & CR338817 & 4.87 & $5.52 \mathrm{E}-04$ \\
\hline 1471 & CNGA4 & 3.19 & $2.30 \mathrm{E}-03$ & 1539 & CPA6 & 4.91 & $3.79 \mathrm{E}-04$ & 1607 & CR338821 & 2.42 & $2.10 \mathrm{E}-02$ \\
\hline 1472 & CNKSR2 & 2.50 & $1.56 \mathrm{E}-02$ & 1540 & CPB1 & 2.70 & $3.87 \mathrm{E}-03$ & 1608 & CR338825 & 3.96 & $8.61 \mathrm{E}-03$ \\
\hline 1473 & CNKSR3 & 2.27 & 7.07E-03 & 1541 & CPED1 & 4.47 & $1.53 \mathrm{E}-04$ & 1609 & CR338826 & 5.12 & $5.89 \mathrm{E}-03$ \\
\hline 1474 & CNN1 & 1.76 & $1.37 \mathrm{E}-02$ & 1542 & CPLX2 & 2.61 & $2.33 \mathrm{E}-03$ & 1610 & CR338827 & 9.00 & 5.00E-03 \\
\hline 1475 & CNOT6L & 1.96 & 7.47E-05 & 1543 & CPLX3 & 3.99 & $1.20 \mathrm{E}-03$ & 1611 & CR338831 & 2.76 & $3.98 \mathrm{E}-03$ \\
\hline 1476 & CNP1 & 3.04 & $1.77 \mathrm{E}-03$ & 1544 & CPLX4 & 6.31 & $3.87 \mathrm{E}-03$ & 1612 & CR338841 & 5.21 & $1.92 \mathrm{E}-03$ \\
\hline 1477 & CNR2 & 4.65 & $3.07 E-04$ & 1545 & CPO & 4.82 & $4.03 \mathrm{E}-03$ & 1613 & CR338845 & 5.09 & $7.77 E-03$ \\
\hline 1478 & CNRIP1 & 1.65 & $6.80 \mathrm{E}-03$ & 1546 & CPSF7 & 2.29 & $4.32 \mathrm{E}-03$ & 1614 & CR338846 & 6.25 & $1.88 \mathrm{E}-03$ \\
\hline 1479 & CNTF & 2.51 & $2.06 \mathrm{E}-03$ & 1547 & CPXM2 & 2.32 & $2.52 \mathrm{E}-03$ & 1615 & CR338848 & 2.37 & $2.17 E-03$ \\
\hline 1480 & CNTFR & 4.46 & $2.02 \mathrm{E}-04$ & 1548 & $\mathrm{CPZ}$ & 3.57 & $1.10 \mathrm{E}-01$ & 1616 & CR338850 & 5.68 & 5.47E-04 \\
\hline 1481 & CNTN2 & 4.61 & $8.38 \mathrm{E}-04$ & 1549 & CR1L & 1.83 & $7.18 \mathrm{E}-03$ & 1617 & CR338856 & 2.84 & $1.70 \mathrm{E}-03$ \\
\hline 1482 & CNTN3 & 3.94 & $6.57 \mathrm{E}-04$ & 1550 & CR2 & 2.17 & $4.48 \mathrm{E}-04$ & 1618 & CR338857 & 2.25 & $2.11 \mathrm{E}-03$ \\
\hline 1483 & CNTN4 & 1.60 & $6.09 \mathrm{E}-04$ & 1551 & CR338678 & 4.99 & $5.29 \mathrm{E}-04$ & 1619 & CR338858 & 1.64 & $2.29 \mathrm{E}-02$ \\
\hline 1484 & CNTN5 & 4.62 & $1.17 \mathrm{E}-03$ & 1552 & CR338681 & 2.21 & $5.77 \mathrm{E}-03$ & 1620 & CR338862 & 3.64 & $1.87 \mathrm{E}-03$ \\
\hline 1485 & CNTN6 & 6.63 & $1.54 \mathrm{E}-02$ & 1553 & CR338682 & 4.04 & $6.72 \mathrm{E}-03$ & 1621 & CR338866 & 2.80 & $1.73 E-03$ \\
\hline 1486 & CNTNAP2 & 5.02 & $9.21 \mathrm{E}-04$ & 1554 & CR338683 & 2.80 & $5.30 \mathrm{E}-03$ & 1622 & CR338867 & 5.87 & $1.70 \mathrm{E}-04$ \\
\hline 1487 & CNTNAP5 & 2.70 & $1.11 \mathrm{E}-02$ & 1555 & CR338684 & 4.73 & $3.31 \mathrm{E}-02$ & 1623 & CR338879 & 4.27 & $6.78 \mathrm{E}-03$ \\
\hline 1488 & CO420084 & 2.05 & $9.31 \mathrm{E}-03$ & 1556 & CR338685 & 4.00 & $3.36 \mathrm{E}-03$ & 1624 & CR338881 & 6.91 & $2.35 \mathrm{E}-03$ \\
\hline 1489 & CO421882 & 4.43 & $2.57 \mathrm{E}-03$ & 1557 & CR338687 & 3.35 & $9.18 \mathrm{E}-03$ & 1625 & CR338884 & 2.71 & $6.29 \mathrm{E}-03$ \\
\hline 1490 & CO421926 & 3.16 & $9.00 \mathrm{E}-03$ & 1558 & CR338689 & 4.76 & $1.03 E-02$ & 1626 & CR338885 & 2.33 & 7.37E-03 \\
\hline 1491 & CO422852 & 4.01 & $3.05 E-04$ & 1559 & CR338690 & 6.08 & $2.15 \mathrm{E}-04$ & 1627 & CR338887 & 1.58 & 3.07E-02 \\
\hline 1492 & CO503335 & 4.07 & $2.06 \mathrm{E}-03$ & 1560 & CR338693 & 5.60 & $2.55 \mathrm{E}-03$ & 1628 & CR338897 & 3.94 & $6.11 \mathrm{E}-03$ \\
\hline 1493 & CO635507 & 5.16 & $1.65 \mathrm{E}-03$ & 1561 & CR338696 & 6.11 & $4.64 \mathrm{E}-05$ & 1629 & CR338898 & 2.14 & $3.37 \mathrm{E}-03$ \\
\hline 1494 & C0635574 & 3.40 & $1.79 \mathrm{E}-03$ & 1562 & CR338697 & 5.61 & $2.22 \mathrm{E}-03$ & 1630 & CR338902 & 4.24 & $1.59 \mathrm{E}-03$ \\
\hline 1495 & CO635711 & 4.84 & $4.30 \mathrm{E}-04$ & 1563 & CR338698 & 3.09 & $2.45 \mathrm{E}-03$ & 1631 & CR338904 & 5.70 & $5.01 \mathrm{E}-06$ \\
\hline
\end{tabular}




\begin{tabular}{|c|c|c|c|c|c|c|c|c|c|c|c|}
\hline 1632 & CR338906 & 5.64 & $3.55 \mathrm{E}-05$ & 1700 & CR352540 & 3.64 & $1.97 \mathrm{E}-03$ & 1768 & CR352969 & 5.03 & $4.05 E-03$ \\
\hline 1633 & CR338908 & 5.95 & $1.68 \mathrm{E}-03$ & 1701 & CR352543 & 4.20 & $1.50 \mathrm{E}-03$ & 1769 & CR352971 & 6.23 & $1.31 \mathrm{E}-04$ \\
\hline 1634 & CR338911 & 2.10 & $3.80 \mathrm{E}-02$ & 1702 & CR352553 & 3.67 & $5.78 \mathrm{E}-03$ & 1770 & CR352972 & 1.28 & $1.32 \mathrm{E}-02$ \\
\hline 1635 & CR338912 & 2.78 & $3.35 \mathrm{E}-03$ & 1703 & CR352561 & 2.67 & $1.33 \mathrm{E}-02$ & 1771 & CR352973 & 1.56 & 4.57E-02 \\
\hline 1636 & CR338918 & 1.16 & $2.32 \mathrm{E}-02$ & 1704 & CR352566 & 6.08 & $3.95 \mathrm{E}-03$ & 1772 & CR352982 & 4.78 & $2.66 \mathrm{E}-02$ \\
\hline 1637 & CR338919 & 3.87 & $3.11 \mathrm{E}-03$ & 1705 & CR352582 & 4.16 & $5.70 \mathrm{E}-04$ & 1773 & CR352986 & 2.27 & 1.17E-04 \\
\hline 1638 & CR338927 & 4.23 & $7.76 E-04$ & 1706 & CR352584 & 2.87 & $4.79 E-03$ & 1774 & CR352993 & 2.40 & $4.36 \mathrm{E}-03$ \\
\hline 1639 & CR338932 & 5.15 & $1.18 \mathrm{E}-02$ & 1707 & CR352589 & 1.70 & $4.00 E-02$ & 1775 & CR353004 & 1.75 & 5.69E- 03 \\
\hline 1640 & CR338934 & 3.20 & $1.03 \mathrm{E}-02$ & 1708 & CR352600 & 4.75 & $3.72 \mathrm{E}-03$ & 1776 & CR353005 & 1.51 & $5.11 \mathrm{E}-02$ \\
\hline 1641 & CR338935 & 5.67 & $9.26 \mathrm{E}-03$ & 1709 & CR352603 & 2.05 & $1.03 E-02$ & 1777 & CR353006 & 5.79 & $6.60 \mathrm{E}-04$ \\
\hline 1642 & CR338937 & 2.86 & $6.02 E-04$ & 1710 & CR352604 & 2.07 & $2.69 \mathrm{E}-03$ & 1778 & CR353008 & 4.63 & $5.50 \mathrm{E}-04$ \\
\hline 1643 & CR338945 & 6.85 & $1.09 \mathrm{E}-03$ & 1711 & CR352614 & 3.52 & $7.03 E-05$ & 1779 & CR353009 & 6.38 & $1.14 \mathrm{E}-02$ \\
\hline 1644 & CR338950 & 1.43 & $1.36 \mathrm{E}-01$ & 1712 & CR352628 & 2.11 & $2.12 \mathrm{E}-03$ & 1780 & CR353012 & 3.17 & $3.03 E-04$ \\
\hline 1645 & CR338951 & 3.07 & $1.39 \mathrm{E}-02$ & 1713 & CR352631 & 3.96 & $8.15 \mathrm{E}-03$ & 1781 & CR353018 & 6.08 & $3.76 E-03$ \\
\hline 1646 & CR338952 & 6.28 & $3.69 \mathrm{E}-04$ & 1714 & CR352644 & 4.64 & $7.35 E-03$ & 1782 & CR353019 & 1.24 & $4.42 \mathrm{E}-03$ \\
\hline 1647 & CR338961 & 1.79 & $4.72 \mathrm{E}-03$ & 1715 & CR352646 & 2.13 & $3.93 \mathrm{E}-03$ & 1783 & CR353020 & 1.87 & $4.20 \mathrm{E}-03$ \\
\hline 1648 & CR338962 & 1.89 & $2.15 \mathrm{E}-02$ & 1716 & CR352649 & 1.24 & $1.22 \mathrm{E}-01$ & 1784 & CR353034 & 4.97 & $2.02 E-03$ \\
\hline 1649 & CR338963 & 4.29 & $2.02 \mathrm{E}-03$ & 1717 & CR352652 & 5.47 & $8.32 \mathrm{E}-04$ & 1785 & CR353037 & 5.78 & 7.44E-04 \\
\hline 1650 & CR338964 & 4.39 & $1.49 \mathrm{E}-03$ & 1718 & CR352669 & 1.52 & $3.10 \mathrm{E}-02$ & 1786 & CR353038 & 4.83 & $1.18 \mathrm{E}-02$ \\
\hline 1651 & CR338967 & 2.91 & $3.47 \mathrm{E}-02$ & 1719 & CR352672 & 3.02 & $3.73 \mathrm{E}-03$ & 1787 & CR353040 & 1.49 & $3.08 \mathrm{E}-02$ \\
\hline 1652 & CR338969 & 5.23 & $5.85 \mathrm{E}-03$ & 1720 & CR352674 & 4.13 & $8.54 \mathrm{E}-03$ & 1788 & CR353059 & 4.44 & 4.39E- 03 \\
\hline 1653 & CR338974 & 2.72 & $2.43 \mathrm{E}-02$ & 1721 & CR352675 & 4.86 & $2.81 \mathrm{E}-03$ & 1789 & CR353073 & 3.41 & 7.66E-03 \\
\hline 1654 & CR338977 & 3.05 & $2.48 \mathrm{E}-03$ & 1722 & CR352676 & 4.40 & $1.17 \mathrm{E}-03$ & 1790 & CR353094 & 2.98 & 4.85E-04 \\
\hline 1655 & CR338988 & 1.55 & $8.83 \mathrm{E}-04$ & 1723 & CR352681 & 5.25 & $3.46 \mathrm{E}-03$ & 1791 & CR353095 & 2.28 & $2.19 \mathrm{E}-01$ \\
\hline 1656 & CR338999 & 6.01 & $7.92 E-04$ & 1724 & CR352684 & 3.05 & 3.55E-02 & 1792 & CR353096 & 2.66 & $6.66 \mathrm{E}-03$ \\
\hline 1657 & CR339002 & 4.13 & $9.36 \mathrm{E}-04$ & 1725 & CR352696 & 4.80 & $6.79 \mathrm{E}-03$ & 1793 & CR353097 & 6.09 & $1.93 \mathrm{E}-03$ \\
\hline 1658 & CR339005 & 2.88 & $3.98 \mathrm{E}-03$ & 1726 & CR352702 & 2.81 & $3.11 \mathrm{E}-03$ & 1794 & CR353105 & 2.42 & $1.90 \mathrm{E}-03$ \\
\hline 1659 & CR339007 & 5.00 & $5.80 \mathrm{E}-05$ & 1727 & CR352703 & 4.40 & $1.95 \mathrm{E}-03$ & 1795 & CR353113 & 1.83 & $9.21 \mathrm{E}-03$ \\
\hline 1660 & CR339009 & 3.53 & $2.76 \mathrm{E}-03$ & 1728 & CR352706 & 1.23 & $1.01 \mathrm{E}-02$ & 1796 & CR353123 & 5.29 & 5.03E- 03 \\
\hline 1661 & CR339011 & 5.23 & $1.19 \mathrm{E}-02$ & 1729 & CR352711 & 3.77 & $1.18 \mathrm{E}-02$ & 1797 & CR353138 & 5.48 & $8.65 \mathrm{E}-03$ \\
\hline 1662 & CR339013 & 1.34 & $2.50 \mathrm{E}-02$ & 1730 & CR352718 & 3.93 & $2.03 \mathrm{E}-03$ & 1798 & CR353140 & 6.04 & $5.70 E-03$ \\
\hline 1663 & CR339018 & 2.22 & $1.26 \mathrm{E}-02$ & 1731 & CR352721 & 1.43 & $1.57 \mathrm{E}-02$ & 1799 & CR353142 & 2.04 & $7.55 E-03$ \\
\hline 1664 & CR339021 & 1.88 & $2.44 \mathrm{E}-03$ & 1732 & CR352734 & 3.91 & $1.62 \mathrm{E}-02$ & 1800 & CR353150 & 1.58 & $1.08 \mathrm{E}-01$ \\
\hline 1665 & CR339023 & 5.67 & $1.12 \mathrm{E}-03$ & 1733 & CR352736 & 2.54 & $1.44 \mathrm{E}-02$ & 1801 & CR353156 & 4.41 & $4.59 \mathrm{E}-03$ \\
\hline 1666 & CR339026 & 3.61 & $6.12 E-04$ & 1734 & CR352756 & 1.43 & $3.18 \mathrm{E}-02$ & 1802 & CR353163 & 6.20 & $8.25 \mathrm{E}-04$ \\
\hline 1667 & CR339027 & 3.08 & $2.27 \mathrm{E}-02$ & 1735 & CR352773 & 2.19 & $2.21 \mathrm{E}-02$ & 1803 & CR353167 & 5.63 & $1.75 E-02$ \\
\hline 1668 & CR339028 & 6.99 & $2.23 \mathrm{E}-03$ & 1736 & CR352774 & 3.76 & $1.42 \mathrm{E}-02$ & 1804 & CR353174 & 2.20 & 7.88E-03 \\
\hline 1669 & CR339029 & 2.50 & $5.44 \mathrm{E}-04$ & 1737 & CR352791 & 1.84 & $3.53 \mathrm{E}-03$ & 1805 & CR353175 & 2.06 & $7.17 E-03$ \\
\hline 1670 & CR339031 & 6.37 & $3.18 \mathrm{E}-05$ & 1738 & CR352792 & 3.10 & 4. $45 \mathrm{E}-03$ & 1806 & CR353176 & 4.04 & $1.89 \mathrm{E}-03$ \\
\hline 1671 & CR339032 & 3.45 & 7.77E-04 & 1739 & CR352795 & 5.79 & $7.55 \mathrm{E}-04$ & 1807 & CR353183 & 1.95 & 3.37E-03 \\
\hline 1672 & CR352343 & 5.53 & 5.07E-04 & 1740 & CR352804 & 6.21 & $9.45 \mathrm{E}-05$ & 1808 & CR353191 & 3.82 & $1.15 \mathrm{E}-03$ \\
\hline 1673 & CR352344 & 4.44 & $2.40 \mathrm{E}-02$ & 1741 & CR352809 & 5.15 & $9.42 \mathrm{E}-03$ & 1809 & CR353199 & 2.17 & $1.42 \mathrm{E}-02$ \\
\hline 1674 & CR352350 & 5.53 & $6.83 \mathrm{E}-04$ & 1742 & CR352825 & 4.27 & $3.46 \mathrm{E}-03$ & 1810 & CR353201 & 4.63 & $1.08 \mathrm{E}-02$ \\
\hline 1675 & CR352354 & 3.39 & $1.07 \mathrm{E}-02$ & 1743 & CR352836 & 2.49 & $2.09 \mathrm{E}-02$ & 1811 & CR353204 & 2.91 & $1.31 \mathrm{E}-02$ \\
\hline 1676 & CR352355 & 2.18 & $3.32 \mathrm{E}-04$ & 1744 & CR352838 & 1.28 & $3.33 \mathrm{E}-02$ & 1812 & CR353205 & 2.91 & $1.15 \mathrm{E}-02$ \\
\hline 1677 & CR352365 & 2.14 & $5.97 \mathrm{E}-02$ & 1745 & CR352839 & 4.60 & $5.23 \mathrm{E}-03$ & 1813 & CR353210 & 5.59 & $3.76 \mathrm{E}-03$ \\
\hline 1678 & CR352405 & 3.67 & $9.06 \mathrm{E}-03$ & 1746 & CR352840 & 5.89 & 3.06E-05 & 1814 & CR353220 & 2.67 & $6.97 E-03$ \\
\hline 1679 & CR352424 & 1.62 & $4.54 \mathrm{E}-03$ & 1747 & CR352841 & 1.56 & $3.13 \mathrm{E}-03$ & 1815 & CR353226 & 1.68 & $1.97 \mathrm{E}-02$ \\
\hline 1680 & CR352428 & 4.37 & $3.34 \mathrm{E}-02$ & 1748 & CR352858 & 3.56 & $5.79 \mathrm{E}-03$ & 1816 & CR353227 & 2.31 & $1.74 E-02$ \\
\hline 1681 & CR352430 & 3.20 & 7.15E-03 & 1749 & CR352859 & 1.90 & $2.45 \mathrm{E}-02$ & 1817 & CR353240 & 4.00 & $2.18 \mathrm{E}-03$ \\
\hline 1682 & CR352431 & 1.29 & $7.48 \mathrm{E}-04$ & 1750 & CR352865 & 5.79 & $3.65 \mathrm{E}-03$ & 1818 & CR353241 & 2.36 & 9.76E-03 \\
\hline 1683 & CR352436 & 1.48 & $5.39 \mathrm{E}-02$ & 1751 & CR352867 & 5.18 & $1.71 \mathrm{E}-03$ & 1819 & CR353243 & 2.16 & $2.58 \mathrm{E}-03$ \\
\hline 1684 & CR352442 & 2.58 & $1.35 \mathrm{E}-02$ & 1752 & CR352868 & 3.76 & $2.71 \mathrm{E}-03$ & 1820 & CR353250 & 2.89 & $1.90 \mathrm{E}-02$ \\
\hline 1685 & CR352443 & 4.44 & $5.95 \mathrm{E}-03$ & 1753 & CR352869 & 3.90 & $6.83 \mathrm{E}-03$ & 1821 & CR353255 & 2.57 & $5.96 \mathrm{E}-03$ \\
\hline 1686 & CR352444 & 2.16 & $1.98 \mathrm{E}-02$ & 1754 & CR352871 & 4.72 & $1.48 \mathrm{E}-03$ & 1822 & CR353259 & 3.74 & $1.04 E-03$ \\
\hline 1687 & CR352451 & 2.42 & $4.29 \mathrm{E}-03$ & 1755 & CR352872 & 2.97 & $8.48 \mathrm{E}-04$ & 1823 & CR353261 & 1.77 & $3.78 \mathrm{E}-03$ \\
\hline 1688 & CR352455 & 4.34 & $2.50 \mathrm{E}-02$ & 1756 & CR352875 & 2.00 & $7.89 \mathrm{E}-03$ & 1824 & CR353267 & 3.56 & $2.06 \mathrm{E}-02$ \\
\hline 1689 & CR352458 & 3.64 & $2.64 \mathrm{E}-03$ & 1757 & CR352879 & 1.20 & $5.60 \mathrm{E}-03$ & 1825 & CR353270 & 2.33 & 4.53E-02 \\
\hline 1690 & CR352464 & 2.02 & $7.83 \mathrm{E}-03$ & 1758 & CR352881 & 5.00 & $1.09 \mathrm{E}-03$ & 1826 & CR353274 & 4.91 & $2.73 E-04$ \\
\hline 1691 & CR352466 & 2.72 & $5.15 \mathrm{E}-02$ & 1759 & CR352904 & 1.78 & $3.94 \mathrm{E}-02$ & 1827 & CR353276 & 2.64 & $4.13 \mathrm{E}-03$ \\
\hline 1692 & CR352468 & 4.70 & $4.80 E-04$ & 1760 & CR352908 & 5.06 & $2.24 \mathrm{E}-03$ & 1828 & CR353278 & 1.72 & $8.18 \mathrm{E}-03$ \\
\hline 1693 & CR352469 & 1.98 & $2.40 \mathrm{E}-02$ & 1761 & CR352930 & 1.19 & $1.66 \mathrm{E}-03$ & 1829 & CR353279 & 6.06 & $2.17 E-03$ \\
\hline 1694 & CR352472 & 1.98 & $1.10 \mathrm{E}-02$ & 1762 & CR352936 & 2.55 & $1.72 \mathrm{E}-01$ & 1830 & CR353282 & 4.46 & $5.61 \mathrm{E}-03$ \\
\hline 1695 & CR352475 & 5.85 & $1.53 \mathrm{E}-02$ & 1763 & CR352940 & 1.82 & $1.57 \mathrm{E}-02$ & 1831 & CR353288 & 2.81 & 6.44E-04 \\
\hline 1696 & CR352479 & 2.97 & $2.09 \mathrm{E}-02$ & 1764 & CR352948 & 2.64 & $2.47 \mathrm{E}-02$ & 1832 & CR353289 & 2.70 & 1.73E-02 \\
\hline 1697 & CR352498 & 5.48 & $2.80 \mathrm{E}-05$ & 1765 & CR352949 & 4.08 & $2.59 \mathrm{E}-03$ & 1833 & CR353292 & 2.84 & $1.78 \mathrm{E}-03$ \\
\hline 1698 & CR352527 & 2.33 & $2.50 \mathrm{E}-02$ & 1766 & CR352952 & 3.03 & $8.95 \mathrm{E}-04$ & 1834 & CR353302 & 4.85 & 7.48E-03 \\
\hline 1699 & CR352535 & 3.04 & $1.10 \mathrm{E}-02$ & 1767 & CR352964 & 2.65 & $3.89 \mathrm{E}-03$ & 1835 & CR353304 & 2.74 & $1.29 \mathrm{E}-04$ \\
\hline
\end{tabular}




\begin{tabular}{|c|c|c|c|c|c|c|c|c|c|c|c|}
\hline 1836 & CR353316 & 5.04 & $2.82 \mathrm{E}-03$ & 1904 & CR353760 & 7.03 & $2.87 \mathrm{E}-04$ & 1972 & CR354216 & 2.07 & $9.12 E-03$ \\
\hline 1837 & CR353319 & 1.75 & $3.37 \mathrm{E}-02$ & 1905 & CR353764 & 6.55 & $2.93 \mathrm{E}-03$ & 1973 & CR354217 & 6.08 & $9.71 E-04$ \\
\hline 1838 & CR353323 & 2.82 & $8.48 \mathrm{E}-03$ & 1906 & CR353771 & 3.08 & $6.29 \mathrm{E}-02$ & 1974 & CR354220 & 1.59 & $1.25 \mathrm{E}-02$ \\
\hline 1839 & CR353341 & 6.12 & $6.53 \mathrm{E}-04$ & 1907 & CR353773 & 3.24 & $3.41 \mathrm{E}-03$ & 1975 & CR354224 & 2.83 & $8.46 \mathrm{E}-03$ \\
\hline 1840 & CR353347 & 1.48 & $1.39 \mathrm{E}-03$ & 1908 & CR353795 & 3.64 & $1.29 \mathrm{E}-02$ & 1976 & CR354227 & 2.67 & $8.68 \mathrm{E}-04$ \\
\hline 1841 & CR353355 & 4.25 & $5.20 \mathrm{E}-03$ & 1909 & CR353796 & 4.90 & 7.65E-03 & 1977 & CR354233 & 6.29 & $1.30 \mathrm{E}-02$ \\
\hline 1842 & CR353358 & 2.86 & $1.44 \mathrm{E}-03$ & 1910 & CR353801 & 6.14 & $1.82 \mathrm{E}-03$ & 1978 & CR354238 & 1.36 & $1.62 \mathrm{E}-03$ \\
\hline 1843 & CR353361 & 3.97 & $4.75 \mathrm{E}-03$ & 1911 & CR353808 & 2.21 & $2.03 \mathrm{E}-03$ & 1979 & CR354243 & 4.05 & $1.48 \mathrm{E}-03$ \\
\hline 1844 & CR353374 & 1.80 & $5.64 \mathrm{E}-03$ & 1912 & CR353815 & 4.16 & $2.19 \mathrm{E}-03$ & 1980 & CR354254 & 1.85 & $2.10 \mathrm{E}-03$ \\
\hline 1845 & CR353391 & 4.63 & $3.62 \mathrm{E}-03$ & 1913 & CR353816 & 1.76 & $2.40 \mathrm{E}-02$ & 1981 & CR354261 & 2.24 & $8.44 \mathrm{E}-04$ \\
\hline 1846 & CR353392 & 3.64 & $5.44 \mathrm{E}-03$ & 1914 & CR353818 & 5.02 & $7.49 \mathrm{E}-03$ & 1982 & CR354264 & 3.80 & $1.87 \mathrm{E}-04$ \\
\hline 1847 & CR353394 & 1.33 & 3.05E-03 & 1915 & CR353837 & 6.23 & $2.01 \mathrm{E}-04$ & 1983 & CR354267 & 2.51 & $7.81 \mathrm{E}-02$ \\
\hline 1848 & CR353395 & 2.20 & $9.88 \mathrm{E}-04$ & 1916 & CR353839 & 5.90 & $3.97 \mathrm{E}-03$ & 1984 & CR354270 & 2.14 & $2.39 E-03$ \\
\hline 1849 & CR353396 & 1.51 & $2.47 \mathrm{E}-03$ & 1917 & CR353844 & 7.16 & $1.14 \mathrm{E}-03$ & 1985 & CR354277 & 4.30 & 4.66E-03 \\
\hline 1850 & CR353399 & 5.59 & $2.93 \mathrm{E}-04$ & 1918 & CR353849 & 3.16 & $1.35 \mathrm{E}-02$ & 1986 & CR354282 & 4.87 & $1.79 \mathrm{E}-03$ \\
\hline 1851 & CR353402 & 2.09 & $1.29 \mathrm{E}-03$ & 1919 & CR353851 & 2.68 & $1.45 \mathrm{E}-02$ & 1987 & CR354290 & 3.84 & $4.17 \mathrm{E}-03$ \\
\hline 1852 & CR353405 & 1.41 & $6.30 \mathrm{E}-04$ & 1920 & CR353852 & 3.66 & $1.29 \mathrm{E}-03$ & 1988 & CR354302 & 5.02 & $2.15 \mathrm{E}-02$ \\
\hline 1853 & CR353409 & 1.85 & $4.95 \mathrm{E}-02$ & 1921 & CR353860 & 3.94 & $2.16 \mathrm{E}-04$ & 1989 & CR354304 & 4.51 & $4.06 \mathrm{E}-03$ \\
\hline 1854 & CR353431 & 1.49 & $8.35 \mathrm{E}-03$ & 1922 & CR353861 & 4.47 & $5.25 \mathrm{E}-04$ & 1990 & CR354344 & 4.90 & $1.45 \mathrm{E}-03$ \\
\hline 1855 & CR353432 & 5.19 & $1.56 \mathrm{E}-02$ & 1923 & CR353864 & 1.86 & $5.66 \mathrm{E}-02$ & 1991 & CR354356 & 4.26 & $3.17 E-03$ \\
\hline 1856 & CR353433 & 1.65 & $4.31 \mathrm{E}-02$ & 1924 & CR353868 & 1.77 & $1.80 \mathrm{E}-02$ & 1992 & CR382424 & 6.08 & $1.75 E-03$ \\
\hline 1857 & CR353451 & 5.23 & $6.41 \mathrm{E}-03$ & 1925 & CR353871 & 1.75 & $2.31 \mathrm{E}-02$ & 1993 & CR385094 & 2.98 & $5.22 \mathrm{E}-03$ \\
\hline 1858 & CR353455 & 3.59 & 4.05E-04 & 1926 & CR353876 & 5.00 & $4.18 \mathrm{E}-03$ & 1994 & CR385097 & 1.69 & $3.23 \mathrm{E}-03$ \\
\hline 1859 & CR353462 & 3.96 & $1.29 \mathrm{E}-03$ & 1927 & CR353882 & 1.25 & $9.05 \mathrm{E}-03$ & 1995 & CR385099 & 3.97 & $4.53 \mathrm{E}-04$ \\
\hline 1860 & CR353463 & 2.25 & $1.78 \mathrm{E}-02$ & 1928 & CR353883 & 5.56 & $1.00 \mathrm{E}-02$ & 1996 & CR385101 & 3.64 & $2.51 E-04$ \\
\hline 1861 & CR353467 & 2.97 & $1.01 \mathrm{E}-03$ & 1929 & CR353885 & 3.38 & $8.45 \mathrm{E}-03$ & 1997 & CR385107 & 2.92 & $2.10 \mathrm{E}-04$ \\
\hline 1862 & CR353469 & 1.86 & $5.95 \mathrm{E}-02$ & 1930 & CR353887 & 5.65 & $1.59 \mathrm{E}-03$ & 1998 & CR385114 & 5.16 & $3.71 \mathrm{E}-04$ \\
\hline 1863 & CR353472 & 4.65 & $1.49 \mathrm{E}-03$ & 1931 & CR353899 & 3.13 & $1.46 \mathrm{E}-03$ & 1999 & CR385128 & 4.36 & $3.99 \mathrm{E}-03$ \\
\hline 1864 & CR353474 & 2.95 & 4.62E-02 & 1932 & CR353908 & 2.81 & $1.89 \mathrm{E}-02$ & 2000 & CR385134 & 1.36 & $7.41 \mathrm{E}-02$ \\
\hline 1865 & CR353477 & 2.33 & $1.79 \mathrm{E}-02$ & 1933 & CR353914 & 3.12 & $1.93 \mathrm{E}-04$ & 2001 & CR385140 & 2.88 & $3.85 \mathrm{E}-03$ \\
\hline 1866 & CR353521 & 2.50 & $4.12 \mathrm{E}-03$ & 1934 & CR353929 & 2.90 & $3.62 \mathrm{E}-02$ & 2002 & CR385163 & 3.88 & $3.70 \mathrm{E}-02$ \\
\hline 1867 & CR353532 & 1.56 & $5.39 \mathrm{E}-02$ & 1935 & CR353933 & 3.98 & $6.64 \mathrm{E}-03$ & 2003 & CR385167 & 1.42 & 2.65E-02 \\
\hline 1868 & CR353570 & 1.58 & 7.73E-03 & 1936 & CR353941 & 5.62 & $3.13 \mathrm{E}-03$ & 2004 & CR385172 & 5.84 & $3.12 \mathrm{E}-04$ \\
\hline 1869 & CR353571 & 2.91 & $1.40 \mathrm{E}-02$ & 1937 & CR353946 & 1.39 & $8.50 \mathrm{E}-03$ & 2005 & CR385174 & 3.67 & $4.51 \mathrm{E}-03$ \\
\hline 1870 & CR353593 & 7.08 & $8.34 \mathrm{E}-06$ & 1938 & CR353987 & 3.83 & $3.44 \mathrm{E}-03$ & 2006 & CR385179 & 3.64 & $1.89 \mathrm{E}-02$ \\
\hline 1871 & CR353594 & 2.36 & $3.51 \mathrm{E}-03$ & 1939 & CR354018 & 1.43 & $4.95 \mathrm{E}-02$ & 2007 & CR385181 & 3.12 & $6.33 \mathrm{E}-03$ \\
\hline 1872 & CR353617 & 4.83 & $1.47 \mathrm{E}-02$ & 1940 & CR354020 & 4.77 & $6.88 \mathrm{E}-04$ & 2008 & CR385182 & 4.25 & $4.94 \mathrm{E}-04$ \\
\hline 1873 & CR353618 & 6.07 & $1.58 \mathrm{E}-02$ & 1941 & CR354027 & 5.01 & $3.02 \mathrm{E}-03$ & 2009 & CR385185 & 1.42 & $6.55 \mathrm{E}-02$ \\
\hline 1874 & CR353620 & 1.58 & $1.68 \mathrm{E}-02$ & 1942 & CR354034 & 3.70 & $2.43 \mathrm{E}-02$ & 2010 & CR385192 & 1.29 & 3.17E-02 \\
\hline 1875 & CR353623 & 1.23 & $3.20 \mathrm{E}-02$ & 1943 & CR354036 & 1.96 & $3.56 \mathrm{E}-02$ & 2011 & CR385200 & 5.08 & $5.55 \mathrm{E}-03$ \\
\hline 1876 & CR353626 & 4.93 & $8.86 \mathrm{E}-02$ & 1944 & CR354047 & 2.79 & $5.86 \mathrm{E}-03$ & 2012 & CR385201 & 1.33 & $3.11 \mathrm{E}-02$ \\
\hline 1877 & CR353632 & 3.83 & $1.96 \mathrm{E}-02$ & 1945 & CR354051 & 2.59 & $1.80 \mathrm{E}-03$ & 2013 & CR385203 & 5.83 & $2.48 \mathrm{E}-03$ \\
\hline 1878 & CR353634 & 3.81 & $4.38 \mathrm{E}-04$ & 1946 & CR354052 & 4.03 & 3.32E-04 & 2014 & CR385204 & 3.25 & $2.15 \mathrm{E}-02$ \\
\hline 1879 & CR353635 & 2.77 & $1.48 \mathrm{E}-03$ & 1947 & CR354053 & 1.81 & $2.38 \mathrm{E}-02$ & 2015 & CR385207 & 5.03 & $3.25 \mathrm{E}-02$ \\
\hline 1880 & CR353636 & 2.10 & $9.23 \mathrm{E}-03$ & 1948 & CR354055 & 4.15 & $1.62 \mathrm{E}-03$ & 2016 & CR385212 & 2.77 & $1.14 \mathrm{E}-02$ \\
\hline 1881 & CR353637 & 2.10 & $9.74 \mathrm{E}-04$ & 1949 & CR354059 & 3.44 & $2.58 \mathrm{E}-04$ & 2017 & CR385213 & 1.39 & $3.01 E-02$ \\
\hline 1882 & CR353649 & 2.52 & $3.88 \mathrm{E}-03$ & 1950 & CR354061 & 2.65 & $9.69 \mathrm{E}-03$ & 2018 & CR385214 & 2.54 & $1.94 \mathrm{E}-02$ \\
\hline 1883 & CR353655 & 3.13 & $1.99 \mathrm{E}-02$ & 1951 & CR354065 & 1.89 & $1.08 \mathrm{E}-03$ & 2019 & CR385215 & 2.46 & $1.52 E-02$ \\
\hline 1884 & CR353672 & 1.47 & $3.91 \mathrm{E}-03$ & 1952 & CR354071 & 4.32 & $1.43 \mathrm{E}-04$ & 2020 & CR385219 & 1.61 & $1.16 \mathrm{E}-02$ \\
\hline 1885 & CR353673 & 2.83 & $1.88 \mathrm{E}-03$ & 1953 & CR354085 & 5.53 & $1.11 \mathrm{E}-02$ & 2021 & CR385221 & 1.62 & $5.55 \mathrm{E}-02$ \\
\hline 1886 & CR353674 & 4.05 & $1.58 \mathrm{E}-02$ & 1954 & CR354095 & 1.70 & $3.11 \mathrm{E}-02$ & 2022 & CR385231 & 2.48 & $3.18 \mathrm{E}-04$ \\
\hline 1887 & CR353679 & 1.86 & $5.48 \mathrm{E}-03$ & 1955 & CR354104 & 5.41 & $8.52 \mathrm{E}-03$ & 2023 & CR385234 & 5.78 & $1.45 \mathrm{E}-03$ \\
\hline 1888 & CR353681 & 3.17 & $9.11 \mathrm{E}-03$ & 1956 & CR354113 & 2.09 & $9.30 \mathrm{E}-03$ & 2024 & CR385237 & 3.07 & $1.42 \mathrm{E}-02$ \\
\hline 1889 & CR353682 & 3.13 & $1.64 \mathrm{E}-03$ & 1957 & CR354126 & 3.95 & $8.76 \mathrm{E}-03$ & 2025 & CR385239 & 2.18 & $4.62 \mathrm{E}-03$ \\
\hline 1890 & CR353686 & 3.20 & $6.12 \mathrm{E}-03$ & 1958 & CR354136 & 5.29 & $3.14 \mathrm{E}-03$ & 2026 & CR385242 & 2.85 & $1.41 \mathrm{E}-03$ \\
\hline 1891 & CR353687 & 4.98 & $3.83 \mathrm{E}-03$ & 1959 & CR354142 & 4.32 & $2.31 \mathrm{E}-03$ & 2027 & CR385248 & 4.45 & $5.39 E-04$ \\
\hline 1892 & CR353696 & 2.12 & $7.42 \mathrm{E}-03$ & 1960 & CR354148 & 3.06 & $1.40 \mathrm{E}-02$ & 2028 & CR385249 & 1.72 & $8.51 E-02$ \\
\hline 1893 & CR353697 & 3.37 & $8.49 \mathrm{E}-03$ & 1961 & CR354161 & 6.47 & $1.64 \mathrm{E}-06$ & 2029 & CR385253 & 6.14 & $4.24 E-03$ \\
\hline 1894 & CR353699 & 2.25 & $9.67 \mathrm{E}-04$ & 1962 & CR354165 & 1.81 & $1.03 E-02$ & 2030 & CR385255 & 3.24 & $2.31 E-03$ \\
\hline 1895 & CR353715 & 1.83 & $2.66 \mathrm{E}-02$ & 1963 & CR354166 & 1.86 & $2.44 \mathrm{E}-02$ & 2031 & CR385257 & 1.53 & $4.16 \mathrm{E}-02$ \\
\hline 1896 & CR353729 & 8.74 & 9.59E-04 & 1964 & CR354168 & 4.27 & $5.36 \mathrm{E}-03$ & 2032 & CR385264 & 2.78 & $9.72 \mathrm{E}-03$ \\
\hline 1897 & CR353738 & 3.73 & $3.22 \mathrm{E}-04$ & 1965 & CR354181 & 5.31 & $3.37 \mathrm{E}-04$ & 2033 & CR385270 & 1.93 & $8.01 E-03$ \\
\hline 1898 & CR353741 & 1.38 & $2.81 \mathrm{E}-03$ & 1966 & CR354182 & 1.96 & $1.93 \mathrm{E}-02$ & 2034 & CR385272 & 3.02 & $5.03 E-03$ \\
\hline 1899 & CR353742 & 4.66 & $4.20 \mathrm{E}-03$ & 1967 & CR354194 & 3.15 & $1.96 \mathrm{E}-02$ & 2035 & CR385275 & 5.89 & $6.46 \mathrm{E}-04$ \\
\hline 1900 & CR353747 & 4.80 & $5.21 \mathrm{E}-03$ & 1968 & CR354200 & 1.93 & $2.02 \mathrm{E}-02$ & 2036 & CR385277 & 3.27 & $4.97 \mathrm{E}-03$ \\
\hline 1901 & CR353751 & 1.49 & $2.00 \mathrm{E}-03$ & 1969 & CR354208 & 5.08 & $2.08 \mathrm{E}-03$ & 2037 & CR385281 & 1.58 & $2.70 \mathrm{E}-02$ \\
\hline 1902 & CR353755 & 8.49 & $1.14 \mathrm{E}-03$ & 1970 & CR354211 & 5.39 & $2.05 E-02$ & 2038 & CR385284 & 2.45 & $8.16 \mathrm{E}-03$ \\
\hline 1903 & CR353758 & 5.60 & $7.32 \mathrm{E}-03$ & 1971 & CR354212 & 6.27 & $1.33 \mathrm{E}-04$ & 2039 & CR385291 & 6.77 & $9.31 \mathrm{E}-04$ \\
\hline
\end{tabular}




\begin{tabular}{|c|c|c|c|c|c|c|c|c|c|c|c|}
\hline 2040 & CR385292 & 3.82 & $9.92 \mathrm{E}-05$ & 2108 & CR385678 & 2.12 & $4.35 \mathrm{E}-03$ & 2176 & CR385942 & 2.57 & $1.80 \mathrm{E}-02$ \\
\hline 2041 & CR385294 & 3.64 & $3.26 \mathrm{E}-03$ & 2109 & CR385679 & 3.70 & $7.42 \mathrm{E}-03$ & 2177 & CR385949 & 2.85 & $1.06 \mathrm{E}-02$ \\
\hline 2042 & CR385295 & 3.38 & $2.96 \mathrm{E}-02$ & 2110 & CR385683 & 1.17 & $1.52 \mathrm{E}-03$ & 2178 & CR385966 & 6.21 & $1.04 \mathrm{E}-03$ \\
\hline 2043 & CR385298 & 1.43 & $2.07 E-02$ & 2111 & CR385686 & 1.21 & $1.93 \mathrm{E}-02$ & 2179 & CR385974 & 2.69 & $1.84 \mathrm{E}-02$ \\
\hline 2044 & CR385301 & 2.23 & $4.41 \mathrm{E}-03$ & 2112 & CR385695 & 2.60 & $2.34 \mathrm{E}-03$ & 2180 & CR385981 & 4.17 & $1.70 \mathrm{E}-02$ \\
\hline 2045 & CR385302 & 2.81 & $2.90 \mathrm{E}-03$ & 2113 & CR385699 & 3.51 & $1.18 \mathrm{E}-02$ & 2181 & CR385988 & 3.46 & 5.37E-04 \\
\hline 2046 & CR385304 & 7.20 & $2.32 \mathrm{E}-03$ & 2114 & CR385704 & 6.37 & $4.70 \mathrm{E}-05$ & 2182 & CR385990 & 1.97 & $2.46 \mathrm{E}-02$ \\
\hline 2047 & CR385309 & 4.88 & $5.86 \mathrm{E}-04$ & 2115 & CR385705 & 2.27 & $6.26 \mathrm{E}-03$ & 2183 & CR385994 & 1.77 & $8.43 E-02$ \\
\hline 2048 & CR385331 & 3.65 & $5.92 \mathrm{E}-03$ & 2116 & CR385709 & 6.14 & $1.03 E-05$ & 2184 & CR386003 & 5.80 & 4.99E- 02 \\
\hline 2049 & CR385348 & 2.16 & $6.24 \mathrm{E}-03$ & 2117 & CR385710 & 1.60 & $9.69 \mathrm{E}-02$ & 2185 & CR386006 & 2.08 & $6.44 \mathrm{E}-03$ \\
\hline 2050 & CR385350 & 2.94 & $5.19 \mathrm{E}-03$ & 2118 & CR385711 & 2.86 & $1.26 \mathrm{E}-03$ & 2186 & CR386008 & 2.42 & $4.83 E-03$ \\
\hline 2051 & CR385379 & 2.72 & $3.66 \mathrm{E}-03$ & 2119 & CR385713 & 6.91 & $7.84 \mathrm{E}-04$ & 2187 & CR386010 & 2.38 & $5.02 E-03$ \\
\hline 2052 & CR385380 & 2.89 & $6.79 \mathrm{E}-03$ & 2120 & CR385714 & 4.12 & $4.53 \mathrm{E}-04$ & 2188 & CR386011 & 6.07 & $4.91 \mathrm{E}-03$ \\
\hline 2053 & CR385385 & 3.75 & $3.86 \mathrm{E}-03$ & 2121 & CR385717 & 3.44 & $1.89 \mathrm{E}-03$ & 2189 & CR386014 & 1.34 & $7.68 \mathrm{E}-03$ \\
\hline 2054 & CR385397 & 4.23 & $2.90 \mathrm{E}-03$ & 2122 & CR385732 & 2.24 & $2.10 \mathrm{E}-02$ & 2190 & CR386017 & 2.39 & $6.32 \mathrm{E}-03$ \\
\hline 2055 & CR385402 & 5.81 & $1.39 \mathrm{E}-03$ & 2123 & CR385738 & 3.25 & $3.97 \mathrm{E}-03$ & 2191 & CR386021 & 3.52 & $1.47 \mathrm{E}-03$ \\
\hline 2056 & CR385405 & 6.67 & $4.77 \mathrm{E}-03$ & 2124 & CR385739 & 2.03 & $1.42 \mathrm{E}-03$ & 2192 & CR386022 & 4.42 & $9.76 \mathrm{E}-03$ \\
\hline 2057 & CR385415 & 5.01 & $1.78 \mathrm{E}-04$ & 2125 & CR385742 & 2.42 & $2.27 \mathrm{E}-02$ & 2193 & CR386023 & 2.71 & $1.21 \mathrm{E}-02$ \\
\hline 2058 & CR385426 & 2.21 & $2.03 \mathrm{E}-03$ & 2126 & CR385744 & 4.26 & 7.33E-04 & 2194 & CR386033 & 3.39 & $1.31 \mathrm{E}-04$ \\
\hline 2059 & CR385428 & 4.71 & $2.83 \mathrm{E}-03$ & 2127 & CR385752 & 4.93 & $1.38 \mathrm{E}-02$ & 2195 & CR386035 & 1.50 & $4.02 \mathrm{E}-03$ \\
\hline 2060 & CR385453 & 2.90 & $1.07 \mathrm{E}-03$ & 2128 & CR385753 & 4.15 & $5.03 \mathrm{E}-03$ & 2196 & CR386036 & 3.77 & 9.57E-03 \\
\hline 2061 & CR385469 & 4.02 & $5.47 \mathrm{E}-03$ & 2129 & CR385755 & 2.19 & 3.07E-03 & 2197 & CR386038 & 4.09 & $2.89 \mathrm{E}-03$ \\
\hline 2062 & CR385472 & 2.85 & 4.64E-02 & 2130 & CR385760 & 3.58 & $8.23 \mathrm{E}-03$ & 2198 & CR386042 & 2.90 & $2.28 \mathrm{E}-03$ \\
\hline 2063 & CR385477 & 3.98 & 3.89E-04 & 2131 & CR385762 & 3.74 & $1.55 \mathrm{E}-02$ & 2199 & CR386046 & 2.08 & 3.13E-02 \\
\hline 2064 & CR385480 & 4.14 & $1.02 \mathrm{E}-02$ & 2132 & CR385764 & 4.81 & $1.99 \mathrm{E}-03$ & 2200 & CR386051 & 1.93 & $1.45 \mathrm{E}-02$ \\
\hline 2065 & CR385481 & 2.68 & $1.23 \mathrm{E}-03$ & 2133 & CR385769 & 9.47 & $3.07 \mathrm{E}-03$ & 2201 & CR386057 & 3.93 & $6.28 \mathrm{E}-03$ \\
\hline 2066 & CR385493 & 4.20 & $7.16 \mathrm{E}-03$ & 2134 & CR385771 & 5.51 & $8.32 \mathrm{E}-04$ & 2202 & CR386059 & 1.66 & $2.27 \mathrm{E}-02$ \\
\hline 2067 & CR385501 & 3.01 & $2.49 \mathrm{E}-03$ & 2135 & CR385774 & 5.70 & $1.68 \mathrm{E}-03$ & 2203 & CR386060 & 1.73 & $7.79 \mathrm{E}-03$ \\
\hline 2068 & CR385507 & 2.09 & $7.40 \mathrm{E}-02$ & 2136 & CR385791 & 4.91 & $4.10 \mathrm{E}-03$ & 2204 & CR386064 & 7.20 & $5.13 \mathrm{E}-03$ \\
\hline 2069 & CR385514 & 5.20 & 4. $45 \mathrm{E}-03$ & 2137 & CR385815 & 1.45 & $2.03 E-02$ & 2205 & CR386065 & 3.11 & $1.18 \mathrm{E}-02$ \\
\hline 2070 & CR385516 & 4.22 & $1.60 \mathrm{E}-02$ & 2138 & CR385820 & 6.36 & $6.71 \mathrm{E}-05$ & 2206 & CR386067 & 1.20 & 7.44E- 03 \\
\hline 2071 & CR385523 & 2.98 & $1.96 \mathrm{E}-02$ & 2139 & CR385833 & 1.58 & $6.09 \mathrm{E}-03$ & 2207 & CR386077 & 4.79 & $7.69 \mathrm{E}-03$ \\
\hline 2072 & CR385527 & 1.35 & $2.83 \mathrm{E}-02$ & 2140 & CR385843 & 3.91 & $6.77 \mathrm{E}-04$ & 2208 & CR386086 & 1.88 & $7.02 E-03$ \\
\hline 2073 & CR385533 & 2.52 & $1.54 \mathrm{E}-03$ & 2141 & CR385846 & 3.50 & $2.76 \mathrm{E}-02$ & 2209 & CR386087 & 2.02 & $1.05 \mathrm{E}-02$ \\
\hline 2074 & CR385535 & 4.54 & $7.27 \mathrm{E}-03$ & 2142 & CR385848 & 4.41 & $6.93 \mathrm{E}-03$ & 2210 & CR386089 & 5.06 & $1.94 \mathrm{E}-02$ \\
\hline 2075 & CR385537 & 3.20 & $5.40 \mathrm{E}-04$ & 2143 & CR385849 & 4.45 & $2.95 \mathrm{E}-03$ & 2211 & CR386092 & 6.33 & $9.91 \mathrm{E}-04$ \\
\hline 2076 & CR385538 & 4.47 & $1.32 \mathrm{E}-03$ & 2144 & CR385851 & 3.27 & $6.65 \mathrm{E}-02$ & 2212 & CR386096 & 2.29 & $2.06 \mathrm{E}-02$ \\
\hline 2077 & CR385542 & 2.45 & $3.15 \mathrm{E}-02$ & 2145 & CR385855 & 3.66 & $3.47 \mathrm{E}-03$ & 2213 & CR386098 & 1.56 & $7.08 \mathrm{E}-03$ \\
\hline 2078 & CR385549 & 4.12 & $1.32 \mathrm{E}-03$ & 2146 & CR385861 & 2.58 & $1.04 \mathrm{E}-02$ & 2214 & CR386099 & 3.02 & $8.92 E-05$ \\
\hline 2079 & CR385555 & 3.79 & $2.85 \mathrm{E}-03$ & 2147 & CR385862 & 4.63 & $1.39 \mathrm{E}-02$ & 2215 & CR386105 & 3.19 & $1.79 \mathrm{E}-02$ \\
\hline 2080 & CR385557 & 2.57 & $4.23 \mathrm{E}-02$ & 2148 & CR385863 & 2.97 & 7.15E-03 & 2216 & CR386111 & 1.68 & $2.24 \mathrm{E}-02$ \\
\hline 2081 & CR385568 & 4.26 & $9.17 \mathrm{E}-03$ & 2149 & CR385865 & 1.32 & $4.31 \mathrm{E}-03$ & 2217 & CR386112 & 1.72 & $3.72 \mathrm{E}-02$ \\
\hline 2082 & CR385584 & 1.60 & $1.33 \mathrm{E}-02$ & 2150 & CR385867 & 5.79 & $1.40 \mathrm{E}-02$ & 2218 & CR386121 & 1.79 & $2.72 \mathrm{E}-03$ \\
\hline 2083 & CR385589 & 1.68 & $1.84 \mathrm{E}-02$ & 2151 & CR385868 & 3.64 & $2.50 \mathrm{E}-03$ & 2219 & CR386136 & 2.67 & $1.19 \mathrm{E}-02$ \\
\hline 2084 & CR385591 & 4.02 & $6.34 \mathrm{E}-03$ & 2152 & CR385872 & 2.48 & $1.54 \mathrm{E}-03$ & 2220 & CR386141 & 5.29 & $2.86 \mathrm{E}-03$ \\
\hline 2085 & CR385592 & 2.76 & $5.67 \mathrm{E}-03$ & 2153 & CR385873 & 2.78 & $3.25 \mathrm{E}-02$ & 2221 & CR386147 & 5.85 & $2.68 \mathrm{E}-04$ \\
\hline 2086 & CR385595 & 2.32 & $3.25 \mathrm{E}-02$ & 2154 & CR385877 & 4.28 & $2.82 \mathrm{E}-03$ & 2222 & CR386148 & 3.75 & $6.76 \mathrm{E}-03$ \\
\hline 2087 & CR385596 & 5.63 & $5.58 \mathrm{E}-03$ & 2155 & CR385878 & 2.74 & $1.29 \mathrm{E}-02$ & 2223 & CR386150 & 2.30 & $3.29 \mathrm{E}-02$ \\
\hline 2088 & CR385598 & 6.50 & $3.35 \mathrm{E}-03$ & 2156 & CR385881 & 1.59 & $4.12 \mathrm{E}-02$ & 2224 & CR386151 & 8.23 & 5.95E-05 \\
\hline 2089 & CR385605 & 7.11 & $3.85 \mathrm{E}-03$ & 2157 & CR385895 & 2.78 & $7.48 \mathrm{E}-03$ & 2225 & CR386169 & 3.71 & $1.87 \mathrm{E}-03$ \\
\hline 2090 & CR385612 & 3.21 & $3.91 \mathrm{E}-03$ & 2158 & CR385896 & 1.66 & $2.73 \mathrm{E}-03$ & 2226 & CR386173 & 1.93 & $2.97 \mathrm{E}-02$ \\
\hline 2091 & CR385613 & 6.24 & $1.57 E-03$ & 2159 & CR385899 & 2.19 & $4.27 \mathrm{E}-02$ & 2227 & CR386181 & 1.03 & 2.42E-03 \\
\hline 2092 & CR385618 & 4.91 & $2.50 \mathrm{E}-02$ & 2160 & CR385902 & 4.75 & $1.17 \mathrm{E}-02$ & 2228 & CR386185 & 3.65 & $1.46 \mathrm{E}-02$ \\
\hline 2093 & CR385620 & 3.33 & $1.44 E-02$ & 2161 & CR385905 & 3.73 & $5.59 \mathrm{E}-04$ & 2229 & CR386200 & 3.18 & 4.15E-03 \\
\hline 2094 & CR385624 & 4.95 & $3.60 \mathrm{E}-03$ & 2162 & CR385908 & 1.13 & $9.74 \mathrm{E}-03$ & 2230 & CR386209 & 2.88 & $5.24 \mathrm{E}-03$ \\
\hline 2095 & CR385628 & 4.91 & $6.80 \mathrm{E}-03$ & 2163 & CR385910 & 3.84 & $1.66 \mathrm{E}-02$ & 2231 & CR386225 & 2.34 & 1.67E-02 \\
\hline 2096 & CR385631 & 3.90 & $8.19 E-04$ & 2164 & CR385913 & 1.97 & $9.88 \mathrm{E}-03$ & 2232 & CR386240 & 3.64 & $2.37 E-03$ \\
\hline 2097 & CR385634 & 5.13 & $2.61 E-03$ & 2165 & CR385914 & 6.87 & $1.21 \mathrm{E}-03$ & 2233 & CR386242 & 3.24 & $2.88 \mathrm{E}-02$ \\
\hline 2098 & CR385638 & 2.10 & $7.66 \mathrm{E}-04$ & 2166 & CR385920 & 2.24 & $1.25 \mathrm{E}-03$ & 2234 & CR386243 & 4.63 & $1.49 \mathrm{E}-03$ \\
\hline 2099 & CR385641 & 2.85 & $6.84 \mathrm{E}-03$ & 2167 & CR385924 & 1.66 & $1.05 \mathrm{E}-01$ & 2235 & CR386244 & 2.38 & 2.09E-02 \\
\hline 2100 & CR385650 & 4.39 & $4.15 \mathrm{E}-04$ & 2168 & CR385928 & 4.68 & $3.11 \mathrm{E}-03$ & 2236 & CR386308 & 2.66 & $2.77 E-03$ \\
\hline 2101 & CR385652 & 1.97 & $3.86 \mathrm{E}-03$ & 2169 & CR385929 & 2.01 & $2.52 \mathrm{E}-03$ & 2237 & CR386324 & 6.13 & $1.36 \mathrm{E}-05$ \\
\hline 2102 & CR385653 & 5.45 & $1.90 \mathrm{E}-06$ & 2170 & CR385930 & 3.67 & $2.22 \mathrm{E}-03$ & 2238 & CR386336 & 3.47 & $1.51 \mathrm{E}-04$ \\
\hline 2103 & CR385655 & 1.65 & $4.90 \mathrm{E}-02$ & 2171 & CR385934 & 6.04 & $2.17 \mathrm{E}-03$ & 2239 & CR386340 & 4.55 & $6.97 E-03$ \\
\hline 2104 & CR385658 & 1.97 & $3.81 \mathrm{E}-01$ & 2172 & CR385935 & 1.23 & $3.44 \mathrm{E}-02$ & 2240 & CR386342 & 4.37 & $5.67 E-03$ \\
\hline 2105 & CR385659 & 1.70 & $5.82 \mathrm{E}-03$ & 2173 & CR385936 & 4.75 & $3.08 \mathrm{E}-02$ & 2241 & CR386345 & 2.58 & $7.67 \mathrm{E}-03$ \\
\hline 2106 & CR385668 & 1.47 & $4.91 \mathrm{E}-02$ & 2174 & CR385938 & 5.67 & $3.80 \mathrm{E}-03$ & 2242 & CR386352 & 2.02 & 4.43E-03 \\
\hline 2107 & CR385672 & 3.72 & $5.09 \mathrm{E}-03$ & 2175 & CR385940 & 4.81 & $6.87 \mathrm{E}-03$ & 2243 & CR386355 & 3.14 & $1.39 \mathrm{E}-03$ \\
\hline
\end{tabular}




\begin{tabular}{|c|c|c|c|c|c|c|c|c|c|c|c|}
\hline 2244 & CR386363 & 2.37 & 7.47E-03 & 2312 & CR386757 & 4.20 & 5.33E-04 & 2380 & CR387042 & 3.17 & $1.13 E-02$ \\
\hline 2245 & CR386365 & 4.53 & $1.37 \mathrm{E}-04$ & 2313 & CR386762 & 3.63 & $2.05 E-03$ & 2381 & CR387045 & 7.62 & $4.39 \mathrm{E}-04$ \\
\hline 2246 & CR386366 & 1.66 & $6.89 \mathrm{E}-02$ & 2314 & CR386765 & 2.23 & $3.91 \mathrm{E}-03$ & 2382 & CR387050 & 4.22 & $2.66 \mathrm{E}-03$ \\
\hline 2247 & CR386372 & 2.48 & $3.06 \mathrm{E}-03$ & 2315 & CR386768 & 1.66 & $5.41 \mathrm{E}-02$ & 2383 & CR387061 & 4.33 & $6.62 \mathrm{E}-03$ \\
\hline 2248 & CR386376 & 4.88 & $3.57 \mathrm{E}-04$ & 2316 & CR386773 & 1.74 & $3.91 \mathrm{E}-02$ & 2384 & CR387073 & 1.15 & $8.79 \mathrm{E}-03$ \\
\hline 2249 & CR386378 & 2.30 & $4.94 \mathrm{E}-03$ & 2317 & CR386774 & 2.53 & 7.37E- 03 & 2385 & CR387086 & 2.37 & $3.85 \mathrm{E}-02$ \\
\hline 2250 & CR386380 & 4.95 & $5.07 \mathrm{E}-04$ & 2318 & CR386783 & 3.18 & $1.34 \mathrm{E}-04$ & 2386 & CR387092 & 1.47 & $3.18 \mathrm{E}-02$ \\
\hline 2251 & CR386388 & 5.18 & $1.57 \mathrm{E}-03$ & 2319 & CR386786 & 3.28 & $9.68 \mathrm{E}-03$ & 2387 & CR387095 & 2.56 & $3.03 E-03$ \\
\hline 2252 & CR386394 & 1.21 & $2.78 \mathrm{E}-02$ & 2320 & CR386789 & 3.34 & $5.58 \mathrm{E}-03$ & 2388 & CR387099 & 3.40 & $6.25 \mathrm{E}-03$ \\
\hline 2253 & CR386405 & 4.92 & $3.88 \mathrm{E}-03$ & 2321 & CR386798 & 4.81 & $6.17 E-05$ & 2389 & CR387106 & 2.47 & $1.77 \mathrm{E}-04$ \\
\hline 2254 & CR386409 & 3.31 & $1.87 \mathrm{E}-03$ & 2322 & CR386801 & 2.18 & $2.63 E-03$ & 2390 & CR387113 & 2.34 & $1.70 \mathrm{E}-01$ \\
\hline 2255 & CR386413 & 8.17 & $3.01 \mathrm{E}-03$ & 2323 & CR386804 & 3.52 & $1.51 \mathrm{E}-03$ & 2391 & CR387117 & 4.67 & $3.57 \mathrm{E}-03$ \\
\hline 2256 & CR386419 & 3.88 & $2.28 \mathrm{E}-03$ & 2324 & CR386809 & 2.80 & $5.24 \mathrm{E}-03$ & 2392 & CR387119 & 3.38 & $4.76 \mathrm{E}-03$ \\
\hline 2257 & CR386420 & 4.12 & $3.37 \mathrm{E}-03$ & 2325 & CR386820 & 4.04 & $2.09 \mathrm{E}-03$ & 2393 & CR387120 & 3.42 & $5.64 \mathrm{E}-04$ \\
\hline 2258 & CR386421 & 2.55 & $2.20 \mathrm{E}-02$ & 2326 & CR386823 & 4.68 & $2.93 E-03$ & 2394 & CR387121 & 1.90 & $4.05 E-02$ \\
\hline 2259 & CR386424 & 1.95 & 4.14E-03 & 2327 & CR386824 & 1.06 & $3.29 \mathrm{E}-02$ & 2395 & CR387124 & 2.84 & $2.77 \mathrm{E}-02$ \\
\hline 2260 & CR386426 & 2.46 & $6.79 \mathrm{E}-03$ & 2328 & CR386827 & 5.03 & $1.49 \mathrm{E}-02$ & 2396 & CR387133 & 2.44 & $1.15 \mathrm{E}-02$ \\
\hline 2261 & CR386428 & 2.15 & $1.86 \mathrm{E}-05$ & 2329 & CR386835 & 2.72 & 1.19E-02 & 2397 & CR387137 & 2.03 & $3.65 \mathrm{E}-03$ \\
\hline 2262 & CR386429 & 2.83 & 3.24E-02 & 2330 & CR386841 & 3.34 & $1.51 \mathrm{E}-02$ & 2398 & CR387142 & 6.44 & $8.82 \mathrm{E}-04$ \\
\hline 2263 & CR386433 & 1.31 & $6.99 \mathrm{E}-03$ & 2331 & CR386846 & 2.14 & $1.66 \mathrm{E}-03$ & 2399 & CR387152 & 1.40 & $3.55 \mathrm{E}-02$ \\
\hline 2264 & CR386435 & 2.43 & $6.19 \mathrm{E}-03$ & 2332 & CR386848 & 4.10 & $1.03 \mathrm{E}-02$ & 2400 & CR387153 & 3.10 & $1.21 \mathrm{E}-03$ \\
\hline 2265 & CR386437 & 4.58 & $1.08 \mathrm{E}-02$ & 2333 & CR386851 & 3.03 & $1.18 \mathrm{E}-03$ & 2401 & CR387155 & 1.95 & 2.12E-02 \\
\hline 2266 & CR386442 & 7.09 & $2.87 \mathrm{E}-04$ & 2334 & CR386852 & 2.12 & $6.08 \mathrm{E}-03$ & 2402 & CR387162 & 1.89 & $2.69 \mathrm{E}-03$ \\
\hline 2267 & CR386443 & 6.95 & $6.24 \mathrm{E}-03$ & 2335 & CR386856 & 4.17 & $6.79 \mathrm{E}-04$ & 2403 & CR387168 & 6.31 & $1.96 \mathrm{E}-02$ \\
\hline 2268 & CR386444 & 3.44 & $2.05 E-04$ & 2336 & CR386857 & 2.85 & 1.60E-03 & 2404 & CR387169 & 1.83 & $2.75 \mathrm{E}-02$ \\
\hline 2269 & CR386455 & 4.68 & $7.21 E-04$ & 2337 & CR386871 & 2.06 & $6.89 \mathrm{E}-02$ & 2405 & CR387178 & 2.85 & $1.06 \mathrm{E}-04$ \\
\hline 2270 & CR386460 & 3.06 & $6.30 \mathrm{E}-03$ & 2338 & CR386873 & 2.79 & $2.81 \mathrm{E}-03$ & 2406 & CR387193 & 8.55 & $8.91 \mathrm{E}-04$ \\
\hline 2271 & CR386464 & 4.19 & $1.39 \mathrm{E}-03$ & 2339 & CR386876 & 5.64 & $4.17 E-04$ & 2407 & CR387196 & 5.66 & $7.46 \mathrm{E}-03$ \\
\hline 2272 & CR386471 & 3.72 & $2.19 \mathrm{E}-03$ & 2340 & CR386882 & 5.45 & $2.52 \mathrm{E}-03$ & 2408 & CR387197 & 3.15 & $1.12 \mathrm{E}-02$ \\
\hline 2273 & CR386484 & 2.58 & $3.17 \mathrm{E}-03$ & 2341 & CR386884 & 1.23 & $8.78 \mathrm{E}-03$ & 2409 & CR387199 & 3.71 & $1.52 \mathrm{E}-03$ \\
\hline 2274 & CR386485 & 4.88 & $2.26 \mathrm{E}-03$ & 2342 & CR386885 & 2.75 & 7.10E-03 & 2410 & CR387205 & 3.42 & $5.55 \mathrm{E}-03$ \\
\hline 2275 & CR386487 & 4.80 & 3.32E-04 & 2343 & CR386889 & 5.02 & $1.25 \mathrm{E}-04$ & 2411 & CR387210 & 8.93 & 4.07E-03 \\
\hline 2276 & CR386490 & 3.36 & $9.71 \mathrm{E}-03$ & 2344 & CR386890 & 2.48 & $1.50 \mathrm{E}-03$ & 2412 & CR387211 & 2.95 & $1.08 \mathrm{E}-02$ \\
\hline 2277 & CR386491 & 5.37 & $4.35 \mathrm{E}-04$ & 2345 & CR386893 & 3.90 & $1.33 \mathrm{E}-01$ & 2413 & CR387215 & 2.82 & $1.23 \mathrm{E}-02$ \\
\hline 2278 & CR386496 & 2.29 & $7.28 \mathrm{E}-03$ & 2346 & CR386903 & 3.34 & $1.59 \mathrm{E}-03$ & 2414 & CR387220 & 3.19 & $1.90 \mathrm{E}-02$ \\
\hline 2279 & CR386510 & 4.86 & $2.20 \mathrm{E}-03$ & 2347 & CR386911 & 3.39 & $1.30 \mathrm{E}-02$ & 2415 & CR387223 & 5.84 & $4.94 \mathrm{E}-03$ \\
\hline 2280 & CR386516 & 1.93 & $1.54 \mathrm{E}-03$ & 2348 & CR386913 & 2.32 & $9.38 \mathrm{E}-03$ & 2416 & CR387225 & 5.63 & $1.17 \mathrm{E}-03$ \\
\hline 2281 & CR386529 & 4.09 & $5.20 \mathrm{E}-03$ & 2349 & CR386915 & 4.60 & $8.78 \mathrm{E}-03$ & 2417 & CR387226 & 2.94 & $2.18 \mathrm{E}-02$ \\
\hline 2282 & CR386530 & 4.00 & $2.36 \mathrm{E}-03$ & 2350 & CR386926 & 2.29 & $1.74 \mathrm{E}-02$ & 2418 & CR387234 & 1.35 & $1.33 \mathrm{E}-02$ \\
\hline 2283 & CR386546 & 4.00 & $6.92 \mathrm{E}-03$ & 2351 & CR386929 & 3.60 & $5.11 \mathrm{E}-03$ & 2419 & CR387240 & 1.56 & $1.34 \mathrm{E}-02$ \\
\hline 2284 & CR386552 & 2.39 & $1.05 \mathrm{E}-02$ & 2352 & CR386933 & 2.74 & $1.43 E-03$ & 2420 & CR387244 & 1.99 & $6.87 \mathrm{E}-02$ \\
\hline 2285 & CR386555 & 2.24 & $2.08 \mathrm{E}-03$ & 2353 & CR386934 & 3.21 & $1.86 \mathrm{E}-02$ & 2421 & CR387247 & 2.88 & $4.65 \mathrm{E}-03$ \\
\hline 2286 & CR386556 & 2.80 & $1.07 E-02$ & 2354 & CR386935 & 1.62 & $6.95 \mathrm{E}-03$ & 2422 & CR387251 & 1.45 & $1.68 \mathrm{E}-02$ \\
\hline 2287 & CR386557 & 1.77 & $9.37 \mathrm{E}-02$ & 2355 & CR386937 & 2.30 & $7.05 E-03$ & 2423 & CR387265 & 5.89 & $4.19 \mathrm{E}-04$ \\
\hline 2288 & CR386576 & 4.78 & $2.80 \mathrm{E}-03$ & 2356 & CR386947 & 3.26 & $7.69 \mathrm{E}-03$ & 2424 & CR387268 & 6.17 & $7.12 \mathrm{E}-03$ \\
\hline 2289 & CR386585 & 2.52 & $2.38 \mathrm{E}-03$ & 2357 & CR386950 & 1.60 & $2.64 \mathrm{E}-03$ & 2425 & CR387286 & 1.34 & $5.54 \mathrm{E}-03$ \\
\hline 2290 & CR386588 & 1.86 & $1.55 \mathrm{E}-02$ & 2358 & CR386952 & 2.87 & $3.00 E-02$ & 2426 & CR387291 & 5.34 & $4.08 \mathrm{E}-02$ \\
\hline 2291 & CR386604 & 2.43 & $7.66 \mathrm{E}-03$ & 2359 & CR386960 & 2.15 & $1.98 \mathrm{E}-02$ & 2427 & CR387295 & 6.64 & $1.67 \mathrm{E}-03$ \\
\hline 2292 & CR386615 & 2.24 & $8.48 \mathrm{E}-03$ & 2360 & CR386961 & 7.05 & $3.23 E-03$ & 2428 & CR387301 & 3.69 & $1.21 \mathrm{E}-03$ \\
\hline 2293 & CR386638 & 3.00 & $1.90 \mathrm{E}-03$ & 2361 & CR386971 & 4.31 & $8.41 \mathrm{E}-03$ & 2429 & CR387302 & 3.70 & 4.13E-03 \\
\hline 2294 & CR386653 & 6.08 & $1.84 \mathrm{E}-03$ & 2362 & CR386973 & 5.02 & $2.66 \mathrm{E}-03$ & 2430 & CR387304 & 4.20 & $8.35 \mathrm{E}-05$ \\
\hline 2295 & CR386655 & 3.29 & 7.37E-03 & 2363 & CR386975 & 1.34 & $3.51 \mathrm{E}-02$ & 2431 & CR387306 & 4.24 & $3.34 \mathrm{E}-03$ \\
\hline 2296 & CR386660 & 2.31 & $7.91 \mathrm{E}-04$ & 2364 & CR386977 & 3.92 & $6.18 \mathrm{E}-03$ & 2432 & CR387307 & 2.63 & $2.18 \mathrm{E}-03$ \\
\hline 2297 & CR386669 & 1.26 & $2.72 \mathrm{E}-04$ & 2365 & CR386981 & 5.10 & $1.49 \mathrm{E}-03$ & 2433 & CR387308 & 3.53 & $2.82 \mathrm{E}-01$ \\
\hline 2298 & CR386676 & 2.72 & $8.77 \mathrm{E}-03$ & 2366 & CR386984 & 3.88 & $7.28 \mathrm{E}-03$ & 2434 & CR387317 & 3.59 & $1.68 \mathrm{E}-03$ \\
\hline 2299 & CR386680 & 1.51 & $3.79 \mathrm{E}-02$ & 2367 & CR386992 & 5.03 & $2.53 \mathrm{E}-03$ & 2435 & CR387327 & 4.62 & $1.80 \mathrm{E}-03$ \\
\hline 2300 & CR386681 & 1.18 & $1.30 \mathrm{E}-02$ & 2368 & CR386998 & 2.74 & $2.39 \mathrm{E}-02$ & 2436 & CR387328 & 2.78 & $3.71 \mathrm{E}-03$ \\
\hline 2301 & CR386690 & 3.19 & $1.58 \mathrm{E}-03$ & 2369 & CR387008 & 1.09 & $8.59 \mathrm{E}-02$ & 2437 & CR387332 & 3.39 & $5.11 \mathrm{E}-03$ \\
\hline 2302 & CR386695 & 3.33 & $2.53 \mathrm{E}-02$ & 2370 & CR387012 & 2.60 & $1.28 \mathrm{E}-02$ & 2438 & CR387335 & 2.59 & $1.00 \mathrm{E}-03$ \\
\hline 2303 & CR386696 & 6.28 & $9.73 \mathrm{E}-03$ & 2371 & CR387013 & 1.21 & $7.95 E-03$ & 2439 & CR387336 & 3.01 & $1.61 \mathrm{E}-02$ \\
\hline 2304 & CR386702 & 5.20 & $6.54 \mathrm{E}-04$ & 2372 & CR387014 & 3.72 & $1.24 \mathrm{E}-02$ & 2440 & CR387342 & 4.81 & $1.93 \mathrm{E}-03$ \\
\hline 2305 & CR386705 & 2.83 & $1.50 \mathrm{E}-02$ & 2373 & CR387015 & 4.16 & $2.50 \mathrm{E}-03$ & 2441 & CR387344 & 8.09 & $1.01 \mathrm{E}-02$ \\
\hline 2306 & CR386722 & 1.22 & $1.45 \mathrm{E}-02$ & 2374 & CR387017 & 2.76 & $2.01 E-04$ & 2442 & CR387346 & 3.98 & $2.12 \mathrm{E}-03$ \\
\hline 2307 & CR386725 & 2.61 & $2.33 \mathrm{E}-03$ & 2375 & CR387023 & 3.57 & $2.71 \mathrm{E}-03$ & 2443 & CR387347 & 4.59 & $1.49 \mathrm{E}-03$ \\
\hline 2308 & CR386734 & 2.56 & $1.27 \mathrm{E}-03$ & 2376 & CR387025 & 1.62 & $4.12 \mathrm{E}-02$ & 2444 & CR387348 & 2.50 & $5.39 \mathrm{E}-03$ \\
\hline 2309 & CR386747 & 2.87 & 7.14E-03 & 2377 & CR387026 & 4.04 & $1.05 E-02$ & 2445 & CR387353 & 4.45 & $4.35 \mathrm{E}-05$ \\
\hline 2310 & CR386750 & 2.77 & $2.62 \mathrm{E}-02$ & 2378 & CR387035 & 2.25 & $4.92 E-04$ & 2446 & CR387354 & 1.85 & $1.60 \mathrm{E}-01$ \\
\hline 2311 & CR386752 & 4.23 & $4.10 \mathrm{E}-03$ & 2379 & CR387036 & 1.86 & $1.58 \mathrm{E}-02$ & 2447 & CR387355 & 4.45 & $2.64 \mathrm{E}-02$ \\
\hline
\end{tabular}




\begin{tabular}{|c|c|c|c|c|c|c|c|c|c|c|c|}
\hline 2448 & CR387360 & 2.92 & $1.98 \mathrm{E}-03$ & 2516 & CR387727 & 5.33 & $1.30 \mathrm{E}-05$ & 2584 & CR388579 & 2.98 & $8.78 \mathrm{E}-05$ \\
\hline 2449 & CR387365 & 2.62 & $3.87 \mathrm{E}-02$ & 2517 & CR387728 & 3.64 & $7.02 \mathrm{E}-03$ & 2585 & CR388581 & 3.31 & 7.13E-03 \\
\hline 2450 & CR387368 & 3.29 & $9.40 \mathrm{E}-05$ & 2518 & CR387734 & 2.41 & $4.01 \mathrm{E}-03$ & 2586 & CR388582 & 1.55 & $4.66 \mathrm{E}-02$ \\
\hline 2451 & CR387369 & 5.96 & $9.60 \mathrm{E}-04$ & 2519 & CR387735 & 1.55 & $5.11 \mathrm{E}-03$ & 2587 & CR388593 & 5.34 & $2.61 \mathrm{E}-03$ \\
\hline 2452 & CR387377 & 3.63 & $1.13 \mathrm{E}-03$ & 2520 & CR387736 & 2.61 & $7.63 \mathrm{E}-03$ & 2588 & CR388595 & 1.31 & $2.37 \mathrm{E}-02$ \\
\hline 2453 & CR387379 & 6.58 & $1.51 \mathrm{E}-04$ & 2521 & CR387738 & 3.19 & $3.17 \mathrm{E}-03$ & 2589 & CR388597 & 5.80 & $2.16 \mathrm{E}-03$ \\
\hline 2454 & CR387391 & 7.26 & $5.69 \mathrm{E}-04$ & 2522 & CR387747 & 3.94 & $3.43 \mathrm{E}-02$ & 2590 & CR388605 & 2.37 & $6.41 E-02$ \\
\hline 2455 & CR387398 & 5.91 & $6.32 \mathrm{E}-04$ & 2523 & CR387748 & 2.22 & 2.47E-03 & 2591 & CR388614 & 5.46 & 4.57E- 03 \\
\hline 2456 & CR387399 & 2.05 & $6.61 E-03$ & 2524 & CR387755 & 2.83 & $4.01 \mathrm{E}-02$ & 2592 & CR388624 & 5.52 & $4.36 \mathrm{E}-03$ \\
\hline 2457 & CR387403 & 4.19 & $2.23 \mathrm{E}-02$ & 2525 & CR387765 & 2.09 & $1.73 \mathrm{E}-02$ & 2593 & CR388629 & 3.16 & 2.54E-02 \\
\hline 2458 & CR387441 & 2.22 & $2.08 \mathrm{E}-02$ & 2526 & CR387779 & 2.09 & $8.26 \mathrm{E}-05$ & 2594 & CR388631 & 1.88 & $1.88 \mathrm{E}-02$ \\
\hline 2459 & CR387443 & 6.32 & $6.16 \mathrm{E}-03$ & 2527 & CR387781 & 6.49 & $4.64 \mathrm{E}-03$ & 2595 & CR388635 & 4.65 & $6.55 E-04$ \\
\hline 2460 & CR387445 & 2.04 & $2.08 \mathrm{E}-03$ & 2528 & CR387786 & 4.23 & $5.09 \mathrm{E}-03$ & 2596 & CR388640 & 2.26 & $1.28 \mathrm{E}-02$ \\
\hline 2461 & CR387461 & 1.88 & $1.52 \mathrm{E}-03$ & 2529 & CR387787 & 2.38 & $3.22 \mathrm{E}-03$ & 2597 & CR388646 & 3.14 & $1.19 \mathrm{E}-02$ \\
\hline 2462 & CR387463 & 3.16 & $1.39 \mathrm{E}-02$ & 2530 & CR387791 & 3.70 & 7.05E-03 & 2598 & CR388652 & 3.53 & $6.83 E-03$ \\
\hline 2463 & CR387465 & 3.56 & $5.87 \mathrm{E}-04$ & 2531 & CR387796 & 2.39 & $2.51 \mathrm{E}-03$ & 2599 & CR388653 & 2.03 & $2.06 \mathrm{E}-02$ \\
\hline 2464 & CR387469 & 4.08 & $5.80 \mathrm{E}-04$ & 2532 & CR387813 & 1.73 & $1.10 \mathrm{E}-01$ & 2600 & CR388662 & 2.95 & 7.97E-02 \\
\hline 2465 & CR387470 & 1.86 & $1.11 \mathrm{E}-01$ & 2533 & CR387818 & 1.89 & $2.22 \mathrm{E}-02$ & 2601 & CR388664 & 1.41 & $1.96 \mathrm{E}-03$ \\
\hline 2466 & CR387471 & 1.56 & $1.89 \mathrm{E}-02$ & 2534 & CR387819 & 4.79 & $3.85 \mathrm{E}-03$ & 2602 & CR388674 & 4.54 & $1.07 E-03$ \\
\hline 2467 & CR387472 & 2.13 & $1.94 \mathrm{E}-03$ & 2535 & CR387822 & 2.81 & 7.41E-03 & 2603 & CR388682 & 3.25 & 2.20E-03 \\
\hline 2468 & CR387476 & 3.31 & $1.17 \mathrm{E}-02$ & 2536 & CR387840 & 1.62 & $1.48 \mathrm{E}-02$ & 2604 & CR388687 & 2.14 & $1.02 \mathrm{E}-02$ \\
\hline 2469 & CR387477 & 4.38 & $2.21 \mathrm{E}-03$ & 2537 & CR387846 & 2.02 & $3.86 \mathrm{E}-02$ & 2605 & CR388689 & 1.50 & 4.11E-03 \\
\hline 2470 & CR387481 & 4.56 & $3.86 \mathrm{E}-04$ & 2538 & CR387851 & 3.00 & $2.54 \mathrm{E}-02$ & 2606 & CR388692 & 1.29 & $8.11 E-04$ \\
\hline 2471 & CR387483 & 1.34 & $4.92 \mathrm{E}-03$ & 2539 & CR387852 & 4.78 & $3.63 \mathrm{E}-03$ & 2607 & CR388694 & 3.22 & $3.78 \mathrm{E}-03$ \\
\hline 2472 & CR387521 & 1.79 & $4.11 \mathrm{E}-02$ & 2540 & CR387854 & 3.05 & $3.59 \mathrm{E}-03$ & 2608 & CR388698 & 1.19 & $4.76 E-02$ \\
\hline 2473 & CR387525 & 1.97 & $2.90 \mathrm{E}-02$ & 2541 & CR387857 & 2.06 & $6.12 \mathrm{E}-02$ & 2609 & CR388701 & 1.79 & $1.09 \mathrm{E}-03$ \\
\hline 2474 & CR387547 & 1.60 & $5.76 \mathrm{E}-03$ & 2542 & CR387862 & 4.02 & $5.38 \mathrm{E}-02$ & 2610 & CR388709 & 3.32 & $2.01 \mathrm{E}-03$ \\
\hline 2475 & CR387550 & 4.47 & $3.96 \mathrm{E}-03$ & 2543 & CR387864 & 2.67 & $1.21 \mathrm{E}-02$ & 2611 & CR388710 & 3.97 & $1.07 E-02$ \\
\hline 2476 & CR387552 & 2.53 & $5.25 \mathrm{E}-03$ & 2544 & CR387866 & 3.25 & $8.44 \mathrm{E}-04$ & 2612 & CR388711 & 5.87 & $1.57 \mathrm{E}-03$ \\
\hline 2477 & CR387555 & 3.87 & $2.08 \mathrm{E}-03$ & 2545 & CR387869 & 1.39 & $2.94 \mathrm{E}-02$ & 2613 & CR388712 & 3.92 & $2.95 \mathrm{E}-04$ \\
\hline 2478 & CR387564 & 4.44 & $3.21 E-04$ & 2546 & CR387875 & 2.71 & $2.20 \mathrm{E}-02$ & 2614 & CR388714 & 1.62 & 3.52E-02 \\
\hline 2479 & CR387565 & 3.95 & $1.11 \mathrm{E}-03$ & 2547 & CR387887 & 2.19 & $1.02 \mathrm{E}-02$ & 2615 & CR388715 & 2.35 & $2.39 \mathrm{E}-02$ \\
\hline 2480 & CR387568 & 3.14 & $1.59 \mathrm{E}-03$ & 2548 & CR387897 & 1.75 & $1.62 \mathrm{E}-02$ & 2616 & CR388720 & 2.04 & 3.10E-02 \\
\hline 2481 & CR387570 & 5.24 & $1.59 \mathrm{E}-03$ & 2549 & CR387912 & 3.16 & $2.84 \mathrm{E}-04$ & 2617 & CR388725 & 3.82 & $1.31 \mathrm{E}-02$ \\
\hline 2482 & CR387571 & 4.36 & $4.05 \mathrm{E}-03$ & 2550 & CR387913 & 1.66 & $2.95 \mathrm{E}-03$ & 2618 & CR388726 & 1.99 & $6.54 \mathrm{E}-02$ \\
\hline 2483 & CR387573 & 3.73 & $2.87 \mathrm{E}-03$ & 2551 & CR388424 & 2.43 & $7.25 \mathrm{E}-04$ & 2619 & CR388737 & 2.52 & $5.15 E-02$ \\
\hline 2484 & CR387575 & 4.17 & $2.52 \mathrm{E}-03$ & 2552 & CR388430 & 1.69 & $7.23 \mathrm{E}-02$ & 2620 & CR388740 & 1.95 & $1.64 \mathrm{E}-02$ \\
\hline 2485 & CR387577 & 4.25 & $1.19 \mathrm{E}-02$ & 2553 & CR388437 & 3.34 & $2.71 \mathrm{E}-02$ & 2621 & CR388741 & 2.95 & $7.50 \mathrm{E}-03$ \\
\hline 2486 & CR387579 & 4.15 & 7.44E-04 & 2554 & CR388439 & 3.57 & $6.10 \mathrm{E}-04$ & 2622 & CR388742 & 4.13 & $3.73 \mathrm{E}-03$ \\
\hline 2487 & CR387587 & 6.36 & $9.72 \mathrm{E}-05$ & 2555 & CR388442 & 4.09 & $2.96 \mathrm{E}-04$ & 2623 & CR388743 & 3.76 & $2.44 E-03$ \\
\hline 2488 & CR387588 & 3.03 & $2.61 \mathrm{E}-03$ & 2556 & CR388443 & 4.08 & $7.83 \mathrm{E}-04$ & 2624 & CR388744 & 2.31 & 4.56E- 03 \\
\hline 2489 & CR387592 & 3.71 & $3.42 \mathrm{E}-03$ & 2557 & CR388451 & 3.91 & $8.00 \mathrm{E}-04$ & 2625 & CR388752 & 7.45 & $6.28 \mathrm{E}-04$ \\
\hline 2490 & CR387597 & 4.92 & $2.53 \mathrm{E}-04$ & 2558 & CR388452 & 5.83 & $6.69 \mathrm{E}-04$ & 2626 & CR388753 & 2.37 & $6.69 \mathrm{E}-03$ \\
\hline 2491 & CR387599 & 2.79 & $4.56 \mathrm{E}-04$ & 2559 & CR388455 & 3.51 & $1.05 \mathrm{E}-04$ & 2627 & CR388759 & 3.32 & 1.40E-02 \\
\hline 2492 & CR387600 & 3.45 & $3.31 \mathrm{E}-03$ & 2560 & CR388463 & 3.53 & $8.46 \mathrm{E}-04$ & 2628 & CR388765 & 4.15 & $2.09 \mathrm{E}-02$ \\
\hline 2493 & CR387601 & 6.14 & 3.80E-04 & 2561 & CR388474 & 2.99 & $2.78 \mathrm{E}-01$ & 2629 & CR388772 & 1.44 & $2.96 \mathrm{E}-02$ \\
\hline 2494 & CR387603 & 2.26 & $6.41 \mathrm{E}-03$ & 2562 & CR388476 & 3.11 & $1.40 \mathrm{E}-02$ & 2630 & CR388774 & 5.40 & $1.21 \mathrm{E}-03$ \\
\hline 2495 & CR387605 & 4.96 & $3.61 \mathrm{E}-03$ & 2563 & CR388479 & 2.94 & $1.73 \mathrm{E}-04$ & 2631 & CR388784 & 2.32 & $1.81 \mathrm{E}-02$ \\
\hline 2496 & CR387610 & 5.39 & $6.99 \mathrm{E}-04$ & 2564 & CR388482 & 2.14 & $2.09 \mathrm{E}-02$ & 2632 & CR388792 & 3.59 & 3.74E- 03 \\
\hline 2497 & CR387614 & 6.44 & $2.99 \mathrm{E}-03$ & 2565 & CR388490 & 3.89 & $3.40 \mathrm{E}-03$ & 2633 & CR388811 & 1.18 & $1.21 \mathrm{E}-02$ \\
\hline 2498 & CR387626 & 1.86 & $5.59 \mathrm{E}-02$ & 2566 & CR388493 & 3.00 & $1.98 \mathrm{E}-03$ & 2634 & CR388813 & 4.15 & $1.36 \mathrm{E}-03$ \\
\hline 2499 & CR387629 & 3.35 & $6.03 E-03$ & 2567 & CR388494 & 4.82 & 3.22E-03 & 2635 & CR388821 & 4.20 & $1.87 \mathrm{E}-02$ \\
\hline 2500 & CR387631 & 2.67 & $5.09 \mathrm{E}-03$ & 2568 & CR388499 & 5.56 & $3.05 \mathrm{E}-03$ & 2636 & CR388825 & 3.21 & 1.11E-02 \\
\hline 2501 & CR387635 & 1.87 & $3.72 E-02$ & 2569 & CR388506 & 2.14 & $2.04 \mathrm{E}-04$ & 2637 & CR388827 & 1.37 & $1.78 \mathrm{E}-02$ \\
\hline 2502 & CR387636 & 2.70 & $3.18 \mathrm{E}-04$ & 2570 & CR388517 & 3.91 & $3.62 \mathrm{E}-03$ & 2638 & CR388828 & 4.56 & 4.42E-03 \\
\hline 2503 & CR387644 & 2.15 & $9.58 \mathrm{E}-03$ & 2571 & CR388518 & 5.42 & $9.47 \mathrm{E}-03$ & 2639 & CR388841 & 2.34 & $5.72 \mathrm{E}-02$ \\
\hline 2504 & CR387647 & 4.00 & $4.52 \mathrm{E}-03$ & 2572 & CR388521 & 3.61 & $1.95 \mathrm{E}-03$ & 2640 & CR388842 & 1.74 & $1.46 \mathrm{E}-02$ \\
\hline 2505 & CR387648 & 4.49 & $4.27 E-03$ & 2573 & CR388530 & 1.56 & 3.66E-02 & 2641 & CR388844 & 4.46 & $2.07 E-04$ \\
\hline 2506 & CR387653 & 5.89 & $1.48 \mathrm{E}-03$ & 2574 & CR388537 & 1.94 & $2.24 \mathrm{E}-02$ & 2642 & CR388845 & 2.47 & $5.38 \mathrm{E}-03$ \\
\hline 2507 & CR387659 & 2.59 & $2.25 \mathrm{E}-03$ & 2575 & CR388544 & 4.49 & $1.70 \mathrm{E}-02$ & 2643 & CR388853 & 1.52 & 2.23E-01 \\
\hline 2508 & CR387667 & 5.13 & $5.70 \mathrm{E}-04$ & 2576 & CR388546 & 4.79 & $1.26 \mathrm{E}-03$ & 2644 & CR388862 & 1.30 & $1.61 \mathrm{E}-02$ \\
\hline 2509 & CR387671 & 3.89 & $1.81 \mathrm{E}-03$ & 2577 & CR388553 & 3.26 & $1.01 \mathrm{E}-03$ & 2645 & CR388863 & 4.98 & $4.93 \mathrm{E}-03$ \\
\hline 2510 & CR387693 & 5.26 & $6.36 \mathrm{E}-05$ & 2578 & CR388555 & 6.04 & $1.09 \mathrm{E}-04$ & 2646 & CR388867 & 2.81 & $1.07 \mathrm{E}-03$ \\
\hline 2511 & CR387698 & 7.51 & $1.40 \mathrm{E}-03$ & 2579 & CR388561 & 5.13 & $1.96 \mathrm{E}-02$ & 2647 & CR388877 & 3.46 & $1.41 \mathrm{E}-02$ \\
\hline 2512 & CR387711 & 4.27 & $1.87 \mathrm{E}-03$ & 2580 & CR388562 & 3.93 & $7.26 \mathrm{E}-04$ & 2648 & CR388884 & 1.90 & $2.39 \mathrm{E}-02$ \\
\hline 2513 & CR387712 & 4.40 & $5.93 E-03$ & 2581 & CR388570 & 4.99 & $5.58 \mathrm{E}-03$ & 2649 & CR388892 & 2.57 & $4.32 \mathrm{E}-04$ \\
\hline 2514 & CR387720 & 1.95 & $1.19 \mathrm{E}-03$ & 2582 & CR388574 & 6.70 & $2.52 \mathrm{E}-03$ & 2650 & CR388908 & 5.00 & 7.06E-04 \\
\hline 2515 & CR387721 & 2.45 & $1.53 \mathrm{E}-02$ & 2583 & CR388576 & 3.11 & $1.83 \mathrm{E}-05$ & 2651 & CR388914 & 1.27 & $3.11 \mathrm{E}-02$ \\
\hline
\end{tabular}




\begin{tabular}{|c|c|c|c|c|c|c|c|c|c|c|c|}
\hline 2652 & CR388920 & 3.82 & $4.31 \mathrm{E}-03$ & 2720 & CR389250 & 6.90 & $3.04 \mathrm{E}-03$ & 2788 & CR389576 & 3.64 & $1.67 \mathrm{E}-03$ \\
\hline 2653 & CR388922 & 3.53 & $4.22 \mathrm{E}-03$ & 2721 & CR389251 & 1.36 & $2.82 \mathrm{E}-02$ & 2789 & CR389577 & 2.89 & $4.34 \mathrm{E}-03$ \\
\hline 2654 & CR388923 & 3.35 & $3.11 \mathrm{E}-03$ & 2722 & CR389257 & 4.58 & 3.19E-03 & 2790 & CR389580 & 2.93 & $3.59 \mathrm{E}-03$ \\
\hline 2655 & CR388925 & 1.40 & $4.85 \mathrm{E}-04$ & 2723 & CR389259 & 2.74 & $4.07 E-03$ & 2791 & CR389582 & 4.91 & $8.76 \mathrm{E}-04$ \\
\hline 2656 & CR388926 & 1.70 & $1.01 \mathrm{E}-02$ & 2724 & CR389263 & 4.52 & $9.61 \mathrm{E}-03$ & 2792 & CR389587 & 7.36 & $1.90 \mathrm{E}-03$ \\
\hline 2657 & CR388930 & 2.02 & $9.35 \mathrm{E}-02$ & 2725 & CR389284 & 4.80 & $6.94 \mathrm{E}-03$ & 2793 & CR389594 & 3.65 & $4.04 \mathrm{E}-03$ \\
\hline 2658 & CR388933 & 2.55 & $4.79 \mathrm{E}-05$ & 2726 & CR389289 & 3.68 & $5.73 \mathrm{E}-03$ & 2794 & CR389609 & 4.78 & $6.01 E-03$ \\
\hline 2659 & CR388935 & 4.18 & $6.15 \mathrm{E}-03$ & 2727 & CR389310 & 6.15 & $2.11 \mathrm{E}-02$ & 2795 & CR389625 & 2.60 & $5.52 \mathrm{E}-03$ \\
\hline 2660 & CR388946 & 5.36 & $2.41 \mathrm{E}-03$ & 2728 & CR389311 & 1.44 & $3.84 \mathrm{E}-02$ & 2796 & CR389630 & 6.59 & $6.52 \mathrm{E}-04$ \\
\hline 2661 & CR388955 & 2.37 & $2.03 \mathrm{E}-03$ & 2729 & CR389314 & 8.25 & $1.84 \mathrm{E}-03$ & 2797 & CR389633 & 2.94 & $8.57 \mathrm{E}-03$ \\
\hline 2662 & CR388956 & 4.13 & 3.16E-03 & 2730 & CR389316 & 3.55 & $5.85 \mathrm{E}-02$ & 2798 & CR389637 & 1.80 & $2.32 \mathrm{E}-02$ \\
\hline 2663 & CR388966 & 2.97 & $1.24 \mathrm{E}-02$ & 2731 & CR389319 & 1.62 & $1.59 \mathrm{E}-03$ & 2799 & CR389649 & 8.02 & $1.68 \mathrm{E}-03$ \\
\hline 2664 & CR388975 & 5.13 & $5.09 \mathrm{E}-03$ & 2732 & CR389321 & 3.25 & $5.34 \mathrm{E}-04$ & 2800 & CR389659 & 5.21 & $7.83 \mathrm{E}-03$ \\
\hline 2665 & CR388977 & 2.25 & $3.05 \mathrm{E}-04$ & 2733 & CR389323 & 2.09 & $1.98 \mathrm{E}-02$ & 2801 & CR389675 & 6.46 & $2.55 \mathrm{E}-03$ \\
\hline 2666 & CR388978 & 5.27 & $3.05 \mathrm{E}-03$ & 2734 & CR389329 & 1.28 & $3.34 \mathrm{E}-03$ & 2802 & CR389682 & 2.00 & 1.07E-02 \\
\hline 2667 & CR388983 & 5.06 & $4.57 \mathrm{E}-03$ & 2735 & CR389335 & 2.18 & $1.88 \mathrm{E}-02$ & 2803 & CR389685 & 4.17 & $1.42 \mathrm{E}-03$ \\
\hline 2668 & CR389001 & 1.83 & $1.66 \mathrm{E}-01$ & 2736 & CR389336 & 3.09 & $1.60 \mathrm{E}-03$ & 2804 & CR389692 & 3.02 & $1.77 \mathrm{E}-04$ \\
\hline 2669 & CR389019 & 4.95 & $1.99 \mathrm{E}-03$ & 2737 & CR389338 & 1.78 & $5.06 \mathrm{E}-03$ & 2805 & CR389698 & 2.63 & $6.14 \mathrm{E}-03$ \\
\hline 2670 & CR389024 & 3.84 & $5.13 \mathrm{E}-03$ & 2738 & CR389344 & 5.49 & $3.41 \mathrm{E}-05$ & 2806 & CR389699 & 2.78 & $1.61 \mathrm{E}-02$ \\
\hline 2671 & CR389026 & 3.88 & $1.01 \mathrm{E}-02$ & 2739 & CR389347 & 4.04 & $4.79 \mathrm{E}-03$ & 2807 & CR389701 & 5.49 & $6.36 \mathrm{E}-03$ \\
\hline 2672 & CR389030 & 3.57 & $3.60 \mathrm{E}-03$ & 2740 & CR389348 & 4.34 & $5.76 \mathrm{E}-03$ & 2808 & CR389707 & 1.86 & $1.20 \mathrm{E}-02$ \\
\hline 2673 & CR389032 & 5.59 & $3.73 \mathrm{E}-03$ & 2741 & CR389349 & 2.56 & $1.64 \mathrm{E}-02$ & 2809 & CR389711 & 7.91 & $1.60 \mathrm{E}-03$ \\
\hline 2674 & CR389045 & 4.79 & $9.97 E-04$ & 2742 & CR389357 & 4.17 & 4.69E-04 & 2810 & CR389712 & 2.88 & $7.92 \mathrm{E}-04$ \\
\hline 2675 & CR389051 & 2.27 & $1.09 \mathrm{E}-02$ & 2743 & CR389362 & 2.77 & $3.34 \mathrm{E}-02$ & 2811 & CR389713 & 1.71 & $1.10 \mathrm{E}-01$ \\
\hline 2676 & CR389055 & 3.86 & $3.21 \mathrm{E}-03$ & 2744 & CR389367 & 2.19 & 7.21E-03 & 2812 & CR389714 & 3.87 & $5.67 \mathrm{E}-03$ \\
\hline 2677 & CR389061 & 6.48 & $1.14 \mathrm{E}-03$ & 2745 & CR389368 & 6.00 & $3.88 \mathrm{E}-03$ & 2813 & CR389715 & 3.11 & $6.18 \mathrm{E}-03$ \\
\hline 2678 & CR389063 & 3.11 & $1.84 \mathrm{E}-03$ & 2746 & CR389377 & 4.28 & $1.68 \mathrm{E}-03$ & 2814 & CR389720 & 1.50 & $1.60 \mathrm{E}-02$ \\
\hline 2679 & CR389066 & 4.64 & $1.25 \mathrm{E}-03$ & 2747 & CR389378 & 5.40 & $8.01 \mathrm{E}-05$ & 2815 & CR389725 & 2.14 & $1.49 \mathrm{E}-02$ \\
\hline 2680 & CR389068 & 2.83 & $2.46 \mathrm{E}-02$ & 2748 & CR389383 & 3.21 & $1.60 \mathrm{E}-02$ & 2816 & CR389730 & 4.89 & $1.47 \mathrm{E}-02$ \\
\hline 2681 & CR389075 & 4.40 & $2.08 \mathrm{E}-03$ & 2749 & CR389388 & 2.88 & $5.12 \mathrm{E}-03$ & 2817 & CR389737 & 4.12 & $5.15 \mathrm{E}-03$ \\
\hline 2682 & CR389082 & 6.02 & $1.11 \mathrm{E}-03$ & 2750 & CR389393 & 3.14 & $1.66 \mathrm{E}-03$ & 2818 & CR389746 & 2.13 & $2.50 \mathrm{E}-02$ \\
\hline 2683 & CR389086 & 5.33 & $2.54 \mathrm{E}-03$ & 2751 & CR389400 & 4.29 & $3.26 \mathrm{E}-03$ & 2819 & CR389753 & 4.30 & $5.40 \mathrm{E}-04$ \\
\hline 2684 & CR389087 & 3.06 & $1.36 \mathrm{E}-02$ & 2752 & CR389403 & 4.91 & $8.47 \mathrm{E}-03$ & 2820 & CR389754 & 3.65 & $2.48 \mathrm{E}-03$ \\
\hline 2685 & CR389089 & 3.70 & $5.24 \mathrm{E}-04$ & 2753 & CR389404 & 2.07 & $4.51 \mathrm{E}-03$ & 2821 & CR389758 & 2.84 & $2.21 \mathrm{E}-02$ \\
\hline 2686 & CR389095 & 4.17 & $1.32 \mathrm{E}-02$ & 2754 & CR389413 & 1.92 & $2.16 \mathrm{E}-02$ & 2822 & CR389759 & 2.71 & $2.31 \mathrm{E}-03$ \\
\hline 2687 & CR389097 & 1.42 & $2.92 \mathrm{E}-02$ & 2755 & CR389414 & 3.93 & $1.42 \mathrm{E}-02$ & 2823 & CR389766 & 4.97 & $2.51 \mathrm{E}-03$ \\
\hline 2688 & CR389098 & 1.43 & $8.95 \mathrm{E}-03$ & 2756 & CR389418 & 3.38 & $5.34 \mathrm{E}-03$ & 2824 & CR389768 & 2.82 & $1.20 \mathrm{E}-02$ \\
\hline 2689 & CR389111 & 5.46 & $5.37 \mathrm{E}-03$ & 2757 & CR389425 & 5.95 & $4.82 \mathrm{E}-04$ & 2825 & CR389771 & 1.06 & $5.31 \mathrm{E}-02$ \\
\hline 2690 & CR389112 & 2.04 & $1.21 \mathrm{E}-02$ & 2758 & CR389430 & 5.54 & $1.97 \mathrm{E}-03$ & 2826 & CR389774 & 4.52 & $2.61 \mathrm{E}-03$ \\
\hline 2691 & CR389114 & 5.01 & $9.86 \mathrm{E}-04$ & 2759 & CR389444 & 4.10 & $2.93 \mathrm{E}-04$ & 2827 & CR389778 & 3.55 & $7.13 \mathrm{E}-04$ \\
\hline 2692 & CR389119 & 6.37 & $7.70 \mathrm{E}-04$ & 2760 & CR389450 & 6.56 & $1.16 \mathrm{E}-03$ & 2828 & CR389783 & 2.92 & $2.01 \mathrm{E}-03$ \\
\hline 2693 & CR389120 & 4.11 & $1.50 \mathrm{E}-03$ & 2761 & CR389457 & 4.40 & $1.42 \mathrm{E}-03$ & 2829 & CR389793 & 6.53 & $1.45 \mathrm{E}-03$ \\
\hline 2694 & CR389123 & 1.65 & $8.70 \mathrm{E}-04$ & 2762 & CR389464 & 1.84 & $1.91 \mathrm{E}-02$ & 2830 & CR389794 & 3.76 & $2.17 \mathrm{E}-02$ \\
\hline 2695 & CR389127 & 1.48 & $7.65 \mathrm{E}-03$ & 2763 & CR389466 & 2.73 & $3.69 \mathrm{E}-03$ & 2831 & CR389796 & 4.57 & $2.41 \mathrm{E}-03$ \\
\hline 2696 & CR389129 & 4.30 & $2.28 \mathrm{E}-03$ & 2764 & CR389471 & 3.33 & $1.51 \mathrm{E}-04$ & 2832 & CR389800 & 6.97 & $2.11 \mathrm{E}-05$ \\
\hline 2697 & CR389142 & 3.03 & $1.27 \mathrm{E}-02$ & 2765 & CR389478 & 9.98 & $1.13 \mathrm{E}-03$ & 2833 & CR389803 & 4.68 & $1.14 \mathrm{E}-02$ \\
\hline 2698 & CR389147 & 5.06 & $1.34 \mathrm{E}-03$ & 2766 & CR389479 & 5.17 & $3.69 \mathrm{E}-03$ & 2834 & CR389805 & 4.10 & $1.40 \mathrm{E}-03$ \\
\hline 2699 & CR389148 & 3.69 & $1.07 E-02$ & 2767 & CR389481 & 2.52 & $1.46 \mathrm{E}-02$ & 2835 & CR389813 & 1.54 & $1.69 \mathrm{E}-01$ \\
\hline 2700 & CR389149 & 5.35 & $3.60 \mathrm{E}-03$ & 2768 & CR389482 & 3.87 & $2.52 \mathrm{E}-04$ & 2836 & CR389814 & 3.23 & $5.06 \mathrm{E}-04$ \\
\hline 2701 & CR389151 & 3.42 & $4.02 E-03$ & 2769 & CR389486 & 2.89 & $1.08 \mathrm{E}-02$ & 2837 & CR389815 & 4.39 & $8.92 \mathrm{E}-04$ \\
\hline 2702 & CR389152 & 4.82 & $9.78 \mathrm{E}-03$ & 2770 & CR389492 & 1.79 & $6.47 \mathrm{E}-02$ & 2838 & CR389825 & 3.76 & $2.11 \mathrm{E}-03$ \\
\hline 2703 & CR389171 & 1.76 & $2.31 \mathrm{E}-02$ & 2771 & CR389493 & 5.93 & $1.12 \mathrm{E}-03$ & 2839 & CR389827 & 3.76 & $1.57 \mathrm{E}-03$ \\
\hline 2704 & CR389177 & 1.36 & $1.08 \mathrm{E}-02$ & 2772 & CR389496 & 4.60 & $8.65 \mathrm{E}-03$ & 2840 & CR389828 & 2.55 & $5.28 \mathrm{E}-04$ \\
\hline 2705 & CR389179 & 4.19 & $1.97 \mathrm{E}-03$ & 2773 & CR389507 & 2.05 & $9.11 \mathrm{E}-03$ & 2841 & CR389831 & 3.76 & $1.85 \mathrm{E}-02$ \\
\hline 2706 & CR389181 & 3.18 & $2.39 \mathrm{E}-04$ & 2774 & CR389509 & 1.20 & $1.06 \mathrm{E}-02$ & 2842 & CR389838 & 2.84 & $2.78 \mathrm{E}-02$ \\
\hline 2707 & CR389183 & 7.33 & $1.42 \mathrm{E}-03$ & 2775 & CR389513 & 6.37 & $6.09 \mathrm{E}-04$ & 2843 & CR389839 & 3.00 & $4.23 \mathrm{E}-02$ \\
\hline 2708 & CR389184 & 2.89 & $6.36 \mathrm{E}-03$ & 2776 & CR389515 & 5.36 & $1.65 \mathrm{E}-03$ & 2844 & CR389843 & 7.15 & $1.01 \mathrm{E}-06$ \\
\hline 2709 & CR389185 & 1.93 & $2.17 \mathrm{E}-02$ & 2777 & CR389528 & 3.65 & $3.93 \mathrm{E}-03$ & 2845 & CR389844 & 3.66 & $2.81 \mathrm{E}-04$ \\
\hline 2710 & CR389187 & 4.80 & $4.31 \mathrm{E}-04$ & 2778 & CR389532 & 2.31 & $1.16 \mathrm{E}-02$ & 2846 & CR389846 & 2.49 & $2.27 E-02$ \\
\hline 2711 & CR389192 & 4.63 & $1.48 \mathrm{E}-03$ & 2779 & CR389539 & 6.33 & $4.81 \mathrm{E}-04$ & 2847 & CR389850 & 3.22 & $2.54 \mathrm{E}-02$ \\
\hline 2712 & CR389199 & 3.44 & $3.51 \mathrm{E}-03$ & 2780 & CR389549 & 8.37 & $6.85 \mathrm{E}-03$ & 2848 & CR389851 & 5.20 & $3.46 \mathrm{E}-03$ \\
\hline 2713 & CR389206 & 4.38 & $2.04 \mathrm{E}-03$ & 2781 & CR389550 & 2.23 & $5.26 \mathrm{E}-03$ & 2849 & CR389852 & 2.93 & $2.51 \mathrm{E}-01$ \\
\hline 2714 & CR389207 & 2.71 & $2.47 \mathrm{E}-02$ & 2782 & CR389554 & 2.96 & $1.13 \mathrm{E}-02$ & 2850 & CR389857 & 4.28 & $8.63 \mathrm{E}-03$ \\
\hline 2715 & CR389210 & 3.77 & $4.87 \mathrm{E}-03$ & 2783 & CR389559 & 4.72 & $1.56 \mathrm{E}-03$ & 2851 & CR389860 & 2.51 & $2.10 \mathrm{E}-03$ \\
\hline 2716 & CR389212 & 4.81 & $7.50 \mathrm{E}-06$ & 2784 & CR389563 & 2.30 & $4.81 \mathrm{E}-03$ & 2852 & CR389863 & 6.63 & $3.60 \mathrm{E}-03$ \\
\hline 2717 & CR389215 & 2.25 & $2.02 \mathrm{E}-03$ & 2785 & CR389570 & 3.41 & $1.37 \mathrm{E}-03$ & 2853 & CR389864 & 4.25 & $7.43 \mathrm{E}-04$ \\
\hline 2718 & CR389229 & 5.93 & $5.63 \mathrm{E}-03$ & 2786 & CR389574 & 3.37 & $1.67 \mathrm{E}-03$ & 2854 & CR389865 & 1.60 & $4.72 \mathrm{E}-02$ \\
\hline 2719 & CR389236 & 6.33 & $1.50 \mathrm{E}-02$ & 2787 & CR389575 & 1.85 & $3.77 \mathrm{E}-02$ & 2855 & CR389866 & 4.87 & 1.67E-02 \\
\hline
\end{tabular}




\begin{tabular}{|c|c|c|c|c|c|c|c|c|c|c|c|}
\hline 2856 & CR389869 & 4.06 & $1.79 E-02$ & 2924 & CR390168 & 3.08 & 4.93E-02 & 2992 & CR390457 & 1.08 & $4.50 \mathrm{E}-02$ \\
\hline 2857 & CR389874 & 4.03 & $2.82 \mathrm{E}-04$ & 2925 & CR390178 & 3.58 & 3.49E-02 & 2993 & CR390458 & 5.69 & $7.65 E-03$ \\
\hline 2858 & CR389876 & 3.15 & $1.14 \mathrm{E}-03$ & 2926 & CR390179 & 5.08 & $3.17 \mathrm{E}-03$ & 2994 & CR390459 & 5.39 & $4.79 \mathrm{E}-03$ \\
\hline 2859 & CR389880 & 4.60 & $3.28 \mathrm{E}-03$ & 2927 & CR390184 & 1.44 & $6.24 \mathrm{E}-04$ & 2995 & CR390460 & 3.40 & 4.69E- 03 \\
\hline 2860 & CR389882 & 5.95 & $2.58 \mathrm{E}-04$ & 2928 & CR390186 & 4.10 & $2.23 \mathrm{E}-03$ & 2996 & CR390470 & 1.56 & $1.40 \mathrm{E}-03$ \\
\hline 2861 & CR389888 & 6.11 & $9.81 \mathrm{E}-05$ & 2929 & CR390193 & 3.05 & $2.08 \mathrm{E}-03$ & 2997 & CR390472 & 3.42 & $1.77 E-03$ \\
\hline 2862 & CR389894 & 6.99 & $2.66 \mathrm{E}-03$ & 2930 & CR390203 & 5.74 & $6.58 \mathrm{E}-03$ & 2998 & CR390473 & 2.94 & $1.80 \mathrm{E}-02$ \\
\hline 2863 & CR389895 & 2.87 & $1.08 \mathrm{E}-03$ & 2931 & CR390204 & 1.87 & 3.53E-02 & 2999 & CR390475 & 2.10 & $1.66 \mathrm{E}-02$ \\
\hline 2864 & CR389898 & 4.10 & $3.59 E-03$ & 2932 & CR390210 & 5.16 & $1.34 \mathrm{E}-03$ & 3000 & CR390485 & 1.66 & $6.53 \mathrm{E}-03$ \\
\hline 2865 & CR389905 & 1.76 & $3.43 \mathrm{E}-03$ & 2933 & CR390213 & 5.05 & $4.65 \mathrm{E}-03$ & 3001 & CR390496 & 3.74 & $5.44 \mathrm{E}-03$ \\
\hline 2866 & CR389908 & 4.92 & $1.43 \mathrm{E}-02$ & 2934 & CR390226 & 6.40 & $1.10 \mathrm{E}-02$ & 3002 & CR390500 & 5.53 & $1.34 \mathrm{E}-02$ \\
\hline 2867 & CR389909 & 4.90 & 3.51E-04 & 2935 & CR390231 & 5.88 & $2.82 \mathrm{E}-03$ & 3003 & CR390508 & 5.62 & $5.18 \mathrm{E}-04$ \\
\hline 2868 & CR389914 & 2.19 & $4.74 E-02$ & 2936 & CR390232 & 3.59 & $3.51 \mathrm{E}-03$ & 3004 & CR390509 & 2.25 & $7.27 \mathrm{E}-04$ \\
\hline 2869 & CR389920 & 1.20 & $7.24 \mathrm{E}-03$ & 2937 & CR390233 & 5.36 & $4.19 \mathrm{E}-03$ & 3005 & CR390516 & 1.62 & 9.54E-03 \\
\hline 2870 & CR389924 & 1.56 & $2.72 \mathrm{E}-02$ & 2938 & CR390237 & 1.33 & $8.99 \mathrm{E}-03$ & 3006 & CR390521 & 5.44 & $1.20 \mathrm{E}-03$ \\
\hline 2871 & CR389927 & 2.46 & $3.21 \mathrm{E}-02$ & 2939 & CR390238 & 2.47 & $9.42 \mathrm{E}-03$ & 3007 & CR390532 & 3.37 & $3.22 \mathrm{E}-03$ \\
\hline 2872 & CR389930 & 3.21 & $8.01 E-02$ & 2940 & CR390239 & 5.43 & $7.03 \mathrm{E}-03$ & 3008 & CR390533 & 6.77 & $1.62 \mathrm{E}-02$ \\
\hline 2873 & CR389934 & 3.77 & $1.82 \mathrm{E}-03$ & 2941 & CR390243 & 1.53 & $5.09 \mathrm{E}-04$ & 3009 & CR390536 & 3.90 & $1.14 \mathrm{E}-03$ \\
\hline 2874 & CR389937 & 3.92 & $1.08 \mathrm{E}-03$ & 2942 & CR390247 & 1.58 & 1.69E-03 & 3010 & CR390537 & 2.84 & $5.70 \mathrm{E}-04$ \\
\hline 2875 & CR389939 & 5.16 & $1.54 \mathrm{E}-03$ & 2943 & CR390253 & 3.72 & $1.26 \mathrm{E}-02$ & 3011 & CR390544 & 3.45 & $6.29 \mathrm{E}-03$ \\
\hline 2876 & CR389941 & 2.44 & $9.61 \mathrm{E}-02$ & 2944 & CR390258 & 3.40 & $7.09 \mathrm{E}-04$ & 3012 & CR390546 & 1.16 & $6.08 \mathrm{E}-02$ \\
\hline 2877 & CR389946 & 1.29 & $2.90 \mathrm{E}-02$ & 2945 & CR390261 & 2.33 & $1.00 \mathrm{E}-03$ & 3013 & CR390547 & 2.51 & $6.40 \mathrm{E}-04$ \\
\hline 2878 & CR389951 & 4.29 & $1.07 E-03$ & 2946 & CR390263 & 5.04 & $4.54 \mathrm{E}-03$ & 3014 & CR390548 & 1.98 & $6.75 \mathrm{E}-03$ \\
\hline 2879 & CR389957 & 6.74 & $3.18 \mathrm{E}-03$ & 2947 & CR390266 & 3.65 & $3.15 \mathrm{E}-04$ & 3015 & CR390550 & 2.85 & $2.16 \mathrm{E}-02$ \\
\hline 2880 & CR389959 & 2.37 & $1.78 \mathrm{E}-02$ & 2948 & CR390278 & 2.47 & $4.41 \mathrm{E}-02$ & 3016 & CR390551 & 1.64 & $3.88 \mathrm{E}-02$ \\
\hline 2881 & CR389964 & 3.10 & $1.12 \mathrm{E}-02$ & 2949 & CR390283 & 4.94 & $2.38 \mathrm{E}-05$ & 3017 & CR390552 & 6.13 & $2.01 \mathrm{E}-03$ \\
\hline 2882 & CR389966 & 4.84 & $5.42 \mathrm{E}-04$ & 2950 & CR390285 & 2.17 & $1.60 \mathrm{E}-02$ & 3018 & CR390554 & 2.35 & $6.14 \mathrm{E}-02$ \\
\hline 2883 & CR389972 & 4.39 & $7.82 E-03$ & 2951 & CR390289 & 3.89 & $9.23 \mathrm{E}-04$ & 3019 & CR390560 & 4.21 & 4.65E-03 \\
\hline 2884 & CR389983 & 4.58 & $1.02 \mathrm{E}-02$ & 2952 & CR390290 & 1.72 & $1.10 \mathrm{E}-02$ & 3020 & CR390567 & 4.69 & $1.50 \mathrm{E}-03$ \\
\hline 2885 & CR389986 & 2.09 & $3.31 \mathrm{E}-02$ & 2953 & CR390293 & 3.39 & $2.88 \mathrm{E}-03$ & 3021 & CR390569 & 3.58 & 4.21E-04 \\
\hline 2886 & CR389989 & 3.26 & $1.07 \mathrm{E}-04$ & 2954 & CR390294 & 4.17 & $6.01 \mathrm{E}-03$ & 3022 & CR390570 & 2.06 & 7.68E-02 \\
\hline 2887 & CR389997 & 1.96 & $2.47 \mathrm{E}-02$ & 2955 & CR390295 & 2.08 & $1.07 \mathrm{E}-02$ & 3023 & CR390575 & 4.78 & $1.57 E-03$ \\
\hline 2888 & CR389998 & 5.27 & $1.22 \mathrm{E}-03$ & 2956 & CR390300 & 3.53 & $6.44 \mathrm{E}-03$ & 3024 & CR390576 & 2.97 & $2.47 E-03$ \\
\hline 2889 & CR390006 & 2.91 & $2.41 E-02$ & 2957 & CR390301 & 7.45 & 3.94E-05 & 3025 & CR390579 & 3.25 & 4.12E-02 \\
\hline 2890 & CR390007 & 2.77 & $8.82 \mathrm{E}-03$ & 2958 & CR390303 & 3.40 & $2.64 \mathrm{E}-03$ & 3026 & CR390581 & 3.12 & $1.41 \mathrm{E}-03$ \\
\hline 2891 & CR390014 & 2.23 & $3.60 \mathrm{E}-03$ & 2959 & CR390314 & 2.86 & $1.04 \mathrm{E}-02$ & 3027 & CR390583 & 1.42 & $3.90 \mathrm{E}-02$ \\
\hline 2892 & CR390015 & 4.78 & $2.17 \mathrm{E}-02$ & 2960 & CR390335 & 1.27 & $2.12 \mathrm{E}-02$ & 3028 & CR390594 & 3.32 & 7.07E-03 \\
\hline 2893 & CR390017 & 3.17 & $4.46 \mathrm{E}-04$ & 2961 & CR390344 & 3.44 & $5.88 \mathrm{E}-03$ & 3029 & CR390595 & 5.15 & $3.18 \mathrm{E}-03$ \\
\hline 2894 & CR390018 & 4.51 & 4.44E-05 & 2962 & CR390349 & 2.08 & $1.98 \mathrm{E}-02$ & 3030 & CR390596 & 4.14 & $1.46 \mathrm{E}-02$ \\
\hline 2895 & CR390027 & 5.51 & $3.24 \mathrm{E}-03$ & 2963 & CR390351 & 3.80 & $3.98 \mathrm{E}-03$ & 3031 & CR390597 & 2.77 & $1.40 \mathrm{E}-02$ \\
\hline 2896 & CR390030 & 5.53 & $1.36 \mathrm{E}-02$ & 2964 & CR390352 & 3.09 & $1.44 \mathrm{E}-02$ & 3032 & CR390602 & 1.69 & $1.57 \mathrm{E}-02$ \\
\hline 2897 & CR390032 & 3.97 & $1.56 \mathrm{E}-04$ & 2965 & CR390359 & 2.90 & $2.47 \mathrm{E}-02$ & 3033 & CR390605 & 5.36 & $1.75 \mathrm{E}-03$ \\
\hline 2898 & CR390035 & 3.63 & $2.12 \mathrm{E}-02$ & 2966 & CR390376 & 4.10 & $1.02 \mathrm{E}-02$ & 3034 & CR390613 & 4.87 & $6.71 E-03$ \\
\hline 2899 & CR390037 & 3.39 & $3.27 \mathrm{E}-03$ & 2967 & CR390382 & 2.31 & $1.39 \mathrm{E}-02$ & 3035 & CR390614 & 5.95 & $1.14 \mathrm{E}-02$ \\
\hline 2900 & CR390038 & 1.85 & $3.90 \mathrm{E}-03$ & 2968 & CR390384 & 1.44 & $6.67 \mathrm{E}-04$ & 3036 & CR390620 & 1.78 & $1.37 \mathrm{E}-01$ \\
\hline 2901 & CR390040 & 1.63 & $7.73 \mathrm{E}-02$ & 2969 & CR390385 & 1.63 & $2.04 \mathrm{E}-03$ & 3037 & CR390622 & 5.76 & $1.22 \mathrm{E}-04$ \\
\hline 2902 & CR390041 & 6.19 & $5.59 \mathrm{E}-04$ & 2970 & CR390387 & 1.55 & $1.71 \mathrm{E}-03$ & 3038 & CR390630 & 1.16 & $1.39 \mathrm{E}-02$ \\
\hline 2903 & CR390045 & 4.18 & $9.16 \mathrm{E}-03$ & 2971 & CR390398 & 4.99 & $2.60 \mathrm{E}-03$ & 3039 & CR390645 & 2.98 & $3.00 \mathrm{E}-03$ \\
\hline 2904 & CR390047 & 4.18 & $8.15 \mathrm{E}-03$ & 2972 & CR390402 & 1.76 & $1.79 \mathrm{E}-02$ & 3040 & CR390650 & 1.38 & 4.25E-02 \\
\hline 2905 & CR390050 & 2.91 & $1.41 \mathrm{E}-02$ & 2973 & CR390403 & 3.36 & $5.17 \mathrm{E}-03$ & 3041 & CR390656 & 5.98 & $1.43 E-02$ \\
\hline 2906 & CR390054 & 2.46 & $1.08 \mathrm{E}-02$ & 2974 & CR390405 & 2.93 & $2.65 \mathrm{E}-04$ & 3042 & CR390663 & 6.21 & $3.13 \mathrm{E}-03$ \\
\hline 2907 & CR390056 & 3.13 & $6.72 E-03$ & 2975 & CR390406 & 5.11 & $1.02 \mathrm{E}-03$ & 3043 & CR390665 & 1.62 & 2.69E-02 \\
\hline 2908 & CR390058 & 4.81 & $1.02 \mathrm{E}-03$ & 2976 & CR390409 & 5.32 & $1.67 \mathrm{E}-03$ & 3044 & CR390678 & 2.93 & $6.61 \mathrm{E}-04$ \\
\hline 2909 & CR390063 & 3.73 & $5.29 \mathrm{E}-04$ & 2977 & CR390410 & 3.13 & $2.42 \mathrm{E}-03$ & 3045 & CR390683 & 3.58 & 3.04E-04 \\
\hline 2910 & CR390074 & 5.51 & $4.38 \mathrm{E}-03$ & 2978 & CR390413 & 5.97 & $2.12 \mathrm{E}-03$ & 3046 & CR390694 & 2.12 & 4.74E-03 \\
\hline 2911 & CR390082 & 4.72 & 4.69E-03 & 2979 & CR390416 & 5.63 & $3.39 \mathrm{E}-03$ & 3047 & CR390705 & 2.61 & $2.18 \mathrm{E}-02$ \\
\hline 2912 & CR390084 & 3.32 & $7.35 \mathrm{E}-03$ & 2980 & CR390419 & 3.32 & $2.64 \mathrm{E}-03$ & 3048 & CR390713 & 2.13 & $2.27 \mathrm{E}-03$ \\
\hline 2913 & CR390086 & 5.20 & $1.02 E-02$ & 2981 & CR390423 & 2.29 & $6.18 \mathrm{E}-03$ & 3049 & CR390714 & 4.99 & $2.97 \mathrm{E}-04$ \\
\hline 2914 & CR390089 & 1.27 & $3.21 E-02$ & 2982 & CR390429 & 4.38 & $1.45 \mathrm{E}-03$ & 3050 & CR390715 & 4.44 & $2.62 \mathrm{E}-03$ \\
\hline 2915 & CR390091 & 3.57 & $2.83 \mathrm{E}-02$ & 2983 & CR390431 & 2.70 & $5.94 \mathrm{E}-02$ & 3051 & CR390716 & 2.74 & 1.33E-02 \\
\hline 2916 & CR390095 & 3.44 & $2.62 \mathrm{E}-03$ & 2984 & CR390433 & 2.26 & $6.82 \mathrm{E}-03$ & 3052 & CR390724 & 6.48 & $5.52 \mathrm{E}-03$ \\
\hline 2917 & CR390101 & 3.48 & $2.07 E-03$ & 2985 & CR390437 & 2.39 & $8.36 \mathrm{E}-04$ & 3053 & CR390725 & 2.67 & $1.01 \mathrm{E}-04$ \\
\hline 2918 & CR390102 & 1.98 & $1.53 \mathrm{E}-04$ & 2986 & CR390438 & 3.28 & 4.01E-03 & 3054 & CR390728 & 6.00 & $2.16 \mathrm{E}-04$ \\
\hline 2919 & CR390108 & 2.97 & $2.75 \mathrm{E}-02$ & 2987 & CR390442 & 4.73 & $1.53 \mathrm{E}-03$ & 3055 & CR390729 & 4.40 & 7.07E-03 \\
\hline 2920 & CR390129 & 4.45 & $1.33 \mathrm{E}-02$ & 2988 & CR390444 & 6.41 & $5.04 \mathrm{E}-03$ & 3056 & CR390733 & 3.78 & $6.54 \mathrm{E}-03$ \\
\hline 2921 & CR390135 & 3.60 & $4.82 \mathrm{E}-03$ & 2989 & CR390448 & 2.61 & $2.90 \mathrm{E}-02$ & 3057 & CR390734 & 3.44 & $5.65 \mathrm{E}-03$ \\
\hline 2922 & CR390158 & 5.00 & $5.01 \mathrm{E}-05$ & 2990 & CR390449 & 1.22 & $7.35 \mathrm{E}-03$ & 3058 & CR390735 & 3.08 & 4.45E-03 \\
\hline 2923 & CR390159 & 1.95 & $1.84 \mathrm{E}-02$ & 2991 & CR390451 & 6.52 & $1.47 \mathrm{E}-02$ & 3059 & CR390737 & 5.53 & $1.70 \mathrm{E}-03$ \\
\hline
\end{tabular}




\begin{tabular}{|c|c|c|c|c|c|c|c|c|c|c|c|}
\hline 3060 & CR390741 & 1.69 & $5.58 \mathrm{E}-03$ & 3128 & CR391019 & 3.62 & 4.27E-04 & 3196 & CR391235 & 2.06 & $3.30 \mathrm{E}-02$ \\
\hline 3061 & CR390744 & 1.45 & $1.87 \mathrm{E}-02$ & 3129 & CR391020 & 2.12 & $1.54 \mathrm{E}-02$ & 3197 & CR391237 & 3.73 & $6.33 \mathrm{E}-04$ \\
\hline 3062 & CR390745 & 4.69 & $2.07 E-02$ & 3130 & CR391021 & 4.32 & $2.52 \mathrm{E}-02$ & 3198 & CR391238 & 4.20 & $2.06 \mathrm{E}-02$ \\
\hline 3063 & CR390746 & 2.02 & $5.17 E-02$ & 3131 & CR391023 & 6.58 & $7.66 \mathrm{E}-04$ & 3199 & CR391244 & 3.62 & $1.09 \mathrm{E}-03$ \\
\hline 3064 & CR390747 & 3.33 & $1.38 \mathrm{E}-02$ & 3132 & CR391027 & 1.75 & 3.33E-02 & 3200 & CR391246 & 6.09 & $6.16 \mathrm{E}-04$ \\
\hline 3065 & CR390750 & 4.14 & $1.46 \mathrm{E}-03$ & 3133 & CR391029 & 7.60 & $3.95 E-03$ & 3201 & CR391247 & 4.60 & $6.25 \mathrm{E}-03$ \\
\hline 3066 & CR390755 & 2.41 & $3.45 E-02$ & 3134 & CR391030 & 3.71 & $6.34 \mathrm{E}-03$ & 3202 & CR391248 & 5.44 & $3.82 \mathrm{E}-03$ \\
\hline 3067 & CR390756 & 3.10 & $2.15 \mathrm{E}-03$ & 3135 & CR391031 & 3.29 & 1.64E-02 & 3203 & CR391252 & 3.61 & $3.12 \mathrm{E}-03$ \\
\hline 3068 & CR390765 & 4.39 & $1.65 \mathrm{E}-03$ & 3136 & CR391035 & 3.86 & $2.23 E-03$ & 3204 & CR391258 & 1.14 & $1.19 \mathrm{E}-03$ \\
\hline 3069 & CR390782 & 6.38 & $1.16 \mathrm{E}-03$ & 3137 & CR391041 & 5.67 & $5.00 E-04$ & 3205 & CR391259 & 4.70 & $5.99 \mathrm{E}-03$ \\
\hline 3070 & CR390787 & 4.45 & $6.62 \mathrm{E}-03$ & 3138 & CR391047 & 4.75 & $1.02 \mathrm{E}-02$ & 3206 & CR391261 & 2.78 & $7.19 \mathrm{E}-03$ \\
\hline 3071 & CR390789 & 1.82 & $2.56 \mathrm{E}-02$ & 3139 & CR391053 & 7.24 & 6.37E-04 & 3207 & CR391265 & 4.92 & $2.26 \mathrm{E}-04$ \\
\hline 3072 & CR390790 & 2.66 & $2.74 E-02$ & 3140 & CR391056 & 6.83 & $3.46 E-04$ & 3208 & CR391268 & 6.64 & $4.32 \mathrm{E}-03$ \\
\hline 3073 & CR390792 & 1.78 & $2.89 \mathrm{E}-02$ & 3141 & CR391061 & 5.04 & $2.47 E-04$ & 3209 & CR391272 & 1.59 & $3.68 \mathrm{E}-02$ \\
\hline 3074 & CR390796 & 1.53 & $1.22 \mathrm{E}-02$ & 3142 & CR391063 & 2.78 & $4.92 E-03$ & 3210 & CR391282 & 7.11 & $8.94 \mathrm{E}-05$ \\
\hline 3075 & CR390801 & 6.03 & $3.75 \mathrm{E}-04$ & 3143 & CR391068 & 4.58 & 4.27E-03 & 3211 & CR391283 & 4.30 & $1.26 \mathrm{E}-03$ \\
\hline 3076 & CR390802 & 1.91 & $8.10 \mathrm{E}-03$ & 3144 & CR391071 & 1.96 & $2.71 \mathrm{E}-02$ & 3212 & CR391287 & 3.91 & $4.24 \mathrm{E}-02$ \\
\hline 3077 & CR390810 & 1.36 & $3.07 E-02$ & 3145 & CR391075 & 6.19 & $4.74 \mathrm{E}-03$ & 3213 & CR391289 & 5.01 & $1.58 \mathrm{E}-04$ \\
\hline 3078 & CR390812 & 3.64 & $6.91 \mathrm{E}-03$ & 3146 & CR391080 & 1.79 & $5.67 \mathrm{E}-04$ & 3214 & CR391300 & 1.17 & $2.46 \mathrm{E}-03$ \\
\hline 3079 & CR390824 & 1.17 & $2.82 \mathrm{E}-02$ & 3147 & CR391081 & 6.14 & $1.62 \mathrm{E}-03$ & 3215 & CR391303 & 4.83 & 4.53E-04 \\
\hline 3080 & CR390826 & 1.93 & $9.85 \mathrm{E}-03$ & 3148 & CR391087 & 3.66 & $2.64 \mathrm{E}-03$ & 3216 & CR391306 & 5.03 & $9.83 \mathrm{E}-02$ \\
\hline 3081 & CR390829 & 1.81 & $3.93 \mathrm{E}-02$ & 3149 & CR391089 & 5.91 & $1.61 \mathrm{E}-04$ & 3217 & CR391312 & 5.28 & $2.84 \mathrm{E}-03$ \\
\hline 3082 & CR390832 & 2.23 & $8.32 \mathrm{E}-03$ & 3150 & CR391093 & 3.52 & $1.73 \mathrm{E}-02$ & 3218 & CR391313 & 4.78 & $1.53 \mathrm{E}-04$ \\
\hline 3083 & CR390838 & 5.48 & $2.87 \mathrm{E}-04$ & 3151 & CR391098 & 3.70 & $2.40 \mathrm{E}-03$ & 3219 & CR391314 & 2.48 & $9.73 \mathrm{E}-03$ \\
\hline 3084 & CR390839 & 5.02 & $6.15 \mathrm{E}-03$ & 3152 & CR391100 & 1.93 & $7.15 E-02$ & 3220 & CR391318 & 4.29 & $4.14 \mathrm{E}-03$ \\
\hline 3085 & CR390845 & 2.16 & $5.62 \mathrm{E}-02$ & 3153 & CR391105 & 3.65 & $2.79 \mathrm{E}-03$ & 3221 & CR391319 & 5.19 & $6.95 \mathrm{E}-03$ \\
\hline 3086 & CR390846 & 5.86 & $1.66 \mathrm{E}-03$ & 3154 & CR391106 & 3.46 & $2.24 \mathrm{E}-03$ & 3222 & CR391320 & 1.10 & $2.97 \mathrm{E}-03$ \\
\hline 3087 & CR390847 & 4.99 & $6.23 \mathrm{E}-03$ & 3155 & CR391113 & 4.38 & $6.41 \mathrm{E}-03$ & 3223 & CR391321 & 2.95 & $4.75 \mathrm{E}-03$ \\
\hline 3088 & CR390849 & 4.52 & $3.45 \mathrm{E}-03$ & 3156 & CR391115 & 4.21 & $4.42 \mathrm{E}-03$ & 3224 & CR391329 & 4.33 & $1.37 \mathrm{E}-03$ \\
\hline 3089 & CR390854 & 4.87 & $6.23 \mathrm{E}-03$ & 3157 & CR391119 & 6.45 & 1.17E-05 & 3225 & CR391337 & 3.25 & $1.42 \mathrm{E}-05$ \\
\hline 3090 & CR390860 & 4.60 & $7.94 \mathrm{E}-04$ & 3158 & CR391121 & 1.50 & $1.82 \mathrm{E}-02$ & 3226 & CR391339 & 5.54 & $6.41 \mathrm{E}-04$ \\
\hline 3091 & CR390864 & 5.86 & $2.02 \mathrm{E}-03$ & 3159 & CR391123 & 5.91 & $3.43 E-03$ & 3227 & CR391342 & 2.97 & $5.46 \mathrm{E}-04$ \\
\hline 3092 & CR390866 & 2.15 & $3.61 \mathrm{E}-02$ & 3160 & CR391124 & 6.45 & 8.64E-05 & 3228 & CR391343 & 1.28 & $3.50 \mathrm{E}-02$ \\
\hline 3093 & CR390868 & 3.96 & $1.35 \mathrm{E}-04$ & 3161 & CR391125 & 3.11 & $1.09 \mathrm{E}-02$ & 3229 & CR391345 & 2.00 & $3.26 \mathrm{E}-02$ \\
\hline 3094 & CR390871 & 1.52 & $1.26 \mathrm{E}-02$ & 3162 & CR391126 & 2.61 & $7.86 \mathrm{E}-03$ & 3230 & CR391346 & 5.94 & $1.52 \mathrm{E}-03$ \\
\hline 3095 & CR390874 & 5.40 & $5.98 \mathrm{E}-04$ & 3163 & CR391128 & 4.56 & $3.66 \mathrm{E}-03$ & 3231 & CR391348 & 3.96 & $4.01 \mathrm{E}-03$ \\
\hline 3096 & CR390878 & 2.41 & 3.36E-02 & 3164 & CR391131 & 1.52 & $1.55 \mathrm{E}-02$ & 3232 & CR391349 & 6.19 & $9.40 \mathrm{E}-04$ \\
\hline 3097 & CR390880 & 2.44 & $1.13 \mathrm{E}-02$ & 3165 & CR391139 & 6.56 & $8.88 \mathrm{E}-05$ & 3233 & CR391352 & 5.98 & $1.41 \mathrm{E}-03$ \\
\hline 3098 & CR390881 & 3.67 & $5.19 \mathrm{E}-03$ & 3166 & CR391141 & 3.76 & $3.72 \mathrm{E}-04$ & 3234 & CR391357 & 1.42 & $7.21 \mathrm{E}-03$ \\
\hline 3099 & CR390883 & 2.74 & 2.09E-02 & 3167 & CR391143 & 5.21 & $1.44 \mathrm{E}-02$ & 3235 & CR391358 & 6.38 & $1.10 \mathrm{E}-02$ \\
\hline 3100 & CR390887 & 4.98 & $9.56 \mathrm{E}-05$ & 3168 & CR391147 & 1.68 & $8.98 \mathrm{E}-03$ & 3236 & CR391359 & 3.49 & $1.97 \mathrm{E}-02$ \\
\hline 3101 & CR390889 & 2.62 & $1.09 \mathrm{E}-03$ & 3169 & CR391149 & 4.88 & $6.72 E-04$ & 3237 & CR391362 & 4.45 & $2.37 \mathrm{E}-02$ \\
\hline 3102 & CR390895 & 4.99 & $1.84 \mathrm{E}-03$ & 3170 & CR391150 & 3.84 & $1.44 \mathrm{E}-02$ & 3238 & CR391363 & 7.18 & $1.43 \mathrm{E}-03$ \\
\hline 3103 & CR390896 & 1.52 & $4.18 \mathrm{E}-02$ & 3171 & CR391158 & 3.52 & $1.72 \mathrm{E}-03$ & 3239 & CR391364 & 3.48 & $1.74 \mathrm{E}-03$ \\
\hline 3104 & CR390904 & 3.61 & 7.14E-04 & 3172 & CR391162 & 3.23 & $9.98 \mathrm{E}-03$ & 3240 & CR391370 & 5.45 & 4.87E-05 \\
\hline 3105 & CR390907 & 2.77 & $7.35 \mathrm{E}-03$ & 3173 & CR391163 & 2.39 & $5.80 E-03$ & 3241 & CR391371 & 6.42 & $1.05 \mathrm{E}-02$ \\
\hline 3106 & CR390908 & 3.69 & $1.32 \mathrm{E}-02$ & 3174 & CR391164 & 5.93 & $2.08 \mathrm{E}-04$ & 3242 & CR391373 & 2.83 & $9.74 \mathrm{E}-03$ \\
\hline 3107 & CR390917 & 3.24 & $1.39 \mathrm{E}-02$ & 3175 & CR391173 & 5.42 & $2.73 \mathrm{E}-02$ & 3243 & CR391376 & 5.60 & $2.51 \mathrm{E}-04$ \\
\hline 3108 & CR390921 & 3.65 & $1.44 \mathrm{E}-02$ & 3176 & CR391177 & 4.90 & $1.11 \mathrm{E}-03$ & 3244 & CR391378 & 6.00 & $1.51 \mathrm{E}-05$ \\
\hline 3109 & CR390922 & 2.23 & $1.57 \mathrm{E}-02$ & 3177 & CR391179 & 4.29 & $1.42 \mathrm{E}-02$ & 3245 & CR391379 & 4.32 & $2.19 \mathrm{E}-03$ \\
\hline 3110 & CR390928 & 2.81 & $2.17 \mathrm{E}-02$ & 3178 & CR391185 & 3.76 & $9.41 \mathrm{E}-04$ & 3246 & CR391381 & 2.27 & $8.33 \mathrm{E}-03$ \\
\hline 3111 & CR390933 & 1.24 & $1.30 \mathrm{E}-02$ & 3179 & CR391186 & 7.18 & $1.34 \mathrm{E}-03$ & 3247 & CR391382 & 2.10 & $7.49 \mathrm{E}-03$ \\
\hline 3112 & CR390948 & 2.72 & $6.19 \mathrm{E}-04$ & 3180 & CR391190 & 4.26 & $1.06 E-04$ & 3248 & CR391383 & 4.07 & $1.11 \mathrm{E}-03$ \\
\hline 3113 & CR390957 & 3.00 & $2.52 \mathrm{E}-02$ & 3181 & CR391192 & 4.10 & 4.68E-03 & 3249 & CR391404 & 1.69 & $1.02 \mathrm{E}-03$ \\
\hline 3114 & CR390963 & 2.40 & $3.64 \mathrm{E}-01$ & 3182 & CR391197 & 1.39 & $2.65 \mathrm{E}-02$ & 3250 & CR391416 & 1.26 & $4.64 \mathrm{E}-02$ \\
\hline 3115 & CR390968 & 2.22 & $2.43 \mathrm{E}-03$ & 3183 & CR391199 & 4.87 & $1.49 \mathrm{E}-03$ & 3251 & CR391424 & 1.11 & $1.58 \mathrm{E}-02$ \\
\hline 3116 & CR390971 & 4.65 & $6.14 \mathrm{E}-03$ & 3184 & CR391200 & 5.95 & $2.84 \mathrm{E}-04$ & 3252 & CR391425 & 2.96 & $2.07 E-03$ \\
\hline 3117 & CR390972 & 3.48 & $1.52 \mathrm{E}-03$ & 3185 & CR391203 & 4.19 & $2.41 \mathrm{E}-02$ & 3253 & CR391431 & 2.85 & $2.32 \mathrm{E}-02$ \\
\hline 3118 & CR390975 & 1.88 & 3.13E-02 & 3186 & CR391206 & 1.90 & $1.27 \mathrm{E}-03$ & 3254 & CR391433 & 3.08 & $2.26 \mathrm{E}-03$ \\
\hline 3119 & CR390986 & 2.77 & $5.05 \mathrm{E}-03$ & 3187 & CR391207 & 3.45 & 4.57E-03 & 3255 & CR391438 & 1.16 & $4.47 \mathrm{E}-02$ \\
\hline 3120 & CR390994 & 4.62 & 3.17E-03 & 3188 & CR391211 & 5.75 & $8.05 E-04$ & 3256 & CR391441 & 6.06 & $7.57 \mathrm{E}-03$ \\
\hline 3121 & CR391003 & 1.40 & $2.26 \mathrm{E}-02$ & 3189 & CR391217 & 4.68 & $4.26 \mathrm{E}-03$ & 3257 & CR391458 & 1.49 & $1.86 \mathrm{E}-02$ \\
\hline 3122 & CR391004 & 6.34 & 4.33E-03 & 3190 & CR391221 & 4.74 & $3.18 \mathrm{E}-03$ & 3258 & CR391466 & 2.50 & $3.69 \mathrm{E}-02$ \\
\hline 3123 & CR391005 & 3.09 & $8.71 \mathrm{E}-03$ & 3191 & CR391225 & 2.06 & $9.10 \mathrm{E}-04$ & 3259 & CR391483 & 2.15 & 5.59E-03 \\
\hline 3124 & CR391008 & 6.66 & $8.88 \mathrm{E}-04$ & 3192 & CR391226 & 1.15 & $4.45 \mathrm{E}-02$ & 3260 & CR391494 & 1.73 & $6.25 \mathrm{E}-03$ \\
\hline 3125 & CR391014 & 2.11 & $2.32 \mathrm{E}-03$ & 3193 & CR391227 & 6.03 & $2.34 \mathrm{E}-03$ & 3261 & CR391499 & 5.56 & 7.69E-04 \\
\hline 3126 & CR391015 & 1.44 & 4.23E-02 & 3194 & CR391228 & 3.25 & $1.97 \mathrm{E}-02$ & 3262 & CR391502 & 3.92 & $8.03 \mathrm{E}-03$ \\
\hline 3127 & CR391017 & 2.68 & $1.14 \mathrm{E}-02$ & 3195 & CR391229 & 3.34 & 4.67E-04 & 3263 & CR391511 & 2.93 & $1.35 \mathrm{E}-02$ \\
\hline
\end{tabular}




\begin{tabular}{|c|c|c|c|c|c|c|c|c|c|c|c|}
\hline 3264 & CR391515 & 1.41 & 4.94E-02 & 3332 & CR405847 & 5.05 & 7.17E-04 & 3400 & CR406195 & 5.01 & $2.80 \mathrm{E}-03$ \\
\hline 3265 & CR391521 & 4.12 & $8.21 \mathrm{E}-04$ & 3333 & CR405853 & 3.54 & $1.57 \mathrm{E}-03$ & 3401 & CR406201 & 3.22 & $5.08 \mathrm{E}-03$ \\
\hline 3266 & CR391528 & 1.94 & $5.38 \mathrm{E}-03$ & 3334 & CR405857 & 2.82 & $5.09 \mathrm{E}-03$ & 3402 & CR406205 & 6.92 & $2.26 \mathrm{E}-04$ \\
\hline 3267 & CR391551 & 2.08 & $2.51 \mathrm{E}-02$ & 3335 & CR405859 & 4.31 & $3.75 \mathrm{E}-03$ & 3403 & CR406224 & 3.18 & $8.85 \mathrm{E}-03$ \\
\hline 3268 & CR391557 & 8.01 & $4.18 \mathrm{E}-03$ & 3336 & CR405864 & 2.79 & $2.46 \mathrm{E}-03$ & 3404 & CR406228 & 4.04 & $1.61 \mathrm{E}-03$ \\
\hline 3269 & CR391558 & 1.54 & $1.87 \mathrm{E}-02$ & 3337 & CR405870 & 6.04 & $9.39 \mathrm{E}-04$ & 3405 & CR406233 & 2.13 & $1.70 \mathrm{E}-02$ \\
\hline 3270 & CR391559 & 4.00 & $1.43 \mathrm{E}-02$ & 3338 & CR405872 & 1.89 & $4.34 \mathrm{E}-03$ & 3406 & CR406245 & 2.69 & $9.06 \mathrm{E}-03$ \\
\hline 3271 & CR391575 & 1.95 & $1.71 \mathrm{E}-02$ & 3339 & CR405876 & 1.79 & $3.39 \mathrm{E}-02$ & 3407 & CR406274 & 5.68 & 2.97E-04 \\
\hline 3272 & CR391577 & 1.72 & $2.80 \mathrm{E}-02$ & 3340 & CR405877 & 4.09 & $4.92 \mathrm{E}-04$ & 3408 & CR406285 & 6.76 & $8.18 \mathrm{E}-02$ \\
\hline 3273 & CR391582 & 4.85 & $5.86 \mathrm{E}-03$ & 3341 & CR405878 & 4.12 & $1.08 \mathrm{E}-02$ & 3409 & CR406295 & 1.93 & 4.73E-04 \\
\hline 3274 & CR391583 & 4.45 & $2.26 \mathrm{E}-03$ & 3342 & CR405880 & 6.78 & $6.43 \mathrm{E}-03$ & 3410 & CR406298 & 2.03 & $3.92 \mathrm{E}-02$ \\
\hline 3275 & CR391587 & 3.23 & $9.15 \mathrm{E}-03$ & 3343 & CR405882 & 5.25 & $2.21 \mathrm{E}-03$ & 3411 & CR406300 & 1.79 & 3.19E-03 \\
\hline 3276 & CR391589 & 5.80 & $1.83 \mathrm{E}-03$ & 3344 & CR405883 & 3.94 & $1.52 \mathrm{E}-02$ & 3412 & CR406317 & 4.14 & $1.00 E-04$ \\
\hline 3277 & CR391590 & 1.99 & $4.27 \mathrm{E}-02$ & 3345 & CR405885 & 4.27 & $2.55 \mathrm{E}-02$ & 3413 & CR406320 & 1.40 & $2.56 \mathrm{E}-02$ \\
\hline 3278 & CR391594 & 4.71 & $2.87 \mathrm{E}-03$ & 3346 & CR405889 & 3.54 & $1.15 \mathrm{E}-02$ & 3414 & CR406327 & 2.50 & $6.81 \mathrm{E}-03$ \\
\hline 3279 & CR391599 & 1.09 & $8.49 \mathrm{E}-03$ & 3347 & CR405891 & 4.38 & $5.88 \mathrm{E}-03$ & 3415 & CR406328 & 2.80 & $1.98 \mathrm{E}-02$ \\
\hline 3280 & CR391601 & 2.90 & $2.16 \mathrm{E}-03$ & 3348 & CR405894 & 5.56 & $8.40 \mathrm{E}-04$ & 3416 & CR406351 & 1.43 & $7.51 \mathrm{E}-03$ \\
\hline 3281 & CR391602 & 9.32 & $1.43 \mathrm{E}-03$ & 3349 & CR405897 & 4.98 & $2.61 \mathrm{E}-05$ & 3417 & CR406358 & 3.86 & $2.17 E-02$ \\
\hline 3282 & CR391606 & 1.43 & $3.70 \mathrm{E}-02$ & 3350 & CR405898 & 4.16 & $1.83 \mathrm{E}-04$ & 3418 & CR406361 & 5.99 & 4.96E-04 \\
\hline 3283 & CR391610 & 5.96 & $4.58 \mathrm{E}-03$ & 3351 & CR405900 & 5.47 & $6.01 \mathrm{E}-03$ & 3419 & CR406364 & 5.76 & 8.93E-04 \\
\hline 3284 & CR391612 & 1.27 & $6.77 \mathrm{E}-03$ & 3352 & CR405901 & 5.64 & $8.76 \mathrm{E}-03$ & 3420 & CR406371 & 5.70 & $1.82 \mathrm{E}-03$ \\
\hline 3285 & CR391630 & 1.45 & $7.75 E-02$ & 3353 & CR405904 & 2.62 & $2.34 \mathrm{E}-02$ & 3421 & CR406374 & 3.81 & $1.62 E-03$ \\
\hline 3286 & CR391647 & 4.21 & $9.11 \mathrm{E}-04$ & 3354 & CR405907 & 2.68 & 3.47E-02 & 3422 & CR406379 & 2.83 & $1.40 \mathrm{E}-02$ \\
\hline 3287 & CR391650 & 1.52 & $3.75 \mathrm{E}-03$ & 3355 & CR405914 & 4.27 & $1.78 \mathrm{E}-03$ & 3423 & CR406380 & 2.13 & 7.47E-03 \\
\hline 3288 & CR391655 & 4.81 & $4.06 \mathrm{E}-03$ & 3356 & CR405916 & 4.84 & $3.67 \mathrm{E}-05$ & 3424 & CR406383 & 3.91 & $7.00 \mathrm{E}-03$ \\
\hline 3289 & CR391658 & 1.37 & $1.61 \mathrm{E}-02$ & 3357 & CR405918 & 4.05 & $3.49 \mathrm{E}-03$ & 3425 & CR406388 & 2.84 & $3.18 \mathrm{E}-03$ \\
\hline 3290 & CR391665 & 5.18 & $3.03 E-03$ & 3358 & CR405919 & 1.13 & $6.05 \mathrm{E}-03$ & 3426 & CR406391 & 3.42 & $8.72 \mathrm{E}-04$ \\
\hline 3291 & CR391672 & 1.59 & $5.14 \mathrm{E}-02$ & 3359 & CR405930 & 3.67 & 4.69E-03 & 3427 & CR406392 & 1.13 & $1.85 \mathrm{E}-02$ \\
\hline 3292 & CR391673 & 1.42 & $2.92 \mathrm{E}-02$ & 3360 & CR405931 & 6.38 & $9.90 \mathrm{E}-04$ & 3428 & CR406401 & 3.21 & 5.94E-04 \\
\hline 3293 & CR391674 & 3.07 & $1.04 E-02$ & 3361 & CR405933 & 2.85 & $1.03 \mathrm{E}-03$ & 3429 & CR406417 & 3.67 & $1.43 \mathrm{E}-02$ \\
\hline 3294 & CR391683 & 2.87 & $7.25 \mathrm{E}-03$ & 3362 & CR405943 & 4.63 & $3.27 \mathrm{E}-03$ & 3430 & CR406420 & 3.13 & 2.93E-03 \\
\hline 3295 & CR391685 & 5.21 & $9.05 E-04$ & 3363 & CR405945 & 4.66 & $1.07 E-02$ & 3431 & CR406421 & 1.85 & $6.63 E-04$ \\
\hline 3296 & CR391688 & 4.00 & $4.01 E-03$ & 3364 & CR405949 & 6.45 & $3.51 \mathrm{E}-03$ & 3432 & CR406422 & 2.73 & $2.71 \mathrm{E}-02$ \\
\hline 3297 & CR391691 & 5.56 & 7.09E-04 & 3365 & CR405950 & 1.77 & $2.54 \mathrm{E}-03$ & 3433 & CR406423 & 3.10 & $6.07 E-03$ \\
\hline 3298 & CR391692 & 3.04 & $8.89 \mathrm{E}-03$ & 3366 & CR405951 & 5.94 & $8.57 \mathrm{E}-04$ & 3434 & CR406424 & 3.29 & $4.92 \mathrm{E}-03$ \\
\hline 3299 & CR391693 & 4.20 & $2.35 \mathrm{E}-03$ & 3367 & CR405955 & 4.34 & $1.07 \mathrm{E}-03$ & 3435 & CR406427 & 3.52 & $1.85 \mathrm{E}-02$ \\
\hline 3300 & CR391697 & 3.34 & $1.31 E-02$ & 3368 & CR405959 & 4.99 & $6.47 \mathrm{E}-04$ & 3436 & CR406432 & 4.46 & $1.87 \mathrm{E}-03$ \\
\hline 3301 & CR391699 & 3.94 & $1.52 \mathrm{E}-04$ & 3369 & CR405964 & 4.04 & $7.54 \mathrm{E}-03$ & 3437 & CR406468 & 4.45 & 7.09E-03 \\
\hline 3302 & CR391700 & 4.36 & $9.81 \mathrm{E}-03$ & 3370 & CR405975 & 3.05 & $1.78 \mathrm{E}-03$ & 3438 & CR406472 & 2.38 & $1.41 \mathrm{E}-03$ \\
\hline 3303 & CR391707 & 2.45 & $3.70 \mathrm{E}-02$ & 3371 & CR405979 & 5.69 & $2.34 \mathrm{E}-03$ & 3439 & CR406476 & 2.19 & $6.42 E-03$ \\
\hline 3304 & CR391712 & 4.26 & $1.67 \mathrm{E}-02$ & 3372 & CR405983 & 4.23 & $2.88 \mathrm{E}-03$ & 3440 & CR406477 & 1.22 & $1.43 \mathrm{E}-02$ \\
\hline 3305 & CR391722 & 3.47 & $5.18 \mathrm{E}-03$ & 3373 & CR405985 & 1.16 & $1.99 \mathrm{E}-02$ & 3441 & CR406515 & 3.76 & $5.33 \mathrm{E}-03$ \\
\hline 3306 & CR391728 & 1.99 & $8.63 \mathrm{E}-03$ & 3374 & CR405992 & 1.51 & $4.67 \mathrm{E}-02$ & 3442 & CR406517 & 6.12 & $5.68 \mathrm{E}-06$ \\
\hline 3307 & CR391732 & 2.72 & $1.10 \mathrm{E}-02$ & 3375 & CR405995 & 5.08 & $1.36 \mathrm{E}-03$ & 3443 & CR406522 & 6.57 & $1.25 \mathrm{E}-03$ \\
\hline 3308 & CR391738 & 3.63 & $7.53 \mathrm{E}-04$ & 3376 & CR406005 & 2.67 & $1.90 \mathrm{E}-02$ & 3444 & CR406528 & 2.89 & 3.14E-02 \\
\hline 3309 & CR391740 & 5.67 & $1.74 E-04$ & 3377 & CR406012 & 1.93 & $1.90 \mathrm{E}-03$ & 3445 & CR406529 & 5.53 & $2.26 \mathrm{E}-03$ \\
\hline 3310 & CR391741 & 1.68 & $5.05 \mathrm{E}-03$ & 3378 & CR406023 & 4.79 & $9.37 \mathrm{E}-03$ & 3446 & CR406536 & 3.05 & $1.55 \mathrm{E}-02$ \\
\hline 3311 & CR391742 & 1.79 & $5.42 \mathrm{E}-03$ & 3379 & CR406027 & 3.77 & $1.66 \mathrm{E}-03$ & 3447 & CR406540 & 3.22 & $3.30 \mathrm{E}-02$ \\
\hline 3312 & CR391743 & 1.28 & $2.99 \mathrm{E}-02$ & 3380 & CR406032 & 3.19 & $8.83 \mathrm{E}-03$ & 3448 & CR406545 & 3.42 & $9.44 \mathrm{E}-04$ \\
\hline 3313 & CR391744 & 3.18 & $1.22 \mathrm{E}-03$ & 3381 & CR406034 & 8.48 & $2.29 \mathrm{E}-03$ & 3449 & CR406549 & 2.14 & $3.20 \mathrm{E}-03$ \\
\hline 3314 & CR391750 & 1.87 & $1.38 \mathrm{E}-02$ & 3382 & CR406035 & 3.27 & $9.31 \mathrm{E}-03$ & 3450 & CR406551 & 6.03 & 4.42E-04 \\
\hline 3315 & CR391761 & 4.49 & $2.04 E-03$ & 3383 & CR406060 & 3.09 & $4.98 \mathrm{E}-03$ & 3451 & CR406552 & 2.03 & 7.09E-03 \\
\hline 3316 & CR391762 & 2.23 & $1.65 \mathrm{E}-04$ & 3384 & CR406085 & 3.47 & $1.95 \mathrm{E}-03$ & 3452 & CR406574 & 5.15 & 3.44E-04 \\
\hline 3317 & CR405726 & 6.82 & $4.50 \mathrm{E}-03$ & 3385 & CR406091 & 4.94 & $4.19 \mathrm{E}-02$ & 3453 & CR406576 & 2.81 & $7.30 \mathrm{E}-03$ \\
\hline 3318 & CR405736 & 5.04 & $1.14 \mathrm{E}-03$ & 3386 & CR406102 & 2.25 & $2.75 \mathrm{E}-03$ & 3454 & CR406579 & 5.20 & 3.14E-04 \\
\hline 3319 & CR405739 & 1.64 & $6.23 \mathrm{E}-03$ & 3387 & CR406106 & 2.57 & $6.58 \mathrm{E}-03$ & 3455 & CR406585 & 1.53 & $6.02 \mathrm{E}-02$ \\
\hline 3320 & CR405746 & 7.89 & $3.20 \mathrm{E}-04$ & 3388 & CR406117 & 5.43 & $5.29 \mathrm{E}-03$ & 3456 & CR406587 & 1.91 & $3.05 E-03$ \\
\hline 3321 & CR405750 & 2.92 & $2.07 E-05$ & 3389 & CR406118 & 5.53 & $1.39 \mathrm{E}-03$ & 3457 & CR406589 & 2.51 & $1.56 \mathrm{E}-02$ \\
\hline 3322 & CR405755 & 2.90 & $8.30 \mathrm{E}-03$ & 3390 & CR406135 & 1.71 & $1.42 \mathrm{E}-02$ & 3458 & CR406593 & 2.78 & $3.03 E-03$ \\
\hline 3323 & CR405767 & 2.30 & $2.60 \mathrm{E}-03$ & 3391 & CR406145 & 2.73 & $4.69 \mathrm{E}-03$ & 3459 & CR406597 & 7.64 & 1.12E-04 \\
\hline 3324 & CR405769 & 1.87 & $1.08 \mathrm{E}-03$ & 3392 & CR406159 & 5.76 & $4.54 \mathrm{E}-03$ & 3460 & CR406602 & 6.41 & $5.51 \mathrm{E}-03$ \\
\hline 3325 & CR405785 & 3.32 & $1.08 \mathrm{E}-01$ & 3393 & CR406171 & 2.14 & $1.43 \mathrm{E}-03$ & 3461 & CR406611 & 4.94 & $1.53 \mathrm{E}-03$ \\
\hline 3326 & CR405799 & 1.71 & $1.72 \mathrm{E}-02$ & 3394 & CR406172 & 3.79 & $8.75 \mathrm{E}-03$ & 3462 & CR406613 & 4.70 & $1.95 \mathrm{E}-03$ \\
\hline 3327 & CR405809 & 1.57 & $2.60 \mathrm{E}-02$ & 3395 & CR406174 & 3.37 & $6.63 \mathrm{E}-03$ & 3463 & CR406620 & 1.32 & $8.77 E-03$ \\
\hline 3328 & CR405812 & 4.60 & $4.27 \mathrm{E}-03$ & 3396 & CR406186 & 4.87 & $3.25 \mathrm{E}-03$ & 3464 & CR406624 & 3.80 & $2.62 \mathrm{E}-02$ \\
\hline 3329 & CR405822 & 1.76 & $8.17 \mathrm{E}-03$ & 3397 & CR406187 & 2.89 & $2.63 \mathrm{E}-02$ & 3465 & CR406638 & 1.96 & 2.93E-04 \\
\hline 3330 & CR405838 & 3.85 & $8.24 E-03$ & 3398 & CR406188 & 6.63 & $6.83 \mathrm{E}-05$ & 3466 & CR406640 & 3.63 & $8.85 \mathrm{E}-03$ \\
\hline 3331 & CR405845 & 2.47 & $4.74 \mathrm{E}-03$ & 3399 & CR406189 & 2.82 & $6.72 \mathrm{E}-03$ & 3467 & CR406647 & 5.45 & $8.62 E-04$ \\
\hline
\end{tabular}




\begin{tabular}{|c|c|c|c|c|c|c|c|c|c|c|c|}
\hline 3468 & CR406654 & 2.66 & $8.05 \mathrm{E}-04$ & 3536 & CR406929 & 3.95 & $6.61 \mathrm{E}-03$ & 3604 & CR407204 & 5.11 & $1.02 E-02$ \\
\hline 3469 & CR406655 & 3.60 & $1.43 \mathrm{E}-03$ & 3537 & CR406933 & 5.54 & $3.51 \mathrm{E}-04$ & 3605 & CR407206 & 2.55 & $2.80 \mathrm{E}-03$ \\
\hline 3470 & CR406661 & 2.52 & $2.40 \mathrm{E}-02$ & 3538 & CR406944 & 3.81 & $6.65 \mathrm{E}-02$ & 3606 & CR407212 & 3.54 & $3.33 \mathrm{E}-03$ \\
\hline 3471 & CR406662 & 3.14 & $1.07 \mathrm{E}-03$ & 3539 & CR406947 & 2.57 & $3.28 \mathrm{E}-02$ & 3607 & CR407216 & 7.42 & 5.73E-04 \\
\hline 3472 & CR406666 & 2.45 & $4.71 \mathrm{E}-03$ & 3540 & CR406949 & 1.40 & $5.03 E-02$ & 3608 & CR407219 & 3.20 & $1.17 \mathrm{E}-03$ \\
\hline 3473 & CR406669 & 1.69 & $1.97 \mathrm{E}-02$ & 3541 & CR406955 & 6.08 & $4.22 \mathrm{E}-03$ & 3609 & CR407223 & 2.31 & $5.44 \mathrm{E}-03$ \\
\hline 3474 & CR406670 & 1.83 & 3.05E-03 & 3542 & CR406963 & 4.52 & $1.41 \mathrm{E}-03$ & 3610 & CR407228 & 2.97 & $7.95 \mathrm{E}-03$ \\
\hline 3475 & CR406673 & 2.44 & 7.14E-02 & 3543 & CR406969 & 4.03 & $1.23 \mathrm{E}-02$ & 3611 & CR407231 & 1.81 & $2.27 \mathrm{E}-03$ \\
\hline 3476 & CR406674 & 3.98 & $2.23 \mathrm{E}-03$ & 3544 & CR406970 & 4.71 & $8.81 \mathrm{E}-03$ & 3612 & CR407236 & 1.84 & $4.68 \mathrm{E}-03$ \\
\hline 3477 & CR406675 & 1.23 & $6.73 \mathrm{E}-02$ & 3545 & CR406972 & 3.89 & $6.64 \mathrm{E}-04$ & 3613 & CR407249 & 3.15 & $3.84 \mathrm{E}-03$ \\
\hline 3478 & CR406677 & 2.68 & $1.95 \mathrm{E}-02$ & 3546 & CR406976 & 5.04 & $4.01 \mathrm{E}-03$ & 3614 & CR407253 & 2.63 & $5.41 \mathrm{E}-03$ \\
\hline 3479 & CR406682 & 2.13 & $1.46 \mathrm{E}-02$ & 3547 & CR406977 & 3.34 & $1.70 \mathrm{E}-02$ & 3615 & CR407255 & 1.39 & $3.67 \mathrm{E}-02$ \\
\hline 3480 & CR406690 & 1.65 & $8.79 \mathrm{E}-03$ & 3548 & CR406980 & 3.79 & $5.35 \mathrm{E}-03$ & 3616 & CR407268 & 1.37 & $7.75 E-02$ \\
\hline 3481 & CR406694 & 2.22 & $6.58 \mathrm{E}-03$ & 3549 & CR406981 & 5.37 & 7.50E-02 & 3617 & CR407295 & 2.72 & $1.07 E-02$ \\
\hline 3482 & CR406699 & 1.68 & $4.35 \mathrm{E}-03$ & 3550 & CR406985 & 2.63 & $5.57 \mathrm{E}-03$ & 3618 & CR407299 & 3.58 & $2.10 \mathrm{E}-02$ \\
\hline 3483 & CR406700 & 1.76 & $3.27 \mathrm{E}-03$ & 3551 & CR406988 & 4.08 & $7.91 \mathrm{E}-03$ & 3619 & CR407311 & 3.98 & $1.19 \mathrm{E}-03$ \\
\hline 3484 & CR406709 & 2.25 & $8.48 \mathrm{E}-02$ & 3552 & CR406989 & 5.79 & $6.84 \mathrm{E}-03$ & 3620 & CR407314 & 1.73 & $2.90 \mathrm{E}-03$ \\
\hline 3485 & CR406712 & 1.71 & $1.47 \mathrm{E}-02$ & 3553 & CR406990 & 4.59 & 3.16E-03 & 3621 & CR407317 & 1.45 & $1.13 \mathrm{E}-02$ \\
\hline 3486 & CR406713 & 3.61 & $8.17 \mathrm{E}-03$ & 3554 & CR406994 & 1.29 & $5.05 E-02$ & 3622 & CR407320 & 1.34 & $2.05 \mathrm{E}-04$ \\
\hline 3487 & CR406718 & 1.50 & $2.35 \mathrm{E}-02$ & 3555 & CR406996 & 2.33 & $1.31 \mathrm{E}-03$ & 3623 & CR407324 & 3.32 & $8.40 \mathrm{E}-03$ \\
\hline 3488 & CR406723 & 4.82 & $2.64 \mathrm{E}-03$ & 3556 & CR406998 & 2.67 & $6.66 \mathrm{E}-03$ & 3624 & CR407327 & 3.55 & $1.74 \mathrm{E}-03$ \\
\hline 3489 & CR406734 & 1.25 & $3.35 \mathrm{E}-02$ & 3557 & CR407002 & 1.50 & $4.83 E-02$ & 3625 & CR407329 & 5.00 & $1.95 \mathrm{E}-03$ \\
\hline 3490 & CR406735 & 3.38 & 4.07E-04 & 3558 & CR407003 & 2.81 & $1.28 \mathrm{E}-03$ & 3626 & CR407331 & 4.05 & $8.06 \mathrm{E}-03$ \\
\hline 3491 & CR406736 & 2.66 & $6.58 \mathrm{E}-03$ & 3559 & CR407005 & 10.56 & $8.66 \mathrm{E}-04$ & 3627 & CR407337 & 6.71 & $1.37 \mathrm{E}-03$ \\
\hline 3492 & CR406739 & 4.99 & $4.10 \mathrm{E}-03$ & 3560 & CR407008 & 3.22 & $1.85 \mathrm{E}-03$ & 3628 & CR407349 & 4.96 & $8.27 \mathrm{E}-04$ \\
\hline 3493 & CR406740 & 1.20 & $2.74 \mathrm{E}-02$ & 3561 & CR407010 & 4.04 & $4.87 \mathrm{E}-03$ & 3629 & CR407367 & 2.52 & 5.27E-04 \\
\hline 3494 & CR406747 & 1.65 & $4.20 \mathrm{E}-02$ & 3562 & CR407011 & 4.94 & $1.37 \mathrm{E}-02$ & 3630 & CR407371 & 1.15 & $2.56 \mathrm{E}-01$ \\
\hline 3495 & CR406750 & 3.59 & $3.84 \mathrm{E}-04$ & 3563 & CR407013 & 2.64 & $7.72 \mathrm{E}-03$ & 3631 & CR407372 & 2.89 & 5.37E-02 \\
\hline 3496 & CR406753 & 1.76 & $2.86 \mathrm{E}-03$ & 3564 & CR407017 & 3.86 & 2.03E-03 & 3632 & CR407373 & 3.83 & $1.89 \mathrm{E}-03$ \\
\hline 3497 & CR406754 & 5.20 & $6.64 \mathrm{E}-03$ & 3565 & CR407025 & 3.89 & $1.43 \mathrm{E}-03$ & 3633 & CR407381 & 2.37 & $1.41 \mathrm{E}-03$ \\
\hline 3498 & CR406756 & 3.93 & $1.36 \mathrm{E}-02$ & 3566 & CR407027 & 3.84 & $2.15 \mathrm{E}-03$ & 3634 & CR407382 & 1.72 & $1.11 \mathrm{E}-01$ \\
\hline 3499 & CR406760 & 5.96 & $2.81 E-03$ & 3567 & CR407029 & 1.61 & 4.69E-02 & 3635 & CR407394 & 2.79 & $3.58 \mathrm{E}-03$ \\
\hline 3500 & CR406761 & 1.79 & $2.21 \mathrm{E}-03$ & 3568 & CR407030 & 5.17 & $3.98 \mathrm{E}-03$ & 3636 & CR407395 & 4.66 & $4.68 \mathrm{E}-03$ \\
\hline 3501 & CR406764 & 1.60 & $4.72 \mathrm{E}-03$ & 3569 & CR407033 & 4.48 & $7.42 \mathrm{E}-04$ & 3637 & CR407397 & 2.74 & $1.49 \mathrm{E}-02$ \\
\hline 3502 & CR406765 & 4.73 & $3.63 \mathrm{E}-03$ & 3570 & CR407034 & 3.02 & $2.10 \mathrm{E}-03$ & 3638 & CR407398 & 4.52 & $3.85 \mathrm{E}-03$ \\
\hline 3503 & CR406769 & 1.46 & $1.69 \mathrm{E}-05$ & 3571 & CR407035 & 4.45 & $4.00 \mathrm{E}-03$ & 3639 & CR407402 & 1.73 & $8.39 \mathrm{E}-04$ \\
\hline 3504 & CR406785 & 4.16 & $1.10 \mathrm{E}-03$ & 3572 & CR407036 & 3.67 & $2.80 \mathrm{E}-03$ & 3640 & CR407404 & 3.41 & $1.13 \mathrm{E}-02$ \\
\hline 3505 & CR406787 & 7.94 & $4.90 \mathrm{E}-03$ & 3573 & CR407037 & 5.49 & $6.01 \mathrm{E}-04$ & 3641 & CR407407 & 2.71 & $4.27 \mathrm{E}-03$ \\
\hline 3506 & CR406791 & 1.48 & $8.17 \mathrm{E}-03$ & 3574 & CR407043 & 4.83 & $5.75 \mathrm{E}-03$ & 3642 & CR407412 & 6.77 & $8.04 \mathrm{E}-04$ \\
\hline 3507 & CR406793 & 3.19 & $5.81 \mathrm{E}-03$ & 3575 & CR407047 & 1.90 & $1.00 \mathrm{E}-02$ & 3643 & CR407417 & 1.50 & $4.09 \mathrm{E}-03$ \\
\hline 3508 & CR406794 & 1.91 & $8.54 \mathrm{E}-03$ & 3576 & CR407062 & 4.03 & $3.87 \mathrm{E}-04$ & 3644 & CR407422 & 2.35 & $1.66 \mathrm{E}-02$ \\
\hline 3509 & CR406802 & 1.41 & $2.28 \mathrm{E}-02$ & 3577 & CR407074 & 1.16 & $1.12 \mathrm{E}-02$ & 3645 & CR407423 & 8.67 & $1.32 \mathrm{E}-04$ \\
\hline 3510 & CR406814 & 4.04 & $6.35 \mathrm{E}-03$ & 3578 & CR407086 & 4.91 & $3.31 \mathrm{E}-03$ & 3646 & CR407425 & 4.33 & 5.97E-04 \\
\hline 3511 & CR406817 & 1.62 & $4.80 \mathrm{E}-03$ & 3579 & CR407090 & 1.91 & $1.78 \mathrm{E}-02$ & 3647 & CR407434 & 4.74 & $7.85 \mathrm{E}-04$ \\
\hline 3512 & CR406828 & 3.48 & $7.62 \mathrm{E}-03$ & 3580 & CR407098 & 1.26 & $1.02 \mathrm{E}-02$ & 3648 & CR407435 & 1.57 & $3.44 \mathrm{E}-02$ \\
\hline 3513 & CR406830 & 3.05 & $1.01 E-02$ & 3581 & CR407110 & 2.60 & $4.70 \mathrm{E}-04$ & 3649 & CR407436 & 1.71 & $6.94 \mathrm{E}-02$ \\
\hline 3514 & CR406834 & 2.42 & $3.84 \mathrm{E}-03$ & 3582 & CR407112 & 5.53 & $1.20 \mathrm{E}-05$ & 3650 & CR407448 & 4.82 & $8.16 \mathrm{E}-04$ \\
\hline 3515 & CR406842 & 3.08 & $2.71 \mathrm{E}-03$ & 3583 & CR407117 & 4.79 & $8.87 \mathrm{E}-03$ & 3651 & CR407449 & 2.10 & $6.57 \mathrm{E}-02$ \\
\hline 3516 & CR406854 & 2.18 & $2.30 \mathrm{E}-02$ & 3584 & CR407118 & 3.31 & $1.55 \mathrm{E}-04$ & 3652 & CR407453 & 6.26 & $1.27 \mathrm{E}-02$ \\
\hline 3517 & CR406856 & 5.91 & 7.33E-04 & 3585 & CR407119 & 2.01 & $1.46 \mathrm{E}-02$ & 3653 & CR407471 & 2.23 & $1.98 \mathrm{E}-02$ \\
\hline 3518 & CR406863 & 2.10 & $5.18 \mathrm{E}-03$ & 3586 & CR407125 & 3.23 & $1.04 \mathrm{E}-02$ & 3654 & CR407514 & 4.18 & $2.75 \mathrm{E}-03$ \\
\hline 3519 & CR406871 & 6.80 & $2.44 \mathrm{E}-04$ & 3587 & CR407131 & 2.44 & $2.39 \mathrm{E}-02$ & 3655 & CR407518 & 2.04 & $4.49 \mathrm{E}-02$ \\
\hline 3520 & CR406875 & 1.60 & $1.89 \mathrm{E}-03$ & 3588 & CR407136 & 2.25 & $6.54 \mathrm{E}-02$ & 3656 & CR407519 & 5.48 & $1.15 \mathrm{E}-02$ \\
\hline 3521 & CR406881 & 2.59 & $2.33 \mathrm{E}-03$ & 3589 & CR407138 & 3.94 & $6.77 \mathrm{E}-04$ & 3657 & CR407520 & 2.41 & $2.65 \mathrm{E}-02$ \\
\hline 3522 & CR406882 & 2.53 & $5.22 \mathrm{E}-03$ & 3590 & CR407141 & 4.15 & $5.55 \mathrm{E}-03$ & 3658 & CR407522 & 5.01 & $6.55 \mathrm{E}-05$ \\
\hline 3523 & CR406885 & 2.51 & $3.55 \mathrm{E}-02$ & 3591 & CR407143 & 1.65 & $6.81 \mathrm{E}-02$ & 3659 & CR407523 & 5.34 & $1.31 \mathrm{E}-03$ \\
\hline 3524 & CR406889 & 2.98 & $7.77 \mathrm{E}-02$ & 3592 & CR407145 & 4.68 & $8.96 \mathrm{E}-03$ & 3660 & CR407524 & 2.59 & $3.14 \mathrm{E}-02$ \\
\hline 3525 & CR406892 & 3.25 & $8.24 \mathrm{E}-03$ & 3593 & CR407147 & 2.28 & $2.56 \mathrm{E}-03$ & 3661 & CR407530 & 1.89 & $2.22 \mathrm{E}-02$ \\
\hline 3526 & CR406893 & 3.32 & $1.63 \mathrm{E}-03$ & 3594 & CR407149 & 2.93 & $1.92 \mathrm{E}-02$ & 3662 & CR407535 & 6.54 & $5.00 \mathrm{E}-03$ \\
\hline 3527 & CR406898 & 4.70 & $4.78 \mathrm{E}-04$ & 3595 & CR407150 & 4.57 & $1.88 \mathrm{E}-03$ & 3663 & CR407539 & 2.98 & $3.07 \mathrm{E}-03$ \\
\hline 3528 & CR406901 & 3.85 & $1.55 \mathrm{E}-03$ & 3596 & CR407159 & 3.39 & $1.20 \mathrm{E}-04$ & 3664 & CR407541 & 3.83 & $2.14 \mathrm{E}-03$ \\
\hline 3529 & CR406904 & 6.26 & $5.66 \mathrm{E}-03$ & 3597 & CR407162 & 1.50 & $1.13 \mathrm{E}-02$ & 3665 & CR522887 & 2.25 & $5.60 \mathrm{E}-02$ \\
\hline 3530 & CR406908 & 4.67 & $4.28 \mathrm{E}-03$ & 3598 & CR407175 & 2.88 & $3.98 \mathrm{E}-02$ & 3666 & CR522896 & 1.64 & $1.23 \mathrm{E}-02$ \\
\hline 3531 & CR406914 & 5.49 & $3.66 \mathrm{E}-03$ & 3599 & CR407177 & 3.07 & $8.45 \mathrm{E}-03$ & 3667 & CR522898 & 1.79 & $6.58 \mathrm{E}-04$ \\
\hline 3532 & CR406917 & 4.26 & $5.22 \mathrm{E}-04$ & 3600 & CR407185 & 1.72 & $2.48 \mathrm{E}-03$ & 3668 & CR522914 & 1.82 & $1.40 \mathrm{E}-01$ \\
\hline 3533 & CR406919 & 5.18 & $3.49 \mathrm{E}-03$ & 3601 & CR407188 & 1.29 & $2.40 \mathrm{E}-03$ & 3669 & CR522919 & 5.50 & $1.74 \mathrm{E}-03$ \\
\hline 3534 & CR406922 & 3.25 & $1.20 \mathrm{E}-02$ & 3602 & CR407195 & 1.70 & $4.59 \mathrm{E}-02$ & 3670 & CR522926 & 3.41 & $6.41 \mathrm{E}-03$ \\
\hline 3535 & CR406928 & 1.36 & $1.13 \mathrm{E}-02$ & 3603 & CR407201 & 5.31 & $7.66 \mathrm{E}-04$ & 3671 & CR522927 & 5.81 & 2.37E-04 \\
\hline
\end{tabular}




\begin{tabular}{|c|c|c|c|c|c|c|c|c|c|c|c|}
\hline 3672 & CR522928 & 4.86 & $9.19 \mathrm{E}-04$ & 3740 & CR523236 & 4.69 & $7.03 \mathrm{E}-03$ & 3808 & CR523647 & 4.41 & $2.80 \mathrm{E}-02$ \\
\hline 3673 & CR522932 & 4.47 & $4.67 \mathrm{E}-03$ & 3741 & CR523249 & 3.45 & $2.28 \mathrm{E}-03$ & 3809 & CR523653 & 4.74 & 7.59E-03 \\
\hline 3674 & CR522935 & 1.54 & $8.99 \mathrm{E}-04$ & 3742 & CR523251 & 3.06 & $5.14 \mathrm{E}-03$ & 3810 & CR523656 & 1.87 & $2.10 \mathrm{E}-03$ \\
\hline 3675 & CR522936 & 1.82 & $1.11 \mathrm{E}-02$ & 3743 & CR523253 & 1.88 & $2.51 \mathrm{E}-02$ & 3811 & CR523660 & 1.48 & $1.18 \mathrm{E}-02$ \\
\hline 3676 & CR522939 & 3.43 & $4.86 \mathrm{E}-03$ & 3744 & CR523267 & 3.04 & $8.21 \mathrm{E}-04$ & 3812 & CR523663 & 6.47 & $3.48 \mathrm{E}-03$ \\
\hline 3677 & CR522943 & 5.68 & 7.47E-04 & 3745 & CR523285 & 2.20 & $2.21 \mathrm{E}-02$ & 3813 & CR523665 & 1.62 & $1.04 \mathrm{E}-03$ \\
\hline 3678 & CR522944 & 4.78 & $9.13 \mathrm{E}-03$ & 3746 & CR523309 & 3.24 & $5.58 \mathrm{E}-03$ & 3814 & CR523667 & 1.71 & $3.99 \mathrm{E}-02$ \\
\hline 3679 & CR522945 & 1.99 & $1.86 \mathrm{E}-02$ & 3747 & CR523322 & 6.10 & $3.46 \mathrm{E}-03$ & 3815 & CR523670 & 3.80 & $2.81 \mathrm{E}-02$ \\
\hline 3680 & CR522948 & 4.00 & $3.95 \mathrm{E}-03$ & 3748 & CR523323 & 3.81 & $1.27 \mathrm{E}-02$ & 3816 & CR523671 & 5.09 & $2.81 \mathrm{E}-04$ \\
\hline 3681 & CR522958 & 4.64 & $6.95 \mathrm{E}-03$ & 3749 & CR523333 & 3.67 & $4.63 \mathrm{E}-04$ & 3817 & CR523686 & 3.14 & $2.89 \mathrm{E}-03$ \\
\hline 3682 & CR522964 & 3.73 & $2.28 \mathrm{E}-03$ & 3750 & CR523334 & 2.11 & $4.19 \mathrm{E}-02$ & 3818 & CR523691 & 5.00 & 9.54E-04 \\
\hline 3683 & CR522968 & 1.88 & $2.15 \mathrm{E}-03$ & 3751 & CR523342 & 5.40 & $3.08 \mathrm{E}-03$ & 3819 & CR523697 & 4.34 & $5.46 E-03$ \\
\hline 3684 & CR522969 & 7.49 & $1.98 \mathrm{E}-04$ & 3752 & CR523344 & 5.77 & $1.28 \mathrm{E}-02$ & 3820 & CR523700 & 4.10 & 4.12E-04 \\
\hline 3685 & CR522972 & 2.74 & $8.96 \mathrm{E}-03$ & 3753 & CR523347 & 3.36 & $2.98 \mathrm{E}-05$ & 3821 & CR523702 & 3.79 & $1.99 \mathrm{E}-02$ \\
\hline 3686 & CR522980 & 1.94 & $3.13 \mathrm{E}-02$ & 3754 & CR523348 & 7.05 & $2.60 \mathrm{E}-05$ & 3822 & CR523703 & 6.63 & $4.78 \mathrm{E}-03$ \\
\hline 3687 & CR522982 & 1.98 & $2.22 \mathrm{E}-02$ & 3755 & CR523352 & 3.09 & $1.78 \mathrm{E}-02$ & 3823 & CR523704 & 3.42 & 5.56E-04 \\
\hline 3688 & CR522984 & 1.75 & $3.98 \mathrm{E}-02$ & 3756 & CR523366 & 1.87 & $1.30 \mathrm{E}-03$ & 3824 & CR523706 & 2.21 & 4.42E-03 \\
\hline 3689 & CR522987 & 1.37 & $2.03 \mathrm{E}-02$ & 3757 & CR523370 & 4.82 & $1.40 \mathrm{E}-02$ & 3825 & CR523709 & 3.78 & $2.62 \mathrm{E}-05$ \\
\hline 3690 & CR522989 & 2.11 & $2.80 \mathrm{E}-03$ & 3758 & CR523375 & 2.75 & 3.59E-02 & 3826 & CR523718 & 2.24 & $1.61 \mathrm{E}-02$ \\
\hline 3691 & CR522993 & 3.60 & $1.46 \mathrm{E}-03$ & 3759 & CR523378 & 3.32 & $5.70 \mathrm{E}-05$ & 3827 & CR523721 & 4.01 & $1.73 \mathrm{E}-02$ \\
\hline 3692 & CR522999 & 6.89 & $1.38 \mathrm{E}-05$ & 3760 & CR523385 & 3.41 & $4.13 \mathrm{E}-03$ & 3828 & CR523731 & 4.28 & $1.73 \mathrm{E}-03$ \\
\hline 3693 & CR523010 & 2.69 & $9.86 \mathrm{E}-03$ & 3761 & CR523387 & 1.93 & $1.61 \mathrm{E}-02$ & 3829 & CR523740 & 2.78 & $3.06 E-04$ \\
\hline 3694 & CR523015 & 2.62 & $2.45 \mathrm{E}-03$ & 3762 & CR523389 & 2.96 & $1.74 \mathrm{E}-04$ & 3830 & CR523741 & 2.83 & $5.11 \mathrm{E}-03$ \\
\hline 3695 & CR523019 & 2.35 & $7.06 \mathrm{E}-03$ & 3763 & CR523391 & 1.96 & $2.50 \mathrm{E}-02$ & 3831 & CR523751 & 4.70 & $1.37 \mathrm{E}-02$ \\
\hline 3696 & CR523021 & 1.68 & $9.69 \mathrm{E}-02$ & 3764 & CR523393 & 3.00 & $6.28 \mathrm{E}-03$ & 3832 & CR523752 & 2.75 & $7.67 E-04$ \\
\hline 3697 & CR523024 & 2.86 & $4.67 \mathrm{E}-03$ & 3765 & CR523405 & 2.01 & $3.40 \mathrm{E}-02$ & 3833 & CR523757 & 5.10 & $1.78 \mathrm{E}-02$ \\
\hline 3698 & CR523029 & 3.13 & $6.32 \mathrm{E}-03$ & 3766 & CR523410 & 1.30 & $9.37 \mathrm{E}-03$ & 3834 & CR523769 & 4.77 & $1.66 \mathrm{E}-03$ \\
\hline 3699 & CR523032 & 1.49 & $1.29 \mathrm{E}-02$ & 3767 & CR523417 & 3.11 & $7.60 \mathrm{E}-03$ & 3835 & CR523775 & 3.08 & $1.37 \mathrm{E}-03$ \\
\hline 3700 & CR523040 & 1.77 & $1.64 \mathrm{E}-02$ & 3768 & CR523422 & 4.88 & $3.61 \mathrm{E}-03$ & 3836 & CR523777 & 2.09 & $8.87 E-03$ \\
\hline 3701 & CR523051 & 2.98 & $3.19 \mathrm{E}-03$ & 3769 & CR523430 & 1.58 & $1.93 \mathrm{E}-02$ & 3837 & CR523791 & 1.71 & $6.14 \mathrm{E}-03$ \\
\hline 3702 & CR523054 & 2.39 & $5.53 \mathrm{E}-03$ & 3770 & CR523437 & 1.42 & $5.71 \mathrm{E}-03$ & 3838 & CR523796 & 4.78 & 2.14E-04 \\
\hline 3703 & CR523055 & 5.41 & $1.36 \mathrm{E}-02$ & 3771 & CR523439 & 3.20 & $1.56 \mathrm{E}-02$ & 3839 & CR523797 & 3.63 & $2.14 \mathrm{E}-03$ \\
\hline 3704 & CR523058 & 3.30 & $1.34 \mathrm{E}-03$ & 3772 & CR523440 & 3.91 & $1.05 \mathrm{E}-03$ & 3840 & CR523800 & 3.49 & $2.73 E-03$ \\
\hline 3705 & CR523069 & 1.36 & $3.12 \mathrm{E}-03$ & 3773 & CR523442 & 2.36 & $3.49 \mathrm{E}-03$ & 3841 & CR523804 & 3.52 & $2.62 \mathrm{E}-02$ \\
\hline 3706 & CR523072 & 2.60 & $7.85 \mathrm{E}-03$ & 3774 & CR523462 & 6.31 & $6.64 \mathrm{E}-05$ & 3842 & CR523815 & 3.45 & $1.29 \mathrm{E}-03$ \\
\hline 3707 & CR523075 & 4.72 & $8.70 \mathrm{E}-03$ & 3775 & CR523465 & 3.15 & $4.15 \mathrm{E}-02$ & 3843 & CR523819 & 4.06 & $1.34 \mathrm{E}-03$ \\
\hline 3708 & CR523090 & 2.84 & $1.06 E-02$ & 3776 & CR523478 & 4.16 & $3.30 \mathrm{E}-03$ & 3844 & CR523824 & 3.11 & $1.03 \mathrm{E}-02$ \\
\hline 3709 & CR523091 & 4.21 & $8.32 \mathrm{E}-03$ & 3777 & CR523482 & 4.38 & $2.36 \mathrm{E}-03$ & 3845 & CR523830 & 2.75 & $5.50 \mathrm{E}-03$ \\
\hline 3710 & CR523103 & 5.12 & $1.02 \mathrm{E}-03$ & 3778 & CR523485 & 2.77 & $9.39 \mathrm{E}-03$ & 3846 & CR523834 & 2.47 & $4.42 E-03$ \\
\hline 3711 & CR523104 & 3.53 & $1.05 \mathrm{E}-03$ & 3779 & CR523494 & 2.92 & $3.51 \mathrm{E}-02$ & 3847 & CR523835 & 3.62 & $8.26 \mathrm{E}-02$ \\
\hline 3712 & CR523108 & 2.03 & $1.25 \mathrm{E}-02$ & 3780 & CR523502 & 3.55 & $2.12 \mathrm{E}-02$ & 3848 & CR523838 & 4.27 & $6.81 \mathrm{E}-04$ \\
\hline 3713 & CR523109 & 2.72 & $1.97 \mathrm{E}-02$ & 3781 & CR523509 & 1.96 & $9.05 \mathrm{E}-03$ & 3849 & CR523843 & 5.35 & $5.56 \mathrm{E}-04$ \\
\hline 3714 & CR523110 & 2.80 & $1.22 \mathrm{E}-02$ & 3782 & CR523512 & 4.73 & $2.96 \mathrm{E}-03$ & 3850 & CR523845 & 3.33 & $9.26 \mathrm{E}-03$ \\
\hline 3715 & CR523117 & 3.17 & $1.83 \mathrm{E}-02$ & 3783 & CR523536 & 4.99 & $9.48 \mathrm{E}-03$ & 3851 & CR523851 & 3.81 & 7.69E-04 \\
\hline 3716 & CR523118 & 3.49 & $2.67 \mathrm{E}-03$ & 3784 & CR523544 & 2.08 & $2.81 \mathrm{E}-03$ & 3852 & CR523854 & 6.04 & $2.87 \mathrm{E}-03$ \\
\hline 3717 & CR523129 & 6.36 & $2.04 E-05$ & 3785 & CR523546 & 3.33 & $7.31 \mathrm{E}-03$ & 3853 & CR523855 & 3.66 & $2.02 \mathrm{E}-02$ \\
\hline 3718 & CR523130 & 2.86 & $1.78 \mathrm{E}-04$ & 3786 & CR523551 & 1.65 & $5.63 \mathrm{E}-02$ & 3854 & CR523861 & 3.27 & $3.13 E-02$ \\
\hline 3719 & CR523133 & 1.78 & $7.23 \mathrm{E}-02$ & 3787 & CR523552 & 3.88 & $1.82 \mathrm{E}-03$ & 3855 & CR523865 & 3.28 & $4.95 \mathrm{E}-03$ \\
\hline 3720 & CR523134 & 4.03 & $3.82 E-05$ & 3788 & CR523559 & 3.58 & $5.37 \mathrm{E}-03$ & 3856 & CR523885 & 7.28 & $1.40 \mathrm{E}-03$ \\
\hline 3721 & CR523148 & 5.68 & $1.88 \mathrm{E}-03$ & 3789 & CR523564 & 3.04 & $1.20 \mathrm{E}-03$ & 3857 & CR523887 & 2.74 & $1.77 E-03$ \\
\hline 3722 & CR523151 & 4.41 & $2.77 \mathrm{E}-03$ & 3790 & CR523572 & 2.70 & $4.24 \mathrm{E}-03$ & 3858 & CR523891 & 1.26 & $1.18 \mathrm{E}-03$ \\
\hline 3723 & CR523154 & 3.60 & $5.01 E-03$ & 3791 & CR523578 & 3.33 & $1.31 \mathrm{E}-02$ & 3859 & CR523893 & 2.12 & $4.23 \mathrm{E}-03$ \\
\hline 3724 & CR523157 & 5.20 & 4.50E-04 & 3792 & CR523579 & 5.48 & $1.88 \mathrm{E}-03$ & 3860 & CR523897 & 6.34 & $9.01 \mathrm{E}-04$ \\
\hline 3725 & CR523158 & 5.76 & $1.70 \mathrm{E}-04$ & 3793 & CR523591 & 4.43 & $3.40 \mathrm{E}-03$ & 3861 & CR523898 & 4.41 & $8.58 \mathrm{E}-03$ \\
\hline 3726 & CR523160 & 3.03 & $1.14 \mathrm{E}-02$ & 3794 & CR523604 & 3.13 & $9.54 \mathrm{E}-03$ & 3862 & CR523899 & 2.80 & $2.01 \mathrm{E}-03$ \\
\hline 3727 & CR523175 & 3.55 & $1.32 \mathrm{E}-03$ & 3795 & CR523608 & 2.12 & $1.16 \mathrm{E}-02$ & 3863 & CR523909 & 4.17 & $1.37 \mathrm{E}-02$ \\
\hline 3728 & CR523178 & 7.58 & $4.18 \mathrm{E}-03$ & 3796 & CR523612 & 1.73 & $5.22 \mathrm{E}-04$ & 3864 & CR523910 & 1.42 & $8.02 \mathrm{E}-03$ \\
\hline 3729 & CR523186 & 3.96 & $3.45 \mathrm{E}-03$ & 3797 & CR523614 & 5.92 & $3.35 \mathrm{E}-03$ & 3865 & CR523911 & 4.82 & $4.03 E-03$ \\
\hline 3730 & CR523189 & 4.31 & $6.23 \mathrm{E}-04$ & 3798 & CR523626 & 3.28 & $4.33 \mathrm{E}-04$ & 3866 & CR523912 & 6.21 & $1.41 \mathrm{E}-04$ \\
\hline 3731 & CR523191 & 4.19 & $6.68 \mathrm{E}-03$ & 3799 & CR523627 & 1.90 & $2.36 \mathrm{E}-02$ & 3867 & CR523914 & 4.16 & $1.73 \mathrm{E}-04$ \\
\hline 3732 & CR523197 & 1.05 & $4.83 \mathrm{E}-02$ & 3800 & CR523628 & 4.40 & $2.16 \mathrm{E}-04$ & 3868 & CR523918 & 1.35 & $1.21 \mathrm{E}-02$ \\
\hline 3733 & CR523203 & 3.70 & $1.36 \mathrm{E}-02$ & 3801 & CR523631 & 2.81 & $1.05 \mathrm{E}-02$ & 3869 & CR523920 & 4.81 & 3.97E-03 \\
\hline 3734 & CR523207 & 6.09 & $2.94 \mathrm{E}-03$ & 3802 & CR523633 & 1.95 & $2.29 \mathrm{E}-03$ & 3870 & CR523936 & 1.42 & 4.03E-02 \\
\hline 3735 & CR523218 & 2.59 & $7.47 \mathrm{E}-03$ & 3803 & CR523635 & 2.56 & $2.13 \mathrm{E}-03$ & 3871 & CR523938 & 2.93 & $1.99 \mathrm{E}-02$ \\
\hline 3736 & CR523220 & 5.14 & $3.66 \mathrm{E}-03$ & 3804 & CR523639 & 5.58 & $1.25 \mathrm{E}-02$ & 3872 & CR523948 & 4.34 & $4.21 \mathrm{E}-03$ \\
\hline 3737 & CR523224 & 1.27 & $7.77 \mathrm{E}-03$ & 3805 & CR523641 & 5.56 & $3.92 \mathrm{E}-03$ & 3873 & CR523949 & 3.26 & $1.15 \mathrm{E}-02$ \\
\hline 3738 & CR523226 & 4.66 & $3.35 \mathrm{E}-04$ & 3806 & CR523642 & 1.38 & $6.11 \mathrm{E}-02$ & 3874 & CR523951 & 3.92 & $4.70 \mathrm{E}-04$ \\
\hline 3739 & CR523232 & 4.80 & $9.10 \mathrm{E}-04$ & 3807 & CR523645 & 3.10 & $1.24 \mathrm{E}-03$ & 3875 & CR523958 & 6.95 & 4.03E-04 \\
\hline
\end{tabular}




\begin{tabular}{|c|c|c|c|c|c|c|c|c|c|c|c|}
\hline 3876 & CR523960 & 6.68 & $6.18 \mathrm{E}-04$ & 3944 & CR524271 & 3.31 & $2.98 \mathrm{E}-03$ & 4012 & CR733214 & 4.39 & $2.01 \mathrm{E}-02$ \\
\hline 3877 & CR523963 & 3.40 & $7.97 \mathrm{E}-02$ & 3945 & CR524272 & 2.26 & $3.40 \mathrm{E}-03$ & 4013 & CR733223 & 5.82 & $4.94 \mathrm{E}-03$ \\
\hline 3878 & CR523964 & 5.35 & $1.58 \mathrm{E}-02$ & 3946 & CR524273 & 3.45 & 2.11E-04 & 4014 & CR733252 & 6.31 & $8.29 \mathrm{E}-04$ \\
\hline 3879 & CR523965 & 3.13 & $1.59 \mathrm{E}-02$ & 3947 & CR524279 & 4.74 & $2.26 \mathrm{E}-03$ & 4015 & CR733261 & 6.57 & $4.49 \mathrm{E}-03$ \\
\hline 3880 & CR523966 & 3.57 & $1.37 \mathrm{E}-03$ & 3948 & CR524283 & 5.70 & $4.26 \mathrm{E}-04$ & 4016 & CR733279 & 4.37 & $2.11 \mathrm{E}-03$ \\
\hline 3881 & CR523967 & 5.60 & $1.53 \mathrm{E}-03$ & 3949 & CR524294 & 6.55 & 2.37E-05 & 4017 & CR733289 & 2.35 & $1.62 \mathrm{E}-02$ \\
\hline 3882 & CR523975 & 4.94 & 7.77E-03 & 3950 & CR524296 & 3.89 & $2.00 \mathrm{E}-03$ & 4018 & CR733292 & 1.89 & $1.46 \mathrm{E}-03$ \\
\hline 3883 & CR523976 & 4.10 & $1.58 \mathrm{E}-04$ & 3951 & CR524303 & 1.11 & $1.76 \mathrm{E}-02$ & 4019 & CR733298 & 3.99 & $2.34 \mathrm{E}-03$ \\
\hline 3884 & CR523979 & 3.55 & $1.33 \mathrm{E}-02$ & 3952 & CR524307 & 1.81 & $1.14 \mathrm{E}-02$ & 4020 & CR733327 & 1.62 & $2.90 \mathrm{E}-02$ \\
\hline 3885 & CR523982 & 2.63 & $1.19 \mathrm{E}-02$ & 3953 & CR524323 & 2.66 & $3.84 \mathrm{E}-03$ & 4021 & CR733336 & 3.12 & $1.35 \mathrm{E}-02$ \\
\hline 3886 & CR523987 & 3.51 & $1.97 \mathrm{E}-03$ & 3954 & CR524335 & 2.51 & $4.02 \mathrm{E}-03$ & 4022 & CR733359 & 3.61 & $2.44 \mathrm{E}-04$ \\
\hline 3887 & CR523988 & 5.75 & $2.11 \mathrm{E}-03$ & 3955 & CR524343 & 3.66 & $3.65 \mathrm{E}-02$ & 4023 & CR733361 & 2.64 & $2.45 \mathrm{E}-02$ \\
\hline 3888 & CR523989 & 6.73 & $1.22 \mathrm{E}-03$ & 3956 & CR524344 & 4.44 & $6.34 \mathrm{E}-03$ & 4024 & CR733370 & 2.98 & $4.14 \mathrm{E}-02$ \\
\hline 3889 & CR523995 & 4.92 & $5.19 \mathrm{E}-05$ & 3957 & CR524346 & 1.61 & $4.04 \mathrm{E}-04$ & 4025 & CR733372 & 3.98 & $6.56 \mathrm{E}-04$ \\
\hline 3890 & CR524000 & 4.50 & $5.60 \mathrm{E}-04$ & 3958 & CR524348 & 5.69 & $3.94 \mathrm{E}-04$ & 4026 & CR733375 & 3.92 & 6.93E-04 \\
\hline 3891 & CR524001 & 2.89 & $4.04 \mathrm{E}-02$ & 3959 & CR524353 & 1.40 & $1.07 \mathrm{E}-02$ & 4027 & CR733377 & 2.36 & $1.12 \mathrm{E}-02$ \\
\hline 3892 & CR524007 & 2.00 & $5.76 \mathrm{E}-03$ & 3960 & CR524385 & 2.19 & $3.48 \mathrm{E}-02$ & 4028 & CR733385 & 1.86 & $1.75 \mathrm{E}-03$ \\
\hline 3893 & CR524017 & 3.40 & $2.25 \mathrm{E}-02$ & 3961 & CR524386 & 6.78 & $2.99 \mathrm{E}-03$ & 4029 & CR733401 & 4.65 & $6.74 \mathrm{E}-03$ \\
\hline 3894 & CR524021 & 5.21 & $1.27 \mathrm{E}-02$ & 3962 & CR524392 & 3.81 & $1.76 \mathrm{E}-03$ & 4030 & CR733403 & 4.67 & $8.00 \mathrm{E}-04$ \\
\hline 3895 & CR524022 & 4.69 & $1.92 \mathrm{E}-03$ & 3963 & CR524397 & 1.73 & $2.04 \mathrm{E}-03$ & 4031 & CR733412 & 1.63 & $5.18 \mathrm{E}-03$ \\
\hline 3896 & CR524032 & 3.34 & $5.84 \mathrm{E}-03$ & 3964 & CR524401 & 3.56 & $1.26 \mathrm{E}-02$ & 4032 & CR733428 & 1.64 & 3.37E-02 \\
\hline 3897 & CR524034 & 5.58 & $8.54 \mathrm{E}-04$ & 3965 & CR524405 & 1.51 & $2.97 \mathrm{E}-02$ & 4033 & CREB3L3 & 4.47 & 1.93E-02 \\
\hline 3898 & CR524039 & 4.92 & $1.44 \mathrm{E}-03$ & 3966 & CR524407 & 1.89 & $6.09 \mathrm{E}-03$ & 4034 & CREB5 & 1.57 & $2.76 \mathrm{E}-02$ \\
\hline 3899 & CR524041 & 3.87 & $4.30 \mathrm{E}-04$ & 3967 & CR524411 & 5.92 & $1.66 \mathrm{E}-03$ & 4035 & CRHBP & 2.68 & $3.28 \mathrm{E}-02$ \\
\hline 3900 & CR524044 & 6.51 & $5.23 \mathrm{E}-03$ & 3968 & CR524420 & 3.33 & $1.72 \mathrm{E}-02$ & 4036 & CRHR1 & 4.22 & 2.97E-04 \\
\hline 3901 & CR524059 & 4.42 & $1.35 \mathrm{E}-02$ & 3969 & CR524432 & 5.33 & $1.53 \mathrm{E}-03$ & 4037 & CRHR2 & 1.24 & $2.80 \mathrm{E}-02$ \\
\hline 3902 & CR524060 & 3.86 & $4.78 \mathrm{E}-04$ & 3970 & CR524439 & 3.63 & $9.28 \mathrm{E}-03$ & 4038 & CRIP2 & 1.73 & $2.06 \mathrm{E}-02$ \\
\hline 3903 & CR524076 & 2.41 & $9.87 \mathrm{E}-02$ & 3971 & CR524441 & 2.61 & $1.08 \mathrm{E}-02$ & 4039 & CRISP2 & 4.38 & $4.99 \mathrm{E}-03$ \\
\hline 3904 & CR524085 & 5.65 & $3.48 \mathrm{E}-03$ & 3972 & CR524448 & 1.55 & $5.66 \mathrm{E}-02$ & 4040 & CRMP1 & 1.11 & $6.72 \mathrm{E}-03$ \\
\hline 3905 & CR524088 & 5.15 & $1.27 \mathrm{E}-03$ & 3973 & CR524453 & 1.59 & $2.90 \mathrm{E}-02$ & 4041 & CRNN & 3.89 & $2.40 \mathrm{E}-02$ \\
\hline 3906 & CR524092 & 5.19 & $2.84 \mathrm{E}-03$ & 3974 & CR524454 & 1.94 & $8.46 \mathrm{E}-03$ & 4042 & CRP & 3.93 & $1.42 \mathrm{E}-03$ \\
\hline 3907 & CR524094 & 4.65 & $2.34 \mathrm{E}-03$ & 3975 & CR524456 & 3.59 & $6.97 \mathrm{E}-03$ & 4043 & CRTAC1 & 3.04 & $7.59 \mathrm{E}-04$ \\
\hline 3908 & CR524098 & 5.95 & $1.72 \mathrm{E}-04$ & 3976 & CR524459 & 4.18 & $4.65 \mathrm{E}-03$ & 4044 & CRTAM & 3.97 & $8.73 \mathrm{E}-04$ \\
\hline 3909 & CR524104 & 2.05 & $3.77 \mathrm{E}-04$ & 3977 & CR524460 & 4.68 & $3.02 \mathrm{E}-03$ & 4045 & CRYAA & 2.14 & $2.69 \mathrm{E}-02$ \\
\hline 3910 & CR524114 & 4.83 & $3.35 \mathrm{E}-04$ & 3978 & CR524461 & 1.75 & $1.33 \mathrm{E}-02$ & 4046 & CRYBA4 & 4.94 & 5.00E-03 \\
\hline 3911 & CR524119 & 2.54 & $4.31 \mathrm{E}-04$ & 3979 & CR732747 & 2.12 & $6.10 \mathrm{E}-03$ & 4047 & CRYBB1 & 4.51 & $4.84 \mathrm{E}-03$ \\
\hline 3912 & CR524124 & 4.08 & $1.47 \mathrm{E}-03$ & 3980 & CR732755 & 3.94 & $9.37 \mathrm{E}-03$ & 4048 & CRYBB2 & 2.07 & $6.76 \mathrm{E}-03$ \\
\hline 3913 & CR524134 & 4.35 & $1.27 \mathrm{E}-03$ & 3981 & CR732767 & 2.92 & 1.64E-04 & 4049 & CSAD & 2.89 & $1.02 \mathrm{E}-03$ \\
\hline 3914 & CR524139 & 5.07 & $1.04 \mathrm{E}-04$ & 3982 & CR732772 & 2.36 & $1.97 \mathrm{E}-03$ & 4050 & CSDC2 & 1.74 & $1.94 \mathrm{E}-02$ \\
\hline 3915 & CR524143 & 2.56 & $2.13 \mathrm{E}-02$ & 3983 & CR732796 & 1.40 & $5.48 \mathrm{E}-03$ & 4051 & CSF2RA & 3.90 & $2.66 \mathrm{E}-03$ \\
\hline 3916 & CR524145 & 1.62 & $3.26 \mathrm{E}-02$ & 3984 & CR732808 & 2.39 & $3.90 \mathrm{E}-02$ & 4052 & CSF3 & 4.75 & $7.70 \mathrm{E}-03$ \\
\hline 3917 & CR524146 & 3.53 & $9.81 \mathrm{E}-03$ & 3985 & CR732816 & 1.75 & $4.36 \mathrm{E}-02$ & 4053 & CSF3R & 3.93 & $3.22 \mathrm{E}-03$ \\
\hline 3918 & CR524150 & 6.65 & $7.81 \mathrm{E}-03$ & 3986 & CR732831 & 2.18 & $6.92 \mathrm{E}-03$ & 4054 & CSMD1 & 5.08 & $1.01 \mathrm{E}-02$ \\
\hline 3919 & CR524151 & 3.81 & $6.73 \mathrm{E}-04$ & 3987 & CR732853 & 5.87 & $7.21 \mathrm{E}-03$ & 4055 & CSMD2 & 3.86 & 1.73E- 03 \\
\hline 3920 & CR524168 & 3.06 & $1.07 \mathrm{E}-02$ & 3988 & CR732910 & 5.78 & $1.43 \mathrm{E}-03$ & 4056 & CSPG5 & 2.25 & $5.81 \mathrm{E}-03$ \\
\hline 3921 & CR524183 & 3.09 & $2.71 \mathrm{E}-04$ & 3989 & CR732922 & 1.55 & $9.77 \mathrm{E}-02$ & 4057 & CSRP3 & 1.32 & $3.35 \mathrm{E}-05$ \\
\hline 3922 & CR524190 & 1.69 & $1.05 \mathrm{E}-01$ & 3990 & CR732928 & 4.71 & 7.61E-03 & 4058 & CST7 & 1.67 & $4.74 E-03$ \\
\hline 3923 & CR524200 & 4.28 & $4.48 \mathrm{E}-03$ & 3991 & CR732989 & 3.43 & $2.21 \mathrm{E}-02$ & 4059 & CTLA4 & 5.73 & $1.68 \mathrm{E}-03$ \\
\hline 3924 & CR524203 & 5.30 & $1.65 \mathrm{E}-02$ & 3992 & CR732995 & 5.33 & $2.02 \mathrm{E}-03$ & 4060 & CTNNA2 & 2.99 & $8.84 \mathrm{E}-04$ \\
\hline 3925 & CR524205 & 6.43 & $9.84 \mathrm{E}-03$ & 3993 & CR733001 & 1.22 & $1.16 \mathrm{E}-02$ & 4061 & CTRC & 4.35 & $6.23 \mathrm{E}-05$ \\
\hline 3926 & CR524207 & 1.88 & $7.38 \mathrm{E}-04$ & 3994 & CR733044 & 5.99 & $1.06 \mathrm{E}-03$ & 4062 & CTSC & 2.09 & $1.01 \mathrm{E}-03$ \\
\hline 3927 & CR524208 & 1.34 & $3.19 \mathrm{E}-03$ & 3995 & CR733047 & 3.16 & $5.79 \mathrm{E}-04$ & 4063 & CTSE & 4.80 & $1.15 \mathrm{E}-03$ \\
\hline 3928 & CR524212 & 4.35 & $4.97 \mathrm{E}-03$ & 3996 & CR733067 & 3.87 & $2.74 \mathrm{E}-03$ & 4064 & CTSG & 6.26 & $1.08 \mathrm{E}-03$ \\
\hline 3929 & CR524216 & 3.62 & $6.14 \mathrm{E}-02$ & 3997 & CR733084 & 3.93 & $3.42 \mathrm{E}-03$ & 4065 & CTSO & 1.68 & $1.38 \mathrm{E}-03$ \\
\hline 3930 & CR524220 & 2.10 & $3.21 \mathrm{E}-03$ & 3998 & CR733086 & 1.95 & $4.92 \mathrm{E}-02$ & 4066 & CTXN1 & 3.46 & $3.38 \mathrm{E}-03$ \\
\hline 3931 & CR524223 & 5.86 & $2.33 \mathrm{E}-03$ & 3999 & CR733088 & 3.02 & $1.81 \mathrm{E}-03$ & 4067 & CV856053 & 1.58 & $1.40 \mathrm{E}-02$ \\
\hline 3932 & CR524229 & 2.84 & $2.73 \mathrm{E}-03$ & 4000 & CR733094 & 6.53 & $6.65 \mathrm{E}-03$ & 4068 & CV859296 & 2.52 & $3.52 \mathrm{E}-03$ \\
\hline 3933 & CR524233 & 3.35 & $2.08 \mathrm{E}-02$ & 4001 & CR733099 & 2.58 & $8.14 \mathrm{E}-04$ & 4069 & CV861404 & 1.57 & $6.32 \mathrm{E}-03$ \\
\hline 3934 & CR524237 & 3.05 & $5.87 \mathrm{E}-04$ & 4002 & CR733107 & 1.84 & 4.14E-02 & 4070 & CV861894 & 3.53 & 2.18E-04 \\
\hline 3935 & CR524238 & 3.78 & $3.20 \mathrm{E}-03$ & 4003 & CR733123 & 3.16 & $8.36 \mathrm{E}-03$ & 4071 & CV862058 & 1.90 & 4.97E-03 \\
\hline 3936 & CR524245 & 5.71 & $2.76 \mathrm{E}-05$ & 4004 & CR733125 & 5.13 & 3.03E-03 & 4072 & CV862243 & 2.44 & $6.15 \mathrm{E}-03$ \\
\hline 3937 & CR524246 & 2.83 & $1.42 \mathrm{E}-02$ & 4005 & CR733129 & 5.97 & $1.42 \mathrm{E}-04$ & 4073 & CV862686 & 1.81 & $9.62 E-03$ \\
\hline 3938 & CR524247 & 3.82 & $1.36 \mathrm{E}-03$ & 4006 & CR733136 & 3.41 & $6.98 \mathrm{E}-03$ & 4074 & CX3CR1 & 2.53 & 9.50E-04 \\
\hline 3939 & CR524253 & 3.63 & $2.82 \mathrm{E}-03$ & 4007 & CR733141 & 1.71 & $8.54 \mathrm{E}-03$ & 4075 & CXCL12 & 1.51 & $1.47 \mathrm{E}-03$ \\
\hline 3940 & CR524256 & 6.36 & $2.98 \mathrm{E}-04$ & 4008 & CR733158 & 3.06 & $3.38 \mathrm{E}-03$ & 4076 & CXCL14 & 3.73 & $1.60 \mathrm{E}-03$ \\
\hline 3941 & CR524258 & 2.91 & $1.54 \mathrm{E}-03$ & 4009 & CR733173 & 1.55 & $3.66 \mathrm{E}-02$ & 4077 & CXCR2 & 1.79 & $5.14 \mathrm{E}-04$ \\
\hline 3942 & CR524262 & 1.69 & $1.53 \mathrm{E}-03$ & 4010 & CR733195 & 1.54 & $5.67 \mathrm{E}-02$ & 4078 & CXCR4 & 4.49 & $9.38 \mathrm{E}-05$ \\
\hline 3943 & CR524269 & 3.85 & $2.19 \mathrm{E}-03$ & 4011 & CR733199 & 7.74 & 7.33E-04 & 4079 & CXCR5 & 3.22 & $3.04 \mathrm{E}-04$ \\
\hline
\end{tabular}




\begin{tabular}{|c|c|c|c|c|c|c|c|c|c|c|c|}
\hline 4080 & CYFIP2 & 6.41 & $4.87 \mathrm{E}-04$ & 4148 & DLK1 & 5.93 & $1.05 E-03$ & 4216 & DR424050 & 2.44 & $1.84 \mathrm{E}-03$ \\
\hline 4081 & CYGB & 4.18 & $2.66 \mathrm{E}-03$ & 4149 & DLK2 & 6.93 & $4.62 \mathrm{E}-03$ & 4217 & DR424309 & 4.20 & $7.78 \mathrm{E}-03$ \\
\hline 4082 & CYP19A1 & 2.81 & $6.44 E-03$ & 4150 & DLL4 & 5.36 & $5.78 \mathrm{E}-03$ & 4218 & DR425157 & 3.60 & $6.20 \mathrm{E}-04$ \\
\hline 4083 & CYP1A2 & 3.45 & $7.32 \mathrm{E}-03$ & 4151 & DLX1 & 4.60 & $9.71 \mathrm{E}-03$ & 4219 & DR426245 & 2.56 & $8.02 E-03$ \\
\hline 4084 & CYP1B1 & 2.24 & $1.02 \mathrm{E}-02$ & 4152 & DLX3 & 3.26 & $1.31 \mathrm{E}-02$ & 4220 & DR427573 & 4.61 & $1.59 \mathrm{E}-02$ \\
\hline 4085 & CYP21A2 & 1.43 & $4.22 \mathrm{E}-03$ & 4153 & DMBT1 & 2.32 & $6.72 \mathrm{E}-03$ & 4221 & DR429513 & 3.91 & $3.35 \mathrm{E}-03$ \\
\hline 4086 & CYP26B1 & 3.35 & $5.68 \mathrm{E}-03$ & 4154 & DMBX1 & 3.74 & $1.06 \mathrm{E}-03$ & 4222 & DR429796 & 2.83 & 3.34E-04 \\
\hline 4087 & CYP26C1 & 2.17 & $7.97 \mathrm{E}-03$ & 4155 & DMP1 & 4.29 & $1.41 \mathrm{E}-04$ & 4223 & DR430205 & 3.66 & 2.19E-03 \\
\hline 4088 & CYP27A1 & 2.73 & $1.17 \mathrm{E}-03$ & 4156 & DMRT2 & 2.12 & $2.02 \mathrm{E}-02$ & 4224 & DR430295 & 2.61 & $5.86 \mathrm{E}-02$ \\
\hline 4089 & CYP2A13 & 4.53 & $9.45 \mathrm{E}-03$ & 4157 & DNAJB5 & 3.79 & $3.76 E-04$ & 4225 & DR431997 & 1.48 & $1.62 \mathrm{E}-02$ \\
\hline 4090 & СYР2B7Р1 & 3.90 & $7.45 E-03$ & 4158 & DNAJC22 & 4.35 & $1.41 E-02$ & 4226 & DRD1 & 3.91 & $1.42 \mathrm{E}-03$ \\
\hline 4091 & CYP2C45 & 6.03 & $1.27 \mathrm{E}-03$ & 4159 & DNAJC6 & 4.43 & $1.91 \mathrm{E}-03$ & 4227 & DRD2 & 1.95 & $5.15 \mathrm{E}-03$ \\
\hline 4092 & CYP2H1 & 2.86 & $5.86 \mathrm{E}-03$ & 4160 & DNAJC7 & 3.33 & $7.36 \mathrm{E}-03$ & 4228 & DRGX & 4.43 & $3.07 E-04$ \\
\hline 4093 & CYP2R1 & 4.21 & $3.78 \mathrm{E}-03$ & 4161 & DNASE1 & 4.61 & $5.00 \mathrm{E}-04$ & 4229 & DSCAM & 1.98 & $1.69 \mathrm{E}-02$ \\
\hline 4094 & CYP2W1 & 2.38 & $2.64 \mathrm{E}-02$ & 4162 & DNASE1L2 & 6.29 & $1.48 \mathrm{E}-03$ & 4230 & DSCAML1 & 3.44 & $6.74 \mathrm{E}-04$ \\
\hline 4095 & CYP3А4 & 1.93 & $4.94 \mathrm{E}-02$ & 4163 & DNASE2B & 2.00 & $1.94 \mathrm{E}-03$ & 4231 & DSE & 3.15 & $4.55 \mathrm{E}-03$ \\
\hline 4096 & CYP3А7 & 5.98 & $7.33 \mathrm{E}-04$ & 4164 & DND1 & 1.84 & $9.77 \mathrm{E}-03$ & 4232 & DSEL & 1.90 & 7.57E-04 \\
\hline 4097 & CYP4B1 & 2.11 & $1.01 \mathrm{E}-03$ & 4165 & DNMT3B & 2.52 & $2.04 \mathrm{E}-03$ & 4233 & DTX4 & 1.83 & $1.18 \mathrm{E}-03$ \\
\hline 4098 & CYP4V2 & 4.79 & $1.43 \mathrm{E}-02$ & 4166 & DNTT & 6.60 & $6.84 \mathrm{E}-05$ & 4234 & DUOX2 & 1.64 & $8.04 E-03$ \\
\hline 4099 & CYR61 & 1.76 & $4.61 \mathrm{E}-02$ & 4167 & DOCK10 & 1.58 & $1.12 \mathrm{E}-02$ & 4235 & DUOXA1 & 3.19 & $1.19 \mathrm{E}-02$ \\
\hline 4100 & CYSLTR1 & 3.81 & $1.61 \mathrm{E}-03$ & 4168 & DOCK11 & 1.71 & $1.08 \mathrm{E}-02$ & 4236 & DUSP13 & 4.57 & 3.55E-03 \\
\hline 4101 & CYSLTR2 & 7.59 & $6.48 \mathrm{E}-03$ & 4169 & DOCK3 & 1.22 & $5.40 \mathrm{E}-03$ & 4237 & DUSP16 & 4.45 & 7.14E-03 \\
\hline 4102 & CYTH4 & 3.44 & $1.02 E-02$ & 4170 & DOCK4 & 2.63 & $2.68 \mathrm{E}-03$ & 4238 & DUSP26 & 1.19 & $3.70 \mathrm{E}-02$ \\
\hline 4103 & CYTIP & 1.74 & $1.18 \mathrm{E}-03$ & 4171 & DOCK5 & 2.42 & $7.78 \mathrm{E}-03$ & 4239 & DUSP27 & 1.70 & $4.03 E-03$ \\
\hline 4104 & CYTL1 & 6.45 & $4.97 \mathrm{E}-05$ & 4172 & DOCK8 & 1.41 & $1.15 \mathrm{E}-03$ & 4240 & DUSP28 & 1.91 & $9.91 \mathrm{E}-03$ \\
\hline 4105 & CZH9orf125 & 2.54 & $1.55 \mathrm{E}-03$ & 4173 & DOK3 & 3.58 & $1.13 \mathrm{E}-03$ & 4241 & DUSP4 & 4.20 & 4.16E-03 \\
\hline 4106 & CZH9orf3 & 1.51 & $4.16 \mathrm{E}-02$ & 4174 & DOK6 & 4.61 & $7.89 \mathrm{E}-03$ & 4242 & DUSP8 & 2.56 & $4.20 \mathrm{E}-03$ \\
\hline 4107 & CZH9orf72 & 1.54 & $5.62 \mathrm{E}-03$ & 4175 & DOK7 & 1.90 & $2.61 \mathrm{E}-03$ & 4243 & DVL3 & 1.59 & $2.25 \mathrm{E}-03$ \\
\hline 4108 & D70821 & 3.39 & $5.03 E-03$ & 4176 & DOLPP1 & 5.53 & $1.01 \mathrm{E}-03$ & 4244 & DYSF & 3.84 & $1.21 \mathrm{E}-02$ \\
\hline 4109 & DAB1 & 3.82 & $5.20 \mathrm{E}-05$ & 4177 & DPEP1 & 4.67 & $4.08 \mathrm{E}-04$ & 4245 & DYSFIP1 & 3.03 & $3.91 \mathrm{E}-03$ \\
\hline 4110 & DACT1 & 1.39 & $5.61 \mathrm{E}-03$ & 4178 & DPEP2 & 2.24 & $6.71 \mathrm{E}-03$ & 4246 & E2F2 & 6.81 & $4.40 \mathrm{E}-03$ \\
\hline 4111 & DAG1 & 1.35 & $4.31 \mathrm{E}-03$ & 4179 & DPF1 & 4.49 & $6.61 \mathrm{E}-03$ & 4247 & E2F3 & 2.45 & $9.78 \mathrm{E}-04$ \\
\hline 4112 & DAPK1 & 4.47 & $3.96 \mathrm{E}-03$ & 4180 & DPF3 & 3.12 & $1.29 \mathrm{E}-03$ & 4248 & E2F7 & 2.77 & $1.03 E-02$ \\
\hline 4113 & DAPK2 & 1.06 & $5.60 \mathrm{E}-04$ & 4181 & DPP10 & 2.72 & $8.87 \mathrm{E}-03$ & 4249 & EAF2 & 1.66 & $6.83 \mathrm{E}-03$ \\
\hline 4114 & DBC1 & 1.43 & $5.83 \mathrm{E}-02$ & 4182 & DPP6 & 1.96 & $8.36 \mathrm{E}-03$ & 4250 & EBF3 & 1.21 & $5.09 \mathrm{E}-03$ \\
\hline 4115 & DBF4B & 2.95 & $9.89 \mathrm{E}-03$ & 4183 & DPP9 & 3.07 & $4.06 \mathrm{E}-03$ & 4251 & ECHDC1 & 1.29 & $2.45 \mathrm{E}-03$ \\
\hline 4116 & DBH & 4.97 & $6.88 \mathrm{E}-04$ & 4184 & DPYD & 3.50 & $2.92 \mathrm{E}-03$ & 4252 & EDA2R & 2.08 & 5.37E- 04 \\
\hline 4117 & DBN1 & 1.62 & $1.90 \mathrm{E}-02$ & 4185 & DQX1 & 1.43 & $2.42 \mathrm{E}-02$ & 4253 & EDAR & 5.81 & $6.75 \mathrm{E}-03$ \\
\hline 4118 & DCAF6 & 2.27 & $1.71 E-02$ & 4186 & DR410295 & 3.35 & $3.90 \mathrm{E}-03$ & 4254 & EDN1 & 1.09 & $4.14 \mathrm{E}-02$ \\
\hline 4119 & DCBLD1 & 1.34 & $1.04 \mathrm{E}-02$ & 4187 & DR410506 & 2.55 & $5.04 \mathrm{E}-03$ & 4255 & EDN2 & 1.28 & $1.72 \mathrm{E}-02$ \\
\hline 4120 & DCLK2 & 1.25 & $2.21 \mathrm{E}-02$ & 4188 & DR411377 & 2.91 & $2.42 \mathrm{E}-03$ & 4256 & EDN3 & 1.66 & $1.71 \mathrm{E}-02$ \\
\hline 4121 & DCP2 & 5.80 & $4.88 \mathrm{E}-05$ & 4189 & DR412676 & 7.35 & $1.59 \mathrm{E}-02$ & 4257 & EDNRB2 & 4.50 & $7.73 E-04$ \\
\hline 4122 & DCT & 2.53 & $1.11 \mathrm{E}-02$ & 4190 & DR413358 & 1.95 & $3.90 \mathrm{E}-03$ & 4258 & EEF1A2 & 3.93 & $2.71 \mathrm{E}-02$ \\
\hline 4123 & DCTN2 & 1.65 & $6.08 \mathrm{E}-02$ & 4191 & DR413521 & 2.42 & $4.61 \mathrm{E}-03$ & 4259 & EF051348 & 3.36 & $1.25 \mathrm{E}-04$ \\
\hline 4124 & DDO & 1.33 & $2.14 \mathrm{E}-03$ & 4192 & DR413962 & 3.09 & $4.76 \mathrm{E}-03$ & 4260 & EF117325 & 2.57 & $1.94 \mathrm{E}-03$ \\
\hline 4125 & DDR2 & 1.19 & $1.80 \mathrm{E}-02$ & 4193 & DR414690 & 3.22 & $8.76 \mathrm{E}-02$ & 4261 & EF117327 & 2.06 & $2.24 \mathrm{E}-02$ \\
\hline 4126 & DDX28 & 4.13 & $4.61 E-04$ & 4194 & DR415530 & 3.26 & $9.80 \mathrm{E}-03$ & 4262 & EF193863 & 5.29 & $8.25 \mathrm{E}-03$ \\
\hline 4127 & DDX41 & 2.02 & $7.25 \mathrm{E}-03$ & 4195 & DR416881 & 1.93 & $2.03 E-03$ & 4263 & EF422212 & 5.13 & $2.76 \mathrm{E}-03$ \\
\hline 4128 & DEF6 & 2.19 & $2.13 \mathrm{E}-02$ & 4196 & DR417069 & 3.28 & $1.11 \mathrm{E}-02$ & 4264 & EF554710 & 1.28 & $1.07 E-01$ \\
\hline 4129 & DEFB1 & 3.66 & $9.73 \mathrm{E}-04$ & 4197 & DR417267 & 1.84 & $1.06 \mathrm{E}-02$ & 4265 & EFNA2 & 2.80 & $3.70 \mathrm{E}-02$ \\
\hline 4130 & DENND2D & 2.68 & $7.88 \mathrm{E}-04$ & 4198 & DR417559 & 1.61 & $2.17 \mathrm{E}-02$ & 4266 & EFNB1 & 2.75 & $8.68 \mathrm{E}-03$ \\
\hline 4131 & DENND3 & 2.99 & $3.45 \mathrm{E}-03$ & 4199 & DR417905 & 3.31 & $7.85 \mathrm{E}-03$ & 4267 & EFNB2 & 2.80 & $6.17 \mathrm{E}-03$ \\
\hline 4132 & DEPDC6 & 6.45 & 7.97E-03 & 4200 & DR418021 & 1.50 & $1.99 \mathrm{E}-02$ & 4268 & EFR3B & 2.22 & $1.00 \mathrm{E}-01$ \\
\hline 4133 & DEXI & 2.65 & $1.10 \mathrm{E}-02$ & 4201 & DR418364 & 3.22 & $5.59 \mathrm{E}-02$ & 4269 & EGF & 2.64 & $1.94 \mathrm{E}-02$ \\
\hline 4134 & DFNB59 & 6.75 & $9.99 \mathrm{E}-04$ & 4202 & DR418610 & 3.10 & $2.42 \mathrm{E}-03$ & 4270 & EGFL11 & 5.96 & $1.47 E-04$ \\
\hline 4135 & DGCR2 & 3.10 & $6.20 \mathrm{E}-03$ & 4203 & DR418638 & 4.02 & $5.37 \mathrm{E}-03$ & 4271 & EGR4 & 8.30 & $3.04 E-03$ \\
\hline 4136 & DGKB & 3.36 & $7.26 \mathrm{E}-03$ & 4204 & DR418885 & 1.45 & $2.02 \mathrm{E}-02$ & 4272 & EHHADH & 2.46 & $4.32 \mathrm{E}-03$ \\
\hline 4137 & DGKG & 6.48 & $3.19 \mathrm{E}-03$ & 4205 & DR419609 & 1.84 & $8.31 \mathrm{E}-03$ & 4273 & $\mathrm{EIF} 2 \mathrm{C} 1$ & 1.98 & $5.68 \mathrm{E}-03$ \\
\hline 4138 & DGKI & 3.63 & $5.98 \mathrm{E}-03$ & 4206 & DR419982 & 2.49 & $7.03 \mathrm{E}-02$ & 4274 & EIF4ENIF1 & 1.67 & $8.50 \mathrm{E}-03$ \\
\hline 4139 & DHRS4 & 1.25 & $6.38 \mathrm{E}-03$ & 4207 & DR420033 & 2.22 & $2.67 \mathrm{E}-03$ & 4275 & ELF2 & 2.24 & $1.61 \mathrm{E}-03$ \\
\hline 4140 & DHRS7C & 1.10 & $7.86 \mathrm{E}-02$ & 4208 & DR420041 & 2.39 & $6.49 \mathrm{E}-04$ & 4276 & ELF3 & 3.52 & $3.73 \mathrm{E}-03$ \\
\hline 4141 & DIO1 & 2.44 & $1.23 \mathrm{E}-02$ & 4209 & DR420103 & 3.49 & $2.11 \mathrm{E}-02$ & 4277 & ELF5 & 3.90 & $8.04 \mathrm{E}-04$ \\
\hline 4142 & DIP2C & 2.93 & $8.76 \mathrm{E}-03$ & 4210 & DR420117 & 2.55 & $1.61 \mathrm{E}-03$ & 4278 & ELFN1 & 3.87 & $1.17 E-02$ \\
\hline 4143 & DISP2 & 3.92 & $5.66 \mathrm{E}-04$ & 4211 & DR420136 & 1.37 & $9.00 \mathrm{E}-03$ & 4279 & ELFN2 & 4.49 & $3.64 \mathrm{E}-03$ \\
\hline 4144 & DKK1 & 3.75 & $4.73 \mathrm{E}-03$ & 4212 & DR420323 & 2.99 & 3.90E-02 & 4280 & ELK4 & 1.59 & $2.23 \mathrm{E}-03$ \\
\hline 4145 & DKK2 & 2.57 & $3.22 \mathrm{E}-03$ & 4213 & DR420361 & 3.40 & $1.15 \mathrm{E}-04$ & 4281 & ELMO3 & 2.57 & $4.13 E-03$ \\
\hline 4146 & DLC1 & 1.25 & $1.21 \mathrm{E}-02$ & 4214 & DR420543 & 1.61 & $2.35 \mathrm{E}-02$ & 4282 & ELMOD1 & 1.62 & $5.04 E-02$ \\
\hline 4147 & DLGAP3 & 1.61 & $2.55 \mathrm{E}-02$ & 4215 & DR420559 & 1.86 & $4.08 \mathrm{E}-02$ & 4283 & ELMSAN1 & 3.87 & $1.39 \mathrm{E}-03$ \\
\hline
\end{tabular}




\begin{tabular}{|c|c|c|c|c|c|c|c|c|c|c|c|c|}
\hline & 4284 & ELN & 5.14 & $1.02 \mathrm{E}-03$ & 4352 & ENSGALT00000001069 & 2.62 & $1.42 \mathrm{E}-01$ & 4420 & ENSGALT00000002638 & 2.23 & $2.22 \mathrm{E}-03$ \\
\hline & 4285 & ELOVL2 & 2.20 & $3.24 \mathrm{E}-05$ & 4353 & ENSGALT00000001082 & 6.45 & $1.86 \mathrm{E}-04$ & 4421 & ENSGALTO00000002644 & 3.71 & $1.19 \mathrm{E}-03$ \\
\hline & 4286 & ELOVL3 & 4.25 & $2.51 \mathrm{E}-02$ & 4354 & ENSGALTO0000001090 & 2.92 & $4.14 \mathrm{E}-03$ & 4422 & ENSGALT00000002658 & 2.07 & $7.54 \mathrm{E}-03$ \\
\hline & 4287 & ELOVL4 & 1.67 & $5.59 \mathrm{E}-03$ & 4355 & ENSGALTO0000001104 & 3.32 & $4.23 \mathrm{E}-03$ & 4423 & ENSGALT00000002697 & 1.30 & 4.55E-02 \\
\hline & 4288 & ELOVL7 & 2.40 & $5.15 \mathrm{E}-03$ & 4356 & ENSGALT00000001115 & 2.51 & $1.16 \mathrm{E}-03$ & 4424 & ENSGALT00000002769 & 3.41 & $2.30 \mathrm{E}-03$ \\
\hline & 4289 & ELTD1 & 5.64 & $3.42 \mathrm{E}-04$ & 4357 & ENSGALT00000001119 & 1.68 & $1.65 \mathrm{E}-02$ & 4425 & ENSGALTO0000002910 & 3.06 & $1.54 \mathrm{E}-03$ \\
\hline & 4290 & EMID2 & 2.16 & $2.17 \mathrm{E}-02$ & 4358 & ENSGALT00000001164 & 5.75 & $1.30 \mathrm{E}-04$ & 4426 & ENSGALT00000002927 & 7.44 & $5.90 \mathrm{E}-04$ \\
\hline & 4291 & EMILIN3 & 4.17 & $1.69 \mathrm{E}-03$ & 4359 & ENSGALTO0000001200 & 6.56 & $1.76 \mathrm{E}-03$ & 4427 & ENSGALTO0000002951 & 2.24 & $1.91 \mathrm{E}-02$ \\
\hline & 4292 & EMP1 & 2.96 & $1.12 \mathrm{E}-02$ & 4360 & ENSGALTO0000001226 & 1.75 & $1.51 \mathrm{E}-02$ & 4428 & ENSGALTO00000002972 & 3.18 & $2.14 \mathrm{E}-02$ \\
\hline & 4293 & EMX2 & 3.70 & $2.35 \mathrm{E}-03$ & 4361 & ENSGALTO0000001286 & 5.59 & $9.14 \mathrm{E}-05$ & 4429 & ENSGALT00000002981 & 1.91 & $2.09 \mathrm{E}-02$ \\
\hline & 4294 & ENC1 & 1.51 & $8.09 \mathrm{E}-03$ & 4362 & ENSGALT00000001321 & 3.58 & 4.11E-05 & 4430 & ENSGALTO0000003009 & 4.06 & $3.05 E-04$ \\
\hline & 4295 & ENDOD1 & 3.73 & $6.94 \mathrm{E}-04$ & 4363 & ENSGALT00000001322 & 5.25 & $1.43 \mathrm{E}-02$ & 4431 & ENSGALT00000003060 & 3.07 & $8.95 \mathrm{E}-04$ \\
\hline & 4296 & ENG & 2.22 & $1.40 \mathrm{E}-02$ & 4364 & ENSGALTO0000001343 & 1.12 & $7.40 \mathrm{E}-02$ & 4432 & ENSGALTO0000003079 & 3.20 & $3.16 \mathrm{E}-03$ \\
\hline & 4297 & ENGASE & 3.45 & $4.25 \mathrm{E}-03$ & 4365 & ENSGALT00000001359 & 4.33 & $1.97 \mathrm{E}-04$ & 4433 & ENSGALT00000003099 & 5.49 & $3.59 \mathrm{E}-03$ \\
\hline & 4298 & ENO2 & 1.53 & $2.12 \mathrm{E}-03$ & 4366 & ENSGALT00000001379 & 3.51 & $1.32 \mathrm{E}-02$ & 4434 & ENSGALT00000003102 & 5.72 & $9.79 \mathrm{E}-04$ \\
\hline & 4299 & ENO3 & 4.03 & $1.53 \mathrm{E}-02$ & 4367 & ENSGALT00000001381 & 3.70 & $5.43 \mathrm{E}-02$ & 4435 & ENSGALTO00000003112 & 7.06 & 3.37E-03 \\
\hline & 4300 & ENOX1 & 1.26 & $3.08 \mathrm{E}-02$ & 4368 & ENSGALTO0000001404 & 3.21 & $1.11 \mathrm{E}-02$ & 4436 & ENSGALTO0000003124 & 2.89 & $2.70 \mathrm{E}-03$ \\
\hline & 4301 & ENPEP & 3.65 & $2.45 \mathrm{E}-03$ & 4369 & ENSGALT00000001405 & 1.69 & $6.62 \mathrm{E}-04$ & 4437 & ENSGALT00000003150 & 6.29 & $7.96 \mathrm{E}-05$ \\
\hline & 4302 & ENPP3 & 3.30 & $1.39 \mathrm{E}-02$ & 4370 & ENSGALT00000001471 & 4.25 & $1.11 \mathrm{E}-03$ & 4438 & ENSGALT00000003220 & 3.23 & $1.82 \mathrm{E}-03$ \\
\hline & 4303 & ENPP6 & 1.20 & $2.75 \mathrm{E}-02$ & 4371 & ENSGALT00000001491 & 2.48 & $1.71 \mathrm{E}-02$ & 4439 & ENSGALTO00000003225 & 4.39 & $6.01 \mathrm{E}-03$ \\
\hline & 4304 & ENPP7 & 3.65 & $9.92 \mathrm{E}-04$ & 4372 & ENSGALT00000001512 & 1.38 & $7.16 \mathrm{E}-03$ & 4440 & ENSGALT00000003234 & 5.29 & $5.27 \mathrm{E}-03$ \\
\hline & 4305 & ENS-1 & 5.13 & $2.19 \mathrm{E}-04$ & 4373 & ENSGALTO0000001517 & 6.73 & $3.73 \mathrm{E}-04$ & 4441 & ENSGALT00000003315 & 5.15 & $1.50 \mathrm{E}-03$ \\
\hline & 4306 & ENS-3 & 5.69 & $1.02 \mathrm{E}-03$ & 4374 & ENSGALT00000001524 & 2.06 & $3.46 \mathrm{E}-03$ & 4442 & ENSGALT00000003330 & 2.06 & $2.89 \mathrm{E}-03$ \\
\hline 4307 & & ENSGALT00000000002 & 1.90 & $5.80 \mathrm{E}-02$ & 4375 & ENSGALT00000001533 & 3.83 & $4.82 \mathrm{E}-02$ & 4443 & ENSGALTO00000003398 & 5.16 & $1.04 \mathrm{E}-03$ \\
\hline 4308 & & ENSGALT00000000023 & 6.57 & $8.16 \mathrm{E}-05$ & 4376 & ENSGALT00000001603 & 4.97 & $1.15 \mathrm{E}-03$ & 4444 & ENSGALT00000003448 & 5.03 & $1.30 \mathrm{E}-04$ \\
\hline 4309 & & ENSGALT00000000075 & 2.56 & $2.08 \mathrm{E}-02$ & 4377 & ENSGALT00000001623 & 5.24 & $9.53 \mathrm{E}-04$ & 4445 & ENSGALT00000003452 & 4.64 & $1.00 \mathrm{E}-03$ \\
\hline 4310 & & ENSGALT00000000078 & 8.23 & $5.69 \mathrm{E}-04$ & 4378 & ENSGALT00000001645 & 2.42 & $4.94 \mathrm{E}-03$ & 4446 & ENSGALT00000003471 & 2.39 & $1.15 \mathrm{E}-03$ \\
\hline 4311 & & ENSGALT00000000097 & 1.79 & $1.00 \mathrm{E}-03^{-}$ & 4379 & ENSGALT00000001709 & 4.55 & $1.57 \mathrm{E}-03$ & 4447 & ENSGALT00000003541 & 7.64 & $1.94 \mathrm{E}-03$ \\
\hline 4312 & & ENSGALT00000000103 & 2.31 & $5.49 \mathrm{E}-03$ & 4380 & ENSGALT00000001710 & 5.25 & $1.96 \mathrm{E}-03$ & 4448 & ENSGALTO0000003550 & 8.38 & $1.88 \mathrm{E}-06$ \\
\hline 4313 & & ENSGALTO0000000104 & 3.88 & $9.58 \mathrm{E}-03$ & 4381 & ENSGALT00000001711 & 6.75 & $4.54 \mathrm{E}-04$ & 4449 & ENSGALT00000003596 & 5.77 & $1.76 \mathrm{E}-04$ \\
\hline 4314 & & ENSGALT00000000126 & 8.60 & $3.19 \mathrm{E}-03$ & 4382 & ENSGALT00000001713 & 3.48 & $5.18 \mathrm{E}-03$ & 4450 & ENSGALT00000003711 & 4.77 & $1.93 \mathrm{E}-04$ \\
\hline 4315 & & ENSGALT00000000128 & 1.49 & $2.64 \mathrm{E}-02$ & 4383 & ENSGALT00000001735 & 2.69 & $2.35 \mathrm{E}-03$ & 4451 & ENSGALT00000003737 & 2.55 & $1.56 \mathrm{E}-05$ \\
\hline 4316 & & ENSGALT00000000134 & 6.32 & $8.74 \mathrm{E}-04$ & 4384 & ENSGALTO0000001743 & 3.53 & $1.41 \mathrm{E}-03$ & 4452 & ENSGALTO0000003760 & 3.36 & $2.05 E-06$ \\
\hline 4317 & & ENSGALT00000000176 & 5.70 & $1.98 \mathrm{E}-02$ & 4385 & ENSGALT00000001817 & 5.98 & $2.21 \mathrm{E}-02$ & 4453 & ENSGALTO0000003843 & 4.03 & $9.18 \mathrm{E}-03$ \\
\hline 4318 & & ENSGALT00000000192 & 3.51 & $6.67 \mathrm{E}-03$ & 4386 & ENSGALT00000001836 & 1.65 & $1.37 E-02$ & 4454 & ENSGALT00000003852 & 2.26 & $1.28 \mathrm{E}-03$ \\
\hline 4319 & & ENSGALT00000000194 & 1.67 & $4.39 \mathrm{E}-02$ & 4387 & ENSGALT00000001838 & 4.81 & $1.50 \mathrm{E}-04$ & 4455 & ENSGALT00000003869 & 4.08 & $1.34 \mathrm{E}-03$ \\
\hline 4320 & & ENSGALTO0000000208 & 9.15 & $1.48 \mathrm{E}-03$ & 4388 & ENSGALT00000001860 & 3.98 & $3.16 \mathrm{E}-04$ & 4456 & ENSGALT00000003872 & 1.63 & $6.13 \mathrm{E}-03$ \\
\hline 4321 & & ENSGALT00000000218 & 3.44 & $1.33 \mathrm{E}-02$ & 4389 & ENSGALT00000001886 & 2.96 & $1.94 \mathrm{E}-03$ & 4457 & ENSGALT00000003879 & 3.02 & $2.11 \mathrm{E}-03$ \\
\hline 4322 & & ENSGALT00000000238 & 5.11 & $8.56 \mathrm{E}-05^{-}$ & 4390 & ENSGALT00000001899 & 2.80 & $3.53 \mathrm{E}-03$ & 4458 & ENSGALT00000003967 & 2.87 & $4.41 \mathrm{E}-03$ \\
\hline 4323 & & ENSGALT00000000245 & 2.75 & $7.35 \mathrm{E}-04$ & 4391 & ENSGALTO0000001910 & 3.09 & $1.20 \mathrm{E}-02$ & 4459 & ENSGALTO0000003968 & 2.79 & $1.11 \mathrm{E}-01$ \\
\hline 4324 & & ENSGALTO0000000318 & 1.29 & $2.45 \mathrm{E}-02$ & 4392 & ENSGALT00000001936 & 1.70 & $2.91 \mathrm{E}-03$ & 4460 & ENSGALTO0000003994 & 2.24 & $9.06 \mathrm{E}-03$ \\
\hline 4325 & & ENSGALT00000000329 & 2.43 & $8.97 \mathrm{E}-03$ & 4393 & ENSGALTO0000001991 & 7.02 & $6.66 \mathrm{E}-04$ & 4461 & ENSGALTO0000004021 & 5.69 & $8.53 \mathrm{E}-04$ \\
\hline 4326 & & ENSGALT00000000384 & 3.93 & $4.10 \mathrm{E}-03$ & 4394 & ENSGALT00000002017 & 7.98 & $1.04 \mathrm{E}-03$ & 4462 & ENSGALTO0000004047 & 4.54 & $1.47 \mathrm{E}-03$ \\
\hline 4327 & & ENSGALT00000000445 & 6.30 & $3.61 \mathrm{E}-04$ & 4395 & ENSGALT00000002048 & 4.96 & $3.71 \mathrm{E}-02$ & 4463 & ENSGALT00000004053 & 5.22 & $4.91 \mathrm{E}-03$ \\
\hline 4328 & & ENSGALT00000000493 & 3.70 & $5.55 \mathrm{E}-03$ & 4396 & ENSGALT00000002056 & 5.56 & $4.63 \mathrm{E}-03$ & 4464 & ENSGALTO0000004079 & 5.69 & $6.82 \mathrm{E}-03$ \\
\hline 4329 & & ENSGALT00000000509 & 4.56 & $2.66 \mathrm{E}-02$ & 4397 & ENSGALT00000002086 & 2.70 & $3.47 \mathrm{E}-03$ & 4465 & ENSGALT00000004086 & 1.40 & $6.87 \mathrm{E}-02$ \\
\hline 4330 & & ENSGALT00000000567 & 3.90 & $1.45 \mathrm{E}-02$ & 4398 & ENSGALT00000002107 & 3.98 & $3.22 \mathrm{E}-03$ & 4466 & ENSGALTO0000004111 & 4.73 & $9.34 \mathrm{E}-04$ \\
\hline 4331 & & ENSGALT00000000601 & 6.11 & $5.44 \mathrm{E}-05^{-}$ & 4399 & ENSGALTO0000002112 & 5.85 & $8.77 \mathrm{E}-04$ & 4467 & ENSGALTO0000004112 & 5.16 & $3.88 \mathrm{E}-03$ \\
\hline 4332 & & ENSGALT00000000609 & 3.44 & $5.38 \mathrm{E}-03$ & 4400 & ENSGALTO00000002133 & 2.81 & $5.80 \mathrm{E}-02$ & 4468 & ENSGALTO0000004142 & 1.55 & $7.46 \mathrm{E}-03$ \\
\hline 4333 & & ENSGALT00000000625 & 4.93 & $4.79 \mathrm{E}-03$ & 4401 & ENSGALT00000002139 & 8.86 & $2.67 \mathrm{E}-05$ & 4469 & ENSGALT00000004153 & 1.36 & $3.05 \mathrm{E}-03$ \\
\hline 4334 & & ENSGALT00000000643 & 4.79 & $5.89 \mathrm{E}-04$ & 4402 & ENSGALT00000002173 & 2.29 & $3.38 \mathrm{E}-03$ & 4470 & ENSGALT00000004175 & 2.36 & $9.02 \mathrm{E}-03$ \\
\hline 4335 & & ENSGALT00000000747 & 2.82 & $8.42 E-05$ & 4403 & ENSGALTO0000002205 & 2.82 & $7.49 \mathrm{E}-04$ & 4471 & ENSGALTO0000004177 & 3.25 & $5.29 \mathrm{E}-04$ \\
\hline 4336 & & ENSGALT00000000760 & 1.72 & $4.02 \mathrm{E}-03$ & 4404 & ENSGALT00000002208 & 5.37 & $1.20 \mathrm{E}-03$ & 4472 & ENSGALTO0000004192 & 3.21 & $1.61 \mathrm{E}-03$ \\
\hline 4337 & & ENSGALT00000000796 & 5.26 & $2.71 \mathrm{E}-04$ & 4405 & ENSGALT00000002213 & 5.27 & $3.88 \mathrm{E}-03$ & 4473 & ENSGALTO0000004228 & 2.55 & $3.74 \mathrm{E}-03$ \\
\hline 4338 & & ENSGALT00000000808 & 7.04 & $2.72 \mathrm{E}-03$ & 4406 & ENSGALT00000002259 & 1.28 & $5.58 \mathrm{E}-03$ & 4474 & ENSGALTO0000004299 & 2.78 & $2.80 \mathrm{E}-03$ \\
\hline 4339 & & ENSGALT00000000813 & 4.12 & $3.88 \mathrm{E}-03$ & 4407 & ENSGALT00000002274 & 3.00 & $1.66 \mathrm{E}-02$ & 4475 & ENSGALT00000004341 & 2.31 & $1.58 \mathrm{E}-02$ \\
\hline 4340 & & ENSGALT00000000817 & 3.10 & $1.17 \mathrm{E}-02$ & 4408 & ENSGALT00000002308 & 3.85 & $6.95 \mathrm{E}-03$ & 4476 & ENSGALTO0000004346 & 3.96 & $3.47 \mathrm{E}-03$ \\
\hline 4341 & & ENSGALT00000000819 & 2.21 & $5.14 \mathrm{E}-04$ & 4409 & ENSGALT00000002310 & 2.86 & $4.14 \mathrm{E}-03$ & 4477 & ENSGALT00000004369 & 3.14 & $5.52 \mathrm{E}-04$ \\
\hline 4342 & & ENSGALTO0000000842 & 2.63 & $7.31 \mathrm{E}-03$ & 4410 & ENSGALTO00000002392 & 3.49 & $3.92 E-04$ & 4478 & ENSGALTO0000004444 & 1.55 & $1.28 \mathrm{E}-02$ \\
\hline 4343 & & ENSGALT00000000915 & 2.45 & $4.12 \mathrm{E}-03$ & 4411 & ENSGALT00000002421 & 2.35 & $7.15 E-04$ & 4479 & ENSGALTO0000004451 & 2.93 & $1.44 \mathrm{E}-03$ \\
\hline 4344 & & ENSGALT00000000936 & 3.61 & $5.30 \mathrm{E}-03$ & 4412 & ENSGALTO0000002497 & 7.78 & $5.10 \mathrm{E}-04$ & 4480 & ENSGALTO0000004461 & 8.11 & $1.21 \mathrm{E}-05$ \\
\hline 4345 & & ENSGALT00000000938 & 2.89 & $4.14 \mathrm{E}-02$ & 4413 & ENSGALTO0000002498 & 1.73 & $1.42 \mathrm{E}-03$ & 4481 & ENSGALT00000004481 & 3.63 & $1.80 \mathrm{E}-04$ \\
\hline 4346 & & ENSGALT00000000949 & 4.25 & $2.55 \mathrm{E}-03$ & 4414 & ENSGALT00000002532 & 5.67 & $9.95 \mathrm{E}-03$ & 4482 & ENSGALT00000004496 & 1.27 & $1.77 \mathrm{E}-02$ \\
\hline 4347 & & ENSGALT00000000975 & 1.46 & $1.29 \mathrm{E}-02$ & 4415 & ENSGALT00000002539 & 6.37 & $2.17 E-03$ & 4483 & ENSGALT00000004499 & 2.03 & $5.30 \mathrm{E}-03$ \\
\hline 4348 & & ENSGALT00000000989 & 3.31 & $4.65 \mathrm{E}-03$ & 4416 & ENSGALT00000002596 & 2.80 & $3.49 \mathrm{E}-03$ & 4484 & ENSGALTO0000004618 & 3.67 & $9.53 \mathrm{E}-04$ \\
\hline 4349 & & ENSGALT00000001035 & 3.90 & $5.01 \mathrm{E}-03$ & 4417 & ENSGALTO0000002620 & 5.03 & $1.22 \mathrm{E}-03$ & 4485 & ENSGALTO0000004639 & 4.15 & $9.24 \mathrm{E}-03$ \\
\hline 4350 & & ENSGALT00000001043 & 2.02 & $1.76 \mathrm{E}-02$ & 4418 & ENSGALTO0000002625 & 6.96 & $1.46 \mathrm{E}-04$ & 4486 & ENSGALT00000004669 & 3.12 & $3.02 \mathrm{E}-02$ \\
\hline 4351 & & ENSGALT00000001051 & 1.88 & $2.55 \mathrm{E}-03^{-}$ & 4419 & ENSGALT00000002632 & 3.44 & $8.23 \mathrm{E}-04$ & 4487 & ENSGALT00000004670 & 7.17 & $2.84 \mathrm{E}-03$ \\
\hline
\end{tabular}




\begin{tabular}{|c|c|c|c|c|c|c|c|c|c|c|c|}
\hline 4488 & ENSGALTO0000004743 & 4.55 & $9.36 \mathrm{E}-03 \quad 4$ & 4556 & ENSGALT00000006552 & 2.21 & $2.36 \mathrm{E}-02$ & 4624 & ENSGALTO0000008424 & 5.60 & $4.72 E-03$ \\
\hline 4489 & ENSGALTO0000004757 & 2.87 & $4.93 E-02 \quad 4$ & 4557 & ENSGALTO0000006567 & 7.61 & $5.86 \mathrm{E}-04$ & 4625 & ENSGALTO0000008478 & 2.38 & $1.36 \mathrm{E}-02$ \\
\hline 4490 & ENSGALTO0000004777 & 4.98 & $3.58 \mathrm{E}-04 \quad 4$ & 4558 & ENSGALTO0000006604 & 5.08 & $1.02 E-04$ & 4626 & ENSGALTO0000008573 & 5.50 & $8.30 \mathrm{E}-03$ \\
\hline 4491 & ENSGALTO0000004804 & 2.78 & $3.65 \mathrm{E}-02-4$ & 4559 & ENSGALTO0000006613 & 3.00 & $1.16 \mathrm{E}-03$ & 4627 & ENSGALTO0000008607 & 3.30 & $5.72 \mathrm{E}-03$ \\
\hline 4492 & ENSGALTO0000004805 & 1.56 & $5.06 \mathrm{E}-02 \quad 4$ & 4560 & ENSGALTO0000006628 & 2.51 & $3.94 \mathrm{E}-03 \mathrm{C}$ & 4628 & ENSGALTO0000008642 & 3.77 & $5.65 \mathrm{E}-03$ \\
\hline 4493 & ENSGALTO0000004821 & 2.67 & $1.70 \mathrm{E}-03 \frac{-4}{4}$ & 4561 & ENSGALTO0000006673 & 3.27 & $2.37 \mathrm{E}-03$ & 4629 & ENSGALTO0000008679 & 4.34 & $6.37 E-04$ \\
\hline 4494 & ENSGALT00000004910 & 2.69 & $5.63 \mathrm{E}-03 \quad 4$ & 4562 & ENSGALTO0000006699 & 1.39 & $7.80 \mathrm{E}-04 \mathrm{4}$ & 4630 & ENSGALTO0000008752 & 3.93 & $1.23 \mathrm{E}-03$ \\
\hline 4495 & ENSGALTO0000004926 & 3.59 & $7.52 \mathrm{E}-03 \overline{4}$ & 4563 & ENSGALTO0000006721 & 2.46 & $2.23 \mathrm{E}-02$ & 4631 & ENSGALTO0000008780 & 2.62 & $9.02 E-04$ \\
\hline 4496 & ENSGALTO0000004939 & 4.49 & $7.15 \mathrm{E}-04 \quad 4$ & 4564 & ENSGALT00000006774 & 6.04 & $3.62 \mathrm{E}-03$ & 4632 & ENSGALTO0000008802 & 1.35 & $6.14 E-03$ \\
\hline 4497 & ENSGALTO0000004942 & 3.87 & $8.96 \mathrm{E}-04 \overline{4}$ & 4565 & ENSGALT00000006828 & 3.71 & $8.83 \mathrm{E}-03$ & 4633 & ENSGALTO0000008892 & 4.74 & $9.57 \mathrm{E}-04$ \\
\hline 4498 & ENSGALT00000004963 & 4.15 & $1.45 \mathrm{E}-03 \quad 4$ & 4566 & ENSGALTO0000006850 & 3.78 & $9.82 \mathrm{E}-04$ & 4634 & ENSGALTO0000008914 & 3.54 & $8.02 E-04$ \\
\hline 4499 & ENSGALTO0000005006 & 4.73 & $1.14 \mathrm{E}-02-4$ & 4567 & ENSGALTO00000006888 & 6.25 & $2.18 \mathrm{E}-03 \mathrm{C}$ & 4635 & ENSGALTO0000008925 & 4.51 & $3.86 \mathrm{E}-04$ \\
\hline 4500 & ENSGALTO0000005037 & 3.21 & $6.39 \mathrm{E}-03 \overline{4}$ & 4568 & ENSGALTO0000006904 & 5.34 & $4.36 \mathrm{E}-03 \mathrm{C}$ & 4636 & ENSGALTO0000008940 & 2.18 & $2.84 \mathrm{E}-03$ \\
\hline 4501 & ENSGALT00000005059 & 1.83 & $5.63 E-03 \overline{4}$ & 4569 & ENSGALTO0000006911 & 2.80 & $7.70 E-044$ & 4637 & ENSGALTO0000008974 & 3.72 & $7.65 \mathrm{E}-03$ \\
\hline 4502 & ENSGALT00000005080 & 2.60 & $7.04 E_{-03} 4$ & 4570 & ENSGALTO0000006915 & 1.78 & $1.87 \mathrm{E}-02$ & 4638 & ENSGALTO0000009037 & 1.03 & $5.62 \mathrm{E}-02$ \\
\hline 4503 & ENSGALT00000005086 & 2.99 & $1.28 \mathrm{E}-03 \quad 4$ & 4571 & ENSGALTO0000006942 & 2.71 & $7.06 E-03$ & 4639 & ENSGALT00000009085 & 7.92 & $7.81 \mathrm{E}-03$ \\
\hline 4504 & ENSGALTO0000005115 & 6.62 & $7.04 \mathrm{E}-03 \frac{4}{4}$ & 4572 & ENSGALTO0000006958 & 5.29 & $8.06 E-064$ & 4640 & ENSGALTO0000009211 & 2.99 & $6.37 \mathrm{E}-03$ \\
\hline 4505 & ENSGALTO0000005125 & 2.80 & $6.29 \mathrm{E}-03 \overline{4}$ & 4573 & ENSGALTO0000007004 & 4.45 & $5.66 \mathrm{E}-04$ & 4641 & ENSGALTO0000009224 & 5.27 & $1.84 \mathrm{E}-03$ \\
\hline 4506 & ENSGALT00000005126 & 3.81 & $8.11 \mathrm{E}-05_{4}^{4}$ & 4574 & ENSGALTO0000007044 & 5.16 & $3.07 E-03$ & 4642 & ENSGALTO0000009229 & 5.86 & $2.94 \mathrm{E}-03$ \\
\hline 4507 & ENSGALTO0000005210 & 1.18 & $2.86 \mathrm{E}-02 \frac{4}{4}$ & 4575 & ENSGALTO00000007057 & 4.70 & $2.66 \mathrm{E}-03$ & 4643 & ENSGALTO0000009242 & 5.95 & $3.69 \mathrm{E}-04$ \\
\hline 4508 & ENSGALTO0000005214 & 4.11 & $2.86 \mathrm{E}-03 \frac{4}{4}$ & 4576 & ENSGALT00000007066 & 2.99 & $9.59 \mathrm{E}-04$ & 4644 & ENSGALTO0000009303 & 5.58 & $2.73 \mathrm{E}-03$ \\
\hline 4509 & ENSGALT00000005219 & 2.56 & $1.74 \mathrm{E}-04 \overline{4}$ & 4577 & ENSGALTO0000007096 & 2.42 & $7.52 \mathrm{E}-03$ & 4645 & ENSGALTO0000009416 & 3.92 & $1.50 \mathrm{E}-02$ \\
\hline 4510 & ENSGALT00000005232 & 5.17 & $1 . 3 9 \mathrm { E } - 0 2 \longdiv { 4 }$ & 4578 & ENSGALTO0000007125 & 4.32 & $8.18 \mathrm{E}-04$ & 4646 & ENSGALTO00000009436 & 5.60 & $2.45 \mathrm{E}-03$ \\
\hline 4511 & ENSGALT00000005255 & 4.15 & $8.91 \mathrm{E}-04 \overline{4}$ & 4579 & ENSGALTO0000007138 & 7.08 & $3.03 E-03$ & 4647 & ENSGALTO00000009444 & 6.68 & $2.27 E-03$ \\
\hline 4512 & ENSGALT00000005279 & 1.62 & $7.31 \mathrm{E}-03 \overline{4}$ & 4580 & ENSGALTO0000007142 & 2.68 & $1.40 \mathrm{E}-03$ & 4648 & ENSGALTO0000009448 & 4.97 & $3.78 \mathrm{E}-03$ \\
\hline 4513 & ENSGALT000000005280 & 2.63 & $6.51 \mathrm{E}-044_{4}$ & 4581 & ENSGALTO0000007151 & 1.25 & $1.52 \mathrm{E}-02$ & 4649 & ENSGALTO0000009480 & 1.67 & $2.60 \mathrm{E}-04$ \\
\hline 4514 & ENSGALT000000005325 & 3.10 & $6.31 \mathrm{E}-04 \frac{4}{4}$ & 4582 & ENSGALTO0000007173 & 4.37 & $4.48 \mathrm{E}-04$ & 4650 & ENSGALTO0000009524 & 4.80 & $6.40 \mathrm{E}-03$ \\
\hline 4515 & ENSGALT000000005335 & 3.03 & $1.29 \mathrm{E}-02 \overline{4}$ & 4583 & ENSGALT00000007209 & 2.67 & $1.41 \mathrm{E}-02$ & 4651 & ENSGALTO0000009555 & 5.51 & $5.80 \mathrm{E}-03$ \\
\hline 4516 & ENSGALTO00000005350 & 3.68 & $1.54 \mathrm{E}-04 \overline{4}$ & 4584 & ENSGALT00000007222 & 6.05 & $7.50 \mathrm{E}-04$ & 4652 & ENSGALT00000009559 & 1.94 & $1.27 \mathrm{E}-02$ \\
\hline 4517 & ENSGALTO0000005376 & 1.52 & $6.51 E-04 \frac{-1}{4}$ & 4585 & ENSGALTO00000007237 & 5.09 & $1.00 \mathrm{E}-02$ & 4653 & ENSGALTO0000009564 & 4.79 & $6.29 \mathrm{E}-03$ \\
\hline 4518 & ENSGALT00000005378 & 2.97 & $2.65 E-044$ & 4586 & ENSGALTO0000007292 & 3.61 & $5.44 \mathrm{E}-03$ & 4654 & ENSGALTO0000009570 & 2.02 & $3.52 \mathrm{E}-03$ \\
\hline 4519 & ENSGALTO0000005394 & 3.97 & $3.85 E-044$ & 4587 & ENSGALTO0000007296 & 5.73 & $9.45 \mathrm{E}-06$ & 4655 & ENSGALTO0000009580 & 4.14 & $1.15 \mathrm{E}-05$ \\
\hline 4520 & ENSGALT00000005397 & 3.03 & $8.46 E-044$ & 4588 & ENSGALTO0000007301 & 1.89 & $1.61 \mathrm{E}-03$ & 4656 & ENSGALTO0000009599 & 5.21 & $3.10 \mathrm{E}-03$ \\
\hline 4521 & ENSGALTO0000005402 & 1.33 & $2.11 \mathrm{E}-03 \frac{4}{4}$ & 4589 & ENSGALTO0000007319 & 3.93 & $1.74 \mathrm{E}-02$ & 4657 & ENSGALTO00000096662 & 1.88 & $1.59 \mathrm{E}-02$ \\
\hline 4522 & ENSGALT00000005514 & 2.33 & $1.62 E-03 \frac{4}{4}$ & 4590 & ENSGALTO0000007323 & 6.53 & $1.86 \mathrm{E}-03$ & 4658 & ENSGALT00000009708 & 5.09 & $3.73 E-04$ \\
\hline 4523 & ENSGALTO0000005529 & 3.79 & $2.66 \mathrm{E}-024$ & 4591 & ENSGALTO0000007324 & 2.27 & $1.15 \mathrm{E}-02$ & 4659 & ENSGALTO0000009768 & 3.32 & $1.85 \mathrm{E}-03$ \\
\hline 4524 & ENSGALTO00000005592 & 2.21 & $6.46 \mathrm{E}-03 \overline{4}$ & 4592 & ENSGALT00000007335 & 5.10 & $1.67 \mathrm{E}-04$ & 4660 & ENSGALTO0000009788 & 1.43 & $1.18 \mathrm{E}-02$ \\
\hline 4525 & ENSGALTO00000005654 & 4.77 & $3.17 \mathrm{E}-03 \frac{4}{4}$ & 4593 & ENSGALTO0000007348 & 1.78 & $9.67 \mathrm{E}-03$ & 4661 & ENSGALTO0000009806 & 2.71 & $4.68 \mathrm{E}-03$ \\
\hline 4526 & ENSGALTO00000005671 & 6.36 & $4.09 \mathrm{E}-03 \overline{4}$ & 4594 & ENSGALTO0000007459 & 2.84 & $1.42 \mathrm{E}-02$ & 4662 & ENSGALT00000009868 & 2.55 & $2.70 E-04$ \\
\hline 4527 & ENSGALT00000005711 & 4.35 & $4.46 E-04 \quad 4$ & 4595 & ENSGALTO0000007519 & 4.61 & $1.63 \mathrm{E}-03$ & 4663 & ENSGALTO0000009896 & 5.43 & $4.27 E-03$ \\
\hline 4528 & ENSGALTO0000005719 & 6.35 & $1.04 E-03 \frac{4}{4}$ & 4596 & ENSGALTO0000007547 & 3.07 & $2.21 \mathrm{E}-03$ & 4664 & ENSGALTO0000009920 & 6.76 & $1.23 \mathrm{E}-03$ \\
\hline 4529 & ENSGALT00000005755 & 2.58 & $2.73 E-024$ & 4597 & ENSGALTO0000007552 & 1.20 & $3.61 \mathrm{E}-02$ & 4665 & ENSGALTO0000009939 & 3.23 & $1.10 \mathrm{E}-03$ \\
\hline 4530 & ENSGALTO0000005781 & 2.98 & $4.78 E-03 \frac{7}{4}$ & 4598 & ENSGALTO0000007557 & 7.07 & $7.12 \mathrm{E}-05$ & 4666 & ENSGALTO0000009955 & 5.36 & $1.02 \mathrm{E}-03$ \\
\hline 4531 & ENSGALTO0000005789 & 4.72 & $2.41 E-03 \frac{4}{4}$ & 4599 & ENSGALTO0000007567 & 1.84 & $1.82 \mathrm{E}-02$ & 4667 & ENSGALTO0000009979 & 2.55 & $1.90 \mathrm{E}-03$ \\
\hline 4532 & ENSGALTO0000005838 & 3.88 & $2.19 \mathrm{E}-024$ & 4600 & ENSGALTO0000007577 & 5.10 & $7.94 \mathrm{E}-04$ & 4668 & ENSGALTO0000010070 & 4.99 & $1.08 \mathrm{E}-02$ \\
\hline 4533 & ENSGALTO0000005938 & 1.35 & $1.21 \mathrm{E}-024$ & 4601 & ENSGALTO0000007608 & 2.44 & $4.78 \mathrm{E}-02$ & 4669 & ENSGALTO0000010131 & 4.88 & $5.35 \mathrm{E}-03$ \\
\hline 4534 & ENSGALTO0000006013 & 3.94 & $8.07 E-03 \frac{4}{4}$ & 4602 & ENSGALTO0000007643 & 5.39 & $4.19 \mathrm{E}-03$ & 4670 & ENSGALTO0000010137 & 2.57 & $6.15 \mathrm{E}-03$ \\
\hline 4535 & ENSGALTO0000006033 & 5.22 & $2.85 \mathrm{E}-04 \quad 4$ & 4603 & ENSGALTO00000007652 & 3.55 & $1.62 \mathrm{E}-03$ & 4671 & ENSGALTO0000010145 & 3.57 & $1.65 \mathrm{E}-02$ \\
\hline 4536 & ENSGALTO00000006065 & 5.42 & $3.02 E-03 \frac{4}{4}$ & 4604 & ENSGALTO0000007752 & 2.20 & $1.38 \mathrm{E}-03$ & 4672 & ENSGALTO0000010154 & 4.91 & $5.87 E-04$ \\
\hline 4537 & ENSGALT000000006090 & 3.73 & $1.76 E-02 \frac{4}{4}$ & 4605 & ENSGALTO0000007766 & 3.40 & $2.51 \mathrm{E}-03$ & 4673 & ENSGALTO0000010165 & 3.43 & $6.37 E-03$ \\
\hline 4538 & ENSGALTO0000006105 & 1.70 & $2.46 E-02 \frac{4}{4}$ & 4606 & ENSGALTO00000007807 & 3.09 & $1.06 \mathrm{E}-02$ & 4674 & ENSGALTO0000010189 & 5.55 & $2.74 E-05$ \\
\hline 4539 & ENSGALT000000006106 & 4.20 & $3.00 \mathrm{E}-03 \frac{4}{4}$ & 4607 & ENSGALTO0000007819 & 4.46 & $2.97 \mathrm{E}-03$ & 4675 & ENSGALTO0000010226 & 1.60 & $5.22 \mathrm{E}-02$ \\
\hline 4540 & ENSGALTO0000006113 & 5.35 & $1.15 \mathrm{E}-04$ & 4608 & ENSGALTO0000007821 & 5.86 & $1.62 \mathrm{E}-03$ & 4676 & ENSGALTO0000010238 & 2.52 & $3.81 \mathrm{E}-03$ \\
\hline 4541 & ENSGALT000000006128 & 5.23 & 9.68E-04 & 4609 & ENSGALT00000007843 & 8.47 & $5.34 \mathrm{E}-05$ & 4677 & ENSGALTO00000010247 & 3.30 & $8.49 \mathrm{E}-04$ \\
\hline 4542 & ENSGALTO00000006186 & 3.47 & $2.97 \mathrm{E}-04^{-}$ & 4610 & ENSGALT00000007883 & 2.46 & $7.98 \mathrm{E}-03$ & 4678 & ENSGALTO00000010269 & 5.24 & $1.03 E-02$ \\
\hline 4543 & ENSGALT000000006228 & 1.97 & $1.32 \mathrm{E}-02$ & 4611 & ENSGALTO0000007887 & 3.13 & $3.20 \mathrm{E}-02$ & 4679 & ENSGALTO0000010282 & 5.45 & $4.89 \mathrm{E}-04$ \\
\hline 4544 & ENSGALT00000006258 & 4.54 & $3.00 \mathrm{E}-03$ & 4612 & ENSGALTO0000007904 & 1.65 & $1.09 \mathrm{E}-02$ & 4680 & ENSGALTO00000010294 & 9.15 & $1.89 \mathrm{E}-03$ \\
\hline 4545 & ENSGALT00000006276 & 3.61 & $8.01 E-03 \frac{}{4}$ & 4613 & ENSGALTO0000007973 & 4.43 & $2.92 \mathrm{E}-02$ & 4681 & ENSGALTO0000010368 & 2.44 & $1.07 E-02$ \\
\hline 4546 & ENSGALTO0000006313 & 3.40 & $1.92 E-04$ & 4614 & ENSGALT00000007999 & 3.14 & $1.24 \mathrm{E}-02$ & 4682 & ENSGALTO0000010402 & 3.48 & $1.94 E-03$ \\
\hline 4547 & ENSGALT000000006344 & 3.35 & $1.82 \mathrm{E}-03$ & 4615 & ENSGALTO00000008018 & 4.78 & $7.49 \mathrm{E}-03$ & 4683 & ENSGALTO0000010411 & 2.12 & $2.58 \mathrm{E}-02$ \\
\hline 4548 & ENSGALTO0000006350 & 2.88 & $5.79 \mathrm{E}-03 \overline{4}$ & 4616 & ENSGALTO0000008040 & 1.07 & $8.95 \mathrm{E}-02$ & 4684 & ENSGALTO00000010483 & 4.47 & $1.21 \mathrm{E}-02$ \\
\hline 4549 & ENSGALT000000006388 & 3.05 & $5.14 \mathrm{E}-02$ & 4617 & ENSGALT00000008061 & 4.59 & $1.88 \mathrm{E}-03$ & 4685 & ENSGALTO00000010495 & 3.93 & $9.26 \mathrm{E}-03$ \\
\hline 4550 & ENSGALT00000006413 & 4.47 & $2.23 \mathrm{E}-02$ & 4618 & ENSGALTO0000008074 & 1.41 & $9.69 \mathrm{E}-03$ & 4686 & ENSGALTO00000010510 & 4.50 & $8.06 E-04$ \\
\hline 4551 & ENSGALT000000006421 & 1.79 & $3.43 \mathrm{E}-02$ & 4619 & ENSGALTO0000008139 & 2.18 & $1.90 \mathrm{E}-03$ & 4687 & ENSGALTO00000010562 & 2.16 & $1.67 E-02$ \\
\hline 4552 & ENSGALT000000006433 & 1.77 & $8.02 \mathrm{E}-02 \mathrm{C}$ & 4620 & ENSGALTO0000008218 & 1.13 & $6.49 \mathrm{E}-02$ & 4688 & ENSGALTO00000010573 & 1.86 & $2.18 \mathrm{E}-02$ \\
\hline 4553 & ENSGALT000000006473 & 3.09 & $5.75 \mathrm{E}-03$ & 4621 & ENSGALT00000008266 & 4.08 & $2.81 \mathrm{E}-03$ & 4689 & ENSGALT00000010578 & 3.08 & $1.92 \mathrm{E}-03$ \\
\hline 4554 & ENSGALTO0000006487 & 2.74 & $2.71 \mathrm{E}-03$ & 4622 & ENSGALTO0000008309 & 3.56 & $4.62 \mathrm{E}-03$ & 4690 & ENSGALTO0000010583 & 6.73 & $1.85 \mathrm{E}-03$ \\
\hline 4555 & ENSGALTO0000006507 & 1.93 & $8.14 \mathrm{E}-03$ & 4623 & ENSGALT00000008310 & 5.56 & $4.68 \mathrm{E}-05$ & 4691 & ENSGALTO0000010585 & 5.16 & $1.48 \mathrm{E}-0 \mathrm{z}$ \\
\hline
\end{tabular}




\begin{tabular}{|c|c|c|c|c|c|c|c|c|c|c|c|}
\hline 4692 & ENSGALTO0000010590 & 3.49 & $3.79 \mathrm{E}-03 \quad 4$ & 4760 & ENSGALTO0000012332 & 4.36 & $1.41 \mathrm{E}-02$ & 4828 & ENSGALTO0000014353 & 6.92 & $6.17 \mathrm{E}-03$ \\
\hline 4693 & ENSGALT00000010633 & 3.04 & $2.98 \mathrm{E}-03 \overline{4}$ & 4761 & ENSGALTO0000012368 & 1.86 & $2.95 \mathrm{E}-02$ & 4829 & ENSGALTO0000014354 & 5.91 & $4.08 \mathrm{E}-05$ \\
\hline 4694 & ENSGALTO0000010640 & 1.28 & $7.75 \mathrm{E}-04 \frac{-1}{4}$ & 4762 & ENSGALTO0000012413 & 2.79 & $9.45 \mathrm{E}-03$ & 4830 & ENSGALTO0000014392 & 1.90 & $3.09 \mathrm{E}-03$ \\
\hline 4695 & ENSGALTO0000010648 & 10.51 & $3.29 \mathrm{E}-03 \overline{4}$ & 4763 & ENSGALTO0000012451 & 5.23 & $1.33 \mathrm{E}-02$ & 4831 & ENSGALTO0000014426 & 4.14 & $3.58 \mathrm{E}-03$ \\
\hline 4696 & ENSGALTO0000010664 & 1.37 & $6.28 \mathrm{E}-03 \frac{4}{4}$ & 4764 & ENSGALTO0000012463 & 5.35 & $1.23 \mathrm{E}-02$ & 4832 & ENSGALTO0000014429 & 2.10 & $5.92 \mathrm{E}-05$ \\
\hline 4697 & ENSGALTO0000010721 & 3.05 & $3.52 \mathrm{E}-03 \frac{4}{4}$ & 4765 & ENSGALTO0000012508 & 2.89 & $2.86 \mathrm{E}-03$ & 4833 & ENSGALTO0000014496 & 6.03 & $2.54 \mathrm{E}-03$ \\
\hline 4698 & ENSGALT00000010732 & 1.37 & $2.95 \mathrm{E}-03 \overline{4}$ & 4766 & ENSGALTO0000012509 & 1.89 & $4.14 \mathrm{E}-02$ & 4834 & ENSGALTO0000014498 & 2.31 & $1.45 \mathrm{E}-03$ \\
\hline 4699 & ENSGALTO0000010736 & 2.93 & $2.21 \mathrm{E}-03 \overline{4}$ & 4767 & ENSGALTO00000012514 & 1.92 & $3.38 \mathrm{E}-03$ & 4835 & ENSGALTO0000014512 & 2.41 & $6.13 \mathrm{E}-03$ \\
\hline 4700 & ENSGALTO0000010795 & 3.69 & $6.31 \mathrm{E}-03 \frac{4}{4}$ & 4768 & ENSGALT00000012528 & 4.26 & $1.00 \mathrm{E}-03$ & 4836 & ENSGALTO0000014537 & 4.10 & $3.21 \mathrm{E}-03$ \\
\hline 4701 & ENSGALT00000010816 & 6.15 & $1.18 \mathrm{E}-02-4$ & 4769 & ENSGALTO00000012554 & 4.99 & $1.18 \mathrm{E}-03$ & 4837 & ENSGALTO0000014554 & 6.37 & $5.30 \mathrm{E}-03$ \\
\hline 4702 & ENSGALT00000010852 & 2.30 & $3.56 \mathrm{E}-02 \frac{4}{4}$ & 4770 & ENSGALTO0000012557 & 6.07 & $8.29 \mathrm{E}-05$ & 4838 & ENSGALTO0000014555 & 2.93 & $1.49 \mathrm{E}-03$ \\
\hline 4703 & ENSGALTO0000010863 & 2.57 & $2.75 \mathrm{E}-03 \frac{4}{4}$ & 4771 & ENSGALTO00000012664 & 3.08 & $2.86 \mathrm{E}-03$ & 4839 & ENSGALTO0000014575 & 4.65 & $2.27 E-05$ \\
\hline 4704 & ENSGALT00000010867 & 3.90 & $7.26 \mathrm{E}-03 \overline{4}$ & 4772 & ENSGALTO0000012674 & 3.08 & $1.12 \mathrm{E}-03$ & 4840 & ENSGALTO0000014610 & 5.48 & $2.44 E-03$ \\
\hline 4705 & ENSGALT00000010872 & 2.38 & $1.53 \mathrm{E}-02-\frac{4}{4}$ & 4773 & ENSGALTO0000012676 & 6.46 & $4.33 \mathrm{E}-04$ & 4841 & ENSGALTO0000014626 & 5.30 & $1.46 \mathrm{E}-03$ \\
\hline 4706 & ENSGALT00000010901 & 1.48 & $5.74 \mathrm{E}-03 \quad 4$ & 4774 & ENSGALTO0000012682 & 2.85 & $1.33 \mathrm{E}-03$ & 4842 & ENSGALTO0000014630 & 2.79 & $9.45 \mathrm{E}-04$ \\
\hline 4707 & ENSGALT00000010905 & 3.70 & $1.77 \mathrm{E}-03 \overline{4}$ & 4775 & ENSGALTO0000012704 & 4.45 & $4.61 \mathrm{E}-04$ & 4843 & ENSGALTO0000014637 & 2.26 & $7.54 E-05$ \\
\hline 4708 & ENSGALTO0000010959 & 4.36 & $5.34 \mathrm{E}-02-4$ & 4776 & ENSGALTO0000012743 & 4.31 & $1.62 \mathrm{E}-03$ & 4844 & ENSGALTO0000014649 & 3.62 & $3.32 E-04$ \\
\hline 4709 & ENSGALTO0000010966 & 4.74 & $1.64 \mathrm{E}-04 \overline{4}$ & 4777 & ENSGALTO0000012744 & 9.38 & $2.20 \mathrm{E}-04$ & 4845 & ENSGALTO0000014662 & 2.00 & $7.63 \mathrm{E}-03$ \\
\hline 4710 & ENSGALT00000010967 & 1.34 & $5.12 \mathrm{E}-03 \overline{4}$ & 4778 & ENSGALTO0000012753 & 4.47 & $3.35 E-03$ & 4846 & ENSGALTO0000014735 & 3.18 & $5.00 E-04$ \\
\hline 4711 & ENSGALTO0000011010 & 4.65 & $1.27 \mathrm{E}-02 \quad 4$ & 4779 & ENSGALTO0000012754 & 2.34 & $1.06 E-02$ & 4847 & ENSGALTO0000014739 & 1.58 & $1.94 \mathrm{E}-02$ \\
\hline 4712 & ENSGALTO0000011018 & 3.62 & $1.21 \mathrm{E}-03 \frac{4}{4}$ & 4780 & ENSGALTO0000012782 & 3.95 & $2.94 \mathrm{E}-03$ & 4848 & ENSGALTO0000014741 & 3.89 & $2.31 E-03$ \\
\hline 4713 & ENSGALT00000011022 & 1.08 & $3.50 \mathrm{E}-02 \frac{4}{4}$ & 4781 & ENSGALTO0000012786 & 5.04 & $4.84 \mathrm{E}-04$ & 4849 & ENSGALTO0000014759 & 3.92 & $3.35 \mathrm{E}-02$ \\
\hline 4714 & ENSGALT00000011032 & 2.19 & $6.89 \mathrm{E}-03 \overline{4}$ & 4782 & ENSGALTO0000012861 & 2.69 & $2.08 \mathrm{E}-03$ & 4850 & ENSGALTO00000014769 & 2.47 & $4.78 \mathrm{E}-03$ \\
\hline 4715 & ENSGALTO0000011145 & 3.27 & $4.62 \mathrm{E}-03 \overline{4}$ & 4783 & ENSGALTO0000012864 & 4.18 & $4.15 E-03$ & 4851 & ENSGALTO0000014791 & 5.37 & $8.35 E-04$ \\
\hline 4716 & ENSGALT00000011151 & 2.25 & $1.21 \mathrm{E}-03 \overline{4}$ & 4784 & ENSGALTO0000012908 & 2.45 & $7.34 \mathrm{E}-03$ & 4852 & ENSGALTO0000014816 & 5.57 & $6.05 \mathrm{E}-03$ \\
\hline 4717 & ENSGALT00000011227 & 1.66 & $5.91 \mathrm{E}-03 \quad 4$ & 4785 & ENSGALT00000012961 & 1.67 & $3.46 \mathrm{E}-03$ & 4853 & ENSGALTO0000014835 & 3.14 & $4.81 E-05$ \\
\hline 4718 & ENSGALT00000011281 & 3.10 & $1.74 \mathrm{E}-03 \frac{4}{4}$ & 4786 & ENSGALTO0000012965 & 2.89 & $1.86 \mathrm{E}-02$ & 4854 & ENSGALTO0000014843 & 4.97 & $8.12 \mathrm{E}-04$ \\
\hline 4719 & ENSGALTO00000011318 & 5.31 & $9.17 \mathrm{E}-04 \overline{4}$ & 4787 & ENSGALT00000012990 & 2.27 & $6.98 \mathrm{E}-03$ & 4855 & ENSGALTO0000014847 & 6.59 & $5.57 \mathrm{E}-03$ \\
\hline 4720 & ENSGALTO0000011319 & 2.68 & $2.27 \mathrm{E}-07 \overline{4}$ & 4788 & ENSGALTO0000013028 & 2.35 & $7.77 \mathrm{E}-03$ & 4856 & ENSGALTO0000014850 & 3.66 & $1.30 \mathrm{E}-04$ \\
\hline 4721 & ENSGALTO00000011341 & 1.75 & $2.30 \mathrm{E}-024$ & 4789 & ENSGALT00000013031 & 3.11 & $6.26 \mathrm{E}-04$ & 4857 & ENSGALTO0000014859 & 1.68 & $1.14 \mathrm{E}-01$ \\
\hline 4722 & ENSGALT00000011377 & 4.80 & $1.03 \mathrm{E}-04 \quad 4$ & 4790 & ENSGALTO0000013058 & 5.80 & $1.20 \mathrm{E}-03$ & 4858 & ENSGALTO0000014871 & 2.04 & $4.26 \mathrm{E}-02$ \\
\hline 4723 & ENSGALTO0000011378 & 2.02 & $1.48 \mathrm{E}-02 \frac{4}{4}$ & 4791 & ENSGALTO0000013120 & 3.89 & $5.86 \mathrm{E}-06$ & 4859 & ENSGALTO0000014889 & 3.54 & $7.16 \mathrm{E}-04$ \\
\hline 4724 & ENSGALTO0000011379 & 4.62 & $3.53 \mathrm{E}-03 \frac{4}{4}$ & 4792 & ENSGALTO0000013148 & 4.33 & $4.15 \mathrm{E}-03$ & 4860 & ENSGALTO0000014930 & 5.92 & $1.34 \mathrm{E}-03$ \\
\hline 4725 & ENSGALTO0000011380 & 2.59 & $5.35 \mathrm{E}-03 \overline{4}$ & 4793 & ENSGALTO0000013205 & 1.96 & $1.30 \mathrm{E}-02$ & 4861 & ENSGALTO0000014934 & 1.98 & $2.82 \mathrm{E}-03$ \\
\hline 4726 & ENSGALTO0000011413 & 4.20 & $2.38 \mathrm{E}-03 \overline{4}$ & 4794 & ENSGALTO0000013206 & 2.08 & $2.98 \mathrm{E}-03$ & 4862 & ENSGALTO0000014945 & 7.38 & $2.58 \mathrm{E}-03$ \\
\hline 4727 & ENSGALT00000011421 & 3.29 & $3.10 \mathrm{E}-03 \frac{4}{4}$ & 4795 & ENSGALTO0000013232 & 4.22 & $2.11 \mathrm{E}-03$ & 4863 & ENSGALTO0000014963 & 10.38 & $9.47 \mathrm{E}-04$ \\
\hline 4728 & ENSGALTO00000011429 & 2.14 & $4.37 \mathrm{E}-04 \overline{4}$ & 4796 & ENSGALT00000013266 & 1.04 & $1.22 \mathrm{E}-03$ & 4864 & ENSGALT00000014968 & 5.68 & $5.88 \mathrm{E}-04$ \\
\hline 4729 & ENSGALTO0000011522 & 6.65 & $8.31 E-03 \frac{4}{4}$ & 4797 & ENSGALTO0000013296 & 5.65 & $1.25 \mathrm{E}-03$ & 4865 & ENSGALTO0000014980 & 6.44 & $1.33 \mathrm{E}-04$ \\
\hline 4730 & ENSGALTO0000011537 & 5.97 & $7.05 E-04 \frac{4}{4}$ & 4798 & ENSGALTO0000013312 & 4.01 & $2.18 \mathrm{E}-03$ & 4866 & ENSGALTO0000014994 & 4.67 & $2.26 \mathrm{E}-03$ \\
\hline 4731 & ENSGALT00000011558 & 4.57 & $8.11 \mathrm{E}-04 \mathrm{4}$ & 4799 & ENSGALTO0000013353 & 8.93 & $2.80 \mathrm{E}-05$ & 4867 & ENSGALTO0000014995 & 3.06 & $3.39 E-03$ \\
\hline 4732 & ENSGALTO0000011570 & 1.36 & $2.11 E-024$ & 4800 & ENSGALTO0000013389 & 2.29 & $3.08 \mathrm{E}-02$ & 4868 & ENSGALTO0000015007 & 5.52 & $4.79 E-03$ \\
\hline 4733 & ENSGALT00000011599 & 1.19 & $6.35 E-03 \frac{4}{4}$ & 4801 & ENSGALTO0000013408 & 2.76 & $1.14 \mathrm{E}-02$ & 4869 & ENSGALTO0000015039 & 1.35 & $2.91 \mathrm{E}-02$ \\
\hline 4734 & ENSGALTO0000011627 & 4.20 & $4.67 \mathrm{E}-04 \mathrm{4}$ & 4802 & ENSGALTO0000013443 & 4.68 & $2.10 \mathrm{E}-03$ & 4870 & ENSGALTO0000015042 & 4.35 & $1.32 \mathrm{E}-03$ \\
\hline 4735 & ENSGALTO0000011659 & 3.45 & $1.19 \mathrm{E}-03 \frac{4}{4}$ & 4803 & ENSGALTO0000013502 & 2.42 & $3.56 \mathrm{E}-03$ & 4871 & ENSGALTO0000015057 & 3.10 & $2.92 \mathrm{E}-03$ \\
\hline 4736 & ENSGALTO0000011664 & 7.21 & $5.07 \mathrm{E}-03 \frac{4}{4}$ & 4804 & ENSGALTO0000013533 & 1.70 & $2.92 \mathrm{E}-03$ & 4872 & ENSGALTO0000015193 & 2.74 & $9.17 \mathrm{E}-03$ \\
\hline 4737 & ENSGALTO0000011701 & 1.36 & $2.52 E-03 \frac{4}{4}$ & 4805 & ENSGALTO0000013580 & 2.14 & $2.18 \mathrm{E}-02$ & 4873 & ENSGALTO0000015238 & 1.97 & $2.12 \mathrm{E}-02$ \\
\hline 4738 & ENSGALTO0000011702 & 2.09 & $1.44 \mathrm{E}-01 \frac{1}{4}$ & 4806 & ENSGALTO0000013594 & 1.46 & $1.63 \mathrm{E}-03$ & 4874 & ENSGALTO0000015339 & 3.45 & $5.02 E-03$ \\
\hline 4739 & ENSGALTO0000011721 & 7.22 & $1.10 \mathrm{E}-03 \frac{4}{4}$ & 4807 & ENSGALTO0000013619 & 4.94 & $4.62 \mathrm{E}-04$ & 4875 & ENSGALTO0000015366 & 1.06 & $8.66 \mathrm{E}-03$ \\
\hline 4740 & ENSGALTO00000011730 & 3.86 & $3.90 \mathrm{E}-03 \frac{4}{4}$ & 4808 & ENSGALTO0000013653 & 1.39 & $2.66 \mathrm{E}-02$ & 4876 & ENSGALTO0000015476 & 3.52 & $6.83 \mathrm{E}-03$ \\
\hline 4741 & ENSGALTO00000011736 & 1.98 & $7.71 E-02 \frac{4}{4}$ & 4809 & ENSGALT00000013764 & 1.67 & $1.82 \mathrm{E}-02$ & 4877 & ENSGALTO00000015540 & 1.96 & $2.15 \mathrm{E}-02$ \\
\hline 4742 & ENSGALTO00000011741 & 3.74 & $9.17 \mathrm{E}-04 \overline{4}$ & 4810 & ENSGALT00000013765 & 2.16 & $8.29 \mathrm{E}-03$ & 4878 & ENSGALTO0000015543 & 3.28 & $9.24 E-04$ \\
\hline 4743 & ENSGALT000000011823 & 2.42 & $2.33 \mathrm{E}-02 \mathrm{4}$ & 4811 & ENSGALT00000013799 & 2.64 & $6.00 \mathrm{E}-03$ & 4879 & ENSGALTO0000015557 & 2.09 & $1.47 \mathrm{E}-03$ \\
\hline 4744 & $\begin{array}{l}\text { ENSGALT00000011839 } \\
\end{array}$ & 4.27 & $3.28 \mathrm{E}-04 \mathrm{4}$ & 4812 & ENSGALT00000013826 & 5.85 & $2.34 \mathrm{E}-05$ & 4880 & ENSGALTO0000015602 & 2.67 & $1.39 \mathrm{E}-02$ \\
\hline 4745 & ENSGALTO00000011840 & 2.78 & $3.97 \mathrm{E}-03 \overline{4}$ & 4813 & ENSGALTO0000013845 & 6.64 & $2.49 \mathrm{E}-04$ & 4881 & ENSGALT00000015618 & 1.68 & $3.09 E-02$ \\
\hline 4746 & ENSGALTO00000011913 & 3.59 & $1.94 \mathrm{E}-04 \overline{4}$ & 4814 & ENSGALTO0000013846 & 6.02 & $3.33 \mathrm{E}-03$ & 4882 & ENSGALTO0000015629 & 2.79 & $4.17 \mathrm{E}-02$ \\
\hline 4747 & ENSGALTO00000011947 & 3.65 & $1.12 \mathrm{E}-02 \overline{4}$ & 4815 & ENSGALTO0000013855 & 2.22 & $2.91 \mathrm{E}-03$ & 4883 & ENSGALTO0000015642 & 7.22 & $6.26 \mathrm{E}-03$ \\
\hline 4748 & ENSGALTO00000011994 & 5.23 & $1.22 \mathrm{E}-03 \overline{4}$ & 4816 & ENSGALTO0000013915 & 2.27 & $1.64 \mathrm{E}-03$ & 4884 & ENSGALT00000015652 & 2.34 & $4.56 \mathrm{E}-04$ \\
\hline 4749 & ENSGALT00000012029 & 2.86 & $1.01 E-024$ & 4817 & ENSGALTO0000013922 & 1.50 & $7.20 \mathrm{E}-03$ & 4885 & ENSGALTO0000015699 & 7.33 & $2.11 \mathrm{E}-03$ \\
\hline 4750 & ENSGALT000000012032 & 3.61 & $2.29 \mathrm{E}-03 \frac{4}{4}$ & 4818 & ENSGALTO0000014008 & 1.46 & $3.58 \mathrm{E}-03$ & 4886 & ENSGALTO00000015710 & 5.36 & $8.88 \mathrm{E}-03$ \\
\hline 4751 & ENSGALT00000012148 & 3.25 & $3 . 1 0 \mathrm { E } - 0 3 \longdiv { 4 }$ & 4819 & ENSGALTO0000014059 & 6.31 & $4.27 \mathrm{E}-03$ & 4887 & ENSGALTO00000015729 & 4.25 & $1.26 \mathrm{E}-03$ \\
\hline 4752 & ENSGALT00000012208 & 3.53 & $1 . 6 6 \mathrm { E } - 0 3 \longdiv { 4 }$ & 4820 & ENSGALTO0000014074 & 1.95 & $3.09 \mathrm{E}-02$ & 4888 & ENSGALTO0000015759 & 7.45 & $2.23 \mathrm{E}-05$ \\
\hline 4753 & ENSGALT000000012209 & 4.90 & $3 . 0 6 \mathrm { E } - 0 3 \longdiv { 4 }$ & 4821 & ENSGALTO0000014078 & 5.01 & $1.73 \mathrm{E}-03$ & 4889 & ENSGALTO00000015763 & 6.43 & $1.52 \mathrm{E}-04$ \\
\hline 4754 & ENSGALT00000012214 & 3.15 & $2.16 \mathrm{E}-04 \overline{4}$ & 4822 & ENSGALTO0000014148 & 5.25 & $2.12 \mathrm{E}-03$ & 4890 & ENSGALTO0000015779 & 4.73 & $5.51 E-04$ \\
\hline 4755 & ENSGALT00000012236 & 5.13 & $2.41 \mathrm{E}-05^{-4}$ & 4823 & ENSGALTO0000014161 & 2.13 & $3.95 \mathrm{E}-04$ & 4891 & ENSGALTO00000015783 & 6.35 & $3.77 E-03$ \\
\hline 4756 & ENSGALT00000012256 & 3.42 & $5.36 \mathrm{E}-04 \overline{4}$ & 4824 & ENSGALTO0000014168 & 4.94 & $3.90 \mathrm{E}-04$ & 4892 & ENSGALTO0000015804 & 1.26 & $6.28 \mathrm{E}-02$ \\
\hline 4757 & ENSGALTO00000012298 & 3.36 & $1.44 \mathrm{E}-02 \overline{4}$ & 4825 & ENSGALT00000014188 & 8.33 & $3.77 \mathrm{E}-04$ & 4893 & ENSGALT00000015817 & 1.59 & $6.61 \mathrm{E}-03$ \\
\hline 4758 & ENSGALTO00000012309 & 6.58 & $2.39 \mathrm{E}-03 \frac{4}{4}$ & 4826 & ENSGALT00000014193 & 1.22 & $7.51 \mathrm{E}-02$ & 4894 & ENSGALTO00000015850 & 1.61 & $1.68 \mathrm{E}-03$ \\
\hline 4759 & ENSGALT00000012318 & 2.02 & $1.11 \mathrm{E}-024$ & 4827 & ENSGALT00000014259 & 1.78 & 3.26E-02 & 4895 & ENSGALTO0000015914 & 3.71 & $2.81 \mathrm{E}-03$ \\
\hline
\end{tabular}




\begin{tabular}{|c|c|c|c|c|c|c|c|c|c|c|c|}
\hline 4896 & ENSGALTO0000015931 & 9.00 & $2.38 \mathrm{E}-03$ & 4964 & ENSGALTO0000018212 & 1.71 & $5.58 \mathrm{E}-025$ & 5032 & ENSGALTO0000020394 & 2.97 & $1.01 \mathrm{E}-02$ \\
\hline 4897 & ENSGALTO0000016120 & 3.46 & $8.38 \mathrm{E}-03^{-}$ & 4965 & ENSGALT00000018322 & 3.22 & $4.35 \mathrm{E}-035$ & 5033 & ENSGALTO0000020399 & 3.40 & $5.56 \mathrm{E}-04$ \\
\hline 4898 & ENSGALTO0000016140 & 5.77 & $2.24 \mathrm{E}-03$ & 4966 & ENSGALTO0000018334 & 6.80 & $3.47 \mathrm{E}-05 \mathrm{~S}$ & 5034 & ENSGALTO0000020409 & 4.56 & $5.74 \mathrm{E}-04$ \\
\hline 4899 & ENSGALTO0000016183 & 3.23 & $8.62 \mathrm{E}-03$ & 4967 & ENSGALTO0000018383 & 1.50 & $1.76 E-035$ & 5035 & ENSGALTO0000020413 & 2.97 & $1.55 \mathrm{E}-03$ \\
\hline 4900 & ENSGALTO0000016206 & 3.41 & $8.92 E-04^{-1}$ & 4968 & ENSGALTO0000018384 & 4.76 & $1.98 \mathrm{E}-03 \mathrm{~S}$ & 5036 & ENSGALTO0000020428 & 7.00 & $2.00 \mathrm{E}-03$ \\
\hline 4901 & ENSGALTO00000016238 & 4.86 & $1.47 \mathrm{E}-03$ & 4969 & ENSGALT00000018451 & 2.92 & $7.55 \mathrm{E}-045$ & 5037 & ENSGALTO0000020467 & 1.67 & $8.55 \mathrm{E}-03$ \\
\hline 4902 & ENSGALTO0000016251 & 4.90 & $4.23 \mathrm{E}-03$ & 4970 & ENSGALTO0000018496 & 6.67 & $1.94 \mathrm{E}-035$ & 5038 & ENSGALT00000020468 & 5.82 & $6.02 \mathrm{E}-03$ \\
\hline 4903 & ENSGALTO0000016265 & 2.72 & $4.98 \mathrm{E}-03$ & 4971 & ENSGALT00000018508 & 3.05 & $2.19 \mathrm{E}-035$ & 5039 & ENSGALTO0000020505 & 6.52 & $7.58 \mathrm{E}-03$ \\
\hline 4904 & ENSGALT00000016413 & 6.16 & $1.11 \mathrm{E}-04$ & 4972 & ENSGALTO00000018524 & 5.06 & $3.99 \mathrm{E}-045$ & 5040 & ENSGALTO0000020541 & 6.50 & $3.42 E-04$ \\
\hline 4905 & ENSGALTO0000016416 & 8.08 & $2.74 \mathrm{E}-04$ & 4973 & ENSGALTO0000018549 & 4.72 & $2.84 \mathrm{E}-045$ & 5041 & ENSGALTO0000020586 & 3.79 & $6.21 \mathrm{E}-03$ \\
\hline 4906 & ENSGALTO0000016444 & 2.05 & $3.07 E-02$ & 4974 & ENSGALTO0000018567 & 5.05 & $1.25 \mathrm{E}-025$ & 5042 & ENSGALTO0000020616 & 4.22 & $3.06 \mathrm{E}-03$ \\
\hline 4907 & ENSGALTO0000016463 & 6.36 & 3.25E-04 & 4975 & ENSGALTO0000018618 & 3.65 & $9.76 E-045$ & 5043 & ENSGALT00000020806 & 1.67 & $2.29 \mathrm{E}-02$ \\
\hline 4908 & ENSGALTO0000016495 & 1.72 & $1.56 \mathrm{E}-03^{3}$ & 4976 & ENSGALT00000018619 & 6.03 & $1.36 \mathrm{E}-045$ & 5044 & ENSGALTO0000020824 & 3.91 & $2.00 E-04$ \\
\hline 4909 & ENSGALTO00000016549 & 3.03 & $1.16 \mathrm{E}-03^{-}$ & 4977 & ENSGALTO0000018733 & 3.49 & $5.49 \mathrm{E}-03 \mathrm{5}$ & 5045 & ENSGALTO0000020948 & 5.40 & $4.80 \mathrm{E}-03$ \\
\hline 4910 & ENSGALTO00000016605 & 2.89 & $2.06 E-02$ & 4978 & ENSGALTO0000018740 & 4.17 & $2.92 \mathrm{E}-035$ & 5046 & ENSGALTO0000020979 & 2.26 & $1.47 \mathrm{E}-03$ \\
\hline 4911 & ENSGALTO0000016608 & 1.02 & $6.94 \mathrm{E}-04$ & 4979 & ENSGALTO0000018784 & 2.81 & $2.70 \mathrm{E}-035$ & 5047 & ENSGALTO0000021084 & 7.48 & $1.70 \mathrm{E}-05$ \\
\hline 4912 & ENSGALT00000016609 & 3.10 & $2.92 E-03$ & 4980 & ENSGALTO0000018829 & 4.04 & $4.36 \mathrm{E}-045$ & 5048 & ENSGALTO0000021109 & 3.62 & $2.68 \mathrm{E}-03$ \\
\hline 4913 & ENSGALTO00000016646 & 3.83 & $5.38 \mathrm{E}-03^{-}$ & 4981 & ENSGALTO0000018841 & 2.98 & $5.05 E-035$ & 5049 & ENSGALTO0000021120 & 2.79 & $1.62 \mathrm{E}-03$ \\
\hline 4914 & ENSGALTO0000016654 & 4.06 & $2.07 E-03$ & 4982 & ENSGALTO0000018891 & 3.76 & $2.79 \mathrm{E}-03 \mathrm{~S}$ & 5050 & ENSGALTO0000021121 & 4.56 & $4.32 \mathrm{E}-03$ \\
\hline 4915 & ENSGALTO00000016672 & 5.65 & $2.59 \mathrm{E}-03$ & 4983 & ENSGALTO0000018906 & 3.19 & $7.86 \mathrm{E}-035$ & 5051 & ENSGALTO0000021187 & 3.68 & $1.23 E-02$ \\
\hline 4916 & ENSGALTO0000016676 & 3.57 & $4.83 \mathrm{E}-03$ & 4984 & ENSGALTO0000018918 & 6.11 & $2.78 \mathrm{E}-035$ & 5052 & ENSGALTO0000021236 & 7.55 & $1.08 \mathrm{E}-03$ \\
\hline 4917 & ENSGALT00000016692 & 1.70 & $2.54 \mathrm{E}-03^{3}$ & 4985 & ENSGALTO0000018922 & 2.61 & $3.75 E-035$ & 5053 & ENSGALTO0000021245 & 5.94 & $9.31 \mathrm{E}-04$ \\
\hline 4918 & ENSGALTO0000016740 & 3.27 & $1.81 \mathrm{E}-03$ & 4986 & ENSGALTO0000018935 & 1.26 & $6.17 \mathrm{E}-045$ & 5054 & ENSGALTO0000021249 & 2.42 & $9.90 \mathrm{E}-04$ \\
\hline 4919 & ENSGALTO0000016901 & 6.75 & $7.11 \mathrm{E}-05$ & 4987 & ENSGALTO0000018953 & 3.88 & $1.04 E-045$ & 5055 & ENSGALTO0000021262 & 3.49 & $2.55 \mathrm{E}-03$ \\
\hline 4920 & ENSGALTO0000016904 & 5.81 & $1.32 \mathrm{E}-02$ & 4988 & ENSGALTO0000019071 & 3.34 & $1.39 \mathrm{E}-025$ & 5056 & ENSGALTO0000021290 & 2.00 & $5.38 \mathrm{E}-04$ \\
\hline 4921 & ENSGALTO00000016913 & 1.57 & $4.90 \mathrm{E}-02$ & 4989 & ENSGALTO0000019133 & 1.59 & $2.72 E-025$ & 5057 & ENSGALTO0000021316 & 2.93 & $3.75 \mathrm{E}-03$ \\
\hline 4922 & ENSGALTO0000016927 & 6.18 & $1.23 \mathrm{E}-03$ & 4990 & ENSGALTO0000019151 & 3.71 & $3.47 \mathrm{E}-035$ & 5058 & ENSGALTO0000021320 & 2.23 & $7.08 E-03$ \\
\hline 4923 & ENSGALTO00000017050 & 2.50 & $2.07 E-02$ & 4991 & ENSGALTO0000019218 & 2.08 & $1.29 \mathrm{E}-025$ & 5059 & ENSGALTO0000021349 & 4.21 & $9.91 \mathrm{E}-03$ \\
\hline 4924 & ENSGALTO0000017079 & 5.35 & $1.45 \mathrm{E}-02$ & 4992 & ENSGALTO0000019240 & 8.33 & $1.43 E-045$ & 5060 & ENSGALTO0000021357 & 1.99 & $1.84 \mathrm{E}-02$ \\
\hline 4925 & ENSGALTO0000017193 & 1.58 & $1.44 \mathrm{E}-02$ & 4993 & ENSGALTO0000019269 & 1.61 & $9.11 \mathrm{E}-035$ & 5061 & ENSGALTO0000021368 & 3.07 & $5.61 \mathrm{E}-03$ \\
\hline 4926 & ENSGALTO0000017213 & 1.93 & $1.57 \mathrm{E}-01$ & 4994 & ENSGALTO0000019298 & 5.71 & $3.15 \mathrm{E}-035$ & 5062 & ENSGALTO0000021463 & 2.47 & $1.53 E-02$ \\
\hline 4927 & ENSGALTO00000017233 & 3.86 & $1.48 \mathrm{E}-03^{-}$ & 4995 & ENSGALTO0000019456 & 4.97 & $4.88 \mathrm{E}-03 \mathrm{~s}$ & 5063 & ENSGALTO0000021510 & 2.46 & $6.91 \mathrm{E}-04$ \\
\hline 4928 & ENSGALTO0000017236 & 3.41 & $2.22 \mathrm{E}-03^{-}$ & 4996 & ENSGALTO0000019506 & 3.67 & $9.81 E-045$ & 5064 & ENSGALTO0000021522 & 6.35 & $1.20 \mathrm{E}-02$ \\
\hline 4929 & ENSGALTO0000017344 & 6.06 & $3.97 \mathrm{E}-02$ & 4997 & ENSGALTO00000019540 & 10.03 & $1.91 \mathrm{E}-055$ & 5065 & ENSGALTO00000021527 & 2.95 & $1.28 \mathrm{E}-03$ \\
\hline 4930 & ENSGALTO0000017347 & 3.86 & $2.50 \mathrm{E}-03$ & 4998 & ENSGALTO0000019592 & 3.22 & $4.74 \mathrm{E}-03$ & 5066 & ENSGALTO0000021595 & 3.43 & $6.12 E-06$ \\
\hline 4931 & ENSGALT000000017386 & 1.26 & $3.04 E-02$ & 4999 & ENSGALTO0000019597 & 1.99 & $4.79 \mathrm{E}-02$ & 5067 & ENSGALTO0000021597 & 3.81 & $2.00 \mathrm{E}-03$ \\
\hline 4932 & ENSGALTO0000017451 & 4.03 & $1.10 \mathrm{E}-02$ & 5000 & ENSGALT000000019607 & 3.59 & $2.43 \mathrm{E}-03$ & 5068 & ENSGALTO0000021600 & 1.16 & $3.36 \mathrm{E}-02$ \\
\hline 4933 & ENSGALTO00000017476 & 2.42 & $4.66 E-03^{-7}$ & 5001 & ENSGALTO00000019630 & 4.22 & $7.69 \mathrm{E}-04$ & 5069 & ENSGALTO0000021603 & 2.05 & $9.81 \mathrm{E}-03$ \\
\hline 4934 & ENSGALTO00000017489 & 4.35 & $2.90 \mathrm{E}-03^{-}$ & 5002 & ENSGALTO0000019656 & 4.67 & $1.96 \mathrm{E}-03$ & 5070 & ENSGALTO0000021604 & 3.39 & $4.11 \mathrm{E}-03$ \\
\hline 4935 & ENSGALTO00000017518 & 6.92 & $7.34 \mathrm{E}-05^{-5}$ & 5003 & ENSGALTO0000019661 & 2.12 & $2.99 \mathrm{E}-03$ & 5071 & ENSGALTO00000021606 & 2.96 & $4.14 \mathrm{E}-03$ \\
\hline 4936 & ENSGALTO00000017572 & 7.28 & 3.40E-04 & 5004 & ENSGALTO0000019669 & 1.69 & $3.51 \mathrm{E}-02$ & 5072 & ENSGALTO0000021609 & 2.82 & $2.83 \mathrm{E}-03$ \\
\hline 4937 & ENSGALTO0000017611 & 5.20 & $7.56 \mathrm{E}-05$ & 5005 & ENSGALTO0000019725 & 2.92 & $1.20 \mathrm{E}-04$ & 5073 & ENSGALTO0000021615 & 4.26 & $8.26 \mathrm{E}-03$ \\
\hline 4938 & ENSGALTO00000017612 & 4.47 & $1.97 \mathrm{E}-03^{-}$ & 5006 & ENSGALTO0000019735 & 6.55 & $1.89 \mathrm{E}-04$ & 5074 & ENSGALTO0000021628 & 2.54 & $2.84 \mathrm{E}-02$ \\
\hline 4939 & ENSGALTO00000017672 & 5.80 & $4.12 \mathrm{E}-03$ & 5007 & ENSGALTO0000019758 & 3.38 & $4.11 \mathrm{E}-02$ & 5075 & ENSGALTO0000021642 & 8.36 & $1.08 \mathrm{E}-03$ \\
\hline 4940 & ENSGALTO0000017675 & 2.62 & $2.11 \mathrm{E}-02$ & 5008 & ENSGALTO0000019775 & 5.86 & $4.44 \mathrm{E}-03$ & 5076 & ENSGALTO0000021678 & 4.10 & $1.15 \mathrm{E}-03$ \\
\hline 4941 & ENSGALTO0000017676 & 1.45 & $1.19 \mathrm{E}-02$ & 5009 & ENSGALTO0000019833 & 9.03 & $1.19 \mathrm{E}-04$ & 5077 & ENSGALTO0000021682 & 2.34 & $2.35 \mathrm{E}-03$ \\
\hline 4942 & ENSGALT00000017686 & 6.86 & $1.83 \mathrm{E}-03$ & 5010 & ENSGALTO0000019836 & 3.17 & $4.15 \mathrm{E}-03$ & 5078 & ENSGALTO0000021689 & 2.60 & $6.74 \mathrm{E}-03$ \\
\hline 4943 & ENSGALT00000017697 & 6.09 & $2.94 \mathrm{E}-03$ & 5011 & ENSGALTO0000019944 & 1.12 & $6.67 \mathrm{E}-03$ & 5079 & ENSGALTO0000021696 & 1.67 & $9.65 \mathrm{E}-03$ \\
\hline 4944 & ENSGALTO0000017734 & 3.26 & $8.14 \mathrm{E}-04$ & 5012 & ENSGALTO0000019953 & 4.60 & $9.00 \mathrm{E}-03$ & 5080 & ENSGALTO0000021745 & 3.27 & $4.70 \mathrm{E}-03$ \\
\hline 4945 & ENSGALTO0000017738 & 5.02 & 3.49E-04 & 5013 & ENSGALTO0000019963 & 1.15 & $4.71 \mathrm{E}-03$ & 5081 & ENSGALTO0000021749 & 5.80 & $2.05 E-05$ \\
\hline 4946 & ENSGALTO0000017748 & 2.37 & $6.13 \mathrm{E}-03$ & 5014 & ENSGALTO0000019997 & 6.68 & $9.91 E-04$ & 5082 & ENSGALTO0000021756 & 8.28 & $4.34 \mathrm{E}-03$ \\
\hline 4947 & ENSGALTO0000017750 & 1.26 & $1.41 \mathrm{E}-02$ & 5015 & ENSGALTO0000020003 & 6.14 & $4.84 \mathrm{E}-03$ & 5083 & ENSGALTO0000021758 & 3.02 & $2.88 \mathrm{E}-03$ \\
\hline 4948 & ENSGALTO0000017789 & 1.86 & $9.65 \mathrm{E}-03$ & 5016 & ENSGALTO0000020040 & 4.50 & $4.53 \mathrm{E}-03$ & 5084 & ENSGALTO0000021774 & 1.28 & $2.47 \mathrm{E}-02$ \\
\hline 4949 & ENSGALTO0000017810 & 4.53 & $1.59 \mathrm{E}-03$ & 5017 & ENSGALTO0000020052 & 4.58 & $1.27 \mathrm{E}-02$ & 5085 & ENSGALTO0000021784 & 7.03 & $1.85 \mathrm{E}-04$ \\
\hline 4950 & ENSGALTO0000017815 & 1.83 & $3.88 \mathrm{E}-03$ & 5018 & ENSGALTO0000020055 & 8.83 & $1.99 \mathrm{E}-03$ & 5086 & ENSGALTO0000021794 & 4.82 & $1.35 \mathrm{E}-03$ \\
\hline 4951 & ENSGALTO0000017818 & 3.81 & $1.84 \mathrm{E}-04$ & 5019 & ENSGALTO0000020058 & 5.22 & $2.70 \mathrm{E}-03$ & 5087 & ENSGALTO0000021838 & 3.56 & $5.01 E-03$ \\
\hline 4952 & ENSGALTO00000017904 & 3.50 & $1.44 \mathrm{E}-03$ & 5020 & ENSGALTO0000020117 & 1.14 & $1.15 \mathrm{E}-01$ & 5088 & ENSGALTO0000021841 & 5.37 & $9.39 E-03$ \\
\hline 4953 & ENSGALTO00000017918 & 2.31 & $3.22 E-03$ & 5021 & ENSGALTO0000020127 & 2.16 & $2.57 \mathrm{E}-02$ & 5089 & ENSGALTO0000021842 & 4.10 & $5.96 \mathrm{E}-03$ \\
\hline 4954 & ENSGALTO0000017921 & 5.37 & $1.42 \mathrm{E}-03$ & 5022 & ENSGALTO0000020173 & 3.83 & $6.53 \mathrm{E}-03$ & 5090 & ENSGALTO0000021853 & 1.11 & $2.59 \mathrm{E}-02$ \\
\hline 4955 & ENSGALTO00000017970 & 2.86 & $3.83 \mathrm{E}-03$ & 5023 & ENSGALTO0000020179 & 5.15 & $1.48 \mathrm{E}-01$ & 5091 & ENSGALTO0000021862 & 4.74 & $1.50 \mathrm{E}-03$ \\
\hline 4956 & ENSGALTO00000018040 & 2.17 & $6.22 E-04$ & 5024 & ENSGALTO0000020220 & 5.37 & $4.60 \mathrm{E}-03$ & 5092 & ENSGALTO0000021878 & 7.02 & $7.37 E-04$ \\
\hline 4957 & ENSGALTO00000018055 & 7.83 & $9.93 E-04$ & 5025 & ENSGALT00000020227 & 5.59 & $1.40 \mathrm{E}-03$ & 5093 & ENSGALT00000021903 & 4.69 & $6.29 \mathrm{E}-03$ \\
\hline 4958 & ENSGALTO00000018056 & 1.49 & $2.31 \mathrm{E}-02$ & 5026 & ENSGALTO0000020255 & 3.72 & $2.03 \mathrm{E}-03$ & 5094 & ENSGALTO0000021923 & 4.16 & $2.18 \mathrm{E}-04$ \\
\hline 4959 & ENSGALTO0000018117 & 4.27 & 5.21E-04 & 5027 & ENSGALTO0000020296 & 3.32 & $1.50 \mathrm{E}-03$ & 5095 & ENSGALTO0000021960 & 5.20 & $1.25 \mathrm{E}-03$ \\
\hline 4960 & ENSGALTO0000018128 & 2.13 & $2.13 \mathrm{E}-02$ & 5028 & ENSGALT00000020313 & 3.53 & $2.24 \mathrm{E}-03$ & 5096 & ENSGALTO0000021976 & 4.46 & $1.16 \mathrm{E}-05$ \\
\hline 4961 & ENSGALT000000018143 & 7.58 & $1.75 E-03^{-7}$ & 5029 & ENSGALTO0000020342 & 5.18 & $1.59 \mathrm{E}-02$ & 5097 & ENSGALTO0000021991 & 5.27 & $4.49 \mathrm{E}-03$ \\
\hline 4962 & ENSGALT00000018191 & 4.29 & $1.49 \mathrm{E}-02$ & 5030 & ENSGALT00000020381 & 2.68 & $2.13 \mathrm{E}-03$ & 5098 & ENSGALTO0000022007 & 2.37 & $1.14 \mathrm{E}-04$ \\
\hline 4963 & ENSGALTO0000018194 & 6.81 & $1.78 \mathrm{E}-03$ & 5031 & ENSGALTO00000020385 & 4.97 & $3.97 \mathrm{E}-05$ & 5099 & ENSGALTO0000022016 & 8.35 & \\
\hline
\end{tabular}




\begin{tabular}{|c|c|c|c|c|c|c|c|c|c|c|c|}
\hline 5100 & ENSGALTO0000022019 & 1.78 & $2.01 \mathrm{E}-02 \quad 5$ & 5168 & ENSGALTO0000023666 & 2.33 & $1.35 E-03 \quad 52$ & & ENSGALTO0000025358 & 4.25 & $1.04 \mathrm{E}-02$ \\
\hline 5101 & ENSGALTO0000022024 & 8.59 & $6.77 \mathrm{E}-03 \overline{5}$ & 5169 & ENSGALT00000023689 & 3.09 & $3.10 \mathrm{E}-03 \quad 52$ & 5237 & ENSGALTO0000025359 & 3.02 & $1.62 \mathrm{E}-03$ \\
\hline 5102 & ENSGALTO0000022037 & 2.73 & $3.44 \mathrm{E}-03-5$ & 5170 & ENSGALTO0000023709 & 2.90 & $2.48 \mathrm{E}-03 \quad 52$ & 5238 & ENSGALTO0000025375 & 1.88 & $1.46 \mathrm{E}-02$ \\
\hline 5103 & ENSGALTO0000022067 & 1.59 & $3.32 \mathrm{E}-02 \overline{5}$ & 5171 & ENSGALT00000023730 & 4.28 & $5.46 \mathrm{E}-03 \quad 52$ & 5239 & ENSGALTO0000025383 & 6.53 & $9.72 \mathrm{E}-04$ \\
\hline 5104 & ENSGALTO0000022146 & 2.11 & $3.47 \mathrm{E}-03 \overline{5}$ & 5172 & ENSGALTO0000023760 & 5.30 & $1.02 E-03 \quad 52$ & 5240 & ENSGALTO0000025435 & 2.43 & $4.44 \mathrm{E}-02$ \\
\hline 5105 & ENSGALTO0000022175 & 5.39 & $1.85 \mathrm{E}-03 \overline{5}$ & 5173 & ENSGALTO0000023784 & 3.62 & $2.08 \mathrm{E}-03 \quad 52$ & 5241 & ENSGALTO0000025472 & 4.17 & $8.19 \mathrm{E}-03$ \\
\hline 5106 & ENSGALTO00000022226 & 5.36 & $2.50 \mathrm{E}-025$ & 5174 & ENSGALTO0000023791 & 4.69 & $1.94 \mathrm{E}-03 \quad 52$ & 5242 & ENSGALTO0000025504 & 3.96 & $7.88 \mathrm{E}-02$ \\
\hline 5107 & ENSGALTO00000022247 & 1.45 & $9.57 \mathrm{E}-04 \overline{5}$ & 5175 & ENSGALTO0000023798 & 4.24 & $4.79 \mathrm{E}-04 \quad 52$ & 5243 & ENSGALTO0000025595 & 3.21 & $5.88 \mathrm{E}-03$ \\
\hline 5108 & ENSGALTO00000022260 & 1.96 & $2.59 \mathrm{E}-035$ & 5176 & ENSGALTO0000023805 & 4.31 & $2.31 E-03 \quad 52$ & 5244 & ENSGALTO0000025606 & 2.20 & $1.90 \mathrm{E}-02$ \\
\hline 5109 & ENSGALTO00000022276 & 2.99 & $1.83 \mathrm{E}-02 \overline{5}$ & 5177 & ENSGALTO0000023808 & 3.21 & $5.14 E-03 \quad 52$ & 5245 & ENSGALTO0000025610 & 1.27 & $6.16 \mathrm{E}-04$ \\
\hline 5110 & ENSGALTO00000022320 & 2.20 & $5.79 \mathrm{E}-03 \overline{5}$ & 5178 & ENSGALTO0000023811 & 3.97 & $3.08 \mathrm{E}-03 \quad 52$ & 5246 & ENSGALTO0000025615 & 5.13 & $1.84 \mathrm{E}-03$ \\
\hline 5111 & ENSGALT000000022328 & 3.92 & $3.64 \mathrm{E}-04-5$ & 5179 & ENSGALT000000023856 & 3.01 & $1.87 \mathrm{E}-03 \quad 52$ & 5247 & ENSGALT00000025682 & 8.40 & $8.25 \mathrm{E}-04$ \\
\hline 5112 & ENSGALT000000022338 & 1.33 & $2.23 \mathrm{E}-02-5$ & 5180 & ENSGALT00000023885 & 4.21 & $1.06 \mathrm{E}-02 \quad 52$ & 5248 & ENSGALTO0000025710 & 2.59 & $4.21 \mathrm{E}-03$ \\
\hline 5113 & ENSGALTO0000022347 & 2.66 & $2.51 \mathrm{E}-03 \overline{5}$ & 5181 & ENSGALTO0000023927 & 3.05 & $7.02 E-03 \quad 52$ & 5249 & ENSGALTO0000025722 & 1.81 & $2.80 \mathrm{E}-03$ \\
\hline 5114 & ENSGALTO0000022354 & 2.28 & $1.67 \mathrm{E}-025$ & 5182 & ENSGALT00000023928 & 2.17 & $4.86 \mathrm{E}-02 \quad 52$ & 5250 & ENSGALTO0000025740 & 2.76 & $4.56 \mathrm{E}-03$ \\
\hline 5115 & ENSGALT00000022355 & 6.24 & $2.81 \mathrm{E}-03 \overline{5}$ & 5183 & ENSGALTO0000023929 & 1.54 & $1.22 \mathrm{E}-03 \quad 52$ & 5251 & ENSGALTO0000025781 & 4.11 & $3.93 \mathrm{E}-03$ \\
\hline 5116 & ENSGALTO0000022391 & 8.74 & $2.72 \mathrm{E}-05 \overline{5}$ & 5184 & ENSGALTO0000023965 & 5.59 & $2.09 \mathrm{E}-03 \quad 52$ & 5252 & ENSGALTO0000025808 & 2.57 & $1.25 \mathrm{E}-02$ \\
\hline 5117 & ENSGALTO0000022427 & 3.52 & $3.29 \mathrm{E}-03 \overline{5}$ & 5185 & ENSGALTO0000023971 & 3.00 & $5.75 E-03 \quad 52$ & 5253 & ENSGALTO0000025879 & 3.03 & $5.58 \mathrm{E}-03$ \\
\hline 5118 & ENSGALTO00000022442 & 2.53 & $1.07 \mathrm{E}-04 \overline{5}$ & 5186 & ENSGALT00000023998 & 4.62 & $1.86 \mathrm{E}-02 \quad 52$ & 5254 & ENSGALTO00000025906 & 1.50 & $2.76 \mathrm{E}-02$ \\
\hline 5119 & ENSGALTO00000022448 & 3.42 & $3.07 \mathrm{E}-03 \overline{5}$ & 5187 & ENSGALTO0000024019 & 5.04 & $1.97 \mathrm{E}-03 \quad 52$ & 5255 & ENSGALTO0000025913 & 4.34 & $2.64 \mathrm{E}-03$ \\
\hline 5120 & ENSGALTO00000022458 & 3.45 & $9.05 \mathrm{E}-04 \overline{5}$ & 5188 & ENSGALTO0000024069 & 1.77 & $2.45 \mathrm{E}-03 \quad 52$ & 5256 & ENSGALTO0000025921 & 3.51 & $8.93 \mathrm{E}-04$ \\
\hline 5121 & ENSGALTO0000022529 & 3.01 & $5.98 \mathrm{E}-02-5$ & 5189 & ENSGALTO0000024084 & 7.53 & $2.63 \mathrm{E}-03 \quad 52$ & 5257 & ENSGALTO0000025935 & 2.24 & $2.30 \mathrm{E}-03$ \\
\hline 5122 & ENSGALTO00000022557 & 2.53 & $8.40 \mathrm{E}-025$ & 5190 & ENSGALTO0000024089 & 1.10 & $1.00 \mathrm{E}-02 \quad 52$ & 5258 & ENSGALTO0000025944 & 6.71 & $2.38 \mathrm{E}-03$ \\
\hline 5123 & ENSGALTO0000022577 & 4.72 & $2.60 \mathrm{E}-03 \overline{5}$ & 5191 & ENSGALTO0000024112 & 1.91 & $3.41 \mathrm{E}-03 \quad 52$ & 5259 & ENSGALTO0000025946 & 4.86 & $1.42 \mathrm{E}-03$ \\
\hline 5124 & ENSGALT000000022602 & 3.63 & $5.30 \mathrm{E}-02 \overline{5}$ & 5192 & ENSGALTO0000024133 & 4.89 & $4.51 \mathrm{E}-03 \quad 52$ & 5260 & ENSGALTO0000025964 & 3.40 & $5.84 \mathrm{E}-03$ \\
\hline 5125 & ENSGALTO00000022620 & 1.82 & $8.22 \mathrm{E}-03 \overline{5}$ & 5193 & ENSGALTO0000024157 & 4.21 & $1.79 E-05 \quad 52$ & 5261 & ENSGALTO0000025983 & 1.57 & $3.17 \mathrm{E}-03$ \\
\hline 5126 & ENSGALT00000022627 & 3.08 & $8.97 \mathrm{E}-04-\overline{5}$ & 5194 & ENSGALTO0000024173 & 4.33 & $8.11 \mathrm{E}-03 \quad 52$ & 5262 & ENSGALTO0000025998 & 3.49 & $1.46 \mathrm{E}-03$ \\
\hline 5127 & ENSGALT00000022645 & 4.91 & $8.65 \mathrm{E}-04 \overline{5}$ & 5195 & ENSGALTO0000024193 & 4.27 & $7.65 \mathrm{E}-03 \quad 52$ & 5263 & ENSGALTO0000026024 & 3.78 & $6.73 \mathrm{E}-03$ \\
\hline 5128 & ENSGALT00000022661 & 4.20 & $6.43 \mathrm{E}-04 \overline{5}$ & 5196 & ENSGALTO0000024228 & 2.48 & $6.29 \mathrm{E}-03 \quad 52$ & 5264 & ENSGALTO0000026028 & 3.54 & $5.02 E-02$ \\
\hline 5129 & ENSGALTO00000022690 & 5.82 & $5.05 \mathrm{E}-04-5$ & 5197 & ENSGALT00000024242 & 3.90 & $5.89 \mathrm{E}-04 \quad 52$ & 5265 & ENSGALTO0000026030 & 1.99 & $2.27 \mathrm{E}-02$ \\
\hline 5130 & ENSGALT000000022692 & 4.14 & $1.78 \mathrm{E}-035$ & 5198 & ENSGALT00000024261 & 2.91 & $2.40 E-02 \quad 52$ & 5266 & ENSGALTO00000026059 & 1.66 & $1.72 E-02$ \\
\hline 5131 & ENSGALTO00000022695 & 1.20 & $1.65 \mathrm{E}-035$ & 5199 & ENSGALTO0000024294 & 1.19 & $6.93 \mathrm{E}-03 \quad 52$ & 5267 & ENSGALTO0000026076 & 5.34 & $1.15 \mathrm{E}-02$ \\
\hline 5132 & ENSGALT000000022720 & 4.07 & $2.03 \mathrm{E}-045$ & 5200 & ENSGALT00000024317 & 4.34 & $4.24 E-03 \quad 52$ & 5268 & ENSGALTO0000026105 & 2.84 & $1.62 \mathrm{E}-03$ \\
\hline 5133 & ENSGALTO0000022721 & 6.44 & $6.96 \mathrm{E}-04-5$ & 5201 & ENSGALTO0000024326 & 3.48 & $1.32 \mathrm{E}-03 \quad 52$ & 5269 & ENSGALTO0000026118 & 3.30 & $2.20 \mathrm{E}-02$ \\
\hline 5134 & ENSGALT00000022758 & 5.21 & $1.19 \mathrm{E}-03 \overline{5}$ & 5202 & ENSGALTO0000024352 & 4.26 & $4.84 \mathrm{E}-03 \quad 52$ & 5270 & ENSGALTO0000026121 & 4.60 & $1.42 \mathrm{E}-03$ \\
\hline 5135 & ENSGALTO00000022788 & 1.15 & $2.55 \mathrm{E}-025$ & 5203 & ENSGALTO0000024363 & 1.92 & $2.09 \mathrm{E}-03 \quad 52$ & 5271 & ENSGALTO0000026135 & 7.53 & $5.12 \mathrm{E}-03$ \\
\hline 5136 & ENSGALT00000022821 & 3.96 & $8.94 \mathrm{E}-055$ & 5204 & ENSGALTO0000024380 & 5.42 & $5.12 \mathrm{E}-03 \quad 52$ & 5272 & ENSGALTO0000026159 & 1.44 & $3.99 \mathrm{E}-02$ \\
\hline 5137 & ENSGALTO0000022825 & 4.48 & $2.59 \mathrm{E}-04 \sqrt{5}$ & 5205 & ENSGALTO0000024480 & 1.13 & $6.83 \mathrm{E}-04 \quad 52$ & 5273 & ENSGALTO0000026201 & 5.86 & $1.90 \mathrm{E}-02$ \\
\hline 5138 & ENSGALTO00000022869 & 9.68 & $2.38 \mathrm{E}-04 \overline{5}$ & 5206 & ENSGALTO00000024500 & 2.18 & $2.99 \mathrm{E}-03 \quad 52$ & 5274 & ENSGALT00000026217 & 1.94 & $2.44 \mathrm{E}-03$ \\
\hline 5139 & ENSGALTO00000022920 & 4.65 & $3.61 E-035$ & 5207 & ENSGALTO0000024511 & 4.02 & $5.17 \mathrm{E}-03 \quad 52$ & 5275 & ENSGALTO0000026224 & 4.83 & $1.24 \mathrm{E}-04$ \\
\hline 5140 & ENSGALT00000022965 & 5.95 & $3.18 \mathrm{E}-04 \overline{5}$ & 5208 & ENSGALTO0000024524 & 6.98 & $9.32 E-03 \quad 52$ & 5276 & ENSGALTO0000026259 & 3.95 & $4.97 \mathrm{E}-03$ \\
\hline 5141 & ENSGALTO0000022973 & 1.43 & $3.54 \mathrm{E}-04 \overline{5}$ & 5209 & ENSGALTO0000024563 & 1.25 & $5.76 \mathrm{E}-03 \quad 52$ & 5277 & ENSGALTO0000026271 & 6.44 & $7.08 E-04$ \\
\hline 5142 & ENSGALTO00000023059 & 2.91 & $2.26 \mathrm{E}-03-5$ & 5210 & ENSGALT00000024595 & 6.01 & $2.52 \mathrm{E}-05 \quad 52$ & 5278 & ENSGALTO0000026280 & 5.05 & $6.07 \mathrm{E}-04$ \\
\hline 5143 & ENSGALTO0000023064 & 2.14 & $3.68 \mathrm{E}-03 \overline{5}$ & 5211 & ENSGALTO0000024649 & 3.81 & $1.37 \mathrm{E}-03 \quad 52$ & 5279 & ENSGALTO0000026281 & 3.96 & $6.40 \mathrm{E}-03$ \\
\hline 5144 & ENSGALT00000023076 & 5.90 & $4.64 \mathrm{E}-045$ & 5212 & ENSGALTO0000024665 & 6.11 & $8.71 \mathrm{E}-04 \quad 52$ & 5280 & ENSGALTO0000026305 & 2.16 & $5.87 \mathrm{E}-03$ \\
\hline 5145 & ENSGALTO00000023079 & 4.61 & $3.92 E-03-5$ & 5213 & ENSGALTO0000024685 & 5.88 & $2.86 \mathrm{E}-04 \quad 52$ & 5281 & ENSGALTO0000026362 & 1.87 & $4.31 \mathrm{E}-03$ \\
\hline 5146 & ENSGALTO00000023100 & 2.78 & $1.26 \mathrm{E}-025$ & 5214 & ENSGALTO0000024704 & 4.27 & $4.95 \mathrm{E}-03 \quad 52$ & 5282 & ENSGALTO0000026381 & 3.40 & $4.08 \mathrm{E}-03$ \\
\hline 5147 & ENSGALTO0000023109 & 1.89 & $6.34 \mathrm{E}-025$ & 5215 & ENSGALT00000024728 & 2.56 & $1.61 \mathrm{E}-03 \quad 52$ & 5283 & ENSGALT00000026392 & 3.19 & $9.95 \mathrm{E}-03$ \\
\hline 5148 & ENSGALTO00000023171 & 2.18 & $2.65 \mathrm{E}-02 \sqrt{5}$ & 5216 & ENSGALTO0000024816 & 6.03 & $3.76 \mathrm{E}-03 \quad 52$ & 5284 & ENSGALTO0000026423 & 1.16 & $3.80 \mathrm{E}-03$ \\
\hline 5149 & ENSGALTO00000023224 & 3.65 & $6.33 \mathrm{E}-03 \overline{5}$ & 5217 & ENSGALTO0000024824 & 2.61 & $4.57 \mathrm{E}-04 \quad 52$ & 5285 & ENSGALTO0000026604 & 6.29 & $1.56 \mathrm{E}-03$ \\
\hline 5150 & ENSGALTO00000023226 & 5.95 & $3.64 \mathrm{E}-04 \sqrt{5}$ & 5218 & ENSGALTO0000024845 & 1.36 & $2.68 \mathrm{E}-02 \quad 52$ & 5286 & ENSGALT00000026605 & 6.78 & $1.84 \mathrm{E}-03$ \\
\hline 5151 & ENSGALTO0000023253 & 1.72 & $2.76 E-03 \sqrt{5}$ & 5219 & ENSGALTO0000024912 & 2.82 & $5.95 \mathrm{E}-04 \quad 52$ & 5287 & ENSGALTO0000026657 & 4.10 & $5.12 \mathrm{E}-03$ \\
\hline 5152 & ENSGALTO00000023264 & 1.66 & $4.54 \mathrm{E}-02 \sqrt{5}$ & 5220 & ENSGALTO0000024996 & 3.74 & $6.49 \mathrm{E}-03 \quad 52$ & 5288 & ENSGALTO0000026660 & 2.95 & $6.13 \mathrm{E}-03$ \\
\hline 5153 & ENSGALTO00000023275 & 4.71 & $4.52 \mathrm{E}-04 \overline{5}$ & 5221 & ENSGALT00000024997 & 6.58 & $\begin{array}{ll}1.18 \mathrm{E}-03 \quad 52 \\
\end{array}$ & 5289 & ENSGALTO0000026733 & 2.22 & $1.64 \mathrm{E}-02$ \\
\hline 5154 & ENSGALTO0000023285 & 3.40 & $2.35 \mathrm{E}-03 \overline{5}$ & 5222 & ENSGALTO0000024999 & 4.74 & $1.09 \mathrm{E}-03 \quad 52$ & 5290 & ENSGALTO0000026739 & 5.68 & $1.98 \mathrm{E}-03$ \\
\hline 5155 & ENSGALTO0000023340 & 2.29 & $9.18 \mathrm{E}-03 \sqrt{5}$ & 5223 & ENSGALTO00000025015 & 1.54 & $2.48 \mathrm{E}-03 \quad 52$ & 5291 & ENSGALTO0000026782 & 5.25 & $5.63 \mathrm{E}-04$ \\
\hline 5156 & ENSGALTO0000023352 & 3.18 & $1.29 \mathrm{E}-03 \overline{5}$ & 5224 & ENSGALTO0000025019 & 5.97 & $1.40 \mathrm{E}-02 \quad 52$ & 5292 & ENSGALTO0000026785 & 4.49 & $2.67 \mathrm{E}-03$ \\
\hline 5157 & ENSGALTO0000023358 & 2.27 & $1.49 \mathrm{E}-02 \overline{5}$ & 5225 & ENSGALTO0000025042 & 2.50 & $2.09 \mathrm{E}-02 \quad 52$ & 5293 & ENSGALTO0000026795 & 2.10 & $1.01 \mathrm{E}-02$ \\
\hline 5158 & ENSGALTO0000023413 & 1.80 & 1.70E-02 5 & 5226 & ENSGALTO0000025091 & 2.93 & $7.36 \mathrm{E}-03 \quad 52$ & 5294 & ENSGALTO0000026815 & 1.38 & $5.90 \mathrm{E}-03$ \\
\hline 5159 & ENSGALTO0000023455 & 3.31 & $3.28 \mathrm{E}-03$ & 5227 & ENSGALTO00000025094 & 2.26 & $1.90 \mathrm{E}-04 \quad 52$ & 5295 & ENSGALTO0000026873 & 1.46 & $4.67 \mathrm{E}^{-02}$ \\
\hline 5160 & ENSGALTO00000023479 & 2.90 & $2.78 \mathrm{E}-03 \overline{5}$ & 5228 & ENSGALTO00000025103 & 4.59 & $3.37 \mathrm{E}-03 \quad 52$ & 5296 & ENSGALTO0000026914 & 1.18 & $1.03 E-01$ \\
\hline 5161 & ENSGALT00000023486 & 2.70 & $5.45 \mathrm{E}-05 \sqrt{5}$ & 5229 & ENSGALTO0000025131 & 4.50 & $1.92 \mathrm{E}-04 \quad 52$ & 5297 & ENSGALTO0000026942 & 3.90 & $4.14 \mathrm{E}-03$ \\
\hline 5162 & ENSGALTO0000023498 & 5.79 & $1.57 \mathrm{E}-03 \overline{5}$ & 5230 & ENSGALT00000025150 & 2.34 & $5.32 \mathrm{E}-03 \quad 52$ & 5298 & ENSGALTO0000027110 & 1.60 & $1.21 \mathrm{E}-02$ \\
\hline 5163 & ENSGALTO00000023518 & 1.34 & $4.44 E-03 \frac{5}{5}$ & 5231 & ENSGALTO00000025160 & 2.96 & $3.38 \mathrm{E}-03 \quad 52$ & 5299 & ENSGALTO0000027134 & 4.87 & $7.77 E-04$ \\
\hline 5164 & ENSGALT00000023520 & 5.96 & $4.51 \mathrm{E}-04 \overline{5}$ & 5232 & ENSGALTO0000025161 & 2.42 & $2.30 \mathrm{E}-03 \quad 53$ & 5300 & ENSGALTO0000027168 & 3.09 & $3.95 \mathrm{E}-03$ \\
\hline 5165 & ENSGALTO00000023577 & 1.46 & $7.54 \mathrm{E}-03 \frac{5}{5}$ & 5233 & ENSGALT00000025163 & 1.21 & $6.42 \mathrm{E}-03 \quad 53$ & 5301 & ENSGALTO00000027291 & 6.18 & $1.98 \mathrm{E}-04$ \\
\hline 5166 & ENSGALTO0000023596 & 2.53 & $1.08 \mathrm{E}-035$ & 5234 & ENSGALTO0000025182 & 1.39 & $1.98 \mathrm{E}-02 \quad 53$ & 5302 & ENSGALTO0000027325 & 5.87 & $1.30 \mathrm{E}-04$ \\
\hline 5167 & ENSGALTO0000023622 & 1.20 & $1.26 \mathrm{E}-025$ & 5235 & ENSGALTO00000025241 & 1.86 & $1.31 \mathrm{E}-02 \quad 53$ & 5303 & ENSGALTO0000027481 & 3.30 & $7.79 \mathrm{E}-03$ \\
\hline
\end{tabular}




\begin{tabular}{|c|c|c|c|c|c|c|c|c|c|c|c|}
\hline 5304 & ENSGALTO0000027515 & 1.69 & $3.15 \mathrm{E}-04 \quad 5$ & 5372 & ENSGALTO0000029542 & 8.83 & $1.20 \mathrm{E}-04 \mathrm{~s}$ & 5440 & ENSGALTO0000030732 & 2.17 & $3.08 \mathrm{E}-02$ \\
\hline 5305 & ENSGALTO0000027604 & 3.13 & $1.68 \mathrm{E}-03 \overline{5}$ & 5373 & ENSGALT00000029557 & 4.26 & $2.88 \mathrm{E}-035$ & 5441 & ENSGALTO0000030753 & 6.05 & $1.54 \mathrm{E}-04$ \\
\hline 5306 & ENSGALTO0000027611 & 1.44 & $1.55 \mathrm{E}-025$ & 5374 & ENSGALTO0000029575 & 4.59 & $4.67 \mathrm{E}-03 \mathrm{~s}$ & 5442 & ENSGALTO0000030760 & 5.58 & $1.56 \mathrm{E}-02$ \\
\hline 5307 & ENSGALTO0000027705 & 3.84 & $8.37 \mathrm{E}-03 \overline{5}$ & 5375 & ENSGALTO0000029588 & 2.90 & $9.19 \mathrm{E}-03 \mathrm{5}$ & 5443 & ENSGALTO0000030764 & 4.20 & $8.36 \mathrm{E}-03$ \\
\hline 5308 & ENSGALTO0000027722 & 1.27 & $5.33 \mathrm{E}-03-5$ & 5376 & ENSGALTO0000029589 & 6.52 & $8.16 \mathrm{E}-04 \mathrm{5}$ & 5444 & ENSGALTO0000030790 & 1.69 & $2.67 \mathrm{E}-04$ \\
\hline 5309 & ENSGALTO0000027729 & 4.50 & $2.59 \mathrm{E}-02 \overline{5}$ & 5377 & ENSGALTO0000029605 & 3.14 & $1.18 \mathrm{E}-025$ & 5445 & ENSGALTO0000030800 & 2.57 & $9.42 \mathrm{E}-03$ \\
\hline 5310 & ENSGALT00000027761 & 5.00 & $6.00 \mathrm{E}-03 \overline{5}$ & 5378 & ENSGALTO0000029606 & 6.72 & $6.27 \mathrm{E}-03 \mathrm{~S}$ & 5446 & ENSGALT00000030804 & 2.23 & $1.12 \mathrm{E}-01$ \\
\hline 5311 & ENSGALTO00000027776 & 2.76 & $2.71 \mathrm{E}-04 \overline{5}$ & 5379 & ENSGALTO0000029619 & 7.23 & $2.40 \mathrm{E}-04 \mathrm{~S}$ & 5447 & ENSGALTO0000030819 & 1.88 & $7.07 E-03$ \\
\hline 5312 & ENSGALTO0000027812 & 1.23 & $6.50 \mathrm{E}-025$ & 5380 & ENSGALTO0000029625 & 6.86 & $4.67 \mathrm{E}-035$ & 5448 & ENSGALTO0000030832 & 5.00 & $4.20 \mathrm{E}-05$ \\
\hline 5313 & ENSGALT00000027864 & 7.10 & $5.94 \mathrm{E}-04 \overline{5}$ & 5381 & ENSGALTO0000029642 & 5.65 & $3.11 \mathrm{E}-035$ & 5449 & ENSGALTO00000030849 & 3.80 & $2.57 \mathrm{E}-03$ \\
\hline 5314 & ENSGALTO0000027891 & 4.52 & $5.71 \mathrm{E}-03 \overline{5}$ & 5382 & ENSGALTO0000029644 & 9.22 & $7.32 \mathrm{E}-05 \mathrm{~s}$ & 5450 & ENSGALTO0000030856 & 2.38 & $1.75 \mathrm{E}-02$ \\
\hline 5315 & ENSGALTO0000027911 & 2.07 & $4.43 \mathrm{E}-03 \overline{5}$ & 5383 & ENSGALTO0000029648 & 4.56 & $4.30 \mathrm{E}-055$ & 5451 & ENSGALTO0000030857 & 5.58 & $1.45 \mathrm{E}-03$ \\
\hline 5316 & ENSGALTO0000027916 & 1.64 & $1.23 \mathrm{E}-01 \overline{5}$ & 5384 & ENSGALTO0000029662 & 1.26 & $2.53 \mathrm{E}-03 \mathrm{~s}$ & 5452 & ENSGALTO0000030864 & 4.19 & $2.74 E-02$ \\
\hline 5317 & ENSGALTO0000027921 & 3.04 & $4.19 \mathrm{E}-03 \overline{5}$ & 5385 & ENSGALT00000029675 & 4.52 & $3.33 \mathrm{E}-03 \mathrm{5}$ & 5453 & ENSGALTO0000030905 & 7.25 & $1.55 \mathrm{E}-04$ \\
\hline 5318 & ENSGALTO0000027936 & 3.15 & $1.93 \mathrm{E}-03 \overline{5}$ & 5386 & ENSGALTO0000029681 & 4.59 & $6.64 \mathrm{E}-03 \mathrm{~s}$ & 5454 & ENSGALTO0000030922 & 4.56 & $4.04 E-03$ \\
\hline 5319 & ENSGALTO0000027947 & 4.84 & $3.53 \mathrm{E}-04 \overline{5}$ & 5387 & ENSGALTO0000029688 & 1.57 & $5.14 \mathrm{E}-035$ & 5455 & ENSGALTO0000030941 & 4.17 & $8.32 E-03$ \\
\hline 5320 & ENSGALT00000027961 & 2.23 & $7.01 E-03 \frac{5}{5}$ & 5388 & ENSGALTO0000029699 & 3.89 & $7.07 \mathrm{E}-04 \mathrm{~S}$ & 5456 & ENSGALTO0000030944 & 3.96 & $7.56 \mathrm{E}-03$ \\
\hline 5321 & ENSGALTO00000027981 & 3.28 & $4.79 \mathrm{E}-04 \overline{5}$ & 5389 & ENSGALTO0000029799 & 11.39 & $1.25 \mathrm{E}-03 \mathrm{~S}$ & 5457 & ENSGALT00000030960 & 3.48 & $6.98 \mathrm{E}-03$ \\
\hline 5322 & ENSGALTO0000027994 & 3.40 & $2.08 \mathrm{E}-03 \overline{5}$ & 5390 & ENSGALTO0000029806 & 2.41 & $6.13 \mathrm{E}-02 \mathrm{~s}$ & 5458 & ENSGALTO0000030969 & 4.32 & $2.81 \mathrm{E}-02$ \\
\hline 5323 & ENSGALTT00000028008 & 4.10 & $1.14 \mathrm{E}-02 \overline{5}$ & 5391 & ENSGALTO0000029811 & 5.03 & $4.86 \mathrm{E}-045$ & 5459 & ENSGALTO0000031023 & 4.39 & $3.24 E-03$ \\
\hline 5324 & ENSGALTO0000028013 & 3.55 & $1.51 \mathrm{E}-01 \overline{5}$ & 5392 & ENSGALT00000029844 & 6.58 & $2.22 E-045$ & 5460 & ENSGALTO0000031037 & 1.37 & $1.71 \mathrm{E}-03$ \\
\hline 5325 & $\begin{array}{l}\text { ENSGALTO00000028019 } \\
\end{array}$ & 2.27 & $4.81 \mathrm{E}-03 \frac{-1}{5}$ & 5393 & ENSGALT00000029851 & 5.79 & $1.21 \mathrm{E}-03 \mathrm{5}$ & 5461 & ENSGALT00000031083 & 5.27 & $1.90 \mathrm{E}-03$ \\
\hline 5326 & ENSGALTO00000028020 & 4.57 & $1.57 \mathrm{E}-03-5$ & 5394 & ENSGALT00000029861 & 4.33 & $1.62 \mathrm{E}-03 \mathrm{5}$ & 5462 & ENSGALT00000031085 & 2.67 & $3.49 \mathrm{E}-04$ \\
\hline 5327 & ENSGALTO0000028061 & 1.31 & $2.47 \mathrm{E}-03 \overline{5}$ & 5395 & ENSGALTO0000029865 & 6.84 & $2.48 \mathrm{E}-045$ & 5463 & ENSGALTO0000031098 & 6.03 & $3.14 \mathrm{E}-03$ \\
\hline 5328 & $\begin{array}{l}\text { ENSGALT00000028066 } \\
\end{array}$ & 3.65 & $2.25 \mathrm{E}-02-5$ & 5396 & ENSGALT00000029868 & 8.88 & $1.53 \mathrm{E}-03 \mathrm{5}$ & 5464 & ENSGALTO0000031100 & 4.47 & $6.32 \mathrm{E}-04$ \\
\hline 5329 & ENSGALTO0000028092 & 2.44 & $3.23 \mathrm{E}-03 \overline{5}$ & 5397 & ENSGALTO0000029878 & 1.49 & $1.59 \mathrm{E}-025$ & 5465 & ENSGALTO0000031128 & 1.16 & $3.43 E-02$ \\
\hline 5330 & ENSGALTO0000028093 & 1.59 & $7.74 \mathrm{E}-025$ & 5398 & ENSGALTO0000029890 & 7.24 & $2.08 \mathrm{E}-035$ & 5466 & ENSGALTO0000031150 & 2.13 & $1.82 E-02$ \\
\hline 5331 & ENSGALTO0000028155 & 7.82 & $2.04 \mathrm{E}-04-5$ & 5399 & ENSGALTO0000029897 & 8.47 & $9.01 E-045$ & 5467 & ENSGALTO0000031178 & 4.29 & $2.59 \mathrm{E}-03$ \\
\hline 5332 & ENSGALTO0000028198 & 9.33 & $1.25 \mathrm{E}-03 \overline{5}$ & 5400 & ENSGALTO0000029899 & 3.76 & $8.79 E-045$ & 5468 & ENSGALTO0000031203 & 2.29 & $3.19 \mathrm{E}-02$ \\
\hline 5333 & ENSGALTO0000028209 & 3.86 & $1.94 \mathrm{E}-03 \overline{5}$ & 5401 & ENSGALTO0000029908 & 6.70 & $7.24 \mathrm{E}-045$ & 5469 & ENSGALTO0000031213 & 9.40 & $1.79 E-04$ \\
\hline 5334 & ENSGALTO0000028251 & 7.79 & $1.18 \mathrm{E}-03 \overline{5}$ & 5402 & ENSGALTO0000029916 & 3.05 & $2.91 E-025$ & 5470 & ENSGALTO0000031230 & 2.94 & $2.25 \mathrm{E}-03$ \\
\hline 5335 & ENSGALTO00000028252 & 1.13 & $9.57 \mathrm{E}-03 \overline{5}$ & 5403 & ENSGALTO0000029929 & 8.39 & $8.29 \mathrm{E}-04 \mathrm{5}$ & 5471 & ENSGALTO0000031237 & 1.28 & $5.15 \mathrm{E}-02$ \\
\hline 5336 & ENSGALTO0000028253 & 8.84 & $7.90 \mathrm{E}-04 \overline{5}$ & 5404 & ENSGALTO0000029983 & 1.22 & $3.67 E-035$ & 5472 & ENSGALTO0000031243 & 2.94 & $1.14 \mathrm{E}-02$ \\
\hline 5337 & ENSGALTO0000028260 & 4.37 & $4.30 \mathrm{E}-03 \overline{5}$ & 5405 & ENSGALTO00000029993 & 7.65 & $7.86 \mathrm{E}-04 \mathrm{5}$ & 5473 & ENSGALTO00000031257 & 2.82 & $5.64 \mathrm{E}-03$ \\
\hline 5338 & ENSGALT000000028263 & 8.58 & $1.21 E-035$ & 5406 & ENSGALT000000029996 & 1.86 & $3.23 \mathrm{E}-02$ & 5474 & ENSGALTO0000031282 & 3.20 & $1.14 \mathrm{E}-02$ \\
\hline 5339 & ENSGALT000000028295 & 6.49 & $1.14 \mathrm{E}-03 \frac{5}{5}$ & 5407 & ENSGALTO0000030013 & 3.66 & $7.51 \mathrm{E}-03$ & 5475 & ENSGALTO0000031306 & 2.08 & $8.37 E-03$ \\
\hline 5340 & ENSGALTO00000028298 & 3.01 & $1.94 \mathrm{E}-03-5$ & 5408 & ENSGALTO0000030046 & 4.85 & $5.85 \mathrm{E}-04$ & 5476 & ENSGALTO0000031316 & 3.92 & $1.06 \mathrm{E}-04$ \\
\hline 5341 & ENSGALTO00000028323 & 5.54 & $1.88 \mathrm{E}-03-5$ & 5409 & ENSGALTO00000030050 & 5.40 & $4.62 \mathrm{E}-04$ & 5477 & ENSGALTO0000031336 & 2.97 & $4.11 \mathrm{E}-03$ \\
\hline 5342 & ENSGALTO00000028342 & 4.60 & $2.05 E-03 \sqrt{5}$ & 5410 & ENSGALTO0000030053 & 3.17 & $1.17 \mathrm{E}-02$ & 5478 & ENSGALTO0000031356 & 3.63 & $1.82 \mathrm{E}-02$ \\
\hline 5343 & $\begin{array}{l}\text { ENSGALT00000028361 } \\
\end{array}$ & 1.41 & $4.49 \mathrm{E}-03-5$ & 5411 & ENSGALTO0000030057 & 5.50 & $6.12 \mathrm{E}-03$ & 5479 & ENSGALTO0000031418 & 2.23 & $3.54 E-04$ \\
\hline 5344 & ENSGALT000000028363 & 6.78 & $2.32 \mathrm{E}-03 \frac{5}{5}$ & 5412 & ENSGALTO0000030065 & 2.62 & $3.24 \mathrm{E}-04$ & 5480 & ENSGALTO0000031437 & 5.18 & $1.09 E-02$ \\
\hline 5345 & ENSGALT000000028372 & 7.81 & $2.46 \mathrm{E}-045$ & 5413 & ENSGALTO00000030076 & 5.46 & $3.84 \mathrm{E}-03$ & 5481 & ENSGALTO00000031440 & 7.18 & $2.88 \mathrm{E}-03$ \\
\hline 5346 & ENSGALT00000028385 & 2.43 & $5.24 \mathrm{E}-02-5$ & 5414 & ENSGALTO0000030090 & 5.43 & $2.92 \mathrm{E}-03$ & 5482 & ENSGALTO00000031441 & 4.70 & $1.61 \mathrm{E}-05$ \\
\hline 5347 & ENSGALTO0000028403 & 6.04 & $1.09 \mathrm{E}-03 \overline{5}$ & 5415 & ENSGALT00000030094 & 1.25 & $9.19 \mathrm{E}-04$ & 5483 & ENSGALTO0000031442 & 4.68 & $9.73 \mathrm{E}-03$ \\
\hline 5348 & ENSGALTO0000028409 & 7.51 & $6.59 \mathrm{E}-045$ & 5416 & ENSGALTO0000030129 & 6.36 & $1.68 \mathrm{E}-03$ & 5484 & ENSGALTO0000031444 & 3.06 & $1.32 E-02$ \\
\hline 5349 & ENSGALTO0000028427 & 3.82 & $1.77 \mathrm{E}-035$ & 5417 & ENSGALTO0000030168 & 4.73 & $1.79 \mathrm{E}-03$ & 5485 & ENSGALTO0000031449 & 5.53 & $2.51 E-04$ \\
\hline 5350 & ENSGALTO0000029147 & 3.22 & $8.87 \mathrm{E}-04 \overline{5}$ & 5418 & ENSGALTO0000030181 & 5.73 & $2.35 \mathrm{E}-04$ & 5486 & ENSGALTO0000031464 & 2.21 & $5.10 \mathrm{E}-03$ \\
\hline 5351 & ENSGALTO0000029170 & 4.56 & $4.97 \mathrm{E}-03 \overline{5}$ & 5419 & ENSGALT00000030183 & 2.42 & $6.08 \mathrm{E}-03$ & 5487 & ENSGALTO0000031473 & 4.62 & $1.73 \mathrm{E}-03$ \\
\hline 5352 & ENSGALTO0000029174 & 2.35 & $5.52 \mathrm{E}-03 \overline{5}$ & 5420 & ENSGALTO0000030260 & 3.99 & $5.26 \mathrm{E}-02$ & 5488 & ENSGALTO0000031497 & 5.61 & $1.21 \mathrm{E}-03$ \\
\hline 5353 & ENSGALTO0000029178 & 7.31 & $3.14 \mathrm{E}-045$ & 5421 & ENSGALTO0000030299 & 4.78 & $6.19 \mathrm{E}-05$ & 5489 & ENSGALTO0000031498 & 3.18 & $1.80 \mathrm{E}-04$ \\
\hline 5354 & ENSGALTO0000029210 & 3.85 & $3.28 \mathrm{E}-03 \overline{5}$ & 5422 & ENSGALTO0000030310 & 2.75 & $7.22 \mathrm{E}-03$ & 5490 & ENSGALTO00000031509 & 4.99 & $5.94 \mathrm{E}-03$ \\
\hline 5355 & ENSGALTO00000029220 & 8.08 & $3.16 \mathrm{E}-04 \overline{5}$ & 5423 & ENSGALTO0000030324 & 1.66 & $2.51 \mathrm{E}-02$ & 5491 & ENSGALTO0000031558 & 2.91 & $1.28 \mathrm{E}-04$ \\
\hline 5356 & ENSGALTO00000029245 & 8.71 & $1.37 \mathrm{E}-04 \overline{5}$ & 5424 & ENSGALTO0000030341 & 3.91 & $8.63 \mathrm{E}-03$ & 5492 & ENSGALT00000031564 & 7.67 & $2.30 \mathrm{E}-05$ \\
\hline 5357 & ENSGALTO0000029253 & 8.65 & $1.62 \mathrm{E}-04 \overline{5}$ & 5425 & ENSGALTO0000030403 & 4.15 & $2.67 \mathrm{E}-04$ & 5493 & ENSGALTO0000031572 & 3.86 & $7.30 \mathrm{E}-03$ \\
\hline 5358 & ENSGALTO0000029259 & 6.32 & $9.82 \mathrm{E}-04 \overline{5}$ & 5426 & ENSGALTO0000030489 & 4.67 & $1.63 \mathrm{E}-03$ & 5494 & ENSGALTO0000031581 & 6.15 & $2.42 E-04$ \\
\hline 5359 & ENSGALTO0000029275 & 4.43 & $1.10 \mathrm{E}-035$ & 5427 & ENSGALTO0000030519 & 4.56 & $3.43 \mathrm{E}-04$ & 5495 & ENSGALTO0000031598 & 4.25 & $7.27 E-03$ \\
\hline 5360 & ENSGALTO0000029299 & 4.14 & $1.16 \mathrm{E}-01 \overline{5}$ & 5428 & ENSGALTO0000030524 & 5.00 & $1.53 \mathrm{E}-03$ & 5496 & ENSGALTO0000031640 & 4.02 & $1.84 \mathrm{E}-03$ \\
\hline 5361 & ENSGALTO00000029301 & 5.49 & $2.02 E-045$ & 5429 & ENSGALTO0000030525 & 7.32 & $3.11 \mathrm{E}-05$ & 5497 & ENSGALTO0000031676 & 4.79 & $1.37 E-03$ \\
\hline 5362 & ENSGALTO00000029310 & 7.90 & $3.14 \mathrm{E}-045$ & 5430 & ENSGALTO0000030553 & 5.90 & $5.78 \mathrm{E}-04$ & 5498 & ENSGALTO0000031685 & 1.35 & $1.02 E-02$ \\
\hline 5363 & ENSGALTO00000029356 & 4.01 & $1.59 \mathrm{E}-03 \overline{5}$ & 5431 & ENSGALTO0000030566 & 2.32 & $9.47 \mathrm{E}-04$ & 5499 & ENSGALT00000031707 & 6.39 & $7.18 \mathrm{E}-04$ \\
\hline 5364 & ENSGALTO00000029387 & 7.31 & $6.93 \mathrm{E}-03 \overline{5}$ & 5432 & ENSGALTO0000030571 & 4.46 & $6.61 \mathrm{E}-03$ & 5500 & ENSGALTO0000031724 & 3.76 & $2.79 E-04$ \\
\hline 5365 & ENSGALTO00000029406 & 4.78 & $8.66 \mathrm{E}-03 \overline{5}$ & 5433 & ENSGALT00000030585 & 3.94 & $1.01 \mathrm{E}-02$ & 5501 & ENSGALT00000031727 & 1.57 & $3.95 \mathrm{E}-02$ \\
\hline 5366 & ENSGALTO00000029412 & 4.39 & $7.75 E-03 \sqrt{5}$ & 5434 & ENSGALTO0000030587 & 5.75 & $5.00 \mathrm{E}-06$ & 5502 & ENSGALTO0000031745 & 2.33 & $1.74 E-03$ \\
\hline 5367 & ENSGALTO0000029418 & 8.57 & $7.84 \mathrm{E}-05 \overline{5}$ & 5435 & ENSGALTO0000030633 & 1.18 & $1.83 \mathrm{E}-02$ & 5503 & ENSGALTO0000031751 & 10.51 & $6.84 \mathrm{E}-03$ \\
\hline 5368 & ENSGALTO0000029433 & 5.66 & $7.79 \mathrm{E}-04 \overline{5}$ & 5436 & ENSGALT00000030657 & 2.72 & $4.25 \mathrm{E}-03$ & 5504 & ENSGALTO0000031754 & 5.41 & $6.41 \mathrm{E}-03$ \\
\hline 5369 & ENSGALTO00000029440 & 2.41 & $1.86 \mathrm{E}-02 \sqrt{5}$ & 5437 & ENSGALTO0000030666 & 6.90 & $2.05 \mathrm{E}-03$ & 5505 & ENSGALTO0000031777 & 5.29 & $2.58 \mathrm{E}-03$ \\
\hline 5370 & ENSGALTO0000029500 & 8.47 & $2.79 \mathrm{E}-03 \quad 5$ & 5438 & ENSGALTO0000030690 & 3.72 & $8.24 \mathrm{E}-04$ & 5506 & ENSGALTO0000031803 & 5.41 & $2.61 \mathrm{E}-03$ \\
\hline 5371 & ENSGALTO0000029531 & 1.47 & $5.76 E-02 \sqrt{5}$ & 5439 & ENSGALT00000030727 & 3.36 & 1.69E-03 & 5507 & ENSGALTO0000031816 & 3.68 & $8.23 \mathrm{E}-0$ \\
\hline
\end{tabular}




\begin{tabular}{|c|c|c|c|c|c|c|c|c|c|c|}
\hline 5508 & ENSGALT00000031853 & 4.91 & $1.50 \mathrm{E}-035$ & 5576 & ENSGALTO0000033309 & 5.11 & $2.84 \mathrm{E}-02 \quad 5644$ & ENSGALT00000034651 & 2.13 & $6.05 E-03$ \\
\hline 5509 & ENSGALTO0000031874 & 3.03 & $9.47 \mathrm{E}-03 \overline{5}$ & 5577 & ENSGALT00000033380 & 3.00 & $9.91 E-04 \quad 5645$ & ENSGALTO0000034670 & 2.74 & $1.12 \mathrm{E}-03$ \\
\hline 5510 & ENSGALTO0000031889 & 3.45 & $7.82 E-03 \frac{5}{5}$ & 5578 & ENSGALT00000033417 & 2.12 & $5.54 \mathrm{E}-02 \quad 5646$ & ENSGALT00000034679 & 2.24 & $3.95 \mathrm{E}-02$ \\
\hline 5511 & ENSGALTO0000031894 & 2.35 & $1.33 \mathrm{E}-02 \sqrt{5}$ & 5579 & ENSGALT00000033425 & 6.33 & $1.25 \mathrm{E}-04 \quad 5647$ & ENSGALT00000034695 & 1.69 & $8.26 \mathrm{E}-03$ \\
\hline 5512 & ENSGALTO0000031932 & 1.46 & $1.50 \mathrm{E}-03 \frac{5}{5}$ & 5580 & ENSGALT00000033458 & 4.54 & $2.56 \mathrm{E}-03 \quad 5648$ & ENSGALTO0000034753 & 3.87 & $6.01 E-04$ \\
\hline 5513 & ENSGALTO0000031960 & 1.44 & $6.82 \mathrm{E}-03$ & 5581 & ENSGALT00000033471 & 2.64 & $2.84 \mathrm{E}-03 \quad 5649$ & ENSGALT00000034766 & 2.43 & $5.70 \mathrm{E}-03$ \\
\hline 5514 & ENSGALTO0000032002 & 3.48 & $1.48 \mathrm{E}-02 \overline{5}$ & 5582 & ENSGALTO0000033473 & 1.43 & $1.93 \mathrm{E}-02 \quad 5650$ & ENSGALTO0000034773 & 4.58 & $7.58 \mathrm{E}-03$ \\
\hline 5515 & ENSGALTO0000032003 & 1.61 & $2.44 \mathrm{E}-02 \sqrt{5}$ & 5583 & ENSGALTO0000033481 & 1.37 & $4.04 \mathrm{E}-02 \quad 5651$ & ENSGALTO0000034777 & 4.69 & $9.46 \mathrm{E}-04$ \\
\hline 5516 & ENSGALTO0000032078 & 5.20 & $7.74 E-035$ & 5584 & ENSGALTO0000033559 & 1.73 & $9.13 \mathrm{E}-03 \quad 5652$ & ENSGALTO0000034784 & 5.89 & $5.70 E-05$ \\
\hline 5517 & ENSGALT00000032082 & 7.21 & $2.89 \mathrm{E}-04 \sqrt{5}$ & 5585 & ENSGALTO0000033587 & 2.03 & $1.12 \mathrm{E}-02 \quad 5653$ & ENSGALT00000034792 & 6.49 & $2.57 \mathrm{E}-03$ \\
\hline 5518 & ENSGALTO0000032184 & 1.29 & $6 . 8 2 E - 0 3 \longdiv { 5 }$ & 5586 & ENSGALTO0000033602 & 1.45 & $5.70 \mathrm{E}-02 \quad 5654$ & ENSGALT000000034802 & 4.86 & $1.64 \mathrm{E}-03$ \\
\hline 5519 & ENSGALTO0000032192 & 4.16 & $3.84 \mathrm{E}-025$ & 5587 & ENSGALT00000033613 & 4.78 & $6.20 \mathrm{E}-04 \quad 5655$ & ENSGALTO0000034855 & 5.00 & $8.84 \mathrm{E}-03$ \\
\hline 5520 & ENSGALTO0000032205 & 6.24 & $8.47 \mathrm{E}-04-5$ & 5588 & ENSGALT00000033643 & 4.08 & $1.02 \mathrm{E}-03 \quad 5656$ & ENSGALTO0000034916 & 5.79 & $5.36 \mathrm{E}-03$ \\
\hline 5521 & ENSGALTO0000032210 & 2.96 & $3.58 \mathrm{E}-04 \overline{5}$ & 5589 & ENSGALTO0000033644 & 5.74 & $1.65 \mathrm{E}-03 \quad 5657$ & ENSGALTO0000034946 & 2.35 & $5.02 E-03$ \\
\hline 5522 & ENSGALTO0000032247 & 2.09 & $1.15 \mathrm{E}-025$ & 5590 & ENSGALT00000033697 & 4.14 & $4.14 \mathrm{E}-03 \quad 5658$ & ENSGALTO0000034962 & 1.21 & $2.73 \mathrm{E}-02$ \\
\hline 5523 & ENSGALT00000032250 & 3.70 & $2.89 \mathrm{E}-03 \overline{5}$ & 5591 & ENSGALTO0000033713 & 6.71 & 4.84E-04 5659 & ENSGALTO0000034973 & 2.38 & $1.83 \mathrm{E}-02$ \\
\hline 5524 & ENSGALT00000032331 & 3.38 & $1.67 \mathrm{E}-02 \overline{5}$ & 5592 & ENSGALTO0000033720 & 2.38 & $5.08 \mathrm{E}-04 \quad 5660$ & ENSGALTO0000034986 & 1.59 & $1.72 E-02$ \\
\hline 5525 & ENSGALTO0000032334 & 6.56 & $4.23 E-04-5$ & 5593 & ENSGALTO0000033721 & 3.58 & $1.55 \mathrm{E}-03 \quad 5661$ & ENSGALT00000034987 & 5.04 & $7.24 E-03$ \\
\hline 5526 & ENSGALTO00000032340 & 4.81 & $1.91 \mathrm{E}-03$ & 5594 & ENSGALTO0000033734 & 3.99 & $7.13 \mathrm{E}-04 \quad 5662$ & ENSGALT00000035005 & 4.81 & $5.52 \mathrm{E}-03$ \\
\hline 5527 & ENSGALTO00000032341 & 6.39 & $6.89 \mathrm{E}-03$ & 5595 & ENSGALTO0000033738 & 1.22 & $1.93 \mathrm{E}-02 \quad 5663$ & ENSGALTO0000035033 & 5.90 & $2.07 E-03$ \\
\hline 5528 & ENSGALT000000032362 & 3.67 & $3.24 \mathrm{E}-03$ & 5596 & ENSGALTO0000033758 & 2.87 & $\begin{array}{ll}1.40 E-04 \quad 5664 \\
\end{array}$ & ENSGALT00000035050 & 3.49 & $2.48 \mathrm{E}-03$ \\
\hline 5529 & ENSGALTO0000032376 & 5.74 & $3.43 E-055$ & 5597 & ENSGALTO0000033778 & 3.17 & $1.53 \mathrm{E}-02 \quad 5665$ & ENSGALTO0000035083 & 6.06 & $1.62 E-03$ \\
\hline 5530 & ENSGALTO0000032405 & 2.92 & $6.82 E-03=5$ & 5598 & ENSGALTO0000033799 & 3.52 & $3.00 \mathrm{E}-03 \quad 5666$ & ENSGALT00000035094 & 3.37 & $4.32 E-03$ \\
\hline 5531 & ENSGALTO00000032414 & 2.42 & $4.79 \mathrm{E}-03$ & 5599 & ENSGALT00000033803 & 4.55 & $6.99 \mathrm{E}-04 \quad 5667$ & ENSGALTO00000035116 & 4.20 & $5.86 \mathrm{E}-03$ \\
\hline 5532 & ENSGALT00000032415 & 3.86 & $1.06 \mathrm{E}-025$ & 5600 & ENSGALTO0000033826 & 4.62 & $1.24 \mathrm{E}-02 \quad 5668$ & ENSGALTO00000035120 & 3.31 & $1.81 \mathrm{E}-03$ \\
\hline 5533 & ENSGALT00000032425 & 3.43 & $7.46 \mathrm{E}-045$ & 5601 & ENSGALTO0000033907 & 4.61 & $1.82 \mathrm{E}-03 \quad 5669$ & ENSGALT000000035129 & 3.69 & $5.65 \mathrm{E}-03$ \\
\hline 5534 & ENSGALTO0000032455 & 4.57 & $8.39 \mathrm{E}-055$ & 5602 & ENSGALTO0000033939 & 2.61 & $6.54 \mathrm{E}-03 \quad 5670$ & ENSGALT00000035157 & 2.09 & $5.27 \mathrm{E}-03$ \\
\hline 5535 & ENSGALTO0000032456 & 4.77 & $1.18 \mathrm{E}-04-5$ & 5603 & ENSGALTO0000033945 & 3.71 & $\begin{array}{ll}7.97 E-02 \quad 5671 \\
\end{array}$ & ENSGALT000000035159 & 4.81 & $6.68 \mathrm{E}-04$ \\
\hline 5536 & ENSGALTO0000032482 & 6.45 & $9.93 \mathrm{E}-04-5$ & 5604 & ENSGALTO0000033954 & 1.35 & $\begin{array}{ll}7.48 \mathrm{E}-03 \quad 5672 \\
\end{array}$ & ENSGALT000000035352 & 4.23 & $2.81 E-04$ \\
\hline 5537 & ENSGALTO0000032500 & 4.97 & $1.08 \mathrm{E}-03-5$ & 5605 & ENSGALTO0000033971 & 5.62 & $9.05 E-05 \quad 5673$ & ENSGALT00000035354 & 3.10 & $7.33 E-04$ \\
\hline 5538 & ENSGALTO00000032501 & 2.16 & $6.66 \mathrm{E}-03^{3} 5$ & 5606 & ENSGALTO0000033999 & 3.03 & $2.75 E-04 \quad 5674$ & ENSGALT00000035356 & 1.93 & $4.12 E-03$ \\
\hline 5539 & ENSGALTO00000032503 & 3.82 & $1.55 \mathrm{E}-035$ & 5607 & ENSGALTO0000034024 & 6.12 & 3.20E-03 5675 & ENSGALT00000035358 & 5.50 & $7.16 \mathrm{E}-05$ \\
\hline 5540 & ENSGALTO0000032560 & 2.49 & $2.54 \mathrm{E}-03$ & 5608 & ENSGALTO0000034031 & 4.80 & $1.32 \mathrm{E}-02 \quad 5676$ & ENSGALTO0000035359 & 3.68 & $2.46 E-04$ \\
\hline 5541 & ENSGALTO0000032583 & 1.19 & $4.98 \mathrm{E}-03$ & 5609 & ENSGALTO0000034032 & 5.30 & $4.80 \mathrm{E}-03 \quad 5677$ & ENSGALTO0000035360 & 2.09 & $9.26 \mathrm{E}-04$ \\
\hline 5542 & ENSGALTO00000032601 & 2.45 & $4.27 \mathrm{E}-04$ & 5610 & ENSGALTO0000034034 & 4.69 & $\begin{array}{ll}8.11 \mathrm{E}-03 \quad 5678 \\
\end{array}$ & ENSGALTO0000035361 & 1.92 & $1.16 \mathrm{E}-01$ \\
\hline 5543 & ENSGALTO00000032637 & 2.67 & $6.63 \mathrm{E}-03$ & 5611 & ENSGALTO0000034043 & 3.90 & $1.70 \mathrm{E}-03 \quad 5679$ & ENSGALTO0000035365 & 6.13 & $5.25 \mathrm{E}-03$ \\
\hline 5544 & ENSGALTO0000032646 & 1.87 & $1.21 \mathrm{E}-015$ & 5612 & ENSGALTO0000034106 & 4.48 & $4.78 \mathrm{E}-05 \quad 5680$ & ENSGALTO0000035367 & 2.88 & $\begin{array}{c}9.19 \mathrm{E}-03 \\
\end{array}$ \\
\hline 5545 & ENSGALTO00000032700 & 3.20 & $3.24 \mathrm{E}-03$ & 5613 & ENSGALTO0000034137 & 2.15 & $3.00 \mathrm{E}-02 \quad 5681$ & ENSGALTO0000035371 & 2.88 & $1.27 E-02$ \\
\hline 5546 & ENSGALTO0000032705 & 1.25 & $5.45 E-03-5$ & 5614 & ENSGALTO0000034143 & 5.77 & $1.67 \mathrm{E}-03 \quad 5682$ & ENSGALT00000035372 & 4.24 & $5.48 \mathrm{E}-03$ \\
\hline 5547 & ENSGALT00000032739 & 5.45 & $2.29 \mathrm{E}-03$ & 5615 & ENSGALTO0000034187 & 2.77 & $\begin{array}{ll}7.14 \mathrm{E}-03 \quad 5683 \\
\end{array}$ & ENSGALTO0000035374 & 4.24 & $2.62 \mathrm{E}-03$ \\
\hline 5548 & ENSGALTO0000032742 & 3.44 & $2.10 \mathrm{E}-03^{-5}$ & 5616 & ENSGALTO0000034200 & 4.92 & $1.41 \mathrm{E}-03 \quad 5684$ & ENSGALTO0000035376 & 1.27 & $1.68 \mathrm{E}-01$ \\
\hline 5549 & ENSGALTO0000032756 & 5.25 & $3.97 \mathrm{E}-03$ & 5617 & ENSGALTO0000034240 & 3.09 & $4.22 \mathrm{E}-01 \quad 5685$ & ENSGALTO0000035377 & 4.34 & $8.92 E-04$ \\
\hline 5550 & ENSGALTO00000032834 & 1.38 & $8.12 \mathrm{E}-03 \mathrm{E}$ & 5618 & ENSGALTO0000034268 & 1.49 & $9.50 \mathrm{E}-03 \quad 5686$ & ENSGALT000000035380 & 6.80 & $3.09 E-04$ \\
\hline 5551 & ENSGALTO0000032836 & 1.55 & $1.12 \mathrm{E}-02 \mathrm{5}$ & 5619 & ENSGALTO0000034293 & 2.11 & $7.40 \mathrm{E}-03 \quad 5687$ & ENSGALT00000035381 & 3.38 & $1.17 \mathrm{E}-03$ \\
\hline 5552 & ENSGALTO0000032837 & 2.31 & $7.76 \mathrm{E}-03$ & 5620 & ENSGALTO0000034294 & 5.61 & $\begin{array}{ll}3.08 \mathrm{E}-03 & 5688\end{array}$ & ENSGALT00000035382 & 5.55 & $7.41 E-04$ \\
\hline 5553 & ENSGALTO0000032872 & 4.80 & $3.53 \mathrm{E}-03$ & 5621 & ENSGALTO0000034308 & 4.41 & $7.79 \mathrm{E}-04 \quad 5689$ & ENSGALTO0000035384 & 2.94 & $4.28 \mathrm{E}-03$ \\
\hline 5554 & ENSGALT00000032885 & 3.40 & $6.24 \mathrm{E}-03$ & 5622 & ENSGALTO0000034321 & 1.35 & 5.27E-04 5690 & ENSGALTO0000035389 & 4.56 & $8.98 \mathrm{E}-03$ \\
\hline 5555 & ENSGALTO0000032889 & 5.82 & $1.65 \mathrm{E}-03$ & 5623 & ENSGALT00000034335 & 3.72 & $3.83 \mathrm{E}-03 \quad 5691$ & ENSGALTO0000035390 & 4.15 & $7.45 \mathrm{E}-03$ \\
\hline 5556 & ENSGALTO00000032925 & 4.29 & $1.12 \mathrm{E}-025$ & 5624 & ENSGALTO0000034340 & 5.04 & $1.02 \mathrm{E}-03 \quad 5692$ & ENSGALTO0000035391 & 5.74 & $6.45 \mathrm{E}-05$ \\
\hline 5557 & ENSGALTO00000032948 & 4.67 & $1.83 \mathrm{E}-045$ & 5625 & ENSGALTO0000034358 & 1.76 & $2.31 \mathrm{E}-03 \quad 5693$ & ENSGALTO0000035392 & 2.28 & $9.79 \mathrm{E}-03$ \\
\hline 5558 & ENSGALTO0000032981 & 1.18 & $6.67 \mathrm{E}-03-5$ & 5626 & ENSGALTO0000034371 & 5.35 & $\begin{array}{ll}7.24 \mathrm{E}-03 & 5694\end{array}$ & ENSGALT00000035397 & 6.49 & $8.36 \mathrm{E}-03$ \\
\hline 5559 & ENSGALTO00000032982 & 1.35 & $2.24 E-02-5$ & 5627 & ENSGALTO0000034387 & 8.89 & $2.47 \mathrm{E}-04 \quad 5695$ & ENSGALTO0000035400 & 6.00 & $9.19 \mathrm{E}-03$ \\
\hline 5560 & ENSGALT00000032989 & 4.42 & $1.84 \mathrm{E}-02$ & 5628 & ENSGALTO0000034402 & 3.13 & $9.98 \mathrm{E}-04 \quad 5696$ & ENSGALTO0000035403 & 5.24 & $1.33 E-03$ \\
\hline 5561 & ENSGALTO0000033005 & 3.31 & 4.36E-04 & 5629 & ENSGALTO0000034415 & 4.42 & $1.49 \mathrm{E}-02 \quad 5697$ & ENSGALT00000035405 & 4.29 & $1.04 E-03$ \\
\hline 5562 & ENSGALT000000033009 & 2.44 & $4.57 \mathrm{E}-02$ & 5630 & ENSGALTO0000034419 & 5.44 & $\begin{array}{ll}8.69 E-05 \quad 5698 \\
\end{array}$ & ENSGALTO0000035406 & 5.01 & $7.16 \mathrm{E}-03$ \\
\hline 5563 & ENSGALT000000033026 & 6.22 & $1.34 \mathrm{E}-02$ & 5631 & ENSGALTO0000034446 & 4.18 & $1.37 \mathrm{E}-03 \quad 5699$ & ENSGALTO0000035407 & 2.15 & $4.44 \mathrm{E}-02$ \\
\hline 5564 & ENSGALTO0000033063 & 3.98 & 7.45E-04 & 5632 & ENSGALTO0000034491 & 2.71 & $3.14 \mathrm{E}-04 \quad 5700$ & ENSGALTO00000035408 & 4.57 & $4.38 \mathrm{E}-03$ \\
\hline 5565 & ENSGALTO0000033099 & 1.32 & $7.60 \mathrm{E}-02$ & 5633 & ENSGALTO0000034493 & 3.79 & $1.54 \mathrm{E}-03 \quad 5701$ & ENSGALTO0000035413 & 4.06 & $1.48 \mathrm{E}-04$ \\
\hline 5566 & ENSGALTO0000033108 & 4.60 & 6.63E- -04 & 5634 & ENSGALTO0000034494 & 1.68 & $2.97 \mathrm{E}-02 \quad 5702$ & ENSGALTO0000035414 & 2.30 & $3.46 \mathrm{E}-02$ \\
\hline 5567 & ENSGALT00000033125 & 9.67 & $2.17 \mathrm{E}-04$ & 5635 & ENSGALTO0000034520 & 1.90 & $1.18 \mathrm{E}-01 \quad 5703$ & ENSGALTO00000035415 & 3.57 & $1.76 \mathrm{E}-03$ \\
\hline 5568 & ENSGALTO0000033147 & 6.51 & $3.50 \mathrm{E}-03$ & 5636 & ENSGALTO00000034523 & 4.80 & $1.33 \mathrm{E}-02 \quad 5704$ & ENSGALT00000035418 & 3.22 & $1.04 E-02$ \\
\hline 5569 & ENSGALTO0000033193 & 4.99 & $7.47 \mathrm{E}-04$ & 5637 & ENSGALTO0000034586 & 5.52 & $5.74 \mathrm{E}-04 \quad 5705$ & ENSGALT00000035420 & 2.54 & $4.14 E-04$ \\
\hline 5570 & ENSGALTO0000033215 & 3.23 & $5.96 \mathrm{E}-02$ & 5638 & ENSGALTO0000034591 & 1.63 & $9.57 \mathrm{E}-03 \quad 5706$ & ENSGALTO0000035421 & 1.12 & $5.30 \mathrm{E}-03$ \\
\hline 5571 & ENSGALTO0000033226 & 4.87 & $1.42 E-03=$ & 5639 & ENSGALTO0000034608 & 4.46 & 1.07E-04 5707 & ENSGALTO0000035422 & 2.40 & $5.19 \mathrm{E}-03$ \\
\hline 5572 & ENSGALTO00000033255 & 4.96 & $2.38 \mathrm{E}-03^{-}$ & 5640 & ENSGALTO0000034611 & 3.58 & $1.22 \mathrm{E}-03 \quad 5708$ & ENSGALT00000035424 & 1.44 & $1.07 E-02$ \\
\hline 5573 & ENSGALTO0000033270 & 3.59 & $7.37 \mathrm{E}-04$ & 5641 & ENSGALTO0000034623 & 4.39 & $2.61 \mathrm{E}-03 \quad 5709$ & ENSGALT00000035426 & 5.99 & $2.08 \mathrm{E}-03$ \\
\hline 5574 & ENSGALTO0000033279 & 2.81 & $1.43 \mathrm{E}-02$ & 5642 & ENSGALTO0000034624 & 2.02 & 3.46E-03 5710 & ENSGALT00000035432 & 3.43 & $1.39 \mathrm{E}-04$ \\
\hline 5575 & ENSGALTO0000033303 & 5.91 & $5.17 \mathrm{E}-03$ & 5643 & ENSGALT00000034628 & 3.29 & $5.09 \mathrm{E}-03 \quad 5711$ & ENSGALT00000035435 & 7.58 & $5.666-03$ \\
\hline
\end{tabular}




\begin{tabular}{|c|c|c|c|c|c|c|c|c|c|c|c|}
\hline 5712 & ENSGALTO0000035437 & 4.55 & $2.18 \mathrm{E}-02$ & 5780 & ENSGALTO0000035643 & 7.57 & $8.37 \mathrm{E}-04$ & 5848 & ENSGALTO0000035871 & 8.72 & $1.41 \mathrm{E}-03$ \\
\hline 5713 & ENSGALTO0000035438 & 5.26 & $1.45 \mathrm{E}-03$ & 5781 & ENSGALT00000035644 & 9.42 & $2.14 \mathrm{E}-03$ & 5849 & ENSGALTO0000035875 & 8.40 & $8.15 \mathrm{E}-04$ \\
\hline 5714 & ENSGALTO0000035440 & 3.27 & $4.69 \mathrm{E}-04$ & 5782 & ENSGALTO0000035650 & 1.79 & $9.18 \mathrm{E}-02$ & 5850 & ENSGALTO0000035876 & 7.74 & $1.55 \mathrm{E}-03$ \\
\hline 5715 & ENSGALTO0000035441 & 2.70 & $6.95 \mathrm{E}-03$ & 5783 & ENSGALTO0000035654 & 3.12 & $6.27 \mathrm{E}-02$ & 5851 & ENSGALT00000035877 & 6.95 & $6.99 \mathrm{E}-04$ \\
\hline 5716 & ENSGALTO0000035447 & 5.42 & $1.52 \mathrm{E}-03^{-}$ & 5784 & ENSGALTO0000035659 & 1.09 & $1.05 \mathrm{E}-01$ & 5852 & ENSGALT00000035879 & 3.65 & $2.41 \mathrm{E}-03$ \\
\hline 5717 & ENSGALTO0000035448 & 2.98 & $5.94 \mathrm{E}-03$ & 5785 & ENSGALTO0000035663 & 2.61 & $1.30 \mathrm{E}-02$ & 5853 & ENSGALTO0000035883 & 7.49 & $3.38 E-05$ \\
\hline 5718 & ENSGALTO0000035449 & 2.15 & $1.98 \mathrm{E}-02$ & 5786 & ENSGALTO0000035664 & 7.14 & $2.28 \mathrm{E}-03$ & 5854 & ENSGALTO0000035884 & 5.45 & $2.61 \mathrm{E}-03$ \\
\hline 5719 & ENSGALTO0000035497 & 4.34 & $2.31 \mathrm{E}-04$ & 5787 & ENSGALTO0000035671 & 8.78 & $1.22 \mathrm{E}-06$ & 5855 & ENSGALTO0000035885 & 5.94 & $3.73 E-04$ \\
\hline 5720 & ENSGALTO0000035499 & 3.72 & $3.53 \mathrm{E}-03$ & 5788 & ENSGALTO0000035679 & 4.57 & $4.93 \mathrm{E}-03$ & 5856 & ENSGALTO0000035892 & 3.06 & $1.50 \mathrm{E}-01$ \\
\hline 5721 & ENSGALT00000035501 & 7.70 & $1.49 \mathrm{E}-03^{-}$ & 5789 & ENSGALTO0000035681 & 4.69 & $8.22 \mathrm{E}-04$ & 5857 & ENSGALTO00000035894 & 4.37 & $1.14 \mathrm{E}-02$ \\
\hline 5722 & ENSGALTO0000035502 & 6.17 & $2.97 \mathrm{E}-03^{-5}$ & 5790 & ENSGALTO0000035686 & 3.89 & $8.27 \mathrm{E}-04$ & 5858 & ENSGALTO0000035899 & 6.04 & $1.26 \mathrm{E}-03$ \\
\hline 5723 & ENSGALTO0000035503 & 2.60 & 1.60E-03 & 5791 & ENSGALT00000035688 & 4.66 & $4.19 \mathrm{E}-03$ & 5859 & ENSGALTO0000035901 & 4.34 & $5.21 \mathrm{E}-03$ \\
\hline 5724 & ENSGALTO00000035507 & 3.47 & 4.03E-03 & 5792 & ENSGALTO0000035689 & 7.80 & $4.82 \mathrm{E}-04$ & 5860 & ENSGALTO0000035905 & 5.14 & $3.95 \mathrm{E}-03$ \\
\hline 5725 & ENSGALTO0000035511 & 2.96 & $7.90 \mathrm{E}-03$ & 5793 & ENSGALT00000035691 & 8.44 & $1.29 \mathrm{E}-03$ & 5861 & ENSGALTO0000035906 & 1.78 & $2.19 \mathrm{E}-02$ \\
\hline 5726 & ENSGALTO00000035516 & 1.56 & $6.44 \mathrm{E}-03$ & 5794 & ENSGALTO0000035692 & 5.64 & $3.26 \mathrm{E}-02$ & 5862 & ENSGALTO0000035907 & 6.62 & $5.19 \mathrm{E}-04$ \\
\hline 5727 & ENSGALTO0000035521 & 8.66 & $9.86 \mathrm{E}-03$ & 5795 & ENSGALTO0000035696 & 2.78 & $2.25 \mathrm{E}-03$ & 5863 & ENSGALTO0000035908 & 7.98 & $9.26 \mathrm{E}-05$ \\
\hline 5728 & ENSGALTO00000035523 & 1.46 & $1.27 \mathrm{E}-03$ & 5796 & ENSGALTO0000035698 & 9.06 & $1.27 \mathrm{E}-03$ & 5864 & ENSGALTO0000035913 & 5.13 & $1.30 \mathrm{E}-03$ \\
\hline 5729 & ENSGALTO00000035525 & 7.95 & $6.04 \mathrm{E}-04$ & 5797 & ENSGALTO0000035700 & 6.39 & $1.42 \mathrm{E}-03$ & 5865 & ENSGALTO0000035915 & 2.50 & $4.06 E-03$ \\
\hline 5730 & ENSGALTO00000035526 & 3.07 & $2.64 \mathrm{E}-02$ & 5798 & ENSGALTO0000035702 & 3.87 & $2.30 \mathrm{E}-04$ & 5866 & ENSGALTO0000035922 & 7.55 & $3.25 \mathrm{E}-03$ \\
\hline 5731 & ENSGALTO00000035534 & 7.06 & $3.90 \mathrm{E}-04$ & 5799 & ENSGALTO0000035707 & 2.65 & $8.14 \mathrm{E}-04$ & 5867 & ENSGALTO0000035928 & 8.52 & $1.42 E-04$ \\
\hline 5732 & ENSGALTO0000035535 & 3.75 & 3.68E-05 & 5800 & ENSGALT00000035709 & 5.11 & $5.92 \mathrm{E}-03$ & 5868 & ENSGALTO0000035933 & 5.45 & $5.61 \mathrm{E}-03$ \\
\hline 5733 & ENSGALTO00000035536 & 1.15 & $1.43 E-02$ & 5801 & ENSGALTO00000035710 & 8.28 & $7.06 \mathrm{E}-04$ & 5869 & ENSGALTO0000035934 & 5.30 & $6.34 \mathrm{E}-03$ \\
\hline 5734 & ENSGALTO00000035537 & 2.85 & $6.01 E-03^{-1}$ & 5802 & ENSGALTO00000035714 & 2.17 & $5.84 \mathrm{E}-04$ & 5870 & ENSGALT00000035939 & 6.56 & $2.49 \mathrm{E}-03$ \\
\hline 5735 & ENSGALT000000035539 & 4.00 & $5.89 \mathrm{E}-03^{-7}$ & 5803 & $\begin{array}{l}\text { ENSGALT00000035718 } \\
\end{array}$ & 6.33 & $2.72 \mathrm{E}-04$ & 5871 & ENSGALTO0000035942 & 2.60 & $1.36 \mathrm{E}-02$ \\
\hline 5736 & ENSGALTO0000035540 & 6.92 & $9.01 E-04$ & 5804 & ENSGALT00000035733 & 4.30 & $1.20 \mathrm{E}-03$ & 5872 & ENSGALTO0000035943 & 7.67 & $1.41 \mathrm{E}-04$ \\
\hline 5737 & ENSGALTO00000035542 & 3.91 & $1.09 \mathrm{E}-03$ & 5805 & ENSGALTO0000035734 & 2.75 & $5.99 \mathrm{E}-04$ & 5873 & ENSGALTO0000035947 & 3.78 & $2.95 \mathrm{E}-02$ \\
\hline 5738 & ENSGALTO0000035543 & 3.37 & $3.73 \mathrm{E}-02$ & 5806 & ENSGALTO0000035745 & 3.91 & $2.73 \mathrm{E}-03$ & 5874 & ENSGALTO0000035950 & 3.34 & $1.16 \mathrm{E}-03$ \\
\hline 5739 & ENSGALTO0000035546 & 4.50 & $1.88 \mathrm{E}-03$ & 5807 & ENSGALTO0000035747 & 6.33 & $1.43 \mathrm{E}-04$ & 5875 & ENSGALTO0000035953 & 2.26 & $1.67 \mathrm{E}-03$ \\
\hline 5740 & ENSGALTO0000035549 & 10.14 & $7.28 E-04$ & 5808 & ENSGALTO0000035748 & 5.34 & $5.81 \mathrm{E}-04$ & 5876 & ENSGALTO0000035963 & 4.42 & $7.25 \mathrm{E}-05$ \\
\hline 5741 & ENSGALTO0000035551 & 2.85 & $7.18 \mathrm{E}-03$ & 5809 & ENSGALTO0000035750 & 4.21 & $1.14 \mathrm{E}-02$ & 5877 & ENSGALTO0000035968 & 7.26 & $2.96 \mathrm{E}-03$ \\
\hline 5742 & ENSGALTO0000035555 & 1.45 & $1.03 E-02$ & 5810 & ENSGALTO0000035752 & 2.95 & $2.11 \mathrm{E}-02$ & 5878 & ENSGALTO0000035979 & 8.60 & $9.32 E-04$ \\
\hline 5743 & ENSGALTO00000035557 & 5.02 & $2.02 E-03$ & 5811 & ENSGALTO0000035755 & 8.93 & $5.11 \mathrm{E}-04$ & 5879 & ENSGALTO0000035980 & 8.32 & $7.42 E-03$ \\
\hline 5744 & ENSGALTO00000035559 & 7.36 & $5.44 \mathrm{E}-05^{-1}$ & 5812 & ENSGALTO00000035756 & 8.60 & $8.22 \mathrm{E}-04$ & 5880 & ENSGALTO0000035982 & 1.47 & $3.52 \mathrm{E}-02$ \\
\hline 5745 & ENSGALTO0000035563 & 8.99 & $9.05 E-03$ & 5813 & ENSGALTO00000035758 & 1.04 & $4.64 \mathrm{E}-03$ & 5881 & ENSGALTO0000035984 & 5.71 & $4.91 \mathrm{E}-03$ \\
\hline 5746 & ENSGALT000000035569 & 1.35 & $5.15 E-03$ & 5814 & ENSGALT00000035760 & 7.82 & $8.45 \mathrm{E}-04$ & 5882 & ENSGALTO0000035988 & 8.45 & $6.52 \mathrm{E}-03$ \\
\hline 5747 & ENSGALT000000035571 & 4.80 & $6.58 \mathrm{E}-03^{-}$ & 5815 & ENSGALTO0000035761 & 8.24 & $6.91 \mathrm{E}-03$ & 5883 & ENSGALTO0000035997 & 8.50 & $3.38 \mathrm{E}-04$ \\
\hline 5748 & ENSGALT000000035575 & 4.60 & $8.73 E-04^{-1}$ & 5816 & ENSGALTO00000035767 & 8.95 & $1.71 \mathrm{E}-03$ & 5884 & ENSGALTO0000035998 & 6.62 & $1.63 \mathrm{E}-03$ \\
\hline 5749 & ENSGALTO00000035579 & 1.39 & $7.35 \mathrm{E}-03^{-}$ & 5817 & ENSGALTO0000035772 & 8.66 & $5.01 \mathrm{E}-04$ & 5885 & ENSGALTO0000036001 & 7.06 & $1.56 \mathrm{E}-03$ \\
\hline 5750 & ENSGALTO00000035581 & 4.67 & $8.06 E-04$ & 5818 & ENSGALTO0000035773 & 6.74 & $1.53 \mathrm{E}-02$ & 5886 & ENSGALTO0000036003 & 3.76 & $1.08 \mathrm{E}-02$ \\
\hline 5751 & $\begin{array}{l}\text { ENSGALTO00000035582 } \\
\end{array}$ & 6.94 & $2.57 \mathrm{E}-04$ & 5819 & ENSGALTO0000035774 & 6.42 & $6.00 \mathrm{E}-04$ & 5887 & ENSGALTO0000036004 & 8.69 & $4.60 \mathrm{E}-05$ \\
\hline 5752 & ENSGALT000000035583 & 3.15 & $2.25 \mathrm{E}-03$ & 5820 & ENSGALTO0000035775 & 6.36 & $3.76 \mathrm{E}-03$ & 5888 & ENSGALTO0000036011 & 3.96 & $8.84 E-04$ \\
\hline 5753 & ENSGALT000000035584 & 2.47 & $2.99 \mathrm{E}-02$ & 5821 & ENSGALTO00000035777 & 1.11 & $1.85 \mathrm{E}-03$ & 5889 & ENSGALTO00000036015 & 8.69 & $1.95 \mathrm{E}-04$ \\
\hline 5754 & ENSGALT00000035588 & 6.35 & $2.43 \mathrm{E}-04$ & 5822 & ENSGALTO0000035779 & 3.74 & $1.26 \mathrm{E}-02$ & 5890 & ENSGALTO00000036019 & 6.62 & $2.78 \mathrm{E}-03$ \\
\hline 5755 & ENSGALTO0000035589 & 1.93 & $1.07 E-01$ & 5823 & ENSGALTO0000035781 & 7.31 & $3.17 \mathrm{E}-05$ & 5891 & ENSGALT00000036020 & 3.58 & $1.49 \mathrm{E}-01$ \\
\hline 5756 & ENSGALTO0000035590 & 4.76 & $4.30 \mathrm{E}-03^{2}$ & 5824 & ENSGALTO0000035784 & 9.16 & $8.43 \mathrm{E}-03$ & 5892 & ENSGALTO0000036024 & 9.55 & $1.47 E-06$ \\
\hline 5757 & ENSGALTO00000035592 & 9.07 & $5.34 \mathrm{E}-04$ & 5825 & ENSGALTO0000035796 & 3.47 & $4.61 \mathrm{E}-03$ & 5893 & ENSGALTO0000036028 & 8.70 & $1.89 \mathrm{E}-03$ \\
\hline 5758 & ENSGALT00000035595 & 7.04 & $3.18 \mathrm{E}-03$ & 5826 & ENSGALT00000035797 & 2.45 & $1.43 \mathrm{E}-02$ & 5894 & ENSGALTO0000036034 & 1.54 & $3.01 E-02$ \\
\hline 5759 & ENSGALTO0000035596 & 6.84 & $2.71 \mathrm{E}-03$ & 5827 & ENSGALT00000035799 & 2.09 & $6.27 \mathrm{E}-02$ & 5895 & ENSGALTO0000036036 & 8.82 & $5.79 E-04$ \\
\hline 5760 & ENSGALTO0000035597 & 1.13 & $1.23 \mathrm{E}-03$ & 5828 & ENSGALTO0000035807 & 6.34 & $1.01 \mathrm{E}-03$ & 5896 & ENSGALTO00000036040 & 8.94 & $1.20 \mathrm{E}-04$ \\
\hline 5761 & ENSGALTO0000035598 & 7.39 & $4.18 \mathrm{E}-03^{3}$ & 5829 & ENSGALT00000035808 & 7.29 & $7.04 \mathrm{E}-05$ & 5897 & ENSGALTO0000036042 & 8.34 & $2.52 E-06$ \\
\hline 5762 & ENSGALTO0000035600 & 2.68 & $1.41 \mathrm{E}-02$ & 5830 & ENSGALTO0000035809 & 8.23 & $2.05 \mathrm{E}-03$ & 5898 & ENSGALTO00000036047 & 8.32 & $1.86 \mathrm{E}-04$ \\
\hline 5763 & ENSGALTO00000035601 & 8.14 & $5.29 \mathrm{E}-04$ & 5831 & ENSGALTO0000035811 & 2.67 & $1.75 \mathrm{E}-03$ & 5899 & ENSGALTO0000036050 & 7.45 & $5.53 \mathrm{E}-03$ \\
\hline 5764 & ENSGALT00000035603 & 2.48 & $2.35 \mathrm{E}-03$ & 5832 & ENSGALTO0000035812 & 2.64 & $2.38 \mathrm{E}-03$ & 5900 & ENSGALTO0000036051 & 11.58 & $4.72 \mathrm{E}-02$ \\
\hline 5765 & ENSGALTO00000035604 & 8.53 & $1.77 \mathrm{E}-02$ & 5833 & ENSGALTO0000035815 & 1.80 & $1.63 \mathrm{E}-02$ & 5901 & ENSGALTO0000036053 & 8.95 & $3.30 \mathrm{E}-04$ \\
\hline 5766 & ENSGALTO00000035608 & 2.58 & $1.77 \mathrm{E}-02$ & 5834 & ENSGALTO0000035820 & 4.14 & $4.72 \mathrm{E}-03$ & 5902 & ENSGALTO0000036054 & 8.50 & $4.92 \mathrm{E}-03$ \\
\hline 5767 & ENSGALTO0000035610 & 8.17 & $1.19 \mathrm{E}-04$ & 5835 & ENSGALT00000035825 & 7.14 & $1.19 \mathrm{E}-02$ & 5903 & ENSGALTO0000036055 & 7.30 & $8.92 E-03$ \\
\hline 5768 & ENSGALTO00000035612 & 1.53 & $1.07 E-02$ & 5836 & ENSGALTO0000035828 & 7.96 & $2.49 \mathrm{E}-04$ & 5904 & ENSGALT00000036056 & 9.44 & $3.08 E-05$ \\
\hline 5769 & ENSGALTO00000035616 & 4.43 & $2.57 \mathrm{E}-02$ & 5837 & ENSGALTO0000035830 & 3.85 & $2.89 \mathrm{E}-03$ & 5905 & ENSGALTO0000036057 & 7.97 & $9.07 E-04$ \\
\hline 5770 & ENSGALTO00000035620 & 2.09 & $1.49 \mathrm{E}-02$ & 5838 & ENSGALTO0000035833 & 3.39 & $2.70 \mathrm{E}-03$ & 5906 & ENSGALT00000036062 & 4.85 & $2.92 E-03$ \\
\hline 5771 & ENSGALTO00000035622 & 4.81 & $5.35 \mathrm{E}-03$ & 5839 & ENSGALTO0000035834 & 5.72 & $1.81 \mathrm{E}-02$ & 5907 & ENSGALT00000036066 & 2.18 & $6.27 E-04$ \\
\hline 5772 & ENSGALTT00000035624 & 3.58 & $3.94 \mathrm{E}-04$ & 5840 & ENSGALTO0000035842 & 6.13 & $1.84 \mathrm{E}-04$ & 5908 & ENSGALTO0000036067 & 3.94 & $9.98 E-04$ \\
\hline 5773 & ENSGALTO00000035628 & 9.35 & $2.24 E-04$ & 5841 & ENSGALT00000035844 & 6.87 & $1.24 \mathrm{E}-03$ & 5909 & ENSGALT00000036068 & 6.38 & $2.49 \mathrm{E}-03$ \\
\hline 5774 & ENSGALTO00000035630 & 6.12 & 4.24E-03 & 5842 & ENSGALTO0000035851 & 1.59 & $3.63 \mathrm{E}-03$ & 5910 & ENSGALT00000036069 & 5.37 & $1.83 \mathrm{E}-02$ \\
\hline 5775 & ENSGALTO00000035631 & 3.54 & $1.01 E-03$ & 5843 & ENSGALTO0000035852 & 5.64 & $5.19 \mathrm{E}-02$ & 5911 & ENSGALTO0000036071 & 8.60 & $6.89 \mathrm{E}-04$ \\
\hline 5776 & ENSGALTO00000035635 & 2.18 & $5.72 \mathrm{E}-03$ & 5844 & ENSGALT00000035857 & 7.05 & $8.88 \mathrm{E}-04$ & 5912 & ENSGALTO0000036073 & 2.75 & $2.33 \mathrm{E}-03$ \\
\hline 5777 & ENSGALTO00000035637 & 4.44 & $3.77 \mathrm{E}-02$ & 5845 & ENSGALTO0000035859 & 5.61 & $4.54 \mathrm{E}-03$ & 5913 & ENSGALTO0000036074 & 2.52 & $2.46 \mathrm{E}-02$ \\
\hline 5778 & ENSGALTO0000035638 & 3.52 & $3.86 \mathrm{E}-04$ & 5846 & ENSGALTO0000035865 & 5.67 & $7.76 \mathrm{E}-06$ & 5914 & ENSGALTO0000036077 & 8.30 & $3.49 \mathrm{E}-05$ \\
\hline 5779 & ENSGALTO0000035642 & 4.71 & $8.89 \mathrm{E}-04$ & 5847 & ENSGALTO0000035869 & 6.67 & 4.17E-04 & 5915 & ENSGALTO0000036080 & 7.17 & \\
\hline
\end{tabular}




\begin{tabular}{|c|c|c|c|c|c|c|c|c|c|c|c|}
\hline 5916 & ENSGALTO0000036081 & 3.97 & $2.64 \mathrm{E}-03$ & 5984 & ENSGALTO0000036291 & 2.97 & $4.45 \mathrm{E}-02 \mathrm{E}$ & 6052 & ENSGALTO0000036814 & 11.82 & $5.88 \mathrm{E}-04$ \\
\hline 5917 & ENSGALTO0000036082 & 2.10 & $2.79 \mathrm{E}-03^{3}$ & 5985 & ENSGALT00000036293 & 5.59 & $6.54 \mathrm{E}-04$ & 6053 & ENSGALTO0000036818 & 3.73 & $1.91 \mathrm{E}-03$ \\
\hline 5918 & ENSGALTO0000036085 & 5.45 & $3.74 \mathrm{E}-03$ & 5986 & ENSGALTO0000036314 & 5.58 & $2.17 \mathrm{E}-04 \mathrm{E}$ & 6054 & ENSGALTO0000036820 & 1.40 & $2.00 \mathrm{E}-03$ \\
\hline 5919 & ENSGALT00000036088 & 1.38 & $4.42 E-03$ & 5987 & ENSGALTO0000036332 & 4.92 & $3.10 \mathrm{E}-04 \mathrm{E}$ & 6055 & ENSGALT00000036821 & 5.34 & $2.40 \mathrm{E}-03$ \\
\hline 5920 & ENSGALTO0000036089 & 9.34 & $1.55 \mathrm{E}-04$ & 5988 & ENSGALTO0000036343 & 5.09 & $4.26 \mathrm{E}-03 \mathrm{E}$ & 6056 & ENSGALTO0000036823 & 3.07 & $3.38 \mathrm{E}-03$ \\
\hline 5921 & ENSGALTO0000036094 & 4.28 & $1.12 \mathrm{E}-03$ & 5989 & ENSGALTO0000036351 & 2.79 & $1.05 E-04 E$ & 6057 & ENSGALTO00000036824 & 4.90 & $1.54 \mathrm{E}-04$ \\
\hline 5922 & ENSGALTO0000036095 & 4.29 & $3.80 \mathrm{E}-03$ & 5990 & ENSGALTO0000036361 & 2.65 & $6.92 \mathrm{E}-03 \mathrm{E}$ & 6058 & ENSGALTO0000036840 & 1.52 & $9.74 \mathrm{E}-03$ \\
\hline 5923 & ENSGALTO0000036097 & 1.71 & $2.39 \mathrm{E}-02$ & 5991 & ENSGALTO0000036362 & 1.25 & $1.37 \mathrm{E}-02 \mathrm{E}$ & 6059 & ENSGALTO0000036844 & 5.01 & $2.12 \mathrm{E}-03$ \\
\hline 5924 & ENSGALT00000036101 & 7.14 & $1.75 \mathrm{E}-03$ & 5992 & ENSGALT00000036363 & 3.67 & $2.04 \mathrm{E}-02 \mathrm{E}$ & 6060 & ENSGALTO0000036856 & 3.73 & $7.94 \mathrm{E}-03$ \\
\hline 5925 & ENSGALTO0000036102 & 6.81 & $3.73 \mathrm{E}-04$ & 5993 & ENSGALTO0000036379 & 2.63 & $4.44 \mathrm{E}-03 \mathrm{E}$ & 6061 & ENSGALTO0000036880 & 5.48 & $9.20 \mathrm{E}-03$ \\
\hline 5926 & ENSGALTO0000036104 & 7.80 & 3.16E- -03 & 5994 & ENSGALTO0000036381 & 1.68 & $2.22 \mathrm{E}-03 \mathrm{E}$ & 6062 & ENSGALTO0000036888 & 2.05 & $2.02 \mathrm{E}-02$ \\
\hline 5927 & ENSGALT00000036107 & 5.93 & $1.52 \mathrm{E}-03^{-7}$ & 5995 & ENSGALT00000036387 & 1.68 & $1.99 \mathrm{E}-02 \mathrm{E}$ & 6063 & ENSGALTO0000036891 & 5.60 & $1.75 E-05$ \\
\hline 5928 & ENSGALTO0000036109 & 9.59 & $5.14 \mathrm{E}-05^{-1}$ & 5996 & ENSGALTO0000036392 & 4.17 & $2.00 \mathrm{E}-04$ & 6064 & ENSGALTO0000036900 & 3.66 & $5.83 \mathrm{E}-03$ \\
\hline 5929 & ENSGALTO0000036110 & 6.51 & $1.65 \mathrm{E}-03^{-7}$ & 5997 & ENSGALTO0000036393 & 3.61 & $3.10 \mathrm{E}-03 \mathrm{E}$ & 6065 & ENSGALTO0000036919 & 1.59 & $1.33 E-04$ \\
\hline 5930 & ENSGALTO0000036116 & 1.16 & $1.60 \mathrm{E}-03^{-}$ & 5998 & ENSGALTO0000036395 & 5.71 & $4.29 \mathrm{E}-04$ & 6066 & ENSGALTO0000036931 & 2.91 & $1.02 E-02$ \\
\hline 5931 & ENSGALTO0000036117 & 1.89 & $8.14 \mathrm{E}-04$ & 5999 & ENSGALTO0000036399 & 1.41 & $7.69 \mathrm{E}-03 \mathrm{E}$ & 6067 & ENSGALTO0000036935 & 2.17 & $4.12 E-04$ \\
\hline 5932 & ENSGALTO0000036119 & 2.22 & $1.86 \mathrm{E}-03^{-}$ & 6000 & ENSGALTO0000036412 & 1.26 & $8.41 E-03$ & 6068 & ENSGALTO0000036938 & 8.30 & $9.21 \mathrm{E}-04$ \\
\hline 5933 & ENSGALTO0000036120 & 10.54 & $4.58 \mathrm{E}-04$ & 6001 & ENSGALTO0000036441 & 3.18 & $2.32 \mathrm{E}-03 \mathrm{E}$ & 6069 & ENSGALTO0000036948 & 1.47 & $3.04 E-02$ \\
\hline 5934 & ENSGALTO00000036122 & 8.05 & $4.56 \mathrm{E}-04$ & 6002 & ENSGALTO0000036442 & 1.17 & $3.80 \mathrm{E}-03 \mathrm{E}$ & 6070 & ENSGALT00000036983 & 2.09 & $7.11 \mathrm{E}-03$ \\
\hline 5935 & ENSGALTO0000036136 & 8.41 & $4.49 \mathrm{E}-05^{-}$ & 6003 & ENSGALTO0000036447 & 2.91 & $9.24 \mathrm{E}-03 \mathrm{E}$ & 6071 & ENSGALTO0000037006 & 5.02 & $5.30 \mathrm{E}-03$ \\
\hline 5936 & ENSGALTO0000036138 & 7.45 & $1.35 \mathrm{E}-03^{-}$ & 6004 & ENSGALT00000036456 & 3.40 & $3.26 \mathrm{E}-03 \mathrm{E}$ & 6072 & ENSGALT00000037024 & 2.61 & $1.03 E-03$ \\
\hline 5937 & ENSGALTO0000036144 & 6.37 & $3.33 \mathrm{E}-04$ & 6005 & ENSGALTO0000036460 & 5.30 & $1.56 \mathrm{E}-03 \mathrm{E}$ & 6073 & ENSGALTO0000037054 & 2.75 & $1.11 \mathrm{E}-03$ \\
\hline 5938 & ENSGALTO0000036147 & 3.56 & $1.47 \mathrm{E}-03$ & 6006 & ENSGALTO0000036462 & 4.27 & $1.04 \mathrm{E}-03 \mathrm{E}$ & 6074 & ENSGALTO0000037057 & 5.36 & $2.03 E-03$ \\
\hline 5939 & ENSGALTO0000036149 & 6.46 & $3.39 \mathrm{E}-03^{-7}$ & 6007 & ENSGALTO0000036471 & 4.89 & $2.31 \mathrm{E}-03 \mathrm{E}$ & 6075 & ENSGALTO0000037067 & 4.45 & $7.64 \mathrm{E}-03$ \\
\hline 5940 & ENSGALTO0000036150 & 6.50 & $6.93 E-03$ & 6008 & ENSGALTO00000036473 & 3.55 & $3.43 \mathrm{E}-03 \mathrm{G}$ & 6076 & ENSGALTO0000037069 & 4.46 & $3.95 \mathrm{E}-03$ \\
\hline 5941 & ENSGALTO0000036151 & 8.80 & $3.25 E-05^{-5}$ & 6009 & ENSGALT00000036478 & 3.02 & $1.10 \mathrm{E}-03 \mathrm{E}$ & 6077 & ENSGALTO0000037079 & 4.88 & $2.36 \mathrm{E}-04$ \\
\hline 5942 & ENSGALTO0000036154 & 7.05 & $3.77 E-04$ & 6010 & ENSGALTO0000036482 & 4.51 & $6.97 E-05$ & 6078 & ENSGALTO0000037081 & 1.75 & $6.15 \mathrm{E}-03$ \\
\hline 5943 & ENSGALTO00000036160 & 1.36 & $1.49 \mathrm{E}-02^{-}$ & 6011 & ENSGALTO0000036506 & 2.43 & $5.40 \mathrm{E}-04 \mathrm{G}$ & 6079 & ENSGALTO0000037083 & 7.10 & $1.04 E-03$ \\
\hline 5944 & ENSGALTO0000036161 & 5.67 & $2.71 \mathrm{E}-03$ & 6012 & ENSGALTO0000036518 & 6.17 & $8.00 \mathrm{E}-06 \mathrm{G}$ & 6080 & ENSGALTO0000037085 & 5.45 & $9.48 \mathrm{E}-03$ \\
\hline 5945 & ENSGALTO00000036165 & 3.76 & $1.58 \mathrm{E}-02$ & 6013 & ENSGALTO0000036524 & 3.58 & $1.57 \mathrm{E}-02 \mathrm{E}$ & 6081 & ENSGALT00000037094 & 1.23 & $2.77 \mathrm{E}-02$ \\
\hline 5946 & $\begin{array}{l}\text { ENSGALTO00000036168 } \\
\end{array}$ & 4.10 & $1.19 \mathrm{EE}-03^{\mathrm{T}}$ & 6014 & ENSGALT000000036525 & 3.06 & $7.04 \mathrm{E}-03 \mathrm{G}$ & 6082 & ENSGALTO0000037095 & 1.34 & $4.19 \mathrm{E}-02$ \\
\hline 5947 & ENSGALTO00000036171 & 8.95 & $3.83 \mathrm{E}-04^{-}$ & 6015 & ENSGALTO00000036527 & 6.13 & $1.49 \mathrm{E}-03 \mathrm{E}$ & 6083 & ENSGALT00000037096 & 2.08 & $6.94 E-03$ \\
\hline 5948 & ENSGALTO0000036173 & 9.71 & $2.90 \mathrm{E}-04^{-}$ & 6016 & ENSGALTO0000036538 & 2.04 & $1.88 \mathrm{E}-02 \mathrm{E}$ & 6084 & ENSGALTO0000037102 & 4.57 & $8.19 \mathrm{E}-04$ \\
\hline 5949 & ENSGALTO00000036175 & 6.00 & $1.41 \mathrm{E}-03$ & 6017 & ENSGALTO0000036540 & 4.63 & $1.47 \mathrm{E}-03 \mathrm{E}$ & 6085 & ENSGALTO0000037111 & 3.68 & $7.79 \mathrm{E}-03$ \\
\hline 5950 & ENSGALTO00000036176 & 1.41 & $1.38 \mathrm{E}-02$ & 6018 & ENSGALTO0000036541 & 8.18 & $6.60 \mathrm{E}-05$ & 6086 & ENSGALTO0000037112 & 3.15 & $1.51 \mathrm{E}-02$ \\
\hline 5951 & ENSGALTO0000036177 & 3.65 & $4.18 \mathrm{E}-03$ & 6019 & ENSGALTO0000036545 & 5.18 & $2.70 \mathrm{E}-03$ & 6087 & ENSGALTO0000037116 & 1.77 & $5.25 \mathrm{E}-03$ \\
\hline 5952 & ENSGALTO0000036181 & 7.39 & $3.50 \mathrm{E}-04$ & 6020 & ENSGALTO0000036546 & 6.29 & $2.02 \mathrm{E}-02$ & 6088 & ENSGALTO0000037125 & 3.25 & $2.00 \mathrm{E}-02$ \\
\hline 5953 & ENSGALTO00000036182 & 8.11 & $2.75 \mathrm{E}-03$ & 6021 & ENSGALTO0000036550 & 1.23 & $3.80 \mathrm{E}-02$ & 6089 & ENSGALTO0000037134 & 2.85 & $3.84 E-04$ \\
\hline 5954 & ENSGALTO0000036184 & 8.86 & $5.11 \mathrm{E}-03^{-}$ & 6022 & ENSGALTO00000036564 & 3.02 & $2.23 \mathrm{E}-04$ & 6090 & ENSGALT00000037136 & 8.89 & $5.38 \mathrm{E}-03$ \\
\hline 5955 & ENSGALTO0000036185 & 4.45 & $7.45 E-03$ & 6023 & ENSGALTO0000036587 & 3.84 & $1.62 \mathrm{E}-03$ & 6091 & ENSGALTO0000037141 & 8.15 & $4.69 \mathrm{E}-03$ \\
\hline 5956 & ENSGALTO0000036187 & 6.69 & $2.62 E-04$ & 6024 & ENSGALTO0000036594 & 4.72 & $2.21 \mathrm{E}-03$ & 6092 & ENSGALTO0000037142 & 7.05 & $4.56 \mathrm{E}-03$ \\
\hline 5957 & ENSGALTO0000036192 & 1.84 & $1.92 E-04$ & 6025 & ENSGALTO0000036607 & 4.28 & $1.32 \mathrm{E}-02$ & 6093 & ENSGALTO0000037148 & 2.94 & $7.04 E-04$ \\
\hline 5958 & ENSGALTO0000036193 & 1.41 & $3.31 \mathrm{E}-03$ & 6026 & ENSGALTO0000036608 & 2.42 & $3.60 \mathrm{E}-03$ & 6094 & ENSGALTO0000037151 & 4.62 & $2.42 \mathrm{E}-03$ \\
\hline 5959 & ENSGALT00000036201 & 3.98 & $1.62 \mathrm{E}-02$ & 6027 & ENSGALTO0000036610 & 4.44 & $1.45 \mathrm{E}-02$ & 6095 & ENSGALTO00000037155 & 2.56 & $2.94 E-03$ \\
\hline 5960 & ENSGALT00000036204 & 1.06 & $5.72 \mathrm{E}-02$ & 6028 & ENSGALTO0000036613 & 2.71 & $2.54 \mathrm{E}-03$ & 6096 & ENSGALTO0000037166 & 5.55 & $1.08 \mathrm{E}-03$ \\
\hline 5961 & ENSGALTO0000036206 & 6.21 & $3.98 \mathrm{E}-03$ & 6029 & ENSGALTO0000036617 & 1.32 & $1.54 \mathrm{E}-02$ & 6097 & ENSGALTO0000037174 & 3.98 & $1.01 E-04$ \\
\hline 5962 & ENSGALTO0000036207 & 8.38 & $6.27 \mathrm{E}-04$ & 6030 & ENSGALTO0000036620 & 4.44 & $2.05 \mathrm{E}-03$ & 6098 & ENSGALTO0000037214 & 1.69 & $4.42 E-03$ \\
\hline 5963 & ENSGALTO0000036208 & 2.06 & $2.41 \mathrm{E}-02$ & 6031 & ENSGALT00000036621 & 2.45 & $1.66 \mathrm{E}-02$ & 6099 & ENSGALT00000037219 & 4.28 & $7.29 \mathrm{E}-03$ \\
\hline 5964 & ENSGALTO0000036210 & 5.42 & $2.37 \mathrm{E}-03$ & 6032 & ENSGALTO00000036655 & 1.78 & $1.63 \mathrm{E}-03$ & 6100 & ENSGALT00000037222 & 4.46 & $2.37 E-03$ \\
\hline 5965 & ENSGALTO0000036211 & 3.17 & $1.32 \mathrm{E}-03^{-}$ & 6033 & ENSGALTO0000036679 & 4.44 & $1.51 \mathrm{E}-04$ & 6101 & ENSGALTO0000037224 & 4.12 & $9.88 \mathrm{E}-06$ \\
\hline 5966 & ENSGALTO0000036217 & 1.90 & $3.95 \mathrm{E}-02$ & 6034 & ENSGALT00000036691 & 3.95 & $6.16 \mathrm{E}-05$ & 6102 & ENSGALTO0000037230 & 3.60 & $6.22 \mathrm{E}-03$ \\
\hline 5967 & ENSGALTO0000036219 & 5.54 & $9.58 \mathrm{E}-03$ & 6035 & ENSGALTO0000036692 & 3.94 & $4.18 \mathrm{E}-03$ & 6103 & ENSGALTO0000037231 & 3.21 & $5.05 E-03$ \\
\hline 5968 & ENSGALTO0000036223 & 6.05 & $4.72 \mathrm{E}-05$ & 6036 & ENSGALTO0000036699 & 5.16 & $3.40 \mathrm{E}-03$ & 6104 & ENSGALTO0000037236 & 4.06 & $5.28 \mathrm{E}-03$ \\
\hline 5969 & ENSGALTO0000036229 & 6.38 & $1.71 \mathrm{E}-05^{-1}$ & 6037 & ENSGALTO0000036700 & 2.72 & $2.35 \mathrm{E}-04$ & 6105 & ENSGALTO0000037246 & 5.81 & $6.64 E-04$ \\
\hline 5970 & ENSGALTO0000036232 & 2.21 & $3.86 \mathrm{E}-03^{-7}$ & 6038 & ENSGALTO0000036703 & 3.93 & $6.69 \mathrm{E}-03$ & 6106 & ENSGALTO0000037254 & 3.43 & $9.93 \mathrm{E}-05$ \\
\hline 5971 & ENSGALTO0000036235 & 3.94 & $2.20 \mathrm{E}-03$ & 6039 & ENSGALTO0000036724 & 2.94 & $7.35 \mathrm{E}-03$ & 6107 & ENSGALTO0000037260 & 1.37 & $9.48 \mathrm{E}-03$ \\
\hline 5972 & ENSGALTO0000036244 & 3.35 & $3.04 \mathrm{E}-03$ & 6040 & ENSGALTO0000036737 & 5.97 & $3.08 \mathrm{E}-03$ & 6108 & ENSGALT00000037290 & 1.88 & $2.20 \mathrm{E}-03$ \\
\hline 5973 & ENSGALTO0000036245 & 4.90 & $4.20 \mathrm{E}-03^{-}$ & 6041 & ENSGALTO0000036738 & 5.56 & $1.94 \mathrm{E}-04$ & 6109 & ENSGALTO0000037291 & 4.31 & $2.56 \mathrm{E}-03$ \\
\hline 5974 & ENSGALTO0000036246 & 2.34 & $1.69 \mathrm{E}-03^{-}$ & 6042 & ENSGALTO0000036739 & 2.14 & $5.49 \mathrm{E}-04$ & 6110 & ENSGALT00000037298 & 1.19 & $4.26 \mathrm{E}-02$ \\
\hline 5975 & ENSGALTO00000036250 & 3.77 & $1.22 \mathrm{E}-03$ & 6043 & ENSGALTO0000036748 & 4.79 & $3.11 \mathrm{E}-04$ & 6111 & ENSGALT00000037328 & 5.79 & $6.19 \mathrm{E}-04$ \\
\hline 5976 & ENSGALTO00000036252 & 4.48 & $8.20 \mathrm{E}-03^{-}$ & 6044 & ENSGALT00000036759 & 3.94 & $1.51 \mathrm{E}-03$ & 6112 & ENSGALTO00000037334 & 2.32 & $8.47 E-05$ \\
\hline 5977 & ENSGALTO0000036265 & 3.16 & $4.48 \mathrm{E}-03$ & 6045 & ENSGALTO0000036776 & 4.73 & $2.70 \mathrm{E}-03$ & 6113 & ENSGALT00000037335 & 3.45 & $1.87 \mathrm{E}-03$ \\
\hline 5978 & ENSGALTO00000036266 & 5.81 & $6.00 E-04$ & 6046 & ENSGALTO00000036795 & 2.07 & $1.97 \mathrm{E}-02$ & 6114 & ENSGALTO00000037346 & 5.58 & $1.69 \mathrm{E}-03$ \\
\hline 5979 & ENSGALTO00000036268 & 2.20 & $6.31 \mathrm{E}-02$ & 6047 & ENSGALTO0000036796 & 2.59 & $8.87 \mathrm{E}-03$ & 6115 & ENSGALTO0000037362 & 3.24 & $2.65 \mathrm{E}-03$ \\
\hline 5980 & ENSGALTO0000036269 & 3.19 & $4.32 \mathrm{E}-03$ & 6048 & ENSGALT00000036804 & 1.69 & $5.56 \mathrm{E}-02$ & 6116 & ENSGALTO00000037370 & 3.66 & $2.11 \mathrm{E}-03$ \\
\hline 5981 & ENSGALTO0000036279 & 3.58 & $5.37 \mathrm{E}-03$ & 6049 & ENSGALTO0000036805 & 7.34 & $6.66 \mathrm{E}-04$ & 6117 & ENSGALTO0000037371 & 2.86 & $2.05 \mathrm{E}-02$ \\
\hline 5982 & ENSGALTO0000036288 & 5.15 & $6.27 \mathrm{E}-04$ & 6050 & ENSGALTO0000036807 & 5.57 & $8.77 \mathrm{E}-05$ & 6118 & ENSGALTO0000037375 & 3.55 & $5.08 E-04$ \\
\hline 5983 & ENSGALTO0000036289 & 2.34 & $9.13 \mathrm{E}-03$ & 6051 & ENSGALTO0000036810 & 3.18 & $1.59 \mathrm{E}-02$ & 6119 & ENSGALTO0000037377 & 3.06 & $3.70 \mathrm{E}-0$ \\
\hline
\end{tabular}




\begin{tabular}{|c|c|c|c|c|c|c|c|c|c|c|}
\hline 6120 & ENSGALTO0000037378 & 3.70 & 2.00E-03 6188 & ENSGALTO0000037820 & 5.75 & $3.70 \mathrm{E}-03$ & 6256 & ENSGALTO0000038330 & 3.55 & $1.83 E-04$ \\
\hline 6121 & ENSGALTO0000037381 & 1.99 & $4.51 E-02-6189$ & ENSGALTO00000037835 & 5.55 & $3.21 \mathrm{E}-04$ & 6257 & ENSGALTO0000038334 & 3.83 & $8.84 \mathrm{E}-04$ \\
\hline 6122 & ENSGALTO0000037384 & 1.78 & $6.15 \mathrm{E}-03 \quad 6190$ & ENSGALTO0000037836 & 4.95 & $1.60 \mathrm{E}-03$ & 6258 & ENSGALTO0000038339 & 4.63 & $2.05 \mathrm{E}-03$ \\
\hline 6123 & ENSGALTO0000037393 & 2.08 & $6.54 \mathrm{E}-02 \quad 6191$ & ENSGALTO0000037851 & 3.28 & $2.01 \mathrm{E}-03$ & 6259 & ENSGALTO0000038346 & 2.62 & $1.57 \mathrm{E}-03$ \\
\hline 6124 & ENSGALT00000037395 & 5.69 & $1.44 E-046192$ & ENSGALTO0000037852 & 2.87 & $2.48 \mathrm{E}-04$ & 6260 & ENSGALTO0000038353 & 2.78 & $4.38 \mathrm{E}-03$ \\
\hline 6125 & ENSGALTO0000037396 & 4.46 & $2.77 E-03 \quad 6193$ & ENSGALTO0000037855 & 2.32 & $6.88 \mathrm{E}-02$ & 6261 & ENSGALTO0000038360 & 4.36 & $1.42 \mathrm{E}-03$ \\
\hline 6126 & ENSGALTO0000037412 & 5.95 & $9.77 E-03 \quad 6194$ & ENSGALTO0000037861 & 5.31 & $2.15 \mathrm{E}-04$ & 6262 & ENSGALTO0000038361 & 6.33 & $1.99 E-05$ \\
\hline 6127 & ENSGALT00000037422 & 2.15 & $9.81 \mathrm{E}-03 \overline{6195}$ & ENSGALTO00000037870 & 1.53 & $3.04 E-02$ & 6263 & ENSGALTO0000038366 & 4.30 & $8.86 E-04$ \\
\hline 6128 & ENSGALTO0000037425 & 5.26 & $8.68 \mathrm{E}-04-6196$ & ENSGALT00000037875 & 1.13 & $1.92 \mathrm{E}-02$ & 6264 & ENSGALTO0000038389 & 2.57 & $4.33 E-04$ \\
\hline 6129 & ENSGALT00000037432 & 1.91 & $5.90 \mathrm{E}-03 \quad 6197$ & ENSGALT00000037883 & 6.04 & $1.38 \mathrm{E}-03$ & 6265 & ENSGALTO0000038394 & 6.11 & $4.35 E-04$ \\
\hline 6130 & ENSGALT00000037448 & 1.55 & $9.11 \mathrm{E}-04 \quad 6198$ & ENSGALTO0000037884 & 4.49 & $3.89 \mathrm{E}-03$ & 6266 & ENSGALTO0000038395 & 8.49 & $1.65 \mathrm{E}-04$ \\
\hline 6131 & ENSGALTO0000037456 & 6.33 & $7.13 \mathrm{E}-04 \quad 6199$ & ENSGALTO00000037885 & 3.11 & $3.95 \mathrm{E}-04$ & 6267 & ENSGALTO0000038396 & 1.94 & $3.16 \mathrm{E}-02$ \\
\hline 6132 & ENSGALTO0000037457 & 7.52 & $1.29 E-03 \quad 6200$ & ENSGALTO0000037900 & 5.63 & $3.89 \mathrm{E}-04$ & 6268 & ENSGALTO0000038398 & 4.88 & $1.77 \mathrm{E}-03$ \\
\hline 6133 & ENSGALT00000037458 & 9.76 & $1 . 3 7 \mathrm { E } - 0 3 \longdiv { 6 2 0 1 }$ & ENSGALTO0000037905 & 1.53 & $1.55 \mathrm{E}-02$ & 6269 & ENSGALTO0000038402 & 1.47 & $6.39 \mathrm{E}-03$ \\
\hline 6134 & ENSGALTO0000037459 & 7.23 & $2.43 E-03 \quad 6202$ & ENSGALTO0000037911 & 2.79 & $3.78 \mathrm{E}-03$ & 6270 & ENSGALTO0000038407 & 5.32 & $3.12 \mathrm{E}-02$ \\
\hline 6135 & ENSGALT00000037462 & 3.44 & $4.22 E-04 \quad 6203$ & ENSGALT00000037918 & 4.01 & $6.63 \mathrm{E}-03$ & 6271 & ENSGALTO0000038408 & 2.39 & $1.42 \mathrm{E}-03$ \\
\hline 6136 & ENSGALTO0000037463 & 8.75 & $2.95 \mathrm{E}-03 \quad 6204$ & ENSGALTO0000037921 & 2.43 & $1.72 \mathrm{E}-02$ & 6272 & ENSGALTO0000038411 & 2.87 & $1.01 E-02$ \\
\hline 6137 & ENSGALTO0000037464 & 3.76 & $3 . 9 1 E - 0 4 \longdiv { 6 2 0 5 }$ & ENSGALTO0000037924 & 2.26 & $1.02 E-02$ & 6273 & ENSGALTO0000038426 & 4.96 & $1.86 \mathrm{E}-03$ \\
\hline 6138 & ENSGALT00000037465 & 9.12 & $4.10 \mathrm{E}-04 \overline{6206}$ & ENSGALTO0000037927 & 4.11 & $3.61 \mathrm{E}-03$ & 6274 & ENSGALTO0000038430 & 5.72 & $1.47 \mathrm{E}-03$ \\
\hline 6139 & ENSGALTO0000037466 & 6.10 & $3.25 \mathrm{E}-03 \quad 6207$ & ENSGALTO0000037937 & 4.21 & $3.04 E-03$ & 6275 & ENSGALTO0000038431 & 6.17 & $1.92 \mathrm{E}-03$ \\
\hline 6140 & ENSGALTO00000037468 & 6.73 & $5.43 E-04 \quad 6208$ & ENSGALTO0000037945 & 4.81 & $4.22 \mathrm{E}-03$ & 6276 & ENSGALTO0000038435 & 4.49 & $8.17 \mathrm{E}-04$ \\
\hline 6141 & ENSGALT00000037487 & 4.00 & $5.51 \mathrm{E}-04 \overline{6209}$ & ENSGALTO0000037973 & 5.03 & $6.18 \mathrm{E}-03$ & 6277 & ENSGALTO0000038443 & 4.04 & $2.89 \mathrm{E}-03$ \\
\hline 6142 & ENSGALT00000037493 & 4.14 & $5 . 0 7 E - 0 4 \longdiv { 6 2 1 0 }$ & ENSGALTO0000037976 & 1.77 & $1.03 E-01$ & 6278 & ENSGALTO00000038445 & 4.03 & $3.97 E-03$ \\
\hline 6143 & ENSGALT00000037498 & 4.64 & $3.53 \mathrm{E}-03 \quad 6211$ & ENSGALTO0000037989 & 4.53 & $2.91 E-03$ & 6279 & ENSGALTO0000038448 & 3.96 & $2.75 \mathrm{E}-03$ \\
\hline 6144 & ENSGALT00000037501 & 2.04 & $5.51 \mathrm{E}-04 \quad 6212$ & ENSGALTO0000038007 & 5.31 & $1.10 \mathrm{E}-02$ & 6280 & ENSGALT00000038458 & 5.65 & $6.61 \mathrm{E}-04$ \\
\hline 6145 & ENSGALT00000037513 & 3.10 & $2.77 \mathrm{E}-03 \quad 6213$ & ENSGALTO0000038009 & 4.92 & $4.81 E-03$ & 6281 & ENSGALT00000038467 & 1.97 & $8.64 E-02$ \\
\hline 6146 & ENSGALTO00000037516 & 2.49 & $2.33 \mathrm{E}-03 \quad 6214$ & ENSGALTO0000038012 & 2.98 & $1.12 \mathrm{E}-02$ & 6282 & ENSGALT00000038468 & 7.40 & $2.39 E-03$ \\
\hline 6147 & ENSGALTO00000037518 & 7.64 & $1.69 \mathrm{E}-04 \overline{6215}$ & ENSGALTO0000038015 & 4.64 & $8.53 \mathrm{E}-03$ & 6283 & ENSGALTO0000038473 & 3.08 & $2.87 \mathrm{E}-03$ \\
\hline 6148 & ENSGALTO0000037519 & 5.10 & $8.78 \mathrm{E}-04 \overline{6216}$ & ENSGALTO0000038030 & 2.89 & $1.14 \mathrm{E}-03$ & 6284 & ENSGALTO0000038475 & 4.97 & $6.15 \mathrm{E}-03$ \\
\hline 6149 & ENSGALTO00000037521 & 2.63 & $2.61 \mathrm{E}-03 \quad 6217$ & ENSGALT00000038032 & 4.69 & $1.76 \mathrm{E}-03$ & 6285 & ENSGALTO0000038477 & 5.37 & $3.33 E-03$ \\
\hline 6150 & ENSGALT00000037535 & 5.08 & $3.53 \mathrm{E}-03 \frac{3}{6218}$ & ENSGALTO0000038033 & 4.54 & $1.85 \mathrm{E}-03$ & 6286 & ENSGALTO0000038480 & 5.56 & $2.68 \mathrm{E}-03$ \\
\hline 6151 & ENSGALTO0000037538 & 2.28 & $1.51 E-03 \quad 6219$ & ENSGALTO0000038043 & 6.12 & $3.80 \mathrm{E}-03$ & 6287 & ENSGALTO0000038485 & 3.84 & $9.39 E-04$ \\
\hline 6152 & ENSGALTO0000037546 & 5.44 & $8.43 \mathrm{E}-04 \quad 6220$ & ENSGALTO0000038053 & 2.40 & $3.36 \mathrm{E}-03$ & 6288 & ENSGALTO0000038493 & 6.21 & $2.09 \mathrm{E}-05$ \\
\hline 6153 & ENSGALTO0000037548 & 2.88 & $4.11 \mathrm{E}-04-6221$ & ENSGALTO0000038098 & 3.45 & $1.57 \mathrm{E}-03$ & 6289 & ENSGALTO0000038517 & 2.46 & $6.20 \mathrm{E}-03$ \\
\hline 6154 & ENSGALT00000037549 & 2.82 & $4.53 \mathrm{E}-02 \quad 6222$ & ENSGALTO0000038099 & 5.38 & $3.25 \mathrm{E}-02$ & 6290 & ENSGALTO00000038519 & 2.23 & $1.34 E-03$ \\
\hline 6155 & ENSGALTO0000037554 & 3.31 & $1.06 \mathrm{E}-03 \quad 6223$ & ENSGALTO0000038118 & 4.85 & $1.84 \mathrm{E}-03$ & 6291 & ENSGALTO0000038521 & 2.24 & $2.03 \mathrm{E}-03$ \\
\hline 6156 & ENSGALT000000037555 & 5.73 & $6.28 \mathrm{E}-04 \overline{6224}$ & ENSGALTO00000038133 & 3.45 & $7.97 \mathrm{E}-05$ & 6292 & ENSGALTO0000038524 & 5.06 & $6.86 \mathrm{E}-03$ \\
\hline 6157 & ENSGALT00000037561 & 4.09 & $2.27 \mathrm{E}-03 \overline{6225}$ & ENSGALTO0000038134 & 3.75 & $3.10 \mathrm{E}-03$ & 6293 & ENSGALTO0000038533 & 3.65 & $3.42 \mathrm{E}-03$ \\
\hline 6158 & ENSGALTO0000037563 & 2.36 & $1 . 5 5 \mathrm { E } - 0 2 \longdiv { 6 2 2 6 }$ & ENSGALTO0000038137 & 2.51 & $7.37 \mathrm{E}-03$ & 6294 & ENSGALT00000038534 & 6.09 & $1.44 E-02$ \\
\hline 6159 & ENSGALT00000037564 & 5.45 & $9.09 E-04 \quad 6227$ & ENSGALTO0000038143 & 3.71 & $8.31 \mathrm{E}-04$ & 6295 & ENSGALTO0000038535 & 2.30 & $3.30 \mathrm{E}-03$ \\
\hline 6160 & ENSGALTO0000037567 & 1.52 & $2.64 E-03 \quad 6228$ & ENSGALTO0000038144 & 3.68 & $3.44 \mathrm{E}-03$ & 6296 & ENSGALTO0000038536 & 5.61 & $5.76 \mathrm{E}-03$ \\
\hline 6161 & ENSGALT00000037568 & 3.16 & $5.40 \mathrm{E}-03 \quad 6229$ & ENSGALTO0000038156 & 3.84 & $2.32 \mathrm{E}-03$ & 6297 & ENSGALTO00000038540 & 5.17 & $1.26 \mathrm{E}-03$ \\
\hline 6162 & ENSGALTO0000037572 & 4.69 & $4.53 \mathrm{E}-03 \overline{6230}$ & ENSGALTO0000038161 & 4.11 & $3.65 \mathrm{E}-03$ & 6298 & ENSGALTO0000038541 & 6.12 & $4.89 \mathrm{E}-03$ \\
\hline 6163 & ENSGALTO0000037573 & 4.62 & $7.63 E-03 \quad 6231$ & ENSGALTO00000038168 & 7.31 & $2.21 \mathrm{E}-03$ & 6299 & ENSGALTO0000038550 & 3.41 & $1.85 \mathrm{E}-02$ \\
\hline 6164 & ENSGALTO0000037578 & 2.64 & $6 . 7 7 E - 0 3 \longdiv { 6 2 3 2 }$ & ENSGALTO0000038169 & 2.78 & $3.48 \mathrm{E}-03$ & 6300 & ENSGALTO0000038554 & 4.12 & $4.56 \mathrm{E}-03$ \\
\hline 6165 & ENSGALTO0000037593 & 4.82 & $1.06 \mathrm{E}-02 \quad 6233$ & ENSGALTO0000038173 & 3.64 & $8.25 \mathrm{E}-03$ & 6301 & ENSGALTO0000038561 & 5.60 & $8.44 E-03$ \\
\hline 6166 & ENSGALTO0000037600 & 4.24 & $3.78 \mathrm{E}-03 \quad 6234$ & ENSGALTO0000038182 & 6.40 & $2.60 \mathrm{E}-03$ & 6302 & ENSGALTO0000038563 & 5.77 & $1.41 \mathrm{E}-03$ \\
\hline 6167 & ENSGALTO0000037645 & 1.95 & $4.03 E-02 \quad 6235$ & ENSGALTO0000038185 & 3.67 & $3.46 \mathrm{E}-03$ & 6303 & ENSGALTO0000038575 & 2.76 & $5.05 E-03$ \\
\hline 6168 & ENSGALTO00000037647 & 2.75 & $1 . 3 9 E - 0 3 \longdiv { 6 2 3 6 }$ & ENSGALTO0000038191 & 5.03 & $2.06 \mathrm{E}-03$ & 6304 & ENSGALTO0000038581 & 2.84 & $1.13 \mathrm{E}-05$ \\
\hline 6169 & ENSGALT000000037668 & 3.97 & $6.13 \mathrm{E}-03 \quad 6237$ & ENSGALTO0000038202 & 2.92 & $2.63 \mathrm{E}-03$ & 6305 & ENSGALTO0000038585 & 3.73 & $6.89 \mathrm{E}-05$ \\
\hline 6170 & ENSGALTO00000037670 & 5.01 & $3.34 \mathrm{E}-04 \quad 6238$ & ENSGALTO0000038213 & 5.86 & $9.08 \mathrm{E}-04$ & 6306 & ENSGALT00000038588 & 1.85 & $4.39 E-03$ \\
\hline 6171 & ENSGALTO00000037694 & 2.72 & $4.83 \mathrm{E}-03 \frac{3}{6239}$ & ENSGALTO0000038224 & 6.08 & $3.92 \mathrm{E}-03$ & 6307 & ENSGALTO0000038589 & 5.01 & $1.85 \mathrm{E}-03$ \\
\hline 6172 & $\begin{array}{l}\text { ENSGALTO00000037706 } \\
\end{array}$ & 4.57 & $3.26 \mathrm{E}-03 \frac{3}{6240}$ & ENSGALTO0000038227 & 3.94 & $9.71 \mathrm{E}-04$ & 6308 & ENSGALTO0000038594 & 7.03 & $1.19 \mathrm{E}-03$ \\
\hline 6173 & ENSGALTO00000037708 & 3.41 & $1.99 \mathrm{E}-04 \overline{6241}$ & ENSGALT00000038250 & 2.65 & $1.31 \mathrm{E}-03$ & 6309 & ENSGALTO0000038598 & 3.35 & $2.49 \mathrm{E}-03$ \\
\hline 6174 & ENSGALT00000037709 & 4.13 & $5.12 E-03 \overline{6242}$ & ENSGALTO00000038251 & 1.46 & $3.17 \mathrm{E}-02$ & 6310 & ENSGALT00000038609 & 2.97 & $4.73 \mathrm{E}-03$ \\
\hline 6175 & ENSGALTO0000037718 & 3.65 & $1 . 4 8 \mathrm { E } - 0 2 \longdiv { 6 2 4 3 }$ & ENSGALTO0000038267 & 2.08 & $2.27 \mathrm{E}-02$ & 6311 & ENSGALTO0000038636 & 1.57 & $5.34 E-02$ \\
\hline 6176 & ENSGALTO0000037725 & 4.29 & $1.24 \mathrm{E}-02 \quad 6244$ & ENSGALTO0000038275 & 5.80 & $4.31 \mathrm{E}-03$ & 6312 & ENSGALTO0000038640 & 4.85 & $3.82 E-04$ \\
\hline 6177 & ENSGALTO0000037729 & 2.50 & $4.89 E-03 \quad 6245$ & ENSGALTO0000038277 & 2.61 & $8.05 E-04$ & 6313 & ENSGALTO0000038642 & 4.48 & $1.78 \mathrm{E}-03$ \\
\hline 6178 & ENSGALTO0000037750 & 3.32 & $2.88 \mathrm{E}-03 \quad 6246$ & ENSGALTO0000038280 & 3.59 & $1.51 \mathrm{E}-04$ & 6314 & ENSGALTO0000038645 & 5.75 & $2.67 \mathrm{E}-03$ \\
\hline 6179 & ENSGALTO00000037751 & 1.40 & $2.94 \mathrm{E}-03 \frac{3}{6247}$ & ENSGALTO00000038282 & 1.43 & $7.02 E-03$ & 6315 & ENSGALT00000038657 & 3.71 & $2.40 \mathrm{E}-03$ \\
\hline 6180 & ENSGALTO00000037755 & 5.42 & $5.89 \mathrm{E}-04 \overline{6248}$ & ENSGALTO00000038286 & 2.22 & $2.03 \mathrm{E}-02$ & 6316 & ENSGALT00000038664 & 4.01 & $3.41 E-04$ \\
\hline 6181 & ENSGALTO0000037771 & 3.62 & $2 . 2 7 \mathrm { E } - 0 3 \longdiv { 6 2 4 9 }$ & ENSGALTO0000038288 & 2.03 & $1.15 \mathrm{E}-02$ & 6317 & ENSGALTO0000038667 & 4.73 & $8.86 E-04$ \\
\hline 6182 & ENSGALT00000037772 & 2.50 & $2.24 \mathrm{E}-03 \overline{6250}$ & ENSGALTO0000038294 & 2.65 & $7.68 \mathrm{E}-05$ & 6318 & ENSGALTO0000038670 & 3.30 & $1.33 \mathrm{E}-03$ \\
\hline 6183 & ENSGALT00000037787 & 3.40 & $1.41 \mathrm{E}-02 \quad 6251$ & ENSGALTO0000038298 & 1.48 & $2.94 \mathrm{E}-03$ & 6319 & ENSGALTO0000038675 & 5.51 & $4.76 E-03$ \\
\hline 6184 & ENSGALT00000037797 & 5.57 & $2.17 \mathrm{E}-03 \overline{6252}$ & ENSGALTO0000038310 & 2.94 & $8.88 \mathrm{E}-04$ & 6320 & ENSGALTO0000038680 & 4.01 & $7.98 \mathrm{E}-03$ \\
\hline 6185 & ENSGALTO0000037798 & 3.42 & $2.94 \mathrm{E}-02 \overline{6253}$ & ENSGALT00000038312 & 2.78 & $4.80 \mathrm{E}-03$ & 6321 & ENSGALT00000038688 & 4.05 & $1.08 \mathrm{E}-02$ \\
\hline 6186 & ENSGALT000000037801 & 5.66 & $1.41 E-03 \quad 6254$ & ENSGALTO00000038324 & 6.41 & $1.21 \mathrm{E}-03$ & 6322 & ENSGALTO0000038690 & 1.59 & $1.23 \mathrm{E}-01$ \\
\hline 6187 & ENSGALTO0000037802 & 5.50 & $1.50 \mathrm{E}-036255$ & ENSGALT00000038325 & 5.11 & 3.70E-05 & 6323 & ENSGALTO0000038694 & 5.66 & $1.46 \mathrm{E}-03$ \\
\hline
\end{tabular}




\begin{tabular}{|c|c|c|c|c|c|c|c|c|c|c|c|}
\hline 6324 & ENSGALTO0000038695 & 4.75 & $4.05 \mathrm{E}-03 \quad 6$ & 6392 & ENSGALTO0000039039 & 6.50 & $4.21 \mathrm{E}-04$ & 6460 & ENSGALTO0000039457 & 5.83 & $2.05 E-03$ \\
\hline 6325 & ENSGALT00000038696 & 4.65 & $3.80 \mathrm{E}-03 \overline{6}$ & 6393 & ENSGALTO0000039046 & 2.69 & $6.34 \mathrm{E}-03$ & 6461 & ENSGALT00000039461 & 7.18 & $6.68 \mathrm{E}-04$ \\
\hline 6326 & ENSGALTO0000038697 & 6.95 & $1.54 \mathrm{E}-04-6$ & 6394 & ENSGALTO0000039054 & 1.68 & $4.56 \mathrm{E}-03$ & 6462 & ENSGALT00000039463 & 4.26 & $1.09 \mathrm{E}-03$ \\
\hline 6327 & ENSGALTO0000038702 & 4.50 & $8.49 \mathrm{E}-03 \overline{6}$ & 6395 & ENSGALTO0000039055 & 1.46 & $2.43 \mathrm{E}-02$ & 6463 & ENSGALTO0000039470 & 3.56 & $2.86 \mathrm{E}-04$ \\
\hline 6328 & ENSGALTO0000038704 & 7.69 & $2.11 \mathrm{E}-03 \frac{6}{6}$ & 6396 & ENSGALTO0000039058 & 4.80 & $3.90 \mathrm{E}-03$ & 6464 & ENSGALT00000039472 & 6.10 & $1.70 \mathrm{E}-03$ \\
\hline 6329 & ENSGALTO0000038708 & 3.84 & $1.03 E-036$ & 6397 & ENSGALTO0000039061 & 1.53 & $2.52 \mathrm{E}-03$ & 6465 & ENSGALTO0000039479 & 6.13 & $2.13 \mathrm{E}-04$ \\
\hline 6330 & ENSGALTO0000038718 & 5.35 & $1.79 \mathrm{E}-03 \overline{6}$ & 6398 & ENSGALTO0000039071 & 3.15 & $7.48 \mathrm{E}-03$ & 6466 & ENSGALT00000039482 & 2.47 & $2.43 \mathrm{E}-03$ \\
\hline 6331 & ENSGALTO0000038719 & 5.29 & $1.41 \mathrm{E}-04 \overline{6}$ & 6399 & ENSGALTO0000039074 & 3.29 & $3.04 \mathrm{E}-03$ & 6467 & ENSGALT00000039483 & 5.71 & $1.47 \mathrm{E}-04$ \\
\hline 6332 & ENSGALT00000038724 & 2.67 & $8.52 \mathrm{E}-03 \overline{6}$ & 6400 & ENSGALTO0000039083 & 6.35 & $7.43 \mathrm{E}-04$ & 6468 & ENSGALT00000039488 & 4.85 & $9.70 \mathrm{E}-04$ \\
\hline 6333 & ENSGALT00000038725 & 3.37 & $2.47 \mathrm{E}-03 \frac{1}{6}$ & 6401 & ENSGALT00000039087 & 1.70 & $2.19 \mathrm{E}-03$ & 6469 & ENSGALT00000039495 & 1.25 & $2.98 \mathrm{E}-02$ \\
\hline 6334 & ENSGALT00000038728 & 6.87 & $1.62 \mathrm{E}-03 \overline{6}$ & 6402 & ENSGALTO0000039099 & 3.23 & $7.87 \mathrm{E}-03$ & 6470 & ENSGALTO0000039498 & 4.62 & $5.02 E-04$ \\
\hline 6335 & ENSGALTO0000038729 & 2.43 & $6.82 \mathrm{E}-03 \frac{-6}{6}$ & 6403 & ENSGALTO0000039101 & 6.59 & $2.87 \mathrm{E}-04$ & 6471 & ENSGALTO0000039516 & 4.66 & $2.76 \mathrm{E}-03$ \\
\hline 6336 & ENSGALTO0000038730 & 1.51 & $5.90 \mathrm{E}-02-6$ & 6404 & ENSGALTO0000039109 & 2.81 & $6.76 \mathrm{E}-03$ & 6472 & ENSGALTO0000039526 & 4.21 & $3.75 \mathrm{E}-03$ \\
\hline 6337 & ENSGALT00000038733 & 3.47 & $9.32 \mathrm{E}-04 \overline{6}$ & 6405 & ENSGALTO0000039110 & 2.34 & $6.02 \mathrm{E}-03$ & 6473 & ENSGALT00000039528 & 1.87 & $3.34 E-03$ \\
\hline 6338 & ENSGALT00000038736 & 3.13 & $1.89 \mathrm{E}-02-6$ & 6406 & ENSGALTO0000039116 & 2.02 & $1.42 \mathrm{E}-02$ & 6474 & ENSGALTO0000039534 & 2.58 & $7.27 E-03$ \\
\hline 6339 & ENSGALT00000038737 & 2.64 & $9.82 \mathrm{E}-04-\frac{6}{6}$ & 6407 & ENSGALTO0000039130 & 8.43 & $8.95 \mathrm{E}-03$ & 6475 & ENSGALT00000039548 & 1.96 & $1.38 \mathrm{E}-03$ \\
\hline 6340 & ENSGALTO0000038753 & 2.93 & $2.55 \mathrm{E}-03 \frac{6}{6}$ & 6408 & ENSGALTO0000039133 & 2.39 & $1.65 \mathrm{E}-02$ & 6476 & ENSGALT00000039562 & 4.96 & $2.62 \mathrm{E}-03$ \\
\hline 6341 & ENSGALTO0000038754 & 2.95 & $8.29 \mathrm{E}-04-6$ & 6409 & ENSGALTO0000039143 & 1.46 & $2.21 \mathrm{E}-02$ & 6477 & ENSGALT00000039563 & 2.11 & $1.53 \mathrm{E}-03$ \\
\hline 6342 & ENSGALT00000038764 & 4.91 & $9.33 \mathrm{E}-04-6$ & 6410 & ENSGALTO0000039148 & 1.68 & $6.54 \mathrm{E}-03$ & 6478 & ENSGALT00000039593 & 2.79 & $1.96 \mathrm{E}-02$ \\
\hline 6343 & ENSGALTO0000038775 & 4.05 & $9.09 \mathrm{E}-03 \overline{6}$ & 6411 & ENSGALTO0000039150 & 4.59 & $2.87 \mathrm{E}-04$ & 6479 & ENSGALTO0000039597 & 1.24 & $9.25 \mathrm{E}-03$ \\
\hline 6344 & ENSGALTO0000038778 & 5.19 & $2.36 \mathrm{E}-04-6$ & 6412 & ENSGALTO0000039162 & 3.06 & $9.92 \mathrm{E}-04$ & 6480 & ENSGALT00000039604 & 3.66 & $8.33 \mathrm{E}-03$ \\
\hline 6345 & ENSGALTO00000038781 & 6.12 & $9.61 \mathrm{E}-04-\frac{-1}{6}$ & 6413 & ENSGALT00000039167 & 2.66 & $2.48 E-03$ & 6481 & ENSGALT00000039605 & 1.55 & $1.00 E-03$ \\
\hline 6346 & ENSGALTO00000038782 & 4.39 & $6.67 \mathrm{E}-04-\frac{1}{6}$ & 6414 & ENSGALTO00000039170 & 4.55 & $1.52 \mathrm{E}-03$ & 6482 & ENSGALT00000039606 & 4.09 & $6.83 \mathrm{E}-03$ \\
\hline 6347 & ENSGALT00000038783 & 5.97 & $6.04 E-03 \overline{6}$ & 6415 & ENSGALTO0000039178 & 3.46 & $3.66 \mathrm{E}-03$ & 6483 & ENSGALT00000039626 & 3.88 & $3.71 E-03$ \\
\hline 6348 & ENSGALT00000038784 & 2.52 & $5.65 \mathrm{E}-03 \overline{6}$ & 6416 & ENSGALTO0000039181 & 5.36 & $1.19 \mathrm{E}-03$ & 6484 & ENSGALT000000039630 & 3.66 & $5.02 E-04$ \\
\hline 6349 & ENSGALTO0000038790 & 4.00 & $4.89 \mathrm{E}-03 \frac{6}{6}$ & 6417 & ENSGALT00000039182 & 3.30 & $5.83 \mathrm{E}-03$ & 6485 & ENSGALTO00000039649 & 6.76 & $1.34 E-03$ \\
\hline 6350 & ENSGALT00000038791 & 2.84 & $6.42 \mathrm{E}-03-\overline{6}$ & 6418 & ENSGALTO0000039189 & 1.78 & $3.68 \mathrm{E}-04$ & 6486 & ENSGALT00000039651 & 4.55 & $1.87 \mathrm{E}-03$ \\
\hline 6351 & ENSGALTO0000038793 & 5.65 & $1.27 \mathrm{E}-05 \frac{6}{6}$ & 6419 & ENSGALT00000039199 & 6.09 & $1.32 \mathrm{E}-03$ & 6487 & ENSGALT000000039655 & 1.86 & $2.00 \mathrm{E}-02$ \\
\hline 6352 & ENSGALT00000038802 & 1.76 & $2.15 \mathrm{E}-03 \overline{6}$ & 6420 & ENSGALTO0000039203 & 5.10 & $2.00 E-03$ & 6488 & ENSGALT00000039656 & 3.64 & $7.32 E-04$ \\
\hline 6353 & ENSGALTO00000038812 & 4.52 & $2.26 \mathrm{E}-03-6$ & 6421 & ENSGALT00000039204 & 2.56 & $2.48 \mathrm{E}-02$ & 6489 & ENSGALT00000039658 & 2.21 & $2.23 \mathrm{E}-02$ \\
\hline 6354 & ENSGALT00000038818 & 3.58 & $1.01 \mathrm{E}-03 \frac{1}{6}$ & 6422 & ENSGALTO0000039213 & 1.77 & $4.59 \mathrm{E}-03$ & 6490 & ENSGALT00000039680 & 4.25 & $1.01 E-03$ \\
\hline 6355 & ENSGALTO0000038824 & 4.08 & $1.37 \mathrm{E}-03 \overline{6}$ & 6423 & ENSGALTO0000039229 & 6.68 & $5.35 \mathrm{E}-03$ & 6491 & ENSGALT00000039681 & 2.81 & $1.87 \mathrm{E}-02$ \\
\hline 6356 & ENSGALT00000038827 & 5.10 & $2.30 \mathrm{E}-03 \frac{6}{6}$ & 6424 & ENSGALTO0000039235 & 3.17 & $7.76 \mathrm{E}-04$ & 6492 & ENSGALT00000039684 & 2.45 & $7.32 E-03$ \\
\hline 6357 & ENSGALTO0000038828 & 3.79 & $2.90 \mathrm{E}-03 \overline{6}$ & 6425 & ENSGALTO0000039237 & 1.88 & $2.79 \mathrm{E}-03$ & 6493 & ENSGALT00000039685 & 3.24 & $7.02 E-03$ \\
\hline 6358 & ENSGALT00000038831 & 6.12 & $2.03 \mathrm{E}-03 \overline{6}$ & 6426 & ENSGALTO0000039245 & 7.25 & $1.94 \mathrm{E}-03$ & 6494 & ENSGALT000000039686 & 3.33 & $4.68 \mathrm{E}-03$ \\
\hline 6359 & ENSGALTO0000038842 & 4.24 & $9.43 E-04 \frac{-6}{6}$ & 6427 & ENSGALTO0000039251 & 2.33 & $3.39 \mathrm{E}-03$ & 6495 & ENSGALTO0000039707 & 2.38 & $1.96 \mathrm{E}-02$ \\
\hline 6360 & ENSGALTO0000038847 & 6.18 & $1.99 \mathrm{E}-03 \frac{}{6}$ & 6428 & ENSGALTO0000039257 & 6.14 & $1.82 \mathrm{E}-03$ & 6496 & ENSGALT00000039712 & 2.52 & $2.55 \mathrm{E}-03$ \\
\hline 6361 & ENSGALTO0000038854 & 4.08 & $6.41 E-04 \frac{6}{6}$ & 6429 & ENSGALTO0000039258 & 4.09 & $1.06 \mathrm{E}-03$ & 6497 & ENSGALT00000039714 & 1.86 & $2.16 \mathrm{E}-02$ \\
\hline 6362 & ENSGALTO0000038856 & 2.02 & $5.16 \mathrm{E}-05 \frac{6}{6}$ & 6430 & ENSGALT00000039272 & 3.95 & $1.49 \mathrm{E}-03$ & 6498 & ENSGALTO0000039721 & 7.45 & $8.35 \mathrm{E}-03$ \\
\hline 6363 & ENSGALT00000038861 & 5.88 & $9.10 \mathrm{E}-05 \overline{6}$ & 6431 & ENSGALT00000039275 & 2.39 & $2.85 \mathrm{E}-03$ & 6499 & ENSGALT00000039729 & 6.52 & $1.09 E-03$ \\
\hline 6364 & ENSGALTO0000038871 & 2.99 & $2.24 \mathrm{E}-03 \frac{\pi}{6}$ & 6432 & ENSGALTO0000039277 & 1.19 & $1.09 \mathrm{E}-02$ & 6500 & ENSGALT00000039733 & 2.61 & $1.38 \mathrm{E}-03$ \\
\hline 6365 & ENSGALTO0000038874 & 3.24 & $7.38 \mathrm{E}-03 \overline{6}$ & 6433 & ENSGALTO0000039281 & 5.49 & $2.86 \mathrm{E}-03$ & 6501 & ENSGALTO0000039736 & 2.90 & $8.74 E-03$ \\
\hline 6366 & ENSGALTO0000038877 & 1.20 & $2.05 E-02 \frac{7}{6}$ & 6434 & ENSGALTO0000039286 & 1.60 & $2.53 \mathrm{E}-02$ & 6502 & ENSGALT00000039738 & 2.70 & $1.47 \mathrm{E}-02$ \\
\hline 6367 & ENSGALT00000038880 & 1.80 & $1.21 \mathrm{E}-02 \overline{6}$ & 6435 & ENSGALTO0000039295 & 3.15 & $2.72 \mathrm{E}-03$ & 6503 & ENSGALT00000039745 & 5.16 & $2.71 \mathrm{E}-03$ \\
\hline 6368 & ENSGALTO0000038883 & 1.20 & $1.79 \mathrm{E}-02 \frac{}{6}$ & 6436 & ENSGALTO0000039300 & 2.24 & $2.36 \mathrm{E}-03$ & 6504 & ENSGALTO0000039751 & 1.80 & $8.03 E-04$ \\
\hline 6369 & ENSGALTO0000038888 & 3.33 & $5.46 E-04 \frac{6}{6}$ & 6437 & ENSGALTO0000039309 & 1.27 & $1.60 \mathrm{E}-02$ & 6505 & ENSGALT00000039774 & 4.03 & $3.13 \mathrm{E}-03$ \\
\hline 6370 & ENSGALTO0000038901 & 6.38 & $7.84 \mathrm{E}-04 \frac{6}{6}$ & 6438 & ENSGALTO0000039310 & 1.92 & $2.32 \mathrm{E}-03$ & 6506 & ENSGALT00000039777 & 1.83 & $2.86 \mathrm{E}-03$ \\
\hline 6371 & ENSGALTO0000038905 & 4.08 & $1.41 E-03 \frac{6}{6}$ & 6439 & ENSGALTO0000039311 & 1.20 & $1.00 \mathrm{E}-01$ & 6507 & ENSGALT00000039782 & 3.04 & $7.87 \mathrm{E}-03$ \\
\hline 6372 & ENSGALTO0000038906 & 3.39 & $1.56 \mathrm{E}-046$ & 6440 & ENSGALTO0000039325 & 2.60 & $3.33 \mathrm{E}-02$ & 6508 & ENSGALT00000039788 & 4.68 & $3.04 E-04$ \\
\hline 6373 & ENSGALT000000038909 & 6.09 & $1.64 \mathrm{E}-03 \frac{16}{6}$ & 6441 & ENSGALTO0000039327 & 5.35 & $2.52 \mathrm{E}-04$ & 6509 & ENSGALT00000039797 & 3.11 & $3.30 \mathrm{E}-04$ \\
\hline 6374 & ENSGALTO00000038912 & 5.82 & $1.30 \mathrm{E}-03 \frac{}{6}$ & 6442 & ENSGALT00000039335 & 3.09 & $4.33 \mathrm{E}-03$ & 6510 & ENSGALT00000039805 & 4.81 & $6.27 \mathrm{E}-03$ \\
\hline 6375 & ENSGALTO0000038922 & 1.12 & $3.26 \mathrm{E}-02-6$ & 6443 & ENSGALT00000039338 & 3.96 & $1.54 \mathrm{E}-03$ & 6511 & ENSGALT000000039810 & 3.34 & $6.51 \mathrm{E}-03$ \\
\hline 6376 & $\begin{array}{l}\text { ENSGALTO00000038925 } \\
\end{array}$ & 3.72 & $3.12 \mathrm{E}-03^{-} 6$ & 6444 & ENSGALT00000039344 & 3.74 & $4.75 \mathrm{E}-04$ & 6512 & ENSGALTO0000039813 & 3.43 & $1.01 \mathrm{E}-03$ \\
\hline 6377 & ENSGALTO00000038934 & 1.38 & $9.94 \mathrm{E}-02 \overline{6}$ & 6445 & ENSGALTO0000039355 & 2.65 & $2.95 E-02$ & 6513 & ENSGALT00000039820 & 3.21 & $1.79 \mathrm{E}-03$ \\
\hline 6378 & ENSGALTO00000038936 & 1.16 & $7.99 \mathrm{E}-03 \overline{6}$ & 6446 & ENSGALTO0000039358 & 2.71 & $6.71 \mathrm{E}-04$ & 6514 & ENSGALT00000039822 & 4.66 & $1.58 \mathrm{E}-02$ \\
\hline 6379 & ENSGALTO00000038950 & 2.65 & $6.77 \mathrm{E}-03 \overline{6}$ & 6447 & ENSGALTO0000039369 & 2.84 & $1.97 \mathrm{E}-02$ & 6515 & ENSGALT00000039824 & 5.88 & $6.04 E-04$ \\
\hline 6380 & ENSGALTO0000038951 & 3.26 & $2.02 E-026$ & 6448 & ENSGALT00000039371 & 2.87 & $6.45 \mathrm{E}-03$ & 6516 & ENSGALT00000039838 & 4.29 & $2.33 \mathrm{E}-03$ \\
\hline 6381 & ENSGALTO00000038952 & 3.94 & $4.80 \mathrm{E}-03 \frac{6}{6}$ & 6449 & ENSGALTO0000039373 & 2.14 & $3.14 \mathrm{E}-03$ & 6517 & ENSGALTO0000039842 & 6.94 & $3.31 E-05$ \\
\hline 6382 & ENSGALT00000038963 & 5.39 & $6.38 \mathrm{E}-03 \frac{1}{6}$ & 6450 & ENSGALT00000039389 & 5.29 & $6.68 \mathrm{E}-04$ & 6518 & ENSGALTO0000039843 & 3.19 & $5.39 E-04$ \\
\hline 6383 & ENSGALT000000038985 & 5.85 & $5.49 \mathrm{E}-05 \overline{6}$ & 6451 & ENSGALTO00000039394 & 2.31 & $5.27 \mathrm{E}-03$ & 6519 & ENSGALTO0000039849 & 2.04 & $5.29 \mathrm{E}-04$ \\
\hline 6384 & ENSGALTO00000038986 & 2.24 & $3.61 \mathrm{E}-046$ & 6452 & $\begin{array}{l}\text { ENSGALTO00000039395 } \\
\end{array}$ & 4.66 & $4.05 \mathrm{E}-03$ & 6520 & ENSGALT00000039850 & 4.31 & $2.71 \mathrm{E}-03$ \\
\hline 6385 & ENSGALTO00000038989 & 6.47 & $6.73 \mathrm{E}-04 \overline{6}$ & 6453 & ENSGALTO0000039399 & 3.07 & $6.62 \mathrm{E}-03$ & 6521 & ENSGALT000000039852 & 5.16 & $4.69 \mathrm{E}-03$ \\
\hline 6386 & ENSGALT00000038996 & 3.93 & $2.15 \mathrm{E}-03 \overline{6}$ & 6454 & ENSGALTO0000039404 & 5.34 & $8.15 \mathrm{E}-04$ & 6522 & ENSGALT000000039854 & 4.25 & $6.98 \mathrm{E}-03$ \\
\hline 6387 & ENSGALT00000039001 & 2.24 & $1.32 \mathrm{E}-02-6$ & 6455 & ENSGALTO0000039409 & 4.28 & $5.83 \mathrm{E}-04$ & 6523 & ENSGALT000000039858 & 1.86 & $1.53 \mathrm{E}-01$ \\
\hline 6388 & ENSGALT000000039010 & 5.05 & $3.08 \mathrm{E}-03 \overline{6}$ & 6456 & ENSGALTO0000039436 & 4.45 & $5.39 \mathrm{E}-05$ & 6524 & ENSGALT000000039863 & 3.96 & $3.21 \mathrm{E}-03$ \\
\hline 6389 & ENSGALT00000039013 & 5.42 & $2.51 \mathrm{E}-04 \overline{6}$ & 6457 & ENSGALT00000039447 & 3.76 & $8.65 \mathrm{E}-06$ & 6525 & ENSGALT00000039867 & 4.68 & $7.92 \mathrm{E}-03$ \\
\hline 6390 & ENSGALT00000039015 & 2.38 & $6.05 \mathrm{E}-03 \frac{-6}{6}$ & 6458 & ENSGALT00000039451 & 4.02 & $1.30 \mathrm{E}-02$ & 6526 & ENSGALT00000039869 & 2.96 & $9.31 E-04$ \\
\hline 6391 & ENSGALT00000039038 & 5.10 & $2.14 \mathrm{E}-05 \overline{6}$ & 6459 & ENSGALT00000039452 & 4.79 & $1.07 \mathrm{E}-03$ & 6527 & ENSGALT00000039874 & 5.50 & $5.59 \mathrm{E}-03$ \\
\hline
\end{tabular}




\begin{tabular}{|c|c|c|c|c|c|c|c|c|c|c|}
\hline 6528 & ENSGALTO0000039880 & 2.87 & 4.99E-03 6596 & ENSGALTO0000040284 & 1.73 & $8.08 \mathrm{E}-03$ & 6664 & ENSGALTO0000040593 & 2.81 & $5.72 \mathrm{E}-04$ \\
\hline 6529 & ENSGALT00000039882 & 2.69 & $2 . 0 3 E - 0 2 \longdiv { 6 5 9 7 }$ & ENSGALT00000040285 & 4.59 & $3.03 \mathrm{E}-03$ & 6665 & ENSGALTO0000040598 & 6.25 & $8.48 \mathrm{E}-04$ \\
\hline 6530 & ENSGALTO0000039911 & 3.65 & $2.27 E-03 \quad 6598$ & ENSGALTO0000040287 & 3.45 & $2.36 \mathrm{E}-03$ & 6666 & ENSGALTO0000040607 & 1.98 & $3.09 \mathrm{E}-03$ \\
\hline 6531 & ENSGALTO0000039912 & 2.78 & $9.62 E-03 \quad 6599$ & ENSGALTO0000040289 & 3.73 & $1.98 \mathrm{E}-02$ & 6667 & ENSGALT00000040609 & 1.75 & $7.11 \mathrm{E}-02$ \\
\hline 6532 & ENSGALTO0000039915 & 1.84 & $9.48 \mathrm{E}-03 \quad 6600$ & ENSGALTO0000040302 & 5.74 & $1.42 \mathrm{E}-03$ & 6668 & ENSGALTO0000040614 & 5.09 & $2.22 E-02$ \\
\hline 6533 & ENSGALTO0000039916 & 3.69 & $1 . 9 7 E - 0 3 \longdiv { 6 6 0 1 }$ & ENSGALTO0000040306 & 4.98 & $2.19 \mathrm{E}-03$ & 6669 & ENSGALTO0000040615 & 2.94 & $3.78 E-04$ \\
\hline 6534 & ENSGALT00000039932 & 5.79 & $2.40 E-04 \quad 6602$ & ENSGALTO0000040308 & 2.73 & $3.10 \mathrm{E}-03$ & 6670 & ENSGALT00000040617 & 4.71 & $1.76 \mathrm{E}-05$ \\
\hline 6535 & ENSGALTO0000039935 & 5.65 & $1 . 4 2 E - 0 2 \longdiv { 6 6 0 3 }$ & ENSGALTO0000040311 & 4.29 & $2.35 \mathrm{E}-04$ & 6671 & ENSGALTO0000040620 & 2.94 & $1.01 \mathrm{E}-03$ \\
\hline 6536 & ENSGALTO0000039940 & 5.26 & $7.99 E-04 \quad 6604$ & ENSGALTO0000040315 & 4.22 & $9.48 \mathrm{E}-04$ & 6672 & ENSGALTO0000040622 & 4.26 & $4.32 E-04$ \\
\hline 6537 & ENSGALTO0000039941 & 5.08 & $1 . 1 4 E - 0 3 \longdiv { 6 6 0 5 }$ & ENSGALTO0000040321 & 4.62 & $6.62 \mathrm{E}-03$ & 6673 & ENSGALTO00000040629 & 5.08 & $5.74 E-03$ \\
\hline 6538 & ENSGALTO0000039943 & 2.53 & $1 . 2 5 E - 0 2 \longdiv { 6 6 0 6 }$ & ENSGALTO0000040322 & 2.89 & $8.18 \mathrm{E}-04$ & 6674 & ENSGALTO00000040630 & 3.67 & $7.966-04$ \\
\hline 6539 & ENSGALTO0000039945 & 2.71 & $1 . 9 8 \mathrm { E } - 0 3 \longdiv { 6 6 0 7 }$ & ENSGALTO0000040325 & 1.56 & $2.58 \mathrm{E}-03$ & 6675 & ENSGALTO0000040632 & 2.54 & $1.14 \mathrm{E}-02$ \\
\hline 6540 & ENSGALTO0000039955 & 1.90 & $8 . 0 5 E - 0 3 \longdiv { 6 6 0 8 }$ & ENSGALTO0000040326 & 6.46 & $3.84 \mathrm{E}-03$ & 6676 & ENSGALTO0000040644 & 6.55 & $1.36 \mathrm{E}-03$ \\
\hline 6541 & ENSGALTO0000039963 & 1.30 & $1 . 6 6 \mathrm { E } - 0 2 \longdiv { 6 6 0 9 }$ & ENSGALT00000040329 & 3.73 & $7.50 \mathrm{E}-03$ & 6677 & ENSGALTO0000040646 & 3.13 & $5.36 \mathrm{E}-04$ \\
\hline 6542 & ENSGALTO0000039964 & 3.06 & $9.78 \mathrm{E}-04 \overline{6610}$ & ENSGALTO0000040341 & 1.98 & $1.99 \mathrm{E}-03$ & 6678 & ENSGALTO0000040647 & 1.48 & $3.01 E-03$ \\
\hline 6543 & ENSGALTO0000039973 & 2.34 & $3 . 2 0 \mathrm { E } - 0 3 \longdiv { 6 6 1 1 }$ & ENSGALTO0000040342 & 2.48 & $2.06 \mathrm{E}-02$ & 6679 & ENSGALTO0000040665 & 3.40 & $2.50 \mathrm{E}-03$ \\
\hline 6544 & ENSGALTO0000039975 & 3.29 & $4 . 3 1 E - 0 5 \longdiv { 6 6 1 2 }$ & ENSGALTO0000040346 & 1.22 & $7.91 \mathrm{E}-02$ & 6680 & ENSGALTO0000040668 & 2.85 & $1.18 \mathrm{E}-03$ \\
\hline 6545 & ENSGALTO0000039987 & 6.74 & $3 . 6 8 \mathrm { E } - 0 3 \longdiv { 6 6 1 3 }$ & ENSGALT00000040348 & 1.60 & $1.28 \mathrm{E}-02$ & 6681 & ENSGALTO0000040677 & 2.30 & $7.09 E-02$ \\
\hline 6546 & ENSGALTO00000039990 & 3.32 & $2 . 8 7 E - 0 3 \longdiv { 6 6 1 4 }$ & ENSGALTO0000040354 & 2.24 & $1.47 \mathrm{E}-02$ & 6682 & ENSGALT00000040679 & 2.31 & $4.97 E-04$ \\
\hline 6547 & ENSGALTO0000039994 & 4.14 & $2 . 2 9 E - 0 3 \longdiv { 6 6 1 5 }$ & ENSGALTO0000040358 & 5.92 & $1.78 \mathrm{E}-03$ & 6683 & ENSGALTO0000040684 & 1.80 & $1.29 \mathrm{E}-02$ \\
\hline 6548 & ENSGALTO00000039997 & 2.53 & $1.18 \mathrm{E}-02-6616$ & ENSGALT00000040375 & 6.28 & $1.04 \mathrm{E}-02$ & 6684 & ENSGALT00000040689 & 6.01 & $1.10 \mathrm{E}-02$ \\
\hline 6549 & ENSGALTO0000040013 & 3.27 & $2.45 E-02 \quad 6617$ & ENSGALTO0000040377 & 8.72 & $1.62 \mathrm{E}-04$ & 6685 & ENSGALTO0000040693 & 1.85 & $1.55 \mathrm{E}-02$ \\
\hline 6550 & ENSGALTO0000040015 & 5.94 & $1.00 E-02-6618$ & ENSGALTO00000040379 & 8.88 & $2.85 \mathrm{E}-04$ & 6686 & ENSGALTO0000040694 & 2.97 & $1.88 \mathrm{E}-03$ \\
\hline 6551 & ENSGALTO0000040016 & 3.54 & $1 . 6 6 \mathrm { E } - 0 2 \longdiv { 6 6 1 9 }$ & $\begin{array}{l}\text { ENSGALTO00000040381 } \\
\end{array}$ & 5.02 & $5.78 \mathrm{E}-05$ & 6687 & ENSGALTO0000040699 & 2.13 & $2.25 \mathrm{E}-03$ \\
\hline 6552 & ENSGALT00000040021 & 4.64 & $3 . 3 4 \mathrm { E } - 0 3 \longdiv { 6 6 2 0 }$ & ENSGALTO0000040387 & 6.03 & $1.05 \mathrm{E}-03$ & 6688 & ENSGALTO0000040704 & 5.60 & $2.63 \mathrm{E}-03$ \\
\hline 6553 & ENSGALTO0000040032 & 6.66 & $1 . 1 1 \mathrm { E } - 0 2 \longdiv { 6 6 2 1 }$ & ENSGALTO0000040388 & 4.51 & $1.03 \mathrm{E}-03$ & 6689 & ENSGALTO0000040710 & 2.50 & $7.48 \mathrm{E}-03$ \\
\hline 6554 & ENSGALTO0000040035 & 2.61 & $2 . 3 6 \mathrm { E } - 0 3 \longdiv { 6 6 2 2 }$ & ENSGALTO0000040391 & 4.90 & $2.52 \mathrm{E}-02$ & 6690 & ENSGALTO0000040715 & 2.82 & $9.51 \mathrm{E}-03$ \\
\hline 6555 & ENSGALTO0000040044 & 2.63 & $8.00 E-03 \quad 6623$ & ENSGALTO0000040394 & 3.24 & $8.28 \mathrm{E}-03$ & 6691 & ENSGALTO0000040716 & 4.71 & $2.02 E-04$ \\
\hline 6556 & ENSGALTO0000040048 & 7.35 & $9 . 8 4 E - 0 6 \longdiv { 6 6 2 4 }$ & ENSGALTO0000040397 & 2.85 & $3.25 \mathrm{E}-03$ & 6692 & ENSGALTO0000040717 & 3.90 & $3.51 \mathrm{E}-03$ \\
\hline 6557 & ENSGALTO00000040051 & 2.23 & $7 . 5 1 E - 0 3 \longdiv { 6 6 2 5 }$ & ENSGALTO0000040408 & 4.23 & $3.31 \mathrm{E}-03$ & 6693 & ENSGALTO0000040728 & 4.80 & $2.78 \mathrm{E}-03$ \\
\hline 6558 & ENSGALTO0000040055 & 3.66 & $8 . 7 4 E - 0 4 \longdiv { 6 6 2 6 }$ & ENSGALTO0000040413 & 2.14 & $1.66 \mathrm{E}-02$ & 6694 & ENSGALTO0000040730 & 4.38 & $1.56 \mathrm{E}-03$ \\
\hline 6559 & ENSGALTO00000040067 & 1.67 & $1 . 6 7 \mathrm { E } - 0 2 \longdiv { 6 6 2 7 }$ & ENSGALTO0000040419 & 5.88 & $5.45 \mathrm{E}-04$ & 6695 & ENSGALTO0000040731 & 3.32 & $1.37 \mathrm{E}-03$ \\
\hline 6560 & ENSGALTO00000040069 & 4.34 & $1 . 0 4 E - 0 3 \longdiv { 6 6 2 8 }$ & ENSGALTO0000040432 & 8.29 & $1.74 \mathrm{E}-02$ & 6696 & ENSGALTO0000040734 & 2.59 & $1.15 \mathrm{E}-01$ \\
\hline 6561 & ENSGALTO00000040078 & 2.83 & $1 . 2 3 \mathrm { E } - 0 3 \longdiv { 6 6 2 9 }$ & ENSGALTO0000040433 & 3.48 & $1.17 \mathrm{E}-03$ & 6697 & ENSGALTO00000040747 & 2.58 & $1.72 \mathrm{E}-03$ \\
\hline 6562 & ENSGALTO00000040080 & 5.60 & $1 . 7 7 E - 0 4 \longdiv { 6 6 3 0 }$ & ENSGALTO0000040434 & 4.60 & $9.16 \mathrm{E}-04$ & 6698 & ENSGALTO0000040757 & 4.49 & $3.16 \mathrm{E}-03$ \\
\hline 6563 & ENSGALT000000040089 & 1.32 & $2.04 E-03 \frac{3}{6631}$ & ENSGALTO00000040451 & 1.09 & $2.91 \mathrm{E}-03$ & 6699 & ENSGALTO0000040765 & 5.07 & $1.19 \mathrm{E}-03$ \\
\hline 6564 & ENSGALTO00000040107 & 3.96 & $2 . 0 3 E - 0 2 \longdiv { 6 6 3 2 }$ & ENSGALTO0000040454 & 3.34 & $3.35 \mathrm{E}-03$ & 6700 & ENSGALTO0000040767 & 4.46 & $3.63 \mathrm{E}-05$ \\
\hline 6565 & ENSGALTO00000040114 & 1.99 & $9 . 7 7 E - 0 3 \longdiv { 6 6 3 3 }$ & ENSGALTO0000040455 & 3.65 & $1.19 \mathrm{E}-02$ & 6701 & ENSGALTO0000040768 & 1.89 & $3.28 \mathrm{E}-03$ \\
\hline 6566 & ENSGALTO00000040119 & 5.07 & $3 . 2 7 E - 0 4 \longdiv { 6 6 3 4 }$ & ENSGALTO00000040456 & 1.72 & $9.44 \mathrm{E}-04$ & 6702 & ENSGALTO0000040771 & 1.86 & $2.32 \mathrm{E}-02$ \\
\hline 6567 & ENSGALTO00000040125 & 2.46 & 5.15E-02 6635 & ENSGALTO0000040461 & 2.77 & $1.30 \mathrm{E}-04$ & 6703 & ENSGALTO00000040776 & 7.30 & $1.71 \mathrm{E}-03$ \\
\hline 6568 & ENSGALTO00000040126 & 1.79 & $5 . 5 1 E - 0 2 \longdiv { 6 6 3 6 }$ & ENSGALTO0000040465 & 3.40 & $7.44 \mathrm{E}-03$ & 6704 & ENSGALTO0000040778 & 6.98 & $3.73 E-04$ \\
\hline 6569 & ENSGALTO00000040142 & 1.79 & $1 . 9 0 \mathrm { E } - 0 3 \longdiv { 6 6 3 7 }$ & ENSGALTO0000040466 & 4.00 & $4.87 \mathrm{E}-03$ & 6705 & ENSGALTO0000040781 & 3.73 & $5.99 \mathrm{E}-03$ \\
\hline 6570 & ENSGALTO0000040144 & 1.82 & $3.17 \mathrm{E}-02 \quad 6638$ & ENSGALTO0000040468 & 1.41 & $1.19 \mathrm{E}-01$ & 6706 & ENSGALTO0000040782 & 7.25 & $1.48 \mathrm{E}-03$ \\
\hline 6571 & ENSGALTO00000040165 & 5.92 & $1 . 0 3 E - 0 3 \longdiv { 6 6 3 9 }$ & ENSGALTO00000040469 & 5.61 & $7.57 \mathrm{E}-04$ & 6707 & ENSGALTO0000040786 & 1.94 & $2.40 \mathrm{E}-02$ \\
\hline 6572 & ENSGALTO0000040176 & 1.65 & $4 . 7 8 \mathrm { E } - 0 3 \longdiv { 6 6 4 0 }$ & ENSGALTO0000040478 & 2.85 & $3.09 \mathrm{E}-02$ & 6708 & ENSGALTO0000040790 & 4.73 & $4.95 E-04$ \\
\hline 6573 & ENSGALTO0000040181 & 3.43 & $2 . 3 3 E - 0 3 \longdiv { 6 6 4 1 }$ & ENSGALTO0000040481 & 3.60 & $6.38 \mathrm{E}-03$ & 6709 & ENSGALTO0000040798 & 2.16 & $1.27 E-02$ \\
\hline 6574 & ENSGALTO0000040188 & 2.20 & $1.71 E-03 \quad 6642$ & ENSGALTO0000040486 & 5.92 & $1.19 \mathrm{E}-02$ & 6710 & ENSGALTO0000040801 & 3.33 & $2.58 \mathrm{E}-02$ \\
\hline 6575 & ENSGALTO0000040189 & 3.37 & $3 . 4 7 E - 0 4 \longdiv { 6 6 4 3 }$ & ENSGALTO0000040491 & 1.40 & $2.68 \mathrm{E}-02$ & 6711 & ENSGALTO0000040802 & 6.62 & $3.18 \mathrm{E}-04$ \\
\hline 6576 & ENSGALTO00000040198 & 1.79 & $2 . 2 0 \mathrm { E } - 0 2 \longdiv { 6 6 4 4 }$ & ENSGALTO0000040498 & 3.42 & $5.67 \mathrm{E}-03$ & 6712 & ENSGALTO0000040803 & 4.96 & $5.24 \mathrm{E}-03$ \\
\hline 6577 & ENSGALTO0000040199 & 4.99 & $1.05 E-026645$ & ENSGALTO0000040504 & 2.40 & $4.60 \mathrm{E}-03$ & 6713 & ENSGALTO0000040811 & 8.56 & $2.30 \mathrm{E}-03$ \\
\hline 6578 & ENSGALTO0000040201 & 4.36 & $1 . 0 6 \mathrm { E } - 0 3 \longdiv { 6 6 4 6 }$ & ENSGALTO0000040510 & 3.06 & $1.14 \mathrm{E}-03$ & 6714 & ENSGALTO00000040813 & 2.11 & $4.77 E_{-}-02$ \\
\hline 6579 & ENSGALTO0000040205 & 5.80 & $1 . 5 1 E - 0 4 \longdiv { 6 6 4 7 }$ & ENSGALTO0000040517 & 5.73 & $3.87 \mathrm{E}-03$ & 6715 & ENSGALTO00000040814 & 4.21 & $1.84 \mathrm{E}-03$ \\
\hline 6580 & ENSGALTO0000040217 & 3.41 & $7 . 6 4 E - 0 3 \longdiv { 6 6 4 8 }$ & ENSGALTO0000040520 & 5.02 & $2.48 \mathrm{E}-04$ & 6716 & ENSGALTO0000040821 & 5.97 & $1.76 \mathrm{E}-03$ \\
\hline 6581 & ENSGALTO00000040220 & 1.66 & $1.13 \mathrm{E}-01 \overline{6649}$ & ENSGALTO0000040521 & 3.65 & $1.85 \mathrm{E}-03$ & 6717 & ENSGALTO0000040825 & 5.58 & $7.42 E-05$ \\
\hline 6582 & ENSGALTO0000040224 & 6.14 & $7 . 8 4 E - 0 4 \longdiv { 6 6 5 0 }$ & ENSGALTO0000040530 & 1.71 & $1.51 \mathrm{E}-02$ & 6718 & ENSGALTO0000040827 & 4.02 & $7.97 E-04$ \\
\hline 6583 & ENSGALTO0000040228 & 4.27 & 3.46E-03 6651 & ENSGALTO0000040532 & 1.78 & $2.33 \mathrm{E}-02$ & 6719 & ENSGALTO0000040833 & 1.92 & $3.20 \mathrm{E}-02$ \\
\hline 6584 & ENSGALTO00000040232 & 7.36 & $1.19 \mathrm{E}-03 \quad 6652$ & ENSGALTO0000040539 & 3.12 & $8.08 \mathrm{E}-03$ & 6720 & ENSGALT00000040835 & 4.21 & $5.54 E-04$ \\
\hline 6585 & ENSGALTO00000040241 & 3.03 & $1 . 7 0 E - 0 3 \longdiv { 6 6 5 3 }$ & ENSGALTO0000040540 & 2.48 & $1.88 \mathrm{E}-03$ & 6721 & ENSGALTO0000040837 & 5.42 & $9.50 \mathrm{E}-03$ \\
\hline 6586 & ENSGALTO00000040252 & 1.91 & $1.04 E-016654$ & ENSGALTO0000040545 & 4.00 & $5.02 \mathrm{E}-03$ & 6722 & ENSGALTO0000040838 & 3.67 & $2.71 \mathrm{E}-02$ \\
\hline 6587 & ENSGALTO00000040254 & 1.92 & $2 . 2 6 \mathrm { E } - 0 3 \longdiv { 6 6 5 5 }$ & ENSGALTO0000040551 & 3.46 & $1.06 \mathrm{E}-03$ & 6723 & ENSGALT00000040839 & 1.64 & $3.09 E-03$ \\
\hline 6588 & ENSGALTT00000040256 & 4.01 & $1 . 3 9 \mathrm { E } - 0 2 \longdiv { 6 6 5 6 }$ & ENSGALTO0000040565 & 1.13 & $2.20 \mathrm{E}-02$ & 6724 & ENSGALTO0000040840 & 7.04 & $3.61 \mathrm{E}-04$ \\
\hline 6589 & ENSGALTO00000040257 & 3.62 & $1 . 6 4 E - 0 4 \longdiv { 6 6 5 7 }$ & ENSGALT00000040566 & 3.30 & $5.97 \mathrm{E}-02$ & 6725 & ENSGALT00000040866 & 6.22 & $9.99 E-04$ \\
\hline 6590 & ENSGALTO00000040261 & 2.10 & $1.60 \mathrm{E}-01 \overline{6658}$ & ENSGALTO0000040567 & 1.93 & $4.45 \mathrm{E}-03$ & 6726 & ENSGALT00000040868 & 6.05 & $3.23 E-04$ \\
\hline 6591 & ENSGALTO0000040263 & 4.72 & $1.62 E-03 \quad 6659$ & ENSGALTO0000040571 & 4.51 & $1.21 \mathrm{E}-02$ & 6727 & ENSGALTO00000040871 & 3.06 & $9.67 \mathrm{E}-02$ \\
\hline 6592 & ENSGALTO00000040270 & 4.83 & $3.21 \mathrm{E}-04 \overline{6660}$ & ENSGALT00000040579 & 5.03 & $2.02 \mathrm{E}-03$ & 6728 & ENSGALTO0000040875 & 7.87 & $2.07 E-04$ \\
\hline 6593 & ENSGALTO00000040273 & 2.04 & $3 . 3 2 E - 0 2 \longdiv { 6 6 6 1 }$ & ENSGALTO0000040585 & 2.57 & $7.82 \mathrm{E}-03$ & 6729 & ENSGALTO0000040879 & 4.18 & $1.05 \mathrm{E}-02$ \\
\hline 6594 & ENSGALTO0000040274 & 3.59 & $6.52 \mathrm{E}-03 \quad 6662$ & ENSGALT00000040588 & 7.47 & $8.21 \mathrm{E}-04$ & 6730 & ENSGALTO0000040880 & 2.85 & $1.15 \mathrm{E}-02$ \\
\hline 6595 & ENSGALTO0000040281 & 3.78 & $2 . 2 6 \mathrm { E } - 0 2 \longdiv { 6 6 6 3 }$ & ENSGALTO00000040592 & 4.49 & $2.30 \mathrm{E}-03$ & 6731 & ENSGALTO0000040883 & 6.48 & \\
\hline
\end{tabular}




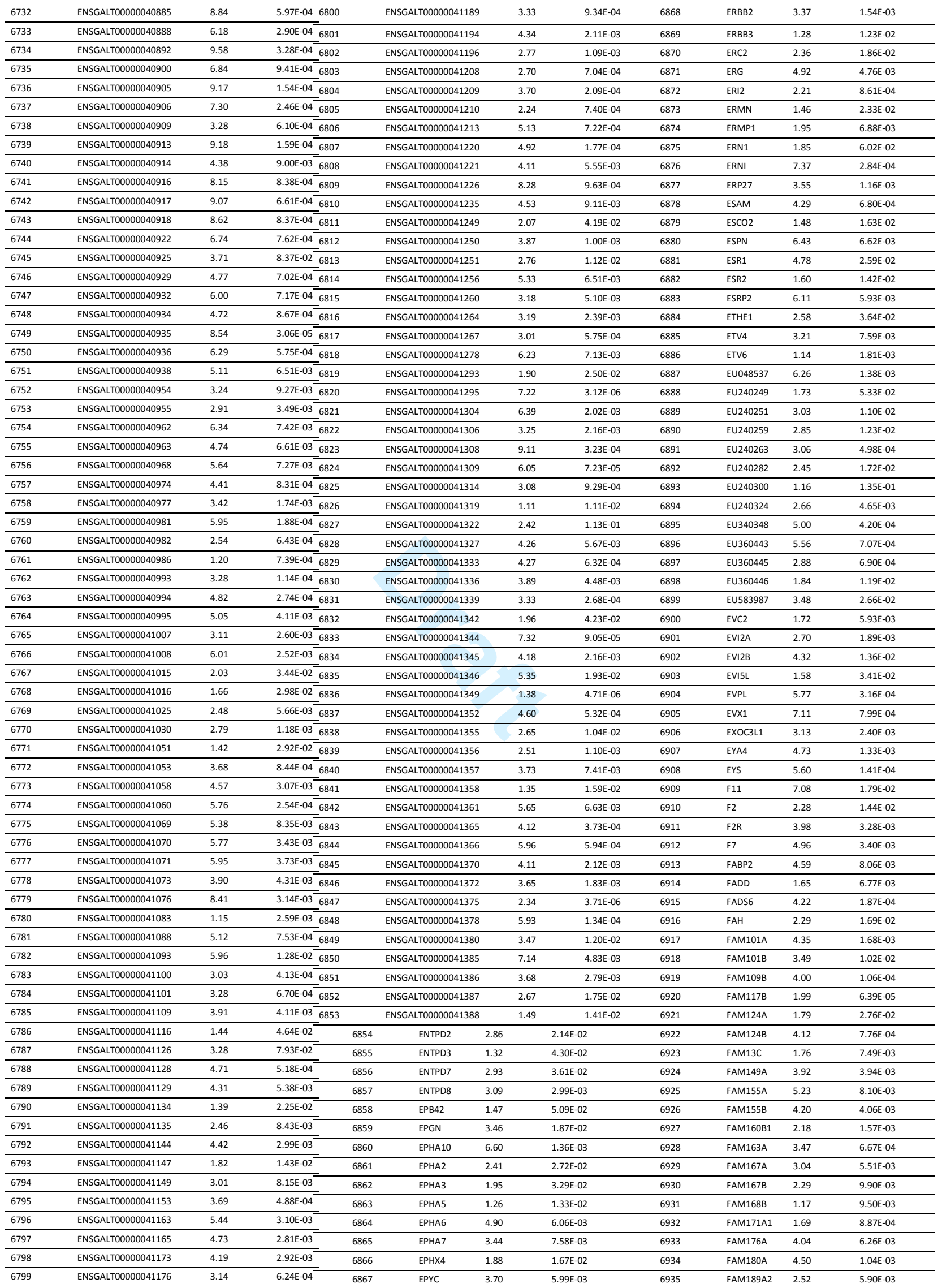




\begin{tabular}{|c|c|c|c|c|c|c|c|c|c|c|c|}
\hline 6936 & FAM190B & 1.92 & $1.82 \mathrm{E}-03$ & 7004 & FKBP15 & 1.55 & $1.37 \mathrm{E}-02$ & 7072 & FZD7 & 1.24 & $1.54 \mathrm{E}-03$ \\
\hline 6937 & FAM193B & 1.67 & $1.71 \mathrm{E}-03$ & 7005 & F-KER & 6.44 & $5.38 \mathrm{E}-05$ & 7073 & GAA & 3.53 & $2.54 \mathrm{E}-03$ \\
\hline 6938 & FAM19A2 & 4.12 & 7.14E-04 & 7006 & FKTN & 1.50 & $2.98 \mathrm{E}-02$ & 7074 & GABBR2 & 7.16 & $2.78 \mathrm{E}-03$ \\
\hline 6939 & FAM19A3 & 2.99 & 4.63E-03 & 7007 & FLII & 1.35 & $7.87 E-02$ & 7075 & GABRA2 & 5.01 & $3.79 \mathrm{E}-03$ \\
\hline 6940 & FAM208B & 1.19 & $1.54 \mathrm{E}-02$ & 7008 & FLNA & 2.28 & $7.76 \mathrm{E}-03$ & 7076 & GABRA6 & 2.99 & $1.51 \mathrm{E}-04$ \\
\hline 6941 & FAM $20 \mathrm{C}$ & 1.08 & $1.35 \mathrm{E}-02$ & 7009 & FLNC & 2.61 & $1.07 \mathrm{E}-02$ & 7077 & GABRB2 & 1.13 & $1.44 \mathrm{E}-03$ \\
\hline 6942 & FAM211A & 4.29 & 4.06E-02 & 7010 & FLRT2 & 2.09 & $2.42 \mathrm{E}-03$ & 7078 & GABRD & 1.99 & $6.14 \mathrm{E}-03$ \\
\hline 6943 & FAM26D & 5.62 & $3.60 \mathrm{E}-03$ & 7011 & FLT1 & 1.84 & $2.59 \mathrm{E}-04$ & 7079 & GABRE & 3.39 & $9.89 \mathrm{E}-04$ \\
\hline 6944 & FAM26E & 4.82 & $1.79 E-04$ & 7012 & FLT4 & 2.91 & $2.16 \mathrm{E}-03$ & 7080 & GABRG1 & 3.04 & 5.77E-03 \\
\hline 6945 & FAM26F & 5.37 & $1.11 \mathrm{E}-03$ & 7013 & FLVCR1 & 1.48 & $1.62 \mathrm{E}-02$ & 7081 & GABRG2 & 3.47 & $3.20 \mathrm{E}-04$ \\
\hline 6946 & FAM38B & 5.05 & $2.22 \mathrm{E}-03$ & 7014 & FLVCR2 & 2.92 & $1.52 \mathrm{E}-03$ & 7082 & GABRP & 2.80 & $7.50 \mathrm{E}-02$ \\
\hline 6947 & FAM3D & 6.47 & 9.58E- 03 & 7015 & FLYWCH2 & 3.90 & 7.27E-03 & 7083 & GABRQ & 4.39 & $1.60 \mathrm{E}-02$ \\
\hline 6948 & FAM43A & 5.00 & $3.45 \mathrm{E}-04$ & 7016 & FM200235 & 6.43 & $6.07 \mathrm{E}-04$ & 7084 & GABRR1 & 6.37 & $5.38 \mathrm{E}-03$ \\
\hline 6949 & FAM46B & 2.73 & $5.02 E-05$ & 7017 & FM200248 & 6.48 & $3.41 \mathrm{E}-04$ & 7085 & GAD2 & 2.99 & $5.22 \mathrm{E}-03$ \\
\hline 6950 & FAM46C & 1.93 & 7.01E-04 & 7018 & FM200260 & 6.56 & $4.58 \mathrm{E}-03$ & 7086 & GAL & 3.18 & $3.48 \mathrm{E}-04$ \\
\hline 6951 & FAM55C & 3.47 & $5.42 E-03$ & 7019 & FM200272 & 6.68 & 3.03E-04 & 7087 & GAL1 & 3.89 & 3.83E-04 \\
\hline 6952 & FAM5B & 5.75 & $2.25 \mathrm{E}-03$ & 7020 & FM200317 & 5.50 & $2.67 \mathrm{E}-03$ & 7088 & GAL10 & 7.00 & 4.83E-04 \\
\hline 6953 & FAM5C & 2.90 & $4.25 \mathrm{E}-04$ & 7021 & FM200318 & 5.92 & $3.13 \mathrm{E}-03$ & 7089 & GAL11 & 2.89 & $2.96 \mathrm{E}-03$ \\
\hline 6954 & FAM78B & 1.30 & $3.15 E-03$ & 7022 & FM200329 & 7.05 & $5.55 \mathrm{E}-04$ & 7090 & GAL12 & 4.07 & $3.86 \mathrm{E}-04$ \\
\hline 6955 & FAM82A1 & 3.02 & $8.78 \mathrm{E}-04$ & 7023 & FM200393 & 5.82 & $1.22 \mathrm{E}-03$ & 7091 & GAL13 & 4.31 & 5.39E-04 \\
\hline 6956 & FAM83B & 2.33 & $2.18 \mathrm{E}-03$ & 7024 & FM955443 & 2.97 & $3.32 \mathrm{E}-03$ & 7092 & GAL3ST1 & 4.17 & $2.83 \mathrm{E}-04$ \\
\hline 6957 & FAM83F & 2.64 & $1.08 \mathrm{E}-02$ & 7025 & FMN2 & 4.30 & $2.46 \mathrm{E}-03$ & 7093 & GAL3ST4 & 6.24 & 4.44E-04 \\
\hline 6958 & FAM83G & 4.55 & $1.32 \mathrm{E}-03$ & 7026 & FMNL2 & 2.55 & $4.32 \mathrm{E}-02$ & 7094 & GAL4 & 3.33 & $1.60 \mathrm{E}-03$ \\
\hline 6959 & FAM84B & 3.16 & $2.05 E-03$ & 7027 & FMO4 & 5.89 & 7.75E-04 & 7095 & GAL5 & 3.40 & $2.04 \mathrm{E}-03$ \\
\hline 6960 & FAR2 & 2.45 & $1.27 \mathrm{E}-03$ & 7028 & FNDC1 & 3.87 & $1.97 \mathrm{E}-03$ & 7096 & GAL6 & 6.39 & $1.44 \mathrm{E}-03$ \\
\hline 6961 & FASLG & 1.71 & $1.21 \mathrm{E}-02$ & 7029 & FNDC3B & 1.78 & 7.65E-03 & 7097 & GAL7 & 3.44 & $1.25 \mathrm{E}-02$ \\
\hline 6962 & FASTKD5 & 4.18 & $3.38 \mathrm{E}-03$ & 7030 & FNDC4 & 3.53 & $8.36 \mathrm{E}-04$ & 7098 & GAL8 & 3.42 & $6.53 \mathrm{E}-03$ \\
\hline 6963 & FAT2 & 5.60 & $7.33 \mathrm{E}-04$ & 7031 & FNDC5 & 3.96 & $8.86 \mathrm{E}-03$ & 7099 & GAL9 & 3.65 & $8.98 \mathrm{E}-04$ \\
\hline 6964 & FAT3 & 3.69 & $3.28 \mathrm{E}-03$ & 7032 & FNIP1 & 2.89 & $3.16 \mathrm{E}-04$ & 7100 & GALE & 3.49 & $1.32 \mathrm{E}-04$ \\
\hline 6965 & FBLN5 & 2.53 & 7.96E-03 & 7033 & FNIP2 & 1.20 & $6.31 \mathrm{E}-03$ & 7101 & GALK1 & 2.27 & $5.54 \mathrm{E}-02$ \\
\hline 6966 & FBLN7 & 1.58 & 9.82E-04 & 7034 & FOSL2 & 4.30 & 7.09E-05 & 7102 & GALNT10 & 2.82 & $9.14 \mathrm{E}-03$ \\
\hline 6967 & FBN1 & 1.65 & 3.57E-03 & 7035 & FOXA2 & 6.25 & $1.03 \mathrm{E}-03$ & 7103 & GALNT12 & 5.80 & $9.00 \mathrm{E}-03$ \\
\hline 6968 & FBN3 & 3.34 & $1.03 E-05$ & 7036 & FOXC2 & 5.03 & $1.28 \mathrm{E}-03$ & 7104 & GALNT13 & 3.45 & $4.71 \mathrm{E}-05$ \\
\hline 6969 & FBP2 & 2.36 & $8.78 \mathrm{E}-03$ & 7037 & FOXD1 & 2.86 & $6.91 \mathrm{E}-03$ & 7105 & GALNT14 & 2.66 & $5.60 \mathrm{E}-03$ \\
\hline 6970 & FBXL12 & 2.21 & $2.30 \mathrm{E}-02$ & 7038 & FOXD3 & 4.71 & 7.43E-03 & 7106 & GALNT6 & 2.04 & 2.97E-03 \\
\hline 6971 & FBXL15 & 3.47 & $5.03 E-03$ & 7039 & FOXF1 & 3.47 & $4.72 \mathrm{E}-03$ & 7107 & GALNTL6 & 2.17 & $1.92 \mathrm{E}-02$ \\
\hline 6972 & FBXL16 & 4.33 & $6.13 \mathrm{E}-03$ & 7040 & FOXG1 & 1.93 & $2.11 \mathrm{E}-02$ & 7108 & GALR1 & 5.58 & $1.97 \mathrm{E}-02$ \\
\hline 6973 & $\mathrm{FBXO2}$ & 2.47 & $1.09 \mathrm{E}-03$ & 7041 & FOXI1 & 5.42 & $4.20 \mathrm{E}-03$ & 7109 & GALR2 & 4.69 & $2.30 \mathrm{E}-04$ \\
\hline 6974 & $\mathrm{FBXO40}$ & 1.25 & $6.16 \mathrm{E}-02$ & 7042 & FOXI3 & 5.39 & $3.43 \mathrm{E}-03$ & 7110 & GALR3 & 4.83 & $7.35 \mathrm{E}-03$ \\
\hline 6975 & FBXW2 & 2.70 & 7.15E-04 & 7043 & FOXL1 & 6.45 & $3.54 \mathrm{E}-04$ & 7111 & GAP43 & 2.93 & $1.92 \mathrm{E}-03$ \\
\hline 6976 & FCHSD1 & 5.36 & $4.72 E-03$ & 7044 & FOXL2 & 3.51 & $1.43 \mathrm{E}-03$ & 7112 & GAS2L2 & 5.97 & 4.48E-04 \\
\hline 6977 & FCRL2 & 4.08 & $1.14 \mathrm{E}-03$ & 7045 & FOXN1 & 3.65 & $5.33 \mathrm{E}-03$ & 7113 & GATA2 & 3.87 & $2.57 \mathrm{E}-03$ \\
\hline 6978 & FERD3L & 6.07 & $2.81 \mathrm{E}-03$ & 7046 & FOXO1 & 2.38 & $1.39 \mathrm{E}-03$ & 7114 & GATA3 & 5.96 & $6.50 \mathrm{E}-04$ \\
\hline 6979 & FET1 & 4.86 & 4.55E-04 & 7047 & FOXO3 & 1.79 & $1.84 \mathrm{E}-02$ & 7115 & GATA4 & 1.16 & $2.29 \mathrm{E}-02$ \\
\hline 6980 & FEZ1 & 1.88 & $1.83 \mathrm{E}-03$ & 7048 & FOXO4 & 2.46 & $4.24 \mathrm{E}-04$ & 7116 & GATA5 & 3.35 & $5.84 \mathrm{E}-03$ \\
\hline 6981 & FGF1 & 1.13 & $6.49 \mathrm{E}-02$ & 7049 & FOXP1 & 1.70 & $3.00 \mathrm{E}-06$ & 7117 & GATSL3 & 3.82 & $2.26 \mathrm{E}-03$ \\
\hline 6982 & FGF10 & 4.85 & $8.56 \mathrm{E}-04$ & 7050 & FPGS & 3.14 & $5.17 \mathrm{E}-03$ & 7118 & GBP7 & 2.58 & $1.44 \mathrm{E}-03$ \\
\hline 6983 & FGF13 & 2.83 & $7.03 E-03$ & 7051 & FRAS1 & 3.70 & $5.38 \mathrm{E}-04$ & 7119 & GBX1 & 1.75 & $2.47 \mathrm{E}-03$ \\
\hline 6984 & FGF16 & 2.96 & $8.10 \mathrm{E}-03$ & 7052 & FRK & 5.26 & $2.56 \mathrm{E}-03$ & 7120 & GBX2 & 4.01 & $1.46 \mathrm{E}-03$ \\
\hline 6985 & FGF20 & 4.39 & $6.69 \mathrm{E}-03$ & 7053 & FRMD4B & 2.59 & $4.51 \mathrm{E}-03$ & 7121 & GCFC2 & 3.02 & $6.75 E-04$ \\
\hline 6986 & FGF23 & 5.52 & $2.80 \mathrm{E}-03$ & 7054 & FRMD5 & 2.66 & $4.56 \mathrm{E}-03$ & 7122 & GCHFR & 1.61 & $1.87 \mathrm{E}-02$ \\
\hline 6987 & FGF3 & 4.55 & $5.02 E-03$ & 7055 & FRMD6 & 1.70 & 7.62E-03 & 7123 & GCK & 1.21 & 2.97E-02 \\
\hline 6988 & FGF4 & 4.99 & $3.43 \mathrm{E}-03$ & 7056 & FRMD7 & 3.67 & $3.99 \mathrm{E}-03$ & 7124 & GCM1 & 2.21 & $1.47 \mathrm{E}-02$ \\
\hline 6989 & FGF6 & 6.18 & 4.69E-04 & 7057 & FRMPD3 & 4.60 & $1.81 \mathrm{E}-02$ & 7125 & GCM2 & 5.17 & $1.74 \mathrm{E}-02$ \\
\hline 6990 & FGF7 & 4.32 & $3.29 E-04$ & 7058 & FSD2 & 1.65 & $6.26 \mathrm{E}-03$ & 7126 & GCN1L1 & 4.88 & $2.69 \mathrm{E}-03$ \\
\hline 6991 & FGF8 & 2.92 & $1.32 \mathrm{E}-02$ & 7059 & FSHB & 5.55 & $3.78 \mathrm{E}-04$ & 7127 & GCNT1 & 2.69 & $8.76 \mathrm{E}-04$ \\
\hline 6992 & FGF9 & 2.09 & $7.53 \mathrm{E}-03$ & 7060 & FST & 1.64 & $3.71 \mathrm{E}-02$ & 7128 & GDA & 2.33 & $4.28 \mathrm{E}-03$ \\
\hline 6993 & FGFR1 & 3.80 & $1.97 \mathrm{E}-03$ & 7061 & FSTL1 & 1.46 & $1.38 \mathrm{E}-03$ & 7129 & GDAP1 & 1.46 & 4.67E-03 \\
\hline 6994 & FGFR4 & 3.56 & 4.16E-05 & 7062 & FTSJD1 & 4.55 & $7.28 \mathrm{E}-04$ & 7130 & GDAP1L1 & 1.88 & $3.22 \mathrm{E}-04$ \\
\hline 6995 & FHF-2 & 5.17 & $6.35 \mathrm{E}-04$ & 7063 & FTSJD2 & 2.42 & $5.79 \mathrm{E}-03$ & 7131 & GDF5 & 1.84 & $3.48 \mathrm{E}-02$ \\
\hline 6996 & FHL5 & 3.83 & $6.31 \mathrm{E}-03$ & 7064 & FUK & 3.49 & $4.12 \mathrm{E}-03$ & 7132 & GDF7 & 4.67 & $6.65 \mathrm{E}-04$ \\
\hline 6997 & FHOD3 & 3.18 & $1.46 \mathrm{E}-02$ & 7065 & FURIN & 1.91 & $2.28 \mathrm{E}-02$ & 7133 & GDF9 & 5.28 & $4.71 \mathrm{E}-04$ \\
\hline 6998 & FIBCD1 & 3.80 & $5.55 \mathrm{E}-03$ & 7066 & FUT4 & 5.83 & $6.99 \mathrm{E}-03$ & 7134 & GDNF & 3.00 & $8.68 \mathrm{E}-03$ \\
\hline 6999 & FIGNL2 & 6.66 & $6.01 E-04$ & 7067 & FUT5 & 3.60 & $5.64 \mathrm{E}-04$ & 7135 & GDPD4 & 4.69 & $2.63 \mathrm{E}-03$ \\
\hline 7000 & FILIP1L & 3.60 & $1.54 \mathrm{E}-02$ & 7068 & FUT7 & 4.76 & $3.81 \mathrm{E}-04$ & 7136 & GDPD5 & 1.30 & $2.13 \mathrm{E}-03$ \\
\hline 7001 & FJ548639 & 3.60 & $7.02 E-03$ & 7069 & FUT9 & 6.77 & $7.60 \mathrm{E}-05$ & 7137 & GEMIN2 & 3.08 & 7.95E-03 \\
\hline 7002 & FJ605355 & 4.57 & $1.19 \mathrm{E}-02$ & 7070 & FZD10 & 3.82 & $1.27 \mathrm{E}-02$ & 7138 & GFAP & 4.96 & $4.42 \mathrm{E}-04$ \\
\hline 7003 & FJ605357 & 2.61 & 2.66E-02 & 7071 & FZD2 & 1.73 & $3.18 \mathrm{E}-02$ & 7139 & GFI1B & 4.75 & $4.71 \mathrm{E}-03$ \\
\hline
\end{tabular}




\begin{tabular}{|c|c|c|c|c|c|c|c|c|c|c|c|}
\hline 7140 & GFOD1 & 1.68 & 2.63E-02 & 7208 & GPR142 & 3.33 & 4.46E-03 & 7276 & GSG1 & 5.00 & $1.39 \mathrm{E}-04$ \\
\hline 7141 & GFRA1 & 1.78 & $3.64 \mathrm{E}-03$ & 7209 & GPR146 & 3.03 & $3.13 \mathrm{E}-03$ & 7277 & GSG1L & 2.92 & 5.83E-04 \\
\hline 7142 & GFRA2 & 5.24 & $8.58 \mathrm{E}-04$ & 7210 & GPR15 & 3.14 & $2.97 \mathrm{E}-04$ & 7278 & GTF3C4 & 3.40 & $1.49 \mathrm{E}-02$ \\
\hline 7143 & GGCT & 1.24 & $4.98 \mathrm{E}-03$ & 7211 & GPR151 & 3.26 & $4.52 \mathrm{E}-03$ & 7279 & GUCA1B & 3.41 & $4.23 \mathrm{E}-03$ \\
\hline 7144 & GGT5 & 2.52 & $4.05 \mathrm{E}-03$ & 7212 & GPR157 & 3.54 & $3.38 \mathrm{E}-03$ & 7280 & GUCA1C & 4.79 & $1.62 \mathrm{E}-02$ \\
\hline 7145 & GGT7 & 1.41 & $1.83 \mathrm{E}-02$ & 7213 & GPR17 & 4.75 & 3.50E-03 & 7281 & GUCY1A2 & 1.20 & $1.09 \mathrm{E}-01$ \\
\hline 7146 & $\mathrm{GH}$ & 5.10 & $4.45 \mathrm{E}-03$ & 7214 & GPR171 & 4.22 & $5.16 \mathrm{E}-04$ & 7282 & GUCY1A3 & 1.79 & $2.54 \mathrm{E}-02$ \\
\hline 7147 & GHDC & 4.15 & $1.65 \mathrm{E}-02$ & 7215 & GPR174 & 1.82 & $8.67 \mathrm{E}-03$ & 7283 & GUCY2F & 2.86 & $1.65 \mathrm{E}-02$ \\
\hline 7148 & GHRH & 2.17 & $6.29 \mathrm{E}-03$ & 7216 & GPR183 & 4.08 & $1.30 \mathrm{E}-04$ & 7284 & GVINP1 & 6.33 & $1.79 \mathrm{E}-03$ \\
\hline 7149 & GHRH-LR & 4.47 & $3.60 \mathrm{E}-03$ & 7217 & GPR2O & 3.92 & 3.06E-05 & 7285 & GYLTL1B & 4.32 & $3.45 \mathrm{E}-04$ \\
\hline 7150 & GHRHR & 4.56 & 3.63E-03 & 7218 & GPR22 & 3.35 & $1.01 \mathrm{E}-03$ & 7286 & GYPC & 1.45 & $8.33 \mathrm{E}-03$ \\
\hline 7151 & GHSR & 6.72 & $1.56 \mathrm{E}-03$ & 7219 & GPR25 & 2.16 & $8.72 \mathrm{E}-03$ & 7287 & GYS1 & 1.58 & $9.52 \mathrm{E}-02$ \\
\hline 7152 & GIMAP5 & 3.50 & $1.16 \mathrm{E}-04$ & 7220 & GPR26 & 5.61 & $6.69 \mathrm{E}-03$ & 7288 & GZMA & 2.41 & $1.06 \mathrm{E}-03$ \\
\hline 7153 & GIMAP8 & 4.35 & $3.73 \mathrm{E}-05$ & 7221 & GPR27 & 1.53 & 1.69E-02 & 7289 & H1FOO & 2.54 & $1.11 \mathrm{E}-02$ \\
\hline 7154 & GIMD1 & 8.46 & $3.74 \mathrm{E}-04$ & 7222 & GPR35 & 4.62 & $5.21 \mathrm{E}-03$ & 7290 & H6PD & 4.41 & 7.06E-04 \\
\hline 7155 & GIPC2 & 2.35 & $2.85 \mathrm{E}-02$ & 7223 & GPR37L1 & 5.21 & $4.55 \mathrm{E}-03$ & 7291 & HAND1 & 3.89 & $1.01 \mathrm{E}-02$ \\
\hline 7156 & GIT1 & 1.94 & 3.57E-02 & 7224 & GPR52 & 4.40 & $1.17 \mathrm{E}-03$ & 7292 & HAND2 & 1.92 & $1.76 \mathrm{E}-02$ \\
\hline 7157 & GJA3 & 4.44 & $1.41 \mathrm{E}-03$ & 7225 & GPR55 & 3.83 & $1.37 \mathrm{E}-03$ & 7293 & HAPLN1 & 1.43 & $2.05 \mathrm{E}-02$ \\
\hline 7158 & GJA4 & 1.45 & $2.44 \mathrm{E}-02$ & 7226 & GPR56 & 3.65 & $3.02 \mathrm{E}-03$ & 7294 & HAPLN2 & 5.36 & $2.35 \mathrm{E}-03$ \\
\hline 7159 & GJA5 & 2.91 & $7.20 \mathrm{E}-03$ & 7227 & GPR6 & 4.69 & $9.73 \mathrm{E}-03$ & 7295 & HAPLN3 & 5.59 & $5.78 \mathrm{E}-03$ \\
\hline 7160 & GJA8 & 3.58 & $5.46 \mathrm{E}-04$ & 7228 & GPR68 & 3.18 & $6.56 \mathrm{E}-04$ & 7296 & HARS2 & 2.10 & 4.70E-04 \\
\hline 7161 & GJB2 & 3.41 & 4.31E-04 & 7229 & GPR75 & 1.77 & $6.68 \mathrm{E}-03$ & 7297 & HAS3 & 2.09 & $1.48 \mathrm{E}-01$ \\
\hline 7162 & GJB5 & 2.47 & $5.37 \mathrm{E}-03$ & 7230 & GPR78 & 7.42 & 7.56E-06 & 7298 & HAVCR1 & 1.39 & $3.23 \mathrm{E}-03$ \\
\hline 7163 & GJD2 & 2.98 & $8.73 \mathrm{E}-02$ & 7231 & GPR83 & 1.17 & $1.02 \mathrm{E}-02$ & 7299 & HBE1 & 5.16 & 4.07E-04 \\
\hline 7164 & GJD4 & 5.22 & $9.69 \mathrm{E}-05$ & 7232 & GPR83-L & 3.27 & $6.57 \mathrm{E}-03$ & 7300 & HBEGF & 4.25 & $9.83 \mathrm{E}-04$ \\
\hline 7165 & GKN1 & 2.39 & $1.51 \mathrm{E}-02$ & 7233 & GPR85 & 1.54 & $6.01 \mathrm{E}-04$ & 7301 & HBG1 & 3.05 & $3.38 \mathrm{E}-03$ \\
\hline 7166 & GKN2 & 1.51 & $1.50 \mathrm{E}-02$ & 7234 & GPR87 & 2.15 & $3.40 \mathrm{E}-02$ & 7302 & HBXIP & 1.52 & $1.66 \mathrm{E}-02$ \\
\hline 7167 & GLA & 1.55 & $2.27 \mathrm{E}-03$ & 7235 & GPR88 & 3.70 & $5.17 \mathrm{E}-04$ & 7303 & $\mathrm{HCK}$ & 2.05 & $3.57 \mathrm{E}-04$ \\
\hline 7168 & GLCE & 5.85 & $6.07 \mathrm{E}-03$ & 7236 & GPR89B & 2.41 & $5.63 \mathrm{E}-02$ & 7304 & HCLS1 & 2.23 & $1.18 \mathrm{E}-02$ \\
\hline 7169 & GLDC & 1.37 & $3.55 \mathrm{E}-03$ & 7237 & GPR97 & 3.61 & $1.05 E-02$ & 7305 & HCRT & 4.96 & $5.51 \mathrm{E}-03$ \\
\hline 7170 & GLI1 & 1.73 & $1.92 \mathrm{E}-02$ & 7238 & GPRC5A & 2.52 & $1.17 \mathrm{E}-03$ & 7306 & HCRTR2 & 2.98 & $1.78 \mathrm{E}-02$ \\
\hline 7171 & GLI2 & 2.37 & $7.94 \mathrm{E}-04$ & 7239 & GPRC5C & 3.78 & $2.21 \mathrm{E}-04$ & 7307 & HDAC7 & 1.78 & $9.39 \mathrm{E}-02$ \\
\hline 7172 & GLIPR1 & 3.36 & $4.98 \mathrm{E}-04$ & 7240 & GPRC6A & 5.40 & $1.27 \mathrm{E}-02$ & 7308 & HDAC9 & 1.54 & $3.25 \mathrm{E}-02$ \\
\hline 7173 & GLIS2 & 3.07 & $2.85 \mathrm{E}-02$ & 7241 & GPRIN2 & 3.26 & $2.23 \mathrm{E}-02$ & 7309 & HDC & 1.60 & $1.05 \mathrm{E}-02$ \\
\hline 7174 & GLOD5 & 7.31 & $2.36 \mathrm{E}-05$ & 7242 & GPRIN3 & 4.06 & $2.57 \mathrm{E}-03$ & 7310 & HEBP2 & 4.48 & 3.37E-03 \\
\hline 7175 & GLP2R & 2.19 & $5.64 \mathrm{E}-03$ & 7243 & GRAMD1B & 1.10 & 7.12E-03 & 7311 & HECA & 1.35 & $1.84 \mathrm{E}-02$ \\
\hline 7176 & GLRA1 & 7.14 & $2.17 \mathrm{E}-03$ & 7244 & GRAP & 2.46 & $1.02 \mathrm{E}-02$ & 7312 & HELZ2 & 3.51 & $8.16 \mathrm{E}-04$ \\
\hline 7177 & GLRA2 & 3.25 & $1.20 \mathrm{E}-02$ & 7245 & GRAP2 & 6.02 & $5.44 \mathrm{E}-03$ & 7313 & HEP21 & 4.27 & $1.02 \mathrm{E}-04$ \\
\hline 7178 & GLRA3 & 4.04 & 3.90E-03 & 7246 & GREB1 & 3.96 & $1.45 \mathrm{E}-02$ & 7314 & HEPACAM & 1.66 & $4.52 E-02$ \\
\hline 7179 & GLRA4 & 1.44 & 7.42E-02 & 7247 & GREB1L & 2.84 & $7.72 \mathrm{E}-03$ & 7315 & HEPACAM2 & 5.83 & $6.23 \mathrm{E}-03$ \\
\hline 7180 & GLRB & 1.88 & $2.40 \mathrm{E}-03$ & 7248 & GREM1 & 4.87 & $6.32 \mathrm{E}-03$ & 7316 & HEPH & 1.41 & $4.63 \mathrm{E}-02$ \\
\hline 7181 & GLTSCR1L & 2.47 & $3.74 \mathrm{E}-03$ & 7249 & GREM2 & 4.43 & $1.51 \mathrm{E}-03$ & 7317 & HES1 & 2.93 & 5.96E-03 \\
\hline 7182 & GLYCTK & 2.29 & $9.47 \mathrm{E}-03$ & 7250 & GRHL3 & 1.41 & $4.52 \mathrm{E}-02$ & 7318 & HES5 & 5.56 & $1.09 \mathrm{E}-04$ \\
\hline 7183 & GMIP & 1.71 & $5.35 \mathrm{E}-04$ & 7251 & GRIA1 & 5.98 & $9.67 \mathrm{E}-04$ & 7319 & HES7 & 2.34 & 1.57E-02 \\
\hline 7184 & GNA14 & 4.31 & $8.12 \mathrm{E}-05$ & 7252 & GRIA2 & 1.50 & $2.90 \mathrm{E}-02$ & 7320 & HEY2 & 4.75 & $1.64 \mathrm{E}-02$ \\
\hline 7185 & GNAT1 & 3.88 & $2.25 \mathrm{E}-03$ & 7253 & GRIA3 & 2.93 & $6.73 \mathrm{E}-03$ & 7321 & HGF & 2.94 & $2.13 E-03$ \\
\hline 7186 & GNB4 & 1.47 & $6.15 \mathrm{E}-03$ & 7254 & GRIA4 & 4.66 & $1.05 \mathrm{E}-03$ & 7322 & HGFAC & 1.63 & $5.88 \mathrm{E}-02$ \\
\hline 7187 & GNG2 & 1.27 & $4.19 \mathrm{E}-02$ & 7255 & GRID1 & 3.62 & $3.48 \mathrm{E}-03$ & 7323 & HHAT & 6.23 & $1.27 \mathrm{E}-03$ \\
\hline 7188 & GNOT1 & 3.06 & $6.17 \mathrm{E}-04$ & 7256 & GRID2 & 5.01 & $8.98 \mathrm{E}-04$ & 7324 & HHATL & 3.04 & $2.55 \mathrm{E}-03$ \\
\hline 7189 & GNRHR & 2.05 & 3.59E-02 & 7257 & GRID2IP & 2.89 & $9.50 \mathrm{E}-03$ & 7325 & HHEX & 3.30 & $7.92 \mathrm{E}-03$ \\
\hline 7190 & GOLPH3L & 5.15 & $1.18 \mathrm{E}-03$ & 7258 & GRIFIN & 5.22 & 7.43E-03 & 7326 & HHIP & 3.52 & $9.84 \mathrm{E}-03$ \\
\hline 7191 & GP1BA & 5.04 & $9.74 \mathrm{E}-04$ & 7259 & GRIK1 & 2.16 & $1.28 \mathrm{E}-02$ & 7327 & HHIPL1 & 6.50 & $2.28 \mathrm{E}-03$ \\
\hline 7192 & GP1BB & 4.19 & $6.89 \mathrm{E}-03$ & 7260 & GRIK2 & 7.03 & $2.48 \mathrm{E}-04$ & 7328 & HHIPL2 & 3.13 & $7.20 \mathrm{E}-03$ \\
\hline 7193 & GP9 & 4.06 & $3.50 \mathrm{E}-03$ & 7261 & GRIK3 & 3.88 & $1.22 \mathrm{E}-02$ & 7329 & HIC2 & 3.29 & $1.18 \mathrm{E}-02$ \\
\hline 7194 & GPATCH2 & 2.41 & $5.13 \mathrm{E}-04$ & 7262 & GRIK4 & 1.98 & $8.30 \mathrm{E}-02$ & 7330 & HIF1AN & 2.53 & $1.04 \mathrm{E}-02$ \\
\hline 7195 & GPC3 & 2.21 & $4.60 \mathrm{E}-03$ & 7263 & GRIN1 & 3.48 & $2.81 \mathrm{E}-03$ & 7331 & HIPK1 & 1.47 & $1.69 \mathrm{E}-02$ \\
\hline 7196 & GPC5 & 5.27 & $1.58 \mathrm{E}-03$ & 7264 & GRIN3A & 3.69 & $6.61 \mathrm{E}-03$ & 7332 & HIRA & 1.17 & $1.31 \mathrm{E}-02$ \\
\hline 7197 & GPER & 5.04 & $3.41 \mathrm{E}-03$ & 7265 & GRIP1 & 2.28 & $3.41 \mathrm{E}-03$ & 7333 & HK2 & 2.50 & $1.73 \mathrm{E}-03$ \\
\hline 7198 & GPHB5 & 4.56 & $1.09 \mathrm{E}-02$ & 7266 & GRM1 & 4.08 & $7.29 \mathrm{E}-03$ & 7334 & HKDC1 & 2.59 & $1.15 \mathrm{E}-02$ \\
\hline 7199 & GPR1 & 2.15 & $1.11 \mathrm{E}-03$ & 7267 & GRM2 & 6.47 & $5.21 \mathrm{E}-04$ & 7335 & HMGA2 & 2.17 & $8.30 \mathrm{E}-04$ \\
\hline 7200 & GPR112 & 2.68 & $1.40 \mathrm{E}-03$ & 7268 & GRM5 & 3.16 & $6.85 \mathrm{E}-03$ & 7336 & HMOX1 & 1.45 & $2.48 \mathrm{E}-02$ \\
\hline 7201 & GPR116 & 2.85 & $2.35 \mathrm{E}-03$ & 7269 & GRM7 & 5.00 & $5.75 \mathrm{E}-05$ & 7337 & HMX1 & 8.72 & $7.91 E-03$ \\
\hline 7202 & GPR119 & 6.87 & $2.96 \mathrm{E}-04$ & 7270 & GRPR & 7.10 & $2.07 \mathrm{E}-04$ & 7338 & HMX3 & 2.59 & $2.99 \mathrm{E}-03$ \\
\hline 7203 & GPR12 & 1.77 & $4.36 \mathrm{E}-02$ & 7271 & GRRP1 & 1.10 & $1.46 \mathrm{E}-02$ & 7339 & HN1L & 2.06 & $3.40 \mathrm{E}-02$ \\
\hline 7204 & GPR126 & 1.83 & $1.68 \mathrm{E}-02$ & 7272 & GRXCR1 & 6.69 & 1.19E-04 & 7340 & HNF1A & 3.09 & $4.81 E-05$ \\
\hline 7205 & GPR137C & 2.47 & $1.57 \mathrm{E}-03$ & 7273 & GRXCR2 & 2.43 & $3.24 \mathrm{E}-03$ & 7341 & HNF1B & 3.73 & $3.25 \mathrm{E}-04$ \\
\hline 7206 & GPR139 & 3.17 & $1.62 \mathrm{E}-03$ & 7274 & GSC & 4.62 & $6.67 \mathrm{E}-03$ & 7342 & HNF4A & 5.21 & $6.37 \mathrm{E}-03$ \\
\hline 7207 & GPR141 & 5.89 & $8.97 \mathrm{E}-05$ & 7275 & GSDMA & 1.40 & $2.49 \mathrm{E}-02$ & 7343 & HNF4beta & 4.49 & $4.36 \mathrm{E}-03$ \\
\hline
\end{tabular}




\begin{tabular}{|c|c|c|c|c|c|c|c|c|c|c|c|}
\hline 7344 & HNF4G & 4.03 & $3.19 \mathrm{E}-02$ & 7412 & IBSP & 4.73 & $1.71 \mathrm{E}-03$ & 7480 & INSR & 1.27 & 1.66E-02 \\
\hline 7345 & HNMT & 2.45 & $1.60 \mathrm{E}-03$ & 7413 & $\operatorname{Icos}$ & 2.33 & $2.91 \mathrm{E}-03$ & 7481 & INTS3 & 3.33 & $1.45 \mathrm{E}-02$ \\
\hline 7346 & HNRNPAO & 1.19 & $2.71 \mathrm{E}-02$ & 7414 & ICOSLG & 2.41 & $1.18 \mathrm{E}-03$ & 7482 & IPP & 2.23 & $1.92 \mathrm{E}-03$ \\
\hline 7347 & HOGA1 & 6.13 & $1.03 \mathrm{E}-03$ & 7415 & ID3 & 1.87 & $2.86 \mathrm{E}-02$ & 7483 & IQCJ & 3.40 & $3.47 \mathrm{E}-03$ \\
\hline 7348 & HOMER2 & 4.59 & $5.10 \mathrm{E}-03$ & 7416 & IFI30 & 3.61 & $2.12 \mathrm{E}-01$ & 7484 & IQGAP2 & 4.68 & $1.94 \mathrm{E}-03$ \\
\hline 7349 & HOXA1 & 4.36 & $8.79 \mathrm{E}-04$ & 7417 & IFITM10 & 5.14 & $8.88 \mathrm{E}-03$ & 7485 & IQSEC1 & 2.73 & $1.30 \mathrm{E}-02$ \\
\hline 7350 & HOXA10 & 4.18 & $2.09 \mathrm{E}-03$ & 7418 & IFNA3 & 5.42 & $1.22 \mathrm{E}-03$ & 7486 & IQSEC3 & 3.92 & 2.87E-03 \\
\hline 7351 & HOXA11 & 7.18 & $1.67 \mathrm{E}-03$ & 7419 & IFNAR1 & 1.84 & $5.53 \mathrm{E}-03$ & 7487 & IRF4 & 1.28 & $1.28 \mathrm{E}-02$ \\
\hline 7352 & HOXA13 & 5.08 & $1.41 \mathrm{E}-03$ & 7420 & IFNAR2 & 2.97 & $8.25 \mathrm{E}-03$ & 7488 & IRF5 & 2.28 & $5.15 \mathrm{E}-04$ \\
\hline 7353 & HOXA2 & 3.73 & $4.71 \mathrm{E}-03$ & 7421 & IFNB & 5.97 & $5.14 \mathrm{E}-04$ & 7489 & IRF8 & 2.06 & $9.00 \mathrm{E}-03$ \\
\hline 7354 & HOXA3 & 2.96 & $4.52 \mathrm{E}-03$ & 7422 & IFNG & 3.50 & $4.18 \mathrm{E}-04$ & 7490 & IRS1 & 2.85 & $8.55 \mathrm{E}-02$ \\
\hline 7355 & HOXA4 & 2.32 & $4.71 \mathrm{E}-03$ & 7423 & IGDCC4 & 3.78 & $9.40 \mathrm{E}-04$ & 7491 & IRS2 & 1.95 & $6.76 \mathrm{E}-03$ \\
\hline 7356 & HOXA5 & 3.34 & $1.44 \mathrm{E}-02$ & 7424 & IGF1 & 5.02 & $2.01 \mathrm{E}-02$ & 7492 & IRX1 & 3.80 & $5.26 \mathrm{E}-04$ \\
\hline 7357 & HOXA6 & 2.24 & $1.00 \mathrm{E}-03$ & 7425 & IGF2BP1 & 3.44 & $2.12 \mathrm{E}-02$ & 7493 & IRX2 & 4.90 & $8.72 \mathrm{E}-05$ \\
\hline 7358 & HOXА9 & 4.36 & $6.38 \mathrm{E}-03$ & 7426 & IGFALS & 5.86 & $3.56 \mathrm{E}-04$ & 7494 & IRX5 & 4.32 & $7.30 \mathrm{E}-03$ \\
\hline 7359 & HOXB1 & 4.11 & $3.71 E-04$ & 7427 & IGFBP1 & 3.92 & $8.52 \mathrm{E}-03$ & 7495 & IRX6 & 6.11 & $1.65 \mathrm{E}-03$ \\
\hline 7360 & HOXB13 & 4.70 & $1.67 \mathrm{E}-03$ & 7428 & IGFN1 & 1.18 & $1.51 \mathrm{E}-02$ & 7496 & ISL1 & 7.17 & $8.18 \mathrm{E}-03$ \\
\hline 7361 & HOXB3 & 2.54 & $6.35 \mathrm{E}-03$ & 7429 & IGJ & 1.78 & $1.05 \mathrm{E}-01$ & 7497 & ISLR & 3.45 & 4.49E-03 \\
\hline 7362 & HOXB4 & 3.95 & $7.28 \mathrm{E}-04$ & 7430 & IGSF1 & 1.50 & 3.40E-03 & 7498 & ISLR2 & 1.83 & $9.46 \mathrm{E}-03$ \\
\hline 7363 & HOXB5 & 2.69 & $9.46 \mathrm{E}-03$ & 7431 & IGSF3 & 3.40 & $1.13 \mathrm{E}-02$ & 7499 & ISM2 & 5.46 & $2.40 \mathrm{E}-02$ \\
\hline 7364 & HOXB7 & 2.63 & $1.49 \mathrm{E}-03$ & 7432 & IGSF5 & 3.36 & $2.13 \mathrm{E}-02$ & 7500 & ITGA11 & 1.43 & $4.92 \mathrm{E}-02$ \\
\hline 7365 & HОХв8 & 2.52 & $8.63 \mathrm{E}-03$ & 7433 & IGSF6 & 2.42 & $1.91 \mathrm{E}-02$ & 7501 & ITGA4 & 3.58 & $1.03 E-02$ \\
\hline 7366 & НОХв9 & 6.62 & $2.84 \mathrm{E}-04$ & 7434 & IGSF9 & 1.59 & $1.59 \mathrm{E}-02$ & 7502 & ITGA5 & 4.19 & $7.54 \mathrm{E}-02$ \\
\hline 7367 & HOXC10 & 5.96 & $1.40 \mathrm{E}-05$ & 7435 & IGSF9B & 4.18 & $6.23 \mathrm{E}-04$ & 7503 & ITGA8 & 2.81 & $5.52 \mathrm{E}-02$ \\
\hline 7368 & HoxC11 & 1.65 & $2.51 \mathrm{E}-02$ & 7436 & $\mathrm{IHH}$ & 3.84 & $2.14 \mathrm{E}-03$ & 7504 & ITGA9 & 1.43 & $1.56 \mathrm{E}-02$ \\
\hline 7369 & HOXC12 & 8.25 & $1.59 \mathrm{E}-03$ & 7437 & IKBKE & 3.34 & $1.41 \mathrm{E}-02$ & 7505 & ITGB3 & 2.74 & $2.09 \mathrm{E}-02$ \\
\hline 7370 & HOXC6 & 2.31 & $7.35 \mathrm{E}-04$ & 7438 & IKZF3 & 4.67 & $5.74 \mathrm{E}-04$ & 7506 & ITGB6 & 5.20 & 7.96E-06 \\
\hline 7371 & HOXC8 & 4.13 & $2.21 \mathrm{E}-03$ & 7439 & IL10 & 6.15 & $5.47 \mathrm{E}-03$ & 7507 & ITGBL1 & 2.02 & 3.09E-02 \\
\hline 7372 & HOXC9 & 5.31 & $2.86 \mathrm{E}-03$ & 7440 & IL10RA & 5.66 & $4.18 \mathrm{E}-03$ & 7508 & ITIH2 & 1.39 & $6.58 \mathrm{E}-03$ \\
\hline 7373 & HOXD1 & 2.59 & $5.15 \mathrm{E}-03$ & 7441 & IL12A & 3.29 & $1.12 \mathrm{E}-02$ & 7509 & ITK & 3.75 & $7.51 \mathrm{E}-03$ \\
\hline 7374 & HOXD12 & 6.16 & $2.71 \mathrm{E}-04$ & 7442 & IL12B & 2.35 & $8.96 \mathrm{E}-02$ & 7510 & ITM2C & 1.14 & 3.61E-01 \\
\hline 7375 & HOXD13 & 4.16 & $2.58 \mathrm{E}-03$ & 7443 & IL13 & 3.59 & $8.10 \mathrm{E}-04$ & 7511 & ITPKA & 1.61 & 9.64E-03 \\
\hline 7376 & HOXD3 & 1.72 & $7.61 \mathrm{E}-03$ & 7444 & IL13RA1 & 3.38 & $4.56 \mathrm{E}-03$ & 7512 & ITPR1 & 1.31 & $6.02 \mathrm{E}-03$ \\
\hline 7377 & HOXD4 & 1.97 & $2.04 \mathrm{E}-02$ & 7445 & IL15RA & 2.28 & $2.74 \mathrm{E}-03$ & 7513 & ITPRIP & 4.88 & 4.64E-03 \\
\hline 7378 & HOXD8 & 3.47 & $1.49 \mathrm{E}-02$ & 7446 & IL17F & 3.39 & $2.55 \mathrm{E}-03$ & 7514 & IYD & 5.62 & $5.38 \mathrm{E}-04$ \\
\hline 7379 & HPCA & 5.13 & $3.56 \mathrm{E}-03$ & 7447 & IL17REL & 5.22 & $1.75 \mathrm{E}-03$ & 7515 & J00914 & 1.25 & $1.15 \mathrm{E}-02$ \\
\hline 7380 & HPDL & 2.53 & $5.50 \mathrm{E}-02$ & 7448 & IL18R1 & 1.38 & $5.57 \mathrm{E}-03$ & 7516 & JAG2 & 1.40 & $1.47 \mathrm{E}-02$ \\
\hline 7381 & HPGD & 5.36 & $9.14 \mathrm{E}-04$ & 7449 & IL18RAP & 3.23 & $1.73 \mathrm{E}-02$ & 7517 & JAK2 & 2.89 & $1.82 \mathrm{E}-04$ \\
\hline 7382 & HPS1 & 3.17 & $8.24 \mathrm{E}-03$ & 7450 & IL1B & 3.20 & $5.18 \mathrm{E}-03$ & 7518 & JAKMIP1 & 3.36 & $5.29 \mathrm{E}-03$ \\
\hline 7383 & HPSE & 3.26 & $2.88 \mathrm{E}-02$ & 7451 & IL1RAPL1 & 5.49 & $8.96 \mathrm{E}-04$ & 7519 & JDP2 & 1.69 & $3.40 \mathrm{E}-03$ \\
\hline 7384 & HPSE2 & 3.52 & $4.22 \mathrm{E}-03$ & 7452 & IL2 & 4.39 & $1.83 \mathrm{E}-04$ & 7520 & JF936801 & 7.77 & $2.64 \mathrm{E}-03$ \\
\hline 7385 & HRH2 & 5.65 & $3.87 \mathrm{E}-03$ & 7453 & IL2ORA & 3.92 & $2.50 \mathrm{E}-03$ & 7521 & JQ780447 & 6.09 & $6.11 \mathrm{E}-03$ \\
\hline 7386 & HRH3 & 5.39 & $9.31 \mathrm{E}-03$ & 7454 & IL21 & 4.49 & $2.65 \mathrm{E}-04$ & 7522 & JQ927550 & 5.78 & $3.00 \mathrm{E}-03$ \\
\hline 7387 & HRH4 & 2.81 & $1.02 \mathrm{E}-03$ & 7455 & IL21R & 2.23 & $3.84 \mathrm{E}-04$ & 7523 & JQ927551 & 10.41 & 7.27E-05 \\
\hline 7388 & HS3ST4 & 5.17 & $1.91 \mathrm{E}-03$ & 7456 & IL22 & 1.66 & $5.25 \mathrm{E}-03$ & 7524 & JX483813 & 3.75 & $3.78 \mathrm{E}-02$ \\
\hline 7389 & HS3ST5 & 2.00 & $1.09 \mathrm{E}-02$ & 7457 & IL22RA1 & 3.88 & $7.58 \mathrm{E}-03$ & 7525 & K01481 & 2.97 & $4.86 \mathrm{E}-03$ \\
\hline 7390 & HS3ST6 & 2.90 & $6.01 \mathrm{E}-03$ & 7458 & IL22RA2 & 3.90 & $6.79 \mathrm{E}-03$ & 7526 & K123 & 4.50 & $1.85 \mathrm{E}-03$ \\
\hline 7391 & HSD11B1 & 6.47 & $5.80 \mathrm{E}-03$ & 7459 & IL28B & 6.62 & $5.09 \mathrm{E}-03$ & 7527 & KAL1 & 4.42 & $1.31 \mathrm{E}-03$ \\
\hline 7392 & HSD17B1 & 4.58 & $9.02 E-04$ & 7460 & IL2RA & 5.80 & $1.89 \mathrm{E}-03$ & 7528 & KALRN & 2.80 & $1.72 \mathrm{E}-02$ \\
\hline 7393 & HSD3B2 & 1.86 & $1.61 \mathrm{E}-01$ & 7461 & IL3 & 5.64 & $5.08 \mathrm{E}-05$ & 7529 & KANK4 & 2.81 & $4.24 \mathrm{E}-05$ \\
\hline 7394 & HSD3B7 & 5.05 & $7.61 E-04$ & 7462 & IL36RN & 3.23 & $8.32 \mathrm{E}-03$ & 7530 & KBP & 1.92 & $8.64 \mathrm{E}-03$ \\
\hline 7395 & HSF4 & 1.37 & $6.82 \mathrm{E}-03$ & 7463 & IL4 & 5.66 & $3.00 \mathrm{E}-04$ & 7531 & KBTBD11 & 2.70 & $8.91 \mathrm{E}-03$ \\
\hline 7396 & HSPA12A & 2.95 & $1.83 \mathrm{E}-03$ & 7464 & IL5 & 4.18 & $4.93 \mathrm{E}-03$ & 7532 & KBTBD12 & 4.70 & $8.18 \mathrm{E}-03$ \\
\hline 7397 & HSPB3 & 3.44 & $1.48 \mathrm{E}-03$ & 7465 & IL5RA & 2.18 & $3.54 \mathrm{E}-04$ & 7533 & KBTBD13 & 1.83 & $1.68 \mathrm{E}-02$ \\
\hline 7398 & HSPB7 & 2.67 & $3.79 \mathrm{E}-03$ & 7466 & IL6 & 5.21 & $4.82 \mathrm{E}-05$ & 7534 & KBTBD5 & 3.65 & $2.88 \mathrm{E}-03$ \\
\hline 7399 & HSPG2 & 3.04 & $8.71 \mathrm{E}-04$ & 7467 & IL6RA & 1.86 & $4.12 \mathrm{E}-03$ & 7535 & KCNA1 & 2.54 & 2.13E-03 \\
\hline 7400 & HTR1B & 3.34 & $1.94 \mathrm{E}-02$ & 7468 & IL7 & 1.87 & $2.53 \mathrm{E}-03$ & 7536 & KCNA2 & 3.86 & $1.40 \mathrm{E}-02$ \\
\hline 7401 & HTR1E & 4.88 & $3.36 \mathrm{E}-04$ & 7469 & IL7R & 3.47 & $2.86 \mathrm{E}-03$ & 7537 & KCNA3 & 3.20 & $3.41 E-03$ \\
\hline 7402 & HTR1F & 3.14 & $5.56 \mathrm{E}-03$ & 7470 & IL8 & 4.47 & $1.47 \mathrm{E}-03$ & 7538 & KCNA4 & 4.99 & $1.74 \mathrm{E}-03$ \\
\hline 7403 & HTR2A & 4.81 & $1.13 \mathrm{E}-04$ & 7471 & IL9R & 6.52 & $6.60 \mathrm{E}-03$ & 7539 & KCNA5 & 3.33 & $3.64 \mathrm{E}-03$ \\
\hline 7404 & HTR3A & 3.99 & $1.86 \mathrm{E}-03$ & 7472 & IMP3 & 2.86 & $1.78 \mathrm{E}-01$ & 7540 & KCNAB2 & 3.03 & $5.83 \mathrm{E}-03$ \\
\hline 7405 & HTR4 & 3.99 & $2.05 \mathrm{E}-03$ & 7473 & IMPG1 & 4.98 & $5.33 \mathrm{E}-04$ & 7541 & KCNB1 & 4.68 & $1.25 \mathrm{E}-02$ \\
\hline 7406 & HTR5A & 4.38 & $1.14 \mathrm{E}-02$ & 7474 & INDOL1 & 5.38 & $3.32 \mathrm{E}-03$ & 7542 & KCNC1 & 6.51 & 9.53E-04 \\
\hline 7407 & HTR6 & 4.08 & $8.37 \mathrm{E}-04$ & 7475 & INHBA & 3.67 & $1.09 \mathrm{E}-02$ & 7543 & $\mathrm{KCNC4}$ & 4.69 & $4.74 \mathrm{E}-03$ \\
\hline 7408 & HTR7 & 3.68 & $3.90 \mathrm{E}-03$ & 7476 & INPP5A & 1.14 & $9.74 \mathrm{E}-03$ & 7544 & KCND2 & 3.66 & $1.26 \mathrm{E}-02$ \\
\hline 7409 & HYAL2 & 2.43 & $6.64 \mathrm{E}-03$ & 7477 & INPP5J & 1.27 & $2.43 \mathrm{E}-02$ & 7545 & KCND3 & 2.71 & 7.02E-04 \\
\hline 7410 & HYALP1 & 3.43 & $1.58 \mathrm{E}-03$ & 7478 & INS & 2.96 & $2.03 \mathrm{E}-03$ & 7546 & KCNE1 & 6.20 & 2.70E-03 \\
\hline 7411 & IAPP & 1.79 & $9.74 \mathrm{E}-03$ & 7479 & INSC & 1.76 & $8.89 \mathrm{E}-02$ & 7547 & KCNE2 & 3.71 & $1.95 E-03$ \\
\hline
\end{tabular}




\begin{tabular}{|c|c|c|c|c|c|c|c|c|c|c|c|}
\hline 7548 & KCNE3 & 4.34 & $1.32 \mathrm{E}-03$ & 7616 & KLHDC8A & 6.40 & $8.08 \mathrm{E}-05$ & 7684 & LCP2 & 1.32 & 7.93E-03 \\
\hline 7549 & KCNE4 & 5.34 & $2.68 \mathrm{E}-04$ & 7617 & KLHL12 & 1.15 & $7.00 \mathrm{E}-02$ & 7685 & LDB1 & 2.62 & $3.96 \mathrm{E}-03$ \\
\hline 7550 & KCNF1 & 3.86 & $4.79 \mathrm{E}-03$ & 7618 & KLHL17 & 5.33 & $4.68 \mathrm{E}-04$ & 7686 & LDLRAD1 & 1.66 & $3.61 \mathrm{E}-02$ \\
\hline 7551 & KCNG1 & 3.08 & $7.53 \mathrm{E}-03$ & 7619 & KLHL25 & 4.78 & $1.43 \mathrm{E}-03$ & 7687 & LEAP2 & 3.38 & $4.22 \mathrm{E}-03$ \\
\hline 7552 & KCNG3 & 4.55 & $4.77 \mathrm{E}-03$ & 7620 & KLHL29 & 1.37 & $2.73 \mathrm{E}-03$ & 7688 & LECT1 & 4.19 & $2.56 \mathrm{E}-03$ \\
\hline 7553 & $\mathrm{KCNH} 4$ & 6.94 & $2.07 E-05$ & 7621 & KLHL30 & 3.60 & $8.37 \mathrm{E}-04$ & 7689 & LECT2 & 2.95 & $1.01 \mathrm{E}-03$ \\
\hline 7554 & KCNH8 & 5.93 & $2.69 \mathrm{E}-05$ & 7622 & KLHL31 & 1.14 & $9.46 \mathrm{E}-03$ & 7690 & LEF1 & 1.75 & $3.64 \mathrm{E}-04$ \\
\hline 7555 & KCNIP2 & 4.92 & $7.43 \mathrm{E}-03$ & 7623 & KLHL34 & 5.46 & $2.04 \mathrm{E}-04$ & 7691 & LEPR & 2.05 & $1.26 \mathrm{E}-03$ \\
\hline 7556 & KCNJ1 & 5.16 & $3.16 \mathrm{E}-04$ & 7624 & KLHL35 & 4.18 & $7.58 \mathrm{E}-04$ & 7692 & LFNG & 3.02 & $5.47 \mathrm{E}-02$ \\
\hline 7557 & KCNJ11 & 4.25 & $4.78 \mathrm{E}-03$ & 7625 & KLHL38 & 5.12 & $1.35 \mathrm{E}-03$ & 7693 & LGALS3 & 1.47 & $5.04 \mathrm{E}-03$ \\
\hline 7558 & KCNJ15 & 5.61 & $4.36 \mathrm{E}-03$ & 7626 & KLHL4 & 2.95 & 3.37E-03 & 7694 & LGR6 & 3.98 & $2.59 \mathrm{E}-03$ \\
\hline 7559 & KCNJ16 & 4.09 & $2.30 \mathrm{E}-04$ & 7627 & KMO & 2.57 & $1.90 \mathrm{E}-03$ & 7695 & LGSN & 4.07 & $8.96 \mathrm{E}-04$ \\
\hline 7560 & KCNJ2 & 5.78 & $1.46 \mathrm{E}-02$ & 7628 & KNG1 & 3.60 & $1.15 \mathrm{E}-04$ & 7696 & LHFPL2 & 2.36 & $1.45 \mathrm{E}-04$ \\
\hline 7561 & KCNJ3 & 3.26 & $1.59 \mathrm{E}-04$ & 7629 & KPNA7 & 4.15 & 7.99E-04 & 7697 & LHX1 & 2.22 & $5.13 \mathrm{E}-02$ \\
\hline 7562 & KCNJ4 & 5.64 & $7.41 \mathrm{E}-05$ & 7630 & KREMEN1 & 3.84 & $1.16 \mathrm{E}-03$ & 7698 & LHX3 & 4.42 & $3.57 \mathrm{E}-03$ \\
\hline 7563 & KCNJ6 & 5.69 & $2.57 \mathrm{E}-03$ & 7631 & KRT12 & 5.50 & $5.79 \mathrm{E}-04$ & 7699 & LHX4 & 5.17 & $1.33 \mathrm{E}-03$ \\
\hline 7564 & KCNJ8 & 1.88 & $1.57 \mathrm{E}-02$ & 7632 & KRT19 & 2.59 & 7.81E-04 & 7700 & LHX5 & 4.76 & $1.07 E-02$ \\
\hline 7565 & KCNK12 & 1.33 & $1.29 \mathrm{E}-02$ & 7633 & KRT20 & 2.13 & $5.37 \mathrm{E}-03$ & 7701 & LHX6 & 7.06 & $6.15 \mathrm{E}-03$ \\
\hline 7566 & KCNK13 & 3.72 & $5.24 \mathrm{E}-04$ & 7634 & KRT5 & 3.78 & $1.68 \mathrm{E}-03$ & 7702 & LHX8 & 1.79 & $3.18 \mathrm{E}-03$ \\
\hline 7567 & KCNK15 & 6.30 & $9.66 \mathrm{E}-04$ & 7635 & KRT75 & 3.56 & $1.69 \mathrm{E}-05$ & 7703 & LHX9 & 2.82 & $1.92 \mathrm{E}-03$ \\
\hline 7568 & KCNK17 & 3.31 & $1.38 \mathrm{E}-02$ & 7636 & KRT8 & 4.48 & $1.56 \mathrm{E}-02$ & 7704 & LIF & 6.80 & $1.26 \mathrm{E}-03$ \\
\hline 7569 & KCNK2 & 6.30 & $5.86 \mathrm{E}-03$ & 7637 & KRTAP10-4 & 5.05 & $4.98 \mathrm{E}-03$ & 7705 & LIG1 & 1.15 & $3.34 \mathrm{E}-02$ \\
\hline 7570 & KCNMA1 & 4.48 & 2.19E-03 & 7638 & KRTCAP3 & 2.63 & 7.32E-04 & 7706 & LIMD2 & 4.54 & 4.28E-03 \\
\hline 7571 & KCNMB2 & 4.20 & $1.70 \mathrm{E}-04$ & 7639 & $\mathrm{KY}$ & 3.45 & $1.59 \mathrm{E}-03$ & 7707 & LIMS2 & 2.22 & $2.40 \mathrm{E}-02$ \\
\hline 7572 & KCNQ1 & 3.78 & $6.63 \mathrm{E}-04$ & 7640 & L12565 & 3.52 & $1.41 \mathrm{E}-03$ & 7708 & LIN28A & 2.58 & $9.26 \mathrm{E}-03$ \\
\hline 7573 & KCNQ2 & 4.29 & $4.12 \mathrm{E}-03$ & 7641 & L1CAM & 3.11 & $1.07 E-03$ & 7709 & LIN7A & 1.91 & $3.73 E-02$ \\
\hline 7574 & KCNQ4 & 3.21 & $9.89 \mathrm{E}-04$ & 7642 & L3MBTL1 & 5.09 & $5.96 \mathrm{E}-03$ & 7710 & LINGO2 & 3.08 & $6.57 \mathrm{E}-03$ \\
\hline 7575 & KCNQ5 & 2.04 & $2.16 \mathrm{E}-03$ & 7643 & L48877 & 3.01 & $2.22 \mathrm{E}-04$ & 7711 & LINGO3 & 5.76 & $2.57 \mathrm{E}-03$ \\
\hline 7576 & KCNRG & 1.52 & $2.54 \mathrm{E}-03$ & 7644 & L48878 & 3.71 & $1.29 \mathrm{E}-02$ & 7712 & LIX1 & 3.86 & $2.23 \mathrm{E}-03$ \\
\hline 7577 & KCNS1 & 5.52 & $8.18 \mathrm{E}-04$ & 7645 & L48880 & 4.71 & $1.40 \mathrm{E}-03$ & 7713 & LL & 5.43 & $5.75 \mathrm{E}-03$ \\
\hline 7578 & KCNS2 & 4.81 & $2.91 \mathrm{E}-03$ & 7646 & L48881 & 5.56 & $5.06 \mathrm{E}-05$ & 7714 & LMCD1 & 3.27 & $1.60 \mathrm{E}-02$ \\
\hline 7579 & KCNT1 & 2.65 & $2.36 \mathrm{E}-03$ & 7647 & L48884 & 6.84 & $5.65 \mathrm{E}-03$ & 7715 & LMNA & 1.83 & $3.21 \mathrm{E}-02$ \\
\hline 7580 & KCTD12 & 1.99 & $2.18 \mathrm{E}-03$ & 7648 & L48885 & 3.93 & $2.62 \mathrm{E}-03$ & 7716 & LMNB1 & 2.75 & $1.96 \mathrm{E}-03$ \\
\hline 7581 & KCTD17 & 3.57 & $8.16 \mathrm{E}-05$ & 7649 & L48886 & 4.16 & $8.01 \mathrm{E}-03$ & 7717 & LMO3 & 1.18 & $9.63 \mathrm{E}-02$ \\
\hline 7582 & KCTD21 & 5.08 & $7.27 \mathrm{E}-03$ & 7650 & L48890 & 3.29 & $9.35 \mathrm{E}-03$ & 7718 & LMOD2 & 2.25 & $4.92 \mathrm{E}-03$ \\
\hline 7583 & KCTD4 & 3.31 & $1.59 \mathrm{E}-03$ & 7651 & L48892 & 4.66 & $2.61 \mathrm{E}-03$ & 7719 & LMOD3 & 5.94 & $1.26 \mathrm{E}-04$ \\
\hline 7584 & KDELR3 & 1.66 & $1.53 \mathrm{E}-02$ & 7652 & L48893 & 3.76 & $1.85 \mathrm{E}-02$ & 7720 & LMX1A & 1.46 & $7.29 \mathrm{E}-02$ \\
\hline 7585 & KDM1B & 2.50 & $3.39 \mathrm{E}-03$ & 7653 & L48895 & 1.77 & $2.30 \mathrm{E}-03$ & 7721 & LNPEP & 2.09 & $4.38 \mathrm{E}-03$ \\
\hline 7586 & KDM4B & 3.51 & $3.87 \mathrm{E}-03$ & 7654 & L48898 & 4.41 & $2.18 \mathrm{E}-02$ & 7722 & LNX1 & 2.45 & $3.90 \mathrm{E}-03$ \\
\hline 7587 & KERA & 5.44 & $6.53 \mathrm{E}-04$ & 7655 & L48899 & 4.20 & $2.96 \mathrm{E}-04$ & 7723 & LNX2 & 2.74 & $1.50 \mathrm{E}-03$ \\
\hline 7588 & KIAA0195 & 2.24 & $5.97 \mathrm{E}-03$ & 7656 & L48900 & 6.62 & $3.71 \mathrm{E}-03$ & 7724 & LOC100857153 & 6.41 & $8.37 \mathrm{E}-04$ \\
\hline 7589 & KIAA0319 & 1.89 & $2.03 \mathrm{E}-02$ & 7657 & L48902 & 1.58 & $6.33 \mathrm{E}-03$ & 7725 & LOC100857386 & 5.36 & $2.81 \mathrm{E}-03$ \\
\hline 7590 & KIAA0889 & 2.91 & $3.12 \mathrm{E}-03$ & 7658 & L48903 & 10.37 & $1.12 \mathrm{E}-03$ & 7726 & LOC100857399 & 6.15 & $1.74 E-04$ \\
\hline 7591 & KIAA1024 & 3.37 & $1.27 \mathrm{E}-04$ & 7659 & L48908 & 4.33 & $5.78 \mathrm{E}-03$ & 7727 & LOC100857586 & 1.54 & $1.01 \mathrm{E}-02$ \\
\hline 7592 & KIAA1045 & 2.10 & $1.42 \mathrm{E}-02$ & 7660 & L48909 & 4.28 & $5.23 \mathrm{E}-03$ & 7728 & LOC100857703 & 1.46 & $2.85 \mathrm{E}-03$ \\
\hline 7593 & KIAA1147 & 3.43 & $1.72 \mathrm{E}-03$ & 7661 & L48912 & 4.32 & $4.10 \mathrm{E}-03$ & 7729 & LOC100858295 & 1.80 & $7.94 \mathrm{E}-03$ \\
\hline 7594 & KIAA1161 & 3.92 & $1.29 \mathrm{E}-04$ & 7662 & L48914 & 1.93 & 3.63E-03 & 7730 & LOC100858345 & 5.70 & $9.54 \mathrm{E}-05$ \\
\hline 7595 & KIAA1211 & 3.72 & $1.21 \mathrm{E}-02$ & 7663 & L48916 & 4.59 & $1.44 \mathrm{E}-02$ & 7731 & LOC100858701 & 4.38 & $1.17 \mathrm{E}-03$ \\
\hline 7596 & KIAA1217 & 1.06 & $2.08 \mathrm{E}-03$ & 7664 & L48917 & 2.75 & $8.60 \mathrm{E}-03$ & 7732 & LOC100858771 & 2.01 & $8.29 \mathrm{E}-02$ \\
\hline 7597 & KIAA1328 & 2.33 & $2.44 \mathrm{E}-02$ & 7665 & L48919 & 4.85 & $1.26 \mathrm{E}-03$ & 7733 & LOC100858837 & 6.39 & $1.20 \mathrm{E}-03$ \\
\hline 7598 & KIAA1409 & 2.37 & $1.34 \mathrm{E}-04$ & 7666 & L48923 & 4.12 & 3.17E-04 & 7734 & LOC100858914 & 3.53 & $1.74 \mathrm{E}-03$ \\
\hline 7599 & KIAA1598 & 1.66 & 3.33E-04 & 7667 & L48924 & 5.92 & $1.85 \mathrm{E}-04$ & 7735 & LOC100858942 & 5.23 & $3.05 \mathrm{E}-03$ \\
\hline 7600 & KIAA1841 & 1.70 & $3.36 \mathrm{E}-03$ & 7668 & LACC1 & 1.42 & $3.36 \mathrm{E}-01$ & 7736 & LOC100859006 & 2.86 & 4.17E-04 \\
\hline 7601 & KIAA1919 & 3.23 & $2.20 \mathrm{E}-03$ & 7669 & LAD1 & 1.98 & $1.82 \mathrm{E}-02$ & 7737 & LOC100859029 & 4.89 & $2.81 \mathrm{E}-03$ \\
\hline 7602 & KIAA1958 & 1.15 & $1.64 \mathrm{E}-02$ & 7670 & LAG3 & 1.43 & $8.93 \mathrm{E}-04$ & 7738 & LOC100859072 & 9.65 & $8.41 \mathrm{E}-04$ \\
\hline 7603 & KIAA2022 & 2.47 & $1.90 \mathrm{E}-02$ & 7671 & LAMA1 & 3.32 & $1.88 \mathrm{E}-05$ & 7739 & LOC100859161 & 2.06 & $2.21 \mathrm{E}-02$ \\
\hline 7604 & KIF21B & 5.48 & $1.16 \mathrm{E}-04$ & 7672 & LAMC2 & 5.89 & $2.42 \mathrm{E}-04$ & 7740 & LOC100859492 & 1.50 & $2.83 \mathrm{E}-02$ \\
\hline 7605 & $\mathrm{KIF} 26 \mathrm{~A}$ & 3.13 & $1.18 \mathrm{E}-03$ & 7673 & LAMC3 & 2.57 & $5.39 \mathrm{E}-03$ & 7741 & LOC100859665 & 2.44 & $1.70 \mathrm{E}-02$ \\
\hline 7606 & KIFC3 & 3.56 & $1.09 \mathrm{E}-02$ & 7674 & LAMP3 & 2.71 & $4.28 \mathrm{E}-02$ & 7742 & LOC100859696 & 2.21 & $1.09 \mathrm{E}-02$ \\
\hline 7607 & KITLG & 2.33 & $7.31 \mathrm{E}-03$ & 7675 & LANCL3 & 1.59 & $6.91 \mathrm{E}-03$ & 7743 & LOC100859709 & 3.95 & $3.63 \mathrm{E}-03$ \\
\hline 7608 & KK34 & 4.25 & $5.76 \mathrm{E}-03$ & 7676 & LAPTM4B & 2.99 & $7.18 \mathrm{E}-03$ & 7744 & LOC100859811 & 4.40 & $3.32 \mathrm{E}-04$ \\
\hline 7609 & $\mathrm{KL}$ & 3.71 & $4.92 \mathrm{E}-05$ & 7677 & LARGE & 2.48 & $1.08 \mathrm{E}-03$ & 7745 & LOC100859887 & 1.31 & $4.95 \mathrm{E}-02$ \\
\hline 7610 & KLF15 & 1.81 & $2.39 \mathrm{E}-04$ & 7678 & LAT2 & 3.86 & $9.80 \mathrm{E}-03$ & 7746 & LOC100859897 & 3.26 & $2.19 \mathrm{E}-02$ \\
\hline 7611 & KLF4 & 4.36 & $1.67 \mathrm{E}-03$ & 7679 & LBFABP & 2.21 & $4.10 \mathrm{E}-03$ & 7747 & LOC378902 & 2.37 & 9.97E-03 \\
\hline 7612 & KLF6 & 1.63 & $4.16 \mathrm{E}-03$ & 7680 & LBX1 & 4.95 & $1.58 \mathrm{E}-02$ & 7748 & LOC386576 & 2.79 & $3.18 \mathrm{E}-02$ \\
\hline 7613 & KLF7 & 1.41 & $2.70 \mathrm{E}-04$ & 7681 & LCK & 4.46 & $5.84 \mathrm{E}-04$ & 7749 & LOC395095 & 6.56 & 1.13E-02 \\
\hline 7614 & KLHDC10 & 2.91 & $1.57 \mathrm{E}-02$ & 7682 & LCN15 & 3.46 & $2.15 \mathrm{E}-03$ & 7750 & LOC395100 & 1.07 & $1.87 \mathrm{E}-02$ \\
\hline 7615 & KLHDC5 & 1.26 & $3.86 \mathrm{E}-03$ & 7683 & LCOR & 1.37 & $1.56 \mathrm{E}-02$ & 7751 & LOC395160 & 7.41 & $1.96 \mathrm{E}-03$ \\
\hline
\end{tabular}




\begin{tabular}{|c|c|c|c|c|c|c|c|c|c|c|c|}
\hline 7752 & LOC395325 & 4.10 & $1.66 \mathrm{E}-02$ & 7820 & LOC417848 & 1.67 & $1.52 \mathrm{E}-03$ & 7888 & LOC421270 & 4.86 & $1.85 \mathrm{E}-04$ \\
\hline 7753 & LOC395379 & 2.94 & $3.91 \mathrm{E}-03$ & 7821 & LOC417937 & 2.94 & $1.64 \mathrm{E}-05$ & 7889 & LOC421285 & 6.16 & 3.80E-04 \\
\hline 7754 & LOC395628 & 2.32 & $1.88 \mathrm{E}-03$ & 7822 & LOC417962 & 1.22 & $1.92 \mathrm{E}-02$ & 7890 & LOC421296 & 2.10 & $1.41 \mathrm{E}-02$ \\
\hline 7755 & LOC395772 & 2.77 & $3.87 \mathrm{E}-04$ & 7823 & LOC418020 & 1.14 & $1.19 \mathrm{E}-01$ & 7891 & LOC421394 & 4.98 & $9.28 \mathrm{E}-03$ \\
\hline 7756 & LOC395884 & 1.07 & $6.28 \mathrm{E}-03$ & 7824 & LOC418109 & 3.76 & $5.19 \mathrm{E}-03$ & 7892 & LOC421441 & 3.12 & $2.22 \mathrm{E}-03$ \\
\hline 7757 & LOC395892 & 2.08 & $3.59 \mathrm{E}-03$ & 7825 & LOC418168 & 3.91 & $9.88 \mathrm{E}-03$ & 7893 & LOC421512 & 4.04 & $2.36 \mathrm{E}-03$ \\
\hline 7758 & LOC395927 & 2.12 & $4.05 E-02$ & 7826 & LOC418298 & 2.57 & $1.60 \mathrm{E}-03$ & 7894 & LOC421515 & 2.09 & $6.18 \mathrm{E}-03$ \\
\hline 7759 & LOC395933 & 5.00 & $7.08 \mathrm{E}-03$ & 7827 & LOC418390 & 1.34 & $2.84 \mathrm{E}-02$ & 7895 & LOC421560 & 3.48 & $1.59 \mathrm{E}-03$ \\
\hline 7760 & LOC396098 & 3.11 & $1.14 \mathrm{E}-02$ & 7828 & LOC418414 & 3.64 & $6.24 \mathrm{E}-04$ & 7896 & LOC421675 & 4.26 & $1.23 \mathrm{E}-04$ \\
\hline 7761 & LOC396151 & 3.70 & 3.47E-03 & 7829 & LOC418416 & 6.04 & $1.22 \mathrm{E}-04$ & 7897 & LOC421690 & 1.66 & $5.22 \mathrm{E}-02$ \\
\hline 7762 & LOC396318 & 3.84 & $8.28 \mathrm{E}-02$ & 7830 & LOC418421 & 5.97 & $5.75 \mathrm{E}-03$ & 7898 & LOC421712 & 4.00 & $3.06 \mathrm{E}-03$ \\
\hline 7763 & LOC396365 & 3.91 & $1.05 \mathrm{E}-01$ & 7831 & LOC418423 & 5.46 & $1.78 \mathrm{E}-03$ & 7899 & LOC421740 & 5.18 & $8.75 E-05$ \\
\hline 7764 & LOC396419 & 6.03 & $1.66 \mathrm{E}-03$ & 7832 & LOC418424 & 2.52 & $3.14 \mathrm{E}-03$ & 7900 & LOC421956 & 4.14 & $1.17 \mathrm{E}-03$ \\
\hline 7765 & LOC396443 & 3.61 & $1.39 \mathrm{E}-03$ & 7833 & LOC418465 & 6.84 & $4.60 \mathrm{E}-05$ & 7901 & LOC421965 & 4.19 & $8.60 \mathrm{E}-04$ \\
\hline 7766 & LOC396454 & 4.70 & $2.05 \mathrm{E}-03$ & 7834 & LOC418543 & 1.19 & $6.33 \mathrm{E}-02$ & 7902 & LOC421982 & 1.55 & $1.85 \mathrm{E}-02$ \\
\hline 7767 & LOC396520 & 2.51 & $1.06 E-02$ & 7835 & LOC418554 & 4.39 & $1.13 \mathrm{E}-03$ & 7903 & LOC421996 & 3.70 & 7.40E-03 \\
\hline 7768 & LOC396525 & 1.83 & $1.67 \mathrm{E}-03$ & 7836 & LOC418707 & 4.00 & $8.36 \mathrm{E}-04$ & 7904 & LOC422039 & 5.58 & 1.09E-03 \\
\hline 7769 & LOC408038 & 2.75 & $1.60 \mathrm{E}-04$ & 7837 & LOC418771 & 4.26 & $5.15 \mathrm{E}-03$ & 7905 & LOC422046 & 4.85 & $1.54 \mathrm{E}-05$ \\
\hline 7770 & LOC415312 & 3.43 & $2.06 E-02$ & 7838 & LOC419016 & 3.93 & 3.41E-03 & 7906 & LOC422075 & 2.29 & $6.33 \mathrm{E}-04$ \\
\hline 7771 & LOC415314 & 5.91 & $3.57 \mathrm{E}-03$ & 7839 & LOC419032 & 2.41 & $4.52 \mathrm{E}-03$ & 7907 & LOC422147 & 4.40 & $1.77 \mathrm{E}-03$ \\
\hline 7772 & LOC415324 & 2.11 & $1.17 \mathrm{E}-03$ & 7840 & LOC419065 & 4.33 & $2.39 \mathrm{E}-04$ & 7908 & LOC422150 & 3.38 & $1.10 \mathrm{E}-03$ \\
\hline 7773 & LOC415325 & 5.48 & $1.99 \mathrm{E}-03$ & 7841 & LOC419113 & 4.68 & $5.46 \mathrm{E}-03$ & 7909 & LOC422171 & 3.55 & $6.35 \mathrm{E}-04$ \\
\hline 7774 & LOC415414 & 4.84 & $8.29 \mathrm{E}-04$ & 7842 & LOC419144 & 6.33 & $5.33 \mathrm{E}-04$ & 7910 & LOC422179 & 2.07 & $7.43 \mathrm{E}-03$ \\
\hline 7775 & LOC415418 & 3.61 & $1.16 \mathrm{E}-03$ & 7843 & LOC419182 & 1.68 & $5.61 \mathrm{E}-02$ & 7911 & LOC422264 & 3.05 & $6.80 \mathrm{E}-02$ \\
\hline 7776 & LOC415472 & 3.31 & $5.57 \mathrm{E}-04$ & 7844 & LOC419250 & 3.39 & $1.06 \mathrm{E}-02$ & 7912 & LOC422276 & 5.07 & $2.56 \mathrm{E}-03$ \\
\hline 7777 & LOC415554 & 1.36 & $2.50 \mathrm{E}-02$ & 7845 & LOC419328 & 2.70 & $5.96 \mathrm{E}-03$ & 7913 & LOC422284 & 2.14 & $1.40 \mathrm{E}-02$ \\
\hline 7778 & LOC415661 & 2.98 & $2.93 \mathrm{E}-04$ & 7846 & LOC419333 & 4.31 & $8.95 \mathrm{E}-03$ & 7914 & LOC422298 & 1.89 & $6.04 \mathrm{E}-03$ \\
\hline 7779 & LOC415671 & 2.48 & $4.61 \mathrm{E}-03$ & 7847 & LOC419335 & 6.05 & $9.06 \mathrm{E}-04$ & 7915 & LOC422307 & 6.68 & 4.11E-04 \\
\hline 7780 & LOC415713 & 4.40 & $2.58 \mathrm{E}-04$ & 7848 & LOC419389 & 2.56 & $7.44 \mathrm{E}-03$ & 7916 & LOC422308 & 2.05 & $1.71 \mathrm{E}-02$ \\
\hline 7781 & LOC415836 & 3.83 & $6.03 E-03$ & 7849 & LOC419425 & 4.49 & $3.78 \mathrm{E}-05$ & 7917 & LOC422319 & 1.96 & $1.38 \mathrm{E}-02$ \\
\hline 7782 & LOC415844 & 2.82 & $3.58 \mathrm{E}-03$ & 7850 & LOC419429 & 1.54 & $1.35 \mathrm{E}-02$ & 7918 & LOC422323 & 3.14 & 5.99E-03 \\
\hline 7783 & LOC415852 & 3.33 & $5.68 \mathrm{E}-03$ & 7851 & LOC419540 & 1.74 & $1.08 \mathrm{E}-02$ & 7919 & LOC422340 & 3.84 & 5.47E-03 \\
\hline 7784 & LOC415922 & 2.08 & $5.14 \mathrm{E}-03$ & 7852 & LOC419677 & 4.62 & $2.38 \mathrm{E}-03$ & 7920 & LOC422393 & 6.46 & 3.44E-05 \\
\hline 7785 & LOC415932 & 3.71 & $5.17 \mathrm{E}-02$ & 7853 & LOC419726 & 3.28 & $2.61 \mathrm{E}-03$ & 7921 & LOC422511 & 3.49 & $2.62 \mathrm{E}-04$ \\
\hline 7786 & LOC416053 & 2.27 & $2.34 \mathrm{E}-02$ & 7854 & LOC419741 & 2.62 & $5.92 \mathrm{E}-03$ & 7922 & LOC422643 & 2.81 & $1.14 \mathrm{E}-02$ \\
\hline 7787 & LOC416082 & 2.16 & $4.73 \mathrm{E}-03$ & 7855 & LOC419749 & 3.02 & $1.88 \mathrm{E}-03$ & 7923 & LOC422654 & 1.49 & $7.23 E-03$ \\
\hline 7788 & LOC416086 & 3.95 & $3.04 E-04$ & 7856 & LOC419833 & 4.37 & $4.27 \mathrm{E}-03$ & 7924 & LOC422725 & 3.60 & $2.34 \mathrm{E}-03$ \\
\hline 7789 & LOC416090 & 6.89 & $8.24 \mathrm{E}-04$ & 7857 & LOC419834 & 4.53 & $1.18 \mathrm{E}-03$ & 7925 & LOC422737 & 9.30 & $1.81 \mathrm{E}-03$ \\
\hline 7790 & LOC416106 & 6.02 & $4.07 E-03$ & 7858 & LOC419840 & 1.66 & $7.63 \mathrm{E}-03$ & 7926 & LOC422811 & 4.46 & $3.56 \mathrm{E}-04$ \\
\hline 7791 & LOC416146 & 7.14 & $3.16 \mathrm{E}-03$ & 7859 & LOC419842 & 5.00 & $6.67 \mathrm{E}-04$ & 7927 & LOC422853 & 4.25 & $1.15 \mathrm{E}-04$ \\
\hline 7792 & LOC416169 & 3.65 & $3.16 \mathrm{E}-03$ & 7860 & LOC419888 & 2.41 & $1.67 \mathrm{E}-04$ & 7928 & LOC422872 & 4.63 & $6.40 \mathrm{E}-05$ \\
\hline 7793 & LOC416178 & 1.20 & $3.09 E-02$ & 7861 & LOC420039 & 5.58 & $3.33 \mathrm{E}-04$ & 7929 & LOC422924 & 3.92 & $7.06 \mathrm{E}-03$ \\
\hline 7794 & LOC416179 & 3.93 & $3.14 \mathrm{E}-03$ & 7862 & LOC420043 & 4.28 & $4.05 \mathrm{E}-03$ & 7930 & LOC422926 & 1.40 & $6.58 \mathrm{E}-02$ \\
\hline 7795 & LOC416197 & 4.26 & $3.25 \mathrm{E}-05$ & 7863 & LOC420052 & 6.57 & $1.09 \mathrm{E}-03$ & 7931 & LOC422929 & 2.78 & $2.27 \mathrm{E}-03$ \\
\hline 7796 & LOC416292 & 1.36 & 5.67E-04 & 7864 & LOC420093 & 5.71 & $1.88 \mathrm{E}-03$ & 7932 & LOC423247 & 1.44 & $1.35 \mathrm{E}-03$ \\
\hline 7797 & LOC416541 & 2.09 & $1.87 \mathrm{E}-03$ & 7865 & LOC420107 & 2.46 & $2.19 \mathrm{E}-03$ & 7933 & LOC423393 & 8.50 & $3.32 \mathrm{E}-03$ \\
\hline 7798 & LOC416629 & 1.27 & $1.22 \mathrm{E}-02$ & 7866 & LOC420111 & 2.99 & $2.10 \mathrm{E}-03$ & 7934 & LOC423478 & 3.18 & $9.24 \mathrm{E}-03$ \\
\hline 7799 & LOC416633 & 2.44 & $1.53 \mathrm{E}-02$ & 7867 & LOC420156 & 1.57 & $3.13 \mathrm{E}-03$ & 7935 & LOC423523 & 3.13 & $1.57 \mathrm{E}-02$ \\
\hline 7800 & LOC416655 & 2.72 & $9.12 \mathrm{E}-02$ & 7868 & LOC420160 & 2.03 & $2.69 \mathrm{E}-02$ & 7936 & LOC423605 & 4.38 & $1.29 \mathrm{E}-02$ \\
\hline 7801 & LOC416752 & 5.24 & $5.19 \mathrm{E}-03$ & 7869 & LOC420300 & 4.52 & $6.50 \mathrm{E}-04$ & 7937 & LOC423629 & 1.90 & 3.36E-02 \\
\hline 7802 & LOC416755 & 1.77 & $5.34 \mathrm{E}-03$ & 7870 & LOC420301 & 4.30 & $5.79 \mathrm{E}-05$ & 7938 & LOC423653 & 2.04 & $1.88 \mathrm{E}-02$ \\
\hline 7803 & LOC416923 & 1.82 & $3.78 \mathrm{E}-03$ & 7871 & LOC420352 & 2.63 & $1.62 \mathrm{E}-02$ & 7939 & LOC423719 & 4.00 & $1.98 \mathrm{E}-03$ \\
\hline 7804 & LOC416927 & 4.09 & $1.23 \mathrm{E}-03$ & 7872 & LOC420381 & 3.20 & $6.24 \mathrm{E}-03$ & 7940 & LOC423731 & 2.02 & 7.33E-02 \\
\hline 7805 & LOC416935 & 4.09 & $1.06 \mathrm{E}-03$ & 7873 & LOC420403 & 4.62 & $5.75 \mathrm{E}-04$ & 7941 & LOC423752 & 2.79 & 3.33E-05 \\
\hline 7806 & LOC416959 & 3.94 & $2.44 \mathrm{E}-02$ & 7874 & LOC420543 & 2.43 & $7.17 \mathrm{E}-04$ & 7942 & LOC423822 & 3.96 & $2.76 \mathrm{E}-03$ \\
\hline 7807 & LOC417013 & 1.33 & $2.29 \mathrm{E}-03$ & 7875 & LOC420609 & 1.71 & $7.36 \mathrm{E}-02$ & 7943 & LOC423891 & 2.83 & 2.60E-03 \\
\hline 7808 & LOC417028 & 6.50 & $1.28 \mathrm{E}-04$ & 7876 & LOC420770 & 2.41 & $8.02 \mathrm{E}-04$ & 7944 & LOC423904 & 8.83 & $1.02 E-03$ \\
\hline 7809 & LOC417094 & 2.42 & $1.87 \mathrm{E}-02$ & 7877 & LOC420789 & 4.05 & 4.47E-04 & 7945 & LOC423919 & 4.03 & 3.35E-03 \\
\hline 7810 & LOC417113 & 1.68 & $6.17 \mathrm{E}-03$ & 7878 & LOC421070 & 1.39 & $1.30 \mathrm{E}-02$ & 7946 & LOC423922 & 6.22 & $3.92 \mathrm{E}-03$ \\
\hline 7811 & LOC417130 & 4.44 & $6.70 \mathrm{E}-03$ & 7879 & LOC421081 & 6.50 & $1.22 \mathrm{E}-04$ & 7947 & LOC423938 & 4.21 & $9.75 E-04$ \\
\hline 7812 & LOC417142 & 3.12 & $9.16 \mathrm{E}-04$ & 7880 & LOC421091 & 6.09 & $2.72 \mathrm{E}-04$ & 7948 & LOC423943 & 2.94 & $1.60 \mathrm{E}-03$ \\
\hline 7813 & LOC417192 & 3.53 & $1.10 \mathrm{E}-03$ & 7881 & LOC421118 & 2.00 & $5.29 \mathrm{E}-02$ & 7949 & LOC424007 & 2.62 & $1.62 \mathrm{E}-03$ \\
\hline 7814 & LOC417291 & 4.45 & $1.06 \mathrm{E}-03$ & 7882 & LOC421123 & 5.02 & $3.72 \mathrm{E}-03$ & 7950 & LOC424033 & 1.41 & $2.39 \mathrm{E}-03$ \\
\hline 7815 & LOC417372 & 5.82 & $2.56 \mathrm{E}-03$ & 7883 & LOC421229 & 4.01 & $1.04 \mathrm{E}-04$ & 7951 & LOC424098 & 3.41 & $2.81 \mathrm{E}-04$ \\
\hline 7816 & LOC417447 & 1.72 & $1.60 \mathrm{E}-02$ & 7884 & LOC421235 & 2.12 & $7.43 \mathrm{E}-03$ & 7952 & LOC424199 & 4.32 & $1.37 \mathrm{E}-02$ \\
\hline 7817 & LOC417459 & 2.73 & $5.58 \mathrm{E}-03$ & 7885 & LOC421238 & 4.34 & $2.82 \mathrm{E}-03$ & 7953 & LOC424261 & 1.15 & $6.50 \mathrm{E}-03$ \\
\hline 7818 & LOC417507 & 1.64 & $1.97 \mathrm{E}-02$ & 7886 & LOC421241 & 1.45 & $1.09 \mathrm{E}-02$ & 7954 & LOC424265 & 2.16 & $1.12 \mathrm{E}-03$ \\
\hline 7819 & LOC417537 & 4.12 & $1.13 \mathrm{E}-02$ & 7887 & LOC421253 & 1.59 & $2.92 \mathrm{E}-02$ & 7955 & LOC424334 & 5.07 & $1.34 \mathrm{E}-03$ \\
\hline
\end{tabular}




\begin{tabular}{|c|c|c|c|c|c|c|c|c|c|c|c|}
\hline 7956 & LOC424428 & 3.35 & $1.07 E-02$ & 8024 & LOC427977 & 1.98 & 7.40E-03 & 8092 & LOC430475 & 8.35 & $1.65 \mathrm{E}-03$ \\
\hline 7957 & LOC424473 & 1.74 & $4.92 \mathrm{E}-02$ & 8025 & LOC428008 & 4.02 & $1.96 \mathrm{E}-03$ & 8093 & LOC430520 & 3.66 & $7.90 \mathrm{E}-03$ \\
\hline 7958 & LOC424511 & 4.14 & $7.17 \mathrm{E}-04$ & 8026 & LOC428024 & 5.12 & 3.31E-03 & 8094 & LOC430594 & 8.22 & $1.83 \mathrm{E}-04$ \\
\hline 7959 & LOC424523 & 5.24 & $5.31 \mathrm{E}-03$ & 8027 & LOC428073 & 4.19 & $1.83 \mathrm{E}-02$ & 8095 & LOC430596 & 8.63 & $2.61 \mathrm{E}-06$ \\
\hline 7960 & LOC424588 & 2.96 & $6.68 \mathrm{E}-04$ & 8028 & LOC428094 & 3.40 & $3.16 \mathrm{E}-03$ & 8096 & LOC430604 & 8.12 & $6.53 \mathrm{E}-04$ \\
\hline 7961 & LOC424593 & 6.92 & $4.71 \mathrm{E}-04$ & 8029 & LOC428111 & 1.20 & $3.59 \mathrm{E}-02$ & 8097 & LOC430610 & 4.30 & $1.62 \mathrm{E}-03$ \\
\hline 7962 & LOC424620 & 3.24 & $3.85 \mathrm{E}-02$ & 8030 & LOC428118 & 6.55 & $3.48 \mathrm{E}-03$ & 8098 & LOC430658 & 7.60 & $1.45 \mathrm{E}-04$ \\
\hline 7963 & LOC424679 & 5.46 & $1.96 \mathrm{E}-02$ & 8031 & LOC428119 & 4.95 & $3.05 E-03$ & 8099 & LOC430661 & 6.30 & $1.91 \mathrm{E}-04$ \\
\hline 7964 & LOC424892 & 2.34 & $2.78 \mathrm{E}-02$ & 8032 & LOC428144 & 4.41 & $7.18 \mathrm{E}-05$ & 8100 & LOC430677 & 7.84 & $5.55 \mathrm{E}-04$ \\
\hline 7965 & LOC424917 & 1.34 & $1.55 \mathrm{E}-02$ & 8033 & LOC428183 & 1.78 & $6.48 \mathrm{E}-02$ & 8101 & LOC430730 & 9.92 & $1.69 \mathrm{E}-03$ \\
\hline 7966 & LOC424919 & 1.22 & $1.20 \mathrm{E}-02$ & 8034 & LOC428212 & 5.31 & $3.19 \mathrm{E}-03$ & 8102 & LOC430735 & 3.46 & $3.91 \mathrm{E}-03$ \\
\hline 7967 & LOC424944 & 4.88 & $6.35 \mathrm{E}-03$ & 8035 & LOC428244 & 4.32 & $6.99 \mathrm{E}-03$ & 8103 & LOC430778 & 8.13 & $2.11 \mathrm{E}-03$ \\
\hline 7968 & LOC424998 & 5.80 & $5.14 \mathrm{E}-04$ & 8036 & LOC428258 & 1.75 & $2.33 \mathrm{E}-03$ & 8104 & LOC430792 & 8.24 & $6.31 E-05$ \\
\hline 7969 & LOC425076 & 7.71 & $3.15 \mathrm{E}-03$ & 8037 & LOC428278 & 3.24 & $4.83 \mathrm{E}-03$ & 8105 & LOC430804 & 9.40 & $3.12 \mathrm{E}-04$ \\
\hline 7970 & LOC425113 & 3.75 & $8.19 \mathrm{E}-03$ & 8038 & LOC428289 & 4.34 & $1.60 \mathrm{E}-02$ & 8106 & LOC430853 & 9.19 & $2.69 \mathrm{E}-03$ \\
\hline 7971 & LOC425137 & 1.56 & $3.90 \mathrm{E}-02$ & 8039 & LOC428290 & 7.84 & $1.25 \mathrm{E}-04$ & 8107 & LOC430861 & 8.24 & $9.25 \mathrm{E}-04$ \\
\hline 7972 & LOC425224 & 2.06 & $9.65 \mathrm{E}-03$ & 8040 & LOC428291 & 7.06 & $7.41 \mathrm{E}-04$ & 8108 & LOC430862 & 4.85 & $3.23 \mathrm{E}-03$ \\
\hline 7973 & LOC425238 & 5.13 & $4.33 \mathrm{E}-03$ & 8041 & LOC428322 & 6.57 & 2.14E-05 & 8109 & LOC430987 & 6.75 & $3.44 \mathrm{E}-03$ \\
\hline 7974 & LOC425261 & 3.64 & $3.47 \mathrm{E}-04$ & 8042 & LOC428323 & 2.45 & $1.49 \mathrm{E}-02$ & 8110 & LOC431045 & 3.52 & $1.04 \mathrm{E}-01$ \\
\hline 7975 & LOC425299 & 2.71 & 3.43E-03 & 8043 & LOC428383 & 5.73 & $1.54 \mathrm{E}-02$ & 8111 & LOC431090 & 4.24 & $1.81 \mathrm{E}-03$ \\
\hline 7976 & LOC425347 & 2.41 & $4.31 \mathrm{E}-03$ & 8044 & LOC428538 & 3.10 & 4.64E-03 & 8112 & LOC431147 & 5.87 & $1.69 \mathrm{E}-04$ \\
\hline 7977 & LOC425462 & 3.79 & $9.87 \mathrm{E}-04$ & 8045 & LOC428541 & 1.27 & 4.18E-02 & 8113 & LOC431205 & 6.71 & $1.21 \mathrm{E}-03$ \\
\hline 7978 & LOC425534 & 3.65 & $2.77 \mathrm{E}-03$ & 8046 & LOC428618 & 1.21 & $2.78 \mathrm{E}-03$ & 8114 & LOC431250 & 5.08 & $2.51 \mathrm{E}-04$ \\
\hline 7979 & LOC425540 & 3.26 & $3.18 \mathrm{E}-02$ & 8047 & LOC428693 & 5.10 & $8.85 \mathrm{E}-05$ & 8115 & LOC431251 & 3.65 & $2.80 \mathrm{E}-04$ \\
\hline 7980 & LOC425562 & 2.31 & $9.47 \mathrm{E}-03$ & 8048 & LOC428714 & 3.75 & $1.19 \mathrm{E}-03$ & 8116 & LOC431270 & 8.89 & $4.00 \mathrm{E}-03$ \\
\hline 7981 & LOC425725 & 3.02 & $5.11 \mathrm{E}-03$ & 8049 & LOC428720 & 2.88 & $1.36 \mathrm{E}-02$ & 8117 & LOC431320 & 9.37 & $2.10 \mathrm{E}-03$ \\
\hline 7982 & LOC425742 & 4.78 & $1.10 \mathrm{E}-04$ & 8050 & LOC428812 & 3.02 & $1.37 \mathrm{E}-03$ & 8118 & LOC431321 & 5.53 & $4.22 \mathrm{E}-04$ \\
\hline 7983 & LOC425757 & 5.26 & $2.99 \mathrm{E}-04$ & 8051 & LOC428815 & 6.02 & $5.77 \mathrm{E}-04$ & 8119 & LOC431323 & 3.62 & $8.16 \mathrm{E}-03$ \\
\hline 7984 & LOC425795 & 3.16 & $3.10 \mathrm{E}-03$ & 8052 & LOC428824 & 1.70 & $6.75 \mathrm{E}-03$ & 8120 & LOC431324 & 7.61 & $6.95 \mathrm{E}-04$ \\
\hline 7985 & LOC426037 & 7.40 & $8.86 \mathrm{E}-05$ & 8053 & LOC428827 & 6.64 & $1.14 \mathrm{E}-03$ & 8121 & LOC431350 & 7.64 & $5.42 \mathrm{E}-04$ \\
\hline 7986 & LOC426062 & 8.36 & $3.18 \mathrm{E}-03$ & 8054 & LOC428867 & 4.92 & $3.84 \mathrm{E}-03$ & 8122 & LOC431422 & 8.64 & $5.12 \mathrm{E}-04$ \\
\hline 7987 & LOC426070 & 5.25 & $3.80 \mathrm{E}-03$ & 8055 & LOC428949 & 6.57 & $8.38 \mathrm{E}-04$ & 8123 & LOC431525 & 5.87 & $2.30 \mathrm{E}-03$ \\
\hline 7988 & LOC426093 & 3.28 & $2.24 \mathrm{E}-02$ & 8056 & LOC428958 & 5.69 & $4.15 \mathrm{E}-03$ & 8124 & LOC431615 & 4.18 & $6.90 \mathrm{E}-05$ \\
\hline 7989 & LOC426218 & 4.48 & $9.79 \mathrm{E}-05$ & 8057 & LOC428961 & 3.31 & $7.43 E-04$ & 8125 & LOC431653 & 4.91 & $9.88 \mathrm{E}-03$ \\
\hline 7990 & LOC426220 & 2.89 & $1.90 \mathrm{E}-02$ & 8058 & LOC429032 & 4.91 & $7.58 \mathrm{E}-05$ & 8126 & LOC431656 & 2.42 & $8.41 \mathrm{E}-03$ \\
\hline 7991 & LOC426229 & 8.74 & $1.75 \mathrm{E}-03$ & 8059 & LOC429098 & 6.48 & $5.76 \mathrm{E}-03$ & 8127 & LOC431660 & 1.85 & $5.62 \mathrm{E}-03$ \\
\hline 7992 & LOC426324 & 2.09 & $4.57 \mathrm{E}-03$ & 8060 & LOC429152 & 5.37 & $8.98 \mathrm{E}-04$ & 8128 & LOC693258 & 4.06 & $2.68 \mathrm{E}-03$ \\
\hline 7993 & LOC426330 & 7.91 & $1.63 \mathrm{E}-03$ & 8061 & LOC429153 & 4.22 & $1.31 \mathrm{E}-02$ & 8129 & LOC768350 & 5.56 & $8.03 \mathrm{E}-02$ \\
\hline 7994 & LOC426456 & 4.66 & $1.28 \mathrm{E}-03$ & 8062 & LOC429244 & 8.85 & $2.82 \mathrm{E}-04$ & 8130 & LOC768484 & 5.92 & $5.52 \mathrm{E}-04$ \\
\hline 7995 & LOC426640 & 4.42 & $3.64 \mathrm{E}-04$ & 8063 & LOC429266 & 5.32 & $5.55 \mathrm{E}-03$ & 8131 & LOC768491 & 3.52 & $3.03 \mathrm{E}-03$ \\
\hline 7996 & LOC426731 & 4.58 & $5.21 \mathrm{E}-03$ & 8064 & LOC429288 & 8.45 & $3.39 \mathrm{E}-03$ & 8132 & LOC768535 & 3.89 & $1.43 \mathrm{E}-03$ \\
\hline 7997 & LOC426743 & 4.21 & $1.50 \mathrm{E}-03$ & 8065 & LOC429329 & 7.33 & $1.46 \mathrm{E}-04$ & 8133 & LOC768537 & 3.98 & $5.58 \mathrm{E}-03$ \\
\hline 7998 & LOC426821 & 5.18 & $3.79 \mathrm{E}-03$ & 8066 & LOC429348 & 2.69 & 3.45E-03 & 8134 & LOC768596 & 5.09 & $3.11 \mathrm{E}-03$ \\
\hline 7999 & LOC426834 & 6.45 & $8.88 \mathrm{E}-05$ & 8067 & LOC429356 & 8.49 & $6.12 \mathrm{E}-04$ & 8135 & LOC768634 & 1.58 & $6.31 \mathrm{E}-03$ \\
\hline 8000 & LOC426881 & 5.33 & $3.70 \mathrm{E}-04$ & 8068 & LOC429395 & 8.41 & $2.16 \mathrm{E}-04$ & 8136 & LOC768665 & 4.96 & $4.00 \mathrm{E}-03$ \\
\hline 8001 & LOC426897 & 2.40 & $9.06 \mathrm{E}-03$ & 8069 & LOC429440 & 3.27 & $2.66 \mathrm{E}-03$ & 8137 & LOC768676 & 3.12 & $3.86 \mathrm{E}-03$ \\
\hline 8002 & LOC426902 & 2.98 & $8.71 \mathrm{E}-04$ & 8070 & LOC429451 & 2.24 & $4.58 \mathrm{E}-02$ & 8138 & LOC768768 & 2.64 & $2.96 \mathrm{E}-03$ \\
\hline 8003 & LOC426913 & 7.30 & $1.08 \mathrm{E}-03$ & 8071 & LOC429492 & 5.85 & $7.92 \mathrm{E}-04$ & 8139 & LOC768786 & 2.52 & $7.92 \mathrm{E}-03$ \\
\hline 8004 & LOC426957 & 6.30 & $9.95 \mathrm{E}-04$ & 8072 & LOC429522 & 6.14 & $2.04 \mathrm{E}-03$ & 8140 & LOC768846 & 8.19 & $9.00 \mathrm{E}-03$ \\
\hline 8005 & LOC427027 & 7.63 & $1.25 \mathrm{E}-03$ & 8073 & LOC429532 & 8.36 & $2.03 E-05$ & 8141 & LOC768941 & 6.59 & $1.23 \mathrm{E}-03$ \\
\hline 8006 & LOC427029 & 2.55 & $8.92 \mathrm{E}-03$ & 8074 & LOC429558 & 1.38 & $4.18 \mathrm{E}-04$ & 8142 & LOC768958 & 6.20 & $3.26 \mathrm{E}-03$ \\
\hline 8007 & LOC427221 & 4.69 & $6.00 \mathrm{E}-04$ & 8075 & LOC429636 & 8.32 & $2.05 \mathrm{E}-04$ & 8143 & LOC768967 & 7.47 & $6.71 \mathrm{E}-04$ \\
\hline 8008 & LOC427240 & 4.24 & $4.99 \mathrm{E}-03$ & 8076 & LOC429670 & 8.02 & $3.64 \mathrm{E}-03$ & 8144 & LOC768983 & 5.33 & $6.90 \mathrm{E}-04$ \\
\hline 8009 & LOC427292 & 3.20 & $8.22 \mathrm{E}-02$ & 8077 & LOC429682 & 3.64 & $4.34 \mathrm{E}-04$ & 8145 & LOC769002 & 4.21 & $9.29 \mathrm{E}-04$ \\
\hline 8010 & LOC427362 & 2.91 & $1.49 \mathrm{E}-03$ & 8078 & LOC429693 & 3.45 & $3.04 \mathrm{E}-03$ & 8146 & LOC769103 & 7.54 & $1.26 \mathrm{E}-02$ \\
\hline 8011 & LOC427400 & 3.58 & $2.06 \mathrm{E}-03$ & 8079 & LOC429721 & 8.22 & $5.98 \mathrm{E}-05$ & 8147 & LOC769114 & 5.79 & $4.06 \mathrm{E}-05$ \\
\hline 8012 & LOC427470 & 2.06 & $5.34 \mathrm{E}-03$ & 8080 & LOC429798 & 7.69 & $1.46 \mathrm{E}-04$ & 8148 & LOC769139 & 5.18 & $9.86 \mathrm{E}-05$ \\
\hline 8013 & LOC427522 & 3.89 & $9.28 \mathrm{E}-04$ & 8081 & LOC429800 & 5.47 & $1.28 \mathrm{E}-03$ & 8149 & LOC769174 & 4.76 & $2.24 \mathrm{E}-03$ \\
\hline 8014 & LOC427545 & 1.85 & $4.71 \mathrm{E}-03$ & 8082 & LOC429812 & 7.90 & $4.33 \mathrm{E}-03$ & 8150 & LOC769207 & 4.38 & $6.70 \mathrm{E}-03$ \\
\hline 8015 & LOC427547 & 1.31 & $1.71 \mathrm{E}-02$ & 8083 & LOC429893 & 8.00 & $5.28 \mathrm{E}-04$ & 8151 & LOC769214 & 6.01 & $5.89 \mathrm{E}-03$ \\
\hline 8016 & LOC427665 & 4.18 & $9.76 \mathrm{E}-03$ & 8084 & LOC429924 & 8.05 & $5.76 \mathrm{E}-04$ & 8152 & LOC769226 & 6.40 & $3.76 \mathrm{E}-03$ \\
\hline 8017 & LOC427700 & 2.00 & $2.24 \mathrm{E}-01$ & 8085 & LOC429936 & 6.26 & $2.58 \mathrm{E}-05$ & 8153 & LOC769232 & 4.45 & $2.43 \mathrm{E}-03$ \\
\hline 8018 & LOC427773 & 4.06 & $2.18 \mathrm{E}-02$ & 8086 & LOC429973 & 8.52 & $2.08 \mathrm{E}-03$ & 8154 & LOC769242 & 4.36 & $4.22 \mathrm{E}-03$ \\
\hline 8019 & LOC427775 & 4.93 & $1.05 E-03$ & 8087 & LOC430014 & 3.71 & $9.46 \mathrm{E}-03$ & 8155 & LOC769251 & 1.66 & $2.28 \mathrm{E}-02$ \\
\hline 8020 & LOC427799 & 2.87 & $9.02 \mathrm{E}-03$ & 8088 & LOC430062 & 5.96 & $1.66 \mathrm{E}-03$ & 8156 & LOC769267 & 6.56 & $2.15 \mathrm{E}-03$ \\
\hline 8021 & LOC427816 & 3.79 & $3.34 \mathrm{E}-03$ & 8089 & LOC430228 & 8.21 & $3.09 \mathrm{E}-04$ & 8157 & LOC769270 & 3.57 & $2.46 \mathrm{E}-03$ \\
\hline 8022 & LOC427923 & 3.89 & $1.90 \mathrm{E}-03$ & 8090 & LOC430334 & 6.90 & $6.07 \mathrm{E}-04$ & 8158 & LOC769357 & 3.29 & $3.21 \mathrm{E}-04$ \\
\hline 8023 & LOC427948 & 5.24 & $4.48 \mathrm{E}-03$ & 8091 & LOC430434 & 9.63 & $1.87 \mathrm{E}-04$ & 8159 & LOC769366 & 5.45 & $1.27 \mathrm{E}-03$ \\
\hline
\end{tabular}




\begin{tabular}{|c|c|c|c|c|c|c|c|c|c|c|c|}
\hline 8160 & LOC769371 & 3.92 & $4.10 \mathrm{E}-03$ & 8228 & LOC771422 & 4.41 & $1.66 \mathrm{E}-03$ & 8296 & LRP2 & 3.15 & $1.20 \mathrm{E}-03$ \\
\hline 8161 & LOC769416 & 8.21 & 5.57E-04 & 8229 & LOC771437 & 5.70 & $3.24 \mathrm{E}-04$ & 8297 & LRP3 & 1.46 & $2.99 \mathrm{E}-02$ \\
\hline 8162 & LOC769517 & 5.45 & $2.31 \mathrm{E}-03$ & 8230 & LOC771494 & 3.90 & $1.40 \mathrm{E}-03$ & 8298 & LRP4 & 2.46 & $1.23 \mathrm{E}-03$ \\
\hline 8163 & LOC769577 & 3.59 & $1.09 \mathrm{E}-03$ & 8231 & LOC771545 & 2.70 & $3.67 \mathrm{E}-03$ & 8299 & LRRC10 & 4.58 & $3.46 E-02$ \\
\hline 8164 & LOC769590 & 2.06 & $1.12 \mathrm{E}-01$ & 8232 & LOC771573 & 4.66 & $2.09 \mathrm{E}-04$ & 8300 & LRRC10B & 1.03 & $1.27 \mathrm{E}-03$ \\
\hline 8165 & LOC769608 & 3.54 & $7.62 E-03$ & 8233 & LOC771598 & 4.70 & $9.55 \mathrm{E}-03$ & 8301 & LRRC14B & 4.56 & $3.76 \mathrm{E}-03$ \\
\hline 8166 & LOC769670 & 2.61 & $1.42 \mathrm{E}-02$ & 8234 & LOC771607 & 3.12 & $6.91 \mathrm{E}-03$ & 8302 & LRRC15 & 5.70 & $4.35 \mathrm{E}-03$ \\
\hline 8167 & LOC769676 & 2.55 & $1.84 \mathrm{E}-02$ & 8235 & LOC771612 & 3.73 & $1.34 \mathrm{E}-03$ & 8303 & LRRC17 & 1.81 & 3.70E-02 \\
\hline 8168 & LOC769726 & 3.21 & $5.17 \mathrm{E}-05$ & 8236 & LOC771647 & 3.15 & $2.57 \mathrm{E}-04$ & 8304 & LRRC2 & 5.21 & $1.85 \mathrm{E}-03$ \\
\hline 8169 & LOC769731 & 3.81 & $1.93 \mathrm{E}-03$ & 8237 & LOC771657 & 3.65 & $4.75 \mathrm{E}-03$ & 8305 & LRRC26 & 4.79 & 2.59E-03 \\
\hline 8170 & LOC769741 & 5.66 & $2.34 \mathrm{E}-03$ & 8238 & LOC771758 & 3.85 & $1.55 \mathrm{E}-03$ & 8306 & LRRC30 & 3.70 & $4.86 \mathrm{E}-04$ \\
\hline 8171 & LOC769749 & 4.11 & $1.21 \mathrm{E}-02$ & 8239 & LOC771761 & 4.85 & $1.64 \mathrm{E}-02$ & 8307 & LRRC32 & 3.28 & $2.56 \mathrm{E}-03$ \\
\hline 8172 & LOC769834 & 5.42 & $2.55 \mathrm{E}-04$ & 8240 & LOC771782 & 3.51 & $1.41 \mathrm{E}-02$ & 8308 & LRRC33 & 4.24 & $6.97 \mathrm{E}-02$ \\
\hline 8173 & LOC769837 & 3.02 & $4.34 \mathrm{E}-03$ & 8241 & LOC771856 & 4.75 & $3.58 \mathrm{E}-02$ & 8309 & LRRC38 & 1.61 & $9.20 \mathrm{E}-04$ \\
\hline 8174 & LOC769841 & 6.43 & $4.76 \mathrm{E}-04$ & 8242 & LOC771877 & 1.45 & $7.65 \mathrm{E}-02$ & 8310 & LRRC39 & 4.94 & 2.14E-04 \\
\hline 8175 & LOC769869 & 5.35 & $2.14 \mathrm{E}-04$ & 8243 & LOC771911 & 4.34 & $6.98 \mathrm{E}-03$ & 8311 & LRRC41 & 2.23 & $1.10 \mathrm{E}-02$ \\
\hline 8176 & LOC769885 & 1.57 & $1.19 \mathrm{E}-02$ & 8244 & LOC771972 & 4.78 & $1.75 \mathrm{E}-03$ & 8312 & LRRC58 & 1.73 & 4.11E-03 \\
\hline 8177 & LOC770026 & 5.37 & $2.19 \mathrm{E}-03$ & 8245 & LOC771995 & 3.15 & $1.97 \mathrm{E}-03$ & 8313 & LRRC8A & 4.68 & $2.44 \mathrm{E}-03$ \\
\hline 8178 & LOC770117 & 9.12 & $4.14 \mathrm{E}-04$ & 8246 & LOC772055 & 2.27 & 7.09E-03 & 8314 & LRRK1 & 1.79 & $6.54 \mathrm{E}-03$ \\
\hline 8179 & LOC770119 & 1.62 & $3.47 \mathrm{E}-03$ & 8247 & LOC772080 & 4.07 & $9.71 \mathrm{E}-04$ & 8315 & LRRN1 & 2.56 & $1.83 \mathrm{E}-03$ \\
\hline 8180 & LOC770127 & 2.10 & $6.03 \mathrm{E}-03$ & 8248 & LOC772096 & 3.60 & $2.20 \mathrm{E}-04$ & 8316 & LRRN2 & 7.55 & $1.50 \mathrm{E}-03$ \\
\hline 8181 & LOC770154 & 3.44 & $2.80 \mathrm{E}-03$ & 8249 & LOC772117 & 4.85 & $2.51 \mathrm{E}-03$ & 8317 & LRRN3 & 2.88 & $4.77 E-03$ \\
\hline 8182 & LOC770184 & 2.83 & $1.36 \mathrm{E}-02$ & 8250 & LOC772190 & 1.41 & $4.53 \mathrm{E}-03$ & 8318 & LRRTM1 & 2.24 & $4.90 \mathrm{E}-02$ \\
\hline 8183 & LOC770201 & 2.86 & $1.12 \mathrm{E}-03$ & 8251 & LOC772198 & 3.00 & $3.67 \mathrm{E}-03$ & 8319 & LRRTM3 & 3.84 & 2.06E-04 \\
\hline 8184 & LOC770206 & 2.85 & $6.95 \mathrm{E}-03$ & 8252 & LOC772212 & 4.70 & $1.48 \mathrm{E}-03$ & 8320 & LRRTM4 & 5.09 & 2.15E-05 \\
\hline 8185 & LOC770260 & 2.10 & $2.25 \mathrm{E}-02$ & 8253 & LOC772218 & 1.25 & $2.82 \mathrm{E}-03$ & 8321 & LSP1 & 1.32 & $5.05 E-03$ \\
\hline 8186 & LOC770265 & 1.58 & $1.52 \mathrm{E}-02$ & 8254 & LOC772304 & 8.50 & $6.39 \mathrm{E}-05$ & 8322 & LTB4R & 2.26 & 7.77E-04 \\
\hline 8187 & LOC770282 & 8.80 & $2.70 \mathrm{E}-04$ & 8255 & LOC772391 & 1.73 & $1.30 \mathrm{E}-02$ & 8323 & LTBP2 & 2.34 & $3.71 \mathrm{E}-02$ \\
\hline 8188 & LOC770284 & 4.04 & $1.31 E-02$ & 8256 & LOC776018 & 5.76 & $1.00 \mathrm{E}-03$ & 8324 & LTF & 1.58 & $4.42 \mathrm{E}-04$ \\
\hline 8189 & LOC770295 & 4.78 & $1.12 \mathrm{E}-03$ & 8257 & LOC776174 & 7.19 & $1.74 \mathrm{E}-03$ & 8325 & LY86 & 2.29 & 1.17E-03 \\
\hline 8190 & LOC770305 & 1.99 & $2.72 \mathrm{E}-02$ & 8258 & LOC776232 & 6.33 & $2.84 \mathrm{E}-02$ & 8326 & LY96 & 3.79 & $4.78 \mathrm{E}-03$ \\
\hline 8191 & LOC770309 & 2.92 & 3.77E-04 & 8259 & LOC776236 & 8.41 & $8.91 \mathrm{E}-03$ & 8327 & LYG2 & 3.50 & $2.56 \mathrm{E}-03$ \\
\hline 8192 & LOC770340 & 3.28 & $3.31 \mathrm{E}-04$ & 8260 & LOC776271 & 2.30 & $6.11 \mathrm{E}-04$ & 8328 & LYPD2 & 1.57 & $4.22 \mathrm{E}-03$ \\
\hline 8193 & LOC770352 & 1.81 & $2.82 \mathrm{E}-02$ & 8261 & LOC776507 & 3.12 & $6.82 \mathrm{E}-04$ & 8329 & LYSMD4 & 1.20 & $8.78 \mathrm{E}-03$ \\
\hline 8194 & LOC770371 & 1.98 & $8.51 \mathrm{E}-03$ & 8262 & LOC776568 & 8.65 & $1.18 \mathrm{E}-02$ & 8330 & LZTS1 & 4.55 & 1.13E-05 \\
\hline 8195 & LOC770392 & 3.65 & $8.39 \mathrm{E}-03$ & 8263 & LOC776703 & 7.71 & $3.39 \mathrm{E}-04$ & 8331 & LZTS2 & 3.29 & $6.48 \mathrm{E}-03$ \\
\hline 8196 & LOC770446 & 3.53 & $1.48 \mathrm{E}-02$ & 8264 & LOC776799 & 2.92 & $2.07 E-02$ & 8332 & M24367 & 5.31 & $3.72 \mathrm{E}-03$ \\
\hline 8197 & LOC770458 & 3.49 & $3.04 E-04$ & 8265 & LOC776865 & 4.79 & $5.40 \mathrm{E}-02$ & 8333 & M27668 & 6.66 & 3.89E-02 \\
\hline 8198 & LOC770503 & 4.06 & $2.14 \mathrm{E}-03$ & 8266 & LOC777021 & 8.44 & $5.33 \mathrm{E}-03$ & 8334 & M30347 & 6.86 & $3.26 \mathrm{E}-03$ \\
\hline 8199 & LOC770506 & 7.49 & $5.13 \mathrm{E}-05$ & 8267 & LOC777022 & 8.11 & $3.33 \mathrm{E}-04$ & 8335 & M31063 & 4.67 & 4.29E-04 \\
\hline 8200 & LOC770512 & 3.60 & $5.84 \mathrm{E}-03$ & 8268 & LOC777032 & 6.10 & $1.19 \mathrm{E}-04$ & 8336 & M31065 & 4.50 & $1.97 \mathrm{E}-05$ \\
\hline 8201 & LOC770548 & 5.16 & $8.25 \mathrm{E}-03$ & 8269 & LOC777037 & 6.11 & $6.97 \mathrm{E}-04$ & 8337 & M31105 & 1.59 & 4.07E-02 \\
\hline 8202 & LOC770619 & 2.91 & $7.78 \mathrm{E}-03$ & 8270 & LOC777039 & 6.04 & $5.43 \mathrm{E}-02$ & 8338 & M32731 & 5.63 & $3.87 \mathrm{E}-03$ \\
\hline 8203 & LOC770639 & 9.92 & $4.80 E-04$ & 8271 & LOC777052 & 7.90 & $2.36 \mathrm{E}-04$ & 8339 & M35195 & 3.00 & 1.17E-02 \\
\hline 8204 & LOC770649 & 3.76 & $4.83 \mathrm{E}-03$ & 8272 & LOC777125 & 7.04 & $8.88 \mathrm{E}-04$ & 8340 & M63068 & 5.56 & $6.38 \mathrm{E}-03$ \\
\hline 8205 & LOC770711 & 1.19 & $7.24 \mathrm{E}-03$ & 8273 & LOC777210 & 9.50 & $6.92 \mathrm{E}-04$ & 8341 & M63081 & 3.46 & $9.08 \mathrm{E}-03$ \\
\hline 8206 & LOC770718 & 2.45 & $6.87 \mathrm{E}-03$ & 8274 & LOC777291 & 8.62 & $1.26 \mathrm{E}-04$ & 8342 & M81150 & 4.20 & $2.66 \mathrm{E}-03$ \\
\hline 8207 & LOC770723 & 8.21 & $3.24 E-04$ & 8275 & LOC777386 & 5.91 & $2.02 \mathrm{E}-03$ & 8343 & M88280 & 3.31 & $1.15 \mathrm{E}-03$ \\
\hline 8208 & LOC770745 & 3.89 & $9.64 \mathrm{E}-05$ & 8276 & LOC777408 & 7.66 & $6.24 \mathrm{E}-04$ & 8344 & MAB21L1 & 6.57 & $5.25 \mathrm{E}-03$ \\
\hline 8209 & LOC770802 & 2.58 & $1.35 \mathrm{E}-02$ & 8277 & LOX & 3.78 & $2.08 \mathrm{E}-03$ & 8345 & MAB21L2 & 5.40 & 4.49E-03 \\
\hline 8210 & LOC770833 & 6.25 & $9.90 \mathrm{E}-04$ & 8278 & LOXL3 & 3.75 & $2.72 \mathrm{E}-03$ & 8346 & MAB21L3 & 2.94 & $7.70 \mathrm{E}-03$ \\
\hline 8211 & LOC770881 & 3.78 & $8.69 E-03$ & 8279 & LOXL4 & 3.02 & $1.11 \mathrm{E}-02$ & 8347 & MACC1 & 5.84 & $3.24 \mathrm{E}-03$ \\
\hline 8212 & LOC770890 & 3.14 & $4.65 \mathrm{E}-02$ & 8280 & LPAR3 & 2.07 & $5.59 \mathrm{E}-06$ & 8348 & MACF1 & 3.86 & $1.00 \mathrm{E}-02$ \\
\hline 8213 & LOC770930 & 2.72 & $8.69 \mathrm{E}-04$ & 8281 & LPAR4 & 1.41 & $4.58 \mathrm{E}-03$ & 8349 & MADPRT & 7.51 & $6.74 \mathrm{E}-03$ \\
\hline 8214 & LOC770936 & 5.04 & $1.30 \mathrm{E}-03$ & 8282 & LPAR5 & 4.38 & $7.22 \mathrm{E}-03$ & 8350 & MAF & 2.02 & $3.42 \mathrm{E}-02$ \\
\hline 8215 & LOC770996 & 1.27 & $4.53 \mathrm{E}-02$ & 8283 & LPHN3 & 4.79 & $1.89 \mathrm{E}-03$ & 8351 & MAFA & 4.95 & 3.27E-04 \\
\hline 8216 & LOC771012 & 4.87 & $1.21 \mathrm{E}-03$ & 8284 & LPIN1 & 1.92 & $5.41 \mathrm{E}-03$ & 8352 & MAFB & 1.53 & $3.44 E-02$ \\
\hline 8217 & LOC771028 & 5.11 & $1.56 \mathrm{E}-04$ & 8285 & LPL & 3.50 & $6.57 \mathrm{E}-04$ & 8353 & MAFF & 3.80 & $2.72 \mathrm{E}-02$ \\
\hline 8218 & LOC771030 & 5.21 & $1.34 \mathrm{E}-02$ & 8286 & LPP & 2.09 & 4.97E-03 & 8354 & MAFG & 1.73 & $1.01 \mathrm{E}-01$ \\
\hline 8219 & LOC771085 & 4.51 & $1.75 \mathrm{E}-03$ & 8287 & LPPR4 & 6.03 & $1.13 \mathrm{E}-02$ & 8355 & MAGI1 & 2.64 & $1.69 \mathrm{E}-02$ \\
\hline 8220 & LOC771107 & 4.86 & $6.16 \mathrm{E}-03$ & 8288 & LPXN & 2.25 & $1.14 \mathrm{E}-01$ & 8356 & MAGI2 & 2.50 & $4.87 E-03$ \\
\hline 8221 & LOC771121 & 3.98 & $8.05 E-03$ & 8289 & LRAT & 3.20 & $1.53 \mathrm{E}-02$ & 8357 & MAL & 1.58 & $1.78 \mathrm{E}-02$ \\
\hline 8222 & LOC771141 & 4.14 & $2.06 \mathrm{E}-02$ & 8290 & LRCH3 & 2.21 & $5.26 \mathrm{E}-04$ & 8358 & MALL & 1.89 & $2.28 \mathrm{E}-03$ \\
\hline 8223 & LOC771171 & 4.09 & $6.13 \mathrm{E}-03$ & 8291 & LRFN2 & 6.46 & $1.55 \mathrm{E}-03$ & 8359 & MALT1 & 3.10 & $4.20 \mathrm{E}-03$ \\
\hline 8224 & LOC771268 & 2.34 & $9.09 \mathrm{E}-03$ & 8292 & LRFN5 & 1.93 & $3.16 \mathrm{E}-02$ & 8360 & MAML2 & 3.10 & $1.52 \mathrm{E}-02$ \\
\hline 8225 & LOC771321 & 4.58 & $2.29 \mathrm{E}-02$ & 8293 & LRIT1 & 5.04 & $2.71 \mathrm{E}-05$ & 8361 & MAML3 & 2.20 & $2.13 \mathrm{E}-03$ \\
\hline 8226 & LOC771349 & 2.40 & $1.40 \mathrm{E}-02$ & 8294 & LRP1 & 2.43 & $1.49 \mathrm{E}-03$ & 8362 & MAMLD1 & 2.17 & $3.04 \mathrm{E}-03$ \\
\hline 8227 & LOC771366 & 1.39 & $1.15 \mathrm{E}-02$ & 8295 & LRP1B & 1.18 & $2.32 \mathrm{E}-02$ & 8363 & MAN1A1 & 1.38 & $1.19 \mathrm{E}-03$ \\
\hline
\end{tabular}




\begin{tabular}{|c|c|c|c|c|c|c|c|c|c|c|c|}
\hline 8364 & MANEAL & 3.03 & $2.03 \mathrm{E}-03$ & 8432 & MLLT1 & 1.33 & $3.22 \mathrm{E}-03$ & 8500 & MYH6 & 2.13 & $1.38 \mathrm{E}-04$ \\
\hline 8365 & MAOB & 1.64 & $1.66 \mathrm{E}-02$ & 8433 & MLNR & 1.40 & $6.33 \mathrm{E}-03$ & 8501 & MYH7 & 3.16 & $8.05 E-03$ \\
\hline 8366 & МАРЗК14 & 1.57 & $9.73 \mathrm{E}-03$ & 8434 & MLPH & 5.03 & $8.81 \mathrm{E}-04$ & 8502 & MYL2 & 3.76 & 2.66E-04 \\
\hline 8367 & MAP3K5 & 3.28 & $1.33 \mathrm{E}-02$ & 8435 & MLXIPL & 5.48 & $3.90 \mathrm{E}-06$ & 8503 & MYLK4 & 4.13 & $1.72 \mathrm{E}-03$ \\
\hline 8368 & МАР3К8 & 3.02 & $1.19 \mathrm{E}-02$ & 8436 & MMD2 & 4.78 & $6.11 \mathrm{E}-04$ & 8504 & MYLPF & 3.91 & $8.16 \mathrm{E}-03$ \\
\hline 8369 & MAP4 & 3.12 & $7.19 \mathrm{E}-03$ & 8437 & MMEL1 & 4.16 & 7.45E-04 & 8505 & MYO16 & 2.36 & $5.22 \mathrm{E}-03$ \\
\hline 8370 & MAP6 & 2.33 & $8.22 \mathrm{E}-04$ & 8438 & MMP1 & 3.98 & $3.40 \mathrm{E}-03$ & 8506 & MYO18B & 1.69 & $1.67 \mathrm{E}-02$ \\
\hline 8371 & MAP7D1 & 4.21 & $1.11 \mathrm{E}-01$ & 8439 & MMP10 & 2.50 & $2.14 \mathrm{E}-02$ & 8507 & MYO1D & 1.78 & 2.55E-03 \\
\hline 8372 & MAPK10 & 3.62 & 9.03E-02 & 8440 & MMP14 & 4.15 & $4.50 \mathrm{E}-03$ & 8508 & MY01G & 2.59 & $1.50 \mathrm{E}-03$ \\
\hline 8373 & MAPK13 & 2.69 & $1.34 \mathrm{E}-02$ & 8441 & MMP16 & 2.25 & $2.01 \mathrm{E}-03$ & 8509 & MY03A & 3.42 & $4.52 \mathrm{E}-03$ \\
\hline 8374 & MAPK8 & 1.49 & $1.35 \mathrm{E}-02$ & 8442 & MMP17 & 2.71 & $2.20 \mathrm{E}-03$ & 8510 & MYO7A & 6.29 & $6.77 E-03$ \\
\hline 8375 & MARCO & 2.74 & $1.73 \mathrm{E}-02$ & 8443 & MMP27 & 3.68 & $1.14 \mathrm{E}-02$ & 8511 & MYOC & 1.46 & $1.12 \mathrm{E}-01$ \\
\hline 8376 & MARS & 2.58 & $2.63 \mathrm{E}-02$ & 8444 & MMP28 & 2.41 & $9.41 \mathrm{E}-04$ & 8512 & MYOCD & 3.11 & 3.87E-03 \\
\hline 8377 & MARVELD3 & 1.72 & 3.36E-03 & 8445 & MMP7 & 4.98 & $1.00 \mathrm{E}-04$ & 8513 & MYOD1 & 3.68 & $1.39 \mathrm{E}-02$ \\
\hline 8378 & MASP1 & 1.77 & $9.99 \mathrm{E}-02$ & 8446 & MMP9 & 1.64 & $8.81 \mathrm{E}-03$ & 8514 & MYOG & 3.76 & $1.68 \mathrm{E}-03$ \\
\hline 8379 & MAST3 & 1.80 & $1.27 \mathrm{E}-02$ & 8447 & MMRN1 & 1.98 & 4.73E-02 & 8515 & MYOM1 & 1.33 & $1.99 \mathrm{E}-02$ \\
\hline 8380 & MATN1 & 1.99 & $1.07 E-02$ & 8448 & MNR2 & 2.55 & $1.19 \mathrm{E}-03$ & 8516 & MYOZ1 & 5.03 & $7.28 \mathrm{E}-03$ \\
\hline 8381 & $\mathrm{MB}$ & 3.68 & $8.45 \mathrm{E}-04$ & 8449 & MNT & 3.70 & 4.53E-04 & 8517 & MYOZ3 & 2.91 & $1.53 \mathrm{E}-02$ \\
\hline 8382 & MBL2 & 1.90 & $1.41 \mathrm{E}-02$ & 8450 & МОВКL2C & 6.18 & $1.10 \mathrm{E}-03$ & 8518 & MYT1 & 4.94 & $1.30 \mathrm{E}-03$ \\
\hline 8383 & MBOAT1 & 3.06 & $2.91 \mathrm{E}-03$ & 8451 & MOCOS & 4.95 & 7.15E-03 & 8519 & MYT1L & 2.35 & $6.23 \mathrm{E}-04$ \\
\hline 8384 & MBOAT4 & 3.38 & $3.11 \mathrm{E}-04$ & 8452 & MOCS1 & 1.26 & $2.34 \mathrm{E}-02$ & 8520 & N4BP3 & 3.86 & $9.75 \mathrm{E}-05$ \\
\hline 8385 & MBP & 5.69 & $4.23 \mathrm{E}-03$ & 8453 & MOG & 1.65 & $2.96 \mathrm{E}-02$ & 8521 & NADKD1 & 1.31 & $7.83 \mathrm{E}-03$ \\
\hline 8386 & MC1R & 4.16 & $2.41 \mathrm{E}-03$ & 8454 & MOGAT2 & 2.71 & $1.89 \mathrm{E}-03$ & 8522 & NANOG & 4.11 & $2.54 \mathrm{E}-03$ \\
\hline 8387 & MC4R & 5.36 & $3.23 \mathrm{E}-03$ & 8455 & MOS & 4.39 & $8.24 \mathrm{E}-04$ & 8523 & NAT & 3.11 & $1.83 \mathrm{E}-02$ \\
\hline 8388 & MC5R & 6.20 & $2.95 \mathrm{E}-03$ & 8456 & MPO & 4.92 & $1.55 \mathrm{E}-02$ & 8524 & NAT8 & 1.41 & $8.51 \mathrm{E}-04$ \\
\hline 8389 & MCAM & 2.04 & $1.24 \mathrm{E}-03$ & 8457 & MPP2 & 1.46 & $1.44 \mathrm{E}-02$ & 8525 & NAT8L & 2.69 & $3.10 \mathrm{E}-02$ \\
\hline 8390 & MCTP1 & 5.67 & $2.14 \mathrm{E}-05$ & 8458 & MPZL2 & 2.32 & $6.93 \mathrm{E}-03$ & 8526 & NBEA & 1.06 & $2.03 \mathrm{E}-02$ \\
\hline 8391 & $\mathrm{MCU}$ & 2.39 & $8.65 \mathrm{E}-03$ & 8459 & MRAP & 2.22 & $1.53 \mathrm{E}-02$ & 8527 & NBEAL2 & 2.99 & $1.26 \mathrm{E}-05$ \\
\hline 8392 & MDFI & 4.12 & $4.08 \mathrm{E}-04$ & 8460 & MRC2 & 3.31 & $1.88 \mathrm{E}-04$ & 8528 & NCAM2 & 2.09 & $4.08 \mathrm{E}-04$ \\
\hline 8393 & MED13 & 2.91 & $1.43 \mathrm{E}-03$ & 8461 & MREG & 2.60 & $2.47 \mathrm{E}-03$ & 8529 & NCAPH2 & 2.31 & $6.41 \mathrm{E}-03$ \\
\hline 8394 & MED13L & 2.12 & $1.18 \mathrm{E}-03$ & 8462 & MRGPRH & 2.59 & $1.13 \mathrm{E}-04$ & 8530 & NCF1 & 2.67 & $5.03 \mathrm{E}-02$ \\
\hline 8395 & MEF2B & 3.02 & $1.72 \mathrm{E}-02$ & 8463 & MSGN1 & 3.41 & $9.00 \mathrm{E}-05$ & 8531 & NCF2 & 2.26 & $2.55 \mathrm{E}-04$ \\
\hline 8396 & MEF2C & 2.59 & $1.80 \mathrm{E}-03$ & 8464 & MSLNL & 5.53 & 2.52E-03 & 8532 & NCF4 & 3.98 & 7.02E-04 \\
\hline 8397 & MEIS1 & 1.40 & 3.37E-02 & 8465 & MSMB & 1.82 & $1.64 \mathrm{E}-02$ & 8533 & NCKAP1L & 3.02 & $3.29 \mathrm{E}-02$ \\
\hline 8398 & MEIS2 & 1.42 & $4.04 \mathrm{E}-04$ & 8466 & MST1R & 5.76 & $2.18 \mathrm{E}-03$ & 8534 & NCOR2 & 3.04 & $1.16 \mathrm{E}-03$ \\
\hline 8399 & MEOX1 & 4.28 & $1.48 \mathrm{E}-03$ & 8467 & MSTN & 7.27 & $8.33 \mathrm{E}-04$ & 8535 & NDNF & 1.95 & $3.00 \mathrm{E}-03$ \\
\hline 8400 & MEPE & 3.33 & $3.27 \mathrm{E}-03$ & 8468 & MSTO1 & 1.19 & $1.40 \mathrm{E}-02$ & 8536 & NDRG4 & 4.49 & $1.92 \mathrm{E}-03$ \\
\hline 8401 & MERTK & 1.04 & $2.47 \mathrm{E}-03$ & 8469 & MSX1 & 1.30 & $9.47 \mathrm{E}-03$ & 8537 & NDST1 & 3.03 & 6.61E-04 \\
\hline 8402 & MESP2 & 3.07 & $1.79 \mathrm{E}-02$ & 8470 & MSX2 & 8.23 & $2.93 \mathrm{E}-03$ & 8538 & NDST2 & 3.09 & $1.25 \mathrm{E}-02$ \\
\hline 8403 & MET & 3.64 & $2.00 \mathrm{E}-03$ & 8471 & MTCH1 & 2.13 & $9.92 \mathrm{E}-03$ & 8539 & NDST4 & 2.62 & $1.06 \mathrm{E}-02$ \\
\hline 8404 & METTL11B & 2.67 & $1.30 \mathrm{E}-03$ & 8472 & MTHFD1L & 1.43 & $1.99 \mathrm{E}-02$ & 8540 & NECAB2 & 3.22 & $2.52 \mathrm{E}-03$ \\
\hline 8405 & METTL21C & 1.89 & $2.46 \mathrm{E}-03$ & 8473 & MTMR12 & 2.26 & $6.82 \mathrm{E}-03$ & 8541 & NEDD9 & 4.08 & $1.31 \mathrm{E}-03$ \\
\hline 8406 & MEX3A & 3.38 & $4.60 \mathrm{E}-03$ & 8474 & MTMR4 & 2.62 & $1.16 \mathrm{E}-03$ & 8542 & NEK4 & 4.74 & $5.01 \mathrm{E}-04$ \\
\hline 8407 & MFAP3L & 3.17 & $3.44 \mathrm{E}-04$ & 8475 & MTMR7 & 2.97 & $1.49 \mathrm{E}-02$ & 8543 & NEK8 & 3.52 & 9.60E-05 \\
\hline 8408 & MFAP5 & 5.32 & $2.49 \mathrm{E}-04$ & 8476 & MTMR9LP & 3.19 & $1.57 \mathrm{E}-03$ & 8544 & NEK9 & 1.89 & $5.21 \mathrm{E}-03$ \\
\hline 8409 & MFHAS1 & 1.83 & $9.69 \mathrm{E}-04$ & 8477 & MTNR1A & 5.89 & $5.17 \mathrm{E}-04$ & 8545 & NES & 3.60 & $5.19 \mathrm{E}-04$ \\
\hline 8410 & MFSD12 & 3.38 & $2.45 \mathrm{E}-05$ & 8478 & MTNR1B & 2.10 & $2.52 \mathrm{E}-02$ & 8546 & NETO1 & 3.18 & $4.52 \mathrm{E}-03$ \\
\hline 8411 & MFSD2B & 3.56 & $4.19 \mathrm{E}-03$ & 8479 & MTSS1L & 3.39 & $1.34 \mathrm{E}-03$ & 8547 & NETO2 & 1.68 & $1.39 \mathrm{E}-02$ \\
\hline 8412 & MFSD4 & 3.65 & $2.53 \mathrm{E}-03$ & 8480 & MUC1 & 1.48 & $1.05 \mathrm{E}-02$ & 8548 & NEURL2 & 3.52 & $2.29 \mathrm{E}-03$ \\
\hline 8413 & MFSD5 & 1.67 & $1.32 \mathrm{E}-03$ & 8481 & MUC13 & 3.08 & 7.71E-03 & 8549 & NEUROD4 & 4.55 & 4.90E-05 \\
\hline 8414 & MFSD6 & 1.65 & $1.08 \mathrm{E}-03$ & 8482 & MUC2 & 3.61 & 2.90E-05 & 8550 & NEUROD6 & 5.62 & 2.05E-04 \\
\hline 8415 & MFSD7 & 3.40 & $8.40 \mathrm{E}-03$ & 8483 & MUC6 & 2.92 & $2.21 \mathrm{E}-04$ & 8551 & NEUROG1 & 7.59 & $1.31 \mathrm{E}-03$ \\
\hline 8416 & MGAT4C & 2.29 & $5.42 \mathrm{E}-03$ & 8484 & MURC & 5.90 & $7.29 \mathrm{E}-04$ & 8552 & NFATC1 & 2.65 & $1.51 \mathrm{E}-02$ \\
\hline 8417 & MGAT5B & 5.54 & $4.33 \mathrm{E}-03$ & 8485 & MUSK & 3.49 & $1.91 \mathrm{E}-02$ & 8553 & NFATC2 & 5.80 & $1.68 \mathrm{E}-03$ \\
\hline 8418 & MICAL1 & 1.73 & $4.78 \mathrm{E}-03$ & 8486 & MUTYH & 3.52 & $6.64 \mathrm{E}-04$ & 8554 & NFIB & 1.23 & $1.11 \mathrm{E}-02$ \\
\hline 8419 & MICAL3 & 1.69 & $7.56 \mathrm{E}-03$ & 8487 & MVD & 4.10 & $1.14 \mathrm{E}-02$ & 8555 & NFKB2 & 2.96 & $7.22 \mathrm{E}-04$ \\
\hline 8420 & MID1 & 1.70 & $4.62 \mathrm{E}-03$ & 8488 & MVK & 3.81 & $1.42 \mathrm{E}-03$ & 8556 & NGB & 2.29 & $1.42 \mathrm{E}-03$ \\
\hline 8421 & MID2 & 3.82 & $2.21 \mathrm{E}-03$ & 8489 & $M \times 1$ & 2.04 & $5.16 \mathrm{E}-03$ & 8557 & NGF & 2.97 & $1.34 \mathrm{E}-02$ \\
\hline 8422 & MIER2 & 3.23 & $3.33 \mathrm{E}-03$ & 8490 & MXRA5 & 1.88 & $2.00 \mathrm{E}-02$ & 8558 & NGFR & 2.63 & $4.94 \mathrm{E}-03$ \\
\hline 8423 & MILR1 & 1.78 & $4.90 \mathrm{E}-03$ & 8491 & MYADML2 & 2.59 & $9.78 \mathrm{E}-03$ & 8559 & NHLH1 & 3.34 & $3.65 \mathrm{E}-03$ \\
\hline 8424 & MIP & 2.55 & $8.21 \mathrm{E}-02$ & 8492 & MYB & 1.53 & $1.07 \mathrm{E}-02$ & 8560 & NHLH2 & 6.21 & $1.02 \mathrm{E}-03$ \\
\hline 8425 & MITF & 1.88 & $1.20 \mathrm{E}-02$ & 8493 & MYBPC1 & 3.31 & $2.02 E-04$ & 8561 & NHLRC1 & 1.72 & $1.63 \mathrm{E}-03$ \\
\hline 8426 & MIXL1 & 2.86 & $2.97 \mathrm{E}-02$ & 8494 & MYBPC2 & 2.74 & $8.15 E-03$ & 8562 & NHS & 4.05 & $1.51 \mathrm{E}-02$ \\
\hline 8427 & MKL2 & 2.76 & $3.61 \mathrm{E}-03$ & 8495 & МҮВРСЗ & 3.65 & $5.66 \mathrm{E}-04$ & 8563 & NHSL1 & 3.03 & $5.25 \mathrm{E}-04$ \\
\hline 8428 & MKNK2 & 3.71 & $1.58 \mathrm{E}-03$ & 8496 & MYBPH & 3.15 & $7.76 \mathrm{E}-04$ & 8564 & NID2 & 1.79 & $2.91 \mathrm{E}-03$ \\
\hline 8429 & MKX & 3.85 & $6.18 \mathrm{E}-03$ & 8497 & MYC & 2.87 & $2.77 E-03$ & 8565 & NIN & 3.77 & $6.27 \mathrm{E}-04$ \\
\hline 8430 & MLANA & 2.06 & $3.91 \mathrm{E}-03$ & 8498 & MYCL1 & 4.68 & $1.10 \mathrm{E}-02$ & 8566 & NIPAL4 & 4.58 & $1.99 \mathrm{E}-03$ \\
\hline 8431 & MLL2 & 4.01 & $3.06 \mathrm{E}-03$ & 8499 & MYCT1 & 2.83 & $1.04 \mathrm{E}-02$ & 8567 & NKAIN1 & 3.14 & $8.65 \mathrm{E}-04$ \\
\hline
\end{tabular}




\begin{tabular}{|c|c|c|c|c|c|c|c|c|c|c|c|}
\hline 8568 & NKAIN2 & 3.49 & $1.24 \mathrm{E}-02$ & 8636 & NP9665218 & 5.78 & $9.94 \mathrm{E}-04$ & 8704 & NP9666023 & 6.64 & $3.19 \mathrm{E}-03$ \\
\hline 8569 & NKX2-1 & 5.11 & $3.30 \mathrm{E}-03$ & 8637 & NP9665230 & 5.69 & $6.57 \mathrm{E}-04$ & 8705 & NP9666029 & 9.15 & 1.47E-05 \\
\hline 8570 & NKX2-2 & 4.36 & $2.03 \mathrm{E}-02$ & 8638 & NP9665238 & 2.98 & $2.72 \mathrm{E}-03$ & 8706 & NP9666036 & 5.92 & $3.62 \mathrm{E}-02$ \\
\hline 8571 & NKX2-5 & 3.22 & $3.91 \mathrm{E}-02$ & 8639 & NP9665240 & 10.72 & $3.07 E-06$ & 8707 & NP9666041 & 5.26 & $6.30 \mathrm{E}-04$ \\
\hline 8572 & NKX2-6 & 3.44 & $3.24 \mathrm{E}-03$ & 8640 & NP9665243 & 6.68 & $1.07 \mathrm{E}-03$ & 8708 & NP9666048 & 4.74 & $6.81 \mathrm{E}-03$ \\
\hline 8573 & NKX6-2 & 3.11 & $2.97 \mathrm{E}-02$ & 8641 & NP9665277 & 3.97 & $1.15 \mathrm{E}-01$ & 8709 & NP9666054 & 5.09 & 7.61E-03 \\
\hline 8574 & NKX6-3 & 1.69 & $6.92 \mathrm{E}-03$ & 8642 & NP9665312 & 5.51 & $3.98 \mathrm{E}-03$ & 8710 & NP9666084 & 9.38 & $1.27 \mathrm{E}-03$ \\
\hline 8575 & NLE1 & 3.69 & $5.72 \mathrm{E}-04$ & 8643 & NP9665347 & 6.19 & $1.74 \mathrm{E}-03$ & 8711 & NP9666085 & 4.60 & $3.35 \mathrm{E}-02$ \\
\hline 8576 & NLGN1 & 1.43 & $5.66 \mathrm{E}-02$ & 8644 & NP9665364 & 2.79 & $5.89 \mathrm{E}-02$ & 8712 & NP9666087 & 2.20 & 5.55E- 02 \\
\hline 8577 & NLGN3 & 3.23 & 4.77E-03 & 8645 & NP9665371 & 3.43 & $1.51 \mathrm{E}-03$ & 8713 & NP9666154 & 3.63 & $2.28 \mathrm{E}-02$ \\
\hline 8578 & NLGN4X & 2.63 & $7.31 \mathrm{E}-03$ & 8646 & NP9665374 & 5.70 & $1.75 \mathrm{E}-03$ & 8714 & NP9666189 & 2.81 & $6.01 E-04$ \\
\hline 8579 & NLRC5 & 4.19 & $7.84 E-04$ & 8647 & NP9665382 & 3.05 & $7.43 \mathrm{E}-03$ & 8715 & NP9666191 & 5.93 & $1.95 \mathrm{E}-03$ \\
\hline 8580 & NMNAT2 & 3.14 & $2.00 \mathrm{E}-03$ & 8648 & NP9665383 & 4.18 & $7.53 \mathrm{E}-03$ & 8716 & NP9666194 & 3.26 & $6.23 \mathrm{E}-04$ \\
\hline 8581 & NMU & 4.05 & $3.98 \mathrm{E}-04$ & 8649 & NP9665389 & 6.83 & $3.83 \mathrm{E}-04$ & 8717 & NP9666229 & 7.96 & $2.56 \mathrm{E}-03$ \\
\hline 8582 & NMUR1 & 1.81 & $3.18 \mathrm{E}-04$ & 8650 & NP9665406 & 1.59 & $5.31 \mathrm{E}-02$ & 8718 & NP9666246 & 11.05 & $1.76 \mathrm{E}-03$ \\
\hline 8583 & NMUR2 & 8.56 & $6.86 \mathrm{E}-04$ & 8651 & NP9665431 & 4.12 & $2.84 \mathrm{E}-03$ & 8719 & NP9666249 & 3.45 & $8.56 \mathrm{E}-02$ \\
\hline 8584 & $\mathrm{NOC} 2 \mathrm{~L}$ & 3.96 & 7.33E-03 & 8652 & NP9665438 & 8.35 & 3.42E-04 & 8720 & NP9666251 & 7.64 & $1.25 \mathrm{E}-03$ \\
\hline 8585 & NOD1 & 3.21 & $6.23 \mathrm{E}-03$ & 8653 & NP9665452 & 5.43 & $2.54 \mathrm{E}-03$ & 8721 & NP9666252 & 2.30 & $2.81 \mathrm{E}-03$ \\
\hline 8586 & NODAL & 2.97 & $1.18 \mathrm{E}-01$ & 8654 & NP9665457 & 8.93 & $1.77 \mathrm{E}-03$ & 8722 & NP9666258 & 4.18 & $1.09 \mathrm{E}-02$ \\
\hline 8587 & NOG2 & 4.06 & $1.16 \mathrm{E}-02$ & 8655 & NP9665462 & 8.20 & $3.20 \mathrm{E}-03$ & 8723 & NP9666272 & 5.05 & $6.54 \mathrm{E}-03$ \\
\hline 8588 & NOL4 & 2.79 & $6.24 \mathrm{E}-03$ & 8656 & NP9665465 & 8.57 & $3.65 \mathrm{E}-03$ & 8724 & NP9666273 & 4.40 & $1.23 \mathrm{E}-02$ \\
\hline 8589 & NOL8 & 2.80 & $5.13 \mathrm{E}-03$ & 8657 & NP9665472 & 7.70 & $4.64 \mathrm{E}-03$ & 8725 & NP9666274 & 5.02 & $9.83 \mathrm{E}-03$ \\
\hline 8590 & NOS3 & 4.21 & $3.21 \mathrm{E}-04$ & 8658 & NP9665481 & 1.44 & $2.38 \mathrm{E}-02$ & 8726 & NP9666285 & 4.17 & 2.19E-02 \\
\hline 8591 & NOTCH2 & 1.61 & $2.29 \mathrm{E}-03$ & 8659 & NP9665532 & 3.55 & $8.17 \mathrm{E}-04$ & 8727 & NP9666313 & 8.81 & 3.30E-03 \\
\hline 8592 & NOX1 & 2.10 & $4.68 \mathrm{E}-02$ & 8660 & NP9665534 & 1.75 & $2.41 \mathrm{E}-03$ & 8728 & NP9666317 & 7.32 & $4.65 \mathrm{E}-03$ \\
\hline 8593 & NOX3 & 4.10 & $1.12 \mathrm{E}-02$ & 8661 & NP9665546 & 4.38 & $1.04 \mathrm{E}-03$ & 8729 & NP9666340 & 5.08 & $6.14 \mathrm{E}-03$ \\
\hline 8594 & NOX4 & 2.79 & $7.28 \mathrm{E}-02$ & 8662 & NP9665562 & 3.61 & $2.18 \mathrm{E}-02$ & 8730 & NP9666376 & 1.53 & $4.75 \mathrm{E}-02$ \\
\hline 8595 & NOX5 & 5.78 & $1.42 \mathrm{E}-04$ & 8663 & NP9665563 & 4.17 & $1.99 \mathrm{E}-02$ & 8731 & NP9666382 & 5.31 & 7.53E-04 \\
\hline 8596 & NOXO1 & 2.98 & $9.89 \mathrm{E}-04$ & 8664 & NP9665590 & 3.82 & $5.34 \mathrm{E}-03$ & 8732 & NP9666400 & 7.90 & $7.31 \mathrm{E}-03$ \\
\hline 8597 & NP12999553 & 1.98 & $1.42 E-02$ & 8665 & NP9665591 & 2.08 & $1.03 \mathrm{E}-01$ & 8733 & NP9666417 & 8.93 & $8.20 \mathrm{E}-04$ \\
\hline 8598 & NP12999571 & 6.10 & $7.80 \mathrm{E}-03$ & 8666 & NP9665599 & 5.21 & $1.11 \mathrm{E}-02$ & 8734 & NP9666446 & 3.22 & $2.27 \mathrm{E}-03$ \\
\hline 8599 & NP12999578 & 7.09 & $1.08 \mathrm{E}-03$ & 8667 & NP9665601 & 5.06 & $1.40 \mathrm{E}-03$ & 8735 & NP9666452 & 2.96 & $4.69 \mathrm{E}-03$ \\
\hline 8600 & NP12999579 & 3.28 & $4.48 \mathrm{E}-03$ & 8668 & NP9665603 & 1.36 & $2.62 \mathrm{E}-03$ & 8736 & NP9666461 & 7.79 & $2.90 \mathrm{E}-03$ \\
\hline 8601 & NP12999587 & 3.63 & $2.68 \mathrm{E}-03$ & 8669 & NP9665618 & 2.75 & $7.66 \mathrm{E}-02$ & 8737 & NP9666462 & 8.87 & $7.43 \mathrm{E}-04$ \\
\hline 8602 & NP12999592 & 5.61 & $3.25 \mathrm{E}-04$ & 8670 & NP9665630 & 7.35 & $3.62 \mathrm{E}-05$ & 8738 & NP9666463 & 3.31 & $1.27 \mathrm{E}-03$ \\
\hline 8603 & NP12999596 & 4.63 & $4.50 \mathrm{E}-03$ & 8671 & NP9665633 & 2.64 & $8.68 \mathrm{E}-02$ & 8739 & NP9666475 & 8.92 & $1.38 \mathrm{E}-03$ \\
\hline 8604 & NP9664788 & 7.53 & $2.66 \mathrm{E}-03$ & 8672 & NP9665640 & 4.82 & $6.22 \mathrm{E}-03$ & 8740 & NP9666513 & 9.82 & $1.43 \mathrm{E}-03$ \\
\hline 8605 & NP9664794 & 5.54 & $1.22 \mathrm{E}-03$ & 8673 & NP9665644 & 5.28 & $1.25 \mathrm{E}-03$ & 8741 & NP9666514 & 9.73 & $2.22 \mathrm{E}-03$ \\
\hline 8606 & NP9664803 & 5.17 & $3.15 \mathrm{E}-03$ & 8674 & NP9665680 & 5.22 & $3.77 \mathrm{E}-03$ & 8742 & NP9666529 & 7.85 & $1.19 \mathrm{E}-03$ \\
\hline 8607 & NP9664811 & 5.50 & $1.65 \mathrm{E}-03$ & 8675 & NP9665682 & 4.34 & $4.56 \mathrm{E}-02$ & 8743 & NP9666532 & 3.54 & $6.06 \mathrm{E}-02$ \\
\hline 8608 & NP9664816 & 2.86 & $4.24 E-03$ & 8676 & NP9665710 & 2.94 & $4.24 \mathrm{E}-03$ & 8744 & NP9666540 & 4.24 & $1.77 \mathrm{E}-02$ \\
\hline 8609 & NP9664839 & 6.81 & $6.90 \mathrm{E}-03$ & 8677 & NP9665725 & 3.57 & $6.83 \mathrm{E}-03$ & 8745 & NP9666542 & 5.19 & $1.96 \mathrm{E}-03$ \\
\hline 8610 & NP9664843 & 5.65 & $3.89 \mathrm{E}-03$ & 8678 & NP9665731 & 6.71 & $1.97 \mathrm{E}-03$ & 8746 & NP9666543 & 6.97 & $8.92 E-04$ \\
\hline 8611 & NP9664861 & 5.12 & $1.54 E-02$ & 8679 & NP9665744 & 6.63 & $4.33 \mathrm{E}-03$ & 8747 & NP9666547 & 4.02 & $1.00 \mathrm{E}-03$ \\
\hline 8612 & NP9664879 & 2.33 & $7.36 \mathrm{E}-03$ & 8680 & NP9665748 & 5.27 & $5.68 \mathrm{E}-03$ & 8748 & NP9666549 & 2.30 & $2.20 \mathrm{E}-02$ \\
\hline 8613 & NP9664897 & 6.08 & $8.17 \mathrm{E}-03$ & 8681 & NP9665779 & 2.29 & $1.94 \mathrm{E}-01$ & 8749 & NP9666555 & 2.71 & $2.21 \mathrm{E}-03$ \\
\hline 8614 & NP9664900 & 3.35 & $1.98 \mathrm{E}-02$ & 8682 & NP9665814 & 4.03 & $1.20 \mathrm{E}-03$ & 8750 & NP9666561 & 1.67 & 9.07E-03 \\
\hline 8615 & NP9664901 & 6.64 & $1.21 \mathrm{E}-02$ & 8683 & NP9665820 & 3.94 & $1.47 \mathrm{E}-02$ & 8751 & NP9666563 & 7.23 & $1.86 \mathrm{E}-03$ \\
\hline 8616 & NP9664904 & 3.47 & $2.82 \mathrm{E}-03$ & 8684 & NP9665849 & 4.38 & $1.09 \mathrm{E}-02$ & 8752 & NP9666585 & 6.66 & $7.31 \mathrm{E}-04$ \\
\hline 8617 & NP9664943 & 3.11 & $3.48 \mathrm{E}-03$ & 8685 & NP9665876 & 4.90 & 7.91E-03 & 8753 & NP9666599 & 3.15 & $6.53 \mathrm{E}-05$ \\
\hline 8618 & NP9664952 & 6.07 & $1.88 \mathrm{E}-03$ & 8686 & NP9665887 & 4.98 & $5.41 \mathrm{E}-03$ & 8754 & NP9666600 & 3.74 & 2.34E-02 \\
\hline 8619 & NP9664960 & 5.03 & $6.33 \mathrm{E}-05$ & 8687 & NP9665891 & 3.98 & $2.18 \mathrm{E}-03$ & 8755 & NP9666601 & 3.72 & 2.63E- 02 \\
\hline 8620 & NP9664963 & 7.03 & $2.61 \mathrm{E}-03$ & 8688 & NP9665893 & 7.44 & $3.04 \mathrm{E}-02$ & 8756 & NP9666604 & 5.82 & $1.25 \mathrm{E}-02$ \\
\hline 8621 & NP9665008 & 7.14 & $1.75 \mathrm{E}-03$ & 8689 & NP9665906 & 7.16 & $1.38 \mathrm{E}-03$ & 8757 & NP9666612 & 7.85 & $1.45 \mathrm{E}-03$ \\
\hline 8622 & NP9665019 & 8.12 & $2.88 \mathrm{E}-03$ & 8690 & NP9665913 & 9.93 & $9.26 \mathrm{E}-04$ & 8758 & NP9666620 & 7.67 & $2.83 \mathrm{E}-04$ \\
\hline 8623 & NP9665025 & 2.98 & $3.10 \mathrm{E}-04$ & 8691 & NP9665914 & 5.08 & $6.25 \mathrm{E}-03$ & 8759 & NP9666637 & 3.52 & $6.10 \mathrm{E}-02$ \\
\hline 8624 & NP9665046 & 9.34 & $2.57 \mathrm{E}-03$ & 8692 & NP9665952 & 5.98 & $1.04 \mathrm{E}-03$ & 8760 & NP9666642 & 4.47 & $1.75 \mathrm{E}-02$ \\
\hline 8625 & NP9665054 & 1.61 & $2.62 E-02$ & 8693 & NP9665961 & 7.74 & $3.89 \mathrm{E}-03$ & 8761 & NP9666644 & 2.64 & $3.51 \mathrm{E}-03$ \\
\hline 8626 & NP9665060 & 5.13 & $8.88 \mathrm{E}-03$ & 8694 & NP9665964 & 4.27 & $1.24 \mathrm{E}-02$ & 8762 & NP9666656 & 5.82 & $3.60 \mathrm{E}-03$ \\
\hline 8627 & NP9665086 & 5.86 & $6.83 \mathrm{E}-03$ & 8695 & NP9665967 & 3.46 & $2.02 \mathrm{E}-04$ & 8763 & NP9666692 & 4.15 & $1.38 \mathrm{E}-03$ \\
\hline 8628 & NP9665099 & 2.57 & $2.20 \mathrm{E}-01$ & 8696 & NP9665971 & 3.21 & $2.37 \mathrm{E}-02$ & 8764 & NP9666703 & 3.00 & $8.36 \mathrm{E}-02$ \\
\hline 8629 & NP9665100 & 3.61 & $2.52 \mathrm{E}-03$ & 8697 & NP9665981 & 2.75 & $7.65 \mathrm{E}-03$ & 8765 & NP9666748 & 4.58 & $3.35 \mathrm{E}-03$ \\
\hline 8630 & NP9665102 & 3.09 & $4.26 \mathrm{E}-03$ & 8698 & NP9665986 & 2.20 & $6.18 \mathrm{E}-02$ & 8766 & NP9666766 & 6.71 & 3.10E-04 \\
\hline 8631 & NP9665112 & 6.00 & $9.68 \mathrm{E}-04$ & 8699 & NP9665989 & 6.24 & $2.74 \mathrm{E}-03$ & 8767 & NP9666783 & 8.18 & $1.24 \mathrm{E}-03$ \\
\hline 8632 & NP9665135 & 5.95 & $4.54 \mathrm{E}-04$ & 8700 & NP9665998 & 3.48 & $2.89 \mathrm{E}-04$ & 8768 & NP9666784 & 3.86 & $1.08 \mathrm{E}-02$ \\
\hline 8633 & NP9665148 & 9.50 & $1.37 \mathrm{E}-03$ & 8701 & NP9666005 & 5.29 & $4.37 \mathrm{E}-06$ & 8769 & NP9666785 & 6.34 & $1.72 \mathrm{E}-01$ \\
\hline 8634 & NP9665202 & 3.73 & $9.77 \mathrm{E}-03$ & 8702 & NP9666006 & 3.88 & $4.66 \mathrm{E}-03$ & 8770 & NP9666797 & 2.05 & $6.62 \mathrm{E}-02$ \\
\hline 8635 & NP9665208 & 4.00 & $5.77 \mathrm{E}-02$ & 8703 & NP9666015 & 3.01 & $1.73 \mathrm{E}-02$ & 8771 & NP9666824 & 7.51 & $3.41 \mathrm{E}-03$ \\
\hline
\end{tabular}




\begin{tabular}{|c|c|c|c|c|c|c|c|c|c|c|c|}
\hline 8772 & NP9666833 & 3.11 & $1.37 \mathrm{E}-02$ & 8840 & NP9667900 & 7.24 & $4.18 \mathrm{E}-03$ & 8908 & NP9669015 & 3.58 & $4.37 \mathrm{E}-03$ \\
\hline 8773 & NP9666838 & 10.06 & $1.32 \mathrm{E}-03$ & 8841 & NP9667919 & 6.89 & $5.02 \mathrm{E}-03$ & 8909 & NP9669033 & 1.96 & $7.23 \mathrm{E}-03$ \\
\hline 8774 & NP9666842 & 4.41 & $4.30 \mathrm{E}-03$ & 8842 & NP9667932 & 5.16 & $9.38 \mathrm{E}-04$ & 8910 & NP9669061 & 3.85 & $7.77 \mathrm{E}-03$ \\
\hline 8775 & NP9666851 & 5.17 & $1.77 \mathrm{E}-03$ & 8843 & NP9667938 & 3.25 & $8.10 \mathrm{E}-04$ & 8911 & NP9669072 & 2.99 & $1.24 \mathrm{E}-02$ \\
\hline 8776 & NP9666879 & 4.35 & $7.22 \mathrm{E}-03$ & 8844 & NP9667945 & 4.07 & $2.60 \mathrm{E}-03$ & 8912 & NP9669075 & 4.07 & $7.63 \mathrm{E}-03$ \\
\hline 8777 & NP9666916 & 7.91 & $4.75 \mathrm{E}-04$ & 8845 & NP9667966 & 3.10 & $8.09 \mathrm{E}-03$ & 8913 & NP9669080 & 3.59 & $3.00 \mathrm{E}-03$ \\
\hline 8778 & NP9666917 & 1.64 & $8.58 \mathrm{E}-03$ & 8846 & NP9667982 & 2.15 & $5.06 \mathrm{E}-03$ & 8914 & NP9669116 & 2.98 & $1.77 \mathrm{E}-03$ \\
\hline 8779 & NP9666932 & 4.61 & $3.52 \mathrm{E}-03$ & 8847 & NP9667999 & 7.26 & $7.12 \mathrm{E}-04$ & 8915 & NP9669128 & 9.76 & $1.83 \mathrm{E}-04$ \\
\hline 8780 & NP9666947 & 2.67 & $7.56 \mathrm{E}-02$ & 8848 & NP9668017 & 3.86 & $3.29 \mathrm{E}-03$ & 8916 & NP9669130 & 3.92 & $1.05 E-03$ \\
\hline 8781 & NP9666948 & 4.73 & $6.84 \mathrm{E}-03$ & 8849 & NP9668021 & 6.03 & $6.03 E-03$ & 8917 & NP9669133 & 7.25 & $4.63 \mathrm{E}-03$ \\
\hline 8782 & NP9666962 & 5.01 & 4.14E-04 & 8850 & NP9668031 & 2.66 & $2.75 \mathrm{E}-02$ & 8918 & NP9669144 & 2.60 & $1.69 \mathrm{E}-03$ \\
\hline 8783 & NP9666967 & 5.59 & $1.70 \mathrm{E}-03$ & 8851 & NP9668058 & 5.48 & $7.14 \mathrm{E}-04$ & 8919 & NP9669152 & 10.57 & $6.11 \mathrm{E}-04$ \\
\hline 8784 & NP9666973 & 4.90 & $7.27 \mathrm{E}-03$ & 8852 & NP9668131 & 6.85 & $5.62 \mathrm{E}-03$ & 8920 & NP9669153 & 2.24 & $5.82 \mathrm{E}-03$ \\
\hline 8785 & NP9666979 & 8.76 & $2.18 \mathrm{E}-04$ & 8853 & NP9668133 & 1.74 & $1.84 \mathrm{E}-02$ & 8921 & NP9669172 & 1.68 & $8.38 \mathrm{E}-04$ \\
\hline 8786 & NP9666988 & 5.53 & $1.02 \mathrm{E}-03$ & 8854 & NP9668158 & 2.95 & $2.17 \mathrm{E}-03$ & 8922 & NP9669190 & 9.69 & 5.86E-03 \\
\hline 8787 & NP9666996 & 2.60 & $4.18 \mathrm{E}-02$ & 8855 & NP9668162 & 1.23 & $2.95 \mathrm{E}-02$ & 8923 & NP9669199 & 3.99 & $6.05 \mathrm{E}-03$ \\
\hline 8788 & NP9666998 & 2.19 & $4.46 \mathrm{E}-03$ & 8856 & NP9668190 & 1.57 & $5.53 \mathrm{E}-02$ & 8924 & NP9669230 & 10.41 & $5.76 \mathrm{E}-04$ \\
\hline 8789 & NP9667024 & 4.64 & $1.41 \mathrm{E}-03$ & 8857 & NP9668219 & 1.61 & $1.28 \mathrm{E}-03$ & 8925 & NP9669232 & 6.76 & $6.22 \mathrm{E}-03$ \\
\hline 8790 & NP9667060 & 2.33 & $4.06 \mathrm{E}-02$ & 8858 & NP9668223 & 4.76 & $9.80 \mathrm{E}-02$ & 8926 & NP9669240 & 2.92 & $2.04 \mathrm{E}-03$ \\
\hline 8791 & NP9667063 & 4.27 & $4.85 \mathrm{E}-03$ & 8859 & NP9668233 & 1.65 & $4.91 \mathrm{E}-03$ & 8927 & NP9669254 & 3.53 & $1.57 \mathrm{E}-02$ \\
\hline 8792 & NP9667087 & 2.01 & $1.86 \mathrm{E}-02$ & 8860 & NP9668243 & 4.54 & 3.03E-02 & 8928 & NP9669275 & 2.24 & $1.80 \mathrm{E}-03$ \\
\hline 8793 & NP9667090 & 3.70 & $1.17 \mathrm{E}-02$ & 8861 & NP9668307 & 3.10 & $8.48 \mathrm{E}-03$ & 8929 & NP9669283 & 8.66 & $1.38 \mathrm{E}-03$ \\
\hline 8794 & NP9667103 & 5.22 & $5.37 \mathrm{E}-04$ & 8862 & NP9668325 & 3.92 & $1.91 \mathrm{E}-02$ & 8930 & NP9669303 & 6.24 & $2.18 \mathrm{E}-03$ \\
\hline 8795 & NP9667109 & 4.04 & $9.76 \mathrm{E}-04$ & 8863 & NP9668346 & 4.66 & $1.10 \mathrm{E}-03$ & 8931 & NP9669313 & 6.64 & 2.87E-04 \\
\hline 8796 & NP9667128 & 7.80 & $2.30 \mathrm{E}-04$ & 8864 & NP9668369 & 5.01 & $4.23 \mathrm{E}-03$ & 8932 & NP9669340 & 3.19 & $6.03 \mathrm{E}-03$ \\
\hline 8797 & NP9667135 & 3.92 & $2.03 \mathrm{E}-04$ & 8865 & NP9668379 & 6.28 & $6.45 \mathrm{E}-05$ & 8933 & NP9669360 & 5.08 & $3.90 \mathrm{E}-03$ \\
\hline 8798 & NP9667169 & 3.30 & $5.49 \mathrm{E}-04$ & 8866 & NP9668402 & 1.60 & $2.07 E-02$ & 8934 & NP9669364 & 6.39 & $1.94 \mathrm{E}-03$ \\
\hline 8799 & NP9667188 & 1.91 & $3.86 \mathrm{E}-03$ & 8867 & NP9668404 & 5.52 & $1.53 \mathrm{E}-04$ & 8935 & NP9669365 & 3.66 & $4.03 \mathrm{E}-03$ \\
\hline 8800 & NP9667204 & 7.22 & $6.70 \mathrm{E}-05$ & 8868 & NP9668409 & 2.81 & $1.51 \mathrm{E}-02$ & 8936 & NP9669391 & 2.76 & $4.17 \mathrm{E}-03$ \\
\hline 8801 & NP9667219 & 6.93 & $1.43 \mathrm{E}-03$ & 8869 & NP9668428 & 3.99 & $9.30 \mathrm{E}-04$ & 8937 & NP9669400 & 3.70 & $1.17 \mathrm{E}-03$ \\
\hline 8802 & NP9667252 & 6.90 & $7.70 \mathrm{E}-02$ & 8870 & NP9668430 & 4.69 & $9.69 \mathrm{E}-04$ & 8938 & NP9669404 & 3.66 & $4.60 \mathrm{E}-04$ \\
\hline 8803 & NP9667258 & 8.66 & $7.96 \mathrm{E}-04$ & 8871 & NP9668440 & 3.97 & $2.09 \mathrm{E}-03$ & 8939 & NP9669410 & 6.12 & $5.98 \mathrm{E}-03$ \\
\hline 8804 & NP9667278 & 5.16 & $1.79 \mathrm{E}-03$ & 8872 & NP9668455 & 6.54 & $4.21 \mathrm{E}-03$ & 8940 & NP9669423 & 3.98 & $8.21 \mathrm{E}-04$ \\
\hline 8805 & NP9667292 & 6.30 & $7.45 \mathrm{E}-03$ & 8873 & NP9668520 & 5.28 & $8.90 \mathrm{E}-04$ & 8941 & NP9669426 & 2.86 & $3.75 \mathrm{E}-03$ \\
\hline 8806 & NP9667303 & 5.54 & $1.82 \mathrm{E}-03$ & 8874 & NP9668554 & 6.29 & $6.83 \mathrm{E}-04$ & 8942 & NP9669429 & 1.47 & $9.37 \mathrm{E}-02$ \\
\hline 8807 & NP9667315 & 4.11 & $5.41 \mathrm{E}-03$ & 8875 & NP9668568 & 4.22 & $7.97 \mathrm{E}-04$ & 8943 & NP9669439 & 5.98 & $2.66 \mathrm{E}-03$ \\
\hline 8808 & NP9667326 & 1.54 & $1.71 \mathrm{E}-02$ & 8876 & NP9668572 & 4.11 & $1.98 \mathrm{E}-02$ & 8944 & NP9669441 & 6.02 & $9.83 \mathrm{E}-03$ \\
\hline 8809 & NP9667343 & 4.08 & $2.53 \mathrm{E}-03$ & 8877 & NP9668578 & 1.84 & $2.62 \mathrm{E}-02$ & 8945 & NP9669457 & 5.15 & $8.60 \mathrm{E}-04$ \\
\hline 8810 & NP9667347 & 6.68 & $6.05 E-03$ & 8878 & NP9668602 & 2.48 & $2.22 \mathrm{E}-03$ & 8946 & NP9669459 & 2.65 & 1.77E-04 \\
\hline 8811 & NP9667384 & 4.09 & $7.23 \mathrm{E}-04$ & 8879 & NP9668651 & 3.19 & $1.71 \mathrm{E}-01$ & 8947 & NP9669471 & 6.40 & $6.54 \mathrm{E}-04$ \\
\hline 8812 & NP9667412 & 6.61 & $1.93 \mathrm{E}-03$ & 8880 & NP9668653 & 4.19 & $3.21 \mathrm{E}-03$ & 8948 & NP9669472 & 2.93 & $1.52 \mathrm{E}-02$ \\
\hline 8813 & NP9667437 & 3.35 & $4.81 \mathrm{E}-03$ & 8881 & NP9668655 & 8.87 & $1.51 \mathrm{E}-03$ & 8949 & NP9669486 & 1.89 & $2.75 E-02$ \\
\hline 8814 & NP9667438 & 1.83 & $5.53 \mathrm{E}-03$ & 8882 & NP9668656 & 10.63 & $3.29 \mathrm{E}-04$ & 8950 & NP9669513 & 3.20 & $3.09 \mathrm{E}-03$ \\
\hline 8815 & NP9667443 & 2.97 & $2.47 \mathrm{E}-03$ & 8883 & NP9668673 & 8.72 & $3.02 \mathrm{E}-03$ & 8951 & NP9669542 & 1.74 & $6.01 E-02$ \\
\hline 8816 & NP9667459 & 3.51 & $2.10 \mathrm{E}-03$ & 8884 & NP9668695 & 2.91 & $4.73 E-03$ & 8952 & NP9669566 & 2.99 & $6.65 \mathrm{E}-03$ \\
\hline 8817 & NP9667498 & 3.82 & $1.10 \mathrm{E}-02$ & 8885 & NP9668704 & 5.58 & $4.77 \mathrm{E}-04$ & 8953 & NP9669571 & 3.40 & $1.59 \mathrm{E}-02$ \\
\hline 8818 & NP9667502 & 2.77 & $2.79 \mathrm{E}-02$ & 8886 & NP9668708 & 4.04 & $1.34 \mathrm{E}-02$ & 8954 & NP9669581 & 5.78 & $3.57 \mathrm{E}-03$ \\
\hline 8819 & NP9667520 & 7.07 & $3.91 \mathrm{E}-04$ & 8887 & NP9668717 & 7.60 & $9.01 \mathrm{E}-04$ & 8955 & NP9669585 & 1.59 & $2.21 \mathrm{E}-04$ \\
\hline 8820 & NP9667540 & 2.50 & $3.13 \mathrm{E}-02$ & 8888 & NP9668730 & 4.03 & $5.28 \mathrm{E}-03$ & 8956 & NP9669605 & 3.62 & $4.92 \mathrm{E}-02$ \\
\hline 8821 & NP9667554 & 3.44 & $1.38 \mathrm{E}-03$ & 8889 & NP9668740 & 4.36 & $3.60 \mathrm{E}-04$ & 8957 & NP9669609 & 4.92 & 3.97E-03 \\
\hline 8822 & NP9667577 & 2.35 & $1.22 \mathrm{E}-01$ & 8890 & NP9668764 & 6.13 & $1.38 \mathrm{E}-03$ & 8958 & NP9669616 & 4.10 & $4.68 \mathrm{E}-05$ \\
\hline 8823 & NP9667610 & 3.04 & $1.97 \mathrm{E}-02$ & 8891 & NP9668784 & 8.72 & $3.80 \mathrm{E}-03$ & 8959 & NP9669622 & 4.52 & $3.24 \mathrm{E}-04$ \\
\hline 8824 & NP9667637 & 1.29 & $3.02 \mathrm{E}-03$ & 8892 & NP9668791 & 9.39 & $1.32 \mathrm{E}-03$ & 8960 & NP9669623 & 5.94 & $9.13 \mathrm{E}-04$ \\
\hline 8825 & NP9667658 & 5.38 & $3.52 \mathrm{E}-03$ & 8893 & NP9668794 & 3.47 & $7.69 \mathrm{E}-02$ & 8961 & NP9669625 & 4.33 & $1.63 \mathrm{E}-04$ \\
\hline 8826 & NP9667679 & 4.89 & $5.44 \mathrm{E}-03$ & 8894 & NP9668795 & 4.69 & $5.50 \mathrm{E}-03$ & 8962 & NP9669629 & 3.54 & $1.15 \mathrm{E}-02$ \\
\hline 8827 & NP9667683 & 2.61 & $1.87 \mathrm{E}-01$ & 8895 & NP9668799 & 4.01 & $2.38 \mathrm{E}-04$ & 8963 & NP9669676 & 4.15 & $1.34 \mathrm{E}-03$ \\
\hline 8828 & NP9667685 & 2.65 & $1.12 \mathrm{E}-02$ & 8896 & NP9668862 & 3.02 & $3.85 \mathrm{E}-03$ & 8964 & NP9669722 & 5.04 & $6.97 \mathrm{E}-03$ \\
\hline 8829 & NP9667703 & 4.24 & $2.70 \mathrm{E}-03$ & 8897 & NP9668872 & 4.78 & $7.93 \mathrm{E}-04$ & 8965 & NP9669723 & 2.56 & $1.65 \mathrm{E}-02$ \\
\hline 8830 & NP9667706 & 6.40 & $4.31 \mathrm{E}-04$ & 8898 & NP9668888 & 7.41 & $1.06 \mathrm{E}-03$ & 8966 & NP9669726 & 5.53 & $1.79 E-03$ \\
\hline 8831 & NP9667708 & 3.37 & $3.77 \mathrm{E}-03$ & 8899 & NP9668901 & 6.15 & $3.25 \mathrm{E}-05$ & 8967 & NP9669751 & 3.98 & $1.11 \mathrm{E}-02$ \\
\hline 8832 & NP9667729 & 7.23 & 3.90E-05 & 8900 & NP9668902 & 10.87 & $1.61 \mathrm{E}-03$ & 8968 & NP9669788 & 4.49 & $1.38 \mathrm{E}-03$ \\
\hline 8833 & NP9667759 & 6.36 & $4.16 \mathrm{E}-04$ & 8901 & NP9668903 & 1.74 & $1.63 \mathrm{E}-02$ & 8969 & NP9669792 & 5.47 & $8.83 \mathrm{E}-03$ \\
\hline 8834 & NP9667769 & 6.23 & $2.62 \mathrm{E}-03$ & 8902 & NP9668920 & 9.12 & $1.06 \mathrm{E}-02$ & 8970 & NP9669807 & 3.31 & $3.21 \mathrm{E}-04$ \\
\hline 8835 & NP9667812 & 6.70 & $7.44 \mathrm{E}-04$ & 8903 & NP9668931 & 4.95 & $4.01 \mathrm{E}-03$ & 8971 & NP9669823 & 4.43 & 5.47E-03 \\
\hline 8836 & NP9667820 & 3.63 & $3.65 \mathrm{E}-03$ & 8904 & NP9668964 & 3.03 & $1.13 \mathrm{E}-02$ & 8972 & NP9669839 & 2.74 & $4.06 \mathrm{E}-03$ \\
\hline 8837 & NP9667841 & 6.64 & $8.19 \mathrm{E}-04$ & 8905 & NP9668968 & 8.75 & $2.13 \mathrm{E}-04$ & 8973 & NP9669849 & 4.71 & $2.36 \mathrm{E}-03$ \\
\hline 8838 & NP9667869 & 5.68 & $8.69 \mathrm{E}-04$ & 8906 & NP9668981 & 3.57 & $2.17 \mathrm{E}-03$ & 8974 & NP9669901 & 4.02 & $6.55 \mathrm{E}-04$ \\
\hline 8839 & NP9667898 & 2.80 & $1.07 E-02$ & 8907 & NP9669003 & 6.28 & $5.23 \mathrm{E}-03$ & 8975 & NP9669941 & 2.31 & $6.93 \mathrm{E}-02$ \\
\hline
\end{tabular}




\begin{tabular}{|c|c|c|c|c|c|c|c|c|c|c|c|}
\hline 8976 & NP9669973 & 2.04 & $1.97 E-02$ & 9044 & NP9671645 & 3.88 & $1.15 \mathrm{E}-02$ & 9112 & NP9673040 & 6.34 & $8.00 \mathrm{E}-04$ \\
\hline 8977 & NP9669991 & 2.36 & $2.72 \mathrm{E}-02$ & 9045 & NP9671675 & 3.26 & $5.20 \mathrm{E}-03$ & 9113 & NP9673071 & 5.77 & $1.94 \mathrm{E}-03$ \\
\hline 8978 & NP9670005 & 2.86 & $4.75 \mathrm{E}-03$ & 9046 & NP9671719 & 3.80 & $7.12 \mathrm{E}-03$ & 9114 & NP9673076 & 4.97 & $1.31 \mathrm{E}-03$ \\
\hline 8979 & NP9670041 & 5.00 & $6.96 \mathrm{E}-03$ & 9047 & NP9671738 & 5.27 & $1.83 \mathrm{E}-04$ & 9115 & NP9673081 & 4.88 & $1.32 \mathrm{E}-03$ \\
\hline 8980 & NP9670043 & 5.11 & $3.68 \mathrm{E}-04$ & 9048 & NP9671765 & 4.77 & $1.76 \mathrm{E}-03$ & 9116 & NP9673093 & 4.28 & $5.05 E-04$ \\
\hline 8981 & NP9670046 & 7.15 & $5.24 \mathrm{E}-03$ & 9049 & NP9671814 & 6.54 & $3.75 \mathrm{E}-03$ & 9117 & NP9673125 & 6.07 & $6.19 \mathrm{E}-03$ \\
\hline 8982 & NP9670057 & 3.25 & $5.48 \mathrm{E}-03$ & 9050 & NP9671824 & 3.01 & $2.48 \mathrm{E}-02$ & 9118 & NP9673152 & 4.28 & $4.72 \mathrm{E}-04$ \\
\hline 8983 & NP9670075 & 3.68 & $6.00 \mathrm{E}-03$ & 9051 & NP9671825 & 3.70 & $9.26 \mathrm{E}-03$ & 9119 & NP9673163 & 2.84 & $6.29 \mathrm{E}-03$ \\
\hline 8984 & NP9670099 & 3.53 & $8.82 \mathrm{E}-04$ & 9052 & NP9671885 & 5.41 & $1.92 \mathrm{E}-03$ & 9120 & NP9673182 & 2.11 & $1.88 \mathrm{E}-01$ \\
\hline 8985 & NP9670105 & 2.04 & $1.85 \mathrm{E}-02$ & 9053 & NP9671917 & 3.01 & $1.73 \mathrm{E}-02$ & 9121 & NP9673217 & 3.25 & 2.06E-03 \\
\hline 8986 & NP9670118 & 5.54 & $1.03 \mathrm{E}-03$ & 9054 & NP9671919 & 5.26 & $4.37 \mathrm{E}-04$ & 9122 & NP9673254 & 2.36 & 5.54E- 03 \\
\hline 8987 & NP9670156 & 1.72 & $1.30 \mathrm{E}-02$ & 9055 & NP9671920 & 2.81 & $6.01 \mathrm{E}-02$ & 9123 & NP9673261 & 3.10 & 3.93E-03 \\
\hline 8988 & NP9670248 & 5.14 & $9.55 \mathrm{E}-04$ & 9056 & NP9671924 & 2.98 & $7.66 \mathrm{E}-03$ & 9124 & NP9673275 & 3.89 & 9.55E-04 \\
\hline 8989 & NP9670250 & 8.36 & $3.54 \mathrm{E}-04$ & 9057 & NP9671973 & 7.27 & $5.56 \mathrm{E}-05$ & 9125 & NP9673346 & 3.67 & $6.68 \mathrm{E}-04$ \\
\hline 8990 & NP9670251 & 8.17 & $1.20 \mathrm{E}-04$ & 9058 & NP9672006 & 5.11 & $5.29 \mathrm{E}-02$ & 9126 & NP9673362 & 3.41 & 7.23E-05 \\
\hline 8991 & NP9670256 & 8.37 & $1.43 \mathrm{E}-03$ & 9059 & NP9672047 & 7.07 & $1.22 \mathrm{E}-02$ & 9127 & NP9673388 & 2.44 & $2.45 \mathrm{E}-02$ \\
\hline 8992 & NP9670274 & 8.09 & $7.80 \mathrm{E}-05$ & 9060 & NP9672059 & 3.79 & $5.20 \mathrm{E}-03$ & 9128 & NP9673392 & 4.30 & $1.41 \mathrm{E}-03$ \\
\hline 8993 & NP9670284 & 9.14 & $6.14 \mathrm{E}-04$ & 9061 & NP9672086 & 3.31 & $1.76 \mathrm{E}-02$ & 9129 & NP9673419 & 5.44 & $4.70 \mathrm{E}-03$ \\
\hline 8994 & NP9670298 & 4.70 & $1.03 \mathrm{E}-03$ & 9062 & NP9672119 & 4.35 & $1.29 \mathrm{E}-03$ & 9130 & NP9673421 & 5.10 & 4.65E- 03 \\
\hline 8995 & NP9670340 & 5.42 & $5.02 E-04$ & 9063 & NP9672123 & 4.68 & 3.90E-02 & 9131 & NP9673435 & 6.08 & $7.62 \mathrm{E}-03$ \\
\hline 8996 & NP9670354 & 4.84 & $1.11 \mathrm{E}-03$ & 9064 & NP9672127 & 4.53 & $3.62 \mathrm{E}-05$ & 9132 & NP9673443 & 1.34 & $5.71 \mathrm{E}-02$ \\
\hline 8997 & NP9670398 & 4.64 & $6.90 \mathrm{E}-04$ & 9065 & NP9672171 & 3.41 & $1.16 \mathrm{E}-02$ & 9133 & NP9673447 & 4.57 & $2.86 \mathrm{E}-03$ \\
\hline 8998 & NP9670462 & 4.67 & $7.78 \mathrm{E}-03$ & 9066 & NP9672174 & 2.66 & $2.87 \mathrm{E}-04$ & 9134 & NP9673449 & 3.98 & $5.06 \mathrm{E}-03$ \\
\hline 8999 & NP9670468 & 2.97 & $8.49 \mathrm{E}-02$ & 9067 & NP9672232 & 4.47 & $3.64 \mathrm{E}-04$ & 9135 & NP9673450 & 1.26 & 3.22E- 02 \\
\hline 9000 & NP9670499 & 3.20 & $8.07 E-03$ & 9068 & NP9672262 & 2.93 & $4.32 \mathrm{E}-03$ & 9136 & NP9673464 & 2.52 & $2.06 \mathrm{E}-03$ \\
\hline 9001 & NP9670504 & 3.79 & $3.12 \mathrm{E}-04$ & 9069 & NP9672287 & 5.34 & $6.91 \mathrm{E}-04$ & 9137 & NP9673497 & 2.17 & 3.33E-03 \\
\hline 9002 & NP9670505 & 3.85 & $5.96 \mathrm{E}-04$ & 9070 & NP9672295 & 2.36 & $7.08 \mathrm{E}-03$ & 9138 & NP9673504 & 1.04 & $1.87 \mathrm{E}-02$ \\
\hline 9003 & NP9670510 & 2.17 & $3.61 \mathrm{E}-03$ & 9071 & NP9672304 & 5.28 & $1.16 \mathrm{E}-02$ & 9139 & NP9673507 & 4.38 & $1.26 \mathrm{E}-01$ \\
\hline 9004 & NP9670512 & 9.48 & $2.04 \mathrm{E}-03$ & 9072 & NP9672314 & 1.66 & $1.82 \mathrm{E}-02$ & 9140 & NP9673550 & 3.89 & $8.64 \mathrm{E}-04$ \\
\hline 9005 & NP9670548 & 6.03 & $4.93 \mathrm{E}-03$ & 9073 & NP9672316 & 3.97 & $6.31 \mathrm{E}-03$ & 9141 & NP9673584 & 3.93 & $8.06 \mathrm{E}-02$ \\
\hline 9006 & NP9670578 & 5.40 & $1.39 \mathrm{E}-02$ & 9074 & NP9672331 & 3.83 & $1.28 \mathrm{E}-03$ & 9142 & NP9673605 & 2.64 & $1.62 \mathrm{E}-02$ \\
\hline 9007 & NP9670650 & 3.87 & $1.51 \mathrm{E}-03$ & 9075 & NP9672359 & 2.44 & $1.14 \mathrm{E}-01$ & 9143 & NP9673613 & 2.82 & $2.43 E-03$ \\
\hline 9008 & NP9670705 & 5.18 & $5.49 \mathrm{E}-04$ & 9076 & NP9672365 & 3.89 & $2.45 \mathrm{E}-04$ & 9144 & NP9673630 & 2.36 & $4.00 E-03$ \\
\hline 9009 & NP9670708 & 3.34 & $3.27 \mathrm{E}-04$ & 9077 & NP9672368 & 2.69 & $1.45 \mathrm{E}-02$ & 9145 & NP9673637 & 6.63 & 3.29E-03 \\
\hline 9010 & NP9670778 & 2.83 & $8.85 \mathrm{E}-03$ & 9078 & NP9672369 & 4.50 & $6.04 \mathrm{E}-03$ & 9146 & NP9673688 & 5.57 & $5.23 \mathrm{E}-04$ \\
\hline 9011 & NP9670813 & 1.71 & $7.84 \mathrm{E}-04$ & 9079 & NP9672411 & 4.05 & $2.06 \mathrm{E}-03$ & 9147 & NP9673715 & 2.58 & $6.65 E-02$ \\
\hline 9012 & NP9670842 & 5.67 & $2.63 \mathrm{E}-04$ & 9080 & NP9672431 & 1.99 & $1.43 \mathrm{E}-02$ & 9148 & NP9673732 & 3.67 & $1.37 \mathrm{E}-03$ \\
\hline 9013 & NP9670852 & 6.74 & $1.61 \mathrm{E}-03$ & 9081 & NP9672438 & 5.80 & 3.30E-02 & 9149 & NP9673756 & 2.88 & $1.27 E-02$ \\
\hline 9014 & NP9670858 & 5.73 & $1.63 \mathrm{E}-04$ & 9082 & NP9672444 & 6.35 & $6.30 \mathrm{E}-05$ & 9150 & NP9673763 & 4.39 & $1.35 \mathrm{E}-03$ \\
\hline 9015 & NP9670911 & 6.31 & $1.22 \mathrm{E}-05$ & 9083 & NP9672456 & 2.18 & $5.81 \mathrm{E}-03$ & 9151 & NP9673772 & 2.53 & $1.72 \mathrm{E}-03$ \\
\hline 9016 & NP9670917 & 4.32 & $6.24 \mathrm{E}-03$ & 9084 & NP9672461 & 4.73 & $1.98 \mathrm{E}-03$ & 9152 & NP9673781 & 2.56 & $2.28 \mathrm{E}-01$ \\
\hline 9017 & NP9670972 & 4.39 & $1.29 \mathrm{E}-02$ & 9085 & NP9672470 & 5.44 & $4.03 \mathrm{E}-03$ & 9153 & NP9673811 & 6.90 & $1.75 \mathrm{E}-03$ \\
\hline 9018 & NP9670979 & 4.37 & $3.46 \mathrm{E}-03$ & 9086 & NP9672543 & 1.93 & $8.07 \mathrm{E}-03$ & 9154 & NP9673833 & 2.15 & $1.85 \mathrm{E}-01$ \\
\hline 9019 & NP9671035 & 3.14 & $3.63 \mathrm{E}-02$ & 9087 & NP9672546 & 2.64 & $3.13 \mathrm{E}-02$ & 9155 & NP9673850 & 5.82 & $9.71 \mathrm{E}-04$ \\
\hline 9020 & NP9671041 & 3.62 & $1.01 \mathrm{E}-02$ & 9088 & NP9672653 & 5.00 & $7.79 \mathrm{E}-05$ & 9156 & NP9673855 & 2.78 & $1.32 \mathrm{E}-02$ \\
\hline 9021 & NP9671049 & 4.61 & $1.75 \mathrm{E}-03$ & 9089 & NP9672688 & 1.85 & $9.29 \mathrm{E}-02$ & 9157 & NP9673866 & 3.08 & $2.36 \mathrm{E}-02$ \\
\hline 9022 & NP9671136 & 3.95 & $1.21 \mathrm{E}-04$ & 9090 & NP9672701 & 3.41 & 3.03E-03 & 9158 & NP9673893 & 4.50 & $2.94 \mathrm{E}-03$ \\
\hline 9023 & NP9671185 & 4.24 & $1.40 \mathrm{E}-02$ & 9091 & NP9672709 & 3.93 & $6.68 \mathrm{E}-03$ & 9159 & NP9673948 & 2.00 & $3.26 \mathrm{E}-03$ \\
\hline 9024 & NP9671203 & 4.12 & $9.86 \mathrm{E}-03$ & 9092 & NP9672718 & 1.65 & $1.86 \mathrm{E}-03$ & 9160 & NP9674001 & 3.05 & 5.97E-02 \\
\hline 9025 & NP9671218 & 4.60 & $3.53 \mathrm{E}-02$ & 9093 & NP9672722 & 4.19 & $3.35 \mathrm{E}-03$ & 9161 & NP9674009 & 5.38 & $2.17 E-03$ \\
\hline 9026 & NP9671242 & 7.06 & $6.73 \mathrm{E}-04$ & 9094 & NP9672752 & 4.70 & $4.41 \mathrm{E}-04$ & 9162 & NP9674025 & 3.64 & $1.48 \mathrm{E}-02$ \\
\hline 9027 & NP9671243 & 1.09 & $3.30 \mathrm{E}-02$ & 9095 & NP9672766 & 2.86 & $2.13 \mathrm{E}-03$ & 9163 & NP9674029 & 2.57 & $7.53 \mathrm{E}-03$ \\
\hline 9028 & NP9671266 & 5.78 & $1.28 \mathrm{E}-03$ & 9096 & NP9672828 & 4.71 & $2.77 \mathrm{E}-03$ & 9164 & NP9674039 & 6.34 & 4.31E-04 \\
\hline 9029 & NP9671285 & 2.60 & $5.59 \mathrm{E}-03$ & 9097 & NP9672830 & 7.34 & $5.39 \mathrm{E}-03$ & 9165 & NP9674045 & 2.27 & $5.11 \mathrm{E}-03$ \\
\hline 9030 & NP9671301 & 4.34 & $3.46 \mathrm{E}-03$ & 9098 & NP9672840 & 5.55 & $5.31 \mathrm{E}-03$ & 9166 & NP9674056 & 2.11 & 7.03E-03 \\
\hline 9031 & NP9671324 & 4.87 & $6.09 E-03$ & 9099 & NP9672861 & 4.22 & 3.83E-03 & 9167 & NP9674057 & 2.73 & $2.11 \mathrm{E}-02$ \\
\hline 9032 & NP9671337 & 3.21 & $2.23 \mathrm{E}-02$ & 9100 & NP9672867 & 2.66 & $3.85 \mathrm{E}-03$ & 9168 & NP9674083 & 2.48 & $9.89 \mathrm{E}-03$ \\
\hline 9033 & NP9671345 & 2.34 & $7.50 E-04$ & 9101 & NP9672870 & 4.24 & $1.27 \mathrm{E}-02$ & 9169 & NP9674085 & 4.03 & 4.07E-03 \\
\hline 9034 & NP9671403 & 1.97 & $2.19 \mathrm{E}-02$ & 9102 & NP9672879 & 4.37 & $5.08 \mathrm{E}-04$ & 9170 & NP9674086 & 6.29 & $2.29 \mathrm{E}-03$ \\
\hline 9035 & NP9671405 & 3.93 & $1.38 \mathrm{E}-01$ & 9103 & NP9672959 & 4.25 & $4.88 \mathrm{E}-03$ & 9171 & NP9674143 & 2.60 & $1.87 E-03$ \\
\hline 9036 & NP9671429 & 6.06 & $5.13 \mathrm{E}-03$ & 9104 & NP9672963 & 1.65 & $5.98 \mathrm{E}-02$ & 9172 & NP9674172 & 2.38 & $1.16 \mathrm{E}-02$ \\
\hline 9037 & NP9671431 & 2.94 & $2.13 \mathrm{E}-02$ & 9105 & NP9672964 & 3.08 & $4.99 \mathrm{E}-03$ & 9173 & NP9674176 & 4.05 & $8.51 \mathrm{E}-03$ \\
\hline 9038 & NP9671472 & 2.63 & $1.45 \mathrm{E}-03$ & 9106 & NP9672998 & 8.34 & $2.15 \mathrm{E}-06$ & 9174 & NP9674186 & 6.88 & $8.26 \mathrm{E}-05$ \\
\hline 9039 & NP9671515 & 5.31 & $4.54 \mathrm{E}-03$ & 9107 & NP9673009 & 1.37 & $1.88 \mathrm{E}-02$ & 9175 & NP9674193 & 5.09 & $9.80 \mathrm{E}-04$ \\
\hline 9040 & NP9671521 & 3.31 & $9.96 \mathrm{E}-03$ & 9108 & NP9673017 & 4.26 & $7.62 \mathrm{E}-04$ & 9176 & NP9674206 & 2.25 & $1.57 E-02$ \\
\hline 9041 & NP9671566 & 2.42 & $1.45 \mathrm{E}-03$ & 9109 & NP9673018 & 2.49 & $1.02 \mathrm{E}-02$ & 9177 & NP9674209 & 3.66 & $6.97 \mathrm{E}-03$ \\
\hline 9042 & NP9671572 & 3.19 & $2.49 \mathrm{E}-02$ & 9110 & NP9673021 & 3.64 & $1.13 \mathrm{E}-06$ & 9178 & NP9674247 & 3.84 & $4.89 \mathrm{E}-03$ \\
\hline 9043 & NP9671634 & 1.40 & $1.72 E-02$ & 9111 & NP9673024 & 7.07 & $1.16 \mathrm{E}-04$ & 9179 & NP9674260 & 1.89 & $1.05 E-02$ \\
\hline
\end{tabular}




\begin{tabular}{|c|c|c|c|c|c|c|c|c|c|c|c|}
\hline 9180 & NP9674309 & 2.81 & $7.56 \mathrm{E}-03$ & 9248 & NP9675499 & 1.66 & $2.44 \mathrm{E}-02$ & 9316 & NP9676786 & 2.05 & $1.21 E-02$ \\
\hline 9181 & NP9674340 & 2.51 & $3.53 \mathrm{E}-02$ & 9249 & NP9675511 & 4.95 & $6.31 \mathrm{E}-04$ & 9317 & NP9676788 & 2.06 & $2.68 \mathrm{E}-02$ \\
\hline 9182 & NP9674360 & 2.08 & 4.07E-02 & 9250 & NP9675515 & 5.67 & 7.42E-04 & 9318 & NP9676790 & 3.44 & $4.30 \mathrm{E}-03$ \\
\hline 9183 & NP9674379 & 3.24 & $9.91 \mathrm{E}-03$ & 9251 & NP9675533 & 6.03 & $3.37 \mathrm{E}-03$ & 9319 & NP9676796 & 3.48 & $6.86 \mathrm{E}-03$ \\
\hline 9184 & NP9674383 & 5.97 & $2.36 \mathrm{E}-04$ & 9252 & NP9675608 & 6.40 & $5.72 \mathrm{E}-03$ & 9320 & NP9676800 & 6.38 & 4.89E-05 \\
\hline 9185 & NP9674405 & 2.60 & 3.69E-03 & 9253 & NP9675645 & 2.75 & 7.27E-05 & 9321 & NP9676820 & 2.64 & 7.04E-04 \\
\hline 9186 & NP9674416 & 4.30 & $1.08 \mathrm{E}-02$ & 9254 & NP9675663 & 6.24 & $4.94 \mathrm{E}-04$ & 9322 & NP9676824 & 4.20 & $1.28 \mathrm{E}-03$ \\
\hline 9187 & NP9674421 & 3.10 & $1.12 \mathrm{E}-03$ & 9255 & NP9675677 & 3.88 & $2.91 \mathrm{E}-03$ & 9323 & NP9676842 & 2.09 & $2.54 \mathrm{E}-03$ \\
\hline 9188 & NP9674477 & 5.08 & $2.24 \mathrm{E}-03$ & 9256 & NP9675716 & 4.03 & $2.16 \mathrm{E}-02$ & 9324 & NP9676858 & 3.96 & $3.56 \mathrm{E}-04$ \\
\hline 9189 & NP9674497 & 5.61 & $4.62 \mathrm{E}-04$ & 9257 & NP9675730 & 5.92 & $5.99 \mathrm{E}-03$ & 9325 & NP9676896 & 3.12 & $9.23 \mathrm{E}-03$ \\
\hline 9190 & NP9674524 & 2.19 & $1.43 \mathrm{E}-02$ & 9258 & NP9675760 & 2.81 & $7.76 \mathrm{E}-02$ & 9326 & NP9676958 & 4.87 & $3.72 \mathrm{E}-04$ \\
\hline 9191 & NP9674526 & 2.50 & $2.80 \mathrm{E}-03$ & 9259 & NP9675767 & 3.30 & $3.12 \mathrm{E}-02$ & 9327 & NP9676970 & 3.43 & $3.03 \mathrm{E}-03$ \\
\hline 9192 & NP9674556 & 5.58 & $1.93 \mathrm{E}-02$ & 9260 & NP9675781 & 2.02 & $2.97 \mathrm{E}-02$ & 9328 & NP9676975 & 5.48 & 7.17E-03 \\
\hline 9193 & NP9674562 & 1.96 & $1.76 \mathrm{E}-02$ & 9261 & NP9675782 & 3.16 & $1.46 \mathrm{E}-02$ & 9329 & NP9676989 & 6.88 & $2.00 E-03$ \\
\hline 9194 & NP9674605 & 2.33 & $5.89 \mathrm{E}-03$ & 9262 & NP9675783 & 4.26 & 2.09E-04 & 9330 & NP9676993 & 6.96 & $1.11 \mathrm{E}-02$ \\
\hline 9195 & NP9674632 & 6.17 & $4.51 \mathrm{E}-05$ & 9263 & NP9675784 & 5.41 & $1.31 \mathrm{E}-03$ & 9331 & NP9677003 & 1.88 & $3.91 \mathrm{E}-04$ \\
\hline 9196 & NP9674646 & 3.45 & $3.12 \mathrm{E}-02$ & 9264 & NP9675795 & 1.43 & $1.32 \mathrm{E}-02$ & 9332 & NP9677005 & 2.15 & $3.95 \mathrm{E}-02$ \\
\hline 9197 & NP9674712 & 5.16 & $4.74 \mathrm{E}-03$ & 9265 & NP9675799 & 1.91 & 2.43E-02 & 9333 & NP9677018 & 3.93 & $5.93 \mathrm{E}-03$ \\
\hline 9198 & NP9674725 & 6.63 & $1.56 \mathrm{E}-04$ & 9266 & NP9675879 & 2.73 & $8.17 \mathrm{E}-04$ & 9334 & NP9677021 & 4.69 & $4.77 E-03$ \\
\hline 9199 & NP9674730 & 3.94 & $1.23 \mathrm{E}-03$ & 9267 & NP9675904 & 5.17 & $7.54 \mathrm{E}-03$ & 9335 & NP9677036 & 1.72 & $2.13 \mathrm{E}-03$ \\
\hline 9200 & NP9674735 & 3.18 & $3.46 \mathrm{E}-03$ & 9268 & NP9675909 & 2.59 & 7.32E-03 & 9336 & NP9677049 & 2.28 & $1.18 \mathrm{E}-03$ \\
\hline 9201 & NP9674742 & 5.45 & $6.89 \mathrm{E}-04$ & 9269 & NP9675916 & 3.21 & $1.06 E-02$ & 9337 & NP9677120 & 1.66 & $5.35 \mathrm{E}-02$ \\
\hline 9202 & NP9674748 & 1.31 & $2.83 \mathrm{E}-03$ & 9270 & NP9675929 & 4.18 & $7.36 \mathrm{E}-05$ & 9338 & NP9677130 & 4.20 & $3.58 \mathrm{E}-03$ \\
\hline 9203 & NP9674762 & 6.14 & $4.25 \mathrm{E}-04$ & 9271 & NP9675951 & 4.07 & $8.88 \mathrm{E}-03$ & 9339 & NP9677149 & 5.79 & $2.86 \mathrm{E}-03$ \\
\hline 9204 & NP9674776 & 6.03 & $2.14 \mathrm{E}-03$ & 9272 & NP9675956 & 3.35 & 4.34E-03 & 9340 & NP9677154 & 1.01 & $1.50 \mathrm{E}-03$ \\
\hline 9205 & NP9674794 & 4.51 & $5.02 \mathrm{E}-03$ & 9273 & NP9676003 & 2.73 & $3.50 \mathrm{E}-03$ & 9341 & NP9677169 & 3.78 & $1.57 \mathrm{E}-03$ \\
\hline 9206 & NP9674795 & 3.75 & $1.21 \mathrm{E}-02$ & 9274 & NP9676007 & 2.49 & $8.47 \mathrm{E}-03$ & 9342 & NP9677187 & 4.38 & $8.31 \mathrm{E}-04$ \\
\hline 9207 & NP9674796 & 2.86 & $6.10 \mathrm{E}-03$ & 9275 & NP9676028 & 4.94 & $1.59 \mathrm{E}-02$ & 9343 & NP9677195 & 6.63 & $1.83 \mathrm{E}-04$ \\
\hline 9208 & NP9674807 & 4.14 & $1.52 E-03$ & 9276 & NP9676059 & 4.08 & $3.25 \mathrm{E}-03$ & 9344 & NP9677219 & 5.42 & $5.78 \mathrm{E}-03$ \\
\hline 9209 & NP9674818 & 4.45 & $5.06 \mathrm{E}-03$ & 9277 & NP9676064 & 6.41 & $1.77 \mathrm{E}-03$ & 9345 & NP9677223 & 4.05 & $3.06 \mathrm{E}-03$ \\
\hline 9210 & NP9674852 & 4.96 & $1.10 \mathrm{E}-02$ & 9278 & NP9676083 & 5.22 & $3.63 \mathrm{E}-02$ & 9346 & NP9677237 & 5.26 & $3.51 E-04$ \\
\hline 9211 & NP9674856 & 2.53 & $1.34 \mathrm{E}-02$ & 9279 & NP9676087 & 4.81 & $2.45 \mathrm{E}-03$ & 9347 & NP9677265 & 6.29 & $6.98 \mathrm{E}-04$ \\
\hline 9212 & NP9674874 & 2.09 & $7.72 \mathrm{E}-02$ & 9280 & NP9676093 & 6.72 & $1.83 \mathrm{E}-03$ & 9348 & NP9677269 & 3.21 & $2.74 \mathrm{E}-03$ \\
\hline 9213 & NP9674893 & 3.39 & $2.43 \mathrm{E}-04$ & 9281 & NP9676123 & 2.25 & $1.44 \mathrm{E}-02$ & 9349 & NP9677274 & 1.95 & $1.02 \mathrm{E}-03$ \\
\hline 9214 & NP9674898 & 3.17 & $3.18 \mathrm{E}-04$ & 9282 & NP9676143 & 4.50 & $2.50 \mathrm{E}-03$ & 9350 & NP9677303 & 6.92 & $2.40 \mathrm{E}-04$ \\
\hline 9215 & NP9674919 & 5.60 & $2.32 \mathrm{E}-03$ & 9283 & NP9676146 & 3.36 & $4.61 \mathrm{E}-04$ & 9351 & NP9677390 & 2.29 & $3.92 \mathrm{E}-02$ \\
\hline 9216 & NP9674926 & 4.75 & $1.89 \mathrm{E}-03$ & 9284 & NP9676169 & 3.05 & $2.01 \mathrm{E}-03$ & 9352 & NP9677462 & 3.22 & $2.25 \mathrm{E}-03$ \\
\hline 9217 & NP9674927 & 4.64 & $1.63 \mathrm{E}-03$ & 9285 & NP9676176 & 4.37 & $5.07 \mathrm{E}-04$ & 9353 & NP9677470 & 1.43 & $8.36 \mathrm{E}-02$ \\
\hline 9218 & NP9674933 & 6.43 & $2.47 \mathrm{E}-04$ & 9286 & NP9676185 & 2.73 & $1.06 \mathrm{E}-01$ & 9354 & NP9677475 & 2.73 & $1.09 \mathrm{E}-02$ \\
\hline 9219 & NP9674951 & 4.54 & $1.20 \mathrm{E}-02$ & 9287 & NP9676186 & 4.05 & $1.74 \mathrm{E}-02$ & 9355 & NP9677477 & 3.11 & $2.07 \mathrm{E}-03$ \\
\hline 9220 & NP9674955 & 1.21 & $6.85 \mathrm{E}-04$ & 9288 & NP9676188 & 2.74 & $1.45 \mathrm{E}-02$ & 9356 & NP9677502 & 2.18 & $2.12 \mathrm{E}-02$ \\
\hline 9221 & NP9674969 & 5.08 & $2.96 \mathrm{E}-04$ & 9289 & NP9676191 & 3.34 & $3.35 \mathrm{E}-03$ & 9357 & NP9677524 & 2.74 & $2.64 E-02$ \\
\hline 9222 & NP9674999 & 6.79 & $2.35 \mathrm{E}-03$ & 9290 & NP9676192 & 1.73 & $5.51 \mathrm{E}-02$ & 9358 & NP9677565 & 3.13 & $2.96 \mathrm{E}-02$ \\
\hline 9223 & NP9675021 & 4.47 & $1.91 \mathrm{E}-03$ & 9291 & NP9676203 & 3.18 & $6.62 \mathrm{E}-03$ & 9359 & NP9677569 & 2.26 & $1.65 \mathrm{E}-02$ \\
\hline 9224 & NP9675059 & 6.22 & $5.54 \mathrm{E}-03$ & 9292 & NP9676217 & 4.89 & $1.75 \mathrm{E}-03$ & 9360 & NP9677582 & 4.13 & $4.28 \mathrm{E}-03$ \\
\hline 9225 & NP9675083 & 3.49 & $1.81 E-02$ & 9293 & NP9676240 & 2.80 & $4.58 \mathrm{E}-03$ & 9361 & NP9677599 & 4.92 & $2.05 \mathrm{E}-03$ \\
\hline 9226 & NP9675091 & 5.79 & $3.84 \mathrm{E}-03$ & 9294 & NP9676314 & 2.98 & $1.08 \mathrm{E}-03$ & 9362 & NP9677658 & 4.69 & $3.98 \mathrm{E}-03$ \\
\hline 9227 & NP9675097 & 3.67 & $1.57 \mathrm{E}-04$ & 9295 & NP9676323 & 4.52 & $3.40 \mathrm{E}-04$ & 9363 & NP9677693 & 6.33 & $7.80 \mathrm{E}-04$ \\
\hline 9228 & NP9675187 & 4.52 & 7.40E-03 & 9296 & NP9676342 & 2.08 & $7.27 \mathrm{E}-02$ & 9364 & NP9677702 & 5.75 & $3.70 \mathrm{E}-02$ \\
\hline 9229 & NP9675193 & 5.30 & $1.35 \mathrm{E}-03$ & 9297 & NP9676360 & 4.42 & $3.26 \mathrm{E}-04$ & 9365 & NP9677712 & 3.91 & $6.78 \mathrm{E}-03$ \\
\hline 9230 & NP9675209 & 3.29 & $7.76 \mathrm{E}-02$ & 9298 & NP9676388 & 4.38 & 3.83E-03 & 9366 & NP9677720 & 2.15 & $2.85 \mathrm{E}-02$ \\
\hline 9231 & NP9675216 & 3.77 & $9.72 \mathrm{E}-03$ & 9299 & NP9676390 & 1.48 & $1.61 \mathrm{E}-02$ & 9367 & NP9677742 & 4.60 & $3.07 E-03$ \\
\hline 9232 & NP9675233 & 3.64 & $9.30 \mathrm{E}-04$ & 9300 & NP9676401 & 4.86 & $5.45 \mathrm{E}-03$ & 9368 & NP9677759 & 5.50 & $8.19 \mathrm{E}-04$ \\
\hline 9233 & NP9675258 & 6.52 & $8.36 \mathrm{E}-04$ & 9301 & NP9676425 & 3.52 & $1.04 \mathrm{E}-03$ & 9369 & NP9677814 & 4.93 & $9.21 \mathrm{E}-04$ \\
\hline 9234 & NP9675270 & 4.72 & $6.83 \mathrm{E}-04$ & 9302 & NP9676449 & 1.61 & $9.70 \mathrm{E}-02$ & 9370 & NP9677903 & 3.96 & $6.39 \mathrm{E}-02$ \\
\hline 9235 & NP9675279 & 4.88 & $4.42 \mathrm{E}-03$ & 9303 & NP9676464 & 4.89 & $1.99 \mathrm{E}-03$ & 9371 & NP9677904 & 4.23 & $3.96 \mathrm{E}-03$ \\
\hline 9236 & NP9675286 & 1.77 & $5.02 \mathrm{E}-02$ & 9304 & NP9676499 & 5.44 & $1.30 \mathrm{E}-02$ & 9372 & NP9677910 & 7.97 & $7.76 \mathrm{E}-04$ \\
\hline 9237 & NP9675305 & 3.01 & $8.42 \mathrm{E}-04$ & 9305 & NP9676525 & 1.95 & $1.26 \mathrm{E}-01$ & 9373 & NP9677937 & 1.63 & $2.96 \mathrm{E}-02$ \\
\hline 9238 & NP9675309 & 1.38 & $6.14 \mathrm{E}-02$ & 9306 & NP9676534 & 4.09 & $1.10 \mathrm{E}-02$ & 9374 & NP9677950 & 6.16 & 4.61E-04 \\
\hline 9239 & NP9675326 & 1.98 & $1.15 \mathrm{E}-03$ & 9307 & NP9676540 & 7.18 & $2.80 \mathrm{E}-04$ & 9375 & NP9677953 & 5.28 & $1.13 \mathrm{E}-03$ \\
\hline 9240 & NP9675355 & 3.72 & $2.66 \mathrm{E}-03$ & 9308 & NP9676568 & 3.98 & $1.09 \mathrm{E}-02$ & 9376 & NP9677964 & 5.10 & $1.27 E-03$ \\
\hline 9241 & NP9675366 & 2.89 & $3.57 \mathrm{E}-03$ & 9309 & NP9676600 & 2.25 & $2.73 E-03$ & 9377 & NP9677976 & 3.38 & $7.80 \mathrm{E}-03$ \\
\hline 9242 & NP9675388 & 3.14 & $4.14 \mathrm{E}-03$ & 9310 & NP9676602 & 4.56 & $3.87 \mathrm{E}-03$ & 9378 & NP9678013 & 3.69 & $5.24 E-04$ \\
\hline 9243 & NP9675414 & 6.30 & $4.07 \mathrm{E}-04$ & 9311 & NP9676666 & 5.52 & $5.91 \mathrm{E}-04$ & 9379 & NP9678017 & 2.53 & $2.88 \mathrm{E}-03$ \\
\hline 9244 & NP9675415 & 3.45 & $3.55 \mathrm{E}-03$ & 9312 & NP9676701 & 2.16 & $7.53 \mathrm{E}-03$ & 9380 & NP9678033 & 3.93 & $9.67 \mathrm{E}-03$ \\
\hline 9245 & NP9675434 & 2.32 & $5.62 \mathrm{E}-04$ & 9313 & NP9676731 & 4.46 & $7.49 \mathrm{E}-04$ & 9381 & NP9678039 & 5.40 & $3.72 \mathrm{E}-04$ \\
\hline 9246 & NP9675452 & 1.96 & $2.31 \mathrm{E}-02$ & 9314 & NP9676732 & 4.81 & $6.64 \mathrm{E}-03$ & 9382 & NP9678051 & 2.44 & $2.63 \mathrm{E}-02$ \\
\hline 9247 & NP9675459 & 4.71 & $7.73 E-04$ & 9315 & NP9676739 & 6.16 & $3.56 \mathrm{E}-04$ & 9383 & NP9678059 & 5.09 & $3.86 \mathrm{E}-04$ \\
\hline
\end{tabular}




\begin{tabular}{|c|c|c|c|c|c|c|c|c|c|c|c|}
\hline 9384 & NP9678062 & 4.55 & $1.18 \mathrm{E}-01$ & 9452 & NP9679433 & 3.13 & $2.63 \mathrm{E}-03$ & 9520 & NP9680618 & 1.64 & $3.70 \mathrm{E}-02$ \\
\hline 9385 & NP9678084 & 4.10 & $2.60 \mathrm{E}-03$ & 9453 & NP9679446 & 4.46 & $2.19 \mathrm{E}-02$ & 9521 & NP9680625 & 4.65 & $1.31 \mathrm{E}-03$ \\
\hline 9386 & NP9678112 & 1.77 & $4.93 \mathrm{E}-03$ & 9454 & NP9679447 & 1.99 & $7.84 \mathrm{E}-03$ & 9522 & NP9680643 & 4.52 & $3.01 \mathrm{E}-04$ \\
\hline 9387 & NP9678124 & 1.79 & $2.08 \mathrm{E}-02$ & 9455 & NP9679455 & 2.57 & $3.50 \mathrm{E}-03$ & 9523 & NP9680772 & 2.48 & $1.44 \mathrm{E}-03$ \\
\hline 9388 & NP9678149 & 4.73 & $1.88 \mathrm{E}-03$ & 9456 & NP9679457 & 4.13 & $1.48 \mathrm{E}-03$ & 9524 & NP9680838 & 3.64 & $6.93 \mathrm{E}-03$ \\
\hline 9389 & NP9678217 & 5.89 & $2.28 \mathrm{E}-03$ & 9457 & NP9679463 & 7.15 & $7.96 \mathrm{E}-04$ & 9525 & NPAS2 & 2.15 & $6.33 \mathrm{E}-03$ \\
\hline 9390 & NP9678234 & 4.56 & $3.34 \mathrm{E}-03$ & 9458 & NP9679482 & 2.27 & $3.17 \mathrm{E}-02$ & 9526 & NPAS3 & 5.33 & $3.94 \mathrm{E}-04$ \\
\hline 9391 & NP9678268 & 2.42 & $5.39 \mathrm{E}-03$ & 9459 & NP9679497 & 3.91 & $1.15 \mathrm{E}-03$ & 9527 & NPBWR1 & 7.58 & $2.23 \mathrm{E}-03$ \\
\hline 9392 & NP9678283 & 3.37 & $2.29 \mathrm{E}-02$ & 9460 & NP9679506 & 2.46 & $2.80 \mathrm{E}-03$ & 9528 & NPDC1 & 2.16 & $5.95 \mathrm{E}-03$ \\
\hline 9393 & NP9678292 & 2.12 & $1.05 \mathrm{E}-03$ & 9461 & NP9679507 & 5.49 & $8.35 \mathrm{E}-04$ & 9529 & NPFFR2 & 5.97 & 7.91E-05 \\
\hline 9394 & NP9678364 & 3.88 & $3.04 \mathrm{E}-03$ & 9462 & NP9679566 & 5.07 & $1.90 \mathrm{E}-04$ & 9530 & NPHP4 & 2.58 & $3.56 \mathrm{E}-03$ \\
\hline 9395 & NP9678373 & 1.56 & $3.29 \mathrm{E}-02$ & 9463 & NP9679595 & 10.35 & $6.81 \mathrm{E}-04$ & 9531 & NPM3 & 3.13 & $4.48 \mathrm{E}-03$ \\
\hline 9396 & NP9678376 & 5.71 & $4.68 \mathrm{E}-03$ & 9464 & NP9679598 & 10.59 & $2.52 \mathrm{E}-03$ & 9532 & NPPB & 3.30 & $5.57 \mathrm{E}-03$ \\
\hline 9397 & NP9678401 & 3.11 & $2.49 \mathrm{E}-02$ & 9465 & NP9679601 & 1.38 & $4.09 \mathrm{E}-03$ & 9533 & NPPC & 3.25 & $4.23 \mathrm{E}-03$ \\
\hline 9398 & NP9678402 & 3.75 & $2.89 \mathrm{E}-02$ & 9466 & NP9679611 & 6.16 & $6.75 \mathrm{E}-04$ & 9534 & NPSR1 & 2.43 & $1.34 \mathrm{E}-02$ \\
\hline 9399 & NP9678405 & 4.11 & $5.30 \mathrm{E}-04$ & 9467 & NP9679628 & 4.73 & $1.26 \mathrm{E}-04$ & 9535 & NPTX2 & 1.22 & 7.95E-03 \\
\hline 9400 & NP9678525 & 7.07 & $2.78 \mathrm{E}-03$ & 9468 & NP9679649 & 2.37 & $2.73 \mathrm{E}-03$ & 9536 & NPTXR & 3.23 & $3.75 \mathrm{E}-03$ \\
\hline 9401 & NP9678534 & 6.66 & $5.41 \mathrm{E}-03$ & 9469 & NP9679655 & 1.53 & $3.43 \mathrm{E}-04$ & 9537 & NPVF & 3.62 & $7.33 \mathrm{E}-03$ \\
\hline 9402 & NP9678541 & 3.96 & $1.49 \mathrm{E}-03$ & 9470 & NP9679670 & 4.84 & $1.44 \mathrm{E}-03$ & 9538 & NPY2R & 6.85 & $1.99 \mathrm{E}-04$ \\
\hline 9403 & NP9678562 & 4.32 & $4.75 E-04$ & 9471 & NP9679681 & 3.44 & $1.24 \mathrm{E}-02$ & 9539 & NPY5R & 1.61 & $1.14 \mathrm{E}-02$ \\
\hline 9404 & NP9678572 & 2.67 & $1.02 \mathrm{E}-02$ & 9472 & NP9679704 & 4.08 & $5.69 \mathrm{E}-04$ & 9540 & NPY6R & 2.11 & $8.60 \mathrm{E}-04$ \\
\hline 9405 & NP9678587 & 1.87 & $1.18 \mathrm{E}-02$ & 9473 & NP9679744 & 6.18 & $5.06 \mathrm{E}-03$ & 9541 & NPY7R & 2.82 & $1.60 \mathrm{E}-03$ \\
\hline 9406 & NP9678613 & 3.13 & $2.55 \mathrm{E}-03$ & 9474 & NP9679771 & 3.19 & $7.57 \mathrm{E}-03$ & 9542 & NQO1 & 1.48 & 7.94E-02 \\
\hline 9407 & NP9678627 & 3.89 & $2.55 \mathrm{E}-04$ & 9475 & NP9679772 & 4.55 & $7.88 \mathrm{E}-03$ & 9543 & $\mathrm{NR} 1 \mathrm{H} 4$ & 3.16 & 3.03E-03 \\
\hline 9408 & NP9678660 & 4.26 & $1.24 \mathrm{E}-02$ & 9476 & NP9679783 & 5.46 & $2.00 \mathrm{E}-03$ & 9544 & NR113 & 3.38 & $8.21 \mathrm{E}-03$ \\
\hline 9409 & NP9678717 & 6.25 & $5.27 \mathrm{E}-04$ & 9477 & NP9679784 & 5.81 & $4.39 \mathrm{E}-05$ & 9545 & NR2E3 & 2.03 & 4.19E-02 \\
\hline 9410 & NP9678730 & 2.69 & $1.44 \mathrm{E}-03$ & 9478 & NP9679801 & 5.62 & $4.80 \mathrm{E}-03$ & 9546 & NR3C1 & 3.03 & 1.40E-04 \\
\hline 9411 & NP9678732 & 3.51 & $1.70 \mathrm{E}-02$ & 9479 & NP9679824 & 6.36 & $7.66 \mathrm{E}-02$ & 9547 & NR3C2 & 2.36 & $2.48 \mathrm{E}-02$ \\
\hline 9412 & NP9678742 & 5.66 & $2.17 \mathrm{E}-03$ & 9480 & NP9679861 & 1.96 & $1.69 \mathrm{E}-03$ & 9548 & NR4A2 & 3.91 & $1.11 \mathrm{E}-02$ \\
\hline 9413 & NP9678746 & 4.41 & $2.82 \mathrm{E}-02$ & 9481 & NP9679875 & 5.34 & $2.90 \mathrm{E}-04$ & 9549 & NRAP & 3.99 & $6.40 \mathrm{E}-03$ \\
\hline 9414 & NP9678769 & 4.85 & $5.60 \mathrm{E}-04$ & 9482 & NP9679899 & 1.56 & $1.91 \mathrm{E}-03$ & 9550 & NRG1 & 3.56 & $3.19 \mathrm{E}-05$ \\
\hline 9415 & NP9678793 & 3.15 & $8.28 \mathrm{E}-03$ & 9483 & NP9679904 & 3.35 & $3.30 \mathrm{E}-02$ & 9551 & NRIP3 & 5.02 & $5.97 E-03$ \\
\hline 9416 & NP9678807 & 2.33 & $1.36 \mathrm{E}-02$ & 9484 & NP9679923 & 1.23 & $2.69 \mathrm{E}-03$ & 9552 & NRN1 & 6.66 & $2.19 \mathrm{E}-03$ \\
\hline 9417 & NP9678814 & 1.68 & $1.02 \mathrm{E}-01$ & 9485 & NP9679942 & 3.20 & $4.14 \mathrm{E}-03$ & 9553 & NRP1 & 1.33 & 7.30E-02 \\
\hline 9418 & NP9678820 & 1.80 & $2.98 \mathrm{E}-03$ & 9486 & NP9679962 & 4.38 & $1.70 \mathrm{E}-03$ & 9554 & NRP2 & 4.15 & $5.85 \mathrm{E}-04$ \\
\hline 9419 & NP9678829 & 2.69 & $1.67 \mathrm{E}-03$ & 9487 & NP9679993 & 2.29 & $1.25 \mathrm{E}-02$ & 9555 & NRTN & 3.14 & $2.96 \mathrm{E}-02$ \\
\hline 9420 & NP9678870 & 4.40 & $2.31 \mathrm{E}-03$ & 9488 & NP9680050 & 3.09 & $8.40 \mathrm{E}-03$ & 9556 & NSD1 & 2.70 & $3.61 \mathrm{E}-03$ \\
\hline 9421 & NP9678904 & 6.89 & $8.66 \mathrm{E}-04$ & 9489 & NP9680151 & 2.85 & $1.04 \mathrm{E}-03$ & 9557 & NT5C1A & 2.63 & $1.16 \mathrm{E}-02$ \\
\hline 9422 & NP9678953 & 2.78 & $1.63 \mathrm{E}-02$ & 9490 & NP9680195 & 3.11 & $1.77 \mathrm{E}-03$ & 9558 & NT5DC3 & 1.15 & $1.23 \mathrm{E}-02$ \\
\hline 9423 & NP9678964 & 6.81 & $1.71 E-04$ & 9491 & NP9680311 & 2.81 & $1.06 \mathrm{E}-03$ & 9559 & NTF3 & 1.71 & $9.13 \mathrm{E}-03$ \\
\hline 9424 & NP9679007 & 3.86 & $3.58 \mathrm{E}-03$ & 9492 & NP9680344 & 2.96 & $2.57 \mathrm{E}-02$ & 9560 & NTN1 & 3.86 & $1.81 \mathrm{E}-03$ \\
\hline 9425 & NP9679071 & 6.17 & $3.38 \mathrm{E}-03$ & 9493 & NP9680352 & 3.46 & $1.85 \mathrm{E}-01$ & 9561 & NTNG1 & 1.46 & $1.50 \mathrm{E}-02$ \\
\hline 9426 & NP9679081 & 3.03 & 7.41E-03 & 9494 & NP9680357 & 3.00 & $5.58 \mathrm{E}-03$ & 9562 & NTRK1 & 4.83 & $1.05 \mathrm{E}-03$ \\
\hline 9427 & NP9679091 & 4.09 & $7.65 E-03$ & 9495 & NP9680378 & 5.53 & $3.35 \mathrm{E}-03$ & 9563 & NTRK3 & 1.40 & $5.87 \mathrm{E}-03$ \\
\hline 9428 & NP9679099 & 8.27 & $8.72 E-03$ & 9496 & NP9680381 & 5.10 & $1.30 \mathrm{E}-03$ & 9564 & NTS & 5.26 & $5.57 E-04$ \\
\hline 9429 & NP9679115 & 2.71 & $1.41 \mathrm{E}-01$ & 9497 & NP9680397 & 7.01 & $1.01 \mathrm{E}-03$ & 9565 & NUAK1 & 4.85 & $8.50 \mathrm{E}-03$ \\
\hline 9430 & NP9679179 & 2.52 & $4.46 \mathrm{E}-03$ & 9498 & NP9680400 & 3.80 & $1.10 \mathrm{E}-02$ & 9566 & NUAK2 & 2.30 & $3.26 \mathrm{E}-03$ \\
\hline 9431 & NP9679186 & 1.50 & $2.35 \mathrm{E}-03$ & 9499 & NP9680407 & 2.59 & $3.00 \mathrm{E}-05$ & 9567 & NUDT3 & 1.05 & $8.34 \mathrm{E}-03$ \\
\hline 9432 & NP9679194 & 4.32 & $2.34 \mathrm{E}-03$ & 9500 & NP9680436 & 5.54 & $3.10 \mathrm{E}-03$ & 9568 & NUFIP2 & 1.21 & 4.39E-03 \\
\hline 9433 & NP9679206 & 3.00 & $1.61 \mathrm{E}-02$ & 9501 & NP9680441 & 3.93 & $1.29 \mathrm{E}-03$ & 9569 & NUMA1 & 2.62 & $3.11 \mathrm{E}-03$ \\
\hline 9434 & NP9679227 & 7.04 & $2.07 E-05$ & 9502 & NP9680462 & 3.48 & $2.67 \mathrm{E}-03$ & 9570 & NXN & 1.56 & $1.59 \mathrm{E}-02$ \\
\hline 9435 & NP9679228 & 3.86 & $7.85 E-03$ & 9503 & NP9680478 & 6.83 & $9.20 \mathrm{E}-03$ & 9571 & NXNL1 & 4.29 & $2.31 \mathrm{E}-03$ \\
\hline 9436 & NP9679233 & 3.89 & $3.41 \mathrm{E}-03$ & 9504 & NP9680484 & 3.36 & $3.89 \mathrm{E}-03$ & 9572 & NXNL2 & 1.66 & $1.70 \mathrm{E}-03$ \\
\hline 9437 & NP9679240 & 6.67 & $2.16 \mathrm{E}-03$ & 9505 & NP9680489 & 3.64 & $1.62 \mathrm{E}-02$ & 9573 & NXPH1 & 1.43 & $2.08 \mathrm{E}-02$ \\
\hline 9438 & NP9679241 & 4.37 & $7.82 E-04$ & 9506 & NP9680495 & 6.96 & $1.12 \mathrm{E}-06$ & 9574 & NXPH3 & 5.91 & $2.02 \mathrm{E}-03$ \\
\hline 9439 & NP9679246 & 4.53 & $1.40 \mathrm{E}-02$ & 9507 & NP9680498 & 3.02 & $2.48 \mathrm{E}-03$ & 9575 & NYX & 4.58 & 4.57E-03 \\
\hline 9440 & NP9679276 & 5.65 & $2.37 \mathrm{E}-03$ & 9508 & NP9680515 & 4.25 & $1.90 \mathrm{E}-04$ & 9576 & OASL & 2.19 & $1.83 \mathrm{E}-02$ \\
\hline 9441 & NP9679288 & 9.09 & $2.70 \mathrm{E}-03$ & 9509 & NP9680518 & 2.11 & $1.82 \mathrm{E}-02$ & 9577 & OC3 & 3.24 & 7.98E-04 \\
\hline 9442 & NP9679312 & 4.49 & $7.49 E-04$ & 9510 & NP9680519 & 2.04 & $1.77 \mathrm{E}-02$ & 9578 & OC90 & 4.30 & 9.73E-04 \\
\hline 9443 & NP9679319 & 3.18 & $9.87 \mathrm{E}-03$ & 9511 & NP9680568 & 1.68 & $7.49 \mathrm{E}-02$ & 9579 & OCM & 5.98 & $1.53 \mathrm{E}-03$ \\
\hline 9444 & NP9679352 & 5.81 & $1.21 \mathrm{E}-03$ & 9512 & NP9680577 & 3.98 & $6.56 \mathrm{E}-04$ & 9580 & ODZ1 & 2.83 & $2.26 \mathrm{E}-03$ \\
\hline 9445 & NP9679369 & 4.03 & $2.18 \mathrm{E}-03$ & 9513 & NP9680580 & 4.09 & $1.32 \mathrm{E}-04$ & 9581 & ODZ4 & 3.17 & $2.05 E-03$ \\
\hline 9446 & NP9679391 & 3.15 & $2.23 \mathrm{E}-03$ & 9514 & NP9680582 & 6.63 & $2.29 \mathrm{E}-03$ & 9582 & OGFOD1 & 1.39 & 3.83E-03 \\
\hline 9447 & NP9679411 & 7.54 & $1.32 \mathrm{E}-03$ & 9515 & NP9680590 & 7.87 & $1.89 \mathrm{E}-03$ & 9583 & OGFR & 1.43 & $1.77 E-02$ \\
\hline 9448 & NP9679412 & 7.69 & $1.00 \mathrm{E}-03$ & 9516 & NP9680591 & 9.41 & $6.36 \mathrm{E}-03$ & 9584 & OGG1 & 4.88 & $4.16 \mathrm{E}-04$ \\
\hline 9449 & NP9679415 & 4.07 & $9.53 \mathrm{E}-06$ & 9517 & NP9680606 & 6.38 & $3.58 \mathrm{E}-05$ & 9585 & OGN & 1.53 & $1.24 \mathrm{E}-02$ \\
\hline 9450 & NP9679423 & 5.00 & $2.42 \mathrm{E}-02$ & 9518 & NP9680615 & 4.48 & $5.79 \mathrm{E}-03$ & 9586 & OІT3 & 2.15 & $3.32 \mathrm{E}-03$ \\
\hline 9451 & NP9679427 & 6.40 & $6.11 \mathrm{E}-04$ & 9519 & NP9680616 & 5.29 & $1.01 \mathrm{E}-03$ & 9587 & OLFM1 & 2.61 & $1.29 \mathrm{E}-02$ \\
\hline
\end{tabular}




\begin{tabular}{|c|c|c|c|c|c|c|c|c|c|c|c|}
\hline 9588 & OLFM4 & 5.10 & $5.22 \mathrm{E}-04$ & 9656 & PARK2 & 1.99 & $9.23 \mathrm{E}-02$ & 9724 & PIGH & 1.54 & 5.87E-03 \\
\hline 9589 & OLFML2B & 2.13 & $4.14 \mathrm{E}-03$ & 9657 & PARM1 & 1.82 & $1.16 \mathrm{E}-02$ & 9725 & PIGO & 3.18 & $1.75 \mathrm{E}-03$ \\
\hline 9590 & OLIG3 & 4.17 & $1.31 \mathrm{E}-02$ & 9658 & PARP3 & 1.83 & 1.40E-02 & 9726 & PIGR & 4.63 & $1.33 \mathrm{E}-02$ \\
\hline 9591 & OPCML & 1.75 & $1.73 \mathrm{E}-02$ & 9659 & PAX2 & 5.27 & $7.72 \mathrm{E}-03$ & 9727 & PIGU & 2.21 & $4.41 \mathrm{E}-04$ \\
\hline 9592 & OPN1LW & 4.61 & $2.96 \mathrm{E}-04$ & 9660 & PAX3 & 2.55 & $9.43 \mathrm{E}-03$ & 9728 & PIK3CG & 2.66 & $7.84 \mathrm{E}-04$ \\
\hline 9593 & OPN3 & 1.83 & $7.90 \mathrm{E}-03$ & 9661 & PAX5 & 3.52 & $1.77 \mathrm{E}-03$ & 9729 & PIK3R2 & 1.61 & $9.71 \mathrm{E}-03$ \\
\hline 9594 & OPN4 & 4.73 & $1.76 \mathrm{E}-03$ & 9662 & PAX6 & 7.44 & $2.54 \mathrm{E}-03$ & 9730 & PIK3R5 & 1.44 & $3.92 \mathrm{E}-02$ \\
\hline 9595 & OPRD1 & 4.98 & $5.97 \mathrm{E}-02$ & 9663 & PAX7 & 1.91 & $1.09 \mathrm{E}-02$ & 9731 & PIK3R6 & 2.66 & $6.17 \mathrm{E}-04$ \\
\hline 9596 & OPRK1 & 3.80 & $2.86 \mathrm{E}-03$ & 9664 & PAX9 & 1.95 & $5.19 \mathrm{E}-03$ & 9732 & PIP5K1A & 2.06 & 5.37E-03 \\
\hline 9597 & OPRL1 & 2.93 & $8.86 \mathrm{E}-03$ & 9665 & PBX4 & 1.41 & $9.48 \mathrm{E}-04$ & 9733 & PIT 54 & 5.92 & $1.20 \mathrm{E}-03$ \\
\hline 9598 & OPRM1 & 6.62 & $7.27 \mathrm{E}-03$ & 9666 & РСBP3 & 1.25 & $6.85 \mathrm{E}-03$ & 9734 & PITX1 & 7.61 & $8.22 \mathrm{E}-04$ \\
\hline 9599 & OPTC & 3.63 & $7.10 \mathrm{E}-04$ & 9667 & PCDH1 & 3.72 & $2.00 \mathrm{E}-03$ & 9735 & PITX3 & 4.45 & $3.05 \mathrm{E}-03$ \\
\hline 9600 & OR10A7 & 7.72 & $5.80 \mathrm{E}-03$ & 9668 & $\mathrm{PCDH} 10$ & 1.44 & $8.82 \mathrm{E}-03$ & 9736 & PKD1 & 4.59 & $3.84 \mathrm{E}-03$ \\
\hline 9601 & OR12D2 & 6.91 & $8.98 \mathrm{E}-05$ & 9669 & PCDH12 & 2.89 & 3.53E-02 & 9737 & PKD2L1 & 4.45 & $1.54 \mathrm{E}-02$ \\
\hline 9602 & OR51M1 & 5.11 & $6.52 \mathrm{E}-03$ & 9670 & PCDH17 & 2.18 & $3.93 \mathrm{E}-03$ & 9738 & PKHD1 & 1.51 & $4.45 \mathrm{E}-02$ \\
\hline 9603 & OR52R1 & 4.62 & $6.82 \mathrm{E}-04$ & 9671 & PCDH19 & 2.53 & $1.08 \mathrm{E}-02$ & 9739 & PKIA & 1.93 & $2.05 \mathrm{E}-02$ \\
\hline 9604 & OR6B3 & 3.55 & $7.63 \mathrm{E}-03$ & 9672 & $\mathrm{PCDH7}$ & 4.70 & $9.01 \mathrm{E}-03$ & 9740 & PKN3 & 6.37 & $1.93 \mathrm{E}-03$ \\
\hline 9605 & OR8D4 & 5.41 & 3.32E-04 & 9673 & $\mathrm{PCDH} 8$ & 3.13 & $1.00 \mathrm{E}-03$ & 9741 & PKP1 & 1.16 & $6.69 \mathrm{E}-03$ \\
\hline 9606 & OR9Q1 & 5.97 & $3.92 \mathrm{E}-03$ & 9674 & PCDH9 & 2.00 & $2.56 \mathrm{E}-02$ & 9742 & PLA1A & 1.46 & $9.29 \mathrm{E}-03$ \\
\hline 9607 & ORMDL3 & 1.29 & $4.39 \mathrm{E}-03$ & 9675 & PCDHA3 & 4.20 & $8.85 \mathrm{E}-04$ & 9743 & PLA2G12A & 2.33 & $3.69 \mathrm{E}-03$ \\
\hline 9608 & OSBP2 & 2.81 & $4.15 \mathrm{E}-02$ & 9676 & PCDHAC2 & 5.16 & $2.54 \mathrm{E}-04$ & 9744 & PLA2G12B & 4.22 & $2.31 \mathrm{E}-03$ \\
\hline 9609 & OSMR & 1.43 & $1.42 \mathrm{E}-03$ & 9677 & PCDHB4 & 7.22 & $5.81 \mathrm{E}-04$ & 9745 & PLA2G15 & 1.11 & $1.51 \mathrm{E}-02$ \\
\hline 9610 & OSR1 & 6.31 & $2.94 \mathrm{E}-03$ & 9678 & PCIF1 & 1.75 & $1.40 \mathrm{E}-02$ & 9746 & PLA2G2A & 2.62 & $3.39 \mathrm{E}-02$ \\
\hline 9611 & OSR2 & 2.69 & $1.01 \mathrm{E}-02$ & 9679 & PCK1 & 3.02 & $1.34 \mathrm{E}-02$ & 9747 & PLA2G2E & 2.24 & $1.76 \mathrm{E}-02$ \\
\hline 9612 & OSTN & 1.44 & $1.45 \mathrm{E}-01$ & 9680 & PCK2 & 2.28 & $2.80 \mathrm{E}-02$ & 9748 & PLA2G4E & 3.94 & $1.48 \mathrm{E}-03$ \\
\hline 9613 & отоF & 3.28 & $2.71 \mathrm{E}-03$ & 9681 & PCLO & 1.17 & $3.43 E-02$ & 9749 & PLA2G5 & 4.95 & $7.40 \mathrm{E}-03$ \\
\hline 9614 & OTOL1 & 3.93 & $5.16 \mathrm{E}-03$ & 9682 & PCMTD2 & 1.46 & $1.13 \mathrm{E}-02$ & 9750 & PLA2G6 & 3.20 & $4.42 \mathrm{E}-03$ \\
\hline 9615 & ОTOP2 & 4.19 & $1.68 \mathrm{E}-04$ & 9683 & PCSK2 & 3.98 & $1.23 \mathrm{E}-03$ & 9751 & PLAC9 & 2.63 & $8.64 \mathrm{E}-02$ \\
\hline 9616 & ОТОР3 & 5.82 & $1.55 \mathrm{E}-02$ & 9684 & PCSK6 & 1.52 & $1.73 \mathrm{E}-03$ & 9752 & PLAG1 & 3.19 & 3.63E-03 \\
\hline 9617 & OTOR & 3.46 & $4.90 \mathrm{E}-03$ & 9685 & PCSK9 & 2.87 & $2.82 \mathrm{E}-02$ & 9753 & PLAGL1 & 2.21 & $1.04 \mathrm{E}-02$ \\
\hline 9618 & OTP & 4.18 & $3.57 \mathrm{E}-03$ & 9686 & $\mathrm{PCX}$ & 3.01 & $3.07 \mathrm{E}-03$ & 9754 & PLAT & 1.23 & $1.02 \mathrm{E}-02$ \\
\hline 9619 & OTUD7A & 1.56 & 7.13E-02 & 9687 & PDC & 6.24 & $7.19 \mathrm{E}-04$ & 9755 & PLCD1 & 1.93 & $1.09 \mathrm{E}-01$ \\
\hline 9620 & OTX2 & 7.44 & $2.71 \mathrm{E}-03$ & 9688 & PDCD1 & 2.15 & $1.49 \mathrm{E}-02$ & 9756 & PLCH1 & 1.91 & $2.51 \mathrm{E}-03$ \\
\hline 9621 & OXGR1 & 3.16 & $4.25 \mathrm{E}-05$ & 9689 & PDE1A & 2.07 & $1.09 \mathrm{E}-02$ & 9757 & PLCL2 & 2.17 & $6.86 \mathrm{E}-04$ \\
\hline 9622 & OXTR & 2.71 & $4.99 \mathrm{E}-03$ & 9690 & PDE3A & 5.01 & $5.88 \mathrm{E}-04$ & 9758 & PLCXD1 & 4.23 & $3.39 \mathrm{E}-03$ \\
\hline 9623 & $\mathrm{P} 22$ & 4.90 & $2.94 \mathrm{E}-04$ & 9691 & PDE4A & 1.96 & 1.20E-03 & 9759 & PLD4 & 4.43 & $1.21 \mathrm{E}-02$ \\
\hline 9624 & P2RX3 & 3.93 & $2.63 \mathrm{E}-03$ & 9692 & PDE6B & 3.69 & $7.85 \mathrm{E}-03$ & 9760 & PLEK2 & 1.69 & $2.47 \mathrm{E}-02$ \\
\hline 9625 & P2RX7 & 5.23 & $6.60 \mathrm{E}-04$ & 9693 & PDE6H & 5.30 & $8.44 E-04$ & 9761 & PLEKHA2 & 3.27 & $3.30 \mathrm{E}-03$ \\
\hline 9626 & P2RY1 & 6.72 & $3.29 \mathrm{E}-03$ & 9694 & PDE7B & 2.16 & $5.75 \mathrm{E}-03$ & 9762 & PLEKHA7 & 1.70 & $4.81 \mathrm{E}-03$ \\
\hline 9627 & P2RY10 & 5.71 & $6.23 \mathrm{E}-03$ & 9695 & PDE8B & 2.40 & $4.66 \mathrm{E}-03$ & 9763 & PLEKHF1 & 3.89 & $1.13 \mathrm{E}-02$ \\
\hline 9628 & P2RY14 & 3.77 & $8.94 \mathrm{E}-03$ & 9696 & PDE9A & 1.54 & $9.60 \mathrm{E}-03$ & 9764 & PLEKHG1 & 3.02 & $1.38 \mathrm{E}-03$ \\
\hline 9629 & P2RY2 & 5.11 & $1.72 \mathrm{E}-03$ & 9697 & PDGFB & 2.69 & $5.39 \mathrm{E}-02$ & 9765 & PLEKHG5 & 4.24 & $1.50 \mathrm{E}-05$ \\
\hline 9630 & P2RY6 & 5.57 & $3.05 \mathrm{E}-03$ & 9698 & PDGFC & 2.02 & $8.65 \mathrm{E}-03$ & 9766 & PLEKHG7 & 2.05 & $8.79 \mathrm{E}-03$ \\
\hline 9631 & P2RY8 & 5.28 & $3.75 \mathrm{E}-03$ & 9699 & PDGFRA & 1.77 & $1.99 \mathrm{E}-02$ & 9767 & PLEKHH2 & 3.56 & 4.77E-02 \\
\hline 9632 & P4HA3 & 4.33 & $3.91 \mathrm{E}-03$ & 9700 & PDGFRB & 3.97 & $2.16 \mathrm{E}-03$ & 9768 & PLEKHM2 & 2.24 & $4.84 \mathrm{E}-03$ \\
\hline 9633 & PABPC1L & 2.78 & $2.56 \mathrm{E}-03$ & 9701 & PDPN & 1.48 & $1.88 \mathrm{E}-02$ & 9769 & PLEKHO2 & 3.62 & $2.04 \mathrm{E}-02$ \\
\hline 9634 & PABPN1 & 1.71 & $8.97 \mathrm{E}-02$ & 9702 & PDPR & 2.35 & $2.58 \mathrm{E}-02$ & 9770 & PLG & 2.32 & $6.11 \mathrm{E}-03$ \\
\hline 9635 & PACS1 & 2.48 & $5.05 \mathrm{E}-03$ & 9703 & PDX1 & 2.89 & $8.98 \mathrm{E}-03$ & 9771 & PLIN1 & 3.72 & $8.16 \mathrm{E}-04$ \\
\hline 9636 & PACSIN1 & 4.14 & $2.31 \mathrm{E}-02$ & 9704 & PDZK1IP1 & 2.14 & $3.75 \mathrm{E}-03$ & 9772 & PLIN3 & 4.49 & $6.25 \mathrm{E}-03$ \\
\hline 9637 & PADI2 & 3.38 & $9.01 \mathrm{E}-04$ & 9705 & PDZRN4 & 2.34 & 7.40E-03 & 9773 & PLK2 & 7.35 & $6.22 \mathrm{E}-03$ \\
\hline 9638 & PADI3 & 1.25 & $1.25 \mathrm{E}-02$ & 9706 & PEMT & 2.37 & $5.22 \mathrm{E}-02$ & 9774 & PLK3 & 2.00 & $6.13 \mathrm{E}-03$ \\
\hline 9639 & PAG1 & 2.35 & $9.52 \mathrm{E}-03$ & 9707 & PERP & 2.85 & $4.72 \mathrm{E}-03$ & 9775 & PLLP & 2.61 & $3.41 \mathrm{E}-03$ \\
\hline 9640 & PAH & 1.64 & $1.67 \mathrm{E}-02$ & 9708 & PFKFB2 & 1.56 & $4.04 \mathrm{E}-02$ & 9776 & PLN & 3.12 & $6.50 \mathrm{E}-03$ \\
\hline 9641 & PAK6 & 2.39 & $3.15 \mathrm{E}-03$ & 9709 & PFKL & 1.40 & $6.47 \mathrm{E}-03$ & 9777 & PLXNA1 & 2.83 & $2.03 \mathrm{E}-02$ \\
\hline 9642 & PAK7 & 2.43 & $9.55 \mathrm{E}-03$ & 9710 & PGLYRP2 & 4.27 & $5.42 \mathrm{E}-03$ & 9778 & PLXND1 & 3.83 & $6.82 \mathrm{E}-04$ \\
\hline 9643 & PALD1 & 1.15 & 4.67E-02 & 9711 & PGM3 & 1.97 & $1.43 \mathrm{E}-02$ & 9779 & PM5 & 4.70 & 2.37E-03 \\
\hline 9644 & PALM & 1.73 & $2.75 \mathrm{E}-03$ & 9712 & PHEX & 4.76 & $1.65 \mathrm{E}-04$ & 9780 & PMCH & 4.01 & $1.33 \mathrm{E}-02$ \\
\hline 9645 & PALMD & 2.07 & 4.37E-04 & 9713 & PHF12 & 5.47 & $6.09 \mathrm{E}-03$ & 9781 & PMEL & 3.72 & $5.26 \mathrm{E}-03$ \\
\hline 9646 & PAMR1 & 2.99 & $1.35 \mathrm{E}-03$ & 9714 & PHF16 & 1.58 & $1.62 \mathrm{E}-02$ & 9782 & PML & 5.08 & $1.29 \mathrm{E}-04$ \\
\hline 9647 & PANX1 & 1.28 & $2.24 \mathrm{E}-02$ & 9715 & PHGDH & 2.66 & $1.09 \mathrm{E}-02$ & 9783 & PMM1 & 3.05 & $3.61 \mathrm{E}-04$ \\
\hline 9648 & PANX2 & 3.12 & $1.04 \mathrm{E}-03$ & 9716 & PHLDA2 & 2.02 & $1.98 \mathrm{E}-02$ & 9784 & PMP2 & 3.68 & $2.10 \mathrm{E}-03$ \\
\hline 9649 & PANX3 & 2.65 & $4.56 \mathrm{E}-03$ & 9717 & PHOX2A & 5.29 & $1.86 \mathrm{E}-02$ & 9785 & PNAT10 & 2.07 & $4.22 \mathrm{E}-02$ \\
\hline 9650 & PAPD4 & 2.69 & $1.18 \mathrm{E}-02$ & 9718 & PHOX2B & 4.66 & $2.41 \mathrm{E}-02$ & 9786 & PNAT3 & 3.38 & 5.03E-04 \\
\hline 9651 & PAPPA & 1.71 & $9.25 \mathrm{E}-03$ & 9719 & PI15 & 4.93 & $2.61 \mathrm{E}-02$ & 9787 & PNKD & 5.52 & 7.42E-04 \\
\hline 9652 & PAPPA2 & 7.02 & $8.77 \mathrm{E}-03$ & 9720 & PI16 & 2.01 & $1.75 \mathrm{E}-03$ & 9788 & PNLIP & 3.77 & $3.09 \mathrm{E}-03$ \\
\hline 9653 & PAPSS2 & 2.38 & $1.54 \mathrm{E}-03$ & 9721 & PI4K2A & 2.30 & $8.75 E-03$ & 9789 & PNOC & 4.24 & $5.28 \mathrm{E}-03$ \\
\hline 9654 & PAQR5 & 3.05 & $1.86 \mathrm{E}-03$ & 9722 & PI4KB & 2.32 & $8.46 \mathrm{E}-04$ & 9790 & PNPLA1 & 2.88 & $5.09 \mathrm{E}-03$ \\
\hline 9655 & PARD6G & 2.90 & $2.79 \mathrm{E}-03$ & 9723 & PIDD & 3.37 & $1.88 \mathrm{E}-03$ & 9791 & PNPLA2 & 2.98 & $1.94 \mathrm{E}-03$ \\
\hline
\end{tabular}




\begin{tabular}{|c|c|c|c|c|c|c|c|c|c|c|c|}
\hline 9792 & PNPLA6 & 4.50 & 4.99E-04 & 9860 & PROM1 & 2.11 & $3.91 \mathrm{E}-03$ & 9928 & RAMP3 & 1.30 & 4.11E-02 \\
\hline 9793 & PODN & 5.38 & $8.98 \mathrm{E}-04$ & 9861 & PROP1 & 1.87 & 3.10E-02 & 9929 & RAP1GAP2 & 1.12 & $1.34 \mathrm{E}-02$ \\
\hline 9794 & POLH & 2.60 & $2.20 \mathrm{E}-02$ & 9862 & PROX1 & 1.39 & $1.92 \mathrm{E}-02$ & 9930 & RAP2B & 3.95 & $2.39 \mathrm{E}-03$ \\
\hline 9795 & POLK & 1.18 & $5.41 \mathrm{E}-03$ & 9863 & PROZ & 4.22 & $8.85 E-04$ & 9931 & RARA & 2.95 & $7.06 \mathrm{E}-03$ \\
\hline 9796 & POLR1B & 1.61 & $5.10 \mathrm{E}-03$ & 9864 & PRPH2 & 1.53 & $3.65 \mathrm{E}-03$ & 9932 & RAR-GAMMA2 & 3.03 & $6.17 \mathrm{E}-03$ \\
\hline 9797 & POLR3C & 4.25 & $5.67 \mathrm{E}-03$ & 9865 & PRR16 & 5.62 & $1.10 \mathrm{E}-02$ & 9933 & RARRES2 & 1.52 & $7.34 \mathrm{E}-03$ \\
\hline 9798 & POM121 & 2.41 & $4.41 \mathrm{E}-03$ & 9866 & PRR5L & 4.13 & $5.47 \mathrm{E}-03$ & 9934 & RASA4 & 4.02 & $3.34 \mathrm{E}-04$ \\
\hline 9799 & POMC & 10.17 & $3.00 \mathrm{E}-03$ & 9867 & PRRG3 & 3.45 & $3.82 \mathrm{E}-04$ & 9935 & RASAL1 & 2.95 & $1.05 \mathrm{E}-03$ \\
\hline 9800 & POMP & 3.41 & $3.39 \mathrm{E}-03$ & 9868 & PRRX1 & 1.75 & $6.99 \mathrm{E}-04$ & 9936 & RASD2 & 4.96 & $5.45 \mathrm{E}-05$ \\
\hline 9801 & POMT2 & 2.87 & $1.51 \mathrm{E}-03$ & 9869 & PRRX2 & 6.12 & $1.14 \mathrm{E}-03$ & 9937 & RASGEF1C & 2.57 & $3.50 \mathrm{E}-03$ \\
\hline 9802 & POPDC2 & 2.23 & 4.24E- 03 & 9870 & PRSS2 & 5.39 & $1.84 \mathrm{E}-03$ & 9938 & RASL10B & 3.60 & $3.97 \mathrm{E}-03$ \\
\hline 9803 & POR & 1.55 & $8.39 \mathrm{E}-03$ & 9871 & PRSS23 & 1.68 & $1.37 \mathrm{E}-02$ & 9939 & RASL11A & 3.90 & $2.59 \mathrm{E}-02$ \\
\hline 9804 & POU1F1 & 1.68 & $2.91 \mathrm{E}-04$ & 9872 & PRSS3 & 6.63 & $6.19 \mathrm{E}-04$ & 9940 & RASL12 & 1.94 & $2.80 \mathrm{E}-02$ \\
\hline 9805 & POU2AF1 & 5.75 & 4.40E-02 & 9873 & PRSS56 & 3.07 & $1.78 \mathrm{E}-04$ & 9941 & RASSF2 & 2.75 & $2.13 \mathrm{E}-02$ \\
\hline 9806 & POU2F3 & 3.56 & $1.19 \mathrm{E}-02$ & 9874 & PRSSL1 & 5.93 & $7.90 \mathrm{E}-03$ & 9942 & RASSF5 & 2.75 & $5.73 \mathrm{E}-03$ \\
\hline 9807 & POU3F1 & 3.00 & $3.02 E-03$ & 9875 & PRTFDC1 & 1.51 & $4.82 \mathrm{E}-03$ & 9943 & RASSF7 & 4.62 & $9.24 \mathrm{E}-03$ \\
\hline 9808 & POU4F1 & 4.38 & 2.57E-03 & 9876 & PRTG & 1.96 & 4.47E-04 & 9944 & RASSF9 & 1.34 & $8.32 \mathrm{E}-03$ \\
\hline 9809 & POU4F2 & 3.67 & $1.65 \mathrm{E}-03$ & 9877 & PRUNE & 2.32 & $1.06 \mathrm{E}-03$ & 9945 & RAX & 2.01 & 4.56E-04 \\
\hline 9810 & POU4F3 & 6.07 & $9.37 \mathrm{E}-03$ & 9878 & PSD & 3.51 & $9.31 \mathrm{E}-03$ & 9946 & RBL1 & 1.71 & $1.01 \mathrm{E}-03$ \\
\hline 9811 & POU6F2 & 3.93 & $5.35 \mathrm{E}-03$ & 9879 & PSKH1 & 3.05 & $2.08 \mathrm{E}-02$ & 9947 & RBM20 & 3.47 & $1.05 \mathrm{E}-02$ \\
\hline 9812 & POUV & 3.19 & $1.00 \mathrm{E}-03$ & 9880 & PSMD11 & 1.63 & $1.16 \mathrm{E}-02$ & 9948 & RBM47 & 3.35 & $2.55 \mathrm{E}-03$ \\
\hline 9813 & PPAP2B & 1.13 & $2.08 \mathrm{E}-02$ & 9881 & PSTK & 1.39 & $2.92 \mathrm{E}-02$ & 9949 & RBMS3 & 3.31 & $6.59 \mathrm{E}-03$ \\
\hline 9814 & PPAPDC1A & 3.29 & $5.30 \mathrm{E}-03$ & 9882 & PSTPIP1 & 3.59 & $3.69 \mathrm{E}-03$ & 9950 & RBP2 & 4.88 & 7.40E-04 \\
\hline 9815 & PPAPDC3 & 3.61 & $1.43 \mathrm{E}-04$ & 9883 & PSTPIP2 & 3.22 & 1.46E-03 & 9951 & RBP3 & 3.52 & $2.36 \mathrm{E}-02$ \\
\hline 9816 & PPARGC1A & 4.51 & $2.61 \mathrm{E}-03$ & 9884 & PTAFR & 4.64 & 3.11E-04 & 9952 & RBPJL & 1.88 & $1.41 \mathrm{E}-02$ \\
\hline 9817 & PPARGC1B & 2.82 & $5.06 \mathrm{E}-03$ & 9885 & PTAR1 & 1.41 & $4.23 \mathrm{E}-02$ & 9953 & $\mathrm{RCC2}$ & 2.16 & $2.40 \mathrm{E}-03$ \\
\hline 9818 & PPEF2 & 2.17 & $5.06 \mathrm{E}-03$ & 9886 & РTBP2 & 1.51 & $2.91 \mathrm{E}-03$ & 9954 & RCOR3 & 2.51 & $6.83 \mathrm{E}-03$ \\
\hline 9819 & PPFIA4 & 2.87 & $1.67 \mathrm{E}-02$ & 9887 & PTCH1 & 4.69 & $2.28 \mathrm{E}-04$ & 9955 & RCSD1 & 1.22 & $7.31 \mathrm{E}-03$ \\
\hline 9820 & PPIP5K1 & 3.41 & $9.10 \mathrm{E}-04$ & 9888 & PTCH2 & 3.09 & $1.23 \mathrm{E}-02$ & 9956 & $\mathrm{RDH} 8$ & 1.65 & $2.20 \mathrm{E}-02$ \\
\hline 9821 & PPL & 3.45 & $9.01 \mathrm{E}-03$ & 9889 & PTCHD2 & 3.70 & $1.85 \mathrm{E}-03$ & 9957 & REG4 & 2.42 & $2.51 \mathrm{E}-04$ \\
\hline 9822 & PPM1F & 1.81 & 7.69E-03 & 9890 & PTCHD4 & 4.58 & 3. $48 \mathrm{E}-03$ & 9958 & RELL2 & 5.43 & $1.04 \mathrm{E}-02$ \\
\hline 9823 & PPM1J & 3.18 & $1.84 \mathrm{E}-03$ & 9891 & PTGER2 & 3.25 & $8.51 \mathrm{E}-03$ & 9959 & REPS2 & 2.05 & 4.06E-04 \\
\hline 9824 & PPP1R1C & 1.97 & 5.19E- 03 & 9892 & PTGER3 & 2.15 & $6.16 \mathrm{E}-04$ & 9960 & RET & 1.82 & $1.70 \mathrm{E}-02$ \\
\hline 9825 & PPP1R3B & 3.90 & $1.37 \mathrm{E}-03$ & 9893 & PTGFR & 3.75 & $2.86 \mathrm{E}-03$ & 9961 & RFWD2 & 3.76 & $5.96 \mathrm{E}-03$ \\
\hline 9826 & PPP1R3C & 4.90 & $1.13 \mathrm{E}-03$ & 9894 & PTGFRN & 2.52 & $5.53 \mathrm{E}-03$ & 9962 & RFX2 & 1.90 & $8.64 \mathrm{E}-03$ \\
\hline 9827 & PPP1R3D & 2.57 & $2.79 \mathrm{E}-03$ & 9895 & PTGS1 & 4.32 & $2.84 \mathrm{E}-04$ & 9963 & RFX4 & 1.40 & $5.36 \mathrm{E}-02$ \\
\hline 9828 & PPP2R5D & 1.52 & $6.87 \mathrm{E}-03$ & 9896 & PTGS2 & 1.52 & $2.93 \mathrm{E}-03$ & 9964 & RFXAP & 2.28 & $4.88 \mathrm{E}-04$ \\
\hline 9829 & РPР3СB & 1.95 & $2.86 \mathrm{E}-02$ & 9897 & PTH & 5.25 & $1.32 \mathrm{E}-03$ & 9965 & RFXDC2 & 5.52 & 7.14E-04 \\
\hline 9830 & PPY & 3.05 & 5.09E-03 & 9898 & PTPN22 & 4.28 & $1.26 \mathrm{E}-02$ & 9966 & RGMA & 4.23 & $2.68 \mathrm{E}-03$ \\
\hline 9831 & PPYR1 & 5.99 & $1.99 \mathrm{E}-03$ & 9899 & PTPN7 & 4.31 & $8.69 \mathrm{E}-04$ & 9967 & RGS17 & 4.16 & 4.94E-04 \\
\hline 9832 & PQLC3 & 1.06 & $1.66 \mathrm{E}-02$ & 9900 & PTPN9 & 1.78 & $7.72 \mathrm{E}-03$ & 9968 & RGS18 & 2.08 & $7.28 \mathrm{E}-03$ \\
\hline 9833 & PRAM1 & 1.89 & $7.26 \mathrm{E}-03$ & 9901 & PTPRF & 1.95 & 3.24E-03 & 9969 & RGS19 & 2.95 & $9.06 \mathrm{E}-04$ \\
\hline 9834 & PRCP & 1.58 & 3.25E-02 & 9902 & PTPRO & 2.83 & $1.15 \mathrm{E}-02$ & 9970 & RGS20 & 1.36 & $9.47 \mathrm{E}-03$ \\
\hline 9835 & PRDM12 & 4.17 & $1.06 \mathrm{E}-03$ & 9903 & PTPRR & 2.23 & $2.80 \mathrm{E}-03$ & 9971 & RGS21 & 2.62 & 5.05E-03 \\
\hline 9836 & PRDM6 & 3.64 & $2.71 E-03$ & 9904 & PTPRU & 4.15 & 4.60E-05 & 9972 & RGS5 & 1.66 & $1.35 \mathrm{E}-01$ \\
\hline 9837 & PRDM9 & 3.98 & $2.32 \mathrm{E}-04$ & 9905 & PTPRZ1 & 1.49 & $2.82 \mathrm{E}-03$ & 9973 & RGSL1 & 1.17 & $2.40 \mathrm{E}-02$ \\
\hline 9838 & PRELP & 3.43 & 5.35E-04 & 9906 & PTX3 & 2.36 & $2.31 \mathrm{E}-02$ & 9974 & RHAG & 4.39 & $9.57 \mathrm{E}-03$ \\
\hline 9839 & PRF1 & 4.92 & $1.14 \mathrm{E}-02$ & 9907 & PVALB & 1.62 & $1.93 \mathrm{E}-02$ & 9975 & RHBG & 1.50 & $1.63 \mathrm{E}-02$ \\
\hline 9840 & PRICKLE1 & 1.10 & $4.37 \mathrm{E}-03$ & 9908 & PWWP2A & 1.34 & $6.12 \mathrm{E}-03$ & 9976 & RHO & 2.34 & $3.54 \mathrm{E}-02$ \\
\hline 9841 & PRICKLE2 & 4.32 & $8.06 \mathrm{E}-03$ & 9909 & PXDN & 1.18 & $1.37 \mathrm{E}-02$ & 9977 & $\mathrm{RHOH}$ & 6.70 & $1.03 \mathrm{E}-04$ \\
\hline 9842 & PRIMA1 & 3.90 & $1.43 \mathrm{E}-02$ & 9910 & PXDNL & 5.51 & $2.31 \mathrm{E}-02$ & 9978 & RHOU & 2.03 & $1.05 \mathrm{E}-02$ \\
\hline 9843 & PRKAA2 & 3.27 & $2.17 \mathrm{E}-02$ & 9911 & PYROXD2 & 3.19 & $8.23 \mathrm{E}-02$ & 9979 & RHOV & 3.54 & $3.03 \mathrm{E}-03$ \\
\hline 9844 & PRKAG3 & 5.06 & $1.55 \mathrm{E}-02$ & 9912 & QRFPR & 2.27 & $1.92 \mathrm{E}-03$ & 9980 & RIC8A & 1.62 & $9.59 \mathrm{E}-03$ \\
\hline 9845 & PRKCB & 2.85 & $5.92 \mathrm{E}-03$ & 9913 & QSOX1 & 1.84 & $4.75 E-02$ & 9981 & RIMKLB & 1.61 & $1.22 \mathrm{E}-03$ \\
\hline 9846 & PRKCE & 2.82 & $2.90 \mathrm{E}-03$ & 9914 & RAB26 & 4.97 & $1.04 \mathrm{E}-03$ & 9982 & RIMS3 & 2.71 & $3.92 \mathrm{E}-03$ \\
\hline 9847 & PRKCH & 1.93 & $6.35 \mathrm{E}-02$ & 9915 & RAB39B & 3.15 & $1.26 \mathrm{E}-03$ & 9983 & RIMS4 & 2.93 & 2.13E- 04 \\
\hline 9848 & PRKD1 & 2.86 & $1.25 \mathrm{E}-02$ & 9916 & RAB3C & 3.63 & $1.03 \mathrm{E}-02$ & 9984 & RIPK1 & 1.18 & $1.24 \mathrm{E}-02$ \\
\hline 9849 & PRKDC & 1.80 & $6.80 \mathrm{E}-04$ & 9917 & RAB3IL1 & 2.83 & $1.10 \mathrm{E}-03$ & 9985 & RIPK2 & 2.55 & 3.67E-02 \\
\hline 9850 & PRKG1 & 7.69 & $5.70 \mathrm{E}-03$ & 9918 & RAB $40 C$ & 2.39 & 4.17E-03 & 9986 & RIPK4 & 1.67 & $1.34 \mathrm{E}-03$ \\
\hline 9851 & PRKG2 & 4.31 & $1.61 \mathrm{E}-03$ & 9919 & RAB43 & 1.67 & $3.85 \mathrm{E}-03$ & 9987 & RIPPLY1 & 3.42 & $4.01 \mathrm{E}-03$ \\
\hline 9852 & PRL & 4.32 & $1.03 E-02$ & 9920 & RAB44 & 4.88 & $7.92 \mathrm{E}-05$ & 9988 & RIT2 & 7.00 & $1.13 \mathrm{E}-03$ \\
\hline 9853 & PRLH & 2.63 & $1.40 \mathrm{E}-01$ & 9921 & RAB7L1 & 2.01 & $2.54 \mathrm{E}-03$ & 9989 & RLTPR & 4.21 & $2.87 \mathrm{E}-03$ \\
\hline 9854 & PRLHR & 5.17 & $1.99 \mathrm{E}-04$ & 9922 & RAB9B & 4.08 & $8.71 \mathrm{E}-04$ & 9990 & RMI2 & 2.25 & $1.14 \mathrm{E}-03$ \\
\hline 9855 & PRLHR2 & 2.13 & $2.27 \mathrm{E}-03$ & 9923 & RABIF & 1.25 & $1.35 \mathrm{E}-02$ & 9991 & RNASEL & 3.00 & $9.61 \mathrm{E}-04$ \\
\hline 9856 & PRODH & 1.50 & $2.34 \mathrm{E}-02$ & 9924 & RAC2 & 2.19 & $2.27 \mathrm{E}-03$ & 9992 & RND2 & 3.07 & $3.62 \mathrm{E}-05$ \\
\hline 9857 & PROK1 & 2.38 & $2.67 \mathrm{E}-03$ & 9925 & RAD9A & 2.83 & $2.25 \mathrm{E}-04$ & 9993 & RNF123 & 2.51 & $2.24 \mathrm{E}-03$ \\
\hline 9858 & PROK2 & 1.45 & $1.18 \mathrm{E}-02$ & 9926 & RALY & 4.03 & $6.85 \mathrm{E}-04$ & 9994 & RNF165 & 3.46 & 7.52E-03 \\
\hline 9859 & PROKR2 & 6.35 & $7.65 \mathrm{E}-04$ & 9927 & RALYL & 3.37 & $1.76 \mathrm{E}-03$ & 9995 & RNF182 & 3.10 & $1.70 \mathrm{E}-04$ \\
\hline
\end{tabular}




\begin{tabular}{|c|c|c|c|c|c|c|c|c|c|c|c|}
\hline 9996 & RNF208 & 4.65 & $1.79 \mathrm{E}-03$ & 10064 & SCT & 3.25 & $2.02 \mathrm{E}-02$ & 10132 & SHISA6 & 1.41 & $1.23 \mathrm{E}-01$ \\
\hline 9997 & RNF222 & 4.49 & $3.70 \mathrm{E}-05$ & 10065 & SCUBE3 & 2.78 & $1.88 \mathrm{E}-03$ & 10133 & SHMT1 & 2.27 & $1.26 \mathrm{E}-02$ \\
\hline 9998 & RNF223 & 3.75 & $6.08 \mathrm{E}-03$ & 10066 & SDK2 & 4.68 & $2.36 \mathrm{E}-04$ & 10134 & SHOX & 6.28 & $3.57 \mathrm{E}-03$ \\
\hline 9999 & RNFT2 & 1.46 & $4.59 \mathrm{E}-03$ & 10067 & SEBOX & 4.85 & $1.56 \mathrm{E}-03$ & 10135 & SHROOM1 & 1.37 & $9.22 \mathrm{E}-03$ \\
\hline 10000 & RORB & 1.43 & $8.17 \mathrm{E}-03$ & 10068 & SEC16B & 1.43 & $1.57 E-02$ & 10136 & SHROOM2 & 2.21 & $4.19 \mathrm{E}-03$ \\
\hline 10001 & ROS1 & 3.93 & $1.79 \mathrm{E}-03$ & 10069 & SELP & 2.41 & $1.14 \mathrm{E}-02$ & 10137 & $\mathrm{SI}$ & 3.19 & $3.48 \mathrm{E}-03$ \\
\hline 10002 & RPP25 & 4.67 & $3.79 \mathrm{E}-04$ & 10070 & SEMA3A & 1.68 & $7.96 \mathrm{E}-03$ & 10138 & SIGIRR & 4.97 & 4.04E-04 \\
\hline 10003 & RPRML & 6.86 & $7.49 \mathrm{E}-03$ & 10071 & SEMA3B & 3.46 & $3.75 \mathrm{E}-04$ & 10139 & SIGMAR1 & 3.64 & $1.21 \mathrm{E}-03$ \\
\hline 10004 & RPUSD1 & 4.28 & $6.79 \mathrm{E}-03$ & 10072 & SEMA3C & 1.96 & $2.39 \mathrm{E}-03$ & 10140 & SIK2 & 3.07 & 5.97E-03 \\
\hline 10005 & RPUSD2 & 2.71 & $5.43 \mathrm{E}-03$ & 10073 & SEMA3E & 5.07 & $1.54 \mathrm{E}-03$ & 10141 & SIM1 & 6.39 & $2.90 \mathrm{E}-03$ \\
\hline 10006 & RRH & 3.05 & $3.83 \mathrm{E}-02$ & 10074 & SEMA3F & 4.11 & $1.05 \mathrm{E}-02$ & 10142 & SIM2 & 3.52 & $1.85 \mathrm{E}-03$ \\
\hline 10007 & RRP12 & 1.84 & $3.50 \mathrm{E}-02$ & 10075 & SEMA3G & 3.63 & $1.57 \mathrm{E}-04$ & 10143 & SIN3A & 1.33 & 4.67E-06 \\
\hline 10008 & RSAD2 & 5.70 & $3.20 \mathrm{E}-03$ & 10076 & SEMA4G & 1.73 & 3.88E-02 & 10144 & SIRPA & 3.37 & $9.02 \mathrm{E}-03$ \\
\hline 10009 & RSPO1 & 4.26 & $2.95 \mathrm{E}-02$ & 10077 & SEMA5A & 1.37 & 2.62E-04 & 10145 & SIX1 & 4.95 & 3.59E-03 \\
\hline 10010 & RSPO2 & 2.53 & $4.22 \mathrm{E}-02$ & 10078 & SEMA5B & 1.81 & $2.53 E-02$ & 10146 & SIX2 & 4.89 & $2.09 \mathrm{E}-03$ \\
\hline 10011 & RSRC2 & 8.23 & 7.99E-04 & 10079 & SEMA6A & 2.46 & $4.55 \mathrm{E}-03$ & 10147 & SIX3 & 3.58 & $1.37 \mathrm{E}-03$ \\
\hline 10012 & RTBDN & 1.76 & $6.18 \mathrm{E}-03$ & 10080 & SEMA6B & 4.31 & $1.35 \mathrm{E}-03$ & 10148 & SIX6 & 2.17 & $3.99 \mathrm{E}-03$ \\
\hline 10013 & RTN4R & 3.88 & $5.99 \mathrm{E}-03$ & 10081 & SENP2 & 4.41 & $3.86 \mathrm{E}-04$ & 10149 & SKIDA1 & 2.19 & $1.80 \mathrm{E}-02$ \\
\hline 10014 & RTN4RL1 & 3.95 & $6.55 \mathrm{E}-03$ & 10082 & SERGEF & 1.66 & $2.75 \mathrm{E}-02$ & 10150 & SLA & 1.17 & $1.13 \mathrm{E}-01$ \\
\hline 10015 & RTN4RL2 & 2.80 & $1.11 \mathrm{E}-03$ & 10083 & SERINC2 & 2.29 & $1.21 \mathrm{E}-02$ & 10151 & SLAIN2 & 1.67 & $8.70 \mathrm{E}-04$ \\
\hline 10016 & RUNX2 & 6.58 & $2.26 \mathrm{E}-03$ & 10084 & SERPINA1 & 6.58 & $1.22 \mathrm{E}-02$ & 10152 & SLAMF8 & 5.73 & 5.57E-03 \\
\hline 10017 & RUNX3 & 3.76 & $2.01 \mathrm{E}-03$ & 10085 & SERPINA10 & 1.25 & $2.42 E-03$ & 10153 & SLC10A2 & 7.25 & $2.61 \mathrm{E}-03$ \\
\hline 10018 & RXFP1 & 2.57 & $6.91 \mathrm{E}-04$ & 10086 & SERPINA3 & 5.66 & $1.89 \mathrm{E}-04$ & 10154 & SLC10A4 & 1.52 & $1.05 \mathrm{E}-02$ \\
\hline 10019 & RXFP3 & 7.15 & $5.53 \mathrm{E}-04$ & 10087 & SERPINA4 & 5.94 & $1.96 \mathrm{E}-05$ & 10155 & SLC11A1 & 3.51 & $4.62 \mathrm{E}-04$ \\
\hline 10020 & RXRA & 1.50 & $7.40 \mathrm{E}-03$ & 10088 & SERPINA5 & 4.73 & $1.39 \mathrm{E}-03$ & 10156 & SLC11A2 & 2.28 & $3.30 \mathrm{E}-02$ \\
\hline 10021 & RXRG & 3.28 & $9.95 \mathrm{E}-04$ & 10089 & SERPINA9 & 5.80 & $7.71 \mathrm{E}-03$ & 10157 & SLC12A9 & 3.55 & $9.89 \mathrm{E}-04$ \\
\hline 10022 & S100A9 & 3.06 & 3.95E-02 & 10090 & SERPINB1 & 2.03 & $9.44 \mathrm{E}-03$ & 10158 & SLC13A1 & 2.36 & 2.67E-04 \\
\hline 10023 & S100z & 5.17 & $5.16 \mathrm{E}-03$ & 10091 & SERPINB10 & 4.22 & $2.39 \mathrm{E}-04$ & 10159 & SLC13A2 & 2.84 & $2.42 \mathrm{E}-03$ \\
\hline 10024 & S1PR1 & 3.90 & $4.83 \mathrm{E}-04$ & 10092 & SERPINB12 & 4.60 & $1.10 \mathrm{E}-03$ & 10160 & SLC13A4 & 6.68 & $1.63 \mathrm{E}-03$ \\
\hline 10025 & S1PR3 & 4.01 & 2.49E-03 & 10093 & SERPINB14 & 2.60 & $6.41 \mathrm{E}-03$ & 10161 & SLC15A2 & 1.64 & $5.74 \mathrm{E}-02$ \\
\hline 10026 & S1PR4 & 3.45 & $8.41 \mathrm{E}-04$ & 10094 & SERPINB14B & 4.04 & $2.94 \mathrm{E}-03$ & 10162 & SLC15A4 & 1.34 & $1.06 \mathrm{E}-02$ \\
\hline 10027 & S71050 & 1.23 & $3.12 \mathrm{E}-03$ & 10095 & SERPINB2 & 2.89 & $1.23 \mathrm{E}-03$ & 10163 & SLC15A5 & 1.82 & $1.13 \mathrm{E}-02$ \\
\hline 10028 & SACS & 1.79 & $1.89 \mathrm{E}-03$ & 10096 & SERPINB5 & 2.19 & $1.09 \mathrm{E}-02$ & 10164 & SLC16A10 & 2.06 & $5.83 \mathrm{E}-03$ \\
\hline 10029 & SALL3 & 3.06 & $1.52 \mathrm{E}-02$ & 10097 & SERPINBP1 & 4.49 & $5.86 \mathrm{E}-03$ & 10165 & SLC16A12 & 5.60 & $2.03 \mathrm{E}-03$ \\
\hline 10030 & SAMD10 & 3.99 & $1.32 \mathrm{E}-03$ & 10098 & SERPINC1 & 5.58 & $6.25 \mathrm{E}-04$ & 10166 & SLC16A13 & 6.11 & $2.62 \mathrm{E}-03$ \\
\hline 10031 & SAMD5 & 2.39 & $2.15 \mathrm{E}-02$ & 10099 & SERPIND1 & 2.56 & $5.60 \mathrm{E}-04$ & 10167 & SLC16A2 & 2.73 & $2.61 \mathrm{E}-03$ \\
\hline 10032 & SAMD7 & 5.15 & $2.11 \mathrm{E}-03$ & 10100 & SERPING1 & 1.33 & $5.07 E-02$ & 10168 & SLC16A4 & 3.53 & $4.46 \mathrm{E}-04$ \\
\hline 10033 & SAMD9L & 3.16 & $2.88 \mathrm{E}-03$ & 10101 & SERTAD2 & 1.38 & $2.54 \mathrm{E}-02$ & 10169 & SLC16A7 & 1.93 & $8.67 \mathrm{E}-03$ \\
\hline 10034 & SAMSN1 & 1.26 & $1.19 \mathrm{E}-02$ & 10102 & SESN2 & 2.20 & $9.70 \mathrm{E}-03$ & 10170 & SLC16A8 & 1.90 & $2.29 \mathrm{E}-03$ \\
\hline 10035 & SARDH & 3.22 & $5.48 \mathrm{E}-03$ & 10103 & SESN3 & 1.76 & $1.13 E-02$ & 10171 & SLC17A6 & 7.70 & $2.15 \mathrm{E}-03$ \\
\hline 10036 & SARM1 & 4.03 & $2.94 \mathrm{E}-03$ & 10104 & SETBP1 & 4.55 & $2.12 \mathrm{E}-03$ & 10172 & SLC18A1 & 3.05 & $1.01 \mathrm{E}-02$ \\
\hline 10037 & SASH3 & 2.83 & $2.19 \mathrm{E}-02$ & 10105 & SEZ6 & 3.92 & $3.38 \mathrm{E}-02$ & 10173 & SLC18A3 & 6.37 & $5.95 \mathrm{E}-03$ \\
\hline 10038 & SATB1 & 2.99 & $3.02 E-03$ & 10106 & SEZ6L & 2.39 & $2.87 \mathrm{E}-03$ & 10174 & SLC19A2 & 1.91 & $8.66 \mathrm{E}-03$ \\
\hline 10039 & SATB2 & 2.41 & $6.80 \mathrm{E}-03$ & 10107 & SFI1 & 1.27 & $1.82 \mathrm{E}-02$ & 10175 & SLC19A3 & 2.03 & 5.09E- 03 \\
\hline 10040 & SBF1 & 1.90 & $2.12 \mathrm{E}-03$ & 10108 & SFN & 5.16 & $2.20 \mathrm{E}-03$ & 10176 & SLC1A1 & 3.39 & $4.63 \mathrm{E}-04$ \\
\hline 10041 & SBF2 & 1.46 & $1.79 \mathrm{E}-02$ & 10109 & SFRP1 & 2.03 & $2.40 \mathrm{E}-03$ & 10177 & SLC1A2 & 2.54 & $2.25 \mathrm{E}-03$ \\
\hline 10042 & SBK1 & 4.62 & $1.52 \mathrm{E}-03$ & 10110 & SFRP5 & 2.37 & $5.57 \mathrm{E}-02$ & 10178 & SLC1A3 & 4.66 & $1.48 \mathrm{E}-03$ \\
\hline 10043 & SBK2 & 2.64 & $2.11 \mathrm{E}-02$ & 10111 & SFTPA1 & 2.84 & $5.26 \mathrm{E}-03$ & 10179 & SLC1A6 & 3.99 & $1.21 \mathrm{E}-02$ \\
\hline 10044 & SBNO2 & 1.65 & $7.54 \mathrm{E}-03$ & 10112 & $\mathrm{SGCD}$ & 5.50 & $1.36 \mathrm{E}-03$ & 10180 & SLC20A1 & 1.24 & $1.87 \mathrm{E}-02$ \\
\hline 10045 & SBSPON & 1.15 & $2.65 \mathrm{E}-02$ & 10113 & SGCG & 2.57 & $6.05 \mathrm{E}-03$ & 10181 & SLC22A13 & 2.63 & $1.18 \mathrm{E}-03$ \\
\hline 10046 & SCAMP4 & 2.72 & $5.35 \mathrm{E}-03$ & 10114 & SGCZ & 5.02 & $2.92 \mathrm{E}-04$ & 10182 & SLC22A15 & 5.40 & $1.78 \mathrm{E}-03$ \\
\hline 10047 & SCARA5 & 2.02 & $8.09 \mathrm{E}-03$ & 10115 & SGIP1 & 4.49 & $8.51 \mathrm{E}-03$ & 10183 & SLC22A18 & 4.24 & $1.67 \mathrm{E}-03$ \\
\hline 10048 & SCARB1 & 2.07 & $1.60 \mathrm{E}-03$ & 10116 & SGK2 & 2.63 & $1.94 \mathrm{E}-03$ & 10184 & SLC22A2 & 3.02 & $7.34 \mathrm{E}-03$ \\
\hline 10049 & SCARF1 & 10.32 & $3.25 \mathrm{E}-03$ & 10117 & SGPP1 & 1.68 & $4.62 \mathrm{E}-03$ & 10185 & SLC22A3 & 1.37 & $1.82 \mathrm{E}-02$ \\
\hline 10050 & $S C D$ & 1.68 & $1.14 \mathrm{E}-03$ & 10118 & SGPP2 & 2.07 & $1.09 \mathrm{E}-02$ & 10186 & SLC22A4 & 2.99 & $1.13 \mathrm{E}-03$ \\
\hline 10051 & SCFD2 & 2.10 & $2.37 \mathrm{E}-03$ & 10119 & SGSH & 3.12 & $5.90 \mathrm{E}-04$ & 10187 & SLC23A1 & 3.00 & $1.68 \mathrm{E}-02$ \\
\hline 10052 & SCG2 & 1.27 & 7.91E-03 & 10120 & SH2B2 & 4.56 & $6.51 \mathrm{E}-04$ & 10188 & SLC23A3 & 3.78 & $1.40 \mathrm{E}-03$ \\
\hline 10053 & SCGN & 4.25 & $8.45 \mathrm{E}-04$ & 10121 & SH3BP1 & 5.04 & $9.01 \mathrm{E}-04$ & 10189 & SLC24A2 & 4.57 & $1.68 \mathrm{E}-03$ \\
\hline 10054 & SCIN & 1.07 & $3.54 \mathrm{E}-04$ & 10122 & SH3GL2 & 2.16 & $1.11 \mathrm{E}-02$ & 10190 & SLC24A6 & 1.60 & $9.55 \mathrm{E}-03$ \\
\hline 10055 & SCML4 & 4.04 & $1.02 \mathrm{E}-06$ & 10123 & SH3PXD2A & 1.44 & $5.83 \mathrm{E}-03$ & 10191 & SLC25A1 & 1.92 & 2.63E-02 \\
\hline 10056 & SCN3A & 4.58 & $4.27 \mathrm{E}-05$ & 10124 & SH3RF1 & 1.67 & $7.21 \mathrm{E}-03$ & 10192 & SLC25A10 & 3.46 & $1.83 \mathrm{E}-04$ \\
\hline 10057 & $\mathrm{SCN} 3 \mathrm{~B}$ & 2.18 & $1.73 \mathrm{E}-02$ & 10125 & SH3RF3 & 2.96 & $5.32 \mathrm{E}-04$ & 10193 & SLC25A30 & 1.48 & $8.61 \mathrm{E}-02$ \\
\hline 10058 & SCN4B & 2.42 & $2.13 \mathrm{E}-03$ & 10126 & SH3TC2 & 6.44 & $1.43 E-04$ & 10194 & SLC25A38 & 2.93 & $1.07 \mathrm{E}-02$ \\
\hline 10059 & SCN8A & 1.62 & $3.27 \mathrm{E}-02$ & 10127 & SHB & 1.17 & $1.63 \mathrm{E}-02$ & 10195 & SLC25A47 & 5.95 & $2.74 \mathrm{E}-03$ \\
\hline 10060 & SCNN1B & 3.06 & $2.19 \mathrm{E}-03$ & 10128 & SHC1 & 1.48 & $7.88 \mathrm{E}-03$ & 10196 & SLC26A4 & 1.80 & $1.49 \mathrm{E}-03$ \\
\hline 10061 & SCNN1G & 2.35 & $2.35 \mathrm{E}-02$ & 10129 & SHC2 & 2.43 & $3.94 \mathrm{E}-02$ & 10197 & SLC26A7 & 1.73 & $7.04 \mathrm{E}-02$ \\
\hline 10062 & SCRG1 & 4.95 & $6.05 \mathrm{E}-04$ & 10130 & SHF & 1.56 & $2.40 \mathrm{E}-04$ & 10198 & SLC26A9 & 3.51 & $1.43 \mathrm{E}-03$ \\
\hline 10063 & SCRN2 & 4.18 & $4.26 \mathrm{E}-03$ & 10131 & SHH & 5.67 & $1.08 \mathrm{E}-03$ & 10199 & SLC28A2 & 1.02 & $5.81 \mathrm{E}-02$ \\
\hline
\end{tabular}




\begin{tabular}{|c|c|c|c|c|c|c|c|c|c|c|c|}
\hline 10200 & SLC29A1 & 3.01 & $5.52 E-03$ & 10268 & SMCR7L & 1.39 & $1.10 \mathrm{E}-02$ & 10336 & SSTR2 & 4.15 & $8.38 \mathrm{E}-05$ \\
\hline 10201 & SLC29A3 & 3.35 & $6.23 \mathrm{E}-03$ & 10269 & SMG5 & 1.96 & $1.72 \mathrm{E}-02$ & 10337 & SSTR3 & 4.97 & $2.81 \mathrm{E}-03$ \\
\hline 10202 & SLC29A4 & 6.45 & $3.38 \mathrm{E}-02$ & 10270 & SMOX & 3.68 & $3.51 \mathrm{E}-02$ & 10338 & SSTR4 & 4.26 & 3.30E-03 \\
\hline 10203 & SLC2A11 & 1.46 & $1.31 \mathrm{E}-02$ & 10271 & SMPD3 & 4.28 & $8.91 \mathrm{E}-03$ & 10339 & SSTR5 & 6.79 & 5.33E- 05 \\
\hline 10204 & SLC2A2 & 3.83 & $1.68 \mathrm{E}-03$ & 10272 & SMPDL3B & 2.03 & $2.78 \mathrm{E}-02$ & 10340 & ST18 & 3.98 & $1.95 \mathrm{E}-03$ \\
\hline 10205 & SLC2A6 & 5.09 & $1.71 \mathrm{E}-03$ & 10273 & SMPX & 1.99 & $5.48 \mathrm{E}-03$ & 10341 & ST3GAL4 & 5.23 & $1.48 \mathrm{E}-03$ \\
\hline 10206 & SLC30A2 & 3.64 & $1.73 \mathrm{E}-03$ & 10274 & SMUG1 & 8.04 & $5.78 \mathrm{E}-04$ & 10342 & ST6GAL2 & 5.38 & $1.50 \mathrm{E}-03$ \\
\hline 10207 & SLC30A8 & 1.58 & $2.97 \mathrm{E}-02$ & 10275 & SMURF1 & 2.78 & $3.43 \mathrm{E}-03$ & 10343 & ST6GALNAC4 & 5.82 & $4.18 \mathrm{E}-03$ \\
\hline 10208 & SLC31A2 & 1.70 & $2.75 \mathrm{E}-03$ & 10276 & SNAI1 & 3.02 & $2.93 \mathrm{E}-03$ & 10344 & ST6GALNAC5 & 1.63 & $4.54 \mathrm{E}-03$ \\
\hline 10209 & SLC32A1 & 5.55 & $5.97 \mathrm{E}-03$ & 10277 & SNAP25 & 3.99 & $2.52 \mathrm{E}-04$ & 10345 & ST8SIA1 & 2.41 & 7.02E-04 \\
\hline 10210 & SLC34A2 & 1.61 & $3.25 \mathrm{E}-03$ & 10278 & SNAP91 & 1.95 & $1.17 \mathrm{E}-02$ & 10346 & ST8SIA4 & 2.51 & $1.04 \mathrm{E}-02$ \\
\hline 10211 & SLC35A4 & 5.15 & $2.98 \mathrm{E}-03$ & 10279 & SNCB & 3.58 & $5.53 \mathrm{E}-03$ & 10347 & ST8SIA5 & 1.94 & $1.32 \mathrm{E}-01$ \\
\hline 10212 & SLC35B2 & 4.15 & $8.60 \mathrm{E}-04$ & 10280 & SNCG & 1.53 & $2.85 \mathrm{E}-02$ & 10348 & STAC & 2.89 & $1.43 \mathrm{E}-03$ \\
\hline 10213 & SLC35D1 & 2.65 & $8.72 \mathrm{E}-03$ & 10281 & SND1 & 4.57 & $1.77 \mathrm{E}-02$ & 10349 & STAR & 2.23 & $9.89 \mathrm{E}-02$ \\
\hline 10214 & SLC38A1 & 1.59 & $7.81 \mathrm{E}-07$ & 10282 & SNED1 & 4.40 & 7.01E-04 & 10350 & STARD10 & 5.31 & 4.68E-04 \\
\hline 10215 & SLC38A4 & 5.00 & $6.58 \mathrm{E}-05$ & 10283 & SNTB2 & 4.65 & $1.03 \mathrm{E}-03$ & 10351 & STAT1 & 1.93 & $5.00 \mathrm{E}-03$ \\
\hline 10216 & SLC38A7 & 2.73 & $2.77 \mathrm{E}-04$ & 10284 & SNTG1 & 1.14 & $2.10 \mathrm{E}-04$ & 10352 & STEAP1 & 2.27 & $2.08 \mathrm{E}-03$ \\
\hline 10217 & SLC39A10 & 1.15 & $2.55 \mathrm{E}-03$ & 10285 & SNTG2 & 2.36 & $1.59 \mathrm{E}-03$ & 10353 & STEAP2 & 3.14 & $7.21 \mathrm{E}-03$ \\
\hline 10218 & SLC39A11 & 3.51 & $5.97 \mathrm{E}-03$ & 10286 & SNTN & 2.06 & $7.60 \mathrm{E}-05$ & 10354 & STEAP3 & 2.79 & $1.25 \mathrm{E}-03$ \\
\hline 10219 & SLC39A14 & 2.31 & $8.57 E-03$ & 10287 & SNX12 & 1.68 & 3.17E-02 & 10355 & STEAP4 & 3.37 & $2.39 \mathrm{E}-03$ \\
\hline 10220 & SLC41A1 & 2.51 & $3.07 E-04$ & 10288 & SNX13 & 2.53 & $2.59 \mathrm{E}-05$ & 10356 & STK32B & 5.64 & $1.19 \mathrm{E}-03$ \\
\hline 10221 & SLC41A3 & 2.00 & $1.25 \mathrm{E}-02$ & 10289 & SNX18 & 3.38 & $8.20 \mathrm{E}-03$ & 10357 & STOML3 & 4.76 & $6.00 \mathrm{E}-03$ \\
\hline 10222 & SLC43A2 & 2.87 & $1.28 \mathrm{E}-02$ & 10290 & SNX8 & 3.81 & $6.69 \mathrm{E}-04$ & 10358 & STRA6 & 1.45 & $1.06 \mathrm{E}-02$ \\
\hline 10223 & SLC43A3 & 3.00 & $1.01 E-03$ & 10291 & socs2 & 1.47 & 3.56E-05 & 10359 & STRADB & 1.65 & $8.75 \mathrm{E}-03$ \\
\hline 10224 & SLC45A2 & 1.56 & $5.74 \mathrm{E}-03$ & 10292 & SOGA3 & 1.92 & $1.25 \mathrm{E}-03$ & 10360 & STS & 1.75 & $2.85 \mathrm{E}-02$ \\
\hline 10225 & SLC45A3 & 3.42 & $2.22 \mathrm{E}-03$ & 10293 & SOHO-1 & 8.96 & 4.11E-03 & 10361 & STX11 & 3.05 & $1.46 \mathrm{E}-03$ \\
\hline 10226 & SLC46A1 & 3.37 & $6.11 \mathrm{E}-03$ & 10294 & SOLH & 5.81 & $1.72 \mathrm{E}-03$ & 10362 & STX19 & 1.22 & 4.03E-04 \\
\hline 10227 & SLC47A1 & 2.71 & $1.40 \mathrm{E}-03$ & 10295 & SON & 1.12 & $2.27 \mathrm{E}-03$ & 10363 & STX1B & 4.37 & 4.93E-03 \\
\hline 10228 & SLC47A2 & 4.25 & $1.46 \mathrm{E}-04$ & 10296 & SORCS3 & 4.12 & $2.80 \mathrm{E}-04$ & 10364 & STXBP1 & 1.98 & $2.79 \mathrm{E}-02$ \\
\hline 10229 & SLC4A1 & 3.54 & $8.76 \mathrm{E}-03$ & 10297 & SOST & 1.82 & $2.15 \mathrm{E}-02$ & 10365 & SUCNR1 & 6.26 & 4.39E-03 \\
\hline 10230 & SLC4A2 & 3.12 & $3.00 \mathrm{E}-04$ & 10298 & SOSTDC1 & 2.04 & $2.24 \mathrm{E}-02$ & 10366 & SUGP2 & 1.72 & 4.73E-03 \\
\hline 10231 & SLC4A7 & 1.39 & $6.89 \mathrm{E}-03$ & 10299 & Sox1 & 5.55 & $8.85 \mathrm{E}-03$ & 10367 & SULT1B1 & 2.05 & 3.40E-02 \\
\hline 10232 & SLC5A1 & 1.29 & $4.76 \mathrm{E}-04$ & 10300 & SOX10 & 1.47 & $1.85 \mathrm{E}-03$ & 10368 & SULT1C3 & 3.25 & $1.07 \mathrm{E}-01$ \\
\hline 10233 & SLC5A10 & 3.84 & $5.04 \mathrm{E}-05$ & 10301 & SOX11 & 1.69 & $2.89 \mathrm{E}-03$ & 10369 & SUPT6H & 1.26 & $6.91 \mathrm{E}-04$ \\
\hline 10234 & SLC5A11 & 2.98 & $6.05 E-05$ & 10302 & SOX14 & 7.10 & $3.96 \mathrm{E}-03$ & 10370 & SUPT7L & 1.38 & $1.36 \mathrm{E}-01$ \\
\hline 10235 & SLC5A7 & 4.53 & $2.41 E-03$ & 10303 & Sox17 & 1.83 & $1.65 \mathrm{E}-02$ & 10371 & SUSD2 & 2.10 & $5.77 \mathrm{E}-03$ \\
\hline 10236 & SLC5A9 & 3.20 & $3.43 \mathrm{E}-03$ & 10304 & SOX18 & 2.27 & $7.24 \mathrm{E}-02$ & 10372 & SV2B & 1.37 & $7.96 \mathrm{E}-03$ \\
\hline 10237 & SLC6A1 & 2.06 & $2.28 \mathrm{E}-03$ & 10305 & SoX2 & 2.81 & $2.00 \mathrm{E}-03$ & 10373 & $\mathrm{SV} 2 \mathrm{C}$ & 3.99 & 2.33E-03 \\
\hline 10238 & SLC6A15 & 7.33 & $1.39 \mathrm{E}-04$ & 10306 & sox3 & 5.05 & 2.07E-03 & 10374 & SVEP1 & 4.08 & $5.12 \mathrm{E}-03$ \\
\hline 10239 & SLC6A4 & 5.73 & $3.75 \mathrm{E}-03$ & 10307 & SoX4 & 2.25 & $5.65 \mathrm{E}-03$ & 10375 & SVOPL & 1.75 & $6.33 \mathrm{E}-03$ \\
\hline 10240 & SLC6A5 & 5.56 & $1.92 \mathrm{E}-03$ & 10308 & SoX7 & 3.71 & $2.79 \mathrm{E}-02$ & 10376 & SYDE2 & 1.99 & $1.22 \mathrm{E}-03$ \\
\hline 10241 & SLC6A6 & 3.55 & $7.13 \mathrm{E}-03$ & 10309 & SOX8 & 2.57 & $1.29 \mathrm{E}-02$ & 10377 & SYK & 1.88 & $5.02 \mathrm{E}-03$ \\
\hline 10242 & SLC7A10 & 6.17 & $2.96 \mathrm{E}-03$ & 10310 & SPAM1 & 2.38 & $1.33 \mathrm{E}-02$ & 10378 & SYN2 & 1.47 & $9.07 E-02$ \\
\hline 10243 & SLC7A11 & 5.32 & $6.55 \mathrm{E}-03$ & 10311 & SPARCL1 & 4.53 & $1.37 \mathrm{E}-03$ & 10379 & SYNC & 1.86 & $2.06 \mathrm{E}-02$ \\
\hline 10244 & SLC7A14 & 2.47 & $1.33 \mathrm{E}-02$ & 10312 & SPATA13 & 3.07 & $1.72 \mathrm{E}-04$ & 10380 & SYNDIG1 & 4.38 & $6.47 \mathrm{E}-04$ \\
\hline 10245 & SLC7A2 & 2.48 & $2.96 \mathrm{E}-03$ & 10313 & SPATA2O & 3.14 & $2.72 \mathrm{E}-02$ & 10381 & SYNDIG1L & 5.77 & 3.19E-04 \\
\hline 10246 & SLC7A3 & 3.05 & $2.39 \mathrm{E}-04$ & 10314 & SPDEF & 5.20 & $1.20 \mathrm{E}-03$ & 10382 & SYNJ2 & 2.59 & $8.69 \mathrm{E}-03$ \\
\hline 10247 & SLC7A4 & 1.91 & $1.12 \mathrm{E}-03$ & 10315 & SPEG & 7.00 & $4.01 \mathrm{E}-05$ & 10383 & SYNPO2 & 4.04 & $2.68 \mathrm{E}-03$ \\
\hline 10248 & SLC8A1 & 2.85 & 4.55E-03 & 10316 & SPG20 & 1.11 & $6.60 \mathrm{E}-04$ & 10384 & SYNPR & 5.01 & 2.96E-04 \\
\hline 10249 & SLC8A3 & 5.19 & $6.88 \mathrm{E}-03$ & 10317 & SPHK1 & 2.40 & $5.15 \mathrm{E}-03$ & 10385 & SYT1 & 2.90 & 2.09E-02 \\
\hline 10250 & SLC9A1 & 3.24 & $8.62 E-06$ & 10318 & SPI1 & 4.57 & $1.44 \mathrm{E}-04$ & 10386 & SYT10 & 5.79 & $4.45 \mathrm{E}-03$ \\
\hline 10251 & SLC9A3 & 2.99 & $5.97 \mathrm{E}-03$ & 10319 & SPIC & 2.25 & $1.94 \mathrm{E}-02$ & 10387 & SYT12 & 3.68 & $3.16 \mathrm{E}-03$ \\
\hline 10252 & SLC9A4 & 3.01 & $8.62 E-03$ & 10320 & SPINK7 & 3.13 & 7.10E-04 & 10388 & SYT13 & 1.98 & $1.27 \mathrm{E}-02$ \\
\hline 10253 & SLC9A6 & 2.24 & $6.87 \mathrm{E}-04$ & 10321 & SPIRE2 & 3.99 & $7.26 \mathrm{E}-04$ & 10389 & SYT16 & 1.54 & $5.88 \mathrm{E}-03$ \\
\hline 10254 & SLC9A9 & 2.38 & $1.60 \mathrm{E}-02$ & 10322 & SPNS2 & 1.30 & $1.94 \mathrm{E}-02$ & 10390 & SYT17 & 3.60 & $4.00 E-04$ \\
\hline 10255 & SLCO1A2 & 3.00 & $8.92 E-03$ & 10323 & SPP1 & 3.85 & $9.42 \mathrm{E}-04$ & 10391 & SYT2 & 5.82 & $1.45 \mathrm{E}-04$ \\
\hline 10256 & SLCO1B1 & 2.98 & $2.78 \mathrm{E}-03$ & 10324 & SPRY2 & 3.11 & $7.64 \mathrm{E}-03$ & 10392 & SYT4 & 7.94 & $3.41 \mathrm{E}-03$ \\
\hline 10257 & SLCO1C1 & 4.27 & $1.97 \mathrm{E}-02$ & 10325 & SPRY3 & 3.35 & $5.26 \mathrm{E}-03$ & 10393 & SYT6 & 3.23 & $4.21 \mathrm{E}-03$ \\
\hline 10258 & SLCO2A1 & 2.35 & $1.67 \mathrm{E}-03$ & 10326 & SPRY4 & 5.98 & $2.12 \mathrm{E}-04$ & 10394 & SYT7 & 5.03 & 4.60E-03 \\
\hline 10259 & SLCO3A1 & 1.32 & $1.85 \mathrm{E}-02$ & 10327 & SPTSSB & 3.22 & $1.05 \mathrm{E}-02$ & 10395 & SYTL1 & 1.60 & 3.37E-02 \\
\hline 10260 & SLCO5A1 & 2.87 & $1.21 E-03$ & 10328 & SREBF1 & 4.77 & $6.03 \mathrm{E}-03$ & 10396 & SYTL4 & 1.32 & $1.31 \mathrm{E}-05$ \\
\hline 10261 & SLIT2 & 3.29 & $9.29 \mathrm{E}-03$ & 10329 & SRGAP3 & 2.29 & $9.49 \mathrm{E}-03$ & 10397 & SZT2 & 2.49 & $1.86 \mathrm{E}-02$ \\
\hline 10262 & SLITRK1 & 6.18 & $7.19 \mathrm{E}-04$ & 10330 & SRMS & 2.96 & $2.25 \mathrm{E}-03$ & 10398 & TAAR1 & 5.11 & $2.19 \mathrm{E}-04$ \\
\hline 10263 & SLITRK4 & 4.01 & $1.26 \mathrm{E}-02$ & 10331 & SRRD & 2.59 & 3.57E-04 & 10399 & TAAR5 & 6.31 & $7.84 \mathrm{E}-04$ \\
\hline 10264 & SMAD5 & 1.10 & 3.37E-02 & 10332 & SRRM3 & 1.82 & $7.48 \mathrm{E}-04$ & 10400 & TAC1 & 4.35 & $1.51 \mathrm{E}-03$ \\
\hline 10265 & SMAD7 & 3.03 & $1.46 \mathrm{E}-02$ & 10333 & SSPN & 3.18 & $6.41 \mathrm{E}-03$ & 10401 & TACR1 & 3.35 & $3.51 \mathrm{E}-03$ \\
\hline 10266 & SMAD7B & 2.45 & $3.29 \mathrm{E}-02$ & 10334 & SSPO & 2.31 & 1.16E-02 & 10402 & TACR2 & 1.63 & $8.15 \mathrm{E}-02$ \\
\hline 10267 & SMAD9 & 1.35 & $5.38 \mathrm{E}-04$ & 10335 & SSTR1 & 5.98 & $2.29 \mathrm{E}-04$ & 10403 & TAL1 & 3.74 & 4.74E-03 \\
\hline
\end{tabular}




\begin{tabular}{|c|c|c|c|c|c|c|c|c|c|c|c|}
\hline 10404 & TAL2 & 3.92 & 4.16E-03 & 10472 & TC378276 & 2.06 & $3.88 \mathrm{E}-03$ & 10540 & TC379835 & 2.38 & $1.09 \mathrm{E}-03$ \\
\hline 10405 & TAP1 & 1.18 & $2.76 E-02$ & 10473 & TC378282 & 1.35 & $8.15 \mathrm{E}-03$ & 10541 & TC379838 & 3.39 & $2.59 \mathrm{E}-04$ \\
\hline 10406 & TAPBPL & 2.74 & $1.68 \mathrm{E}-03$ & 10474 & TC378300 & 3.99 & $3.60 \mathrm{E}-03$ & 10542 & TC379864 & 2.55 & $7.42 \mathrm{E}-03$ \\
\hline 10407 & TAS1R1 & 2.16 & 9.13E-03 & 10475 & TC378302 & 1.81 & $6.23 E-03$ & 10543 & TC379886 & 3.86 & $2.71 \mathrm{E}-03$ \\
\hline 10408 & TAS2R7 & 1.99 & $1.23 \mathrm{E}-02$ & 10476 & TC378369 & 3.57 & $8.50 E-03$ & 10544 & TC379912 & 5.08 & $3.19 \mathrm{E}-03$ \\
\hline 10409 & TBC1D10A & 2.36 & $9.69 \mathrm{E}-04$ & 10477 & TC378370 & 2.43 & 3.87E-04 & 10545 & TC379931 & 3.43 & $5.84 \mathrm{E}-04$ \\
\hline 10410 & TBC1D16 & 1.21 & $3.83 \mathrm{E}-03$ & 10478 & TC378392 & 1.62 & $1.63 \mathrm{E}-01$ & 10546 & TC379936 & 4.34 & $1.28 \mathrm{E}-03$ \\
\hline 10411 & TBC1D20 & 2.59 & $6.93 \mathrm{E}-04$ & 10479 & TC378420 & 1.20 & $1.17 \mathrm{E}-03$ & 10547 & TC379944 & 1.78 & $3.23 \mathrm{E}-02$ \\
\hline 10412 & TBC1D30 & 3.46 & $1.88 \mathrm{E}-03$ & 10480 & TC378457 & 3.34 & $2.05 E-03$ & 10548 & TC379976 & 2.39 & $8.81 \mathrm{E}-02$ \\
\hline 10413 & TBCEL & 3.11 & $7.13 \mathrm{E}-03$ & 10481 & TC378475 & 2.92 & $1.51 \mathrm{E}-02$ & 10549 & TC379990 & 4.33 & $1.09 \mathrm{E}-03$ \\
\hline 10414 & TBX10 & 3.23 & $8.69 \mathrm{E}-03$ & 10482 & TC378485 & 1.89 & $2.73 E-03$ & 10550 & TC379991 & 5.92 & $1.72 \mathrm{E}-03$ \\
\hline 10415 & TBX15 & 5.23 & $5.64 \mathrm{E}-03$ & 10483 & TC378488 & 3.69 & $4.08 \mathrm{E}-03$ & 10551 & TC379993 & 1.31 & 2.07E-02 \\
\hline 10416 & TBX18 & 1.99 & $3.03 E-02$ & 10484 & TC378495 & 2.19 & $5.84 \mathrm{E}-03$ & 10552 & TC380057 & 1.76 & $3.75 \mathrm{E}-03$ \\
\hline 10417 & TBX19 & 3.36 & $2.73 \mathrm{E}-02$ & 10485 & TC378508 & 5.63 & $5.35 \mathrm{E}-03$ & 10553 & TC380101 & 2.95 & $3.26 \mathrm{E}-03$ \\
\hline 10418 & TBX2 & 3.67 & $1.14 \mathrm{E}-03$ & 10486 & TC378552 & 1.47 & $5.29 \mathrm{E}-03$ & 10554 & TC380107 & 5.56 & $4.36 \mathrm{E}-05$ \\
\hline 10419 & TBX20 & 4.54 & 4.11E-03 & 10487 & TC378597 & 1.18 & $1.48 \mathrm{E}-02$ & 10555 & TC380108 & 2.06 & $9.33 \mathrm{E}-03$ \\
\hline 10420 & TBX22 & 4.46 & $6.32 \mathrm{E}-02$ & 10488 & TC378629 & 2.78 & $1.89 \mathrm{E}-03$ & 10556 & TC380134 & 3.95 & $6.17 \mathrm{E}-04$ \\
\hline 10421 & $\mathrm{~TB} \times 3$ & 3.31 & $1.53 \mathrm{E}-03$ & 10489 & TC378635 & 4.83 & $3.00 E-03$ & 10557 & TC380141 & 3.06 & $1.55 \mathrm{E}-03$ \\
\hline 10422 & $\mathrm{TBX} 4$ & 6.09 & $2.26 \mathrm{E}-04$ & 10490 & TC378656 & 2.77 & $5.02 E-04$ & 10558 & TC380154 & 3.22 & $2.67 \mathrm{E}-04$ \\
\hline 10423 & TBX6 & 3.74 & $6.92 \mathrm{E}-03$ & 10491 & TC378664 & 3.92 & 4.04E-03 & 10559 & TC380171 & 2.00 & $2.15 \mathrm{E}-02$ \\
\hline 10424 & TBXAS1 & 3.22 & $7.80 \mathrm{E}-04$ & 10492 & TC378669 & 2.84 & 4.68E-02 & 10560 & TC380180 & 3.86 & $1.30 \mathrm{E}-03$ \\
\hline 10425 & TC373795 & 6.32 & $1.13 \mathrm{E}-02$ & 10493 & TC378682 & 2.17 & $8.50 E-03$ & 10561 & TC380207 & 2.16 & $7.24 \mathrm{E}-03$ \\
\hline 10426 & TC374008 & 2.47 & $8.55 \mathrm{E}-03$ & 10494 & TC378695 & 6.04 & $3.59 E-03$ & 10562 & TC380216 & 6.72 & $6.10 \mathrm{E}-06$ \\
\hline 10427 & TC374201 & 7.26 & $1.56 \mathrm{E}-03$ & 10495 & TC378725 & 4.82 & $1.69 \mathrm{E}-03$ & 10563 & TC380219 & 2.58 & $1.74 \mathrm{E}-02$ \\
\hline 10428 & TC374259 & 1.09 & $1.08 \mathrm{E}-02$ & 10496 & TC378739 & 4.78 & $2.76 E-03$ & 10564 & TC380227 & 3.69 & $5.10 \mathrm{E}-04$ \\
\hline 10429 & TC374302 & 1.93 & $3.46 \mathrm{E}-04$ & 10497 & TC378751 & 3.02 & $3.38 \mathrm{E}-03$ & 10565 & TC380245 & 1.74 & $2.29 \mathrm{E}-02$ \\
\hline 10430 & TC375081 & 4.76 & $9.60 \mathrm{E}-04$ & 10498 & TC378821 & 2.08 & $1.90 \mathrm{E}-02$ & 10566 & TC380285 & 5.27 & $4.46 \mathrm{E}-03$ \\
\hline 10431 & TC375871 & 3.83 & $1.53 \mathrm{E}-04$ & 10499 & TC378830 & 6.08 & $8.00 E-04$ & 10567 & TC380322 & 2.75 & $7.73 E-03$ \\
\hline 10432 & TC376542 & 3.50 & $1.39 \mathrm{E}-03$ & 10500 & TC378876 & 2.78 & $1.08 \mathrm{E}-02$ & 10568 & TC380324 & 2.92 & $2.29 \mathrm{E}-03$ \\
\hline 10433 & TC376555 & 4.89 & $5.95 \mathrm{E}-04$ & 10501 & TC378906 & 1.48 & 4.63E-03 & 10569 & TC380332 & 1.05 & $1.92 \mathrm{E}-04$ \\
\hline 10434 & TC376560 & 2.59 & $5.29 \mathrm{E}-02$ & 10502 & TC378922 & 2.50 & $1.27 \mathrm{E}-02$ & 10570 & TC380391 & 3.64 & $6.32 \mathrm{E}-02$ \\
\hline 10435 & TC376878 & 8.36 & $1.58 \mathrm{E}-05$ & 10503 & TC378940 & 2.16 & $6.72 \mathrm{E}-03$ & 10571 & TC380415 & 2.11 & $8.82 \mathrm{E}-04$ \\
\hline 10436 & TC376885 & 7.09 & $2.47 \mathrm{E}-03$ & 10504 & TC378949 & 2.63 & $4.82 \mathrm{E}-03$ & 10572 & TC380447 & 2.41 & $1.09 \mathrm{E}-02$ \\
\hline 10437 & TC377007 & 1.43 & $6.43 \mathrm{E}-03$ & 10505 & TC378954 & 3.22 & $1.96 \mathrm{E}-03$ & 10573 & TC380456 & 2.19 & $1.33 \mathrm{E}-03$ \\
\hline 10438 & TC377104 & 2.03 & 1.11E-02 & 10506 & TC378979 & 4.22 & $1.64 \mathrm{E}-02$ & 10574 & TC380490 & 1.38 & $1.53 \mathrm{E}-03$ \\
\hline 10439 & TC377318 & 1.98 & $1.52 \mathrm{E}-03$ & 10507 & TC379025 & 4.43 & $1.71 \mathrm{E}-03$ & 10575 & TC380511 & 2.82 & $1.24 \mathrm{E}-03$ \\
\hline 10440 & TC377321 & 3.49 & $4.59 \mathrm{E}-04$ & 10508 & TC379027 & 1.84 & $1.23 \mathrm{E}-04$ & 10576 & TC380527 & 1.45 & $4.90 \mathrm{E}-02$ \\
\hline 10441 & TC377357 & 4.48 & $2.17 \mathrm{E}-03$ & 10509 & TC379058 & 6.19 & $8.10 \mathrm{E}-05$ & 10577 & TC380592 & 1.45 & $9.26 \mathrm{E}-03$ \\
\hline 10442 & TC377373 & 2.85 & $1.01 \mathrm{E}-03$ & 10510 & TC379061 & 5.69 & $3.27 \mathrm{E}-03$ & 10578 & TC380598 & 3.48 & $4.68 \mathrm{E}-04$ \\
\hline 10443 & TC377379 & 5.46 & $4.53 \mathrm{E}-04$ & 10511 & TC379086 & 4.01 & $4.24 E-03$ & 10579 & TC380601 & 2.76 & $1.08 \mathrm{E}-02$ \\
\hline 10444 & TC377389 & 3.89 & $5.09 \mathrm{E}-03$ & 10512 & TC379094 & 4.24 & $1.24 \mathrm{E}-04$ & 10580 & TC380613 & 2.14 & $1.08 \mathrm{E}-01$ \\
\hline 10445 & TC377401 & 8.33 & $6.04 \mathrm{E}-04$ & 10513 & TC379107 & 1.39 & $6.48 \mathrm{E}-03$ & 10581 & TC380622 & 3.24 & $7.77 \mathrm{E}-04$ \\
\hline 10446 & TC377509 & 2.86 & $1.37 \mathrm{E}-02$ & 10514 & TC379168 & 2.61 & $1.20 \mathrm{E}-01$ & 10582 & TC380638 & 2.99 & $5.48 \mathrm{E}-04$ \\
\hline 10447 & TC377747 & 3.95 & $7.00 \mathrm{E}-04$ & 10515 & TC379171 & 2.97 & $5.26 \mathrm{E}-03$ & 10583 & TC380644 & 2.65 & $3.34 \mathrm{E}-04$ \\
\hline 10448 & TC377764 & 5.44 & $4.76 \mathrm{E}-03$ & 10516 & TC379210 & 3.80 & $4.25 E-03$ & 10584 & TC380677 & 2.74 & $5.62 \mathrm{E}-04$ \\
\hline 10449 & TC377769 & 1.06 & $8.51 \mathrm{E}-04$ & 10517 & TC379277 & 1.36 & 3.67E-02 & 10585 & TC380692 & 4.77 & $4.57 \mathrm{E}-06$ \\
\hline 10450 & TC377791 & 3.00 & $1.68 \mathrm{E}-03$ & 10518 & TC379287 & 1.87 & $1.82 \mathrm{E}-03$ & 10586 & TC380693 & 6.03 & $3.17 \mathrm{E}-04$ \\
\hline 10451 & TC377955 & 1.01 & $3.93 \mathrm{E}-03$ & 10519 & TC379341 & 2.36 & $1.72 \mathrm{E}-02$ & 10587 & TC380711 & 1.63 & $8.69 \mathrm{E}-03$ \\
\hline 10452 & TC377966 & 5.86 & $2.82 \mathrm{E}-03$ & 10520 & TC379344 & 4.80 & $8.79 E-03$ & 10588 & TC380721 & 3.76 & $4.65 \mathrm{E}-03$ \\
\hline 10453 & TC377981 & 3.68 & $1.28 \mathrm{E}-02$ & 10521 & TC379355 & 1.82 & $1.06 \mathrm{E}-03$ & 10589 & TC380754 & 2.18 & $1.57 \mathrm{E}-02$ \\
\hline 10454 & TC377982 & 6.05 & $1.16 \mathrm{E}-02$ & 10522 & TC379420 & 1.86 & $3.93 E-03$ & 10590 & TC380761 & 2.56 & $1.45 \mathrm{E}-02$ \\
\hline 10455 & TC377998 & 1.71 & $4.72 \mathrm{E}-03$ & 10523 & TC379436 & 3.04 & $6.46 \mathrm{E}-04$ & 10591 & TC380785 & 2.38 & $1.46 \mathrm{E}-03$ \\
\hline 10456 & TC378008 & 3.27 & $1.86 \mathrm{E}-03$ & 10524 & TC379480 & 1.19 & $4.91 E-02$ & 10592 & TC380793 & 2.53 & $1.88 \mathrm{E}-03$ \\
\hline 10457 & TC378011 & 4.38 & $1.47 \mathrm{E}-03$ & 10525 & TC379499 & 3.58 & $5.48 \mathrm{E}-03$ & 10593 & TC380819 & 4.07 & $1.32 \mathrm{E}-03$ \\
\hline 10458 & TC378052 & 3.41 & $7.12 \mathrm{E}-03$ & 10526 & TC379514 & 4.16 & $4.30 E-04$ & 10594 & TC380835 & 1.75 & $3.85 \mathrm{E}-03$ \\
\hline 10459 & TC378080 & 2.24 & $3.62 \mathrm{E}-02$ & 10527 & TC379525 & 1.80 & $8.23 E-04$ & 10595 & TC380850 & 1.99 & $3.06 \mathrm{E}-02$ \\
\hline 10460 & TC378086 & 4.94 & $4.77 E-04$ & 10528 & TC379535 & 2.96 & $1.01 E-02$ & 10596 & TC380855 & 1.35 & $4.35 \mathrm{E}-02$ \\
\hline 10461 & TC378087 & 2.06 & $2.03 \mathrm{E}-02$ & 10529 & TC379541 & 4.47 & $7.88 \mathrm{E}-05$ & 10597 & TC380920 & 6.42 & $1.74 \mathrm{E}-03$ \\
\hline 10462 & TC378089 & 3.50 & $9.08 \mathrm{E}-03$ & 10530 & TC379598 & 3.40 & $4.15 E-03$ & 10598 & TC380923 & 3.85 & $3.26 \mathrm{E}-03$ \\
\hline 10463 & TC378129 & 6.24 & 4.49E-03 & 10531 & TC379634 & 3.28 & $3.10 \mathrm{E}-03$ & 10599 & TC380930 & 3.12 & $6.63 \mathrm{E}-03$ \\
\hline 10464 & TC378132 & 4.87 & $2.60 \mathrm{E}-04$ & 10532 & TC379683 & 2.82 & $3.70 E-03$ & 10600 & TC381010 & 5.10 & $5.47 \mathrm{E}-03$ \\
\hline 10465 & TC378144 & 2.25 & $1.34 \mathrm{E}-02$ & 10533 & TC379702 & 2.67 & $1.22 \mathrm{E}-03$ & 10601 & TC381020 & 3.38 & $1.30 \mathrm{E}-02$ \\
\hline 10466 & TC378154 & 4.82 & $1.53 \mathrm{E}-03$ & 10534 & TC379711 & 2.69 & $5.32 \mathrm{E}-03$ & 10602 & TC381054 & 1.63 & $2.17 \mathrm{E}-02$ \\
\hline 10467 & TC378183 & 2.48 & $1.13 \mathrm{E}-02$ & 10535 & TC379753 & 5.35 & $8.32 E-04$ & 10603 & TC381091 & 6.36 & $5.19 \mathrm{E}-06$ \\
\hline 10468 & TC378207 & 1.57 & $8.88 \mathrm{E}-03$ & 10536 & TC379761 & 3.08 & $7.31 \mathrm{E}-03$ & 10604 & TC381121 & 2.18 & $6.86 \mathrm{E}-03$ \\
\hline 10469 & TC378220 & 2.36 & $3.58 \mathrm{E}-03$ & 10537 & TC379770 & 5.76 & $3.24 E-04$ & 10605 & TC381146 & 3.08 & $1.07 \mathrm{E}-02$ \\
\hline 10470 & TC378232 & 1.18 & $2.16 \mathrm{E}-03$ & 10538 & TC379801 & 4.97 & $4.41 E-03$ & 10606 & TC381177 & 4.45 & 3.99E-03 \\
\hline 10471 & TC378237 & 1.41 & $1.15 \mathrm{E}-01$ & 10539 & TC379834 & 3.90 & $1.25 \mathrm{E}-04$ & 10607 & TC381188 & 5.45 & $1.66 \mathrm{E}-03$ \\
\hline
\end{tabular}




\begin{tabular}{|c|c|c|c|c|c|c|c|c|c|c|c|}
\hline 10608 & TC381207 & 4.49 & $8.12 \mathrm{E}-04$ & 10676 & TC383242 & 4.31 & $1.12 \mathrm{E}-03$ & 10744 & TC385885 & 1.31 & $2.95 \mathrm{E}-04$ \\
\hline 10609 & TC381214 & 1.29 & $7.63 \mathrm{E}-03$ & 10677 & TC383247 & 2.16 & $2.45 \mathrm{E}-03$ & 10745 & TC385919 & 1.97 & $8.16 E-03$ \\
\hline 10610 & TC381226 & 3.97 & $8.55 \mathrm{E}-04$ & 10678 & TC383259 & 4.79 & $2.97 \mathrm{E}-04$ & 10746 & TC386050 & 5.80 & $1.31 \mathrm{E}-03$ \\
\hline 10611 & TC381228 & 6.08 & $2.22 \mathrm{E}-04$ & 10679 & TC383294 & 3.81 & $8.53 \mathrm{E}-03$ & 10747 & TC386082 & 1.61 & $1.72 \mathrm{E}-02$ \\
\hline 10612 & TC381237 & 1.95 & $8.20 \mathrm{E}-02$ & 10680 & TC383322 & 3.59 & $2.26 \mathrm{E}-03$ & 10748 & TC386103 & 2.86 & $2.75 \mathrm{E}-03$ \\
\hline 10613 & TC381249 & 3.89 & $5.61 \mathrm{E}-03$ & 10681 & TC383365 & 3.09 & $6.59 \mathrm{E}-03$ & 10749 & TC386117 & 4.48 & $3.15 \mathrm{E}-03$ \\
\hline 10614 & TC381264 & 3.47 & $3.93 \mathrm{E}-03$ & 10682 & TC383420 & 4.29 & $8.91 \mathrm{E}-04$ & 10750 & TC386135 & 2.94 & $1.47 E-03$ \\
\hline 10615 & TC381266 & 5.04 & $3.20 \mathrm{E}-04$ & 10683 & TC383426 & 3.19 & $6.75 \mathrm{E}-03$ & 10751 & TC386230 & 1.62 & $4.34 \mathrm{E}-03$ \\
\hline 10616 & TC381356 & 3.56 & $4.65 \mathrm{E}-05$ & 10684 & TC383564 & 4.27 & $1.49 \mathrm{E}-02$ & 10752 & TC386249 & 5.35 & $2.77 E-03$ \\
\hline 10617 & TC381453 & 1.28 & $2.37 \mathrm{E}-03$ & 10685 & TC383595 & 1.33 & $9.16 \mathrm{E}-03$ & 10753 & TC386285 & 5.94 & $8.36 \mathrm{E}-04$ \\
\hline 10618 & TC381454 & 1.31 & $1.12 \mathrm{E}-02$ & 10686 & TC383598 & 5.46 & $5.17 \mathrm{E}-04$ & 10754 & TC386289 & 2.31 & $1.83 \mathrm{E}-02$ \\
\hline 10619 & TC381503 & 1.94 & $1.14 \mathrm{E}-02$ & 10687 & TC383625 & 2.18 & $1.87 \mathrm{E}-02$ & 10755 & TC386300 & 2.59 & 4.24E-04 \\
\hline 10620 & TC381504 & 2.23 & $2.90 \mathrm{E}-04$ & 10688 & TC383654 & 1.79 & $1.56 \mathrm{E}-02$ & 10756 & TC386323 & 1.57 & $5.35 \mathrm{E}-02$ \\
\hline 10621 & TC381512 & 3.60 & $1.25 \mathrm{E}-02$ & 10689 & TC383686 & 3.94 & $1.35 \mathrm{E}-02$ & 10757 & TC386354 & 2.89 & $2.13 \mathrm{E}-02$ \\
\hline 10622 & TC381538 & 3.08 & $3.04 \mathrm{E}-03$ & 10690 & TC383775 & 4.55 & $6.12 \mathrm{E}-04$ & 10758 & TC386404 & 5.92 & 7.44E-05 \\
\hline 10623 & TC381556 & 3.00 & $1.62 \mathrm{E}-02$ & 10691 & TC383823 & 4.79 & $6.68 \mathrm{E}-05$ & 10759 & TC386482 & 4.61 & 2.14E-02 \\
\hline 10624 & TC381568 & 4.83 & $5.12 \mathrm{E}-03$ & 10692 & TC383903 & 2.10 & $1.77 \mathrm{E}-02$ & 10760 & TC386643 & 8.75 & $1.91 \mathrm{E}-03$ \\
\hline 10625 & TC381586 & 2.66 & $1.68 \mathrm{E}-02$ & 10693 & TC383906 & 5.89 & $7.51 \mathrm{E}-04$ & 10761 & TC386663 & 2.41 & $3.99 \mathrm{E}-03$ \\
\hline 10626 & TC381617 & 5.44 & $3.27 \mathrm{E}-04$ & 10694 & TC383927 & 5.25 & $9.28 \mathrm{E}-04$ & 10762 & TC386720 & 4.59 & $9.97 \mathrm{E}-04$ \\
\hline 10627 & TC381679 & 3.85 & $6.54 \mathrm{E}-03$ & 10695 & TC383934 & 5.55 & $6.38 \mathrm{E}-04$ & 10763 & TC386785 & 2.61 & $1.09 \mathrm{E}-03$ \\
\hline 10628 & TC381702 & 5.26 & $2.21 \mathrm{E}-03$ & 10696 & TC384017 & 6.75 & $2.77 \mathrm{E}-05$ & 10764 & TC386830 & 4.56 & $6.66 \mathrm{E}-03$ \\
\hline 10629 & TC381709 & 5.61 & $5.90 \mathrm{E}-02$ & 10697 & TC384024 & 4.73 & $3.19 \mathrm{E}-01$ & 10765 & TC386845 & 4.30 & $1.21 \mathrm{E}-02$ \\
\hline 10630 & TC381710 & 4.54 & $1.97 \mathrm{E}-03$ & 10698 & TC384109 & 6.62 & $2.88 \mathrm{E}-03$ & 10766 & TC386857 & 1.33 & $3.01 E-02$ \\
\hline 10631 & TC381729 & 3.19 & $1.19 \mathrm{E}-02$ & 10699 & TC384114 & 2.36 & $1.75 \mathrm{E}-02$ & 10767 & TC386876 & 5.35 & $1.39 \mathrm{E}-03$ \\
\hline 10632 & TC381748 & 5.20 & $3.09 \mathrm{E}-03$ & 10700 & TC384117 & 2.29 & $1.50 \mathrm{E}-03$ & 10768 & TC386937 & 3.52 & $1.44 \mathrm{E}-02$ \\
\hline 10633 & TC381832 & 3.95 & $4.95 \mathrm{E}-03$ & 10701 & TC384118 & 4.59 & $1.25 \mathrm{E}-03$ & 10769 & TC387096 & 4.73 & $1.35 \mathrm{E}-03$ \\
\hline 10634 & TC381841 & 4.29 & $1.91 \mathrm{E}-03$ & 10702 & TC384255 & 5.50 & $1.37 \mathrm{E}-02$ & 10770 & TC387118 & 5.87 & $1.53 \mathrm{E}-04$ \\
\hline 10635 & TC381873 & 2.67 & $1.77 \mathrm{E}-03$ & 10703 & TC384306 & 3.25 & $3.87 \mathrm{E}-04$ & 10771 & TC387153 & 4.68 & $8.08 \mathrm{E}-04$ \\
\hline 10636 & TC381889 & 1.53 & $9.10 \mathrm{E}-02$ & 10704 & TC384359 & 4.07 & $1.52 \mathrm{E}-02$ & 10772 & TC387214 & 1.51 & $1.11 \mathrm{E}-04$ \\
\hline 10637 & TC381903 & 4.44 & $5.53 \mathrm{E}-04$ & 10705 & TC384421 & 3.38 & $1.04 \mathrm{E}-03$ & 10773 & TC387278 & 9.62 & $6.53 \mathrm{E}-04$ \\
\hline 10638 & TC381905 & 1.38 & $2.11 \mathrm{E}-02$ & 10706 & TC384422 & 3.02 & $1.28 \mathrm{E}-02$ & 10774 & TC387347 & 6.39 & 4.00E-04 \\
\hline 10639 & TC381930 & 3.71 & $3.44 \mathrm{E}-03$ & 10707 & TC384441 & 3.73 & $1.76 \mathrm{E}-03$ & 10775 & TC387507 & 1.75 & $6.96 \mathrm{E}-02$ \\
\hline 10640 & TC381965 & 1.21 & $5.01 \mathrm{E}-02$ & 10708 & TC384452 & 1.65 & $7.10 \mathrm{E}-02$ & 10776 & TC387558 & 1.49 & $1.55 \mathrm{E}-02$ \\
\hline 10641 & TC381970 & 2.37 & $1.13 \mathrm{E}-02$ & 10709 & TC384463 & 3.68 & $1.02 \mathrm{E}-03$ & 10777 & TC387600 & 5.19 & 1.23E-02 \\
\hline 10642 & TC381997 & 2.88 & $8.13 \mathrm{E}-04$ & 10710 & TC384488 & 1.13 & $2.87 \mathrm{E}-02$ & 10778 & TC387613 & 5.49 & $1.88 \mathrm{E}-05$ \\
\hline 10643 & TC382002 & 5.34 & $4.01 E-05$ & 10711 & TC384548 & 3.12 & $6.63 \mathrm{E}-02$ & 10779 & TC387641 & 4.45 & $2.28 \mathrm{E}-03$ \\
\hline 10644 & TC382036 & 1.88 & $2.27 \mathrm{E}-03$ & 10712 & TC384595 & 5.66 & $2.82 \mathrm{E}-05$ & 10780 & TC387721 & 2.97 & $3.91 \mathrm{E}-03$ \\
\hline 10645 & TC382119 & 5.89 & $8.04 E-05$ & 10713 & TC384629 & 2.37 & $4.01 \mathrm{E}-03$ & 10781 & TC387778 & 1.66 & $2.35 \mathrm{E}-03$ \\
\hline 10646 & TC382128 & 3.51 & $3.68 \mathrm{E}-03$ & 10714 & TC384631 & 4.43 & $8.45 \mathrm{E}-04$ & 10782 & TC387783 & 1.42 & $9.21 \mathrm{E}-02$ \\
\hline 10647 & TC382195 & 4.53 & $3.37 \mathrm{E}-03$ & 10715 & TC384743 & 5.88 & $2.46 \mathrm{E}-03$ & 10783 & TC387829 & 4.60 & $2.43 \mathrm{E}-02$ \\
\hline 10648 & TC382231 & 1.76 & $4.27 \mathrm{E}-03$ & 10716 & TC384802 & 1.25 & $1.61 \mathrm{E}-02$ & 10784 & TC387883 & 4.02 & $6.58 \mathrm{E}-04$ \\
\hline 10649 & TC382320 & 9.17 & $5.33 \mathrm{E}-05$ & 10717 & TC384805 & 3.72 & $3.26 \mathrm{E}-03$ & 10785 & TC387930 & 5.52 & $3.96 \mathrm{E}-03$ \\
\hline 10650 & TC382332 & 3.49 & $6.85 \mathrm{E}-05$ & 10718 & TC384861 & 2.04 & $3.25 \mathrm{E}-03$ & 10786 & TC387972 & 3.00 & $7.40 \mathrm{E}-03$ \\
\hline 10651 & TC382352 & 1.51 & $1.69 \mathrm{E}-02$ & 10719 & TC384868 & 5.90 & $5.53 \mathrm{E}-03$ & 10787 & TC388019 & 2.58 & $1.52 \mathrm{E}-01$ \\
\hline 10652 & TC382361 & 1.74 & $1.62 E-02$ & 10720 & TC384872 & 3.77 & $1.21 \mathrm{E}-03$ & 10788 & TC388086 & 2.13 & $2.62 E-03$ \\
\hline 10653 & TC382375 & 4.62 & $3.76 \mathrm{E}-03$ & 10721 & TC384929 & 5.54 & $2.10 \mathrm{E}-02$ & 10789 & TC388124 & 4.09 & $9.14 \mathrm{E}-04$ \\
\hline 10654 & TC382399 & 2.47 & $1.93 \mathrm{E}-02$ & 10722 & TC384956 & 3.15 & $1.01 \mathrm{E}-03$ & 10790 & TC388204 & 3.93 & $2.83 \mathrm{E}-01$ \\
\hline 10655 & TC382467 & 2.63 & $9.08 \mathrm{E}-04$ & 10723 & TC385004 & 6.34 & $1.35 \mathrm{E}-03$ & 10791 & TC388294 & 4.78 & $8.28 \mathrm{E}-05$ \\
\hline 10656 & TC382488 & 3.43 & $2.50 \mathrm{E}-03$ & 10724 & TC385011 & 4.20 & $2.93 \mathrm{E}-03$ & 10792 & TC388298 & 1.89 & $2.98 \mathrm{E}-03$ \\
\hline 10657 & TC382495 & 2.09 & $2.49 \mathrm{E}-02$ & 10725 & TC385029 & 1.58 & $7.72 \mathrm{E}-03$ & 10793 & TC388323 & 5.85 & $3.76 \mathrm{E}-03$ \\
\hline 10658 & TC382536 & 3.50 & $8.93 \mathrm{E}-04$ & 10726 & TC385108 & 5.57 & $5.41 \mathrm{E}-03$ & 10794 & TC388369 & 3.16 & $2.90 \mathrm{E}-03$ \\
\hline 10659 & TC382578 & 2.72 & $4.00 E-05$ & 10727 & TC385136 & 3.10 & $4.85 \mathrm{E}-03$ & 10795 & TC388473 & 1.57 & $1.59 \mathrm{E}-03$ \\
\hline 10660 & TC382606 & 2.19 & $1.70 \mathrm{E}-03$ & 10728 & TC385154 & 6.24 & $6.69 \mathrm{E}-03$ & 10796 & TC388478 & 5.11 & $3.90 \mathrm{E}-04$ \\
\hline 10661 & TC382607 & 1.96 & $1.05 E-02$ & 10729 & TC385168 & 4.94 & $1.78 \mathrm{E}-03$ & 10797 & TC388507 & 4.35 & $2.34 \mathrm{E}-03$ \\
\hline 10662 & TC382617 & 4.60 & $4.99 \mathrm{E}-03$ & 10730 & TC385215 & 2.76 & $1.11 \mathrm{E}-02$ & 10798 & TC388629 & 6.70 & $2.90 \mathrm{E}-03$ \\
\hline 10663 & TC382679 & 5.97 & $1.03 \mathrm{E}-04$ & 10731 & TC385232 & 4.51 & $1.95 \mathrm{E}-03$ & 10799 & TC388671 & 2.48 & 3.93E-03 \\
\hline 10664 & TC382691 & 2.65 & $5.47 \mathrm{E}-03$ & 10732 & TC385241 & 4.93 & $4.09 \mathrm{E}-03$ & 10800 & TC388730 & 2.25 & 2.63E-02 \\
\hline 10665 & TC382695 & 3.19 & $1.64 \mathrm{E}-04$ & 10733 & TC385296 & 2.10 & $6.83 \mathrm{E}-02$ & 10801 & TC388731 & 1.38 & $2.01 \mathrm{E}-02$ \\
\hline 10666 & TC382709 & 3.05 & $6.39 \mathrm{E}-03$ & 10734 & TC385319 & 2.45 & $4.32 \mathrm{E}-03$ & 10802 & TC388783 & 6.38 & 5.19E-04 \\
\hline 10667 & TC382741 & 4.10 & 3.47E-04 & 10735 & TC385410 & 2.53 & $1.74 \mathrm{E}-02$ & 10803 & TC388836 & 3.17 & $1.78 \mathrm{E}-04$ \\
\hline 10668 & TC382941 & 5.23 & $2.31 \mathrm{E}-01$ & 10736 & TC385420 & 4.21 & $2.51 \mathrm{E}-02$ & 10804 & TC388861 & 2.22 & $2.81 \mathrm{E}-03$ \\
\hline 10669 & TC383030 & 6.79 & $6.95 \mathrm{E}-04$ & 10737 & TC385427 & 3.34 & $3.59 \mathrm{E}-03$ & 10805 & TC388926 & 1.36 & $2.30 \mathrm{E}-02$ \\
\hline 10670 & TC383058 & 3.09 & $5.48 \mathrm{E}-03$ & 10738 & TC385640 & 2.46 & $8.42 \mathrm{E}-03$ & 10806 & TC388936 & 2.96 & 3.63E-03 \\
\hline 10671 & TC383085 & 4.82 & $2.91 \mathrm{E}-03$ & 10739 & TC385657 & 4.02 & $1.74 \mathrm{E}-05$ & 10807 & TC388968 & 2.98 & $1.49 \mathrm{E}-02$ \\
\hline 10672 & TC383088 & 4.79 & $1.91 \mathrm{E}-03$ & 10740 & TC385708 & 3.08 & $2.73 \mathrm{E}-03$ & 10808 & TC388974 & 1.57 & $1.40 \mathrm{E}-02$ \\
\hline 10673 & TC383160 & 4.56 & $1.00 E-02$ & 10741 & TC385753 & 3.82 & $1.66 \mathrm{E}-03$ & 10809 & TC388986 & 1.51 & $1.32 \mathrm{E}-03$ \\
\hline 10674 & TC383180 & 3.03 & $1.10 \mathrm{E}-02$ & 10742 & TC385774 & 1.67 & $5.66 \mathrm{E}-02$ & 10810 & TC389001 & 6.88 & $3.16 \mathrm{E}-04$ \\
\hline 10675 & TC383240 & 4.63 & $5.36 \mathrm{E}-04$ & 10743 & TC385828 & 1.45 & $1.52 \mathrm{E}-02$ & 10811 & TC389048 & 1.25 & $1.83 \mathrm{E}-02$ \\
\hline
\end{tabular}




\begin{tabular}{|c|c|c|c|c|c|c|c|c|c|c|c|}
\hline 10812 & TC389049 & 2.73 & $3.39 \mathrm{E}-03$ & 10880 & TC393449 & 2.62 & $6.99 \mathrm{E}-03$ & 10948 & TC400321 & 3.93 & $3.11 \mathrm{E}-03$ \\
\hline 10813 & TC389172 & 3.75 & $1.26 \mathrm{E}-03$ & 10881 & TC393460 & 1.17 & $8.60 \mathrm{E}-03$ & 10949 & TC400352 & 4.64 & $2.75 \mathrm{E}-03$ \\
\hline 10814 & TC389210 & 1.69 & $2.74 \mathrm{E}-02$ & 10882 & TC393550 & 2.89 & $7.32 \mathrm{E}-03$ & 10950 & TC400376 & 4.39 & $1.58 \mathrm{E}-03$ \\
\hline 10815 & TC389247 & 7.39 & $2.30 \mathrm{E}-04$ & 10883 & TC393579 & 5.95 & $5.55 \mathrm{E}-05$ & 10951 & TC400407 & 3.35 & $7.65 \mathrm{E}-04$ \\
\hline 10816 & TC389287 & 4.36 & $5.37 \mathrm{E}-03$ & 10884 & TC393783 & 5.15 & $1.34 \mathrm{E}-04$ & 10952 & TC400468 & 3.13 & $1.06 \mathrm{E}-02$ \\
\hline 10817 & TC389303 & 6.80 & $1.64 \mathrm{E}-01$ & 10885 & TC393786 & 6.19 & 2.63E-05 & 10953 & TC400588 & 4.18 & 4.05E-04 \\
\hline 10818 & TC389316 & 2.73 & $1.07 \mathrm{E}-01$ & 10886 & TC393801 & 6.17 & $2.27 \mathrm{E}-03$ & 10954 & TC400923 & 4.84 & $2.04 \mathrm{E}-03$ \\
\hline 10819 & TC389363 & 3.02 & $1.53 \mathrm{E}-03$ & 10887 & TC393991 & 4.10 & $2.78 \mathrm{E}-04$ & 10955 & TC400973 & 2.23 & $6.02 \mathrm{E}-03$ \\
\hline 10820 & TC389423 & 3.12 & $1.08 \mathrm{E}-03$ & 10888 & TC394242 & 4.55 & $1.29 \mathrm{E}-03$ & 10956 & TC401175 & 3.56 & $8.94 \mathrm{E}-03$ \\
\hline 10821 & TC389440 & 5.09 & $1.21 \mathrm{E}-02$ & 10889 & TC394339 & 2.89 & $3.69 \mathrm{E}-04$ & 10957 & TC401381 & 5.80 & $3.11 \mathrm{E}-04$ \\
\hline 10822 & TC389638 & 2.57 & $4.97 \mathrm{E}-03$ & 10890 & TC394619 & 1.15 & $2.36 \mathrm{E}-02$ & 10958 & TC401494 & 4.71 & $2.48 \mathrm{E}-03$ \\
\hline 10823 & TC389665 & 3.43 & $1.99 \mathrm{E}-03$ & 10891 & TC394628 & 6.21 & $6.35 \mathrm{E}-04$ & 10959 & TC401629 & 3.61 & $5.22 \mathrm{E}-03$ \\
\hline 10824 & TC389722 & 4.60 & $3.75 \mathrm{E}-03$ & 10892 & TC394698 & 5.44 & $3.75 \mathrm{E}-03$ & 10960 & TC402122 & 5.32 & $2.30 \mathrm{E}-02$ \\
\hline 10825 & TC389748 & 2.97 & $4.98 \mathrm{E}-03$ & 10893 & ТC394730 & 6.76 & 3.68E-02 & 10961 & TC402258 & 4.92 & $9.94 \mathrm{E}-03$ \\
\hline 10826 & TC389797 & 3.43 & $2.08 \mathrm{E}-03$ & 10894 & TC394794 & 5.11 & $1.99 \mathrm{E}-03$ & 10962 & TC402329 & 3.83 & $2.62 \mathrm{E}-03$ \\
\hline 10827 & TC390041 & 5.32 & $6.32 \mathrm{E}-04$ & 10895 & TC394799 & 4.68 & $1.19 \mathrm{E}-03$ & 10963 & TC402375 & 1.85 & $8.84 \mathrm{E}-03$ \\
\hline 10828 & TC390082 & 4.44 & $1.00 \mathrm{E}-02$ & 10896 & TC394875 & 6.95 & $1.46 \mathrm{E}-03$ & 10964 & TC402403 & 4.85 & $6.31 \mathrm{E}-03$ \\
\hline 10829 & TC390108 & 2.73 & $2.72 \mathrm{E}-03$ & 10897 & TC394958 & 1.69 & $5.84 \mathrm{E}-03$ & 10965 & TC402486 & 3.83 & $2.10 \mathrm{E}-04$ \\
\hline 10830 & TC390114 & 1.87 & $1.72 \mathrm{E}-02$ & 10898 & TC395006 & 3.78 & $2.56 \mathrm{E}-03$ & 10966 & TC402659 & 3.24 & $9.46 \mathrm{E}-04$ \\
\hline 10831 & TC390190 & 3.69 & $4.34 \mathrm{E}-03$ & 10899 & TC395423 & 1.86 & $3.63 \mathrm{E}-03$ & 10967 & TC402671 & 6.95 & 4.54E-04 \\
\hline 10832 & TC390201 & 7.28 & $3.83 \mathrm{E}-04$ & 10900 & TC395642 & 4.81 & 3.17E-03 & 10968 & TC402716 & 1.27 & 4.24E-02 \\
\hline 10833 & TC390294 & 3.15 & $1.99 \mathrm{E}-03$ & 10901 & TC395794 & 1.77 & $3.88 \mathrm{E}-03$ & 10969 & TC402745 & 2.21 & $3.15 \mathrm{E}-03$ \\
\hline 10834 & TC390382 & 1.32 & 3.03E-02 & 10902 & TC396337 & 7.12 & $3.74 \mathrm{E}-03$ & 10970 & TC402805 & 5.65 & $5.29 \mathrm{E}-03$ \\
\hline 10835 & TC390397 & 2.73 & $1.36 \mathrm{E}-02$ & 10903 & TC396384 & 1.74 & $2.87 \mathrm{E}-02$ & 10971 & TC402828 & 6.33 & $3.23 \mathrm{E}-04$ \\
\hline 10836 & TC390521 & 1.36 & $4.40 \mathrm{E}-02$ & 10904 & TC396587 & 3.60 & $4.33 \mathrm{E}-04$ & 10972 & TC402858 & 5.85 & $1.07 \mathrm{E}-04$ \\
\hline 10837 & TC390530 & 5.05 & $1.47 \mathrm{E}-03$ & 10905 & TC396628 & 4.97 & $2.37 \mathrm{E}-03$ & 10973 & TC402944 & 3.04 & $1.59 \mathrm{E}-02$ \\
\hline 10838 & TC390606 & 5.32 & $1.16 \mathrm{E}-03$ & 10906 & TC396869 & 1.47 & $2.74 \mathrm{E}-02$ & 10974 & TC402960 & 1.55 & $2.65 \mathrm{E}-02$ \\
\hline 10839 & TC390653 & 3.25 & $4.74 \mathrm{E}-03$ & 10907 & TC396961 & 5.57 & $1.16 \mathrm{E}-03$ & 10975 & TC403029 & 5.55 & $4.43 \mathrm{E}-03$ \\
\hline 10840 & TC390684 & 2.31 & $3.00 \mathrm{E}-03$ & 10908 & TC397063 & 3.32 & $1.72 \mathrm{E}-03$ & 10976 & TC403098 & 5.46 & $2.75 \mathrm{E}-03$ \\
\hline 10841 & TC390696 & 5.57 & $4.68 \mathrm{E}-05$ & 10909 & TC397176 & 4.38 & $1.04 \mathrm{E}-02$ & 10977 & TC403455 & 7.05 & $2.21 \mathrm{E}-03$ \\
\hline 10842 & TC390775 & 2.66 & $7.06 \mathrm{E}-03$ & 10910 & TC397374 & 1.07 & $1.03 \mathrm{E}-03$ & 10978 & TC403596 & 3.86 & $1.58 \mathrm{E}-03$ \\
\hline 10843 & TC390952 & 3.07 & $2.41 \mathrm{E}-02$ & 10911 & TC397378 & 3.78 & $1.87 \mathrm{E}-03$ & 10979 & TC403915 & 3.25 & $4.11 \mathrm{E}-03$ \\
\hline 10844 & TC390963 & 3.84 & $3.62 \mathrm{E}-03$ & 10912 & TC397445 & 3.01 & $2.61 \mathrm{E}-03$ & 10980 & TC403917 & 2.25 & $1.38 \mathrm{E}-03$ \\
\hline 10845 & TC391039 & 1.43 & $2.30 \mathrm{E}-02$ & 10913 & TC397622 & 3.28 & $2.06 \mathrm{E}-03$ & 10981 & TC404023 & 2.55 & $1.15 \mathrm{E}-02$ \\
\hline 10846 & TC391169 & 4.41 & $1.44 \mathrm{E}-02$ & 10914 & TC397624 & 2.43 & $3.88 \mathrm{E}-03$ & 10982 & TC404025 & 4.49 & $6.32 \mathrm{E}-03$ \\
\hline 10847 & TC391173 & 6.85 & $4.90 \mathrm{E}-03$ & 10915 & TC397693 & 1.95 & $1.09 \mathrm{E}-02$ & 10983 & TC404138 & 7.64 & $1.60 \mathrm{E}-03$ \\
\hline 10848 & TC391199 & 1.92 & $2.63 \mathrm{E}-02$ & 10916 & TC397714 & 3.46 & $1.11 \mathrm{E}-04$ & 10984 & TC404369 & 5.10 & $6.06 \mathrm{E}-04$ \\
\hline 10849 & TC391238 & 7.06 & $3.02 \mathrm{E}-05$ & 10917 & TC397821 & 4.71 & $1.42 \mathrm{E}-03$ & 10985 & TC404573 & 3.61 & $1.42 \mathrm{E}-03$ \\
\hline 10850 & TC391250 & 1.43 & $1.77 \mathrm{E}-02$ & 10918 & TC397842 & 3.65 & $9.81 \mathrm{E}-03$ & 10986 & TC404615 & 3.42 & 4.03E-03 \\
\hline 10851 & TC391254 & 2.56 & $2.28 \mathrm{E}-03$ & 10919 & TC397920 & 4.72 & $4.26 \mathrm{E}-03$ & 10987 & TC404681 & 5.90 & $3.35 \mathrm{E}-04$ \\
\hline 10852 & TC391286 & 3.89 & $5.41 \mathrm{E}-03$ & 10920 & TC397986 & 2.81 & $2.10 \mathrm{E}-03$ & 10988 & TC404683 & 3.95 & $1.13 \mathrm{E}-02$ \\
\hline 10853 & TC391302 & 7.20 & $2.52 \mathrm{E}-03$ & 10921 & TC398246 & 3.80 & $1.94 \mathrm{E}-03$ & 10989 & TC404843 & 6.12 & $6.86 \mathrm{E}-05$ \\
\hline 10854 & TC391502 & 2.30 & $4.83 \mathrm{E}-03$ & 10922 & TC398335 & 3.62 & $4.66 \mathrm{E}-03$ & 10990 & TC404848 & 4.88 & $2.99 \mathrm{E}-03$ \\
\hline 10855 & TC391669 & 4.16 & $1.12 \mathrm{E}-03$ & 10923 & TC398341 & 4.24 & $5.82 \mathrm{E}-05$ & 10991 & TC404870 & 4.30 & $8.62 \mathrm{E}-03$ \\
\hline 10856 & TC391741 & 8.52 & $2.03 E-04$ & 10924 & TC398388 & 2.13 & $1.03 E-03$ & 10992 & TC404943 & 3.87 & $4.94 \mathrm{E}-03$ \\
\hline 10857 & TC391897 & 4.67 & $1.34 \mathrm{E}-03$ & 10925 & TC398424 & 2.24 & $4.18 \mathrm{E}-02$ & 10993 & TC404980 & 2.45 & $5.18 \mathrm{E}-02$ \\
\hline 10858 & TC391959 & 6.76 & $1.13 \mathrm{E}-03$ & 10926 & TC398443 & 1.55 & $1.39 \mathrm{E}-02$ & 10994 & TC405295 & 2.98 & $5.92 \mathrm{E}-03$ \\
\hline 10859 & TC391968 & 2.08 & $2.15 \mathrm{E}-03$ & 10927 & TC398664 & 4.37 & $6.19 \mathrm{E}-04$ & 10995 & TC405372 & 5.63 & $1.98 \mathrm{E}-03$ \\
\hline 10860 & TC391977 & 2.74 & $7.92 \mathrm{E}-02$ & 10928 & TC398676 & 4.85 & $4.20 \mathrm{E}-03$ & 10996 & TC405388 & 4.10 & $5.89 \mathrm{E}-04$ \\
\hline 10861 & TC391990 & 2.42 & $1.31 \mathrm{E}-04$ & 10929 & TC398714 & 2.76 & $7.61 \mathrm{E}-04$ & 10997 & TC405550 & 4.84 & $1.78 \mathrm{E}-03$ \\
\hline 10862 & $\begin{array}{l}\text { TC392121 } \\
\end{array}$ & 2.01 & $6.10 \mathrm{E}-03$ & 10930 & TC398787 & 6.29 & $1.42 \mathrm{E}-05$ & 10998 & TC405646 & 1.72 & $5.14 \mathrm{E}-02$ \\
\hline 10863 & TC392140 & 2.52 & $1.37 \mathrm{E}-02$ & 10931 & TC398849 & 2.99 & $1.06 \mathrm{E}-02$ & 10999 & TC405679 & 3.05 & $7.25 \mathrm{E}-04$ \\
\hline 10864 & TC392238 & 6.31 & $3.60 \mathrm{E}-03$ & 10932 & TC398925 & 6.22 & $3.10 \mathrm{E}-03$ & 11000 & TC405822 & 5.32 & $5.75 \mathrm{E}-02$ \\
\hline 10865 & TC392276 & 4.16 & $8.58 \mathrm{E}-04$ & 10933 & TC399137 & 3.10 & $2.36 \mathrm{E}-03$ & 11001 & TC405917 & 4.34 & $2.48 \mathrm{E}-03$ \\
\hline 10866 & TC392304 & 2.81 & $5.45 \mathrm{E}-03$ & 10934 & TC399165 & 3.77 & $2.63 \mathrm{E}-03$ & 11002 & TC406091 & 2.46 & $3.97 \mathrm{E}-02$ \\
\hline 10867 & TC392327 & 3.43 & $9.08 \mathrm{E}-04$ & 10935 & TC399193 & 5.17 & $7.01 \mathrm{E}-03$ & 11003 & TC406099 & 4.00 & $2.29 \mathrm{E}-03$ \\
\hline 10868 & TC392345 & 2.30 & $7.53 \mathrm{E}-03$ & 10936 & TC399252 & 4.63 & $1.01 \mathrm{E}-02$ & 11004 & TC406110 & 2.05 & $2.68 \mathrm{E}-03$ \\
\hline 10869 & TC392410 & 1.84 & $9.78 \mathrm{E}-03$ & 10937 & TC399300 & 6.03 & $1.28 \mathrm{E}-02$ & 11005 & TC406133 & 5.21 & $7.24 \mathrm{E}-03$ \\
\hline 10870 & TC392492 & 3.32 & $3.69 \mathrm{E}-02$ & 10938 & TC399400 & 2.81 & $1.24 \mathrm{E}-03$ & 11006 & TC406208 & 5.96 & $1.02 E-05$ \\
\hline 10871 & TC392506 & 5.45 & $1.73 \mathrm{E}-03$ & 10939 & TC399408 & 5.00 & $3.95 \mathrm{E}-04$ & 11007 & TC406295 & 7.64 & $3.83 \mathrm{E}-03$ \\
\hline 10872 & TC392628 & 2.88 & $1.51 \mathrm{E}-03$ & 10940 & TC399413 & 2.46 & $6.43 \mathrm{E}-03$ & 11008 & TC406396 & 2.31 & $1.33 \mathrm{E}-02$ \\
\hline 10873 & TC392837 & 1.15 & $8.19 \mathrm{E}-03$ & 10941 & TC399416 & 1.08 & $2.64 \mathrm{E}-02$ & 11009 & TC406563 & 4.43 & $2.19 \mathrm{E}-03$ \\
\hline 10874 & TC392880 & 5.74 & $2.45 \mathrm{E}-03$ & 10942 & TC399509 & 2.78 & $1.55 \mathrm{E}-01$ & 11010 & TC406696 & 2.96 & $3.67 \mathrm{E}-03$ \\
\hline 10875 & TC392954 & 4.36 & $7.49 \mathrm{E}-04$ & 10943 & TC399566 & 2.29 & $1.58 \mathrm{E}-02$ & 11011 & TC406841 & 2.24 & $6.39 \mathrm{E}-04$ \\
\hline 10876 & TC392958 & 4.61 & $1.40 \mathrm{E}-02$ & 10944 & TC399597 & 4.68 & $1.11 \mathrm{E}-02$ & 11012 & TC406845 & 3.27 & $1.44 \mathrm{E}-04$ \\
\hline 10877 & TC392966 & 3.56 & $5.20 \mathrm{E}-03$ & 10945 & TC399664 & 5.03 & $5.14 \mathrm{E}-04$ & 11013 & TC407148 & 2.91 & $9.70 \mathrm{E}-03$ \\
\hline 10878 & TC393051 & 4.92 & 4.59E-03 & 10946 & TC399706 & 3.05 & $1.88 \mathrm{E}-02$ & 11014 & TC407161 & 4.18 & $5.70 \mathrm{E}-03$ \\
\hline 10879 & TC393251 & 1.84 & $2.47 \mathrm{E}-03$ & 10947 & TC400181 & 4.90 & $5.90 \mathrm{E}-03$ & 11015 & TC407337 & 3.27 & 5.13E-03 \\
\hline
\end{tabular}




\begin{tabular}{|c|c|c|c|c|c|c|c|c|c|c|c|}
\hline 11016 & TC407575 & 2.44 & $3.49 \mathrm{E}-03$ & 11084 & TC417062 & 3.94 & $3.77 \mathrm{E}-03$ & 11152 & TC424017 & 1.93 & $2.82 \mathrm{E}-02$ \\
\hline 11017 & TC407733 & 1.96 & $2.43 \mathrm{E}-02$ & 11085 & TC417106 & 2.97 & $8.69 \mathrm{E}-03$ & 11153 & TC424171 & 3.16 & $1.90 \mathrm{E}-03$ \\
\hline 11018 & TC407848 & 3.74 & $4.36 \mathrm{E}-03$ & 11086 & TC417117 & 1.49 & $3.93 \mathrm{E}-04$ & 11154 & TC424193 & 7.24 & 5.49E-03 \\
\hline 11019 & TC407923 & 4.51 & $8.73 \mathrm{E}-03$ & 11087 & TC417374 & 4.81 & $1.70 \mathrm{E}-03$ & 11155 & TC424339 & 3.63 & $1.03 E-02$ \\
\hline 11020 & TC408091 & 6.08 & 7.13E-04 & 11088 & TC417469 & 2.15 & $6.12 \mathrm{E}-03$ & 11156 & TC424396 & 3.15 & $2.27 E-03$ \\
\hline 11021 & TC408117 & 5.30 & $1.65 \mathrm{E}-04$ & 11089 & TC417784 & 1.58 & $1.83 \mathrm{E}-03$ & 11157 & TC424550 & 4.19 & $5.36 \mathrm{E}-03$ \\
\hline 11022 & TC408177 & 1.49 & $2.46 \mathrm{E}-03$ & 11090 & TC417786 & 5.71 & $5.25 \mathrm{E}-04$ & 11158 & TC424644 & 4.12 & $7.21 E-02$ \\
\hline 11023 & TC408201 & 2.89 & $7.57 \mathrm{E}-03$ & 11091 & TC417795 & 2.68 & $1.79 \mathrm{E}-03$ & 11159 & TC424693 & 4.83 & $2.41 \mathrm{E}-03$ \\
\hline 11024 & TC408509 & 2.11 & $1.23 \mathrm{E}-03$ & 11092 & TC417831 & 3.18 & $3.71 \mathrm{E}-02$ & 11160 & TC424740 & 1.51 & $2.54 \mathrm{E}-02$ \\
\hline 11025 & TC408548 & 2.87 & $5.52 \mathrm{E}-03$ & 11093 & TC417861 & 3.71 & $2.52 \mathrm{E}-04$ & 11161 & TC424791 & 3.85 & $1.80 \mathrm{E}-03$ \\
\hline 11026 & TC408809 & 1.75 & $2.86 \mathrm{E}-02$ & 11094 & TC417943 & 2.98 & $2.20 \mathrm{E}-02$ & 11162 & TC424832 & 4.43 & 9.79E-04 \\
\hline 11027 & TC409252 & 5.87 & $5.87 \mathrm{E}-03$ & 11095 & TC417994 & 2.57 & $2.38 \mathrm{E}-01$ & 11163 & TC424896 & 9.44 & $5.73 E-04$ \\
\hline 11028 & TC409342 & 5.08 & $1.02 \mathrm{E}-02$ & 11096 & TC418012 & 2.36 & $2.33 \mathrm{E}-03$ & 11164 & TC425114 & 8.03 & 3.65E-05 \\
\hline 11029 & TC409661 & 7.17 & $3.24 \mathrm{E}-05$ & 11097 & TC418032 & 3.34 & $8.39 \mathrm{E}-03$ & 11165 & TC425252 & 2.79 & $7.41 E-03$ \\
\hline 11030 & TC409909 & 4.27 & $1.85 \mathrm{E}-05$ & 11098 & TC418068 & 3.82 & $9.96 \mathrm{E}-03$ & 11166 & TC425295 & 6.14 & 3.04E-03 \\
\hline 11031 & TC410014 & 5.47 & $2.09 E-03$ & 11099 & TC418183 & 3.17 & $1.59 \mathrm{E}-03$ & 11167 & TC425372 & 3.90 & $6.59 \mathrm{E}-04$ \\
\hline 11032 & TC410161 & 4.84 & $4.61 E-04$ & 11100 & TC418606 & 6.45 & $4.95 \mathrm{E}-03$ & 11168 & TC425612 & 6.71 & $5.01 \mathrm{E}-05$ \\
\hline 11033 & TC410238 & 2.94 & $2.04 \mathrm{E}-03$ & 11101 & TC418656 & 3.14 & $5.34 \mathrm{E}-03$ & 11169 & TC425752 & 3.70 & $3.12 \mathrm{E}-03$ \\
\hline 11034 & TC410278 & 5.32 & $1.17 \mathrm{E}-05$ & 11102 & TC418686 & 4.19 & 3.41E-04 & 11170 & TC425877 & 5.73 & $1.36 \mathrm{E}-03$ \\
\hline 11035 & TC410377 & 3.09 & 3.63E-04 & 11103 & TC418883 & 5.26 & $1.71 \mathrm{E}-03$ & 11171 & TC425896 & 1.88 & $1.23 \mathrm{E}-01$ \\
\hline 11036 & TC410379 & 3.72 & $8.30 \mathrm{E}-03$ & 11104 & TC418964 & 1.95 & 7.05E-03 & 11172 & TC425919 & 2.29 & $2.28 \mathrm{E}-03$ \\
\hline 11037 & TC410459 & 4.83 & $6.61 \mathrm{E}-03$ & 11105 & TC419187 & 5.98 & $1.99 \mathrm{E}-03$ & 11173 & TC426094 & 6.36 & $9.58 \mathrm{E}-03$ \\
\hline 11038 & TC410499 & 2.20 & $2.76 \mathrm{E}-03$ & 11106 & TC419215 & 5.34 & $2.28 \mathrm{E}-03$ & 11174 & TC426117 & 1.79 & $7.59 \mathrm{E}-03$ \\
\hline 11039 & TC410561 & 3.70 & $9.11 \mathrm{E}-03$ & 11107 & TC419376 & 5.30 & $8.63 \mathrm{E}-05$ & 11175 & TC426191 & 4.36 & $8.54 \mathrm{E}-03$ \\
\hline 11040 & TC410608 & 2.83 & $3.91 \mathrm{E}-04$ & 11108 & TC419404 & 3.19 & $1.64 \mathrm{E}-03$ & 11176 & TC426551 & 2.74 & $1.54 \mathrm{E}-01$ \\
\hline 11041 & TC410613 & 3.38 & $7.27 \mathrm{E}-04$ & 11109 & TC419826 & 5.90 & $7.27 \mathrm{E}-04$ & 11177 & TC426560 & 3.99 & $9.25 \mathrm{E}-03$ \\
\hline 11042 & TC410725 & 4.36 & $2.01 E-03$ & 11110 & TC419910 & 6.47 & $5.65 \mathrm{E}-04$ & 11178 & TC426589 & 1.53 & $1.51 \mathrm{E}-03$ \\
\hline 11043 & TC410803 & 1.43 & $1.56 \mathrm{E}-01$ & 11111 & TC420063 & 5.38 & $2.31 \mathrm{E}-04$ & 11179 & TC426600 & 3.56 & 8.41E-04 \\
\hline 11044 & TC410859 & 3.72 & $1.20 \mathrm{E}-03$ & 11112 & TC420076 & 3.84 & $2.78 \mathrm{E}-02$ & 11180 & TC426607 & 3.07 & $1.61 \mathrm{E}-03$ \\
\hline 11045 & TC411043 & 1.99 & $4.36 \mathrm{E}-03$ & 11113 & TC420082 & 1.49 & $7.20 \mathrm{E}-03$ & 11181 & TC426660 & 3.63 & 3.33E-03 \\
\hline 11046 & TC411159 & 5.71 & $1.79 \mathrm{E}-03$ & 11114 & TC420185 & 7.16 & $8.16 \mathrm{E}-04$ & 11182 & TC426668 & 4.78 & $8.55 E-04$ \\
\hline 11047 & TC411198 & 4.91 & $6.59 \mathrm{E}-03$ & 11115 & TC420385 & 5.35 & $5.14 \mathrm{E}-04$ & 11183 & TC426685 & 3.01 & $3.50 \mathrm{E}-03$ \\
\hline 11048 & TC411345 & 1.56 & $8.02 E-04$ & 11116 & TC420425 & 5.63 & $4.90 \mathrm{E}-03$ & 11184 & TC426704 & 2.54 & $3.98 \mathrm{E}-03$ \\
\hline 11049 & TC411379 & 2.27 & $1.25 \mathrm{E}-02$ & 11117 & TC420758 & 2.48 & $1.52 \mathrm{E}-03$ & 11185 & TC426931 & 4.64 & $2.48 \mathrm{E}-03$ \\
\hline 11050 & TC411536 & 5.64 & $1.25 \mathrm{E}-03$ & 11118 & TC421008 & 5.82 & $2.65 \mathrm{E}-04$ & 11186 & TC426934 & 8.39 & 4.49E-05 \\
\hline 11051 & TC411560 & 2.79 & $4.89 \mathrm{E}-03$ & 11119 & TC421108 & 4.85 & $4.92 \mathrm{E}-04$ & 11187 & TC426939 & 3.39 & $1.93 \mathrm{E}-04$ \\
\hline 11052 & TC411740 & 3.84 & $2.31 \mathrm{E}-04$ & 11120 & TC421140 & 2.38 & $1.49 \mathrm{E}-02$ & 11188 & TC427172 & 2.39 & $4.12 \mathrm{E}-03$ \\
\hline 11053 & TC411776 & 8.28 & $5.68 \mathrm{E}-03$ & 11121 & TC421171 & 5.94 & $8.14 \mathrm{E}-03$ & 11189 & TC427209 & 8.44 & $9.38 \mathrm{E}-03$ \\
\hline 11054 & TC411935 & 6.92 & $9.87 \mathrm{E}-04$ & 11122 & TC421182 & 2.29 & $3.28 \mathrm{E}-03$ & 11190 & TC427263 & 1.21 & $4.25 \mathrm{E}-03$ \\
\hline 11055 & TC412057 & 5.13 & $2.92 \mathrm{E}-03$ & 11123 & TC421207 & 3.67 & $7.41 \mathrm{E}-03$ & 11191 & TC427306 & 6.35 & $7.65 \mathrm{E}-04$ \\
\hline 11056 & TC412082 & 1.68 & $1.25 \mathrm{E}-02$ & 11124 & TC421232 & 4.82 & $1.69 \mathrm{E}-03$ & 11192 & TC427329 & 3.80 & 7.44E-03 \\
\hline 11057 & TC412090 & 5.65 & $6.33 \mathrm{E}-05$ & 11125 & TC421248 & 1.98 & $4.26 \mathrm{E}-02$ & 11193 & TC427563 & 5.72 & $1.17 E-03$ \\
\hline 11058 & TC412374 & 1.79 & $8.35 \mathrm{E}-02$ & 11126 & TC421383 & 3.40 & $3.31 \mathrm{E}-03$ & 11194 & TC427868 & 5.60 & $8.52 E-05$ \\
\hline 11059 & TC412531 & 6.72 & $8.06 E-03$ & 11127 & TC421514 & 2.43 & $2.33 \mathrm{E}-03$ & 11195 & TC427987 & 5.88 & $1.74 \mathrm{E}-03$ \\
\hline 11060 & TC412636 & 3.34 & $4.17 E-03$ & 11128 & TC421600 & 3.98 & $1.25 \mathrm{E}-03$ & 11196 & TC428060 & 3.57 & $2.14 E-03$ \\
\hline 11061 & TC413164 & 2.38 & $6.27 \mathrm{E}-03$ & 11129 & TC421671 & 1.09 & $1.93 \mathrm{E}-02$ & 11197 & TC428166 & 2.14 & $3.03 E-02$ \\
\hline 11062 & TC413171 & 4.33 & $1.01 \mathrm{E}-02$ & 11130 & TC422017 & 5.00 & $2.73 \mathrm{E}-02$ & 11198 & TC428374 & 1.56 & 7.74E-03 \\
\hline 11063 & TC413356 & 1.33 & $4.64 E-02$ & 11131 & TC422049 & 2.87 & $8.27 \mathrm{E}-04$ & 11199 & TC428652 & 4.35 & $5.70 \mathrm{E}-06$ \\
\hline 11064 & TC413373 & 1.91 & $3.72 E-04$ & 11132 & TC422103 & 3.04 & $4.37 \mathrm{E}-03$ & 11200 & TC428708 & 4.72 & $9.47 E-04$ \\
\hline 11065 & TC413403 & 2.82 & $2.81 \mathrm{E}-02$ & 11133 & TC422195 & 1.32 & 7.02E-01 & 11201 & TC428742 & 4.07 & $8.87 E-03$ \\
\hline 11066 & TC413436 & 5.13 & $1.39 E-05$ & 11134 & TC422248 & 2.77 & $1.83 \mathrm{E}-03$ & 11202 & TC428838 & 5.82 & $8.28 \mathrm{E}-04$ \\
\hline 11067 & TC413629 & 2.84 & $4.50 \mathrm{E}-03$ & 11135 & TC422292 & 2.45 & $1.57 \mathrm{E}-03$ & 11203 & TC428983 & 4.15 & $1.91 \mathrm{E}-03$ \\
\hline 11068 & TC413716 & 4.14 & $5.49 \mathrm{E}-05$ & 11136 & TC422326 & 2.46 & $1.48 \mathrm{E}-02$ & 11204 & TC428999 & 1.61 & $2.90 \mathrm{E}-03$ \\
\hline 11069 & TC413777 & 5.61 & $6.12 \mathrm{E}-03$ & 11137 & TC422418 & 1.92 & $4.36 \mathrm{E}-02$ & 11205 & TC429194 & 2.95 & $5.61 \mathrm{E}-04$ \\
\hline 11070 & TC414270 & 7.31 & $5.35 \mathrm{E}-06$ & 11138 & TC422598 & 4.19 & $3.75 \mathrm{E}-03$ & 11206 & TC429222 & 6.97 & $2.50 \mathrm{E}-03$ \\
\hline 11071 & TC414602 & 5.64 & $4.63 \mathrm{E}-03$ & 11139 & TC422754 & 3.66 & $9.87 \mathrm{E}-03$ & 11207 & TC429556 & 2.27 & 5.05E-03 \\
\hline 11072 & TC414857 & 3.52 & $1.29 \mathrm{E}-03$ & 11140 & TC422845 & 2.77 & $1.09 \mathrm{E}-03$ & 11208 & TC429575 & 4.21 & $1.49 \mathrm{E}-04$ \\
\hline 11073 & TC414894 & 5.54 & $2.11 \mathrm{E}-04$ & 11141 & TC423091 & 4.61 & $7.84 \mathrm{E}-04$ & 11209 & TC429602 & 9.44 & $1.06 \mathrm{E}-04$ \\
\hline 11074 & TC415011 & 3.39 & $5.03 E-03$ & 11142 & TC423100 & 3.05 & 4.03E-03 & 11210 & TC429740 & 3.23 & 4.63E-04 \\
\hline 11075 & TC415190 & 2.68 & $1.04 E-02$ & 11143 & TC423122 & 7.09 & $4.26 \mathrm{E}-03$ & 11211 & TC429843 & 3.02 & $5.76 \mathrm{E}-03$ \\
\hline 11076 & TC415322 & 3.34 & $1.62 \mathrm{E}-04$ & 11144 & TC423218 & 3.74 & $4.06 \mathrm{E}-02$ & 11212 & TC429883 & 3.30 & 7.18E-03 \\
\hline 11077 & TC415447 & 2.00 & $5.33 \mathrm{E}-03$ & 11145 & TC423369 & 5.37 & $2.21 \mathrm{E}-03$ & 11213 & TC429933 & 5.32 & 7.95E-04 \\
\hline 11078 & TC415557 & 5.13 & $3.64 \mathrm{E}-04$ & 11146 & TC423457 & 2.28 & $9.70 \mathrm{E}-03$ & 11214 & TC429940 & 9.97 & 3.69E-03 \\
\hline 11079 & TC415779 & 2.56 & $2.18 \mathrm{E}-03$ & 11147 & TC423515 & 1.38 & $1.69 \mathrm{E}-02$ & 11215 & TC430142 & 2.52 & $3.18 \mathrm{E}-03$ \\
\hline 11080 & TC416410 & 7.54 & $4.29 \mathrm{E}-04$ & 11148 & TC423722 & 5.55 & $2.67 \mathrm{E}-05$ & 11216 & TC430426 & 3.43 & $2.84 \mathrm{E}-03$ \\
\hline 11081 & TC416447 & 5.06 & $6.72 E-03$ & 11149 & TC423814 & 3.46 & $1.06 \mathrm{E}-02$ & 11217 & TC430602 & 6.39 & 4.55E-03 \\
\hline 11082 & TC416795 & 5.11 & $8.50 \mathrm{E}-04$ & 11150 & TC423944 & 5.13 & $1.55 \mathrm{E}-05$ & 11218 & TC430629 & 4.59 & $8.35 \mathrm{E}-03$ \\
\hline 11083 & TC417017 & 2.52 & $4.70 E-03$ & 11151 & TC423960 & 4.43 & $6.94 \mathrm{E}-04$ & 11219 & TC430917 & 4.42 & $3.64 \mathrm{E}-03$ \\
\hline
\end{tabular}




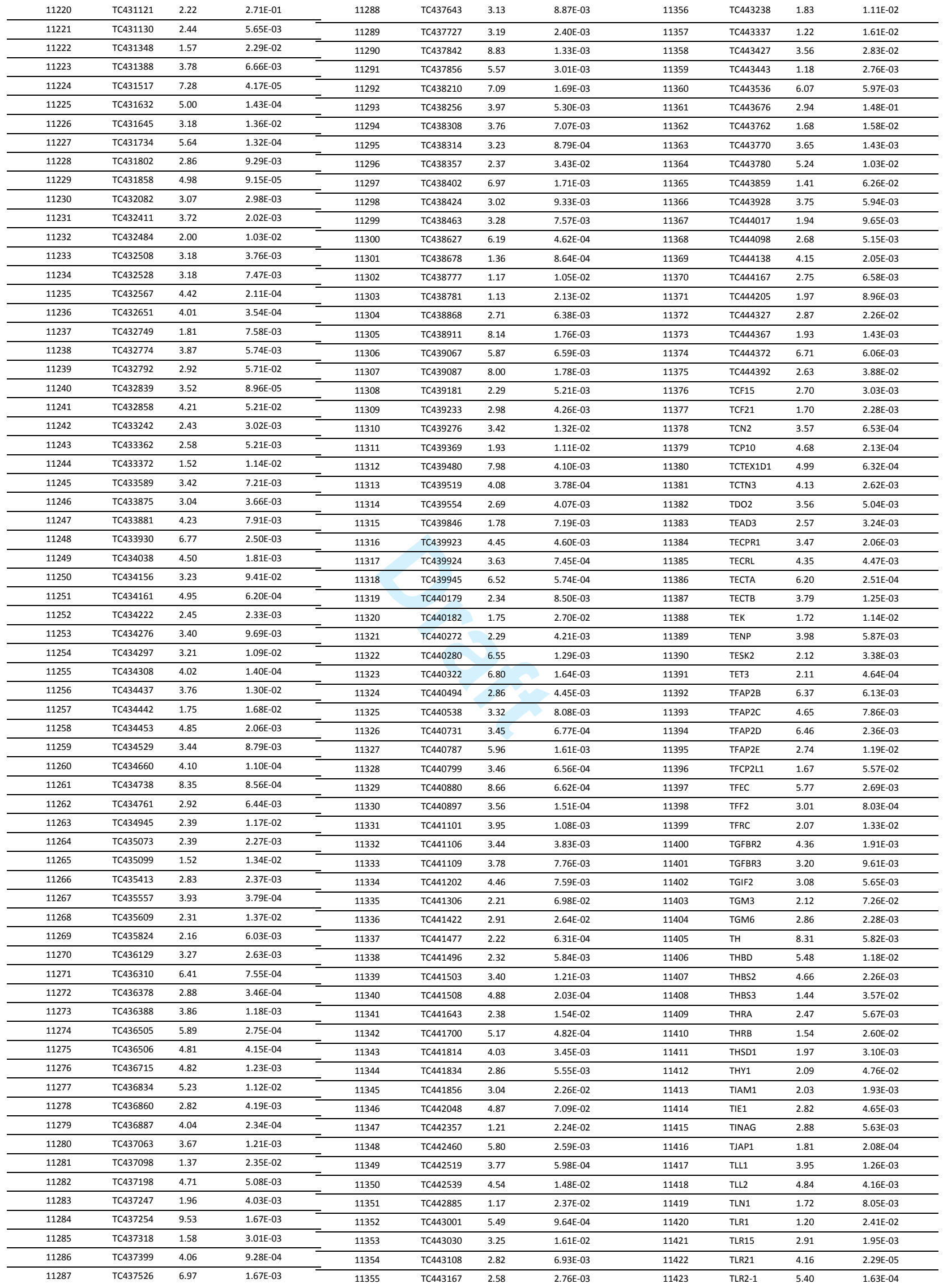




\begin{tabular}{|c|c|c|c|c|c|c|c|c|c|c|c|}
\hline 11424 & TLR2-2 & 1.90 & $1.63 \mathrm{E}-04$ & 11492 & TNC & 3.83 & $1.02 \mathrm{E}-03$ & 11560 & TSNARE1 & 1.38 & $2.93 E-02$ \\
\hline 11425 & TLR3 & 4.42 & $3.36 \mathrm{E}-03$ & 11493 & TNFAIP2 & 4.16 & $2.74 \mathrm{E}-04$ & 11561 & TSPAN10 & 2.73 & 3.07E-03 \\
\hline 11426 & TLR7 & 3.78 & $3.47 \mathrm{E}-03$ & 11494 & TNFAIP3 & 3.24 & $3.46 \mathrm{E}-03$ & 11562 & TSPAN18 & 1.39 & $3.50 \mathrm{E}-02$ \\
\hline 11427 & TLX1 & 2.33 & $2.05 \mathrm{E}-02$ & 11495 & TNFRSF10B & 3.98 & $9.83 \mathrm{E}-04$ & 11563 & TSPAN8 & 2.15 & $9.56 \mathrm{E}-03$ \\
\hline 11428 & TM4SF1 & 2.11 & $3.20 \mathrm{E}-03$ & 11496 & TNFRSF13B & 2.10 & $1.32 \mathrm{E}-03$ & 11564 & TSPO2 & 2.72 & $3.08 \mathrm{E}-03$ \\
\hline 11429 & TM4SF19 & 1.28 & $3.42 \mathrm{E}-02$ & 11497 & TNFRSF13C & 4.19 & $2.41 \mathrm{E}-03$ & 11565 & тТСз9С & 1.79 & $5.12 \mathrm{E}-03$ \\
\hline 11430 & TM7SF4 & 4.91 & $2.55 \mathrm{E}-03$ & 11498 & TNFRSF25 & 3.09 & $1.39 \mathrm{E}-02$ & 11566 & TTLL3 & 1.87 & 5.04E-03 \\
\hline 11431 & TM9SF4 & 2.01 & $6.46 \mathrm{E}-03$ & 11499 & TNFRSF6B & 4.21 & $3.37 \mathrm{E}-04$ & 11567 & TTN & 2.23 & $3.29 \mathrm{E}-03$ \\
\hline 11432 & TMC2 & 2.77 & $1.18 \mathrm{E}-03$ & 11500 & TNFSF11 & 1.32 & $2.16 \mathrm{E}-02$ & 11568 & $T T R$ & 1.67 & $1.47 \mathrm{E}-03$ \\
\hline 11433 & TMC3 & 2.73 & $8.95 \mathrm{E}-03$ & 11501 & TNFSF13B & 2.18 & $1.73 \mathrm{E}-04$ & 11569 & TUBA3C & 1.07 & $5.48 \mathrm{E}-02$ \\
\hline 11434 & TMC6 & 1.90 & $5.95 \mathrm{E}-03$ & 11502 & TNFSF15 & 1.27 & $9.08 \mathrm{E}-03$ & 11570 & TULP1 & 2.12 & $2.61 \mathrm{E}-04$ \\
\hline 11435 & TMED6 & 6.34 & $2.76 \mathrm{E}-03$ & 11503 & TNFSF4 & 4.94 & $3.77 \mathrm{E}-04$ & 11571 & TUSC5 & 4.46 & $2.86 \mathrm{E}-03$ \\
\hline 11436 & TMEFF1 & 3.00 & $4.03 E-03$ & 11504 & TNFSF8 & 3.33 & $9.45 \mathrm{E}-04$ & 11572 & TWF1 & 3.57 & $1.34 \mathrm{E}-03$ \\
\hline 11437 & TMEM100 & 2.09 & $9.90 \mathrm{E}-05$ & 11505 & TNIP1 & 2.49 & $9.19 \mathrm{E}-03$ & 11573 & TWIST1 & 4.78 & $1.88 \mathrm{E}-02$ \\
\hline 11438 & TMEM108 & 1.35 & $2.29 \mathrm{E}-02$ & 11506 & TNIP2 & 1.70 & $3.80 \mathrm{E}-03$ & 11574 & TWIST2 & 3.57 & $1.81 \mathrm{E}-03$ \\
\hline 11439 & TMEM115 & 2.62 & $4.61 \mathrm{E}-02$ & 11507 & TNK2 & 3.07 & $2.76 \mathrm{E}-03$ & 11575 & TWIST3 & 2.91 & $1.29 \mathrm{E}-03$ \\
\hline 11440 & TMEM117 & 1.46 & $6.49 \mathrm{E}-03$ & 11508 & TNMD & 2.48 & 3.40E-03 & 11576 & TXK & 3.45 & $8.84 \mathrm{E}-03$ \\
\hline 11441 & TMEM119 & 3.26 & $2.15 \mathrm{E}-02$ & 11509 & TNNC2 & 3.54 & $2.38 \mathrm{E}-03$ & 11577 & TYK2 & 3.24 & $1.13 \mathrm{E}-02$ \\
\hline 11442 & TMEM121 & 5.57 & $1.22 \mathrm{E}-02$ & 11510 & TNNI1 & 5.84 & 3.46E-03 & 11578 & TYR & 4.13 & $2.42 \mathrm{E}-03$ \\
\hline 11443 & TMEM125 & 4.54 & $6.08 \mathrm{E}-03$ & 11511 & TNN12 & 2.30 & $4.25 \mathrm{E}-03$ & 11579 & TYRP1 & 1.93 & $1.53 \mathrm{E}-02$ \\
\hline 11444 & TMEM132A & 4.42 & $4.65 \mathrm{E}-04$ & 11512 & TNNT2 & 3.88 & $1.18 \mathrm{E}-03$ & 11580 & TYSND1 & 2.35 & $2.51 \mathrm{E}-03$ \\
\hline 11445 & TMEM132D & 2.32 & $3.07 E-03$ & 11513 & TNR & 4.79 & $4.24 \mathrm{E}-04$ & 11581 & U04612 & 6.94 & $1.00 \mathrm{E}-03$ \\
\hline 11446 & TMEM132E & 2.69 & $2.10 \mathrm{E}-02$ & 11514 & TNXB & 1.87 & $8.17 \mathrm{E}-03$ & 11582 & U04613 & 9.08 & $2.06 \mathrm{E}-03$ \\
\hline 11447 & TMEM136 & 4.40 & $1.39 \mathrm{E}-05$ & 11515 & TOP1MT & 2.89 & $1.41 \mathrm{E}-02$ & 11583 & U78211 & 8.57 & $6.87 \mathrm{E}-04$ \\
\hline 11448 & TMEM150A & 4.39 & $5.36 \mathrm{E}-03$ & 11516 & TOPBP1 & 2.74 & $5.42 \mathrm{E}-03$ & 11584 & U78230 & 8.66 & $8.21 \mathrm{E}-03$ \\
\hline 11449 & TMEM151B & 4.11 & $2.93 \mathrm{E}-03$ & 11517 & TOR1AIP2 & 3.77 & $3.80 \mathrm{E}-03$ & 11585 & U78233 & 8.55 & $1.60 \mathrm{E}-02$ \\
\hline 11450 & TMEM154 & 1.34 & $2.69 \mathrm{E}-03$ & 11518 & TOR3A & 4.55 & $2.79 \mathrm{E}-03$ & 11586 & U78234 & 8.17 & $6.72 \mathrm{E}-03$ \\
\hline 11451 & TMEM169 & 1.80 & $1.25 \mathrm{E}-02$ & 11519 & TOX & 4.86 & $6.81 \mathrm{E}-03$ & 11587 & U78236 & 4.08 & $1.55 \mathrm{E}-03$ \\
\hline 11452 & TMEM174 & 3.95 & $6.98 \mathrm{E}-03$ & 11520 & TOX3 & 4.20 & $1.72 \mathrm{E}-02$ & 11588 & U78241 & 6.32 & $1.68 \mathrm{E}-04$ \\
\hline 11453 & TMEM179 & 7.01 & $1.53 \mathrm{E}-04$ & 11521 & TP53 & 2.06 & $8.90 \mathrm{E}-02$ & 11589 & U78243 & 8.36 & $6.07 E-03$ \\
\hline 11454 & TMEM181 & 1.87 & $8.99 \mathrm{E}-03$ & 11522 & TP53I11 & 2.87 & $8.92 \mathrm{E}-03$ & 11590 & U78288 & 3.42 & $5.00 E-04$ \\
\hline 11455 & TMEM182 & 2.92 & $4.22 \mathrm{E}-03$ & 11523 & TPBG & 2.25 & $9.75 \mathrm{E}-03$ & 11591 & UBA7 & 1.09 & $2.06 \mathrm{E}-02$ \\
\hline 11456 & TMEM184A & 3.73 & $2.01 \mathrm{E}-03$ & 11524 & TPD52L1 & 5.62 & $1.90 \mathrm{E}-03$ & 11592 & UBAP1L & 1.75 & $3.04 \mathrm{E}-02$ \\
\hline 11457 & TMEM194A & 4.00 & $8.68 \mathrm{E}-03$ & 11525 & TPO & 1.21 & $7.19 \mathrm{E}-02$ & 11593 & UBAP2L & 2.72 & 4.66E-04 \\
\hline 11458 & TMEM196 & 5.72 & $4.92 \mathrm{E}-04$ & 11526 & TPRXL & 2.10 & $1.70 \mathrm{E}-03$ & 11594 & UBASH3A & 3.00 & $1.14 \mathrm{E}-03$ \\
\hline 11459 & TMEM20 & 2.53 & $1.13 \mathrm{E}-03$ & 11527 & TRABD2A & 3.34 & $4.01 \mathrm{E}-03$ & 11595 & UBE3B & 1.25 & $4.66 \mathrm{E}-02$ \\
\hline 11460 & TMEM200A & 1.41 & $5.06 E-02$ & 11528 & TRAIL-LIKE & 3.15 & $4.24 \mathrm{E}-03$ & 11596 & UBTD1 & 1.55 & $7.73 E-04$ \\
\hline 11461 & TMEM201 & 2.14 & $2.28 \mathrm{E}-03$ & 11529 & TRAM2 & 2.60 & 3.70E-04 & 11597 & UCK2 & 3.09 & $2.86 \mathrm{E}-02$ \\
\hline 11462 & TMEM204 & 3.35 & $8.02 \mathrm{E}-03$ & 11530 & TRAT1 & 2.83 & $1.85 \mathrm{E}-03$ & 11598 & UCN3 & 4.09 & $2.86 \mathrm{E}-04$ \\
\hline 11463 & TMEM207 & 6.88 & $3.15 \mathrm{E}-05$ & 11531 & TRBV6-5 & 5.40 & $5.11 \mathrm{E}-04$ & 11599 & UCP3 & 3.70 & $7.52 E-04$ \\
\hline 11464 & TMEM213 & 2.78 & $2.84 \mathrm{E}-03$ & 11532 & TREM2 & 2.08 & $5.28 \mathrm{E}-03$ & 11600 & UGT2A 3 & 4.40 & 9.99E-04 \\
\hline 11465 & TMEM230 & 5.13 & $2.06 \mathrm{E}-03$ & 11533 & TRHR & 6.23 & $1.20 \mathrm{E}-03$ & 11601 & UHRF1BP1 & 2.72 & $3.79 E-04$ \\
\hline 11466 & TMEM $30 \mathrm{C}$ & 3.86 & $5.73 \mathrm{E}-03$ & 11534 & TRIB1 & 3.17 & $1.68 \mathrm{E}-03$ & 11602 & ULK1 & 2.56 & $5.18 \mathrm{E}-03$ \\
\hline 11467 & TMEM37 & 2.48 & $4.17 \mathrm{E}-03$ & 11535 & TRIL & 3.72 & $3.40 \mathrm{E}-05$ & 11603 & ULK3 & 3.07 & $4.02 E-03$ \\
\hline 11468 & TMEM39A & 5.18 & $2.67 \mathrm{E}-03$ & 11536 & TRIM13 & 2.73 & $3.77 \mathrm{E}-03$ & 11604 & ULK4 & 2.11 & 5.05E-03 \\
\hline 11469 & TMEM45A & 3.85 & $7.35 \mathrm{E}-03$ & 11537 & TRIM27 & 2.90 & $1.35 \mathrm{E}-03$ & 11605 & UNC119 & 2.68 & $3.98 \mathrm{E}-03$ \\
\hline 11470 & TMEM45B & 6.29 & $1.23 \mathrm{E}-03$ & 11538 & TRIM27.2 & 4.41 & $3.69 \mathrm{E}-03$ & 11606 & UNC13D & 4.61 & $5.92 \mathrm{E}-03$ \\
\hline 11471 & TMEM51 & 3.45 & $4.78 \mathrm{E}-04$ & 11539 & TRIM28 & 3.07 & $6.08 \mathrm{E}-03$ & 11607 & UNC5A & 4.69 & 5.33E-05 \\
\hline 11472 & TMEM52 & 3.64 & $4.49 \mathrm{E}-03$ & 11540 & TRIM39 & 4.38 & $7.66 \mathrm{E}-04$ & 11608 & UNC5B & 3.01 & $4.62 \mathrm{E}-03$ \\
\hline 11473 & TMEM52B & 4.85 & $3.43 \mathrm{E}-03$ & 11541 & TRIM41 & 3.09 & $2.94 \mathrm{E}-04$ & 11609 & UNC93A & 2.34 & $6.67 E-03$ \\
\hline 11474 & TMEM56 & 2.09 & 4.63E-03 & 11542 & TRIM50 & 3.45 & $3.15 \mathrm{E}-03$ & 11610 & UNC93B1 & 2.94 & 9.07E-04 \\
\hline 11475 & TMEM61 & 1.90 & $7.21 E-02$ & 11543 & TRIM8 & 1.66 & $5.46 \mathrm{E}-02$ & 11611 & UNCX & 4.09 & $5.45 \mathrm{E}-03$ \\
\hline 11476 & TMEM63B & 2.03 & $3.82 \mathrm{E}-03$ & 11544 & TRIM9 & 3.29 & $6.30 \mathrm{E}-04$ & 11612 & UNK & 1.07 & $6.55 \mathrm{E}-03$ \\
\hline 11477 & TMEM82 & 1.94 & $1.49 \mathrm{E}-02$ & 11545 & TRIML1 & 6.61 & $3.97 \mathrm{E}-03$ & 11613 & UPK1B & 2.19 & $1.51 \mathrm{E}-02$ \\
\hline 11478 & TMEM86A & 2.30 & $2.92 \mathrm{E}-02$ & 11546 & TROJAN & 3.52 & $1.42 \mathrm{E}-02$ & 11614 & UPK3A & 4.17 & $1.66 \mathrm{E}-03$ \\
\hline 11479 & TMEM88 & 3.10 & $4.19 \mathrm{E}-03$ & 11547 & TRPA1 & 1.38 & $2.51 \mathrm{E}-02$ & 11615 & UPK3BL & 2.28 & $5.10 \mathrm{E}-03$ \\
\hline 11480 & TMEM8C & 2.92 & $7.34 \mathrm{E}-03$ & 11548 & TRPC4 & 5.46 & $2.86 \mathrm{E}-03$ & 11616 & USF1 & 3.21 & $5.74 E-03$ \\
\hline 11481 & TMIGD1 & 3.31 & $1.32 \mathrm{E}-03$ & 11549 & TRPC5 & 2.35 & $1.27 \mathrm{E}-02$ & 11617 & USH1G & 4.85 & $2.29 \mathrm{E}-03$ \\
\hline 11482 & TMOD4 & 2.62 & $7.40 E-03$ & 11550 & TRPM3 & 4.08 & $1.13 \mathrm{E}-02$ & 11618 & USH2A & 2.77 & $1.33 \mathrm{E}-03$ \\
\hline 11483 & TMPRSS11F & 1.95 & $4.09 E-03$ & 11551 & TRPM8 & 1.97 & $2.17 \mathrm{E}-02$ & 11619 & USP 35 & 3.87 & $2.12 \mathrm{E}-03$ \\
\hline 11484 & TMPRSS13 & 5.15 & $8.61 \mathrm{E}-05$ & 11552 & TRPV2 & 3.23 & $2.29 \mathrm{E}-03$ & 11620 & USP 36 & 2.48 & $1.16 \mathrm{E}-04$ \\
\hline 11485 & TMPRSS15 & 7.40 & $3.83 \mathrm{E}-03$ & 11553 & TRPV3 & 3.17 & $3.14 \mathrm{E}-03$ & 11621 & UST & 2.38 & $1.50 \mathrm{E}-03$ \\
\hline 11486 & TMPRSS2 & 4.05 & $6.80 \mathrm{E}-03$ & 11554 & TRPV4 & 3.12 & $1.50 \mathrm{E}-03$ & 11622 & UTP14A & 1.11 & $1.18 \mathrm{E}-02$ \\
\hline 11487 & TMPRSS3 & 4.72 & $1.53 \mathrm{E}-02$ & 11555 & TRPV6 & 2.66 & $9.63 \mathrm{E}-03$ & 11623 & UTS2 & 1.77 & $5.87 E-03$ \\
\hline 11488 & TMPRSS4 & 2.38 & $1.40 \mathrm{E}-02$ & 11556 & TSC2 & 2.22 & $1.16 \mathrm{E}-02$ & 11624 & UTS2D & 3.84 & $5.79 \mathrm{E}-03$ \\
\hline 11489 & TMPRSS9 & 3.15 & $2.09 \mathrm{E}-02$ & 11557 & TSEN54 & 1.59 & $6.83 \mathrm{E}-03$ & 11625 & UTS2R & 3.75 & $1.31 \mathrm{E}-02$ \\
\hline 11490 & TMSB10 & 2.19 & $1.35 \mathrm{E}-02$ & 11558 & TSHZ1 & 1.76 & $2.61 \mathrm{E}-02$ & 11626 & VANGL2 & 2.10 & $1.45 \mathrm{E}-02$ \\
\hline 11491 & TMTC4 & 1.52 & $1.26 \mathrm{E}-02$ & 11559 & TSKU & 3.24 & $1.27 \mathrm{E}-03$ & 11627 & VAT1 & 2.80 & $2.58 \mathrm{E}-03$ \\
\hline
\end{tabular}




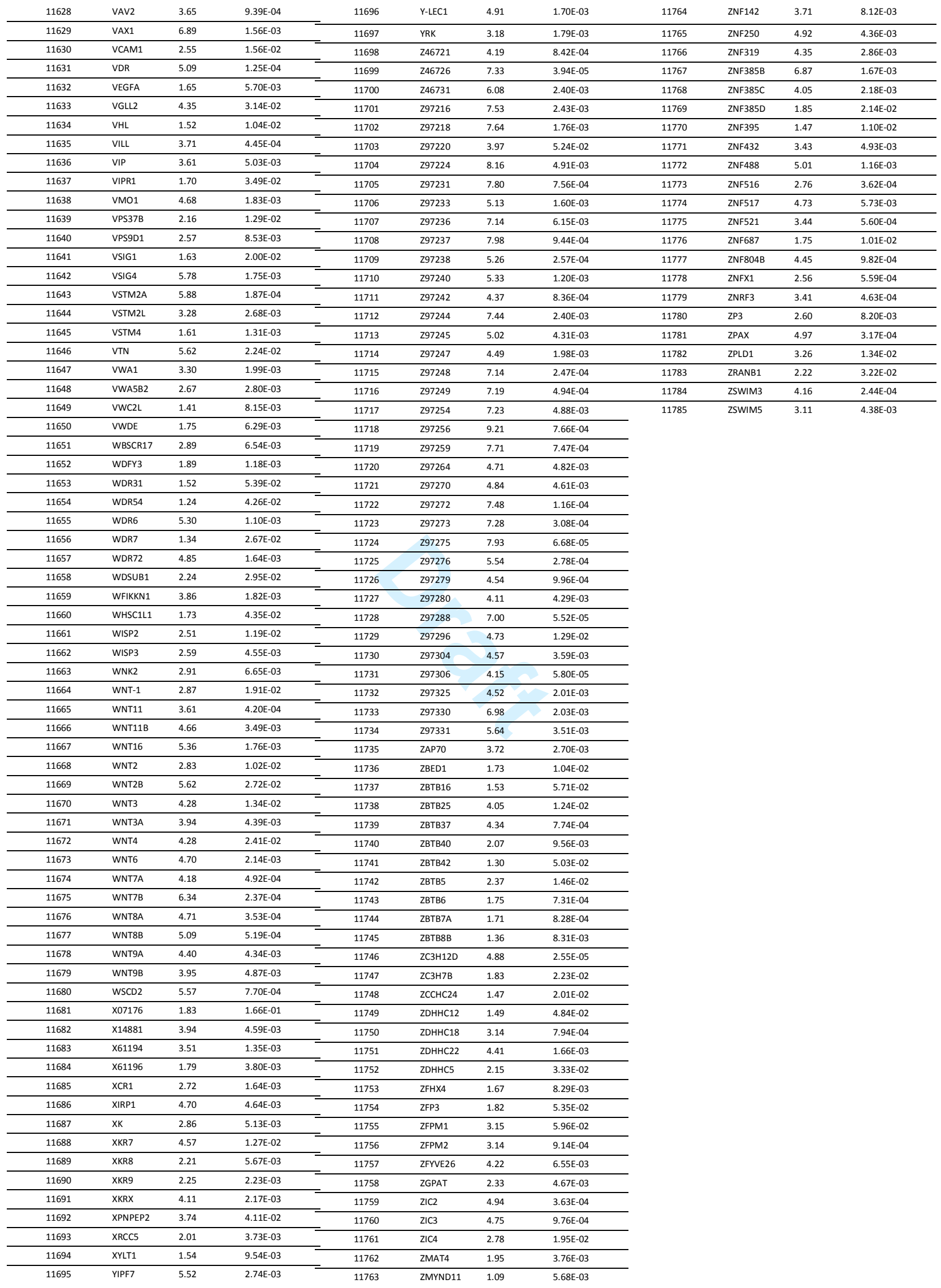

